COLUMBIA LIBRARIES OFFSITE

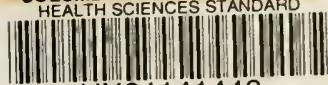

HX64141446
QP514.H31 1916 Practical physiologi

RECAP 

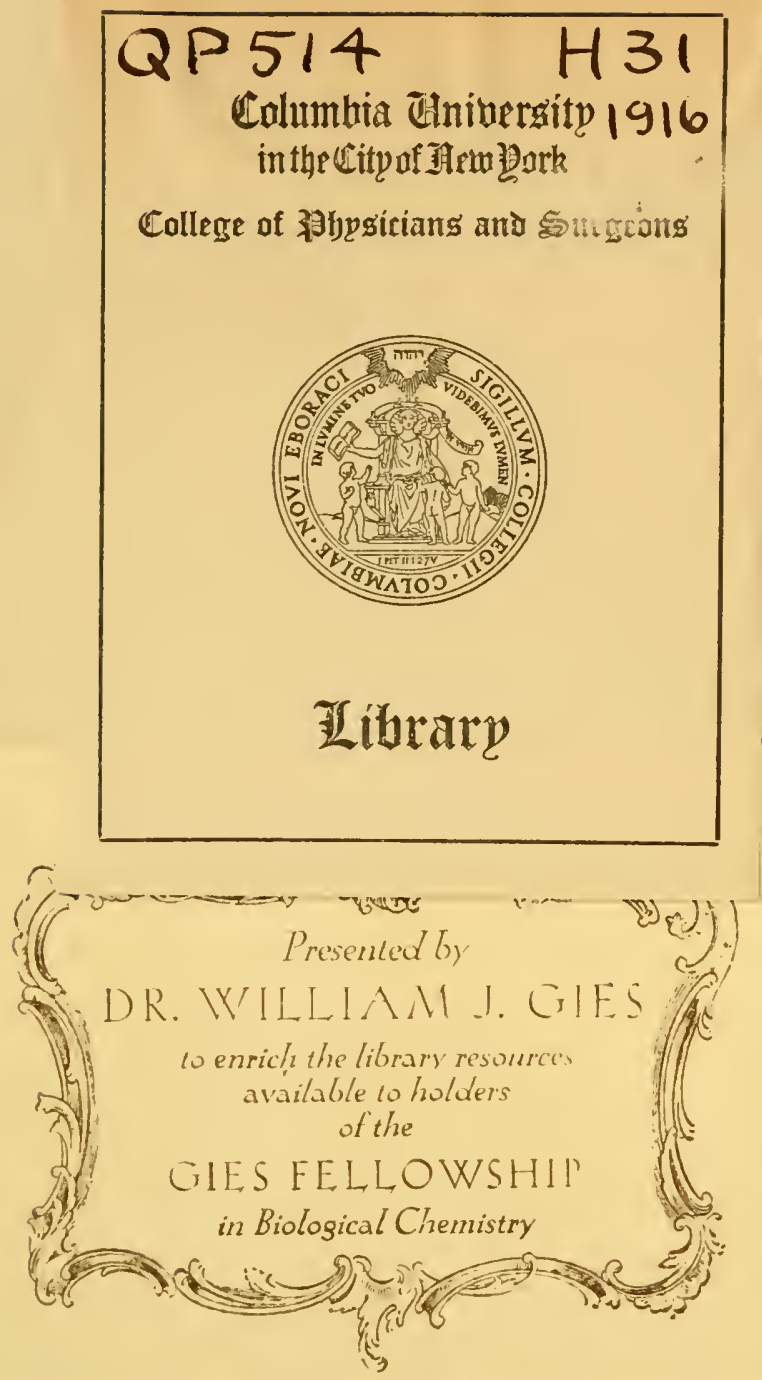


Digitized by the Internet Archive in 2010 with funding from Columbia University Libraries 



\section{PRACTICAL \\ PHYSIOLOGICAL CHEMISTRY}

H A W K 




\section{Absorption Spectra.}

\section{plate I.}

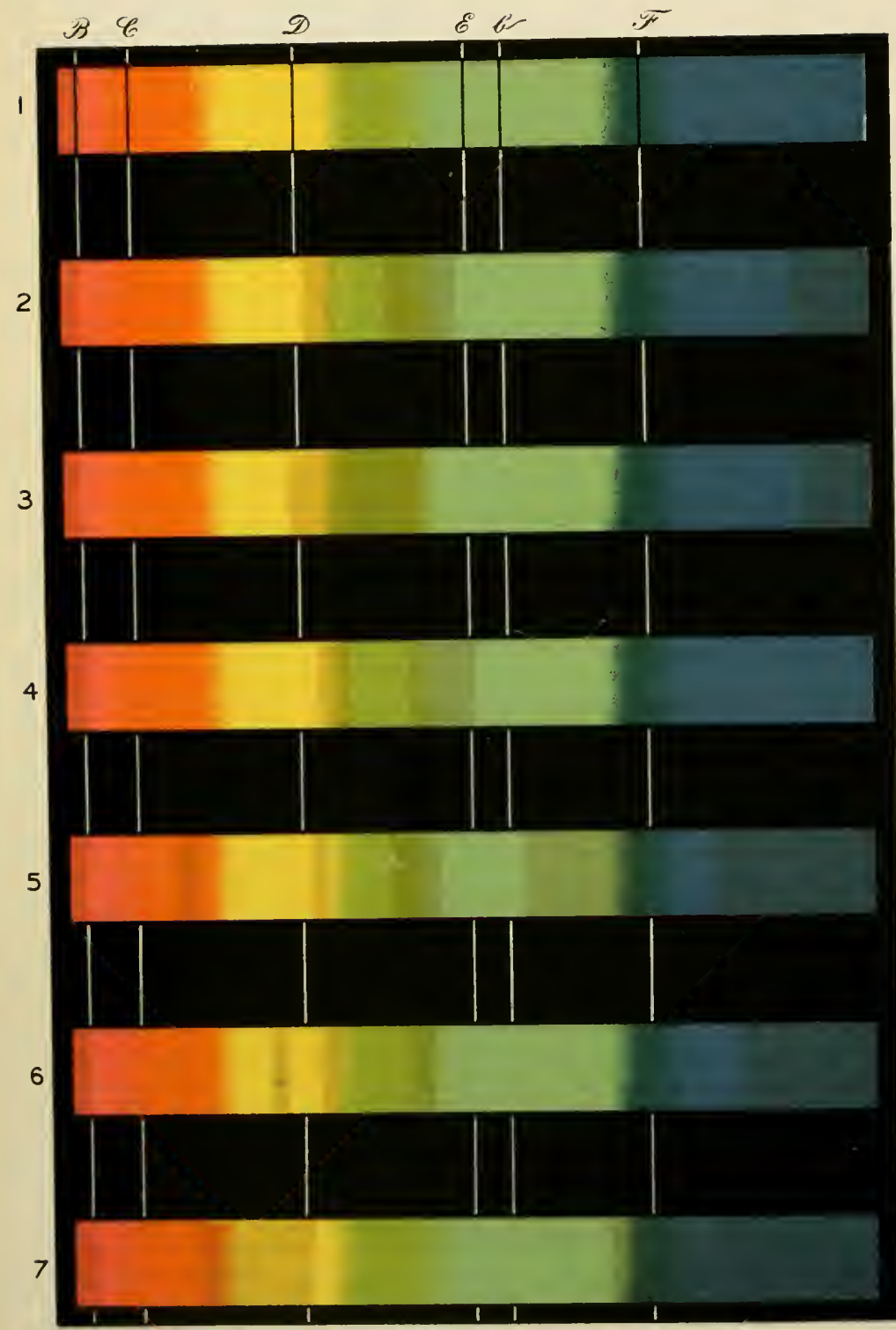

Oxyhaemoglobin.

Haemoglobin.

Carboxy-

haemogiobin.

Neutral Met-

haemoglobin.

Aikallne Met-

haemogiobin.

Aikali

Haematin. 


\section{Absorption Spectra.}

\section{plate II.}

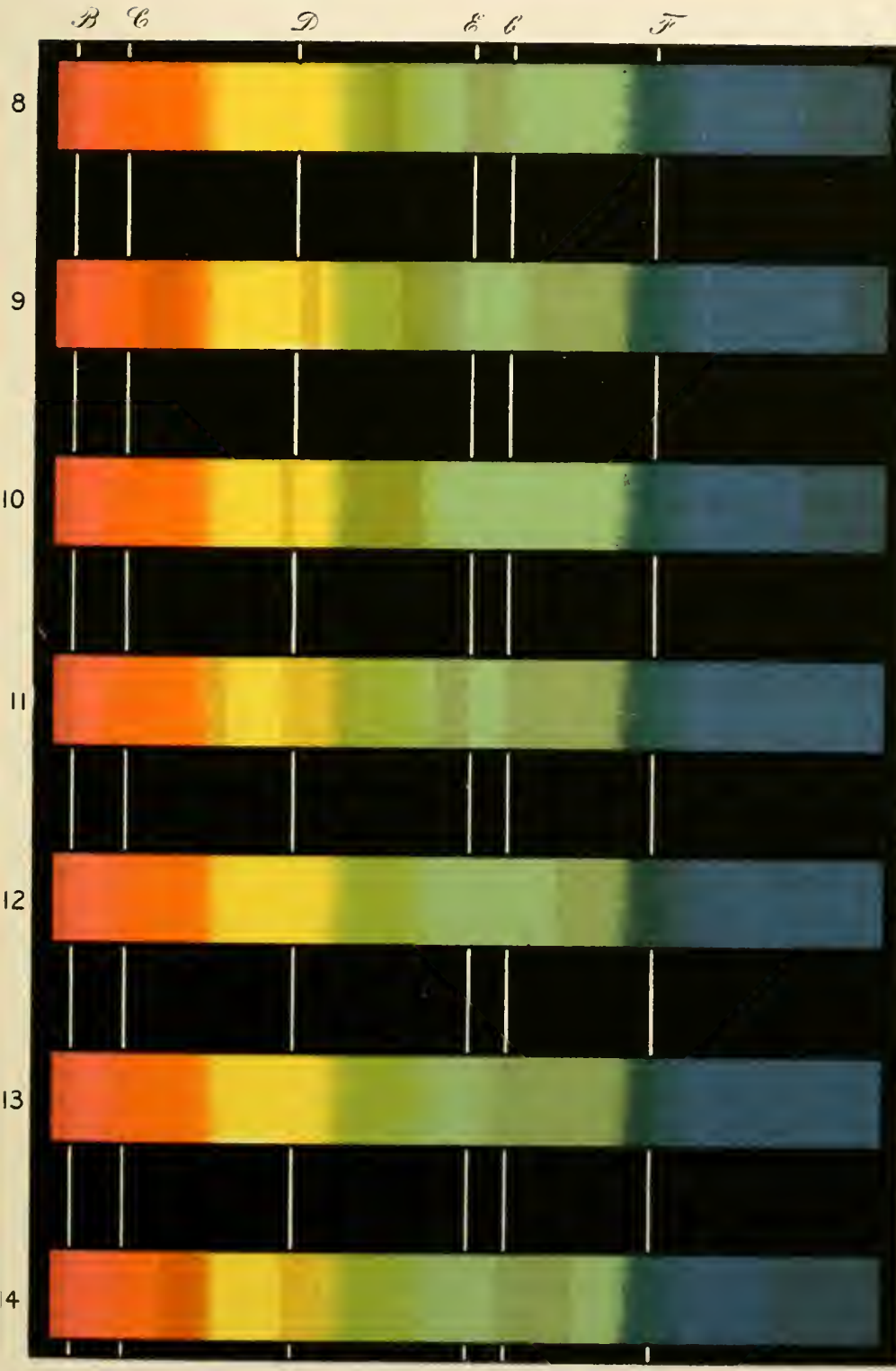

Reduced Alkal

Haematln or

Haemochromogen.

Acld Haematin in ethereal solution.

Acld Haemato-

porphyrin.

\section{Alkallne}

Haematopor -

phyrin.

Urobllin or Hydrobllirubin in acld solution.

Urobllin or Hydrobllirubin in alkallne solution after the addition of zinc chlorlde solution.

Blllcyanin or Cholecyanin in alkallne solution. 



\section{PRACTICAL}

\section{PHYSIOLOGICAL CHEMISTRY}

A BOOK DESIGNED FOR USE IN COURSES IN PRACTICAL PHYSIOLOGICAL CHEMISTRY IN SCHOOLS OF MEDICINE AND OF SCIENCE

BY

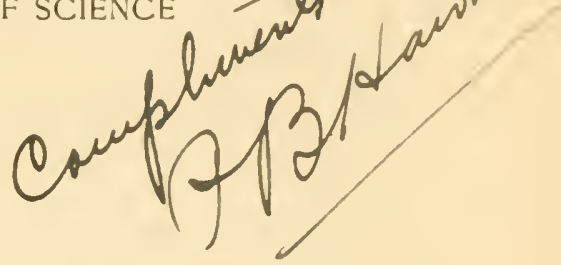

PHILIP B. HAWK, M. S., Ph. D.

PROFESSOR OF PHYSIOLOGICAL CHEMISTRY AND TOXICOLOGY IN THE JEFFERSON MEDICAL COLLEGE OF PHILADELPHIA

FIFTH EDITION, REVISED AND ENLARGED

WITH TWO FLLL-PAGE PLATES OF ABSORPTION SPECTRA IN COLORS FOUR ADDITIONAL FULL-PAGE COLOR PLATES AND ONE HUNDRED AND SEVENTY-TWO FIGURES OF WHICH TWELVE ARE IN COLORS

PHILADELPHIA

P. BLAKISTON'S SON \& CO.

1012 WALNUT STREET

1916 
First Edition, Copyright, I907, by P. Blakiston's Son \& Co. Second Edition, Copyright, I909, By P. Blakiston's Son \& Co. Third Edition, Copyright, igio, By P. Blakiston's Son \& Co. Fourth Edition, Copyright, igi2, By P. Blakiston's Son \& Co. REPRINTED, OCTOBER, I9I3 AND MaY, I9I 5

Fifth Edition, Copyright, igi6, by P. Blakiston's Son \& Co. 
THESE PAGES ARE

AFFECTIONATELY DEDICATED

TO

MY PARENTS

$1866-1916$ 



\section{PREFACE TO FIFTH EDITION}

The book has been thoroughly revised and in part rewritten. Five additional chapters have been inserted, in order to increase the usefulness of the volume and to keep thoroughly abreast with recent developments in Physiological Chemistry. The new Chapters are, Chapter VI on Nucleic Acids and Nucleoproteins, Chapter VIII on Gastric Analysis, Chapter XI on Intestinal Digestion, Chapter XVI on Blood Analysis and Chapter XXVII on Metabolism.

The Chapter on Metabolism consists in large part of directions for typical metabolism tests which may be conducted by the student in order to demonstrate important principles of metabolism.

The Chapter on Blood Analysis includes the most recent methods which have played such an important part in adding to our knowledge regarding the composition of the blood under normal and pathological conditions.

The Chapter on Gastric Analysis includes a general discussion of titratable acidity and hydrogen-ion concentration as well as an exposition of the Fractional Method of Gastric Analysis which was elaborated in the author's laboratory by Dr. Martin E. Rehfuss, and which has been very widely adopted.

The Chapter on Nucleic Acids and Nucleoproteins is a brief discussion of basic facts accompanied by experiments suitable for student use.

In order to facilitate the selection of tests and methods for courses in which but little time is devoted to the subject, the actual laboratory procedure involved in such tests and methods as the author considers most important has been printed in black face type to difierentiate such tests and methods from those which he considers of less importance, which are set in small type. In certain instances, however, tests and methods which the author believes to be of first importance, but which are not so readily adapted to student use, have been printed in small type.

The latest and best methods of quantitative analysis have been introduced throughout the volume. The nephelometer has been discussed and certain nephelometric methods described.

Certain tests and methods which had obviously outlived their usefulness have been omitted from this edition. Others which the 
author believes might well have been omitted have been retained in small type. The author believes that it is preferable that each individual instructor shall use his own judgment as to which tests and methods he will omit from his course. For this reason, in connection with certain quantitative procedures more than a single approved method has been given.

Thirty-five new illustrations have been incorporated.

The author takes pleasure in acknowledging the important part played by Dr. Olaf Bergeim in the preparation of this new edition. Dr. Bergeim's suggestions and assistance have been invaluable in connection with all phases of the revision, as well as in the preparation of new material for introduction. To Dr. Martin E. Rehfuss the author is indebted for the drawings of the microscopical constituents of the feces and gastric contents and the curves which are introduced in the Chapter on Gastric Analysis, as well as for timely suggestions as:'to certain clinical aspects of fecal and gastric analysis. Other members of the author's staff who have aided in certain phases of the revision are Drs. H. Rodell Fishback,-Melvin A. Saylor and Clarence A. Smith.

To Professors Lafayette B. Mendel, Paul E. Howe, Marshall P. Cram, A. P. Sy and J. C. Blake, the author is also indebted for helpful suggestions, and to Professors William J. Gies and Walter Jones for permission to insert selected experiments from their laboratory directions. The author is under further obligation to Professor Jones for crystals of hypoxanthine chloride and guanine chloride from which were prepared the microphotographs appearing in the Chapter on Nucleic Acids and Nucleoproteins. He also wishes to thank Professor Chester C. Fowler for permission to insert unpublished material.

The author is very appreciative of the unfailing courtesy and consideration of the publishing house of P. Blakiston's Son \& Co.; the timely suggestions of Mr. C. V. Brownlow, Mr. I. A. Hagy and Mr. Horace G. White have been very helpful. 


\section{CONTENTS}

CHAPTER I

Enzymes and Their Action

CHAPTER II

Carbohydrates

CHAPTER III

Salivary Digestion

CHAPTER IV

Proteins: Their Decomposition and Synthesis .

CHAPTER V

Proteins: Their Classification and Properties

CHAPTER VI

Nucleic Acids and Nucleoproteins

CHAPTER III

Gastric Digestion.

CHAPTER IIII

Gastric Analysis

CHAPTER IX

FATS

CHAPTER X

Pancreatic Digestion .

CHAPTER XI

Intestinal Digestion

CHAPTER XII

BILE . . . . . . . . . . . . . 202

CHAPTER XIII

Putrefaction Products

CHAPTER XIY

FECES . 
BLOOD AND LyMPH .

CHAPTER XVI

Blood ANALYSis. 270

CHAPTER XVII

MrLK

CHAPTER IVIII

Epithelial and Connectine Tissues

CHAPTER XIX

Muscular Tissue

CHAPTER XI

Nervous Tissue.

CHAPTER XXI

Urine: General Characteristics of Normal and Pathological URINE.

\section{CHAPTER XXII}

Urine: Physiological Constituents 369

CHAPTER XXIII

Urine: Pathological Constituents $4 I_{2}$

CHAPTER XXIV

Urine: Organized and Unorganized Sediments .

CHAPTER XXV

URine: Calculi .

CHAPTER XXVI

Urine: Quantitative Analysis.

CHAPTER XXVII

Metabolisar . 564

Reagents and Solutions. 594

INDEX . 


\section{LIST OF ILLUSTRATIONS}

Plate

I. Absorption Spectra

II. Absorption Spectra

Frontispiece

III. Osazones.

IV. Normal Erythrocytes and Leucocytes

Opposite page 22

Opposite page 249

V. Uric Acid Crystals .

Opposite page 380

VI. Ammonium Urate.

Opposite page 462

Figure

PAGE

r. Apparatus for Quantitative Determination of Catalase . . . . I7

2. Dialyzing Apparatus for Students' Use . . . . . . . . . 24

3. Einhorn Saccharometer . . . . . . . . . . 3I

4. Illustrating Different Stages in Fermentation . . . . . . 3I

5. One Form of Laurent Polariscope. . . . . . . . . . . . . 32

6. Diagrammatic Representation of the Course of the Light through the Laurent Polariscope.

7. Polariscope (Schmidt and Hänsch Model) . . . . . . . 34

8. Iodoform . . . . . . . . . . . . . . 42

9. Potato Starch . . . . . . . . . . . . . . . 44

Iо. Bean Starch. . . . . . . . . . . . 44 44

Ir. Arrowroot Starch. . . . . . . . . . 44

I2. Rye Starch . . . . . . . . . . . . . . . . . 44

I3. Barley Starch . . . . . . . . . . . . . . 44

I4. Oat Starch . . . . . . . . . . . . 44 44

I5. Buckwheat Starch . . . . . . . . . . . . 44

r6. Maize Starch . . . . . . . . . . . . . 44

17. Rice Starch . . . . . . . . . . . . 44 44

18. Pea Starch . . . . . . . . . . . . . 44

19. Wheat Starch . . . . . . . . . . . . . . 44

20. Microscopical Constituents of Saliva . . . . . . . . . 58

2I. Glycocoll Ester Hydrochloride . . . . . . . . . . . . . . . $7_{2}^{2}$

22. Serine. . . . . . . . . . . . . . 73

23. Phenylalanine . . . . . . . . . . . . . 74

24. Fischer Apparatus . . . . . . . . . . . . 75

25. Tyrosine . . . . . . . . . . . . 76

26. Cystine . . . . . . . . . . . . . 76

27. Histidine Dichloride . . . . . . . . . . is $^{\mathrm{S}}$

28. Leucine . . . . . . . . . . . So

29. Lysine Picrate . . . . . . . . . . . SI

30. Aspartic Acid . . . . . . . . . . . . . . . S S

3r. Glutamic Acid. . . . . . . . . . . . $S_{3}$

32. Levo- $\alpha$-Proline. . . . . . . . . . . . $S_{4}$

33. Copper Salt of Proline . . . . . . . . . $\$_{4}$ 
Figure

34. Van Slyke Amino Nitrogen Apparatus . . . . . . . . . 88

35. Section of Van Slyke Apparatus . . . . . . . . . . . 88

36. Coagulation Temperature Apparatus . . . . . . . . . 106

37. Edestin . . . . . . . . . . . . . 109

38. Excelsin, the Protein of the Brazil Nut . . . . . . . r ro

39. Guanine Chloride ... . . . . . . . . . I35

40. Hypoxanthine Chloride . . . . . . . . . . . . . I36

4I. Normal and Pathological Curves after an Ewald Meal . . . . I48

42. Rehfuss Stomach Tube . . . . . . . . . . . . . 149

43. Influence of Acid Introduced into the Normal Human Stomach . $5_{50}$

44. Hydrogen Ion Concentration Chart. . . . . . . . . . 157

45. Acidity Curves of Normal Human Stomach . . . . . . . . I63

46. Acidity Curves from a Case of Hyperacidity . . . . . . . . . 164

47. Acidity and Protein Curves in Gastric Carcinoma . . . . . . . I64

48. Total Acidity and Protein Curves in Benign Achylia . . . . . 165

49. Microscopical Constituents of the Gastric Contents. . . . . . . I72

50. Beef Fat . . . . . . . . . . . . 176

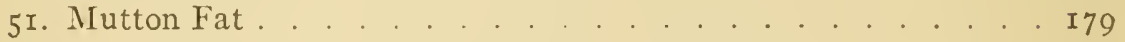

52. Pork Fat . . . . . . . . . . . . . . . I8I

53. Palmitic Acid . . . . . . . . . . . . . . . I 82

54. Melting-point Apparatus . . . . . . . . . . . . 183

55. Bile Salts . . . . . . . . . . . . . . 205

56. Bilirubin (Hematoidin) . . . . . . . . . . . . . 205

57. Cholesterol . . . . . . . . . . . . . . . 210

58. Taurine . . . . . . . . . . . . . 2II

59. Glycocoll . . . . . . . . . . . . . . . 2II

60. Ammonium Chloride . . . . . . . . . 2I6

6r. Hematoidin Crystals from Acholic Stools . . . . . . . . 222

62. Charcot-Leyden Crystals . . . . . . . . . 225

63. Boas' Sieve . . . . . . . . . . . . . . . 229

64-69. Microscopical Constituents of Feces. . . . . . . 230-3I

70. Oxyhemoglobin Crystals from Blood of the Guinea-pig . . . . 25I

71. Oxyhemoglobin Crystals from Blood of the Rat . . . . . . . . 25I

72. Oxyhemoglobin Crystals from Blood of the Horse . . . . . . . $25^{2}$

73. Oxyhemoglobin Crystals from Blood of the Squirrel. . . . . 252

74. Oxyhemoglobin Crystals from Blood of the Dog . . . . . . . 253

75. Oxyhemoglobin Crystals from Blood of the Cat . . . . . . . 253

76. Oxyhemoglobin Crystals from Blood of the Necturus . . . . . 254

77. Effect of Water on Erythrocytes . . . . . . . . . . . 26I

78. Hemin Crystals from Human Blood . . . . . . . . . . . 265

79. Hemin Crystals from Sheep Blood . . . . . . . . . . . . 265

8o. Sodium Chloride. . . . . . . . . . . . . . . . . . 267

8I. Bang Reduction Flask . . . . . . . . . . . . . . 28I

82. Apparatus for Epstein's Sugar Method . . . . . . . . . 283

83. Bloor's Nephelometer. . . . . . . . . . . 29r 
84. Nephelometer in Position, Showing Relation to Source of Light . 292

85. Lenzmann-Kober Nephelometer . . . . . . . . . . . . 293

86. Direct-vision Spectroscope. . . . . . . . . . . . . . . . . . 296

87. Angular-vision Spectroscope Arranged for Absorption Analysis . 297

88. Diagram of Angular-vision Spectroscope. . . . . . . . . . . . 297

89. Fleischl's Hemometer. . . . . . . . . . . . . . 300

90. Pipette of Fleischl's Hemometer . . . . . . . . . . . . . . . 30I

91. Colored Glass Wedge of Fleischl's Hemometer. . . . . . . . 30r

92. Dare's Hemoglobinometer. . . . . . . . . . . . . . . . 302

93. Horizontal Section of Dare's Hemoglobinometer . . . . . . . . 303

94. Method of Filling the Capillary Observation Cell of Dare's Hemoglobinometer. . . . . . . . . . . . . . . 303

95. Thoma-Zeiss Counting Chamber . . . . . . . . . . . . 304

96. Thoma-Zeiss Capillary Pipettes . . . . . . . . . . . . 305

97. Ordinary Ruling of Thoma-Zeiss Counting Chamber . . . . . 306

98. Zappert's Modified Ruling of Thoma-Zeiss Counting Chamber . . 307

99. Bürker's Pipettes, Mixing Flasks, and Counting Chamber. . . 309

roo. Ruling of Bürker Counting Chamber . . . . . . . . . . . . . 3ro

IоI. Schema . . . . . . . . . . . . . 3II

I02. Bürker Counting Chamber . . . . . . . . . . . . . 312

ro3. Normal Milk and Colostrum . . . . . . . . . . 3I5

ro4. Lactose . . . . . . . . . . . . . . . . 3I8

ro5. Calcium Phosphate. . . . . . . . . . . . . . . . 322

ıo6. Centrifuge Tube used in Babcock Fat Method . . . . . . 324

ro7. Croll's Fat Apparatus. . . . . . . . . . . . . . . 325

ro8. Soxhlet Apparatus . . . . . . . . . . . . . . . . 326

rog. Feser's Lactoscope . . . . . . . . . . . . . . . . . . . 326

I Iо. Creatine . . . . . . . . . . . . . . . . 342

Ir. Xanthine . . . . . . . . . . . . . . . . . 344

I r2. Hypoxan thine Silver Nitrate. . . . . . . . . . . . . 35

I 3. Xanthine Silver Nitrate. . . . . . . . . . . . . . 35I

I 4. Deposit in Ammoniacal Fermentation . . . . . . . . . . 362

I 5. Deposit in Acid Fermentation . . . . . . . . . . . . . 363

I 6. Urinometer and Cylinder . . . . . . . . . . . . 364

II7. Beckmann-Heidenhain Freezing-point Apparatus . . . $\quad 365$

Ir8. Urea . . . . . . . . . . . . . . . . . . . . . . . 2

Ir9. Urea Nitrate. . . . . . . . . . . . 374

I20. Melting-point Tubes Fastened to Bulb of Thermometer * 375

I 2 I. Urea Oxalate . . . . . . . . . . . . . . . 376

I22. Pure Uric Acid . . . . . . . . . . . 3So

I23. Creatinine. . . . . . . . . . $3 \mathrm{~S}_{2}$

I 24. Creatinine-Zinc Chloride . . . . . . ${ }_{3} S_{5}$

I25. Hippuric Acid . . . . . . . . . . . . 3 39

I 26. Allantoïn from Cat's Urine . . . . . . . 302

I27. Benzoic Acid. . . . . . . . . . . . . 396 
I28. Calcium Sulphate

I29. "Triple Phosphate"

408

I30. Albumoscope.

\31. Marsh Apparatus

I32. The Purdy Electric Centrifuge.

133. Sediment Tube for the Purdy Electric Centrifuge . . . . . 457

134. Calcium Oxalate . . . . . . . . . . . . . . . . . . . . . . . 459

I35. Calcium Carbonate.

459

136. Various Forms of Uric Acid . . . . . . . . . . . 46r

r37. Acid Sodium Urate. . . . . . . . . . . . . . . . 4662

138. Cystine . . . . . . . . . . . . . . 462

I39. Crystals of Impure Leucine . . . . . . . . . . . 463

I40. Epithelium from Different Areas of the Urinary Tract . . . . 466

I4I. Pus Corpuscles. . . . . . . . . . . . . . . . . . . . 467

I42. Hyaline Casts . . . . . . . . . . . . . . . 468

I43. Granular Casts.

I44. Granular Casts. . . . . . . . . . . . . . . . . 470

145. Epithelial Casts . . . . . . . . . . . . . 470

I46. Blood, Pus, Hyaline and Epithelial Casts . . . . . . . 470

I47. Fatty Casts . . . . . . . . . . . . . . . . . 47 I

I48. Fatty and Waxy Casts . . . . . . . . . . . . . 47 47

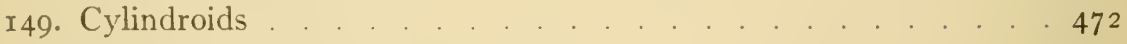

I 50. Crenated Erythrocytes .

151. Human Spermatozoa . . . . . . . . . . . . 474

I52. Folin Fume Absorber . . . . . . . . . . . . . . . . . 483

I53. Duboscq Colorimeter . . . . . . . . . . 486

I54, I55. Forms of Apparatus used in Methods of Folin and Associates for Determination of Total Nitrogen, Urea and Ammonia . . . 487

r56. Bock and Benedict Apparatus. . . . . . . . . . . . 489

I57. Gulick Micro-oxidation Flask. . . . . . . . . . . . . . . 490

I58. Van Slyke and Cullen Apparatus. . . . . . . . 492

r59. Folin's Urea Apparatus . . . . . . . . . . . . . . . . . . . 496

I60. Doremus-Hinds Ureometer . . . . . . . . . . . . . 497

I6r. Marshall's Urea Apparatus . . . . . . . . . . . . . 498

r62. Hüfner's Urea Apparatus . . . . . . . . . . . . . . . 498

163. Folin's Ammonia Apparatus . . . . . . . . . . . . . . . . . . 499

I64. Folin Improved Absorption Tube . . . . . . . . . . . . 500

I65. Ruhemann's Uricometer . . . . . . . . . . . 513

I66. Hall's Purinometer . . . . . . . . . . . . . . . . 517

167. Esbach's Albuminometer . . . . . . . . . . . 532

I68. Scott-Wilson Apparatus. . . . . . . . . . . . . . . 536

169. Blood Sugar as Influenced by Diet . . . . . . . . . . 566

170. Influence of Protein Ingestion on Endogenous Uric Acid Output 573

x71. The Endogenous Uric Acid Output during Fasting . . . . . 573

172. Berthelot-Atwater Bomb Calorimeter. . . . . . . . $5^{86}$ 


\section{PHYSIOLOGICAL CHEMISTRY}

\section{CHAPTER I}

\section{ENZYMES AND THEIR ACTION}

According to the old classification ferments were divided into two classes, the organized ferments and the unorganized ferments. As organized ferments or true ferments there were grouped such substances as yeast and certain bacteria which were supposed to act by virtue of vital processes, whereas the unorganized ferments included salivary amylase (ptyalin), gastric protease (pepsin), pancreatic protease (trypsin), etc.. which were described as "non-living unorganized substances of a chemical nature." Kühne designated this latter class of substances as

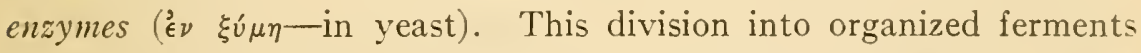
(true ferments) and unorganized ferments (enzymes) was generally accepted and was practically unquestioned until Buchner overthrew it in the year 1897 by his epoch-making investigations on zymase. Previous to this time many writers had expressed the opinion that the action of the ferment organisms was similar to that of the unorganized ferments or enzymes and that therefore the activity of the former was possibly due to the production of a substance in the cell, which was in nature similar to an enzyme. Investigation after investigation, however, failed to isolate any such principle from an active cell and the exponents of the "vital" theory became strengthened in their belief that certain fermentative processes brought about by living cells could not occur apart from the biological activity of such cells. However, as early as 1858 , Traube had enunciated, in substance, the principles which were destined to be fundamental in our modern theory of fermentation. He expressed the belief that the yeast cell produced a product in its metabolic activities which had the property of reacting with sugar with the production of carbon dioxide and alcohol, and further that this reaction between the product of the metabolism of the yeast cell and the sugar occurred without aid from the original cell. It was not until i $S_{9}$. however, that this theory was placed upon a firm experimental basis. This was brought about through the efforts of Buchner, who succeeded in isolating from the living yeast cells a substance (zymase) which, when freed from the last trace of organized cellular material, was able to bring 
about the identical fermentative processes which, up to this time, had been deemed possible only in the presence of the active, living yeast cell.

Buchner's manipulation of the yeast cells consisted in first grinding them with sand and infusorial earth, after which the finely divided material was subjected to great pressure (300 atmospheres) and yielded a liquid which possessed the fermentative activity of the unchanged yeast cell. ${ }^{1}$ This liquid contained zymase, the principal enzyme of the yeast cell. Later the lactic-acid- and acetic-acid-producing bacteria were subjected by Buchner to treatment similar to that accorded the yeast cells, and the active intracellular enzymes were obtained. Many other instances are on record in which a soluble, active agent has been isolated from ferment cells, with the result that it is pretty well established that all the so-called organized ferments elaborate substances of this character.

Enzymes act by catalysis and hence may be termed catalyzers or catalysts. A simple rough definition of a catalyst is "a substance which alters the velocity of a chemical reaction without undergoing any apparent physical or chemical change itself and without becoming a part of the product formed." It is a well-known fact that the velocity of the greater number of chemical reactions may be changed through the presence of some catalyst. For example, take the case of hydrogen peroxide. It spontaneously decomposes slowly into water and oxygen. In the presence of colloidal platinum,${ }^{2}$ however, the decomposition is much accelerated and ceases only when the destruction of the hydrogen peroxide is complete. Without multiplying instances, suffice it to say that there is a close analogy between inorganic catalysts and enzymes, the main point of difference between the enzymes and most of the inorganic catalysts being that the enzymes are colloids. The great majority of enzymes are hydrolytic in character.

We may define an enżyme as an organic catalyst which is elaborated by an animal or vegetable cell and whose activity is entirely independent of any of the life processes of such a cell. According to this definition the enzyme zymase elaborated by the yeast cell is entirely comparable to the enzyme pepsin elaborated by the cells of the stomach mucosa. One is derived from a vegetable cell, the other from an animal cell, yet the activity of neither is dependent upon the integrity of the cell.

Inasmuch as each of the enzymes has an action which is more or less specific in character, and since it is a fairly simple matter, ordinarily, to determine the character of that action, the classification of the enzymes

\footnotetext{
1 In later investigations the process was improved by freezing the ground cells with liquid air and finely pulverizing them before applying the pressure.

${ }_{2}$ Produced by the passage of electric sparks between two platinum terminals immersed in distilled water, thus liberating ultra-microscopic particles.
} 
is not attended with very great difficulties. They are ordinarily classified according to the nature of the substrate ${ }^{l}$ or according to the type of reaction they bring about. Thus we have various classes of enzymes, such as amylolytic, ${ }^{2}$ proteolytic, lipolytic, glycolytic, uricolytic, autolytic, oxidizing, reducing, inverting, protein-coagulating, deamidizing, etc. In every instance the class name indicates the individual type of enzymatic activity which the enzymes included in that class are capable of accomplishing. For example, amylolytic enzymes facilitate the hydrolysis of starch (amylum) and related substances, lipolytic enzymes facilitate the hydrolysis of fats ( $\lambda \iota \pi$ os), whereas through the agency of uricolytic enzymes uric acid is broken down. There is a tendency, at the present time, to harmonize the nomenclature of the enzymes by the use of the termination -ase. According to this system of nomenclature, all starch-transforming enzymes, or so-called amylolytic enzymes, are called amylases; all fat-splitting enzymes are called lipases, etc. Thus ptyalin, the amylolytic enzyme of the saliva, would be termed salivary amylase in order to distinguish it from pancreatic amylase (amylopsin) and vegetable amylases (diastase, etc.). According to the same system, the fat-splitting enzyme of the gastric juice would be termed gastric lipase to differentiate it fro"i pancreatic lipase (steapsin), the fat-splitting enzyme of the pancreatic juice.

Defensive (protective) enzymes are those beiieved to be manufactured by certain cells (perhaps the leucocytes) and passed into the circulating blood in order to digest any foreign material of endogenous or exogenous origin that may have found its way into the circulation. The most important defensive enzymes are proteolytic enzymes. Abderhalden $^{3}$ claims that the parenteral introduction of any foreign protein into the animal body will be followed by the appearance in the blood of a defensive enzyme capable of digesting that protein. He also claims that in pregnancy the passage into the blood of protein material in the form of cells and fragments of chorionic villi will cause the appearance in the blood of a defensive proteolytic enzyme capable of digesting placenta protein. The Abdcrhalden reaction for pregnancy is based upon this hypothesis. The reaction has been widely employed and much has been said both for and against its accuracy. ${ }^{4}$ Modifications of the reaction have been employed as an aid in the diagnosis of various disorders, e.g., cancer, tuberculosis, dementia precox, etc. Taylor ${ }^{5}$

${ }^{1}$ Substance acted upon. See Lippmann: Bcr.d. Deutsch. Chem. Ges., 36, 33 I, I903.

2 Armstrong suggests the use of the termination "clastic" instead of "lytic." He calls attention to the fact that amylolytic, in analogy with clectrolytic, means "decomposition by" means of starch" and is therefore a misnomer. He suggests the use of amyloclastic, proteoclastic, etc.

${ }^{3}$ Abwehrfermente des tierischen Organismus, 5 th Ed., Berlin, 19 $x_{5}$, Springer.

"Bronfenbrenner: Jour. Am. Aled. Assn., 65, r 268, Igr5. Van Slyke: New I"ork 3 red. Jour., 103, 219 , 1916.

5 Taylor: Jour. Biol. Chcm., 22, 59, x915. 
has very recently shown that "the rabbit does not form a protective ferment in response to the injection of the protamine of the salmon." He, however. points out the fact that this does not prove that a defensive enzyme is not formed in response to the passage of placental protein into the blood.

Our knowledge regarding the distribution of enzymes has been wonderfully broadened in recent years. Up to within a few years, the real scientific information as to the enzymes of the animal organism, for example, was limited, in the main, to a rather crude understanding of the enzymes intimately connected with the main digestive functions of the organism. We now have occasion to believe that enzymes are doubtless present in every animal cell and are actively associated with.all vital phenomena. As a preëminent example of such cellular activity may be cited the liver cell with its reputed complement of $\mathrm{I} 5-20$ or more enzymes.

A list of the more important enzymes together with their class, distribution, substrate and end-products is given below.

\section{CLASSIFICATION OF ENZYMES}

Name and Class

Distribution

Substrate

End-products
Carbohydrases

I. A mylases.

(a) Pancreatic

(b) Salitary.

(ptyalin)

(c) Vegetable.

. Liver, muscles? ...........

3. Inulase.

4. Lactase........

5. Maltase........
Pancrearic juice

Saliva

Malt, rice fungus, etc

Fungi, other plants..

Intestinal juice and mucosa

pancreatic and intestinal juices and lymph.

\begin{tabular}{|c|c|c|c|}
\hline $\begin{array}{l}\text { 6. Sucrase......... } \\
\text { (invertase) }\end{array}$ & Intestinal juice and mucosa. & Sucrose.. & Glucose and fructose. \\
\hline 7. Zymase........ & Yeast................ & 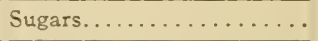 & Alcohol, $\mathrm{CO}_{2}$, etc. \\
\hline Carboxylase. & Yeast. & $\begin{array}{l}\mathrm{COOH} \text { group of aliphatic } \\
\text { acids. }\end{array}$ & Carbon dioxide. \\
\hline Deaminases....... & $\cdots$ & Amino compounds ........ & \\
\hline I. Adenase........ & Animal tissues.......... & Adenine..... & Hypoxanthine. \\
\hline 2. Arginase. & $\begin{array}{l}\text { Intestine, liver, kidney, } \\
\text { spleen, etc. }\end{array}$ & Arginine. & Ornithine and urea. \\
\hline 3. Guanase.... & Animal tissues........... & Guanin........ & Xanthine. \\
\hline 4. Crease. & $\begin{array}{l}\text { Micrococcus urea, soy } \\
\text { bean, etc. }\end{array}$ & Urea. & $\begin{array}{l}\text { Carbon dioxide and am- } \\
\text { monia. }\end{array}$ \\
\hline $\begin{array}{l}\text { Glucosidases. } \\
\text { I. Emulsin. } \\
\text { 2. Invertase }\end{array}$ & $\begin{array}{l}\text { Plants........... } \\
\text { Yeast, etc... . }\end{array}$ & 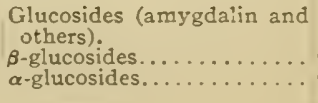 & $\begin{array}{l}\text { Glucose, etc. } \\
\text { Glucose, etc. }\end{array}$ \\
\hline
\end{tabular}

Carbohydrates.

Starch, dextrin, etc

Starch, dextrin, etc.

Starch, dextrin, etc.

Starch, dextrin, etc.

Maltose.

IIaltose.

Maltose.

Dextrin and maltose (glucose?)

Fructose.

Glucose and galactose.

Maltose.............. Glucose. 


\section{CLASSIFICATION OF EXZYMES.-Conlinued}

Name and Class

Lipases.

1. Autolytic

2. Pancrealic

(steapsin)

3. Vegetable

Nucleases....

1. Nucleicacidase.

2. Nucleotidase...

3. Nucleosidase..

Oxidases.

I. Laccase...

2. Peroxidase.

3. Catalase.

4. Purine-oxidases.

(a) Hypoxanthine Animal tissues.

oxidase.

(b) Uricase.

(c) Xanthine oxidase.

5. Tyrosinase.

$\frac{}{\text { Peptases........... }}$

Phytase.

Proleases.

I. Coagulases.

(a) Rennin.

(gastric)

(b) Rennin.

(pancreatic)

(c) Thrombin.

2. Pepsin. (acid-protease) 3. Trypsin.......... Pancreatic juice.

4. Vegetab

(a) Bromelin ..... Pineapple..
(b) Papain. ... Pawpaw...

Animal tissues.
Animal tissues.

Plant and animal tissues.

Intestinal mucosa and juice. Polypeptids.

other tissues.

Rice bran, liver, blood. .

Gastric juice.

Pancreatic juice.

Blood.

Gastric juice.

Purinases(see Purine

Oxidases and Pur-

ine Deaminases).
Substrate

End-products

Patty acid and glycerol. Fatty acid and ylycerril.

Fats.

Fats.

Fatty acid and klycersi.

Fats..

Fatty acid and glycerol.

Iucleic acidand derivatives.

Nucleic acid.. ........ Nuclentides.

Nucleotides.

Nucleosides.

Phosphoric acirl and nucleosides.

Carbohydrate and bases.

Polyhydric para-phenols as Oxidation products.

hydroquinol and pyro-

gallol.

Organic peroxides.

Oxygen or oxidation products.

Oxy"genor oxidation products.

Purines.

Hypoxanthine. .... Xanthine.

Uric acid...... A Hlantoin.

Xanthine. ....... Uric acid.

Trrosine.

Homogentisic acid, etc. Polypeptids.

Simpler peptids and amino acids.

Inositol and phosphric acid.

Proteins.

Proteins in solution.

Casein.

Paracasein.

Casein.

Fibrinogen

Proteins.

Paracasein.

Proteins.

Fibrin.

Proteoses, pepinnes, and peptides.

Proteoses, peptones, peptides, amino-acids.

Proteins. Proteins.

Proteoses, peptones, etc. Proteoses, pepiones. etc.

In text-book discussions of the enzymes it is customary to say that very little is known regarding the chemical characteristics of these substances since no member of the enzyme group has, up to the present time, been prepared in an absolutely pure condition. Apparently, however, from the nature of the facts in the case, it would be much more accurate to say that we absolutely do not know' whether a specific enzyme has, or has not, been prepared in a pure state. (Some authors, like Arthus, have assumed that enzymes are not chemical individuals, but properties conferred upon bodies.) The enzymes are very difficult to prepare in anything like a condition approximating purity. since the are very prone to change their nature during the process by which the 
investigator is attempting to isolate them. For this reason we have absolutely no proof that the final product obtained is, or is not, in the same state of purity it possessed in the original cell. Some of the enzymes are more or less closely associated with the proteins from the fact that they are both formed in every cell as the result of the cellular activity, both may be removed from solution by "salting-out," both are for the most part non-diffusible and are probably very similar as regards elementary composition. Hence in the preparation of some enzymes it is extremely difficult to make an absolute separation from the protein. Most of the evidence points to the protein character of enzymes. ${ }^{1} \quad$ Under certain conditions enzymes are readily adsorbed by shredded protein material, such as fibrin, and may successfully resist the most prolonged attempts at washing them free. We may summarize some of the properties of the great body of enzymes as follows: Enzymes are soluble in dilute glycerol, sodium chloride solution, dilute alcohol and water, and precipitable by ammonium sulphate and strong alcohol. Their presence may be proven from the nature of the end-products of their action and not through the agency of any chemical test. They are colloidal and non-diffusible, and occur closely associated with protein material with which they generally possess many properties in common. Each enzyme shows the greatest activity at a certain temperature called the optimum temperature; there is also a minimum and a maximum temperature for each specific enzyme. Their action is inhibited by sufficiently lowering the temperature, although some activity may be shown at $0^{\circ}$ or even at lower temperatures and freezing does not, in most cases, permanently injure enzymes. Most enzymes, if in solution, are entirely destroyed by subjecting them to a temperature of $70-100^{\circ} \mathrm{C}$. The best known enzymes, whether derived from warm-blooded or cold-blooded animals, are most active between $35^{\circ}-45^{\circ} \mathrm{C}$. The nature of the surrounding media alters the velocity of the enzymatic action, some enzymes being more active in acid solution whereas others require an alkaline fluid.

Many of the more important enzymes do not occur preformed within the cell, but are present in the form of a zymogen or mothersubstance. In order to yield the active enzyme this zymogen must be transformed in a certain specific manner and by a certain specific substance. This transformation of the inactive zymogen into the active enzyme is termed activation. For instance, the zymogen of the enzyme pepsin of the gastric juice, termed pepsinogen, is activated by the hydrochloric acid secreted by the gastric cells (see page $\mathrm{r} 4 \mathrm{I}$ ), whereas the activation of the trypsinogen of the pancreatic juice is brought about by a

${ }^{1}$ Others seem to be like the substrate on which they act, e.g., carbohydrate. 
substance termed enterokinase (sec page $x_{96}$ ). These are examples of many well-known activation processes going on continually within the animal organism. The agency which is instrumental in activating a zymogen is generally termed a zymo-exciter or a kinase. In the cases cited hydrochloric acid would be termed a zymo-exciter and enterokinase would be termed a kinase.

After filtering yeast juice, prepared by the Buchner process (see page 2), through a Martin gelatin filter, Harden and Young showed that the colloids left behind and the filtrate were both inactive fermentatively. Upon treating the colloid material (enzyme) with some of the filtrate, however, the mixture was shown to be able to bring about pronounced fermentation. It is believed that a co-enzyme present in the filtrate was the efficient agent in the transformation of the inactive enzyme. It is necessary to make frequent renewals of the co-enzyme in order to maintain continuous fermentation. It was further shown that this co-enzyme, in addition to being diffusible, was not destroyed by boiling and that it disappeared from yeast juice when this latter was fermented or allowed to undergo autolysis. The exact nature of this co-enzyme of zymase is unknown. The co-enzyme action, in this case, is probably dependent upon the presence of two individual agencies, one of which is phosphates.

It has been shown by Loevenhart that the property of acting as a pancreatic lipase co-enzyme is vested in bile salts, and Magnus has further shown that the synthetic salts are as efficient in this regard as the natural ones. A few other instances of co-enzyme demonstrations have been reported.

Electrolytes are very important factors in facilitating or inhibiting enzyme action. ${ }^{2}$ For example, the $\mathrm{Cl}$ ion in proper amount facilitates the action of amylases. ${ }^{3}$ In fact the presence of the $\mathrm{Cl}$ or $\mathrm{Br}$ ion is apparently absolutely essential to the activity of pancreatic amylase, inasmuch as dialysis renders this enzyme inactive, the activity returning on the addition of sodium chloride. ${ }^{4}$ The acidity or hydrogen ion concentration of the solution also exerts much influence on the activity of enzymes. It has been demonstrated in the case of certain enzymes. at least, that the continuous vibration or shaking of their solutions tends to produce a destruction of the enzyme. Ultraviolet light also has a destructive action on enzymes.

The so-called "specificity" of enzyme action is an interesting and important fact. That enzymes are very specific as to the character of

${ }^{1}$ According to Delezenne, trypsinogen may be rapidly activated by soluble calcium salts.

${ }^{2}$ For literature, see Kendall and Sherman: Jour. Am. Chcm. Soc., 32, 1087, 19ro.

3 Wohlgemuth: Biochemische Zeitschrifl, 9 , ro, rgos.

4 Bierry: Ibid., 40, 357, 1912. 
the substrate, or substance acted upon, is well known. Emil Fischer investigated this problem of specificity extensively in connection with the fermentation of sugars and reached the conclusion that enzymes, with the possible exception of certain oxidases, can act only upon such substances as have a specific stereo-isomeric relationship to themselves. He considers that the enzyme and its substrate must have an interrelation, such as the key has to the lock, or the reaction does not occur. Fischer was able to predict, in certain definite cases, from a knowledge of the constitution and stereo-chemical relationships of a substance, whether or not it would be acted upon by a certain enzyme. An application of this specificity of enzyme action may be seen in the well-known facts that certain enzymes act on carbohydrates, others on fats, and others on protein; and, moreover, that the group of those which transform carbohydrates, for example, is further subdivided into specific enzymes each of which has the power of acting alone upon some one sugar.

It has been conclusively shown, in the case of certain enzymes, ${ }^{1}$ at least, that their action is a reversible one and is, in all its main features, directly analogous to the reversible reactions produced by chemical means. For instance, in the saponification of ethyl-butyrate by means of pancreatic lipase, it has been shown that upon the formation of the end-products of the reaction, i.e., butyric acid and ethyl alcohol, there is reversion ${ }^{2}$ and the reaction is stationary. This does not mean there are no chemical changes going on, but simply indicates that chemical equilibrium has been established, and that the change in one direction is counterbalanced by the change in the opposite direction. Pancreatic lipase was one of the first enzymes to have the reversibility of its reaction clearly demonstrated. ${ }^{3}$ A knowledge of the fact that lipase possesses this reversibility of action is of extreme physiological importance and aids us materially in the explanation of the processes involved in the digestion, absorption, and deposition of fats in the animal organism (see page 178 ).

Euler ${ }^{4}$ claims that enzymatic cleavage and synthesis are often brought about by two different components of an enzyme preparation. $\mathrm{He}$ would indicate this fact by giving the termination -ese to those enzymes exerting a synthetic function. For example, the enzyme which catalyzes the formation of nitriles Euler would call nitrilese in distinction from nitrilase which splits nitriles. He would further designate as phos-

1 This is probably a general condition.

${ }^{2}$ The re-synthesis of ethyl-butyrate from its hydrolysis products. This may be indicated thus:

$$
\mathrm{C}_{3} \mathrm{H}_{7} \mathrm{COO} . \mathrm{C}_{2} \mathrm{H}_{5}+\mathrm{H}_{2} \mathrm{O} \leftrightarrows \mathrm{C}_{3} \mathrm{H}_{7} \mathrm{COOH}+\mathrm{C}_{2} \mathrm{H}_{5} \mathrm{OH} \text {. }
$$

$$
\text { Ethyl-butyrate. Butyric acid. Ethyl alcohol. }
$$

${ }^{3}$ This principle was first demonstrated in connection with the enzyme maltase (see p. 57 ).

4 Euler: Zeilschrift fïr physiologische chemie, 74, I3, I9I I. 
phatese the enzyme which builds up phosphoric acid esters of carbohydrates in distinction from phosphatase which causes their cleavage. In the same way he would differentiate the lipolytic enzymes into lipases and lipeses.

In respect to many enzymes it has been found that the law governing the action of inorganic catalyzers is directly applicable, i.e., that the intensity is almost directly proportional to the concentration of the enzyme. In the case of enzymes, however, there is a difference in that a maximum intensity is soon reached and that subsequent concentration of the enzyme is productive of no further increase in intensity. The enzymes which have been shown to obey this linear law are lipase. sucrase, rennin, and trypsin. In certain instances, where this law of direct proportionality between the intensity of action and the concentration of enzymes does not hold, it has been found that the Schiitz-Borissow law', first experimentally demonstrated by E. Schütz, was applicable. This is to the effect that the intensity is directly proportional to the square root of the concentration, or conversely; that the relutice concentrations of enzyme preparations are directly proportional to the squares of the intensities. ${ }^{1}$

It has been shown that there are certain substances which possess the property of directly inhibiting or preventing the action of a catalyzer. These are called anti-catalyzers or paralyzers and have been compared to the anti-toxins. Related to this class of anti-catalytic agents stand the anti-enzymes. The first anti-enzyme to be reported was the antirennin of Morgenroth. This was produced by injecting into an animal increasing doses of rennet solution, whereupon an "anti" substance was subsequently found both in the serum and in the milk. which prevented the enzyme rennin from exerting its normal activity in the presence of casein. In other words, anti-rennin had been formed in the serum of the animal, ${ }^{2}$ through the repeated injections of rennet solution. Since the discovery of this anti-enzyme, anti-bodies have been demonstrated for pepsin, trypsin, lipase, urease, amylase. laccase, tyrosinase, emulsin, papain, and thrombin. According to Weinland, the reason why the stomach does not digest itself is, that during life there is present in the mucous membrane of the stomach an anti-cnzyme (anti-pepsin) which has the property of inhibiting the action of pepsin. A similar substance (anti-trypsin) is present in the intestinal mucosa as well as in the tissues of various intestinal worms. Some investigators are not inclined to accept the enzyme nature of these inhibitory agents as proven.

1 This Schütz-Borissow law is not generally applicable.

${ }^{2}$ Serum is normally anli-tryplic. 
The investigations of Ehrlich ${ }^{1}$ and of Neuberg ${ }^{2}$ have served to cause a complete revision of our ideas regarding yeast fermentation. Ehrlich, for example, has shown that yeast will liberate ammonia from amino acids and leave behind a non-nitrogenous complex. Among these complexes amyl alcohol, succinic acid and others may be mentioned. Thus, amyl alcohol results from the fermentation of leucine, whereas ethyl alcohol results from the fermentation of sugar. Neuberg has demonstrated the presence in the yeast of an enzyme termed carboxylase which has the property of splitting off carbon dioxide from the carboxyl group of amino and other aliphatic acids. The findings mentioned above constitute the basis for much important work on so-called "sugar-free fermentation."

For a more extended consideration of enzymes the student is referred to the following sources:

Bayliss.-The Nature of Enzyme Action, Third Edition, Longmans, Green and Co., New York and London.

Cohnheim.-Enzymes, Wiley and Sons, New York, igr 2.

Duclaux.-Traité de Microbiologie, Masson and Co., Paris.

Effront.-Enzymes and their Applications, Translated by Prescott, Wiley and Sons, New York.

Euler.-(a) Allgemeine Chemie der Enzyme, Bergmann, Wiesbaden, I910. (b) Ergebnisse der Physiologie, I909-10. (c) General Chemistry of the Enzymes, Translated by Pope, Wiley and Sons, I9I 2 .

Oppenheimer.-Die Fermente und Thre Wirkungen, Vierte Auflage, Vogel, Leipzig.

Samuely.-Handbuch der Biochemie des Menschen und der Thiere (Oppenheimer), Gustav Fischer, Jena, igro-r r.

Wohlgemuth.-Grundriss der Fermentmethoden, r9r3, Berlin, Springer.

\section{EXPERIMENTS ON ENZYMES AND ANTI-ENZYMES}

\section{A. Experiments on Enzymes ${ }^{3}$}

\section{AMYLASES}

I. Demonstration of Salivary Amylase. L-To 25 c.c. of a I per cent starch paste in a small beaker, add 5 drops of saliva and stir thoroughly. At intervals of a minute remove a drop of the solution to one of the depressions of a test-tablet

${ }^{3}$ Ehrlich: Biochemische Zeilschrift, 36, 477, I91 r.

${ }^{2}$ Neuberg and Collaborators: Biochemische Zeitschrifl, 31, r70; 32, 323; $36(60,68$, and 76), I9I I.

${ }^{3}$ If it is deemed advisable by the instructor to give all the practical work upon enzymes at this point in the course, additional experiments will be found in Chapters III, VI, VII, $\mathrm{X}$ and XI.

${ }^{4}$ For a discussion of this enzyme see p. 55. 
and test by the iodine test. ${ }^{1}$ If the blue color with iodine still forms after five minutes, add another 5 drops of saliva.

The opalescence of the starch solution should soon disappear, indicating the formation of soluble starch (amidulin) which gives a blue color with iodine. This body should soon be transformed into erythrodextrin which gives a red color with iodine, and this, in turn, should pass into achroodextrin which gives no color with iodine. This point is called the achromic point. When this point is reached test by Fehling's test $^{2}$ to show the production of a reducing substance (maltose). A positive Fehling's test may be obtained while the solution still reacts red with iodine, inasmuch as some sugar is formed from the soluble starch coincidently with the formation of the erythrodextrin. For further discussion of the transformation of starch see page 56 .

2. Demonstration of Pancreatic Amylase. ${ }^{3}$ - Proceed exactly as indicated above in the Demonstration of Salivary Amylase except that the saliva is replaced by 5 c.c. of pancreatic extract prepared as described on p. I89. ${ }^{4}$ Pancreatic amylase transforms the starch in a manner entirely analogous to the transformation resulting from the action of salivary amylase.

3. Preparation of Vegetable Amylase.-Extract finely ground malt with water, filter and subject the filtrate to alcoholic fermentation by means of yeast. When fermentation is complete filter off the yeast and precipitate the amylase from the filtrate by the addition of alcohol. The precipitate may be filtered off and obtained in the form of a fine white powder.

A purer preparation ${ }^{5}$ is obtained if the solution is dialyzed against water at about $10^{\circ} \mathrm{C}$. (in the ice-box) for 24 hours, filtered and precipitated with alcohol or acetone. First alcohol or acetone to make a 50 per cent solution is added, the precipitate thus formed being rejected, while the precipitate formed on the addition of sufficient alcohol or ace tone to make a final concentration of $65-7$ o per cent is preserved, and dried in a vacuum desiccator at a low temperature.

4. Demonstration of Vegetable Amylase.-This enzyme may be demonstrated according to the directions given under Demonstration of Salivary Amylase, page Io, with the exception that the saliva used in that experiment is replaced by an aqueous solution of the vegetable amylase powder prepared as described above. ${ }^{6}$

1 See p. 45 .

${ }^{2}$ See p. 26 .

${ }^{3}$ For a discussion of this enzyme see p. 187 .

- Commercial preparations of pancreatic amylase may be substituted for the pancreatic extract.

'Sherman and Schlesinger: I. Am. Ch. Soc., 35, I6r7, igr 5

${ }^{6}$ If desired the first aqueous extract of the original malt may be used in this demonstration. Commercial taka-diastase may also be employed. 


\section{PROTEASES}

I. Preparation of Gastric Protease. ${ }^{1-}$ Treat the finely comminuted mucosa of a pig's stomach with 0.4 per cent hydrochloric acid and extract at $38^{\circ} \mathrm{C}$. for 24-48 hours. The filtrate from this mixture constitutes a very satisfactory acid extract of gastric protease (see page I44).

2. Demonstration of Gastric Protease.-Introduce some protein material (fibrin, coagulated egg-white, or washed lean beef) into the acid extract of gastric protease prepared as above described, ${ }^{2}$ add an equal volume of 0.4 per cent hydrochloric acid and place the mixture at $38^{\circ} \mathrm{C}$. for $2-3$ days. Identify the products of digestion according to directions given on page 144 .

Carmine-fibrin may also be used in this test. This is prepared by running fibrin through a meat chopper washing carefully and placing in a $1 / 2$ per cent ammoniacal carmine solution (very little excess ammonia should be present) until the maximum coloration of the fibrin (a dark red) is obtained. 'The fibrin is then washed in water and water acidified with acetic acid. It is preserved under glycerol.

To $5_{5}$ c.c. of the solution to be tested add a small amount of the carmine fibrin and allow to digest at room temperature. Digestion will be shown by the setting free of carmine with coloration of the solution. This is a delicate test for pepsin. A control should be run using acid of same strength as that of enzyme solution tested.

3. Preparation of Pancreatic Protease. ${ }^{3-A}$ satisfactory extract of this enzyme may be made from the pancreas of a pig or sheep according to the directions given on page 189 .

4. Demonstration of Pancreatic Protease.-Into an alkaline extract of pancreatic protease ${ }^{4}$ prepared as directed on page 189 , introduce some fibrin, coagulated egg-white or lean beef and place the mixture at $38^{\circ} \mathrm{C}$. for $2-5$ days. ${ }^{5}$ At the end of that period separate and identify the end-products of the action of pancreatic protease according to the directions given on page 189 .

Congo-red fibrin may be used in this test. This may be prepared by placing fibrin in faintly alkaline congo-red solution and heating to $80^{\circ} \mathrm{C}$. The fibrin is then washed. A small amount of this colored fibrin is placed in the slightly alkaline solution of the enzyme. Digestion is shown by a red coloration of the solution due to setting free of congo red.

${ }^{1}$ Also called pepsin, pepsase, gastric protease and acid protease. For a discussion of this enzyme see p. I $4 \mathrm{I}$.

${ }^{2}$ If so desired. a solution of commercial pepsin powder in 0.2 per cent hydrochloric acid may be substituted.

3 Also called trypsin, trypsase, pancreatic protease and alkali protease. For a discussion of this enzyme see p. I 86.

${ }^{4} \mathrm{~A} 0.25$ per cent sodium carbonate solution of commercial trypsin or pancreatin may be substituted.

${ }^{5} \mathrm{~A}$ few c.c. of toluol or an alcoholic solution of thymol should be added to prevent putrefaction. 
5. Demonstration of a Vegetable Protease. - A commercial preparation of papain (papayotin, carase or papase), the protease of the fruit of the pawpaw (carica papaya), may be used in this connection. Follow the same procedure as that described under Gastric Protease (sce p. I2).

It has been demonstrated by Mendel and $\mathrm{Blood}^{1}$ that the presence of $\mathrm{HCN}$ will accelerate the proteolytic activity of papain. It is suggested that the HCN acts as a so-called co-enzyme (sec page 7 ).

Vines" believes that "papain" consists of a mixture of two enzymes, a pepsin and an erepsin. Mendel and Blood do not consider the evidence on this point as conclusive.

\section{LIPASES}

I. Preparation of Pancreatic Lipase. ${ }^{3}$-An extract of this enzyme may be prepared from the pancreas of the pig or sheep according to the directions given on page $189 .^{4}$

2. Demonstration of Pancreatic Lipase.-Into each of two test-tubes introduce Io c.c. of milk and a small amount of litmus powder. To the contents of one tube add 3 c.c. of a neutral extract of pancreatic lipase and to the contents of the other tube add 3 c.c. of a boiled neutral extract of pancreatic lipase. Keep the tubes at $38^{\circ} \mathrm{C}$. and watch for color changes.

The blue color of the litmus powder will gradually give place to a red. This change in color of the litmus from blue to red has been brought about by the fatty acid which has been produced through the lipolytic action exercised by the lipase upon the milk fats.

3. Preparation of Vegetable Lipase.-This enzyme may be readily prepared from castor beans, several months' old, by the following procedure: ${ }^{5}$ Grind the shelled beans very fine ${ }^{6}$ and extract for twenty-four-hour periods with alcohol-ether and ether, in turn. Reduce the semi-fat-free material to the finest possible consistency by means of mortar and pestle and pass this material through a sieve of very fine mesh. Place this material in a Soxhlet extractor and extract for one week. This fat-free powder may then be used to demonstrate the action of vegetable lipase. Powder prepared as described may be used in quantitative tests. For ordinary qualitative tests it is not necessary to remove the last traces of fat and therefore the extraction period in the Soxhlet apparatus may be much shortened.

4. Demonstration of Vegetable Lipase.-The lipolytic action of the lipase prepared from the castor bean, as just described, may be demonstrated in a manner entirely analogous to that used in the Demonstration of Pancreatic Lipase, sec above. Proceed as indicated in that experiment and substitute the vegetable lipase powder for the neutral extract of pancreatic lipase. The type of action is entirely analogous in the two instances.

1 Mendel and Blood: Journal of Biological Chemistry, S, 177, igro.

2 Vines: Annals of Bolany, 19, 174, 1905.

${ }^{3}$ Also called steapsin. For a discussion of the enzyme see p. r8S. A very active lipolytic extract may also be prepared from the liver.

"If preferred, a glycerol cxtract may be prepared according to the directions given by Kanitz (Zeilschrift fïr physiologische Chemie, 1906, 46, p. 482) or commercial pancreatin may be employed.

5 A. E. Taylor: On Fermentation; C'nizersity of California Publications, $x 0^{-}$.

${ }^{6}$ The shells should be removed without the use of water. These beans are poisonons, due to their content of ricin. 
An experiment similar to Experiment 2, page I93, may also be tried if desired. In this experiment 0.2 c.c. of either ethyl butyrate or amyl acetate may be employed.

\section{INVERTASES ${ }^{1}$}

r. Preparation of Vegetable Sucrase. ${ }^{2}$ - Thoroughly grind about roo grams of brewer's yeast in a mortar with sand. Spread the ground yeast in thin layers on glass or porous plates and dry it rapidly in a current of dry, warm air. Powder this dry yeast, extract it with distilled water and filter. Pour the filtrate into acetone, stir and after permitting the acetone mixture to stand for a few minutes filter on a Buchner funnel. The resulting precipitate, after drying and pulverizing, may be used to demonstrate vegetable sucrase.

2. Demonstration of Vegetable Sucrase.-To about 5 c.c. of a I per cent solution of sucrose in a test-tube add a small amount of the sucrase powder prepared as directed above. Place the tube at $38^{\circ} \mathrm{C}$. for $24-72$ hours and at the end of that period test the solution by Fehling's test. Reduction indicates that the active sucrase powder has transformed the non-reducing sucrose into glucose and fructose, and these sugars, in turn, have reduced the Fehling solution.

For other experiments on Invertases, see Chapter XI.

\section{OXIDASES ${ }^{3}$}

I. Demonstration of Oxidase.-Oxidases or oxidizing enzymes constitute a very important group of intracellular enzymes. They are intimately connected with the oxidation processes in the plant and animal organisms.

I. Cut a thin slice from a freshly pared potato, place it on a watch glass and examine at intervals during the laboratory exercise. Note that the colorless potato gradually becomes brown.

This is due to the oxidation of para-oxyphenyl substances such as tyrosin, in the cells and in the intracellular juice of the potato. Two oxidases which have the power of accelerating the oxidation of paraoxyphenyl compounds are called tyrosinase and laccase.

2. Preparation of Potato Extract.-Scrape a pared potato by means of a knife or scalpel or comminute the potato substance by means of a grater. Extract the macerated potato substance by means of water. Strain through cheese cloth and filter the extract. Make an iodine test on the solid substance (see Starch, page 45), and save the water extract for use in the following experiments.

3. Oxidation of Para-oxyphenyl Compounds by Potato Oxidases.-Introduce 5 c.c. of filtered potato extract prepared as indicated above, into each of six testtubes. Introduce additional reagents into the tubes according to the following series:

(a) Potato extract +5 drops of toluol (control).

(b) Potato extract +5 drops of ether (control).

1 The inverting enzymes of the alimentary tract; Mendel and Mitchell: American Journal of Physiology, 20, 8r, 1907-08.

${ }^{2}$ For a discussion of this enzyme see p. 195.

3 These experiments have been adapted from directions contained in the Laboratory Notes of Professor Gies of the College of Physicians and Surgeons, New York. 
(c) Potato extract +5 drops of I per cent phenol solution.

(d) Potato extract +5 drops of I per cent "tri-cresol " solution.

(e) Potato extract (boiled and cooled) +5 drops of I per cent phenol solution.

(f) Potato extract (boiled and cooled) + 5 drops of I per cent "tri-cresol" solution.

Shake the contents of the six tubes thoroughly. Are there any immediate color changes? Place the tubes in your rack, and examine them at the next laboratory exercise.

4. Experiments with Typical Oxidase Reagents.--Introduce 5 c.c. of filtered potato extract into each of four test-tubes. Add oxidase reagents as follows:

(a) Potato extract + ro drops of guaiac solution. ${ }^{1}$

(b) Potato extract + Io drops of $\alpha$-naphthol solution. ${ }^{2}$

(c) Potato extract + Io drops of para-phenylenediamine hydrochloride solution. ${ }^{3}$

(d) Potato extract +5 drops of $\alpha$-naphthol solution +5 drops of paraphenylenediamine hydrochloride solution +5 drops of ro per cent sodium carbonate (Indophenol Test).

Shake the contents of each tube thoroughly and note immediate color changes. Place the tubes in the rack and leave them undisturbed until the end of the laboratory exercise. Note any changes or peculiarities in the coloration effects, especially at the surface of the liquid.

In tube (a) the guaiaconic acid of the guaiac resin has been oxidized and formed guaiac blue.

In tube (b) a violet coloration due to the production of di-naphthol appears. The oxidase has oxidized the $\alpha$-naphthol.

In tube (c) we have a change whose chemistry is not well known.

In tube (d) we have the production of indophenol from the $\alpha$-naphthol and the para-phenylenediamine hydrochloride under the influence of oxidase. The indophenol is soluble in the alkaline solution. The color gradually changes from red to purple as the indophenol accumulates.

The production of the above colors does not possess any biological significance. These colors simply serve to indicate that certain reactions are taking place which occur normally in living cells, although in the latter case they are of course unaccompanied by any color change. Intracellular oxidase favors the utilization of oxygen by a cell, just as the potato oxidase has facilitated the oxidation of the chromogens in the above tests.

\section{CATALASE}

Demonstration of Catalase.--The various animal tissues as liver, kidney, blood, lung, muscle and brain contain an enzyme called catalase or peroxidase which possesses the property of decomposing hydrogen

1 Made by dissolving $0.5 \mathrm{gram}$ of guaiac resin in 30 c.c. of 95 per cent alcohol.

2 Made by dissolving I gram of $\alpha$-naphthol in roo c.c. of 95 per cent alcohol.

${ }^{3}$ Dissolve I gram of para-phenylene diamine hydrochloride in roo c.c. of water. 
peroxide as well as certain biological peroxides with the liberation of oxygen. Catalase is an indirect oxidizing enzyme, that is, it brings about oxidations only in the presence of a peroxide. Catalase is also found in many plant tissues and an extract of it may be prepared from potatoes.

I. Vegetable Catalase. - Into each of four test-tubes place 5 c.c. of filtered potato extract prepared as in Experiment 2, page I4. Prepare a second series of four tubes (see 4, p. I5), but use a boiled potato extract. Prepare also a third series using water instead of potato extract. Now add to each of the twelve tubes 5 drops of a 3 per cent solution of hydrogen peroxide. While the resultant lively effervescence is in progress add to each series the four "Typical Oxidase Reagents" in the order and quantities specified in the preceding experiment(4). Allow the tubes to remain undisturbed and carefully note comparative effects during the remainder of the laboratory exercise. ${ }^{1}$

2. Animal Catalase.-The presence of this enzyme may also be demonstrated as follows: Introduce into a low, broad, wide-mouthed bottle some pulped liver tissue and a porcelain crucible containing neutral hydrogen peroxide. ${ }^{2}$ Connect the bottle with a eudiometer filled with water, upset the crucible of hydrogen peroxide upon the liver pulp and note the collection of gas in the eudiometer. This gas is oxygen which has been liberated from the hydrogen peroxide through the action of the catalase of the liver tissue.

See next experiment for a method for the quantitative determination of catalase based on the above principle.

3. Quantitative Determination of Catalase. ${ }^{3}$-In the determination of the catalase values of tissues weighed portions of the tissue under examination should be ground with sand in a mortar then treated with four volumes of chloroform water and permitted to extract for 24 hours at room temperature. An apparatus such as that shown in Fig. I may be employed in determining the catalase values. The main features of the apparatus are based upon those of a delivery funnel for introducing liquids under increased or diminished pressure.

In making a determination introduce a measured volume ( $\mathrm{I}-4$ c.c.) of the filtered extract ${ }^{4}$ into the small flask and insert the modified Johnson burette graduated to 5 c.c. and containing 50 c.c. of hydrogen peroxide (Oakland dioxygen neutral ${ }^{5}$ to Congo red) into the neck of the flask. Fill the eudiometer with water and place in position. Close cocks $\mathrm{A}$ and $\mathrm{C}$ and open cocks $\mathrm{B}$ and $\mathrm{D}$ thus permitting 5 c.c. of the peroxide to flow into the flask. Shake the contents of the flask briskly ${ }^{6}$ and record the volume of oxygen evolved in a two-minute period taking readings at intervals of fifteen seconds.

Calculation.-When a series of comparative tests are made on different tissues or on the same tissue under different conditions it is considered satisfactory to make a comparison of the catalase values upon the basis of the volume of oxygen evolved

1 This experiment has been adapted from one contained in the Laboratory Notes of Professor Gies of the College of Physicians and Surgeons, New York.

${ }^{2}$ Mendel and Leavenworth: American Journal of Physiology, 21, 85, 1908.

${ }^{3}$ Hawk: Journal of American Chemical Sociely, 33, 425, I9I.

4 If less than 4 c.c. of extract are used the volume should be made up to 4 c.c. by the addition of distilled water.

${ }_{5}^{5}$ An acid reaction modifies the rate of the oxygen evolution. (See Mendel and Leavenworth, American Journal of Physiology, 21, 85, 1908.)

${ }^{6}$ In making a series of comparative tests it is essential that the shaking process should be uniform from determination to determination. 
in a period of two minutes from 5 c.c. of neutral hydrogen peroxide by means of I c.c. of a I : 4 chloroform-waler extract of the tissue.

\section{B. Experiments on Anti-Enzymes}

I. Preparation of an Extract of Anti-Pepsin. ${ }^{1}$ - Grind up a number of intestinal worms (ascaris) ${ }^{2}$ with quartz sand in a mortar. Subject this mass to high pressure, filter the resultant juice and treat it with alcohol until a concentration of 60 per cent is reached. If any precipitate forms it should be filtered of ${ }^{3}$ and alcohol added to the filtrate until the concentration of alcohol is 85 per cent, or over. The anti-enzyme is precipitated by this concentration. Permit this precipitate to stand

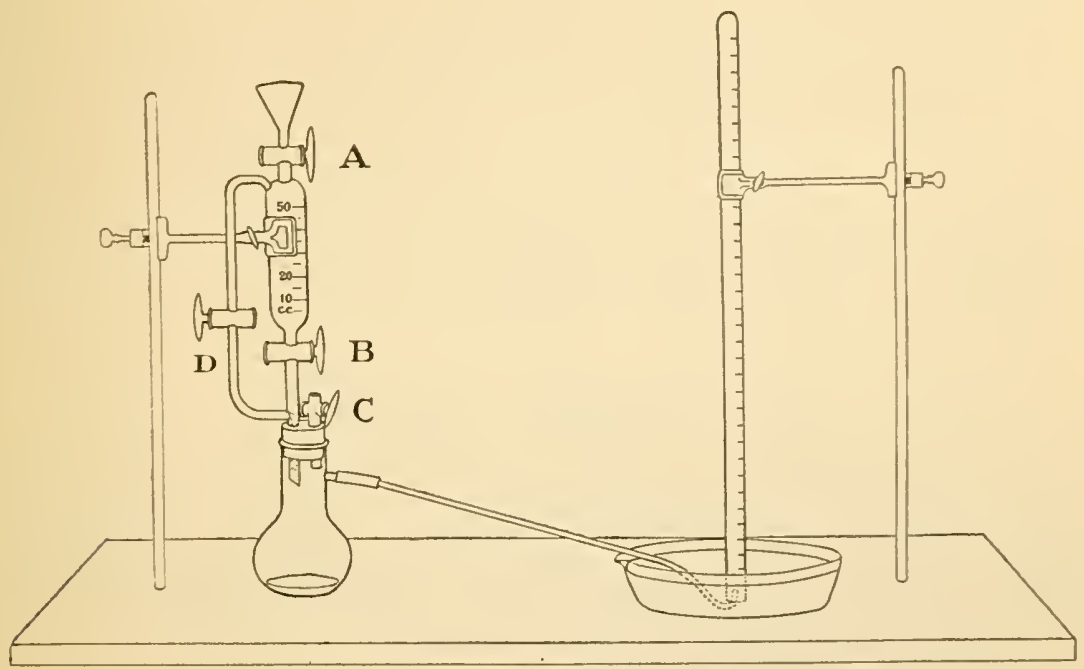

Fig. I.-Apparates for Quantitative Determination of Catalase.

for twenty-four hours, then filter it off, wash it with 95 per cent alcohol, absolute alcohol, and ether, in turn, and finally dry the substance over sulphuric acid. The sticky powder which results may be used in this form or may be dissolved in water as desired and the aqueous solution used. ${ }^{4}$

2. Demonstration of Anti-Pepsin. ${ }^{5}$-Introduce into a test-tube a few fibrin shreds and equal volumes of pepsin-hydrochloric acid ${ }^{6}$ and ascaris extract made as indicated above. Prepare a control tube in which the ascaris extract is replaced by water. Place the tubes at $38^{\circ} \mathrm{C}$. Ordinarily in one hour the fibrin in the control tube will be completely digested. The fibrin in the tube containing the ascaris extract may, however, remain unchanged for diys, thus indicating the inhibitory influence exerted by the anti-enzyme present in this extract.

1 Anti-gastric-proteatse or anti-acid-protease.

2 These may be readily obtained from pigs at a slaughter house.

3 This precipitate consists of impurities, the anti-enzyme not being precipitated until a higher concentration of alcohol is reached.

4 The original ascaris extract possesses much greater activity than either the powder or the aqueous solution.

${ }^{5}$ Martin H. Fischer: Physiology of Alimentalion. 1007, p. 134.

${ }^{6}$ Made by bringing 0.015 gram of pepsin into solution in 7 c.c. of water and 0.23 gram of concentrated hydrochloric acid. 
3. Preparation of an Extract of Anti-Trypsin. ${ }^{1}$ - The extract may be prepared from the intestinal worm, ascaris, according to the directions given on page 17 .

4. Demonstration of Anti-Trypsin. - Introduce into a test-tube a few shreds of fibrin and equal volumes of an artificial tryptic solution ${ }^{2}$ and the ascaris extract made as described on page I7. Prepare a control tube in which the ascaris extract is replaced by water. Place the two tubes at $38^{\circ} \mathrm{C}$.

Ordinarily the fibrin in the control tube will be completely digested in two hours. The fibrin in the tube containing the ascaris extract may, however, remain unchanged for days, thus indicating the inhibitory influence of the anti-enzyme.

Blood serum also contains anti-trypsin. This may be demonstrated as follows: Introduce equal volumes of serum and artificial tryptic solution (prepared as described above) into a test-tube and add a few shreds of fibrin. Prepare a control tube containing boiled serum. Place the two tubes at $38^{\circ} \mathrm{C}$. It will be observed that the fibrin in the tube containing the boiled serum digests, whereas that in the other tube does not digest. The anti-trypsin present in the unboiled serum has exerted an inhibitory influence upon the action of the trypsin.

\section{Quantitative Applications}

Methods for the quantitative determination of various enzymes will be found in the following chapters: Amylase (Chapter $\mathrm{X}$ ); Erepsin (Chapter XI); Pepsin (Chapter VIII); Trypsin (Chapter X); Urease (Chapter XXVI).

${ }^{1}$ Anti-pancreatic-protease or anti-alkali-protease.

${ }^{2}$ Made by dissolving 0.04 gram of sodium carbonate and 0.015 pram of trypsin in 8 c.c. of water. 


\section{CHAP'TER II}

\section{CARBOHYDRATES}

The name carbohydrates is given to a class of bodies which are an especially prominent constituent of plants and which are found also in the animal body either free or as an integral part of various proteins. They are called carbohydrates because they contain the elements $\mathrm{C}, \mathrm{H}$ and $\mathrm{O}$; the $\mathrm{H}$ and $\mathrm{O}$ being present in the proportion to form water. The term is not strictly appropriate inasmuch as there are bodies, such as acetic acid, lactic acid and inositol, which have $\mathrm{H}$ and $\mathrm{O}$ present in the proportion to form water, but which are not carbohydrates, and there are also true carbohydrates which do not have $\mathrm{H}$ and $\mathrm{O}$ present in this proportion, e.g., rhamnose, $\mathrm{C}_{6} \mathrm{H}_{12} \mathrm{O}_{5}$.

Chemically considered, the carbohydrates are aldehyde or ketone derivatives of complex alcohols. Treated from this standpoint, the aldehyde derivatives are spoken of as aldoses, and the ketone derivatives are spoken of as ketoses. The carbohydrates are also frequently named according to the number of oxygen atoms present in the molecule, e.g., trioses, pentoses, and hexoses.

The more common carbohydrates may be classified as follows:

I. Monosaccharides.

I. Pentoses, $\mathrm{C}_{5} \mathrm{H}_{10} \mathrm{O}_{5}$.

(a) Arabinose.

(b) Xylose.

(c) Rhamnose (Methyl-pentose), $\mathrm{C}_{6} \mathrm{H}_{12} \mathrm{O}_{5}$.

2. Hexoses, $\mathrm{C}_{6} \mathrm{H}_{12} \mathrm{O}_{6}$.

(a) Glucose.

(b) Fructose.

(c) Galactose.

II. Disaccharides, $\mathrm{C}_{18} \mathrm{H}_{22} \mathrm{O}_{11}$.

I. Maltose.

2. Lactose.

3. Iso-Maltose.

4. Sucrose.

- III. Trisaccharides, $\mathrm{C}_{18} \mathrm{H}_{32} \mathrm{O}_{16}$.

I. Raffinose. 
IV. Polysaccharides, $\left(\mathrm{C}_{6} \mathrm{H}_{10} \mathrm{O}_{5}\right)_{8}$.

I. Gum and Vegetable Mucilage Group.

(a) Dextrin.

(b) Vegetable Gums.

2. Starch Group.

(a) Starch.

(b) Inulin.

(c) Glycogen.

(d) Lichenin.

3. Cellulose Group.

(a) Cellulose.

(b) Hemicelluloses.

(I) Pentosans.

Gum Arabic.

(2) Hexosans.

Galactans.

Agar-agar.

Each member of the above carbohydrate classes, except the members of the pentose group, may be supposed to contain the group $\mathrm{C}_{6} \mathrm{H}_{10} \mathrm{O}_{5}$, called the saccharide group. The polysaccharides consist of this group alone taken a large number of times, whereas the disaccharides may be supposed to contain two such groups plus a molecule of water, and the monosaccharides to contain one such group plus a molecule of water. Thus, $\left(\mathrm{C}_{6} \mathrm{H}_{10} \mathrm{O}_{5}\right)_{\mathrm{x}}=$ polysaccharide, $\left(\mathrm{C}_{6} \mathrm{H}_{10} \mathrm{O}_{5}\right)_{2}+\mathrm{H}_{2} \mathrm{O} \rightarrow$ disaccharide, $\mathrm{C}_{6} \mathrm{H}_{10} \mathrm{O}_{5}+\mathrm{H}_{2} \mathrm{O} \rightarrow$ monosaccharide. In a general way the solubility of the carbohydrates varies with the number of saccharide groups present, the substances containing the largest number of these groups being the least soluble. This means simply that, as a class, the monosaccharides (hexoses) are the most soluble and the polysaccharides (starches and cellulose) are the least soluble.

\section{MONOSACCHARIDES}

\section{Hexoses, $\mathrm{C}_{6} \mathrm{H}_{12} \mathrm{O}_{6}$}

The hexoses are monosaccharides containing six oxygen atoms in a molecule. They are the most important of the simple sugars, and two of the principal hexoses, glucose and fructose, occur widely distributed in plants and fruits. Of these two hexoses, glucose results from the hydrolysis of starch, whereas both glucose and fructose are formed in the hydrolysis of sucrose. Galactose, which with glucose results from the hydrolysis of lactose, is also an important hexose. These three hexoses are fermentable by yeast, and yield levulinic acid upon heating 
with dilute mineral acids. They reduce metallic oxides in alkaline solution, are optically active and extremely soluble. With phenylhydrazine they form characteristic osazones.

\section{$\mathrm{CH}_{2} \mathrm{OH}$ \\ GLU COSE $(\mathrm{CHOH})_{4}$ \\ $\mathrm{CHO}$}

Glucose, also called dextrose or grape sugar, is present in the blood in small amount and also occurs in traces in normal urine. ${ }^{1}$ After the ingestion of large amounts of sucrose, lactose or glucose, causing the assimilation limit to be exceeded, an alimentary glycosuria may arise. In diabetes mellitus very large amounts of glucose are excreted in the urine. The following structural formula has been suggested by Victor Meyer for $d$-glucose:<smiles>O=C[C@H](O)[C@H](O)[C@H](O)[C@H](O)CO</smiles>

(For further discussion of glucose see section on Hexoses, page 20.)

\section{Experiments on Glucose}

The following tests are made on glucose as a typical carbohydrate and are not specific for this sugar. A specific test for glucose is the Phenylhydrazine Reaction (3) in the absence of a positive ResorcinolHydrochloric Acid Reaction (see page 35).

I. Solubility.-Test the solubility of glucose in the "ordinary solvents" and in alcobol. (In the solubility test throughout the book we shall designate the following solvents as the "ordinary solvents:" $\mathrm{H}_{2} \mathrm{O}$; ro per cent $\mathrm{NaCl}$; 0.5 per cent $\mathrm{Na}_{2} \mathrm{CO}_{3} ; 0.2$ per cent $\mathrm{HCl}$; concentrated $\mathrm{KOH}$; concentrated $\mathrm{HCl}$.)

2. $\alpha$-Naphthol Reaction (Molisch).- Place approximately 5 c.c. of concentrated $\mathrm{H}_{2} \mathrm{SO}_{4}$ in a test-tube. Incline the tube and slowly pour down the inner side of it approximately 5 c.c. of the sugar solution to which 2 drops of Molisch's reagent (a I5 per cent alcoholic solution of $\alpha$-naphthol) has been added, so that the sugar solution will not mix with the acid. A reddish-violet zone is produced at the point of contact.

${ }^{1}$ See Folin's test for sugar in normal urine (Jour. Biol. Chem., 22, 32 $\overline{7}, 1915$. 
The reaction is due to the formation of furfural, ${ }^{1}$

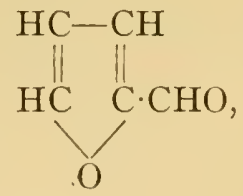

by the acid. The test is given by all bodies containing a carbohydrate group and is therefore not specific and, in consequence, of very little practical importance.

3. Phenylhydrazine Reaction.-Test according to one of the following methods: (a) To a small amount of phenylhydrazine mixture (enough to fill the rounded portion of a small test-tube) furnished by the instructor, ${ }^{2}$ add 5 c.c. of the sugar solution, shake well and heat on a boiling water-bath for one-half to three-quarters of an hour. Allow the tube to cool slowly (not under the tap) and examine the crystals microscopically (Plate III, opposite).

If the solution has become too concentrated in the boiling process it will be light red in color and no crystals will separate until it is diluted with water.

Yellow crystalline bodies called osazones are formed from certain sugars under these conditions, in general each individual sugar giving rise to an osazone of a definite crystalline form which is typical for that sugar. It is important to remember in this connection that of the simple sugars of interest in physiological chemistry, glucose and fructose yield the same osazone. Each osazone has a definite melting-point and as a further and more accurate means of identification it may be recrystallized and identified by the determination of its melting-point and nitrogen content. The reaction taking place in the formation of phenylghucosazone is as follows:
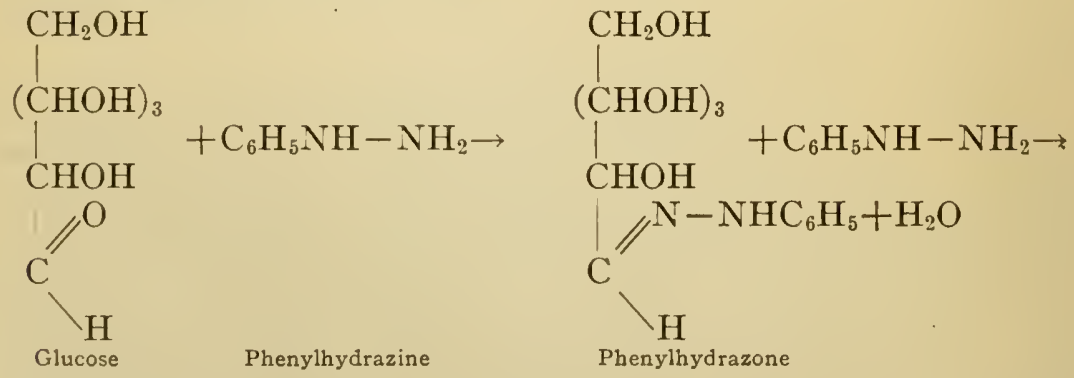

${ }^{1}$ According to v. Ekenstein and Blanksma (Ber.d.d. chem. Gesell., 43, 2358, I9 10), oxymethylfurfural is formed.

2 This mixture is prepared by combining one part of phenylhydrazine hydrochloride and two parts of sodium acetate by weight. These are thoroughly mixed in a mortar. 
PLATE III.
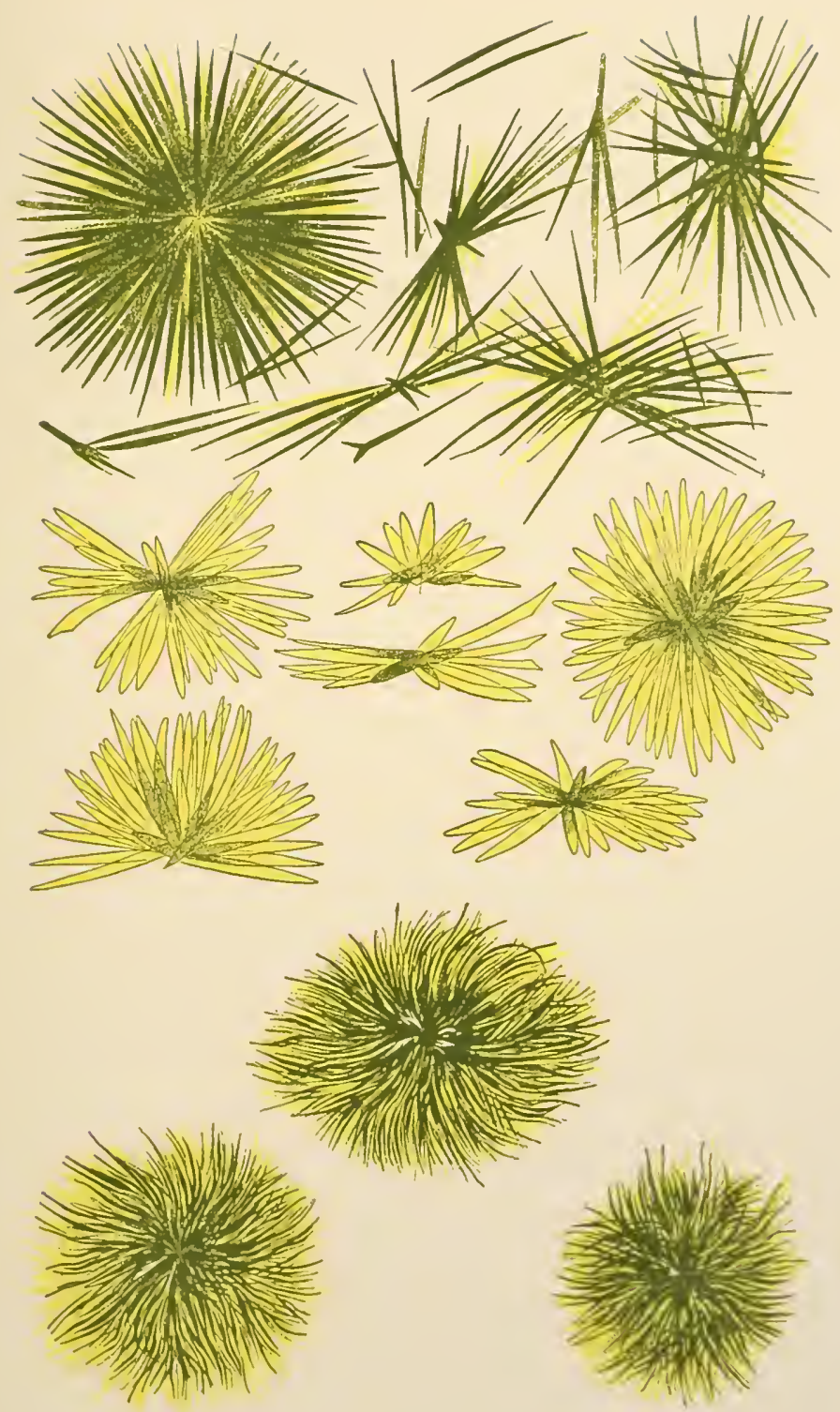

Osazoses.

Upper form, dextrosazone; central form, maltosazone; lower form, lactosazone. 



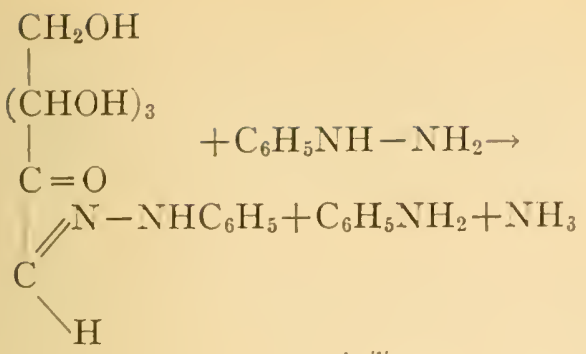

Aniline
$\mathrm{CH}_{2} \mathrm{OH}$

$(\mathrm{CHOH})_{3}$

$\mathrm{C}=\mathrm{N}-\mathrm{NHC}_{6} \mathrm{H}_{5}+\mathrm{H}_{2} \mathrm{O}$

${ }_{\mathrm{C}}^{\mathrm{N}-\mathrm{NHC}_{6} \mathrm{H}_{5}}$

$\mathrm{H}$

Glucosazone

(b) Place 5 c.c. of the sugar solution in a test-tube, add I c.c. of the phenylhydrazine-acetate solution furnished by the instructor, ${ }^{1}$ and heat on a boiling water-bath for one-half to three-quarters of an hour. Allow the liquid to cool slowly and examine the crystals microscopically (Plate III, opposite p. 22).

The phenylhydrazine test has been so modified by Cipollina as to be of use as a rapid clinical test. The directions for this test are given in the next experiment.

4. Cipollina's Test.-Thoroughly mix 4 c.c. of dextrose solution, 5 drops of phenylhydrazine (the base) and $1 / 2$ c.c. of glacial acetic acid in a test-tube. Heat the mixture for about one minute over a low flame, shaking the tube continually to prevent loss of fluid by bumping. Add $4-5$ drops of sodium hydroxide (sp. gr. I. I6), being certain that the fluid in the test-tube remains acid, heat the mixture again for a moment and then cool the contents of the tube. Ordinarily the crystals form at once, especially if the sugar solution possesses a low specific gravity. If they do not appear immediately allow the tuhe to stand at least 20 minutes before deciding upon the absence of sugar.

Examine the crystals under the microscope and compare them with those shown in Plate III, opposite page 22.

5. Riegler's Reaction. ${ }^{2}$-Introduce o.I gram of phenylhydrazine-hydrochloride and 0.25 gram of sodium acetate into a test-tube, add 20 drops of the solution under examination and heat the mixture to boiling. Now introduce ro c.c. of a 3 per cent solution of potassium hydroxide and gently shake the tube and contents. If the solution under examination contains dextrose the liquid in the tube will assume a red color.

One per cent dextrose yields an immediate color, whereas 0.05 per cent yields the color only after the lapse of a period of one-half hour from the time the alkali is added. In urinary examination if the color appears after the thirty-minute interval the color change is without significance, inasmuch as sugar-free urine will respond thus. The reaction is given by all aldehydes and therefore the test cannot be safely employed in testing urines preserved by formaldehyde. Albumin does not interfere with the test.

6. Bottu's Test. ${ }^{3}$-To 8 c.c. of Bottu's reagent ${ }^{4}$ in a test-tube add I c.c. of the

1 This solution is prepared by mixing one part by volume, in each case, of glacial acetic acid, one part of water and two parts of phenylhydrazine (the base).

${ }^{2}$ Riegler: Compt. rend. soc. biol., 66, p. 795.

3 Bottu: Compt. rend. soc. biol., 66, p. 972.

- This reagent contains 3.5 grams of 0 -nitrophenylpropiolic acid and 5 c.c. of a freshly prepared Io per cent solution of sodium hydroxide per liter. 
solution under examination and mix the liquids by gentle shaking. Now heat the upper portion of the mixture to boiling, add an additional I c.c. of the solution and heat the mixture again immediately. The appearance of a blue color accompanied by the precipitation of small particles of indigo blue indicates the presence of dextrose in the solution under examination. The test will serve to detect the presence of o.I per cent of dextrose.

7. Precipitation by Alcohol.- To ro c.c. of 95 per cent alcohol add about 2 c.c. of dextrose solution. Compare the result with that obtained under Dextrin, 5, page 48 .

8. Iodine Test.-Make the regular iodine test as given under Starch, 5, page 45, and keep this result in mind for comparison with the results obtained later with starch and with dextrin.

9. Diffusibility of Glucose.-Test the diffusibility of glucose solution through animal membrane or parchment paper, making a dialyzer like one of the models shown in Fig. 2.

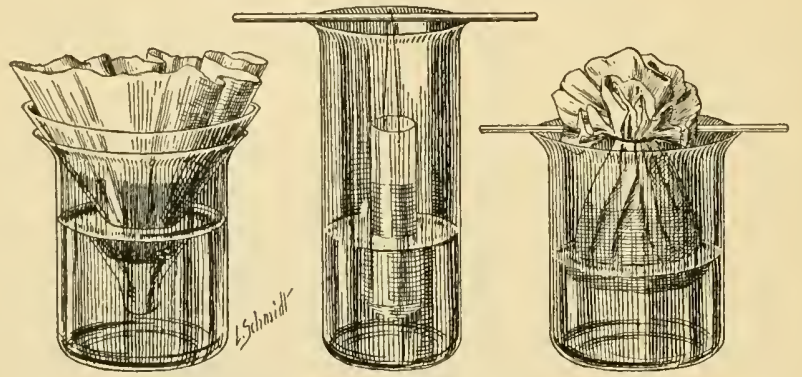

Fig. 2.-Dialyzing Apparatus for Students' Use.

A most satisfactory dialyzing bag may be made of collodion as follows: Pour a solution of collodion into a clean dry Erlenmeyer flask or test-tube. While rotating the vessel on its longitudinal axis, gradually pour out the collodion, at the same time being careful that the interior surface of the flask is completely coated with the solution. Continue the rotation in the inverted position until the collodion ceases to flow. After the solution has evaporated such that the collodion skin on the rim is dry and stiff, cut or loosen it around the edge of the rim. With a pipette or wash bottle run in a few cubic centimeters of water between the membrane and the wall of the flask or test-tube. Shake the inclined vessel while rotating on its longitudinal axis, thus detaching the membrane. Now withdraw the detached bag and fill with water, to determine whether or not it contains defects. ${ }^{1}$

All monosaccharides and disaccharides are diffusible, but many polysaccharides are not.

10. Moore's Test.-To $2-3$ c.c. of sugar solution in a test-tube add an equal volume of concentrated $\mathrm{KOH}$ or $\mathrm{NaOH}$, and boil. The solution darkens and finally assumes a brown color. At this point the odor of caramel may be detected.

This is an exceedingly crude test and is of little practical value. The alkali brings about condensation and decomposition. The brown color is due to the formation of condensation products. Among the decomposition products are the potassium or sodium salts of certain organic acids.

${ }^{1}$ Gies: Quoted by Clark. Bioch. Bull., I, 198, I911. 
II. Reduction Tests. - To their aldehyde or ketone structure many sugars owe the property of readily reducing alkaline solutions of the oxides of metals like copper, bismuth and mercury; they also possess the property of reducing ammoniacal silver solutions with the separation of metallic silver. Upon this property of reduction the most widely used tests for sugars are based. When whitish-blue cupric hydroxide in suspension in an alkaline liquid is heated it is converted into insoluble black cupric oxide, but if a reducing agent like certain sugars be present the cupric hydroxide is reduced to insoluble yellow cuprous hydroxide, which in turn. on further heating, may be converted into brownish-red or red cuprous oxide. These changes are indicated as follows:

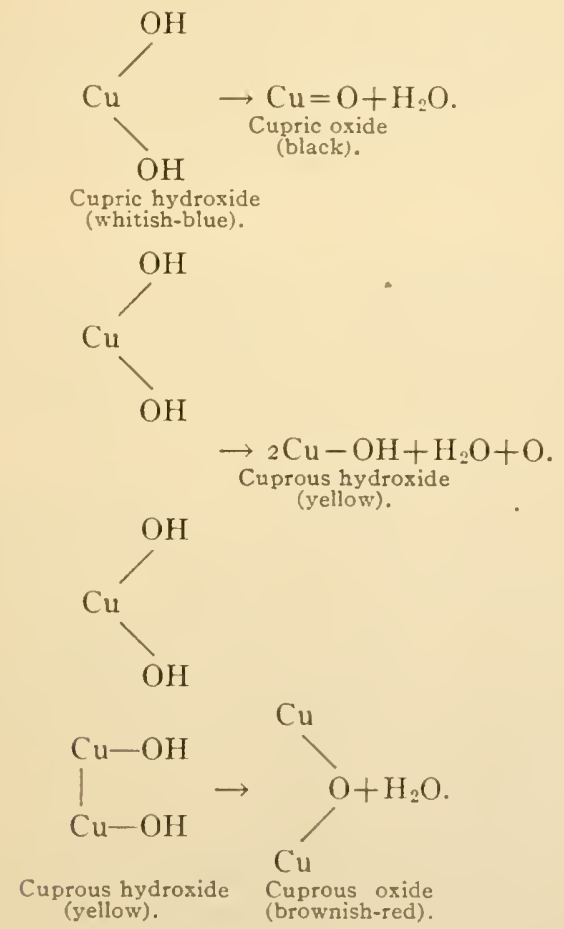

The chemical equations here discussed are exemplified in Trommer's and Fehling's tests.

(a) Trommer's Test.-To $\overrightarrow{5}$ c.c. of sugar solution in a test-tube add one-half its volume of $\mathrm{KOH}$ or $\mathrm{NaOH}$. Mix thoroughly and add, drop by drop, a cery diluli solution of copper sulphate. Continue the addition until there is a slight permanent precipitate of cupric hydroxide and in consequence the solution is slighty turbicl. Heat, and the cupric hyclroxide is reduced to yellow cuprous hydroxide or to brownish-red cuprous oxide.

If the solution of copper sulphate used is too strong a small brownish-red precipitate produced in a weak sugar solution may be entirely masked. On the other 
hand, particularly in testing for sugar in the urine, if too little copper sulphate is used a light-colored precipitate formed by uric acid and purine bases may obscure the brownish-red precipitate of cuprous oxide. The action of $\mathrm{KOH}$ or $\mathrm{NaOH}$ in the presence of an excess of sugar and insufficient copper will produce a brownish color. Phosphates of the alkaline earths may also be precipitated in the alkaline solution and be mistaken for cuprous hydroxide. Trommer's test is not very satisfactory.

Salkowski has proposed a modification of the Trommer procedure which he claims is a very accurate sugar test.

(b) Fehling's Test.-To about I c.c. of Fehling's solution ${ }^{2}$ in a test-tube add about 4 c.c. of water, and boil. ${ }^{3}$ [The cupric hydroxide is held in solution by the sodium potassium tartrate (Rochelle salt).] This is done to determine whether the solution will of itself cause the formation of a precipitate of brownish-red cuprous oxide. If such a precipitate forms, the Fehling's solution must not be used. Add sugar solution to the warm Fehling's solution a few drops at a time and heat the mixture after each addition. The production of yellow cuprous hy droxide or brownish-red cuprous oxide indicates that reduction has taken place. The yellowish precipitate is more likely to occur if the sugar solution is added rapidly and in large amount, whereas with a less rapid addition of smaller amounts of sugar solution the brownish-red precipitate is generally formed.

'This is a much more satisfactory test than Trommer's, but even this test is not entirely reliable when used to detect sugar in the urine. Such bodies as conjugate glycuronates, uric acid, mucleoprotein and homogentisic acid when present in sufficient amount may produce a result similar to that produced by sugar. Phosphates of the alkaline earths may be precipitated by the alkali of the Fehling's solution and in appearance may be mistaken for cuprous hydroxide. Cupric hydroxide may also be reduced to cuprous oxide and this in turn be dissolved by creatinine, a normal urinary constituent. This will give the urine under examination a greenish tinge and may obscure the sugar reaction even when a considerable amount of sugar is present. According to Laird, ${ }^{4}$ even small amounts of creatinine will retard the reaction velocity of reducing sugars with Fehling's solution.

In testing urine preserved by chloroform a positive test may be obtained in the absence of sugar. This is due to the fact that the hot alkali produces formic acid (a reducing fatty acid) from the chloroform.

1 Salkowski: Zeit.physiol. Chem., 79, г64, I912.

${ }^{2}$ Fehling's solution is composed of two definite solutions-a copper sulphate solution and an alkaline tartrate solution-which may be prepared as follows:

Copper sulphate solution $=34.65$ grams of copper sulphate dissolved in water and made up to 500 c.c.

Alkaline tarirate solution $=\mathrm{I} 25$ grams of potassium hydroxide and $\mathrm{I} 73$ grams of Rochelle salt dissolved in water and made up to 500 c.c.

These solutions should be preserved separately in rubber-stoppered bottles and mixed in equal volumes when needed for use. This is done to prevent deterioration.

${ }^{3}$ More dilute Fehling's solution should be used in testing very dilute sugar solutions. In case of concentrated sugar solutions it may sometimes be desirable to use a larger volume of the Fehling's solution.

${ }^{4}$ Laird: Journal of Pathology and Bacteriology, 16, 398, 191 2. 
Ammonium salts also interfere with Fehling's test. If present in excess the solution (e.g., urine) should be made alkaline and boiled in order to decompose the ammonium salts.

If the solution under examination by Fehling's test is acid in reaction it must be neutralized or made alkaline before applying the test.

(c) Benedict's Modifications of Fehling's Test.-First Modification.-To 2 c.c. of Benedict's solution ${ }^{1}$ in a test-tube add 6 c.c. of distilled water and 7-9 drops (not more) of the solution under examination. Boil the mixture vigorously for about ${ }^{15-30}$ seconds and permit it to cool to room temperature spontaneously.

If sugar is present in the solution a precipitate will form which is of ten bluish-green or green at first, especially if the percentage of sugar is low, and which usually becomes yellowish upon standing. If the sugar present exceeds 0.06 per cent this precipitate generally forms at or below the boiling-point, whereas if less than 0.06 per cent of sugar is present the precipitate forms more slowly and generally only after the solution has cooled.

Benedict claims, whereas the original Fehling test will not serve to detect sugar when present in a concentration of less than o.r per cent, that the above modification will serve to detect sugar when present in as small quantity as $0.015^{-0.02}$ per cent. Corroboration of this claim of increased delicacy has recently been submitted by Harrison. ${ }^{2}$

The modified Fehling solution used in the above test differs from the original Fehling solution in that roo grams of sodium carbonate is substituted for the I 25 grams of potassium hydroxide ordinarily used, thus forming a Fehling solution which is considerably less alkaline than the original. This alteration in the composition of the Fehling solution is of advantage in the detection of sugar in the urine inasmuch as the strong alkalinity of the ordinary Fehling solution has a tendency, when the reagent is boiled with a urine containing a small amount of dextrose, to decompose sufficient of the sugar to render the detection of the remaining portion exceedingly difficult by the usual technic. Benedict claims that for this reason the use of this modified solution permits the detection of much smaller amounts of sugar than does the use of the ordinarily Fehling solution.

\footnotetext{
${ }^{1}$ Benedict's modified Fehling solution consists of two definite solutions-a copper sulphate solution and an alkaline tartrate solution, which may be prepared as follows:

Copper sulphate solution $=34.65$ grams of copper sulphate dissolved in water and made up to 500 c.c.

Alkaline tarlrate solution $=100$ grams of anhydrous sodium carbonate and 173 grams of Rochelle salt dissolved in water and made up to 500 c.c.

These solutions should be preserved separately in rubber-stoppered bottles and mixed in equal volumes when needed for use. This is done to prevent deterioration.

2 Harrison: Pharm. Jour., 87, 746, I911.
} 
Second Modification. ${ }^{1-B e n e d i c t ~ h a s ~ f u r t h e r ~ m o d i f i e d ~ h i s ~ s o l u t i o n ~ a n d ~ h a s ~}$ succeeded in obtaining one which does not deteriorate upon long standing. ${ }^{2}$ The following is the procedure for the detection of glucose in solution: To 5 c.c. of the reagent in a test-tube add 8 (not more) drops of the solution under examination. Boil the mixture vigorously for from one to two minutes and then allow the fluid to cool spontaneously. In the presence of dextrose the entire body of the solution will be filled with a precipitate, which may be red, yellow or green in color, depending upon the amount of sugar present. If no glucose is present, the solution will remain perfectly clear. (If urine is being tested, it may show a very faint turbidity, due to precipitated urates.)

Even very small quantities of glucose (o. I per cent) yield precipitates of surprising bulk with this reagent, and the positive reaction for glucose is the filling of the entire body of the solution with a precipitate, so that the solution becomes opaque. Since amount rather than color of the precipitate is made the basis of this test, it may be applied even for the detection of small quantities of glucose, as readily in artificial light as in daylight. Chloroform does not interfere with this test nor do uric acid or creatinine interfere to such an extent as in the case of Fehling's test.

Mercuric Oxide Reduction Test (Cramer). ${ }^{3}$ - This test depends on the reduction of mercuric oxide in a weakly alkaline solution, with the formation of metallic mercury. The degree of alkalinity is an important factor, as the test becomes more sensitive but less specific the greater the alkalinity of the reagent.

For the detection of reducing sugar in aqueous solution proceed as follows: Introduce 3 c.c. of Cramer's " 2.5 reagent" into a test-tube 4 and heat to boiling. The solution remains clear, but turns slightly yellow. Add 3 c.c. of the sugar solution and again heat to boiling. Remove the tube from the flame and note that the mixture becomes turbid, darkens, and that on standing a precipitate of finely divided mercury settles to the bottom of the tube.

1 Benedict: Jour. Am. Med. Ass'n, 57, i r93, r9 г .

${ }^{2}$ Benedict's new solution has the following composition:

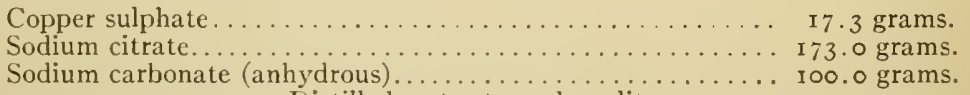

Distilled water to make 1 liter.

With the aid of heat dissolve the sodium citrate and carbonate in about $600 \mathrm{c} . \mathrm{c}$ of water. Pour (through a folded filter paper if necessary) into a glass graduate and make up to 850 c.c. Dissolve the copper sulphate in about Ioo c.c. of water and make up to I50 c.c. Pour the carbonate-citrate solution into a large beaker or casserole and add the copper sulphate solution slowly, with constant stirring. The mixed solution is ready for use and does not deteriorate upon long standing.

${ }^{3}$ Cramer: Bioch. Jour., 9, I 56, r9 5.

"Cramer's " 2.5 Reagent."- -4 gram mercuric oxide (red or yellow) and 6 grams potassium iodide are dissolved in 100 c.c. water. This solution is weakly alkaline. The alkalinity must now be adjusted so that io c.c. of the reagent are neutralized by 2.5 C.c. of $\mathrm{N}$ / Io acid, using phenolphthalein as indicator. This is done by titrating Io c.c. of the reagent with $\mathrm{N} / \mathrm{I} 0$ acid and, after the alkalinity of the reagent has thus been determined, adding the requisite a mount of $\mathrm{N}$ / Io acid or alkali to the bulk of the reagent. The reagent is a clear colorless solution which turns slightly yellow on heating and becomes colorless again on cooling. It must remain clear on boiling. 
This test is positive with such sugars as give other reduction tests, e.g., glucose, fructose, lactose, maltose, arabinose, etc. If the reducing sugar be present in aqueous solution a slight reduction may be obtained with I $\mathrm{mg}$. of glucose. If the reagent be made strongly alkaline, it ceases to be specific for reducing sugars and chemically allied substances and is reduced by other organic substances, e.g., creatinine.

It is claimed that this test is free from fallacies inherent in Fehling's test as the result of the reducing action of uric acid and creatinine.

The test is more sensitive than the Fehling's or Nylander's reactions, and is particularly suitable for the examination of urines in which the amount of sugar present exceeds the normal amount only slightly.

(e) Bismuth Reduction Test (Boettger).-To 5 c.c. of sugar solution in a test-tube add I c.c. of $\mathrm{KOH}$ or $\mathrm{NaOH}$ and a very small amount of bismuth subnitrate, and boil. The solution will gradually darken and finally assume a black color due to reduced bismuth. If the test is made on urine containing albumin this must be removed, by boiling and filtering, before applying the test, since with albumin a similar change of color is produced (bismuth sulphide).

(f) Bismuth Reduction Test (Nylander).-To 5 c.c. of sugar solution in a testtube add one-tenth its volume of Nylander's reagent ${ }^{1}$ and heat for five minutes in a boiling water-bath. ${ }^{2}$ The solution will darken if reducing sugar is present, and upon standing for a few moments a black color will appear.

This color is due to the precipitation of bismuth. If the test is made on urine containing albumin this must be removed, by boiling and filtering, before applying the test. since with albumin a similar change of color is produced. Glucose when present to the extent of 0.08 per cent may be easily detected by this reaction $\left(\mathrm{Rabe}^{3}\right.$ claims that o.or per cent sugar may be so detected). Uric acid and creatinine which interfere with the Fehling's test do not interfere with the Nylander test. It is claimed by Bechold that the bismuth reduction tests give a negative reaction with solutions containing sugar when mercuric chloride or chloroform is present. Other observers ${ }^{4}$ have failed to verify the inhibitory action of mercuric chloride and have shown that the inhibitory influence of chloroform may be overcome by raising the temperature of the urine to the boiling-point for a period of five minutes previous to making the test. Urines rich in indican, urochrome, uroerythrin or hematoporphyrin, as well as urines excreted after the ingestion of large amounts of certain medicinal substances, may give a darkening of

1 Nylander's reagent is prepared by digesting 2 grams of bismuth subnitrate and 4 grams of Rochelle salt in 100 c.c. of a ro per cent potassium hydroxide solution. The reagent is then cooled and filtered.

${ }^{2}$ Hammarsten suggests that the mixture should be boiled 2-5 minutes (according to the sugar content) over a free flame and the tube then permitted to stand 5 minutes before drawing conclusions.

${ }^{3}$ Rabe: A polh. Zlg., 29, 554, i 14.

4 Rehfuss and Hawk: Journal of Biological Chemistry; 7.207 , 1010; also Zeidlitz: Épsala Lakürforen Forh., N. F., in, igo6. 
Nylander's reagent similar to that of a true sugar reaction. It is a disputed point whether the urine after the administration of urotropin will reduce Nylander's reagent. ${ }^{1}$ Strausz ${ }^{2}$ has recently shown that the urine of diabetics to whom "Iothion" (diiodohydroxypropane) has been administered will give a negative Nylander-Almén reaction and respond positively to the Fehling and polariscopic tests. "Iothion" also interferes with the Nylander-Almén test in vitro whereas KI and I do not.

According to Rustin and Otto, the addition of $\mathrm{PtCl}_{4}$ increases the delicacy of Nylander-Almén reaction. They claim that this procedure causes the sugar to be converted quantitatively. No quantitative method has yet been devised, however, based upon this principle.

Bohmansson ${ }^{3}$ before testing the urine under examination treats it (IO c.c.) with $1 / 5$ volume of 25 per cent hydrochloric acid and about $1 / 2$ volume of boneblack. This mixture is shaken one minute; then filtered and the neutralized filtrate tested by Nylander-Almén reaction. Bohmansson claims that this procedure removes certain interfering substances, in particular urochrome.

A positive bismuth reduction test is probably due to the following reactions:

(a) $\mathrm{Bi}(\mathrm{OH})_{2} \mathrm{NO}_{3}+\mathrm{KOH} \rightarrow \mathrm{Bi}(\mathrm{OH})_{3}+\mathrm{KNO}_{3}$.

(b) $\quad 2 \mathrm{Bi}(\mathrm{OH})_{3}-3 \mathrm{O} \rightarrow \mathrm{Bi}_{2}+{ }_{3} \mathrm{H}_{2} \mathrm{O}$.

(g). Barfoed's Test.-Place about 5 c.c. of Barfoed's solution ${ }^{4}$ in a test-tube and heat to boiling. Add glucose solution slowly, a few drops at a time, heating after each addition. Reduction is indicated by the formation of a red precipitate of cuprous oxide. If the precipitate does not form upon continued boiling allow the tube to stand a few minutes and examine.

According to Welker ${ }^{5}$ chlorides interfere pronouncedly with the reaction causing the formation of a green precipitate.

Barfoed's test is not a specific test for glucose as is frequently stated, but simply serves to detect monosaccharides. Disaccharides will also respond to the test, under proper conditions of acidity. ${ }^{6}$ Also if the sugar solution is boiled sufficiently long, in contact with the reagent, to hydrolyze the disaccharide through the action of the acetic acid present in the Barfoed's solution a positive test results. ${ }^{7}$ Barfoed's is a copper

\footnotetext{
1 Abt: Archives of Pcdiatrics, 24, 275, I907; also Weitbrecht: Schweiz. Wochschr., 47, 577, Ig09.

2 Strausz: Münch. med. Woch., 59, 85, 1912.

${ }^{3}$ Bohmansson: Biochem. Zeit., I9, p. 28I.

${ }^{4}$ Barfoed's solution is prepared as follows: Dissolve 4.5 grams of neutral crystallized copper acetate in 100 c.c. of water and add I.2 c.c. of 50 per cent acetic acid. This solution should be freshly made.

${ }^{5}$ Welker: Jour. Am. Chem. Soc., 37, 2227, 1915.

${ }^{6}$ Mathews and McGuigan: Am. Jour. Physiol., 19, 1 75, 1907.

${ }^{7}$ Hinkle and Sherman: Jour. Am. Chem. Soc., 29, 1744, 1907.
} 
reduction test, but differs from Fehling's and other reduction tests in that the reduction is brought about in an acid solution. It is unsuited for the detection of sugar in urine.

I2. Fermentation Test. - "Rub up" in a mortar about 20 c.c. of the sugar solution with a small piece of compressed yeast. Transfer the mixture to a saccharometer (shown in Fig. 3) and stand it aside in a warm place for about twelve hours. If the sugar is fermentable, alcoholic fermentation will occur and carbon dioxide will collect as a gas in the upper portion of the tube (see Fig. 4). On the completion of fermentation introduce a little potassium hydroxide solution into the graduated portion by means of a bent pipette, place the thumb tightly over the opening in the apparatus and invert the saccharometer. $\mathrm{Re}-$

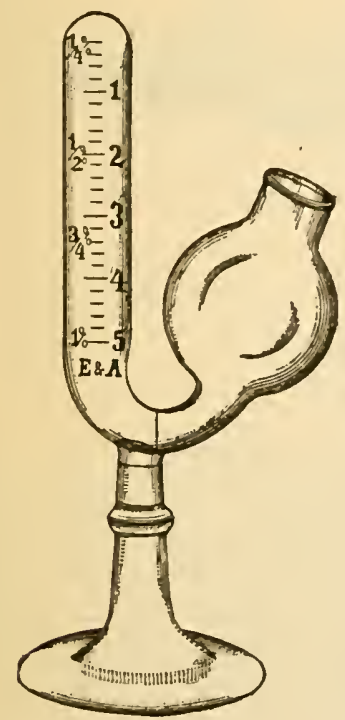

Fig. 3.-Einhorn Saccharometer. membering that $\mathrm{KOH}$ has the power to absorb $\mathrm{CO}_{2}$ how do you explain the result? ${ }^{1}$

13. Formation of Caramel.-Gently heat a small amount of pulverized dextrose in a test-tube. After the sugar has melted and turned brown, allow the tube to cool, add water and warm. The coloring matter produced is known as caramel.

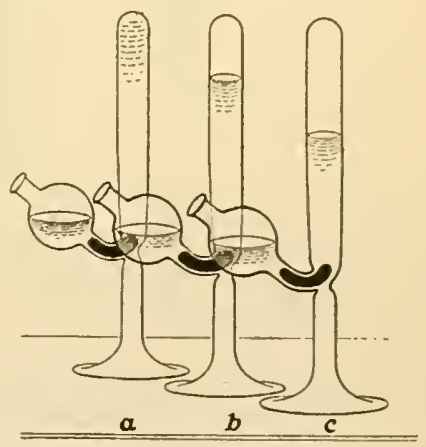

Fig. 4.-Illustrating Different Stages in Fermextation.

14. Demonstration of Optical Activity.-A demonstration of the use of the polariscope, by the instructor, each student being required to take readings and compute the "specific rotation."

\section{USE OF THE Polariscope}

For a detailed description of the different forms of polariscopes, the method of manipulation and the principles involved, the student is referred to any standard text-book of physics. A brief description follows: An ordinary ray of light vibrates in every direction. If such a

1 The findings of Neuberg and associates ${ }^{2}$ indicate that the liberation of carbon dioxide by yeast is not necessarily a criterion of the presence of sugar. The presence of an enzyme called carboxylase has been demonstrated in yeast which has the power of splitting off $\mathrm{CO}_{2}$ from the carboxyl group of amino- and other aliphatic acids.

2 Neuberg and Associates: Biochem. Zcilsch., 31, r;0; 32, 323; 36, $(60,68,-6)$, I9I. 
ray is caused to pass through a "polarizing" Nicol prism it is resolved into two rays, one of which vibrates in every direction as before and a second ray which vibrates in one plane only. This latter ray is said to be polarized. Many organic substances (sugar, proteins, etc.) have the power of twisting or rotating this plane of polarized light, the extent to which the plane is rotated depending upon the number of molecules which the polarized light passes. Substances which possess this power are said to be "optically active." The specific rotation of a substance is the rotation expressed in degrees which is afforded by I gram of sub-

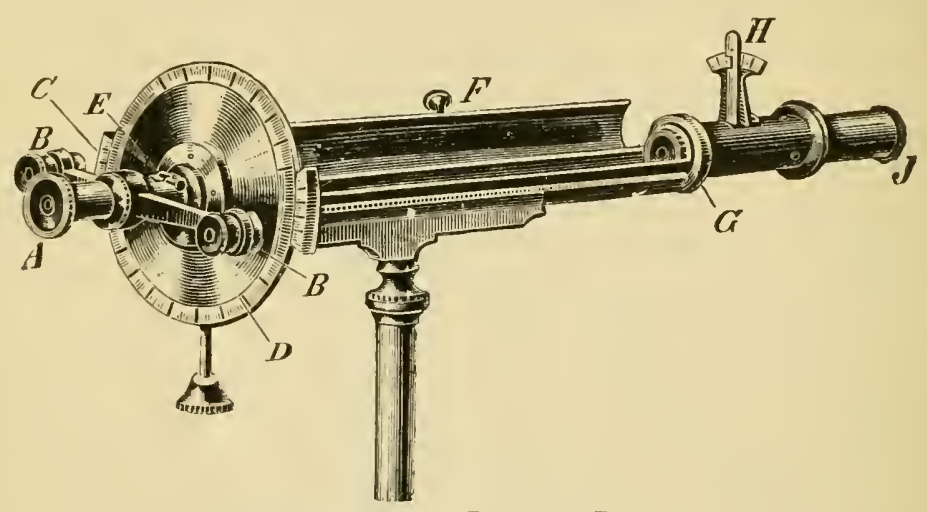

Fig. 5.- One Form of Laurent Polariscope.

$B$, Microscope for reading the scale; $C$, a vernier; $E$, position of the analyzing Nicol prism; II, polarizing Nicol prism in the tube below this point.

stance dissolved in I c.c. of water in a tube one decimeter in length. The specific rotation, $(\alpha)_{\mathrm{D}}$, may be calculated by means of the following formula:

$$
(\alpha)_{D}=\frac{\alpha}{p \cdot l},
$$

in which

$D=$ sodium light.

$\alpha=$ observed rotation in degrees.

$p=$ grams of substance dissolved in I c.c. of liquid.

$l=$ length of the tube in decimeters.

If the specific rotation has been determined and it is desired to ascertain the per cent of the substance in solution, this may be obtained by the use of the following formula,

$$
p=\frac{\alpha}{(a)_{D} l}
$$

The value of $p$ multiplied by roo will be the percentage of the substance in solution. 
SPECIFIC ROTATIONS OF MORE COMMON CARBOHYDRATES I

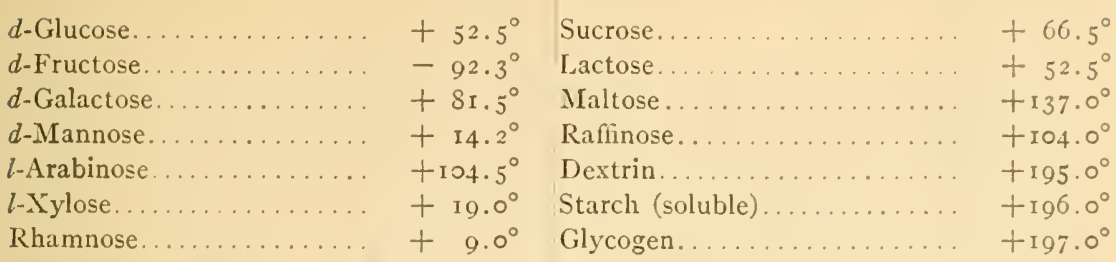

An instrument by means of which the extent of the rotation may be determined is called a polariscope or polarimeter. Such an instrument designed especially for the examination of sugar solutions is termed a saccharimeter or polarizing saccharimeter. The form of polariscope in Fig. 5, page 32, consists essentially of a long barrel provided with a Nicol prism at either end (Fig. 6,). The solution under examination is contained in a tube which is placed between these two prisms.

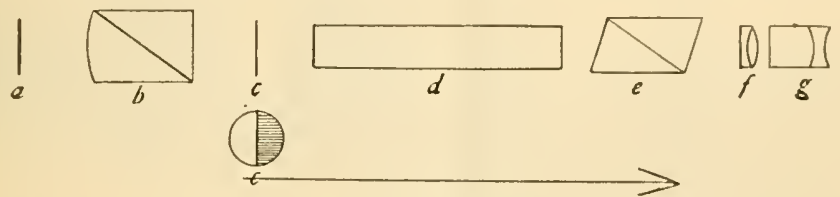

Fic. 6.-Diagramatic Representation of the Covrse of the Light throlgh the

L.IUREAT POLARISCOPE. (The direction is reversed from that of Fig. 5, p. 32.)

$a$, Bichromate plate to purify the light; $b$, the polarizing Nicol prism; $c$, a thin quartz plate covering one-half the field and essential in producing a second polarized plane; $d$, tube to contain the liquid under examination; $e$, the analyzing Nicol prism; $f$ and $g$, ocular lenses.

At the front end of the instrument is an adjusting eyepiece for focusing and a large recording disc which registers in degrees and fractions of a degree. The light is admitted into the far end of the instrument and is polarized by passing through a Nicol prism. This polarized ray then traverses the column of liquid within the tube mentioned above and if the substance is optically active the plane of the polarized ray is rotated to the right or left. Bodies rotating the ray to the right are called dextro-rotatory and those rotating it to the left levo-rotatory.

Within the apparatus is a disc which is so arranged as to be without lines and uniformly light at zero. Upon placing the optically active substance in position, however, the plane of polarized light is rotated or turned and it is necessary to rotate the disc through a certain number of degree in order to secure the normal conditions, i.c., "without lines

1 The specific rotation varies with the temperature and concentration of the solution. The figures here given are for concentrations of about ro per cent and temperatures of about $20^{\circ} \mathrm{C}$. Fresh solutions may give markedly different values due to mutarotation, the figures here given representing the constant values obtained on standing. 
and uniformly light." The difference between this reading and the zero is $\alpha$ or the observed rotation in degrees.

Polarizing saccharimeters are also constructed by which the percentage of sugar in solution is determined by making an observation and multiplying the value of each division on a horizontal sliding scale by the value of the division expressed in terms of dextrose. This factor may vary according to the instrument.

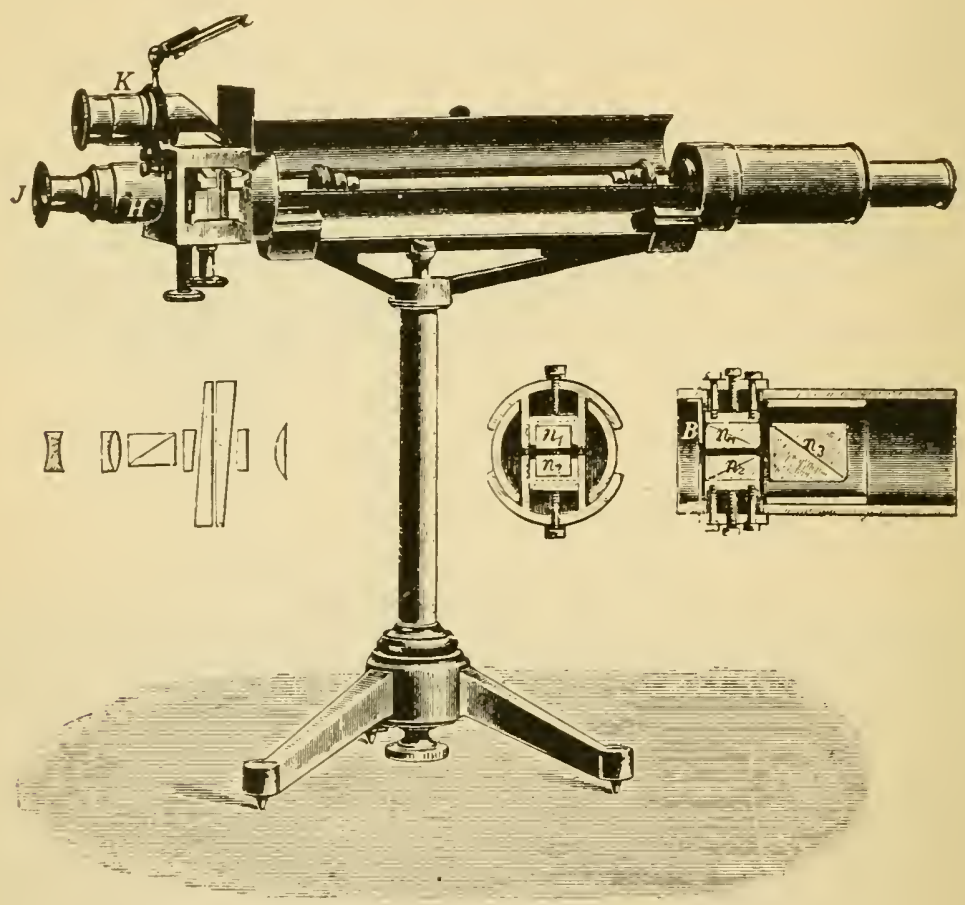

Fig. 7-Polariscope (Schmidt and Hänsch Model).

"Optical" methods embracing the determination of the optical activity are being utilized in recent years in many "quantitative"

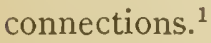

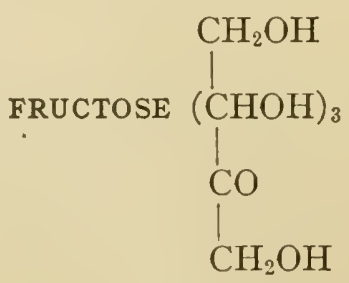

${ }^{1}$ Abderhalden and Schmidt: "Determination of blood content by means of the optical method," Zeit. physiol. Chem.,66, I20, I910; also C. Neuberg: "Determination of nucleic acid cleavage by polarization," Biochemische Zeitschrift, 30, 505, I9I I. 
As already stated, fructose, sometimes called levulose or fruit sugar, occurs widely disseminated throughout the plant kingdom in company with glucose. Its reducing power is somewhat weaker than that of dextrose. Fructose does not ordinarily occur in the urine in diabetes mellitus, but has been found in exceptional cases. With phenylhydrazine it forms the same osazone as glucose. With methylphenylhydrazine, levulose forms a characteristic methylphenylfructosazone.

(For a further discussion of fructose see the section on Hexoses, page 20.)

\section{EXPERIMENTS ON FRUCTOSE}

I-6. Repeat Solubility, Fehling's, Phenylhydrazine, Barfoed's, Nylander's, and Fermentation tests as given under Glucose, pages 2I-3I.

7. Resorcinol-Hydrochloric Acid Reaction (Seliwanoff).-To 5 c.c. of Seliwanoff's reagent ${ }^{1}$ in a test-tube add a few drops of a fructose solution and heat the mixture to boiling. A positive reaction is indicated by the production of a red color and the separation of a brown-red precipitate. The latter may be dissolved in alcohol to which it will impart a striking red color.

If the boiling be prolonged a similar reaction may be obtained with solutions of glucose or maltose. This has been explained ${ }^{2}$ in the case of glucose as due to the transformation of the glucose into fructose by the catalytic action of the hydrochloric acid. The precautions necessary for a positive test for levulose are as follows: The concentration of the hydrochloric acid must not be more than $\mathrm{r} 2$ per cent. The reaction (red color) and the precipitate must be observed after not more than 20-30 seconds boiling. Glucose must not be present in amounts exceeding 2 per cent. The precipitate must be soluble in alcohol with a bright red color.

8. Borchardt's Reaction.--To about 5 c.c. of a solution of fructose in a testtube add an equal volume of 25 per cent hydrochloric acid and a few crystals of resorcinol. Heat to boiling and after the production of a red color, cool the tube under running water and transfer to an evaporating dish or beaker. Make the mixture slightly alkaline with solid potassium hydroxide, return it to a test-tube, add $2-3$ c.c. of acetic ether and shake the tube vigorously. In the presence of levulose, the acetic ether is colored yellow. (For further discussion of the test see Chapter XXIII.)

9. Formation of Methylphenylfructosazone.-To a solution of I.S grams of levulose in Io c.c. of water add 4 grams $^{3}$ of methylphenylhydrazine and enough alcohol to clarify the solution. Introduce 4 c.c. of 50 per cent acetic acid and heat the mixture for 5-10 minutes on a boiling water-bath. ${ }^{4}$ On standing I $_{5}$ minutes

${ }^{1}$ Seliwanoff's reagent may be prepared by dissolving 0.05 gram of resorcinol in 100 c.c. of dilute $(\mathrm{r}: 2)$ hydrochloric acid.

2 Koenigsfeld: Bioch. Zeil., 3S, 3 II, rg1 2.

${ }^{3} 3.66$ grams if absolutely pure.

Longer heating is to be avoided. 
at room temperature, crystallization begins and is complete in two hours. By scratching the sides of the flask or by inoculation, the solution quickly congeals to form a thick paste of reddish-yellow silky needles. These are the crystals of methylphenylfructosazone. They may be recrystallized from hot 95 per cent alcohol and melt at $533^{\circ} \mathrm{C}$.

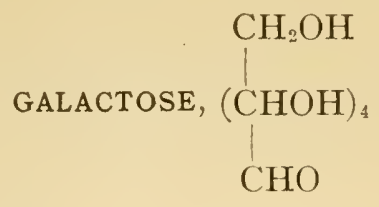

Galactose occurs with glucose as one of the products of the hydrolysis of lactose. It is dextro-rotatory, forms an osazone with phenylhydrazine and ferments slowly with yeast. Upon oxidation with nitric acid galactose yields mucic acid, thus differentiating this monosaccharide from glucose and fructose. Lactose also yields mucic acid under these conditions. The mucic acid test may be used in urine examination to differentiate lactose and galactose from other reducing sugars.

\section{Experiments on Galactose}

I. Phloroglucinol-Hydrochloric Acid Reaction (Tollens).-To equal volumes of galactose solution and hydrochloric acid (sp. gr. 1.09) add a little phloroglucinol, and heat the mixture on a boiling water-bath. Galactose, pentose and glycuronic acid will be indicated by the appearance of a red color. Galactose may be differentiated from the two latter substances in that its solutions exhibit no absorption bands upon spectroscopical examination.

2. Mucic Acid Test.-Treat roo c.c. of the solution containing galactose with 20 c.c. of concentrated nitric acid (sp. gr. I.4) and evaporate the mixture in a broad, shallow glass vessel on a boiling water-bath until the volume of the mixture has been reduced to about 20 c.c. At this point the fluid should be clear, and a fine white precipitate of mucic acid should form.

If the percentage of galactose present is low it may be necessary to cool the solution and permit it to stand for some time before the precipitate will form. It is impossible to differentiate between galactose and lactose by this test, but the reaction serves to differentiate these two sugars from all other reducing sugars. Differentiate lactose from galactose by means of Barfoed's test (page 30).

3. Phenylhydrazine Reaction. - Make the test according to directions given under Glucose, 3 or 4 , pages 22 and 23 .

\section{Pentoses, $\mathrm{C}_{5} \mathrm{H}_{10} \mathrm{O}_{5}$}

In plants, and more particularly in certain gums, very complex carbohydrates, called pentosans (see page 50), occur. These pentosans 
through hydrolysis by acids may be transformed into pentoses. Pentoses do not ordinarily occur in the animal organism, but have been found in the urine of morphine habitués and others, their occurrence sometimes being a persistent condition without known cause. They may be obtained from the hydrolysis of nucleoproteins being present in the nucleic acid complex of the molecule. Pentoses are non-fermentable, have strong reducing power and form osazones with phenylhydrazine. Pentoses are an important constituent of the dictary of herbivorous animals. Glycogen is said to be formed after the ingestion of these sugars containing five oxygen atoms. This, however, has not been conclusively proven. On distillation with strong hydrochloric acid pentoses and pentosans yield furfurol, which can be detected by its characteristic red reaction with aniline-acetate paper.

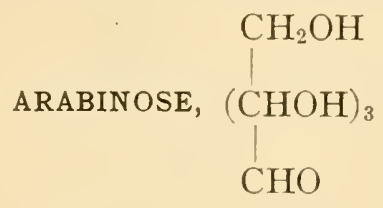

Arabinose is one of the most important of the pentoses. The l-arabinose may be obtained from gum arabic, plum or cherry gum by boiling for ro minutes with concentrated hydrochloric acid. This pentose is dextro-rotatory, forms an osazone and has reducing power, but does not ferment. The $i$-arabinose has been isolated from the urine and yiclds an osazone which melts at $166^{\circ}-168^{\circ} \mathrm{C}$.

\section{ExPERIMENTS ON ARABINOSE}

I. Orcinol-Hydrochloric Acid Reaction (Bial). ${ }^{1-}-$ To 5 c.c. of Bial's reagent ${ }^{2}$ in a test-tube add 2-3 c.c. of the arabinose solution and heat the mixture gently until the first bubbles rise to the surface. Immediately or upon cooling the solution becomes green and a flocculent precipitate of the same color may form. (For further discussion see Chapter XXIII.) The test may also be performed by adding the pentose to the hot reagent.

It is claimed that this test is more delicate than the original orcinol test (see 3) and more accurate, since menthol, kreosotal, ctc., respond to the original orcinol test but not to Bial's. Sachs ${ }^{3}$ has offered suggestions as to modification of the test in order to aroid confusion with glycuronic acid.

${ }^{1}$ Bial: Dcul. med. Woch., 28, 252, 1002, and Berl. klin. Hoch.. No. 18, 1903.

2 Orcinol. . I. 5 gram.

Fuming $\mathrm{HICl}$ 500 grams.

Ferric chloride (ro per cent) $20-30$ drops

${ }^{3}$ Sachs: Bioch. Zcil., $\mathrm{x}, 3 \mathrm{~s}_{3}, \mathrm{1} 906$, and 2,245 , r 906. 
2. Phloroglucinol-Hydrochloric Acid Reaction (Tollens).-To equal volumes of arabinose solution and hydrochloric acid (sp. gr. I.09) add a little phloroglucinol and heat the mixture on a boiling water-bath. Galactose, pentose or glycuronic acid will be indicated by the appearance of a red color. To differentiate between these bodies make a spectroscopic examination and look for the absorption band between $D$ and $E$ given by pentoses and glycuronic acid. Differentiate between the two latter bodies by the melting-points of their osazones.

Compare the reaction with that obtained with galactose (page 36 ).

3. Orcinol Test.-Repeat 2, using orcinol instead of phloroglucinol. A succession of colors from red through reddish blue to green is produced. A green precipitate is formed which is soluble in amyl alcohol and has absorption bands between $C$ and $D$.

4. Phenylhydrazine Reaction.-Make this test on the arabinose solution according to directions given under Glucose, 3 or 4, pages 22 and 23.

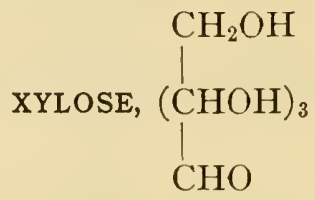

Xylose, or wood sugar, is obtained by boiling wood gums with dilute acids as explained under Arabinose, page 37. It is dextro-rotatory, forms an osazone and has reducing power, but does not ferment.

\section{Experiments on Xylose}

I-4. Same as for arabinose (see above).

\section{RHAMNOSE, $\mathrm{C}_{6} \mathrm{H}_{12} \mathrm{O}_{5}$}

Rhamnose or methyl-pentose is an example of a true carbohydrate which does not have the $\mathrm{H}$ and $\mathrm{O}$ atoms present in the proportion to form water. Its formula is $\mathrm{C}_{6} \mathrm{H}_{12} \mathrm{O}_{5}$. It has been found that rhamnose when ingested by rabbits or hens has a positive influence upon the formation of glycogen in those organisms.

\section{DISACCHARIDES, $\mathrm{C}_{12} \mathrm{H}_{22} \mathrm{O}_{11}$}

The disaccharides as a class may be divided into two rather distinct groups. The first group would include those disaccharides which are found in nature as such, e.g., sucrose and lactose, and the second group would include those disaccharides formed in the hydrolysis of more complex carbohydrates, e.g., maltose and iso-maltose.

The disaccharides have the general formula $\mathrm{C}_{12} \mathrm{H}_{22} \mathrm{O}_{11}$, to which, in the process of hydrolysis, a molecule of water is added causing the 
single disaccharide molecule to split into two monosaccharide (hexose) molecules. The products of the hydrolysis of the more common disaccharides are as follows:

\section{Maltose $=$ glucose + glucose . \\ Lactose $=$ glucose + galactose. \\ Sucrose $=$ glucose + fructose.}

All of the more common disaccharides except sucrose have the power of reducing certain metallic oxides in alkaline solution, notably those of copper and bismuth. This reducing power is due to the presence of the aldehyde group ( $-\mathrm{CHO})$ in the sugar molecule.

\section{MALTOSE, $\mathrm{C}_{12} \mathrm{H}_{22} \mathrm{O}_{11}$}

Maltose or malt sugar is formed in the hydrolysis of starch through the action of an enzyme, vegetable amylase (diastase), contained in sprouting barley or malt. Certain enzymes in the saliva and in the pancreatic juice may also cause a similar hydrolysis. Maltose is also an intermediate product of the action of dilute mineral acids upon starch. It is strongly dextro-rotatory, reduces metallic oxides in alkaline solution and is fermentable by yeast after being inverted (see Polysaccharides, page 43) by the enzyme maltase of the yeast. In common with the other disaccharides, maltose may be hydrolyzed with the formation of two molecules of monosaccharide. In this instance the products are two molecules of glucose. With phenylhydrazine maltose forms an osazone, maltosazone. The following formula represents the probable structure of maltose:

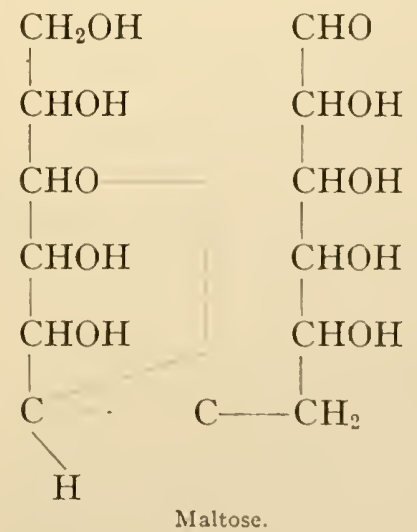

EXPERIMENTS ON MALTOSE

I-6. Repeat Solubility, Fehling's, Nylander's, Phenylhydrazine, Barfoed's and Fermentation tests as given under Glucose, pages 2I-3I. 


\section{ISO-MALTOSE, $\mathrm{C}_{12} \mathrm{H}_{22} \mathrm{O}_{11}$}

Iso-maltose, an isomeric form of maltose, is formed, along with maltose by the action of diastase upon starch paste, and also by the action of hydrochloric acid upon glucose. It also occurs with maltose as one of the products of salivary digestion. It is dextro-rotatory and with phenylhydrazine gives an osazone which is characteristic. Iso-maltose is very soluble and reduces the oxides of bismuth and copper in alkaline solution. Pure iso-maltose is probably only slightly fermentable.

\section{LACTOSE, $\mathrm{C}_{12} \mathrm{H}_{22} \mathrm{O}_{11}$}

Lactose or milk sugar occurs ordinarily only in milk, but has often been found in the urine of women during pregnancy and lactation. It may also occur in the urine of normal persons after the ingestion of unusually large amounts of lactose in the food. It has a strong reducing power, is dextro-rotatory and forms an osazone with phenylhydrazine. Upon hydrolysis lactose yields one molecule of glucose and one molecule of galactose.

In the souring of milk the bacterium lactis and certain other microorganisms bring about lactic acid fermentation by transforming the lactose of the milk into lactic acid,

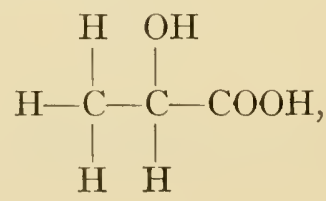

and alcohol. This same reaction may occur in the alimentary canal as the result of the action of putrefactive bacteria. In the preparation of kephyr and koumyss the lactose of the milk undergoes alcoholic fermentation, through the action of ferments other than yeast, and at the same time lactic acid is produced. Lactose and galactose yield mucic acid on oxidation with nitric acid. This fact is made use of in urine analysis to facilitate the differentiation of these sugars from other reducing sugars.

Lactose is not fermentable by pure yeast.

\section{EXPERIMENTS ON LACTOSE}

I-6. Repeat Solubility, Fehling's, Phenylhydrazine, Barfoed's, Nylander's and Fermentation tests as given under Glucose, pages 2I-3I.

7. Mucic Acid Test.-Treat roo c.c. of the solution containing lactose with 20 c.c. of concentrated nitric acid (sp. gr. I.4) and evaporate the mixture in a broad, shallow glass vessel on a boiling water-bath, until the volume of the mixture has been reduced to about 20 c.c. At this point the fluid should be clear, and a fine white precipitate of mucic acid should form. 
If the percentage of lactose present is low it may be necessary to cool the solution and permit it to stand for some time before the precipitate will appear. It is impossible to differentiate between lactose and galactose by this test, but the reaction serves to differentiate these two sugars from all other reducing sugars.

Differentiate lactose from galactose by means of Barfoed's test, page 30.

\section{SUCROSE, $\mathrm{C}_{12} \mathrm{H}_{22} \mathrm{O}_{11}$}

Sucrose, also called saccharose or cane sugar, is one of the most important of the sugars and occurs very extensively distributed in plants, particularly in the sugar cane, sugar beet, sugar millet and in certain palms and maples.

Sucrose is dextro-rotatory and upon hydrolysis, as before mentioned, the molecule of sucrose takes on a molecule of water and breaks down in to two molecules of monosaccharide. The monosaccharides formed in this instance are glucose and fructose. This is the reaction:

$$
\underset{\text { Sucrose }}{\mathrm{C}_{12} \mathrm{H}_{22} \mathrm{O}_{11}}+\mathrm{H}_{2} \mathrm{C} \rightarrow \underset{\text { Glucose }}{\mathrm{C}_{6} \mathrm{H}_{12} \mathrm{O}_{6}}+\underset{\text { Fructose }}{\mathrm{C}_{6} \mathrm{H}_{12} \mathrm{O}_{6}} \text {. }
$$

This process is called inversion and may be produced by bacteria, enzymes, and certain weak acids. After this inversion the previously strongly dextro-rotatory solution becomes levo-rotatory. This is due to the fact that the fructose molecule is more strongly levo-rotatory than the glucose molecule is dextro-rotatory. The product of this inversion is called invert sugar.

Sucrose does not reduce metallic oxides in alkaline solution and forms no osazone with phenylhydrazine. It is not fermentable directly by yeast, but must first be inverted by the enzyme sucrase (inverlase or invertin) contained in the yeast. The probable structure of sucrose may be represented by the following formula. Note the absence of any free ketone or aldehyde group.

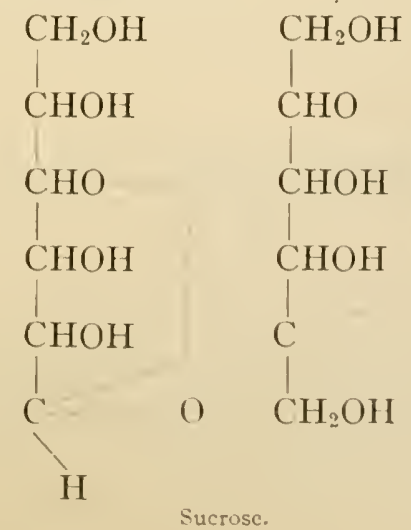




\section{EXPERIMENTS ON SUCROSE}

I-6. Repeat Solubility, Fehling's, Nylander's, Barfoed's, Phenylhydrazine and Fermentation tests according to the directions given under Glucose, pages $2 \mathrm{I}-3 \mathrm{I}$.

7. Inversion of Sucrose.-To 25 c.c. of sucrose solution in a beaker add 5 drops of concentrated $\mathrm{H}_{2} \mathrm{SO}_{4}$ and boil one minute. Cool the solution and render neutral with saturated barium hydroxide. Filter off the precipitate of basium sulphate and upon the resulting fluid repeat the phenylhydrazine, Fehling, Nylander's and Barfoed's reactions as given under Glucose, pp. 26, 29 and 30; the Resorcinol-Hydrochloric Acid Reaction (Seliwanoff), as given under Fructose, page 35. Explain the results.

8. Alcoholic Fermentation.-Prepare 500 c.c. of a concentrated (ro-20 per cent) solution of sucrose, add a small amount of egg albumin or commercial

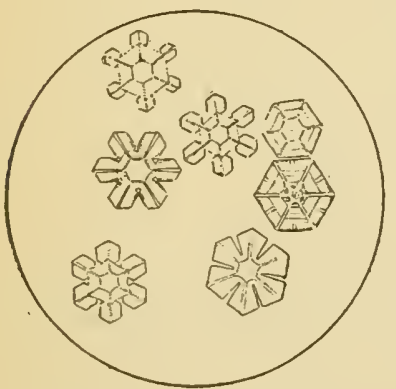

FIG. 8.-IODOFORM. (Autenrieth.) peptone and introduce the mixture into a liter flask. Add yeast, and by means of a bent tube connect this flask with a second flask containing a solution of barium hydroxide protected from the air by a sodalime tube in the stopper. Place the flasks in a warm place and note the passage of gas bubbles into the barium hydroxide solution. As these gas bubbles $\left(\mathrm{CO}_{2}\right)$ enter, a white precipitate of barium carbonate will form. The sucrase has been changed to glucose and fructose and these sugars have been fermented according to the following equation:

$$
\mathrm{C}_{6} \mathrm{H}_{12} \mathrm{O}_{6} \rightarrow 2 \mathrm{C}_{2} \mathrm{H}_{5} \mathrm{OH}+\mathrm{CO}_{2}
$$

When the activity of yeast has practically ceased, connect the fermentation flask with a condenser and distil the mixture. Catch the first portion of the distillate separately and test for alcohol by one of the following reactions:

(a) Iodoform Test.-Render 2-3 c.c. of the distillate alkaline wtih potassium hydroxide solution and add a few drops of iodine solution. Heat gently and note the formation of iodoform crystals. Examine these crystals under the microscope and compare them with those in Fig. 8.

(b) Aldehyde Test.-Place 5 c.c. of the distillate in a test-tube, add a few drops of potassium dichromate solution, $\mathrm{K}_{2} \mathrm{Cr}_{2} \mathrm{O}_{7}$, and render it acid with dilute sulphuric acid. Boil the acid solution and note the odor of aldehyde changing to that of acetic acid.

\section{TRISACCHARIDES, $\mathrm{C}_{18} \mathrm{H}_{32} \mathrm{O}_{16}$}

\section{RAFFINOSE}

This trisaccharide, also called melitose, or melitriose occurs in cotton seed, Australian manna, and in the molasses from the preparation of beet sugar. It is dextro-rotatory, does not reduce Fehling's solution, and is only partly fermentable by yeast.

Raffinose may be hydrolyzed by weak acids the same as the poly- 
saccharides are hydrolyzed, the products being fructose and melibiose; further hydrolysis of the melibiose yields glucose and galactose.

\section{POLYSACCHARIDES, $\left(\mathrm{C}_{6} \mathrm{H}_{10} \mathrm{O}_{5}\right)_{x}$}

In general the polysaccharides are amorphous bodies, a few, however, are crystallizable. Through the action of certain enzymes or weak acids the polysaccharides may be hydrolyzed with the formation of monosaccharides. As a class the polysaccharides are quite insoluble and are non-fermentable until inverted. By inversion is meant the hydrolysis of disaccharide or polysaccharide sugars to form monosaccharides, as indicated in the following equations:

$$
\begin{gathered}
\mathrm{C}_{12} \mathrm{H}_{22} \mathrm{O}_{11}+\mathrm{H}_{2} \mathrm{O} \rightarrow 2\left(\mathrm{C}_{6} \mathrm{H}_{12} \mathrm{O}_{6}\right) . \\
\mathrm{C}_{6} \mathrm{H}_{10} \mathrm{O}_{5}+\mathrm{H}_{2} \mathrm{O} \rightarrow \mathrm{C}_{6} \mathrm{H}_{12} \mathrm{O}_{6} .
\end{gathered}
$$

\section{STARCH, $\left(\mathrm{C}_{6} \mathrm{H}_{10} \mathrm{O}_{15}\right)_{\mathrm{x}}$}

Starch is widely distributed throughout the vegetable kingdom, occurring in grains, fruits, and tubers. It occurs in granular form, the microscopical appearance being typical for each individual starch. The granules, which differ in size according to the source, are composed of alternating concentric rings of granulose and cellulose. Ordinary starch is insoluble in cold water, but if boiled with water the cell walls are ruptured and slarch paste results. In general starch gives a blue color with iodine.

Starch is acted upon by amylases, e.g., salivary amylase (ptyalin) and pancreatic amylase (amylopsin), with the formation of soluble starch, erythro-dextrin, achroo-dextrins and maltose (see Salivary Digestion, page 56 ). Maltose is the principal end-product of this enzyme action. Upon boiling a starch solution with a dilute mineral acid a series of similar bodies is formed, but under these conditions glucose is the principal end-product.

Soluble starch may be prepared by the action of dilute hydrochloric acid on ordinary starch for several weeks, or at higher temperatures for a shorter period. By precipitation with alcohol this may be obtained in a dry form readily soluble in cold water. ${ }^{1}$

\section{Experiments on Starci}

I. Preparation of Potato Starch.-Pare a raw potato, comminute it upon a fine grater, mix with water, and "whip up" the pulped material vigorously before straining it through cheese cloth or gauze to remove the coarse particles. The

${ }^{1}$ Fernbach: Proceedings sih Inl. Cong. Appl. Chem., I3, I3I, I9I 2.

Chapin: Jour. Ind. and Eng. Chem., 6, 649, I9I4. 


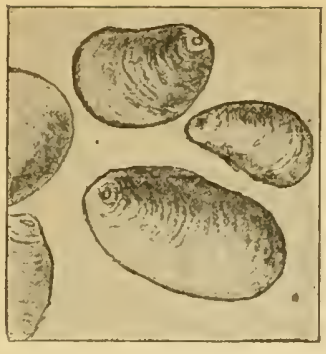

Fig. 9.-Potato.

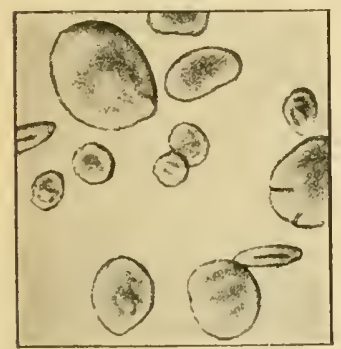

FIG. I2.-RYE.

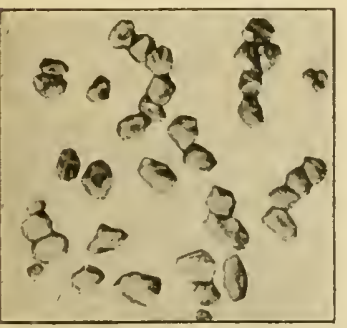

Fig. I5.-BUCKWHEAT.

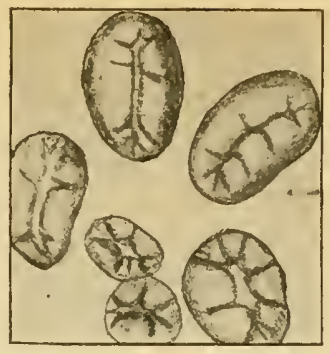

FIG, IO.-BEAN.

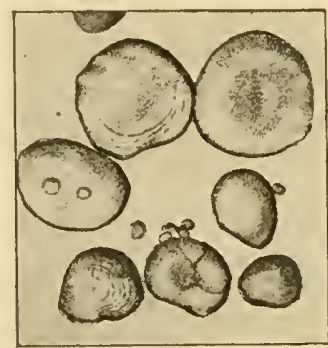

FIG. 13.-BARLEY.

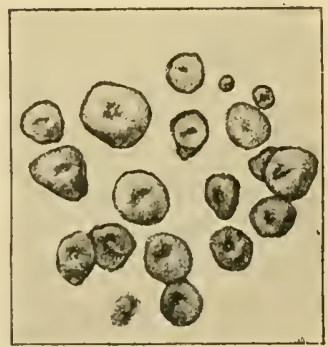

Fig, i6.-Maize.

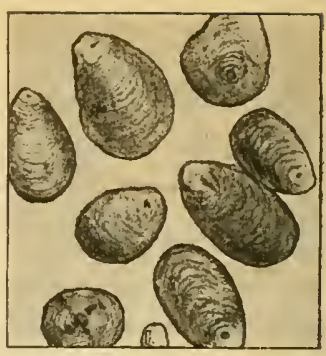

FIG. II.-.IRROWROOT.

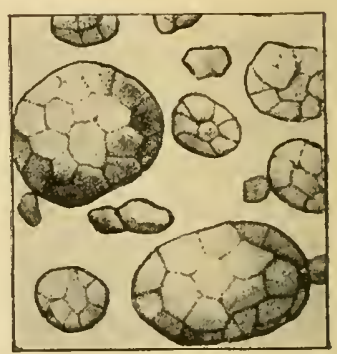

FIG. I4.-OAT.

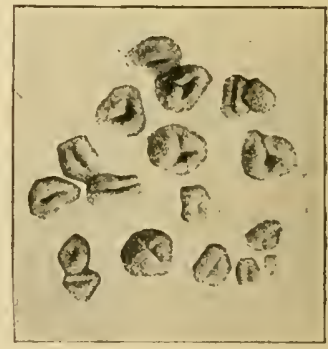

FIG. I7.-RICE.

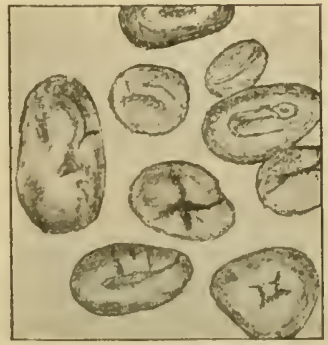

Fig. I8.-PEA.

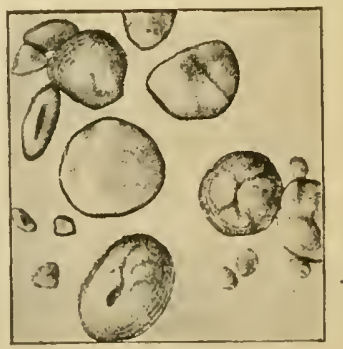

Fig. I9.- IVheat.

Starci Granules from Various Sources. (Leffmann and Beam.) 
starch rapidly settles to the bottom and can be washed by repeated decantation. Allow the compact mass of starch to drain thoroughly and spread it out on a watch glass to dry in the air. If so desired this preparation may be used in the experiments which follow.

2. Microscopical Examination.-Examine microscopically the granules of the various starches submitted and compare them with those shown in Figs. 9-I9, page 44. The suspension of the granules in a drop of water will facilitate the microscopical examination.

3. Solubility.-Try the solubility of one form of starch in each of the ordinary solvents (see page $2 \mathrm{I}$ ). If uncertain regarding the solubility in any reagent, filter and test the filtrate with iodine solution as given under 5 below. The production of a blue color would indicate that the starch had been dissolved by the solvent.

4. Iodine Test.-Place a few granules of starch in one of the depressions of a porcelain test-tablet and treat with a drop of a dilute solution of iodine in potassium iodide. The granules are colored blue due to the formation of so-called iodide of starch. The cellulose of the granule is not stained as may be seen by examining microscopically.

5. Iodine Test on Starch Paste. ${ }^{1-}$ Repeat the iodine test using the starch paste. Place $2-3$ c.c. of starch paste ${ }^{2}$ in a test-tube, add a drop of the dilute iodine solution and observe the production of a blue color. Heat the tube and note the disappearance of the color. It reappears on cooling.

In similar tests note the influence of alcohol and of alkali upon the so-called iodide of starch.

The composition of the iodide of starch is not definitely known. In performing this test the solution must always be neutral or acid in reaction.

6. Fehling's Test.-On starch paste (see page 26).

7. Hydrolysis of Starch.-Place about 25 c.c. of starch paste in a small beaker, add rodrops of concentrated $\mathrm{HCl}$, and boil. By means of a small pipette, at the end of each minute; remove a drop of the solution to the test-tablet and make the regular iodine test. As the testing proceeds the blue color should gradually fade and finally disappear. At this point, after cooling and neutralizing with solid $\mathrm{KOH}$, Fehling's test (see page 26 ) should give a positive result due to the formation of a reducing sugar from the starch. Make the phenylhydrazine test upon some of the hydrolyzed starch. What sugar has been formed?

8. Influence of Tannic Acid.- Add an excess of tannic acid solution to a small amount of starch paste in a test-tube. The liquid will become strongly opaque and ordinarily a yellowish-white precipitate is produced. Compare this result with the result of the similar experiment on dextrin (page ${ }_{4} S$ ).

9. Diffusibility of Starch Paste.-Test the diffusibility of starch pastc through animal membrane, parchment paper or collodion, making a dialyzer like one of the models shown in Fig. 2, page 24 .

1 Preparation of Starch Pusle.-Grind 2 grams of starch poweler in a mortar with a small a mount of cold water. Bring 200 c.c. of water to the boiling-point and add the starch mixture from the mortar with continuous stirring. Bring again to the boiling-point and allow it to cool. 'This makes an approximate I per cent starch paste which is a very satisfactory" strength for general use.

2 For this particular test a starch paste of very satisfactory strength may be made by mixing I c.c. of a I per cent starch paste with roo c.c. of water. 


\section{INULIN, $\left(\mathrm{C}_{6} \mathrm{H}_{10} \mathrm{O}_{5}\right)_{\mathbf{x}}$}

Inulin is a polysaccharide which may be obtained as a white, odorless, tasteless powder from the tubers of the artichoke, elecampane, or dahlia. It has also been prepared from the roots of chicory, dandelion, and burdock. It is very slightly soluble in cold water and quite easily soluble in hot water. In cold alcohol of 60 per cent or over it is practically insoluble. Inulin gives a negative reaction with iodine solution. The "yellow" color reaction with iodine mentioned in many books is doubtless merely the normal color of the iodine solution. It is very difficult to prepare inulin which does not reduce Fehling's solution slightly. This reducing power may be due to an impurity. Practically all commercial preparations of inulin possess considerable reducing power.

Inulin is levo-rotatory and upon hydrolysis by acids or by the enzyme imulase it yields the monosaccharide fructose which readily reduces Fehling's solution. The ordinary amylolytic enzymes occurring in the animal body do not digest inulin. A small part of the ingested inulin may be hydrolyzed by the acid gastric juice, but Lewis ${ }^{1}$ has recently shown that "the value of inulin as a significant source of energy in human dietaries must be questioned."

\section{Experiments ON INULIN}

I. Solubility.-Try the solubility of inulin powder in hot and cold water and alcohol. If uncertain regarding the solubility in any reagent, filter and neutralize the filtrate if it is alkaline in reaction. Add a drop of concentrated hydrochloric acid to the filtrate and boil it for one minute. Render the solution neutral or slightly alkaline with solid potassium hydroxide and try Fehling's test. What is the significance of a positive Fehling's test in this connection?

2. Iodine Test.-(a) Place 2-3 c.c. of the inulin solution in a test-tube and add a drop of dilute iodine solution. What do you observe?

(b) Place a small amount of inulin powder in one of the depressions of a testtablet and add a drop of dilute iodine solution. Is the effect any different from that observed above?

3. Molisch's Reaction.--Repeat this test according to directions given under Glucose, 2, page $2 \mathrm{I}$.

4. Fehling's Test.-Make this test on the inulin solution according to the instructions given under Glucose, page 26. Is there any reduction??

5. Hydrolysis of Inulin.-Place 5 c.c. of inulin solution in a test-tube, add a drop of concentrated hydrochloric acid and boil it for one minute. Now cool the solution, neutralize it with concentrated $\mathrm{KOH}$ and test the reducing action of $I$ c.c. of the solution upon I c.c. of diluted $(\mathrm{I}: 4)$ Fehling's solution. Also

${ }^{1}$ Lewis: Journal American Medical Ass'n., 58, I I 76, I9I 2.

${ }^{2}$ See the discussion of the properties of inulin, above. 
try the Resorcinol-Hydrochloric Acid reaction as given on p. 35. Explain the result. ${ }^{1}$

GLYCOGEN, $\left(\mathrm{C}_{6} \mathrm{H}_{10} \mathrm{O}^{5}\right)_{x}$

(For discussion and experiments see Muscular Tissue, Chapter XIX.)

LICHENIN, $\left(\mathrm{C}_{6} \mathrm{H}_{10} \mathrm{O}_{5}\right)$

Lichenin may be obtained from Cetraria islandica (Iceland moss). It forms a difficultly soluble jelly in cold water and an opalescent solution in hot water. It is optically inactive and gives no color with iodine. Upon hydrolysis with dilute mineral acids lichenin yields dextrins and glucose. It is said to be most nearly related chemically to starch. Saliva, pancreatic juice, malt diastase and gastric juice have no noticeable action on lichenin.

DEXTRIN, $\left(\mathrm{C}_{6} \mathrm{H}_{10} \mathrm{O}_{5}\right)_{\mathrm{x}}$

The dextrins are the bodies formed midway in the stages of the hydrolysis of starch by weak acids or an enzyme. They are amorphous bodies which are easily soluble in water, acids, and alkalis, but are insoluble in alcohol or ether. Dextrins are dextro-rotatory and are not fermentable by yeast.

The dextrins may be hydrolyzed by dilute acids to form glucose and by amylases to form maltose. With iodine one form of dextrin (erythro-dextrin) gives a red color. Their power to reduce Fehling's solution is questioned. The lower members of the dextrin series probably reduce.

\section{EXPERIMENTS ON DEXTRIN}

I. Solubility.- - Test the solubility of pulverized dextrin in hot and cold water. Dextrin forms a clear solution in hot water, distinguishing it from glycogen which gives an opalescent solution.

2. Iodine Test.-Place a drop of dextrin solution in one of the depressions of the test-tablet and add a dilute solution of iodine in potassium iodide. A red color results due to the formation of the red iodide of dextrin. Ordinary dextrin preparations contain some starch and in the presence of starch it is necessary to have an excess of iodine present. If the reaction is not sufficiently pronounced make a stronger solution from pulverized dextrin and repeat the test. The solution should be slightly acid to secure the best results.

Make proper tests to show that the red iodide of dextrin is influenced by

${ }^{1}$ If the inulin solution gave a positive Fchling test in the last experiment it will be necessary to check the hydrolysis experiment as follows: To 5 c.c. of the inulin solution in a testtube add one drop of concentrated hydrochloric acid, neutralize with concentrated $\mathrm{KOH}$ solution and test the reducing action of I c.c. of the resulting solution upon I c.c. of diluted $(I: 4)$ Fehling's solution. This will show the normal reducing power of the inulin solution. In case the inulin was hydrolyzed, the Fehling's test in the hydrolysis experiment should show a more pronounced reduction than that observed in the check experiment. 
heat, alkali, and alcohol in a similar manner to the blue iodide of starch (see page 45 ).

The color in the case of dextrin does not reappear as readily on cooling as in the case of starch.

3. Fehling's Test.-See if the dextrin solution will reduce Fehling's solution.

4. Hydrolysis of Dextrin.-Take 25 c.c. of dextrin solution in a small beaker, add 5 drops of dilute hydrochloric acid, and boil. By means of a small pipette, at the end of each minute, remove a drop of the solution to one of the depressions of the test-tablet and make the iodine test. The power of the solution to produce a color with iodine should rapidly disappear. When a negative reaction is obtained cool the solution and neutralize it with concentrated potassium hydroxide. Try Fehling's test (see page 26). This reaction is now strongly positive, due to the formation of a reducing sugar. Determine the nature of the sugar by means of the phenylhydrazine test (see pages 22 and 23).

5. Precipitation by Alcohol. -To about 50 c.c. of 95 per cent alcohol in a small beaker add about ro c.c. of a concentrated dextrin solution. Dextrin is thrown out of solution as a gummy white precipitate. Compare the result with that obtained under Glucose, 7, page 24 .

6. Infiuence of Tannic Acid.-Add an excess of tannic acid solution to a small amount of dextrin solution in a test-tube. No precjpitate forms. This result differs from the result of the similar experiment upon starch (see Starch, 8, page 45$)$.

7. Diffusibility of Dextrin.--(See Starch, 9, page 45.)

\section{CELlulose, $\left(\mathrm{C}_{5} \mathrm{H}_{10} \mathrm{O}_{5}\right)_{\mathrm{x}}$}

This complex polysaccharide forms a large portion of the cell wall of plants. It is extremely insoluble and its molecule is much more complex than the starch molecule. The best quality of filter paper and the ordinary absorbent cotton are good types of cellulose.

At one time there was but a single known solvent for cellulose. Recent investigation has, however, revealed a long list of cellulose solvents. (See Experiment 7.)

Cellulose is not hydrolyzed by boiling with dilute mineral acids. It may be hydrolyzed, however, by treating with concentrated sulphuric acid then subsequently diluting the solution with water and boiling. The product of this hydrolysis is glucose.

There is some difference of opinion as to the exact extent to which cellulose is utilized in the animal organism. It is no doubt, more efficiently utilized by herbivora than by carnivora or by man. It is claimed that about 25 per cent may be utilized by herbivora, less than 5 per cent by dogs whereas the quantity utilized by man is " too small for it to play a rôle of importance in the diet of a normal individual." In neither man nor the lower animals has there been demonstrated any formation

${ }^{1}$ Swartz: Transactions of the Connecticut Academy of Arts and Sciences, I6, 247, I9 I I. 
of sugar or glycogen from cellulose. ${ }^{1}$ It is probable that the cellulose which disappears from the intestine is transformed for the most part into fatty acids. ${ }^{2}$

\section{Experiantent on Celletose}

I. Solubility.-Test the solubility of cellulose in water, dilute and concentrated acid and alkali.

2. Iodine Test. - Add a drop of dilute iodine solution to a few shreds of cotton on a test-tablet. Cellulose differs from starch and dextrin in giving no color with iodine.

3. Formation of Amyloid.3-Add Io c.c. of dilute and 5 c.c. of concentrated $\mathrm{H}_{2} \mathrm{SO}_{4}$ to some absorbent cotton in a test-tube. When entirely dissolved (without heating) pour one-half of the solution into another test-tube, cool it and dilute with water. Amyloid forms as a gummy precipitate and gives a brown or blue coloration with iodine.

After allowing the second portion of the acid solution of cotton to stand about Io minutes, dilute it with water in a small beaker and boil for $15-30$ minutes. Now cool, neutralize with solid $\mathrm{KOH}$ and test with Fehling's solution. Glucose has been formed from the cellulose by the action of the acid.

4. Ammoniacal Cupric Hydroxide Solubility Test (Schweitzer).-Place a little absorbent cotton in a test-tube, add Schweitzer's reagent, ${ }^{4}$ and stir the cellulose with a glass rod. When completely dissolved acidify the solution with acetic acid. An amorphous precipitate of cellulose is produced.

5. Hydrochloric Acid-Zinc Chloride Solubility Test (Cross and Bevan). ${ }^{5}$ Place a little absorbent cotton in a test-tube, add Cross and Bevan's reagent, ${ }^{6}$ and stir the cellulose with a glass rod. When solution is complete reprecipitate the cellulose with 95 per cent alcohol.

6. Iodine-Zinc Chloride Reaction.-Place a little absorbent cotton or quantitative filter paper in a test-tube and treat it with the iodine-zinc chloride reagent.' A blue color forms on standing. Amyloid has been formed from the cellulose through the action of the $\mathrm{ZnCl}_{2}$ and the iodine solution has stained the amyloid blue.

7. Other Cellulose Solvents.-It has recently been demonstrated by Deming ${ }^{3}$ that there are many excellent solvents for cellulose (filter paper). For example, the concentrated aqueous solutions of certain salts such as antimony trichloride.

${ }^{1}$ Lusk: Amcrican Journal of Physiology, 27. 467 , I9II; also Hoffmann, Inaugural dissertation, Halle-Wittenberg, igro.

2 Tappeiner: Zeilschrift fiir Biologic, 24,105, I 888 .

3 This body derives its name from amylum (starch) and is not to be confounded with amyloid, the glycoprotein.

"Schweitzer's reagent is made by adding potassium hydroxide to a solution of copper sulphate which contains some ammonium chloride 1 precipitate of cupric hydroxide forms and this is filtered off, washed, and 3 grams of the moist cupric hydroxide brought into solution in a liter of 20 per cent ammonium hydroxide

${ }^{5}$ Cross and Bevan: Chemical New's, 63, p. 06.

${ }^{6}$ Cross and Bevan's reagent may be prepared by combining two parts of concentrated hydrochloric acid and one part of zinc chloride, by iicight.

${ }^{7}$ The iodine-zinc chloride reagent as suggested by Nowopokrowsky (Beihefte Bolur. Cenir., 28, 90, 1912) may be made by dissolving 20 grams $\mathrm{ZnCl}$ in 5.5 c.c. Water and when cool introducing the iodine solution ( 3 grans $\mathrm{KI}+\mathrm{r} .5 \mathrm{gram} \mathrm{I}$ in 60 c.c. water) drop by drop until iodine begins to precipitate.

8 Deming: Journal 1 mericun Chomical Socicly, 33, I515, 101 r. 
stannous chloride and zinc bromide. In hydrochloric acid solution the solvent action of the above salts is increased. The following salts are also good solvents in hydrochloric acid solution: mercuric chloride, bismuth chloride, antimony pentachloride, tin telrachloride and titanium tetrachloride. In the case of the last-mentioned salt the swollen, transparent character of the cellulose fibers preliminary to solution can be seen very nicely.

Try selected soivents suggested by the instructor.

\section{HEMICELLULOSES}

The hemicelluloses differ from cellulose in that they may be hydrolyzed upon boiling with dilute mineral acids. They differ from other polysaccharides in not being readily digested by amylases. Hemicellulose may yield pentosans, or hexosans upon hydrolysis.

Pentosans.-Pentosans yield pentoses upon hydrolysis. So far as is known they do not occur in the animal kingdom. They have, however, a very wide distribution in the vegetable kingdom, being present in leaves, roots, seeds and stems of all forms of plants, many times in intimate association or even chemical combination with galactans. In herbivora, pentosans are $40-80$ per cent utilized. ${ }^{1}$ The few tests on record as to the pentosan utilization by $\operatorname{man}^{2}$ indicate that $80-95$ per cent disappear from the intestine. According to Cramer, ${ }^{3}$ bacteria are efficient hemicellulose transformers. It has not yet been demonstrated that pentosans form glycogen in man, and for this reason they must be considered as playing an unimportant part in human nutrition. Gum arabic an important pentosan may be hydrolyzed by concentrated hydrochloric acid if boiled for a short time. The pentose arabinose results from such hydrolysis.

Galactans.- In common with the pentosans the galactans have a very wide distribution in the vegetable kingdom. The pure galactans yield galactose upon hydrolysis. One of the most important members of the galactan group is agar-agar, a product prepared from certain types of Asiatic sea-weed. This galactan is about 50 per cent utilizable by herbivora ${ }^{4}$ and 8-27 per cent utilizable by man. ${ }^{5}$ Agar ingestion has been shown to be a very efficient therapeutic aid in cases of chronic constipation. ${ }^{6.7}$ This is particularly true when the constipation is due to the formation of dry, hard, fecal masses (scybala), a type of fecal formation which frequently follows the ingestion of a diet which is

\footnotetext{
1 Swartz: Transactions of the Connecticut Academy of Arts and Sciences, I6, 247, I9I I.

2 König and Reinhardt: Zeit.f. Untersuchung der Nalirungs u. Genussmittel, 5, I 10, I 902.

${ }^{3}$ Cramer: Inaug. Diss., Halle, I910.

${ }^{4}$ Lohrisch: Zeit.f. exper. Path. u. Pharm., 5, 478, 1908.

5 Saiki: Jour. Biol. Chem., 2, 25I, 1906.

6 Mendel: Zeniralblat f. d. gesammte Phys. u. Path. des Stoffw., No. I 7, I, 1908.

${ }^{7}$ Schmidt: Mïnch. med. Woch., 52, 1970, I905.
} 
very thoroughly digested and absorbed. The agar, because of its relative indigestibility and its property of absorbing water yields a bulky fecal mass which is sufficiently soft to permit of easy evacuation. Agar has been used with good results in the treatment of constipation in children. ${ }^{1}$ The function of agar is not limited to its use in connection with constipation, it may. serve in other capacities as an aid to intestinal therapeutics. ${ }^{2}$

\section{Experiments on a Pentosan}

I. Solubility.-Test the solubility of gum arabic in hot and cold water and alcohol.

2. Iodine Test.-Add a drop of dilute iodine solution to a little gum arabic on a test-tablet. It resembles cellulose in giving no color with iodine.

3. Hydrolysis of Gum Arabic.-Introduce a little gum arabic into a test-tube, add 5-Io c.c. of strong hydrochloric acid (conc. $\mathrm{HCl}$ and water $\mathrm{I}: \mathrm{I}$ ) and heat to boiling for 5-1o minutes. Cool, neutralize with potassium hydroxide and test by the Fehling or some other reduction test. A positive reaction should be obtained indicating that the gum arabic has been hydrolyzed by the acid with the production of a reducing substance. What is this reducing substance? How would you identify it?

\section{EXPERIMENTS ON a GaLACTAN}

I. Solubility.-Test the solubility of agar-agar in hot and cold water. Observe its marked property of imbibing water (see above).

2. Iodine Test.-Add a drop of dilute iodine solution to a little agar-agar on a test-tablet. It resembles cellulose in giving no color with iodine.

3. Hydrolysis of Agar-agar.-Introduce a few pieces of agar-agar into a testtube, add 5-10 c.c. of strong hydrochloric acid (conc. $\mathrm{HCl}$ and water $\mathrm{I}: \mathrm{I}$ ) and heat to boiling for 5-10 minutes. Cool, neutralize with potassium hydroxide and test by the Fehling or some other reduction test. A positive reaction should be obtained indicating that the agar-agar has been hydrolyzed by the acid with the production of a reducing substance. What is this reducing substance? How would you identify it?

\section{REVIEW OF CARBOHYDRATES}

In order to facilitate the student's review of the carbohydrates, the preparation of a chart similar to the appended model is recommended. The signs + and - may be conveniently used to indicate positive and negative reaction. Only those carbohydrates which are of greatest importance from the standpoint of physiological chemistry have been included in the chart.

1 Morse: Journal American Medical Ass'n., 55, 934, r910.

2 Einhorn: Berl. klin. Wóch., 49, I I3, rg12. 
MODEL CHART FOR REVIEW PURPOSES

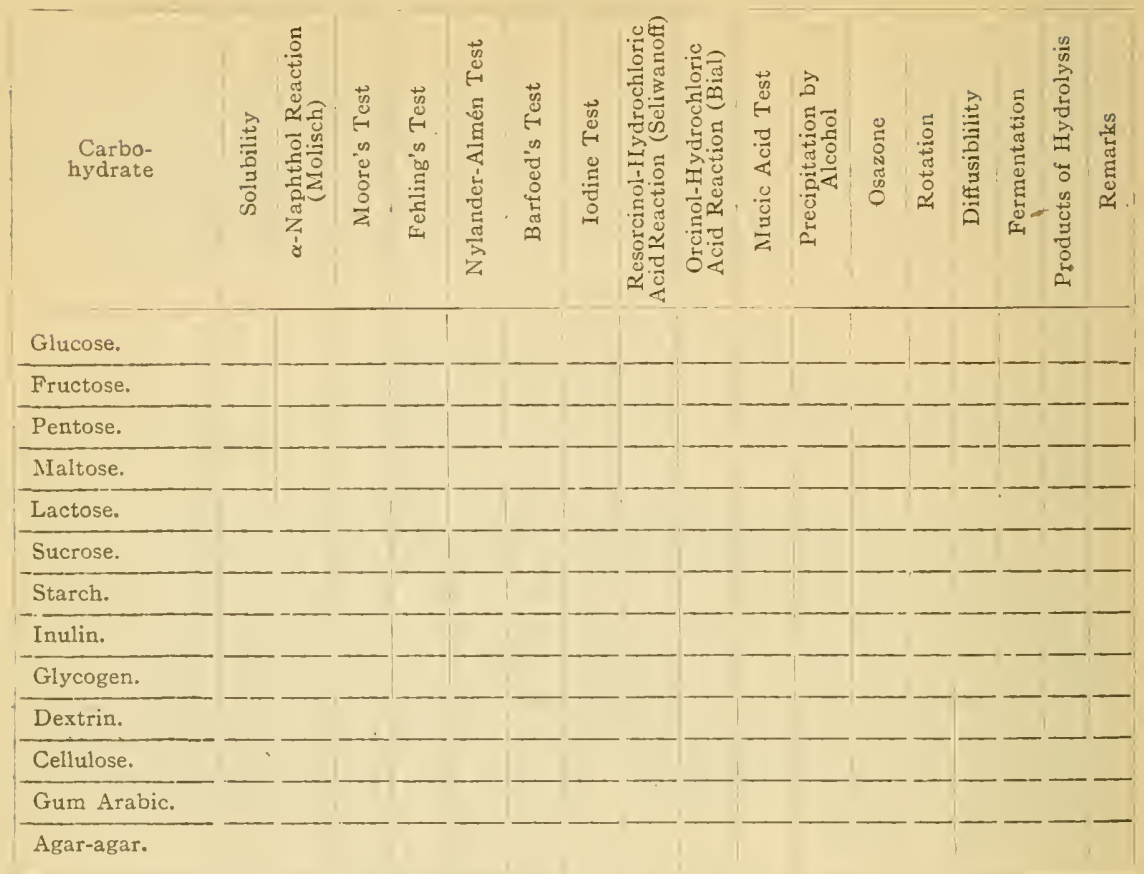

\section{"Unknown" Solutions of Carbohydrates}

At this point the student will be given several "unknown" solutions, each solution containing one or more of the carbohydrates studied. $\mathrm{He}$ will be required to detect, by means of the tests on the preceding pages, each carbohydrate constituent of the several "unknown" solutions and hand in, to the instructor, a written report of his findings, on slips furnished by the laboratory.

The scheme given on page 53 may be of use in this connection. 


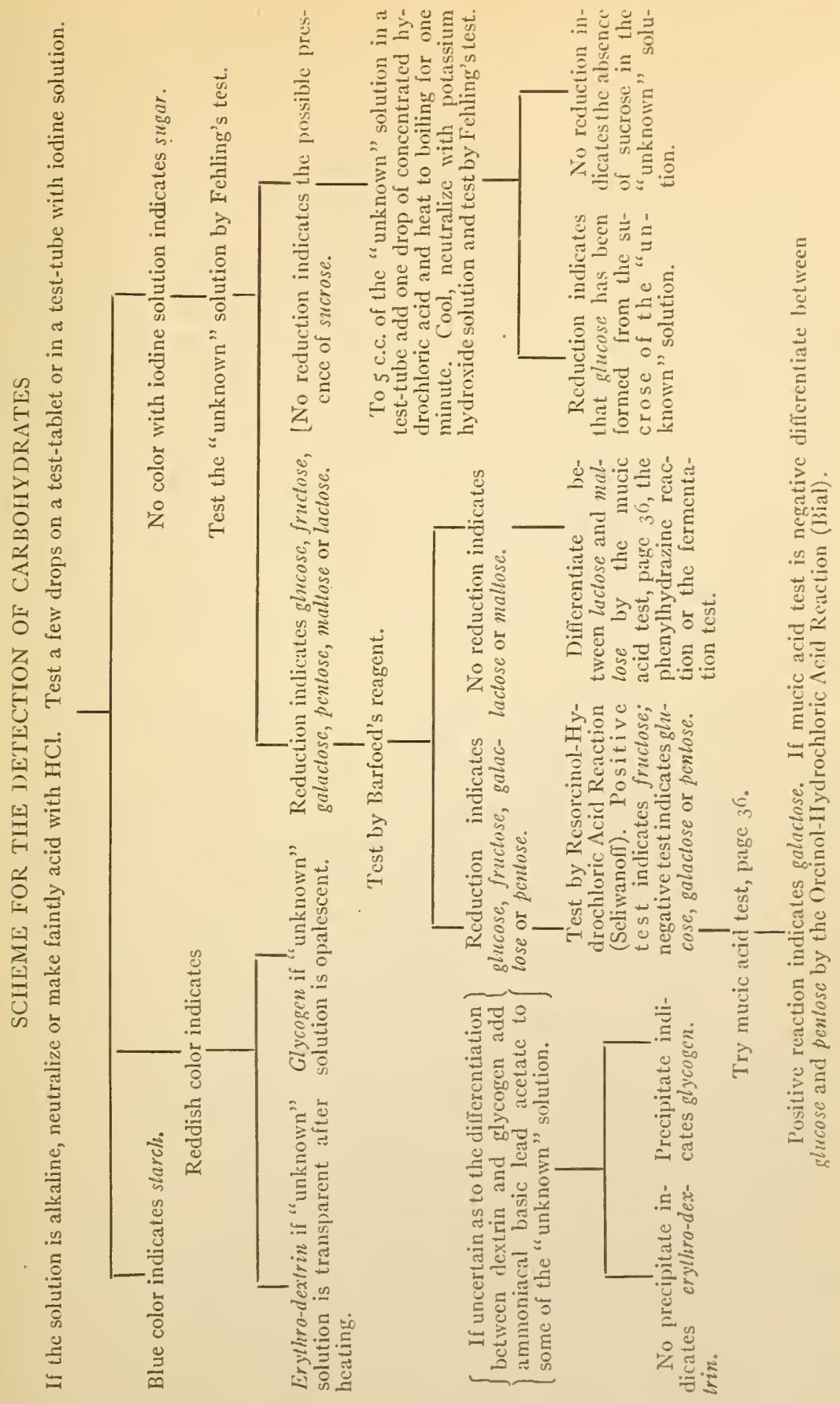




\section{CHAPTER III}

\section{SALIVARY DIGESTION}

THE saliva is secreted by three pairs of glands, the submaxillary, sublingual, and parotid, reinforced by numerous small glands called buccal glands. The saliva secreted by each pair of glands possesses certain definite characteristics peculiar to itself. For instance, in man the parotid glands ordinarily secrete a thin, watery fluid, the submaxillary glands secrete a somewhat thicker fluid containing mucin, while the product of the sublingual glands has a more mucilaginous character. The saliva as collected from the mouth is the combined product of all the glands mentioned. The fact that there are pronounced variations in the composition of different fractions of saliva secreted by the same normal individual on a uniform diet has been emphasized by Lothrop and Gies. ${ }^{1}$

The saliva may be induced to flow by many forms of stimuli, such as chemical, mechanical, electrical, thermal, and psychical, the nature and amount of the secretion depending, to a limited degree, upon the particular class of stimuli employed as well as upon the character of the individual stimulus. For example, in experiments upon dogs it has been found that the mechanical stimulus afforded by dropping several pebbles into the animal's mouth caused the flow of but one or two drops of saliva, whereas the mechanical stimulus afforded by sand thrown into the mouth induced a copious flow of thin watery fluid. Again, when ice-water or snow was placed in the animal's mouth no saliva was seen, while an acid or anything possessing a bitter taste, which the dog wished to reject, caused a free flow of the thin saliva. On the other hand, when articles of food were placed in the dog's mouth the animal secreted a thicker saliva having a higher mucin content-a fluid which would lubricate the food and assist in the passage of the bolus through the esophagus. It was further found that by simply drawing the attention of the animal to any of the substances named above, results were obtained similar to those secured when the substances were actually placed in the animal's mouth. For example, when a pretense was made of throwing sand into the dog's mouth, a watery saliva was secreted, whereas food under the same conditions excited a thicker and more slimy secretion. The exhibition of dry food, in which the dog had no par-

${ }^{1}$ Lothrop and Gies: Journal of the Allied (Dental) Societies, 6, 65, I9I I. 
ticular interest (dry bread), caused the secretion of a large amount of watery saliva, while the presentation of moist food, which was eagerly desired by the animal, called forth a much smaller secretion, slimy in character. These experiments show it to be rather difficult to differentiate between the influence of physiological and psychical stimuli.

The amount of saliva secreted by an adult in 24 hours has been variously placed, as the result of experiment and observation, between 1000 and I500 c.c., the exact amount depending, among other conditions, upon the character of the food.

The saliva of adults ordinarily has a weak, alkaline reaction to litmus, but becomes acid, in some instances, 2-3 hours after a meal or during fasting. The saliva of the newborn is generally neutral to litmus, whereas that of infants, especially those breast-fed, is generally acid. ${ }^{1}$ The alkalinity of saliva is due principally to di-sodium hydrogen phosphate $\left(\mathrm{Na}_{2} \mathrm{HPO}_{4}\right)$ and its average alkalinity may be said to be equivalent to 0.08-o.I per cent sodium carbonate. 'The saliva is the most dilute of all the digestive secretions, having an average specific gravity of 1.005 and containing only 0.5 per cent of solid matter. Among the solids are found albumin, globulin, mucin, urea, the enzymes salivary amylase (ptyalin), maltase, and peptide-splitting enzymes, phosphates, and other inorganic constituents. Potassium thiocyanate, KSCN, is also generally present in the saliva. It has been claimed that this substance is present in greatest amount in the saliva of habitual smokers. The significance of thiocyanate in the saliva is not known; it probably comes from the ingested thiocyanates and from the breaking down of protein material. The attempts to show some relationship between tooth decay and the thiocyanate content of the saliva secreted into the mouth cavity have met with failure. The most recent experiments ${ }^{2}$ indicate a virtual absence of such relationship.

The so-called tartar formation on the teeth is composed almost entirely of calcium phosphate with some calcium carbonate, mucin. epithelial cells, and organic débris derived from the food. The calcium salts are held in solution as acid salts, and are probably precipitated by the ammonia of the breath. The various organic substances just mentioned are carried down in the precipitation of the calcium salts.

The suggestion has been made that mucin is the salivary constituent "which is particularly influential in the development of local conditions favoring the onset of dental decay."

The principal enzyme of the saliva is known as salivary amylase or ptyalin. This is an amylolytic enzyme (sce page 4 ), so called because it

1 Allaria: Monalsschr fïr Kindcrheilkunde, ro, I79, rg r I.

${ }^{2}$ Lothrop and Gies: Journal of the Allied (Dental) Sociclies, 6, 65, rg I I.

${ }^{3}$ Id.: Ibid., 5, No. 4, I9ro. 
possesses the property of transforming complex carbohydrates such as starch and dextrin into simpler bodies. The action of salivary amylase is one of hydrolysis and through this action a series of simpler bodies are formed from the complex starch. The first product of the action of the ptyalin of the saliva upon starch paste is soluble starch (amidulin) and its formation is indicated by the disappearance of the opalescence of the starch solution. This body resembles true starch in giving a blue color with iodine. Next follows the formation, in succession, of a series of dextrins, called erythro-dextrin, $\alpha$-achroo-dextrin, $\beta$-achroo-dextrin, and $\gamma$-achroo-dextrin, the crythro-dextrin being formed directly from soluble starch and later being itself transformed in to $\alpha$-achroo-dextrin from which in turn are produced $\beta$-achroo-dextrin, $\gamma$-achroo-dextrin and perhaps other dextrins. Accompanying each dextrin a small amount of maltose is formed, the quantity of maltose growing gradually larger as the process of transformation progresses. (Erythro-dextrin gives a red color with iodine, the other dextrins give no color.) The next stage is the transformation of the final dextrin into maltose, the latter being the principal end-product of the salivary digestion of starch. At this point a small amount of glucose is formed from the maltose through the action of the enzyme maltase. The above changes may be represented graphically as follows:

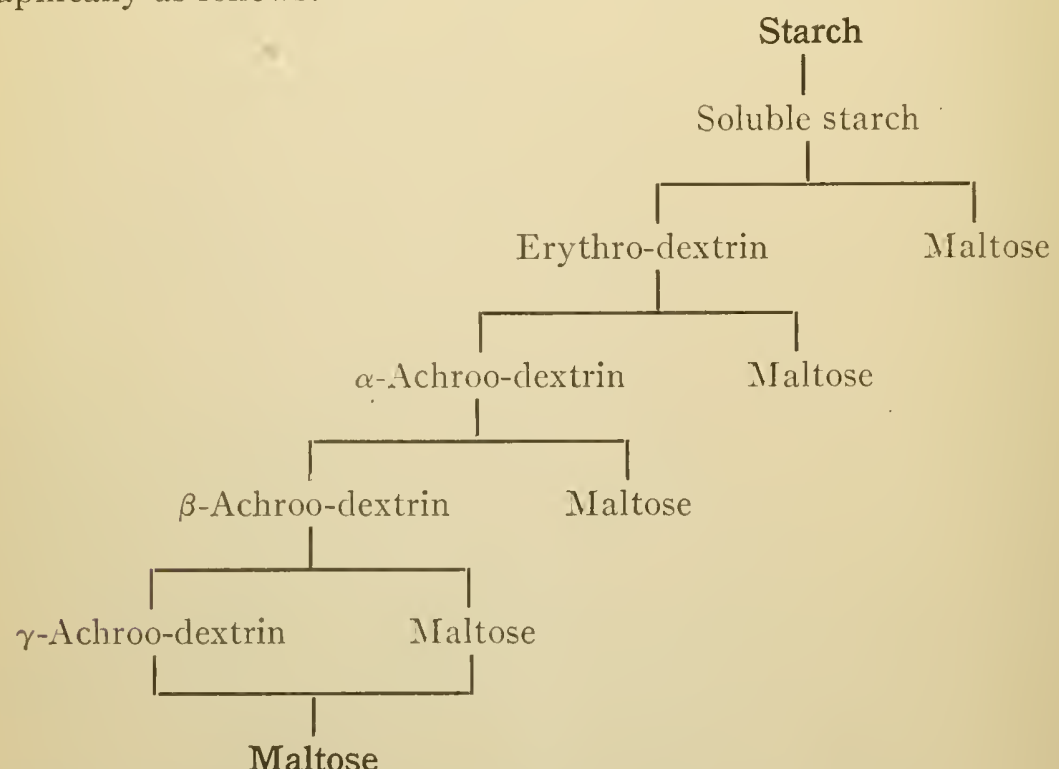

Salivary amylase acts in alkaline, neutral, or combined acid solutions. It will act in the presence of relatively strong combined $\mathrm{HCl}$ (see page I40), whereas a trace ( 0.003 per cent to 0.0006 per cent) of ordinary 
free hydrochloric acid will not only prevent the action but will destroy the enzyme. By sufficiently increasing the alkalinity of the saliva to litmus, the action of the salivary amylase is inhibited.

It has been shown by Cannon that salivary digestion may proceed for a considerable period after the food reaches the stomach, owing to the slowness with which the contents are thoroughly mixed with the acid gastric juice and the consequent tardy destruction of the enzyme. Food in the pyloric end of the stomach is soon mixed with the gastric secretion, but food in the cardiac end is not mixed with the acid gastric juice for a considerable period of time, and in this region during that time salivary digestion may proceed undisturbed.

It has been found that salivary amylase acts more efficiently when the saliva is diluted from 4 to 7 times. ${ }^{1}$

Water softened by lime $e^{2}$ inhibits the action of salivary amylase due to the presence of magnesium hydroxide in this water. ${ }^{3}$ Electrolytes have an important influence upon the action of amylases. The $\mathrm{Cl}$ ion has a pronounced facilitating action (see Pancreatic Amylase).

The question of the adaptation of the salivary secretion to diet is one which has received considerable attention in recent years. It has been claimed, on the basis of experimental evidence, ${ }^{4}$ that the continued feeding of a carbohydrate diet causes the secretion of a saliva which contains a higher concentration of salivary amylase and one which is therefore able to more efficiently digest the carbohydrate fed. On the other hand, strong evidence ${ }^{5}$ has been submitted that the amylase content of the saliva is not increased through the continued feeding of a carbohydrate diet. In general the consensus of opinion is opposed to the adaptation of digestive secretions to diet.

Maltase, sometimes called glucase, is the second enzyme of the saliva. The principal function of maltase is the splitting of maltose into glucose. Besides occurring in the saliva it is also present in the pancreatic and intestinal juices. For experimental purposes the enzyme is ordinarily prepared from corn. The principles of the "reversibility" of enzyme action were first demonstrated in connection with maltase by Croft Hill.

It is claimed that the saliva contains dipeptide- and tripeptidesplitting enzymes. ${ }^{6}$ Leucyl-glycyl-alanine was the tripeptide split,

${ }^{1}$ Bergeim and Hawk: Jour. Am. Chem. Soc., 35, 46I, I913.

${ }^{2}$ Prepared by treating tap water with one-sixth its volume of saturated lime water, allowing to stand 24 hours and filtering.

${ }^{3}$ Bergeim and Hawk: Jour. Am. Chem. Soc., 35, 1049, 1913.

-Neilson and Terry: American Journal of Physiology', I5, 406, I905; Neilson and Lewis: Journal of Biological Chemistry, 4, $50 \mathrm{I}$, rgoS.

'Mendel: Almcrican Journal of the Medical Sciences, Oct., I909; Mendel and Inderhill: Journal of Biological Chemisiry, 3, 135, I907; Mendel, Chapman and Blood: Medical Record, Aug. 27, I910.

'Koelker: Zcilschrift für physiol. Chcm, 76, 27, 19 I r. 
whereas the cleavage of several dipeptids was brought about. The action is similar to that of intestinal erepsin (see Chapter XI). Later investigations (see page I99), apparently have demonstrated that the peptolytic power of saliva, at least in some cases, is due to bacteria.

Microscopical examination of the saliva reveals salivary corpuscles, bacteria, food débris, epithelial cells, mucus, and fungi. In certain pathological conditions of the mouth, pus cells and blood corpuscles may be found in the saliva.

\section{Experiments on SALIVA}

A satisfactory method of obtaining the saliva necessary for the experiments which follow is to chew a small piece of pure paraffin wax, thus stimulating the flow of the secretion, which may be collected in a small beaker. Filtered saliva is to be used in every experiment except for the microscopical examination.

I. Microscopical Examination.-Examine a drop of unfiltered saliva microscopically, after staining with methylene blue, and compare with Fig. I9 below.

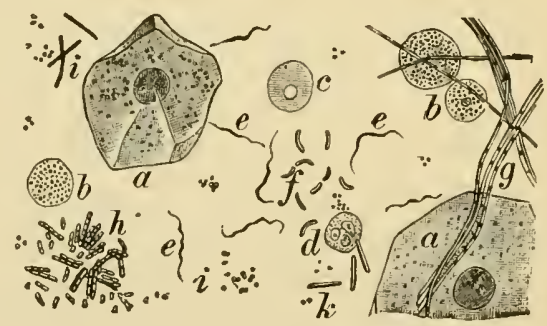

Fig. 20.-Microscopical Constituents of Saliva.

$a$, Epithelial cells; $b$, salivary corpuscles; $c$, fat drops; $d$, leucocytes; $e, f$ and $g$, bacteria; $h, i$, and $k$, fission-fungi.

2. Reaction.-Test the reaction to litmus, phenolphthalein and Congo red.

3. Specific Gravity.--Partially fill a urinometer cylinder with saliva, introduce the urinometer, and observe the reading.

4. Test for Mucin.-To a small amount of saliva in a test-tube add $I-2$ drops of dilute acetic acid. Mucin is precipitated.

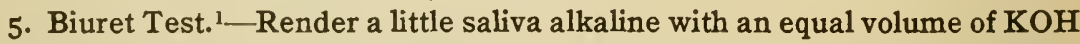
and add a few drops of a very dilute (2-5 drops in a test-tube of water) copper sulphate solution. The formation of a purplish-violet color is due to mucin.

This reaction is given by protein material and simply indicates that mucin is a protein.

6. Millon's Reaction. ${ }^{2-}$-Add a few drops of Millon's reagent to a little saliva. A light yellow precipitate formed by the mucin gradually turns red upon being gently heated.

This reaction indicates the presence of protein (mucin).

1 The significance of this reaction is pointed out on p. 98 .

2 The significance of this reaction is pointed out on p. 97 . 
7. Preparation of Mucin.-Pour 25 c.c. of saliva into I 00 c.c. of 95 per cent alcohol, stirring constantly. Cover the vessel and allow the precipitate to stand at least 12 hours. Pour off the supernatant liquid, collect the precipitate on a filter and wash it, in turn, with alcohol and ether. Finally dry the precipitate, remove it from the paper and make the following tests on the mucin: (a) Test its solubility in the ordinary solvents (see page 2r); (b) Millon's reaction; (c) dissolve a small amount in $\mathrm{KOH}$, and try the biuret test on the solution; (d) boil the remainder, with $10-25$ c.c. of water to which 5 c.c. of dilute $\mathrm{HCl}$ has been added, until the solution becomes brownish. Cool, render alkaline with solid $\mathrm{KOH}$, and test by Fehling's solution. A reduction should take place.

Mucin is what is known as a conjugated protein or glycoprotein (see page II 2) and upon boiling with the acid the carbohydrate group in the molecule has been split off from the protein portion and its presence is indicated by the reduction of Fehling's solution.

8. Inorganic Matter.-Test for chlorides, phosphates, sulphates, and calcium. For chlorides, acidify with $\mathrm{HNO}_{3}$ and add $\mathrm{AgNO}_{3}$. For phosphates, acidify with $\mathrm{HNO}_{3}$, heat and add molybdate solution. ${ }^{1}$ For sulphates, acidify with $\mathrm{HCl}$ and add $\mathrm{BaCl}_{2}$ and warm. For calcium, acidify with acetic acid, $\mathrm{CH}_{3}$ $\mathrm{COOH}$, and add ammonium oxalate, $\left(\mathrm{NH}_{4}\right)_{2} \mathrm{C}_{2} \mathrm{O}_{4}$.

9. Viscosity Test.-Place filter papers in two funnels, and to each add an equal quantity of starch paste (5 c.c.). Add a few drops of saliva to one lot of paste and an equivalent amount of water to the other. Note the progress of filtration in each case. Why does one solution filter more rapidly than the other?

10. Test for Nitrites.-Add I-2 drops of dilute $\mathrm{H}_{2} \mathrm{SO}_{4}$ to a little saliva and thoroughly stir. Now add a few drops of a potassium iodide solution and some starch paste. Nitrous acid is formed which liberates iodine, causing the formation of the blue iodide of starch.

II. Thiocyanate Tests.-(a) Ferric Chloride Test. - To a little saliva in a small porcelain crucible, or dish, add a few drops of dilute ferric chloride and acidify slightly with $\mathrm{HCl}$. Red ferric thiocyanate $\mathrm{Fe}(\mathrm{SCN})_{3}$ forms. To show that the red coloration is not due to iron phosphate add a drop of $\mathrm{HgCl}_{2}$ when colorless mercuric thiocyanate forms.

(b) Solera's Reaction.-This test depends upon the liberation of iodine through the action of thiocyanate upon iodic acid. Moisten a strip of starch paste-iodic acid test paper ${ }^{2}$ with a little saliva. If thiocyanate be present the test paper will assume a blue color, due to the liberation of iodine and the subsequent formation of the socalled iodide of starch.

12. Digestion of Starch Paste.-To 25 c.c. of starch paste in a small beaker, add 5 drops of saliva and stir thoroughly. At intervals of a minute remove a drop of the solution to one of the depressions in a test-tablet and test by the iodine test. If the blue color with iodine still forms after five minutes, add another 5 drops of saliva. The opalescence of the starch solution should soon disappear, indicating the formation of soluble starch which gives a blue color with iodine.

${ }^{1}$ See "Reagents and Solutions."

2 This test paper is prepared as follows: Saturate a good quality of filter paper with 0.5 per cent starch paste to which has been added sufficient iodic acid to make a I per cent solution of iodic acid and allow the paper to dry in the air. Cut it in strips of suitable size and preserve for use. 
This body should soon be transformed into erythro-dextrin which gives a red color with iodine, and this in turn should pass into achroo-dextrin which gives no color with iodine. This is called the achromic point. When this point is reached test by Fehling's test to show the production of a reducing body. A positive Fehling's test may be obtained while the solution still reacts red with iodine inasmuch as some maltose is formed from the soluble starch coincidently with the formation of the erythro-dextrin. How long did it take for a complete transformation of the starch? For a graphic representation of the above changes see page 56.

13. Separation of the Products of Salivary Digestion.-To 25 c.c. of starch paste in a small beaker add I c.c. of saliva and stir thoroughly. At intervals of one minute test a drop of the mixture by the iodine test. If the blue color persists after five minutes add another I c.c. of saliva. When the mixture reacts red with iodine, indicating that erythrodextrin has been formed, add Ioo c.c. of 95 per cent alcohol. Allow to stand until the white precipitate has settled. Filter, evaporate the filtrate to dryness, dissolve the residue in 5-10 c.c. of water and try Fehling's test (page 26) and the phenylhydrazine reaction (see Glucose, 3, page 22). On the dextrin precipitate try the iodine test (page 45). Also hydrolyze the dextrin as given under Dextrin, 4, page 48.

I4. Digestion of Dry Starch.-In a test-tube shake up a small amount of dry starch with a little water. Add a few drops of saliva, mix well, and allow to stand. After ro-20 minutes filter and test the filtrate by Fehling's test. What is the result and why?

Dry starch is very slowly digested by salivary amylase.

I5. Digestion of Inulin.-To 5 c.c. of inulin solution in a test-tube add ro drops of saliva and place the tube in the incubator or water-bath at $40^{\circ} \mathrm{C}$. After onehalf hour test the solution by Fehling's test. ${ }^{1}$ Is any reducing substance present? What do you conclude regarding the salivary digestion of inulin?

I6. Influence of Temperature.-In each of four tubes place about 5 c.c. of starch paste. Immerse one tube in cold water from the faucet, keep a second at room temperature, and place a third in the incubator or the water-bath at $40^{\circ} \mathrm{C}$. (If the temperature of the bath or incubator is allowed to rise to $70^{\circ} \mathrm{C}$. or over the enzyme is destroyed and no digestion takes place.) Now add to the contents of each of these three tubes two drops of saliva and shake well; to the contents of the fourth tube add two drops of boiled saliva. Test frequently by the iodine test, using the test-tablet, and note in which tube the most rapid digestion occurs. Explain the results.

I7. Influence of Dilution. ${ }^{2}$-Take a series of six test-tubes each containing 9 c.c. of water. Add I c.c. of saliva to tube I and shake thoroughly. Remove I c.c. of the solution from tube I to tube 2 and after mixing thoroughly remove I c.c. from tube 2 to tube 3. Continue in this manner until you have 6 saliva solutions of gradually decreasing strength. Now add starch paste in equal amounts to each tube, mix very thoroughly, and place in the incubator or water-

\footnotetext{
${ }^{1}$ If the inulin solution gives a reduction before being acted upon by the saliva it will be necessary to determine the extent of the original reduction by means of a "check" test (see p. 47).

${ }_{2}^{2}$ The technic of Wohlgemuth's method (sce Chapter $\mathrm{X}$ ) may be employed in this test if so desired.
} 
bath at $40^{\circ} \mathrm{C}$. After 10-20 minutes test by both the iodine and Fehling's tests. In how great dilution does your saliva act?

18. Influence of Acids and Alkalis.-(a) Influence of Free Acid.-Prepare a series of six tubes in each of which is placed 4 c.c. of one of the following strengths of free $\mathrm{HCl}: 0.2$ per cent, 0.1 per cent, 0.05 per cent, 0.025 per cent, 0.0125 per cent and 0.006 per cent. Now add 2 c.c. of starch paste to each tube and shake them thoroughly. Complete the solutions by adding 2 c.c. of saliva to each and repeat the shaking. The total acidity of this series would be as follows: 0.I per cent, 0.05 per cent, 0.025 per cent, 0.0125 per cent, 0.006 per cent and 0.003 per cent. Place these tubes on the water-bath at $40^{\circ} \mathrm{C}$. for $10-20$ minutes. Divide the contents of each tube into two parts, testing one part by the iodine test and testing the other, after neutralization, by Fehling's test. What do you find?

(b) Influence of Combined Acid (Protein Salt). - Repeat the first three experiments of the above series using combined hydrochloric acid (see page I40) instead of the free acid. How does the action of the combined acid differ from that of the free acid? (For a discussion of combined acid see page 140.

(c) Influence of Alkali-Repeat the first four experiments under (a) replacing the $\mathrm{HCl}$ by 2 per cent, I per cent, 0.5 per cent and 0.25 per cent $\mathrm{Na}_{2} \mathrm{CO}_{3}$. Neutralize the alkalinity before trying the iodine test (see Starch, 5, page 45).

(d) Nature of the Action of Acid and Alkali.-Place 2 c.c. of saliva and 2 c.c. of 0.2 per cent $\mathrm{HCl}$ in a test-tube and leave for 15 minutes. Neutralize the solution, add 4 c.c. of starch paste and place the tube in the incubator or waterbath at $40^{\circ} \mathrm{C}$. In 10 minutes test by the iodine and Fehling's tests and explain the result. Repeat the experiment, replacing the 0.2 per cent $\mathrm{HCl}$ by 2 per cent $\mathrm{Na}_{2} \mathrm{CO}_{3}$. What do you deduce from these two experiments?

19. Influence of Metallic Salts, étc. - In each of a series of tubes place + c.c. of starch paste and $1 / 2$ c.c. of one of the solutions named below. Shake well, add $1 / 2$ c.c. of saliva to each tube, thoroughly mix, and place in the incubator or waterbath at $40^{\circ} \mathrm{C}$. for $10-20$ minutes. Show the progress of digestion by means of the iodine and Fehling tests. Use the following chemicals: Melallic salts, Io per cent lead acetate, 2 per cent copper sulphate, 5 per cent ferric chloride, 8 per cent mercuric chloride; Neutral salts, ro per cent sodium chloride, to per cent magnesiun sulphate, 3 per cent barium chloride, io per cent Rochelle salt. Also try the influence of 2 per cent carbolic acid, 95 per cent alcohol, and ether and chloroform. What are your conclusions?

Antiseptics do not necessarily inhibit enzyme action.

20. Excretion of Potassium Iodide.-Ingest a small dose of potassium iodide (0.2 gram) contained in a gelatin capsule, quickly rinse out the mouth with water, and then test the saliva at once for iodine. This test should be negative. Make additional tests for iodine at two-minute intervals. The test for iodine is made as follows: Take I c.c. of $\mathrm{NaNO}_{2}$ and I c.c. of dilute $\mathrm{H}_{2} \mathrm{SO}_{4}{ }^{1}$ in a testtube, add a little saliva directly from the mouth, and a small amount of starch paste. The formation of a blue color signifies that the potassium iodide is being excreted through the salivary glands. Note the length of time elapsing between the ingestion of the potassium iodide and the appearance of the first traces of the substance in the saliva. If convenient, the urine may also be tested. The

${ }^{1}$ Instead of this mixture a few drops of $11 \mathrm{NO}_{3}$ possessing a yellowish or brownish color due to the presence of IINO. may be empluyed. 
chemical reactions taking place in this experiment are indicated in the following equations :
(a)
(b)
(c)

$2 \mathrm{NaNO}_{2}+\mathrm{H}_{2} \mathrm{SO}_{4} \rightarrow 2 \mathrm{HNO}_{2}+\mathrm{Na}_{2} \mathrm{SO}_{4}$. $2 \mathrm{KI}+\mathrm{H}_{2} \mathrm{SO}_{4} \rightarrow 2 \mathrm{HI}+\mathrm{K}_{2} \mathrm{SO}_{4}$. ${ }_{2} \mathrm{HNO}_{2}+{ }_{2} \mathrm{HI} \rightarrow \mathrm{I}_{2}+2 \mathrm{H}_{2} \mathrm{O}+2 \mathrm{NO}$. 


\section{CHAPTER IV}

\section{PROTEINS:1 THEIR DECOMPOSITION AND SYNTHESIS}

THE proteins are a class of substances, which in the light of our present knowledge, consist, in the main, of combinations of $\alpha$-amino acids or their derivatives. These protein substances form the chief constituents of many of the fluids of the body, constitute the organic basis of animal tissue, and at the same time occupy a decidedly preeminent position among our organic food-stuffs. They are absolutely necessary to the uses of the animal organism for the continuance of life and they cannot be satisfactorily replaced in the diet of such an organism by any other dietary constituent either organic or inorganic. Such an organism may exist without protein food for a period of time, the length of the period varying according to the specific organism and the nature of the substitution offered for the protein portion of the diet. Such a period is, however, distinctly one of existence rather than one of normal life and one which is consequently not accompanied by such a full and free exercise of the various functions of the organism as would be possible upon an evenly balanced ration, i.e., one containing the requisite amount of protein food. These protein substances are, furthermore, essential constituents of all living cells and therefore without them iegetable life as well as animal life is impossible.

The proteins, which constitute such an important group of substances, differ from carbohydrates and fats very decidedly in elementary composition. In addition to containing carbon, hydrogen, and oxygen, which are present in fats and carbohydrates, the proteins invariably contain nitrogen in their molecule and generally sulphur also. Proteins have also been described which contain phosphorus, iron.copper, iodine, manganese, and zinc. The percentage composition of the more important members of the group of protein substances would fall within the following limits: $\mathrm{C}=50-55$ per cent, $\mathrm{H}=6-7.3$ per cent, $\mathrm{O}=$ I 9-24 per cent, $\mathrm{N}=\mathrm{I}_{5}-\mathrm{I} 9$ per cent, $\mathrm{S}=0.3^{-2.5}$ per cent, $\mathrm{P}=0.4^{-}$ 0.8 per cent when present. When iron, copper, iodine, manganese or zinc are present in the protein molecule they are practically without

1 The term proteid has been very widely used by Lnglish-speaking scientists to signify the class of substances we have called proleins. 
exception present only in traces and with the exception of iodine are probably not constituents of the protein molecule. ${ }^{1}$

Of all the various elements of the protein molecule, nitrogen is by far the most important. The human body needs nitrogen for the continuation of life, but it cannot use the nitrogen of the air or that in various other combinations as we find it in nitrates, nitrites, etc. However, in the protein molecule the nitrogen is present in a form which is utilizable by the body. The nitrogen in the protein molecule occurs in at least four different forms as follows:

I. Monamino acid nitrogen.

II. Diamino acid nitrogen or basic nitrogen.

III. Amide nitrogen.

IV. A guanidine residue.

The actual structure of the protein molecule is still unknown, and we have as yet no means by which its molecular weight can be even approximately established. The many attempts which have been made to determine this have led to very different results, some of which are given in the following table:

$$
\begin{aligned}
& \text { Globin }=I_{5} 000-\simeq 6086 \\
& \text { Oxyhemoglobin }=\mathrm{I}_{4} 800-\mathrm{I} 5000-\mathrm{I} 6655-\mathrm{I} 6730
\end{aligned}
$$

Of these figures, those given for oxyhemoglobin deserve the most consideration, for these are based on the atomic ratios of the sulphur and iron contained in this substance. The simplest formula that can be calculated from analyses of oxyhemoglobin, namely,

$$
\mathrm{C}_{658} \mathrm{H}_{1181} \mathrm{~N}_{207} \mathrm{~S}_{2} \mathrm{FeO}_{210}
$$

serves to show the great complexity of this substance.

The decomposition ${ }^{2}$ of protein substances may be brought about by oxidation or hydrolysis, but inasmuch as the hydrolytic procedure has been productive of the more satisfactory results, that type of decomposition procedure alone is used at present. This hydrolysis of the protein molecule may be accomplished by acids, alkalis, or superheated steam, and in digestion by the action of the proteolytic enzymes. The character of the decomposition products varies according to the method utilized in tearing the molecule apart. Bearing this in mind, we may say that the decomposition products of proteins include proteoses, peptones, peptides, carbon dioxide, ammonia, hydrogen sulphide, and amino

${ }^{1}$ Some investigators regard these elements as contaminations, or constituents of some non-protein substance combined with the protein.

2"The terms "degradation," "dissociation," and "cleavage," are often used in this connection. 
acids. These amino acids ${ }^{1}$ constitute a long list of important substances which contain nuclei belonging either to the aliphatic, carbocyclic, or heterocyclic series. The list includes glycocoll, alanine, serine, phenylalanine, tyrosine, cystine, tryptophane, histidine, valine, arginine, leucine, isolencine, lysine, aspartic acid, glutamic acid, proline, oxyproline, and diaminotrihydroxydodecanoic acid. Of these amino acids, tyrosine and phenylalanine contain carbocyclic nuclei: histidine, proline, and tryptophane contain heterocyclic nuclei: and the remaining members of the list, as given, contain aliphatic nuclei. The amino acids are preëminently the most important class of protein decomposition products. These amino acids are all $\alpha$-amino acids, and, with the exception of glycocoll, are all optically active. Furthermore, they are amphoteric substances and consequently are able to form salts with both bases and acids. These properties are inherent in the $\mathrm{NH}_{2}$ and $\mathrm{COOH}$ groups of the amino acids.

The decomposition products of protein may be grouped as primary and secondary decomposition products. By primary products are meant those which exist as radicals within the protein molecule and which are liberated, upon cleavage of this molecule, with their carbon chains intact and the position of their nitrogen unaltered. The secondary products are those which result from the disintegration of the primary cleavage products. No matter what method is used to decompose a given protein molecule, the primary products are largely the same under all conditions. ${ }^{2}$

In the process of hydrolysis the protein molecule is gradually broken down and less complicated aggregates than the original molecule are formed, which are known as proteoses, peptoncs, and peptides, and which still possess true protein characteristics. Further hydrolysis causes the ultimate transformation of these substances, of a protein nature, into the amino acids of known chemical structure. In this decomposition the protein molecule is not broken down in a regular manner into $1 / 2,1 / 4$, $1 / 8$ portions and the amino acids formed in a group at the termination of the hydrolysis. On the contrary, certain amino acids are formed very early in the process, in fact while the main hydrolytic action has proceeded no further than the proteose stage. Gradually the complexity of the protein portion undergoing decomposition is simplified by the splitting off of the amino acids and finally it is so far decomposed through previous cleavages that it yields only amino acids at the succeeding cleavage. In short, the general plan of the ly-drolysis of the protein molecule is similar to the hydrolysis of starch. In the case

${ }^{1}$ For a discussion of amino acids see Underhill's "Plyysiology of Amino Acids," Tale University Press, Nov., I9r 5.

${ }^{2}$ Alkaline hydrolysis yields urea and ornithine which result from arginine, the product of acid hydrolysis. 
of starch there is formed a series of dextrins of gradually decreasing complexity and coincidently with the formation of each dextrin a small amount of sugar is split off and finally nothing but sugar remains. In the case of protein hydrolysis there is a series of proteins of gradually decreasing complexity produced and coincidently with the formation of each new protein substance amino acids are split off and finally the sole products remaining are amino acids.

Inasmuch as diversity in the method of decomposing a given protein does not result in an equally diversified line of decomposition products, but, on the other hand, yields products which are quite comparable in character, it may be argued that there are probably well-defined lines of cleavage in the individual protein molecule and that no matter what the force brought to bear to tear such a molecule apart, the disintegration, when it comes, will yield in every case certain definite fragments. These fragments may be called the "building stones" of the protein molecule, a term used by some of the German investigators. Take, for example, the decomposition of protein which may be brought about through the action of the enzyme trypsin of the pancreatic juice. When this enzyme is allowed to act upon a given protein, the latter is disintegrated in a series of definite cleavages, resulting in the formation of proteoses, peptones, and peptides in regular order, the peptides being the last of the decomposition products which possess protein characteristics. They are all built up from amino acids and are therefore closely related to these acids on the one side and to peptones on the other. We have di-, tri-, tetra-, penta-, deca-, and poly-peptides which are named according to the number of amino acids included in the peptide molecule. Following the peptides there are a diverse assortment of monamino and diamino acids which constitute the final products of the protein decomposition. These acids are devoid of any protein characteristics and are therefore decidedly different from the original substance from which they were derived. From a protein of huge molecular weight, a typical colloid, perhaps but slightly soluble, and entirely non-diffusible, we have passed by way of proteoses, peptones, and peptides to a class of simpler crystalline substances which are, for the most part, readily soluble and diffusible.

These amino acids after their production in the process of digestion, as just indicated, are synthesized within the cells of the organism to form protein material which goes to build up the tissues of the body. It is thus seen that the amino acids are of prime importance in the animal economy. It was formerly believed that these essential factors in metabolism and nutrition could not be produced within the animal organism from their elements, but were only yielded upon the hydrol- 
ysis of ingested protein of animal or vegetable origin. Experiments, however, by Abderhalden and by Grafe and Schläpfer and others indicate that the nitrogen of food protein may in part be replaced by ammonium salts. Experiments by Osborne and others also indicate amino acid synthesis by animals.

Important data regarding the decomposition products of the protein molecule are given in the tables which follow.

COMPARISON OF THE DECOMPOSITION PRODUCTS OF PROTAMINES, AND OTHER PROTEINS.

\begin{tabular}{|c|c|c|c|c|c|c|c|c|c|c|c|c|c|}
\hline \multirow[b]{2}{*}{$\begin{array}{l}\text { Decomposition } \\
\text { Product. }\end{array}$} & \multicolumn{7}{|c|}{$\begin{array}{c}\text { ProtaniNes. } 1 \\
\text { (Per cent of total nitrogen of } \\
\text { amino acid.) }\end{array}$} & \multicolumn{6}{|c|}{$\begin{array}{c}\text { OTHER Protelis. } \\
\text { (Per cent of amino acids in } \\
\text { proteins.) }\end{array}$} \\
\hline & $\frac{\stackrel{\mathscr{E}}{E}}{\tilde{E}}$ & 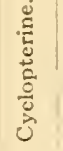 & 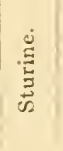 & 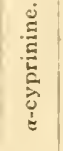 & 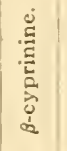 & 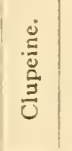 & $\frac{\ddot{g}}{\stackrel{\Xi}{\Xi}}$ & 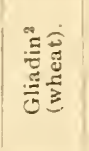 & 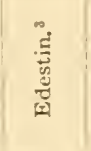 & نُ & 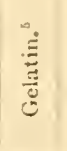 & $\frac{0}{3}$ & ڤั \\
\hline Glycocoll.... & & & & & & . & ... & o & 3.8 & o & 16.5 & 0 & o \\
\hline Alanine........... & + & 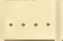 & + & $\ldots$ & 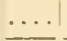 & + & $\cdots$ & 2.00 & 3.6 & 1.5 & 0.8 & 4.2 & 9.79 \\
\hline Valine............... & $\ldots$ & $\cdots$ & $\ldots$ & + & + & +1 & 1.65 & 3.34 & 6.2 & 7.2 & 1.0 & $\cdots \cdots$ & I. 88 \\
\hline Leucine............. & $\cdots$ & $\ldots$ & + & $\ldots$ & $\cdots$ & $\cdots$ & $\cdots$ & 6.62 & $14 \cdot 5$ & 9.4 & 2.1 & 29.0 & 19.55 \\
\hline Proline.............. & 3.8 & $\cdots$ & $\cdots$ & $\because \cdots$ & $\cdots$ & +4 & $4 \cdot 3$ & 13.22 & 4.1 & 6.7 & 5.2 & 2.3 & 9.04 \\
\hline Phenylalanine. . & $\ldots$ & $\ldots$ & $\cdots$ & $\cdots$ & $\cdots$ & $\cdots$ & $\cdots$ & 2.35 & 3.1 & 3.2 & 0.4 & 4.2 & 6.55 \\
\hline Aspartic acid.......... & $\cdots$ & $\cdots$ & $\cdots$ & $\cdots$ & $\ldots$ & $\ldots$ & $\ldots$ & 0.58 & $4 \cdot 5$ & I. 4 & 0.56 & $4 \cdot 4$ & $1.7 I$ \\
\hline Glutamic acid. . & $\ldots$ & $\cdots$ & $\ldots$ & $\cdots$ & $\cdots$ & $\cdots$ & $\cdots$ & 43.66 & 18.74 & II.O & I. 88 & 1.7 & 26.17 \\
\hline Serine........ & $\ldots$ & . & $\ldots$ & $\ldots$ & $\ldots$ & $1+13$ & 3.25 & 0.13 & 0.33 & 0.5 & 0.4 & 0.6 & 1.02 \\
\hline Tyrosine.. & $\ldots$ & 2.2 & $\ldots \ldots$ & + & I. 5 & $\cdots$ & $\ldots$ & 1.20 & 2.1 & $4 \cdot 5$ & $\circ$ & 1.3 & 3.55 \\
\hline Arginine...$\ldots \ldots \ldots$ & 88.8 & 67.7 & $63 \cdot 5$ & 8.7 & 28.0 & 88.0 & 89.2 & 3.16 & 14.2 & 4.84 & 7.62 & 5.4 & $I .55$ \\
\hline Lysine... & $\ldots$ & $\ldots$ & 8.4 & $30 \cdot 3$ & 6.6 & $\cdots$ & $\ldots$ & 0 & I. 7 & 5.95 & 2.75 & $4 \cdot 3$ & 0 \\
\hline Histidine............. & $\ldots$ & $\ldots$ & I I. .8 & $\ldots$ & $\therefore$ & $\therefore$ & $\cdots$ & $0.6 \mathrm{I}$ & 2.2 & 2.50 & 0.40 & 11.0 & 0.82 \\
\hline Tryptophane. & + & +1 & $\therefore$ & $\cdots$ & $\therefore$ & & $\cdots$ & 1.0 & + & $\mathrm{I} .5$ & 0 & + & 0 \\
\hline 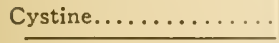 & $\cdots$ & $\cdots$ & $\cdots$ & $\cdots$ & $\cdots$ & $\cdots$ & $\cdots$ & 0.45 & 1.00 & 0.065 & 0 & 0.3 & $?$ \\
\hline Oxyproline............ & $\cdots$ & $\cdots$ & $\cdots$ & $\cdots$ & $\cdots$ & $\cdots \cdots$ & $\cdots$ & $?$ & 2.0 & 0.23 & 6.4 & I. 0 & $?$ \\
\hline $\begin{array}{l}\text { Diaminotrihydroxydo... } \\
\text { decanoic acid. }\end{array}$ & & & & & & & . & .. & $\ldots \ldots$ & 0.75 & & & $?$ \\
\hline Ammonia. & $\cdots$ & & & & & & ${ }^{\circ}$ & 5.22 & 2.3 & 1.61 & & & 3.64 \\
\hline
\end{tabular}

When we examine the formulas of the principal members of the crystalline end-products of protein decomposition we note that they are

- Kossel: Zeit. physiol. Chem., 44, 347, 1905.

${ }^{2}$ Osborne and Guest: Jour. Biol. Chem., 9, 425, I9 I1.

${ }^{3}$ Abderhalden, Kossel and others.

Abderhalden, Fischer, Mörner and others.

Fischer, Levene and Aders: Zeil. physiol. Chem., 35, 70, rgo2; also Levene and Beatty: Ibid., 49, 252, r 906.

'Abderhalden: Zeit. physiol. Chem., 37, 4S4, r 903.

7 Osborne and Liddle, Am. Jour. Physiol., 26, 295, 1910.

* This unique and important protein has probably been more carefully analyzed than any other. 


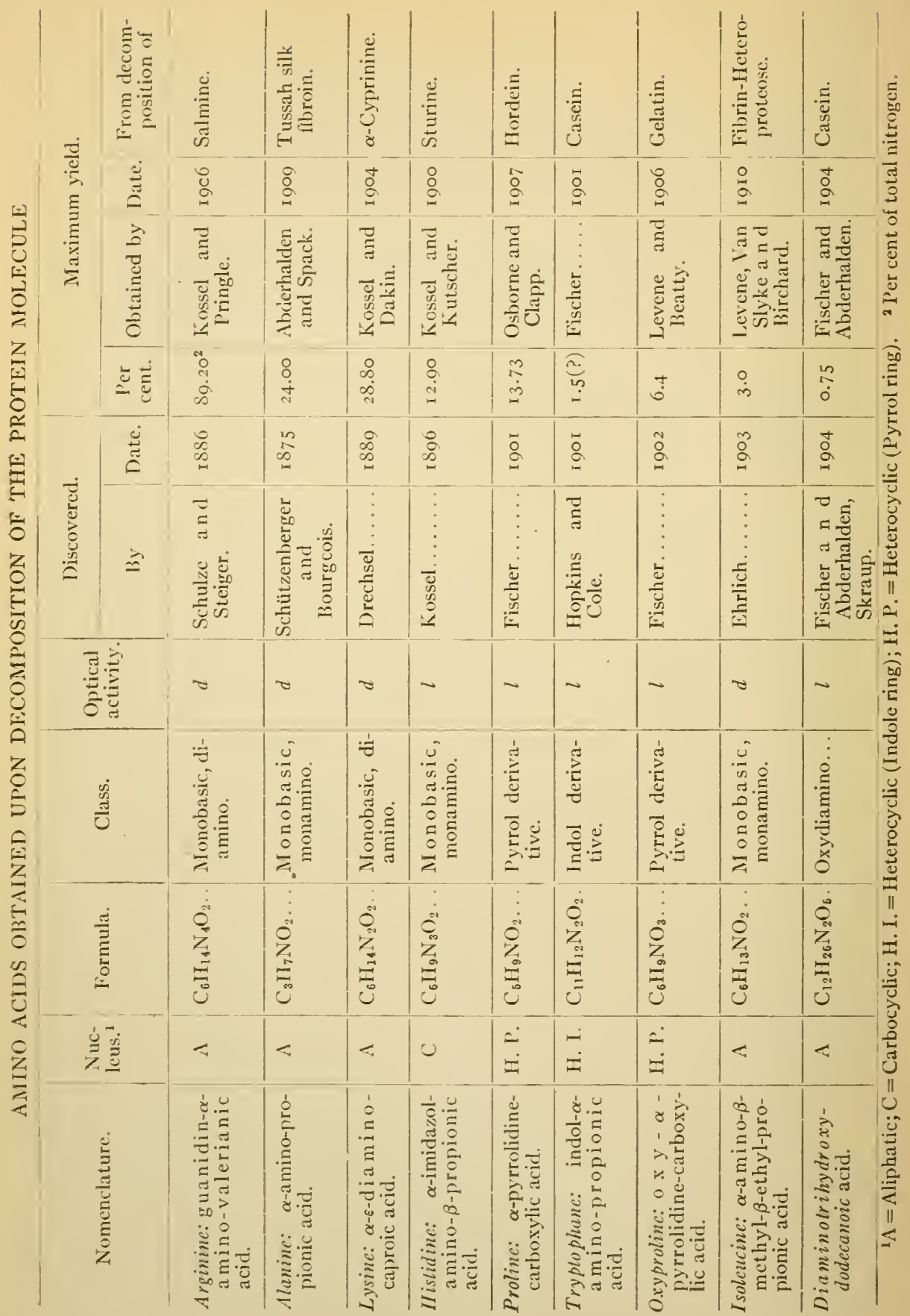




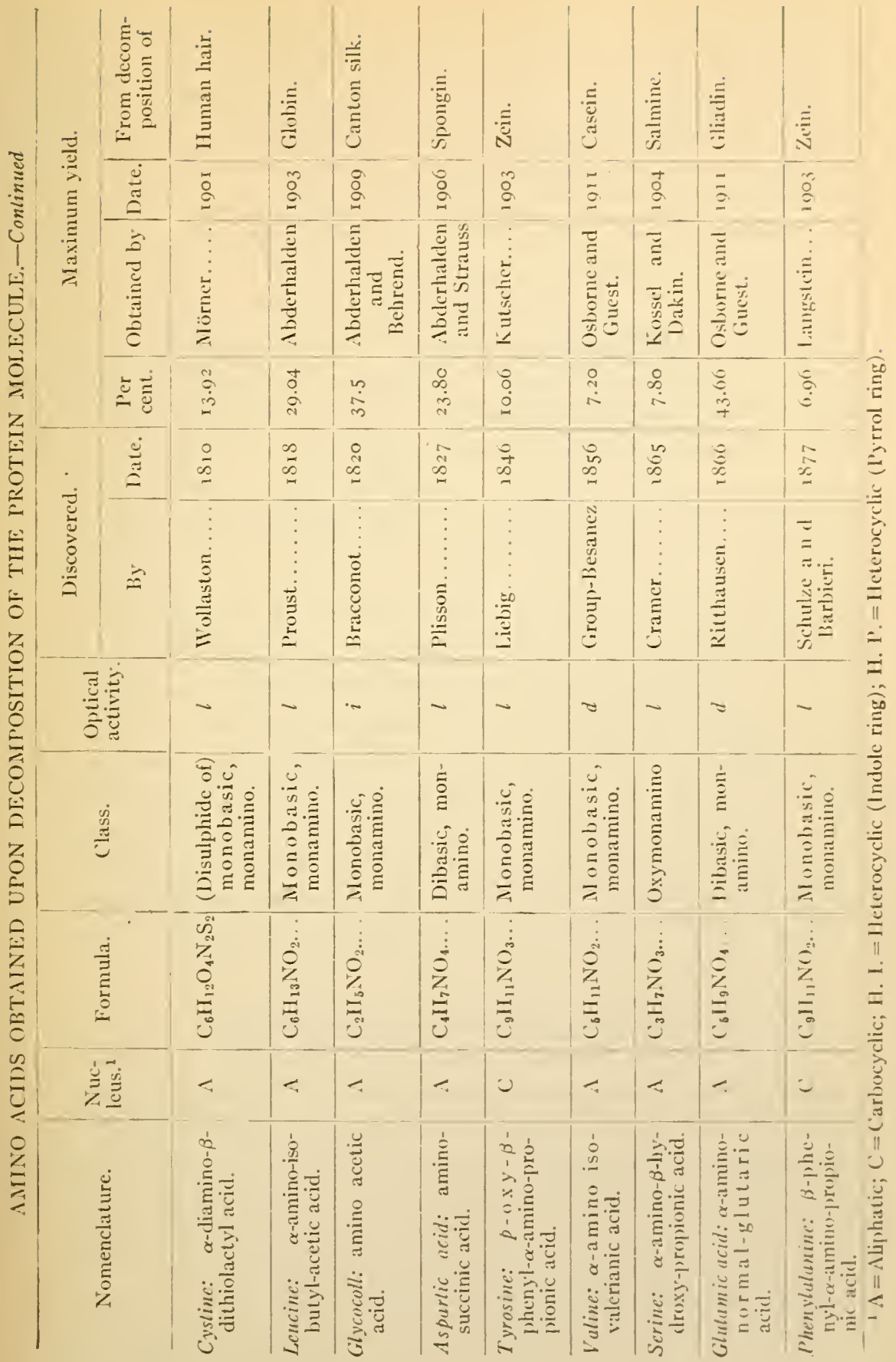


invariably acids, as has already been mentioned, and contain an $\mathrm{NH}_{2}$ group in the $\alpha$ position. This relation of the $\mathrm{NH}_{2}$ group to the acid radical is constant, no matter what other groups or radicals are present. We may have straight chains as in alanine and glutamic acid, the benzene ring as in phenylalanine, or we may have sulphurized bodies as in cystine and still the formula is always of the same type, i.e.,

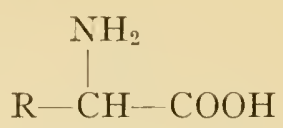

It is seen that this characteristic grouping in the amino acid provides each one of these ultimate fragments of the protein molecule with both a strong acid and a strong basic group. For this reason it is theoretically possible for a large number of these amino acids to combine and the resulting combinations may be very great in number, since there is such a varied assortment of the acids. The protein molecule, which is of such mammoth proportions, is probably constructed on a foundation of this sort. Much valuable data have been collected regarding the synthetic production of protein substances, the leaders in this line of inrestigation being Fischer and Abderhalden. After having gathered a mass of data regarding the final products of the protein decomposition and demonstrating that amino acids were the ultimate results of the various forms of decomposition, these investigators, and notably Fischer, set about in an effort to form, from these amino acids, by synthetic means, substances which should possess protein characteristics. The simplest of these bodies formed in this way was synthesized from two molecules of glycocoll with the liberation of water, thus:

$$
\mathrm{CH}_{2}-\left(\mathrm{NH}_{2}\right)-\mathrm{CO} \text { OH H HN}-\mathrm{CH}_{2}-\mathrm{COOH} \text {. }
$$

The body thus formed is a dipeptide, called glycyl-glycine. In an analogous manner may be produced leucyl-leucine, through the synthesis of two molecules of leucine or leucyl-alanyl-glycine through the union of one molecule of leucine, one of alanine, and one of glycocoll. 'By this procedure Fischer and his pupils have been able to make a large number of peptides containing varied numbers of amino acid radicals, the name polypeptides being given to the whole group of synthetic substances thus formed. One of the most complex polypeptides yet produced is one containing fifteen glycocoll and three leucine residues.

Notwithstanding the fact that most synthetic polypeptides are produced through a union of amino acids by means of their imide bonds, it must not be imagined that the protein molecule is constructed from 
amino acids linked together in straight chains in a manner analogous to the formation of simple peptides, such as glycyl-glycine. 'The molecular structure of the proteins is much too complex to be explained upon any such simple formation as that. There must be a variety of linkings. since there is a varied assortment of decomposition products of totally different structure.

Many of these synthetic bodies respond to the biuret test, are precipitated by phosphotungstic acid, and behave, in other ways, as to leave no doubt as to their protein characteristics. For instance, a number of amino acids each possessing a sweet taste have been synthesized in such a manner as to yield a polypeptide of bitter taste, a well-known characteristic of peptones. From the fact that the polypeptides formed in the manner indicated have free acidic and basic radicals we gather the explanation of the amphoteric character of true proteins.

For the benefit of those especially interested in such matters a photograph of the Fischer apparatus (Fig. 24, page 75) used in the fractional distillation, in vacuo, of the esters of the decomposition products of the proteins, as well as micro-photographs and drawings of preparations of several of these decomposition products (Figs. 2 I to 33 , pages 72 to 84 ) are introduced. For the preparations and the photograph of the apparatus the author is indebted to Dr. T. B. Osborne, of New Haven, Conn., who has made many important observations upon the hydrolysis of proteins. The reproduction of the crystalline form of some of the more recent of the products may be of interest to those viewing the field of physiological chemistry from other than the student's aspect.

An extended discussion of the various decomposition products being out of place in a book of this character, we will simply make a few general statements in connection with the primary decomposition products.

\section{DISCUSSION OF THE PRODUCTS}

Ammonia, $\mathrm{NH}_{3}$.-Ammonia is an important decomposition product of all proteins and probably arises from an amide group combined with a carboxyl group of some of the amino acids. It is possible that the dibasic acids, aspartic and glutamic, furnish most of these carboxyl groups. This is indicated by the more or less close relationship which exists betreen the amount of ammonia and that of the dibasic acids which the several proteins yield upon decomposition. The elimination of the ammonia from proteins under the action of acids and alkalis is very similar to that from amides like asparagine.

Glycocoll, $\mathrm{CH}_{2} \cdot\left(\mathrm{NH}_{2}\right) \cdot \mathrm{COOH}$ - Gilycocoll, or amino acclic acid, is 
the simplest of the amino acids which occurs as a protein decomposition product ${ }^{1}$ and has the following formula:<smiles>NCC(=O)O</smiles>

Glycocoll, as the formula shows, contains no asymmetric carbon atom, and is the only amino acid yielded by protein decomposition which is optically inactive. Glycocoll and leucine were among the first decomposition products of proteins to be discovered. Upon administering benzoic acid to man or lower animals the output of hippuric acid in the

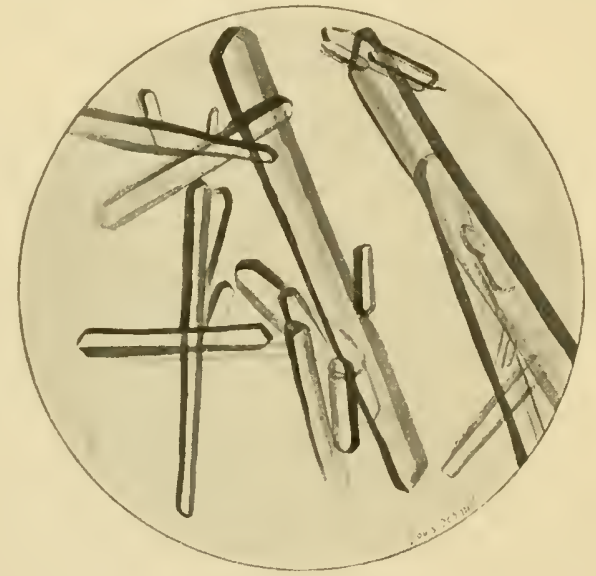

Fig. 21.-Glycocoll Ester Hydrochloride.

urine is greatly increased, thus showing a synthesis of benzoic acid and glycocoll in the organism (see page $5 \delta_{5}$, Chapter XXVII). Glycocoll, ingested in small amount, is excreted in the urine as urea, whereas if administered in excess it appears in part unchanged in the urine. It is usually separated from the mixture of protein decomposition products as the hydrochloride of the ester. The crystalline form of this compound is shown in Fig. $2 \mathrm{I}$.

Alanine, $\mathrm{CH}_{3} \cdot \mathrm{CH}\left(\mathrm{NH}_{2}\right) \cdot \mathrm{COOH}$-- Alanine is $\alpha$-amno-propionic acid, and as such it may be represented structurally as follows:<smiles>CC(N)C(=O)O</smiles>

${ }^{1}$ Amino formic acid (carbamic acid), $\mathrm{NH}_{2} \cdot \mathrm{COOH}$, is the simplest amino acid. 
Obtained from protein substances, alanine is dextro-rotatory; is very soluble in water, and possesses a sweet taste. Tyrosine, phenylalanine, cystine, and serine are derivatives of alanine. This amino acid has been obtained from nearly all proteins examined. Its absence from those proteins from which it has not been obtained has not been proven. Most proteins yield relatively small amounts of alanine.

Serine, $\mathrm{CH}_{2}(\mathrm{OH}) \cdot \mathrm{CH}\left(\mathrm{NH}_{2}\right) \cdot \mathrm{COOH}$.- Serine is $\alpha$-amino- $\beta$ - $h$ y droxypropionic acid and possesses the following structural formula:

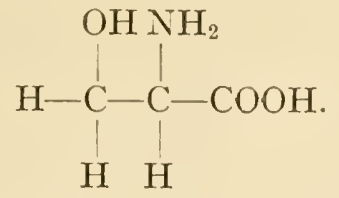

Serine obtained from proteins is levo-rotatory, possesses a sweet taste, and is quite soluble in water. Serine is not obtained in quantity from

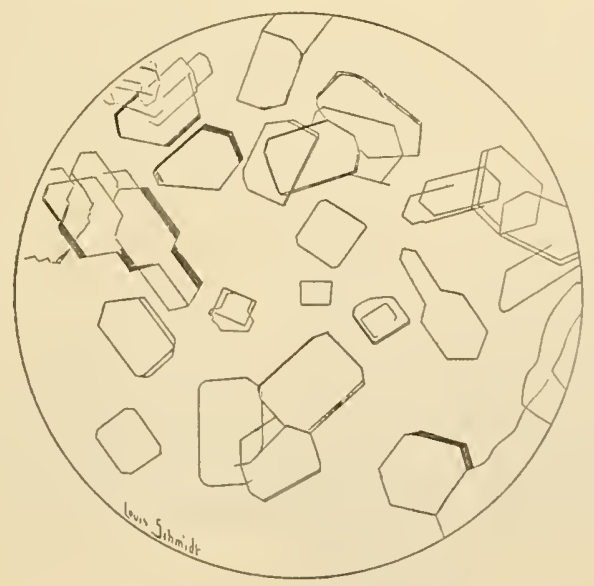

FIG. 22.-SERINE.

most proteins, but is yielded abundantly by silk glue. Owing to the difficulty of separating serine it has not been found in a number of proteins in which it probably occurs. Serine crystals are shown in Fig. 22.

Phenylalanine, $\mathrm{C}_{6} \mathrm{H}_{5} \cdot \mathrm{CH}_{2} \cdot \mathrm{CH}\left(\mathrm{NH}_{2}\right) \cdot \mathrm{COOH}$. This product is $\beta$ phenyl-a-amino-propionic acid, and may be represented graphically as follows:

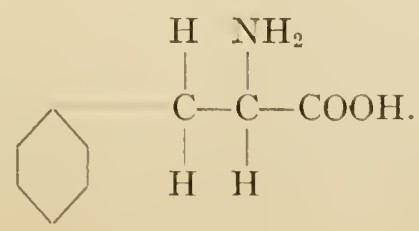


The levo-rotatory form is obtained from proteins. Phenylalanine has been obtained from all the proteins examined except from the protamines and some of the albuminoids. The yield of this body from the decomposition of proteins is frequently greater than the yield of tyrosine. The crystalline form of phenylalanine is shown in Fig. 23.

Tyrosine, $\mathrm{C}_{6} \mathrm{H}_{5}(\mathrm{OH}) \cdot \mathrm{CH}_{2} \cdot \mathrm{CH}\left(\mathrm{NH}_{2}\right) \cdot \mathrm{COOH}$.-Tyrosine, one of the first discovered end-products of protein decomposition, is the amino

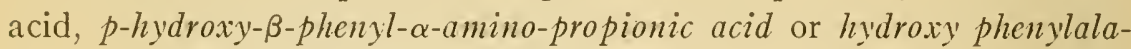
nine. It has the following formula:

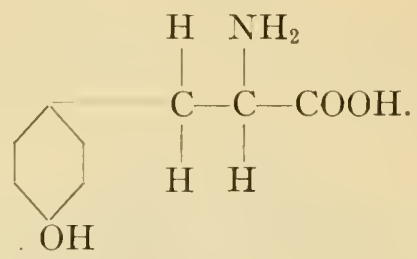

The tyrosine which results from protein decomposition is usually levorotatory. Tyrosine is one of the end-products of tryptic digestion and usually separates in conspicuous amount early in the process of diges-

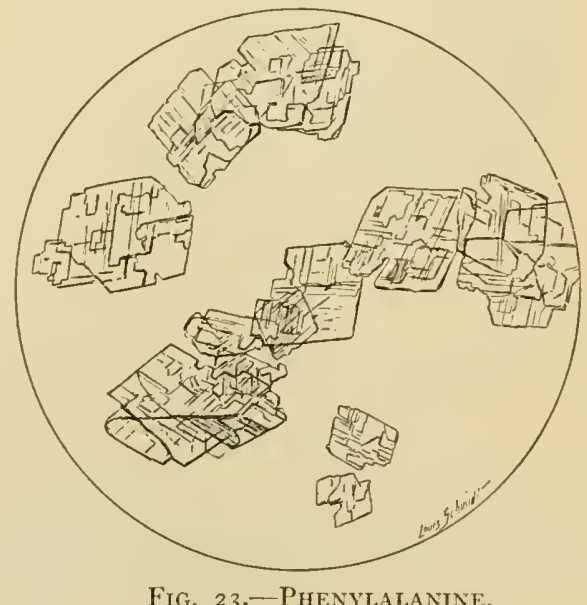

Fig. 23.-Phenylalanine.

tion. It does not occur, however, as an end-product of the decomposition of gelatin.

Tyrosine is found in old cheese, and derives its name from this fact. It crystallizes in tufts, sheaves, or balls of fine needles, which decompose at $295^{\circ} \mathrm{C}$. and are sparingly soluble in cold $(\mathrm{I}-2454)$ water, but much more so in boiling ( $\mathrm{I}-\mathrm{I} 54$ ) water. Tyrosine forms soluble salts with alkalis, ammonia, or mineral acids, and is soluble with difficulty in acetic acid. It responds to Millon's reaction, thus showing the presence 
of the hydroxyphenyl group, but gives no other protein test. The aromatic groups present in tyrosine, phenylalanine, and tryptophane cause proteins to yield a positive xanthoproteic reaction. In severe cases of typhoid fever and smallpox, in acute yellow atrophy of the liver, and in

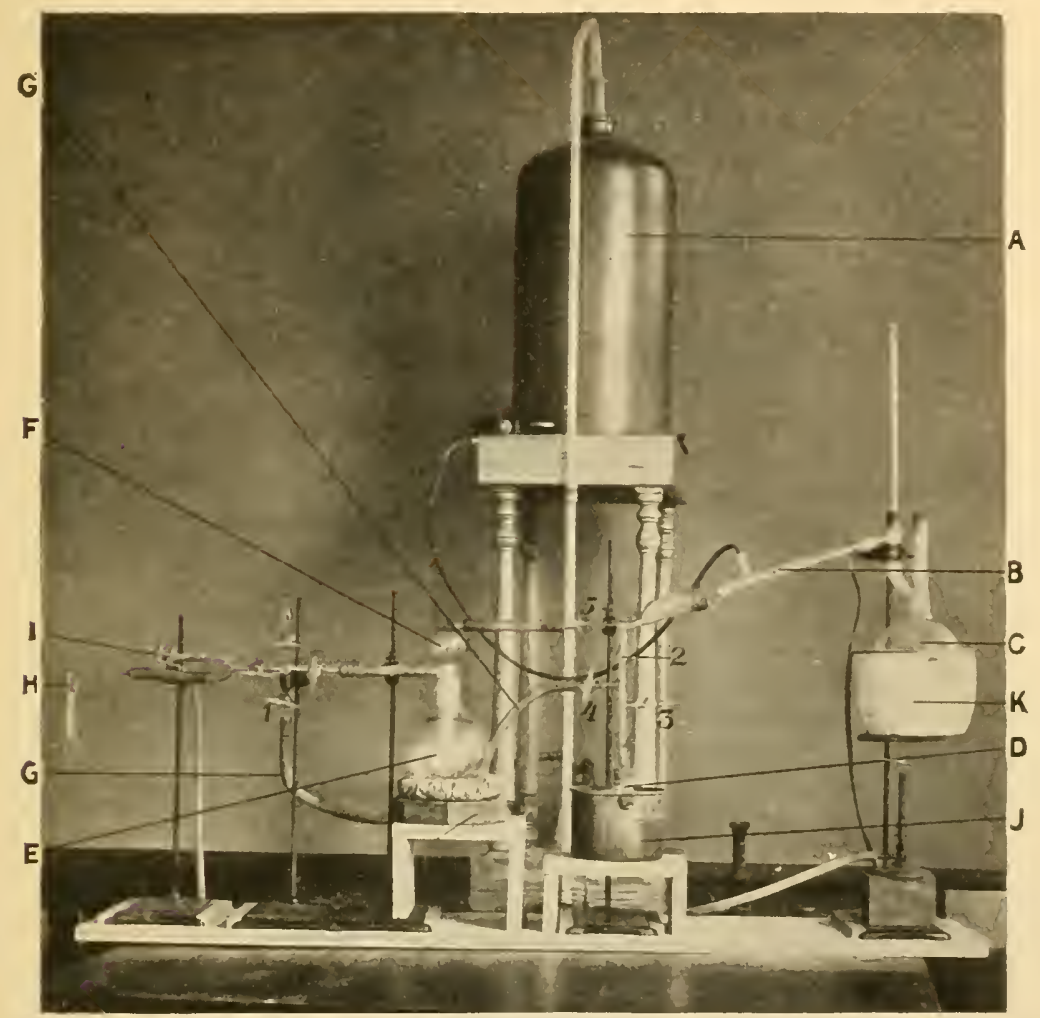

Fig. 24.-Fischer APPARATLS.

Reproduced from a plotograph made by Prof. E. T. Reichert, of the University of Pennsylvania. The negative was furnished by Dr. T. B. Osborne, of New Haven, Conn.

$A$, Tank into which freezing mixture is pumped and from which it flows through the condenser, $B$; $C$, llask from which the esters are distilled, the distillate being collected in $D$; $E$, a Dewar flask containing liquid air serving as a cooler for condensing tube $F ; G$ and $G^{\prime}$, tubes leading to the Geryck pump by which the vacuum is maintained; $I$, tube leading to a McLeod gauge (not shown in figure); $J$, a bath containing freezing mixture in which the receiver $D$ is immersed; $K$, a bath of water during the first part of the distillation and of oil during the last part of the process; $1-5$, stop cocks which permit the cutting out of different parts of the apparatus as the procedure demands.

acute phosphorus poisoning, tyrosine has been found in the urine. Tyrosine crystals are shown in Fig. 25 , page 76 .

Cystine, $\mathrm{C}_{6} \mathrm{H}_{12} \mathrm{O}_{4} \mathrm{~N}_{2} \mathrm{~S}_{2}$. - Friedmann has shown cystine to be $\alpha$-diamino- $\beta$-dithiolactyl acid and to possess the following structural formula : 

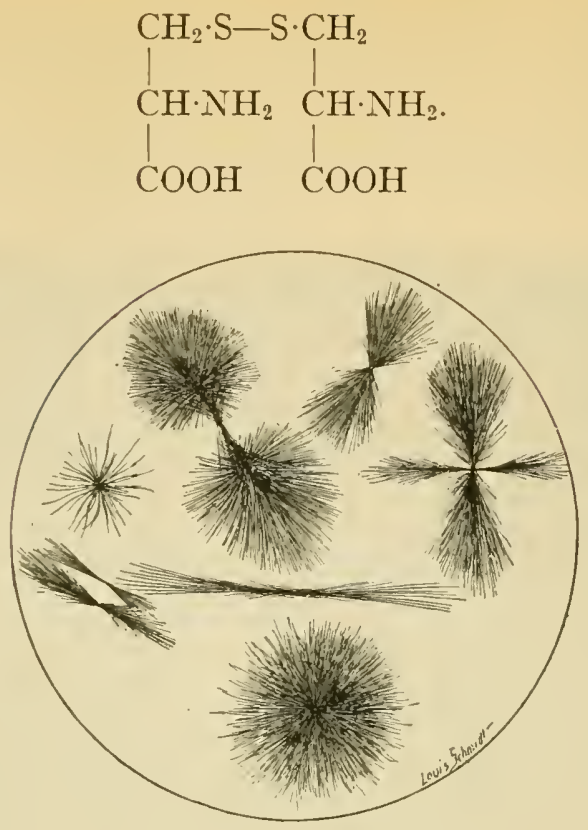

Fig. 25.-TyRosine.

Cystine is the principal sulphur-containing body obtained from the decomposition of protein substances. It is obtained in greatest amount as a decomposition product of keratin-containing tissues as horn, hoof,

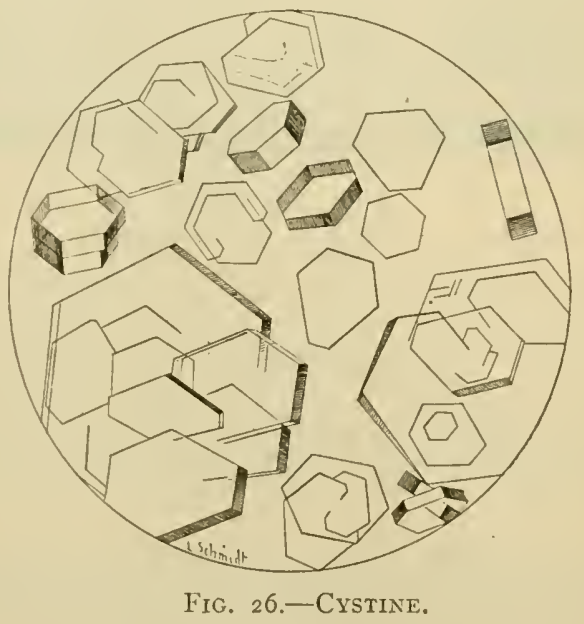

and hair. Cystine occurs in small amount in normal urine and is greatly increased in quantity under certain pathological conditions. It crystallizes in thin, colorless, hexagonal plates which are shown in Fig. 
26. Cystine is very slighlly soluble in water but its salts, with both bases and acids, are readily soluble in water. It is levo-rotatory.

It was formerly claimed that cystine occurred in two forms, i.e., stone-cystine and protein-cystine, and that these two forms are distinct in their properties. This view is incorrect.

For the preparation of cystine from wool or hair see page 87 .

For a discussion of cystine sediments in urine see Chapter XXIV.

Tryptophane, $\mathrm{C}_{8} \mathrm{H}_{6} \mathrm{~N} \cdot \mathrm{CH}_{2} \cdot \mathrm{CH}\left(\mathrm{NH}_{2}\right) \cdot \mathrm{COOH}$.-Recently Ellinger and Flamand have shown that tryptophane possesses the following formula:

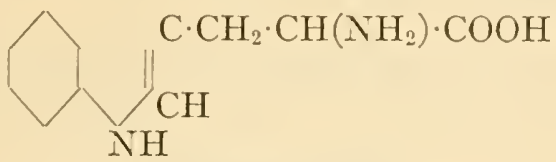

It is therefore $\beta$-indol- $\alpha$-amino-propionic acid. Tyrophane is the molher-substance of indole, skatole, skatole acetic acid and skatole carboxylic acid, all of which are formed as secondary decomposition products of proteins (see Chapter XIII on Putrefaction Products). Its presence in protein substances may be shown by means of the Hopkins-Cole reaction (see page 98). It may be detected in a tryptic digestion mixture through its property of giving a violet color reaction with bromine water. ${ }^{1}$ Tryptophane is yielded by nearly all proteins, but has been shown to be entirely absent from zein, the prolamin (alcohol-soluble protein) of maize and also from gelatin.

According to Osborne and Mendel, ${ }^{2}$ tryptophane is present in maximum amount in lactalbumin. Upon being heated to $285^{\circ} \mathrm{C}$. trytophane decomposes with the evolution of gas.

Histidine, $\mathrm{C}_{3} \mathrm{H}_{3} \mathrm{~N}_{2} \cdot \mathrm{CH}_{2} \cdot \mathrm{CH}\left(\mathrm{NH}_{2}\right) \cdot \mathrm{COOH}$.- Histidine is $\alpha$-amino$\beta$-imidazol-propionic acid or $\beta$-imidazol-alanine with the following structural formula:

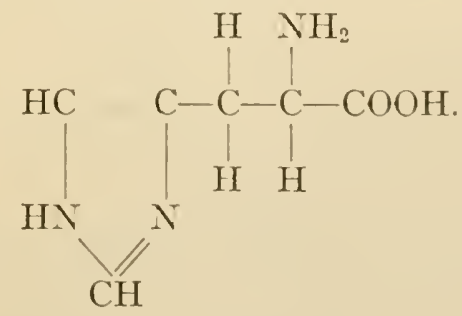

The histidine obtained from proteins is lero-rotatory. It has been obtained from all the proteins thus far examined, the majority of them yielding about 2.5 per cent of the amino acid. Howerer, about I i per

1 Kurajeff: Zeit. physiol. Chem., 36, 501, I 808-99.

${ }^{2}$ Osborne and Mendel: Jour. Biol. Chem., 20, 357, rgi5. 
cent was obtained by Abderhalden from globin, the protein constituent of oxyhemoglobin, and about I 3 per cent by Kossel and Kutscher from the protamine sturine.

Crystals of histidine dichloride are shown in Fig. 27.

Knoop's Color Reaction for Histidine.-To an aqueous solution of histidine or a histidine salt in a test-tube add a little bromine water. A yellow coloration develops in the cold and upon further addition of bromine water becomes permanent. If the tube be heated, ${ }^{1}$ the color will disappear and will shortly be replaced by a faint red coloration which gradually passes into a deep wine red. Usually black, amorphous particles separate out and the solution becomes turbid.

The reaction cannot be obtained in solutions containing free alkali. It is best to use such an amount of bromine as will produce a permanent

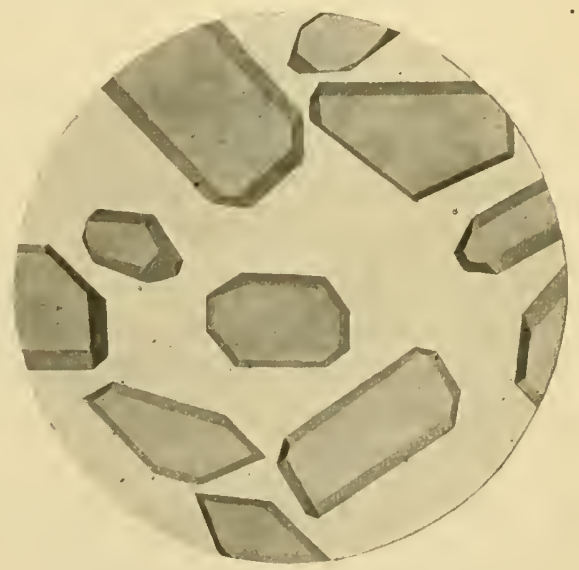

Fig. 27--Histidine Dichloride.

yellow color in the cold. The use of a less amount of bromine than this produces a weak coloration, whereas an excess of bromine prevents the reaction. The test is not very delicate, but a characteristic reaction may always be obtained in I : 1000 solutions. The only histidine derivative which yields a similar coloration is imidazolethylamine, and the reaction in this case is rather weak as compared with the color obtained with histidine or histidine salts.

Valine, $\mathrm{C}_{5} \mathrm{H}_{11} \mathrm{NO}_{2}$. - The amino-valerianic acid obtained from proteins is a-amino-isovalerianic acid, and as such bears the following formula :

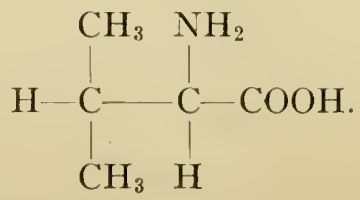

${ }^{2}$ The same reaction will take place in the cold more slowly. 
It closely resembles leucine in many of its properties, but is more soluble in water. It is a difficult matter to identify valine in the presence of leucine and isoleucine inasmuch as these amino acids crystallize together in such a way that the combination persists even after repeated recrystallizations. Valine is dextro-rotatory.

Arginine, $\mathrm{C}_{6} \mathrm{H}_{14} \mathrm{~N}_{4} \mathrm{O}_{2}$.-Arginine is o-guanidine- $\alpha$-amino-íalerianic acid and possesses the following structural formula:

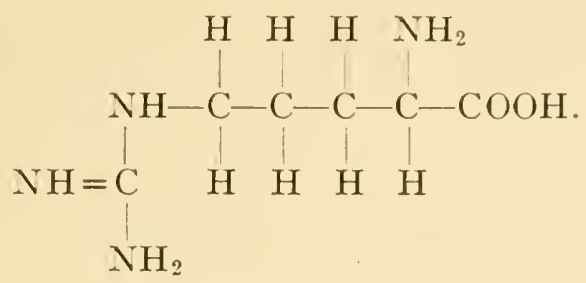

It has been obtained from every protein so far subjected to decomposition. The arginine obtained from proteins is dextro-rotatory, and has pronounced basic properties, reacts strongly alkaline to litmus, and forms stable carbonates. Because of these facts, Kossel considers arginine to be the nucleus of the protein molecule. It is obtained in widely different amounts from different proteins, over 85 per cent of certain protamines having been obtained in the form of this amino acid. It is claimed that in the ordinary metabolic activities of the animal body arginine gives rise to urea. While this claim is probably true, it should, at the same time, be borne in mind that the greater part of the protein nitrogen is eliminated as urea and that, therefore, but a very small part can arise from arginine.

Leucine, $\mathrm{C}_{6} \mathrm{H}_{13} \mathrm{NO}_{2}$.- Leucine is an abundant end-product of the decomposition of protein material, and was one of the first of these products to be discovered. It is $\alpha$-amino-isobutyl-acetic acid, and therefore has the following formula:

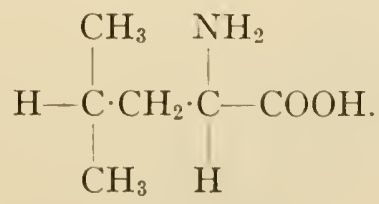

The leucine which results from protein decomposition is l-leucine. Leucine is present normally in the pancreas, thymus, thyroid, spleen, brain, liver, kidneys, and salivary glands. It has been found pathologically in the urine (in acute yellow atrophy of the liver, in acute phosphorus poisoning, and in severe cases of typhoid fever and smallpox), and in the liver, blood, and pus.

Pure leucine crystallizes in thin, white, hexagonal plates. Crystals 
of pure leucine are reproduced in Fig. 28. It is rather easily soluble in water ( 46 parts), alkalis, ammonia, and acids. On rapid heating to $295^{\circ} \mathrm{C}$., leucine decomposes with the formation of carbon dioxide, ammonia, and amylamine. Aqueous solutions of leucine obtained from proteins are levo-rotatory, but its acid or alkaline solutions are dextrorotatory. So-called impure leucine ${ }^{1}$ is a slightly refractive substance, which generally crystallizes in balls having a radial structure, or in aggregations of spherical bodies, Fig. I39, Chapter XXIV.

Isoleucine, $\mathrm{C}_{6} \mathrm{H}_{13} \mathrm{NO}_{2}$. - Isoleucine is $\alpha$-amino- $\beta$-methyl- $\beta$-ethyl-propionic acid, and possesses the following structural formula.
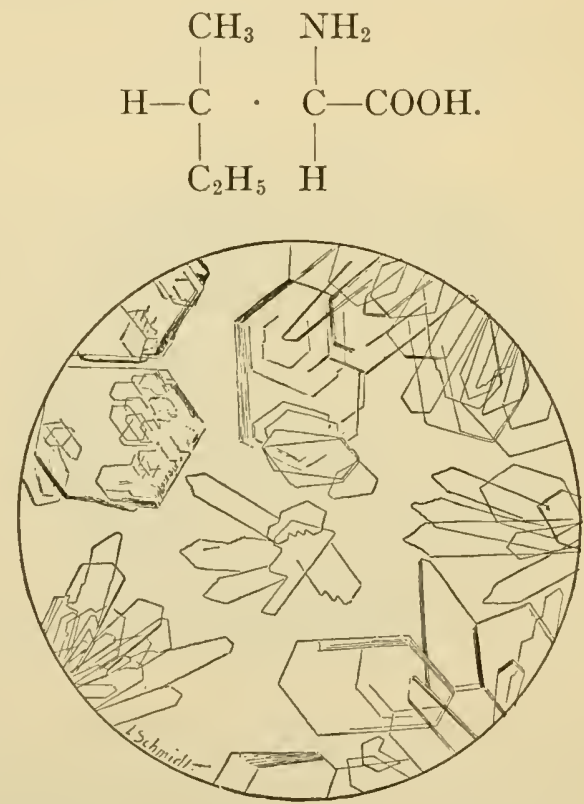

Fig. 28.-LEUCINE.

This amino acid was discovered by Ehrlich in I903. Its presence has been established among the decomposition products of only a few proteins, although it probably occurs among those of many or most of them. Ehrlich has shown that the $d$-amyl alcohol which is produced by yeast fermentation originates from isoleucine and the isoamylalcohol originates from leucine. Isoleucine is dextro-rotatory.

Lysine, $\mathrm{CH}_{2}\left(\mathrm{NH}_{2}\right) \cdot \mathrm{CH}_{2} \cdot \mathrm{CH}_{2} \cdot \mathrm{CH}_{2} \cdot \mathrm{CH}\left(\mathrm{NH}_{2}\right) \cdot \mathrm{COOH}$.-The three bodies, lysine, arginine, and histidine, are frequently classed together as the hexone bases. Lysine was the first of the bases discovered. It is $\alpha$ - - -diamino-caproic acid and hence possesses the following structure:

1 These balls of so-called impure leucine do contain considerable leucine, but inasmuch as they may contain many other things it is a bad practice to allude to them as leucine. 
<smiles>CCCCCC(N)C(=O)O</smiles>

Fig. 29.-Lysine Picrate.

It is dextro-rotatory and is found in relatively large amount in casein and gelatin. Lysine is obtained from nearly all proteins, but is absent from the vegetable proteins which are soluble in strong alcohol. It is

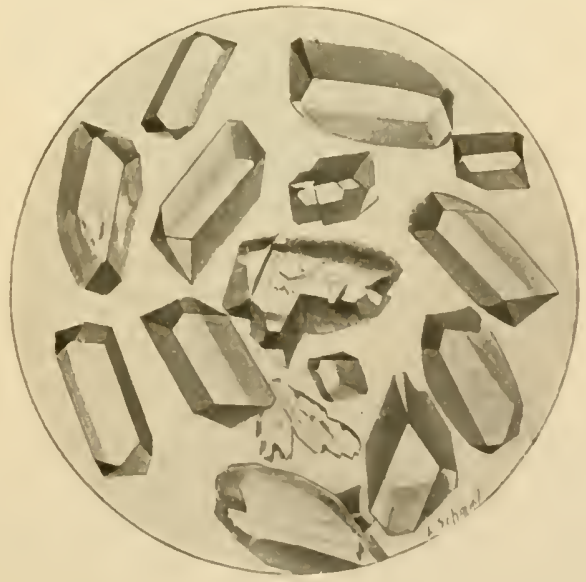

Fig. 30.-Aspartic ACID.

the mother-substance of cadaverin and has never been obtained in crystalline form. Lysine is usually obtained as the picrate which is sparingly soluble in water and crystallizes readily. These crystals are shown in Fig. 29. 
Aspartic Acid, $\mathrm{C}_{4} \mathrm{H}_{7} \mathrm{NO}_{4}$.-Aspartic acid is amino-succinic acid and has the following structural formula:

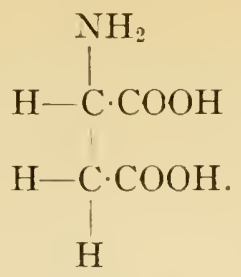

The amide of aspartic acid, asparagine, is very widely distributed in the regetable kingdom. Asparagine has the following formula.

$$
\underset{\mathrm{H}}{\mathrm{H}-\mathrm{C} \cdot \mathrm{COOH}} \stackrel{\mathrm{CH}}{\mathrm{NH}_{2}}
$$

The crystalline form of aspartic acid is exhibited in Fig. 30.

Aspartic acid has been found among the decomposition products of all the proteins examined, except the protamines. It has not been obtained, however, in very large proportion from any of them. The aspartic acid obtained from protein is levo-rotatory.

Glutamic Acid, $\mathrm{C}_{5} \mathrm{H}_{9} \mathrm{NO}_{4}$.-This acid is $\alpha$-amino-normal-glutaric acid and as such bears the following graphic formula:<smiles>CC(C(=O)O)C(N)C(=O)O</smiles>

Glutamic acid is yielded by all the proteins thus far examined, except the protamines, and by most of these in larger amount than any other of their decomposition products. It is yielded in especially large proportion by most of the proteins of seeds, 43.66 per cent having been obtained by Osborne and Guest ${ }^{1}$ by the hydrolysis of gliadin, the prolamin of wheat. This is the largest amount of any single decomposition product yet obtained from any protein except the protamines.

${ }_{1}^{1}$ Osborne and Guest: Jour. Biol. Chem. 9, 425, IgII. 
Glutamic acid and aspartic acid are the only dibasic acids which have thus far been obtained as decomposition products of proteins. As there is an apparent relation between the proportion of these acids and that of ammonia which the different proteins yield it is possible that one of the carboxyl groups of these acids is united with $\mathrm{NH}_{2}$ as an amide, the other carboxyl group being united in polypeptide union (see page 32) with some other amino acid. This might be represented by the following formula:

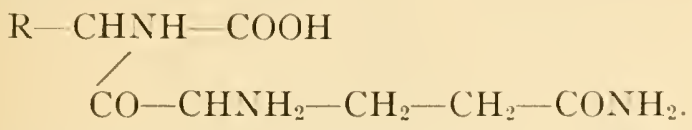

It has been shown by Thierfelder and Sherwin ${ }^{1}$ that the amide, glutamine, is a product of normal metabolism and hence this substance rather than glutamic acid is present in the protein molecule.

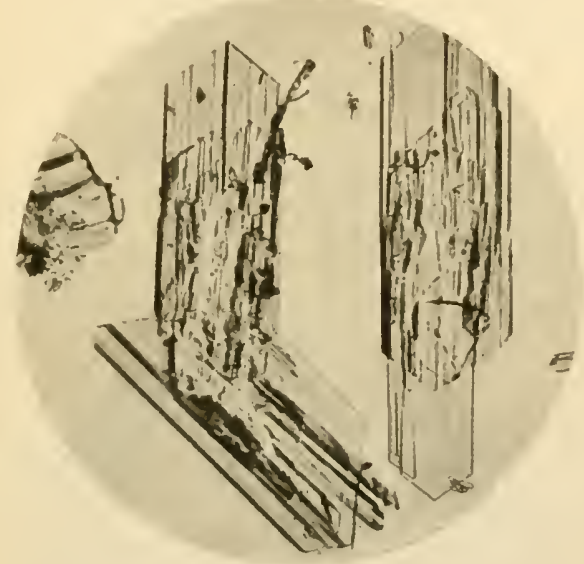

Fig. 31.-Glutamic Acid.

Reproduced from a micro-photograph made by Prof. E. T. Reichert, of the University. of Pennsylvania.

The glutamic acid, yielded by proteins upon hydrolysis, is dextrorotatory. Crystals of glutamic acid are reproduced in Fig. $3 \mathrm{I}$.

Proline, $\mathrm{C}_{5} \mathrm{H}_{9} \mathrm{NO}_{2}$.- Proline is $\alpha$-pyrrolidine-curboxylic acid and possesses the following graphic structure:

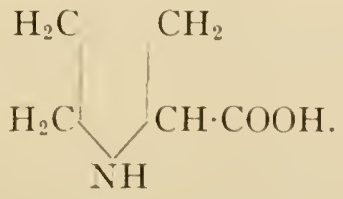

Proline was first obtained as a decomposition product of casein. I'ro-

${ }^{1}$ Thierfelder and Sherwin: Zcil. Physiol. Chemic, 94, 1. 1915. 
line obtained from proteins is levo-rotatory and is the only protein decomposition product which is readily soluble in alcohol. It is also one of the few heterocyclic compounds obtained from proteins. Proline has been found among the decomposition products of all proteins except the

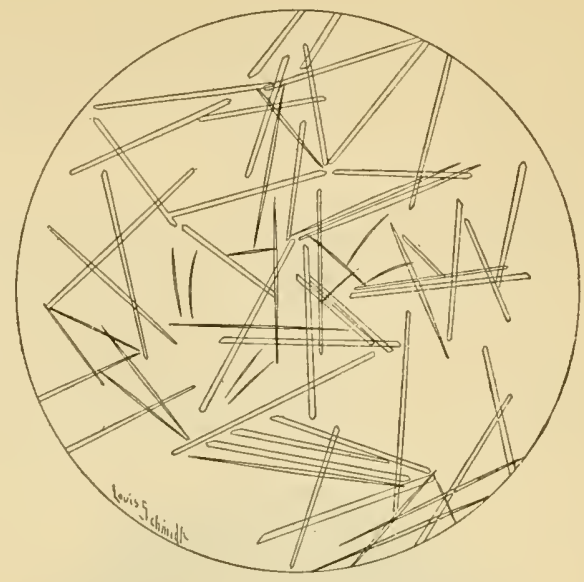

Fig. 32.-LeVo- $\alpha$-Proline.

protamines. The maximum yield reported is $\mathrm{I} 3.73$ per cent obtained by Osborne and Clapp from the hydrolysis of hordein. Fischer and Boehner ${ }^{1}$ have obtained 7.7 per cent from the hydrolysis of gelatin.

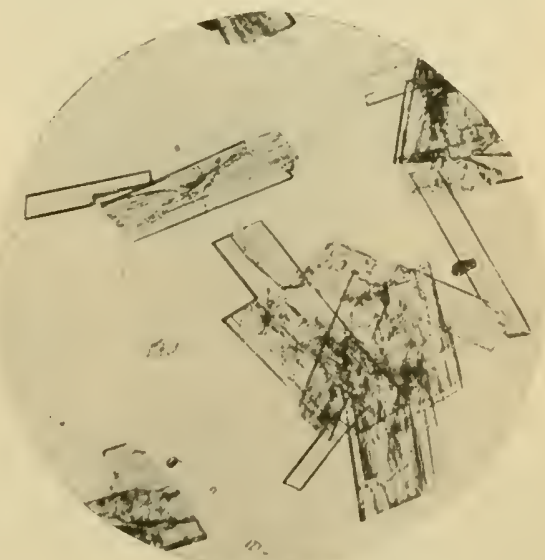

Fig. 33.-Copper Salt of Proline.

Reproduced from a micro-photograph made by Prof. E. T. Reichert, of the University of Pennsylvania.

The crystalline form of levo- $\alpha$-proline is shown in Fig. 32 and the copper salt of proline is represented by a micro-photograph in Fig. 33 . The crystals of the copper salt have a deep blue color, but when they

${ }^{2}$ Fischer and Boehner: Zeit. phys. chem., 65, ir8, rgro. 
lose their water of crystallization they assume a characteristic violet color.

Oxyproline, $\mathrm{C}_{5} \mathrm{H}_{9} \mathrm{NO}_{3}$.-Oxyproline was discovered by Fischer. It has as yet been obtained from only a few proteins, but this may be due to the fact that only a few have been examined for its presence. The position of the hydroxyl group has not yet been established.

Diaminotrihydroxydodecanoic Acid, $\mathrm{C}_{12} \mathrm{H}_{26} \mathrm{~N}_{2} \mathrm{O}_{5}$. - This amino acid was discovered by Fischer and Abderhalden as a product of the hydrolysis of casein. It has thus far been obtained from no other source. It is levo-rotatory and its constitution has not been determined.

\section{EXPERIMENTS}

Protein Decomposition.-While the ordinary courses in physiological chemistry preclude any extended study of the decomposition products of proteins, the manipulation of a simple decomposition and the subsequent isolation and study of a few of the products most easily and quickly obtained will not be without interest. ${ }^{1} \quad$ To this end the student may use the following decomposition procedure.

Treat the protein (coagulated egg albumin) in a large fiask with water containing 3-5 per cent of $\mathrm{H}_{2} \mathrm{SO}_{4}$ and place it on a water-bath until the protein material has been decomposed and there remains a fine, fluffy, insoluble residue. Filter off this residue and neutralize the filtrate with $\mathrm{Ba}(\mathrm{OH})_{2}$ and $\mathrm{BaCO}_{3}$. Filter off the precipitate of $\mathrm{BaSO}_{4}$ which forms and when certain that the fluid is neutral or faintly acid, ${ }^{2}$ concentrate (first on a wire gauze and later on a waterbath) to a syrup. This syrup contains the end-products of the decomposition of the protein, among which are proteoses, peptones, tyrosine, leucine, etc. Add 95 per cent alcohol slowly to the warm syrup until no more precipitate forms, stirring continuously with a glass rod. This precipitate consists of proteoses and peptones. Gather the sticky precipitate on the rod or the sides of the dish and, after warming the solution gently for a few moments, filter it through a filter paper which has not been previously moistened. After dissolving the precipitate of proteoses and peptones in water ${ }^{3}$ the solution may be treated according to the method of separation given on page r20.

The leucine and tyrosine, etc., are in solution in the warm alcoholic filtrate. Concentrate this filtrate on the water-bath to a thin syrup, transfer it to a beaker, and allow it to stand over night in a cool place for crystallization. The tyrosine first crystallizes (Fig. 25, page 76), followed later by the formation of characteristic

1 The procedure here set forth has nothing in common with the procedure by means of which the long line of decomposition products just enumerated are obtained. This latter process is an exceedingly complicated one which is entirely outside the province of any course in physiological chemistry.

${ }^{2}$ If the solution is alkaline in reaction at this point, the amino acids will be broken down and ammonia will be evolved.

${ }^{3}$ At this point the aqueous solution of the proteoses and peptones may be filtered to remove any $\mathrm{BaSO}_{4}$ which may still remain. Tyrosine crystals will also be found here, since it is less soluble than the leucine and may adhere to the proteose-peptone precipitate. Add the crystals of tyrosine to the warm alcohol filtrate. 
crystals of impure leucine (see Fig. I39, Chapter XXIV). After examining these crystals under the microscope, strain off the crystalline material through fine muslin, heat it gently in a little water to dissolve the leucine (the tyrosine will be practically insoluble) and filter. Concentrate the filtrate and allow it to stand in a cool place over night for the crude leucine to crystallize. Filter off the crystals and use them in the tests for leucine given on page 87. The crystals of tyrosine remaining on the paper from the first filtration may be used in the tests for tyrosine as given below. If desired, the tyrosine and leucine may be purified by recrystallizing in the usual manner. Habermann has suggested a method of separating leucine and tyrosine by means of glacial acetic acid.

\section{Experiments on Tyrosine}

Make the following tests with the tyrosine crystals prepared in the above experiments, or upon those obtained during the preparation of cystine (see page 87), or upon some pure tyrosine furnished by the instructor.

1. Microscopical Examination.-Place a minute crystal of tyrosine on a slide, add a drop of water, cover with a cover-glass, and examine microscopically. Now run more water under the cover-glass and warm in a Bunsen flame until the tyrosine has dissolved. Allow the solution to cool slowly, then examine again microscopically, and compare the crystals with those shown in Fig. 25, page 76 .

2. Solubility. - Try the solubility of very small amounts of tyrosine in cold and hot water, cold and hot 95 per cent alcohol, dilute $\mathrm{NH}_{4} \mathrm{OH}$, dilute $\mathrm{KOH}$ and dilute $\mathrm{HCl}$.

3. Sublimation.-Place a little tyrosine in a dry test-tube, heat gently and notice that the material does not sublime. How does this compare with the result of Experiment 3 under Leucine?

4. Hoffman's Reaction. - This is the name given to Millon's reaction when employed to detect tyrosine. Add about 3 c.c. of water and a few drops of Millon's reagent to a little tyrosine in a test-tube. Upon dissolving the tyrosine by heat the solution gradually darkens and may assume a dark red color. What group does this test show to be present in tyrosine?

5. Sulphuric Acid Test (Piria).-Warm a little tyrosine on a watch glass on a boiling water-bath for 20 minutes with 3-5 drops of conc. $\mathrm{H}_{2} \mathrm{SO}_{4}$. Tyrosinesulphuric acid is formed in the process. Cool the solution and wash it into a small beaker with water. Now add $\mathrm{CaCO}_{3}$ in substance slowly with stirring, until the reaction of the solution is no longer acid. Filter, concentrate the filtrate, and add it to a few drops (avoid an excess) of very dilute neutral ferric chloride. A purple or violet color, due to the formation of the ferric salt of tyrosine-sulphuric acid, is produced. This is one of the most satisfactory tests for the identification of tyrosine.

6. Formaldehyde-Sulphuric Acid Test (Mörner).-Add about 3 c.c. of Morner's reagent ${ }^{1}$ to a little tyrosine in a test-tube, and gently raise the temperature to the boiling-point. A green color results.

${ }^{1}$ Mörner's reagent is prepared by thoroughly mixing I volume of formalin, 45 volumes of distilled water, and 55 volumes of concentrated sulphuric acid. 
7. Folin and Denis's Test. ${ }^{1-T o}$ I- 2 c.c. of the solution to be tested add an equal volume of a special reagent (containing ro per cent sodium tungstate, 2 per cent phosphomolybdic acid and ro per cent phosphoric acid) and 3-10 c.c. of a saturated solution of sodium carbonate. A blue color indicates tyrosine. It is said to detect I part in one million.

Abderhalden ${ }^{2}$ claims the reagent also reacts with tryptophane, oxytryptophane and l-oxyproline.

\section{Experments ON LeUCINE}

Make the following tests upon the leucine crystals already prepared or upon some pure leucine furnished by the instructor.

I, 2 and 3. Repeat these experiments according to the directions given under Tyrosine (pages 86 and 87 ).

\section{Preparation of Cystine ${ }^{3}$}

From 50 to 500 grams of wool or hair is pushed into a (Jena) flask and concentrated hydrochloric acid (200 c.c. to each 100 grams of wool) is added. In order to get a part of the acid quickly to the bottom of the flask a part of the acid may be put in first, then the wool, and finally the remaining acid. A condenser consisting only of a glass tube 2 to $3 \mathrm{ft}$. long is inserted and the mixture is boiled until the biuret reaction is entirely negative. The wool dissolves in a few minutes and if much cystine is desired more wool and acid can then be introduced. After three to five hours' boiling with moderate quantities of wool the biuret reaction has usually disappeared.

To the hot acid solution of amino acids so obtained is added at once an excess of solid sodium acetate, i.e., until the Congo red reaction for mineral acids is entirely negative. A dark, heavy precipitate containing practically all the cystine is obtained. After a few hours' standing at room temperature the liquid is filtered off and the precipitate is washed with cold water. (From the mother liquor diluted with the wash water is usually obtained on long standing a second precipitate consisting chiefly of tyrosine.)

The crude cystine is then dissolved in boiling 3-5 per cent hydrochloric acid and the solution is decolorized with good boneblack which should have been previously thoroughly digested with hot, dilute hydrochloric acid and then washed with water in order to remove the calcium phosphate. The hot filtrate from the boneblack should be as clear as water. If it is not perfectly colorless the boneblack treatment should be repeated and if a colorless solution is not then obtained the fault lies with the quality of the boneblack. The last filtrate is heated to boiling and the cystine precipitated by a slow addition of concentrated hot sodium acetate solution.

Large amounts of colorless cystine consisting of typical hexagonal plates can thus be prepared without difficulty and with very little labor. Compare the microscopical appearance of these crystals with those shown in Fig. 26, page 76.

1 Folin and Denis: Jour. Biol. Chem., 12, 245, 1012.

${ }^{2}$ Abderhalden: Zeil. Physiol. Chem., 85, 9r, ror3.

${ }^{3}$ Folin: Jour. Biol. Chem., 8, 9, rgro. 


\section{THE QUANTITATIVE DETERMINATION OF ALIPHATIC AMINO GROUPS}

Method of Van Slyke. ${ }^{1-P r i n c i p l e .-T h i s ~ m e t h o d ~ f o r ~ t h e ~ d e t e r m i n a t i o n ~ o f ~}$ aliphatic amino nitrogen is based on the measurement of the nitrogen gas evolved in the reaction,

$$
\mathrm{RNH}_{2}+\mathrm{HNO}_{2}=\mathrm{ROH}+\mathrm{N}_{2}+\mathrm{H}_{2} \mathrm{O} \text {. }
$$

During the process the following reaction also takes place, the nitrous acid solution decomposing spontaneously with the formation of nitric oxide,

$$
{ }_{2} \mathrm{HNO}_{2}=\mathrm{HNO}_{3}+\mathrm{NO} \text {. }
$$

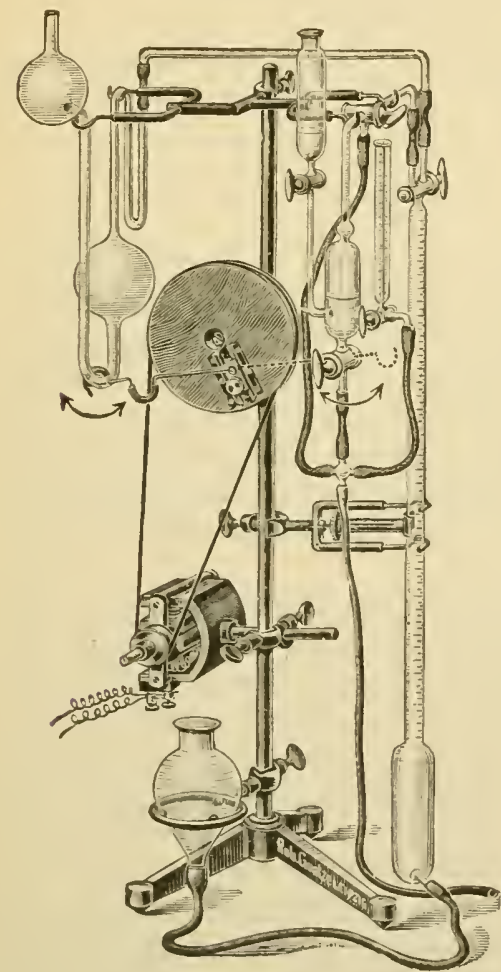

Fig. 34--Van Slyke Amino Nitrogen Apparatus.

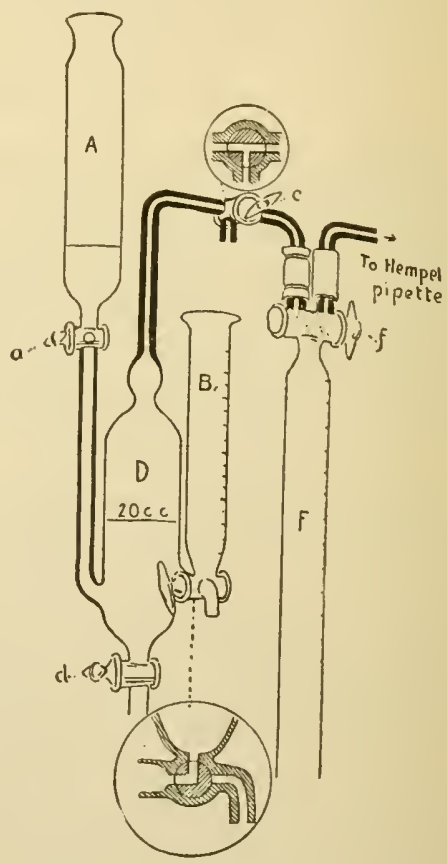

Fig. 35.-Section of VAN SLYKe

This latter reaction is utilized in displacing all the air of the apparatus with nitric oxide. The amino solution is then introduced, evolution of nitrogen mixed with nitric oxide resulting. The oxide is absorbed with alkaline permanganate solution and the pure nitrogen measured in a special gas burette shown in the figure.

Procedure.-The determination is carried out in three stages:

I. Displacement of Air by Vitric Oxide.-Water from F (see Figs. 34 and 35), fills the capillary leading to the Hempel pipette and also the other capillary as far as $c$. Into $A$ one pours a volume of glacial acetic acid sufficient to fill

${ }^{1}$ Van Slyke: Jour. Biol. Chem., r 2, 275, r9 12; 6r, I 2 r and I25, rgr3. 
one-fifth of $D$. For convenience, $A$ is etched with a mark to measure this amount. The acid is run into $D$ cock $c$ being turned so as to let the air escape from $D$. Through $A$ one now pours sodium nitrite solution (30 grams $\mathrm{NaNO}_{2}$ to Ioo c.c. $\mathrm{H}_{2} \mathrm{O}$ ) until $D$ is full of solution and enough excess is present to rise a little above the cock into $A$. It is convenient to mark $A$ for measuring off this amount also. The gas exit from $D$ is now closed at $c$, and, $a$ being open, $D$ is shaken for a few seconds. The nitric oxide, which instantly collects, is let out at $c$, and the shaking repeated. The second crop of nitric oxide which washes out the last portions of air, is also let out at $c . \quad D$ is now connected with the motor and shaken till all but 20 c.c. of the solution have been displaced by nitric oxide and driven back into $A$. A mark on $D$ indicates the 20 c.c. point. One then closes $a$ and turns $c$ and $f$ so that $D$ and $F$ are connected. The above manipulations require between one and two minutes.

2. Decomposition of the Amino Substance.-Of the amino solution to be analyzed Io c.c. or less, as the case may be, are measured off in $B$. Any excess added above the mark can be run off through the outflow tube. The desired amount is then run into $D$, which is already connected with the motor, as shown in Fig. 34 . It is shaken when $\alpha$-amino acids are being analyzed for a period of three to five minutes. With $\alpha$-amino acids, proteins or partially or completely hydrolyzed proteins, we find that at the most five minutes vigorous shaking completes the reaction. Only in the case of some native proteins which, when deaminized form unwieldy coagula that mechanically interfere with the thorough agitation of the mixture, a longer time may be required. In case a viscous solution is being analyzed and the liquid threatens to foam over into $F, B$ is rinsed out and a little caprylic alcohol is added through it. For amino substances such as amino purins, requiring a longer time than five minutes to react, one merely mixes the reacting solutions and lets them stand the required length of time, then shakes about two minutes to drive the nitrogen completely out of solution.

When it is known that the solution to be analyzed is likely to foam violently, it is advisable to add caprylic alcohol through $B$ before the amino solution. $B$ is then rinsed with alcohol and dried with ether or a roll of filter paper before it receives the amino solution.

3. Absorption of Nitric Oxide and Measurement of Nitrogen.-The reaction being completed, all the gas in $D$ is displaced into $F$ by liquid from $A$ and the mixture of nitrogen and nitric oxide is driven from $F$ into the absorption pipette. ${ }^{1}$ The driving rod is then connected with the pipette by lifting the hook from the shoulder of $d$ and placing the other hook, on the opposite side of the driving rod, over the horizontal lower tube of the pipette. The latter is then shaken by the motor for a minute, which, with any but almost completely exhausted permanganate solutions, completes the absorption of nitric oxide. The pure nitrogen is then measured in $F$. During the above operations $a$ is left open, to permit displacement of liquid from $D$ as nitric oxide forms in $D$.

Blank determinations, performed as above except that ro c.c. of distilled water replaces the solution of amino substance, must be performed on every fresh lot of nitrite used. Nitrite giving a much larger correction than 0.3 to 0.4 c.c. should be rejected.

The room temperature and the barometric pressure must be noted. The liter.

${ }_{1}$ The solution in the absorption pipette is 40 grams $\mathrm{KMNO}$ and $25 \mathrm{grams} \mathrm{KOH}$ in a 
MILLIGRAMS OF AMINO NITROGEN CORRESPONDING TO I C.C. OF NITROGEN GAS AT II ${ }^{\circ}-30^{\circ} \mathrm{C}$; $728-772$ MM. PRESSURE

\begin{tabular}{|c|c|c|c|c|c|c|c|c|c|c|c|c|c|}
\hline$\ell$ & 728 & 730 & 732 & 734 & 736 & 738 & 740 & 742 & 744 & 746 & 748 & 750 & $t$ \\
\hline I I $^{\circ}$ & $0.568 \mathrm{o}$ & 0.5695 & 0.5 & 0.5725 & o. 5745 & 0.5760 & 0.5775 & 0.5790 & 0.5805 & 0.5820 & 0.5840 & 0.5855 & I I $^{\circ}$ \\
\hline I $2^{\circ}$ & 0.5655 & 0.5670 & 0.56 & 0.5700 & 0.5720 & 0.5735 & 0.5750 & 0.5765 & 0.5780 & O. 5795 & 0.5815 & 0.5830 & $12^{\circ}$ \\
\hline $13^{\circ}$ & 0.5630 & 0.5645 & o. 5660 & 0.5675 & 0.5695 & 0.5710 & 0.5725 & 0.5740 & 0.5755 & 0.5770 & 0.5785 & 0.5805 & $13^{\circ}$ \\
\hline I $4^{\circ}$ & 0.5605 & 0.5620 & 0.5635 & 0.5650 & 0.5665 & 0.5680 & 0.5 & 0.5715 & 0.5730 & 0.5745 & 0.5760 & .05775 & $14^{\circ}$ \\
\hline I $5^{\circ}$ & o. 5580 & O. 5595 & $0.56 \mathrm{IO}$ & 0.5625 & 0.56 .70 & 0.5655 & 0.5670 & 0.5685 & 0.5705 & 0.5720 & 0.5735 & 0.5750 & $15^{\circ}$ \\
\hline $16^{\circ}$ & O. 5555 & 0.5570 & 0.5585 & 0.5600 & 0.56 I 5 & 0.5630 & 0.5645 & 0.5660 & 0.5675 & 0.5690 & 0.5710 & 0.5725 & $16^{\circ}$ \\
\hline I $7^{\circ}$ & 0.5525 & o. & 0.55 & 0.5575 & 0.5590 & 0.5605 & 0.5620 & 0.5635 & 0.5650 & 0.5665 & 0.5680 & 0.5695 & I $7^{\circ}$ \\
\hline $18^{\circ}$ & 0.5500 & 0.55 I 5 & 0.5530 & 0.5545 & 0.5560 & 0.5580 & 0.5595 & 0.5610 & 0.5625 & 0.5640 & 0.5655 & 0.5670 & $18^{\circ}$ \\
\hline $19^{\circ}$ & o. 5475 & 0.5490 & 0.5505 & 0.5520 & 0.5535 & 0.5550 & 0.5565 & 0. & 0.5 & 0.5610 & 0.5630 & 0.5645 & $19^{\circ}$ \\
\hline $20^{\circ}$ & 0.5445 & 0.5460 & 0.5475 & 0.5495 & 0.5510 & 0.5525 & 0.5540 & 0.5555 & 0.5570 & 0.5585 & 0.5600 & 0.5615 & $20^{\circ}$ \\
\hline $2 \mathrm{I}^{\circ}$ & 0.5420 & 0.5435 & 0.5450 & 0.5465 & 0.5480 & 0.5495 & 0.5510 & 0.5525 & 0.5540 & O. 5555 & O. 5575 & 0.5590 & $2 \mathrm{I}^{\circ}$ \\
\hline $22^{\circ}$ & 0.53 & o. & 0.5425 & 0.5440 & 0.5455 & 0.5470 & 0.5485 & 0.5500 & 0.5 & 0.5530 & 0.5545 & 0.5560 & $22^{\circ}$ \\
\hline $23^{\circ}$ & 0.5365 & 0.5 & 0.5 & 0.5410 & 0.5425 & 0.5 & 0.5455 & 0.5470 & 0.5 & 0.5500 & O. 55 I 5 & 0.5530 & $23^{\circ}$ \\
\hline $24^{\circ}$ & 0.5335 & 0.5350 & 0.5365 & 0.5380 & 0.5400 & 0.5 & 0.5430 & 0.5445 & 0.5460 & 0.5475 & 0.5490 & 0.5505 & $24^{\circ}$ \\
\hline $25^{\circ}$ & 0.5310 & 0.5325 & 0.5340 & 0.5355 & 0.5370 & 0.5385 & 0.5400 & 0.54 I 5 & 0.5430 & 0.5445 & 0.5460 & 0.5475 & $25^{\circ}$ \\
\hline $26^{\circ}$ & 0.5260 & 0.5295 & 0.5310 & 0.5325 & 0.5340 & 0.5355 & 0.5370 & 0.5365 & 0.5400 & 0.5415 & 0.5430 & 0.5445 & $26^{\circ}$ \\
\hline $27^{\circ}$ & 0.5250 & 0.5265 & 0.5280 & 0.5295 & 0.5310 & 0.5325 & 0.5340 & 0.5355 & 0.5370 & 0.5385 & 0.5400 & 0.5415 & $27^{\circ}$ \\
\hline $28^{\circ}$ & 0.5220 & 0.5235 & 0.5250 & 0.5265 & 0.5280 & 0.5295 & $0.53 \mathrm{IO}$ & 0.5325 & 0.5340 & 0.5355 & 0.5370 & 0.5385 & $28^{\circ}$ \\
\hline $29^{\circ}$ & 0.5195 & 0.5210 & 0.5220 & 0.5235 & 0.5250 & 0.5265 & 0.5280 & 0.5295 & 0.5310 & 0.5325 & 0 & 0.5 & $29^{\circ}$ \\
\hline $30^{\circ}$ & 0.5160 & 0.5175 & 0.5190 & 0.5205 & 0.5220 & 0.5235 & 0.5250 & 0.5265 & 0.5280 & 0.5 & 0.5310 & 0.5325 & $30^{\circ}$ \\
\hline$t$ & 728 & 730 & 732 & 734 & 736 & 738 & 740 & 742 & 744 & 746 & 748 & 750 & $l$ \\
\hline
\end{tabular}

\begin{tabular}{|c|c|c|c|c|c|c|c|c|c|c|c|c|}
\hline$t$ & 752 & 754 & 756 & 758 & 760 & 762 & 764 & 766 & 768 & 770 & 772 & $t$ \\
\hline $1 I^{\circ}$ & 0.5870 & 0.5885 & 0.5900 & 0.5915 & 0.5935 & o. 5950 & 0.5965 & 0.5980 & o. 5995 & 0.6010 & 0.6030 & I I ${ }^{\circ}$ \\
\hline $12^{\circ}$ & 0.5845 & 0.5860 & 0.5875 & 0.5800 & 0.5905 & o. 5925 & 0.5940 & 0.5955 & 0.5970 & 0.5985 & 0.6000 & $12^{\circ}$ \\
\hline $13^{\circ}$ & 0.5820 & 0.5835 & 0.5850 & 0.5865 & 0.5880 & 0.5895 & 0.5910 & 0.5930 & 0.5945 & 0.5960 & 0.5975 & $13^{\circ}$ \\
\hline $14^{\circ}$ & 0.5790 & 0.5805 & 0.5825 & 0.5840 & 0.5855 & 0.5870 & 0.5885 & 0.5900 & 0.5915 & 0.5935 & 10.5950 & $14^{\circ}$ \\
\hline $15^{\circ}$ & 0.5765 & 0.5765 & 0.5795 & 0.5810 & 0.5830 & 0.5845 & 0.5860 & 0.5875 & 0.5890 & 0.5905 & 0.5920 & $15^{\circ}$ \\
\hline $16^{\circ}$ & 0.5740 & 0.5755 & 0.5770 & 0.5785 & 0.5800 & 0.5815 & 0.5830 & 0.5850 & o. 5865 & 0.5880 & 0.5895 & $16^{\circ}$ \\
\hline $17^{\circ}$ & 0.5710 & 0.5730 & 0.5745 & 0.5760 & 0.5775 & o. 5790 & 0.5805 & 0.5820 & 0.5825 & 0.5850 & 0.5865 & $17^{\circ}$ \\
\hline $18^{\circ}$ & 0.5685 & 0.5700 & 0.5715 & 0.5730 & 0.5745 & 0.5765 & 0.5780 & 0.5795 & $0.58 \mathrm{IO}$ & 0.5825 & 0.5840 & $18^{\circ}$ \\
\hline $19^{\circ}$ & 0.5660 & 0.5675 & 0.5600 & 0.5705 & 0.5720 & o. 5735 & 0.5750 & 0.5765 & 0.5780 & 0.5795 & $0.58 \mathrm{IO}$ & $19^{\circ}$ \\
\hline $20^{\circ}$ & 0.5630 & 0.5645 & 0.5660 & 0.5675 & 0.5690 & 0.5705 & 0.5725 & 0.5740 & 0.5755 & 0.5770 & 0.5785 & $20^{\circ}$ \\
\hline $2 I^{\circ}$ & 0.5605 & 0.5620 & 0.5635 & 0.5650 & 0.5665 & o. 5680 & 0.5695 & 0.5710 & 0.5725 & 0.5740 & 0.5755 & $2 \mathrm{I}^{\circ}$ \\
\hline $22^{\circ}$ & 0.5575 & 0.5590 & 0.5605 & 0.5620 & 0.5635 & 0.5650 & 0.5665 & 0.5680 & 0.5695 & 0.5715 & 0.5730 & $22^{\circ}$ \\
\hline $23^{\circ}$ & 0.5545 & 0.5560 & 0.5575 & 0.5595 & 0.5610 & 0.5625 & 0.5640 & 0.5655 & 0.5670 & 0.5685 & 0.5700 & $23^{\circ}$ \\
\hline $24^{\circ}$ & 0.5520 & 0.5535 & 0.5550 & 0.5565 & 0.5580 & 0.5595 & 0.5610 & 0.5625 & 0.5640 & 0.5655 & 0.5670 & $24^{\circ}$ \\
\hline $25^{\circ}$ & 0.5490 & 0.5505 & 0.5520 & 0.5535 & 0.5550 & 0.5565 & 0.5580 & 0.5595 & 0.5610 & 0.5625 & 0.5640 & $25^{\circ}$ \\
\hline $26^{\circ}$ & 0.5460 & 0.5475 & 0.5490 & 0.5505 & 0.5520 & 0.5535 & 0.5550 & 0.5565 & 0.5580 & 0.5595 & 0.5610 & $26^{\circ}$ \\
\hline $27^{\circ}$ & 0.5430 & 0.5445 & 0.5460 & 0.5475 & 0.5490 & 0.5505 & 0.5520 & 0.5535 & 0.5550 & 0.5565 & 0.5580 & $27^{\circ}$ \\
\hline $28^{\circ}$ & 0.5400 & 0.54 I 5 & 0.5430 & 0.5445 & 0.5460 & 0.5475 & 0.5490 & 0.5505 & 0.5520 & 0.5535 & 0.5550 & $28^{\circ}$ \\
\hline $29^{\circ}$ & 0.5370 & 0.5385 & 0.5400 & 0.54 I 5 & 0.5430 & 0.5445 & 0.5460 & 0.5475 & 0.5490 & 0.5505 & 0.5520 & $29^{\circ}$ \\
\hline $30^{\circ}$ & O. 5340 & 0.5355 & 0.5370 & 0.5385 & 0.5400 & 0.5415 & 0.5430 & 0.5445 & & 0.5475 & 0.5490 & $30^{\circ}$ \\
\hline$t$ & 752 & 754 & 756 & 758 & 760 & 762 & 764 & 766 & 768 & 770 & 772 & $t$ \\
\hline
\end{tabular}

Journal of Biological Chemistry, 12, 275, I9r2. Van Slyke: The Quantitative Determination of Amino Groups. 
calculation of the weight of nitrogen gas corresponding to the volume obtained is most readily made with the aid of the tables (see page 90 ) devised for this purpose.

The Van Slyke Micro-apparatus. ${ }^{2}$ - In later work Van Slyke has used to a large extent an apparatus which differs from the one described above only in being considerably smiller. More accurate measurements can be made with this and smaller amounts of amino nitrogen determined. In using this only ro c.c. of nitrite solution and 2.5 c.c. of acetic acid are required for an analysis. One-fifth the amount of substance may be analyzed with the same degree of accuracy as with the larger apparatus. Practically the only alteration from the mode of operation already detailed above, is in the speeds at which the deaminizing bulb and the Hempel pipette are shaken. During the first stage of the analysis the deaminizing bulb should be shaken by the motor at a very high rate of speed, about as fast as the eye can follow or an unnecessary amount of time is lost in freeing the apparatus from air. This stage is also much accelerated by warming the nitrite solution to $3.0^{\circ}$ before it is used, in case a low room temperature has reduced the temperature of the solutions below $20^{\circ}$. In the third stage when the nitric oxide is being absorbed by the permanganate, the Hempel pipette should be shaken not faster than twice per second. This is to prevent the breaking off of small gas bubbles.

It is especially necessary that in the first stage the removal of air be complete. This is assured by shaking the solution in the deaminizing bulb back each time, in this stage, until the bulb is two-thirds filled with nitric oxide.

For the determination of total and free amino acid nitrogen in the urine by this method see chapter on Quantitative Analysis of Urine.

\section{ESTIMATION OF AMINO-ACID $\alpha$-NITROGEN}

Method of Harding and MacLean. ${ }^{3}$-Principle.-Amino-acid mixtures when treated with triketohydrindene hydrate give a colored solution which may be compared colorimetrically with a standard.

Procedure.-One c.c. of the solution to be estimated (containing not more than $0.05 \mathrm{mg}$. of amino-acid $\alpha$-nitrogen and neutral to phenolphthalein, is mixed with I c.c. of a Io per cent aqueous solution of pure pyridine and I c.c. of a freshly prepared 2 per cent solution of triketohydrindene hydrate and heated in a rapidy boiling constant-level water-bath for 20 minutes. At the end of that time the test tube is removed, cooled and diluted to a suitable volume, usually roo c.c., but if the amino-acid $\alpha$-nitrogen is very small in amount a correspondingly smaller dilution can be used. 'The solution is compared with a standard in a Duboscq colorimeter. The standard solution is prepared by dissolving 0.3 I $78 \mathrm{gm}$. of pure, freshly crystallized alanine in a liter of distilled water. The solution contains $0.05 \mathrm{mg}$. of $\mathrm{N}$ per c.c. Treat I c.c. of this standard just as above, except that only I c.c. of triketohydrindene is required. The standard solution is stable for three months. Amounts of amino nitrogen from 0.005 to $0.05 \mathrm{mg}$. may be determined. The method is inaccurate for cystine and has not yet been adapted for use with biological fluids other than solutions of protein hydroly'sis products.

${ }^{1}$ See Van Slyke: Jour. Biol. Chem., I 2, 275, rgा 2 or Gattermann: Praxis des orgunischen Chemikers, ninth edition. In using the tables in the latter work or similar tables it should be borne in mind that the volume of nitrogen gas must be divided by two, inasmuch as only one-half of the nitrogen collected comes from the amino groups.

2 Either of these apparatus may be obtained from limil Greiner, 45 Cliti Street, lew York, or from Robert Goetze, Leipzig. Van Slyke has recently described a third form of his apparatus about half of the size of the earlier micro-apparatus. This has a more accurate burette so that the gas volumes can be read to o.00I c.c. (Vin Slyke: Jour. Biol. Chem., 23, 40\%, 1915.)

${ }^{3}$ Harding and MacLean: Jour. Biol. Chem., 20, $21 \%, 1915$. 


\section{CHAPTER V}

\section{PROTEINS: THEIR CLASSIFICATION AND PROPERTIES}

From what has already been said in Chapter IV regarding the protein substances it will be recognized that the grouping of the diverse forms of this class of substances in a logical manner is not an easy task. The fats and carbohydrates may be classified upon the fundamental principles of their stereo-chemical relationships, whereas such a system of classification in the case of the proteins is absolutely impossible since, as we have already stated, the molecular structure of these complex substances is unknown. Because of the diversity of standpoint from which the proteins may be viewed, relative to their grouping in the form of a logically classified series, it is obvious that there is an opportunity for the presentation of classifications of a widely divergent character. The fact that there were until recent years at least a dozen different classifications which were recognized by various groups of English-speaking investigators emphasizes the difflculties in the way of the individual or individuals who would offer a classification which should merit universal adoption. Realizing the great handicap and disadvantage which the great diversity of the protein classifications was forcing upon the workers in this field, the Chemical and Physiological Societies of England drafted a classification which appealed to these groups of scientists as fulfilling all requirements and presented it for the consideration of the American Physiological Society and the American Society of Biological Chemists. The outcome of this has been that there are now only two protein classifications which are recognized by English-speaking scientists, one the British Classification, the other the American Classification. These classifications are very similar and doubtless will ultimately be merged into a single classification. In our consideration of the proteins we shall conform in all details to the American Classification. In this connection we will say, however, that we fell that the English Societies have strong grounds for preferring the use of the term scleroproteins for albuminoids and chromoproteins for hemoglobins. The two classifications are as follows: 


\section{CLASSIFICATION OF PROTEINS ADOPTED BY THE AMERI- CAN PHYSIOLOGICAL SOCIETY AND THE AMERICAN SOCIETY OF BIOLOGICAL CHEMISTS}

\section{SIMPLE PROTEINS}

Protein substances which yield only $\alpha$-amino acids or their derivatives on hydrolysis.

(a) Albumins.-Soluble in pure water and coagulable by heat, e.g., ovalbumin from egg white, serum albumin from blood scrum, lactalbumin from milk, vegetable albumins.

(b) Globulins.- Insoluble in pure water but soluble in neutra] solutions of salts of strong bases with strong acids, ${ }^{1}$ e.g., serum globulin, ovoglobulin from egg yolk, edestin from hemp seed, amandin from almond and peach kernel, and other vegetable globulins.

(c) Glutelins.-Simple proteins insoluble in all neutral solvents, but readily soluble in very dilute acids and alkalis, ${ }^{2}$ e.g., glutenin from wheat.

(d) Alcohol-soluble Proteins (Prolamins). ${ }^{3}$ - Simple proteins soluble in 7o-So per cent alcohol, insoluble in water, absolute alcohol, and other neutral solvents, ${ }^{4}$ e.g., zein from corn, gliadin from wheat and rye, hordein from barley, and bynin from malt.

(e) Albuminoids.-Simple proteins possessing a similar structure to those already mentioned, but characterized by a pronounced insolubility in all neutral solvents, ${ }^{5}$ e.g., elastin from ligament, collagen from tendon, keratin from horn and hoof.

( $f$ ) Histones.-Soluble in water and insoluble in very dilute ammonia, and, in the absence of ammonium salts, insoluble even in excess of ammonia; vield precipitates with solutions of other proteins and a coagulum on heating which is easily soluble in very dilute acids. On hydrolysis they yield a large number of amino acids among which the basic ones predominate. In short, histones are basic proteins which stand between protamines and true proteins, e.g., globin from hemoglobin, scombrone from mackerel sperm, thymus histone.

(g) Protamines.-Simpler polypeptides than the proteins included in the preceding groups. They are soluble in water, uncoagulable by

1 The precipitation limits with ammonium sulphate should not be made a basis for distinguishing the albumins from the globulins.

2 Such substances occur in abundance in the seeds of cereals and doubtless represent a well-defined natural group of simple proteins.

${ }^{3}$ The name prolumins has been suggested for these alcohol-soluble proteins by Inr. Thomas B. Osborne (Science, rgoS, xxviii, p. 4 I 7 ). It is a very fitting term inasmuch as upon hydrolysis they yield particularly large amounts of proline and a mmonia.

t The subclasses defined $(a, b, c, \dot{d}$,) are exemplified by proteins obtained from both plants and animals. The use of appropriate prefixes will suffice to indicate the origin of the compounds, e.g., ovoglobulin, laclalbumin, etc.

${ }^{5}$ These form the principal organic constituents of the skeletal structure of animals and also their external covering and its appendages. This definition does not provide for gelatin which is, however, an artificial derivative of collagen. 
heat, have the property of precipitating aqueous solutions of other proteins, possess strong basic properties and form stable salts with strong mineral acids. They yield comparatively few amino acids, among which the basic ones predominate. They are the simplest natural proteins, e.g., salmine from salmon sperm, sturine from sturgeon sperm, clupeine from herring sperm, scombrine from mackerel sperm.

\section{CONJUGATED PROTEINS}

Substances which contain the protein molecule united to some other molecule or molecules otherwise than as a salt.

(a) Nucleoproteins.- Compounds of one or more protein molecules with nucleic acid, e.g., cytoglobulin from cytoplasm, nucleohistone from nucleus.

(b) Glycoproteins.-Compounds of the protein molecule with a substance or substances containing a carbohydrate group other than a nucleic acid, e.g., mucins and mucoids (osseomucoid from bone, tendomucoid from tendon, ichthulin from carp eggs, helicoprotein from snail).

(c) Phosphoproteins.-Compounds of the protein molecule with some, as yet undefined, phosphorus-containing substances other than a nucleic acid or lecithin, ${ }^{1}$ e.g., casein from milk, avovitellin from egg yolk.

(d) Hemoglobins.-Compounds of the protein molecule with hematin, or some similar substance, e.g., hemoglobin from red blood cells, hemocyanin from blood of invertebrates.

(e) Lecithoproteins.-Compounds of the protein molecule with lecithins.

\section{DERIVED PROTEINS}

\section{Primary Protein Derivatives}

Derivatives of the protein molecule apparently formed through hydrolytic changes which involve only slight alteration of the protein molecule.

(a) Proteans.-Insoluble products which apparently result from the incipient action of water, very dilute acids or enzymes, $e . g$., myosan from myosin, edestan from edestin.

(b) Metaproteins.- Products of the further action of acids and alkalis whereby the molecule is so far altered as to form products soluble in

1 The accumulated chemical evidence distinctly points to the propriety of classifying the phosphoproteins as conjugated compounds, i.e., they are possibly esters of some phosphoric acid or acids and protein. 
very weak acids and alkalis but insoluble in neutral fluids, e.g., acid metaprotein (acid albuminate), alkali metaprotein (alkali albuminate).

(c) Coagulated Proteins. - Insoluble products which result from (I) the action of heat on their solutions, or (2) the action of alcohol on the protein.

\section{Secondary Protein Derivatives ${ }^{1}$}

Products of the further hydrolytic cleavage of the protein molecule.

(a) Proteoses.-Soluble in water, non-coagulable by heat, and precipitated by saturating their solutions with ammonium-or zinc sulphate, ${ }^{2}$ e.g., protoproteose, denteroproteose.

(b) Peptones.--Soluble in water, non-coagulable by heat, but nol precipitaled by saturating their solutions with ammonium sulphate, ${ }^{3}$ e.g., antipeplone, amphopeptone.

(c) Peptides.-Definitaly characterized combinations of two or more amino acids, the carboxyl group of one being united with the amino group of the other with the elimination of a molecule of water, ${ }^{4}$ e.g., dipeptides, tripeptides, tetrapeptides, pentapeptides.

\section{CLASSIFICATION OF PROTEINS ADOPTED BY THE CHEM- ICAL AND PHYSIOLOGICAL SOCIETIES OF ENGLAND}

\section{Simple Proteins}

I. Protamines, e.g., salmine, clupeine.

2. Histones, e.g., globin, scombrone.

3. Albumins, e.g., ovalbumin, serum albumin, vegetable albumins.

4. Globulins, e.g., serum globulin, oroglobulin, vegetable globulins.

5. Glutelins, e.g., glutenin.

6. Alcohol-soluble proteins, e.g., zein, gliadin.

7. Scleroproteins, e.g., elastin, keratin.

8. Phosphoproteins, e.g., casein, vitellin.

\section{Conjugated Proteins}

I. Glucoproteins, e.g., mucins, mucoids.

2. Nucleoproteins, e.g., nuclcohistone, cytoglobu!in.

3. Chromoproteins, e.g., hemoglobin, hemocyanin.

1 The term secondary protein derivatives is used because the formation of the primary derivatives usually precedes the formation of the secondary derivatives.

${ }^{2} \mathrm{As}$ thus defined, this term does not strictly cover all the protein derivatives commonly called proteoses, e.g., heteroproteose and dysproteose.

${ }^{3}$ In this group the kyrines may be included. For the present it is believed that it will be helpful to retain this term as defined, reserving the expression peplide for the simpler compounds of definite structure, such as dipeptides, etc.

${ }^{4}$ The peptones are undoubtedly peptides or mixtures of peptides, the latter term being at present used to designate those of definite structure. 


\section{Products of Protein Hydrolysis}

I. Infraproteins, e.g., acid infraprotein (acid albuminate), alkali infraprotein (alkali albuminate).

2. Proteoses, e.g., protoproteose, heteroproteose, deuteroproteose.

3. Peptones, e.g., amphopeptone, antipeptone.

4. Polypeptides, e.g., dipeptides, tripeptides, tetrapeptides.

\section{CONSIDERATIONS OF THE VARIOUS CLASSES OF PROTEINS}

\section{SIMPLE PROTEINS}

The simple proteins are true protein substances which, upon hydrolysis, yield only $\alpha$-amino acids or their derivatives. "Although no means are at present available whereby the chemical individuality of any protein can be established, a number of simple proteins have been isolated from animal and vegetable tissues which have been so well characterized by constancy of ultimate composition and uniformity of physical properties that they may be treated as chemical individuals until further knowledge makes it possible to characterize them more definitely." Under simple proteins we may class albumins, globulins, glutelins, prolamins, albuminoids, histones and protamines.

\section{ALBUMINS}

Albumins constitute the first class of simple proteins and may be defined as simple proteins which are coagulable by heat and soluble in pure (salt-free) water. Those of animal origin are not precipitated upon saturating their neutral solutions at $30^{\circ} \mathrm{C}$. with sodium chloride or magnesium sulphate, but if a saturated solution of this character be acidified with acetic acid the aibumin precipitates. All albumins of animal origin may be precipitated by saturating their solutions with ammonium sulphate. ${ }^{1}$ They may be thrown out of solution by the addition of a sufficient quantity of a mineral acid, whereas a weak acidity produces a slight precipitate which dissolves upon agitating the solution. Metallic salts also possess the property of precipitating albumins, some of the precipitates being soluble in excess of the reagent, whereas others are insoluble in such an excess. Of those proteins which occur native the albumins contain the highest percentage of sulphur, ranging from 1.6 to 2.5 per cent. Some albumins have been

${ }^{1}$ In this connection, Osborne's observation that there are certain vegetable albumins which are precipitated by saturating their solutions with sodium chloride or magnesium sulphate or by half-saturating with ammonium sulphate, is of interest. 
obtained in crystalline form, notably egg albumin, serum albumin, and lactalbumin, but the fact that they may be obtained in crystalline form does not necessarily prove them to be chemical individuals.

\section{GENERAL COLOR REACTIONS OF PROTEINS}

These color reactions are due to a reaction between some one or more of the constituent radicals or groups of the complex protein molecule and the chemical reagent or reagents used in any given test. Not all proteins contain the same groups and for this reason the various color tests will yield reactions varying in intensity of color according to the nature of the groups contained in the particular protein under examination. Various substances not proteins respond to certain of these color reactions, and it is therefore essential to submit the material under examination to several tests before concluding definitely regarding its nature.

\section{TECHNIC OF THE COLOR REACTIONS}

I. Millon's Reaction.-To 5 c.c. of a dilute solution of egg albumin ${ }^{1}$ in a testtube add a few drops of Millon's reagent. A white precipitate forms which turns red when heated.

This test is a particularly satisfactory one for use on solid proteins, in which case the reagent is added directly to the solid substance and heat applied, which causes the substance to assume a red color. Such proteins as are not precipitated by mineral acids, for example certain of the proteoses and peptones, yield a red solution instead of a red precipitate.

The reaction is due to the presence of the hydroxy-phenyl group, $-\mathrm{C}_{6} \mathrm{H}_{4} \mathrm{OH}$, in the protein molecule and certain non-proteins such as tyrosine, phenol (carbolic acid) and thymol also respond to the reaction. Inasmuch as the tyrosine grouping is the only hydroxyphenyl grouping which has definitely been proven to be present in the protein molecule it is evident that protein substances respond to Millon's reaction because of the presence of this tyrosine complex. The test is not a very satisfactory one for use in solutions containing inorganic salts in large amount, since the mercury of the Nillon's reagent ${ }^{2}$ is thus precipitated and the reagent rendered inert. This reagent is therefore never used for the detection of protein material in the urine. If the solution under

1 This egg albumin solution may be prepared by beating egg-white with 6-10 volumes of water. The precipitate of ovoglobulin is filtered off and the filtrate used in the tests.

${ }^{2}$. Hillon's reagent consists of mercury dissolved in nitric acid containing some nitrous acid. It is prepared by digesting one part (by weight) of mercury with two parts (by weight) of $\mathrm{HNO}_{3}$ (sp. gr. I.42) and diluting the resulting solution with two volumes of water. 
examination is strongly alkaline it should be neutralized inasmuch as the alkali will precipitate yellow or black oxides of mercury.

2. Xanthoproteic Reaction.-To 2-3 c.c. of egg albumin solution in a test-tube add concentrated nitric acid. A white precipitate forms, which upon heating turns yellow and finally dissolves, imparting to the solution a yellow color. Cool the solution and carefully add ammonium hydroxide, potassium hydroxide, or sodium hydroxide in excess. Note that the yellow color deepens into an orange.

This reaction is due to the presence in the protein molecule of the phenyl group $-\mathrm{C}_{6} \mathrm{H}_{5}$, with which the nitric acid forms certain nitro modifications. The particular complexes of the protein molecule which are of especial importance in this connection are those of tyrosine, phenylalanine, and tryptophane. The test is not a satisfactory one for use in urinary examination because of the color of the end-reaction.

3. Glyoxylic Acid Reaction (Hopkins-Cole). ${ }^{1}$-Place I-2 c.c. of egg albumin solution and 3 c.c. of glyoxylic acid, $\mathrm{CHO} . \mathrm{COOH}+\mathrm{H}_{2} \mathrm{O}$ or $\mathrm{CH}(\mathrm{OH})_{2} \mathrm{COOH}$, solution (Hopkins-Cole reagent ${ }^{2}$ ) in a test-tube and mix thoroughly. In a second tube place 5 c.c. of concentrated sulphuric acid. Incline the tube containing sulphuric acid and by means of a pipette allow the albumin-glyoxylic acid solution to flow carefully down the side. When stratified in this manner a reddish-violet color forms at the zone of contact of the two fluids.

This color is due to the presence of the tryptophane group. Gelatin does not respond to this test. For formula of tryptophane see page 77. Benedict ${ }^{3}$ has suggested a new reagent for use in carrying out the Hopkins-Cole reaction. ${ }^{4}$ Nitrates $\left(\mathrm{NaNO}_{3}\right.$ and $\left.\mathrm{KNO}_{3}\right)$ entirely prevent the reaction whereas formaldehyde or nitric acid interfere somewhat. ${ }^{5}$

4. Biuret Test.-To 2-3 c.c. of egg albumin solution in a test-tube add an equal volume of concentrated potassium hydroxide solution, mix thoroughly, and add slowly a very dilute (2-5 drops in a test-tube of water) copper sulphate solution until a purplish-violet or pinkish-violet color is produced. The depth

${ }^{2}$ Hopkins and Cole: Journal of Physiology, 27, 418, 1902.

2 Hopkins-Cole reagent is prepared as follows: To one liter of a saturated solution of oxalic acid add 60 grams of sodium amalgam and allow the mixture to stand until the evolution of gas ceases. Filter and dilute with $2-3$ volumes of water.

${ }^{3}$ Benedict: Journal of Biological Chemistry, 6, 51, I909.

4 Benedict's modified Hopkins-Cole reagent is prepared as follows: Ten grams of powdered magnesium are placed in a large Erlenmeyer flask and shaken up with enough distilled water to liberally cover the magnesium. Two hundred and fifty c.c. of a cold, saturated solution of oxalic acid is now added slowly. The reaction proceeds very rapidly and with the liberation of much heat, so that the flask should be cooled under running water during the addition of the acid. The contents of the flask are shaken after the addition of the last portion of the acid and then poured upon a filter, to remove the insoluble magnesium oxalate. A little wash water is poured through the filter, the filtrate acidified with acetic acid to prevent the partial precipitation of the magnesium on long standing, and made up to a liter with distilled water. This solution contains only the magnesium salt of glyoxylic acid.

- Nathewson: Dissertation (Columbia Univ.), Eschenbach Publishing Co., Easton, Pa., I912. 
of the color depends upon the nature of the protein; proteoses, and peptones giving a decided pink, while the color produced with gelatin is not far removed from a blue.

This reaction is given by those substances which contain two amino groups in their molecule, these groups either being joined directly together or through a single atom of nitrogen or carbon. The amino groups mentioned must either be two $\mathrm{CONH}_{2}$ groups or one $\mathrm{CONH}_{2}$ group and one $\mathrm{CSNH}_{2}, \mathrm{C}(\mathrm{NH}) \mathrm{NH}_{2}$ or $\mathrm{CH}_{2} \mathrm{NH}_{2}$ group. It follows from this fact that substances which are non-protein in character but which contain the necessary groups will respond to the biuret test. As examples of such substances may be cited oxamide,

and biuret,

$$
\begin{aligned}
& \mathrm{CONH}_{2} \\
& \mathrm{CONH}_{2}
\end{aligned}
$$

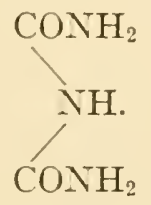

The test derives its name from the fact that this latter substance which is iormed on heating urea to $180^{\circ} \mathrm{C}$ (see page 375 ) will respond to the test. Protein material responds positively since there are two $\mathrm{CONH}_{2}$ groups in the protein molecule.

According to Schiff the end-reaction of the biuret test is dependent upon the formation of a copper-potassium-biuret compound (cupripotassium biuret or biuret potassium cupric hydroxide). This substance was obtained by Schiff in the form of long red needles. It has the following formula:

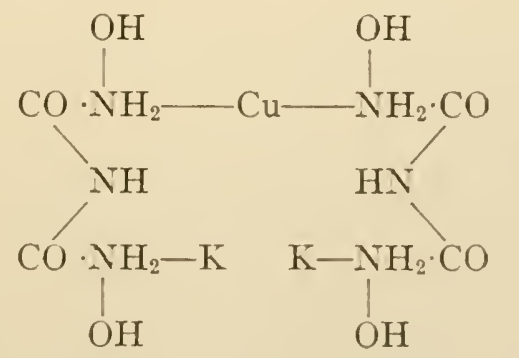

Testing Colored Solutions by Biuret Test.-If the color of the solution is such as to interfere with the end-reaction of the biuret test, proceed as follows: Make the solution strongly alkaline with potassium hydroxide and add a solution of copper sulphate. Shake up the mixture with alcohol and if protein is present the alcohol will assume the typical 
biuret coloration. This procedure is not applicable in case the pigment of the original solution is soluble in alcohol. Excess of the copper salt need not be avoided in this test.

Gies's Biuret Reagent. ${ }^{1}$ - Gies has devised a reagent for use in the biuret test. This reagent consists of to per cent $\mathrm{KOH}$ solution, to which 25 c.c. of 3 per cent $\mathrm{CuSO}_{4}$ solution per liter has been added. This imparts a slight though distinct blue color to the clear liquid. This reagent is of material assistance in performing the biuret test.

Biuret Paper of Kantor and Gies.-According to 'Kantor and Gies² when filter paper is immersed in the above reagent and subsequently dried it forms a very" satisfactory "biuret paper" which may be used in a manner analogous to indicator papers. Moist papers may be used in the examination of powders which are neutral or alkaline in reaction. In preparing the "biuret paper," if the filter paper is left for a sufficient length of time in the reagent all traces of the copper sulphate will be removed from the solution.

5. Ring Biuret Test (Posner).-This test is particularly satisfactory for use on dilute protein solutions, and is carried out as follows. To some dilute egg albumin in a test-tube-add one-half its volume of potassium hydroxide solution. Now hold the tube in an inclined position and allow some very dilute copper sulphate solution, made as suggested on page 98 to flow down the side, being especially careful to prevent the fluids from mixing. At the juncture of the two solutions the typical end-reaction of the biuret test should appear as a colored zone (see Biuret Test, page 98).

6. Liebermann's Reaction.-Add about Io drops of concentrated egg albumin solution (or a little dry egg albumin) to about 5 c.c. of concentrated $\mathrm{HCl}$ in a testtube. Boil the mixture until a pinkish-violet color results. This color was originally supposed to indicate the presence of a carbohydrate group in the protein molecule, the furfural formed through the action of the acid upon the protein reacting with the hydroxyphenyl group of the protein producing the pinkish-violet color. It is now considered uncertain whether the carbohydrate group enters into the reaction. Cole has called attention to the fact that a blue color results if protein material which has been boiled with alcohol and subsequently washed with ether be used in making the test. He believes the blue color to be due to an interaction between the glyoxylic acid, which was present as an impurity in the ether used in washing the protein, and the tryptophane group of the protein molecule which was split off through the action of the acid.

7. Acree-Rosenheim Formaldehyde Reaction.-Add a few drops of a dilute (I : 5000) solution of formaldehyde to 2-3 c.c. of egg albumin solution in a test-tube. Mix thoroughly and after two to three minutes carefully introduce a little concentrated sulphuric acid into the tube in such a manner that the two solutions do not mix. A violet zone will be observed at the point of juncture of the two solutions, especially if the mixture is slightly agitated. This color probably results through the union of the protein and the formaldehyde. If the sulphuric acid is added to the protein before the formaldehyde is added the typical end-reaction is not obtained. So far as is known this is a specific test for proteins. The reaction cannot be applied satisfactorily with concentrated formaldehyde.

${ }^{1}$ Gies: Proceedings of Society of Biological Chemists, Journal of Biological Chemistry, $7,60,1910$.

${ }^{2}$ Kantor and Gies: Proc. Soc. Biol. Chem., p. II, I9ro. 
Rosenheim claims the reaction is due to the presence of oxidizing material in the sulphuric acid and that when pure sulphuric acid is used no reaction is obtained. He advises the use of a slight amount of an oxidizing agent, e.g., ferric chloride or potassium nitrate ( 0.005 gram per roo c.c. of sulphuric acid) in order to facilitate the reaction. Rosenheim further states that proteins respond to the formaldehyde reaction because of the presence of the tryptophane group, a statement which Acree does not accept as proven.

8. Bardach's Reaction. 1--This is one of the most recent tests which have been described for the detection of protein material. The test depends upon the property possessed by protein substances of preventing the formation of typical iodoform crystals through the interaction of an alkaline acetone solution with iodopotassium iodide. Instead of the typical hexagonal plates or stellar formations of iodoform there are produced, under the conditions of the test, fine yellow needles which are apparently some iodine compound other than iodoform. The technic of the test is as follows: Place about 5 c.c. of the protein solution ${ }^{2}$ under examination in a testtube, add 2-3 drops of a 0.5 per cent solution of acetone and sufficient Lugol's solution $^{3}$ to supply a moderate excess of iodine and produce a red-brown coloration. (The amount of Lugol's solution necessary will depend upon the content of protein, sugar, and other iodine-reacting substances in the solution under examination and may vary from one drop to several cubic centimeters.) Add an excess (ordinarily about 3 c.c.) of concentrated ammonium hydroxide and thoroughly mix the solution. Place the tube in the test-tube rack, examine the contents at intervals of five minutes, and when it is evident that crystals have formed, place a drop of the mixture upon a microscopic slice, put a cover-glass in position, and examine the mixture under the microscope. The formation of canary yellow crystals indicates the presence of protein material in the solution examined. The crystals are ordinarily needle-like in appearance and show a tendency to assume rosette or bundlelike formations, but under certain conditions they may show knobbed (nail-like) and branching variations.

If a moderate excess of iodine is used in making the test, a black precipitate of iodonitro compounds is at once formed upon the addition of the ammonium hydroxide, and yellow needles are subsequently deposited upon it. In case just the proper amount of iodine is used, the solution soon assumes a yellow color and the black precipitate formed upon the addition of the ammonium hydroxide is gradually transformed more or less completely into the yellow crystals. In either case the needles ordinarily form within an hour, and frequently in a much shorter time. If too great an excess of iodine is employed the heavy black precipitate may obscure or even prevent the reaction. The presence of insufficient iodine or excess protein may likewise prevent the reaction. In tests in which a concentrated protein solution and an excess of iodine are used, the addition of ammonium hydroxide immediately produces a grayish-green precipitate. In such instances, if the proportions are favorable and the mixture be stirred with a glass rod for a few minutes, the precipitate is gradually transformed into the crystals before mentioned.

It is probable that all soluble proteins will respond to Bardach's reaction, but the relative delicacy of the reaction as weil as the value of the test as compared with

${ }^{1}$ Bardach: Zeitschrift für Physiologische Chemic, 54, 355, 190S; also Seaman and Gies: Proceedings of the Society for Experimental Biology and Ufedicine, 5 , r 25, InoS.

${ }^{2}$ The solution should not contain more than 5 per cent of protein material.

${ }^{3}$ Dissolve + grams of iodine and 6 grams of potassium iodide in roo c.c. of distilled water. 
other protein tests remain to be determined. The only disturbing factor noted thus far is the presence of earthy phosphates in the solution under examination.

\section{PRECIPITATION REACTIONS AND OTHER PROTEIN TESTS}

There are three forms in which proteins may be precipitated, i.e., unaltered, as an albuminate, and as an insoluble salt. An instance of the precipitation in a native or maltered condition is seen in the so-called salting-out experiments. Various salts, notably $\left(\mathrm{NH}_{4}\right)_{2} \mathrm{SO}_{4}, \mathrm{ZnSO}_{4}$, $\mathrm{MgSO}_{4}, \mathrm{Na}_{2} \mathrm{SO}_{4}$ and $\mathrm{NaCl}$, possess the power, when added in solid form to certain definite protein solutions, of rendering the menstruum incapable of holding the protein in solution, thereby causing the protein to be precipitated or salted-out, to use the common term. Mineral acids and alcohol also precipitate proteins unaltered. In the case of concentrated acids the protein is dissolved in the presence of an excess of acid with the formation of a protein salt. Proteins are precipitated as albuminates when treated with certain metallic salts, and precipitated as insoluble salts when weak organic acids such as certain of the alkaloidal reagents are added to their solutions.

If certain acids (picric, phosphotungstic, phosphomolybdic, tannic, or chromic) be added to a neutral albumin solution a precipitate of an insoluble protein salt occurs. If, however, the salts of these acids be added no precipitate occurs. The addition of a small amount of acid, as acetic acid, to such a solution will cause a precipitate to form. ${ }^{2}$

The effect of the addition of the salts of the heavy'metals is in the first instance to cause a precipitation of the protein. In many cases, however, the addition of an excess of such salts causes the solution of the precipitate while a further excess may cause a reprecipitation. The precipitate which is first formed in a protein solution by the addition of the salts of the heavy metals may be redissolved not only by an excess of such salts but by an.excess of protein as well. ${ }^{2}$

It is generally stated that globulins are precipitated from their solutions upon half saturation with ammonium sulphate and that albumins are precipitated upon complete saturation by this salt. Comparatively few exceptions were found to this rule until proteins of vegetable origin came to be more extensively studied. These studies, furthered especially by Osborne and associates, have demonstrated very clearly that the characterization of a globulin as a protein which is precipitated by half saturation with ammonium sulphate, can no longer hold. Certain vegetable globulins have been isolated which are not precipi-

1 Mathews: Amer. Jour. of Physiology, I, 445, I898.

2 Pauli: Hofmeister's Beitrage, 6, 233, 1904-05; Robertson: Ergebnisse der Physiologie, IO, 290, I9IO. 
tated by this salt until a concentration is reached greater than that secured by half-saturation. As an example of an albumin which does not conform to the definition of an albumin as regards its precipitation by ammonium sulphate may be mentioned the leucosin of the wheat germ, which is precipitated from its solution upon half-saturation with ammonium sulphate. The limits of precipitation by ammonium sulphate, therefore, do not furnish a sufficiently accurate basis for the differentiation of globulins from albumins. It has further been determined that a given protein which is precipitable by ammonium sulphate cannot be "salted-out" by the same concentration of the salt under all conditions.

\section{EXPERIMENTTS}

r. Influence of Concentrated Mineral Acids, Alkalis and Organic Acids.Prepare five test-tubes each containing 5 c.c. of concentrated egg albumin solution. To the first add concentrated $\mathrm{H}_{2} \mathrm{SO}_{4}$, drop by drop, until an excess of the acid has been added. Note any changes which may occur in the solution. Allow the tube to stand for $\mathbf{2 4}$ hours and at the end of that period observe any alteration which may have taken place. Heat the tube and note any further change which may occur. Repeat the experiment in the four remaining tubes with concentrated hydrochloric acid, concentrated nitric acid, concentrated potassium hydroxide and acetic acid. How do strong mineral acids, strong alkalis, and strong organic acids differ in their action toward protein solutions?

2. Precipitation by Metallic Salts.-Prepare four tubes each containing 2-3 c.c. of dilute egg albumin solution. To the first add mercuric chloride, drop by drop slowly, until an excess of the reagent has been added, noting any changes which may occur. If not added very gradually the formation of the precipitate may not be noted, due to its solubility in excess of the reagent. Repeat the experiment with lead acetate, silver nitrate, copper sulphate, ferric chloride, and barium chloride, using very dilute solutions.

Egg albumin is used as an antidote for lead or mercury poisoning. Why? Is it an equally good antidote for the other metallic salts tested?

3. Precipitation by Alkaloidal Reagents.-Prepare six tubes each containing 2-3 c.c. of dilute egg albumin solution. To the first add picric acid drop by drop until an excess of the reagent has been added, noting any changes which may occur. Repeat the experiment with trichloracetic acid, tannic acid, phosphotungstic acid, phosphomolybdic acid, and potassio-mercuric iodide. Are these precipitates soluble in excess of the reagent? Acidify with hydrochloric acid before testing with the last three reagents.

4. Nitric Acid Test (Heller).-Place 5 c.c. of concentrated nitric acid in a test-tube, incline the tube, and by means of a pipette allow the dilute albumin solution to flow slowly down the side. The liquids should stratify with the formation of a white zone of precipitated albumin at the point of juncture. This is a very delicate test and is further discussed on page 423 .

An apparatus called the albumoscope or horismascope has been devised for use in the tests of this character and has met with considerable favor. The method of using the albumoscope is described on p. I04. The instrument is shown in Fig. I30, p. 424 . 
Use of the Albumoscope.-This instrument is intended to facilitate the making of "ring" tests such as Heller's and Roberts'. In making a test about 5 c.c. of the solution under examination is first introduced into the apparatus through the larger arm and the reagent used in the particular test is then introduced through the capil. lary arm and allowed to flow down underneath the solution under examination. If a reasonable amount of care is taken there is no possibility of mixing the two solutions and a definitely defined white "ring" is easily obtained at the zone of contact.

5. Nitric Acid- $\mathrm{MgSO}_{4}$ Test (Roberts).-Place 5 c.c. of Roberts' reagent ${ }^{1}$ in a test-tube, incline the tube, and by means of a pipette allow the albumin solution to flow slowly down the side. The liquids should stratify with the formation of a white zone of precipitated albumin at the point of juncture. This test is a modification of Heller's ring test and is rather more satisfactory. The albumoscope may also be used in making this test (see Fig. 130, page 424).

6. Spiegler's Ring Test.-Place 5 c.c. of Spiegler's reagent ${ }^{2}$ in a test-tube, incline the tube, and by means of a pipette allow 5 c.c. of albumin solution, acidified with acetic acid, to flow slowly down the side. A white zone will form at the point of contact. This is an exceedingly delicate test, in fact too delicate for ordinary clinical purposes, since it serves to detect albumin when present in the merest trace ( $1: 250,000$ ). This test is further discussed on page 424 .

7. Tanret's Test.-To 5 c.c. of albumin solution in a test-tube add Tanret's reagent, ${ }^{3}$ drop by drop, until a turbidity or precipitate forms. This is an exceedingly delicate test. Sometimes the albumin solution is stratified upon the reagent as in Heller's or Roberts' ring tests. In urine examination it is claimed by Repiton that the presence of urates lowers the delicacy of the test. Tanret claims that the removal of urates is not necessary inasmuch as the urate precipitate will disappear on warming and the albumin precipitate will not. He says, however, that mucin interferes with the delicacy of his test and should be removed by acidification with acetic acid and filtration before testing for albumin.

8. Sodium Chloride and Acetic Acid Test.-Mix 2 volumes of albumin solution and I volume of a saturated solution of sodium chloride in a test-tube, acidify with acetic acid, and heat to boiling. The production of a cloudiness or the formation of a precipitate indicates the presence of albumin.

9. Acetic Acid and Potassium Ferrocyanide Test.-To 5 c.c. of dilute egg albumin solution in a test-tube add $5^{-10}$ drops of acetic acid. Mix well and add potassium ferrocyanide, drop by drop, until a precipitate forms. This test is very delicate.

\section{Schmiedl claims that a precipitate of $\mathrm{Fe}(\mathrm{Cn})_{6} \mathrm{~K}_{2} \mathrm{Zn}$ or $\mathrm{Fe}(\mathrm{Cn})_{6^{-}}$} $\mathrm{Zn}_{2}$, is formed when solutions containing zinc are subjected to this test, and that this precipitate resembles the precipitate secured with protein

${ }_{1}^{1}$ Roberts' reagent is composed of I volume of concentrated $\mathrm{HNO}_{3}$ and 5 volumes of a saturated solution of $\mathrm{MgSO}_{4}$.

${ }^{2}$ Spiegler's reagent has the following composition:

Tartaric acid

Mercuric chloride

20 grams.

40 grams.

Sodium chloride............................ 50 grams.

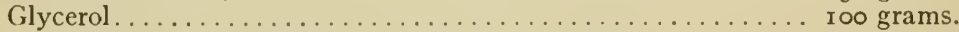

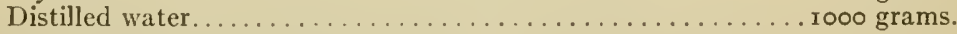

${ }^{3}$ Tanret's reagent is prepared as follows: Dissolve $\mathrm{I} .35$ grams of mercuric chloride in 25 c.c. of water, add to this solution 3.32 grams of potassium iodide dissolved in 25 c.c. of water, then make the total solution up to 60 c.c. with water and add 20 c.c. of glacial acetic acid to the combined solutions. 
solutions. In the case of human urine a reaction was obtained when 0.000022 gram of zinc per cubic centimeter was present. Schmiedl further found that the urine collected from rabbits housed in zinc-lined cages possessed a zinc content which was sufficient to yield a ready response to the test. Zinc is the only interfering substance so far reported.

10. Salting-out Experiments.-(a) To 25 c.c. of egg albumin solution in a small beaker add solid ammonium sulphate to the point of saturation, keeping the temperature of the solution below $40^{\circ} \mathrm{C}$. Filter, test the precipitate by Millon's reaction and the filtrate by the biuret test. What are your conclusions? (b) Repeat the above experiment, making the saturation with solid sodium chloride. How does this result differ from the result of the saturation with ammonium sulphate? Add 2-3 drops of acetic acid. What occurs?

All proteins except peptones are precipitated by saturating their solutions with ammonium sulphate. Globulins are the only proteins precipitated by saturating with sodium chloride (see Globulins, page I08), unless the saturated solution is subsequently acidified, in which event all proteins except peptones are precipitated.

Soaps may be salted-out in a similar manner (see page I $\delta_{I}$ ).

Ix. Coagulation or Boiling Test.-Heat 25 c.c. of dilute egg albumin solution to the boiling-point in a small evaporating dish. The albumin coagulates. Complete coagulation may be obtained by acidifying the solution with $3-5$ drops of acetic acid $^{1}$ at the boiling-point. Test the coagulum by Millon's reaction.

The acid is added to neutralize any possible alkalinity of the solution, to dissolve any substances which are not albumin and to facilitate coagulation (see further discussion on pages II 7 and 424).

I2. Coagulation Temperature.-Prepare four test-tubes each containing 5 c.c of neutral egg albumin solution. To the first add I drop of 0.2 per cent hydrochloric acid, to the second add I drop of 0.5 per cent sodium carbonate solution, to the third add $\mathrm{I}$ drop of Io per cent sodium chloride solution and leave the fourth neutral in reaction. Partly fill a beaker of medium size with water and place it within a second larger beaker which also contains water, the two vessels being separated by pieces of cork. Fasten the four test-tubes compactly together by means of a rubber band, lower them into the water of the inner beaker and suspend them, by means of a clamp attached to one of the tubes, in such a manner that the albumin solutions shall be midway between the upper and lower surfaces of the water. In one of the tubes place a thermometer with its bulb entirely beneath the surface of the albumin solution (Fig. 36). Gently heat the water in the beakers, noting carefully any changes which may occur in the albumin solutions and record the exact temperature at which these changes occur. The first appearance of an opacity in an albumin solution indicates the commencement of coagulation and the temperature at which this occurs should be recorded as the coagulation temperature for that particular albumin solution.

${ }^{1}$ Nitric acid is often used in place of acetic acid in this test. In case nitric acid is used, ordinarily $1-2$ drops are sufficient. 
What is the order in which the four solutions coagulate?

Repeat the experiment, adding to the first tube I drop of acetic acid, to the second I drop of concentrated potassium hydroxide solution, to the third 2 drops of a ro per cent sodium chloride solution and leave the fourth neutral as before.

What is the order of coagulation here? Why? See page II6.

13. Precipitation by Alcohol.-Prepare three test-tubes each containing about ro c.c. of 95 per cent alcohol. To the first add I drop of 0.2 per cent

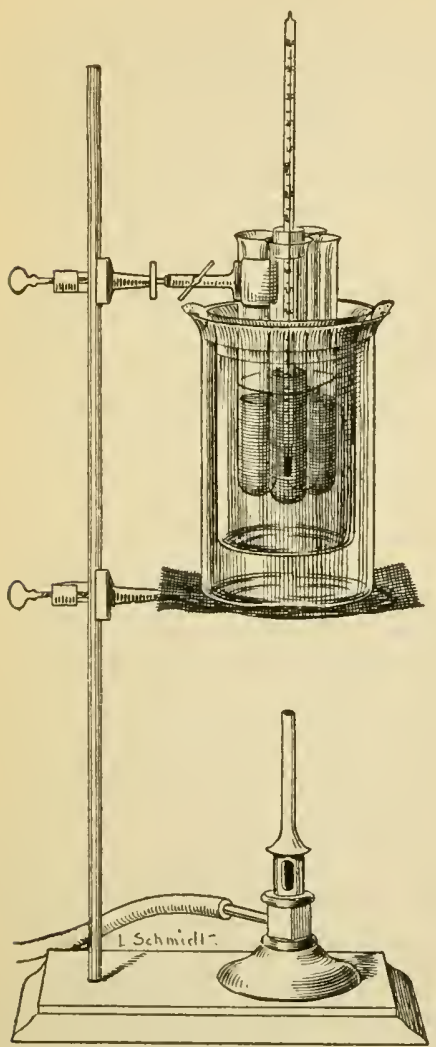

Fig. 36.-Coagulation TEMPERATURE APPARATUS. hydrochloric acid, to the second I drop of potassium hydroxide solution and leave the third neutral in reaction. Add to each tube a few drops of egg albumin solution and note the results. What do you conclude from this experiment?

If in acid or neutral solution alcohol precipitates proteins unaltered, but if allowed to remain under alcohol the protein is transformed. The "fixing" of tissues for histological examination by means of alcohol is an illustration of the application of this transformation produced by alcohol. It apparently is a process of dehydration.

14. Crystallization of Egg Albumin. ${ }^{1-C a r e-}$ fully remove the egg-white from a number of absolutely fresh eggs. ${ }^{2}$ Measure the volume of the egg-white and add an equal volume of saturated ammonium sulphate a small portion at a time, beating the mixture vigorously after each addition. ${ }^{3}$ Filter the mixture through a large pleated filter paper. ${ }^{4}$ Measure the volume of the filtrate. To Ioo c.c. of the filtrate add very carefully a so per cent solution of acetic acid from a burette being certain to note the exact volume of the acid used. The acid should be added drop by drop, the albumin mixture being gently shaken during the process. Add acid until the precipitate, which forms at each addition is no longer dissolved when the albumin is shaken, and an opalescent mixture is secured. (It is generally rather difficult to determine this point, inasmuch as suspended air bubbles may simulate a precipitate.) As soon as the solution is milky, indicating that a permanent precipitate has formed, run in from the burette I c.c. of the acetic acid. This should produce a heavy white precipitate. Now iake the burette reading to determine the exact volume of acid used in the treatment of Ioo c.c. of the albumin mixture.

1 Hopkins and Pinkus: Jour. Physiol., 23.

2 If not perfectly fresh the albumin will not crystallize.

${ }^{3}$ Note the odor of ammonia. What causes it?

4 Sometimes better results are obtained by permitting the mixture to stand several hours before filtering. 
Calculate the exact volume of acid necessary to precipitate the remaining portion of the original albumin mixture and add this calculated quantity. Mix the two portions of albumin and allow to stand over night. Remove a drop of the suspended material to a slide and examine microscopically. Crystals in the form of fine needles will be observed. This is the crystallized egg albumin. To recrystallize, filter off the crystals and dissolve them in the smallest possible volume of water. Filter, and to the filtrate carefully add saturated ammonium sulphate until a faint, permanent precipitate is formed. Allow the mixture to stand several hours and examine as before. The crystals of albumin should be somewhat larger than when first examined.

The above method may also be used for crystallizing serum albumin from the fresh blood serum of the horse, mule or ass.

I5. Preparation of Powdered Egg Albumin.-This may be prepared as follows: Ordinary egg-white finely divided by means of scissors or a beater is treated with 4 volumes of water and filtered. The filtrate is evaporated on a water-bath at about $50^{\circ} \mathrm{C}$. and the residue powdered in a mortar.

r6. Tests on Powdered Egg Albumin.-With powdered albumin prepared as described above (by yourself or furnished by the instructor), try the following tests :

(a) Solubility.-Test the solubility of the albumin in water, sodium chloride, dilute acid and alkali.

(b) Millon's Reaction.

(c) Glyoxylic Acid Reaction (Hopkins-Cole).-When used to detect the presence of protein in solid form this reaction should be conducted as follows: Place 5 c.c. of concentrated sulphuric acid in a test-tube and add carefully, by means of a pipette, 3-5 c.c. of Hopkins-Cole reagent. Introduce a small amount of the solid substance to be tested, agitate the tube slightly, and note that the suspended pieces assume a reddish-violet color, which is the characteristic endreaction of the Hopkins-Cole test; later the solution will also assume the reddishviolet color.

(d) Composition Test.-Heat some of the dry powder in a dry test-tube in which is suspended a strip of moistened red litmus paper and across the mouth of which is placed a piece of filter paper moistened with lead acetate solution. As the powder is heated it chars, indicating the presence of carbon; the fumes of ammonia are evolved, turning the red litmus paper blue and indicating the presence of nitrogen and hydrogen; the lead acetate paper is blackened, indicating the presence of sulphur, and the deposition of moisture on the side of the tube indicates the presence of hydrogen. Moisture indicates hydrogen only in case both powder and test-tube used in the test are absolutely dry.

(e) Coagulation Test.-Immerse a dry test-tube containing a little powdered egg albumin in boiling water for a few moments. Remove and test the solubility of the albumin according to the directions given under (a) above. It is still soluble. Why has it not been coagulated? Repeat the above experiments with powdered serum albumin and see how the results compare with those just obtained.

\section{SULPHUR IN PROTEIN}

Sulphur is believed to be present in two different forms in the protein molecule. The first form, which is present in greatest amount, 
is that loosely combined with carbon and hydrogen. An example of this combination is shown in cystine,

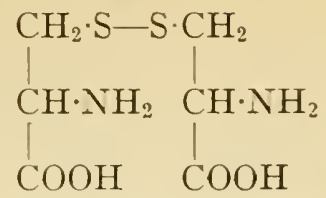

Sulphur in this form is variously termed unoxidized, loosely combined, mercaptan, and lead-blackening sulphur. The second form is combined in a more stable manner with carbon and oxygen and is known as oxidized or acid sulphur. The protamines are the only class of sulphurfree proteins.

\section{TESTS FOR SUlphuR}

I. Tests for Unoxidized Sulphur.-(a) To equal volumes of $\mathrm{KOH}$ and egg albumin solutions in a test-tube add I-2 drops of lead acetate solution and boil the mixture. Unoxidized sulphur is indicated by a darkening of the solution, the color deepening into a black if sufficient sulphur is present. Add hydrochloric acid and note the characteristic odor evolved from the solution. Write the reactions for this test. (b) Place equal volumes of $\mathrm{KOH}$ and egg albumin solutions in a test-tube and boil the mixture vigorously. Cool, make acid with glacial acetic acid and add I-2 drops of lead acetate. A darkening indicates the presence of unoxidized sulphur.

2. Test for Total Sulphur (Unoxidized and Oxidized).-Place the substance to be examined (powdered egg albumin) in a small porcelain crucible, add a suitable amount of solid fusion mixture (sodium carbonate and potassium nitrate mixed in the proportion $2: \mathrm{I}$ ) and heat carefully until a colorless mixture results. (Sodium peroxide may be used in place of this fusion mixture if desired.) Cool, dissolve the cake in a little warm water and filter. Acidify the filtrate with hydrochloric acid, heat it to the boiling-point and add a small amount of barium chloride solution. A white precipitate forms if sulphur is present. What is this precipitate?

\section{GLOBULINS}

Globulins are simple proteins especially predominant in the vegetable kingdom. They are closely related to the albumins and in common with them give all the ordinary protein tests. Globulins differ from the albumins in being insoluble in pure (salt-free) water. They are, however, soluble in neutral solutions of salts of strong bases with strong acids. Most globulins are precipitated from their solutions by saturation with solid sodium chloride or magnesium sulphate. As a class they are much less stable than the albumins, a fact shown by the increasing difficulty with which a globulin dissolves during the course of successive reprecipitations.

We have used an albumin of animal origin (egg albumin), for all 
the protein tests thus far, whereas the globulin to be studied will be prepared from a regetable source. There being no essential difference between animal and regetable proteins, the regetable globulin we shall study may be taken as a true type of all globulins, both animal and vegetable.

\section{Experiments ON Globelin}

Preparation of the Globulin.-Extract 20-30 grams (a handful) of crushed hemp seed with a 5 per cent solution of sodium chloride for one-half hour at $60^{\circ} \mathrm{C}$. Filter while hot through a paper moistened with 5 per cent sodium chloride solution. Place the filtrate in the water-bath at $60^{\circ} \mathrm{C}$. and allow it to stand for 24 hours in order that the globulin may crystallize slowly. In case the filtrate is cloudy it should be warmed to $60^{\circ} \mathrm{C}$. in order to produce a clear solution. The globulin is soluble in hot 5 per cent sodium chloride solution and is thus extracted

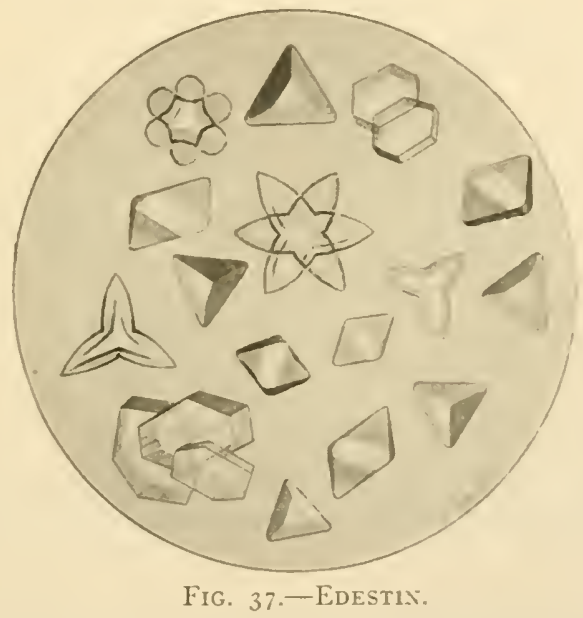

from the hemp seed, but upon cooling this solution much of the globulin separates in crystalline form. This particular globulin is called edestin. It crystallizes in several different forms, chiefly octahedra (see Fig. 37, above). (The crystalline form of excelsin, a protein obtained from the Brazil nut, is shown in Fig. 38 , p. IIo. This vegetable protein crystallizes in the form of hexagonal plates.) Filter off the edestin and make the following tests on the crystalline body and on the filtrate which still contains some of the extracted globulin.

Tests on Crystallized Edestin.-Microscopical examination (see Fig. 37.

(2) Solubility.-Try the solubility in the ordinary solvents (see page $2 \mathrm{I}$ ). Keep these solubilities in mind for comparison with those of edestan, to be made later (see page II5).

(3) Millon's Reaction.

(4) Coagulation Test.-Place a small amount of the globulin in a test-tube, add a little water and boil. Now add dilute hydrochloric acid and note that the protein no longer dissolves. It has been coagulated. 
(5) Dissolve the remainder of the edestin in 0.2 per cent hydrochloric acid and preserve this acid solution for use in the experiments on proteans (see page II5).

Tests on Edestin Filtrate.-(I) Influence of Protein Precipitants.-Try a few protein precipitants such as nitric acid, tannic acid, picric acid, and mercuric chloride.

(2) Biuret Test.

(3) Coagulation Test.-Boil some of the filtrate in a test-tube. What happens?

(4) Saturation with Sodium Chloride.-Saturate some of the filtrate with solid sodium chloride. How does this result differ from that obtained upon saturating egg albumin solution with solid sodium chloride?

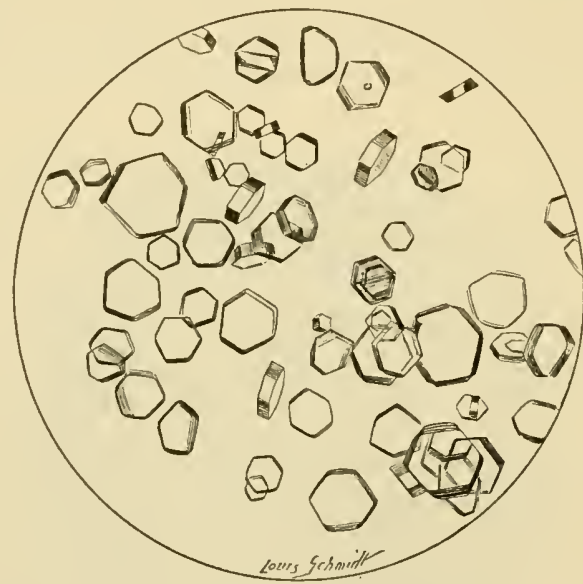

Fig. 38.-Excelsin, The Protein of the Brazil Nut.

(Drawn from crystals furnished by Dr. Thomas B: Osborne, New Haven, Conn.)

(5) Precipitation by Dilution.-Dilute some of the filtrate with ro-I5 volumes of water. Why does the globulin precipitate?

\section{Glutelins}

It has been repeatedly shown, particularly by Osborne, that after extracting the seeds of cereals with water, neutral salt solution, and strong alcohol, there still remains a residue which contains protein material which may be extracted by very dilute acid or alkali. These proteins which are insoluble in all neutral solvents, but readily soluble in very dilute acids and alkalis are called glutelins. The only member of the group which has yet received a name is the glutenin of wheat, a protein which constitutes nearly 50 per cent of the gluten, the remainder being principally gliadin. It is not definitely known whether glutelins occur as constituents of all seeds. 
Gluten: Preparation and Tests. ${ }^{1}$-To about 50 grams of wheat flour in a casserole or evaporating dish, add a little water and mix thoroughly until a stiff dough results. Knead this dough thoroughly and permit it to stand for about a half hour. This is done in order that the maximum quantity of gluten may be obtained. Treat the dough with about 200 c.c. of water and knead it thoroughly. Note the yellowish color of the dough and the milky appearance of the water due to suspended starch granules. (Place a drop of the suspension on a slide, cover with a cover slip, run underneath the slip a drop of iodine solution and observe the stained starch granules under the microscope.) Filter and apply a protein color reaction (see page 97) to the filtrate. It should be positive indicating that water-soluble proteins were present in the flour. Add fresh water to the dough and repeat the kneading process. Continue this procedure with fresh addition of water until practically no starch granules are noted in suspension. To a small piece of the yellow, fibrous gluten apply Millon's Reaction (page 97). This test shows gluten to be protein material. Utilize the remainder of the gluten in the preparation of gliadin (page I12).

Glutenin: Preparation and Tests.-(In the preparation of gliadin (page I12) it is customary to remove this prolamin from the crude gluten by extracting with 70 per cent alcohol. Inasmuch as gluten consists chiefly of gliadin and glutenin the portion of the gluten remaining after the extraction of the alcohol-soluble protein gliadin may be utilized for the preparation of glutenin.)

To the finely divided residue from the preparation of gliadin (page II2) in a flask or bottle add about 250 c.c. of 70 per cent alcohol. Allow to stand for about 48 hours with repeated shaking. This alcohol treatment will remove the gliadin and leave crude glutenin. To purify the glutenin treat it in a mortar, with sufficient 0.2 per cent $\mathrm{NaOH}$ to dissolve it, and filter the liquid through a wet pleated filter. Neutralize the filtrate carefully, with 0.2 per cent $\mathrm{HCl}$ adding the acid drop by drop with thorough mixture after each addition. (The glutenin is soluble in excess of acid.) Filter off the glutenin precipitate and wash several times with 70 per cent. alcohol and finally with water. Apply the following tests:

I. Solubility in water, salt solution, 0.2 per cent $\mathrm{HCl}$ and 0.5 per cent $\mathrm{Na}_{2} \mathrm{CO}_{3}$.

2. Millon's Reaction.

\section{Prolamins (Alcohol-soluble Proteins)}

The term prolamin has been proposed by Osborne for the group of proteins formerly termed "alcohol-soluble proteins." The name is very appropriate inasmuch as these proteins yield, upon hydrolysis, especially large amounts of proline and ammonia. The prolamins are simple proteins which are insoluble in water, absolute alcohol and other neutral solvents, but are soluble in 70 to 80 per cent alcohol and in dilute acids and alkalis. They occur widely distributed, particularly in the vegetable kingdom. The only prolamins yet described are the zein of maize, the hordein of barley, the gliadin of wheat and rye, and the bynin of malt. They yield relatively large amounts of glutamic acid on hy-

1 This experiment as well as those on glutcnin and gliadin which follow have been adapted from directions given in Laboratory Notes of Professor Gies, College of Physicians and Surgeons, New York. 
drolysis but no lysin. The largest percentage of glutamic acid (43.66 per cent) ever obtained as a decomposition product of a protein substance has very recently been obtained by Osborne and Guest from the hydrolysis of the prolamin gliadin. ${ }^{1}$ This yield of glutamic acid is also the largest amount of any single decomposition product yet obtained from any protein except protamines.

Gliadin: Preparation and Tests.-Introduce the finely divided crude gluten as prepared on page III into a flask or bottle, add about $25^{\circ}$ c.c. of 70 per cent alcohol ${ }^{2}$ and allow the mixture to stand 24 hours with occasional shaking. Filter (retaining the undissolved portion for preparation of glutenin, page III), evaporate the filtrate to dryness in a porcelain dish over a water-bath. Pulverize the dry material. Apply the following tests to this gliadin powder:

Solubility and Protein Tests.-Test the solubility in alcohol (30 per cent, 50 per cent and 70 per cent), water, 0.9 per cent $\mathrm{NaCl}, 0.2$ per cent $\mathrm{HCl}$ and 0.5 per cent $\mathrm{Na}_{2} \mathrm{CO}_{3}$. Shake each test repeatedly and filter. To the filtrate apply Coagulation test (page 105) and Biuret test (page 98).

\section{Albuminoids (Scleroproteins)}

The albuminoids yield similar hydrolytic products to those obtained from the other simple proteins already considered, thus indicating that they possess essentially the same chemical structure. They differ from all other proteins, whether simple, conjugated, or derived, in that they are insoluble in all neutral solvents. The albuminoids include "the principal organic constituents of the skeletal structure of animals as well as their external covering and its appendages." Some of the principal albuminoids are keratin, elastin, collagen, reticulin, spongin, and fibroin. Gelatin cannot be classed as an albuminoid although it is a transformation product of collagen. The various albuminoids differ from each other in certain fundamental characteristics which will be considered in detail under Epithelial and Connective Tissue (see Chapter XVIII).

\section{CONJUGATED PROTEINS}

Conjugated proteins consist of a protein molecule united to some other molecule or molecules otherwise than as a salt. We have glycoproteins, mucleoproteins, hemoglobins (chromoproteins), phosphoproteins and lecithoproteins as the five classes of conjugated proteins.

Glycoproteins may be considered as compounds of the protein mole-

${ }^{1}$ Osborne and Guest: Jour. Biol. Chem., 9, 425, I9rr. Up to this time the yield of 4I.32 per cent obtained by Kleinschmitt from hordein was the maximum yield.

${ }_{2}^{2}$ Bailey and Blish claim that 50 per cent alcohol is more satisfactory (Jour. Biol. Chem., 23, 345, I915). 
cule with a substance or substances containing a carbohydrate group other than a nucleic acid. The glycoprolcins yield, upon decomposition, protein and carbohydrate derivatives, notably glucosamine, $\mathrm{CH}_{2} \mathrm{OH}$.$(\mathrm{CHOH})_{3} \cdot \mathrm{CH}\left(\mathrm{NH}_{2}\right) \cdot \mathrm{CHO}$, and galactosamine, $\mathrm{OHCH}_{2} \cdot(\mathrm{CHOH})_{3} \cdot \mathrm{CH}-$ $\left(\mathrm{NH}_{2}\right)$.CHO. The principal glycoproteins are mucoids, mucins, and chondroproteins. By the term mucoid we may in general designate those glycoproteins which occur in tissues, such as lendomucoid from tendinous tissue and osseomucoid from bone. (For the preparation of tendomucoid see Chapter XVIII.) The elementary composition of these typical mucoids is as follows:

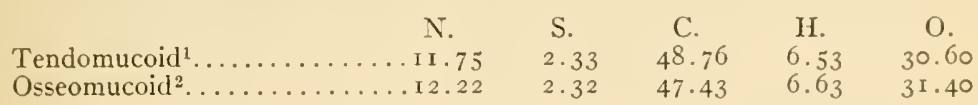

The term mucins may be said in general to include those forms of glycoproteins which occur in the secretions and fluids of the body (For the preparation of salivary mucin see Chapter III.) Chondroproteins are so named because chondromucoid, the principal member of the group, is derived from cartilage (chondrigen). A myloid, ${ }^{3}$ which appears pathologically in the spleen, liver, and kidneys, is also a chondroprotein.

The phosphoproteins are considered to be "compounds of the protein molecule and some, as yet undefined, phosphorus-containing substances other than a nucleic acid or lecithin." The percentage of phosphorus in phosphoproteins is very similar to that in nucleoproteins, but they differ from this latter class of proteins in that they do not yield any purine bases upon hydrolytic cleavage. Two of the common phosphoproteins are the casein of milk and the ovovitellin of the eggyolk. The phosphorus in these, as in all proteins, exists in phosphoric acid radicals. For the preparation of a typical phosphoprotein (casein) see Chapter XVII.

The hemoglobins (chromoproteins) are compounds of the protein molecule with hematin or some similar substance. The principal member of the group is the hemoglobin of the blood. Upon hydrolytic clearage this hemoglobin yields a protein termed globin and a coloring matter termed hemochromogen. The latter substance contains iron and upon coming into contact with oxygen is oxidized to form hematin. Hemocyanin, another member of the class of hemoglobins, occurs in the blood of certain invertebrates, notably cephalopods, gasteropods, and

${ }^{1}$ Chittenden and Gies: Jour. Lxp. Mcd., I, IS6, I 896 .

${ }^{2}$ Hawk and Gies: Imer. Jour. Physiol., 5, 387, I 901.

${ }^{3}$ Not to be confused with the substance amyloid which may be formed from cellulose (see p. 40). 
crustacea. Hemocyanin generally contains either copper, manganese, or zinc in place of the iron of the hemoglobin molecule. For the preparation of hemoglobin in crystalline form see Chapter XV.

The lecithoproteins consist of a protein molecule joined to lecithin. They have been comparatively little studied and may possibly be mixtures of protein and lecithin.

For consideration of mucleoproteins see Chapter VI.

\section{DERIVED PROTEINS}

These substances are derivatives which are formed through hydrolytic changes of the original protein molecule. They may be divided into two groups, the primary protein derivatives and the secondary protein derivatives. The term secondary derivatives is made use of in this connection since the formation of the primary derivatives generally precedes the formation of these secondary derivatives. These derived proteins are obtained from native simple proteins by hydrolyses of various kinds, e.g.. through the action of acids, alkalis, heat, or enzymes. The particular class of derived protein desired regulates the method of treatment to which the native protein is subjected.

\section{Primary Protein Derivatives}

The primary protein derivatives are "apparently formed through hydrolytic changes which involve only slight alterations of the protein molecule." This class includes proteans, metaproteins and coagulated proteins.

\section{PROTEANS}

Proteans are those insoluble protein substances which are produced from proteins originally soluble through the incipient action of water, enzymes, or very dilute acids. It is well known that globulins become insoluble upon repeated reprecipitation and it may possibly be found that the greater number of the proteans are transformed globulins. Osborne, however, believes that nearly all proteins may give rise to proteans. This investigator who has so very thoroughly investigated many of the vegetable proteins claims that the hydrogen ion is the active agent in the transformation. The protein produced from the transformation of $e$ destin is called edestan, that produced from myosin is called myosan, etc. The name protean was first given to this class of proteins by Osborne in I 900 in connection with his studies of edestin. 


\section{Experiments on Proteans}

Preparation and Study of Edestan.-Prepare edestin according to the directions given on page 109. Bring the edestin into solution in 0.2 per cent hydrochloric acid and permit the acid solution to stand for about one-half hour. ${ }^{1}$ Neutralize with a 0.5 per cent. solution of sodium carbonate, filter off the precipitate of edestan and make the following tests:

I. Solubility.-Try the solubility in water, sodium chloride, dilute acid and alkali. Note the altered solubility of the edestan as compared with that of edestin (see page rog).

\section{Millon's Reaction.}

3. Coagulation Test.-Place a small amount of the protean in a test-tube, add a little water and boil. Now add dilute hydrochloric acid and note that the protein no longer dissolves. It has been coagulated.

4. Tests on Edestan Solution.-Dissolve the remainder of the edestan precipitate in 0.2 per cent hydrochloric acid and make the following tests:

(a) Biuret Test.

(b) Infuence of Protein Precipitants. - Try a few protein precipitants such as picric acid and mercuric chloride.

\section{METAPROTEINS}

The metaproteins are formed from the native simple proteins through an action similar to that by which proteans are formed. In the case of the melaproteins, however, the changes in the original protein molecule are more profound. These derived proteins are characterized by being soluble in very weak acids and alkalis, but insoluble in neutral fluids. The metaproteins were formerly termed albuminates, but inasmuch as the termination ate signifies $a$ salt it has always been somewhat of a misnomer.

Two of the principal metaproteins are the acid metaprotein or socalled acid albuminate and the alkali metaprotein or so-called alkali albuminate. They differ from the native simple proteins principally in being insoluble in sodium chloride solution and in not being coagulated except when suspended in neutral fluids. Both forms of metaprotein are precipitated upon the approximate neutralization of their solutions. They are precipitated by saturating their solutions with ammonium sulphate, and by sodium chloride also, provided they are dissolved in an acid solution. Acid metaprotein contains a higher percentage of nitrogen and sulphur than the alkali metaprotein from the same source, since some of the nitrogen and sulphur of the original protein is liberated in the formation of the latter. Because of this fact, it is impossible to transform an alkali metaprotein into an acid metaprotein, while it is possible to reverse the process and transform the acid metaprotein into the alkali modification.

1 The edestan solution preserved from experiment (5), p. 110, may be used. 


\section{Experiments on Metaproteins}

\section{ACID METAPROTEIN (ACID ALBUMINATE)}

Preparation and Study. - Take 25 grams of hashed lean beef washed free from the major.portion of blood and inorganic matter, and place it in a mediumsized beaker with roo c.c. of 0.2 per cent $\mathrm{HCl}$. Place it on a boiling water-bath for one-half hour, filter, cool, and divide the filtrate into two parts. Neutralize the first part with dilute $\mathrm{KOH}$ solution, filter off the precipitate of acid metaprotein and make the following tests :

(I) Solubility.-Solubility in the ordinary solvents (see page $2 \mathrm{I}$ ).

(2) Millon's Reaction.

(3) Coagulation Test.-Suspend a little of the metaprotein in water (neutral solution) and heat to boiling for a few moments. Now add I-2 drops of $\mathrm{KOH}$ solution to the water and see if the metaprotein is still soluble in dilute alkali. What is the result and why?

(4) Test for Unoxidized Sulphur (see page 108).

Subject the second part of the original solution to the following tests:

(5) Coagulation Test.-Heat some of the solution to boiling in a test-tube. Does it coagulate?

(6) Biuret Test.

(7) Influence of Protein Precipitants.-Try a few protein precipitants such as picric acid and mercuric chloride. How do the results obtained compare with those from the experiments on egg albumin? (See page 103.)

\section{ALKALI METAPROTEIN (ALKALI ALBUMINATE)}

Preparation and Study.-Carefully separate the white from the yolk of a hen's egg and place the former in an evaporating dish. Add concentrated potassium hydroxide solution, drop by drop, stirring continuously. The mass gradually thickens and finally assumes the consistency of jelly. This is solid alkali metaprotein or "Lieberkühn's jelly." Do not add an excess of potassium hydroxide or the jelly will dissolve. Cut it into small pieces, place a cloth or wire gauze over the dish, and by means of running water wash the pieces free from adherent alkali. Now add a small amount of water, which forms a weak alkaline solution with the alkali within the pieces, and dissolve the jelly by gentle heat. Cool the solution and divide it into two parts. Proceed as follows with the first part: Neutralize with dilute hydrochloric acid, noting the odor of the liberated hydrogen sulphide as the alkali metaprotein precipitates. Filter off the precipitate and test as for acid metaprotein (tests I, 2, 3 and 4), above, noting particularly the sulphur test. How does this test compare with that given by the acid metaprotein? Make tests on the second part of the solution the same as for acid metaprotein (tests 5,6 and 7 ) above.

\section{Coagulated Proteins}

These derived proteins are produced from unaltered protein materials by heat, by long standing under alcohol, or by the continuous movement of their solutions such as that produced by rapid stirring or shaking. In particular instances, such as the formation of fibrin from 
fibrinogen (sec page 256 ), the coagulation may be produced by enzyme action. Ordinary soluble proteins after having been transformed into the coagulated modification are no longer soluble in the ordinary solvents. Upon being heated in the presence of strong acids or alkalis, coagulated proteins are converted into metaproteins.

Many proteins coagulate at an approximately fixed temperature under definite conditions (see pages I05 and 339). This characteristic may be applied to separate different coagulable proteins from the same solution by fractional coagulation. The coagulation temperature frequently may serve in a measure to identify proteins in a manner similar to the melting-point or boiling-point of many other organic substances. The separation of proteins by fractional coagulation is thus analogous to the separation of volatile substances by means of fractional distillation. This method of separating proteins is not a satisfactory one, however, inasmuch as proteins in solution have different effects upon one another and also because of the fact that the nature of the solvent causes a variation in the temperature at which a given protein coagulates. The nature of the process involved in the coagulation of proteins by heat is not well understood, but it is probable that in addition to the altered arrangement of the component atoms in the molecule, there is a mild hydrolysis which is accompanied by the liberation of minute amounts of hydrogen, nitrogen, and sulphur. The presence of a neutral salt or a trace of a mineral acid may facilitate the coagulation of a protein solution (see page 105), whereas any appreciable amount of acid or alkali will retard or entirely prevent such coagulation.

It has been shown that the coagulation of proteins by heat proceeds in two stages: ${ }^{1}$ first, a reaction between the protein and the hot water (denaturation), and second, an agglutination or separation of the altered protein in particulate form. The concentration of acid, or hydrogen ion, in the solution influences the coagulation of proteins, such that the original protein is acted upon less readily by hot water alone than in the presence of acid. The formation of the coagulum is accompanicd by the disappearance of the frec acid from the solution, indicating the formation of a protein salt. A disturbance of the equilibrium between the hydrolyzed and unhydrolyzed portions of the protein salt, due to the greater rapidity with which the unhydrolyzed portion is precipitated, results in the gradual removal of both protein and acid from the solution. This has been offered as an explanation of the decreasing acidity.

According to Chick and Martin, the addition of neutral salts to the acid solution of the salt-frec protein to be coagulated results in a decreased

${ }^{1}$ Chick and Martin: Journal of Physiology, 43, I, I9Ir. 
rate of coagulation. This is due in part to the decrease in the concentration of the free acid, which results from the disturbance of the equilibrium between the protein and acid and also in part to the direct influence which the salts exert upon the protein. The presence of neutral salts may under certain circumstances facilitate the coagulation of proteins by heat.

The temperature at which egg-white is coagulated causes a difference in the appearance of the coagulum. ${ }^{1}$ Coagulated egg-white which has been immersed in water at a low temperature and then gradually heated to the coagulating temperature is more translucent and has a bluish color, whereas egg-white which has been immersed in water heated to a temperature above the coagulating temperature is creamy white in color. They also possess different digestibilities.

\section{Experiments on Coagulated Protein}

Ordinary coagulated egg-white may be used in the following tests:

I. Solubility.-Try the solubility of small pieces of the coagulated protein in each of the ordinary solvents (see page $2 \mathrm{I}$ ).

2. Millon's Reaction.

3. Xanthoproteic Reaction.-Partly dissolve a medium-sized piece of the protein in concentrated nitric acid. Cool the solution and add an excess of ammonium hydroxide. Both the protein solution and the undissolved protein will be colored orange.

4. Biuret Test.-Partly dissolve a medium-sized piece of the protein in concentrated potassium hydroxide solution. If the proper dilution of copper sulphate solution is now added the white coagulated protein, as well as the protein solution, will assume the characteristic purplish-violet color.

5. Glyoxylic Acid Reaction (Hopkins-Cole).-Conduct this test according to the modification given on page ro7.

\section{Secondary Protein Derivatives}

These derivatives result from a more profound cleavage of the protein molecule than that which occurs in the formation of the primary derivatives. The class includes proteoses, peptones, and peptides.

\section{PROTEOSES AND PEPTONES}

Proteoses are intermediate products in the digestion of proteins by proteolytic enzymes, as well as in the decomposition of proteins by hydrolysis and the putrefaction of proteins through the action of bacteria. Proteoses are called albumoses by some writers, but it seems more logical to reserve the term albumose for the proteose of albumin.

Peptones are formed after the proteoses and it has been customary to

${ }^{1}$ Frank: Journal of Biological Chemistry, 9, 463, I9I I. 
consider them as the last product of the processes before mentioned which still possess true protein characteristics. In other words, ithas been considered that the protein nature of the end-products of the cleavage of the protein molecule ceased with the peptones, and that the simpler bodies formed from peptones were substances of a different nature (see page 65). However, as the end-products have been more carefully studied, it has been found to be no easy matter to designate the exact character of a peptone or to indicate the exact point at which the peptone characteristic ends and the peptide characteristic begins. The situation regarding the proteoses, peptones and peptides is at present a most unsatisfactory one because of the unsettled state of our knowledge regarding them. The exact differences between certain members of the peptone and peptide groups remain to be more accurately established. It has been quite well established that the peptones are peptides or mixtures of peptides, but the term peptide is used at present to designate only those possessing a definite structure.

There are several proteoses (protoproteose, heteroproteose and deuteroproteose), and at least two peptones (amphopeptone and antipeptone), which result from proteolysis. The differentiation of the various proteoses and peptones at present in use is rather unsatisfactory. These compounds are classified according to their varying solubilities, especially in ammonium sulphate solutions of different strengths. The exact differences in composition between the various members of the group remain to be more accurately established. Because of the difficulty attending the separation of these bodies, pure proteose and peptone are not easy to procure. The so-called peptones sold commercially contain a large amount of proteose. As a class the proteoses and peptones are very soluble, diffusible bodies which are non-coagulable by heat. Peptones differ from proteoses in being more diffusible, non-precipitable by $\left(\mathrm{NH}_{4}\right)_{2} \mathrm{SO}_{4}$, and by their failure to give any reaction with potassium ferrocyanide and acelic acid, potassio-mercuric iodide and $\mathrm{HCl}$, picric acid, and trichloracetic acid. Peptones may be precipitated by phosphotungstic acid, phosphomolybdic acid, absolute alcohol and tannic acid, but an excess of the precipitant may dissolve the precipitate. The so-called primary proteoses are precipitated by $\mathrm{HNO}_{3}$ and are the only members of the proteose-peptone group which are so precipitated.

Some of the more general characteristics of the proteose-peptone group may be noted by making the following simple tests on a proteose-peptone powder:

(I) Solubility.- Solubility in hot and cold water and sodium chloride solution. (2) Millon's Reaction.

Dissolve a little of the powder in water and test the solution as follows: 
(I) Precipitation by Picric Acid.-To 5 c.c. of proteose-peptone solution in a test-tube add picric acid until a permanent precipitate forms. The precipitate disappears on heating and returns on cooling.

(2) Precipitation by a Mineral Acid.- Try the precipitation by nitric acid.

(3) Coagulation Test.-Heat a little proteose-peptone solution to boiling. Does it coagulate like the other simple proteins studied?

\section{SEPARATION OF PROTEOSES AND PEPTONES ${ }^{1}$}

Place 50 c.c. of proteose-peptone solution in an evaporating dish or casserole, and half-saturate it with ammonium sulphate solution, which may be accomplished by adding an equal volume of saturated ammonium sulphate solution. At this point note the appearance of a precipitate of the primary proteoses (protoproteose and hetero-proteose). Now heat the half-saturated solution and its suspended precipitate to boiling and saturate the solution with solid ammonium sulphate. At full saturation the secondary proteoses (deuteroproteoses) are precipitated. The peptones remain in solution.

Proceed as follows with the precipitate of proteoses: Collect the sticky precipitate on a rubber-tipped stirring rod or remove it by means of a watch glass to a small evaporating dish and dissolve it in a little water. To remove the ammonium sulphate, which adhered to the precipitate and is now in solution, add barium carbonate, boil, and filter off the precipitate of barium sulphate. Concentrate the proteose solution to a small volume ${ }^{2}$ and make the following tests :

(r) Biuret Test.

(2) Precipitation by Nitric Acid.-What would a precipitate at this point indicate?

(3) Precipitation by Trichloracetic Acid.-This precipitate dissolves on heating and returns on cooling.

(4) Precipitation by Picric Acid.-This precipitate also disappears on heating and returns on cooling.

(5) Precipitation by Potassio-mercuric Iodide and Hydrochloric Acid.

(6) Coagulation Test.-Boil a little in a test-tube. Does it coagulate?

(7) Acetic Acid and Potassium Ferrocyanide Test.

The solution containing the peptones should be cooled and filtered, and the ammonium sulphate in solution removed by boiling with barium carbonate as described above. After filtering off the barium sulphate precipitate, concentrate the peptone filtrate to a small volume and repeat the tests as given under the proteose solution, above. Also try the precipitation by phosphotungstic acid and by tannic acid. In the biuret test the solution should be made very strongly alkaline with solid potassium hydroxide.

\section{PEPTIDES}

The peptides are "definitely characterized combinations of two or more amino acids, the carboxyl ( $\mathrm{COOH})$ group of one being united

1 The separation of proteoses and peptones by means of fractional precipitation with ammonium sulphate does not possess the significance it was once supposed to possess inasmuch as the boundary between these substances and peptides is not well defined (see p. I Ig).

${ }^{2}$ If the proteoses are desired in powder form, this concentrated proteose solution may now be precipitated by alcohol, and this precipitate, after being washed with absolute alcohol and with ether, may be dried and powdered. 
with the amino $\left(\mathrm{NH}_{2}\right)$ group of the other with the elimination of a molecule of water." These peptides are more fully discussed on pages $7^{\circ}$ and Iig.

\section{REVIEW OF PROTEINS}

In order to facilitate the student's review of the proteins, the preparation of a chart similar to the model given is recommended. The signs + and - may be conveniently used to indicate positive and negative reactions.

\section{MODEL CHART FOR REVIEW PURPOSES}

Protein

Albumin

Globulin

Nucleoprotein

Phosphoprotein

Glucoprotein

Acid metaprotein

Alkali metaprotein

Proteose

Peptone

Coagulated protein

\section{"Unknown" Mextures and Solutions of Proteins}

At this point the student's knowledge of the characteristics of the various proteins studied will be tested by requiring him to examine several "unknown" protein mixtures or solutions and make full report upon the same. The scheme given on page 122 may be used in this examination. 


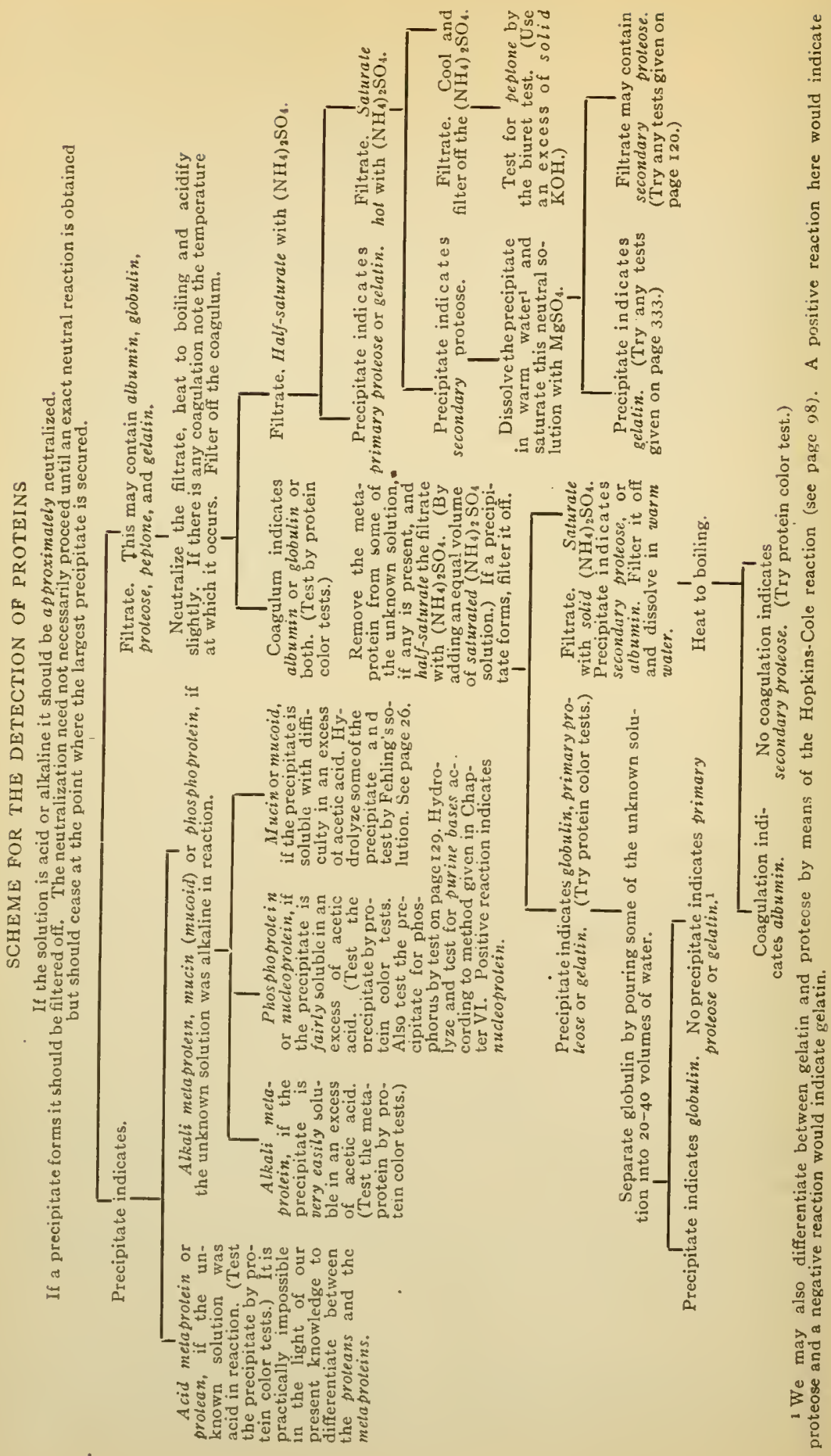




\section{NUCLEIC ACIDS AND NUCLEOPROTEINS ${ }^{1}$}

The Nucleoproteins. - The nucleoproteins occur widely distributed in the animal and plant kingdoms, being found in nearly all cells and particularly in the nuclei of cells. They are found in especially large amounts in glandular tissues such as those of the thymus, pancreas and spleen. The nucleoproteins are combinations of protein with a phosphorus-containing substance known as nucleic acid. As different nucleic acids exist and are found in combination with different proteins, a variety of nucleoproteins exist. The protein combined with the nucleic acid is in certain cases a histone, the conjugated protein in this case being called a nucleohistone.

The nucleoproteins give the ordinary protein color reactions. They are acidic in character and insoluble. in water. They are readily soluble in weak alkali but are precipitated from such solution on the addition of acetic acid in excess of which they dissolve with more or less difficulty although readily soluble in very dilute hydrochloric acid. We distinguish them from mucins, which are likewise precipitated by acetic acid through the fact that the latter give no tests for phosphorus on decomposition.

The nucleoproteins are very complex and unstable substances and one has probably never been prepared in a pure form. Under the action of the gastric juice or of weak acid nucleoproteins lose a portion of their protein content and are transformed into a rather ill-defined class of substances known as mucleins which still possess some protein in combination with the nucleic acid molecule. In most cases the decomposition does not proceed further in gastric digestion. Through the action of the pancreatic juice, however, the remainder of the protein is split off and the nucleic acid set free. The decomposition of nucleoprotein may be diagramatically expressed thus, although the course of decomposition is probably not quite so simple as indicated.

${ }^{1}$ For review of the literature on nucleic acids and nucleases see Monograph on "Nucleic Acids" by Walter Jones, New York, I9I4, Longmans Green \& Co. 


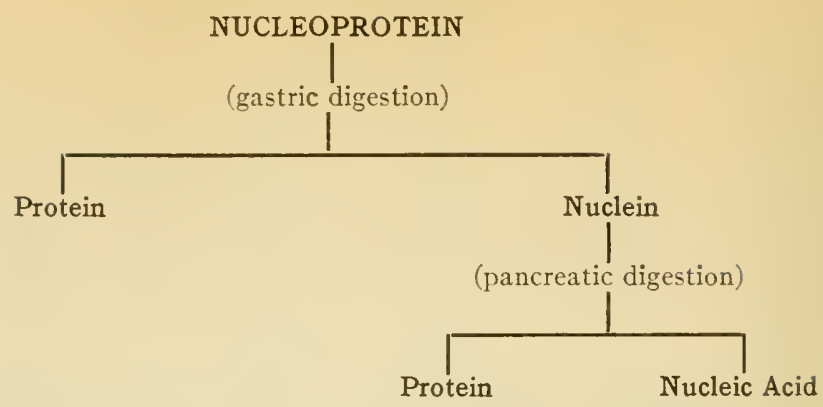

The Nucleic Acids. - The nucleic acids of the animal body occur mainly in combination with protein material in the so-called nucleoproteins of which they form the characteristic radicals (see page I23). The amount and character of the protein with which the nucleic acid molecule is combined varies and the acid may in certain cases be found in cells in a free form. Naturally those tissues are richest in nucleic acid which contain the largest amount of nuclear material and of nucleoprotein. Such are the glandular tissues of the body as the thymus, spleen, pancreas, liver, etc. The heads of the spermatozoa consist almost entirely of nucleic acid in combination with protamine.

The nucleic acids are a distinct class of substances, characterized by their decomposition products. They are strongly acid in reaction and contain considerable phosphorus. They may be divided into two main groups, the animal and the plant nucleic acids. The two classes differ in certain respects but all of the true animal nucleic acids appear to be practically identical in composition. Animal nucleic acid is most readily prepared from the thymus while plant nucleic acid is most readily obtained from yeast.

The nucleic acids are difficultly soluble in cold water, more readily in hot water, insoluble in alcohol, but readily soluble in weak alkali with the formation of the alkali salt. If pure they do not give the protein color reactions. They are optically active. They are precipitated from their alkaline solutions by $\mathrm{HCl}$, but only the plant nucleic acid is precipitated by acetic acid. In weak acid solution they are precipitated by protein the combination being considered a "nuclein." They form insoluble salts with alkaline earth and heavy metals. The sodium salt of animal nucleic acid in 4 per cent solution is liquid while warm but solidifies to a gelatinous mass on cooling. Plant nucleic acid does not do this.

The nucleic acids on hydrolysis yield phosphoric acid, purine and pyrimidine bases, and a carbohydrate or carbohydrate derivative. The composition varies slightly with the type of nucleic acid. Plant nucleic acids contain a pentose group while animal nucleic acids contain a 
hexose group. Both types contain the purine bases, guanine and adenine and the pyrimidine base cytosine. Plant nucleic acid contains also the pyrimidine base uracil, which in the animal nucleic acid is substituted by the base thymine. The nucleic acids are not, however, simple substances whose molecules contain a single phosphoric acid or carbohydrate group. They are apparently combinations of several radicals known as nucleotides each of which contains one carbohydrate group combined with a single base and a single phosphoric acid molecule. Thus the following structural formula has been given to yeast nucleic acid by Levene and Jacobs ${ }^{1}$ indicating that it contains four nucleotide radicals and may hence be called a tetranucleotide.

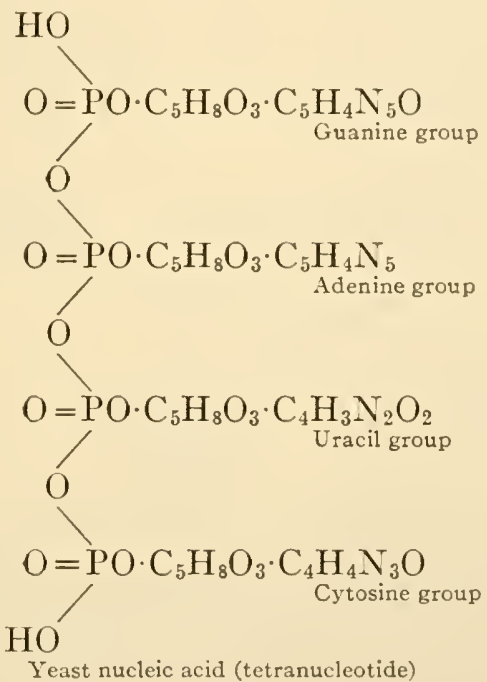

The cleavage of the nucleic acid molecule into its corresponding nucleotides is brought about during digestion by enzymes present in the intestinal juice and intestinal mucosa. Enzymes of similar origin act further on the nuclcotides thus formed and split off the phosphoric acid radicals together with carbohydrate-base compounds which are called nucleosides. The decomposition prior to absorption does not probably proceed further than to the formation of nucleotides and nucleosides. Many tissues however contain enzymes capable of completing the decomposition with liberation of the carbohydrate and basic radicals. The purine bases may also be deaminized while still in combination as nucleosides and further hydrolysis would then lead to the direct liberation of the oxypurines instead of their precursors, the amino-purines.

${ }^{1}$ Levene and Jacobs: Ber. d. deulsch. Chem. Ges., 43, 3151, 1010; 44, 1027, I01 
Jones $^{1}$ has suggested a method by which the course of the decomposition of the nucleic acid molecule can be followed. By this means it is readily shown that phosphoric acid is liberated at very different rates from the different nucleotides.

The following outline will indicate the course of decomposition of a nucleic acid and the enzymes involved in the process.

\section{DECOMPOSITION OF NUCLEIC ACID}

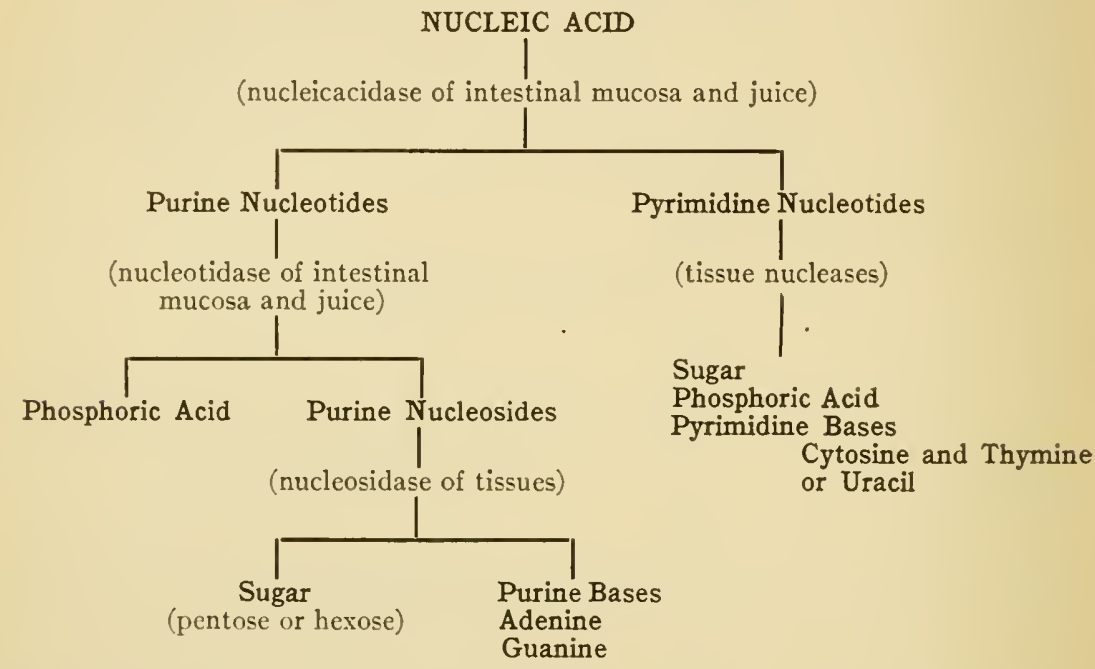

With regard to the fate of the various radicals of the nucleic acids in the body after absorption little is definitely known. The phosphoric acid may of course be built up into phosphorus-containing cell constituents such as nucleoproteins, phosphoproteins or phosphatides, or be eliminated as phosphate in the urine. The carbohydrate portion may undergo the usual transformations of intermediary carbohydrate metabolism. The nucleosides appear to be ordinarily absorbed unchanged from the intestine and may be to a certain extent directly resynthesized in the animal body to nucleoprotein. The excess over body requirement must, however, be decomposed, although a certain portion may possibly be stored up in the individual cells or in certain organs. Enzymes capable of decomposing nucleic acids are found in most of the cells of the body.

The Purine Bases.-As has been indicated the basic substances present in nucleic acid belong to two classes the purine and pyrimidine bases. The purine bases set free on the decomposition of nucleic acid are adenine and guanine belonging to the class of amino purines.

${ }^{1}$ Jones: Presidential address before the Society of Biological Chemists, Boston, Dec. 27, I9I5. 
The fate of the amino purines in the animal body is of considerable interest. It has been shown that certain tissues contain enzymes which transform these amino purines first to corresponding oxypurines known as hypoxanthine and xanthine and finally to uric acid. It is probable that different enzymes enter into the various steps of these transformations leading to the formation of uric acid. Still another enzyme carries the oxidation further with the formation of the compound allantoin. This enzyme is known as uricase. The/purine enzymes are widely distributed in tissues. The transformations brought about are indicated in the following diagrams.
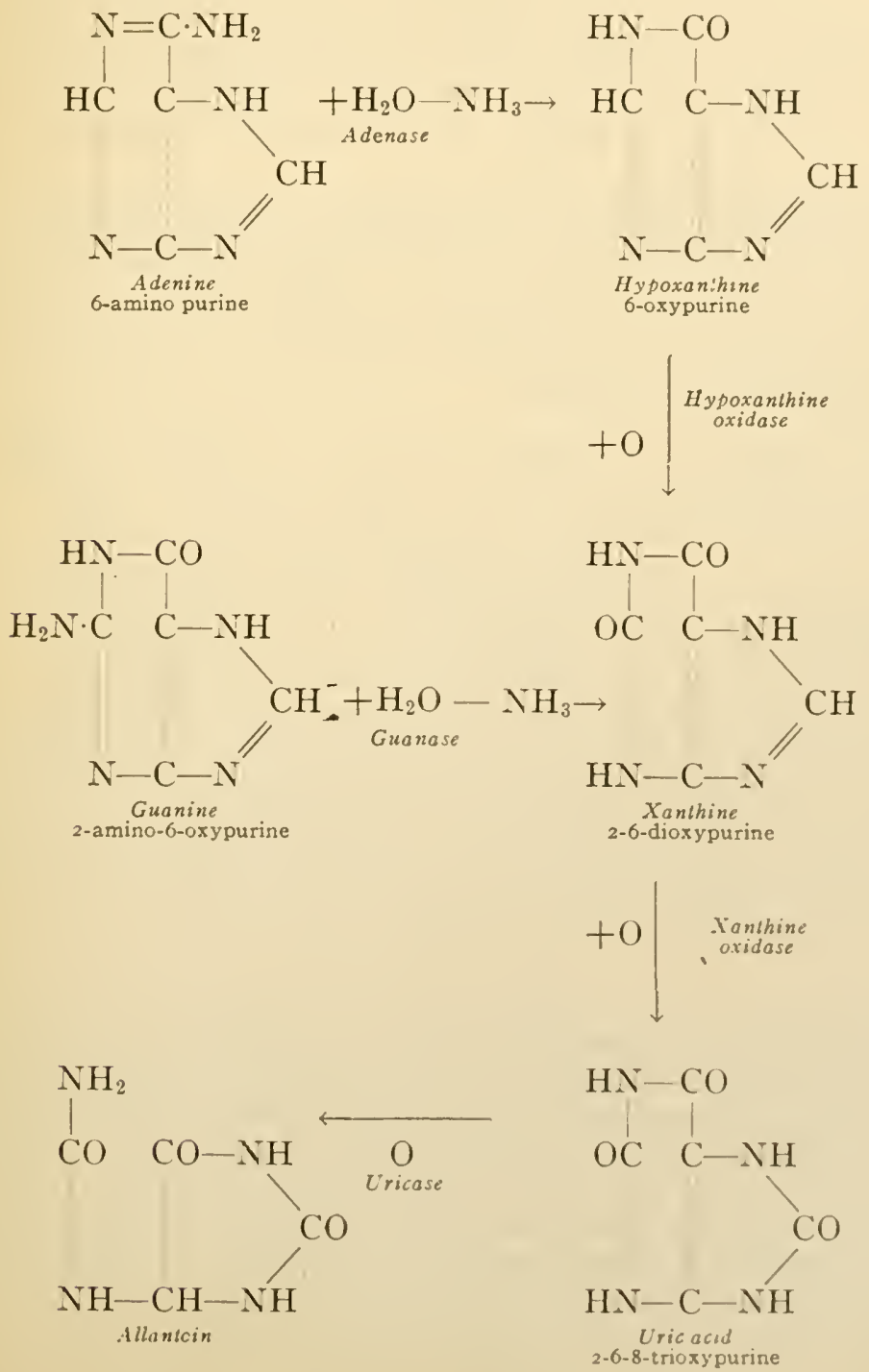
All of the physiologically important purine bodies are precipitated by ammoniacal silver nitrate solution in the cold and by copper sulphate and sodium bisulphite in boiling solutions. Some of them are readily identified by their crystalline forms or the crystalline forms of certain of their salts. Uric acid differs from the other purines in being insoluble in dilute sulphuric acid. The purine bodies may be distinguished to a certain extent also by the reactions which they give when their solutions are evaporated with nitric acid and the residue treated with ammonia. Uric acid gives the characteristic formation of the purple murexide (ammonium purpurate). Potassium hydroxide changes this to a bluish-violet color which disappears on heating. Xanthine and guanine form yellow compounds with nitric acid which turn purple or violet on treating with potassium hydroxide. The color in this case is not lost by heating. Adenine and hypoxanthine do not give a color reaction with nitric acid.

The Pyrimidine Bases.-The pyrimidine bases entering into the composition of nucleic acid are thymine, cytosine and uracil. Cytosine is found in both types of nucleic acid, while thymine is found only in animal nucleic acid and uracil only in plant nucleic acid. They possess the following formulas.
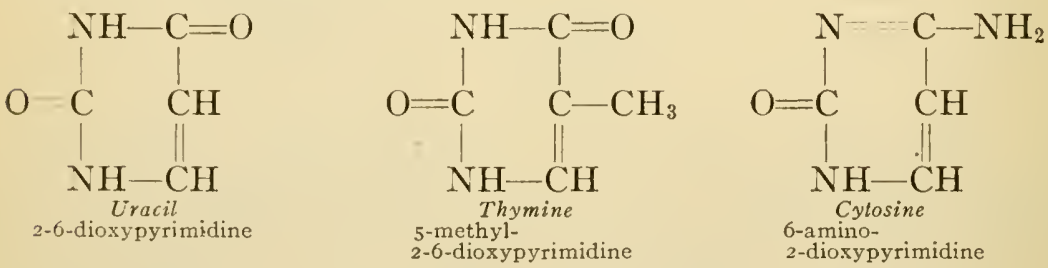

With regard to the fate of pyrimidine bases in metabolism very little is known. When the bases as such are fed they reappear unchanged in the urine. ${ }^{1}$ If nucleic acid is fed this does not occur which indicates that the pyrimidine bases may undergo certain alterations in the animal body while still existing in combination.

\section{Experiments}

I. Preparation of Nucleoprotein from Yeast. ${ }^{2}$ Place two small cakes of ordinary compressed yeast in a mortar. Sprinkle a small horn-spoonful of sand over the yeast, add 5 c.c. of ether and ro c.c. of water and thoroughly triturate the mixture, grinding vigorously. The ether kills the yeast, in which condition the comminution of the cells with sand is more thoroughly affected. Occasionally during the trituration process add I or 2 c.c. of water until the mixture is comparatively fluid. The whole process of maceration can be completed in five

1 Mendel and Myers: $A m$. J.Physiol., 26, 77, r910.

2 All experiments on nucleoprotein of yeast have been taken from Laboratory Notes of Professor II. J. Gies, of College of I'hysicians and Surgeons, New York. 
minutes. Pour the thick liquid into a bottle aiding the transfer with enough 0.4 per cent $\mathrm{NaOH}$ to make a final volume of about I25 c.c. The alkali extracts the nucleoprotein along with the water-soluble proteins of the yeast. Add a little toluol and allow to stand with frequent shaking for 12-24 hours. Filter through a wet, fluted filter. While thoroughly stirring add I drop at a time of ro per cent $\mathrm{HCl}$ cautiously continuing the addition as long as the milkiness of the mixture can be increased. Continue until the protein completely separates and the liquid is practically clear. Note that the solution is now acid in reaction. Excess of acid causes resolution. Filter on a wet, fluted filter. Retain the precipitate on the filter for nucleoprotein tests.

2. Tests on Nucleoprotein.-Try the following tests on the nucleoprotein prepared as above.

(a) Try the xanthoproteic and Millon's tests.

(b) Test the solubility in water, ro per cent $\mathrm{NaCl}$, ro per cent $\mathrm{HCl}$, dilute $\mathrm{KOH}$, and alcohol.

(c) Test for organically combined phosphorus by one of the following methods.

Tests for Phosphorus in Organic Matter.-I. Fusion Test.-To a small amount of the substance in a crucible add about five times its bulk of fusion mixture ( 2 parts of sodium carbonate to $I$ of potassium nitrate). Heat carefully until the resulting mixture is colorless. Cool, dissolve the mass in a little warm water, acidify with nitric acid, heat nearly to boiling and add a few cubic centimeters of molybdate solution. In the presence of phosphorus a yellow precipitate of phosphomolybdate is formed.

Instead of acidifying with nitric acid, the aqueous solution may be approximately neutralized with hydrochloric acid, a few cubic centimeters of magnesia mixture added and then excess of ammonium hydroxide solution. A white precipitate of magnesium ammonium phosphate is formed.

2. Moist Ashing Procedure.--Treat a small amount of the substance in a large test-tube with about I c.c. of concentrated sulphuric acid. Then add drop by drop an equal volume of concentrated nitric acid, and warm gently until a clear solution is obtained. A few more drops of nitric acid may be added if necessary. This treatment with sulphuric and nitric acids must be carried out with the greatest caution particularly when fatty substances are present ; otherwise an explosive reaction may take place. Dilute the acid solution with a little water, make slightly alkaline with ammonia and then acid with nitric acid. Add molybdate solution and warm. A yellow precipitate is formed.

(d) Dissolve a little of the precipitate in very dilute $\mathrm{KOH}$ and then make slightly acid with acetic acid.

(e) Mix a small portion of the nucleoprotein with ro c.c. of alcohol. Filter and wash free from $\mathrm{HCl}$ with more alcohol. (Freedom from $\mathrm{HCl}$ is indicated by absence of $\mathrm{AgNO}_{3}$-chloride reaction in the filtrate.) Wash free from alcohol with a little water. Transfer small particles of the precipitate to moistened red and blue litmus paper on a microscopic slide. What is the reaction of nucleoprotein thus freed from adherent acid?

3. To Show the Presence of Purine Base Radicals in Nucleoprotein.-The nucleic acid portion of the protein molecule contains phosphoric acid, carbohydrate, and purin base radicals (see page I26). Hence on the complete acid hydrolysis of nucleoprotein material these substances will be liberated as well as the decomposition products of the protein part of the molecule. To show their pres- 
ence proceed as follows: Transfer the precipitate of nucleoprotein remaining from the previous experiment to a small flask and add 25-50 c.c. of 5 per cent $\mathrm{H}_{2} \mathrm{SO}_{4}$. Boil for an hour or more to decompose. Maintain the original volume by adding water. The solution becomes brown due to formation of melaninlike substances. The purine bases are set free. Retain one-fourth of the solution for the next experiment. Transfer the remainder to a casserole and add ammonia with thorough mixing, a little at a time, until the fluid is nearly neutral. Then make slightly alkaline with dilute ammonia and filter if not clear. Transfer to a beaker and add about Io c.c. of 5 per cent ammoniacal silver nitrate solution. Purine bases if present will yield a brown flocculent precipitate of their silver compounds. If a precipitate does not appear immediately, examine the solution after it has been allowed to stand for some time undisturbed.

4. To Show the Presence of Protein, Carbohydrate, and Phosphoric Acid Radicals in Nucleoprotein.-Filter the greater portion of the acid liquid which was reserved from the preceding experiment. Apply the following tests to portions of it: (a) The biuret test. (b) The xanthoproteic test. (c) Molisch test. (d) Fehling's test. (e) Test for phosphate.

5. Preparation of Thymus Nucleoprotein.-About Ioo grams of fresh thymus gland (lymphatic glands may also be used) freed as nearly as possible from adherent fat are run through a meat chopper. To this material in a flask add 300 c.c. of 0.9 per cent $\mathrm{NaCl}$ and allow to stand $24-48$ hours in the cold. A little chloroform and toluol should be added as preservatives, and the mixture shaken occasionally during this period. Filter. A milk white liquid is obtained. Precipitate the nucleoprotein from solution by the careful addition of dilute acetic acid. Excess of the acid should be avoided. Ordinarily acetic acid to make a I per cent solution is sufficient. Filter off the precipitate. Wash with alcohol and then with ether and dry.

6. Experiments on Thymus Nucleoprotein.-Repeat the experiments given under Yeast Nucleoprotein (page I 29).

7. Preparation of Yeast Nucleic Acid.-Dilute 50 c.c. of I per cent $\mathrm{NaOH}$ with 250 c.c. of water in a casserole and add to this solution 100 grams of compressed yeast cut in small pieces. Heat on the water-bath for half an hour with occasional stirring. Remove from the bath and filter at once through a folded filter. To the cooled filtrate add acetic acid until faintly acid to litmus. Filter again. Evaporate the solution to roo c.c. or less and filter if necessary. Allow to $\mathrm{cool}$ to $40^{\circ} \mathrm{C}$. or below, then pour with vigorous stirring into $200 \mathrm{c}$.c. of 95 per cent alcohol containing 2 c.c. of concentrated $\mathrm{HCl}$. Allow to settle and wash the precipitate by decantation in a tall vessel, twice with 95 per cent. alcohol and twice with ether. Transfer to a filter paper. Allow to drain and dry at room temperature.

8. Tests on Nucleic Acid from Yeast. ${ }^{1}$-I. Test the solubility of nucleic acid in cold and hot water, in alcohol, and in dilute acid and alkali. To the solution in alkali add dilute $\mathrm{HCl}$ drop by drop until the solution is acid, then add excess of concentrated $\mathrm{HCl}$.

Does nucleic acid coagulate on boiling? Does the solution in hot water gelatinize on cooling?

2. Try xanthoproteic reaction and biuret test.

3. Dissolve a little nucleic acid in water with the aid of heat. Test the re-

${ }^{1}$ A satisfactory preparation of yeast nucleic acid may be obtained from Merck and Co. 
action of different portions of the solution with litmus, alizarin, and Congo red solution.

4. Boil a small portion of the nucleic acid with about io c.c. of Io per cent sulphuric acid for one to two minutes. Divide into three portions.

(a) To one portion apply carbohydrate tests, e.g., the $\alpha$-naphthol (Molisch) reaction and Bial's test. What do these indicate?

(b) To a second portion apply a test for purine bases. Add an excess of ammonia and then a little silver nitrate solution.

(c) To the third portion apply test for phosphate, adding ammonia in slight excess, then making acid with nitric acid, adding molybdic solution and warming.

9. Preparation of Thymus Nucleic Acid. ${ }^{1-}$ "To a boiling mixture of 200 c.c. of water, Io grams of sodium acetate and 3.3 grams of $\mathrm{NaOH}$, is added in small successive portions 100 grams of trimmed and finely ground thymus gland. The tissue usually dissolves completely forming a pale brown liquid, but any resistant portions are either removed or gotten into solution by heating for a short time over a small flame. The vessel containing the products is now immersed in a briskly boiling water-bath where it is allowed to remain with occasional stirring for two hours, when the product is diluted with one-third its volume of water and made faintly but distinctly acid to litmus with 50 per cent acetic acid. The amount of acid required is about Io c.c. but the final additions must be made with care because the fluid will not filter unless the proper condition of acidity is reached. Any difficulty met at this point may be easily overcome by the alternate addition of acetic acid and sodium hydroxide and testing a small portion of the material after each addition on a small flat filter that has been heated with boiling water. When the acidity has finally been obtained which is favorable to rapid filtration, the material is heated to vigorous boiling and filtered with a hot water funnel. Under proper conditions the filtration proceeds with considerable rapidity and continuously leaves a green slime on the filter and gives a pale yellow filtrate which gelatinizes upon cooling. The filtrate and washings are evaporated on a water-bath to about 75 c.c. and while warm the concentrated solution is poured slowly into Ioo c.c. of 95 per cent alcohol. On standing over night the precipitated sodium nucleate settles sharply to a spongy white mass from which the bulk of brown alcoholic fluid can be sharply decanted and the remainder pressed out with a spatula leaving the material in one cohesive mass. The substance is washed by decantation in turn with 80 per cent and 95 per cent alcohol and, after pressing out the last wash fluid as far as possible is transferred to a flask with 30 c.c. of hot water and heated on a water-bath. In half an hour or less, insoluble phosphates will collect leaving a perfectly transparent interstitial fluid which is treated with I c.c. of 20 per cent $\mathrm{NaOH}$ to lower the viscosity and filtered with a hot water funnel. The perfectly transparent yellow filtrate is acidified with acetic acid and poured into 70 c.c. of 95 per cent alcohol when sodium nucleate will be precipitated which can be washed by decantation as before with alcohol of increasing strength and ground in a mortar with absolute alcohol until it has crumbled to a fine white powder. If necessary the absolute alcohol may be decanted and renewed once or twice but not of tener because the nucleate emulsifies with alcohol after the last traces of acetic acid and sodium acetate have been washed away. The material is finally. washed on a filter with absolute alcohol and allowed to dry in a sulphuric acid desiccator. The yield of nucleic acid is about 3.3 grams from 100 grams of gland. The product is a fine white non-hygroscopic powder

${ }^{1}$ From Monograph on "Nucleic Acids" by Walter Jones: Longmans, Green \& Co. 
that can scarcely be improved by any method of purification. It is a soluble sodium salt of thymus nucleic acid but is generally referred to simply as thymus nucleic acid. Very similar or identical substances may be prepared by the same procedure from other animal tissues, rich in cell nuclei such as the pancreas and spleen."

Io. Tests on Thymus Nucleic Acid.-I-4. Repeat the experiments as given under yeast nucleic acid, page 130 . 5. Make a 4 per cent solution of thymus nucleic acid in hot water ( $2 / 5$ gram to 1o c.c.). Allow to cool. What happens? Divide into two portions. To one add a little $\mathrm{NaOH}$ solution; to the other add acetic acid. Then neutralize carefully in each case.

Both acetic acid and $\mathrm{NaOH}$ decrease the viscosity of the nucleate solution. It may be changed back and forth from the gelatinous to the fluid condition by the alternate addition of acid and alkali.

II. Tests on Purine Bases and Derivatives.--(a) Xanthine.-I. Silver Nitrate Reaction.-Dissolve a little xanthine in ammonia and add silver nitrate solution. Examine a little of the precipitate microscopically. (See page 35 r.)

2. Copper Sulphate Reaction.-Dissolve a little of the substance in dilute alkali, make faintly acid with acetic acid. Heat to boiling. Add I c.c. Io per cent $\mathrm{CuSO}_{4}$ and then a few drops at a time of sodium bisulphite (saturated solution) until the precipitate becomes yellowish. All of the purines give this reaction.

3. Nitric Acid Test.-Place a small amount of the substance in a small evaporating dish, add a few drops of concentrated nitric acid, and evaporate to dryness very carefully on a water-bath. The yellow residue upon moistening with caustic potash becomes red in color and upon further heating assumes a purplish-red hue. Now add a few drops of water and warm. A yellow solution results which yields a red residue upon evaporation. Compare with similar reaction on other purine bases and uric acid. (See Murexide test, Chapter XXII.)

4. Weidel's Reaction.-Bring a small amount of the substance into solution in bromine water. Evaporate to dryness on a water-bath. Remove the stopper from an ammonia bottle and by blowing across the mouth of the bottle direct the fumes of ammonia so that they come into contact with the dry residue. Under these conditions the presence of xanthine is shown by the residue assuming a red color. A somewhat brighter color may be obtained by using a trace of nitric acid with the bromine water. By the use of this modification, however, we may get a positive reaction with bodies other than xanthine.

(b) Hypoxanthine.-I. Repeat Experiments I and 3 under Xanthine. Examine the crystals of hypoxanthine silver nitrate under the microscope. (See page 35r.)

2. Dissolve a little of the substance in a very small amount of hot 6 per cent nitric acid and allow to cool. Characteristic whetstone crystals of hypoxanthine nitrate should be formed. Examine under the microscope. (See Fig. 40, page I36.)

(c) Adenine.- I. Warm a few crystals of adenine in a test-tube with a little water. They should become cloudy at $53^{\circ} \mathrm{C}$.

2. Dissolve a little adenine in hot water and add a few drops of picric acid. Examine the pale yellow crystals under the microscope. The picrate crystallizes as needle clusters.

3. Repeat Experiment 3 under Xanthine. 
(d) Guanine.- I. Dissolve a little substance in 20-25 times its weight of boiling 5 per cent alcohol. Allow to cool and examine crystals microscopically.

3. Dissolve a little guanine in 20-25 times its weight of boiling 5 per cent hydrochloric acid. Allow to cool and examine crystals under microscope. (See Fig. 39, page r35.)

3. Perform Experiment 3 under Xanthine.

(e) Uric Acid.- I. On a small amount of uric acid try the test as given under Xanthine number 3. This test on uric acid is called the Murexide test.

2. For other tests on uric acid see Chapter XXII on Urine.

I2. Isolation of Guanine and Adenine from Nucleic Acid (Method of Walter Jones). ${ }^{1}$ - The amino purines may be isolated from yeast or thymus nucleic acid or from glandular tissue (such as the pancreas) after hydrolysis of the material with sulphuric acid.

In the case of yeast nucleic acid, heat Io grams of the substance with 50 c.c. of ro per cent sulphuric acid on a boiling water-bath for about two hours, replacing any water lost, or using a condenser tube. To the hot solution add concentrated ammonia slowly until approximately neutral. Then add enough excess of ammonia to make about a 2 per cent solution. Filter off the precipitate of guanine and wash it with I per cent ammonia. Dissolve in as small an amount of 20 per cent sulphuric acid as possible, add a little animal charcoal and boil. Filter, heat to boiling and precipitate with excess of ammonia. Filter, dry the precipitate at $40^{\circ} \mathrm{C}$. and dissolve it in about 20 parts of boiling 5 per cent hydrochloric acid. As the solution cools guanine chloride separates out as needle-shaped crystals. Filter off, wash with very dilute hydrochloric acid and dry in the air (do not put in desiccator). Perform the nitric acid test on the product.

Combine the ammoniacal filtrates obtained in the isolation and purification of guanine. Filter if necessary. The ammonia may then be boiled off and an excess of picric acid added in which case a ycllow precipitate of adenine picrate is produced which is filtered off and dried. It is better, however, to neutralize the ammonia of the combined filtrates and make faintly acid with sulphuric acid. Then precipitate the adenine as its copper compound (see directions under experiment on Demonstration of Nucleases B) decomposing this with hydrogen sulphide and evaporating the filtrate from the copper sulphide to dryness on the water-bath. Dissolve the residue in hot 5 per cent sulphuric acid and allow to crystallize out. If necessary dissolve in hot water decolorize with a little charcoal and allow to crystallize out again. The compound has the formula $\left(\mathrm{C}_{5} \mathrm{H}_{5} \mathrm{~N}_{5}\right)_{2} \cdot \mathrm{H}_{2} \mathrm{SO}_{4} \cdot 2 \mathrm{H}_{2} \mathrm{O}$. Apply the picric acid and nitric acid tests as given under adenine (page I32).

13. The Pyrimidine Derivatives.-The pyrimidine derivatives, cytosine, thymine, and uracil, are separated from nucleic acid with some difficulty. The following test may be made on a solution of cytosine or uracil. Thymine does not give the test.

Wheeler-Johnson Reaction for Uracil and Cytosine.-To about 5 c.c. of the solution under examination add bromine water until the color is permanent. Avoid the addition of a large excess as this will interfere with the test. In case the solution contains only small quantities of cytosine or uracil it is advisable to remove any excess of bromine by passing a stream of air through the

${ }^{1}$ Sce Walter Jones: Monograph on "Nucleic Icijs," rgr 4 , I Longmans, Green \& Co. 
solution. Now add an excess of an aqueous solution of barium hydroxide and note the appearance of a purple color.

Very dilute solutions do not give the test. Under these conditions the solution should be evaporated to dryness, the residue dissolved in a little bromine water and the excess of bromine removed. Then upon adding an excess of barium hydroxide a decided bluish-pink or lavender color will appear in the presence of as small an amount as $0.00 \mathrm{I}$ gram of uracil.

In testing solutions for cytosine it is preferable to warm or boil the solution with bromine water, and after cooling the solution to apply the test as suggested above, being careful to have a slight excess of bromine present before adding barium hydroxide.

14. Demonstration of Nucleases and Purinases in Tissues. ${ }^{1}$-All glandular tissues contain nucleic acids and enzymes capable of their hydrolysis as well as the transformation of liberated purine bodies. By allowing autolysis (self-digestion) to take place in such a tissue and studying the products formed it is possible to determine what enzymes were present in the tissue under examination. Typical results may be obtained by using ox and pig spleens, which differ in the purine enzymes which they contain. The two experiments should be run in parallel.

A. Preparation of the Material for Digestion.-Run the gland once or twice through a meat chopper. Introduce about 650 grams into a two liter bottle, fill about three-quarters full of water, add 20 c.c. of chloroform, and allow to remain at room temperature for $12-36$ hours with occasional agitation. Strain through linen and replace the turbid extract in the bottle with ro c.c. of chloroform. This solution contains the enzymes and nucleic acid of the tissue. (Reserve 50 c.c. of one of the extracts for the experiment on phosphonuclease (E), which should also be started at this time.) The bottle is tightly closed and allowed to remain in the thermostat 4-5 days.

B. Separation of Purine Derivatives from Other Substances.-Introduce the products into a saucepan or large evaporating dish, heat to brisk boiling, make faintly alkaline with caustic soda, boil a few minutes, make faintly acid with acetic acid, boil vigorously and filter hot. (If desired one-half of the filtrate may be treated with roo c.c. of 20 per cent sulphuric acid per liter and boiled for one hour, keeping at constant volume. At the end of the hydrolysis the sulphuric acid is nearly neutralized with caustic soda and the purine bases of the solution determined as in the other half of the filtrate. This will give information as to the presence of deaminases acting upon the amino purines remaining in combination). To the boiling filtrate add roo c.c. of ro per cent copper sulphate and small successive portions ( $2-5$ c.c.) of sodium bisulphite (commercial saturated solution) until the precipitate takes on a decided yellow color due to precipitation of cuprous oxide. Filter, wash the precipitate with boiling water, pierce the filter and wash the precipitate into a flask. Add I per cent sodium sulphide solution to decom-

${ }^{1}$ From Monograph on "Nucleic Acids" and Laboratory Notes in Physiological Chemstry by Professor WValter Jones of Johns Hopkins University, with additions. 
pose the copper compound continuing the addition until the precipitate becomes uniformly black and then in small successive portions, testing a drop of the product after each addition by bringing it into contact with a drop of lead acetate on a filter paper. To the boiling fluid add acetic acid (in the case of the extract of pig's spleen and other solutions containing guanine the acetic acid should be replaced by dilute sulphuric) until the insoluble copper sulphide collects and filter the hot fluid as quickly as possible.

C. Treatment of the Filtrate from Pig's Spleen.-When the filtrate from the copper sulphide is cold make strongly alkaline with ammonia and precipitate the purine compounds with a slight excess of ammoniacal silver nitrate. Filter, wash thoroughly with cold water. Pierce the paper, wash the precipitate into a flask with boiling water and decompose the silver precipitate with hydrochloric acid. When enough acid has been added the silver chloride will settle as a heavy caseous precipitate leaving clear interstitial fluid. Filter and heat the filtrate to boiling. Treat with an excess of ammonia (enough to make about $\mathrm{I}-2$ per cent).

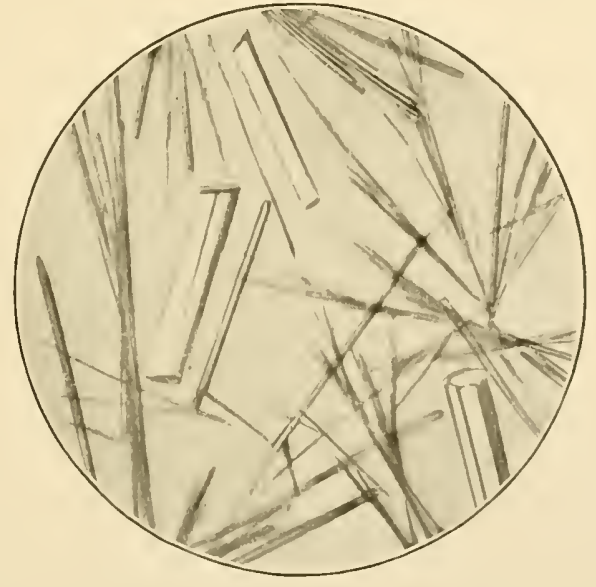

Fig. 39--Guanine Chloride.

(Reproduced from crystals furnished by Professor Walter Jones.)

Allow to cool and filter off the guanine which precipitates. Wash the guanine with I per cent ammonia and then suspend it in a little hot water and add a few drops of 20 per cent sulphuric acid to dissolve it. At the boiling point add a little animal charcoal, boil and filter. Make strongly alkaline with ammonia. Snow white guanine is precipitated. Dissolve the precipitate in 20 volumes of boiling 5 per cent hydrochloric acid. Upon cooling beautiful needle-shaped crystals of guanine chloride separate. (See Fig. 39.)

Evaporate the filtrate from the guanine to dryness on the water-bath to expel ammonia. Moisten with hydrochloric acid and again evaporate. Treat the residue with warm water. Does it dissolve almost completely indicating the absence of xanthine and uric acid? Test a drop of the solution with picric acid. If no precipitate is obtained adenine is absent. Evaporate to dryness, moisten with alcohol and again evaporate. Dissolve the residue in about 30 parts of hot 6 per cent nitric acid. On cooling, characteristic whetstone crystals of hypoxanthine nitrate form. ((See Fig. 40, page 136.) The xanthine nitric acid color test should be practically negative on this product. 
What does the finding of guanine and hypoxanthine but not adenine or xanthine indicate as to the type of purine deaminase present in the pig's spleen?

D. Treatment of Filtrate from Ox Spleen.-The filtrate from the copper sulphide should be evaporated to dryness on the water-bath. Extract with cold water. Test a part of this aqueous solution with picric acid. A lack of precipitate indicates the absence of adenine. To another portion add ammonia (a lack of precipitate indicates the absence of guanine), and then a little ammoniacal silver nitrate solution (lack of appreciable precipitate indicates absence of purines of any kind in more than traces). Dissolve half of the residue, which should consist mainly of uric acid and xanthine, in as few drops of concentrated sulphuric acid as possible and dilute with 4 volumes of water. Stir until the uric acid begins to separate and then let stand for about three hours. The uric acid is completely precipitated. Apply the murexide test. To the remainder of the xanthine-uric acid residue add a little 4 per cent potassium hydroxide solution. Warm and add an equal volume of 30 per cent nitric acid. Allow to cool.

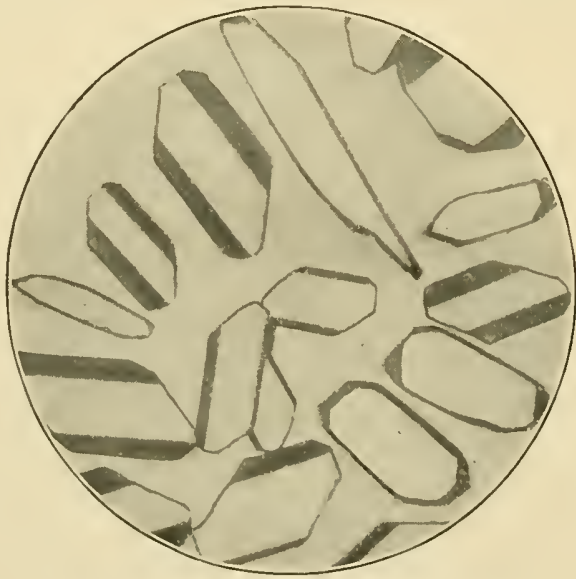

Fig. 40.-Hypoxanthine Chloride. ${ }^{1}$

(Reproduced from crystals furnished by Professor Walter Jones.)

Xanthine nitrate separates out in a granular form, showing characteristic crystals under the microscope. Apply the nitric acid test.

What does the presence of uric acid and xanthine and the absence of guanine and adenine indicate as to the purine enzymes of ox spleen?

E. Demonstration of Nucleotidase (Phosphonuclease).-In this experiment use the 50 c.c. portion of enzyme solution retained from Experiment A preceding. Prepare a 2 per cent solution of yeast nucleic acid aiding the solution by the slow addition of $\mathrm{KOH}$ solution until the reaction, as indicated by a few drops of litmus added, is neutral. Prepare a series of three large test-tubes as follows: In No. I place ro c.c. of enzyme solution and 5 c.c. of nucleic acid. In No. 2 place ro c.c. of enzyme solution and 5 c.c. of water. In No. 3 place ro c.c. of enzyme solution, boiled and 5 c.c. of nucleic acid. To each tube add 2-3 c.c. each of $\mathrm{CHCl}_{3}$ and toluol as preservatives. Place the tubes at $37^{\circ} \mathrm{C}$. for 24 hours. Add I c.c. of litmus solution to each tube and note whether any changes in reaction have taken place. Put the tubes in boiling water for a few minutes to coagulate proteins Then add 5 c.c. of 5 per cent $\mathrm{HCl}$ and let stand for an hour. This precipitates.

${ }^{1}$ Hypoxanthine nitrate crystallizes in similar form. 
any unaltered nucleic acid which may be present. Filter and take an aliquot portion of each filtrate (r5 c.c.). Add magnesia mixture and a few cubic centimeters of strong ammonia. Let stand over night. Any phosphoric acid present will be precipitated as magnesium ammonium phosphate. Observe the relative amounts of phosphate in each case. Has any phosphate been set free from the nucleic acid added? From the nucleic acid of the gland extract?

15. Experiments on Uricase (Uricolytic Enzyme).-A. Preparation of Extract Containing Uricase.-Extract about 50 grams of pulped kidney tissue of the ox with 200 c.c. of toluol or chloroform water at $38^{\circ} \mathrm{C}$. for 24 hours, with occasional shaking. Filter and use the filtrate in the following experiment.

B. Demonstration of Uricase.-Add about 0.1 gram of uric acid to ro c.c. of water and bring the uric acid into solution by the addition of the minimal quantity of KOH. To 5 c.c. of this uric acid solution in a test-tube add 50 c.c. of the uricolytic enzyme extract prepared as described above. Prepare a second tube containing a like amount of the uric acid solution but boil the extract before it is introduced. Place the two tubes at $38^{\circ} \mathrm{C}$. for two days. The vessels should be open to the air and the contents stirred occasionally, or much better, a continuous current of air which has gone through a chloroform wash bottle is passed through the mixture. Make both mixtures faintly acid with acetic acid and boil. Filter and take an aliquot of each filtrate. Evaporate to low volume, make faintly alkaline with ammonia and filter. Add a few cubic centimeters of ammoniacal silver nitrate solution. Any uric acid will be precipitated as silver urate. The control should give a heavy precipitate while the test should show no precipitate or one much lighter than the control, due to uric acid destruction in the latter case.

If it is desired to separate out the pure uric acid the silver-purine precipitate may then be filtered off. It is washed with water and transferred to a beaker with the aid of a little water. To the mixture add a few cubic centimeters of hydrogen sulphide solution and a few drops of $\mathrm{HCl}$ and allow to stand over night. The uric acid should separate out in crystalline form and should be found in less amount in the test than in the control experiment. The uric acid may also be titrated with permanganate as in the Folin-Shaffer method for uric acid in urine. (See Chapter XXVI on Quantitative Analysis of Urine.) This will enable us to determine exactly how much of the uric acid was destroyed through the action of the enzyme extract. 


\section{GASTRIC DIGESTION}

GASTRIC digestion takes place in the stomach and is promoted by the gastric juice, which is secreted by the glands of the stomach mucosa. These glands are of two kinds, fundus glands and pyloric glands which are situated, as their names imply, in the regions of the fundus and pylorus. The principal foods acted upon in gastric digestion are the proteins which are so changed by its processes as to become better prepared for further digestion in the intestine and for their final absorption.

From reliable experiments made upon lower animals it is evident that the gastric juice is secreted as the result of stimuli of two forms, i.e., psychical stimuli and chemical stimuli. The psychical form of stimuli may be produced by the sight, thought, or taste of food, and the chemical stimuli may be produced by certain substances, such as water, milk, the extractives of meat, etc., when coming in contact with the stomach mucosa. The stimulatory power of water has been very strikingly demonstrated. ${ }^{1}$ The claim that the drinking of water with meals is harmful because such a procedure causes a dilution of the gastric juice, has no basis in fact. The drinking of water with meals by normal individuals has been found to be accompanied by a more economical utilization of the ingested proteins, fats and carbohydrates. Various other desirable and no undesirable features have been demonstrated as accompanying or following such a dietary procedure. ${ }^{2}$ No experimental evidence has been submitted which can justly be interpreted as showing any harmful influence to accompany or follow the drinking, by normal persions, of large quantities of water at meal time.

The volume of gastric juice secreted during any given period of digestion varies with the quantity and kind of the food. These conclusions were deduced principally from a series of so-called delusive feeding experiments. A dog was prepared with two esophageal openings and a gastric fistula. When thus prepared and fed foods of various

${ }^{1}$ Foster and Lambert: Journ. Exper. Med., ro, 820, 1908.

Bergeim, Rehfuss and Hawk: Jour. Biol. Chem., 19, 345, I9r 4.

${ }^{2}$ Hawk: University of Pennsylvania Medical Bulletin, $18,1,1905$.

Fowler and Hawk: Jour. Exper. Med., 12, 388, 1910.

Hattrem and Hawk: Arch. Int. Med., 7, 6 ro, rorr.

Mattill and Hawk: Jour. Am. Chem. Soc., 33, pp. 1978, 1999, and 2019, I9II.

Hawk: Arch.Int. Med., 8, 382, I9гі.

Hawk: Proceedings Soc. Exp. Biol. and Med., 8, 36, 1910.

Fairhall and Hawk: Jour. Am. Chem. Soc., 34, 546, r91 2.

Howe and Hawk: Jour. Biol. Chem., I I, I29, I9I2.

Hawk: Biochem. Bull., 3, 420, 1914. 
kinds such as meat and bread, the material instead of passing to the stomach, would invariably find its way out of the animal's body at the upper esophageal opening. Through the medium of the gastric fistula the course of the secretion of gastric juice could be carefully followed. It was found that when the dog ate meat, for example, there was a large secretion of gastric juice notwithstanding no portion of the food eaten had reached the stomach. Further experiments made through the medium of a cul-de-sac formed from the stomach wall have given us many valuable conclusions, among others those regarding the influence of the chemical stimuli. The method followed was to feed the animal certain substances and note the secretion of gastric juice in the miniature stomach while the real process of digestion was taking place in the stomach proper.

Normal gastric juice is a thin, light colored fluid which is acid in reaction and has a specific gravity varying between I.OOI and I.oIO. It contains only 2-3 per cent of solid matter which is made up principally of hydrochloric acid, sodium chloride, potassium chloride, earthy phosphates, mucin and the enzymes pepsin, gastric rennin, and gastric lipase; the hydrochloric acid and the enzymes are of the greatest importance. The acidity of the gastric juice is due to free hydrochloric acid. It was formerly believed that this acid was secreted by the parietal cells of the fundus as well as by the chief cells of both the fundus and pyloric glands. It has been claimed, ${ }^{1}$ however, that the parietal coll is the seat of the formation of the hydrochloric acid. This conclusion is based upon the formation of Prussian blue after the subcutaneous injection of potassium ferrocyanide and ammonium ferric citrate (rabbits and guinea-pigs) and the subsequent ( 3 to 30 hours) microscopical examination of the gastric mucosa. The acid was shown to be present in the lumina of the gland tubules and in the canaliculi of the parietal cells; traces were also apparently present in the cytoplasm. Later Bensley and Harvey ${ }^{2}$ showed by means of dyes which act as vital stains and as indicators very sensitive to alkali that the secretion in the parietal cells is slightly alkaline whereas that in the lumen of the gland proper is very nearly neutral. Therefore, the acid is formed entirely above the level of the gland proper, i.e., in the foveola and on the surface. Hammet $^{3}$ and still more recently Macallum and Collip ${ }^{4}$ have confirmed Miss Fitzgerald's claim that the acid is formed in the parietal cells.

It was believed that hydrochloric acid was generally present in the gastric juice of man to the extent of about 0.2 per cent. When the

${ }^{1}$ Fitzgerald: Proccedings Royal Society (B), $8_{3}, 56$, ig 1о.

${ }^{2}$ Bensley and Marvey: Biological Bullctin, 23, 225, I91 2.

${ }^{3}$ Hammett: Analomical Record, 9, 21, 19ז5.

4 Reported before Society of Biological Chemists, Boston, Dec. 27, I9I5. 
amount of hydrochloric acid varied to any considerable degree from this value a condition of hypoacidity or hyperacidity was said to be established. On the basis of more recent experiments, ${ }^{1}$ however, it appears that the actual acidity of the gastric juice of man as secreted by the glands is 0.4 to 0.5 per cent hydrochloric acid. Boldyreff believes that this acidity is lowered to about 0.2 per cent by regurgitation of alkaline fluid from the intestine (Chapter VIII on Gastric Analysis). Hydrochloric acid has the power of combining with protein substances taken in the food, thus forming so-called combined hydrochloric acid. This combined acid is a less potent germicide than free hydrochloric acid and has less power to destroy the amylolytic enzyme salicary amylase (ptyalin) of the saliva. This last fact explains to a degree the possibility of the continuance of salivary digestion in the stomach.

The term combined hydrochloric acid is really a misnomer. When free hydrochloric acid is treated with a protein the latter functions as a base and a salt is formed. Therefore, instead of having "combined hydrochloric acid" we have a protein salt of hydrochloric acid. This salt ionizes differently from the free acid. This fact explains the variation in the germicidal properties of the two solutions as well as their different action toward enzymes, such, for example, as salivary amylase (see page 6r).

The hydrochloric acid of the gastric juice forms a medium in which the pepsin can most satisfactorily digest the protein food, and at the same time it acts as an antiseptic or germicide which prevents putrefactive processes in the stomach. It also possesses the power of inverting cane sugar, this property being due to the hydrogen ion. When the hydrochloric acid of the gastric juice is diminished in quantity (hypoacidity) or absent, as it may be in many cases of functional or organic disease, there is no check to the growth of micro-organisms in the stomach. There are, however, certain of the more resistant spores which eren the normal acidity of the gastric juice will not destroy. A condition of hypoacidity may also give rise to fermentation with the formation of comparatively large amounts of such substances as lactic acid and butyric acid.

The question of the origin of the hydrochloric acid of the gastric juice is a problem to whose solution many investigators have given much attention. Many theories have been proposed, among them the interaction of sodium chloride with carbonic acid, ${ }^{2}$ with acid phosphate ${ }^{3}$

${ }^{2}$ Babkin: Die äussere Sekretion der Verdauungsdrüsen, Berlin, I9r4, p. Ir 3 .

Boldyreff: Quart. Jour. Exper. Physiol., 8, r, r914.

Carlson: Am. Jour. Physiol., 38, 248, 1915.

${ }^{2}$ Bunge: Physiologic and Pathologic Chemistry, 2nd. Eng. Ed., Philadelphia, 1902, p. 135.

${ }^{3}$ Maly: Zeit. f. physiol. Chem., I, I 74, 1877. Macallum and Collip: Reported before the Society of Biological Chemists, Boston, Dec. 27, I9r5. 
or with organic acids. On the other hand. it is possible that hydrochloric acid is set free from combination with some weak base as ammonia ${ }^{1}$ or that free phosphoric acid valences may be set free by the enzymic hydrolysis of organic phosphoric acid esters. ${ }^{2}$ We cannot go into a discussion of these various theories. That the hydrochloric acid arises from the chlorides of the blood appears to be weil established but the same cannot be said with regard to the immediate or ultimate origin of the hydrogen ions involved in the reaction.

The most important of the enzymes of the gastric juice is the proteolytic enzyme pepsin. The pepsin does not originate as such in the gastric cells but is formed from its precursor, the zymogen or mothersubstance pepsinogen, which is apparently produced by the parietal cells of the fundus as well as by the chief cells of the fundus and pyloric glands. Pepsinogen may be differentiated from pepsin from the fact that it is more resistant to alkali. ${ }^{3}$ Upon coming into contact with the hydrochloric acid of the secretion this pepsinogen is immediately transformed into pepsin. Pepsin is not active in alkaline or neutral solutions, but requires at least a faint acidity before it can exert its power to dissolve and digest proteins. The percentage of hydrochloric acid facilitating the most rapid peptic action varies with the character of the protein acted upon, e.g., 0.08 per cent to o. I per cent for the digestion of fibrin and 0.25 per cent for the digestion of coagulated egg-white. While hydrochloric acid is the acid usually employed to promote artificial peptic proteolysis, other acids, organic and inorganic, will serve the same purpose. Acidity of the liquid is necessary to promote the activity of the pepsin, but the acidity need not necessarily be confined to hydrochloric acid.

In common with many other enzymes pepsin acts best at about $38^{\circ}-40^{\circ} \mathrm{C}$. and its digestive power decreases as the temperature is lowered, the enzyme being only slightly active at $0^{\circ} \mathrm{C}$. Its power is only temporarily inhibited by the application of such low temperatures, however, and the enzyme regains its full proteolytic power upon raising the temperature to $40^{\circ} \mathrm{C}$. As the temperature of a digestive mixture is raised above $40^{\circ} \mathrm{C}$. the pepsin gradually loses its activity until at about $80^{\circ}-100^{\circ} \mathrm{C}$. its proteolytic power is permanently destroyed.

Our ideas regarding the nature of the products formed in the course of peptic proteolysis have undergone considerable revision in recent years. The former view that these products included only acid albuminate (acid metaprotein), proteoses, peptones and peptides is no

1 Mathews: Physiological Chemistry, New York, rgi5, p. 3it.

2 Bergeim: Proc. Soc. Exp. Biol, and Med., I 2, 21, I I I 4 .

${ }^{3}$ Langley: Jour. of Physiol., $3,246$. 
longer tenable. From the investigations of numerous observers we have learned that artificial gastric digestion if permitted to proceed for a sufficiently long period will yield, in addition to proteoses, peptones and peptides, a long list of protein cleavage products which are crystalline in character, including leucine, tyrosine, alanine, phenylalanine, aspartic acid, glutamic acid, proline, leucinimide, valine, and lysine. A similar group of substances may result from the action of the enzyme trypsin (see page I86). The relative amounts of proteoses, peptones, and crystalline substances formed depends to a great extent upon the character of the protein undergoing digestion, e.g., a greater proportion of proteoses results from the digestion of fibrin than from the digestion of coagulated egg-white. We must not be led into the error of thinking that the large number of protein cleavage products just mentioned are formed in the course of normal gastric digestion within the animal organism. They are formed only after comparatively long-continued hydrolysis. In pancreatic digestion, however, there are formed even under normal conditions the large number of cleavage products to which reference has been made. Peptic proteolysis, therefore, within the animal organism differs from tryptic proteolysis (see page I86) in that the former yields larger amounts of proteoses, smaller amounts of peptones and no considerable quantity of crystalline bodies as endproducts in the brief period during which proteins are ordinarilysubjected to gastric digestion. Prolonged hydrolysis with gastric juice does, however, yield considerable quantities of the non-protein endproducts. In cases of cancer of the stomach a peptide-splitting enzyme (erepsin) is said to be present in the stomach contents. This enzyme is believed to be elaborated by the cancer tissue and its identification is of importance in connection with the diagnosis of gastric cancer. The glycyl-trytophane test ${ }^{1}$ is sometimes used for this purpose. This test has been very severely criticized (see page I99).

Abderhalden and Meyer $^{2}$ have shown active pepsin to be present in the contents of all parts of the small intestine. It is suggested that pepsin may be adsorbed in the stomach by such protein substances as pass into the intestine in solid form and that the pepsin thus protected may bring about gastric digestion whenever the reaction of the surrounding intestinal contents is favorable. This fact may be of importance in connection with the profound proteolysis taking place in the intestine. Heretofore, this process was believed to be furthered alone by trypsin and erepsin. The passage of adsorbed pepsin into the intestine may be an efficient aid to the proper digestion of solid proteins

${ }_{1}^{1}$ Neubauer and Fischer: Deut. Arch.f. klin. Med., 97, 499, I909.

2 Abderhalden and Meyer: Zeit. für physiol. Chem., 74, 67, I9I I. 
which are ingested without sufficient mastication ("bolted ") 1 and which consequently, at times, pass into the intestine in rather large pieces (see Chapter XIV on Feces).

Gastric rennin, the second enzyme of the gastric juice, is what is known as a milk curdling or protein coagulating enzyme. Rennin acts upon the casein of the milk, splitting it into a peptone-like body and soluble paracasein. This soluble body, in the presence of calcium salts, combines with calcium, forming calcium paracasein which is insoluble and precipitates. There is some uncertainty regarding the reaction to litmus in which gastric rennin shows the greatest activity. It is, however, said to be active in neutral, alkaline, or acid solution. However, it probably possesses its greatest activity in the presence of a slight acid reaction, as would naturally be expected. It is especially abundant in the gastric mucosa of the calf, and is used to curdle the milk used in cheese making. Gastric rennin is always present normally in the gastric juice, but in certain pathological conditions such as atrophy of the mucosa, chronic catarrh of the stomach, or in carcinoma it may be absent.

The theory that the proteolytic activity and the milk curdling property of the gastric juice reside in a single substance is causing much controversy at the present time. The theory was originally advanced by the Pawlow school. ${ }^{2}$ According to Nencki and Sieber, ${ }^{3}$ the milk curdling and protein hydrolyzing activities reside in definite and distinct side chains of a single mammoth molecule. The view which has rather the strongest support, however, is to the effect that there are two entirely distinct enzymes. Important evidence has been advanced in favor of this view by Hammarsten, ${ }^{4}$ Taylor, ${ }^{5}$ and Hemmeter. ${ }^{6}$ Burge ${ }^{7}$ has reported experiments upon the influence of a direct electric current upon solutions possessing typical rennin and peptic activities. By this means he was able to prepare a solution possessing strong rennin activity but entirely devoid of peptic activity. This furnishes strong evidence against the identity of the two enzymes but does not necessarily deny the accuracy of the side-chain theory.

Gastric lipase, the third enzyme of the gastric juice, is a fat-splitting enzyme. It possesses but slight activity when the gastric juice is of normal acidity, but evinces its action principally at such times as a

2 Foster and Hawk: Procecdings of the Eighth Intcrnational Congress of A pplicd Chemistry, New York, September, I9I 2 .

2 Pawlow and Parastschuk: Zeilschrift fiir Physiologische Chemie, 42, 415, 1904.

${ }^{3}$ Nencki and Sieber: Zcilschrift fiir Phy'siologische Chemie, 23, I9I, I90I.

- Hammarsten: Zeilschrift fiir Physiologische Chemie, 56, IS, 190S; 94, 29r, 19r5.

${ }^{5}$ Taylor: Journal of Biological Chemisiry, 5, 399, 1909.

- Hemmeter: Berliner klinische Wochenschrift, Evald Festnummer, 44, 1905.

${ }^{7}$ Burge: American Journal of Physiology, 29, 1912. 
gastric juice of low acidity is secreted either from physiological or pathological cause. The digestion of fat in the stomach is, however, at most, of but slight importance as compared with the digestion of fat in the intestine through the action of the lipase of the pancreatic juice (see page I88).

Boldyreff ${ }^{1}$ has shown trypsin to be present in stomach contents, due to regurgitation of intestinal contents through the pylorus. This claim has been verified by others ${ }^{2}$ (see Chapter VIII on Gastric Analysis).

\section{PREPARATION OF AN ARTIFICIAL GASTRIC JUICE}

Dissect the mucous membrane of a pig's stomach from the muscular portion and discard the latter. Divide the mucous membrane into two parts $(4 / 5$ and $\mathrm{r} / 5$ ). Cut up the larger portion, place it in a large-sized beaker with 0.4 per cent hydrochloric acid and keep at $38^{\circ}-40^{\circ} \mathrm{C}$. for at least 24 hours. Filter off the residue, consisting of nuclein and other substances, and use the filtrate as an artificial gastric juice. This filtrate contains pepsin, rennin, and the products of the digestion of the stomach tissue, i.e., acid metaprotein (acid albuminate), proteoses, peptones, etc.

\section{Preparation of a Glycerol Extract of Pig's Stomach}

Take the one-fifth portion of the mucous membrane of the pig's stomach not used in the preparation of the artificial gastric juice, cut it up very finely, place it in a small-sized beaker and cover the membrane with glycerol. Stir frequently and allow to stand at room temperature for at least 24 hours. The glycerol will extract the pepsinogen. Separate, with a pipette or by other means, the glycerol from the pieces of mucous membrane and use the glycerol extract as required in the later experiments.

\section{Products of Gastric Digestion}

Into the artificial gastric juice, prepared as above described, place the protein material (fibrin, coagulated egg-white, or lean beef) provided for you by the instructor, add 0.4 per cent hydrochloric acid as suggested by the instructor and keep the digestion mixture at $40^{\circ} \mathrm{C}$. for two to three days. Stir frequently and keep free hydrochloric acid present in the solution (for tests for free hydrochloric acid see page 152 ).

The original protein has been digested and the solution now contains the products of peptic proteolysis, i.e., acid metaprotein (acid albuminate), proteoses, peptones, etc. The insoluble residue may include nuclein and other substances. Filter the digestion mixture and after testing for free hydrochloric acid neutralize the filtrate with potassium hydroxide solution. If any of the acid metaprotein (acid albuminate) is still untransformed into proteoses it will precipitate upon neutralization. If any precipitate forms heat the mixture to boiling, and filter. If no precipitate forms proceed without filtering.

${ }^{1}$ Boldyreff: Quart. Jour. Exper. Physiol., 8, т, г9г4.

${ }^{2}$ Spencer, Meyer, Rehfuss and Hawk: Am. Jour. Physiol, 39, 459, I9r6. 
We now have a solution containing a mixture consisting principally of proteoses and peptones. Separate and identify the proteoses and peptones according to the directions given on pages II 9 and $\mathrm{I}_{2} \mathrm{O}$.

\section{GENERAL EXPERIMENTS ON GASTRIC DIGESTION}

I. Conditions Essential for the Action of Pepsin.-Prepare four test-tubes as follows :

(a) Five c.c. of pepsin solution.

(b) Five c.c. of 0.4 per cent hydrochloric acid.

(c) Five c.c. of pepsin-hydrochloric acid solution.

(d) Two or 3 c.c. of pepsin solution and 2-3 c.c. of 0.5 per cent sodium carbonate solution.

Introduce into each tube a small piece of fibrin and place them in the incubator or water-bath at $40^{\circ} \mathrm{C}$. for one-half hour, carefully noting any changes which occur. ${ }^{1}$ (Carmine-fibrin may be used to advantage in this and the following tests under Gastric Digestion. In this case, however, the experiments should be conducted at room temperature. For directions as to the preparation of carminefibrin see Chapter I.) Now combine the contents of tubes (a) and (b) and see if any further change occurs after standing at $40^{\circ} \mathrm{C}$. for $1^{-20}$ minutes. Explain the results obtained from these five experiments.

2. Influence of Different Temperatures. - In each of four test-tubes place 5 c.c. of pepsin-hydrochloric acid solution. Immerse one tube in cold water from the faucet, keep a second tube at room temperature and place a third in the incubator or water-bath at $40^{\circ} \mathrm{C}$. Boil the contents of the fourth tube for a few moments, then cool and also keep it at $40^{\circ} \mathrm{C}$. Into each tube introduce a small piece of fibrin and note the progress of digestion. In which tube does the most rapid digestion occur? Explain this.

3. The Most Favorable Acidity.-Prepare three tubes as follows:

(a) Five c.c. of 0.2 per cent pepsin-hydrochloric acid solution.

(b) Two or 3 c.c. of 0.2 per cent hydrochloric acid + I c.c. of concentrated hydrochloric acid +5 c.c. pepsin solution.

(c) One c.c. of 0.2 per cent pepsin-hydrochloric acid solution +5 c.c. of water.

Introduce a small piece of fibrin into each tube, keep them at $40^{\circ} \mathrm{C}$, and note the progress of digestion. In which degree of acidity does the fibrin digest the most rapidly? follows :

4. Differentiation between Pepsin and Pepsinogen.-Prepare five tubes as

(a) Few drops of glycerol extract of pepsinogen $+2-3$ c.c. of water.

(b) Few drops of glycerol extract of pepsinogen +5 c.c. of 0.2 per cent hydrochloric acid.

(c) Few drops of glycerol extract of pepsinogen +5 c.c. of 0.5 per cent sodium carbonate.

${ }^{1}$ Digestion of fibrin in a pepsin-hydrochloric acid solution is indicated first by a suelling of the protein due to the action of the acid, and later by a disintegration and dissolving of the fibrin due to the action of the pepsin-hydrochloric acid. If uncertain at any time whether digestion has taken place, the solution under examination may be filtered and the biuret test applied to the filtrate. A positive reaction will signify the presence of acid metaprotein (acid albuminate), proteoses (albumoses), or peptones, the presence of any one of which would indicate that digestion has taken place. 
(d) Two or 3 c.c. of pepsin solution $+2-3$ c.c. of I per cent sodium carbonate.

(e) Few drops of glycerol extract of pepsinogen +5 c.c. of I per cent sodium carbonate.

Add a small piece of fibrin to the contents of each tube, keep the five tubes at $40^{\circ} \mathrm{C}$. for one-half hour and observe any changes which may have occurred. To (a) add an equal volume of 0.4 per cent hydrochloric acid, neutralize (c), (d) and (e) with hydrochloric acid and add an equal volume of 0.4 per cent hydrochloric acid. Place these tubes at $40^{\circ} \mathrm{C}$. again and note any further changes which may occur. What contrast do we find in the results from the last three tubes? On the basis of these tests what is the relative resistance of pepsin and pepsinogen to alkalis?

5. Comparative Digestive Power of Pepsin with Different Acids.-Prepare a series of tubes each containing a $\mathrm{N} / \mathrm{I}$ o solution of one of the following acids: hydrochloric, sulphuric, nitric, combined hydrochloric, acetic, lactic and oxalic. To each acid add a few drops of the glycerol extract of pig's stomach and a small piece of fibrin. Shake well, place at $40^{\circ} \mathrm{C}$., and note the progress of digestion. In which tubes does the most rapid digestion occur?

6. Influence of Metallic Salts, etc.-Prepare a series of tubes and into each tube introduce 4 c.c. of pepsin-hydrochloric acid solution and $1 / 2$ c.c. of one of the chemicals listed in Experiment I 8 under Salivary Digestion, page 6r. Introduce a small piece of fibrin into each of the tubes and keep them at $40^{\circ} \mathrm{C}$. for one-half hour. Note the variations in the progress of digestion. Where has the least rapid digestion occurred?

7. Sahli's Desmoid Reaction.-This is a method for testing gastric function without using the stomach tube. The underlying principle of the test is the fact that raw cagtut may be digested in gastric juice but is entirely indigestible in pancreatic juice. The test is made as follows: A methylene-blue pill is introduced into a small rubber bag and the mouth of the bag subsequently tied with catgut. ${ }^{1}$ The small bag is then ingested immediately after the mid-day meal and the urine examined 5, 7,9 and 18-20 hours later for methylene blue. If methylene blue is present in appreciablequantity, it will impart to the urine a greenishblue color. If not present in sufficient amount to impart this color the urine should be boiled with one-fifth its volume of glacial acetic acid, whereupon a greenishblue color results if the chromogen of methylene blue is present. This contingency seldom arises, however, inasmuch as in most cases of uncolored urine it will be found that the rubber bag has passed through the stomach unopened. If the methylene blue is found in the urine inside of $18-20$ hours a satisfactory gastric function is indicated.

For Einhorn's bead method for the study of digestive function see chapter on Feces.

8. Testing the Motor and Functional Activities of the Stomach.-This test is performed the same as Experiment I9 under Salivary Digestion, page 6r. If the experiment was carried out under salivary digestion it will not be necessary to repeat it here.

1 About 0.05 gram of methylene blue is mixed with sufficient ext. glycyrrhiza to form a pill about 3-4 mm. in diameter. The pill is then placed in the center of a square piece of thin rubber dam and a little bag-like receptacle constructed by a twisting movement. The neck of the bag is then closed by wrapping three turns of catgut about it. The most satisfactory catgut to use is number oo raw catgut which has previously been soaked in water until soft. When ready for use the bag should sink instantly when placed in water and be water-tight. 
9. Influence of Bile.-Prepare three tubes as follows :

(a) Five c.c. of pepsin-hydrochloric acid solution $+I / 2-I$ c.c. of bile.

(b) Five c.c. of pepsin-hydrochloric acid solution +5 c.c. of bile.

(c) Five c.c. of pepsin-hydrochloric acid solution.

Introduce into each tube a small piece of fibrin. Keep the tubes at $40^{\circ} \mathrm{C}$. and note the progress of digestion. Does the bile exert any appreciable influence? How?

10. Influence of Gastric Rennin on Milk.-Prepare a series of five tubes as follows:

(a) Five c.c. of fresh milk +0.2 per cent hydrochloric acid (add slowly until precipitate forms).

(b) Five c.c. of fresh milk +5 drops of rennin solution. ${ }^{1}$ tion.

(c) Five c.c. of fresh milk + Io drops of 0.5 per cent sodium carbonate solu-

(d) Five c.c. of fresh milk + Io drops of a saturated solution of ammonium oxalate.

(e) Five c.c. of fresh milk +5 drops of 0.2 per cent hydrochloric acid. Now to each of the tubes (c), (d), and (e) add 5 drops of rennin solution. Place the whole series of five tubes at $40^{\circ} \mathrm{C}$. and after ro-15 minutes note what is occurring in the different tubes. Give a reason for each particular result. How do ammonium oxalate and sodium carbonate prevent coagulation?

${ }^{1}$ Any good commercial rennin or rennet preparation may be used in preparing this solution. 


\section{CHAPTER VIII}

\section{GASTRIC ANALYSIS}

The method of gastric analysis which has been in vogue clinically for years (see page I74) entails the feeding of a standard test meal, the removal of the complete stomach contents at the end of a onehour period, and the analysis of the material so removed. That this method is inaccurate has been repeatedly demonstrated in the author's laboratory ${ }^{1}$ and elsewhere. ${ }^{2}$ Furthermore, owing to the bulk of the old form of stomach tube and the discomfort occasioned by its use, it is impossible to follow the whole cycle of digestion and estimate, step

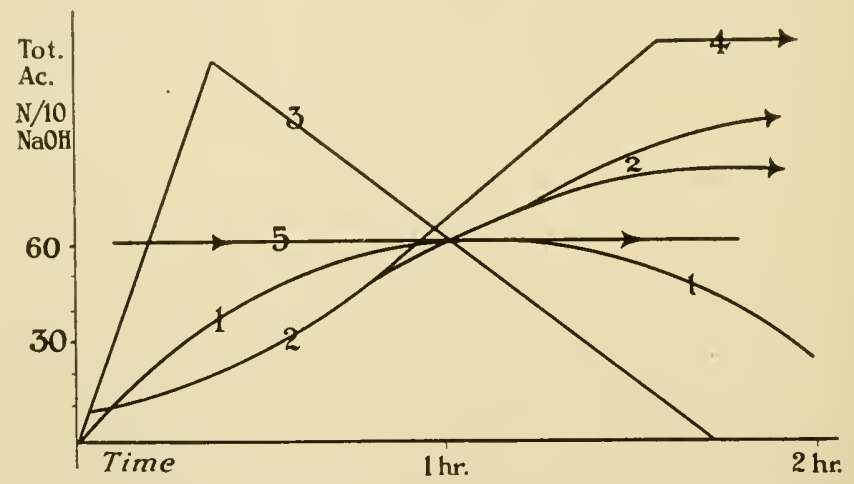

Fig. 4i,-Normal axd Pathological Curves after an Ewald Meal.

I. normal curve; 2 delayed digestion with late hyperacidity; 3 , larval hyperacidity;

4, tardive hyperacidity; 5, marked continued secretion from obstruction.

by step, the exact changes which take place in the stomach after the introduction of definite food mixtures into that organ.

Realizing the inadequacy of the procedure entailed in the old method of gastric analysis, a new procedure has been developed by Dr. Martin E. Rehfuss in the author's laboratory. This so-called "Fractional Method" entails the analysis of samples of material withdrawn from the stomach (by syringe) at short intervals for a period of two hours or more (until stomach is empty) after the ingestion of the test meal. By this means the observer is able to follow the entire cycle of

'Rehfuss: 'Jour. Am. MIed. Ass'n, 64, 569, rgr4.

Rehfuss, Bergeim and Hawk: Jour. Am. Med. Ass'n, 63, 909, r9r4.

Bergeim, Rehfuss and Hawk: Jour. Am. Med. Ass'n, 63, II, I9I4.

${ }^{2}$ Harmer and Dodd: Arch. Int. Med., Nov. 13, I913, p. 488. 
gastric digestion and is not limited, as in the old method, to information derived from the analysis of a single sample of stomach contents withdrawn at the end of one hour. That the acid values obtained by the old method may be grossly misinterpreted and lead to an incorrect diagnosis is indicated by the foregoing diagram (Fig. 4I):

It is set forth in the above diagram that various types of abnormal gastric secretion would be considered normal on the basis of a single examination at the end of one hour whereas the application of the fractional method reveals the abnormality of the secretion and enables a rapid and correct diagnosis. The removal of samples of gastric contents at short intervals, for a period of two hours or more after a test meal, is made possible by the use of a modified stomach tube ${ }^{1}$ of small diameter (No. I 2 French tubing) and fitted with a metal tip.

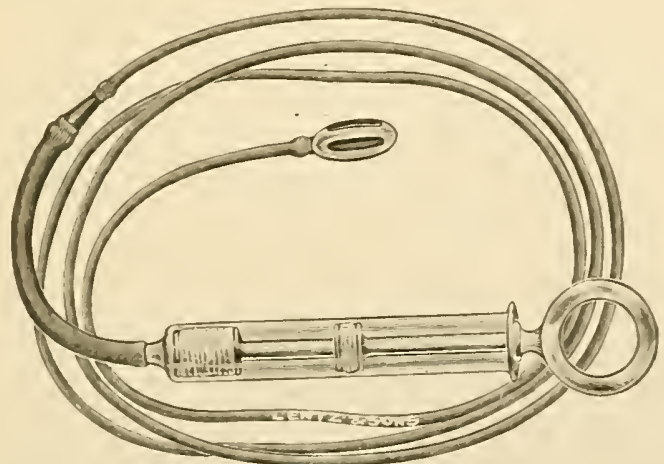

Fig. 42.-Rehfess Stomach Tube.

The tip is slotted with large perforations, the diameter of each being equivalent to the maximum bore of the tubing. Such a tube can be left in the stomach through the entire cycle of gastric digestion without inconvenience to the patient. ${ }^{2}$ A cut of the Rehfuss stomach tube (Fig. 42) is shown above. ${ }^{3}$

The idea of making a fractional examination of gastric contents is not new. Most of such attempts have been made, however, by using the old type of stomach tube and removing the entire stomach contents at different intervals on successive days, e.g., after fifteen minutes the first day, thirty minutes the second day, forty-five minutes the third day, etc. Hayem ${ }^{4}$ was the first to employ this method and later

1 Rehfuss: Am. Jour. LICd. Sci., June, I914.

${ }^{2}$ McClendon has recently suggested the introduction of an electrode in to the stomach in an attempt to follow the consecutive changes in the hydrogen ion concentration of the stomach contents (see Am. Jour. Physiol., 38, i 80,1915 ).

3 This tube is manufactured by Charles Lentz \& Sons, Philadelphia.

"Hayem: Brouardel \& Gilbert's, Traile de Mcdccine, 4, 236, 1905 
Ewald and Boas, ${ }^{1}$ Reichmann, ${ }^{2}$ v. Jaksch, ${ }^{3}$ Kornemann, ${ }^{4}$ Schule ${ }^{5}$ and Gregersen ${ }^{6}$ followed a similar procedure. The first report of data from the entire gastric cycle obtained by means of a small bore tube were made by Ehrenreich. ${ }^{7}$ This investigator used a Nélaton catheter. Skaller ${ }^{8}$ has reported a few experiments in which a small bore stomach tube with a metal tip was employed and the stomach contents subjected to fractional analysis.

Until recent years, the concensus of opinion based principally upon the work of the Pawlow school ${ }^{9}$ was to the effect that the gastric juice

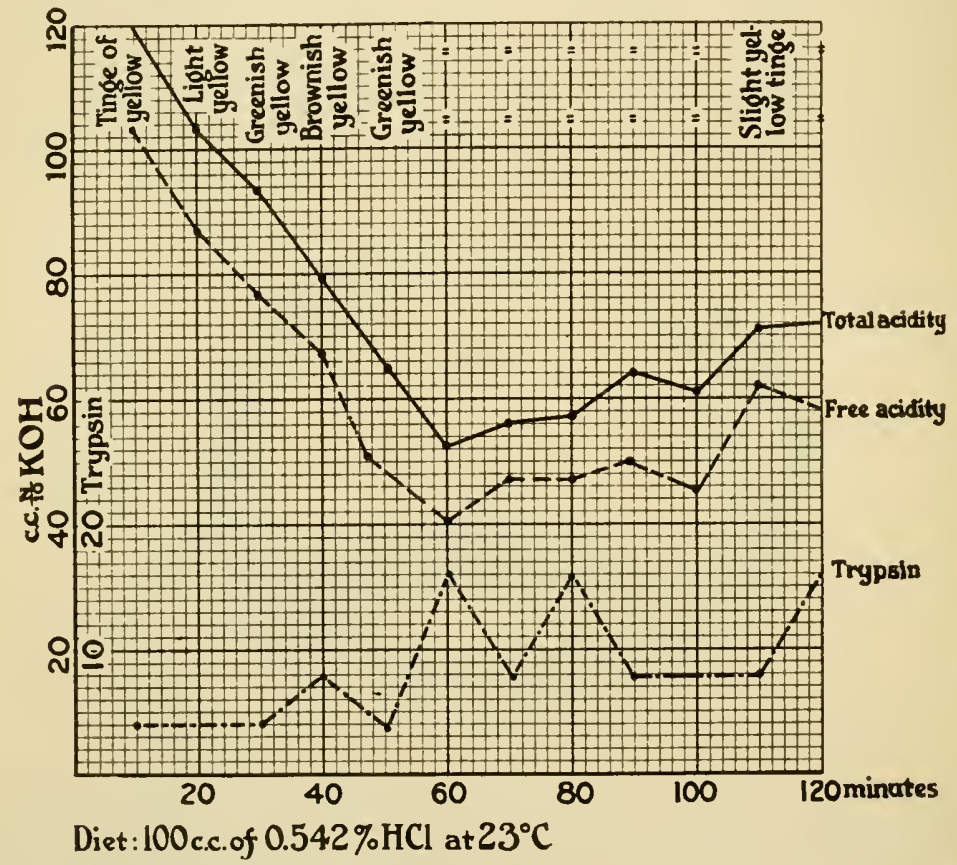

F g. 43.-Influence of Acid Introduced into the Normal Human Stomach. (Spencer, Meyer, Rehfuss and Hawk: American Journal of Physiology, March, Igr6.)

of normal man had an average acid concentration of 0.2 per cent hydrochloric acid, whereas the gastric juice of the dog and cat had an average acid concentration of $0.4-0.5$ per cent hydrochloric acid. These experiments were based principally upon the examination of the pure gastric juice of the lower animals as compared with the stomach

${ }^{1}$ Ewald \& Boas: Virchow's Arch., Ior, 325, 1885.

2 Reichmann: Zeit.f. klin. Med., 24, 565 , 1 885 .

3 V. Jaksch: Zeit. f. klin. Med., 19, 383, I89o.

${ }^{4}$ Kornemann: Arch. $f$. Verdauungskr., p. 369, I9 2.

${ }^{6}$ Schule: Zeit. f. klin. Med., 27, 46r, i 895.

6 Gregersen: Arch. f. Verdaunngskr., 19, 263, I9I3.

7 Ehrenreich: Zeil.f. klin. Med., p. 231, I9I2.

${ }^{8}$ Skaller: Berl. klin. Woch., 50, No. 47, I9r3.

- Pawlow: The Work of the Digestive Glands. Translated by Thompson, Second Edition, I9Io. 
contents of man. Later experiments ${ }^{1}$ have, however, demonstrated that the acid concentration of the freshly secreled gastric juice of man is similar to that of the dog, i.e., 0.4-0.5 per cent. Boldyreff claims that this initial high acidity of the human gastric juice is normally lowered to the "optimum acidity" of $0.15-0.2$ per cent hydrochloric acid by regurgitalion of alkaline fluids (bile, pancreatic and intestinal juices) from the intestine. This claim has been substantiated by experiments made in the author's laboratory ${ }^{2}$ and elsewhere. ${ }^{3}$ Both bile and trypsin are easily identified in the stomach contents of man after the introduction of 0.5 per cent hydrochloric acid into the empty organ. The above points are illustrated by the chart shown in Fig. 43, page I jo. ${ }^{4}$

The composition of human gastric juice and of the residuum (see page 160 ) is given in the following table:

COMPOSITION OF HUMAN GASTRIC JUICE.

\begin{tabular}{|c|c|c|}
\hline Constituent & Appetite juice ${ }^{5}$ & Residuum $^{6}$ \\
\hline Specific gravity................ & 1.007 & I. .006 \\
\hline$\Delta$ degrees $\ldots \ldots \ldots \ldots \ldots \ldots \ldots \ldots$ & -0.55 & -0.47 \\
\hline Total acidity, per cent. . . . . . . . & 0.45 & 0.30 \\
\hline \multicolumn{3}{|c|}{ Per Ioo c.c. of Juice } \\
\hline Total solids, gram............... & 0.55 & $0.98^{7}$ \\
\hline 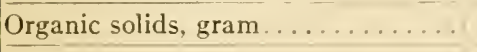 & 0.41 & $0.53^{7}$ \\
\hline Inorganic solids, gram............ & 0.14 & $0.45^{7}$ \\
\hline Total nitrogen, gram ............ & 0.060 & $0.066^{i}$ \\
\hline Total phosphorus, gram......... & & $0.005^{7}$ \\
\hline Total sulphur, gram........... & & $0.007^{i}$ \\
\hline 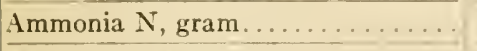 & $0.002-3$ & \\
\hline 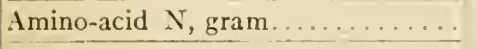 & $0.003-9$ & \\
\hline Chlorides, gram.............. & 0.5 & \\
\hline
\end{tabular}

1 Babkin: Die Äussere Sekretion der Verdauungsdrüsen, Berlin, IgI 4 .

Boldyreff: Transactions of 11 th Congress of Physicians, St. Petersburg, I 909.

Boldyreff: Quarl. Jour. Exp. Med., 8, I, rgrt.

Carlson: Am. Jour. Physiol., 38, 248 , 1915.

Bergeim, Rehfuss \& Hawk: Jour. Biol. Chem., 19, 345, 1914.

${ }^{2}$ Spencer, Meyer, Rehfuss and Hawk: Am. Jour. Physiol., 39, +50. r 910.

'Migai: Diss., St. Petersburg, 1909.

Milosorov: Zent. Physiol., 28, 615 , I9I4.

Zaitzeff: Russky Vrach., I4, No. 29, 1915.

4 Spencer et al: Loc. cit.

'Carlson: Loc. cil.

' Fowler, Rehfuss and Hawk: Loc. cit.

${ }^{7}$ Fowler \& Buchanan: Unpublished. 
The Use of Indicators in Determining the Reaction of Gastric Juice And Other Fiuids

The reaction of the gastric juice and other body fluids is most readily tested by means of indicators, so-called because they show changes of color with differing degrees of acidity or alkalinity of the solution. They behave as though they were weak acids or bases whose ions and unionized molecules have different colors. Modern theories of color in organic compounds however class them as tautomeric substances.

A neutral solution is one in which there are equal numbers of hydrogen and hydroxyl ions. An acid solution has a preponderance of hydrogen ion and an alkaline solution an excess of hydroxyl ion. All indicators do not show changes of color at the true neutral point, but at some fixed degree of acidity (or alkalinity), i.e., at a definite hydrogen or hydroxyl ion concentration. Indicators which change color at the approximate true neutral point are litmus and rosolic acid, while phenolphthalein changes color in a slightly alkaline solution. Congo red, sodium alizarin sulphonate and tropæolin $\mathrm{OO}$ are examples of indicators which change color in an acid solution.

Organic acids in general are not sufficiently strong; i.e., do not dissociate with the production of enough hydrogen ion to cause color changes in dilute solution with indicators of the last-mentioned class. Litmus, rosolic acid and phenolphthalein, however, change at so low a hydrogen ion concentration that they are affected by dilute solutions of organic acids and may be used for their titration. Even very dilute solutions of mineral acids are sufficiently acid to produce color changes with Congo red, alizarin, etc., and hence these indicators may be used in the titration of mineral acid. Phenolphthalein which changes color in a weakly alkaline solution indicates the presence of acid combined with weakly alkaline substances (as protein) as well as other types of acid such as acid salts, and hence, is used in the titration of solutions for their total acidity.

The hydrogen ion concentration of pure water or a neutral solution is approximately $\mathrm{I} \times \mathrm{IO}^{-7}$, being expressed as approximate moles of hydrogen ion per liter. That is water is a $\mathrm{I} / \mathrm{I} 0,000,000 \mathrm{~N}$ solution of hydrogen ions. The concentration of hydroxyl ions in pure water or a neutral solution is exactly equal to that of the hydrogen ions, so that water may be considered to be an N/10,000,000 alkali as well as an $\mathrm{N} / 10,000,000$ acid. Hydrogen ion concentrations are often expressed for the sake of brevity as their logarithms with the sign reversed. For example the logarithm of $1 \times 1 \mathrm{IO}^{-7}$ would be -7.0 and 
according to this notation the $\mathrm{H}$ ion concentration would be expressed as $\mathrm{P}_{\mathrm{H}}=7.0$. The product of the hydrogen ion concentration $\left(\mathrm{H}^{+}\right)$ by the hydroxyl ion concentration $\left(\mathrm{OH}^{-}\right)$is constant at about $\mathrm{I} \times 1 \mathrm{O}^{-14}$ so that as $\left(\mathrm{H}^{+}\right)$increases from $\mathrm{I} \times \mathrm{IO}^{-7}\left(\mathrm{P}_{\mathrm{H}}=7.0\right)$ to $\mathrm{I} \times \mathrm{IO}^{-4}$ $\left(\mathrm{P}_{\mathrm{H}}=4.0\right)$ the $\left(\mathrm{OH}^{-}\right)$falls to $\mathrm{I} \times \mathrm{IO}^{-10}$, and vice versa. It must be borne in mind that higher figures for the logarithmic notation indicate lower figures for $\left(\mathrm{H}^{+}\right)$. The hydrogen ion concentrations at which certain indicators commonly used in titration work change color, are indicated below.

Indicator

Phenolphthalein.

Neutral red.

Rosolic acid.....

Litmus.

Sodium alizarin sulphonate

Congo red..............................

Dimethyl-amino-azobenzene

Methyl orange. .

Tropxolin OO.
Hydrogen ion concentration

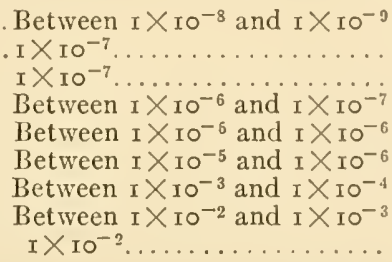

Between $\mathrm{I} \times \mathrm{IO}^{-8}$ and $\mathrm{I} \times \mathrm{IO}^{-9}$

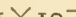

Between $I \times 10^{-6}$ and $\mathrm{I} \times 10^{-7}$

Between $\mathrm{I} \times \mathrm{IO}^{-6}$ and $\mathrm{I} \times \mathrm{IO}^{-6}$

Between $I \times 10^{-5}$ and $I \times 10^{-6}$

Between $\mathrm{I} \times \mathrm{IO}^{-3}$ and $\mathrm{I} \times \mathrm{IO}^{-4}$

Between $\mathrm{I} \times \mathrm{IO}^{-2}$ and $\mathrm{I} \times \mathrm{IO}^{-3}$
True nature

of solution

when the

color changes

Alkaline.

Neutral.

Neutral.

Neutral

Acid.

Acid.

Acid.

Acid.

Acid.

Tests with Indicators.-Prepare a series of solutions of varying acidities as outlined in the following table, page 154 . Introduce 5 or ro c.c. portions of each of these into a series of test-tubes and add to each a few drops of a solution of Tropæolin OO. Make a note of the colors produced, in the spaces left for this purpose. In the same way test out the other indicators mentioned, in order, using in each case a few drops of the indicator solution. The tests using the last three mentioned indicators: Günzberg's, Boas' and Tropæolin (evaporation test) are carried out differently as indicated on page $I_{55}$.

Are the following assumptions on which the use of certain of these indicators in gastric analysis is based borne out by your findings:

I. That Töpfer's reagent (Dimethyl-amino-azo-benzene) gives its characteristic pinkish-red color only in the presence of free $\mathrm{HCl}$.

2. That a blue color with Congo red indicates free hydrochloric (or other mineral acid), a violet color indicates an organic acid, and a brown color indicates combined hydrochloric acid.

3. That Tropæolin $\mathrm{OO}$ and methyl orange are indicators for free mineral acid.

4. That alizarin reacts to free mineral acid, organic acids and acid salts but not to combined $\mathrm{HCl}$.

5. That phenolphthalein can be used in titrating totai acidity, that is, acidity due to mineral and organic acids, acid salts and combined acid.

6. That iodine is liberated from $\mathrm{KI}-\mathrm{KIO}_{3}$ to a relatively slight extent by other than free mineral acid. 


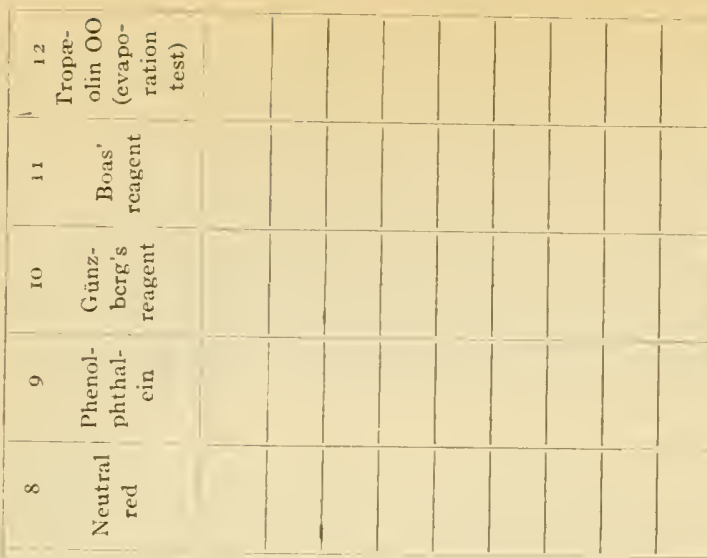

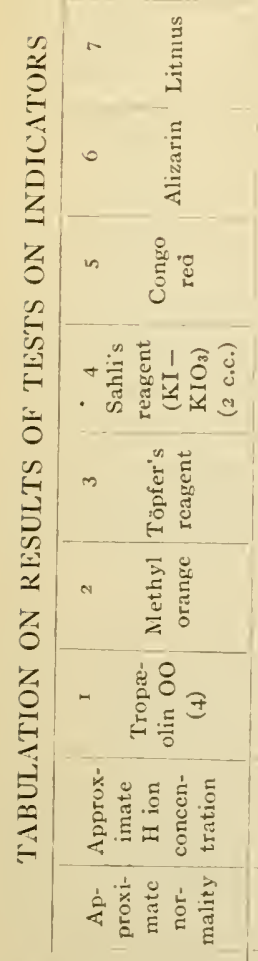

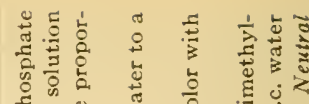

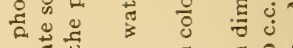

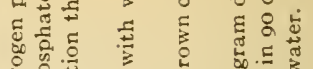
to

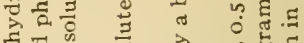

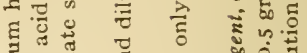

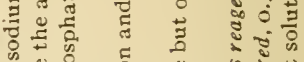
号论

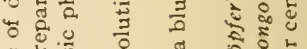
๘ नै.

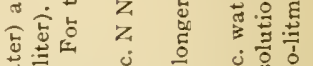
त. ن ऊ

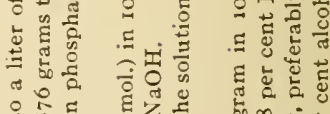

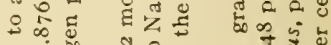

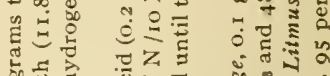

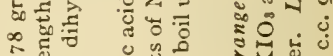

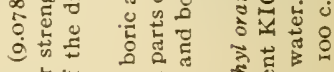

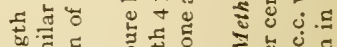

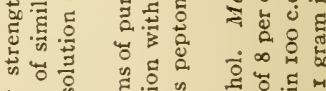
出 0 ह है

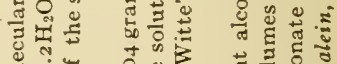

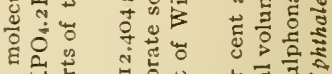

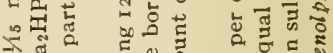

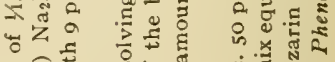

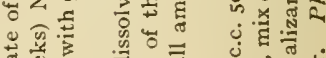

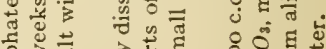

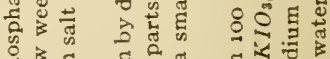
है है है

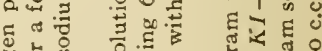
की

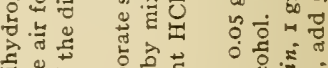
चั घ्g. .5 O

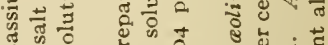

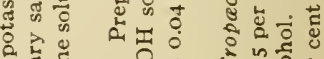

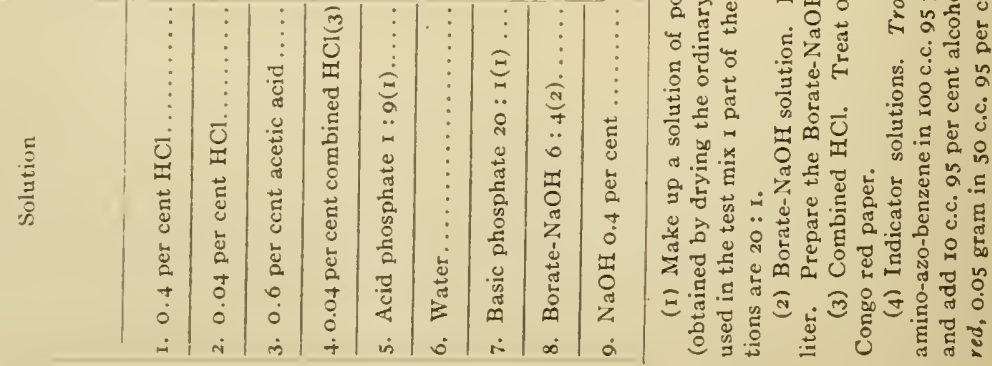


7. That Günzberg's test is the most satisfactory one for free $\mathrm{HCl}$ and that Boas' reagent and Tropaolin $\mathrm{OO}$ are also delicate reagents for free mineral acid.

Special Tests for Free HCl.-Perform the following tests on the solutions as outlined on page 154 and tabulate the results.

r. Guinzberg's Reagent. - Place r-2 drops of the reagent in a small porcelain evaporating dish and carefully evaporate to dryness over a low flame. Insert a glass stirring rod into the mixture to be tested and draw the moist end of the rod through the dried reagent. Warm again gently and note the production of a purplish-red color in the presence of free hydrochloric acid.

2. Boas' Reagent. ${ }^{2-}$ - Perform this test in the same manner as I, above. Free hydrochloric acid is indicated by the production of a rose-red color which becomes less pronounced on cooling.

3. Tropæolin $\mathrm{OO}, 3$

$$
\mathrm{NH}\left(\mathrm{C}_{6} \mathrm{H}_{5}\right)-\mathrm{C}_{6} \mathrm{H}_{4}-\mathrm{N}=\mathrm{N}-\mathrm{C}_{6} \mathrm{H}_{4}-\mathrm{SO}_{3} \mathrm{Na} \text {. }
$$

Place 2 drops of the solution to be tested and $r$ drop of the indicator in an evaporating dish and evaporate to dryness over a low flame. The formation of a reddish-violet color indicates free hydrochloric acid.

This test may also be conducted in the same manner as I, above.

\section{Hydrogex Ion Concentration and Titratable Acidity}

The acidity of a solution may be determined in two different ways by means of indicators. One method is by titration with standard alkali using the indicator to determine the end point of the titration. For this purpose the indicator should be one which gives a sharp color change which is sensitive to the form of acidity which is to be determined, and which is not destroyed by any substance contained in the titration mixture. Thus phenolphthalein can be used for the titration of strong bases and nearly all weak acids, but cannot be used for weak bases, and is unsatisfactory in the presence of ammonium salts. Methyl orange on the other hand is useful for strong acids and weak bases such as ammonia and for the soluble carbonates but cannot be used for weak acids such as carbonic acid or the organic acids. Almost any indicator may be used in the titration of mineral acids against strong bases such as $\mathrm{KOH}$ inasmuch as under these conditions I drop of the standard solution will throw the hydrogen ion concentration so far beyond that of neutrality that the turning point of any common indicator will be passed.

Titration does not, however, enable us to determine in all cases the

${ }^{1}$ Günzberg's reagent is prepared by dissolving 2 grams of phloroglucinol and $x$ gram of vanillin in $100 \mathrm{c.c}$. of 95 per cent alcohol.

${ }^{2}$ Boas' reagent is prepared by dissolving 5 grams of resorcinol and 3 grams of sucrose in I00 c.c. of 50 per cent alcohol.

${ }^{3}$ Prepared by dissolving 0.05 gram of troparolin $\mathrm{OO}$ in $100 \mathrm{c.c}$. of 50 per cent alcohol. 
true acidity of a solution, that is, its hydrogen ion concentration. In the case of strong acids and bases very accurate results for the true acidity may be obtained in this way. In the case of weak acids or bases the titration values may give but slight information as to the true acidity. Thus in the case of a slightly dissociated acid, such as acetic acid, as fast as the acidity due to its dissociated hydrogen ions is neutralized the undissociated acid ionizes further and the titration value finally obtained represents the total acid present at the beginning both ionized and unionized. Salts of strong acids and very weak bases and vice versa also hydrolyze during the course of the titration and the values obtained in no sense represent the true acidity.

Hydrogen ion concentrations may be determined through a certain range by means of indicators. The unknown solution is treated with a few drops of indicator and the color obtained compared with that produced with the same amount of indicator and a solution of known hydrogen ion concentration. If the same tint is produced in both cases the two acidities are the same. This is of course only true when the indicator chosen is a suitable one, that is, one that shows definite color changes in hydrogen ion concentrations in the neighborhood of that of the unknown. The choice of indicators for this purpose is somewhat different than that for titration purposes. For use in the determination of the hydrogen ion concentration of a solution we need an indicator showing a very gradual change in color through a given range, one which is not readily affected by the presence of neutral salts or other substances likely to be present, and the color of which does not fade too rapidly. The ranges through which a number of indicators may be used with satisfactory results for the determination of hydrogen ion concentrations is indicated in the chart (Fig. 44, page I57). Those surrounded by the heavy lines are the most satisfactory.

The chart also indicates how standard solutions of definite hydrogen ion concentrations may be made up from a series of stock solutions, by mixing in various proportions. The stock solutions indicated on the chart were suggested by Sörensen and are as follows: 0.10 $\mathrm{N} \mathrm{HCl}$; ○.10 N NaOH; 7.505 g. glycocoll plus $5.85 \mathrm{gm}$. $\mathrm{NaCl}$ per liter; II.876 g. $\mathrm{Na}_{2} \mathrm{HPO}_{4} .2 \mathrm{H}_{2} \mathrm{O}$ per liter; 9.078 g. $\mathrm{KH}_{2} \mathrm{PO}_{4}$ per liter; 21.008 g. citric acid in I liter of $0.20 \mathrm{~N} \mathrm{NaOH}$; $12.404 \mathrm{~g}$. boric acid in I liter of $0.10 \mathrm{~N} \mathrm{NaOH}$. The other solutions are $0.20 \mathrm{~N}$ sodium acetate and $0.20 \mathrm{~N}$ acetic acid. Solutions of known hydrogen ion concentration are prepared from these by mixing in the proportions indicated on the chart, the abscissæ representing parts of the more alkaline or less acid constituent. Thus a mixture of seven volumes of the sodium acetate stock solution with three volumes of the stock acetic acid solution 


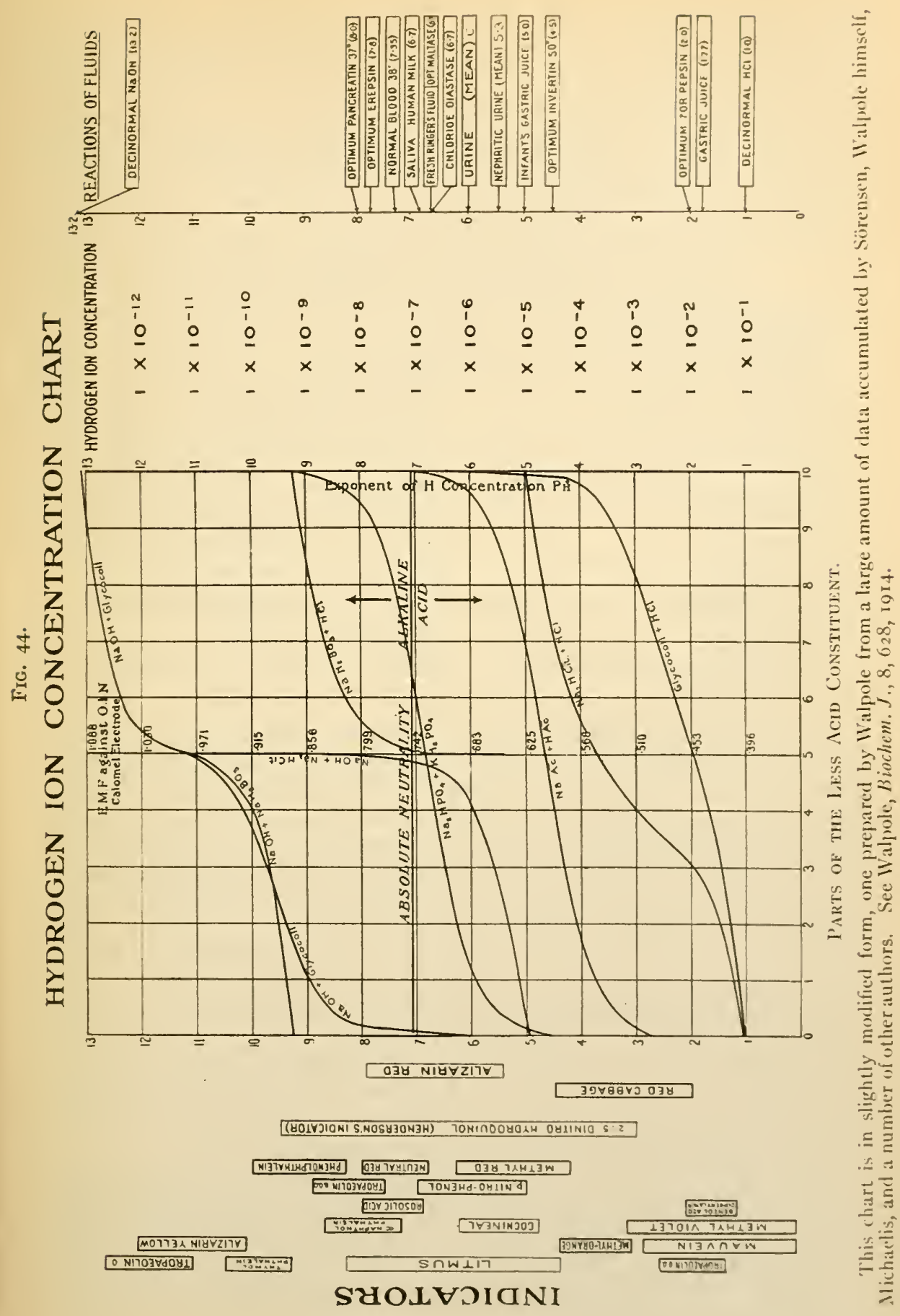


gives a mixture with an hydrogen ion concentration of $I \times 10^{-5}$ (exponent: 5.0). The mixtures are most satisfactory through the ranges where the hydrogen ion concentration changes most gradually, that is, through the flatter portions of the curves.

The amounts of indicator solutions and their strengths to be used in the determinations of hydrogen ion concentrations in Io c.c. portions of unknown solution are indicated below.

\section{INDICATOR SOLUTIONS}

1. Alizarin yellow R ( $\rho$ nitrobenzene-azo-salicylic acid)

2. Azolitmin (litmus)

3. Cochineal

4. 2,5-dinitro-hydroquinone

5. Mauvein

6. Methyl orange

7. Methyl red

8. Methyl violet

9. Neutral red

I0. $\rho$-Nitrophenol

II. Phenolphthalein

I2. Rosolic acid

I3. Thymolphthalein

I4. Tropæolin O

I5. Tropæolin OO

I6. Tropæolin $\mathrm{OOO}$

\begin{tabular}{|c|c|}
\hline Drops & Preparation of solution \\
\hline $10-5$ & $\begin{array}{l}\text { O. I gram to Iooo c.c. water. } \\
\text { Aqueous solution. } \\
\text { Alcoholic solution. }\end{array}$ \\
\hline $5^{-2}$ & I gram to 1000 c.c. alcohol. \\
\hline $8-1$ & 0.5 gram to 1000 c.c. water. \\
\hline $5^{-3}$ & $\begin{array}{l}\text { O. I gram recrystallized salt to I000 c.c. } \\
\text { water. }\end{array}$ \\
\hline $4^{-2}$ & Saturated solution in 50 per cent. alcohol. \\
\hline $8-\mathbf{I}$ & 0.5 gram to I000 c.c. water. \\
\hline $20-10$ & o. I gram in 500 c.c. alcohol, and 500 c.c. water \\
\hline $20-3$ & 0.4 gram to 60 c.c. alcohol, 940 c.c. water. \\
\hline $20-3$ & o. 5 gram to 500 c.c. alcohol, 500 c.c. water. \\
\hline I $5-6$ & 0.4 gram to 400 c.c. alcohol, 600 c.c. water. \\
\hline IO- 3 & 0.4 gram to 500 c.c. alcohol, 500 c.c. water. \\
\hline $10-5$ & o. I gram to I000 c.c. water. \\
\hline $5-3$ & $\begin{array}{l}\text { Of recrystallized salt, o.I gram to rooo c.c. } \\
\text { water. }\end{array}$ \\
\hline $10-4$ & o. I gram to Iooo c.c. water. \\
\hline
\end{tabular}

Determination of Hydrogen Ion Concentration.-Introduce ro c.c. portions of the unknown solution into a series of test-tubes of similar diameter and of clear glass. Test first with litmus paper which changes at about the neutral point. According to whether the reaction is acid or basic to litmus test other indicators on the acid side such as $\rho$-nitrophenol, methyl orange and tropæolin $O O$, or on the basic side as phenolphthalein. Select an indicator which gives with the solution neither its maximum acid or maximum basic color. Note from the chart through what range this indicator exhibits its characteristic change of color. Then to Io c.c. portions of standard solutions of known hydrogen ion concentration (furnished by the instructor), which cover approximately the same range as the indicator add exactly the same number of drops of indicator solution as was added to the standard. Compare colors of unknown and standards until one is found which matches and which consequently possesses the same hydrogen ion concentration. If the unknown is so strongly acid or basic that none of the indicators mentioned can be used directly it will be necessary to dilute it with ro or a greater number of volumes of water before testing further.

In case the unknown solution is slightly colored the standards should likewise be brought to the same tint by the addition of some coloring agent as Bismarck brown, methyl orange, methyl violet, etc., before making the comparison.

For applications of the indicator method for the determination of hydrogen ion concentration to biological fluids see chapters on the quantitative analysis of blood (XVI) and urine (XXVI).

Comparison of $\mathrm{H}$ Ion Concentration and Titratable Acidity.-I. Determine 
colorimetrically the $\mathrm{H}$ ion concentration of an $\mathrm{N} /$ IOo solution of hydrochloric acid using tropæolin $\mathrm{OO}$ as an indicator and of an $\mathrm{N} / 100$ acetic acid using methyl orange as an indicator. Note the great difference between the true acidities of the two solutions.

Titrate ro c.c. portions of $\mathrm{N} / \mathrm{I} 00$ hydrochloric acid and of $\mathrm{N} / \mathrm{I} 00$ acetic acid with $\mathrm{N} / \mathrm{I} 00 \mathrm{KOH}$ using phenolphthalein as an indicator. Note that identical results are obtained for the titratable acidities of the two.

2. Determine colorimetrically the $\mathrm{H}$ ion concentration of an $\mathrm{N} / \mathrm{I} 00 \mathrm{KOH}$ solution using tropæolin $\mathrm{O}$ as an indicator, and of an $\mathrm{N} / \mathrm{roo}$ ammonia solution using phenolphthalein as an indicator. Note the results and then titrate roc.c. portions of both solutions with $\mathrm{N} / 100 \mathrm{HCl}$ using alizarin as an indicator.

3. Mix equal portions of $M / I_{5}$ potassium dihydrogen phosphate and $M / I_{5}$ disodium phosphate (see chart). Note that the mixture is practically neutral to litmus. Titrate one Io c.c. portion of this mixture with $\mathrm{N} /$ I o $\mathrm{KOH}$, using phenolphthalein as an indicator. Titrate another portion with $\mathrm{N} /$ Io $\mathrm{HCl}$ solution, using methyl orange as an indicator.

4. Mix equal volumes of $\mathrm{N} / 5$ sodium acetate solution and $\mathrm{N} / 5$ acetic acid. Note that the mixture is acid to litmus. Titrate one ro c.c. portion with $\mathrm{N} / 10$ $\mathrm{HCl}$ using tropæolin $\mathrm{OO}$ as an indicator. Titrate another portion with $\mathrm{N} / \mathrm{Io}$ $\mathrm{KOH}$ using phenolphthalein as an indicator.

\section{THE FRACTIONAL METHOD OF GASTRIC ANALYSIS}

\section{Procedure in Gastric Analysis by the Fractional Method}

I. Introduction of the stomach tube (see pages $1_{59}$ and I60).

2. Removal of the residuum (see pages 160 and ${ }_{1} 6$ ).

3. Feeding the test meal (see page $16 \mathrm{I}$ ).

4. Feeding the retention meal (in special cases), see page ${ }_{6}$ I. $_{\text {. }}$

5. Removing samples of stomach contents for analysis (see page $16 \mathrm{I}$ ).

6. Examination of the samples for:

(a) Total acidity (see page 162 ).

(b) Free acidity (see page I64).

(c) Pepsin (see page I 65).

(d) Trypsin (not a routine procedure), sec page 160.

(c) Lactic acid (see page I 70).

(f) Occult blood (see page I 7 I).

(g) Bile (see page I $7 \mathrm{I}$ ).

(h) Microscopical constituents (see page I73).

I. Introduction of the Stomach Tube.-Whereas the large tube is directly inserted by propulsion, the Rehfuss tube is swallowed in the natural manner and aided by gravity. The tube may be passed in one of three ways, i.e.: (I) lubricated; (2) with aid of fluid; (3) after throat is cocainized. When passed by the first method the tip of the tube, after thorough lubrication with glycerol or liquid petrolatum, is seized between 
the thumb and forefinger and placed on the tongue. Then with the aid of the forefinger the tip is pushed forward until it reaches the root of the tongue and is engaged in the oropharynx. Then the patient is encouraged to swallow persistently while the tube is slowly fed into the mouth. After slight discomfort in the pharynx and its passage past the level of the cricoid cartilage, practically no discomfort is felt. This method is used when it is essential that the pure gastric secretion or residuum be obtained. Ordinarily, however, it is much easier to swallow the tube by the second method. This method consists in placing the tip in the oropharynx and then giving the patient a measured quantity of water or tea to swallow. The movements induced by the swallowing carry the tube rapidly to the stomach with a minimum of discomfort. When an Ewald meal (see below) is given, part of the tea can be reserved for swallowing the tube. This procedure makes it scarcely more arduous than the swallowing of food. Should the patient, however, be extremely neurotic or the unfortunate possessor of marked pharyngeal hyperesthesia, cocain hydrochloride in 2 per cent aqueous solution can be applied to the throat rendering the passage of the tube practically insensitive. When the tube has entered the stomach, aspiration of the material shows the characteristic gastric contents. Should the tip remain in the esophagus through transient cardiospasm or other cause, aspiration results in the removal of only a very small specimen having all the characteristics of the pharyngeal and esophageal secretions.

2. Removal of Residuum.-If the so-called "empty" stomach is examined in the morning before any food or drink has been taken it will be found to contain considerable material. This is termed residuum. Before a test meal is introduced into the stomach, this organ should be emptied. If this is not done we cannot consider the samples withdrawn after the test meal is eaten as representing the secretory activity of the gastric cells under the influence of the stimulation of the test meal. It has been generally recognized, clinically, that a residuum above 20 c.c. is pathological. ${ }^{1}$ Such a volume has been considered as indicative of hypersecretion, and this in turn in many cases indicates an organic lesion. The observations indicating that a residuum of over 20 c.c. was pathological, were made upon residuums removed by means of the old type of stomach tube which does not completely empty the stomach. ${ }^{2}$ When the residuum is completely removed by means of

${ }^{1}$ Loeper: Leçons de pathologie digestive, 1912, Series 2, pp. 17-19.

Zweig: Magen- und Darmkrankheiten, p. 459.

Kemp: Diseases of the Stomach, Intestines and Pancreas, I912, p. I33.

Wolff: Taschenbuch der Magen- und Darmkrankheiten, p. 22.

${ }^{2}$ Harmer and Dodd: Loc. cil. 
the Rehfuss tube it has been demonstrated that the normal residuum is practically always over 20 c.c. and that the average is about 50 c.c. ${ }^{1}$ The normal residuum has been found to possess all the qualities of a physiologically active gastric juice with an average total acidity of 30 and an average free acidity of 18.5 . The residuum is often colored by bile. This is particularly true if the fluid has a relatively high acidity. Trypsin is also generally present. These findings indicate regurgita-

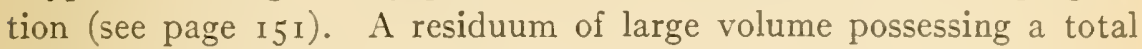
acidity value of 70 or over may indicate ulcer.

Analysis of Residuum.-Remove the residuum as directed under (5), below, and analyze the fluid according to methods outlined on page 162 .

3. The Test Meal.-Before making an analysis of the stomach contents it is customary to introduce something into the stomach which will stimulate the gastric cells. The response to this stimulation is then measured clinically by the determination of total acidity, free acidity and pepsin in the stomach contents. Many forms of test meal have been used.

The test meal most widely employed is the Ewald test meal. This consists of 2 pieces ( 35 grams) of toast and 8 ounces ( 250 c.c.) of tea.

Inasmuch as it was demonstrated in the author's laboratory ${ }^{2}$ that water gave a similar gastric stimulation to that produced by the Ewald meal it was suggested that a simple water meal might be substituted for the Ewald meal. This water meal also has the added advantage of enabling one to determine the presence of food rests and to test more accurately for lactic acid, blood and bile.

4. The Retention Meal.-In order to obtain more information regarding gastric motility than is furnished by the ordinary test meal described above the patient may be fed a so-called relention meal. This meal is fed in place of the regular evening meal and contains substances readily detected. In the morning before breakfast $(7-8 \mathrm{a} . \mathrm{m}$.) remove the stomach contents (residuum, see page 160 ) by aspiration and examine for food rests. The normal stomach should give no evidences of food retention. A satisfactory retention meal consists of 4 ounces each of boiled string beans and rice. ${ }^{3}$ Diets containing prunes, raspberry marmalade, lycopodium powder, etc., have also been employed. In many instances an ordinary mixed diet will serve the purpose.

5. Removal of Samples for Analysis.-At intervals of exactly I5 minutes from the time the test meal is eaten until the stomach is empty

1 Rehfuss, Bergeim and Hawk: Jour. Am. M $e d$. Ass'n, 63, I I, I9I 4 .

Fowler, Rehfuss and Hawk: Jour. Am. Mcd. Ass'n, 65, 102r, Igr5.

${ }^{2}$ Bergeim, Rehfuss, and Hawk: Jour. Biol. Chem., 19, 345, I 9 r 4.

Rehfuss, Bergeim and Hawk: Jour. Am. Med. Ass'n, 63, II, Igr4.

3 Myers and Fine: Essentials of Pathological Chemistry, I913. 
$5^{-6}$ c.c. samples of gastric contents are withdrawn from the stomach by means of aspiration.

In the removal of samples from the stomach, it is essential that very little traction be employed. To completely empty the stomach, aspiration is practised in four positions: (a) on the back; $(b)$ on the stomach; $(c)$ on right side, $(d)$ on left side. This results in complete evacuation of the stomach. Three tests may be employed to determine whether the stomach is empty: (I) No more material can be aspirated in any position; (2) injection of air and auscultation over the stomach with a stethoscope reveals a sticky rale and not a series of gurgling rales such as is heard when there is material in the stomach; (3) lavage or irrigation through the tube which shows the absence of all food in the stomach.

6. Examination of the Samples.- The old methods of gastric analysis involved the collection (by analysis and calculation) of data regarding several types of acidity (see Töpfer's method, page 174). The modern tendency among clinicians is to lay particular emphasis upon the values for total acidity and free acidity. The determination of the peptic activity is also important as well as the demonstration of the presence or absence of occult blood, lactic acid, mucus, food rests, etc.

Procedure.-Strain each sample through a fine-mesh cheese cloth. ${ }^{1}$ Examine the residue for mucus, blood and food rests. Use the strained stomach contents for the determination of total acidity, free acidity and peptic activity by methods which follow.

(a) Determination of Total Acidity.-Principle.-The indicator used is phenolphthalein. Since the indicator reacts with mineral acid, organic acid, combined acid and acid salts the values obtained represent the total acidity of the solution.

Procedure.-Measure I c.c. of the strained stomach contents by means of an Ostwald pipette and introduce it into a low-form 60 c.c. porcelain evaporating dish. Dilute with 15 c.c. of distilled water. Add 2 drops of a I per cent alcoholic solution of phenolphthalein and titrate with $\mathrm{N} /$ roo sodium hydroxide until a faint pink color is obtained and persists for about two minutes. ${ }^{2}$ Take the burette reading and calculate the total acidity.

1 The examination for microscopical constituents (see $(h)$ p. I 73) should be made on the original (unstrained) gastric contents. Tests for occult blood may be made on the sediment if desired.

${ }^{2}$ Procedure for Serial Titrations.-When a series of titrations are to be made the following procedure may be used: Arrange the numbered evaporating dishes in rows on a tray. Introduce I c.c. of the proper sample into each dish, dilute with Io c.c. of water and add the indicator. Add the N/I00 NaOH to contents of dish No. $\mathrm{I}$ at a definite rate until a point is reached at which a faint pink color is obtained, as described above. Return dish No. I to its place in the tray and place dish No. 2 under the burette. Take the burette reading of No. r. Then titrate No. 2 in the same way. Continue the series. This procedure has the advantage of being speedy and accurate. There is a slight error made by the rapid addition of the $\mathrm{NaOH}$ but it is uniform and the results (titrations) are therefore comparable. 
Calculation.- Note the number of cubic centimeters of $\mathrm{N} / \mathrm{I} 00 \mathrm{NaOH}$ required to neutralize I c.c. of stomach contents, and multiply it by ro to obtain the number of cubic centimeters $\mathrm{N} / \mathrm{ro} \mathrm{NaOH}$ necessary to neutralize roo c.c. of stomach contents. This is the method of calculation most widely used. For other forms of expressing total acidity see page I74. Plot your results in a form similar to those shown in Figs. 45 and 46.

Curves Obtained by the Fractional Method.-When an Ewald test meal is given to normal individuals a curve such as indicated below is usually obtained. The curve may vary within certain limits depending on individual idiosyncrasies, but is usually found to follow the curve depicted, and the meal normally leaves the stomach in two and one-

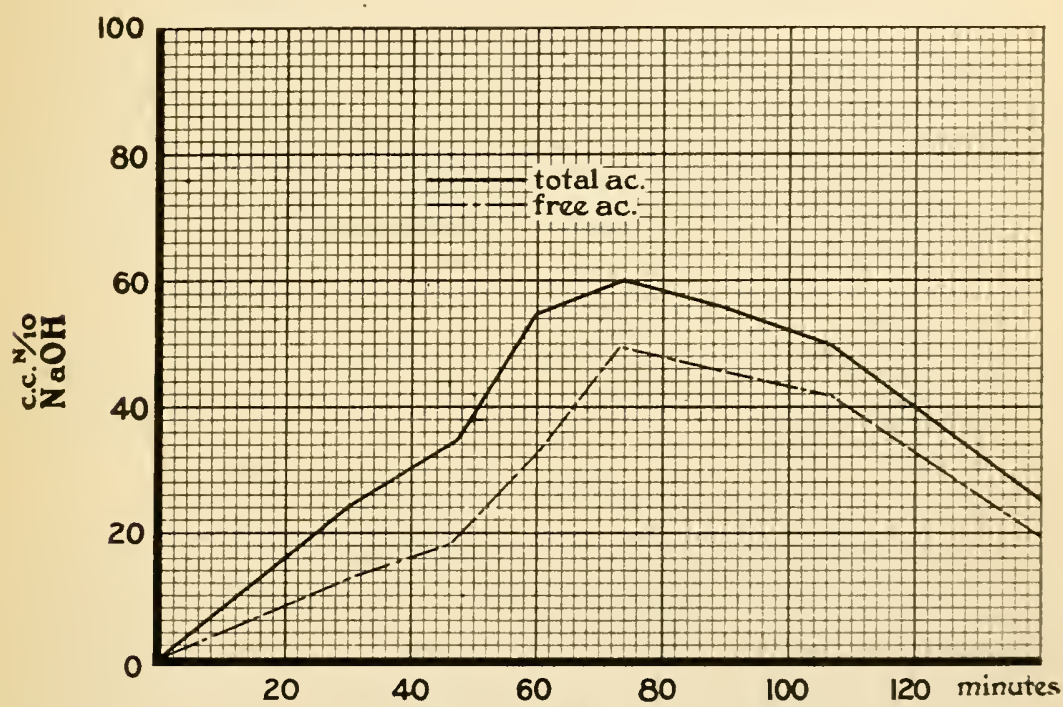

Fig. 45.-Acidity Curves of Normal Human Stomach.

half hours. Pathologically every variation occurs, both in time of evacuation as well as the character of the curve and the quantity of the secretion elaborated. Fig. 4I represents some of the possibilities of pathological cases, but a consideration of their interpretation is outside the purpose of the present volume. It will be evident, however, from a consideration of the figure that the cycle of gastric digestion is a constantly changing one, and no information concerning the trend of digestion can be obtained by an examination of only a single stage of digestion. Marked changes may precede or follow that stage and the possibilities suggested in Fig. 4I are all observed clinically and are of varying significance. Typical curves from cases of hyperacidity, gastric carcinoma and achylia are shown in Figs. 46,47 and $4 \mathrm{~S}$ respectively. 
(b) Determination of Free Acidity.-The reagent most widely used, clinically, for the determination of free hydrochloric acid in stomach

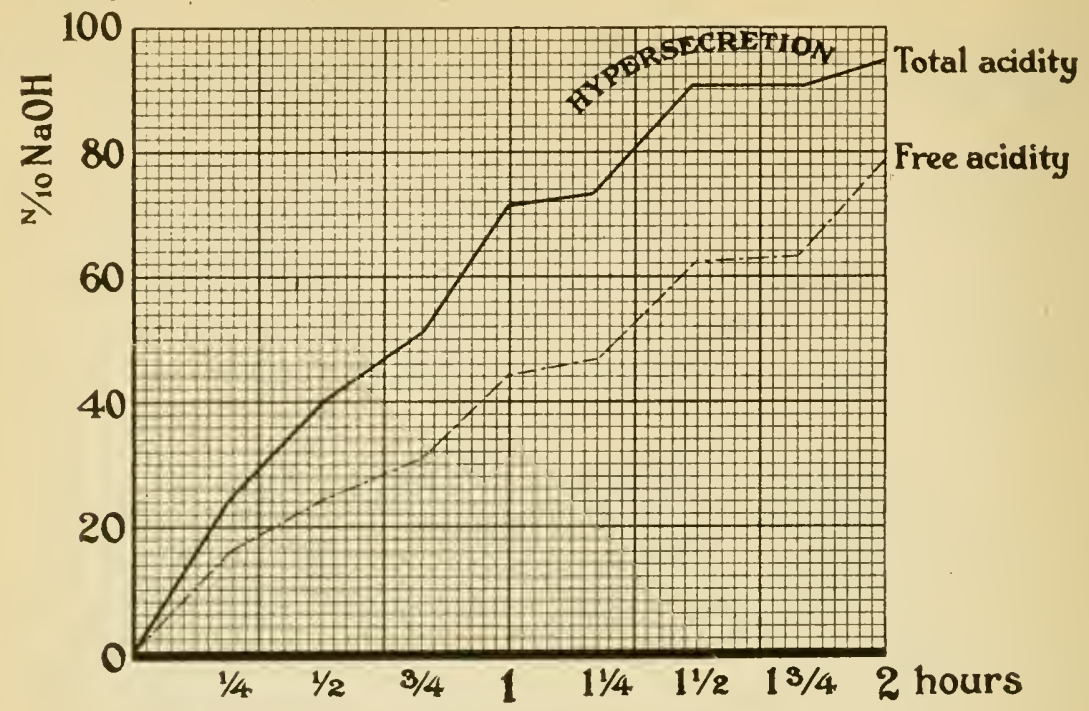

Fig. 46.-Acidity Curves From a Case of Hyperacidity.

contents is Töpfer's reagent (see page I 75). It has been found, however, that this reagent gives rather inaccurate results due to the uncertain

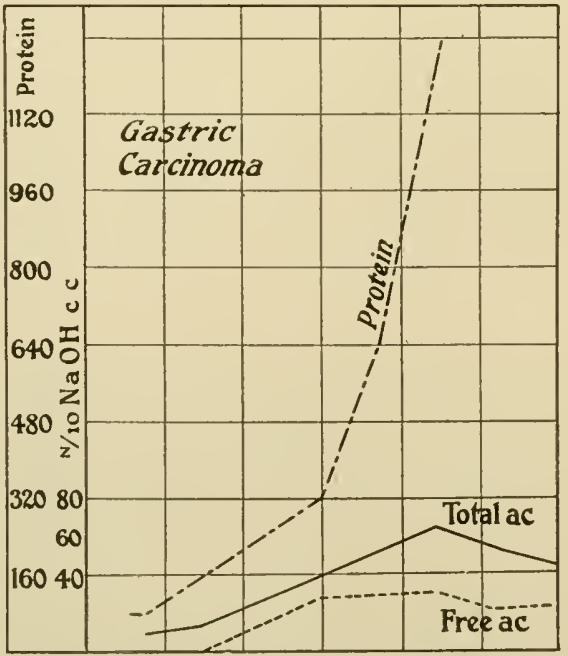

Fig. 47.-Acidity and Protein Curves in Gastric Carcinoma. (Clarke and Rehfuss: Jour. Am. Med. Ass'n, 64, I737, 1915.)

end point. For this reason we have employed Sahli's reagent. ${ }^{1}$ This reagent contains $\mathrm{KI}$ and $\mathrm{KIO}_{3}$ and liberates iodine in the presence of

${ }^{1}$ A mixture of equal parts of a 48 per cent solution of potassium iodide and an 8 per cent solution of potassium iodate. 
free hydrochloric acid. The liberated iodine is titrated by thiosulphate using starch as an indicator. It gives values similar to 'Töpfer's reagent in average acidities. ${ }^{1}$ Acidities other than free hydrochloric react to a certain extent with Sahli's reagent.

Procedure.-Measure $\mathrm{x}$ c.c. of the strained stomach contents by means of an Ostwald pipette and introduce it into a 60 c.c. porcelain evaporating dish. Dilute with Io c.c. of distilled water, and add I c.c. of Sahli's reagent (a mixture of equal parts of 48 per cent $\mathrm{KI}$ and 8 per cent $\mathrm{KIO}_{3}$ ). Allow the stomach contents thus treated to stand for five minutes and then titrate with $\mathrm{N} /$ Ioo sodium thiosulphate until only a faint yellow color remains. Now add 5-10 drops of a I per cent solution of soluble starch and continue the titration until the blue color disappears. In serial titrations the same procedure may be employed as described on page 162 , note 2 .

Calculation.- Note the number of cubic centimeters of $N /$ roo sodium thiosulphate required to titrate I c.c. of stomach contents to the total disappearance of blue color in the presence of starch. Inasmuch as N/roo thiosulphate is equivalent to $\mathrm{N} / \mathrm{x}$ oo alkali, this value indicates the number of cubic centimeters of $\mathrm{N} / \mathrm{I} 00$ sodium hydroxide necessary to neutralize the free hydrochloric acid in

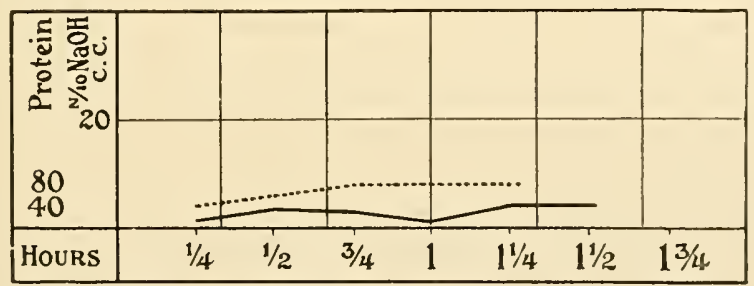

Fig. 48.-Total Acidity and Protein Curves in Benign Achylia (Solid Line RePresents ACIDity). (Clarke and Rehfuss: Jour. Am. Med. Ass'n., 64, r737, r915.)

I c.c. of the stomach contents. Multiply the value by ro to obtain the number of cubic centimeters of $\mathrm{N} / \mathrm{ro} \mathrm{NaOH}$ necessary to neutralize Ioo c.c. of stomach contents. This is the method of calculation most widely used. For other forms of expressing free acidity see page $\mathbf{1 7 4}$. Plot your results in a curve similar to those shown in Figs. 43, 45, and 46, pages 150,163 and $x 64$.

(c) Determination of Peptic Activity.-(I) Method of Mett ${ }^{2}$ as Modified by Nirenstein and Schiff. ${ }^{3}$-Prinicple.-Small glass tubes filled with coagulated egg albumin are introduced into the solution to be tested, and kept for a definite length of time in the incubator. The protein column is digested at both ends of the tube to an extent depending upon the amount of pepsin present. The method is not strictly accurate but is the most satisfactory for clinical purposes on account of its simplicity. Nirenstein and Schiff showed that human gastric juice contained inhibiting substances the effect of which is overcome by the dilution recommended.

${ }^{1}$ Fowler, Bergeim and Hawk: Unpublished data.

2 Mett: Argh.f. Anal. u. Physiol., isot. 6S.

${ }^{3}$ Nirenstein and Schiff: Arch. f. T'crdaunngskrankhcilen, S, 559, 1002. 
Procedure.-Introduce into a small Erlenmeyer flask I c.c. of gastric juice and 15 c.c. of $\mathrm{N} / 20 \mathrm{HCl}$ (=0.18 per cent $\mathrm{HCl}$ ). Add two Mett tubes prepared as indicated below, stopper the flask to prevent evaporation and place in an incubator at $37^{\circ} \mathrm{C}$. for 24 hours. By means of a low power microscope and a millimeter scale (graduated to half millimeters) determine accurately the length of the column of albumin digested at each end of the tubes. It is well to run the determination in duplicate in which case the result is the average of the eight figures obtained. Ordinarily from $2-4 \mathrm{~mm}$. of albumin are digested by normal human gastric juice.

Calculation.-The peptic power is expressed as the square of the number of millimeters of albumin digested. This is based on the Schuitz-Borissow law that the amount of proteolytic enzyme present in a digestion mixture is proportional to the square of the number of millimeters of albumin digested. Therefore a gastric juice which digests $2 \mathrm{~mm}$. of albumin contains four times as much pepsin as one which digests only I mm. of albumin.

Example.- If the microscopic reading gives on an average $2.2 \mathrm{~mm}$. of albumin digested the pepsin value for the diluted juice would be $2.2^{2}=4.84$, and for the pure undiluted juice, $4.84 \times 16=77 \cdot 44$.

Preparation of Mett Tubes (Christiansen's Method). ${ }^{1-}$-The liquid portions of the whites of several eggs are mixed and strained through cheese cloth. The mixture should be homogeneous and free from air bubbles. A number of thin-walled glass tubes of $\mathrm{I}-2 \mathrm{~mm}$. internal diameter are thoroughly cleaned and dried and cut into lengths of about ro inches. These are sucked full of the egg-white and kept in a horizontal position. Into a large evaporating dish or basin 5 -ro liters of water are introduced and heated to boiling. The vessel is then removed from the fire and stirred with a thermometer until the temperature sinks to exactly $85^{\circ} \mathrm{C}$. The tubes filled with egg-white are immediately introduced and left in the water until it has cooled. The tubes thus prepared are soft boiled, more easily digested than hard boiled tubes, and free from air bubbles. The ends are sealed by dipping in melted paraffin or sealing wax (preferably the latter), and the tubes can be kept thus for a long time. When ready for use mark with a file and break into pieces about $3 / 4$ inch long. After cutting, the tubes should be immediately introduced into the digestion mixture or may be kept a short time under water. Tubes whose ends are not squarely broken off must be rejected.

The digestibility of different egg-whites varies widely. Hence in making up a new set of tubes if we wish our results to be comparable these tubes must be standardized against those first prepared. This may be done by running simultaneous tests with tubes from the two series, using the same gastric juice and comparing the lengths of the columns digested in each case. Christiansen's method of preparing tubes of the same digestibility is to be preferred. He proceeds as in the original preparation of the tubes except that as the water cools from $90^{\circ}-80^{\circ} \mathrm{C}$. a single tube containing the new egg-white is dropped in at each degree change of temperature, that is at $90^{\circ}, 89^{\circ}$, etc. Pieces of each of these tubes as well as of the original standard tubes are then allowed to digest simultaneously in portions of the same gastric juice. One of these tubes should show a digestibility equal to that of the standard tubes. For example the tube coagulated at $88^{\circ} \mathrm{C}$. may show the proper digestibility. Then the new series of tubes should be made in the same manner as this one, that is introduced at $88^{\circ} \mathrm{C}$. The tubes thus prepared should be again checked up with the standard to see that no mistake has been made.

${ }^{1}$ Christiansen: Biochem. Zeil., 46, 257, I9I2. 
(2) Fuld and Levison's Method.-This test is founded upon the fact, shown by Osborne, that edestin when brought into solution in dilute acid will change in its solubility, due to the contact with the acid, and that a protean called edestan, which is insoluble in neutral fluid, will be formed. The procedure is as follows: Dilute the gastric juice under examination with 20 volumes of water and introduce gradually decreasing volumes of the diluted juice into a series ${ }^{1}$ of narrow test-tubes about I $\mathrm{cm}$. in diameter. The measurements of gastric juice may conveniently be made with a I c.c. pipette which is accurately graduated in $1 / 100$ c.c. Into the first tube in the series may be introduced I c.c. of gastric juice, and the tubes which follow in the series may receive volumes which differ, in each instance, from the volume introduced into the preceding tube by $1 / 100,1 / 50,1 / 20$, or $1 / 10$ of a cubic centimeter. Now rapidly introduce into each tube the same volume (e.g., 2 c.c.) of a I : 1000 solution of edestin ${ }^{2}$ and place the tubes at $40^{\circ} \mathrm{C}$. for one-half hour. At the end of this time stratify ammonium hydroxide upon the contents of each tube, ${ }^{3}$ place the tubes in position before a black background and examine them carefully. The ammonium hydroxide, by diffusing into the acid fluid, forms a neutral zone and in this zone will be precipitated any undigested edestan which is present. Select the tube in the series which contains the least amount of gastric juice and which exhibits no ring, signifying that the edestan has been completely digested, and calculate the peptic activity of the gastric juice under examination on the basis of the volume of gastric juice used in this particular tube.

Calculation.-Multiply the number of cubic centimeters of edestin solution used by the dilution to which the gastric juice was originally subjected and divide the volume of gastric juice necessary to completely digest the edestan by this product. For example, if 2 c.c. of the edestin solution was completely digested by 0.25 c.c. of a I : 20 gastric juice we would have the following expression: $0.25 \div(20 \times 2)$ or I : I60. This peptic activity may be expressed in several ways, e.g., (a) I : I60 pepsin; (b) I6o pepsin content; (c) I60 parts.

(3) Rose's Modification ${ }^{4}$ of the Jacoby-Solms Method. ${ }^{5}$-Dissolve 0.25 gram of the globulin of the ordinary garden pea, ${ }^{6}$ Pisum sativum, in roo c.c. of ro per cent

${ }^{1}$ The longer the series, the more accurate the deductions which may be drawn.

2 This edestin should be prepared in the usual way (see p. rog), and brought into solution in a dilute hydrochloric acid of approximately the same strength as that which occurs normally in the human stomach. This may be conveniently made by adding 30 c.c. of $\mathrm{N} / \mathrm{ro}$ hydrochloric acid to 70 c.c. of water. Ordinarily it should not take longer than one minute to introduce the edestin solution into the entire series of tubes. However, if the edestin is added to the tubes in the same order as the ammonium hydroxide is afterward stratified, no appreciable error is introduced.

${ }^{3}$ Making the stratification in the same order as the edestin solution was added.

Rose: Archives of Internal Medicine, 5, 459, I9ro.

5olms: Zeitschrift für klinische Medizin, 64, 159, 1907.

- The globulin may be prepared as follows: "The finely ground peas, freed as much as possible from the outer coating, are repeatedly extracted with large quantities of ro per cent sodium chloride solution, the extracts combined, strained through fine bolting-cloth, and allowed to stand over night in large cylinders to deposit insoluble matter. The supernatant fluid is siphoned off and saturated with ammonium sulphate. The precipitate of albumin and globulin is filtered off, suspended in a little water, and dialy'zed in running water for three day's, until the salt has been removed, and the albumins have been dissolved. The globulins are filtered off and washed two or three times to remove the last trace of albumins. To purify further, the precipitate is extracted with ro per cent sodium chloride solution, and filtered until perfectly clear. The resulting solution is neutralized to litmus paper by the cautious addition of dilute sodium hydroxide, and again dialyzed in running water for three days to remove the salts completely. The precipitated globulins are then filtered off and dried on a water-bath at $40^{\circ} \mathrm{C}$. During the entire process of separation the proteins should be preserved with a mixture of alcoholic thy mol and toluol." This dried globulin is used in the clinical procedure. 
sodium chloride solution, warming slightly if necessary. ${ }^{1}$ Filter and introduce I c.c. of the clear filtrate into each of a series of $\operatorname{six}^{2}$ test-tubes about $\mathrm{I} \mathrm{cm}$. in diameter. Introduce into each tube I c.c. of 0.6 per cent hydrochloric acid and permit a period of about five minutes to elapse for the development of the turbidity. Make a known volume of the gastric juice ( 5 -IO c.c. is sufficient) exactly neutral to litmus paper with dilute alkali; and record the volume of the alkali so used. If acid metaprotein precipitates, filter it off; if there is no precipitate proceed without filtration. Dilute the clear neutral solution with a known quantity of distilled water (usually 5 volumes) making proper allowance for the volume of alkali used in the neutralization. Boil 5-Io c.c. of the diluted juice, filter and add the following decreasing volumes (c.c.) to the series of six tubes: $1.0,0.9,0.7,0.5,0.2,0.0$. Make the measurements by means of a I c.c. pipette graduated in o.or c.c. Now rapidly introduce the unboiled, diluted juice in the following increasing volumes (c.c.) in order: 0.0, 0.1, 0.3, 0.5, 0.8, 1.0. Each tube now contains a total volume of 3 c.c. and a total acidity of 0.2 per cent hydrochloric acid. Shake each tube thoroughly and place them at $50-52^{\circ} \mathrm{C}$. for 15 minutes or at $35-36^{\circ} \mathrm{C}$. for one hour. Examine the series of tubes at the end of the digestion period and select that tube which contains the smallest quantity of gastric juice and which shows no turbidity. The volume of the juice used in this tube is taken as the basis for the calculation of the peptic activity.

Calculation.-The peptic activity is expressed in terms of I c.c. of the undiluted juice. For example, if it requires 0.5 c.c. of the diluted juice (five-fold dilution) to clear up the turbidity in $\mathrm{I}$ c.c. of the globulin solution in the proper experimental time interval ( 5 minutes or one hour according to temperature) the peptic activity would be expressed as follows:

$$
(I \div 0.5) \times_{5}=10 \text { (peptic activity) }
$$

According to this scale of pepsin units ro may be considered as "normal" peptic activity. These units are about $1 / 10$ as large as those expressed by the JacobySolms scale.

Inasmuch as it has been shown ${ }^{3}$ that blood serum contains an antipepsin it is advisable to test the gastric juice for blood before determining its proteolytic power.

(4) Given's Modification of Rose's Method. ${ }^{4-T h e}$ gastric contents are strained through cheese cloth. Two c.c. are measured by means of an Ostwald pipette into a 25 c.c. stoppered volumetric cylinder, and diluted to the mark with distilled water. Into each of seven small test-tubes ( $\mathrm{I} \times$ I0 $\mathrm{cm}$.) is measured, with an Ostwald pipette, I c.c. of a 0.25 per cent filtered pea globulin in Io per cent sodium chloride solution. To each tube is added I c.c. of 0.6 per cent hydrochloric acid, also by means of an Ostwald pipette. The tubes are allowed to stand about five minutes, until the maximum turbidity develops. To the first five, distilled water is added as follows: To the first, 0.9 c.c.; to the second, 0.8 c.c.; to the third, 0.7 c.c.; to the fourth, 0.6 c.c.; and to the fifth, 0.2 c.c.; to the sixth and seventh, none. Then there are rapidly added to each test-tube the following amounts of the diluted (I : I 2.5) gastric juice; to the first, O.I c.c.; to the second, 0.2 c.c.; to the third, 0.3 c.c.; to the fourth, 0.5 c.c.; to the fifth, 0.8 c.c.;

1 This solution may be preserved at least two months under toluene.

$2 \mathrm{~A}$ longer series of tubes may be used if desired. However, experience has shown that a series of six ordinarily affords sufficient range for all diagnostic purposes.

${ }^{3}$ Oguro: Biochemische Zeitschrift, 22, 266, rgog.

- Givens: Hygienic Lab. Bull. ror, p. 7 r, August, I9r 5. 
to the sixth, I.O c.c.; and to the seventh, I.O c.c. of the diluted juice boiled. These measurements can be accurately made with a I c.c. pipette graduated in o.or c.c. All tubes are then immersed for I 5 minutes in a water-bath at $50^{\circ}$ to $52^{\circ} \mathrm{C}$. At the end of this time, the tube is selected which is clear and contains the least amount of diluted gastric juice. Upon this basis, the peptic activity is calculated as the number of cubic centimeters of 0.25 per cent globulin digested by I c.c. of undiluted gastric juice. For example, if tube 2 containing 0.3 c.c. of a 12.5 times diluted juice be clear, then the result would be expressed:

$$
\text { Peptic activity }=(\mathrm{I} \div 0.3) \times \mathrm{I} 2.5=4 \mathrm{I} \cdot 2 \text {. }
$$

Ordinarily this scheme of seven tubes is used, though it is not a rule. If the free acidity be high, sometimes a dilution of $1 / 25$ is made. The number of tubes used will depend upon the accuracy desired.

(d) Determination of Tryptic Activity.-Trypsin is not a gastric enzyme but occurs in the pancreatic juice (see page I88). In case of regurgitation of intestinal contents through the pylorus trypsin would be passed into the stomach. This regurgitation is doubtless of frequent occurrence and may even be a normal mechanism by which gastric acidity is regulated (see page $\mathrm{I} 5 \mathrm{I}$ ). Trypsin is, therefore, generally present in the contents of the normal human stomach.

Spencer's Method. ${ }^{-1}$ (a) Prepare five reagent tubes, Nos. I, 2, 3, 4, and 5; more if desired.

To tubes I a nd 2 add 0.5 c.c. of gastric contents (filter if cloudy).

(b) To tubes $2,3,4$, and 5 add 0.5 c.c. of distilled water.

(c) From tube 2 remove 0.5 c.c. of its mixed contents and add to tube 3. Mix thoroughly and add 0.5 c.c. from tube 3 to tube 4 . Repeat for tube 5 .

We now have dilutions of gastric contents of $x, 1 / 2,1 / 4,1 / 8$, and $1 / 16$.

(d) To each tube add one drop of phenolphthalein solution (phenolphthalein I gram; alcohol (95 per cent) roo c.c.); then add drop by drop a 2 per cent sodium bicarbonate solution until a light pink color is produced.

(e) To tubes I, 2, 3, and 4 add 0.5 C.c. of casein solution. Tube 5 must receive I c.c. of casein solution, since it contains I c.c. of the diluted gastric contents. For the casein solution, dissolve 0.4 gram of casein in 40 c.c. of $\mathrm{N} /$ ro $\mathrm{NaOH}$. Add $\mathrm{r}_{30}$ c.c. of distilled water, then 30 c.c. of $\mathrm{N} /$ ro $\mathrm{HCl}$. This leaves the solution alkaline to the extent of ro c.c. of $\mathrm{N} / \mathrm{ro} \mathrm{NaOH}$, minus about 3 c.c. neutralized by the casein.

(f) Incubate for five hours at $40^{\circ} \mathrm{C}$.

(g) Precipitate the undigested casein by dropwise addition of a solution of the following composition: glacial acetic acid I c.c., alcohol (95 per cent) 50 c.c., distilled water 50 c.c. The tubes in which digestion has been complete remain clear; others become turbid.

(h) The tryptic values are expressed in terms of dilution. Thus, complete digestion in tube 3 (a dilution of $1 / 4$ ) shows four times the tryptic power of undiluted gastric juice; taken as a standard as $I$, therefore, its tryptic value is 4 .

${ }^{1}$ Elaborated by Dr. W. H. Spencer (Jour. Biol. Chcm., 21, I65, I9r5) in the author's laboratory for the specific purpose of determining trypsin in gastric juice. For other trypsin methods see Chapter X. 
(i) Controls of boiled gastric contents plus casein solution, and of distilled water plus casein solution, treated as above stated, must show no digestion, and become turbid on addition of the precipitating solution.

(e) Detection of Lactic Acid.-When the acidity of the stomach contents is reduced to a low value there may oceur considerable fermentation of carbohydrates which have been introduced into the stomach in the ingested food. This fermentation yields various organic acids among which lactic acid is particularly prominent. It is important, therefore, in case of low gastric acidity that the stomach contents be examined for lactic acid.

Tests. I. Ether-Ferric Chloride Test (Strauss).-A satisfactory deduction regarding the presence of lactic acid can only be made by removing the lactic acid from disturbing factors (e.g., hydrochloric acid, protein digestion products, etc.) present in the stomach contents. Lactic acid may be extracted from the stomach contents by ether. The following technic not only serves to detect lactic acid but also gives an approximate idea as to the amount of the acid present.

Procedure.-Introduce 5 c.c. of strained stomach contents into a small graduated separatory funnel, add 20 c.c. of ether and shake the mixture thoroughly. Permit the ether to separate, then allow all the fluid to run out of the separatory funnel except the upper 5 c.c. of ether. To this ether extract add 20 c.c. distilled water and 2 drops of a 10 per cent solution of ferric chloride and shake the mixture gently. A slight green color is obtained in the presence of 0.05 per cent lactic acid whereas 0.1 per cent lactic acid yields a very intense yellowish-green color.

2. Ferric Chloride Test (Kelling).-Fill a test-tube with water, add I-2 drops of a Io per cent solution of ferric chloride and mix thoroughly to form a liquid which is very faintly colored. Divide the solution into two parts and keep one part as a control. To the other part add a small amount of the strained gastric contents and to the control tube add a similar volume of water. Lactic acid is indicated by the immediate development of a distinct yellow color in the tube containing the gastric contents.

The color in this test is due to the formation of ferric lactate.

3. Uffelmann's Reaction.-To 5 c.c. of Uffelmann's reagent ${ }^{1}$ in a test-tube add an equal volume of strained gastric juice. A canary yellow or greenishyellow color develops if lactic acid be present to the extent of 0.01 per cent or over.

Other organic acid gives a similar reaction. Mineral acids such as hydrochloric acid discharge the blue coloration leaving a colorless solution. In other words, the color of the reagent is weakened in the presence of an acid reaction.

${ }^{1}$ Uffelmann's reagent is prepared by adding ferric chloride solution to a I per cent solution of carbolic acid until an amethyst-blue color is obtained, due in part to the formation of a ferric salt of carbolic acid and in part to the reduction of some of the iron. 
4. Hopkins' Thiophene Reaction.-Place about 5 c.c. of concentrated sulphuric acid in a test-tube and add I drop of a saturated solution of copper sulphate. ${ }^{1}$ Introduce a few drops of the gastric contents, shake the tube well, and immerse it in the boiling water of a beaker-water-bath for one or two minutes. Now remove the tube, cool it under running water, add 2-3 drops of a dilute alcoholic solution ${ }^{2}$ of thiophene, $\mathrm{C}_{4} \mathrm{H}_{4} \mathrm{~S}$, from a pipette, replace the tube in the beaker and carefully observe any color change which may occur. Lactic acid is indicated by the appearance of a bright cherry-red color which forms rapidly. This color may be made more or less permanent by cooling the tube as soon as the color is produced. Excess of thiophene produces a deep yellow or brown color with sulphuric acid. The test is not wholly specific though the author claims it to be more so than Uffelmann's reaction.

(f) Detection of Occult Blood. ${ }^{3}-$ I. Ortho-tolidin Test (Ruttan and Hardisty). ${ }^{4}$ - To I c.c. of a 4 per cent glacial acetic acid solution of 0 -tolidin ${ }^{5}$ in a test-tube add I c.c. of the gastric juice under examination and I c.c. of 3 per cent hydrogen peroxide. In the presence of blood a bluish color develops (sometimes rather slowly) and persists for some time (several hours in some instances).

This test is said to be as sensitive for the detection of occult blood in feces and stomach contents as is the benzidine reaction. It is also claimed to be more satisfactory for urine than any other blood test. The acetic acid solution may be kept for one month with no reduction in delicacy.

2. Benzidine Reaction.-This is one of the most delicate of the reactions for the detection of blood. Different benzidine preparations vary greatly in their sensitiveness, however. Inasmuch as benzidine solutions change readily upon contact with light it is essential that they be kept in a dark place. The test is performed as follows: To a saturated solution of benzidine in alcohol or glacial acetic acid add an equal volume of 3 per cent hydrogen peroxide and I c.c. of the gastric contents under examination. If the mixture is not already acid render it so with acetic acid, and note the appearance of a blue color. A control test should be made substituting water for the solution under examination.

The sensitiveness of the benzidine reaction is greater when applied to aqueous solutions than when applied to the urine. According to Ascarelli the benzidine reaction serves to detect blood when present in a dilution of $\mathrm{I}: 300,000$. (For further discussion of this test see chapter on Blood.)

(g) Detection of Bile in Stomach Contents.- If we accept Boldyreff's theory as to the automatic regulation of gastric acidity ${ }^{6}$ under normal

1 This is added to catalyze the oxidation which follows.

${ }^{2}$ About ro-20 drops in 100 c.c. af 95 per cent alcohol.

3 These tests may be made upon the strained stomach contents or upon the solid residue.

- Ruttan and Hardisty: Canadian Medicine Ass'n Journal, Nov., rgr 2; also Biochem. Bull., 2, 225, 1913.<smiles>CCCCCC(C)N</smiles>

${ }^{6}$ Boldyreff: Quarl. Jour. Exp. Med., S, I, 19I4. 
conditions by the regurgitation of alkaline material from the intestine, then the presence of bile in the gastric juice does not possess the clinical significance it has been accorded. However, if an ordinary Ewald meal be fed, and bile in any considerable quantity be found throughout the entire course of digestion it may indicate, pathologically, a stenosis below the level of the common bile duct. Frequently samples of gastric contents are encountered which are uncolored and which nevertheless contain bile. It is also true that bile may be adsorbed from stomach contents by mucus and food rests. The regulation technic for bile testing is often inadequate to demonstrate the presence of this

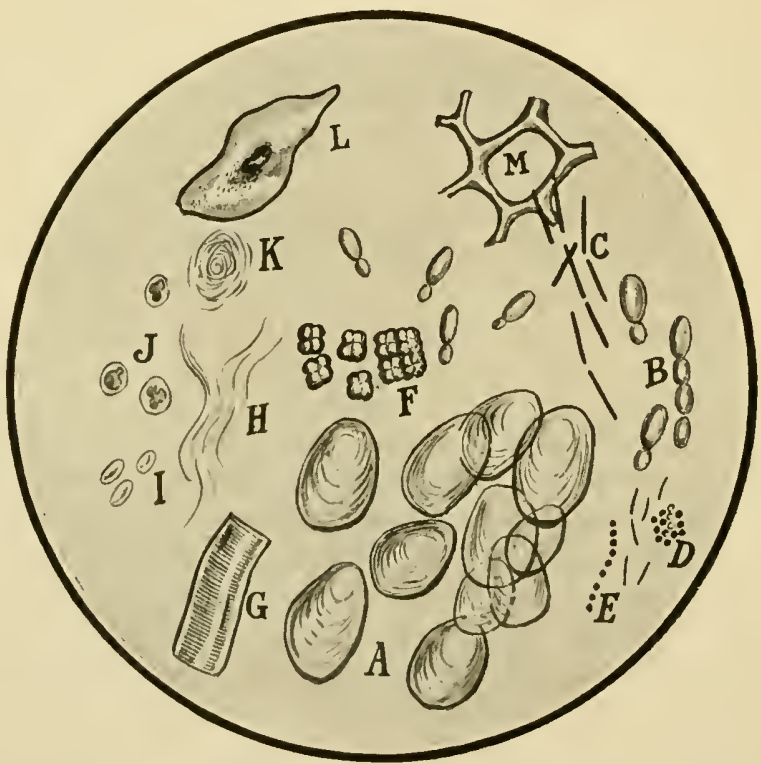

Fig. 49.-Microscopical Constituents of the Gastric Contents.

$A$, Starch cells; $B$, yeast cells; $C$, Oppler-Boas bacilli; $D$, staphylococci; $E$, streptococci; $F$, sarcinæ; $G$, muscle fibre; $H$, mucus; $I$, red blood cells; $J$, leucocytes; $K$, snail-like mucus formations; $L$, squamous epithelial cell; $I I$, cellulose.

fluid in gastric contents. The following procedure based upon the oxidation of the bilirubin with nitric acid forming green biliverdin is delicate and easy of application.

Procedure.-Saturate ro c.c. of the fluid portion of the stomach contents with powdered ammonium sulphate. This may be accomplished by shaking for one to two minutes. It generally requires about $I$ inch of powdered sulphate in the bottom of an ordinary test-tube to obtain full saturation. When the fluid is saturated add I-3 c.c. of acetone and thoroughly mix the contents of the tube by inverting the tube five or six times. (It is better not to shake.) Permit the tube to stand and allow the acetone to rise to the surface. This acetone con- 
tains the bile pigment if any is present in the stomach contents. Allow a drop of yellow nitric acid to flow down the side of the tube and note the green color in the acetone.

This green color is biliverdin which has been produced from the bilirubin by oxidation with nitric acid. If too much acid is added the green color will be oxidized to a purple or red. If the acetone does not rise to the surface promptly the liquid has not been completely saturated with ammonium sulphate.

If the stomach contents contains large amounts of bile as indicated by a deep green color $4-5$ drops of the fluid may be diluted with ro c.c. water and the above test applied.

(h) Microscopy of the Gastric Contents.-A microscopical examination of the gastric contents is a routine clinical procedure.

When an Ewald meal is given the starch granules in various stages of digestion are observed together with epithelia from the pharynx, esophagus, and occasionally the stomach. Gastric and salivary mucus are seen and readily recognized by their ropy appearance. Pathologically various bacteria are seen, sarcinæ, Oppler-Boas bacilli, streptococci, leptothrix, etc. Retained food from previous meals is readily recognized by its histological appearance; meat fibers, vegetable cells, and cellulose may all occur in pathological retention. In certain pathological processes such as ulcer and cancer, red blood cells, pus, and even the cancer cells themselves may be found. For illustrations of the microscopical constituents of gastric contents, see Fig. 49.

Procedure.-Examine a drop of the original (mixed) stomach contents unstained under the low and high powers of the microscope. Compare your findings with the microscopical views shown in Fig. 49.

Wolff Technic for the Protein Concentration of the Gastric Contents. ${ }^{\mathrm{I}}$ Owing to the diagnostic importance of the protein concentration of the gastric secretion, a short note of this test is given here. Under normal conditions the protein concentration follows that of acidity rather closely. In certain cases, however, such as carcinoma (Fig. 47), there is an actual increase in the protein concentration of the gastric juice out of all proportion to the acidity. The test may be made as follows: The regular Ewald test meal is fed and specimeus of the gastric contents are obtained at $x 5$-minute intervals by means of the Rehfuss tube. One c.c. of the filtered juice is then diluted with 9 c.c. of water representing a dilution of $x: 10 ; 5$ c.c. of this mixture is again added to 5 c.c. of water and a dilution of $x: 20$ obtained; this is again repeated using 5 c.c. of the mixturc last obtained and 5 c.c. of distilled water and the dilutions are kept up until a series is obtained representing I: $10,1: 20.1: 40, x:$ o, $x: 160, x: 320$, and if necessary $1: 640$ or more. They are

1 Wolff: Magen- und Darmkrankh., Berlin, 1912, p. 217; also Berl.klin. W"och., May 29, I9I I, and March I8, IgI 2 .

Rolph: Med. Rec., igr3, p. 848 .

Clarke and Rehfuss: Jour. Am. Med. Ass'n, 64, I737, I9I5. 
then stratified with approximately I c.c. of Wolff's reagent, ${ }^{1}$ care being taken that the liquids do not mix. The tubes should be read immediately against a dark background and the tube giving a protein ring at the greatest dilution of gastric juice recorded. A glance at Fig. 47 will show a pronounced case of gastric carcinoma. With normal acid figures the protein concentration evolves proportionally to the acidity. A case of achylia is shown in Fig. 48.

\section{Töpfer's Method of Gastric Analysis}

This method is much less elaborate than many others but is sufficiently accurate for ordinary clinical purposes. The method embraces the volumetric determination of (1) total acidity, (2) free acidity (organic and inorganic), ${ }^{2}$ and (3) free hydrochloric acid, and the subsequent calculation of (4) combined acidity and (5) acidity due to organic acids and acid salts, from the data thus obtained.

Procedure.-Feed the Ewald test meal as directed on page I6r. At the end of one hour remove the entire stomach contents and analyze as directed below. This method of procedure is less accurate than the Fractional Method (see page $\mathrm{r}_{4} 8$ ). Measure the volume of the gastric contents, strain it through cheese cloth and introduce ro c.c. of the strained material into each of three small beakers or porcelain dishes $^{3}$ Label the vessels $A, B$, and $C$, respectively, and proceed with the analysis according to the directions given below. The volume of fluid present in the stomach one hour after an Ewald meal varies under normal conditions between 50 and Ioo c.c. In cases of hypersecretion or defective motility $200-300$ c.c. may be found. Very excessive volumes, e.g., 500-3000 c.c., are indicative of dilatation of the stomach and suggest pyloric stenosis, either benign or malignant.

I. Total Acidity. ${ }^{4}$ - Add 3 drops of a $\mathrm{I}$ per cent alcoholic solution of phenolphthalein ${ }^{5}$ to the contents of vessel $A$ and titrate with $\mathrm{N} /$ ro sodium hydroxide solution until a faint pink color is produced and persists for almost two minutes. Take the burette reading and calculate the total acidity.

Calculation.-The total acidity may be expressed in the following ways:

r. The number of cubic centimeters of $\mathrm{N} /$ Io sodium hydroxide solution necessary to neutralize 100 c.c. of gastric juice.

2. The weight (in grams) of sodium hydroxide necessary to neutralize roo c.c. of gastric juice.

3. The weight (in grams) of hydrochloric acid which the total acidity of 100 c.c. of gastric juice represents, i.e., percentage of hydrochloric acid.

The forms of expression most frequently employed are I and 3 , preference being given to the former, particularly in clinical work.

In making the calculation note the number of cubic centimeters of $\mathrm{N} /$ ro sodium hydroxide required to neutralize ro c.c. of the gastric juice and multiply it by ro to obtain the number of cubic centimeters necessary to neutralize roo c.c. of the fluid.

${ }^{1}$ Phosphotungstic acid........................... 0.3 c.c.

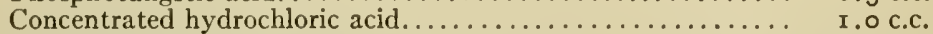

Alcohol 95 per cent.............................. 20.0 c.c.

Distilled water sufficient to make................... I00.0 c.c.

${ }^{2}$ For a discussion of combined acid see chapter on Gastric Digestion.

${ }^{3}$ If sufficient gastric juice is not available it may be diluted with water or a smaller amount, e.g., 5 c.c., taken for each determination.

${ }^{4}$ This includes free and combined acid and acid salts.

${ }^{5}$ One gram of phenolphthalein dissolved in roo c.c. of 95 per cent alcohol. 
If it is desired to express the acidity of 100 c.c. of gastric juice in terms of hydro. chloric acid, by weight, multiply the value just obtained by $0.0036_{5}$. 1

2. Free Acidity (Organic and Inorganic).-Add 3 drops of sodium alizarin sulphonate solution ${ }^{2}$ to the contents of vessel $B$ and titrate with $N$ / ro sodium hydroxide solution until a violet color is produced. In this titration the red color, which appears after the tinge of yellow due to the addition of the indicator has disappeared, must be entirely replaced by a distinct violet color. Take the burette reading and calculate the free acidity due to organic and inorganic acids.

Calculation.-Since the indicator used reacts to both organic and inorganic acids, the number of cubic centimeters of $\mathrm{N} /$ ro sodium hydroxide used indicates the free acidity of Io c.c. of gastric juice. The data for Ioo c.c. of gastric juice may be calculated according to the directions given under Total Acidity, page $\mathrm{r}_{74}$.

3. Free Hydrochloric Acid. ${ }^{3}$-Add 4 drops of di-methyl-amino-azobenzene (Töpfer's reagent) solution ${ }^{4}$ to the contents of the vessel $C$ and titrate with $\mathrm{N} /$ ro sodium hydroxide solution until the initial red color is replaced by orange yellow. Take the burette reading and calculate the free acidity.

Calculation.-The indicator used reacts only to free hydrochloric acid, hence the number of cubic centimeters of $\mathrm{N} /$ ro sodium hydroxide used indicates the volume necessary to neutralize the free hydrochloric acid of Io c.c. of gastric juice. To determine the data for roo c.c. of gastric juice proceed according to the directions given under Total Acidity, page I 74 .

4. Combined Acidity.- This value may be obtained by subtracting the number of cubic centimeters of $\mathrm{N}$ / Io sodium hydroxide used in neutralizing the contents of vessel $B$ from the number of cubic centimeters of $N /$ ro sodium hydroxide used in neutralizing $A$. The data for Ioo c.c. of gastric juice may be calculated according to directions given under Total Acidity, page $\mathbf{1} 74$.

5. Acidity Due to Organic Acids and Acid Salts. - This value may be conveniently calculated by subtracting the number of cubic centimeters of $\mathrm{N} /$ ro sodium hydroxide used in neutralizing the contents of vessel $C$ from the number of cubic centimeters of $\mathrm{N} /$ Io sodium hydroxide solution used in neutralizing the contents of vessel $B$. The remainder indicates the number of cubic centimeters of $\mathrm{N} /$ ro sodium hydroxide solution necessary to neutralize the acidity due to organic acids and acid salts present in ro c.c. of gastric juice. The data for roo c.c. of gastric juice may be calculated according to directions given under Total Acidity, page I 74 .

${ }^{1}$ One c.c. of $\mathrm{N} /$ Io hydrochloric acid contains 0.00365 gram of hydrochloric acid.

${ }^{2}$ One gram of sodium alizarin sulphonate dissolved in roo c.c. of water.

${ }^{3}$ Hydrochloric acid not combined with protein material.

${ }^{4}$ One-half gram dissolved in roo c.c. of 95 per cent alcohol.

${ }^{5}$ If the orange yellow color appears as soon as the indicator is added it denotes the $a b$ sence of free acid. 


\section{CHAPTER IX}

\section{FATS}

Fats occur very widely distributed in the plant and animal kingdoms, and constitute the third general class of food-stuffs. In plant organisms they are to be found in the seeds, roots, and fruit while each individual tissue and organ of an animal organism contains more or less of the substance. In the animal organism fats are especially abundant in the bone marrow and adipose tissue. They contain the same elements as the carbohydrates, i.e., carbon, hydrogen, and oxygen, but the oxygen is present in smaller percentage than in the carbohydrates

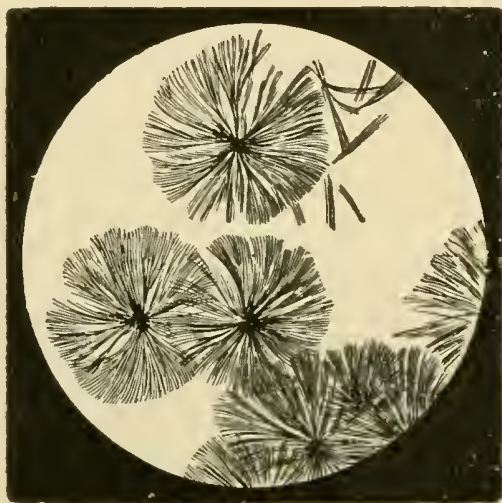

FIG. 50.-BEEF FAT. (Long.)

and the hydrogen and oxygen are not present in the proportion to form water.

Chemically considered the fats are esters ${ }^{1}$ of the tri-atomic alcohol, glycerol, and the mono-basic fatty acids. In the formation of these fats three molecules of water result. This water arises by the replacement of the H's of the carboxyl groups of the three fatty acid molecules by the glycerol radical, thus yielding the following type of formula. In this case the combination is with palmitic acid $\left(\mathrm{C}_{15} \mathrm{H}_{31} \mathrm{COOH}\right)$.

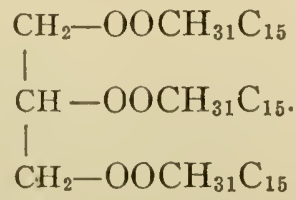

${ }^{1}$ An ester is an oxyacid, one of whose acid hydrogens is replaced by an organic radical. 
The three fatty acid radicals entering into the structure of a neutral fat may be the radicals of the same fatty acid or they may consist of the radicals of three different fatty acids.

By hydrolysis of a neutral fat, i.e., by the addition to the molecule of those elements which are eliminated in the formation of the fat from glycerol and fatty acid, it may be resolved into its component parts, i.e., glycerol and fatty acid. In the case of palmitin the following would be the reaction:

$$
\mathrm{C}_{3} \mathrm{H}_{5}\left(\underset{\text { Palmitin. }}{\mathrm{O} \cdot \mathrm{C}_{15} \mathrm{H}_{31} \mathrm{CO}}\right)_{3}+{ }_{3} \mathrm{H}_{2} \mathrm{O} \rightarrow \underset{\text { Glycerol. }}{\mathrm{C}_{3} \mathrm{H}_{5}}(\mathrm{OH})_{3}+{ }_{3}\left(\underset{\text { Palmitic acid. }}{\mathrm{C}_{15} \mathrm{H}_{31} \mathrm{COOH}}\right) .
$$

This process is called saponification and may be produced by boiling with alkalis; by the action of steam under pressure; by long-continued contact with air and light; by the action of certain bacteria and by fat-splitting enzymes or lipases, e.g., pancreatic lipase (see page I88). The cells forming the walls of the intestines evidently possess the peculiar property of synthesizing the glycerol and fatty acid thus formed so that after absorption these bodies appear in the blood not in their individual form but as neutral fats.

The principal animal fats with which we have to deal are stearin, palmitin, olein, and butyrin. Such less important forms as laurin and myristin may occur abundantly in plant organisms. The older system of nomenclature for these fats was to apply the prefix "tri" in each case (e.g., tri-palmitin) since three fatty acid radicals are contained in the neutral fat molecule.

The fatty acids corresponding to the above-mentioned animal fats are stearic, $\mathrm{CH}_{3}\left(\mathrm{CH}_{2}\right)_{16} \mathrm{COOH}$; palmitic, $\mathrm{CH}_{3}\left(\mathrm{CH}_{2}\right)_{14} \mathrm{COOH}$; oleic, $\mathrm{CH}_{3}\left(\mathrm{CH}_{2}\right)_{7} \mathrm{CH}=\mathrm{CH}\left(\mathrm{CH}_{2}\right)_{7} \mathrm{COOH}$; and butyric, $\mathrm{CH}_{3}\left(\mathrm{CH}_{2}\right)_{2} \mathrm{COOH}$. Stearic, palmitic and butyric acids are saturated fatty acids, whereas oleic acid belongs to the class of unsaturated acids. Linoleic acid is also unsaturated. Upon the presence of these unsaturated fatty acids depends the property which certain fats possess of absorbing or combining with iodine. The determination of this so-called "iodine absorption number" is important in the differentiation of fats and oils. Fats containing the unsaturated acids oleic and linoleic may be transformed by "hydrogenation" into the fats containing the corresponding saturated acid (stearic). The oleic acid is changed thus:

$$
\underset{\text { Oleic acid. }}{\mathrm{C}_{18} \mathrm{H}_{34} \mathrm{O}_{2}}+2 \mathrm{H} \rightarrow \underset{\text { Stearic acid. }}{\mathrm{C}_{18} \mathrm{H}_{36} \mathrm{O}_{2}} \text {. }
$$

Fats occur ordinarily as mixtures of several individual fats. For example, the fat found in animal tissues is a mixture of olein, palmitin

${ }^{1}$ Addition of hydrogen to the molecule, producing a "hydrogenated fat." 
and stearin, the percentage of any one of these fats present depending upon the particular species of animal from whose tissue the fat was derived. Thus the ordinary mutton fat contains more stearin and less olein than the pork fat. Human fat contains from 67 per cent to 85 per cent of olein and, according to Benedict and Osterberg, upon analysis yields 76.08 per cent of carbon and II. 78 per cent of hydrogen. Butter consists in large part of olein and palmitin. Stearin, butyrin, caproin and traces of other fats are also present.

Pure neutral fats are odorless, tasteless, and generally colorless. They are insoluble in the ordinary protein solvents such as water, salt solutions, and dilute acids and alkalis, but are very readily soluble in ether, benzene, chloroform, and boiling alcohol. The neutral fats are non-volatile substances possessing a neutral reaction. If allowed to remain in contact with the air for a sufficient length of time they become yellow in color, assume an acid reaction and are said to be rancid. The neutral fats may be crystallized, some of them with great facility. The crystalline forms of some of the more common fats are reproduced in Figs. 50, 5I and 52 on pages I76, I79 and I8r. Each individual fat possesses a specific melting- or boiling-point (according to whether the body is solid or fluid in character), and this property of melting or boiling at a definite temperature may be used as a means of differentiation in the same way as the coagulation temperature (see page 105) is used for the differentiation for coagulable proteins. When shaken with water, or a solution of albumin, soap, or acacia, the liquid fats are finely divided and assume a condition known as an emulsion. The emulsion with water is transitory, while the emulsions with soap, acacia, or albumin are permanent.

The fat ingested continues essentially unaltered until it reaches the intestine where it is acted upon by pancreatic lipase (steapsin), the fatsplitting enzyme of the pancreatic juice (see page i88), and glycerol and fatty acid are formed. The glycerol is absorbed directly. The fatty acid thus formed unites with the alkalis of the pancreatic juice and forms soluble soaps. These soaps are readily absorbed. That bile is of assistance in the absorption of fat is indicated by the increase of fat in the feces when for any reason bile does not pass into the intestine. Bloor ${ }^{1}$ claims that neither petroleum hydrocarbons nor nonsaponifiable esters, e.g., wool fat (lanolin), are absorbed. He believes that saponification is a necessary preliminary to absorption.

The fat distributed throughout the animal body is formed partly from the ingested fat and partly from carbohydrates and the "carbon

${ }^{1}$ Bloor: Jour. Biol. Chem., 15, 105, 1913. 
moiety" of protein material. The formation of adipocere ${ }^{1}$ and the occurrence of fatty degeneration are sometimes given as proofs of the formation of fat from protein. This is questioned by many investigators. Rather more satisfactory and direct proof of the formation of fat from protein material has been obtained by Hofmann in experimentation with fly-maggots. The normal content of fat in a number of maggots was determined and later the fat content of others which had developed in blood ( 84 per cent of the solid matter of blood plasma is protein material) was determined. The fat content was found to have increased 700 to I roo per cent as a result of the diet of blood proteins.

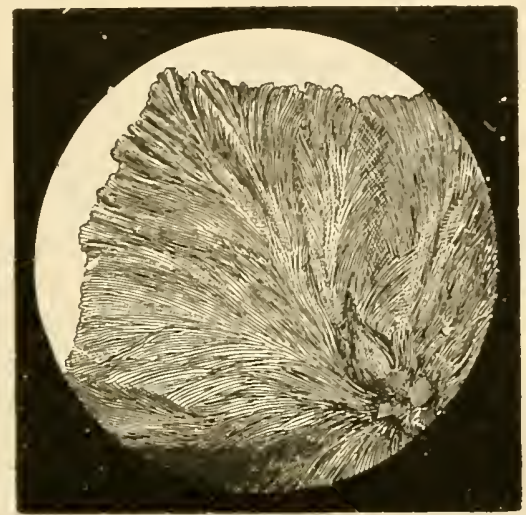

Fig. 51.-Mituton Fat. (Long.)

The celebrated experiments of Pettenkofer and Voit, however, have furnished what is, perhaps, the most substantial positive evidence of the formation of fat from protein. These investigators fed dogs large amounts of lean meat, daily, and through examination of urine, feces and expired air were enabled to account for only part of the ingested carbon, although obtaining a satisfactory nitrogen balance. The discrepancy in the carbon balance was explained upon the theory that the protein of the ingested meat had been split into a nitrogenous and a non-nitrogenous portion in the organism, and that the non-nitrogenous portion, the so-called "carbon moiety" of the protein, had been subsequently transformed into fat and deposited as such in the tissues of the organism. Later evidence in favor of the formation of fat from protein has been furnished by the experiments of Weinland. This investigator worked with the larva of Calliphor $a,{ }^{2}$ these larva being rubbed up in a mortar ${ }^{1}$ with Witte's peptone and water to form a homogeneous

${ }^{1}$ A very complete analysis of adipocere was reported by Ruttan and Marshall before the Society of Biological Chemists, Boston, Dec. 27, I9I 5.

${ }^{2}$ The ordinary "blow-fly." 
mixture. After placing these mixtures at $38^{\circ} \mathrm{C}$. for 24 hours the fat content was found to have increased, as much as 140 per cent in some instances. The active agency in this transformation of fat is the larval tissue, since the tissues of both the dead and living larvæ possess the property. Data are given from control tests which show that the action of bacteria in this transformation of protein was excluded.

Some investigators are not inclined to accept any data regarding the formation of fat from protein as conclusive.

\section{EXPERIMENTS ON FATS}

I. Solubility.-Test the solubility of olive oil in water, dilute acid and alkali and in cold alcohol, hot alcohol, chloroform, ether, and carbon tetrachloride.

2. Formation of a Transparent Spot on Paper.-Place a drop of olive oil upon a piece of ordinary writing paper. Note the transparent appearance of the paper at the point of contact with the fat.

3. Reaction.- Try the reaction of fresh olive oil to litmus, Congo red and phenolphthalein. Repeat the test with rancid olive oil. ${ }^{2}$ What is the reaction of a fresh fat and how does this reaction change upon allowing the fat to stand for some time?

4. Formation of Acrolein.-To a little olive oil in a mortar add some dry potassium bisulphate, $\mathrm{KHSO}_{4}$, and rub up thoroughly. Transfer to a dry test-tube and cautiously heat. Note the irritating odor of acrolein. The glycerol of the fat has been dehydrolyzed and acrylic aldehyde or acrolein has been produced. This is the reaction which takes place:

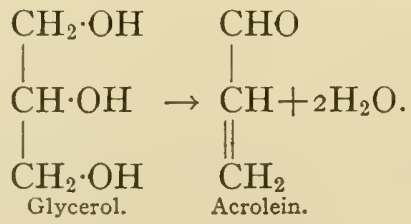

5. Emulsification.-(a) Shake up a drop of neutral s olive oil with a little water in a test-tube. The fat becomes finely divided, forming an emulsion. This is not a permanent emulsion since the fat separates and rises to the top upon standing.

(b) To 5 c.c. of water in a test-tube add 2 or 3 drops of 0.5 per cent $\mathrm{Na}_{2} \mathrm{CO}_{3}$. Introduce into this faintly alkaline solution a drop of neutral olive oil and shake. The emulsion while not permanent is not so transitory as in the caes of water free from sodium carbonate.

(c) Repeat (b) using rancid olive oil. What sort of an emulsion do you get

${ }^{1}$ Intact larvæ were used in some experiments.

2 To prepare rancid olive oil add 5 drops of oleic acid to ro c.c. of olive oil.

${ }^{3}$ Neutral olive oil may be prepared by shaking ordinary olive oil with a ro per cent solution of sodium carbonate. This mixture should then be extracted with ether and the ether removed by evaporation. The residue is neutral olive oil. 
and why? It is impossible to emulsify a highly rancid fat due to the excessive formation of rather insoluble soaps about the oil drops.

(d) Shake a drop of neutral olive oil with dilute albumin solution. What is the nature of this emulsion? Examine it under the microscope.

6. Fat Crystals. -Dissolve a small piece of lard in ether in a test-tube, add an equal volume of alcohol and allow the alcohol-ether mixture to evaporate spontaneously. Examine the crystals under the microscope and compare them with those reproduced in Figs. 50, 51 , and 52 , on pages 176,179 and 181 .

7. Saponification of Bayberry Tallow. ${ }^{1}$-Fill a large casserole two-thirds full of water rendered strongly alkaline with solid potassium hydroxide (a stick one inch in length). Add about Io grams of bayberry tallow and boil, keeping the volume constant by adding water as needed. When saponification is com-

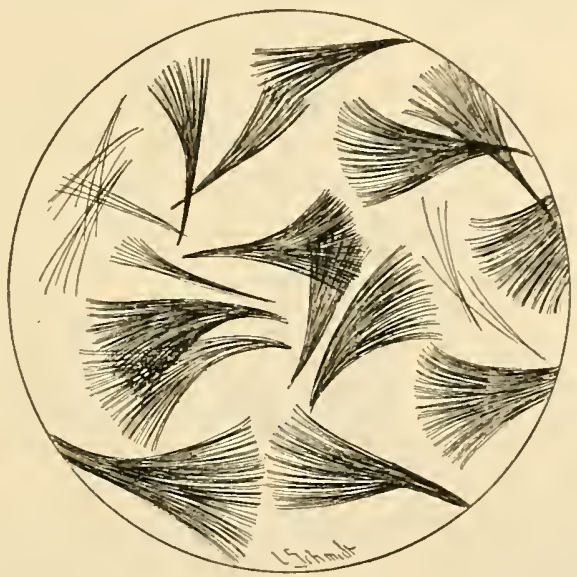

FIG. 52.-PORE FAT.

plete $^{2}$ remove 25 c.c. of the soap solution for use in Experiment 8 and add concentrated hydrochloric acid slowly to the remainder until no further precipitate is produced. ${ }^{3}$ Cool the solution and the precipitate of free fatty acid will rise to the surface and form a cake. In this instance the fatty acid is principally palmitic acid. Remove the cake, break it into small pieces, wash it with water by decantation and transfer to a small beaker by means of 95 per cent alcohol. Heat on a water-bath until the palmitic acid is dissolved, then filter through a dry filter paper and allow the filtrate to cool slowly in order to obtain satisfactory crystals. Write the reactions which have taken place in this experiment.

When the palmitic acid has completely crystallized filter off the alcohol, dry the crystals between filter papers and try the tests given in Experiment 10, p. I 82.

8. Salting-out Experiments.-To 25 c.c. of soap solution, prepared as described above, add solid sodium chloride to the point of saturation, with continual stirring. A menstrum is thus formed in which the soap is insoluble. This

${ }^{1}$ Bayberry tallow is derived from the fatty covering of the berries of the wax myrlc. It is therefore frequently called "myrtle wax" or "bayberry wax."

2 Place 2 or 3 drops in a test-tube full of water. If saponification is complete the products will remain in solution and no oil will separate.

${ }^{3}$ Under some conditions a purer product is obtained if the soap solution is cooled before precipitating the fatty acid. 
salting-out process is entirely analogous to the salting-out of proteins (see page I02).

9. Formation of Insoluble Soaps.-Introduce 5 c.c. of soap solution into each of two test-tubes. To the contents of one tube add a small amount of a solution of calcium chloride and to the contents of the other tube add a small amount of a solution of magnesium sulphate. Note the formation of insoluble soaps of calcium and magnesium.

I0. Palmitic Acid.--(a) Examine the crystals under the microscope and compare them with those shown in Fig. 53, below.

(b) Solubility.-Try the solubility of palmitic acid in the same solvents as used on fats (see page 180 ).

(c) Melting-point.-Determine the melting-point of palmitic acid by one of the methods given on page 183 .

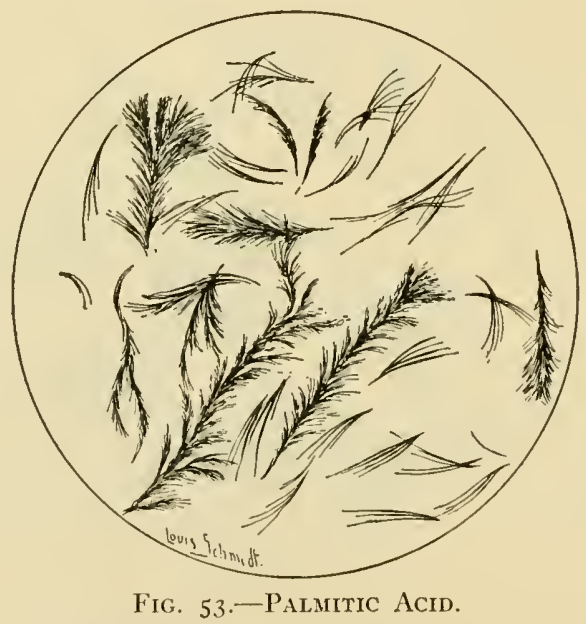

(d) Formation of Transparent Spot on Paper.-Melt a little of the fatty acid and allow a drop to fall upon a piece of ordinary writing paper. How does this compare with the action of a fat under similar circumstances?

(e) Acrolein Test.-Apply the test as given under 4, page 180. Explain the result.

(f) Iodine Absorption Test.-For directions see Experiment 13.

I I. Saponification of Lard.-To 25 grams of lard in a flask add 75 c.c. of alcoholic-potash solution and warm upon a water-bath until saponification is complete. (This point is indicated by the complete solubility of a drop of the solution when allowed to fall into a little water.) Now transfer the solution from the flask to an evaporating dish containing about 100 c.c. of water and heat on a water-bath until all the alcohol has been driven off. Precipitate the fatty acid with hydrochloric acid and cool the solution. Remove the fatty acid which rises to the surface, ${ }^{1}$ neutralize the solution with sodium carbonate and evaporate to dryness. Extract the residue with alcohol, remove the alcohol by evaporation upon a water-bath and on the residue of glycerol thus obtained make the tests as given below.

12. Glycerol. (a) Taste.-What is the taste of glycerol?

${ }^{1}$ After drying the acid make an iodine absorption test as described in Experiment $\times 3$. 
(b) Solubility.--Try the solubility of glycerol in water, alcohol and ether.

(c) Hypochlorite-Orcinol Reaction. '-This is based on the oxidation of glycerol to the corresponding aldose sugar glycerose and the detection of the latter by means of orcinol. Homologues of glycerol as well as the corresponding acids and certain sugars as glucose and mannose give the reaction. The first named occur seldom while the latter may be removed with baryta.

Two to 3 c.c. of a $I$ per cent or $1 / 10$ per cent solution of glycerol in water is treated with exactly 3 drops ( $=0.12$ c.c.) normal $\mathrm{NaOCl}^{2}$ and boiled for a minute. To the liquid while still hot add 3 drops of hydrochloric acid (sp. gr. I.I24) and boil 30-60 seconds to drive off chlorine, a colorless solution being obtained. Then add an equal volume of fuming hydrochloric acid and a small knife-point of orcinol. On boiling the mixture becomes a beautiful violet or green blue. The precipitate formed is soluble in amyl alcohol and may be examined spectroscopically.

(d) Acrolein Test.-Repeat the test as given under 4, page I80.

(e) Borax Fusion Test.-Fuse a little glycerol on a platinum wire with some powdered borax and note the characteristic green flame. This color is due to the glycerol ester of boric acid.

(f) Fehling's Test.-How does this result compare with the results on the sugars?

(g) Solution of $\mathrm{Cu}(\mathrm{OH})_{2}$. Form a little cupric hydroxide by mixing copper sulphate and potassium hydroxide. Add a little glycerol to this suspended precipitate and note what occurs.

13. Iodine Absorption Test.-Dissolve a small amount of an unsaturated organic acid, e.g., oleic acid, in chloroform. Add 2-3 drops of Huibl's iodine solution ${ }^{3}$ and shake. The solution will be decolorized if unsaturated acids are present. This is due to the absorption of the iodine. The test should be controlled by shaking chloroform and iodine solution to which no acid has been added.

14. Melting-point of Fat.-First Method.-

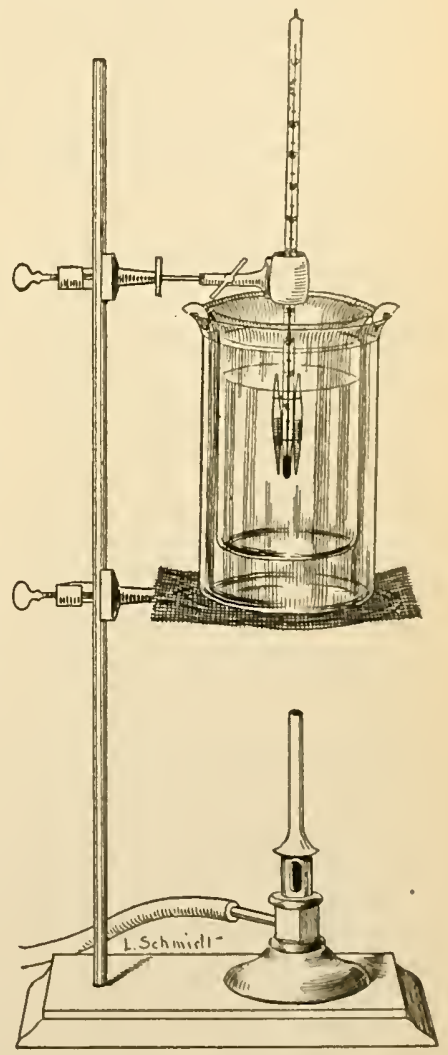

Fig. 54.-MELTING-PONT APPARATUS.

Insert one of the melting-point tubes, furnished by the instructor, into the liquid fat and draw up the fat until the bulb of the tube is about one-half full of the material. Then fuse one end of the tube in the flame of a Bunsen burner

1 Mandel and Neuberg: Bioch. Zcil., 7 I, 214, I015.

2 Made according to Raschig: Ber., 40, 4586 , I907.

${ }^{3}$ Prepared by dissolving 26 grams of iodine and 30 grams of mercuric chloride in one liter of 95 per cent alcohol. 
and fasten the tube to a thermometer by means of a rubber band in such a manner that the bottom of the fat column is on a level with the bulb of the thermometer (Fig. 54, p. 183). Fill a beaker of medium size about two-thirds full of water and place it within a second larger beaker which also contains water, the two vessels being separated by pieces of cork. Immerse the bulb of the thermometer and the attached tube in such a way that the bulb is about midway between the upper and the lower surfaces of the water of the inner beaker. The upper end of the tube being open it must extend above the surface of the surrounding water. Apply gentle heat, stir the water, and note the temperature at which the fat first begins to melt. This point is indicated by the initial transparency. For ordinary fats, raise the temperature very cautiously from $30^{\circ} \mathrm{C}$. To determine the congealing-point remove the flame and note the temperature at which the fat begins to solidify. Record the melting- and congealing-points of the various fats submitted by the instructor.

Second Method.-Fill a small evaporating dish about one-half full of mercury and place it on a water-bath. Put a small drop of the fat under examination on an ordinary cover-glass and place this upon the surface of the mercury. Raise the temperature of the water-bath slowly and by means of a thermometer whose bulb is immersed in the mercury, note the melting-point of the fat. Determine the congealing-point by removing the flame and leaving the fat drop and cover-glass in position upon the mercury. How do the melting-points as determined by this method compare with those as determined by the first method? Which method is the more accurate, and why? 


\section{PANCREATIC DIGESTION}

As soon as the food mixture leaves the stomach it comes into intimate contact with the bile and the pancreatic juice. Since these fluids are alkaline in reaction (see Bile, page 202) there can obviously be no further peptic activity after they have become intimately mixed with the chyme and have neutralized the acidity previously imparted to it by the hydrochloric acid of the gastric juice. The pancreatic juice reaches the intestine through the duct of Wirsung which opens into the intestine near the pylorus.

Normally the secretion of pancreatic juice is brought about by the stimulation produced by the acid chyme as it enters the duodenum. Therefore, any factor which produces an increased flow of gastric juice such, for example, as water ${ }^{2}$ will cause a stimulation of the pancreatic secretion. The secretion of pancreatic juice is probably not due to a nervous reflex as was believed by Pawlow but rather, as Bayliss and Starling have shown, is dependent upon the presence, in the epithelial cells of the duodenum and jejunum of a body known as prosecretin. This body is changed into secretin through the hydrolytic action of the acid present in the chyme. The secretin is then absorbed by the blood, passes to the pancreas and stimulates the pancreatic cells, causing a flow of pancreatic juice. The quantity of juice secreted under these conditions is proportional to the amount of secretin present. The activity of secretin solutions is not diminished by boiling, hence the body does not react like an enzyme. Further study of the body may show it to be a definite chemical individual of relatively low molecular weight. It has not been possible thus far to obtain secretin from any tissues except the mucous membrane of the duodenum and jejunum.

This secretin mentioned above belongs to the class of substances called hormones or chemical messengers. These hormones play a very important part in the coordination of the activities of certain functions and glands. Other important hormones are those elaborated by the thyroids, the adrenals, the pituitary body (hypophysis), the embryo and

${ }_{1}$ Under this head we will consider only such digestive processes as are brought about by enzymes originating in the pancreas. In the following chapter on Intestinal Digestion will be found a consideration of such enzymes as have a true inlestinal origin.

'See chapter on Gastric Digestion. 
the reproductive glands. It is claimed by some that all active organs of the body produce hormones.

The juice as obtained from a permanent fistula differs greatly in its properties from the juice as obtained from a temporary fistula, and neither form of fluid possesses the properties of the normal fluid. Pancreatic juice collected by Glaessner from a natural fistula has been found to be a colorless, clear, strongly alkaline fluid which foams readily. It is further characterized by containing albumin, globulin, proteose, and peptone; nucleoprotein is also present in traces. ${ }^{1}$ The average daily secretion of pancreatic juice is 650 c.c. and its specific gravity is I.oo8. The fluid contains I.3 per cent of solid matter and the freezingpoint is $-0.47^{\circ} \mathrm{C}$. The normal pancreatic secretion contains at least four distinct enzymes. They are trypsin, a proteolytic enzyme; pancreatic amylase (amylopsin), an amylolytic enzyme; pancreatic lipase (steapsin), a fat-splitting enzyme; and pancreatic rennin, a milk-coagulating enzyme.

The most important of the four enzymes of the pancreatic juice is the proteolytic enzyme trypsin. This enzyme resembles pepsin in so far as each has the power of breaking down protein material, but the trypsin has much greater digestive power and is able to cause a more complete decomposition of the complex protein molecule. In the process of normal digestion the protein constituents of the diet are for the most part transformed into proteoses (albumoses) and peptones before coming in contact with the enzyme trypsin. This is not absolutely essential, however, since trypsin possesses digestive activity suffcient to transform unaltered native proteins and to produce from their complex molecules comparatively simple fragments. Among the products of tryptic digestion are proteoses, peptones, peptides, leucine, tyrosine, aspartic acid, glutamic acid, alanine, phenylalanine, glycocoll, cystine, serine, valine, proline, oxyproline, isoleucine, arginine, lysine, histidine, and tryptophane. (The crystalline forms of many of these products are reproduced in Chapter IV.) Trýpsin does not occur preformed in the gland, but exists there as a,zymogen called trypsinogen which bears the same relation to trypsin that pepsinogen does to pepsin. Trypsin has never been obtained in a pure form and therefore very little can be stated definitely as to its nature. The enzyme is the most active in alkaline solution but is also active in neutral or slightly acid solutions. Trypsin is destroyed by mineral acids and may also be destroyed by comparatively weak alkali ( 2 per cent sodium carbonate) if left in contact for a sufficiently long time. Trypsinogen, on the other hand, is more resistant to the action of alkalis. In pancreatic digestion the pro-

${ }^{1}$ Glaessner: Zeitschrift für physiologische Chemie, 40, 476, r904. 
tein does not swell as is the case in gastric digestion, but becomes more or less "honey-combed" and finally disintegrates.

The presence of active pepsin in the contents of the intestine has been demonstrated by Abderhalden and Meyer. ${ }^{1}$ It may possibly be that pepsin may play a part in the profound intestinal proteolysis which has up to this time been assigned to trypsin and erepsin (see chapter on Gastric Digestion).

The pancreatic juice which is collected by means of a fistula possesses practically no power to digest protein matter. A body called enterokinase occurs in the intestinal juice and has the power of converting trypsinogen into trypsin. This process is known as the "activation" of trypsinogen and through it a juice which is incapable of digesting protein may be made active. (For further discussion of enterokinase see chapter on Intestinal Digestion.) Mendel and Rettger ${ }^{2}$ and others have demonstrated that activation of trypsinogen into trypsin may be brought about in the gland as well as in the intestine of the living organism. The manner of the activation in the gland and the nature of the body causing it are unknown at present. Prym ${ }^{3}$ denies that such an activation occurs.

Delezenne claims that trypsinogen may be activated by soluble calcium salts. He reports experiments which indicate that proteolytically inactive pancreatic juice, obtained directly from the duct, when treated with salts of this character, assumes the property of digesting protein material. This process by which the trypsinogen is activated through the instrumentality of calcium salts is very rapid and is designated by Delezenne as an "explosion." The suggestion of Mays that there may possibly be several precursors of trypsin one of which is activated by enterokinase and the others by other agents, is of interest in this connection.

Boldyreff ${ }^{4}$ has demonstrated the presence of trypsin in the stomach due to the regurgitation of duodenal contents through the pylorus (see Chapters VII and VIII). Others ${ }^{5}$ have confirmed this finding (see chapter on Gastric Analysis).

Pancreatic amylase (amylopsin), the second of the pancreatic enzymes, is an amylolytic enzyme which possesses somewhat greater digestive power than the salivary amylase (ptyalin) of the saliva. As its name implies, its activity is confined to the starches, and the products of its amylolytic action are dextrins and sugar. The sugar is principally

${ }^{1}$ Abderhalden and Meyer: Zeit. physiol. Chem., 74, 67, I9I1.

2 Mendel and Rettger: American Journal of Physiology, 7.

${ }^{3}$ Prym: Pfliger's Archiv, $\mathrm{IO}_{4}$ and 107.

"Boldyreff: Transactions of the I Ith Pirogoff's Congress of Physicians, St. Petersburg, I910.

'Spencer, Meyer, Rehfuss and Hawk: American Jour. Physiol., 39, 459, IgI6. 
maltose and this by the further action of an inverting enzyme (maltase) is transformed into glucose.

It is possible that the saliva as a digestive fluid is not absolutely essential. The salivary amylase (ptyalin) is destroyed by the hydrochloric acid of the gastric juice and is therefore inactive when the chyme reaches the intestine. Should undigested starch be present at this point, however, it would be quickly transformed by the active pancreatic amylase. This enzyme is not present in the pancreatic juice of infants during the first few weeks of life, thus showing very clearly that a starchy diet is not normal for this period.

The pronounced influence of electrolytes upon the action of pancreatic amylase and other amylases has been demonstrated many times. ${ }^{1}$ In fact the removal of electrolytes from pancreatic juice by dialysis yields a juice which possesses no power to split starch. It also appears that the $\mathrm{Cl}$ or $\mathrm{Br}$ ion is absolutely essential to the activity of animal amylases. ${ }^{2}$

It has been claimed that pancreatic amylase has a slight digestive action upon unboiled starch.

The extent to which amylase is present in the feces has been taken as the index of pancreatic activity.

The third enzyme of the pancreatic juice is called pancreatic lipase (steapsin) and is a fat-splitting enzyme. It has the power of splitting the neutral fats of the food by hydrolysis, into fatty acid and glycerol. A typical reaction would be as follows:

$$
\left.\mathrm{C}_{3} \mathrm{H}_{5}\left(\underset{\text { Palmitin. }}{\mathrm{O} \cdot \mathrm{C}_{15} \mathrm{H}_{31} \mathrm{CO}}\right)_{3}+{ }_{3} \mathrm{H}_{2} \mathrm{O} \rightarrow 3 \underset{\text { Palmitic acid. }}{\left(\mathrm{C}_{15} \mathrm{H}_{31} \mathrm{COOH}\right.}\right)+\underset{\text { Glycerol. }}{\mathrm{C}_{3} \mathrm{H}_{5}}(\mathrm{OH})_{3} .
$$

Recent researches make it probable that fats undergo saponification to a certain extent prior to their absorption. The fatty acids formed unite with the alkalis of the pancreatic juice and intestinal secretion to form soluble soaps which are readily absorbed. It was formerly believed that the fats could also be absorbed in emulsiona condition promoted by the presence of the soluble soaps. After absorption the fatty acids are resynthesized to form neutral fats with glycerol.

Bloor ${ }^{3}$ has reported experiments which "make it extremely probable that fats can be absorbed only in water-soluble form and that saponification is a necessary preliminary to absorption." Petroleum hydrocarbons and non-saponifiable esters, e.g., wool fat (lanolin) were un-

${ }^{1}$ For the literature see Kendall and Sherman: Jour. Am. Chem. Soc., 32, ro87, rgro.

${ }^{2}$ Wohlgemuth: Biochem. Zeit., 9, 10, 1908; and Kendall and Sherman: Jour. Am. Chem. Soc., 32, 1087 1910. Bierry: Biochem. Zeit., 40, 357, I91 2.

3 Bloor: Jour. Biol. Chem., I5, 105, 1913. 
absorbed. Bloor further claims ${ }^{1}$ that in the absorption of fats there is a tendency toward the formation of a uniform chyle fat, presumably the characteristic body fat of the animal.

Pancreatic lipase is very unstable and is easily rendered inert by the action of acid. For this reason it is not possible to prepare an extract having a satisfactory fat-splitting power from a pancreas which has been removed from the organism for a sufficiently long time to have become acid in reaction.

The fourth enzyme of the pancreatic juice is called pancreatic rennin. It is a milk-coagulating enzyme whose action is very similar to that of the gastric rennin found in the gastric juice. It is supposed to show its greatest activity at a temperature varying from $60^{\circ}$ to $65^{\circ} \mathrm{C}$.

\section{PREPARATION OF AN ARTIFICIAL PANCREATIC JUICE ${ }^{2}$}

After removing the fat from the pancreas of a pig or sheep, finely divide the organ by means of scissors and grind it in a mortar. If convenient, the use of an ordinary meat chopper is a very satisfactory means of preparing the pancreas.

When finely divided as above the pancreas should be placed in a 500 c.c. flask, about 150 c.c. of 30 per cent alcohol added and the flask and contents shaken frequently for 24 hours. (What is the reaction of this alcoholic extract at the end of this period, and why?) Strain the alcoholic extract through cheese cloth, filter, nearly neutralize with potassium hydroxide solution and then exactly neutralize it with 0.5 per cent sodium carbonate.

\section{Products of Tryptic Digestion}

Take about 200 grams of lean beef which has been freed from fat and finely ground and place it in a large-sized beaker. Introduce equal volumes of the pancreatic extract prepared as above and 0.5 per cent sodium carbonate, add 5 c.c. of an alcoholic solution of thymol to prevent putrefaction, and place the beaker in an incubator at $40^{\circ} \mathrm{C}$. Stir the contents of the beaker frequently and add more thymol if it becomes necessary. Allow digestion to proceed for from two to five days and then separate the products formed as follows: Strain off the undissolved residue through cheese cloth, nearly neutralize the solution with dilute hydrochloric acid and then exactly neutralize it with 0.2 per cent hydrochloric acid. A precipitate at this point would indicate alkali metaprotein (alkali albuminate). Filter off any precipitate and divide the filtrate into two parts, a one-fourth and a three-fourth portion.

Transfer the one-fourth portion to an evaporating dish and make the separation of proteoses and peptones as well as the final tests upon these bodies according to the directions given on page 120.

Place about 5 c.c. of the three-fourth portion in a test-tube and add about I c.c. of bromine water. A violet coloration ${ }^{3}$ indicates the presence of trypto-

${ }^{1}$ Bloor: Jour. Biol. Chem., 16, 517 , 19r4.

2 For other methods of preparation see Karl Mays: Zeitschrift für physiologische Chemie, 38,428 , 1903 .

${ }^{3}$ Kurajeff: Zeit. physiol. Chem., 36, 501, rS9S-99. 
phane (see page 77. Also see glycyl-tryptophane reaction in chapter on Intestinal Digestion). To another 5 c.c. add ro drops of concentrated sulphuric acid and ro c.c. of a ro per cent solution of mercuric sulphate in 5 per cent sulphuric acid. Shake the tube and allow to stand five minutes. A yellow precipitate of a mercury compound of tryptophane forms. ${ }^{1}$ Concentrate ${ }^{2}$ the remainder of the three-fourths portion to a thin syrup and make the separation of leucine and tyrosine according to the directions given on page 85 .

\section{GENERAL EXPERIMENTS ON PANCREATIC DIGESTION}

\section{EXPERIMENTS ON TRYPSIN ${ }^{3}$}

I. The Most Favorable Reaction for Tryptic Digestion.-Prepare seven tubes as follows:

(a) 2-3 c.c. of neutral pancreatic extract $+2-3$ c.c. of water.

(b) 2-3 c.c. of neutral pancreatic extract $+2-3$ c.c. of I per cent sodium carbonate.

(c) 2-3 c.c. of neutral pancreatic extract $+2-3$ c.c. of 0.5 per cent sodium carbonate.

(d) 2-3 c.c. of neutral pancreatic extract $+2-3$ c.c. of 0.2 per cent hydrochloric acid.

(e) 2-3 c.c. of neutral pancreatic extract $+2-3$ c.c. of 0.2 per cent combined hydrochloric acid.

(f) 2-3 c.c. of neutral pancreatic extract $+2-3$ c.c. of 0.4 per cent boric acid.

(g) 2-3 c.c. of neutral pancreatic extract $+2-3$ c.c. of 0.4 per cent acetic acid.

Add a small piece of fibrin ${ }^{4}$ to the contents of each tube and keep them at $40^{\circ} \mathrm{C}$. noting the progress of digestion. In which tube do we find the most satisfactory digestion, and why? How do the indications of the digestion of fibrin by trypsin differ from the indications of the digestion of fibrin by pepsin?

2. The Most Favorable Temperature.-(For this and the following series of experiments under tryptic digestion use the neutral extract plus an equal volume of 0.5 per cent sodium carbonate.) In each of four tubes place 5 c.c. of alkaline pancreatic extract. Immerse one tube in cold water from the faucet, keep a second at room temperature and place a third in the incubator or water-bath at $40^{\circ} \mathrm{C}$. Boil the contents of the fourth for a few moments, then cool and also keep it at $40^{\circ} \mathrm{C}$. Into each tube introduce a small piece of fibrin and note the progress of digestion. In which tube does the most rapid digestion occur? What is the reason?

3. Influence of Bile.-Prepare three tubes as follows:

(a) 5 c.c. of pancreatic extract $+I / 2-I$ c.c. of bile.

(b) 5 c.c. of pancreatic extract +5 c.c. of bile.

${ }^{1}$ It has been claimed that a similar yellow precipitate forms in the presence of tyrosine, cystine and polypeptides. For quantilative estimation of tryptophane see Homer: Jour. Biol. Chem., 22, 369, 19r5.

${ }^{2}$ If the solution is alkaline in reaction, while it is being concentrated, the amino acids will be broken down and ammonia will be liberated.

${ }^{3}$ For these experiments as well as for those on the other pancreatic enzymes commercial preparations of trypsin and pancreatin may be employed.

${ }_{4}$ Congo red fibrin may be used in this and the following tests on tryptic digestion. If used the experiments should be made at room temperature. For preparation of this fibrin see Chapter I. 
(c) 5 c.c. of pancreatic extract.

Introduce into each tube a small piece of fibrin and keep them at $40^{\circ} \mathrm{C}$. Shake the tubes frequently and note the progress of digestion. Does the presence of bile retard tryptic digestion? How do these results agree with those obtained under gastric digestion?

4. Quantitative Determination of Tryptic Activity. '-Gross' Method.-This method is based upon the principle that faintly alkaline solutions of casein are precipitated upon the addition of dilute ( $\mathrm{I}$ per cent) acetic acid whereas its digestion products are not so precipitated. The method follows: Prepare a series of tubes each containing ro c.c. of a 0. I per cent solution of pure, fat-free casein, ${ }^{2}$ which has been heated to a temperature of $40^{\circ} \mathrm{C}$. Add to the contents of the series of tubes increasing amounts of the trypsin solution under examination, ${ }^{3}$ and place them at $40^{\circ} \mathrm{C}$. for fifteen minutes. At the end of this time remove the tubes and acidify the contents of each with a few drops of dilute (I per cent) acetic acid. The tubes in which the casein is completely digested will remain clear when acidified, while those tubes which contain undigested casein will become more or less turbid under these conditions. Select the first tube in the series which exhibits no turbidity upon acidification, thus indicating complete digestion of the casein, and calculate the tryptic activity of the enzyme solution under examination.

Calculation.-The unit of tryptic activity is an expression of the power of I c.c. of the fluid under examination exerted for a period of fifteen minutes on Io c.c. of a 0.1 per cent casein solution. For example, if 0.5 c.c. of a trypsin solution completely digests ro c.c. of a 0.1 per cent solution of casein in fifteen minutes the activity of that solution would be expressed as follows:

$$
\text { Tryptic activity }=1 \div 0.5=2 \text {. }
$$

Such a trypsin solution would be said to possess an activity of 2 . If 0.3 c.c. of the trypsin solution had been required the solution would be said to possess an activity of 3.3 ; i.e., $I \div 0.3=3 \cdot 3$.

\section{Experiments on Pancreatic Amylase}

I. The Most Favorable Reaction.-Prepare seven tubes as follows:

(a) I c.c. of neutral pancreatic extract + I c.c. of starch paste +2 c.c. of water.

(b) I c.c. of neutral pancreatic extract + I c.c. of starch paste +2 c.c. of I per cent sodium carbonate.

(c) I c.c. of neutral pancreatic extract + I c.c. of starch paste +2 c.c. of 0.5 per cent sodium carbonate.

(d) I c.c. of neutral pancreatic extract + I c.c. of starch paste +2 c.c. of 0.2 per cent hydrochloric acid.

Shake each tube thoroughly and place them in the incubator or water-bath at $40^{\circ} \mathrm{C}$. At the end of a half-hour divide the contents of each tube into two parts and test one part by the iodine test and the other part by Fehling's test. Where

${ }^{1}$ For a discussion of Spencer's method for the quantitative determination of trypsin in stomach contents see chapter on Gastric Analysis.

${ }^{2}$ Made by dissolving I gram of Grübler's casein in a liter of o.I per cent sodium carbonate. A little chloroform may be added to prevent bacterial action.

${ }^{3}$ The amount of solution used may vary from O.I-I c.c. The measurements nay conveniently be made by means of a I c.c. graduated pipette. 
do you find the most satisfactory digestion? How do the results compare with those obtained from the similar series under Trypsin, page rgo?

2. The Most Favorable Temperature.-(For this and the following series of experiments upon pancreatic amylase use the neutral extract plus an equalvolume of 0.5 per cent sodium carbonate.) In each of four tubes place 2-3 c.c. of alkaline pancreatic extract. Immerse one tube in cold water from the faucet, keep a second at room temperature, and place a third on the water-bath at $40^{\circ} \mathrm{C}$. Boil the contents of the fourth for a few moments, then cool and also keep it at $40^{\circ} \mathrm{C}$. Into each tube introduce $2-3$ c.c. of starch paste and note the progress of digestion. At the end of one-half hour divide the contents of each tube into two parts and test one part by the iodine test and the other part by Fehling's test. In which tube do you find the most satisfactory digestion? How does this result compare with the result obtained in the similar series of experiments under Trypsin (see page 190)?

3. Influence of Bile.-Prepare three tubes as follows:

(a) 2-3 c.c. of pancreatic extract $+2-3$ c.c. of starch paste $+I / 2-I$ c.c. of bile.

(b) 2-3 c.c. of pancreatic extract $+2-3$ c.c. of starch paste +5 c.c. of bile.

(c) 2-3 c.c. of pancreatic extract $+2-3$ c.c. of starch paste.

Shake the tubes thoroughly and place them in the incubator or water-bath at $40^{\circ} \mathrm{C}$. Note the progress of digestion frequently and at the end of a half-hour divide the contents of each tube into two parts and test one part by the iodine test and the other part by Fehling's test. What are your conclusions regarding the influence of bile upon the action of pancreatic amylase?

4. Digestion of Dry Starch.-To a little dry starch in a test-tube add about 5 c.c. of pancreatic extract and place the tube in the incubator or water-bath at $40^{\circ} \mathrm{C}$. At the end of a half-hour filter and test separate portions of the filtrate by the iodine and Fehling tests. What do you conclude regarding the action of pancreatic amylase upon dry starch? Compare this result with that obtained in the similar experiment under Salivary Digestion (page 6o).

5. Digestion of Inulin.-To 5 c.c. of inulin solution in a test-tube add ro drops of pancreatic extract and place the tube in the incubator or water-bath at $40^{\circ} \mathrm{C}$. After one-half hour test the solution by Fehling's test. ${ }^{1}$ Is any reducing substance present? What do you conclude regarding the digestion of inulin by pancreatic amylase?

6. Quantitative Determination of Amylolytic Activity.-Wohlgemuth's Method. ${ }^{2}$ Arrange a series of test-tubes with diminishing quantities of the enzyme solution under examination, introduce into each tube 5 c.c. of I percent solution of soluble starch $^{3}$ and place each tube at once in a bath of ice-water. ${ }^{4}$

${ }^{1}$ If the inulin solution gives a reduction before being acted upon by the pancreatic juice it will be necessary to determine the extent of the original reduction by means of a "check" test (see p. 47).

2 Wohlgemuth: Biochemische Zeitschrift, 9, r, I908.

3 Kahlbaum's soluble starch is satisfactory. In preparing the I per cent solution, the weighed starch powder should be dissolved in cold distilled water in a casserole and stirred until a homogeneous suspension is obtained. The mixture should then be heated, with constant stirring, until it is clear. This ordinarily takes about 8-Io minutes. A slightly opaque solution is thus obtained which should be cooled and made up to the proper volume before using.

'Ordinarily a series of six tubes is satisfactory, the volumes of the enzyme solution used ranging from I C.c. to O.I C.c. and the measurements being made by means of a I c.c. graduated pipette. Each tube should be placed in the ice-water bath as soon as the starch solution is introduced. It will be found convenient to use a small wire basket to hold the tubes. 
When all the tubes have been prepared in this way and placed in the ice-water bath they are transferred to a water-bath or incubator and kept at $38^{\circ} \mathrm{C}$. for from 30 minutes to an hour. ${ }^{1}$ At the end of this digestion period the tubes are again removed to the bath of ice-water in order that the action of the enzyme may be stopped.

Dilute the contents of each tube, to within about $I / 2$ inch of the top, with water, add one drop of a $\mathrm{N} /$ I 0 solution of iodine and shake the tube and contents thoroughly. A series of colors ranging from dark blue through bluish violet and reddish yellow to yellow, will be formed. ${ }^{2}$ The dark blue color shows the presence of unchanged starch, the bluish-violet indicates a mixture of starch and erythrodextrin, whereas the reddish-yellow signifies that erythrodextrin and maltose are present and the yellow solution denotes the complete transformation of starch into maltose. Examine the tubes carefully before a white background and select the last tube in the series which shows the entire absence of all blue color, thus indicating that the starch has been completely transformed into dextrins and sugar. In case of indecision between two tubes, add an extra drop of the iodine solution, and observe them again, after shaking.

Calculation.- The amylolytic activity ${ }^{3}$ of a given solution is expressed in terms of the activity of I c.c. of such a solution. For example, if it is found that 0.02 c.c. of an amylolytic solution, acting at $38^{\circ} \mathrm{C}$., completely transformed the starch in 5 c.c. of a I per cent starch solution in 30 minutes, the amylolytic activity of such a solution would be expressed as follows:

$$
\mathrm{D}_{30^{\prime}}^{33^{\circ}}=250
$$

This indicates that I c.c. of the solution under examination possesses the power of completely digesting 250 c.c. of I per cent starch solution in 30 minutes at $38^{\circ} \mathrm{C}$.

Wohlgemuth has suggested a slight alteration in the above procedure for use in the determination of the amylase content of the feces. ${ }^{4}$ A modification of the Wohlgemuth procedure ${ }^{5}$ for this purpose is given in the chapter on Feces.

\section{Experiments on Pancreatic Lipase}

I. "Litmus-milk" Test.-Into each of two test-tubes introduce Io c.c. of milk and a small amount of litmus solution. To the contents of one tube add 3 c.c. of neutral pancreatic extract ${ }^{6}$ and to the contents of the other tube add 3 c.c. of water or of boiled neutral pancreatic extract. Keep the tubes at $40^{\circ} \mathrm{C}$. and note any changes which may occur. What is the result and how do you explain it?

2. Ethyl Butyrate Test.-Into each of two test-tubes introduce 4 c.c. of water, 2 c.c. of ethyl butyrate, $\mathrm{C}_{3} \mathrm{H}_{7} \mathrm{COO} . \mathrm{C}_{2} \mathrm{H}_{5}$, and a small amount of litmus powder. To the contents of one tube add 4 c.c. of neutral pancreatic extract and to the contents of the other tube add 4 c.c. of water or of boiled neutral pancreatic ex-

${ }^{1}$ Longer digestion periods may be used where it is deemed advisable. If exceedingly weak solutions are being investigated, it may be most satisfactory to permit the digestion io extend over a period of 24 hours.

${ }^{2}$ See p. 56 .

3 Designated by " $\mathrm{D}$ " the first letter of "diastatic."

-Wohlgemuth: Berliner klinische Wochenschrift, 47, 92, 19:0.

'Hawk: Archizes of Internal Medicine, 8, 552, I9r.

${ }^{6}$ Commercial pancreatin may be used in this test if desired. 
tract. Keep the tubes at $40^{\circ} \mathrm{C}$. and observe any change which may occur. What is the result and how do you explain it? Write the equation for the reaction which has taken place.

\section{Experiments on Pancreatic Rennin}

Prepare four test-tubes as follows:

(a) 5 c.c. of milk + ro drops of neutral pancreatic extract.

(b) 5 c.c. of milk +20 drops of neutral pancreatic extract.

(c) 5 c.c. of milk + ro drops of alkaline pancreatic extract.

(d) 5 c.c. of milk +20 drops of alkaline pancreatic extract.

Place the tubes at $60^{\circ}-65^{\circ} \mathrm{C}$. for a half hour without shaking. Note the formation of a clot. ${ }^{1}$ How does the action of pancreatic rennin compare with the action of the gastric rennin?

I This reaction will not always succeed, owing to conditions which are not well understood. 


\section{CHAPTER XI}

\section{INTESTINAL DIGESTION}

Strictly speaking, all digestive processes which take place in the intestine may be classed under Intestinal Digestion. However, we will consider under Intestinal Digestion only those digestive processes which are brought about by enzymes which have their origin in the intestine. The activities of those enzymes which originate in the pancreas we have considered in Chapter $\mathrm{X}$ under Pancreatic Digestion.

The enzymes of the intestinal juice (succus entericus) are of great importance to the animal organism. These enzymes include erepsin, sucrase, maltase, lactase, nucleases, and enterokinase.

Erepsin is a proteolytic enzyme which has the property of acting upon the proteoses, peptones, and peptides which are formed through the action of trypsin, and further splitting them into amino-acids. Erepsin has no power of digesting any native proteins except caseinogen. histones, and protamines. It possesses its greatest activity in an alkaline solution, although it is slightly active in acid solution. An extract of the intestinal erepsin may be prepared by treating the finely divided intestine of a cat, dog, or pig with toluol- or chloroform-water and permitting the mixture to stand with occasional shaking for $24-72$ hours. Enzymes similar to erepsin occur in various tissues of the organism.

In cases of gastric cancer a peptide-splitting enzyme is claimed to be present in the stomach contents. The glycyl-tryptophane test is sometimes used for its detection. Some investigators claim that the peptide-splitting power of gastric juice in cancer is generally due to the regurgitation of trypsin or erepsin from the intestine or to the presence in the gastric contents of swallowed saliva which possesses peptolytic power. The peptide-splitting power of saliva may be due to a specific enzyme or to the presence of bacteria (see Glycyltryptophane Reaction, page 199).

The three invertases sucrase, maltase, and lactase are also important enzymes of the intestinal mucosa. The sucrase acts upon sucrose and inverts it with the formation of invert sugar (glucose and fructose). Some investigators claim that sucrase is also present in saliva and gastric juice. It probably does not exist normally in either of these 
digestive juices, however, and if found owes its presence to the excretory processes of certain bacteria. Sucrases may also be obtained from several vegetable sources. For investigational purposes it is ordinarily obtained from yeast (see page I4). It exhibits its greatest activity in the presence of a slight acidity, but if the acidity be increased to any extent the reaction is inhibited.

Lactase is an enzyme which inverts lactose with the consequent formation of glucose and galactose. Its action is entirely analogous, in type, to that of sucrase. It has apparently been proven that lactase occurs in the intestinal mucosa of the young of all animals which suckle their offspring. ${ }^{1}$ It may also occur in the intestinal mucosa of certain adult animals if such animals be maintained upon a ration containing more or less lactose. Fischer and Armstrong have demonstrated the reversible action ${ }^{2}$ of lactase.

Maltase possesses the power of splitting maltose, the end-product of the digestion of starch, into glucose. It was first discovered in the urine and shortly after this time its presence was noted in the small intestine and the saliva. Corn is sometimes used as the medium for the preparation of the enzyme for experimental purposes. It occurs in corn in a very active state. It was in connection with maltase that the principles of the "reversibility of enzyme action" were first demonstrated.

Enterokinase possesses the power of "activating" trypsinogen see Chapters $I$ and $X$ ). In other words, trypsinogen as formed by the pancreas has no proteolytic power, but when this inactive trypsinogen reaches the intestine and comes into contact with enterokinase the latter transforms it into active trypsin. Enterokinase is not always present in the intestinal juice since it is secreted only after the pancreatic juice reaches the intestine. It resembles the enzymes in that its activity is destroyed by heat, but differs materially from this class of bodies in that a certain quantity is capable of activating only a definite quantity of trypsinogen. It is, however, generally classified as an enzyme. Enterokinase has been detected in the higher animals, and a kinase possessing similar properties has been shown to be present in bacteria, fungi, impure fibrin, lymph glands, and snake-venom.

The intestinal juice and the epithelium of the intestinal wall contain enzymes capable of hydrolyzing nucleic acids and as these acids are not acted upon by the gastric juice and probably not to any great extent by pure pancreatic juice, the intestine apparently plays the chief rôle in decomposition or digestion of these substances. At least two

1 Mendel and Mitchell: American Journal of Physiology, 20, 8r, I907.

${ }^{2}$ See p. 8 . 
enzymes take part in this digestion process, one decomposing the nucleic acid with formation of simple nucleotides containing a single radical each of phosphoric acid, carbohydrate and base (see chapter on Nucleic Acids). This enzyme may be called nucleicacidase. Another enzyme present in the intestine and intestinal juice decomposes these nucleotides with the liberation of phosphoric acid. This enzyme may be called nucleotidase or phosphomuclease. The intestinal mucosa also decomposes many other organic phosphorus compounds with liberation of their phosphoric acid. ${ }^{I}$ Thus glycero-phosphoric acid and hexose-phosphoric acid as well as phosphoproteins are split in a similar manner, the phosphoric acid they contain thus being absorbed in the free form.

\section{General Experiments on Intestinal Digestion}

Demonstration of Enterokinase.-Trypsinogen may be activated by enterokinase. This activation occurs normally in the intestine. Calcium salts also bring about a similar activation of the trypsinogen.

Procedure.-Prepare an extract of trypsinogen by grinding Io grams of the fresh, fat-free pancreas of the pig with a little sand. Gradually add Ioo c.c. of water during the grinding process. Strain through cheese cloth.

Prepare an extract of enterokinase by grinding 5 grams of fresh, fat-free duodenal mucosa ${ }^{2}$ of the pig with a little sand. Gradually add 50 c.c. of water during the grinding process. Strain through cheese cloth.

Prepare the following series of tubes:

(a) Io c.c. pancreas extract +5 c.c. water.

(b) Io c.c. pancreas extract +5 c.c. duodenal extract.

(c) 5 c.c. duodenal extract + ro c.c. water.

(d) Io c.c. pancreas extract +5 c.c. duodenal extract.

(e) Io c.c. pancreas extract +5 c.c. duodenal extract (boiled).

(f) Io c.c. pancreas extract +5 c.c. of 4 per cent calcium chloride.

Boil the contents of tube (d) for five minutes and cool to $40^{\circ} \mathrm{C}$. Keep all six tubes at $40^{\circ} \mathrm{C}$. for 20 minutes.

To each tube add 5 c.c. of Io per cent sodium carbonate and mix the contents thoroughly and immediately. Introduce into each tube the same quantity (size of a pea) of fresh fibrin. Shake the tubes and place them at $40^{\circ} \mathrm{C}$. Observe the tubes frequently for one hour to note digestive changes. Tubes (b) and (f) should show most rapid digestion. Why?

\section{Experiments on Intestinal Nucleases}

I. Preparation of Intestinal Extract.-Wash thoroughly 100 grams of pig's intestine and run through a meat chopper several times. Introduce into a 500 c.c. mixing cylinder and add normal salt solution to make 500 c.c. Allow to

1 Plimmer: Biochem. $J ., 7,43$, r9r3.

2 The dried mucosa may be substituted if desired. 
stand for 6-24 hours at room temperature, shaking occasionally, toluol being added as a preservative. Strain and filter.

2. Demonstration of Intestinal Nucleases.-Prepare a 2 per cent solution of yeast nucleic acid put in solution with the aid of just sufficient $\mathrm{NaOH}$ solution to make the resulting mixture neutral to litmus. To each of two large test-tubes add 20 c.c. of the intestinal extract prepared as above. Boil one for one to two minutes. To each tube then add ro c.c. of the 2 per cent nucleic acid solution. Add 2-3 c.c. each of toluol and chloroform to each mixture. Keep at $38^{\circ} \mathrm{C}$. for 24 hours.

Heat the tubes to boiling in a water-bath to coagulate protein. Add 5 c.c. of 5 per cent $\mathrm{HCl}$ and allow to stand for one hour. This precipitates any unchanged nucleic acid. Filter and take aliquots of the filtrate (about 20 c.c.). Precipitate the phosphate from each mixture by adding 5 c.c. of magnesia mixture and 5 c.c. of ammonia. Allow to stand over night. A heavy precipitate of magnesium. ammonium phosphate should be found in the test experiment indicating that the phosphoric acid of the nucleic acid had been liberated by the nucleotidase of the intestinal extract. The control should show only a slight precipitate.

If desired the phosphorus of the precipitates may be determined quantitatively by dissolving in 2 per cent $\mathrm{HNO}_{3}$, precipitating as the phosphomolybdate and determining volumetrically according to the Neumann procedure (see 554).

\section{Experiments oN EREpsin}

I. Preparation of Erepsin.-Grind the mucous membrane of the small intestine of a cat, dog, or pig with sand in a mortar. Treat the finely divided membrane with toluol-or chloroform-water and permit the mixture to stand, with occasional shaking, for $24-72$ hours. ${ }^{1}$ Filter the extract thus prepared through cotton and use the filtrate in the following experiment.

2. Demonstration of Erepsin.-To about 5 c.c. of a I per cent solution of Witte's peptone in a test-tube add about I c.c. of the erepsin extract prepared as described above and make the mixture slightly alkaline (o.I per cent) with sodium carbonate. Prepare a second tube containing a like amount of peptone solution but boil the erepsin extract before introducing it. Place the two tubes at $38^{\circ} \mathrm{C}$. for two to three days. At the end of that period heat the contents of each tube to boiling, filter and try the biuret test on each filtrate. In making these tests care should be taken to use like amounts of filtrate, potassium hydroxide and copper sulphate in each test in order that the drawing of correct conclusions may be facilitated. The contents of the tube which contained the boiled extract should show a deep pink color with the biuret test, due to the peptone still present. On the other hand, the biuret test upon the contents of the tube containing the unboiled extract should be negative or exhibit, at the most, a faint pink or blue color, signifying that the peptone, through the influence of the erepsin, has been transformed, in great part at least, into amino-acids which do not respond to the biuret test. ${ }^{2}$

1 The enzyme may also be extracted by means of glycerol or alkaline "physiological" salt solution if desired.

${ }^{2}$ Sirictly speaking, this erepsin demonstration is not adequate unless a control test is made with native protein (except casein, histones and protamines) to show that the extract is trypsin-free and digests peptone but not native protein. 
3. The Glycyl-tryptophane Reaction.-The dipeptide glycyl-tryptophane may be used in place of the peptone solution for the demonstration of erepsin. It is claimed to be of service in the diagnosis of gastric cancer. It is claimed that a peptide-splitting enzyme (erepsin) is present in the stomach contents of individuals suffering from cancer of the stomach, whereas the stomach contents of normal individuals contains no such enzyme. The glycyl-tryptophane test, therefore, may sometimes furnish a means of aiding in the diagnosis of this disorder. As applied to stomach contents, the test is as follows: ${ }^{2}$ Introduce about Io c.c. of the filtrate from the stomach contents into a test-tube, add a little glycyl-tryptophane and a layer of toluol, and place the tube in an incubator at $38^{\circ} \mathrm{C}$. for 24 hours. At the end of this time by means of a pipette transfer 2-3 c.c. of the fluid from beneath the toluol to a test-tube, add a few drops of 3 per cent acetic acid and carefully introduce bromine vapors. Shake the tube and note the production of a red color if tryptophane is present. The tryptophane has, of course, been liberated from the peptide through the action of the peptide-splitting enzyme (erepsin) elaborated by the cancer tissue.

If an excess of bromine is added the color will vanish. If no rose color is noted, add more bromine vapors carefully with shaking until further addition of the vapors causes the production of a yellowish color. This indicates an excess of bromine and constitutes a negative test. Occasionally the rose color indicating a positive test is so transitory as to escape detection unless the test be very carefully performed.

Several fallacies have been pointed out in connection with this test. In the first place the regurgitation of duodenal contents through the pylorus might insure the presence in the stomach of erepsin and trypsin either of which possesses peptide-splitting power. It has also been claimed that saliva contains an enzyme capable of splitting glycyltryptophane. Doubt has, however, been cast upon the dipeptidesplitting agent of the saliva by Smithies ${ }^{3}$ and by Jacque and Woodyatt, ${ }^{4}$ who point to bacteria as the peptolytic agents. In any event saliva contains something which is capable of splitting the glycyltryptophan, thus making the entrance of saliva into the stomach an important source of error, so far as the utility of this test is concerned, as a diagnostic aid. Bacteria may, of course, be removed from the gastric juice by passing the fluid through an effective filter.

\section{EXPERIMENTS ON INTERTASES ${ }^{5}$}

I. Preparation of an Extract of Sucrase.-Treat the finely divided epithelium of the small intestine of a dog, pig, rat, rabbit, or hen with about 3 volumes of a 2 per cent solution of sodium fluoride and permit the mixture to

1 This dipeptide is sold commercially under the name "Ferment Diagnosticon."

${ }^{2}$ Neubauer and Fischer: Deulsches Archiv f. klinische .IC cdisin, 97, 499, I909.

3 Smithies: Arch. Inl. Mcd., Iо, 52 I, ror 2.

"Jacque and Woodyatt: Arch. Inl..Med., Dec., rgr2, p. 560.

"Tle Inverting Enzymes of the Alimentary Tract," IIendel and Mitchell: American Journal of Physiology, 20, Sr, r907-0S. 
stand at room temperature for 24 hours. Strain the extract through cloth or absorbent cotton and use the strained material in the following demonstration.

2. Demonstration of Sucrase.-To about 5 c.c. of a $\mathrm{I}$ per cent solution of sucrose, in a test-tube, add about I c.c. of a 2 per cent sodium fluoride intestinal extract, prepared as described above. Prepare a control tube in which the intestinal extract is boiled before being added to the sugar solution. Place the two tubes at $38^{\circ} \mathrm{C}$. for two hours. ${ }^{1}$ Heat the mixture to boiling to coagulate the protein material, filter, and test the filtrate by Fehling's test (see page 26). The tube containing the boiled extract should give no response to Fehling's test, whereas the tube containing the unboiled extract should reduce the Fehling's solution. This reduction is due to the formation of invert sugar (see page 4I) from the sucrose through the action of the enzyme sucrase which is present in the intestinal epithelium.

For preparation and demonstration of Vegetable Sucrase see Chapter I.

3. Preparation of an Extract of Lactase.- Treat the finely divided epithelium of the small intestine of a kitten, puppy, or pig embryo with about 3 volumes of a 2 per cent solution of sodium fluoride and permit the mixture to stand at room temperature for 24 hours. Strain the extract through cloth or absorbent cotton and use the strained material in the following demonstration.

4. Demonstration of Lactase. ${ }^{2}$ To about 5 c.c. of a I per cent solution of lactose in a test-tube add about I c.c. of a toluol-water or a 2 per cent sodium fluoride extract of the first part of the small intestine ${ }^{3}$ of a kitten, puppy, or pig embryo prepared as described above. Prepare a control tube in which the intestinal extract is boiled before being added to the sugar solution. Place the two tubes at $38^{\circ} \mathrm{C}$. for 24 hours. At the end of this period add I c.c. of the digestion mixture to 5 c.c. of Barfoed's reagent ${ }^{4}$ and place the tubes in a boiling waterbath. ${ }^{5}$ Examine the tubes at the end of three minutes against a black background in a good light. If no cuprous oxide is visible replace the tubes and repeat the examination at the end of the fourth and fifth minutes. If no reduction is then observed permit the tubes to stand at room temperature for 5-10 minutes and examine again. ${ }^{6}$

It has been determined that disaccharide solutions will not reduce Barfoed's reagent until after they have been heated for 9-10 minutes on a boiling waterbath in contact with the reagent. ${ }^{7}$ Therefore in the above test, if the tube containing the unboiled extract exhibits any reduction after being heated as indicated, for a period of five minutes or less, and the control tube containing boiled extract shows no reduction, it may be concluded that lactase was present in the intestinal extract. ${ }^{8}$

${ }^{1}$ If a positive result is not obtained in this time permit the digestion to proceed for a longer period.

2 Roaf: Bio-Chemical Journal, 3, I82, 1908.

${ }^{3}$ Duodenum and first part of jejunum.

${ }^{4}$ To 4.5 grams of neutral crystallized copper acetate in goo c.c. of water add 0.6 c.c. of glacial acetic acid and make the total volume of the solution I liter.

${ }_{5}^{5}$ Care should be taken to see that the water in the bath reaches at least to the upper level of the contents of the tubes.

${ }^{6}$ Sometimes the drawing of conclusions is facilitated by pouring the mixture from the tube and examining the bottom of the tube for adherent cuprous oxide.

${ }_{7}$ The heating for $9^{-10}$ minutes is sufficient to transform the disaccharide into monosaccharide.

${ }^{8}$ The reduction would, of course, be due to the action of the glucose and galactose which had been formed from the lactose through the action of the enzyme lactase. 
5. Preparation of an Extract of Maltase.-Treat the finely divided epithelium of the small intestine of a cat, kitten, or pig (embryo or adult) with about 3 volumes of a 2 per cent solution of sodium fluoride and permit the mixture to stand at room temperature for 24 hours. Strain the extract through cloth and use the strained material in the following demonstration.

6. Demonstration of Maltase.-Proceed exactly as indicated in the demonstration of lactase, p. 200, except that a I per cent solution of maltose is substituted for the lactose solution. The extract used may be prepared from the upper part of the intestine of a cat, kitten, or pig (embryo or adult). In the case of lactase, as indicated, the intestine used should be that of a kitten, puppy, or pig (embryo). 


\section{CHAPTER XII}

\section{BILE}

THE bile is secreted continuously by the liver and passes into the intestine through the common bile duct which opens near the pylorus. Bile is not secreted continuously into the intestine. In a fasting animal no bile enters the intestine, but when food is taken the bile begins to flow; the length of time elapsing between the ingestion of the food and the secretion of the bile as well as the qualitative and quantitative characteristics of the secretion depending upon the nature of the food ingested. Fats, the extractives of meat and the protein end-products of gastric digestion (proteoses and peptones), cause a copious secretion of bile, whereas such substances as water, acids and boiled starch paste fail to do so. In general a rich protein diet is supposed to increase the amount of bile secreted, whereas a carbohydrate diet would cause a much less decided increase and might even tend to decrease the amount. It has been demonstrated by Bayliss and Starling that the secretion of bile is under the control of the same mechanism that regulates the flow of pancreatic juice (see page I85). In other words, the hydrochloric acid of the chyme, as it enters the duodenum transforms prosecretin into secretin and this in turn enters the circulation, is carried to the liver, and stimulates the bile-forming mechanism to increased activity.

We may look upon the bile as an excretion as well as a secretion. In the fulfillment of its excretory function it passes such bodies as lecithin, metallic substances, cholesterol, and the decomposition products of hemoglobin into the intestine and in this way aids in removing them from the organism. The bile assists materially in the absorption of fats from the intestine by its solvent action on the fatty acids formed by the action of the pancreatic juice.

The bile is a ropy, viscid substance which is usually alkaline in reaction to litmus, ${ }^{1}$ and ordinarily possesses a decidedly bitter taste. It varies in color in the different animals, the principal variations being yellow, brown and green. Fresh human bile from the living organism ordinarily has a green or golden-yellow color. Post-mortem bile is variable in color. It is very difficult to determine accurately the amount of normal bile secreted during any given period. For an adult man it

\footnotetext{
${ }^{1}$ It does not contain more than a slight excess of hydroxyl ions, however.
} 
has been variously estimated at from 500 c.c. to I IO0 c.c. for 24 hours. The specific gravity of the bile varies between I.OIO and I.040, and the freezing-point is about $-0.56^{\circ} \mathrm{C}$. As secreted by the liver, the bile is a clear, limpid fluid which contains a relatively low content of solid matter. Such bile would have a specific gravity of approximately I.oro. After it reaches the gall-bladder, however, it becomes mixed with mucous material from the walls of the gall-bladder; and this process coupled with the continuous absorption of water from the bile has a tendency to concentrate the secretion. Therefore the bile as we find it in the gall-bladder ordinarily possesses a higher specific gravity than that of the freshly secreted fluid. The specific gravity under these conditions may run as high as I.040.

The principal constituents of the bile are the salts of the bile acids, bile pigments, neutral fats, lecithin, phosphatides, nucleoprotein, mucin, and cholesterol, besides the salts of iron, copper, calcium, and magnesium. Zinc has also frequently been found in traces.

The quantitative composition of bile varies according to the source of the bile, i.e., whether the bile for analysis is obtained from the gall-

\section{QUANTITATIVE COMPOSITION OF BILE ${ }^{1}$}

(Parts per I000)

Constituent
Bladder bile,

Hammartsen ${ }^{2}$
Biliary fistula, Rosenbloom ${ }^{3}$

\begin{tabular}{|c|c|c|}
\hline $\begin{array}{l}\text { Water } . \ldots \ldots \ldots \ldots \ldots \ldots \ldots \ldots \ldots \ldots \ldots \ldots \ldots \ldots \ldots \ldots \\
\text { Solids } \ldots \ldots \ldots \ldots \ldots \ldots \ldots \ldots\end{array}$ & $\begin{array}{l}829.7 \\
170.3\end{array}$ & $\begin{array}{r}970.2 \\
39.8\end{array}$ \\
\hline Bile salts.................. & 97.0 & 10. I \\
\hline Mucin and pigments........... & 41.9 & 4.86 \\
\hline Cholesterol................ & $9 \cdot 9$ & $2.6 \mathrm{r}^{5}$ \\
\hline Fat............. & 1.9 & $6.8_{5}$ \\
\hline Soaps.............. & II. $2^{4}$ & 2.6 \\
\hline Lecithin..... & 2.2 & 6.42 \\
\hline Inorganic matter.......... & $5 \cdot 1$ & 9.2 \\
\hline 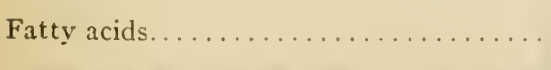 & Included under "soaps" & I. 2 \\
\hline \multicolumn{3}{|c|}{ 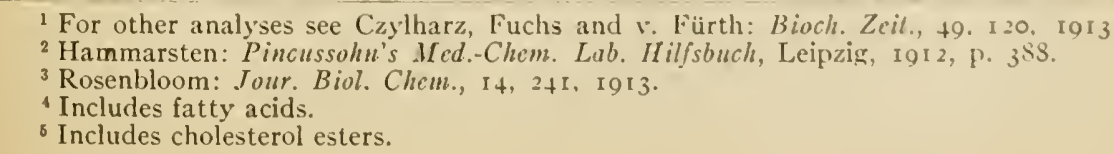 } \\
\hline
\end{tabular}


bladder or by means of a fistula before it reaches the gall-bladder. The variation in the composition of these two types of bile is shown in the preceding selected analyses:

The bile acids, which are elaborated exclusively by the hepatic cells, may be divided into two groups, the glycocholic acid group and the taurocholic acid group. In human bile glycocholic acid predominates, while taurocholic acid is the more abundant in the bile of carnivora. The bile acids are conjugate amino-acids, the glycocholic acid yielding glycocoll,

\title{
$\mathrm{CH}_{2} \cdot \mathrm{NH}_{2}$ \\ $\mathrm{COOH}$
}

and cholic acid upon decomposition, whereas taurocholic acid give rise to taurine,

\author{
$\mathrm{CH}_{2} \cdot \mathrm{NH}_{2}$ \\ $\mathrm{CH}_{2} \cdot \mathrm{SO}_{2} \cdot \mathrm{OH}$
}

and cholic acid under like conditions. Glycocholic acid contains some nitrogen but no sulphur, whereas taurocholic acid contains both these elements. The sulphur of the taurocholic acid is present in the taurine (amino-ethyl-sulphonic-acid), of which it is a characteristic constituent. There are several varieties of cholic acid and therefore we have several forms of glycocholic and taurocholic acids, the variation in constitution depending upon the nature of the cholic acid which enters into the combination. The bile acids are present in the bile as salts of one of the alkalis, generally sodium. The sodium glycocholate and sodium taurocholate may be isolated in crystalline form, either as balls or rosettes of fine needles or in the form of prisms having ordinarily four or six sides (Fig. 55, page 205). The salts of the bile acids are dextro-rotatory. Among other properties these salts have the power of holding the cholesterol and lecithin of the bile in solution.

Hammarsten has demonstrated a third group of bile acids in the bile of the shark. This same group very probably occurs in certain other animals also. These acids are very rich in sulphur and resemble etheral sulphuric acids inasmuch as upon treatment with boiling hydrochloric acid they yield sulphuric acid.

The bile pigments are important and interesting biliary constituents. The following have been isolated: bilirubin, biliverdin, bilifuscin, biliprasin, bilihumin, bilicyanin, choleprasin, and choletelin. Of these, bilirubin and biliverdin are the most important and predominate in normal bile. The colors possessed by the various varieties of normal bile are due almost entirely to these two pigments, the biliverdin being 
the predominant pigment in greenish bile and the bilirubin being the principal pigment in lighter colored bile. The pigments, other than the two just mentioned, have been found almost exclusively in biliary calculi or in altered bile obtained at post-mortem examinations.

Bilirubin, which is perhaps the most important of the bile pigments, is apparently derived from the blood pigment, the iron freed in the

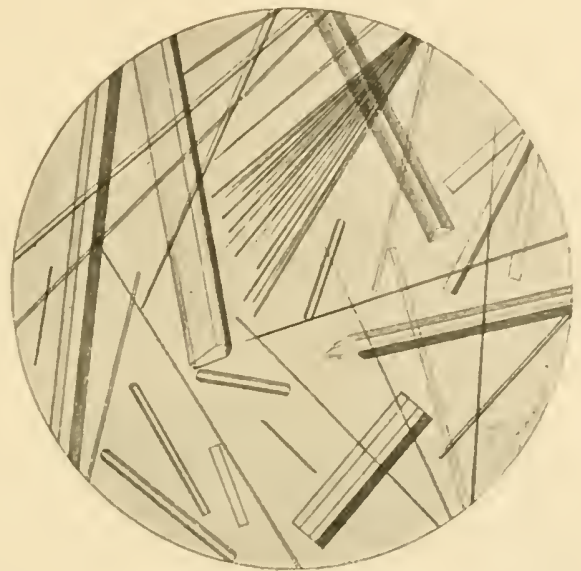

Fig. 55.-Bile Salts.

process being held in the liver. Bilirubin has the same percentage composition as hematoporphyrin, which may be produced from hematin. It is a specific product of the liver cells, but may also be formed in other parts of the body. The pigment may be isolated in the form of a reddish-yellow powder or may be obtained in part, in the form of reddish-

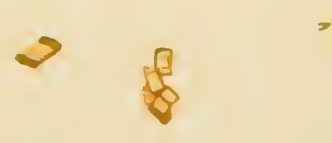

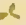

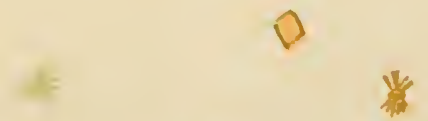

Fig. 56.-Bilirubin (Hesatoidin). (Ogden.)

yellow rhombic plates (Fig. 56) upon the spontaneous evaporation of its chloroform solution. The crystalline form of bilirubin is practically the same as that of hematoidin. It is easily soluble in chloroform, somewhat less soluble in alcohol and only slightly soluble in ether and benzene. Bilirubin has the power of combining with 
certain metals, particularly calcium, to form combinations which are no longer soluble in the solvents of the unaltered pigment. Upon long standing in contact with the air, the reddish-yellow bilirubin is oxidized with the formation of the green biliverdin. Bilirubin occurs in animal fluids as soluble bilirubin-alkali.

Solutions of bilirubin exhibit no absorption bands. If an ammoniacal solution of bilirubin-alkali in water is treated with a solution of zinc chloride, however, it shows bands similar to those of bilicyanin (Absorption Spectra, Plate II), the two bands between $\mathrm{C}$ and $\mathrm{D}$ being rather well defined.

Biliverdin is particularly abundant in the bile of herbivora. It is soluble in alcohol and glacial acetic acid and insoluble in water, chloroform, and ether. Biliverdin in formed from bilirubin upon oxidation. It is an amorphous substance, and in this differs from bilirubin which may be at least partly crystallized under proper conditions. Biliverdin may be obtained in the form of a green powder. In common with bilirubin, it may be converted into hydrobilirubin by nascent hydrogen.

The neutral solution of bilicyanin or cholecyanin is bluish green or steel blue and possesses a blue fluorescence, the alkaline solution is green with no appreciable fluorescence, and the strongly acid solution is violet blue. The alkaline solution exhibits three absorption bands, the first a dark, well-defined band between $\mathrm{C}$ and $\mathrm{D}$, somewhat nearer $\mathrm{C}$; the second a less sharply defined band extending across $\mathrm{D}$ and the third a rather faint band between $\mathrm{E}$ and $\mathrm{F}$, near E (Absorption Spectra, Plate II). The strongly acid solution exhibits two absorption bands, both lying between $\mathrm{C}$ and $\mathrm{E}$ and separated by a narrow space near $\mathrm{D}$. A third band, exceedingly faint, may ordinarily be seen between $\mathrm{b}$ and $\mathrm{F}$.

Bile pigments are converted into urobilinogen (urobilin) in the intestine. This is absorbed, carried to the liver and reconverted into bile pigment. In diseases of the liver the liver cell loses the capacity to convert the urobilinogen and this is then excreted in the urine. The presence of urobilinogen in urine, therefore, may be considered as an index of functional liver incapacity. ${ }^{1}$

Biliary calculi, otherwise designated as biliary concretions or gall stones, are frequently formed in the gall-bladder. These deposits may be divided into three classes, cholesterol calculi, pigment calculi, and calculi made up almost entirely of inorganic material. This last class of calculus is formed principally of the carbonate and phosphate of calcium and is rarely found in man although quite common to cattle. The pigment calculus is also found in cattle, but is more common to man than the inorganic calculus. This pigment calculus ordinarily

${ }^{1}$ Rowntree, Hurwitz and Bloomfield: Johns Hopkins Hospital Bulletin, Nov., I9r3. 
consists principally of bilirubin in combination with calcium; biliverdin is sometimes found in small amount. The cholesterol calculus is the one found most frequently in man. These may be formed almost entirely of cholesterol, in which event the color of the calculus is very light, or they may contain more or less pigment and inorganic matter mixed with the cholesterol, which tends to give us calculi of various colors.

For discussion of cholesterol see page 355 .

\section{Experiments oN Bile}

I. Reaction.-Test the reaction of fresh ox bile to litmus, phenolphthalein and Congo red.

2. Nucleprotein.-Acidify a small amount of bile with dilute acetic acid. A precipitate of nucleoprotein forms. Bile acids will also precipitate here under proper conditions of acidity.

3. Inorganic Constituents.-Test for chlorides, sulphates, and phosphates (see page 59 ).

4. Tests for Bile Pigments.-Practically all of these tests for bile pigments are based on the oxidation of the pigment, by a variety of reagents, with the formation of a series of colored derivatives, e.g., biliverdin (green), bilicyanin (blue), choletelin (yellow).

(a) Gmelin's Test.-To about 5 c.c. of concentrated nitric acid in a test-tube add 2-3 c.c. of diluted bile carefully so that the two fluids do not mix. At the point of contact note the various colored rings, green, blue, violet, red and reddish yellow. Repeat this test with different dilutions of bile and observe its delicacy.

(b) Rosenbach's Modification of Gmelin's Test.-Filter 5 c.c. of diluted bile through a small filter paper. Introduce a drop of concentrated nitric acid into the cone of the paper and note the succession of colors as given in Gmelin's test.

(a) Nakayama's Reaction.--To 5 c.c. of diluted bile in a test-tube add an equal volume of a Io per cent solution of barium chloride, centrifugate the mixture, pour off the supernatant fluid, and heat the precipitate with 2 c.c. of Nakayama's reagent. ${ }^{1}$ In the presence of bile pigments the solution assumes a blue or green color.

(d) Huppert's Reaction.-Thoroughly shake equal volumes of undiluted bile and milk of lime in a test-tube. The pigments unite with the calcium and are precipitated. Filter off the precipitate, wash it with water, and transfer to a small beaker. Add alcohol acidified slightly with hydrochloric acid and warm upon a water-bath until the solution becomes colored an emerald green.

In examining urine for bile pigments, according to Steensma, this procedure may give negative results even in the presence of the pigments, owing to the fact that the acid-alcohol is not a sufficiently strong oxidizing agent. He therefore suggests the addition of a drop of a 0.5 per cent solution of sodium nitrite to the acid-alcohol mixture before warming on the water-bath. Try this modification also.

(e) Hammarsten's Reaction.-To about 5 c.c. of Hammarsten's reagent ${ }^{2}$ in a

1 Prepared by combining 99 c.c. of alcohol and I c.c. of fuming hrdrochloric acid containing 4 grams of ferric chloride per liter.

${ }^{2}$ Hammarsten's reagent is made by mixing I volume of 25 per cent nitric acid and I 9 volumes of 25 per cent hydrochloric acid and then adding I volume of this acid mixture to 4 volumes of 95 per cent alcohol. 
small evaporating dish add a few drops of diluted bile. A green color is produced. If more of the reagent is now added the play of colors as observed in Gmelin's test may be obtained.

(f) Smith's Test.-To $2-3$ c.c. of diluted bile in a test-tube add carefully about 5 c.c. of dilute tincture of iodine ( $\mathrm{I}: \mathrm{ro}$ ) so that the fluids do not mix. A play of colors, green, blue and violet, is observed. In making this test upon the urine ordinarily only the green color is observed.

(g) Salkowski-Schipper's Reaction.-To to c.c. of diluted bile in a test-tube add 5 drops of a 20 per cent solution of sodium carbonate and Io drops of a 20 per cent solution of calcium chloride. Filter off the resultant precipitate upon a hardened filter paper and wash it with water. Remove the precipitate to a small porcelain dish, add 3 c.c. of an acid-alcohol mixture ${ }^{1}$ and a few drops of a dilute solution of sodium nitrite and heat. The production of a green color indicates the presence of bile pigments.

(h) Bonanno's Reaction. ${ }^{2}$ - Place 5-10 c.c. of diluted bile in a small porcelain evaporating dish and add a few drops of Bonanno's reagent. ${ }^{3}$ An emerald-green color will develop.

5. Tests for Bile Acids.-(a) Sucrose- $\mathrm{H}_{2} \mathrm{SO}_{4}$ Test (Pettenkofer).-To 5 c.c of diluted bile in a test-tube add 5 drops of a 5 per cent solution of sucrose. Now run about 2-3 c.c. of concentrated sulphuric acid carefully down the side of the tube and note the red ring at the point of contact. Upon slightly agitating the contents of the tube the whole solution gradually assumes a reddish color. As the tube becomes warm, it should be cooled in running water in order that the temperature of the solution may not rise above $70^{\circ} \mathrm{C}$.

It is claimed that this test is not satisfactory in the presence of protein and chromogenic substances which yield interfering colors with sulphuric acid.

(b) Furfural- $\mathrm{H}_{2} \mathrm{SO}_{4}$ Test.-Mylius's Modification of Pettenkofer's Test.To approximately 5 c.c. of diluted bile in a test-tube add 3 drops of a very dilute (I : I000) aqueous solution of furfural,

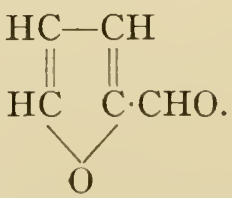

Now run about 2-3 c.c. of concentrated sulphuric acid carefully down the side of the tube and note the red ring as above. In this case, also, upon shaking the tube the whole solution is colored red. Keep the temperature of the solution below $70^{\circ} \mathrm{C}$. as before.

(c) Foam Test (v. Udránsky). - To 5 c.c. of diluted bile in a test-tube add 3-4 drops of a very dilute ( $I:$ rooo) aqueous solution of furfural. Place the thumb over the top of the tube and shake the tube until a thick foam is formed. By means of a small pipette add $2-3$ drops of concentrated sulphuric acid to the foam and note the dark pink coloration produced. alcohol.

1 Made by adding 5 c.c. of concentrated hydrochloric acid to 95 c.c. of 96 percent

${ }_{2}^{2}$ Il Tommasi, 2, No. $2 \mathrm{I}$.

${ }^{3}$ This reagent may be prepared by dissolving 2 grams of sodium nitrite in rooc.c. of concentrated hydrochloric acid. 
(d) Surface Tension Test (Hay).--This test is based upon the principle that bile acids have the property of reducing the surface tension of fluids in which they are contained. The test is performed as follows: Cool about ro c.c. of diluted bile in a test-tube to $17^{\circ} \mathrm{C}$. or lower and sprinkle a little finely pulverized sulphur upon the surface of the fluid. The presence of bile acids is indicated if the sulphur sinks to the bottom of the liquid, the rapidity with which the sulphur sinks depending upon the quantity of bile acids present in the mixture. The test is said to react with bile acids when they are present in the ratio of $\mathrm{I}: \mathrm{I} 20,000$.

(e) Neukomm's Modification of Pettenkofer's Test.-To a few drops of diluted bile in an evaporating dish add a trace of a dilute sucrose solution and one or more drops of dilute sulphuric acid. Evaporate on a water-bath and note the development of a violet color at the edge of the evaporating mixture. Discontinue the evaporation as soon as the color is observed.

(f) Peptone Test (Oliver).-To 5 c.c. of diluted bile add 2-3 drops of acetic acid, filtering if necessary. Add an equal volume of a I per cent solution of Witte's peptone to the acid solution. A precipitate is produced which is insoluble in excess of acetic acid. This precipitate is a compound of protein and bile acids.

6. Crystallization of Bile Salts.-To 25 c.c. of undiluted bile in an evaporating dish add enough animal charcoal to form a paste and evaporate to dryness on a water-bath. Remove the residue, grind it in a mortar, and transfer it to a small fiask. Add about 50 c.c. of 95 per cent alcohol and boil on a water-bath for 20 minutes. Filter, and add ether to the filtrate until there is a slight permanent cloudiness. Cover the vessel and stand it away until crystallization is complete. Examine the crystals under the microscope and compare them with those shown in Fig. 55, page 205. Try one of the tests for bile acids upon some of the crystals.

7. Analysis of Biliary Calculi.-Grind the calculus in a mortar with ro c.c. of ether. Filter.

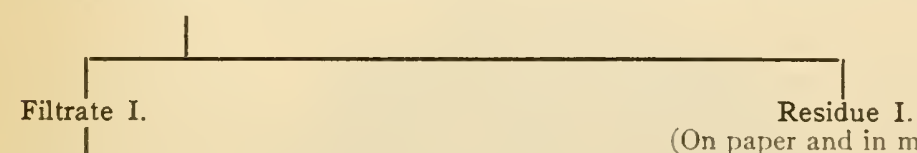

Add an equal volume of 95 per cent alcoholl $^{1}$ to the ether extract, allow the mixture to evaporate and examine for cholesterol crystals (Fig. 57, page 210). (Eor further tests see Experiment 8, p. 2ro.)

Filtrate II.

Test for calcium, phosphales, and iron. Evaporate remainder of filtrate to dryness in porcelain crucible and ignite. Dissolve residue in dilute hydrochloric acid and make alkaline with ammonium hydroxide. Blue color indicates coppcr.

Residue $\boldsymbol{I}$.

(On paper and in mortar.)

Wash with a little water. Dry the tilter paper.

Treat with 5 c.c. chloroform and filter. Treat with dilute hydrochloric acid and filter.

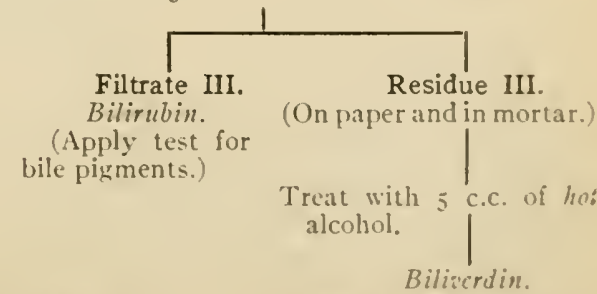

Bilierdin

1 The alcohol is added because of the fact that it is often found that crystallization from pure ether does not yield typical cholesterol crystals. 
8. Tests for Cholesterol.

(a) Microscopical Examination.-Examine the crystals under the microscope and compare them with those shown in Fig. 57, below.

(b) Sulphuric Acid Test (Salkowski).-Dissolve a few crystals of cholesterol in a little chloroform and add an equal volume of concentrated sulphuric acid. A play of colors from bluish-red to cherry-red and purple is noted in the chloroform while the acid assumes a marked green fluorescence.

(c) Acetic Anhydride- $\mathrm{H}_{2} \mathrm{SO}_{4}$ Test (Liebermann-Burchard).-Dissolve a few crystals of cholesterol in 2 c.c. of chloroform in a dry test-tube. Now add ro drops of acetic anhydride and $1-3$ drops of concentrated sulphuric acid. The solution becomes red, then blue, and finally bluish-green in color. This reaction is used in the quantitative determination of cholesterol (see Chapter XVI).

(d) Iodine-sulphuric Acid Test.-Place a few crystals of cholesterol in one of the depressions of a test-tablet and treat with a drop of concentrated sulphuric acid and a drop of a very dilute solution of iodine. A play of colors consisting of violet, blue, green, and red results.

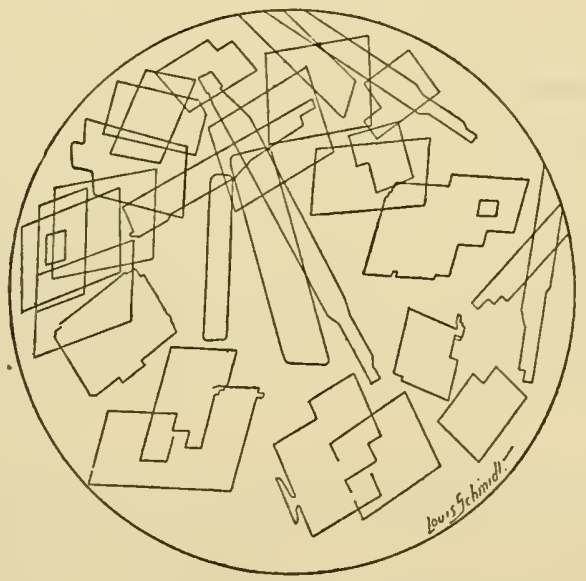

Fig. 57.-Cholesterol.

(e) Schiff's Reaction.-To a little cholesterol in an evaporating dish add a few drops of a reagent made by adding I volume of Io per cent ferric chloride to 3 volumes of concentrated sulphuric acid. Evaporate to dryness over a low flame and observe the reddish-violet residue which changes to a bluish-violet.

9. Preparation of Taurine.-To 300 c.c. of bile in a casserole add roo c.c. of hydrochloric acid and heat until a sticky mass (dyslysin) is formed. This point may be determined by drawing out a thread-like portion of the mass by means of a glass rod, and if it solidifies immediately and assumes a brittle character we may conclude that all the taurocholic and glycoholic acid has been decomposed. Decant the solution and concentrate it to a small volume on the water-bath. Filter the hot solution to remove sodium chloride and other substances which may have separated, and evaporate the filtrate to dryness. Dissolve the residue in 5 per cent hydrochloric acid and precipitate with io volumes of 95 per cent alcohol. Filter off the taurine and recrystallize it from hot water. (Save the alcoholic filtrate for the preparation of glycocoll, p. 2II.) Make the following tests upon the taurine crystals. 
(a) Examine them under the microscope and compare with Fig. 58 .

(b) Heat a crystal upon platinum foil. The taurine at first melts, then turns brown, and finally carbonizes as the temperature is raised. Note the suffocating odor. What is it?

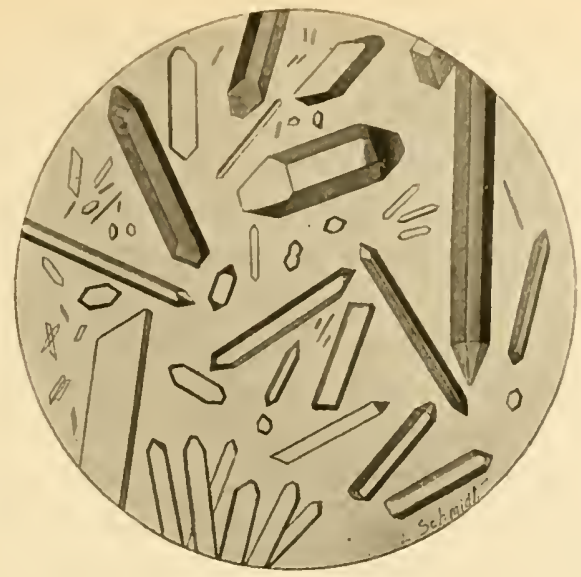

Fig. 58.-TAURINe.

(c) Test the solubility of the crystals in water and in alcohol.

(d) Grind up a crystal with four times its volume of dry sodium carbonate and fuse on platinum foil. Cool the residue, transfer it to a test-tube, and dissolve it in water. Add a little dilute sulphuric acid and note the odor of hydrogen sulphide. Hold a piece of filter paper, moistened with a small amount of lead acetate, over the opening of the test-tube and observe the formation of lead sulphide.

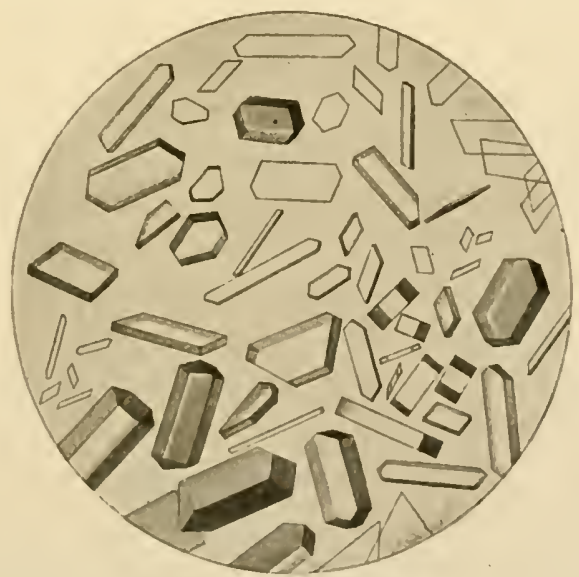

Fig. 59.-Gilicocoll.

Io. Preparation of Glycocoll.-Concentrate the alcoholic filtrate from the last experiment (9) until no more alcohol remains. The glycocoll is present here in the form of an hydrochloride and may be liberated from this combination by the addition of freshly precipitated lead hydroxide or by lead hydroxide solution. Remove the lead by hydrogen sulphide. Filter and decolorize the filtrate by animal charcoal. Filter again, concentrate the filtrate, and set it aside for crystallization. Glycocoll separates as colorless crystals (Fig. 50). 


\section{CHAPTER XIII}

\section{PUTREFACTION PRODUCTS}

THE putrefactive processes in the intestine are the result of the action of bacteria upon the protein material present. This bacterial action which is the combined effort of many forms of micro-organisms is confined almost exclusively to the large intestine. Some of the products of the putrefaction of proteins are identical with those formed in tryptic digestion, although the decomposition of the protein material is much more extensive when subjected to putrefaction. Some of the more important of the putrefaction products are the following: Indole, skatole, paracresol, phenol, para-oxyphenylpropionic acid, para-oxyphenylacetic acid, volatile fatty acids, hydrogen sulphide, methane, methyl mercaptan, hydrogen, and carbon dioxide, besides proteoses, peptones, peptides, ammonia, and amino-acids. Basic substances such as choline, neurine, putrescine and cadaverine are present under certain conditions. Of the putrefaction products the indole, skatole, phenol, and paracresol appear in part in the urine as ethereal sulphuric acids, whereas the oxyacids mentioned pass unchanged into the urine. The potassium indoxyl sulphate (page 386) content of the urine is a rough indicator of the extent of the putrefaction within the intestine.

The portion of the indole which is excreted in the urine is first subjected to a series of changes within the organism and is subsequently eliminated as indican. These changes may be represented thus:

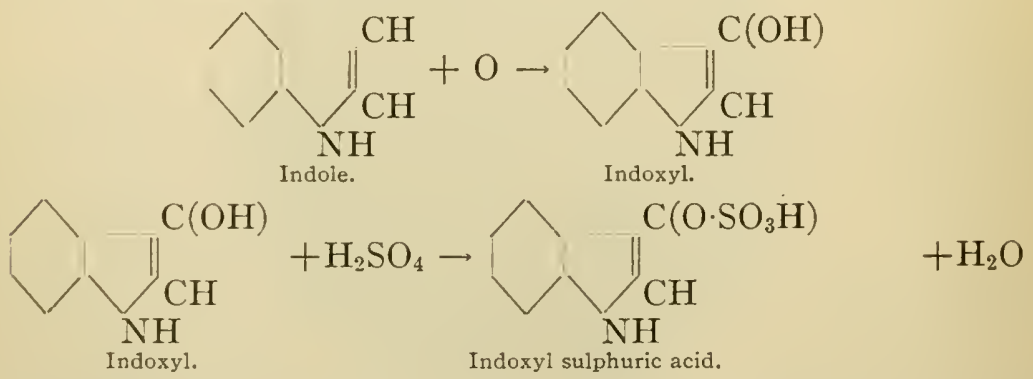

In the presence of potassium salts the indoxyl sulphuric acid is then transformed into indoxyl potassium sulphate (or indican),

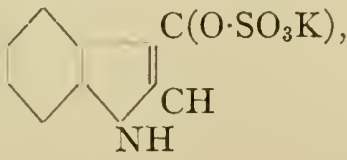

and eliminated as such in the urine. 
Indican may be decomposed by treatment with concentrated hydrochloric acid (see tests on page 387 ) into sulphuric acid and indoxyl. The latter body may then be oxidized to form indigo-blue thus:

2

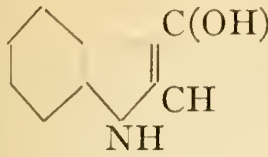

Indoxyl.

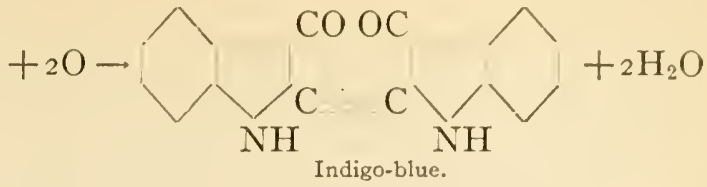

This same reaction may also occur under pathological conditions within the organism, thus giving rise to the appearance of crystals of indigo-blue in the urine.

Skatole or methyl indole possesses the following structure:

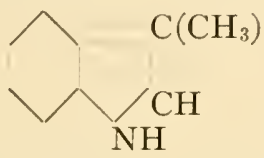

In common with indole it is changed within the organism and eliminated in the form of a chromogenic substance. Skatole is, however, of less importance as a putrefaction product than indole and ordinarily occurs in much smaller amount. The tryptophane group of the protein molecule yields the indole and skatole formed in intestinal putrefaction, but the reasons for the transformation of the major portion of this tryptophane into indole and the minor portion into skatole are not well understood. Indole is more toxic than skatole.

Phenol occurs in fairly large amount in certain abnormal conditions of the organism, but ordinarily the amount is very small. It is probably derived from the tyrosine group of the protein molecule. Phenol is conjugated in the liver to form phenyl potassium sulphate and appears in the urine in this form (Baumann, and Herter). Para-cresol occurs in the urine as cresyl potassium sulphate.

Regarding the claim of Nencki that methyl mercaptan is formed as a gas during intestinal putrefaction it is an important fact that Herter $^{1}$ was unable to detect the mercaptan in fresh feces. He was, therefore, not inclined to accept the theory that methyl mercaptan is formed in ordinary intestinal putrefaction but believed that it may be formed in exceptional cases. Hydrogen sulphide is, however, formed in all cases of intestinal putrefaction.

It has been demonstrated that putrefaction processes in the humin intestine may be retarded by the ingestion of a carbohydrate dict. ${ }^{2}$ The putrefactive organisms are facultatice organisms and prefer a carbo-

1Herter: "Bacterial Infections of the Digestive Tract, p. 227."

${ }^{3}$ Kendall: Jour. Med. Res., 24, 411, 19I1; also Pedialrics, 23, No. 9, I9Io. 
hydrate medium if it is available. These organisms are also unable to exert their maximum activity in an acid medium and therefore the acids resulting from the carbohydrate fermentation would tend to lessen their activity.

It has been shown by Kutscher and his associates ${ }^{1}$ that many acids and bases formed in putrefaction and which have been considered as originating alone from bacterial action, may also be formed in certain phases of metabolism in both the plant and animal kingdoms. These transformation products of amino-acids have been termed "aporrhegmas." The following aporrhegmas may result from putrefaction processes:

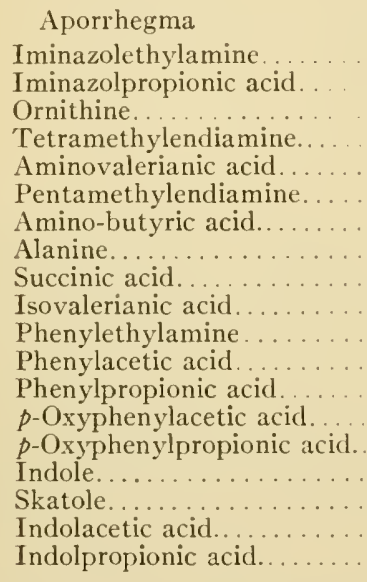

Iminazolethylamine

Iminazolpropionic acid

Ornithine.

Tetramethylendiamine.

Aminovalerianic acid.

Amino-butyric acid.

Alanine.

Isovalerianic acid

Phenylethylamine.

Phenylacetic acid

Phenylpropionic acid

p-Oxyphenylacetic acid

p-Oxyphenylpropionic acid.

Indole... . .

Indolacetic acid.

Indolpropionic acid.

Amino-acid source

Histidine.

Arginine.

Lysine.

Glutamic acid.

Aspartic acid.

Leucine.

Phenylalanine.

Tyrosine.

Tryptophane.

\section{Experiments on Putrefaction Products}

In many courses in physiological chemistry the instructors are so limited for time that no extended study of the products of putrefaction can very well be attempted. Under such conditions the scheme here submitted may be used profitably in the way of demonstration. Where the number of students is not too great, a single large putrefaction máy be started, and, after the initial distillation, both the resulting distillate and residue may be distributed to the members of the class for individual manipulation.

Preparation of Putrefaction Mixture.-Place a weighed mixture of coagulated egg albumin and ground lean meat in a flask or bottle and add approximately 2 liters of water for every kilogram of protein used. Sterilize the vessel and contents, inoculate with the colon bacillus, and keep at $40^{\circ} \mathrm{C}$. for two or three weeks. If cultures of the colon bacillus are not available, add 60 c.c. of a cold saturated

${ }^{1}$ Ackermann and Kutscher: Zeil. physiol. Chem., 69, 265, rgro.

Ackermann: Ibid., 273.

Engeland and Kutscher: Ibid., 282. 
solution of sodium carbonate for every liter of water previously added and inoculate with some putrescent material (pancreas or feces). ${ }^{2}$ Mix the putrefaction mixture very thoroughly by shaking and insert a cork furnished with a glass tube to which is attached a wash bottle containing a 3 per cent solution of mercuric cyanide. This device is for the purpose of collecting the methyl mercaptan, a gas formed during the process of putrefaction. It also serves to diminish the odor arising from the putrefying material. Place the putrefaction mixture at $40^{\circ} \mathrm{C}$. for two or three weeks and at the end of that time make a separation of the products of putrefaction according to the following directions:

Subject the mixture to distillation until the distillate and residue are approximately equal in volume.

\section{PART I}

\section{MANIPULATION OF THE DISTILLATE}

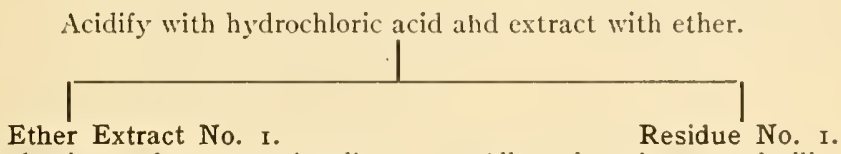

Add an equal volume of water, make alkaline with potassium hydroxide, and shake thoroughly.

Allow the ether to volatilize. Evaporate and detect ammonium chloride crystals (Fig. 6o, page 216 ).

Ether Extract No. 2.

Evaporate spontaneously. Indote and skatol remain. 'Try proper reactions (see pages 2 I 8 and 219).
Alkaline Solution No. I.

Acidify with hydrochloric acid, add sodium carbonate, and extract with ether.

\section{Ether Extract No. 3 .}

Evaporate. Detect phenol and cresol

(paracresol). See page 219 .

Ether Extract No. 4.

Evaporate. Volatile fatty acids remain.
Alkaline Solution No. 2 .
with hydrochloric acid, and Acidify with ether.

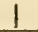

Final Residue.

(Discard.)

\section{DETAILED DIRECTIONS FOR MAKING THE SEPARATIONS INDICATED IN THE SCHEME}

Preliminary Ether Extraction.-This extraction may be conveniently conducted in a separatory funnel. Mix the fluids for extraction in the ratio of two volumes of ether to three volumes of the distillate. Shake very thoroughly for a few moments, then draw off the extracted fluid and add a new portion of the clistillate. Repeat the process until the entire distillate has been extracted. Adel a small amount of fresh ether at each extraction to replace that dissolved by the water in the preceding extraction.

${ }^{1}$ Putrefying protein may be prepared by treating so grams of finely ground lean meat with 100 c.c. of water and 2 c.c. of a saturated solution of sodium carbonate and keeping the mixture at $40^{\circ} \mathrm{C}$. for 24 hours.

- ${ }^{2}$ Concentrated sulphuric acid containing a small amount of isalin may be used as a substitute for mercuric cyanide. When this modification is employed it is necessary to use calcium chloride tubes to exclude moisture from the isatin solution. 
- Residue No. I.- Unite the portions of the distillate extracted as above and allow the ether to volatilize spontaneously. Evaporate until crystallization begins. Examine the crystals under the microscope. Ammonium chloride predominates. Explain its presence.

Ether Extract No. I.-Add equal volume of water, render the mixture alkaline with potassium hydroxide, and shake thoroughly by means of a separatory funnel as before. The volatile fatty acids, contained among the putrefaction products, would be dissolved by the alkaline solution (No. I) whereas any indole or skatole would remain in the ethereal solution (No. 2).

Alkaline Solution No. I.-Acidify with hydrochloric acid and add sodium carbonate solution until the fluid is neutral or slightly acid from the presence of carbonic acid. At this point a portion of the solution, after being heated for a few moments, should possess an alkaline reaction on cooling. Extract the whole mixture with ether in the usual way, using care in the manipulation of the stop cock to

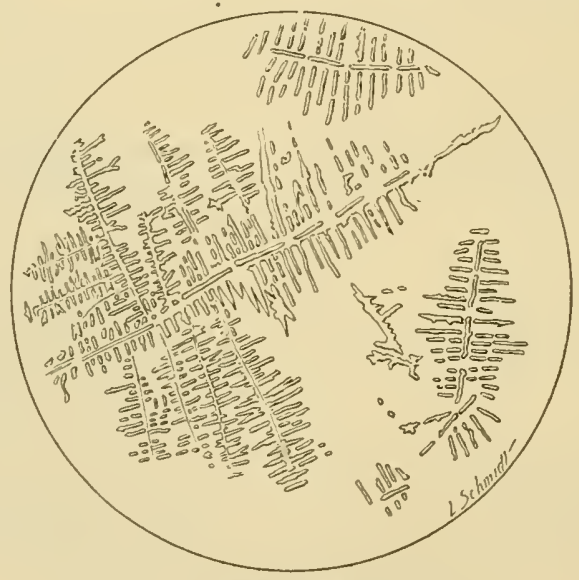

Fig. 60.-AmMonium Chloride.

relieve the pressure due to the evolution of carbon dioxide. The ether (Ether Extract No. 3) removes any phenol or cresol which may be present while the volatile fatty acids will remain in the alkaline solution (No. 2) as alkali salts.

Ether Extract No. 2.-Drive off the major portion of the ether at a low temperature on a water-bath and allow the residue to evaporate spontaneously. Indole and skatole should be present here. Prove the presence of these bodies. For tests for indole and skatole see pages 2 I 8 and 219.

Alkaline Solution No. 2.-Make strongly acid with hydrochloric acid and extract with a small amount of ether, using a separatory funnel. As carbon dioxide is liberated here, care must be used in the manipulation of the stop cock of the funnel in relieving the pressure within the vessel. The volatile fatty acids are dissolved by the ether (Ether Extract No. 4).

Ether Extract No. 3.-Evaporate this ethereal solution on a water-bath. The oily residue contains phenol and cresol. The cresol is present for the most part as paracresol. Add some water to the oily residue and heat it in a flask. Cool and prove the presence of phenol and cresol. For tests for these bodies see page 2 I 9 .

Ether Extract No. 4.-Evaporate on a water-bath. The volatile fatty acids remain in the residue. 
PART II

\section{MANIPULATION OF THE RESIDUE}

Evaporate, filter, and extract with ether.

\section{Ether Extract.}

Evaporate, extract the residue with warm water, and filter.
Consists of a mixture of leucine and tyrosine crystals (Figs. 25, 28 and 139 , pages $76,80$ and 463.$)$
Filtrate No. 2.

Contains oxyacids and skatole-carbonic acid.

\section{Aqueous Solution.}

Evaporate until crystals begin to form. Stand in a cold place until crystallization is complete. Filter.

\section{\begin{tabular}{|l|l|}
\hline & \\
Crystalline Deposit. & Filtrate No. I.
\end{tabular}}

Contains proteose, peplone, aromatic acids, and tryplophane.

\section{DETAUED DIRECTIONS FOR MAKING THE SEPARATIONS INDICATED IN THE SCHEME}

Preliminary Elher Extraction.-This extraction may be conducted in a separatory funnel. In order to make a satisfactory extraction the mixture should be shaken thoroughly. Separate the ethereal solution from the aqueous portion and treat them according to the directions given on page 2 I 5 .

Ether Exlract.-Evaporate this solution on a safety water-bath until the ether has been entirely removed. Extract the residue with warm water and filter.

Aqueous Solution.-Evaporate this solution until crystallization begins. Stand the solution in a cold place until no more crystals form. This crystalline mass consists of impure leucine and tyrosine. Filter off the crystals.

Crystalline Deposit.-Examine the crystals under the microscope and compare them with those reproduced in Figs. 25,28 , and 139 , pages 76 , So, and 463 . Do the forms of the crystals of leucine and tyrosine resemble those previously examined? Make a separation of the leucine and tyrosine and apply typical tests according to directions given on pages $8_{5}$ and 86 .

Fillrale No. I.-Make a test for tryptophane with bromine water (see page ISg). and also with the Hopkins-Cole reagent (see page 98). Use the remainder of the filtrate for the separation of proteoses and peptones. Make the separation according to the directions given on page I 20.

Fillrate No. 2.-This solution contains para-oxyphenylacetic acid, para-oxyphenylpropionic acid and skatole-carbonic acid. Prove the presence of these bodies by appropriate tests. Tests for oxyacids and skatole-carbonic acid are given on page 220 . 


\section{TESTS FOR VARIOUS PUTREFACTION PRODUCTS}

\section{Tests for Indole}

The various tests for indole ond skatole may be carried out upon an aqueous solution of these products or upon an aqueous solution of the residue from Ether Extract No. 2 (see page I72). A distillate secured by distilling a putrefaction mixture first in alkaline and then in acid reaction may also be employed.

I. Herter's $\beta$-Naph thaquinone Reaction.-(a) To a dilute aqueous solution of indole $(\mathrm{I}: 500,000)$ add $\mathrm{I}$ drop of a 2 per cent solution of $\beta$-naphthaquinonesodium-monosulphonate. No reaction occurs. Add a drop of a Io per cent solution of potassium hydroxide and note the gradual development of a blue or blue-green color which fades to green if an excess of the alkali is added. Render the green or blue-green solution acid and note the appearance of a pink color. Heat facilitates the development of the color reaction.

One part of indole in one million parts of water may be detected by means of this test if carefully performed.

(b) If the alkali be added to a more concentrated indole solution before the the introduction of the naphthaquinone the course of the reaction is different, particularly if the indole solution is somewhat more concentrated than that mentioned above and if heat is used. Under these conditions the blue indole compound ultimately forms as fine acicular crystals which rise to the surface.

If we do not wait for the production of the crystalline body but as soon as the blue color forms, shake the aqueous solution with chloroform, the blue color disappears from the solution and the chloroform assumes a pinkish-red hue. This is a distinguishing feature of the indole reaction and facilitates the differentiation of indole from other bodies which yield a similar blue color. A very satisfactory method for the quantitative determination of indole is based upon the principle underlying this test (see chapter on Feces).

2. Formaldehyde Reaction (Konto).- To I c.c. of the material under examination in a test-tube add 3 drops of a 40 per cent solution of formaldehyde and I c.c. of concentrated sulphuric acid. Now agitate the mixture and note the appearance of a violet-red color if a trace of indole is present. The test is said to serve for the detection of indole when present in a dilution of $\mathrm{I}: 700,000$.

Skatole gives a yellow or brown color under the above conditions.

3. Cholera-red Reaction. - To a little of the material under examination in a test-tube add one-tenth its volume of a 0.02 per cent solution of potassium nitrite and mix thoroughly. Carefully run concentrated sulphuric acid down the side of the tube so that it forms a layer at the bottom. Note the purple color. Neutralize with potassium hydroxide and observe the production of a bluish-green color.

4. Nitroprusside Reaction (Legal).-To a small amount of the material under examination in a test-tube add a few drops of a freshly prepared solution of sodium nitroprusside, $\mathrm{Na}_{2} \mathrm{Fe}(\mathrm{CN})_{5} \mathrm{NO}+{ }_{2} \mathrm{H}_{2} \mathrm{O}$. Render alkaline with potassium hydroxide and note the production of a violet color. If the solution is now acidified with glacial acetic acid the violet is transformed into a blue. 
5. Pine Wood Test.-Moisten a pine splinter with concentrated hydrochloric acid and insert it into the material under examination. The woor assumes a cherry-red color.

6. Nitroso-indole Nitrate Test.-Acidify some of the material under examination with nitric acid, add a few drops of a potassium nitrite solution and note the production of a red precipitate of nitroso-indole nitrate. If the residue contains but little indole simply a red coloration will result. Compare this result with the result of the similar test on skatole.

\section{Tests for Skatole}

I. Herter's Para-dimethylaminobenzaldehyde Reaction. '- To 5 c.c. of the distillate or aqueous solution under examination add I c.c. of an acid solution of para-dimethylaminobenzaldehyde 2 and heat the mixture to boiling. A purplishblue coloration is produced ${ }^{3}$ which may be intensified through the addition of a few drops of concentrated hydrochloric acid. If the solution be cooled under running water it loses its purplish tinge of color and becomes a definite blue. The solution at this point may be somewhat opalescent through the separation of uncombined para-dimethylaminobenzaldehyde. Care should be taken not to add an excess of hydrochloric acid inasmuch as the end-reaction has a tendency to fade under the influence of a high acidity.

A rough idea regarding the actual quantity of skatole in a mixture may be obtained by extracting this blue solution with chloroform and subsequently comparing this chloroform solution, by means of a colorimeter (Duboscq), with the maximal reaction, obtained with a skatole solution of known strength.

2. Color Reaction with Hydrochloric Acid.-Acidify some of the residue with concentrated hydrochloric acid. Tote the production of a violet color.

3. Acidify some of the residue with nitric acid and add a few drops of a potassium nitrite solution. Iote the white turbidity. Compare this result with the result of the similar test on indole.

\section{Tests for Phenol and Cresole}

I. Color Test.-Test a little of the solution with Millon's reagent. A red color results. Compare this test with the similar one under Tyrosine (see page 86).

2. Ferric Chloride Test.-Add a few drops of neutral ferric chloride solution to a little of the material under examination. A dirty bluish-gray color is formed.

3. Formation of Bromine Compounds.-Add some bromine water to a little of the fluid under examination. Note the crystalline precipitate of tribromphenol and tribromcresol. The reaction for phenol is as follows:

$$
\underset{\substack{6 \\ \text { Phenol. }}}{\mathrm{C}_{5} \mathrm{OH}}+3 \mathrm{Br}_{2} \rightarrow \underset{\text { Trib:omphenol. }}{\mathrm{C}_{6} \mathrm{H}_{2} \mathrm{Br}_{3} \mathrm{OH}}+{ }_{3} \mathrm{HBr} \text {. }
$$

1.Herter: Bacterial Infections of the Digestise Tract, 190;, n. It $\mathrm{t}$.

2 Made by dissolving 5 grams of para-dimethylaminobenzaldehyde in 100 c.c. of is per cent sulphuric acid.

${ }^{3}$ If the color does not appear add more of the aldehyde solution. 
4. Nitric Acid Test.-Add some nitric acid to some of the material under examination. Heat and note a yellow color due to the production of picric acid (trinitrophenol) from phenol. This is the reaction :

$$
\underset{\text { Phenol. }}{\mathrm{C}_{6} \mathrm{H}_{5} \mathrm{OH}}+\mathrm{HNO}_{3} \rightarrow \underset{\text { Picric acid. }}{\mathrm{C}_{6} \mathrm{H}_{2}\left(\mathrm{NO}_{2}\right)_{3} \mathrm{OH}}+3 \mathrm{H}_{2} \mathrm{O} \text {. }
$$

\section{Tests for Oxyacids}

I. Color Test.-Test a little of the solution with Millon's reagent. A red color results.

2. Bromine Water Test.-Add a few drops of bromine water to some of the filtrate. A turbidity or precipitate is observed.

\section{Test for Skatole-carbonic Acid}

Ferric Chloride Test.-Acidify some of the filtrate with hydrochloric acid, add a few drops of ferric chloride solution, and heat. Compare the end-reaction with that given by phenol. 


\section{CHAP'TER XIV}

\section{FECES}

THE feces are the residual mass of material remaining in the intestine after the full and complete exercise of the digestive and absorptive functions and are ultimately expelled from the body through the rectum.

They may be said to be composed of the following substances:

I. Food residues: (a) those portions of the food which have escaped absorption, and $(b)$ that part of the diet either not digested or incapable of absorption.

2. The remains of the intestinal and digestive secretions not destroyed or reabsorbed.

3. Substances excreted into the intestinal tract, notably salts of calcium. iron, and other metals.

4. The bacterial flora of the intestinal tract.

5. Cellular elements to which may be added, under pathological conditions, blood, pus, mucus, serum, and parasites.

6. Abnormally: enteroliths, gall stones, and pancreatic calculi.

The amount of the fecal discharge varies with the individual and the diet. Upon an ordinary mixed diet various authorities claim that the daily excretion by an adult male will aggregate r IO-I 70 grams with a solid content ranging between 25 and 45 grams; the fecal discharge of such an individual upon a regetable diet will.be much greater and may even be as great as $35^{\circ}$ grams and possess a solid content of 75 grams. In the author's own experience the average daily output of moist feces, calculated on the basis of data secured from the examination of over I000 stools, was about Io० grams. The variation in the normal daily output being so great renders this factor of very little value for diagnostic purposes, except where the composition of the diet is accuratelyknown. Lesions of the digestive tract, a defective absorptive function, or increased peristalsis as well as an admixture of mucus, pus, blood, and pathological products of the intestinal wall may cause the total amount of excrement to be markedly increased. An idea of the rariation of the percentage of dry matter in the feces eracuated after the ingestion of different diets may be gathered from a consideration of the following table. ${ }^{1}$

'Schmidt \& Strasburger: "Die Fäzes des Menschen", Berlin ror 5 
INFLUENCE OF DIET ON FECAL DRY MATTER

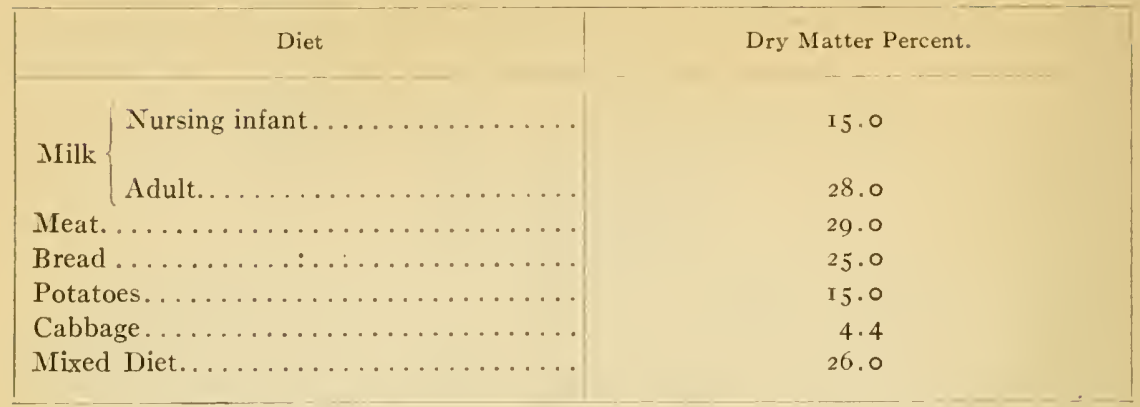

The fecal pigment of the normal adult is hydrobilirubin This. pigment originates from the bilirubin which is secreted into the intestine in the bile, the transformation from bilirubin to hydrobilirubin being brought about through the activity of certain bacteria. Hydro-

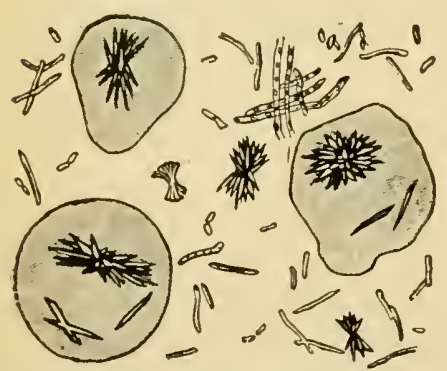

Fig. 6I.-HeMatoidin CrystAls FROM ACHOLIC Stools. (v. Jaksch.)

Color of crystals same as the color of those in Fig. 56, page 205. bilirubin is sometimes called stercobilin and bears a close resemblance to urobilin or may even be identical with that pigment. Neither bilirubin nor biliverdin occurs normally in the fecal discharge of adults, although the former may be detected in the excrement of nursing infants. If these pigments are found in the feces of adults, they indicate an abnormally rapid transit through the large bowel thus preventing their transformation into hydrobilirubin. Frequently, in some way as yet unknown, probably through the agency of certain bacterial processes, colorless hydrobilirubinogen (leucohydrobilirubin) is formed which after the passage of the movement and exposure to air is reconverted into hydrobilirubin. This may explain in some cases the darkening of the stool when exposed to the air. The most important factor in determining the color of the fecal discharge is the diet. A mixed diet, for instance, produces stools which vary in color from light to dark brown, an exclusive meat diet gives rise to a brownish-black stool, whereas the stool resulting from a milk diet is invariably light colored. Certain pigmented foods, such as the chlorophyllic vegetables and various varieties of berries, each afford stools having a characteristic color. Certain drugs act in a similar way to color the fecal discharge. This is well illustrated by the occurrence of green stools following the use of calomel, of black stools after bismuth ingestion, and of yellow stools following the administration of rhubarb, senna or santonin. The green 
color of the calomel stool is generally believed to be due to biliverdin. $\mathrm{v}$. Jaksch, however, claims to have proven this view to be incorrect since he was able to detect hydrobilirubin (or urobilin) but no biliverdn in stools after the administration of calomel. 'The bismuth stool was at one time thought to derive its color from the black sulphide which is formed from the subnitrate of bismuth. We now know that the color is due to the reduction of the bismuth compound (subnitrate) to bismuth suboxide. In cases of biliary obstruction the grayish-white acholic stool is formed.

Under normal conditions the odor of feces is due to skatole and indole, two bodies formed in the course of putrefactive processes occurring within the intestine (see page 212). Such bodies as methane, methyl mercaptan, and hydrogen sulphide may also add to the disagreeable character of the odor. The intensity of the odor depends to a large degree upon the character of the diet, being very marked in stools from a meat diet, much less marked in stools from a vegetable diet, and frequently hardly detectable in stools from a milk diet. Thus the stool of the infant is ordinarily nearly odorless and any decided odor may generally be readily traced to some pathological source.

A neutral reaction ordinarily predominates in normal stools, al though slightly alkaline or even acid stools are met with. The acid reaction is encountered much less frequently than the alkaline, and then commonly only following a vegetable diet.

Experiments in which the actual hydrogen ion concentration of the feces was determined indicate that the reaction of the excreta is uniformly slightly alkaline. ${ }^{2}$ Pronounced dietary changes, e.g. low protein diet, high protein diet, fasting, water drinking with meals, produce at most only minor changes in the reaction of the feces.

The form and consistency of the stool is dependent, in large measure, upon the nature of the diet. Under normal conditions the consistency may vary from a thin, pasty discharge to a firmly formed stool. Stools which are exceedingly thin and watery ordinarily have a pathological significance. In general the feces of the carnivorous animals is of a firmer consistency than that of the herbivora.

The continued ingestion of a diet which is very thoroughly digested and absorbed is frequently accompanied by the formation of dry. hard fecal masses (scybala). Constipation generally results, due to the small bulk of the feces and its lack of moisture. At present the formation of scybala is considered pathological, as an expression of spastic constipation. To counteract this tendency toward constipation the ingestion

${ }^{1}$ Quincke: Mïnch. med. Woch., p. $854,1896$.

${ }^{2}$ Howe and Hawk: Jour. Biol. Chemb., I I, I 29, I9I 2. 
of agar-agar ${ }^{1}$ has been suggested. ${ }^{2}$ This agar is relatively indigestible and readily absorbs water (about 16 times its weight), thus forming a bulky fecal mass which is sufficiently soft to permit of easy evacuation. The function of agar is not limited to its use in connection with constipation; it may serve in other capacities as an aid to intestinal therapeutics by serving as a vehicle for certain drugs. ${ }^{3}$

It is frequently desirable for clinical or experimental purposes to make an examination of the fecal output which constitutes the residual mass from a certain definite diet. Under such conditions, it is customary to cause the person under observation to ingest some substance, at the beginning and end of the period in question, which shall sufficiently differ in color and consistency from the surrounding feces as to render comparatively easy the differentiation of the feces of that period from the feces of the immediately preceding and succeeding periods. One of the most satisfactory methods of making this "separation" is by means of the ingestion of a gelatin capsule containing about 0.2 gram of powdered charcoal at the beginning and end of the period under observation. This procedure causes the appearance of two black zones of charcoal in the fecal mass and thus renders comparatively simple the differentiation of the feces of the intermediate period. Carmine ( 0.3 gram) may be used in a similar manner and forms two dark red zones. Some similar method for the "separation of feces" is universally practised in connection with the scientifically accurate type of nutrition or metabolism experiment which embraces the collection of useful data regarding the income and outgo of nitrogen and other elements.

Among the macroscopical constituents of the feces may be mentioned the following: Intestinal parasites, undigested food particles, gall stones, pathological products of the intestinal wall, enteroliths, intestinal sand, and objects which have been accidentally swallowed.

The fecal constituents which at various times and under different conditions may be detected by the use of the microscope are as follows: Constituents derived from the food, such as muscle fibers, connectivetissue shreds, starch granules, and fat; form elements derived from the intestinal tract, such as epithelium, erythrocytes, and leucocytes; mucus; pus corpuscles; parasites and bacteria. In addition to the constituents named the following crystalline deposits may be detected: cholestcrol, koprosterol, soaps, fatty acid, fat, hematoidin, "Iriple phos-

${ }^{1}$ Agar-agar is a product prepared from certain types of Asiatic sea-weed. It is a carbohydrate and is classified as a galactan in the polysaccharide group.

2 Mendel: Zent.f. ges. Physiol. u. Path. des Stoffw., No. 17, p. 1, x908; Schmidt: Münch. med. Woch., 52, 1970, 1905.

${ }^{3}$ Einhorn: Berl. klin. Woch., 49, I I3, I912. 
phate," Charcot-Leyden crystals, and the oxalate, carbonate, phosphate, sulphate, and lactate of calcium. (See Figs. 64 to 69, pp. 230 and 23r.)

The koprosterol of the feces is similar to cholesterol, and may be formed by the reduction of the latter. It responds to cholesterol color tests and has the same solubility, but possesses a lower melting-point and crystallizes in fine needles instead of plates such as cholesterol forms.

The detection of minute quantities of blood in the feces ("occult blood") has recently become a recognized aid to a correct diagnosis of certain disorders. In these instances the hemorrhage is ordinarily so slight that the identification by means of macroscopical characteristics as well as the microscopical identification through the detection of erythrocytes are both unsatisfactory in their results. Of the tests given for the cletection of "occult blood" the benzidine reaction and the ortho-tolidin and hematein tests (page 233) are probably the most satisfactory. Since "occult blood" occurs with considerable regularity and frequency in gastrointestinal cancer and in gastric and duodenal ulcer, its detection in the feces is of especial value as an aid to a correct diagnosis of these disorders. Certain precautions are essential, such as the establishment of a meat-free diet over a period of time before the specimen is collected. (Feces from a meat diet will give an occult blood reaction with some of the most delicate tests.) Bleeding from the bowel such as is seen in hemorrhoids, as well as the admixture of

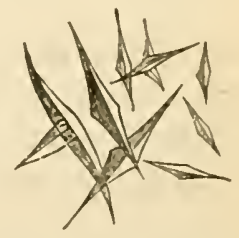
menstrual blood, is to be considered in the interpretation of the result.

It has been quite clearly shown that the intestine of the newly born is sterile. However, this condition is quickly altered and bacteria maybe present in the feces before or after the first ingestion of food. There are three possible means of infecting the intestine, i.e., by way of the mouth or anus or through the blood. The infection by means of the blood seldom occurs except under pathological conditions, thus limiting the general infection to the mouth and anus.

In infants with pronounced constipation two-thirds of the dry substance of the stools has been found to consist of bacteria. In the stools of normal adults probably about one-third of the dry substance is bacteria. ${ }^{1}$ The average excretion of dry bacteria in 24 hours for an adult is about $S$ grams. The output of fecal bacteria has been found to undergo a decrease under the influence of water drinking with meals.

${ }^{1}$ Schittenhelm and Tollens found bacteria to comprise 42 per cent of the dry matter. This value is, however, undoubtedly too high.

${ }^{2}$ Mattill and Hawk: Jour. Am. Chcm. Soc., 33, 1999, I91r; Hlatherwick and Hawk: Bioch. Bull., 3, 28, 1913., 
There was also a decrease in intestinal putrefaction, ${ }^{1}$ a fact which indicates that at least a part of the bacterial deficit was made up of putrefactive organisms. In some cases over 50 per cent of the total nitrogen of feces has been shown to be bacterial nitrogen. ${ }^{2}$

Various enzymes have been detected in the feces. The first one so demonstrated was pancreatic amylase. ${ }^{3}$ The amylase content of the feces is believed to be an index of the activity of the pancreatic function. ${ }^{4}$ The excretion of this enzyme has been found to increase under the influence of water drinking with meals. ${ }^{5}$ Other enzymes which have been found in the feces under various conditions are trypsin, rennin, maltase, sucrase, lactase, nuclease and lipase. ${ }^{6}$ In an abnormally rapid transit of food through the intestinal tract, such as is seen in certain diarrheas, nearly all of these enzymes may be detected.

Some of the more important organisms met with in the feces are the following:" B. coli, B. lactis aërogenes, Bact. Welchii, B. bifidus, and coccal forms. Of these the first three types mentioned are gas-forming organisms. The production of gas by the fecal flora in dextrosebouillon is subject to great variations under pathological conditions; alterations in the diet of normal persons will also cause wide fluctuations. Data as to the production of gas are of considerable importance in a diagnostic way, although the exact cause of the variations is not yet established. It should be borne in mind in this connection that gas volumes are frequently variable with the same individual. For this reason it is necessary in every instance to follow the gas production for a considerable period of time before drawing conclusions. ${ }^{8}$ While the question of the study of bacterial flora of the feces is a question beyond the range of this work, mention may be made here of the character of the organisms observed by Gram staining of the stool after administration of different types of diet. It has been shown that when the diet is markedly protein, the protein type of flora becomes predominant in the stools. Gram-stained smears show a fairly equal distribution of Gram-negative and Gram-positive organisms. Among the latter are largely the subtiloid organisms with some of the Bact. Welchii, together with a moderate number of diplococci and coccoid forms. Most of the Gram-negative organisms resemble the $B$. coli. When the diet is

\footnotetext{
${ }^{1}$ Hattrem and Hawk: Arch. Int. Med., 7, 610, I9I I; Blatherwick, Sherwin and Hawk: loc. cit.

${ }^{2}$ MacNeal, Latzer and Kerr: Jour. Inf. Dis., 6, I 23, 1909; Mattill and Hawk: Jour. Exp. Med., I4, 433, I9II; Blatherwick and Hawk: Biochem. Bull., 3, 28, I9I3.

${ }^{3}$ Wegscheider: Inaug. Diss., Strassburg, 1875 .

4 Wohlgemuth: Berl. klin. Woch., 47, 3, 92, igro.

${ }^{5}$ Hawk: Arch. Int. Med., 8, 382, I9I.

${ }^{6}$ Ury: Biochem. Zeit., 23, I52, 1909.

${ }^{7}$ Herter and Kendall: Journal of Biological Chemistry, 5, 283, 1908.

3 Herter and Kendall: loc. cit.
} 
carbohydrate the field is strongly Gram positive and has a more homogeneous appearance. The bacteria seen consist chiefly of long slender Gram-positive rods belonging to the $B$. acidophilus and $B$. bifidus groups. ${ }^{1}$

The nitrogen present in the feces consists principally of bacieria, unabsorbed intestinal secretions, epithelial cells, mucus material and food residues. In the early days of nutrition study the fecal nitrogen was believed to consist principally of food residues. We now know that such residues ordinarily make up but a small part of the nitrogen quota of the stools of normal individuals who exercise normal mastication. ${ }^{2}$ When meat has been "bolted," however, from 1/2 gram to I 6 grams of macroscopical meat residues has been found in a single stool. ${ }^{3}$ The phrase "metabolic product nitrogen" is frequently used as a designation for all fecal nitrogen except that present as food residues and bacteria. Bacteria cannot logically be classed under "metabolic' nitrogen since they doubtless develop at the expense of food nitrogen as well as at the expense of that in the form of intestinal secretions. In the accurate study of "protein utilization"4 a correction should be made for "metabolic nitrogen." Data regarding the output of metabolic nitrogen may be secured by determining the fecal nitrogen excretion on a diet of proper energy value but containing no nitrogen. ${ }^{5}$ Agar-agar may be utilized advantageously in connection with such a nitrogen-free diet.

Feces are still excreted from the intestine even when no food is ingested. Carefully conducted fasting experiments have demonstrated this. A dog nourished on an ordinary diet to which bone ash has been added will excrete a grey feces. When fasted such an animal will, after a few days, excrete a small amount of a greenish-brown mass, containing no bone ash. These are fasting feces. It is of a pitch-like consistency and turns black on contact with the air. ${ }^{6}$ Adult fasting men have been found to excrete $7^{-8}$ grams of feces per day, the daily nitrogen value being about o.I gram. ${ }^{7}$ No separating medium such as charcoal or carmine (page 238) should be used in differentiating fasting feces.

In recent years the examination of feces for evidences of parasitism (detection of parasites and their ova) has taken on an added importance. The investigation of the hookworm has been particularly developed.

${ }^{1}$ Cammidge: The Feces of Children and Adults, 19I 4 , p. I 26.

2 Kermauner: Zeit. fiir Biol., 35, 316, 189i.

${ }^{3}$ Foster and Hawk: Jour. A m. Chem. Soc., 37. I 347, 1915.

- The percentage of the ingested protein which is absorbed from the intestine. This may be calculated by subtracting the metabolic nitrogen from the total fecal nitrogen and dividing this value by the food nitrogen.

'Tsuboi: Zeil. für Biol., 35, 68, 1 \$97; Mendel and Fine: Jour. Biol. Chem., I I, 5, 1912.

' Howe and Hawk: Jour. Am. Chem. Soc., 33, 215 , I91.

${ }^{7}$ Howe, Mattill and Hawk: Ibid., 33, 568, I9I. 
(For methods and discussion see Bulletin $\mathrm{r} 35$, Bureau of Animal Industry, U. S. Department of Agriculture, Igr r, M. C. Hall.)

For diagnostic purposes the macroscopical and microscopical examinations of the feces ordinarily yield much more satisfactory data than are secured from its chemical examination. Possibly with the exception of certain examinations for occult blood, the most satisfactory data for diagnostic purposes are secured by microscopical examination. This presupposes a knowledge of microscopical technic and the use of certain microchemical tests, by which much information can be obtained. The principle underlying this examination consists in the study of the actual changes which the various food-stuffs have undergone during digestion. A knowledge of the changes which occur in normal digestion and which are seen in normal feces enables one to readily detect pathological variations. One diet widely used for this purpose is the Schmidt diet which is given below. The modification ${ }^{1}$ described is better adapted to American conditions.

The Schmidt intestinal diet is as follows:

In the morning: 0.5 liter of milk, or if milk does not agree 0.5 liter of cocoa (prepared from 20 grams of cocoa porter, Io grams sugar, 400 grams water, and 100 grams milk). To this add 50 grams zwiebach.

In the forenoon: 0.5 liter oatmeal gruel (made from 40 grams oatmeal), ro grams butter, Ioo grams milk, 300 grams water, I egg strained.

At noon: I 25 grams of chopped beef (raw weight) broiled rare with 20 grams of butter, so that the interior will remain raw. To this add 250 grams potato broth (made of 190 grams mashed potatoes, Ioo grams milk, ro grams of butter).

In the afternoon: as in the morning.

In the erening: as in the forenoon.

This diet necessitates five meals a day especially prepared and does not follow the average American dietary. In simple microscopical examinations for food digestion, the following diet as more closely approximating the ordinary dietary regime is suggested. Should chemical determinations for fat be desired all fat containing foods can be eliminated except those in which its specific content is known and a measured amount of fat given. The feces can then be separated by means of carmine.

Breakfasl:

Modified Schmidi Diet

I 00 grams Cream of wheat or oatmeal

60 grams toast

20 grams butter

250 c.c. milk.

${ }^{1}$ Used by Dr. Rehfuss at Jefferson Hospital. 
Luncheon:

Rice soup (chicken broth with rice)

roo grams green vegetable (asparagus)

Ioo grams mashed potato

60 grams toast

20 grams butter

250 c.c. milk.

4 o'clock:

250 c.c. of milk.

\section{Dinner:}

I 50 grams of chopped meat, grilled on the outside and rare in the center

roo grams green vegetable (spinach)

Ioo grams mashed potatoes

60 grams of toast

20 grams of butter

250 c.c. milk

Stewed fruit.

\section{Experiments on Feces}

I. Macroscopical Examination.- - If the stool is watery pour it into a shallow dish and examine directly. If it is firm or pasty it should be treated with water and carefully stirred before the examination for macroscopical constituents is attempted. The macroscopical constituents may be collected very satisfactorily by means of a double layer of cheese cloth.

A Boas sieve (Fig. 63) may also be used to collect the macroscopical constituents of feces. This sieve is constructed of two easily detachable hemispheres which are held together by means of a bayonet catch. In using the apparatus the feces is spread out upon a very fine sieve contained in the lower hemisphere and a stream of water is allowed to play upon it through the medium of an opening in the upper hemisphere. The apparatus is provided with an orifice in the upper hemisphere through which the feces may be stirred by means of a glass rod during the washing process. After $\mathrm{I}_{5}-30$ minutes washing nothing but the coarse fecal constituents remain upon the sieve.

2. Microscopical Examination.-After the ingestion of the test diet (see Schmidt diet above) for several days, a specimen of the movement is collected. Any gross abnormalities are recorded in the form, consistence, and char-

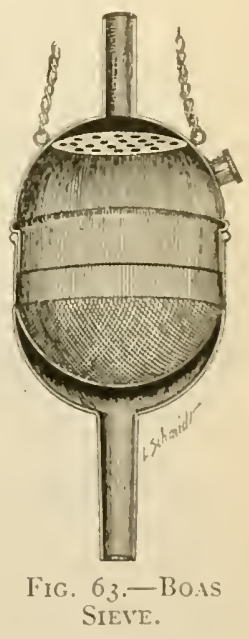
acter of the stool as well as the admixture of certain pathological elements such as pus, blood, mucus, and parasites. The movement is then rubbed out on plates and the presence of undigested food-stuffs sought for. Normally the test diet is almost completely digested and no gross undigested material is found. Therefore the presence of these macroscopic rests is in itself evidence of disturbed digestion. Clean slides and cover-glasses are then prepared and a small representative portion of the movement is placed on each of three slides. The routine clinical method of examination follows: To the first slide 
is added a drop of distilled water and it is then examined with low and high powers.

Meat fibers are readily recognized by their yellowish hyaline appearance possibly with a few strix still visible in the fibers. Should

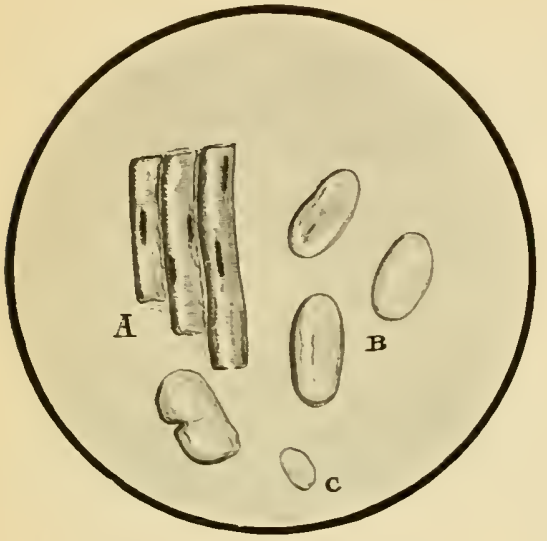

FIG. 64.- $A$, intact undigested meat fibers; $B$, partially digested meat fibers; $C$, almost completely digested meat fibers.

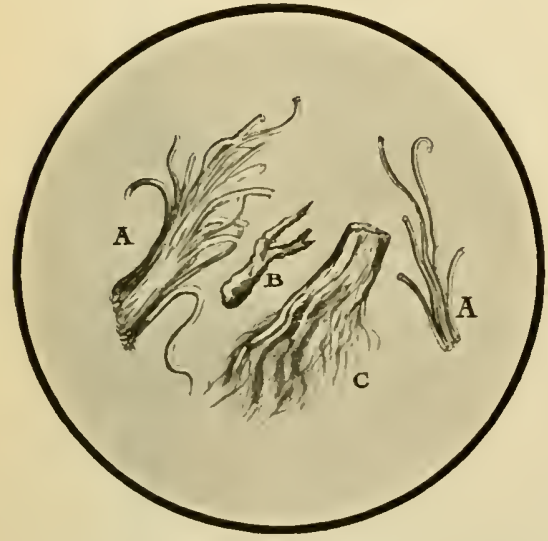

FIg. 66.- $A$, elastic tissue; $B$, white fibrous tissue (macroscopic); $C$, white fibrous tissue (microscopic.)

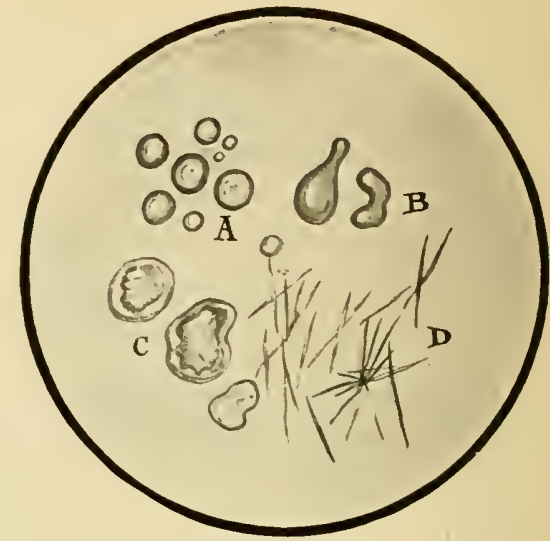

FIG. 65.- $A$, neutral fat; $B$, fatty acid liberated by acetic acid; $C$, soaps; $D$, fatty acid crystals.

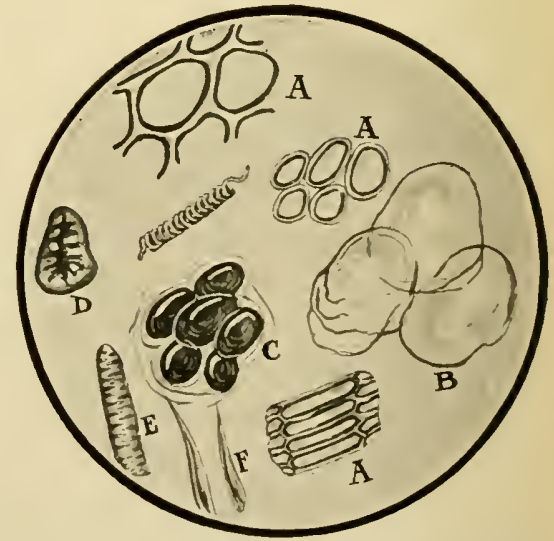

Fig. 6\%- $A$, cellulose remains of vegetables; $B$, empty potato cells; $C$, potato cells filled with starch, and stained with iodine; $D$, hard cells found in pears; $E$, spiral and woody fibers from pith of vegetables; $F$, vegetable hairs.

Figs. 64 to 67.-Microscopical Constituents of Feces.

meat fibers be found bound together by connective tissue or raw connective tissue, either white fibrous or yellow elastic, be noted, it indicates a disturbance of gastric function inasmuch as one of the specific functions of the gastric juice is to dissolve the intercellular tissue 
binding together the fibers. If large numbers of meat fibers are found after a test diet, particularly if the nuclei are still intact in the fibers, the inference of poor or low pancreatic function is justifiable. This is true if it can be demonstrated that the food has been sufficiently. long in its transit through the intestinal tract to permit the pancreatic enzymes to carry on their work. A dilute solution of methylene blue will readily show the nuclei if present.

The second slide is examined for fats and then treated with acetic acid and heated to split any soaps which may be present and form fatty acid.

Fats are met with in three forms (a) neutral fats readily demonstrated by Sudan III, Scharlach R or Osmic acid; (b) fatty acids which are usually found in the form of needle-like crystals soluble in ether, alcohol, and solutions of sodium

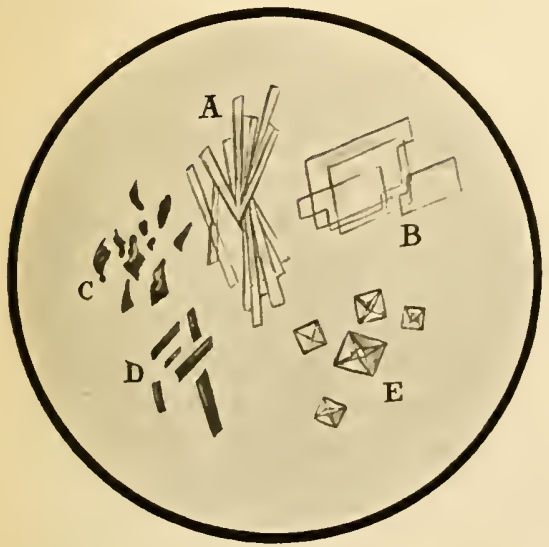

Fig. 68.- $A$, calcium sulphate crystals; $B$, cholesterol crystals; $C$, charcoal detritus; $D$, bismuth sub-oxide crystals; $E$, calcum oxalate crystals.

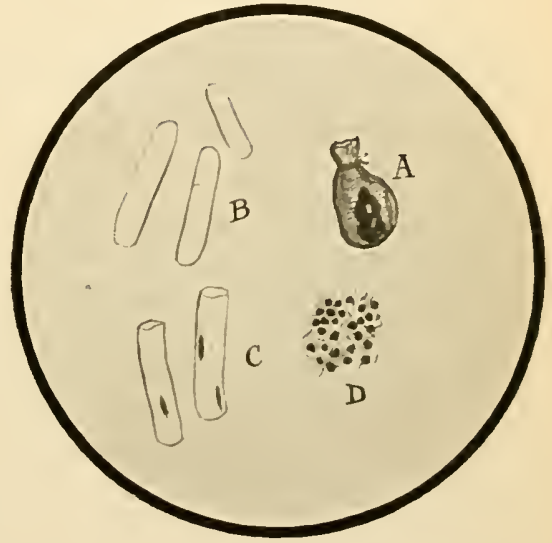

FIG. 69.-A, Schmidt test bag for study: of pancreatic function; $B$, nuclei of meat fibers digested; $C$, nuclei of meat fibers undigested; $D$, undigested stained thymus cells.

Figs. 68 and 69.-Microscopical Cosstituexts of Feces.

hydrate (these crystals do not stain with Sudan III but form drops on being warmed); (c) soaps are usually found in the feces either as amorphous flakes or scallop shell-like formations, but may occasionally occur in crystalline form. The calcium soaps which compose the bulk of the soaps in the feces can be distinguished from the potassium and sodium compounds because of their insolubility in hot water, alcohol, and ether. On heating with 30 per cent acetic acid, fatty acids are set free in drops which crystallize out on cooling.

The estimation of fats is a rather important matter and the trained observer can usually detect disturbances in fat digestion. Normally there are fats present in the movement, but abmormally their quantity is relatively increased either in total fat, or in one of its components. 
While it is true that bacterial activity plays a considerable rôle in the digestion of fats, a marked increase in fat usually indicates pancreatic disease, or a disturbance in pancreatic function. This is, of course, the case only when the amount of fat ingested is not in excess of that which can be readily handled under normal conditions. In cases of pure biliary obstruction without pancreatic involvement, fat-splitting takes place in a normal way, but the fatty acids and soaps formed are not absorbed owing to the absence of bile. Such a movement is full of soaps and fatty acid crystals which on treatment with acetic acid show a marked increase in total fat over normal. Failure of absorption owing to extensive disease of the intestinal mucosa can produce a similar picture but will usually give some cytological evidence of intestinal disease. Pure pancreatic disease gives a marked increase in total and neutral fat with the presence of bile.

Undigested starches are readily recognized by their blue reaction with iodine. This can be studied on the third slide.

This phenomenon is the least frequent among the different forms of pathological digestion and usually indicates food bolting, an excessive ingestion of, or poor preparation of carbohydrate food, or an infection of the bowel with so-called "gärungsdyspepsia" rather than an actual disturbance of pancreatic function inasmuch as the amylolytic function of the pancreas is the most persistent and the last to disappear.

Disturbance in cellulose digestion, the presence of blood, leucocytes, mucus, etc., can all be demonstrated by appropriate technic and represent a chapter in the study of the feces of great diagnostic importance, but one which is beyond the province of this volume. (For further discussion, see page 228. For cuts of fecal constituents found microscopically, see pages 230 and 23I.)

3. Reaction.-Thoroughly mix the feces and apply moist red and blue litmus papers to the surface. If the stool is hard it should be mixed with water before the reaction is taken. Examine the stool as soon after defecation as is convenient, since the reaction may change very rapidly. The reaction of the normal stools of adult man is ordinarily neutral or faintly alkaline to litmus, but seldom acid. Infants' stools are generally acid in reaction. Try the reaction to Congo red paper. Also test the reaction of fecal extract to phenolphthalein.

4. Starch.-If any imperfectly cooked starch-containing food has been ingested it will be possible to detect starch granules by a microscopical examination of the feces. If the granules are not detected by a microscopical examination, the feces should be placed in an evaporating dish or casserole and boiled with water for a few minutes. Filter and test the filtrate by the iodine test in the usual way (see page 45 ).

5. Cholesterol, Koprosterol and Fat.-Introduce about 5 grams of moist feces into a roo c.c. glass-stoppered cylinder. Add 30 c.c. of distilled water and 
25 c.c. of ether, then stopper the cylinder and shake vigorously for five minutes. Allow to separate, pour or pipette off the ethereal solution. Filter and remove the ether by evaporation. The residue contains cholesterol and the mixed fats of the feces. For every gram of fat add about I I/2 grams of solid potassium hydroxide and 25 c.c. of 95 per cent alcohol and boil in a flask on a water-bath for one-half hour, maintaining the volume of alcohol constant. This alcoholicpotash has saponified the mixed fats and we now have a mixture of soaps, cholesterol and koprosterol. Add sodium chloride, in substance, to the mixture and extract with ether to dissolve out the cholesterol and koprosterol. Remove the ether by evaporation and examine the residue microscopically for cholesterol and koprosterol crystals. Try any of the other tests for cholesterol as given on page 2 Io.

6. Blood.-Undecomposed blood may be detected macroscopically. If uncertain, look for erythrocytes under the microscope, and spectroscopically for the spectrum of oxyhemoglobin (see Absorption Spectra, Plate I).

In case the blood has been altered or is present in minute amount ("occult blood"), and cannot be detected by the means just mentioned, the following tests may be tried:

(a) Benzidine Reaction.-Make a thin fecal suspension using about 5 c.c. of distilled water, and heat it to boiling to render oxidizing enzymes inactive. To 2 c.c. of a saturated solution of benzidine in glacial acetic acid add 3 c.c. of 3 per cent hydrogen peroxide and 2-3 drops of the cooled fecal suspension. A clear blue color appears within one to two minutes in the presence of blood. If the mixture is not shaken a ring of color will form at the top. Minute traces of blood are more easily detected by the latter procedure.

Wagner ${ }^{1}$ has simplified the benzidin test so that it can be applied much more conveniently.

Slide Modification.-Take up a little of the solid stool on a match, smear it on an object glass and pour the reagent over it. It turns blue if there is blood present and there is no misleading green tint from fluid. Make the solution as follows: Add a knife-tip of benzidine to 2 c.c. of glacial acetic acid, and add 20 drops of a 3 per cent solution of hydrogen peroxide.

By this dry technic there is no danger of soiling the fingers, and the test is more sensitive than the usual "wet" benzidine test. The smear of stool is either blue or it is not blue. The rapidity of the color change gives some idea as to the proportion of blood in the stool; with much blood present the change to blue is instantaneous.

(b) Ortho-tolidin Test (Ruttan and Hardisty)2 ${ }^{2}$ To I c.c. of a 4 per cent

1 Wagner: Zentbl. fïr Chirurgie, 4 I. No. 2S, ror4.

${ }^{2}$ Ruttan and Hardisty: Canadian Medicul. 1ss'n Journal, Nov., Igr 2, also Biochemical Bull., 2, 225, 1913. 
glacial acetic acid solution of 0 -tolidin ${ }^{1}$ in a test-tube add I c.c. of the solution under examination and I c.c. of 3 per cent hydrogen peroxide. In the presence of blood a bluish color develops (sometimes rather slowly) and persists for some time (several hours in some instances).

This test is said to be as sensitive for the detection of occult blood in feces and stomach contents as is the benzidine reaction. It is also claimed to be more satisfactory for urine than any other blood test. The acetic acid solution may be kept for one month with no reduction in delicacy.

(c) Phenolphthalein Test. ${ }^{2}$-Make a thin fecal suspension using about 5 c.c. of distilled water. Heat to boiling, ${ }^{3}$ cool and add 2 c.c. of the suspension to I c.c. of the phenolphthalein reagent ${ }^{4}$ and a few drops of hydrogen peroxide. A pink or red color promptly forms in the presence of blood.

Schirokauer ${ }^{5}$ makes the statement that a mixture of alcohol and glacial acetic acid will give the phenolphthalein reaction for occult blood. The action of an oxidizing agent will make this reaction more distinct. Von Czylharz and Neustadl ${ }^{6}$ find that a solution of sodium salicylate added to a blood-free extract of feces will give a very deceptive reaction, while feces after the administration of sodium salicylate by mouth gave the same reaction. The same was true of acetyl salicylic acid and other similar drugs. Their studies in clinical cases likewise indicated that the phenolphthalein test was unreliable.

(d) Hematein Reaction for Occult Blood.-Couturier ${ }^{7}$ advises the use of hematein ${ }^{8}$ in testing for occult blood. It is only slightly soluble in water, but gives a pronounced red color. In contact with sodium hydroxide this red solution turns a deep violet blue, giving an insoluble compound of nematein and sodium. This compound, exposed to the air oxidizes after several days, and gives brownish or yellowish compounds, depending on dilution. This change is only hastened a little by the addition of hydrogen peroxide, but if a trace of blood is added to the hydrogen peroxide, it takes place almost instantly. To avoid oxidation, the hematein sodium mixture should be prepared just before use. Three fluids<smiles>CCC[C@H](C)N</smiles>

${ }^{2}$ Boas: Deut. med. Woch., 37, 62, г91 r.

3 Boas suggests using an ether extract of the fecal suspension thus eliminating the necessity of boiling. However, oxidizing enzymes are the main sources of error here and the action is easily and effectively eliminated by boiling. (See White: Boston Medical and Surgical Journal, 164, 876, I91.)

4 Prepared by dissolving $\mathrm{I}-2$ grams of phenolphthalein and 25 grams of $\mathrm{KOH}$ in roo c.c. of distilled water. Add Io grams of powdered zinc and heat gently until the solution is decolorized. Prepared in this way the solution will not deteriorate on standing.

${ }^{5}$ Deutsch. med. Woch., Aug. 6, I9r4.

'Wien. med. Woch., Sept. 5, 19I4.

${ }^{7}$ Lyon Med., 46, 313, I9I4.

${ }^{8}$ Hematein is a brownish-red crystalline substance derived from hematoxylin by the successive action of ammonia and acetic acid. It should not be confused with hematin, the hemoglobin derivative. 
are required: (I) a 0.05 per cent aqueous solution of hematein; (2) a 40 per cent solution of sodium hydroxide and (3) 3 per cent hydrogen peroxide. These will keep almost indefinitely.

The test may be performed as follows: Take $4-5$ c.c. of the liquid specimen in a tube and in another tube take the same amount of material known not to contain blood as a control. To each add 4-5 c.c. of the sodium hydroxide solution and shake. Then to each of the tubes add 2 drops of the hematein solution. A blue color of about equal intensity will develop in both tubes. Then add ro drops of hydrogen peroxide to each tube and compare. If blood is present, the tube containing it will turn very rapidly (in three or four seconds) to violet red, then in twenty seconds to clear brown, in forty seconds to pale yellow while the second tube will not show these changes for several minutes. The reaction is said to detect blood when present in a concentration of I part in 400,000 .

(e) Aloin-turpentine Test.-Mix the stool very thoroughly and take about 5 grams of the mixture for the test. Reduce this sample to a semi-fluid mass by means of distilled water and extract very thoroughly with an equal volume of ether to remove any fat which may be present. Now treat the extracted feces with onethird its volume of glacial acetic acid and ro c.c. of ether and extract very thoroughly as before. The acid-ether extract will rise to the top and may be removed.

Introduce $2-3$ c.c. of this acid-ether solution into a test-tube, add an equal volume of a dilute solution of aloin in 70 per cent alcohol and $2-3$ c.c. of ozonized turpentine and shake the tube gently. If blood is present the entire volume of fluid ordinarily becomes pink and finally cherry red. In some instances the color will be limited to the aloin solution which sinks to the bottom. This color reaction should occur within $\mathrm{I} 5$ minutes in order to indicate a positive test for blood, since the aloin will turn red of itself if allowed to stand for a longer period. The color is ordinarily light yellow in a negative test. Hydrogen peroxide is not a satisfactory substitute for turpentine in the test.

(f) Cowie's Guaiac Test.-To I gram of moist feces add 4-5 c.c. of glacial acetic acid and extract the mixture with 30 c.c. of ether. To I-2 c.c. of the extract add an equal volume of water, agitate the mixture, introduce a few granules of powdered guaiac resin, and after bringing the resin into solution, gradually add 30 drops of old turpentine or hydrogen peroxide. A blue color indicates the presence of blood. Cowie claims that by means of this test an intestinal hemorrhage of I gram can easily be detected by an examination of the feces.

(g) II eber's Guaiac Test.-Mix a little feces with 30 per cent acetic acid to form a fluid mass. Transfer to a test-tube and extract with ether. If blood is present the ether will assume a brownish-red color. Filter off the ether extract and to a portion of the filtrate add an alcoholic solution of guaiac (strength about $1: 60$ ), ${ }^{1}$ drop by drop, until the fluid becomes turbid. Now add hydrogen peroxide or old turpentine. In the presence of blood a blue color is produced (see page $25 S$ ).

(h) Acid-hematin.-Examine some of the ethereal extract from Experiment ( $g$ ) spectroscopically. Note the typical spectrum of acid-hematin (see Absorption Spectra, Plate II).

7. Hydrobilirubin. Schmidt's Test. - Rub up a small amount of feces in a mortar with a concentrated aqueous solution of mercuric chloride. Transfer to a

${ }^{2}$ Buckmaster advises the use of an alcoholic solution of guaiaconic acid instead of an alcoholic solution of guaiac resin. 
shallow, flat-bottomed dish and allow to stand 6-24 hours. The presence of hydrobilirubin will be indicated by a deep red color being imparted to the particles of feces containing this pigment. This red color is due to the formation of hydrobilirubin-mercury. If unaltered bilirubin is present in any portion of the feces that portion will be green in color due to the oxidation of bilirubin to biliverdin.

Another method for the detection of hydrobilirubin is the following: Treat the dry feces with absolute alcohol acidified with sulphuric acid and shake thoroughly. The acidified alcohol extracts the pigment and assumes a reddish color. Examine a little of this fluid spectroscopically and note the typical spectrum of hydrobilirubin (Absorption Spectra, Plate II).

8. Bilitubin. ${ }^{1}$ (a) Gmelin's Test.-Place a few drops of concentrated nitric acid in an evaporating dish or on a porcelain test-tablet and allow a few drops of the feces and water to mix with it. The usual play of colors of Gmelin's test is produced, i.e., green, blue, violet, red, and yellow. If so desired, this test may be executed on a slide and observed under a microscope.

(b) Huppert's Test.- Treat the feces with water to form a semi-fluid mass, add an equal amount of milk of lime, shake thoroughly, and filter. Wash the precipitate with water, then transfer both the paper and the precipitate to a small beaker or flask, add a small amount of 95 per cent alcohol acidified slightly with sulphuric acid, and heat to boiling on a water-bath. The presence of bilirubin is indicated by the alcohol assuming a green color.

Steensma advises the addition of a drop of a 0.5 per cent solution of sodium nitrite to the acid-alcohol mixture before warming on the water-bath. Try this modification also.

9. Bile Acids.-Extract a small amount of feces with alcohol and filter. Evaporate the filtrate on a water-bath to drive off the alcohol and dissolve the residue in water made slightly alkaline with potassium hydroxide. Upon this aqueous solution try any of the tests for bile acids given on page 208 .

I0. Casein.-Extract the fresh feces first with a dilute solution of sodium chloride, and later with water acidified with dilute acetic acid, to remove soluble proteins. Now extract the feces with 0.5 per cent sodium carbonate and filter. Add dilute acetic acid to the filtrate to precipitate the casein, being careful not to add an excess of the reagent as the casein would dissolve. Filter off the casein and test it according to directions given on page $32 \mathrm{I}$. Casein is found principally in the feces of children who have been fed a milk diet. Mucin would also be extracted by the dilute alkali, if present in the feces. What test could you make on the newly precipitated body to differentiate between mucin and casein?

II. Nucleoprotein.-Mix the stool thoroughly with water, transfer to a flask, and add an equal amount of saturated lime water. Shake frequently for a few hours, filter, and precipitate the nucleoprotein with acetic acid. Filter off this precipitate and test it as follows:

(a) Phosphorus.-Test for phosphorus by fusion (see page 129).

(b) Solubility.-Try the solubility in the ordinary solvents.

(c) Protein Color Test.- Try any of the protein color tests.

1 The detection of bilirubin in the feces is comparatively simple provided it is not accompanied by other pigments. When other pigments are present, however, it is difficult to detect the bilirubin and, at times, may be found impossible. 
What proof have you that the above body was not mucin?

What other test can you use to differentiate between nucleoprotein and mucin?

12. Albumin and Globulin.--Extract the fresh feces with a dilute solution of sodium chloride. (The preliminary extract from the preparation of casein (I0), above, may be utilized here.) Filter, and saturate a portion of the filtrate with sodium chloride in substance. A precipitate signifies globulin. Filter off the precipitate and acidify the filtrate slightly with dilute acetic acid. A precipitate at this point signifies albumin. Make a protein color test on each of these bodies.

13. Proteose and Peptone.-Heat to boiling the portion of the sodium chloride extract not used in the last experiment. Filter off the coagulum, if any forms. Acidify the filtrate slightly with acetic acid and saturate with sodium chloride in substance. A precipitate here indicates proteose. Filter it off and test it according to directions given on page I20. Test the filtrate for peptone by the biuret test.

I4. Inorganic Constituents.--Incinerate a small amount of feces in a crucible and dissolve the ash in a small volume of dilute nitric acid. Dilute with water and filter. Make the following tests upon the clear filtrate.

(a) Chlorides.-Acidify with nitric acid and add silver nitrate.

(b) Phosphates.-Acidify with nitric acid, add molybdic solution, and warm gently.

(c) Sulphates.-Acidify with hydrochloric acid, add barium chloride, and warm.

(d) Calcium.-Neutralize with ammonium hydroxide, make slightly acid with acetic acid and add ammonium oxalate. Let stand.

(e) Magnesium.-Neutralize with ammonium hydroxide, and add $\mathrm{Na}_{2} \mathrm{HPO}_{4}$ and excess of $\mathrm{NH}_{4} \mathrm{OH}$. Let stand.

I5. Indole Reactions. - Rub up the stool with water to form a thin paste and distill first in alkaline and then in acid solution. Test the distillate by any of the tests for the detection of indole in putrefaction mixtures (see page 2 I 8 ).

I6. Schmidt's Nuclei Test.-This test serves as an aid to the diagnosis of pancreatic insufficiency. The test is founded upon the theory that cell nuclei are digestible only in pancreatic juice, and therefore that the appearance in the feces of such nuclei indicates insufficiency of pancreatic secretion.

The procedure is as follows: Cubes of fresh beef about $I / 2 \mathrm{~cm}$. square are enclosed in small gauze bags and ingested with a test meal. Subsequently the fecal mass resulting from this test meal is examined, the bag opened, and the condition of the enclosed residue determined. Under normal conditions the nuclei would be digested. Therefore if the nuclei are found to be for the most part undigested, and the intervening period has been sufficient to permit of the full activity of the pancreatic function (at least six hours), it may be considered a sign of pancreatic insufficiency (see Fig. 69, p. 231).

It has been claimed by Steele that under certain conditions the nondigestion of the nuclei may indicate a general lowering of the digestive power rather than a true pancreatic insufficiency.

Kashiwado' has suggested the use of stained cell nuclei in this test. A preparation put out under the name "Gefarbte gewebskerne zur

${ }^{1}$ Kashiwado: Deul. Arch. Klin. Med., ro4, $5^{8}+$, I9r r. 
Pankreasfuntionsprüfung nach Prof. Dr. Schmidt und Dr. Kashiwado" consists of a mixed preparation of thymus cells, the nuclei of which are stained by iron hematoxylin, and lycopodium powder. After administration, the lycopodium, which is readily recognized, is sought for in the stool and when found that portion is examined for the stained thymus cells. Their statement is as follows: If stained nuclei are not found in the feces after an intestinal transit of sufficient duration (at least six hours) normal pancreatic function (external) is indicated. If, however, all or part of the cells are found, a definite disturbance in pancreatic function is present.

17. Influence of Drugs upon the Color of the Stool.-Ingest an ordinary mixed diet, take the indicated dose of one of the following drugs, "separate" the feces (see page 587) and after the "marker" appears note the color of the stools evacuated:

Drug

\begin{tabular}{l} 
Bismuth subnitrate, grams \\
\hline Calomel, mg............ \\
\hline Reduced iron, mg........ \\
\hline Methylene blue, mg....... \\
\hline Manganese dioxide, mg... \\
\hline Hematoxylin, grams...... \\
\hline Rhubarb, c.c. fluid extract.. \\
\hline Senna, c.c. fluid extract... \\
\hline Cambogia, mg........... \\
\hline Santonin, mg............
\end{tabular}

Dose

Color of stool

18. Einhorn's Bead Test. ${ }^{1-T h i s}$ is a method for testing the digestive function. In some respects it is similar to Sahli's desmoid reaction (see Gastric Analysis). The procedure consists in wrapping the material under examination (catgut, fish-bone, raw beef, cooked potatoes, thymus gland or mutton fat, etc.) in gauze to which glass beads of various colors are attached and enclosing gauze and beads in a gelatine capsule. ${ }^{2}$ The gelatine capsule is swallowed and the beads serve to facilitate the separation of the gauze from the feces. The residue

${ }^{1}$ Einhorn: The Post-Graduate, May, 191 2 : Boas' Arch., 12, 26, I906; 13, 35, I907; Ibid., 475; I 5, part 2, I909.

2 Ordinarily two substances are attached to each bead, three beads tied together and enclosed in one capsule. Test capsules may be obtained from Eimer and Amend, New York. 
within the gauze is then examined. If beads appear in much less than 24 hours an accelerated motility is indicated, whereas an interval of 48 hours or over elapsing indicates retarded motility. If gastric function alone is to be studied silk threads are attached to the beads and the latter are withdrawn and examined before they have passed into the intestine.

19. "Separation" of Feces.-In order to become familiar with the method ordinarily utilized in n.etabolism experiments to differentiate the feces which correspond to the food ingested during any given interval, and at the same time to secure data as to the length of time necessary for ingested substances to pass through the alimentary tract proceed as follows: Just before one of the three meals of the day ingest a gelatine capsule (No. oo) containing $0.2-0.3$ of a gram of carmine or charcoal. Make an inspection of all stools subsequently dropped and note the time interval elapsing between the ingestion of the capsule and the appearance of its contents in the feces. Under normal conditions this period is ordinarily 24 hours. This test is thus an index of intestinal motility.

20. Influence of Foods upon the Color of the Stool.-Ingest a diet which contains a liberal quantity of one of the following articles of diet, "separate" the feces (page 587) and after the "marker" appears note the color of the stools evacuated :

Color of stool

Milk.......................... Light yellow or grayish white.

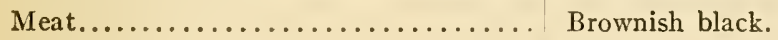

Chlorophyllic vegetables, e.g., spinach.... Greenish.

Non-chlorophyllic vegetables ......... Light brown.

Cherries or blackberries............. Reddish brown.

Cocoa...................... Dark red or chocolate brown.

Coffęe...................... Dark brown.

Corn meal................... Light colored.

21. Quantitative Determination of Fecal Amylase (The Author's ${ }^{1}$ Modification of Wohlgemuth's ${ }^{2}$ Method).-Weigh accurately about 2 grams of fresh feces into a mortar, ${ }^{3}$ add 8 c.c. of a phosphate-chloride solution (O.I mol dihydrogen sodium phosphate and $0.2 \mathrm{~mol}$ disodium hydrogen phosphate per liter of I per cent sodium chloride), 2 c.c. at a time, rubbing the feces mixture to a homogeneous consistency after each addition of the extraction medium. Permit the mixture to stand at room temperature for a half-hour with frequent stirring. We now have a neutral fecal suspension. Transfer this suspension to a 5 c.c. graduated centrifuge tube, being sure to wash the mortar and pestle carefully with the phosphate-chloride solution and add all washings to the suspension in the centrifuge tube. The suspension is now made up to the 15 c.c. mark with the phosphate-chloride solution

${ }^{2}$ Hawk: Arch. Inl. Mcd., 8, 552, I91I.

${ }^{2}$ Wohlgemuth: Berl.klin. Woch., 47, 3, 92, I9 10; also see page i92, this book.

${ }^{3}$ Duplicate determinations should be made. 
and centrifugated for a $\mathrm{I} 5$-minute period, or longer if necessary, to secure satisfactory sedimentation. At this point, read and record the height of the sediment column. Remove the supernatant liquid by means of a bent pipette, transfer it to a 50 c.c. volumetric flask and dilute it to the 50 c.c. mark with the phosphatechloride solution. Mix the fecal extract thoroughly by shaking and determine its amylolytic activity. For this purpose a series of six graduated tubes is prepared, containing volumes of the extract ranging from 2.5 c.c. to 0.078 c.c. Each of the intermediate tubes in this series will thus contain one-half as much fluid as the preceding tube. Now make the contents of each tube 2.5 c.c. by means of the phosphate-chloride solution in order to secure a uniform electrolyte concentration. Introduce 5 c.c. of a I per cent soluble starch solution ${ }^{1}$ and three drops of toluol into each tube, thoroughly mix the contents by shaking, close the tubes by means of stoppers and place them in an incubator at $38^{\circ} \mathrm{C}$. for 24 hours. At the end of this time remove the tubes, fill each to within half an inch of the top with ice-water, add I drop of tenth-normal iodin solution, thoroughly mix the contents and examine the tubes carefully with the aid of a strong light. Select the last tube in the series which shows entire absence of blue color, thus indicating that the starch has been completely transformed into dextrin and sugar, and calculate the amylolytic activity on the basis of this dilution. In case of indecision between two tubes, add an extra drop of the iodin solution and observe them again. ${ }^{2}$

The amylolytic value, Df, of a given stool, may be expressed in terms of i c.c. of the sediment obtained by centrifugation as above described. For example, if it is found that 0.3 I C.c. of the phosphate-chloride extract of the stool acting at $38^{\circ} \mathrm{C}$. for 24 hours completely transformed the starch in 5 C.c. of a I per cent starch solution, then we would have the following proportion:

$$
0.3 \text { I : } 5 \text { (c.c. starch) :: I(c.c. extract) :X }
$$

The value of $\mathrm{X}$ in this case is I6.I, which means that I c.c. of the fecal extract possesses the power of completely digesting I6.I C.C. of a I per cent starch solution in 24 hours at $38^{\circ} \mathrm{C}$.

Inasmuch as stools vary so greatly as to water content, it is essential to an accurate comparison of stools that such comparison be made on the basis of the solid matter. Supposing, for example, that in the above determination we had 6.2 c.c. of sediment. Since the supernatant fluid was removed and made up to

${ }^{1}$ In preparing the I per cent solution, the weighed starch powder should be dissolved in cold distilled water in a casserole and stirred until a homogeneous suspension is obtained. The mixture should then be heated with constant stirring, until it is clear. This ordinarily takes from eight to ten minutes. A slightly opaque solution is thus obtained, which should be cooled and made up to the proper volume before using.

2 Theoretically we would expect the colors to range from a light yellow to a dark blue, with red tubes holding an intermediate position in the series. This color sequence does of ten occur, but its occurrence is far from universal. Many times the first tubes in the series, i.e., those containing the largest quantities of the fecal extract, will exhibit a bluish cast of color which should not be confused with the starch color reaction. When these blue tubes are present, they are generally followed by yellow, red and blue tubes in order, the final blue tube, of course, being the regulation starch reaction. Occasionally greenish colors will be obtained to the left of the red color. It also sometimes happens that it is somewhat difficult to determine in which tube to the right of the red color the starch blue color is first detected, unless the tube be examined carefully before a strong light. In every instance, however, when these blue and green colors are observed, it is noted that tubes possessing the true dextrin red color are always present between these tubes and the tubes possessing the true starch blue color. It is evident, therefore, that these bluish tints in the tubes to the left of the dextrin color cannot be due to the presence of starch. The cause of the blue color reaction in the first tubes of the series has not been ascertained as yet. 
50 c.c. before testing its amylolytic value, it is evident that I c.c. of this sediment is equivalent to 8.r c.c. of extract. Therefore, in order to derive the amylolytic value of I c.c. of sediment, we must multiply the value (I6.I) as obtained above for the extract, by 8.I. This yields I 30.4 and enables us to express the activity as follows:

$$
\mathrm{Df}_{24^{\mathrm{h}}}^{38^{\circ}}=I 30.4
$$

The above method of calculation is that suggested by Wohlgemuth. In case time and facilities permit $\mathrm{cf}$ the determination of the moisture content of the feces, it is much more accurate and satisfactory to place the amylolytic values of the stools on a "gram of dry matter" basis. The amylolytic values of the stools are expressed as the number of cubic centimeters of I per cent starch solution which the amylase content of I gram of dry feces is capable of digesting.

22. Quantitative Determination of Fecal Bacteria. ${ }^{1}$ - The method is a simplification of MacNeal's adaptation of the Strasburger procedure. ${ }^{2}$ About 2 grams of feces are accurately weighed and placed in a 50 c.c. centrifuge tube. To the feces in the tube a few drops of 0.2 per cent hydrochloric acid are added, and the material is mixed to a smooth paste by means of a glass rod. Further amounts of the acid are added with continued crushing and stirring until the material is thoroughly suspended. The tube is then whirled in the centrifuge at high speed for one-half to one minute. The suspension is found sedimented into more or less definite layers, the uppermost of which is fairly free from the larger particles. The upper and more liquid portion of the suspension is now drawn off by means of a pipette and transferred to a beaker. ${ }^{3}$ The sediment remaining in the tube is again rubbed up with the glass rod with the addition of further amounts of dilute acid, and again centrifugalized for one-half to one minute. The supernatant liquid is pipetted off and added to the first, the same pipette being used for the one determination throughout. ${ }^{4}$ A third portion of the dilute acid is then added to the sediment, which is again mixed by stirring and again centrifugalized. All the washings are added to the first one, and during the process care is taken to wash the material from the walls and mouth of the centrifuge tube down into it. Finally; when the sediment is sufficiently free from bacteria, the various remaining particles are visibly clean, and the supernatant liquid after centrifugalization remains almost clear. This is removed to the beaker in which are now practically all the bacteria present in the original portion of feces, together with some solid matter not yet separated. In the centrifuge tubes there is a considerable amount of bacteria-free solid matter.

The suspension is now transferred to the same centrifuge tube, centrifugalized for a minute, and the supernatant liquid transferred to a clean beaker by means of the same pipette. The tube is then refilled from the first beaker and thus all the suspension centrifugalized a second time. The beaker is finally carefully washed with the aid of a rubber-tipped glass rod, the second sediment in the centrifuge tube is washed free of bacteria by means of this wash water and by successive portions of the dilute acid, and the supermatant liquid after centrifugalization is added to the contents of the second beaker. The second clean sediment is added to the

1 Mattill and Hawk: Jour. Exp. Med., It, 433 , I91 I.

2 MacNeal, Latzer and herr, Jour. Inf. Dis., 6, 123, 1909.

3 A 25 c.c. pipette is the most satisfactory size; to facilitate observation, the delivery tube is bent near the bulb to an angle of about I 20 degrees.

- A convenient support for the pipettes is a wire spring on a glass base, such as is used on a desk for pen-holders. The delivery tube, just where it is bent, is inserted between the wires, and any liquid not delivered collects in the bend of the tube. 
first. The bacterial suspension now in the second beaker is again centrifugalized in the same way and a third portion of bacteria-free sediment is separated. Frequently a fourth serial centrifugalization is performed-always if the third sediment is of appreciable quantity. At all stages of the separation, small portions of the dilute hydrochloric acid are used, so that the final suspension shall not be too voluminous. Ordinarily it amounts to 125 to 200 c.c. At the same time, the final amount of fluid should not be too small, as shown by Ehrenpfordt, ${ }^{1}$ because the viscosity accompanying increased concentration prevents proper and complete sedimentation.

To the final bacterial suspension an equal volume of alcohol is added and the beaker set aside to concentrate. A water-bath at $50^{\circ}$ to $60^{\circ} \mathrm{C}$. is very satisfactory. After two or three days, when the liquid is concentrated to about 50 c.c., the beaker is removed and about 200 c.c. of alcohol are added. The beaker is covered and allowed to stand at room temperature for 24 hours. At the end of this time the bacterial substance is generally settled, so that most of the clear supernatant liquid, of dark brown color, can be directly siphoned off without loss of solid matter. The remainder is then transferred to centrifuge tubes, centrifugalized, and the remaining clear liquid pipetted off. ${ }^{2}$ The sediment consists of the bodies of the bacteria, and is transferred to a Kjeldahl flask for nitrogen determination. This is the bacterial nitrogen. Where a determination of bacterial dry substance is desired, the sediment of bacteria is extracted by absolute alcohol and ether in succession, transferred to a weighed porcelain crucible, and dried at $102{ }^{\circ} \mathrm{C}$. to constant weight. This dried sample is then used in the nitrogen determination. Our procedure differs from that of MacNeal in that the bacterial dry matter is not determined. A saving of about seven days' time and of considerable labor is accomplished by this omission.

Inasmuch as it has been shown by various investigators that such bacteria as are present in the feces contain on the average about II per cent of nitrogen, the values for bacterial nitrogen as determined by our method may conveniently serve as a basis for the calculation of the actual output of bacterial substance.

23. Quantitative Determination of Indol in Feces. Bergeim's Modification of the Herter-Foster Method. ${ }^{3}$-Principle.-The feces is distilled from alkaline solution to remove phenols. This distillate are again distilled from acid solution to remove ammonia. The indol in the final distillate is treated with $\beta$-naphthaquinone sodium monosulphonate and alkali and the blue compound formed extracted with chloroform and determined colorimetrically.

Procedure.-Rub 30-50 grams of the fresh, well-mixed feces in a mortar with water to a uniform consistency. Transfer to a wide mouth Kjeldahl flask of about rooo c.c. capacity, rinsing mortar and neck of flask with distilled water to make about 400 c.c. Add 5 c.c. of 10 per cent $\mathrm{KOH}$ solution and about 2 c.c. of paraffin to decrease foaming. Distill with steam using ordinary Kjeldahl distillation apparatus with good stream of water in the condenser. Heat carefully for a few minutes until danger of foaming is past and then allow to boil vigorously. Distill over 500 c.c. of liquid, bringing the volume of the fecal suspension down to about roo c.c. toward the end of the distillation.

${ }^{1}$ Ehrenpfordt: Zeil. exp. Path. Ther., 7, 455, r 909.

In later work (see Blatherwick and Hawk: Biochem. Bull., 3, 28, 1913) it was found advantageous to centrifugalize with alcohol and ether in succession before transferring the bacterial cells to Kjeldahl flasks.

'Herter and Foster: Jour. Biol. Chem., 1, 257, r9o6. Bergeim, Fishback, and Hawk: Unpublished data. 
Transfer the distillate to a clean Kjeldahl flask, add 2 drops of phenolphthalein as an indicator. Make neutral with $N$ sulphuric acid and add I c.c. cxcess. Distill with steam as before, collecting the first 500 c.c. of distillate and bringing the residue finally to about Ioo c.c. Mix distillate well by shaking

Take an aliquot portion of the distillate (roo c.c.); add I c.c. of a 2 per cent solution of $\beta$-naphthaquinone sodium monosulphonate solution. Then add 2 c.c. of 10 per cent $\mathrm{KOH}$. Shake and let stand for $\mathrm{I}_{5}$ minutes. This is best carried out in a 150 c.c. Squibb shape separatory funnel. Extract with chloroform, shaking vigorously, using a ro c.c. and a 7 c.c. portion which will bring the total volume of the extract to the mark of a $I_{5}$ c.c. graduated tube. Mix thoroughly. Run at the same time and in the same way a standard using I c.c. of a solution of indol o. I mg. of indol per c.c. Compare the extract with this standard in a colorimeter, using the standard ordinarily at the $30-\mathrm{mm}$. mark. Calculate the indol to the basis of milligrams of indol per gram of moist feces.

Indol, and naphthaquinone solutions should be freshly prepared or may be kept in the ice-box for some days. Indol distillates should be kept in the ice-box if not used at once, especially in hot weather. The feces must be fresh.

24. Quantitative Determination of Fat in Feces.-Principle.-The determination of fat in dried feces is a more or less tedious process, and one which is somewhat dangerous if applied to pathological feces. Most of the methods for the determination of fat in the moist feces are accurate, but require a long time. Saxon ${ }^{1}$ has proposed a method for the determination of fat in moist feces, which is speedy. convenient. and accurate. The soaps of the feces are converted into free fatty acids by means of hydrochloric acid, and the material is then extracted by shaking with ether. The ether removes the neutral fat, the fatty acids which were present as such, the fatty acids derived from the soaps, and the cholesterol. The ether is removed by distillation, the crude fat purified by means of petroleum ether, and the weight of the total fat obtained. The fat is then dissolved in benzol and titrated with tenthnormal sodium alcoholate solution, using phenolphthalein as an indicator. The fatty acid is calculated, from the titration. to stearic acid.

Procedure.-Place about 5 grams (accurately weighed) of the thoroughly mixed feces in a roo c.c. glass-stoppered graduated cylinder. ${ }^{2}$

Add 20 c.c. of distilled water, I to 2.5 c.c. of concentrated hydrochloric acid (depending upon the amount of the sample) and again, sufficient distilled water to make a total bulk of 30 c.c. Add exactly 20 c.c. of ether, stopper, and shake vigorously for five minutes. Allow to stand for a few seconds, remove the stopper, add exactly 20 c.c. of 95 per cent alcohol, and again shake for five minutes.

Stand the cylinder aside. The ether, containing practically all of the fat, will come to the top as a colored transparent layer. Blow the ether layer off into

'Saxon: J. Biol. Chem., 17, 09. 1914.

2 Care must be taken not to smear the neck of the cylinder. This may be avoided by removing the feces from the weighing bottle by means of a glass rod. the end of which is flattened. and bent in the shape of a hoe, and transferring small bits of the feces from the hoe to the cylinder, using short pieces of glass rod, which are dropped into the cylinder together with the feces. 
a tall $150-200$ c.c. beaker. ${ }^{1}$ The thin layer of ether which remains is diluted with 5 c.c. of ether, the tube slightly agitated, and the ether blown off. This is done in all five times, care being taken each time to wash down the sides of the cylinder. The stopper should also be washed.

Twenty c.c. of ether are again added, and the cylinder shaken for five minutes and set aside. When the ether has nearly stratified, blow it off and wash as before. During the second washing stratification will complete itself.

Evaporate the ether ${ }^{2}$ until no trace of the alcohol, which has been carried over with it, remains. To the residue add 30 c.c. of low-boiling petroleum ether (should boil below $60^{\circ} \mathrm{C}$.), and allow to stand over night. Petroleum for this work should be frequently tested for a residue on evaporation. If a residue is left, the ether should be redistilled.

Filter the petroleum ether solution of the fat, catch the filtrate and washings in a tall, weighed, roo c.c. beaker, evaporate off the solvent, dry the beaker at $90^{\circ} \mathrm{C}$., desiccate and weigh.

After weighing, dissolve the contents of the beaker in 50 c.c. of benzol, heat almost to the boiling-point, add 2 drops of a 0.5 per cent solution of phenolphthalein, and titrate with a decinormal solution of sodium alcoholate. ${ }^{3}$

Calculations. - The weight of total fat is obtained by subtracting the weight of the empty beaker from the weight of the beaker plus the dried fat. The weight of fatty acids (in terms of milligrams of stearic acid) is obtained by multiplying the number of cubic centimeters of decinormal sodium alcoholate solution by the factor 28.4. The difference between the weight of total fat and the weight of fatty acids is the weight of neutral fat in the sample extracted.

A separate determination without the addition of hydrochloric acid may be run upon the sample, for the purpose of determining the weight of neutral fat and free fatty acids. The difference between this weight and the weight of total fat is the weight of fatty acid present in the original sample in the form of soaps.

${ }^{1}$ This is accomplished in the same manner that water is blown from a wash bottle. The submerged end of the delivery tube is bent upward, as in the apparatus used for the determination of fat in milk by Meig's method (see Milk). This avoids upward currents which would disturb the subjacent alcohol-ether-feces layer.

${ }^{2}$ Erlenmeyer flasks of about 200 c.c. capacity may be used, instead of beakers, for the collection of the ether blown from the cylinders. The ether may then be distilled and recovered. The same procedure may be followed in removing the petroleum ether.

${ }^{3}$ In the preparation of the sodium alcoholate solution, absolute alcohol and freshly cut, bright, metallic sodium are used; otherwise the procedure is the same as that for the standardization and preparation of any alkali solution. 


\section{BLOOD AND LYMPH}

BLOOD is composed of four types of form-elements (erythrocytes or red blood corpuscles, leucocytes or white blood corpuscles, blood plates or plaques and blood dust or hemoconein) held in suspension in a fluid called blood plasma. These form-elements compose about 60 per cent of the blood, by wejght. Ordinarily blood is a dark red opaque fluid due to the presence of the red blood corpuscles, but through the action of certain substances, such as water, ether, or chloroform, it may be rendered transparent. Blood so altered was formerly said to be laked. The term hemolysis is now used in this connection and substances which cause such action are spoken of as hemolytic agents. The hemolytic process is simply a liberation of the hemoglobin from the stroma of the red blood corpuscle. Normal blood is alkaline in reaction to litmus, the alkalinity being due principally to sodium carbonate. When examined according to physico-chemical methods the blood is found to be neutral, i.e., it does not contain an excess of hydroxyl ions. Even in cases of the most pronounced acidosis the reaction of the blood is but slightly altered. The specific gravity of the blood of adults ordinarily varies between I.045 and I.075. It varies somewhat with the sex, the blood of males having a rather higher specific gravity than that of females of the same species. Under pathological conditions also the density of the blood may be very greatly altered. The freezingpoint $(\Delta)$ of normal blood is about $-0.56^{\circ} \mathrm{C}$. Variations between $-0.51^{\circ}$ and $-0.62^{\circ} \mathrm{C}$. may be due entirely to dietary conditions, but if any marked variation is noted it can in most cases be traced to a disordered kidney function. The total amount of blood in the body has been variously estimated at from one-twelfth to one-fourteenth of the body weight. Perhaps $\mathrm{I} / \mathrm{I}_{3.5}$ is the most satisfactory figure. Abderhalden and Schmidt ${ }^{1}$ have suggested a unique method for the determination of this value. It is based upon the change in the optical activity of the blood upon injection of a body of known optical activity; such, for example, as dextrin. Keith, Rowntree and Geraghty.2 have recently made use of a dye in the determination of blood volume.

Among the most important constituents of blood plasma are the four

1 Abderhalden and Schmidt: Zeit. physiol. chem., 66, I20, I9 ro.

${ }^{2}$ Keith, Rowntree and Geraghty: Arch. Inl. Med., 16, 509, ror 5. 
protein bodies, fibrinogen, nucleoprotein, serum globulin (euglobulin and pseudo-globulin) and serum albumin. Plasma contains about 8.2 per cent of solids of which the protein constituents named above constitute approximately 84 per cent and the inorganic constituents (mainly chlorides, phosphates and carbonates) approximately io per cent. Among the inorganic constituents sodium chloride predominates. To prevent coagulation, blood plasma is ordinarily studied in the form of an oxalated or salted plasma. The former may be obtained by allowing the blood to flow from an opened artery into an equal volume of 0.2 per cent ammonium oxalate solution, whereas in the preparation of a salted plasma ro per cent sodium chloride solution may be used as the diluting fluid.

Fibrinogen is perhaps the most important of the protein constituents of the plasma. It is also found in lymph and chyle as well as in certain exudates and transudates. Fibrinogen possesses the general properties of the globulins, but differs from serum globulin in being precipitated upon half-saturation with sodium chloride. In the process of coagulation of the blood the fibrinogen is transformed into fibrin. This fibrin is one of the principal constituents of the ordinary blood clot.

The nucleoprotein of blood possesses many of the characteristics of serum globulin. In common with this body it is easily soluble in sodium chloride, and is completely precipitated from its solutions uponsaturation with magnesium sulphate. It is much less soluble in dilute acetic acid than serum globulin, and its solutions coagulate at $65^{\circ}-69^{\circ} \mathrm{C}$.

The body formerly called serum globulin is probably not an individual substance. Recent investigations seem to indicate that it may be resolved into two individual bodies called euglobulin and pseudoglobulin. The euglobulin is practically insoluble in water and may be precipitated in the presence of $28-36$ per cent of saturated ammonium sulphate solution. The pseudo-globulin, on the contrary, is soluble in water and is only precipitated by ammonium sulphate in the presence of from 36 to 44 per cent of saturated ammonium sulphate solution.

In common with serum globulin the body known as serum albumin seems also to consist of more than a single individual substance. The so-called serum albumin may be separated into at least two distinct bodies, one capable of crystallization, the other an amorphous body. The solution of either of these bodies in water gives the ordinary albumin reactions. The coagulation temperature of the serum albumin mixture as it occurs in serum or plasma varies from $70^{\circ}$ to $85^{\circ} \mathrm{C}$. according to the reaction of the solution and its content of inorganic material. Serum albumin differs from egg albumin in being more levorotatory, in being rendered less insoluble by alcohol, and in the fact that when 
precipitated by hydrochloric acid it is more easily soluble in an excess of the reagent.

When blood coagulates and the usual clot forms, a light yellow fluid exudes. This is blood serum. It differs from blood plasma in containing a large amount of fibrin ferment, a body of great importance in the coagulation of the blood, and also in possessing a lower protein content. The protein material present in plasma and not found in serum is the fibrinogen which is transformed into fibrin in the process of coagulation and removed. The specific gravity of the serum of human blood varies between 1.026 and 1.032 . If blood be drawn into a vessel and allowed to remain without stirring or agitation of any sort the major portion of the red corpuscles will sink away from the upper surface. causing this portion of the clot to assume a lighter color due to the predominance of leucocytes. This light-colored portion of the clot is called the "buffy coat."

Beside the protein constituents already mentioned, other bodies which are found in both the plasma and serum are the following: Sugar (glucose), uric acid (urates), urea, fat, amino-acids, enzymes, lecithin. creatine, carbamic acid, cholesterol and its esters, nucleoprotein, acetone bodies, paralactic acid, gases, ammonia, coloring-matter (lutein or lipochrome) and mineral substances. In addition to the substances just named the blood doubtless contains a class of substances called hormones of which adrenaline is the only one thus far definitely identified. Some of the pathological constituents of blood are proteoses, biliary constituents and purine bodies. In many pathological conditions certain normal constituents are present in increased amount.

Normal human blood contains slightly less than o.I per cent of glucose on the average. Strouse ${ }^{1}$ in a very recent series of tests places the average glucose content at 0.084 per cent. That the diet influences the sugar content is shown by the fact that two and one-half to four hours after a meal the sugar content has been found to equal 0.18 per cent. $^{2}$ In case of glycosuria the blood sugar may increase (hyperglycemia) to $0.3-1.0$ per cent. For the quantitative determination of blood sugar see page 279 .

The determination of the cholesterol content of the blood is assuming clinical importance. Normal blood contains I40-ISo mg. per roo grams of blood, or about 0.15 per cent. This value has been found to be increased (hypercholesterolemia) in gall stones, pregnancy, nephritis, diabetes, and arteriosclerosis and syphilis. (See page $28_{5}$ for quantitative methods.)

${ }^{1}$ Strouse: Bull. Johns IIop. IIosp., 26, $21 \mathrm{r}, 1915$.

${ }^{2}$ Hirsch: Zeil. physiol. Chem., 93. 355, tor 5. 
Uric acid is present in normal blood to the extent of about $\mathrm{I}-2 \mathrm{mg}$. per Ioo grams of blood. In gout this value may be increased to $4^{-6}$ mg. The quantitative determination of the uric acid content of blood is of importance as an aid in differentiating gout and certain other disorders exhibiting similar clinical symptoms (for method see page 274).

The non-protein nitrogen of normal blood amounts to about $25-35$ $\mathrm{mg}$. per too grams of blood. The urea forms about 50 per cent of this, creatinine 2 per cent, uric acid 2 per cent, ammonia 0.3 per cent, and amino-acids, etc., about 46 per cent. In nephritis the non-protein nitrogen of the blood is much increased. Myers and Fine ${ }^{1}$ report a fatal case of uremia in which the non-protein nitrogen reached $300 \mathrm{mg}$.

A mino-acids are always present in the blood. They result for the most part from the digestion of protein material in the intestine.

Creatinine occurs in normal blood to the extent of about $\mathrm{I}-2 \mathrm{mg}$. per roo c.c. of blood. In uremia the amount is increased. ${ }^{2}$ Various investigators report the values as ranging from 5 to $33 \mathrm{mg}$. per IO० c.C.

The creatine content of normal blood ranges from 5 to Io mg. per Ioo c.c. of blood. The creatine values have no important pathological significance at the present time.

The acetone (acetone and acetoacetic acid) content of normal blood ranges from o to I $\mathrm{mg}$. per Ioo c.c. of blood. In mild diabetes mellitus the value rises to $5^{-12} \mathrm{mg}$., whereas in severe diabetes mellitus (coma) as much as $20-45 \mathrm{mg}$. per Ioo c.c. of blood serum has been found.

Normal blood contains about 20 per cent of solids and 3 per cent of total nitrogen, whereas chlorides are present to the extent of about 0.65 per cent. In severe diabetes the chlorides are decreased because of the accompanying diuresis.

$\mathrm{Abel}^{3}$ and associates have devised a method by which diffusible substances may be removed from the blood of a living animal. The process is termed vividiffusion and is brought about by permitting the blood from an artery to flow through collodion tubes surrounded by physiological salt solution. The dialyzable substances, except sodium chloride, are removed and the dialyzed blood is returned to the body of the animal by means of a vein. The apparatus has been modified by McGuigan and von Hess. ${ }^{4}$

${ }^{1}$ Myers and Fine: Chemical Composition of the Blood in Health and Disease, ig 5.

${ }^{2}$ Folin and Denis; Jour. Biol. Chim., 17, 487, I914.

Myers and Fine: Jour. Biol. Chem., 20, 391, I9I5.

3 Abel, Rowntree and Turner: Transactions of the Ass'n of American Physicians, I9I3; also Jour. of Pharm. and Exp. Therap., 5, 275, I9I4.

${ }^{4}$ MIcGuigan and Von Hess: Jour. Pharm. and Exp. Therap., vol. 6, 19I4. 
. 
PLATE IV.

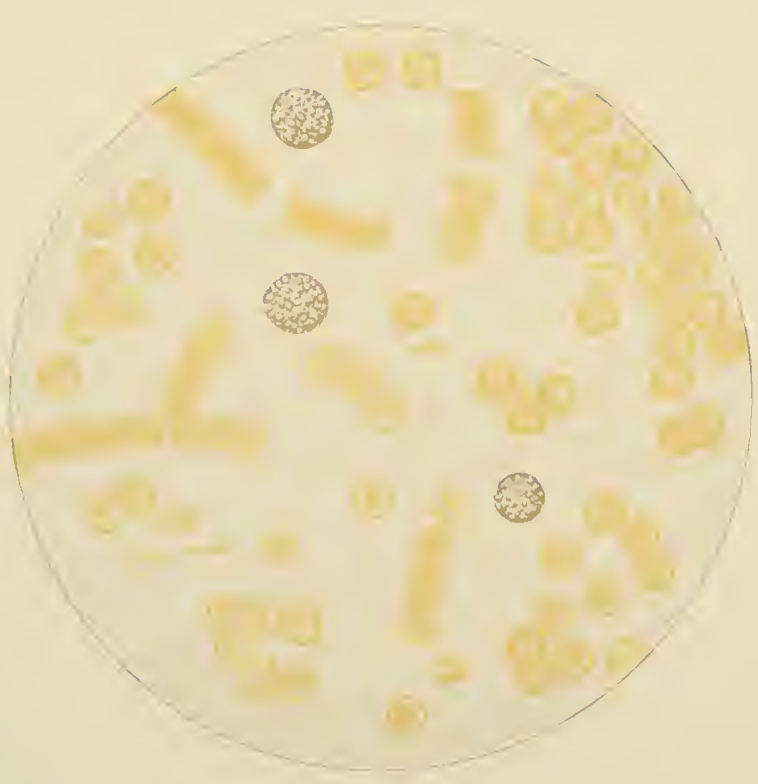

Normal Erythrocytes and Leucocytes. 
In the application of blood-letting or venesection it has been customary to discard both the corpuscles and plasma of the withdrawn blood. Abel ${ }^{1}$ and associates have found it possible to separate the corpuscles from the removed blood by centrifugation and to return them to the body suspended in Locke's solution. They name the procedure plasmapheresis. By this means blood-letting can be carried out repeatedly during a short interval of time without endangering the life of the animal.

There has been considerable controversy regarding the form of the erythrocytes or red blood corpuscles of human blood. It is claimed by some investigators that the cells are bell-shaped or cup-shaped. As the erythrocytes occur normally in the circulation, however, they are probably thin, non-nucleated, biconcave discs. ${ }^{2}$

The blood of most mammals contains erythrocytes similar in form to those of human blood. In the blood of birds, fishes, amphibians and reptiles the erythrocytes are ordinarily more or less elliptical, biconvex and possess a nucleus. The erythrocytes vary in size with the different animals. The average diameter of the erythrocytes of blood from various species is given in the following table: ${ }^{3}$

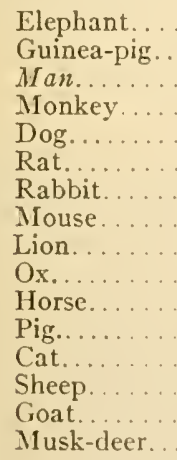

$2 \tau^{1} 3 \bar{x}$ of an inch. $\frac{1}{32.23}$ of an inch. 3250 of an inch. $\frac{1}{38}$ of an inch. $35^{1} \mathrm{~T}$ of an inch. 3652 of an inch. 3653 of an inch. $3 x^{2}+3$ of an inch. $\frac{1}{1+3}$ of an inch. 4219 of an inch. $\frac{x}{4213}$ of an inch. T2t $x$ of an inch. I 3 of an inch. Jy? of an inch. inch. - 2325 of an inch.

The erythrocytes, from whatever source obtained, consist essentially of two parts, the stroma or protoplasmic tissue and its enclosed pigment, hemoglobin. For human blood the number of erythrocytes present in the fluid as obtained from well-developed males in good physical condition is about 5,500,000 per cubic millimeter. ${ }^{4}$ The normal content of the blood of adult females is from $4,000,000$ to $4,500,000$ per cubic

\footnotetext{
'Abel, Rowntree, Turner, Marshall and Lamson (see Abel's Mellon Lecture," 1015): also Abel: Science, 42, 135, 1915.

${ }^{2}$ When examined singly under the microscope, they possess a pale greenish-yellow color (see Plate IV, opposite), whereas when grouped in large masses a reddish tint is noted.

3 Wormley's Micro-Chemistry of Poisons, second edition, p. 733.

1 This statement is based upon observations made upon the blood of athletes in training. See Hawk: Amer. Jour. Physiol.,10, 384 , 1904. It is generally stated in text-books that the blood of males contains about $5,000,000$, per cubic millimeter.
} 
millimeter. The number of erythrocytes varies greatly under different conditions. For instance, the number may be increased after the transfusion of blood of the same species of animal; by residing in a high altitude; or as a result of strenuous physical exercise continued over a short period of time. An increase is also noted in starvation; after partaking of food; after cold or hot baths; after massage, as well as after the administration of certain drugs and accompanying certain diseases, such as cholera, diarrhea, dysentery and yellow atrophy of the liver. A decrease in the number occurs in the different forms of anemia. The number has been known to increase to $7,040,000$ per cubic millimeter as a result of physical exercise, while i I, $, 00,000$ per cubic millimeter have been noted in cases of polycythemia and increases nearly as great in cyanosis. The number has been known to decrease to 500,000 per cubic millimeter or lower in pernicious anemia.

Erythrocytes possess the property, when properly treated, of "clumping" together in masses and precipitating, producing so-called agglutination. Cells other than erythrocytes (e.g., bacteria) possess this property. When spoken of in connection with the blood such action is termed hemagghtination. A substance which will bring about hemagglutination is said to contain hemagglutinins. These hemagglutinins are particularly abundant in the vegetable kingdom. ${ }^{1}$ For a demonstration of hemagglutination see page $26 \mathrm{I}$.

Oxyhemoglobin, the coloring matter of the blood, is a conjugated protein. Through treatment with hydrochloric acid it may be split into a protein body called globin, and hemochromogen, an iron-containing pigment. The latter body is rapidly transformed into hematin in the presence of oxygen, and this in turn gives place to hematin-hydrochloride or hemin (Figs. 78 and 79, page 265). The pigment of arterial blood is for the most part loosely combined with oxygen and is termed oxyhemoglobin, whereas the pigment of venous blood is principally hemoglobin (so-called reduced hemoglobin). Oxyhemoglobin is the oxygen carrier of the body and belongs to the class of bodies known as respiratory pigments. It is held within the stroma of the erythrocyte. The reduction of oxyhemoglobin to form hemoglobin (so-called reduced hemoglobin) occurs in the capillaries. Oxyhemoglobin may be crystallized and a specific form of crystal obtained from the blood of each individual species (see Figs. 70 to 76 , pages $25^{I}$ to 254 ). This fact seems to indicate that there are many varieties of oxyhemoglobin. The interesting findings of Reichert and Brown are of great value in this connection. These investigators prepared oxyhemoglobin crystals from the blood

\footnotetext{
${ }^{1}$ Mendel: Archivio di fisiologia, 7, I68, 1909; also Schneider: Journal of Biological Chem., II, 47, I9I 2.
} 


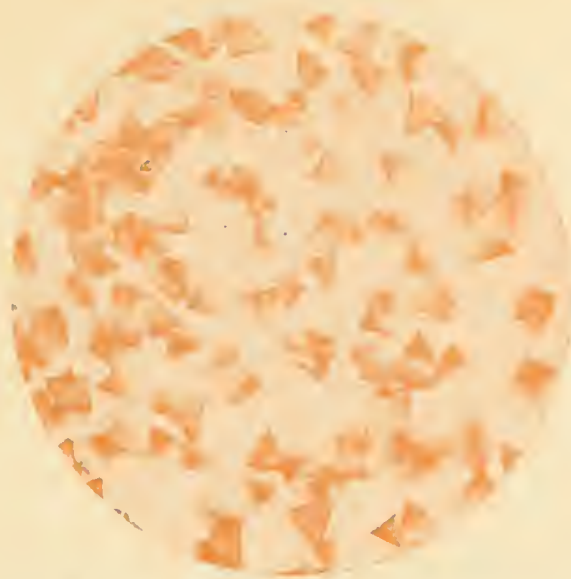

Fig. 70.-Oxyhemoglobin Cristals from Blood of the Guinea-pig. Reproduced from a micro-photograph furnished by Prof. E. T. Reichert, of the University of Pennsylvania.

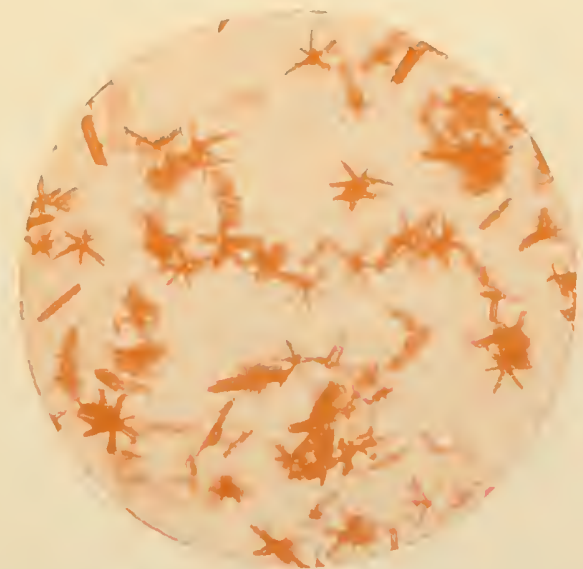

Fig. 71.-Oxyemoglobix Crystals from Blood of the Rat. Reproduced from a micro-photograph furnished by I'rof. K. T. Reichert, of the University. of Pennsylvania. 


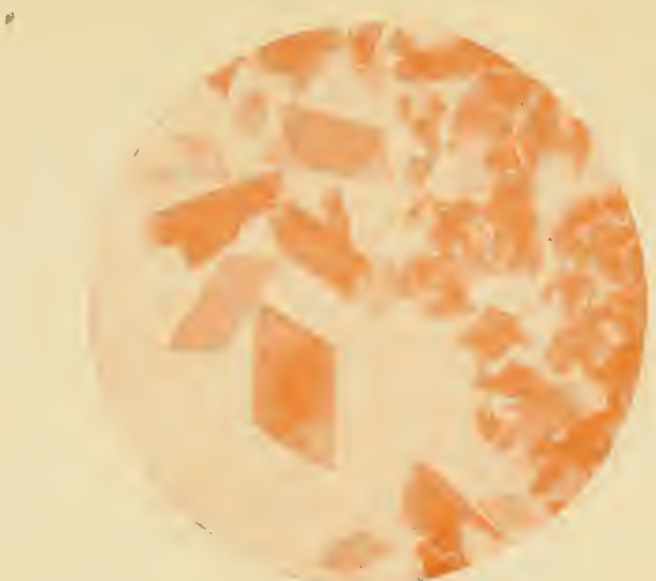

Fig. 72,-Oxyhemoglobin Cristals from Blood of the Horse. Reproduced from a micro-photograph furnished by Prof. E. T. Reichert, of the University of Pennsylvania.

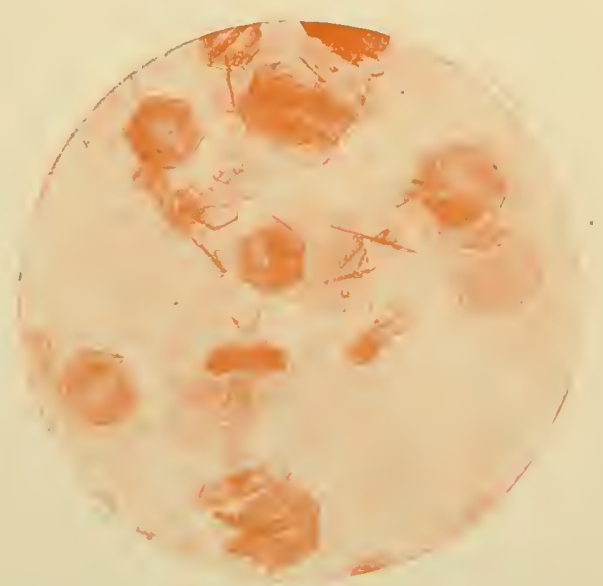

Fig. 73.-Oxyhemoglobin Crystals from Blood of the Squirrel. Reproduced from a micro-photograph furnished by Prof. E. T. Reichert, of the University of Pennsylvania. 


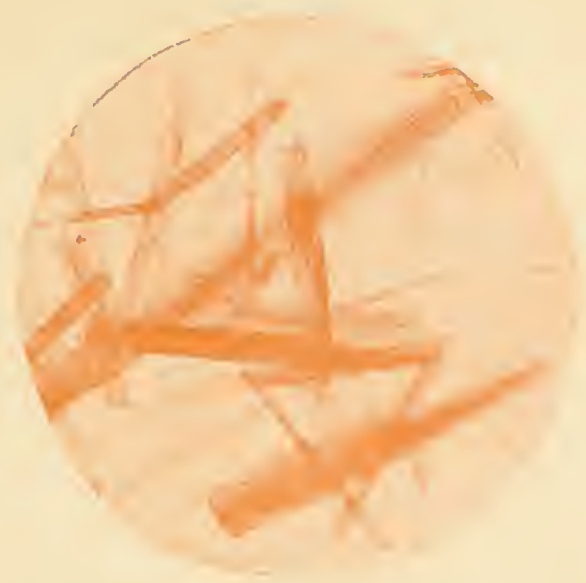

Fig. 74.-Oxyhejroglobin Crystals from Blood of the Dog.

Reproduced from a micro-photograph furnished by Prof. E. 'T. Reichert, of the University of Pennsyriania.

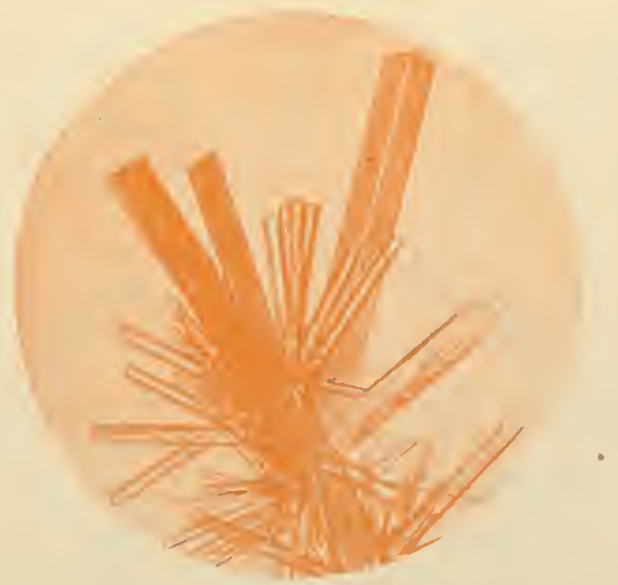

Fig. 75.-Oxyhemoglobin Cristals from Blood of the Cat.

Reproduced from a micro-photograph furnished by Prof. E. T. Reichert, of the University of Pennsyliania. 
of over one hundred species of animal and subsequently studied the characteristics of the crystals very minutely from the standpoint of crystallography. Their findings may prove of importance from the standpoint of heredity and the origin of species.

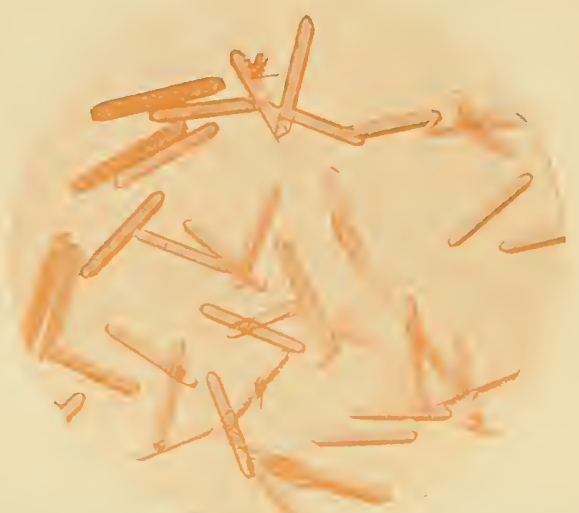

Fig. 76.-Oxyhemoglobin Crystals from Blood of the Necturus.

Reproduced from a micro-photograph furnished by Prof. E. T. Reichert, of the University of Pennsylvania. ${ }^{1}$

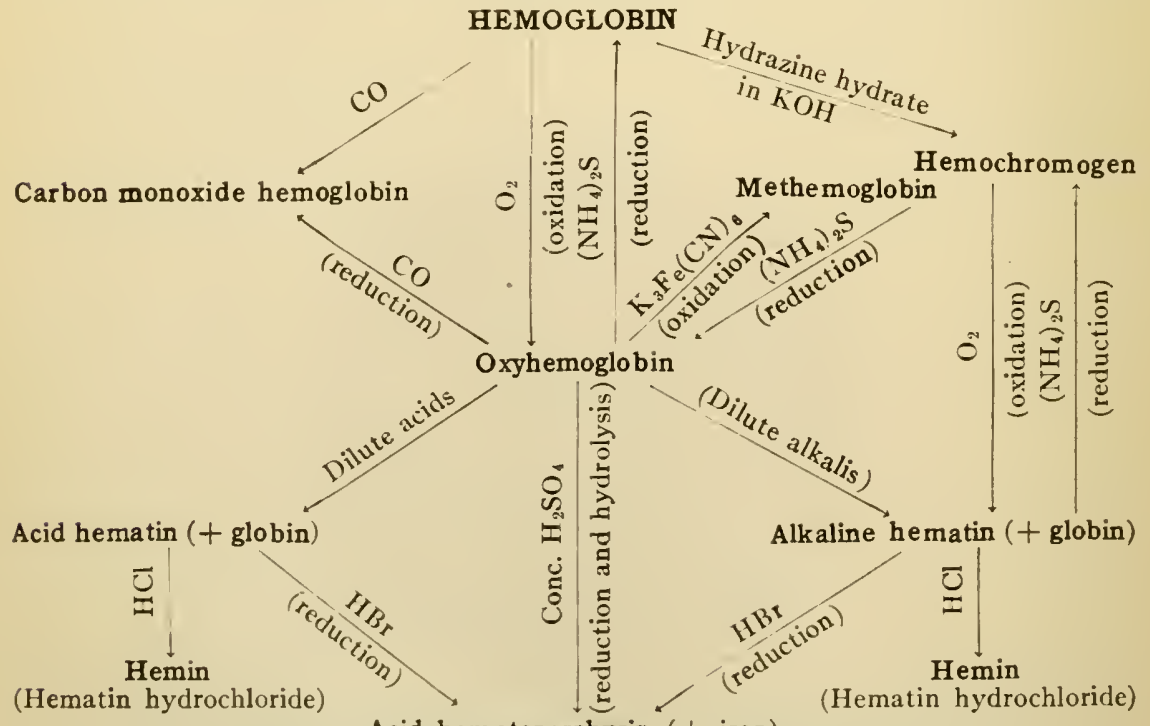

Acid hematoporphyrin ( + iron)

$$
\text { 总 }
$$

Alkaline hematoporphyrin

1 The micro-photographs of oxyhemoglobin (see pages $25 \mathrm{I}-254$ ) and hemin (see page $\left(26_{5}\right)$ are reproduced through the courtesy of Professors $\mathrm{E}$. T. Reichert and Amos P. Brown, of the University of Pennsylvania, who have investigated the crystalline forms of biochemic substances. 
The following bodies may be derived from hemoglobin, and each possesses a specific spectrum which serves as an aid in its detection and identification: Oxyhemoglobin, methemoglobin, carbon-monoxide hemoglobin, nitric-oxide hemoglobin, hemochromogen, hematin, acid-hematin, alkali-hematin and hematoporphyrin (see Absorption Spectra, Plates I and II).

The relationship between hemoglobin and its derivatives may be represented by the scheme shown on page 254 .

The chemical transformations which occur in the blood during respiration are complicated and of great importance. In brief the exchange of oxygen and carbon dioxide may be described as follows: Oxygen from the air passes through the lungs into the blood where it is appropriated for the most part by the red blood corpuscles. The hemoglobin of these corpuscles possesses the property of uniting with oxygen, forming oxyhemoglobin. This oxyhemoglobin possesses a red color and imparts to the arterial blood its bright appearance. The oxygen is thus borne by these blood cells in the circulating blood to all parts of the body. As the blood passes through the capillaries it gives up the major part of its oxygen which is used by the tissues in their varied activities. As the blood loses its oxygen it becomes darker in color due to the fact that the oxyhemoglobin has been transformed into hemoglobin (or reduced hemoglobin). At the same time in the tissue capillaries the blood takes up excretory products from the tissues, the chief of which is carbon dioxide. This carbon dioxide is present in the blood mainly as sodium carbonate and sodium acid carbonate; a small amount is probably combined with the proteins of the plasma. We now have so-called venous blood. This is, in turn, carried to the lungs where the carbon dioxide is exchanged for oxygen and the cycle is repeated.

The white corpuscles (or leucocytes) of human blood differ from the red corpuscles (or erythrocytes) in many particulars, such as being somewhat larger in size, in containing at least a single nucleus and in possessing ameboid movement (see Plate IV, opposite page 249). They are typical animal cells and therefore contain the following bodies which are customarily present in such cells: Protcins, fats, glycogen. purinc bodies, enzymes, phosphatides, lecithin, cholesterol, inorganic salts and water. Compound proteins make up the chief part of the protein quota of leucocytes, the nucleoproteins predominating. Of the enzymes present the proteolytic are the most important. It is claimed ${ }^{1}$ that there are two proteolytic enzymes in leucocytes, one active in alkaline solution and present in the polynuclear cells, ${ }^{2}$ and the other

1 Opie: Jour. of Experimental Med., 8; Opie and Barker: Ibid.. 9.

${ }^{2}$ For discussion of different types of leucocytes see "Da Costa's Clinical Hematology" or some similar volume. 
active in acid medium and present in mononuclear cells. It is claimed that the granular leucocytes originate in the bone marrow, whereas the non-granular leucocytes (lymphocytes) have a lymphatic origin (lymph glands or lymphoid tissue); this matter of origin is uncertain. The normal number of leucocytes in human blood varies between 5000 and Io,000 per cubic millimeter. The ratio between the leucocytes and erythrocytes is about $\mathrm{I}: 350-500$. A leucocytosis is said to exist when the number of leucocytoses is increased for any reason. Leucocytoses may be divided into two general classes, the physiological and the pathological. Under the physiological form would be classed those leucocytoses accompanying pregnancy, parturition and digestion, as well as those due to mechanical and thermal influences. The leucocytoses spoken of as pathological are the inflammatory, infectious, post-hemorrhagic, toxic and experimental forms, as well as the type of leucocytosis which accompanies malignant disease.

The blood plates (platelets or plaques) are round or oval colorless discs which possess a diameter about one-third as great as that of the erythrocytes. Upon treatment with certain reagents, e.g., artificial gastric juice, they may be separated into a homogeneous, non-refractive portion and a granular, refractive portion. The blood plates are associated with the coagulation of the blood. This relationship is not completely understood at present.

The hemoconein or so-called "blood dust" is made up of round granules which usually have a diameter somewhat less than I micron. The serum of normal as well as of pathological blood contains these granules. They were first described by Müller to whom they appeared as highly refractile granules possessed of Brownian movement. The "blood dust" is apparently not concerned with the coagulation of the blood. The granules are insoluble in alcohol, ether and acetic acid and are not blackened by osmic acid. According to Müller, the granules making up the so-called "blood dust" constitute a new organized constituent of the blood, whereas other investigators believe them to be merely free granules from certain of the forms of leucocytes. They appear to possess no clinical significance.

The processes involved in the coagulation of the blood are not fully understood. Several theories have been advanced and each has its adherents. The theory which appears to be fully as firmly founded upon experimental evidence as any is the following: Blood contains a zymogen called prothrombin which combines with the calcium salts present to form an enzyme known as thrombin or fibrin-ferment. When freshly drawn blood comes into contact with the air the fibrin-ferment at once acts upon the fibrinogen present and gives rise to the formation 
of fibrin. This fibrin forms in shreds throughout the blood mass and, holding the form elements of the blood within its meshes, serves to produce the typical blood clot. The fibrin shreds gradually contract, the whole clot assumes a jelly-like appearance and the yellowish serum exudes. If, immediately upon the withdrawal of blood from the body, the fluid be rapidly stirred or thoroughly "whipped" with a bundle of coarse strings, twigs or a specially constructed beater, the fibrin shreds will not form in a network throughout the blood mass but instead will cling to the device used in beating. In this way the fibrin may be removed and the remaining fluid is termed defibrinated blood. The above theory of the coagulation of the blood may be stated briefly as follows:

I. Prothrombin + Calcium Salts $=$ Thrombin (or Fibrin-ferment).

II. Thrombin (or Fibrin-ferment) + Fibrinogen $=$ Fibrin .

Howell ${ }^{1}$ has suggested an ingenious modification of the above theory. He says: "In the circulating blood we find as constant constituents fibrinogen, prothrombin, calcium salts and antithrombin. The last-named substance holds the prothrombin in combination and thus prevents its conversion or activation to thrombin. When the blood is shed, the disintegration of the corpuscles (platelets) furnishes material (thromboplastin) which combines with the antithrombin and liberates the prothrombin; the latter is then activated by the calcium and acts on the fibrinogen. According to this view the actual process of coagulation involves only three factors, fibrinogen, prothrombin and calcium. These three factors exist normally in the circulating blood but are prevented from reacting by the presence of antithrombin."

The question as to whether menstrual blood coagulates has caused much discussion. The most recent investigations seem to show that it does not coagulate because of the removal of fibrin-ferment and fibrinogen from such blood by the endometrium or lining membrane of the uterus. ${ }^{2}$

Among the medico-legal tests for blood are the following: ( $I$ ) Microscopical identification of the erythrocytes, (2) spectroscopic identification of blood solutions, (3) the guaiac test, (4) the benzidine reaction, (5) preparation of hemin crystals. Of these five tests the last two named are generally considered to be the most satisfactory. They give equally reliable results with fresh blood and with blood from clots or stains of long standing, provided the latter have not been exposed to a high temperature or to the rays of the sun for a long period. The technic of the tests is simple and the formation of the dark brown or chocolate-colored crystals of hemin or the production of the green or

${ }^{1}$ Howell: American Journal of Physiology, 29, r87, xgr.

2 Bell: Jour. Path. and Bact., r8, No. 4, rgr 4. 
blue color with benzidine is indisputable proof of the presence of blood in the fluid, clot or stain examined. The weak point of the tests, medico-legally, lies in the fact that they do not differentiate between human blood and that of certain other species of animal.

The guaiac test (see page 262), although generally considered less accurate than the hemin test, is held by some to be a more delicate test than the hemin test if properly performed. One of the most common mistakes in the manipulation of this test is the use of a guaiac solution which is too concentrated and which, when brought into contact with the aqueous blood solution, causes the separation of a voluminous precipitate of a resinous material which may obscure the blue coloration; this is particularly true of the test when used for the examination of blood stains. A solution of guaiac made by dissolving I gram of the resin in 60 c.c. of 95 per cent alcohol is very satisfactory for general use. The test is frequently objected to upon the ground that various other substances, e.g., milk, pus, saliva, etc., respond to the test and that it cannot therefore be considered a specific test for blood and is of value only in a negative sense. We have demonstrated to our own satisfaction, however, that many samples of milk give the blue color upon the addition of an alcoholic solution of guaiac resin without the addition of hydrogen peroxide or old turpentine. It has also been shown ${ }^{1}$ that those milks which respond positively, fail to do so after boiling. In the case of blood the test is positive both before and after boiling the blood for $I_{5}-20$ seconds. Pus does not respond after boiling. Old, partly putrified pus gives the test even without the addition of hydrogen peroxide or old turpentine, whereas fresh pus responds upon the addition of hydrogen peroxide. Saliva gives a positive reaction only in case blood or pus is present. Certain plant extracts give the test before but not after boiling for $\mathrm{I}^{-20}$ seconds. Buckmaster has advocated the use of an alcoholic solution of guaiaconic acid instead of an alcoholic solution of guaiac resin. He claims that he was able to produce the blue color upon the addition of the guaiaconic acid to milk only when the sample of milk tested was brought from the country in sterile bottles, and further, that no sample of London milk which he examined responded to the test. In the application of the guaiac test to the detection of blood, he states that he was able to detect laked blood when present in the ratio $I: 5,000,000$ and unlaked blood when present in the ratio I : I, $, \infty 0, \infty 00$. This author considers the guaiac test to be far more trustworthy than is generally believed.

Up to within recent times it has been impossible to make an absolute differentiation of human blood. The so-called "biological" blood test

\footnotetext{
${ }^{1}$ Leary: Private communication.
} 
has, however, made such a differentiation possible. This test, known as the Bordet reaction, is founded upon the fact that the blood serum of an animal into which has been injected the blood of another animal of different species develops the property of agglutinating and dissolving erythrocytes similar to those injected, but exerts this influence upon the blood from no other species. The antiserum used in this test is prepared by injecting rabbits with 5 -Io c.c. of human defibrinated blood, at intervals of about four days, until a total of between 50 and 80 c.c. has been injected. After a lapse of one or two weeks the animal is bled, the serum collected, placed in sterile tubes and preserved for use as needed. In examining any specific solution for human blood it is simply necessary to combine the antiserum and the solution under examination in the proportion of $\mathrm{I}: 100$ and place the mixture at $37^{\circ} \mathrm{C}$. If human blood is present in the solution a turbidity will be noted and this will change within three hours to a distinctly flocculent precipitate. This antiserum will react thus with no other known substance.

Lymph may be considered as the "middle man" in the transactions between blood and tissues. It is the medium by which the nutritive material and oxygen transported by the blood for the tissues is brought into intimate contact with those tissues and thus utilized. In the further fulfillment of its function, the lymph bears from the tissues water, salts and the products of the activity and catabolism of the tissues and passes these into the blood. Lymph, therefore, exercises the function of a "go-between" for blood and tissues. It bathes every active tissue of the animal body, and is believed to have its origin partly in the blood and partly in the tissues.

In chemical characteristics, lymph resembles blood plasma. In fact, it has been termed "blood without its red corpuscles." Lymph from the thoracic duct of a fasting animal or from a large lymphatic vessel of a well-nourished animal is of a variable color (colorless, yellowish or slightly reddish) and alkaline in reaction to litmus. It contains fibrinogen, fibrin-ferment and leucocytes and coagulates slowly, the clot being less firm and bulky than the blood clot. Serum albumin and serum globulin are both present in lymph, the albumin predominating in a ratio of about 3 or $4: \mathrm{I}$. The principal inorganic salts are sodium salts (chloride and carbonate), whereas the phosphates of potassium, calcium, magnesium and iron are present in smaller amount.

Substances which stimulate the flow of lymph are termed lymphagıgues. Such substances as sugar, urea, certain salts (especially sodium chloride), peptone, egg albumin, extracts of dogs' liver and intestine, crab muscles and blood leeches are included in this class.

In a fasting animal, the lymph coming from the intestine is a clear, 
transparent fluid possessing the characteristics already outlined. After a meal containing fat has been ingested, this intestinal lymph is white or "milky." This is termed chyle and is essentially lymph possessing an abnormally high ( $5-15$ per cent) content of emulsified fat. This chyle is absorbed by the lacteals of the intestine and transported to the lower portion of the thoracic duct. Apart from the fat value, the composition of lymph and chyle are similar.

\section{Experiments ON BLOOD}

\section{Defibrinated Ox-blood}

I. Reaction.-Moisten red and blue litmus papers with Io per cent sodium chloride solution and test the reaction of the defibrinated blood. Test by Congo red paper also.

2. Microscopical Examination.-Examine a drop of defibrinated blood under the microscope. Compare the objects you observe with Plate IV, opposite page 249. Repeat the test with a drop of your own blood.

3. Specific Gravity.-Determine the specific gravity of defibrinated blood by means of an ordinary specific gravity spindle. Compare this result with the specific gravity as determined by Hammerschlag's method in the next experiment.

4. Specific Gravity by Hammerschlag's Method.-Fill an ordinary urinometer cylinder about one-half full of a mixture of chloroform and benzene, having a specific gravity of approximately 1.050. Into this mixture allow a drop of the blood under examination to fall from a pipette or directly from the finger in case fresh blood is being examined. Care must be taken not to use too large a drop of blood and to keep the drop from coming into contact with the walls of the cylinder. If the blood drop sinks to the bottom of the vessel, thus showing it to be of higher specific gravity than the surrounding fluid, add chloroform until the blood drop remains suspended in the mixture. Stir carefully with a glass rod after adding the chloroform. If the blood drop rises to the surface upon being introduced into the mixture, thus showing it to be of lower specific gravity than the surrounding fluid, add benzene until the blood drop remains suspended in the mixture. Stir with a glass rod after the benzene is added. After the blood drop has been brought to a suspended position in the mixture by means of one or more additions of chloroform and benzene this final mixture should be filtered through muslin and its specific gravity accurately determined. What is the specific gravity of the blood under examination?

5. Tests for Various Constituents.-Place Io c.c. of defibrinated blood in an evaporating dish, dilute with Ioo c.c. of water and heat to boiling. Is there any coagulation, and if so what bodies form the coagulum? At the boiling-point acidulate slightly with dilute acetic acid. Filter. The filtrate should be clear and the coagulum dark brown. Reserve this coagulum. What body gives the coagulum this color? Evaporate the filtrate to about 25 c.c., filtering off any precipitate which may form in the process. Make the following tests upon the filtrate:

(a) Fehling's Test.-To 5 c.c. of the neutralized filtrate add 5 drops of Fehling's solution and boil one minute. 
(b) Chlorides.-To a small amount of the filtrate in a test-tube add a few drops of nitric acid and a little silver nitrate. In the presence of chloride, a white precipitate of silver chloride will form.

(c) Phosphates.-Test for phosphates by nitric acid and molybdate solution according to directions given on page 59.

(d) Proteose and Peptone.-Test a small amount of the solution for proteose and peptone by saturating with ammonium sulphate according to directions given on page 120 .

(e) Crystallization of Sodium Chloride.-Place the remainder of the filtrate in a watch glass and evaporate it on a water-bath. Examine the crystals under the microscope and compare them with those in Fig. 80, page 267.

6. Test for Iron.-Incinerate a small portion of the coagulum from the last experiment (5) in a porcelain crucible. Cool, dissolve the residue in dilute hydrochloric acid and test for iron by potassium ferrocyanide or ammonium thiocyanate. Which of the constituents of the blood contains the iron?

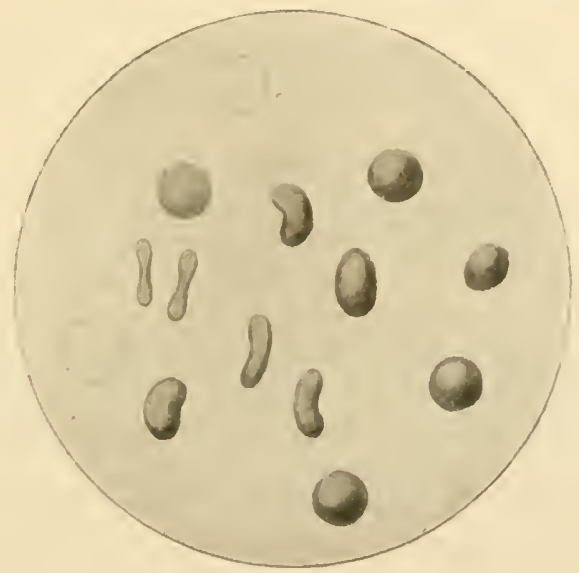

Fig. 77.-EfFect of WATER ON ERithrocites.

7. Hemolysis ("Laky Blood").--Note the opacity of ordinary defibrinated blood. Place a few cubic centimeters of this blood in a test-tube and add water, a little at a time, until the blood is rendered transparent. Hemolysis has taken place. How does the water act in causing this transparency? Examine a drop of hemolyzed blood under the microscope. How does its microscopical appearance differ from that of unaltered blood? What other agents may be used to bring about hemolysis?

8. Osmotic Pressure.-Place a few cubic centimeters of blood in each of three test-tubes. Hemolyze the blood in the first tube according to directions given in the last experiment (7): add an equal volume of isotonic ( 0.9 per cent) sodium chloride to the blood in the second tube, and an equal volume of "1o per cent sodium chloride to the blood in the third tube. Mix thoroughly by shaking and after a few moments examine a drop from each of the three tubes under the microscope (see Figs. 77 and I50, page 473). What do you find and what is your explanation from the standpoint of osmotic pressure? 
9. Hemagglutination.-The common garden bean, such as the Scarlet Runner, ${ }^{1}$ contains a protein substance which exhibits the interesting property of causing a clumping or agglutination of red blood corpuscles. ${ }^{2}$

Dilute defibrinated blood ${ }^{3}$ ten times with physiological sodium chloride solution ( 0.9 per cent) and place I c.c. in each of three small test-tubes.

Grind three beans in a coffee mill, or with mortar and pestle to a fine meal and extract for a few minutes with 0.9 per cent sodium chloride solution. Filter and add 0.05 c.c. (about $2-3$ drops) of the filtered extract to the first of the blood tubes; 0.01 c.c. to the second ; and 0.05 of 0.9 per cent sodium chloride to the third.

Invert each tube to mix the contents thoroughly, and note the rapid agglutination and precipitation of the blood corpuscles in the first tube, a less rapid agglutination in the second, while the third or control tube remains unaltered. In one-half hour the corpuscles in the first tube often are packed solid and one is able to pour off perfectly clear serum.

If the remainder of the bean extract is boiled for a few minutes, the coagulum filtered out and 0.05 c.c. of the filtrate added to the control tube, still no agglutination occurs, indicating that the hemagglutinin has been destroyed or removed by the boiling.

I0. Diffusion of Hemoglobin.-Prepare some hemolyzed ("laky") blood, thus liberating the hemoglobin from the erythrocytes. Test the diffusion of the hemoglobin by preparing a dialyzer like one of the models shown in Fig. 2, page 24. How does hemoglobin differ from other well-known crystallizable bodies?

II. Guaiac Test.-To 5 c.c. of water in a test-tube add 2 drops of blood. By means of a pipette drop an alcoholic solution of guaiac (strength about $I: 60)^{4}$ into the resulting mixture until a turbidity is observed and add old turpentine or hydrogen peroxide, drop by drop, until a blue color is obtained.

In the detection of small amounts of blood the quantity of guaiac used should also be decreased. Do any other substances respond in a similar manner to this test? Is a positive guaiac test a sure indication of the presence of blood? (See discussion on page 258.)

12. Schumm's Modification of the Guaiac Test.-To about 5 c.c. of the solution under examination ${ }^{5}$ in a test-tube add about ro drops of freshly prepared alcoholic solution of guaiac. Agitate the tube gently, add about 20 drops of old turpentine, subject the tube to a thorough shaking and permit it to stand for about two to three minutes. A blue color indicates the presence of blood in the solution under examination. In case there is insufficient blood to yield a blue color under

${ }^{1}$ The Scarlet Runner is a familiar variety purchasable in every seed store. It occurs in two varieties, the white and the red. Ricin, a protein constituent of the castor bean, also possesses pronounced agglutinating properties. Because of its poisonous nature it is, however, not suitable for use in class experiments.

2. Mendel: Archivio di fisiologia, 7, I68, I909; Schneider: Journal Biol. Chem., I I, 47, I9I 2 .

${ }^{3}$ Rabbit's blood is especially desirable (Mendel: loc. cit.) and may be obtained for the purpose by bleeding from a small cut on the animal's ear and defibrinating.

"Buckmaster advises the use of an alcoholic solution of guaiaconic acid instead of an alcoholic solution of guaiac resin.

${ }^{5}$ Alkaline solutions should be made slightly acid with acetic acid, as the blue endreaction is very sensitive to alkali. 
these conditions, a few cubic centimeters of alcohol should be added and the tube gently shaken, whereupon a blue coloration will appear in the upper alcoholturpentine layer.

A control test should always be made, using water in place of the solution under examination. In the detection of very minute traces of blood only $3-5$ drops of the guaiac solution should be employed.

13. Ortho-tolidin Test (Ruttan and Hardisty). ${ }^{1}$-To I c.c. of a 4 per cent glacial acetic acid solution of 0 -tolidin ${ }^{2}$ in a test-tube add I c.c. of the solution under examination and I c.c. of 3 per cent hydrogen peroxide. In the presence of blood a bluish color develops (sometimes rather slowly) and persists for some time (several hours in some instances).

This test is said to be as sensitive for the detection of occult blood in feces and stomach contents as is the benzidine reaction. It is also claimed to be more satisfactory for urine than any other blood test. The acetic acid solution may be kept for one month with no reduction. in delicacy.

I4. Benzidine Reaction.-This is one of the most delicate of the reactions for the detection of blood. Different benzidine preparations vary greatly in their sensitiveness, however. Inasmuch as benzidine solutions change readily upon contact with light it is essential that they be kept in a dark place.

The test is performed as follows: To a saturated solution of benzidine in alcohol or glacial acetic acid add an equal volume of 3 per cent hydrogen peroxide and I c.c. of the solution under examination. If the mixture is not already acid render it so with acetic acid, and note the appearance of a green or blue color. A control test should be made substituting water for the solution under examination.

The hemoglobin decomposes the hydrogen peroxide (catalysis) and the liberated oxygen oxidizes the benzidine. The sensitiveness of the benzidine reaction is greater when applied to aqueous solutions than when applied to the urine. According to Ascarelli ${ }^{3}$ the benzidine reaction serves to detect blood when present in a dilution of $1: 3,000,000$. Walter ${ }^{4}$ has also shown the test to be very delicate, and claims it to be more satisfactory than the guaiac test.

Lyle, Curtman and Marshall ${ }^{j}$ have investigated the benzidine

${ }^{1}$ Ruttan and Hardisty: Canadian Medical Ass'n Journal, Nov., ror z; also Biochemical Bull., 2, 225, 1913.

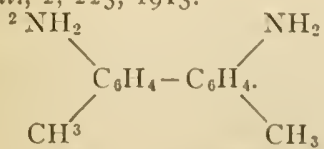

3 Ascarelli: Il policlin sez. pral., 1900.

"Walter: Deul. med. Woch., 36, p. 300.

'Lyle, Curtman and Marshall: Jour. Biol. Chem., In, 445. Ior 4. 
reaction very carefully. They suggest a new procedure in preparing the reagent $t^{1}$ and in conducting the test.

The test follows: Into a perfectly clean dry test-tube introduce r.4 c.c. benzidine solution, ${ }^{1}$ add 0.2 c.c. of water or glacial acetic acid, then I c.c. of the fluid to be tested and finally 0.4 c.c. of 3 per cent hydrogen peroxide. Note the appearance of a blue color, which reaches its maximum in five to six minutes.

The acetic acid keeps the benzidine in solution. An excess diminishes the delicacy of the reagent.

Hydrogen peroxide supplies oxygen for the reaction and also bleaches the blue color. An excess of peroxide interferes with the reaction by destroying the catalytic power of the blood and by reacting with the benzidine itself, with the formation of products which appear to have an inhibitory action. It is very essential that the peroxide be added last.

The benzidine solution should be dilute. Such solutions are exceedingly sensitive and permit the detection of blood when present in ratio I : $5,000,000$.

I5. Hemin Test.-(a) Teichmann's Method.-Place a very small drop of blood on a microscopic slide, add a minute grain of sodium chloride ${ }^{2}$ and carefully evaporate to dryness over a low flame. Put a cover-glass in place, run underneath it a drop of glacial acetic acid and warm gently until the formation of gas bubbles is noted. Add another drop of glacial acetic acid, cool the preparation, examine under the microscope and compare the crystals with those shown in Figs. 78 and 79 .

The hemin crystals result from the decomposition of the hemoglobin of the blood. What are the steps involved in this process? The hemin crystals are also called Teichmann's crystals. Is this an absolute test for blood? Is it possible to differentiate between human blood and the blood of other species by means of the hemin test?

(b) Nippe's Method. ${ }^{3}$-Spread a small drop of blood on a slide in the form of a film and evaporate to dryness over a low flame. Now add 2 drops of a solution containing 0.1 gram each of potassium chloride, iodide and bromide in roo c.c. of glacial acetic acid. Place a cover-glass in position and heat gently over a low flame until gas bubbles form and the solution boils. Run I-2 drops of the reagent underneath the cover-glass and examine under a microscope. Compare the crystals with those shown in Figs. 78 and 79.

\section{This method is more rapid than Teichmann's method and crystals}

${ }^{1}$ Benzidine solution may be prepared as follows: Place 4.33 c.c. of glacial acetic acid in a small Erlenmeyer flask, warm to $50^{\circ}$ and add 0.5 gram of benzidine. Heat the flask for eight to ten minutes in water at $50^{\circ}$. To the resultant solution add I9 c.c. of distilled water. This solution may be kept for several days without deterioration.

2 Buckmaster considers the use of potassium chloride preferable.

${ }^{3}$ Nippe: Deut. med. Woch., 38, 2222 , I912. 


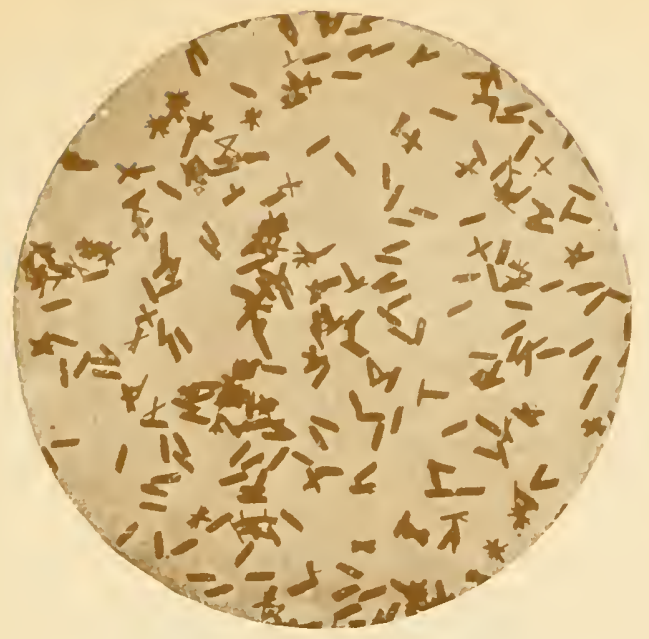

Fig. 78.-Hemin Crystals from Human Blood.

Reproduced from a micro-photograph furnished by Prof. E. T. Reichert, of the University of Pennsylvania.

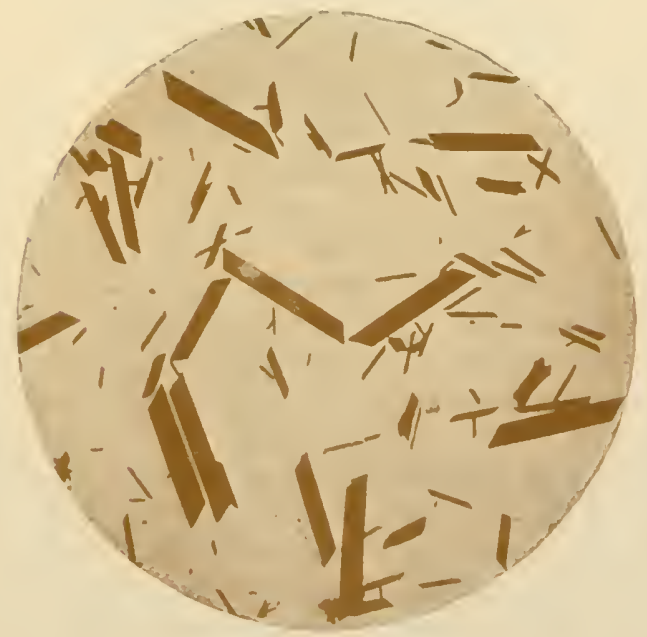

Fig. 79.-Hemin Cristals frou Sheep Blood.

Reproduced from a micro-photograph furnished by Prof. E. T. Reichert, of the University of Pennsylvania. 
of inorganic chlorides are not formed. In Teichmann's method crystals of sodium chloride often obscure the hemin crystals.

(c) Atkinson and Kendall's Method.-Introduce a small amount of the solution under examination into a tube closed at one end, add sodium chloride and glacial acetic acid as in Teichmann's method, ${ }^{1}$ fuse or tightly plug the open end of the tube and heat for fifteen minutes in a boiling water-bath. ${ }^{2}$ Remove the tube and permit it to cool to room temperature spontaneously. When the tube has cooled, break it open, transfer the contents to a watch glass or small evaporating dish and concentrate on a water-bath until the volume of the fluid in the watch glass or dish has been reduced to a few drops. Transfer a drop of this fluid to a slide, cover with a cover slip, allow the slide to stand for a few minutes and examine it under a microscope. Compare the crystals with those shown in Figs. 78 and 79, page 265 . In case crystals of sodium chloride (see Fig. So) obstruct the view of the hemin crystals, dissolve the sodium chloride crystals by running a drop of water under the cover slip.

(d) v. Zeynek and Nencki's Method.-To ro c.c. of defibrinated blood add acetone until no more precipitate forms. Filter off the precipitated protein and extract it with io c.c. of acetone made acid with 2-3 drops of hydrochloric acid. Place a drop of the resulting colored extract on a slide, immediately place a cover-glass in position and examine under the microscope. Upon the evaporation of the acetone, crystals of hemin will form. Larger crystals may be obtained by evaporating the acetone extract about one-half, transferring it to a stoppered vessel and allowing it to remain overnight.

(e) Schalfijew's Method.-Place 20 c.c. of glacial acetic acid in a small beaker and heat to $80^{\circ} \mathrm{C}$. Add 5 c.c. of strained defibrinated blood, again bring the temperature to $80^{\circ} \mathrm{C}$., remove the flame and allow the mixture to cool. Examine the crystals under the microscope and compare them with those reproduced in Figs. 78 and 79 , page 265 .

i6. Catalytic Action.-To about ro drops of blood in a test-tube add twice the volume of hydrogen peroxide, without shaking. The mixture foams. What is the cause of this phenomenon?

17. Crystallization of Oxyhemoglobin. Reichert's Method.-Add to 5 c.c. of the blood of the dog, horse, guinea-pig, or rat, before or after laking, or defibrinating, from I to 5 per cent of ammonium oxalate in substance. Place a drop of this oxalated blood on a slide and examine under the microscope. The crystals of oxyhemoglobin will be seen to form at once near the margin of the drop, and in a few minutes the entire drop may be a solid mass of crystals. Compare the crystals with those shown in Figs. 70 to 76 , pages $25 \mathrm{I}$ to 254.

18. Preparation of Hematin.-Place roo c.c. of hemolyzcd (laked) blood in a beaker and add 95 per cent alcohol until precipitation ceases. What bodies are precipitated? Transfer the precipitate to a flask and boil with 95 per cent alcohol previously acidulated with sulphuric acid. Through the action of the acid the hemoglobin is split into hematin and a protein body called globin. Later the "sulphuric acid ester of hematin" is formed, which is soluble in the alcohol. Continue heating until the precipitate is no longer colored, then filter. Partly saturate the filtrate with sodium chloride and warm. In this process the "hydrochloric acid

${ }^{1}$ Care should be taken not to add too great an excess of these reagents.

${ }^{2}$ This process insures constancy of temperature and strength of reagents. 
ester of hematin" is formed. Filter and dissolve on the filter paper by sodium carbonate. Save this alkaline solution of hematin and make a spectroscopic examination later after becoming familiar with the use of the spectroscope. How does the spectrum of oxyhemoglobin differ from that of the derived alkali hematin?

19. Preparation of Thrombin (Howell). ${ }^{1-P r e p a r e ~ f i b r i n ~ f r o m ~ p i g ' s ~ b l o o d ~}$ according to directions given on page 268 . Wash the fibrin thoroughly in water to remove hemoglobin. Squeeze out the water, mince the fibrin and cover with an 8 per cent sodium chloride solution and allow to stand in the cold for 48 hours. Filter. Precipitate the thrombin (and other proteins) from the filtrate by adding an equal volume of acetone. Filter the mixture rapidly through a number of small (25-50 c.c.) filters. Spread out filter papers and precipitate and dry rapidly in 'a current of cold air. Cut the dried papers into small pieces and treat with a volume of water equivalent to 66 per cent of the 8 per cent $\mathrm{NaCl}$ previously used. Allow to stand one-half hour and filter. Shake the filtrate with chloroform. (I0-I5 C.c. per 100 c.c. filtrate) until on settling no opalescence"is developed by

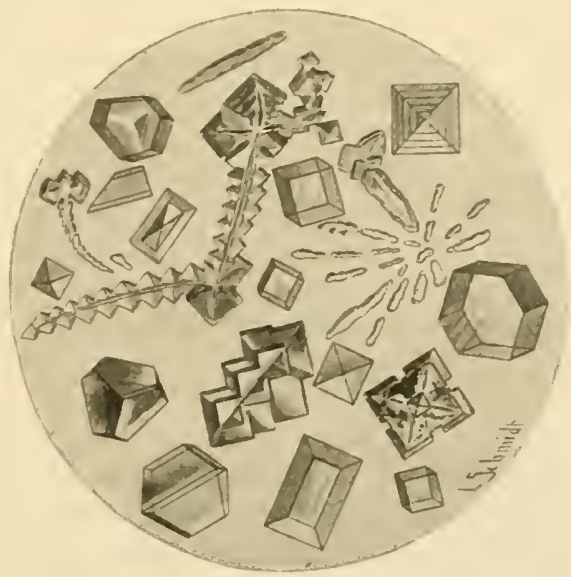

Fig. 80.-Sodiuar Chloride.

heating a portion of the supernatant fluid. Decant the liquid and evaporate on watch glasses (2 c.c. to a watch glass) in a current of air. Thrombin so prepared may be kept indefinitely in a desiccator.

20. Variation in Size of Erythrocytes.-Prepare two small funnels with filter papers such as are used in quantitative analysis. Moisten each paper with physiological (isotonic) salt solution. Into one funnel introduce a small amount of defibrinated ox blood and into the other funnel allow blood to drop directly from a decapitated frog. Note that the filtrate from the ox blood is colored, whereas that from the frog blood is colorless. What deduction do you make regarding the relative size of the erythrocytes in ox and frog blood? Does either filtrate clot? IIhy?

\section{Blood Serum²}

I. Coagulation Temperature.-Place 5 c.c. of undiluted serum in a test-tube and determine its temperature of coagulation according to the method described

1 Howell: Am. Jour. Physiol., 32, 264, igr3.

${ }^{2}$ For directions as to preparation of serum, see "Reagents and Solutions." (Page 593.) 
on page 105. Note the temperature at which a cloudiness occurs as well as the temperature at which coagulation is complete.

2. Precipitation by Alcohol. - To 5 c.c. of serum in a test-tube add twice the amount of 95 per cent alcohol and thoroughly mix by shaking. What is this precipitate? Make a confirmatory test. Test the alcoholic filtrate for protein. Explain the result.

3. Proteins of Blood Serum.-Place about Io c.c. of serum in a small evaporating dish, dilute with 5 c.c. of water and heat to boiling. At the boiling-point acidify slightly with dilute acetic acid. Of what does this coagulum consist? Filter off the coagulum (reserve the filtrate) and test it as follows:

(a) Millon's Reaction.-Make the test according to directions given on page 97 .

(b) Hopkins-Cole Reaction.-Make the test according to directions given on page 98.

4. Sugar in Serum.-To 5 c.c. of the neutralized filtrate from Experiment 3 add 5 drops of Fehling's solution and boil one minute. What do you conclude?

5. Detection of Sodium Chloride.-(a) Test a little of the filtrate from Experiment 3 for chlorides, by the use of nitric acid and silver nitrate. (b) Evapoorate 5 c.c. of the filtrate from Experiment 3 in a watch glass on a water-bath. Examine the crystals and compare them with those reproduced in Fig. 80, p. 267.

6. Separation of Serum Globulin and Serum Albumin.-Place ro c.c. of blood serum in a small beaker and saturate with magnesium sulphate. What is this precipitate? Filter it off and acidify the filtrate slightly with acetic acid. What is this second precipitate? Filter this precipitate off and test the filtrate by the biuret test. What do you conclude?

\section{Blood Plasma}

I. Preparation of Oxalated Plasma.-Allow arterial blood to run into an equal volume of 0.2 per cent ammonium oxalate solution.

2. Preparation of Fibrinogen.-To 25 c.c. of oxalated plasma add an equal volume of saturated sodium chloride solution. Note the precipitation of fibrinogen. Filter off the precipitate (reserve the filtrate) and test it by a protein color test (see page 97).

3. Effect of Calcium Salts.-Place a small amount of oxalated plasma in a test-tube and add a few drops of a 2 per cent calcium chloride solution. What occurs? Explain it.

4. Preparation of Salted Plasma.-Allow arterial blood to run into an equal volume of a saturated solution of sodium sulphate or a ro per cent solution of sodium chloride. Keep the mixture in a cool place for about 24 hours.

5. Effect of Dilution.-Place a few drops of salted plasma in a test-tube and dilute it with I0-I5 volumes of water. What do you observe? Explain it.

\section{Fibrin}

I. Preparation of Fibrin.-Allow blood to flow directly from the animal into a vessel and rapidly whip it by means of a bundle of twigs, a mass of strong cords, or a specially constructed beater. If a pure fibrin is desired it is not best to attempt to manipulate a large volume of blood at one time. After the fibrin has been collected it should be freed from any adhering blood clots and washed in water to remove further traces of blood. The pure product should be very light in color. It may be preserved under glycerol, dilute alcohol, or chloroform water. 
2. Solubility.-Try the solubility of small shreds of freshly prepared fibrin in water, dilute acid and alkali.

3. Millon's Reaction.-Make the test according to directions given on page 97.

4. Glyoxylic Acid Reaction (Hopkins-Cole).-Make the test according to directions given on page 98 .

5. Biuret Test.-Make the test according to directions given on page 98 .

\section{Detection of Blood in Stains on Cloth, Etc.}

I. Identification of Corpuscles. - If the stain under examination is on cloth a portion should be extracted with a few drops of glycerol or physiological (0.9 per cent) sodium chloride solution. A drop of this solution should then be examined under the microscope to determine if corpuscles are present.

2. Tests on Aqueous Extract.-A second portion of the stain should be extracted with a small amount of water and the following tests made upon the aqueous extract:

(a) Hemochromogen.-Make a small amount of the extract alkaline by potassium hydroxide or sodium hydroxide, and heat until a brownish-green color results. Cool and add a few drops of ammonium sulphide or Stokes' reagent (see page 296) and make a spectroscopic examination. Compare the spectrum with that of hemochromogen (see Absorption Spectra, Plate II). Hankin ${ }^{1}$ has suggested a test based upon the formation of cyanhemochromogen and the microspectroscopical demonstration of the spectrum of this compound.

(b) Hemin Test.-Make this test upon a small drop of the aqueous extract according to the directions given on page $26_{4}$.

(c) Guaiac Test.-Make this test on the aqueous extract according to the directions given on page 262. The guaiac solution may also be applied directly to the stain without previous extraction in the following manner: Moisten the stain with water, and after allowing it to stand several minutes, add an alcoholic solution of guaiac (strength about I :60) and a little hydrogen peroxide or old turpentine. The customary blue color will be observed in the presence of blood.

(d) Benzidine Reaction.-Make this test according to directions given on page 263 .

(e) Acid Hematin.-If the stain fails to dissolve in water extract with acid alcohol and examine the spectrum for absorption bands of acid hematin (see Absorption Spectra, Plate II).

${ }^{1}$ Hankin: Brit. Med. Jour., p. I261, 1906.

Sutherland and Mitra: Biochemical Journal, 8, I28, I914. 


\section{CHAPTER XVI}

\section{BLOOD ANALYSIS}

THE study of the composition of the blood under various normal and pathological conditions has received great impetus from the development of methods for blood analysis which require but small amounts of material and yet give accurate results. Many facts of physiological as well as clinical importance have thus been made available. Some typical examples of data obtained in this way are given in the following table:

\section{COMPOSITION OF NORMAL BLOOD AND OF THE BLOOD IN CERTAIN} PATHOLOGICAL CONDITIONS ${ }^{1}$

\begin{tabular}{|c|c|c|c|c|c|c|c|c|c|}
\hline & Normal & $\begin{array}{c}\text { Chronic } \\
\text { nephritis }\end{array}$ & Uremia & $\begin{array}{c}\text { Early } \\
\text { diabetes }\end{array}$ & $\begin{array}{c}\text { Severe } \\
\text { diabetes }\end{array}$ & Gout & Lipemia & $\begin{array}{c}\text { Cholelith- } \\
\text { iasis }\end{array}$ & $\begin{array}{c}\text { Ar- } \\
\text { thritis }\end{array}$ \\
\hline $\begin{array}{l}\text { Total solids, } \\
\text { per cent..... }\end{array}$ & 20.0 & $13-19$ & $12-18$ & $\ldots \ldots \ldots$ & I $7-20$ & $19-21$ & & & \\
\hline $\begin{array}{c}\text { Total N, per } \\
\text { cent......... }\end{array}$ & 3.0 & $2.5-3.0$ & $1.7^{-2} .7$ & $\ldots \ldots \ldots$ & I. $8-2.9$ & & & & \\
\hline $\begin{array}{l}\text { Non-protein } \\
\text { N............ }\end{array}$ & . $25-35$ & $35-90$ & $90-350$ & $\ldots \ldots \ldots$ & $\ldots \ldots \ldots$ & $25-35$ & $\cdots$ & 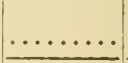 & $60-100$ \\
\hline Urea N....... & $12-23$ & $16-70$ & $70^{-}-300$ & & & & & & \\
\hline Uric acid..... & $\mathrm{I}-3$ & $\mathrm{I}-4$ & $4-27$ & $\ldots \ldots \ldots$ & $\ldots \ldots \ldots$ & $3-6$ & $\ldots \ldots$ & $\ldots \ldots \ldots$ & $3-8$ \\
\hline Creatinine... . & $I-2$ & $I-3$ & 4-33 & & & & & & \\
\hline Creatine...... & $5-9$ & $\ldots \ldots$ & $5-30$ & & & & & & \\
\hline Amino-acidN. & $4-5$ & $\ldots \ldots \ldots$ & $6-16.0$ & & & & & & \\
\hline Ammonia N. & $0.1-0.2$ & $0.1-0.2$ & $0.2-1.0$ & & & & & & \\
\hline Sugar, percent & $0.06-0.11$ & $\ldots \ldots \ldots$ & $0.1-0.2$ & $0.15-0.30$ & $0.3-0.8$ & & & & \\
\hline $\begin{array}{l}\text { Acetone }+ \\
\text { Acetoacetic } \\
\text { acid .......... }\end{array}$ & $0-1.0$ & $\ldots \ldots \ldots$ & $2-25$ & I. 5-12 & $10-40$ & & & & \\
\hline $\begin{array}{l}\text { B-hydroxy- } \\
\text { butyric acid }\end{array}$ & $0-3.0$ & $\ldots \ldots \ldots$ & $5-25$ & $5^{-15}$ & 10-50 & & & & \\
\hline $\begin{array}{l}\text { Cholesterol, } \\
\text { per cent..... }\end{array}$ & $0.14-0.18$ & $0.17-0.35$ & $0.17-0.35$ & $\ldots \ldots \ldots$ & $0.15-0.30$ & $\cdots$ & $0.5-3.6$ & $0.28-0.95$ & \\
\hline $\begin{array}{l}\text { Chlorides as } \\
\mathrm{NaCl} \text {, per cent }\end{array}$ & 0.65 & $0.55-0.75$ & $0.45-0.65$ & $\ldots \ldots \ldots$ & $0.6 n$ & & & & $\dot{-}$ \\
\hline $\begin{array}{r}\text { Acid soluble } \\
\text { phosphorus.. }\end{array}$ & $2-6$ & $3-7$ & $7-21$ & & & & & & \\
\hline $\begin{array}{l}\text { Lipoid phos- } \\
\text { phorus....... }\end{array}$ & $6-12$ & $8-13$ & $8-30$ & & & & & & \\
\hline Fat, per cent & $0.1-0.7^{2}$ & $\cdots$ & . & $\ldots \ldots \ldots$ & $3-18$ & $\cdots \cdots$ & $3-29$ & & \\
\hline
\end{tabular}

${ }^{1}$ Results are expressed as milligrams per roo grams of blood unless otherwise indicated. Some of the figures given are based upon but few analyses and may not be entirely characteristic. The data here tabulated have been compiled from the work of many observers. The following may be particularly mentioned: Myers and Fine: Jour. Biol. Chem., 20, 39I, I9I5; Post-Graduate, I9I4-15; reprinted as "Chemical Composition of the Blood in Health and Disease," New York, I9I5; Folin and Denis: Jour. Biol. Chem., I4, 29, rgI3; 13, 469, I913; I 7, 487, I914; Arch. Int. Med., 16, 33, I915; Christian, Frothingham and Wood: Am.J.Med. Sci., I50, 655, I915; Greenwald: Jour. Biol. Chem., 21, 29, 1915; Van Slyke and Meyer: Jour. Biol. Chem., I2, 399, I9I 2; Bloor: Jour. Biol. Chem., 23, 3 I 7, I915; Marriott: Jour. Biol. Chem., I6, 293, I9I3; 18, 507, I9I4.

${ }^{2} \mathrm{~A}$ short time after a meal rich in fat the blood may contain considerably more fat. 
It will be noted that in chronic nephritis the principal change is in the urea and non-protein nitrogen of the blood which may increase considerably. In severe cases associated with uremia the retention of these forms of nitrogen may be very great and there is a consequent rise in the blood content which may amount to rooo per cent or more. In uremia there is likewise a great increase in other individual nitrogeneous components of the blood such as uric acid, creatinine, creatine, amino-acid nitrogen, and even of ammonia. The increase in creatinine has been shown by Myers and Fine and others to be significant, inasmuch as this increase does not appear to occur in other types of nephritis. Uric acid is greatly increased in uremia and may be very much higher than in gout. Associated with uremia there is ordinarily an acidosis. There may be an increase in the sugar of the blood and a very great increase of the acetone bodies present. An increase is also generally found in cholesterol and in the various forms of phosphorus of the blood.

In diabetes the most noteworthy changes are in the content of glucose and of acetone bodies. Glucose may be increased above the normal (about o.I per cent) to o.I 5-0.80 per cent. The increase in acetone, diacetic acid and hydroxybutyric acid is very marked in comparison with the minute amounts found in normal blood. There may also be an increase in fat and other lipoids in severe diabetes.

In gout the characteristic change is in the uric acid content which is almost always considerably increased. Other forms of nitrogen are affected but little. In arthritis the blood may also be high in uric acid but in this case ordinarily there is a rise in non-protein nitrogen also.

Lipemia is usually associated with an increased sugar content of the blood. The fat content in this condition has been found as high as 29 per cent. There is a correspondingly large increase in the cholesterol of the blood.

In cholelithiasis there appears generally to be a fairly marked increase in the cholesterol content of the blood and this determination is thus of diagnostic aid. Some increase may also be found in other disorders as in nephritis, severe diabetes, pregnancy, alteriosclerosis and syphilis.

\section{Methods}

Non-protein Nitrogen.--(a) Colorimetric Mcthod of Folin and Denis. ${ }^{1}$ -Principle.-This method, which is simple and convenient, depends upon the removal of the proteins from a sample of blood by precipitation with methyl alcohol, and the estimation of nitrogen in the methyl

'Folin and Denis: J. Biol. Chem., II, 527, I912. 
alcohol solution (after the removal of the proteins) by means of oxidation and Nesslerization. The details of the procedure are carried out in the following manner:

Method of Drawing Blood.-Attach, by means of a short piece of pure gum tubing, an hypodermic needle about $\mathrm{I} \mathrm{mm}$. in diameter and $25 \mathrm{~mm}$. in length (previously sterilized and paraffined) to the tip of a 2 or 5 c.c. pipette. Introduce into the upper end of the pipette (which must be perfectly clean and dry) a small pinch of powdered potassium oxalate, and allow it to run down into the tip and the needle. Attach a piece of rubber tubing to the upper end of the pipette, and to this a mouthpiece consisting of a short tapering glass tube. Place a pinchcock over the rubber tube near the top of the pipette. To draw the blood, insert the needle into the vein or artery and regulate the flow by means of the pinchcock and suction. The exact quantity of blood desired is thus obtained without any waste or clotting.

Method of Isolating Non-protein Nitrogen Constituents.-Methyl alcohol and zinc chloride are employed as precipitants for the protein materials of the blood, and the determination of the non-protein nitrogen is then carried out upon a portion of the methyl alcohol extract. The procedure is as follows: Transfer the blood, as soon as drawn, to a measuring flask which is half filled with pure methyl alcohol (must be acetone free). Fill to the mark with methyl alcohol and shake thoroughly. (If 2 c.c. of blood are taken, 25 c.c. flasks are used for the precipitation, while for 5 c.c. of blood 50 c.c. flasks are used.) Allow the flask to stand for at least two hours and at the end of that time, or later, filter the contents through dry filter paper. Add 2-3 drops of a saturated alcoholic solution of zinc chloride to the filtrate and filter again through a dry filter paper after a few minutes. The zinc chloride brings down an appreciable precipitate and the last traces of coloring matter, so that the second filtrate obtained is perfectly colorless and clear. This filtrate is used for the determination of non-protein nitrogen.

Trichloracetic Acid Modification.-Greenwald ${ }^{1}$ has suggested the use of trichloracetic acid as the precipitant for the proteins of the blood, as being more satisfactory than the methyl alcohol and zinc chloride. The objection to the methyl alcohol is that some of the amino-acids (creatine, asparagine, and tyrosine) are insoluble in it and hence precipitated along with the proteins. These acids are not removed by the trichloracetic acid. Certain nitrogenous lipoid substances are precipitated by the trichloracetic acid and not by the methyl alcohol. Greenwald suggests that these substances, even though non-protein in character, should not be included with the non-protein nitrogen of the amino-acids and urea.

Procedure.-Dilute the blood to ten times its original volume with 2.5 per cent trichloracetic acid solution. Let stand 30 minutes and then filter. Shake the filtrate with about 4 grams of kaolin per roo c.c. and filter again. An aliquot of this final filtrate is taken, digested with sulphuric acid and nitrogen determined in the usual way.

${ }^{1}$ Greenwald: J. Biol. Chem., 21, 6I, I9I5. 
Determination of Total Non-protein Nitrogen.-Transfer 5 c.c. of the alcoholic filtrate to a large Jena test-tube of the same kind as is used in urine analysis (see page 485). Add I drop of concentrated sulphuric acid, I drop of kerosene, and a small pebble or glass bead to prevent bumping. Immerse the test-tube in a beaker of boiling water for five or ten minutes to drive off the methyl alcohol. When the alcohol is removed add I c.c. of concentrated sulphuric acid, I gram of potassium sulphate and I drop of copper sulphate solution. Boil, cool, dilute and aerate the solution as described in the determination of total nitrogen in urine (see page 485), except that the ammonia is collected in a large test-tube instead of the roo c.c. flask. Nesslerize the solution, using 7 to 8 c.c. of diluted Nessler reagent (dilution $I: 5$ ), dilute to 25 or 50 c.c. according to the amount of color, and compare with a standard solution containing I $\mathrm{mg}$. of ammonia nitrogen, Nesslerized and diluted to roo c.c. and the colorimeter prism set at $20 \mathrm{~mm}$.

Calculations.-If 5 c.c. of blood are diluted to 50 c.c. and Io c.c. of the alcoholic extract (equivalent to I c.c. of blood) are used for the determination, the amount of non-protein nitrogen (as milligrams per roo c.c. of the blood) can be obtained by use of the formula $\frac{20}{R} \times D$, in which $R$ stands for the reading of the unknown and $D$ represents the volume to which its ammonia has been diluted. If the equivalent of 0.4 c.c. of blood has been taken for the determination the formula ${ }_{\mathrm{R}}^{50} \times \mathrm{D}$ is used, and if the equivalent of 0.5 c.c. of blood has been taken the formula becomes $\frac{40}{R} \times D$.

(b) Colorimetric Method for Finger Blood.-Principle.-Taylor and Hulton $^{1}$ have suggested a modification for the determination of nonprotein nitrogen using from 4 to 8 drops of blood. It is based upon the observation of Gulick ${ }^{2}$ that the presence of small amounts of potassium sulphate does not appreciably interfere with the Nesslerization of solutions containing ammonium salts. The Nesslerization is accordingly carried out directly upon the oxidized material without removal of the ammonia by aspiration or distillation. The authors use a mixture of ether and alcohol for the precipitation of proteins. The authors claim only approximate accuracy for the method and the small amount of lipoid nitrogen included with the other non-protein nitrogen may be disregarded.

Procedure.-Place Io c.c. of a mixture of absolute alcohol and ether $(3: 1)$ in a small Erlenmeyer flask. Stopper and weigh the flask with its contents. Remove the stopper and allow from 4 to 8 drops of blood (depending upon the amount of non-protein nitrogen) to drop from the finger which has been cleaned and pricked with a sharp lance. The blood should drop freely when the hand is held down. Insert the stopper and weigh the flask and contents. The increase in weight is of course the weight of blood. Within a half-hour filter the mixture into the digestion flask and wash the filter with 5 c.c. of alcohol-ether. The filtrate is clear and practically free from protein, although it probably contains traces of nitrogen-containing lipoids. The Kjeldahl digestion is carried out in a

1 A. E. Taylor and F. Hulton: J. Biol. Chem., 22, 63, I915.

${ }^{2}$ Gulick: J. Biol. Chem., I8, 54r, I9I4. 
25 c.c. long-necked, round-bottomed Kjeldahl flask, the neck of which has a crook near the top. Add a small piece of acid potassium sulphate, several glass beads, and drive off the alcohol-ether by heating on a hot plate. When the flask is nearly dry add I c.c. of concentrated sulphuric acid and not more than 300 or $400 \mathrm{mg}$. of potassium sulphate. Then place the flask over the direct flame of a micro-burner (resting the bottom of the flask upon an asbestos board which has a circular perforation a little smaller than the circle of I c.c. of sulphuric acid in the digestion flask) and heat until the mixture is colorless. Transfer the digestion mixture to a Ioo c.c. flask, neutralize the sulphuric acid with about 3 c.c. of a 30 per cent solution of sodium hydroxide, fill the flask to over three-quarters full with ammonia-free water, and add 5 c.c. of Winkler's modification of Nessler's solution (see page 603). Dilute to mark with water and compare the color in the Duboscq colorimeter (see Fig. I53, p. 486) against a standard solution of ammonium sulphate.

2. Urea.-The Urease Method.-Van Slyke and Cullen's ${ }^{1}$ Modification of Marshall's Method.²

Principle.-See Urease Method, Chapter XXVI.

Procedure.-Run 3 c.c. of fresh blood (carefully measured with an accurate pipette) into a roo c.c. test-tube containing I c.c. of a 3 per cent solution of potassium citrate (to prevent clotting). Add 0.5 c.c. of the urease solution ${ }^{3}$ and 2 or 3 drops of caprylic alcohol (to prevent foaming). After ten minutes add I5 c.c. of a saturated solution of potassium carbonate, and drive off the ammonia by aspiration into another tube containing I5 c.c. of hundredth-normal hydrochloric or sulphuric acid. Titrate the excess of acid with hundredth-normal sodium hydroxide or potassium hydroxide ${ }^{4}$ using methyl red or alizarin as indicator.

Calculations.-Each cubic centimeter of acid neutralized indicates 0.01 gram of urea per Ioo c.c. of blood, or 0.0467 gram of urea nitrogen per Ioo c. c. of blood. In case the blood should be one of the rare samples containing over 0.15 per cent of urea, all the'acid will be neutralized, and it will be necessary to repeat the determinations, using in the determination only I c.c. of blood. Fresh blood contains so little ammonia that it may be disregarded. For further discussion of the urease method see Chapter XXVI.

3. Uric Acid.-Colorimetric Microchemical Method.-Principle.Folin and Denis ${ }^{5}$ were the first to apply the technic of their method for the determination of small quantities of uric acid to the quantitative estimation of uric acid in blood. (For a discussion of the principle of the color reaction see the determination of uric acid in urine, page 510.) The procedure necessary for the determination in blood is somewhat different from that used for the determination in urine because of the presence of proteins in the blood. These must be removed first, and then the uric acid may be determined in the protein-free extract, according to the procedure used for the determination in urine. The

${ }^{1}$ Van Slyke and Cullen: J. Am. Med. Ass'n, 62, I558, I9I4.

2 Marshall: Jour. Biol. Chem., I5, 487, I9r3.

3 The enzyme solution is prepared as described under "Reagents and Solutions," p. 594.

${ }^{4}$ Rose and Coleman (Biochem. Bull., 3, 4II, IgI4) suggest the colorimetric determination of the ammonia (see page 492 ).

5 Folin and Denis: J. Biol. Chem., I3, 469, I913. 
proteins are removed from the blood by precipitation with hot dilute acetic acid in the following manner:

(a) Folin-Denis Procedure.-The blood is drawn into small, wide-mouthed bottles previously weighed and containing a small amount (about o.I gram) of finely powdered potassium oxalate. From the subsequent weight of each bottle is obtained the weight of the blood. Five times this weight of $\mathrm{N} / \mathrm{I} 00$ acetic acid solution $^{1}$ is transferred to an ordinary rooo c.c. flask and heated to boiling. The oxalated blood is then poured into this boiling acetic acid solution, stirring constantly, and the heating is continued until the solution has again begun to boil. The mixture is filtered while still hot. The coagulated material on the filter paper is transferred back into the flask (by means of a small spoon or a spatula), about 200 c.c. of boiling water ${ }^{2}$ are poured over it and it is allowed to stand for five minutes. This mixture is then filtered through the same filter as was used for the first filtration. The filtrate in the receiving flask should be very nearly as clear as water, and will be found to be so if the original blood was promptly shaken with the oxalate, so that no clotting has taken place.

If clotting has occurred, the coagulation and washing of the blood is a little more complicated. The clot leads to so much bumping in the boiling acetic acid solution that it is not practical or safe to try to heat the mixture to boiling. The filtration is, therefore, made earlier. The partially coagulated clot is then broken up with a glass rod, transferred to a mortar and there ground into a paste in the presence of hot water. This suspension is then poured on the filter. The protein material on the filter is then washed, as before, with about 200 c.c. of hot water. In this case the combined filtrates are, however, never colorless but more or less reddish. On being heated to boiling a second small coagulum will be obtained and the filtrate will then be practically as clear as water.

The combined filtrate and washings, containing the uric acid and other soluble materials, is further acidified by the addition of 5 c.c. of 50 per cent acetic acid and is evaporated, over a free flame in a suitable dish, ${ }^{3}$ to a very small volume (about 3 c.c.). The liquid is then poured into an ordinary centrifuge tube and the dish washed with two successive portions of o.I per cent lithium carbonate solution, using about 2 c.c. for each rinsing. Any solid material adhering to the sides of the dish is removed by rubbing with a rubber-tipped stirring rod. This solid material can be removed by centrifuging and pouring the supernatant liquid into another tube, washing the sediment with lithium carbonate solution (Smith).

The liquid in the centrifuge tube, which at this stage should not be more than Io c.c. in volume, is then treated as in the method for urine.

(b) Benedict Procedure.-Benedict ${ }^{1}$ has suggested that the precipitation of proteins by the method of Folin and Denis is incomplete, and proposes the use of colloidal iron for the completion of precipitation

${ }^{1}$ Prepared by diluting 0.6 c.c. of glacial acetic acid to I liter.

2 For this washing, water is used rather than $\mathrm{Y} /$ roo acetic acid, because if the latter is used the coagulum will give off more or less of the blood pigment and the filtrates are less clear.

${ }^{3}$ Deep (half globular) dishes $10 \mathrm{~cm}$. in diameter and having a capacity' of 250 c.c. are very good for this purpose. While free llames are the most convenient for concentrating the uric acid solutions, care must, of course, be taken not to char the contents toward the end of the operation. Unless the solution can be watched carefully at this stage, it is safer to finish the concentration on the water-bith.

- Benedict: $J$. Biol. Chem., 20, 629, I915. 
after coagulation in dilute acetic acid. Myers and Fine ${ }^{1}$ suggest the use of aluminium cream for this purpose. Benedict's procedure is as follows:

To 20 c.c. ${ }^{2}$ of oxalated blood are added roo c.c. of boiling $\mathrm{N} / \mathrm{I} 00$ acetic acid in a casserole, and the mixture is heated to boiling for a moment. Remove the casserole from the flame and add 200 c.c. of boiling distilled water. Pour the mixture upon a folded filter and wash the residue with 50 c.c. of boiling water (beated in the same casserole in which the original coagulation took place). Transfer the whole filtrate to a casserole and boil down rapidly to a volume of about 25 c.c. Pour this solution into a small flask roughly marked to indicate a volume of 50 c.c. Transfer the contents of the casserole to the flask quantitatively, with the help of two or three portions of water, heating vigorously to boiling and rubbing the sides of the casserole with a rubber-tipped stirring rod each time. The total volume in the flask should not exceed 50 c.c. after the addition of the washings. Thoroughly cool the turbid solution in the flask under running water, and add 2 c.c. ${ }^{3}$ of colloidal iron solution (Merck's "Dialyzed Iron," 5 per cent solution) while the flask is being gently rotated. Filter the mixture through a small folded filter into a Ioo c.c. Jena Florence flask, and wash the residue twice with distilled water. The filtrate obtained here should be as clear and colorless as distilled water. Boil the solution down to a volume of from I to 2 c.c. (care being taken in the early stages to prevent bumping), then carefully pour into a small centrifuge tube and wash out the flask with three portions of water ( $\mathrm{I}$ or 2 c.c. each), heating each to boiling in the flask and shaking thoroughly prior to transferring it to the centrifuge tube. The volume of liquid in the tube at this point should be from 5 to Io c.c. Cool the liquid, add 20 drops of the ammoniacal silver magnesium solution and proceed with the determination as described under Urine (Chapter XXVI). If the amount of uric acid present is very small the addition of I drop of cyanide solution, I c.c. of uric acid reagent, 5 c.c. of 20 per cent sodium carbonate solution, and dilution to 25 c.c. are carried out rather than using the larger quantities given for the determination in the urine.

4. Creatine and Creatinine. Methods of Folin. ${ }^{4}$-Preformed Creatinine.-Measure ro c.c. of blood into a 50 c.c. volumetric flask or, better, into a 50 c.c. shaking cylinder which can be closed with a glass stopper. Fill to the 50 c.c. mark with saturated picric acid solution and shake a few times. Add about I gram of dry picric acid to the mixture and shake for five minutes. Transfer the mixture to centrifuge tubes, throw down the sediment and precipitate and pour the supernatant liquid through a filter. This is the most economical process where but little blood is available. If desired, however, double quantities of blood and reagents may be taken and filtration carried out without preliminary centrifugation. This process removes the protein materials and leaves the creatine and creatinine in the filtrate which is a satu-

I Myers and Fine: "Blood in Health and Disease," I9I 5, p. I4.

${ }^{2}$ Smaller amounts of blood may be employed, and the quantity of acetic acid and water correspondingly reduced. Unless the quantity of uric acid present is very large, the results are far more accurate when 20 c.c. of blood are used.

${ }^{3}$ With old samples of blood it may be necessary to add 3 or 4 c.c. of the iron solution and a little Io per cent sodium chloride solution. When the precipitate separates in large flocculent masses the right amount of iron has been added. Any excess of iron must be avoided, as it would oxidize some of the uric acid later on in the process.

- Folin: Jour. Biol. Chem., I 7, 475, I914. 
rated picric acid solution. The preformed creatinine is then determined colorimetrically. For this purpose a standard solution of creatinine for comparison is necessary. Prepare this from the standard creatinine stock solution as used in the analysis of urine (see chapter on Quantitative Analysis of Urine) by diluting an amount of this solution equivalent to $\mathrm{I} \mathrm{mg}$. of creatinine to 500 c.c. with saturated picric acid solution. We have then a standard solution containing $0.2 \mathrm{mg}$. of creatinine in roo c.c. Of saturated picric acid solution.

Take 20 c.c. portions each of the filtrate and of the standard solution. To each solution then add exactly I c.c. of Io per cent $\mathrm{NaOH}$ from a burette. (If the blood filtrate becomes turbid on addition of alkali it must be centrifuged or filtered.) Allow to stand for ro minutes and compare the colors directly in the colorimeter without further dilution. The standard creatinine solution may be set advantageously at $20 \mathrm{~mm}$., although this is not necessary.

Calculation.-Since the blood was diluted five times in the precipitation procedure and as the standard for comparison contains $0.2 \mathrm{mg}$. of creatinine per roo c.c., it is merely necessary to divide the reading of the standard by the reading of the unknown to obtain without further calculation the number of milligrams of creatinine in roo c.c. of blood.

Creatine Plus Creatinine.-For determining the total creatinine plus creatine in the blood carry out the preliminary precipitation with picric acid just as in the determination of creatinine above. Take ro c.c. of this filtrate for the determination. Transfer it to a small Erlenmeyer flask or large test-tube. Cover the flask or test-tube with tin foil, transfer to an autoclave and heat to about $120^{\circ} \mathrm{C}$. for about 20 minutes. The autoclave should not be opened until the temperature has fallen below $100^{\circ} \mathrm{C}$. Cool the solution to room temperature, rinse into a 25 c.c. volumetric flask with saturated picric acid solution. Add I.25 C.c. of ro per cent $\mathrm{NaOH}$ for the development of the color.

On account of the variations in the creatine content of normal blood two standard creatinine solutions are used. In working on pathological cases a third standard is desirable. These standards contain $0.5, \mathrm{I}$, and $2 \mathrm{mg}$. of creatinine respectively per 100 c.c. of saturated picric acid solution. To 20 c.c. of each of these solutions in measuring cylinders add I c.c. of ro per cent $\mathrm{NaOH}$ and allow to stand for ro minutes. By inspection determine which standard corresponds most nearly in color with the unknown and use this for comparison. The standard is usually set at ro $\mathrm{mm}$. in the Duboscq colorimeter.

Calculation.-Multiply the reading of the standard by 125 and by $0.5,1$, or 2 , according to which standard is used, and divide by the reading of the unknown in millimeters. The result gives the number of milligrams of creatine + creatinine in roo c.c. of the blood examined.

5. Amino-acid Nitrogen. (a) Method of Van Slyke and Meyer. LPrinciple.The protein of the blood is removed by precipitation with alcohol and the aminoacid nitrogen determined in the filtrate by the nitrous acid method.

Procedure.-Thirty to 50 c.c. of freshly drawn blood are mixed with 9 or ro volumes of 95 per cent alcohol to precipitate the proteins. The volume of the alcohol-blood mixture must be known, but in case it is not convenient to use a graduated cylinder for the mixture, its volume can be taken as the sum of the volumes of the alcohol and blood without essentially affecting the results. The alcohol and blood are thoroughly mixed, the vessel containing them is closed and

${ }^{1}$ Van Slyke and Meyer: Jour. Biol. Chem., I 2, 399, г пा 2. 
24 hours are allowed for precipitation of the proteins to become complete. The solution is filtered through a dry folded filter into a measuring cylinder without washing the precipitate. The volume of filtrate is noted and is taken for analysis as an aliquot part of the total blood-alcohol mixture. The filtrate is then concentrated to a volume of $3-5$ c.c. and used for determination of amino nitrogen by the Van Slyke nitrous acid method (see Chapter IV on Proteins). The use of a few drops of caprylic alcohol to prevent foaming is advisable.

(b) Method of Constantino. ${ }^{1-T h i s}$ is based on the formol titration procedure. One hundred c.c. of blood or serum is mixed with a measured (500 c.c.) volume of 2 per cent mercuric chloride solution containing 0.8 per cent hydrochloric acid. The mixture is shaken vigorously in a stoppered flask and allowed to stand a few hours. Centrifugate for Io minutes, pour the supernatant liquid through a dry filter into a graduated cylinder. An aliquot of the filtrate is taken, the mercury is removed with hydrogen sulphide and the latter by a current of air. The liquid is exactly neutralized and concentrated on the water-bath, or better, at $50^{\circ}$ in a vacuum, $\mathrm{MgO}$ added, and the mixture distilled in a vacuum at $45^{\circ}$ to get rid of ammonia. The volume should now be about 30 c.c. A little solid barium chloride and barium hydroxide are added and I.5 C.c. of 0.5 per cent solution of phenolphthalein. Filter. Neutralize accurately to sensitive litmus paper. Add neutral formalin solution and titrate with $\mathrm{N} / 5 \mathrm{NaOH}$ as described in the chapter on quantitative analysis of urine (page 504).

6. Ammonia.-Method of Folin and Denis. - The determination of ammonia in blood is attended with considerable difficulty because of the fact that it is present only in very small amounts, and to the fact that blood very readily and quickly undergoes changes which are accompanied by the formation of ammonia from the nitrogenous compounds present in the fresh blood.

Of the methods proposed for its quantitative estimation that of Folin and Denis ${ }^{2}$ is perhaps least unsatisfactory.

Principle.-The method is based upon the liberation of the ammonia from fresin blood by aspiration after adding sodium carbonate solution, and the Nesslerization of the solution into which the ammonia is aspirated.

Procedure.-Place ı c.c. of systemic blood or 5 c.c. of portal or mesenteric blood ${ }^{3}$ in a large Jena test-tube. Add 2 or 3 c.c. of a solution composed of $I_{5}$ per cent potassium oxalate and ro per cent sodium carbonate, and about 5 c.c. of toluene. Connect the tube for aspiration as in the method for urea in blood; start the air current, and run as fast as the apparatus will stand for 20 to 30 minutes. Collect the ammonia in another test-tube containing I c.c. of water and 5 or 6 drops of tenth-normal acid.

At the end of the aspiration Nesslerize in the usual manner (see method for nonprotein nitrogen, page 273) but more cautiously, adding in all not over I c.c. of the previously diluted Nessler reagent (dilution I:5). Transfer the contents of the receiver to a Io c.c. volumetric flask, fill to the mark with ammonia-free water, and mix. Fill a $100 \mathrm{~mm}$. polariscope tube with the mixture and close as for ordinary polariscopic work.

Prepare two standard solutions, one containing $0.5 \mathrm{mg}$. and the other $1.0 \mathrm{mg}$. of nitrogen, these being Nesslerized simultaneously with the unknown solution and made up to volume (roo c.c.). Use the standard possessing a tint most similar to that of the unknown. Compare in a Duboscq colorimeter, using the ordinary

${ }^{1}$ Constantino: Bioch. Zeit., 55, 419, I913.

${ }^{2}$ Folin and Denis: J. Biol. Chem., II, 532, I9I5.

${ }^{3}$ The blood must be freshly drawn. For method of obtaining blood, see page 272 . 
cylinder for the standard and replacing the other cylinder and prism by the polariscope tube, containing the unknown, which usually fits properly in place in the colorimeter.

The unknown solution remains stationary (100 mm.) and the standard is adjusted until the colors match. An iris diaphragm, such as is used in microscopical work, must be attached to the side of the colorimeter holding the standard to reduce the light passing through the standard solution. This is necessary in order to obtain two fields of the same tint. The calculation is made in the usual manner for colorimetric determinations, the amount of ammonia in the unknown being directly proportional to the reading of the standard and its concentration.

7. Total Nitrogen.-The total nitrogen of the blood may be readily determined by the regular Kjeldahl 'method (see Chapter XXVI). One c.c. of the blood accurately measured is used in this method. The microchemical method of Folin and Farmer, as outlined in the same chapter, may also be employed. In this case transfer I c.c. of the well-mixed blood to a 25 c.c. flask, make to the mark with distilled water, mix thoroughly and take I c.c. of this diluted blood for the digestion and determination as there given.

\section{Sugar. (a) Method of Lewis and Benedict. ${ }^{1}$ - Principle.-The red} color obtained by heating a glucose solution with picric acid and sodium carbonate is employed as the basis of the colorimetric determination. The blood protein is removed by precipitation with picric acid.

Procedure.-Two c.c. of blood are aspirated through a hypodermic needle 2 and a piece of rubber tubing into an Ostwald pipette, a little powdered potassium oxalate in the tip of the pipette preventing clotting. The blood is drawn up a little above the mark and the end of the pipette is closed with the finger. After the rubber tubing and needle are disconnected, the blood is allowed to flow back to the mark and is discharged at once into a 25 c.c. volumetric flask containing 5 c.c. of water. The contents of the flask are shaken to insure thorough mixing and the consequent hemolysis of the blood. Then 15 c.c. of saturated aqueous solution of picric acid are added, as well as a drop or two of alcohol to dispel any foam, and the contents of the flask are made up to the mark with water and then shaken. After filtration 8 c.c. aliquots are measured out into large Jena testtubes for duplicate determinations. Two c.c. of saturated picric acid solution and exactly I c.c. of Io per cent sodium carbonate are added (as well as two glass beads and 2 or 3 drops of mineral oil), and the contents of the tube are evaporated rapidly over a direct flame until precipitation occurs. About 3 c.c. of water are added, the tube is again heated to boiling to dissolve the precipitate, the contents of the tube are transferred quantitatively to a 1o c.c. volumetric flask, ${ }^{3}$ cooled, made up to the mark, shaken, and then filtered through cotton into the chamber of a Duboscq colorimeter (see Fig. I53, p. 486). The color is compared atonce with that obtained from $0.64 \mathrm{mg}$. of glucose, 5 c.c. of saturated picric acid, and I c.c. of I9 per cent sodium carbonate, when evaporated to precipitation over a free

${ }^{1}$ Lewis and Benedict: Jour. Biol. Chem., 20, 6r, 1915. For modification see Myers \& Bailey: Jour. Biol. Chem., 24, 147, I916.

${ }^{3}$ It may be more convenient to draw about 5 c.c. of blood directly into a test-tube containing a little finely powdered potassium oxalate and removing 2 c.c. portions of this with the Ostwald pipette.

${ }^{3}$ In case of hyperglycemia the final volume of the reaction fluid is made 25 c.c. or 50 c.c., and the results are accordingly multiplied by 2.5 or 5.0 . 
flame and diluted to Io c.c. as was the unknown, or against the picramic acid standard mentioned below. ${ }^{1}$

Calculation.-If directions are followed exactly the calculation is as follows: Milligrams glucose in unknown $=\begin{aligned} & \text { reading of standard } \\ & \text { reading of unknown }\end{aligned} \times$ milligrams of glucose in standard.

(b) Pearce's Modification of Lewis-Benedict Method. ${ }^{2}$ - This modification entails the use of an autoclave instead of the free flame and has the advantages of decreasing danger of loss and making it possible to carry out a large number of estimations at one time. Proceed exactly as in the Lewis-Benedict, but use 6 c.c. of the the picric acid filtrate instead of 8 c.c. and instead of heating over the free flame introduce into an autoclave for $15-30$ minutes at about 20 pounds pressure to the square inch. Compare with standard in a colorimeter. The standard recommended by Lewis and Benedict may be diluted one-fourth or allowed for by calculation, since 6 c.c. of filtrate are used in place of 8 c.c.

(c) Micro-method of Bang.-Principle.-Two or 3 drops of blood are transferred to a small weighed piece of blotting paper and the paper again weighed to determine the amount of blood. The paper is then treated with boiling acidified $\mathrm{KCl}$ solution which coagulates the protein and allows the sugar to diffuse out. The sugar solution thus obtained is boiled with alkaline cupric chloride solution. The amount of cuprous chloride formed by the reducing action of the sugar is determined by titration with standard iodine solution.

Procedure.-Small pieces of good absorbent paper, about $16 \times 28 \mathrm{~mm}$. in size, ${ }^{3}$ weighing about $100 \mathrm{mg}$. and held by a small spring clip, are used. To one of these previously weighed ${ }^{4}$ transfer 2-3 drops (about $120 \mathrm{mg}$.) of blood obtained by piercing the cleansed finger. Weigh again immediately and determine by subtraction the weight of blood taken.

${ }^{1}$ Permanent Standard.-A solution of picramic acid makes a very satisfactory permanent standard. The color is identical in quality with that formed in the method above and its solution keeps perfectly. The formula of the permanent standard is:
Picramic acid
Sodium carbonate (anhydrous)
Water to make
0.064 gram
0. 100 gram
1000.0 c.c.

Dissolve the picramic acid with the aid of heat in 25 to 50 c.c. of distilled water which has been made alkaline with sodium carbonate. Cool and dilute to I liter. This solution has the same intensity of color as that obtained by the proposed method with 0.64 mg. of sugar when the final volume of the reaction fluid is made io c.c. The solution should be standardized against pure glucose.

A satisfactory preparation of picramic acid may be obtained from the J. T. Baker Chemical Co., Phillipsburg, N. J.

2 Pearce: Jour. Biol. Chem., 22, 525, 1915.

${ }^{3}$ Suitable pieces of paper, weighed, ready for use, and with clip attached, may be obtained from Warmbrunn and Quilitz, Berlin. A suitable paper may also be obtained from Griffin and Sons, London, or Grave of Stockholm. Unless specially prepared, the paper should be repeatedly washed with large volumes of hot water acidified with acetic acid to remove impurities.

"The weighing is preferably made on a special torsion micro-balance which, as well as the other apparatus used in this method, may be obtained from either of the firms mentioned in Note 3 . The weighing must be made in a few seconds and with an accuracy of about I $\mathrm{mg}$. 
Coagulation of Blood Protein.-Transfer the piece of paper to a test-tube and add 6.5 c.c. of boiling acid-potassium chloride solution ${ }^{1}$ and let stand half an hour. The clear solution containing the sugar is poured into a 50 c.c. Jena flask the flange of which has been removed. Wash the paper and tube again with 6.5 c.c. of hot salt solution and transfer washings to the flask. Cool.

Reduction of Cupric Chloride.-Attach to the mouth of the flask a piece of tight-fitting rubber tubing about 2 inches long (see Fig. 8I), provided with a clamp which permits of shutting off the contents of the flask from the outside air. Now add to the flask I c.c. of the cupric chloride solution. ${ }^{2}$ Heat so that the solution is brought to a boil in one minute and 30 seconds (an error of five seconds may be disregarded). Allow to boil for exactly two minutes; at the end of this time tighten the clamp over the mouth of the flask. At the same time remove from the flame and cool at once under the tap for about a minute.

Titration of Cuprous Chloride Formed.-The titration is made with $\mathrm{N} / 200$ iodine solution ${ }^{3}$ run in from a very accurate burette (preferably a 2 c.c. burette graduated in $I / 50$ c.c.). Two or 3 drops of starch solution (preferably soluble starch ${ }^{4}$ ) are added as an indicator. During the titration air must be excluded to prevent re-oxidation. This is done by running a slow stream of carbon dioxide from a generating bottle through a small tube which extends nearly to the bottom of the flask. The titration should be carried out against a white background and the end point taken when the blue color persists for 20-30 seconds.

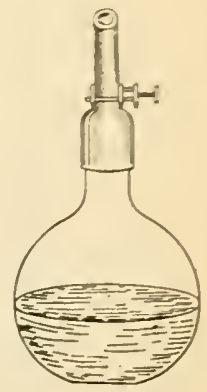

FIG. 8I.BANG REDECTION FLASK.

Calculation.- The copper and other solutions used in the test bind about 0.12 c.c. of the iodine solution. This amount must hence be subtracted from the reading. The corrected reading is then divided by 4 to obtain the number of milligrams of glucose in the sample.

Example.-If 0.68 c.c. of $\mathrm{N} / 200$ I solution were required, $\frac{0.68-0.12}{4}=0.14$ mg. glucose in the amount of blood used. If $140 \mathrm{mg}$. of blood were taken for analysis the per cent of glucose in the blood would be $\frac{1000}{140} \times 0.14 \mathrm{mg} .=0.1$ per cent glucose.

The results obtained by this method are a little higher than those obtained by other reliable methods due to the presence of certain I-binding substances in blood. As these appear to be nearly constant in amount a correction may be applied. To obtain true values for glucose of the blood therefore subtract 0.015 per cent from the value obtained as above. 0.1 per cent -0.015 per cent $=0.085$ per cent glucose.

${ }^{1}$ Consisting of 1360 c.c. of saturated $\mathrm{KCl}$ to which is added 640 c.c. of water and 1.5 c.c. of 25 per cent HCL.

${ }^{2}$ Copper solution. Introduce into a rooo c.c. flask 700 c.c. of boiled and cooled water. Warm to about $30^{\circ} \mathrm{C}$. and add r 60 grams of pure potassium bicarbonate in powder form. When dissolved add 66 grams of pure $\mathrm{KCl}$. Cool and then add roo grams potassium carbonate. Finally add roo c.c. of 4.4 per cent solution of pure crystalline copper sulphate. Let stand a short time, then make to mark with boiled water. Allow to stand a day or so before using.

${ }^{3} \mathrm{~N} / 200$ I solution, made fresh each day. Dilute $\mathrm{N} /$ IO I solution 20 times, or make as follows: Introduce into a roo c.c. flask 2 grams KI, I-2 c.c. of 2 per cent $\mathrm{KIO}_{3}$ solution and 5 c.c. of $\mathrm{N} /$ ro $\mathrm{HCl}$. Make to mark with boiled and cooled distilled water.

'A I per cent solution of Kahlbaum's soluble starch in a saturated $\mathrm{K} \mathrm{Cl}$ solution. 
To secure accurate results the method of Bang must be rigidly controlled, all new solutions and absorbent papers being checked up against pure 0.2 per cent glucose solutions. Taylor and Hulton ${ }^{1}$ also suggest the following precautions. A blank check must be made on the reagents each day an estimation is made. O.IO-O.I5 gram of blood should be taken and must spread smoothly on the paper. The proteins are best coagulated by heating of the blood-impregnated papers in the hot air oven at $100^{\circ}$ (as recommended by Gardner and McLean) ${ }^{2}$ for five minutes with corks of flasks inverted. The solution should be boiled four minutes for complete reduction. The iodine solution must be fresh each day and checked each day. Determinations should be made in triplicate. Results cannot be depended upon to be more accurate than to 0.005 gram glucose in roo c.c. blood. Other authors have recommended that an hour instead of half an hour be allowed for the diffusion of the blood sugar, the fluid being brought to the boiling-point twice during this period or kept in a bath at $40^{\circ} \mathrm{C}$.

Method of Epstein. ${ }^{3-}$-Principle.-This method is a modification of the Lewis and Benedict procedure, being based on the same principle but making possible the determination of reducing sugar in finger blood (0.I-0.2 c.c.) with a sufficient degree of accuracy for clinical purposes, and with little expenditure of time. Instead of a Duboscq colorimeter the less expensive Sahli-Gower hemoglobin colorimeter is recommended.

Procedure.-The apparatus ${ }^{4}$ shown in the illustration (Fig. 82) and the following reagents are necessary:

I. Picric acid, saturated solution.

2. Sodium carbonate, Io per cent solution.

3. Sodium fluorid or potassium oxalate, 2 per cent solution.

Put one or two drops of the fluorid or oxalate solution into the graduated testtube (see illustration). By means of the blood pipette, 3, 0.2 c.c. of blood is obtained from the tip of the finger or the lobe of the ear and is discharged into the tube containing the fluorid solution. The pipette is rinsed two or three times with distilled water and the washings added to the blood in the tube. Distilled water is then added to the r.o c.c. mark. After laking of the blood has taken place, picric acid is added to this (a few drops at a time) up to the 2.5 c.c. mark, shaking the tube gently with each addition of the acid. Precipitation of the blood-proteins takes place; the sugar, together with an excess of picric acid sufficient for the

1 Taylor and Hulton: Jour. Biol. Chem., 22, 63, I915.

${ }^{2}$ Gardner and McLean: Biochem. J., 8, 391, I9I4.

3 Epstein: J.Am.Med. Assn., 63, I667, I914.

The tubes belonging to this hemoglobinometer are not all equally calibrated. With some the 50 per cent mark represents a volume of 1.0 c.c.; with others, I.O c.c. of fluid reaches up to the $43,45,46$ or 47 per cent mark. The error in the calibration is generally below the ro per cent mark; the graduations above this mark are usually correct. By means of the standard I.O c.c. pipette one can readily determine whether or not a given tube is properly calibrated. In order to facilitate a direct reading of the percentage of sugar on these hemoglobinometer tubes, it is essential to have I.O c.c. of fluid stand at mark 50. To overcome a discrepancy (if any exists) in the calibration of a given tube, one may put one, two or three small glass beads in the bottom of the tube, of such size as to raise the meniscus of $\mathrm{r} .0$ c.c. of fluid up to the 50 per cent mark. 
reaction, stays in solution. The tube is finally shaken vigorously (covering the end of the tube with the finger) and the contents filtered through a small filter, or, better still, centrifuged for one or two minutes.

One c.c. of the filtrate or the clear supernatant fluid obtained on centrifugalization is withdrawn, put into the plain test-tube, and heated carefully over the naked flame. The contents of the tube are boiled until all but 2 or 3 drops of the solution is evaporated. One-half c.c. of the ro per cent sodium carbonate solution is then added and the tube heated again until the contents are concentrated to a small volume equal to about 2 or 3 drops. The color of the fluid changes from yellow to deep red or reddish brown and the reaction is completed.

Three or 4 drops of distilled water are added and the tube warmed gently. The contents are then transferred to the graduated tube of the hemoglobinometer. The boiling tube is rinsed several times with water (using only 3 or 4 drops at a time). The tube is warmed with each rinsing before transferring the contents to the graduated tube. The volume of fluid is then made up to the mark jo on the scale.
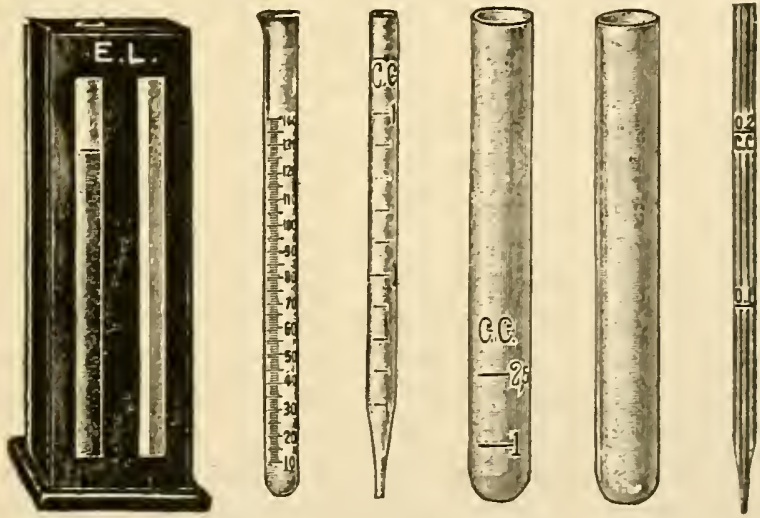

Fig. 82.-Apparatus for Epstemx's Stgar Method.

The color of the resulting solution is compared with that of the two standard tubes, $A$ and $B$ which accompany the instrument. (A solution of picramic acid of the proper strength, prepared as described on p. 280 , may be used as a standard.) If it is darker than standard $A$ (representing 0.05 per cent of sugar) and lighter than standard $B$ (representing o.I per cent), the first standard is used for comparison. In either case the solution in the graduated tube is diluted gradually with water (just as is usually done in hemoglobin estimations) until the colors match.

The percentage of sugar in the blood is then computed thus: Using the lighter standard $A$ the figure on the scale, divided by rooo represents the percentage of sugar in the blood. For example, the tube reads 86 ; then the result is

$$
\frac{86}{1 \mathrm{cco}}=0.086 \text { per cent }
$$

When Standard $B$ is used for comparison, the figure on the scale is multiplied by 2 and divided by 1000 . For example, the tube reads 73 ; then the percentage of sugar is

$$
\underset{1000}{73 \times 2}=0.146 \text { per cent }
$$


With the instructions given, the above formulas may be used for direct computation of the percentage of sugar only, when 0.2 c.c. of blood is used in the determination. When, however, only o.I c.c. of blood is used, the formulas apply as well, but the value obtained must be multiplied by 2 .

It is better, in cases in which a high sugar content in the blood is suspected (in diabetes for example) to use only o.I c.c. of blood for the determination. In all other cases 0.2 c.c. of blood should be used.

8. Acetone Bodies ${ }^{1}$ (Acetone, Diacetic Acid and $\beta$-Hydroxybutyric Acid).Marriott-Scott-Wilson Method. ${ }^{2}$-(a) Acetone and Diacetic Acid.-Draw ro c.c. of blood from a superficial vein by a sterile graduated syringe and run it into about 40 c.c. of 0.5 per cent potassium oxalate solution. Fit up a Kjeldahl distillation apparatus using an 800 c.c. flask, provided with a dropping funnel, the delivery tube of the condenser dipping beneath the surface of the water in a receiving flask. Introduce into the Kjeldahl flask roo c.c. of water and I c.c. of glacial acetic acid. Bring the acidified water to a boil and then run the diluted blood in slowly through the dropping funnel.

Boil for 30 minutes after the last blood is run in. ${ }^{3}$ To the distillate add a little dilute sulphuric acid and redistil. To this distillate add 20 c.c. of hydrogen peroxide solution and a slight excess of alkali and redistil again. The final distillate is caught in small Erlenmeyer flasks containing an excess of the ScottWilson "acetone reagent". which has been recently filtered. ${ }^{4}$ The delivery tube must dip under the surface of the liquid. It is not necessary to distil more than Io minutes to get off all the acetone. Allow to stand for I0-15 minutes. Filter through an asbestos mat ${ }^{5}$ in a separable bottom Gooch crucible. Clear filtrates are more readily obtained if the pores of the filter have been partly closed by filtering through it a suspension of talcum powder in water. If the first portions of the filtrate are turbid, refilter. Wash the precipitate with cold water until the washings are free from silver.

With the aid of a pointed hooked glass rod transfer the precipitate, mat and crucible bottom to a 50 c.c. beaker, any adhering particles of precipitate being washed into the beaker with the aid of about ro c.c. of "acid mixture." 6 Add I c.c. of $\mathrm{N} / 5$ potassium permanganate, cover the beaker with a watch glass and boil until the liquid is colorless. Add more permanganate, a few drops at a time, until a persistent brown color is obtained which does not disappear on boiling for a couple of minutes. The brown color is then discharged by the addition of a few drops of strong yellow nitric acid. The greater the amount of acetone present the more permanganate is required, and it is essential to the accuracy of the method that an excess be added as indicated above, otherwise the results are too low.

Cool the beaker under the tap, add 2 c.c. of saturated ferric alum solution,

${ }^{1}$ For nephelometric method see p. 294.

${ }^{2}$ Scott-Wilson: Jour. of Physiol., 42, 444, I91 1 .

Marriott: Jour. Biol. Chem., I6, 295, I9 3 .

${ }^{3}$ If $\beta$-oxybutyric acid is to be determined the residue in the Kjeldahl flask should be kept and treated as outlined in the latter part of this procedure.

${ }^{4}$ The reagent is made up as follows: Mercuric cyanide, Io grams; sodium hydroxide, I 80 grams; water, I 200 c.c. The solution is agitated in a flask and 400 c.c. of a 0.7268 per cent solution of silver nitrate slowly run in. At least 30 c.c. of the reagent must be taken for each milligram of acetone present.

${ }^{5}$ Filter paper cannot be used as the strong alkali quickly attacks it.

- "Acid mixture:" Nitric acid 40 parts; sulphuric acid, 5 parts; water 55 parts. 
and run in from a burette a standard solution of potassium sulphocyanate (approximately o.I per cent) ${ }^{1}$ until a very faint pinkish-brown color is obtained throughout the solution. The end point which consists in the faintest trace of color, can be detected only when the titration is performed on a pure white surface. A control beaker with I drop excess of sulphocyanate should be on hand for comparison. A whole cubic centimeter of sulphocyanate may be run in after the end point is reached without very greatly darkening the shade.

Calculation.-By this procedure acetone preformed and from diacetic acid are determined together. The amount of acetone and diacetic acid combined, in terms of acetone, may then be calculated by multiplying the number of cubic centimeters of the KSCN solution used by the equivalent of I c.c. of this solution in acetone as determined by standardization. ${ }^{1}$ To obtain the amount of acetone and diacetic acid in roo c.c. of blood the result must of course be multiplied by Io.

(b) Determination of $\beta$-Hydroxybutyric Acid.- The residue in the Kjeldahl flask from the above determination is used in the determination of $\beta$-hydroxybutyric acid. While still hot, precipitate it with about 8 c.c. of ro per cent sodium carbonate, boil a moment, filter on a Büchner funnel and wash with hot water. To the clear filtrate add ${ }_{5}$ c.c. of basic lead acetate (U.S.P.) and Io c.c. of strong ammonia and make to definite volume (I50 c.c.) with water. Allow the precipitate to settle and then filter off on a dry, folded filter. Take an aliquot of the clear filtrate (about 25 c.c.) and boil it to expel the greater part of the ammonia. Cool and add dilute sulphuric acid to precipitate the excess of lead as sulphate and filter. Add ro c.c. of 50 per cent sulphuric acid and transfer the whole to a Kjeldahl flask provided with a dropping funnel. The contents of the flask are distilled and a solution of potassium bichromate is run in from the dropping funnel at such a rate that the liquid always retains some yellow color and the volume remains at about roo c.c. It is rarely necessary to add more than about 0.1 gram of bichromate and an excess is to be avoided. Slow distillation is continued for two hours and about roo c.c. of distillate is collected. The tip of the delivery tube must always remain under the surface of the water in the receiving flask. Add 20 c.c. of hydrogen peroxide, make slightly alkaline with $\mathrm{NaOH}$ and distil again. Catch the distillate in small Erlenmeyer flasks containing an excess of the ScottWilson acetone reagent (at least 30 c.c. for each milligram of acetone) and determine the acetone according to the procedure outlined in the preceding method for acetone and diacetic acid. Calculate the $\beta$-hydroxybutyric acid in terms of acetone and express as milligrams of acetone per Ioo c.c. of blood.

\section{Cholesterol. Method of Autenrieth and Funk. ${ }^{2}-$ Principle.-} The blood or serum is boiled with strong alkali to saponify the fats. The alkaline solution is extracted with chloroform. The chloroform is dried and clarified by means of anhydrous sodium sulphate and filtration and then treated with sulphuric acid and acetic anhydride, and the characteristic color reaction of the Liebermann-Burchard test

1 The sulphocyanate solution should be standardized against pure acetone treated in the same manner as the final distillate above. According to Scott-Wilson it may also be standardized against a pure solution of mercuric nitrate and the equivalent of I c.c. of the KSCN solution in milligrams of $\mathrm{Hg}$ determined. According to this author I mg. of $\mathrm{Hg}$ is equivalent to $0.058 \mathrm{mg}$. of acetone.

2 Autenrieth and Funk: Mïnch. med. Woch., 60, I2+3, I913. has been suggested by Bloor (Jour. Biol. Chem., 23, 317, I915). 
for cholesterol obtained. The color is compared with a standard in a colorimeter.

Procedure. -With an accurate pipette transfer 2 c.c. of whole blood or serum to a Ioo c.c. Erlenmeyer flask, and add 20 c.c. of a 25 per cent potassium hydroxide solution. Heat on the water-bath for two hours shaking frequently and adding a little water if necessary to keep from going to dryness. Pour the undiluted mixture into a separatory funnel and add 25-30 c.c. of chloroform. Shake vigorously for five minutes and separate. Shake out with four more portions of 20 c.c. each of chloroform. The combined chloroform extracts are turbid and of a green or brown color and are clarified by shaking with 5-10 grams of anhydrous sodium sulphate and filtering. Dilute the filtrate to I0o c.c. with chloroform. Transfer 5 c.c. of this extract to a small glass-stoppered bottle of about ro c.c. capacity, add 2 c.c. of acetic anhydride and 0. .I c.c. of concentrated sulphuric acid and shake. Place in a water-bath at $32-35^{\circ} \mathrm{C}$. and keep in the dark 15 minutes. A green color is developed. At the same time a series of standards are prepared and treated in the same manner. The color of the test is compared with that of the most similar standard in a colorimeter (see Fig. 153, p. 486) and its color strength determined.

Five standards are kept, these being prepared by dissolving in 100 c.c. portions of chloroform: (I) 3.2 ; (2) 4.8 ; (3) 6.4 ; (4) 8.0 ; (5) $9.6 \mathrm{mg}$., respectively of pure cholesterol. In preparing the standards for comparison 5 c.c. portions of each of the above solutions are taken, placed in small glass-stoppered bottles and treated as were the unknowns. Each standard then represents a concentration of: $160 \mathrm{mg}$.; $240 \mathrm{mg}$.; $320 \mathrm{mg}$; $400 \mathrm{mg}$.; and $480 \mathrm{mg}$. respectively of cholesterol in roo c.c. of the original blood or serum.

ro. Chlorides. - Method of McLean and I I an Slyke. ${ }^{1}$-The determination requires two steps: (I) removal of proteins and (2) titration of chlorides.

Coagulation of Proteins.- Removal of the proteins may be accomplished in two ways, by coagulation or by ignition. Results are identical by both methods, but coagulation is the simpler. For coagulation 2 c.c. of oxalated plasma (I c.c. may be used if material is limited) are drawn into a 2 c.c. pipette which has been calibrated to contain $2 \pm 0.005$ c.c. From the pipette the plasma is run into a 20 c.c. stoppered volumetric flask which contains ro c.c. of a ro per cent magnesium sulphate solution. The pipette is rinsed twice by drawing up into it the solution from the flask. Two drops of 50 per cent acetic acid are added, the flask is filled to the mark with water, the contents are mixed by inverting the flask, and heated in a bath to $100^{\circ}$ for ten minutes. By keeping the stopper loosely in place evaporation is prevented, and when cool the contents return to their original volume. Ten minutes on the steam bath are sufficient to coagulate the albumin and to distribute the chlorides evenly between the fluid and precipitated albumin. The flask is then allowed to cool, and the contents are poured upon about $0.3 \mathrm{gram}$ of blood char$\mathrm{coal}^{2}$ in a small beaker, and mixed. After a few minutes the liquid is filtered through a dry folded filter, and a water-clear filtrate obtained.

Titration of the Protein-free Filtrate.-In brief, the chlorides are precipitated in the presence of nitric acid by standard silver nitrate solution, the silver chloride is removed by filtration, and the excess silver titrated with standard potassium

1 McLean and Van Slyke: Jour. Biol. Chem., 21, 361, I915.

${ }^{2}$ Merck's "Blood Charcoal Reagent," purified by acid and freefr om chloride, is used. No other form of charcoal has been found to be of service. 
iodide. ${ }^{1}$ The titration is performed in the presence of nitrous acid and starch, so that the first drop of iodide in excess of the silver present is changed to free iodine and gives the blue starch-iodine color. The optimum acidity for the end point is fixed by the addition of trisodium citrate in amount equivalent $(1 / 3 \mathrm{~mol}$.) to the free nitric acid present. Under these conditions I drop of excess $\mathrm{N} / 50$ iodide gives a color preceptible in $\mathbf{I} 50$ c.c. of solution.

In detail, the titration of the protein-free plasma filtrate is carried out as follows: Either ro c.c. of the filtrate, containing the chlorides of I c.c. of plasma, are taken in a pipette for titration or the filtrate is collected directly in a certified 25 c.c. graduated cylinder, where it is measured, so that the entire amount may be taken for titration. In this way $\mathrm{I}_{3}$ to $\mathrm{I} 4$ C.C. of filtrate, corresponding to $\mathrm{I} .3$ to $x .4$ C.c. of plasma, may be obtained for titration.

After either measuring Io c.c. of the filtrate into a 25 c.c. volumetric flask or recording the amount of filtrate obtained in the cylinder, 5 c.c. of the acidified $M I / 29.25$ silver nitrate solution (Solution $\mathrm{I}$ ) are added, and the whole is made to the 25 c.c. mark with water. This will precipitate up to to $\mathrm{mg}$. of $\mathrm{NaCl}$. In samples with high percentage of chloride, only enough filtrate is taken to keep within this limit of ro mg. Two drops of octyl (caprylic) alcohol are added, and the vessel is stoppered and shaken gently by inverting it several times. Immediate coagulation of the silver chloride occurs. After allowing five minutes for complete precipitation to occur, the solution is filtered through a dry folded filter, and a perfectly clear and colorless filtrate again obtained. In aliquot part of the filtrate (20 c.c.) is now taken with a pipette for titration.

Just before titration with the potassium iodide solution (Solution II) one adds a volume of the citrate solution (Solution III) equal to the volume of Solution I represented in the filtrate to be titrated. If, as is usually the case, one has used 5 c.c. of Solution I diluted to 25 c.c., and taken 20 c.c. of the filtrate for titration one has the equivalent of 4 c.c. of Solution I present, and must accordingly add

I The following solutions are required;

I. An acid $M / 29.25$ solution of silver nitrate, I c.c. of which is equivalent to $2 \mathrm{mg}$. of $\mathrm{NaCL}$.
$\mathrm{AgNO}_{3}$.
$\mathrm{HNO}_{3}$ (sp. gr. 1.42 ).
$5.8 \mathrm{r} 2$ grams
IVater to.............

$\begin{aligned} 250 & \text { c.c. } \\ 1000 & \text { c.c. }\end{aligned}$
NaCL.

II. A solution of $M / 58.5$ potassium iodide, I c.c. of which is equivalent to I $\mathrm{mg}$. of
KI..
Water to.
3.0 grams
1000 c.c.

This solution is standardized against the silver solution by adding 5 c.c. of the latter to 5 c.c. of Solution III, and titrating with the iodide solution to the blue end-point. The iodide solution is then cliluted to such a degree that so c.c. are exactly equiralent to 5 c.c. of the silver solution.

III. A solution, for use in the final titration, containing sodium citrate, sodium nitrate, and starch, which substances respectively regulate the acidity, provide an oxidizing agent for the iodide, and serve as indicator.

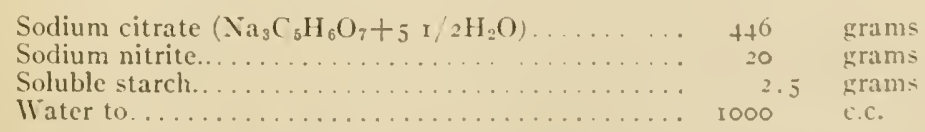

The starch is first dissolved with the aid of heat in about 500 c.c. of water. The citrate and nitrite are then added, and the mixture is heated until all is dissolved. The solution. while still hot is filtered through cotton, the filter washed with hot water, the filtrate allowed to cool, and made up to rooo c.c. Filtration removes insoluble substances occurring chiefly in the nitrite, and cotton filters more rapidly than filter paper. The solution keeps indefinitely. It becomes cloudy on standing, but its efficacy is not impaired. 
4 c.c. of Solution III. On adding Solution III a slight turbidity appears, which in no way interferes with the end point.

The potassium iodide solution is then run in from a burette until the blue end point appears. The first definite blue color is taken as the end point, and with slight practice is unmistakable. An additional drop, which may be used as a control, causes such a deep blue color that it is impossible to make an error of more than one drop in titration. Should the end point be accidentally passed, one may add I c.c. of Solution I, x c.c. of Solution III and retitrate, allowing in the calculation for the extra silver nitrate.

The result may be calculated from the following formula, which applies only when 20 c.c. of the filtrate from silver chloride are titrated:

$$
\text { Grams NaCl per liter }=\frac{\mathrm{r} 2.5(8-\text { c.c. } \mathrm{KI} \text { solution used })}{\text { c.c. blood filtrate taken }} \text {. }
$$

Thus, if $\mathrm{I}_{3}$ c.c. of filtrate are obtained and titrated after precipitation of plasma protein, and r.6o c.c. of KI are used in the final titration,

$$
\text { Grams NaCl per liter }=\frac{I_{2.5}(8-\mathrm{r} .60)}{I_{3}}=6.15 \text {. }
$$

In case only I c.c. of plasma instead of 2 c.c. has been used, the factor 25 replaces I 2.5 in the numerator. The error should be within a limit of I per cent. ${ }^{1}$

I I. Total Solids. - The total solids of the blood are most readily determined by using a type of weighing bottle differing from the usual form merely in having a glass loop attached to the under side of the stopper. ${ }^{2}$ A block of filter paper is suspended from this loop by means of a small wire hook. The bottle and filter block are dried and weighed. From a small pipette $0.3-0.6$ gram of the well-mixed blood is allowed to flow rapidly upon the filter block. The stopper is quickly inserted and the bottle weighed. The stopper is then tilted and the bottle placed in the drying oven at $105^{\circ}$ over night. When convenient the bottle is cooled and again weighed. The total solids are calculated from the loss of moisture.

I2. Relative Hydrogen Ion Concentration of the Blood. Method of Levy, Rowntree, and Marriott. ${ }^{3}$-Principle.-The blood is dialyzed against normal salt solution and the $\mathrm{H}$ ion concentration of the proteinfree dialyzate is determined by the indicator method, using phenolsulphonephthalein.

Procedure.-One to 3 c.c. of clear serum or of blood is run, by means of a blunt-pointed pipette, into a dialyzing $\operatorname{sac}^{4}$ which has been washed outside and

\footnotetext{
${ }_{1}^{1}$ The exactness of the method depends directly on the care with which measurements are made and on the purity of the reagents used. All reagents must, of course, be free from substances precipitated by silver, and are easily tested. As the volumes measured are not large, however, small absolute errors in measurement may cause considerable percentage error in the final result. All glassware must therefore be carefully calibrated by either the weight of water contained (flasks, cylinders, 2 c.c. pipettes) or the weight delivered (other pipettes, burettes), and burettes should be used which are capable of being read to 0.02 c.c. and which deliver small drops. If these precautions are followed, one should uniformly obtain results, which are well within a limit of error of I per cent.

2 Myers and Fine: "Chemical Composition of the Blood in Health and Disease," 1915. The weighing bottles may be obtained from Eimer and Amend, N. Y.

${ }_{3}^{3}$ Levy, Rowntree and Marriott, Arch. Int. Med., I6, 389, I9I 5.

4 Preparation of Sacs.-One ounce of celloidin is dissolved in 500 c.c. of a mixture of equal quantities of ether and ethyl alcohol. The solids swells up and disolves with oc-
} 
inside with salt solution. ${ }^{1}$ The sac is lowered into a small test-tube (100 $\times 10$ mm., inside measurements), containing 3 c.c. of salt solution, until the fluid on the outside of the sac is as high as on the inside. From $5^{-10}$ minutes are allowed for dialysis. The collodion sac is removed and 5 drops of the indicator (o.or per cent solution of phenolsulphonephthalein) are thoroughly mixed with the dialyzate. The tube is then compared with the standards ${ }^{2}$ until the corresponding color is found, which indicates the hydrogen ion concentration present in the dialyzate. Readings should be made immediately against a white background. Results are expressed in logarithmic notation.

Oxalated blood from normal individuals gives a dialyzate with a $\mathrm{P}_{\mathrm{H}}$ varying from 7.4 to 7.6 , while that of serum ranges from 7.6 to 7.8 . In clinical acidosis figures from 7.55 to 7.2 have been noted by this method for serum and for oxalated blood from 7.3 to 7.1 . A rise in the $\mathrm{H}$ ion concentration of the blood is significant because it indicates a failure on the part of the protective mechanism of the body to preserve the proper reaction.

casional gentle shakings, in 48 hours. As a small amount of brown sediment separates out at first, the solution should stand for at least three or four days, after which the clear supernatant solution is ready for use. A small test-tube ( 20 by $9 \mathrm{~mm}$., inside measurement) is filled with this mixture, inverted, and half the contents poured out. The tube is then righted, and the collodion allowed to fill the lower half again. A second time it is inverted and rotated on its axis, the collodion being drained off. Care must be taken to rotate the tube, in order to secure a uniform thickness throughout. The tube is clamped in the inverted position and allowed to stand for ten minutes, until the odor of ether finally disappears. It is filled five or six times with cold water, or it is allowed to soak five minutes in cold water. A knife blade is run around the upper rim, so as to loosen the sac from the rim of the test-tube, and a few cubic centimeters of water are run down between the sac and the glass tube. By gentle pulling the tube is extracted, after which it is preserved by complete immersion in water.

${ }^{1}$ The Salt Solution.- -The blood or serum is dialyzed against an 0.8 per cent sodium chloride solution.

Before applying the test, it is necessary to ascertain that the solution is free from acids other than carbonic. To determine this, a few cubic centimeters of the salt solution are placed in a Jena test-tube and I or 2 drops of the indicator added, whereupon a yellow color appears. On boiling, carbon dioxide is expelled, and the solution loses its lemon color and takes on a slightly brownish tint. In the absence of this change other acids are present, and the salt solution is therefore not suitable. If, on the other hand, on adding the indicator pink at once appears, the solution is alkaline and hence cannot be used.

${ }^{2}$ Preparation of Standard Colors.-Standard phosphate mixtures are prepared according to Sörensen's directions as follows:

$1 / 15 \mathrm{~mol}$. acid or primary potassium phosphate. 9.078 grams of the pure recrystallized salt $\left(\mathrm{K}_{2} \mathrm{PO}_{4}\right)$ is dissolved in freshly distilled water and made up to $\mathrm{I}$ liter.

$1 / 15$ mol. alkaline or secondary sodium phosphate. The pure recrystallized salt $\left(\mathrm{Na}_{2} \mathrm{HPO}_{4} \cdot \mathrm{I}_{2} \mathrm{H}_{2} \mathrm{O}\right)$ is exposed to the air for from ten days to two weeks, protected from dust. Ten molecules of water of crystallization are given off and a salt of the formula $\mathrm{Na}_{2} \mathrm{IPO}_{4} \cdot 2 \mathrm{H}_{2} \mathrm{O}$ is obtained. II.876 grams of this is dissolved in freshly distilled water and made up to I liter. The solution should give a deep rose-red color with phenolphthalein. If only a faint pink color is obtained, the salt is not sufficiently pure.

The solutions are mixed in the proportions indicated below to obtain the desired $\mathrm{P}_{11}$.

TABLE FOR PREPARATION OF STANDARD COLORS

$\mathrm{P}_{H} \ldots \ldots \ldots \ldots \ldots 6.6 .66 .87 .07 .17 .27 .37 .47 .5 \quad 7.6 \quad 7.7 \quad 7.8 \quad 8.0 \quad 8.28 .4$

Primary potassium

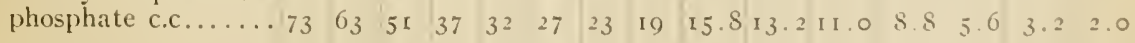

Secondary sodium

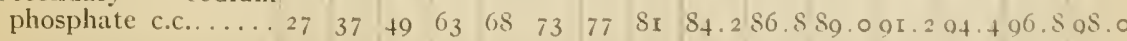


13. The Determination of Epinephrin (Adrenalin) in the Adrenal Glands. ${ }^{1-P}$ Principle.-It has not yet been found possible to determine adrenalin in the blood by chemical methods. In the adrenals it may be determined by the method given below.

The method is based upon the quantitative production of blue color when a solution of adrenalin is treated with phosphotungstic acid. Uric acid and other substances likewise give the reaction but can be disregarded when the determination is made upon adrenal glands.

Procedure.-Rub the weighed gland thoroughly in a mortar with fine sand and $\mathrm{N} / \mathrm{ro}$ hydrochloric acid. The mixture is then rinsed into an Erlenmeyer flask with more $\mathrm{N} / \mathrm{ro}$ acid and water, using in all about 15 c.c. of the acid for each 2 grams of gland and about three times as much water. Heat to boiling to dissolve the epinephrine. Then add 5 c.c. of 10 per cent sodium acetate solution for each 15 c.c. of hydrochloric acid present and heat again to boiling. Transfer the whole mixture (except the sand) to a volumetric flask (capacity roo c.c. for each 2 grams of gland) and make to mark with water. Filter. Pipette 5 c.c. of the clear extract into a roo c.c. measuring flask and I c.c. of a fresh uric acid solution containing I mg. of uric acid into another Ioo c.c. flask. To each flask add 2 c.c. of uric acid reagent (see Folin and Denis' method for uric acid, page 274) and 20 c.c. of saturated sodium carbonate solution. Allow to stand for two to three minutes, dilute to the mark, shake thoroughly, and compare in the usual manner in a Duboscq colorimeter with the uric acid standard set at $20 \mathrm{~mm}$. Calculate as if the solution contained uric acid, and divide the result by three to obtain the equivalent in epinephrine which gives three times as much color as an equal weight of uric acid.

\section{Nephelometric Methods}

The Nephelometer.- The nephelometer is an instrument for measuring the density of precipitates and thus determining the amount of any substance which can be obtained in the form of a suitable suspension. It is somewhat similar in form and principle to a colorimeter. It differs from the latter in that the light which reaches the eye is not transmitted light, which, on the contrary, is excluded, but light reflected from the particles of the suspension. The brightness of the two fields is compared instead of their colors. It is adapted particularly for the determination of substances that in very dilute solution may be precipitated in the form of suspensions which do not agglutinate appreciably in the time required for making readings (ro-20 minutes). The method has been adapted to the determination of proteins in digestion mixtures, milk, urine, etc. ${ }^{2}$ nucleic acids; ${ }^{3}$

${ }^{1}$ Folin, Cannon, and Denis: Jour. Biol. Chem., I3, 477, I9I3.

2 Kober: Jour. Biol. Chem., I3, 485, I913; Jour. Am. Ch. Soc., 35, 1585, 1913; Folin and Denis: Jour. Biol. Chem., I8, 273 , I9I4.

${ }^{3}$ Kober and Graves: Jour. Am. Chem. Soc., 36, 1304, 1914. 
chlorides, ${ }^{1}$ phosphates, and phosphatides in blood, etc. ${ }^{2}$ fats in milk, blood, etc. $;^{3}$ acetone bodies in urine and blood $;{ }^{4}$ uric acid and purine bases; ${ }^{5}$ ammonia $;^{6}$ calcium $;^{7}$ silver, etc., and is continually finding new applications. It is possible to determine very minute amounts of substances, entirely outside of the range of gravimetric methods of analysis, and hence the procedure may be used where the amount of material is very limited. If properly carried out the limits of error of the method are not greater than those of the colorimetric methods commonly used. Below will be found descriptions of and figures representing two satisfactory types of nephelometer.

The Duboscq colorimeter has been adapted for nephelometric purposes by Kober $^{8}$ and by Bloor. ${ }^{9} \quad$ Bloor's nephelometer is illustrated in Figs. 83 and 84 . The brass plate carrying the colorimeter plungers is replaced by the plate $A$ with two slots in which are supported the nephelometer tubes $B$ with their flanges resting on the edges of the slots. The slots are so cut that the center lines of the tubes are exactly in line with the centers of the lower openings of the prism case $E$. If desired they may be countersunk to receive the flanges. The colorimeter cups

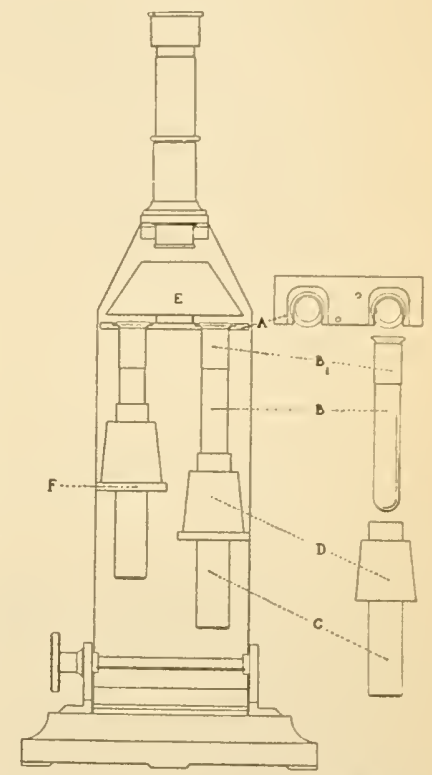

Fig. 83.-BLOOR'S NePHELOMETER. are replaced by the jackets $C$ which project through the holes in the cup supports $F$ and are supported on them by the collars $D$. They move when the cup supports move. The mirror is turned to the horizontal position so that it reflects no light. The light in the nephelometer comes from in front and not from below (see Fig. 84). The nephelometer tubes are small test-tubes I00 $\mathbf{I}_{5} \mathrm{~mm}$., preferably made from the same sample of colorless glass tubing so that they are of exactly the same bore. The flanges at the top should be well made so that the tubes rest firmly and evenly in the slots. The glass should

${ }^{1}$ Richards: Zeilschr. f. anorg. Chem., 7, 269, IS95.

${ }^{2}$ Greenwald: Jour. Biol. Chem., 21, 29, I915; Bloor: Jour. Biol. Chem., 22, I33, I9r5; Kober and Egerer: Jour. Am. Chem. Soc., 37, 2373, I9r 5.

${ }^{3}$ Bloor: Jour. Biol. Chem., I7, 377, I9r4; J. Am. Chem. Soc., 36, I300, 1914.

${ }^{4}$ Folin and Denis: Jour. Biol. Chem., IS, 263, I914; Marriott: same, I6, 2S9, I913.

${ }^{5}$ Graves and Kober: Jour. A m. Chem. Soc., 37, 2430, I9I5.

"Graves: J. Am. Chem. Soc., 37, in 8r, I9I5.

${ }^{7}$ Lyman: Jour. Biol. Chem., 21, 55I, I9I5.

${ }^{8}$ Kober: Jour. Biol. Chem., I3, 485, I913; Jour. Am. Chem. Soc., 35, 15 55, 1913.

9 Bloor: Jour. Biol. Chem., 22, 145, 1915. 
be as free as possible from imperfections and striations. After the tubes are made and fitted into place the jackets are moved up on each tube by means of the rack and pinion until the indicator on the scale is exactly at zero. Marks are made on each tube at the point reached by the top of the jacket and the portion of the tube above that point is made opaque by a ring BI of black paper or paint. Tubes and jackets are then marked right and left and always used on the same side. Since it is rare to find two tubes which when filled with the same solution give exactly the same readings it is necessary to take this fact into account and correct accordingly.

The jackets $C$ are made of tubing (metal or glass) a little larger than the tubes and about the same length (they should clear the mirror when it is turned horizontal), closed at the bottom and made

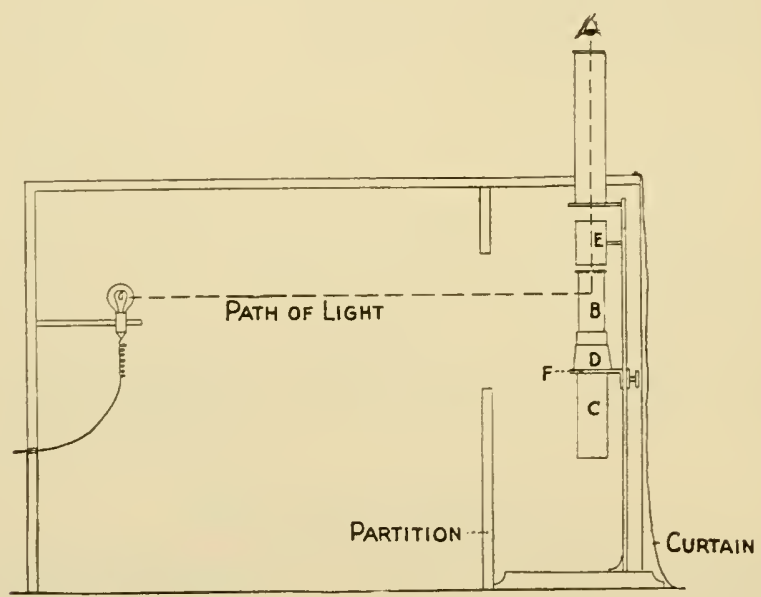

Fig. 84--Nephelometer in Position, Showing Relation to Source of Light.

light tight by black paint or paper. The collars $D$ supporting the jackets may be made of cork or more permanently of metal. A little cotton wool in the bottom of the jackets will prevent breakage if the tubes should fall into the jackets.

The openings in the prism case, particularly the lower ones, should be protected against accidental splashing by thin glass plates (thick cover slips) which are held in place by a little glue.

Artificial light is necessary and the lamp should be enclosed in a tight box into one end of which the nephelometer fits snugly. A partition extending part way up the box as shown in the diagram (Fig. 84) serves the double purpose of shutting off the light from the lower part of the instrument and of providing a stop against which the instrument is pushed, so that its distance from the light 
is kept constant. The box is conveniently made without a buttom and the end closed with a dark curtain after the nephelometer is pushed into place. The inside of the box should be painted black. A dark room is desirable but not necessary; as the instrument may be used satisfactorily in a room darkened by a dark shade or even in a dark corner of the laboratory.

The relations of the nephelometer and the light source may be seen in the diagram, Fig. 84. The lamp used is an ordinary 50watt tungsten ("Mazda") supported by a bracket about $30 \mathrm{~cm}$. from the nephelometer and at the height of the nephelometer tubes. The change from one instrument to the other can be made in one or two

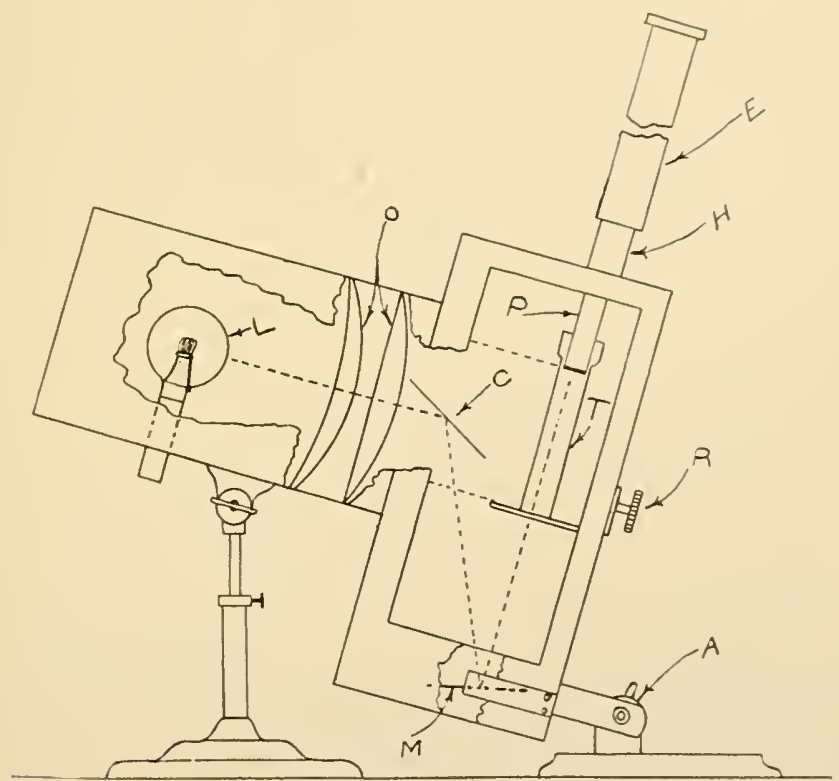

Fig. 85.-Lexzmini-Koerer Nephelometer.

minutes, since it consists essentially only in unscrewing the brass plate carrying the plungers and screwing on the plate to carry the nephelometer tubes. The extra parts needed, plate, tubes. and jackets, are few and can be made if necessary from material at hand in any laboratory and by anyone with a slight degree of mechanical skill. ${ }^{1}$

The above description applies only to the later type of colorimeter where the cups move and the prisms are stationary. The changes required to convert the older type of instrument are more complicated and scarcely to be advised unless the instrument is to have fairly

1 The extra parts necessary for the conversion of the colorimeter into the nephelometer may be obtained from the International Instrument Co. of Cambridge, Mass. 
continuous use as a nephelometer. If the change is desired the nephelometer tubes are to be supported in the same way as above, but the jackets must be carried on special brackets which are made to replace the brackets carrying the plungers. The nephelometer tubes must be stationary, the jackets being the movable parts.

Kober has devised ${ }^{1}$ a combined colorimeter and nephelometer less expensive than the Duboscq apparatus and which may be obtained in this country. ${ }^{2}$ A diagram illustrating the construction of this Lenzmann-Kober nephelometer is given in Fig. 8j, page 293.

Nephelometric Calculations. - The amounts of precipitate in solutions examined nephelometrically is not exactly inversely proportional to the readings of the scale. When the concentration of the unknown and of the standard are within Io per cent of each other (or within about 20 per cent if the readings are made at depths as great as $50-$ $60 \mathrm{~mm}$.) accurate results may however be obtained directly. If the variations are greater than this a correction is necessary. Kober ${ }^{3}$ has proposed an equation to supply this correction and thus make possible very accurate work under conditions of moderate variations of concentration. The equation is as follows:

$$
y=\frac{s}{x}-\frac{(1-x) s k}{x^{2}}
$$

or

$$
x=\frac{s+s k+\frac{\sqrt{(s+s k)^{2}}-4 s k y}{2 y}}{2 y}
$$

where $y=$ height of unknown solution, on the left side of the instrument, when standard solution is kept on the right side at a definite height, $s=$ height of standard solution on the left side and $x=$ the ratio of the concentrations of the two solutions.

$k={ }_{s}^{K}$ where $K=$ a constant, obtained by substitution of standardization values of $s, y$, and $x$. The instrument should be checked up for each series of analyses by reading the standard against itself and determining the potential height of the standard solution by reading the scale on the left side when the solution on the right side is kept at a definite height, and the two are matched.

I. Acetone Bodies.-Nephelometric Methods of Marriott. ${ }^{4}$-Principle.-Acetone in very small amounts forms a cloudy solution with the Scott-Wilson reagent which may be read nephelometrically. By this

${ }^{1}$ Kober: Jour. Ind. and Eng. Chem., 7, 843, I9r5.

2 The instrument is manufactured by Lenz and Naumann Pullman Building, 17 Madison Ave., New York City.

${ }^{3}$ Kober: J. Am. Chem. Soc., 37, 2379, rgr 5; Jour. Biol. Chem., 13, 4 S5, $_{5}$ rgr3.

4 Marriott: Jour. Biol. Chem., I6, 289 and 293, r9r3. 
method it is possible to make a complete analysis for acetone and diacetic acid and hydroxybutyric acid in from $2-5$ c.c. of blood.

Procedure.--Two to 5 c.c. of blood drawn from a superficial arm vein by means of a sterile syringe are run into a small weighed flask containing 50 c.c. of 0.5 per cent potassium oxalate solution. The flask is reweighed. The diluted blood is run into Ioo c.c. of boiling water acidified with I c.c. of glacial acetic acid ${ }^{1}$ contained in an 800 c.c. Kjeldahl distilling flask and the procedure is then carried out as described in the Marriott-Scott-Wilson methods for (a) acetone and diacetic acid and (b) $\beta$-hydroxybutyric acid as outlined on page 284 . The precipitate in the mercury reagent is however estimated nephelemometrically. In this case the distillate which should measure 75-roo c.c. is allowed to stand half an hour, then transferred to a graduated cylinder and diluted until an opalescence that can be conveniently read is obtained. The turbidity occasioned by $0.05 \mathrm{mg}$. of acetone diluted to 100 c.c. is a convenient strength for this purpose, although considerably larger or smaller amounts give good results. With heavy opalescence it is desirable after diluting to a certain volume, say 250 c.c. to remove an aliquot portion with a pipette and dilute this appropriately. A solution containing a known amount of acetone ${ }^{2}$ is distilled into an excess of reagent ${ }^{3}$ and this distillate which is to be used as the standard is diluted as above. Read in the nephelometer against this standard, taking the readings as quickly as possible after filling the tubes as the suspension settles slowly.

If the unknown suspension is diluted so as to be not more than 20 per cent different from the standard and if comparisons are made in considerable depths of solution (50-60 mm.) no corrections are necessary. If the two agree within ro per cent accurate comparison may be made at less depths. If divergences are greater and accurate results are desired, Kober's correction equation must be used (see discussion of nephelometer p. 294).

2. Fat.-Nephelometric Method of Bloor. ${ }^{4}-$ Principle. - The protein is precipitated with alcohol and ether and the fatty acid in the extract determined nephelometrically after saponification.

Procedure.-Extraction.-About 2 c.c. of blood are drawn from the vein with a graduated syringe and run at once with stirring into a weighed graduated flask containing about 40 volumes of a mixture of 3 parts alcohol and I part ether. After again weighing to find the weight of blood added, the solution is raised to boiling in a water-bath, cooled under the tap, made to volume with alcohol-ether mixture, mixed and filtered. The filtrate is water clear and almost colorless.

Determination.-From 5-20 c.c. of the extract (containing about $2 \mathrm{mg}$. of fat) are measured with a pipette into a small beaker and saponified by evaporating nearly but not quite to dryness with 2 c.c. of $\mathrm{N} / \mathrm{x}$ sodium ethylate. The residue is heated just to boiling after the addition of 5 c.c. of alcohol-ether, and 50 c.c. of distilled water are added.

A similar solution of the standard is prepared by adding 5 c.c. of the standard

' Commercial varieties of acetic acid frequently contain substances which behave like acetone. Blank determinations should always be made and corrections made accordingly.

${ }^{2} \mathrm{~A}$ convenient stock solution contains about $0.03 \mathrm{mg}$. acetone perc.c. The strength of such a solution is determined by titration of $200 \mathrm{c.c}$. by the Mlessinger-Huppert method (see Chapter XXVI).

${ }^{3}$ The solution cannot be aclded directly to the reagent as a lower result is obtained than when distilled.

-Bloor: Jour. Biol. Chem., 17, 377, 1914; 23:31\%, I015. 
fatty acid solution ${ }^{1}$ from a pipette with stirring to 50 c.c. of distilled water. To the standard and to the test solutions are added simultaneously from pipettes and with stirring Io c.c. portions of dilute $(1: 3)$ hydrochloric acid and the solutions allowed to stand for five minutes, after which they are transferred to the comparison tubes of the nephelometer (see Fig. 83, p. 29I). Several readings should be taken and averaged. The standard tube should always be on the same side. See discussion of nephelometer (page 290) for details as to reading. The results represent the amount of total fat (fatty acids and cholesterol) in the blood, expressed as oleic acid. The fat of the corpuscles is not completely extracted, and it should be borne in mind that other lipoids as cholesterol are included in the results. Cholesterol may be determined separately and subtracted from the result for total fat. It may also be determined in a part of the blood extract as prepared above by a modified Autenrieth-Funk procedure. ${ }^{2}$ Methods have also been devised for the determination of the phosphatides of blood. ${ }^{3}$

\section{Instruments Used in the Examination of the Blood}

Spectroscope. ${ }^{4-}$ Either the angular-vision spectroscope (Figs. 87 and 88) or the direct-vision spectroscope (Fig. 86) may be used in making the spectroscopic examination of the blood. For a complete description of these instruments the student is referred to any standard text-book of physics.

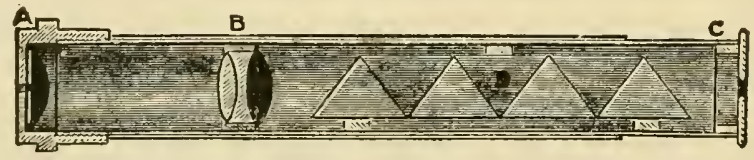

Fig. 86.-Direct-Vision Spectroscope.

I. Oxyhemoglobin.-Examine dilute $(1: 50)$ defibrinated blood spectroscopically. Note the broad absorption band between D and E. Continue the dilution until this single broad band gives place to two narrow bands, the one nearer the $\mathrm{D}$ line being the narrower. These are the typical absorption bands of oxyhemoglobin obtained from dilute solutions of blood. Now dilute the blood very freely and note that the bands gradually become more narrow and, if the dilution is sufficiently great, they finally entirely disappear.

2. Hemoglobin (so-called Reduced Hemoglobin).-To blood which has been diluted sufficiently to show well-defined oxyhemoglobin absorption bands add a small amount of Stokes' reagent. ${ }^{5}$ The blood immediately changes in color from

1 The standard solution used is an alcohol-ether solution of pure oleic acid of which 5 c.c. contain about $2 \mathrm{mg}$. of the acid. The alcohol and ether used for the standard are freshly redistilled absolute alcohol and pure dry ether.

2 Bloor: Jour. Biol. Chom., 23, 317, I9I5.

${ }^{3}$ Greenwald: Jour. Biol. Chem., 21, 29, I915.

Bloor: Jour. Biol. Chem., 22, 133, 1915, 23, 317, 1915.

Kober and Egerer: J. Am. Chem. Soc., 37, 2373, I91 5.

Taylor and Miller: Jour. Biol. Chem., i 8, 2 I5, ig 4.

For other nephelometric methods see Chapters XVII and XXVI.

${ }^{4}$ For Absorption Spectra see Plates I and II.

5 Stokes' reagent is a solution containing 2 per cent ferrous sulphate and 3 per cent tartaric acid. When needed for use a small amount should be placed in a test-tube and ammonium hydroxide added until the precipitate which forms on the first addition of the hydroxide has entirely dissolved. This produces ammonium ferrotartrate which is a reducing agent. 
a bright red to violet-red. The oxyhemoglobin has been reduced through the action of Stokes' reagent and hemoglobin (so-called reduced hemoglobin) has been formed. This has been brought about by the removal of some of the loosely
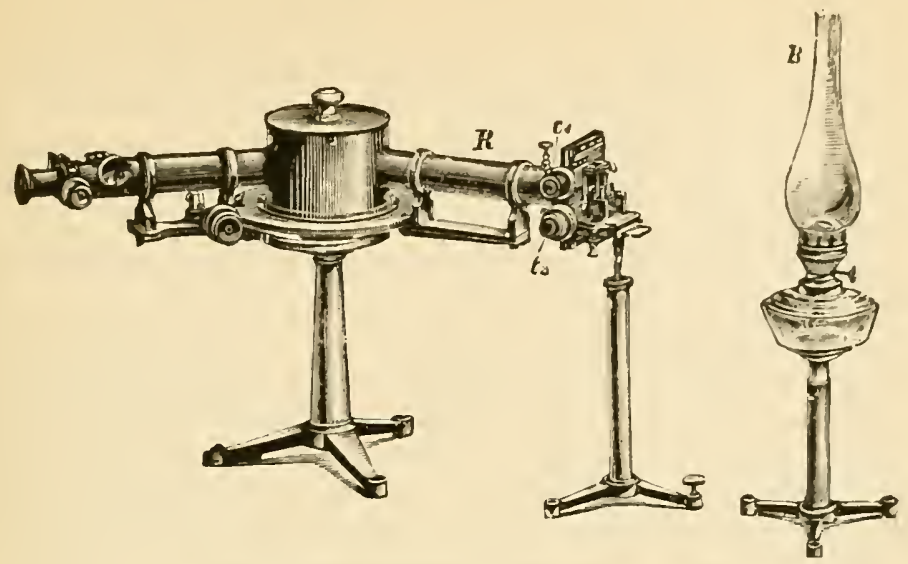

Fig. 87.-ANgular-vision Spectroscope Arranged for Absorption Axilysis.

combined oxygen from the oxyhemoglobin. Examine this hemoglobin spectroscopically. Note that in place of the two absorption bands of oxyhemoglobin we now have a single broad band lying almost entirely between $\mathrm{D}$ and $\mathrm{E}$. This is the typical spectrum of hemoglobin. If the solution showing this spectrum be

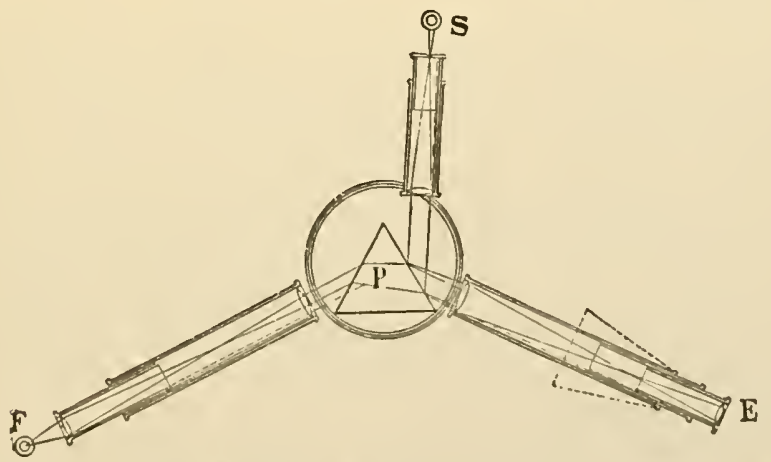

Fig. 88.-DiAgraM of AxgtLar-vision Spectroscope. (Long.)

The white light $F$ enters the collimator tube through a narrow slit and passes to the prism, $P$, which has the power of refracting and dispersing the light. The rays then pass to the double convex lens of the ocular tube and are deflected to the erepiece $E$. The dotted lines show the magnified virtual image which is formed. The third tube contains a scale whose image is reflected into the ocular and shown with the spectrum. Between the light $F$ and the collimator slit is placed a cell to hold the solution undergoing examination.

shaken in the air for a few moments it will again assume the bright red color of oxyhemoglobin and show the characteristic spectrum of that pigment.

3. Carbon Monoxide Hemoglobin.-The preparation of this pigment may be easily accomplished by passing ordinary illuminating gas ${ }^{1}$ through defibrinated

${ }^{1}$ The so-called water gas with which ordinary illuminating gas is diluted contains usually as much as 20 per cent of carbon monoxide $(\mathrm{CO})$. 
ox-blood. Blood thus treated assumes a brighter tint (carmine) than that imparted by oxyhemoglobin. In very dilute solution oxyhemoglobin appears yellowish red whereas carbon monoxide hemoglobin under the same conditions appears bluish red. Examine the carbon monoxide hemoglobin solution spectroscopically. Observe that the spectrum of this body resembles the spectrum of oxyhemoglobin in showing two absorption bands between $\mathrm{D}$ and $\mathrm{E}$. The bands of carbon monoxide hemoglobin, however, are somewhat nearer the violet end of the spectrum. Add some Stokes' reagent to the solution and again examine spectroscopically. Note that the position and intensity of the absorption bands remain unaltered.

The following is a delicate chemical test ${ }^{1}$ for the detection of carbon monoxide hemoglobin :

Tannin Test.-Divide the blood to be tested into two portions and dilute each with 4 volumes of distilled water. Place the diluted blood mixtures in two small flasks or large test-tubes and add 20 drops of a ro per cent solution of potassium ferricyanide. ${ }^{2}$ Allow both solutions to stand for a few minutes, then stopper the vessels and shake one vigorously for I0-I5 minutes, occasionally removing the stopper to permit air to enter the vessel. ${ }^{3}$ Add 5-10 drops of ammonium sulphide (yellow) and ro c.c. of a ro per cent solution of tannin to each flask. The contents of the shaken flask will soon exhibit the formation of a dirty olive-green precipitate, whereas the flask which was not shaken and which, therefore, still contains carbon monoxide hemoglobin, will exhibit a bright red precipitate, characteristic of carbon monoxide hemoglobin. This test is more delicate than the spectroscopic test and serves to detect the presence of as low a content as 5 per cent of carbon monoxide hemoglobin.

4. Neutral Methemoglobin.-Dilute a little defibrinated blood ( $1: 10$ ) and add a few drops of a freshly prepared ro per cent solution of potassium ferricyanide. Shake this mixture and observe that the bright red color of the blood is displaced by a brownish red. Now dilute a little of this solution and examine it spectroscopically. Note the single, very dark absorption band lying to the left of $\mathrm{D}$, and, if the dilution is sufficiently great, also observe the two rather faint bands lying between $\mathrm{D}$ and $\mathrm{E}$ in somewhat similar positions to those occupied by the absorption bands of oxyhemoglobin. Add a few drops of Stokes' reagent to the methemoglobin solution while it is in position before the spectroscope and note the immediate appearance of the oxyhemoglobin spectrum which is quickly followed by that of hemoglobin.

5. Alkaline Methemoglobin.-Render a neutral solution of methemoglobin, such as that used in the last experiment (4), slightly alkaline with a few drops of ammonia. The solution becomes redder in color, due to the formation of alkaline methemoglobin and shows a spectrum different from that of the neutral body. In this case we have a band on either side of $D$, the one nearer the red end of the spectrum being much the fainter. A third band, darker than either of those mentioned, lies between $\mathrm{D}$ and $\mathrm{E}$ somewhat nearer $\mathrm{E}$.

6. Alkali Hematin.-Observe the spectrum of the alkali hematin prepared in Experiment I 8 on page 266. Also make a spectroscopic examination of a freshly

${ }^{1}$ Sand (Ugeskrift for Laeger, 76, I72I, I9I4; Abst. J.A. M.A., Nov. 2I, I9I4) proposes a potassium iodide test for carbon monoxide hemoglobin in blood. He claims 0.ा25 per cent may be detected by his test.

${ }^{2}$ This transforms the oxyhemoglobin into methemoglobin.

${ }^{3}$ This is done to free the blood from carbon monoxide hemoglobin. 
prepared alkali hematin. ${ }^{1}$ The typical spectrum of alkali hematin shows a single absorption band lying across D and mainly toward the red end of the spectrum.

7. Reduced Alkali Hematin or Hemochromogen.-Dilute the alkali hematin solution used in the last experiment (6) to such an extent that it shows no absorption band. Now add a few drops of Stokes' reagent or ammonium sulphide and note that the greenish-brown color of the alkali hematin solution is displaced by a bright red color. This is due to the formation of hemochromogen or reduced alkali hematin. Examine this solution spectroscopically and observe the narrow, dark absorption band lying midway between $\mathrm{D}$ and $\mathrm{E}$. If the dilution is not too great a faint band may be observed in the green extending across $E$ and $b$.

8. Acid Hematin. - To some defibrinated blood add half its volume of glacial acid and an equal volume of ether. Mix thoroughly. The acidified etheral solution of hematin rises to the top and may be poured off and used for the spectroscopic examination. If desired it may be diluted with acidified ether in the ratio of one part of glacial acetic acid to two parts of ether. A distinct absorption band will be noted in the red between $\mathrm{C}$ and $\mathrm{D}$ and lying somewhat nearer $\mathrm{C}$ than the band in the methemoglobin spectrum. Between D and $\mathrm{F}$ may be seen a rather indistinct broad band. Dilute the solution until this band resolves itself into two bands. Of these the more prominent is a broad, dark absorption band lying in the green between $\mathrm{b}$ and $\mathrm{F}$. The second, a narrow band of faint outline, lies in the light green to the red side of $\mathrm{E}$. A fourth very faint band may be observed lying on the violet side of $\mathrm{D}$.

9. Acid Hematoporphyrin.-To 5 c.c. of concentrated sulphuric acid in a testtube add 2 drops of blood, mixing thoroughly by agitation after the addition of each drop. A wine-red solution is produced. Examine this solution spectroscopically. Acid hematoporphyrin gives a spectrum with an absorption band on either side of $\mathrm{D}$, the one nearer the red end of the spectrum being the narrower.

Io. Alkaline Hematoporphyrin.-Introduce the acid hematoporphyrin solution just examined into an excess of distilled water. Cool the solution and add potassium hydroxide slowly until the reaction is but slightly acid. A colored precipitate forms which includes the principal portion of the hematoporphyrin. The presence of sodium acetate facilitates the formation of this precipitate. Filter off the precipitate and dissolve it in a small amount of dilute potassium hydroxide. Alkaline hematoporphyrin prepared in this way forms a bright red solution and possesses four absorption.bands. The first is a very faint, narrow band in the red, midway between $\mathrm{C}$ and $\mathrm{D}$; the second is a broader, darker band lying across $\mathrm{D}$, principally to the violet side. The third absorption band lies principally between $\mathrm{D}$ and $\mathrm{E}$, extending for a short distance across $\mathrm{E}$ to the violet side, and the fourth band is broad and dark and lies between $\mathrm{b}$ and $\mathrm{F}$. The first band mentioned is the faintest of the four and is the first to disappear when the solution is diluted.

I. Fleischl's Hemometer (Fig. 89, p. 300).-This is an instrument used quite extensively clinically, for the quantitative determination of hemoglobin. The instrument consists of a small cylinder which is provided with a fixed glass bottom and a movable glass cover, and which is

${ }^{1}$ Alkali hematin may be prepared by mixing one volume of a concentrated potassium hydroxide or sodium hydroxide solution and two volumes of dilute ( $r: 5$ ) detibrinated blood. This mixture should be heated gradually almost to boiling, then cooled and shaken for a few moments in the air before examination. 
divided, by means of a metal septum, into two compartments of equal capacity. This cylinder is supported in a vertical position by means of a mechanism which resembles the base and stage of an ordinary microscope. Underneath the stage is placed a colored glass wedge (see Fig. 9I), so arranged as to run immediately beneath the glass bottom of one of the compartments of the cylinder and ground in such a manner that each part of the wedge corresponds in color to a solution

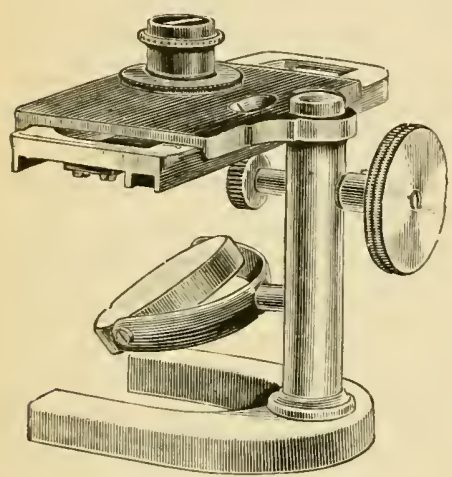

Fig. 80.-Fleischl's Hemometer. (Da Costa.) of hemoglobin of some definite percentage. The glass wedge is held in a metal frame and may be moved backward or forward by means of a rack and pinion arrangement. A scale along the side of this frame indicates the percentage of the normal amount of hemoglobin which each particular variation in the depth of color of the ground wedge represents, taking the normal hemoglobin content as roo. ${ }^{1}$ In a position corresponding to the position of the mirror on the ordinary microscope is attached a light-colored opaque plate which serves to reflect the light upward through the colored wedge and the cylinder to the eye of the observer.

In making a determination of the percentage of hemoglobin by this instrument the procedure is as follows: Fill each compartment about three-fourths full of distilled water. Puncture the finger-tip or lobe of the ear of the subject by means of a sterile needle or scalpel and, as soon as a drop of blood appears, place one end of the capillary pipette (Fig. 90), which accompanies the instrument, against the drop and allow it to fill by capillary attraction. To prevent the blood from adhering to the exterior of the tube, and so render the determination inaccurate, it is customary to apply a very thin coating of mutton fat to the outer surface before using or to wrap the tube in a piece of oily chamois when not in use. As soon as the tube has been accurately filled with blood it should be dipped into the water of one of the compartments of the cylinder and all traces of the blood washed out with water by means of a small dropper which accompanies the instrument. If the blood is not well distributed throughout the compartment and does not form a homogeneous solution the contents of the compartment should be mixed thoroughly by means of the metal handle of the capillary measuring pipette. When this has been done each compartment should be completely filled with distilled water and the glass cover adjusted, care being taken that the contents of the two compartments do not mix. Now adjust the cylinder so that the compartment containing the pure distilled water is immediately above the colored glass wedge. By means of the rack and pinion arrangement manipulate the colored wedge until a portion of it is found which corresponds in color with the diluted blood. When this agreement in color has been secured the point on the scale cor-

1 The scale of the ordinary instrument is usually too high. 
responding to this particular color should be read and the actual percentage of hemoglobin computed. For instance, if the scale reading is 90 it means that the blood under examination contains 90 per cent of the normal quantity of hemoglobin, i.e., 90 per cent of 14 per cent.

2. Fleischl-Miescher Hemometer.-The apparatus of Fleischl has been modified by Miescher. If all precautions are taken, the margin of error in the absolute quantity of hemoglobin determined by this instrument does not exceed $0.15^{-0.22}$ per cent by weight of the blood. Detailed directions for the manipulation of the Fleischl-Miescher hemometer accompany the instrument. In brief Miescher modified the instrument as follows: (I) The scale of each instrument is supplied with a caliber table of absolute hemoglobin values, expressed in milligrams: the scale of Fleischl's hemometer shows the percentage of hemoglobin in relation to an average selected somewhat

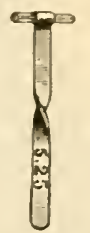

Fig. 90.-Pipette OF FIEISCHL'S HEMOMETER arbitrarily. Thus many errors arising from the irregular coloring of the glass wedge of the older apparatus are avoided in the instrument as modified. (2) Each instrument is accompanied by a measuring pipette (melangeur) which allows of a more accurate measurement of the blood than was possible with the capillary tubes of the older apparatus. (3) With the aid of the measuring pipette mentioned above blood of varying degrees of concentration may be compared. In this way the individual examinations are controlled and a check upon the

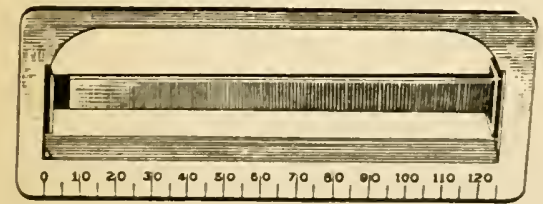

Fig. 91.-Colored Glass Wedge of Fleischl's HeMometer. (Da Costa.) accuracy of the graduation in the color of the glass wedge is also afforded. This wedge is much more evenly and accurately colored than in the unmodified apparatus of Fleischl. (4) Before reading the percentage as indicated by the scale, the chamber is covered with a glass and a diaphragm which sharply define the field on all sides without the formation of a meniscus.

The measuring pipette is constructed essentially the same as the pipettes which accompany the Thoma-Zeiss apparatus (see page 305 ). The capillary portion, however, is graduated, $I, 2 / 3$ and $I / 2$ which enables the observer to dilute the blood sample in the proportion of I $: 200, I: 300$ or $I: 400$ as he may desire. If there is difficulty in drawing in the blood exactly to one of the graduations just mentioned the amount of blood above or below the volume indicated by the graduation may be determined by means of certain delicate cross-lines which 
are placed directly above and below the graduation. Each cross-line corresponds to I/ I o of the volume of the capillary tube from the tip to the I graduation.

A O.I per cent solution of sodium carbonate is used to dissolve the stroma of the erythrocytes and so render the blood solution perfectly clear. If this is not done the color of the blood solution invariably ap-

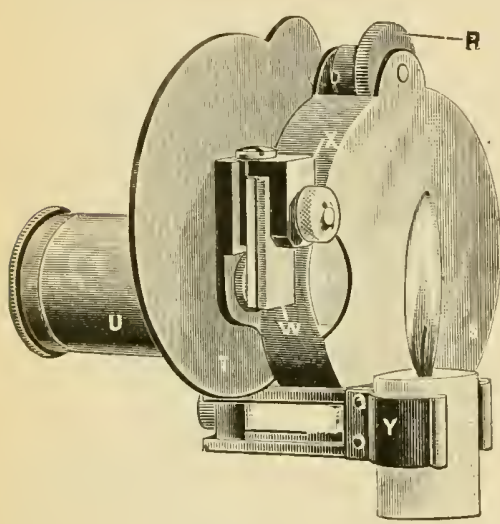

Fig. 92.-Dare's Hemoglobinometer. (Da Costa.)

$\mathrm{R}$, Milled wheel acting by a friction bearing on the rim of the color disc; $\mathrm{S}$, case inclosing color disc, and provided with a stage to which the blood chamber is fitted; T, movable wing which is swung outward during the observation, to serve as a screen for the observer's eyes, and which acts as a cover to inclose the color disc when the instrument is not in use; $\mathrm{U}$, telescoping camera tube, in position for examination; $\mathrm{V}$, aperture admitting light for illumination of the color disc; $\mathrm{X}$, capillary blood chamber adjusted to stage of instrument, the slip of opaque glass, $\mathrm{W}$, being nearest to the source of light; Y, detachable candle-holder; Z, rectangular slot through which the hemoglobin scale indicated on the rim of the color disc is read. to illuminate portions of the capillary observation cell and the colored wedge, (5) a small telescope used in the examination of the areas illuminated by the candle flame, (6) a scale graduated in percentages of the normal amount of hemoglobin, (7) a hard-rubber case, (8) a movable screen attached to the case.

The capillary observation cell is formed of two small, polished rectangular plates of glass, one being transparent and the other opaque. When held in position on the instrument, by means of a small metal bracket, the opaque portion of the cell is nearer the candle and thus serves to soften the glare of light when an observation is being made. 
The transparent portion of the cell is directly over a circular opening in the case, through which the blood specimen is viewed by means of the small telescope.

The semicircular colored glass wedge is so ground that each particular shade of color corresponds to that possessed by fresh blood which contains some definite percentage of hemoglobin. It is mounted upon a disc which may be manipulated by the milled wheel in such a manner as to bring successive portions of the wedge in position to be viewed through a circular opening contiguous to the opening through which the blood specimen is viewed. For a further description of the instrument see Figs. 92, 93, and 94.

In using the Dare hemoglobinometer proceed as follows: Puncture the finger-tip or lobe of the ear of the subject by means of a needle or scalpel and, after a drop of blood of good proportions has formed, place the flat capillary observation cell in contact with the drop and allow it to fill by cap-

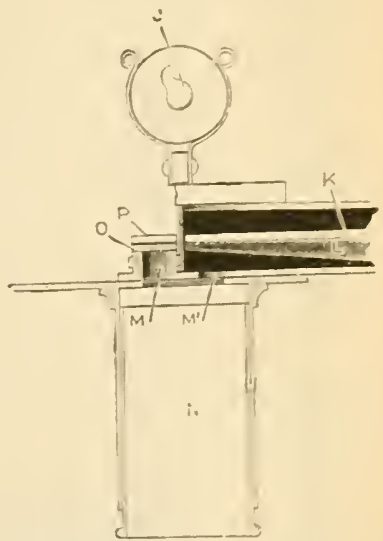

Fig. 93.-Horizontal SeCTION OF DARE'S HEMOGLOBINOMETER. (Da Cosia.) illary attraction (Fig. 94). Replace the cell in its proper place on the instrument. When in position, a portion of this cell may be observed through a small telescope attached to the apparatus. It is viewed through a circular opening and near this circle is a second one through which a portion of a semicircular colored glass wedge is visible. These two circles are illuminated simultaneously by means of the flame of a candle. The colored glass

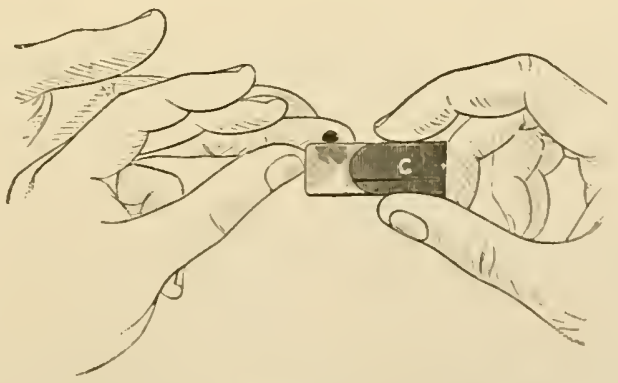

Fig. 94.-Method of Fillixg tile Capillakl Obserration Cell of Dare's HemoGLOBINOMETER. (Da Cosla.)

may be rotated by means of a milled wheel and the point of agreement of the color of the adjoining discs may be determined in the same way as in Fleischl's hemometer. The scale reading gives the percentage of the normal quantity of hemoglobin which the blood sample under examination contains. Compute the actual hemoglobin content in the same manner as from the scale reading of the Fleischl hemometer (see page 299). 
4. Tallquist's Hemoglobin Scale.-This consists essentially of a series of ten colors corresponding to stains produced by blood containing varying percentages of hemoglobin.

In using this scale a drop of blood is allowed to fall on a small section of filter paper and the resulting color is compared with the ten colors of the scale. When the color in the scale is found which corresponds to the color of the blood stain the accompanying hemoglobin value is read off directly.

This is a very convenient method for determining hemoglobin at the bedside. There is a possibility of the colors being inaccurately printed, however, and even if originally correct in tint, under the continued influence of air and light they must eventually alter somewhat.

5. Thoma-Zeiss Hemocytometer.-This is an instrument used in "blood counting," i.e., in determining the number of erythrocytes and leucocytes. The instrument consists of a microscopic slide constructed of heavy glass and provided with a central counting cell (see Fig. 95,

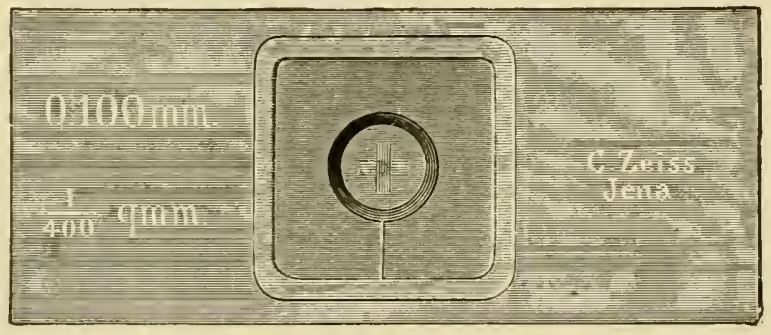

Fig. 95.-Thoma-Zeiss Counting Chamber. (Da Costa.)

below). This cell, with the cover glass in position, is exactly o.I $\mathrm{mm}$. deep. The floor of the cell is divided by delicate lines into squares each of which is I/ $400 \mathrm{sq}$. mm. in area (see Fig. 97, page 306). The volume of blood, therefore, between any particular square and the cover glass above must be $\mathrm{I} / 4000 \mathrm{cu}$. mm. Accompanying each instrument are two capillary pipettes (Fig. 96), each constructed with a mixing bulb in its upper portion. Each bulb is further provided with an enclosed glass bead which is of great assistance in mixing the contents of the chamber. The stem of each pipette is graduated in tenths from the tip to the bulb. The final graduation at the upper end of the bulb is ror on the pipette used in mixing the blood sample in which the erythrocytes are counted (erythrocytometer, see Fig. 96), and II on the pipette used in mixing the blood sample for the leucocyte count (leucocytometer, see Fig. 96). In making "blood counts" with the hemocytometer it is necessary to use some diluting fluid. Two very satisfactory forms of fluid for this purpose are Toison's and Sher- 
rington's solutions. ${ }^{1} \quad$ When either of these solutions is used as the diluting fluid it is possible to make a very satisfactory count of both the erythrocytes and leucocytes from the same prepara. tion, since the leucocytes are stained by the methylviolet or methylene-blue.

In counting the erythrocytes by means of the hemocytometer, proceed as follows: Thoroughly cleanse the tip of the finger or lobe of the ear of the subject by the use of soap and water, alcohol and ether applied in the sequence just given. Puncture the skin by means of a needle or scalpel and allow the blood drop to form without pressure. Place the tip of the pipette in contact with the blood drop, being careful to avoid touching the skin, and draw blood into the pipette up to the point marked 0.5 or I according to the desired dilution. Rapidly wipe the tip of the pipette and immediately fill it to the point marked ror with Toison's or Sherrington's solution. Now thoroughly mix the blood and diluting fluid within the mixing chamber by tapping the pipette gently against the finger, or by shaking it while held securely with the thumb at one end and the middle finger at the other. After the two fluids have been thoroughly mixed the diluting fluid contained in the capillary-tube below the bulb should be discarded in order to insure the collection of a drop of the thoroughly mixed blood and diluting solution for examination. Transfer a drop from the pipette to the ruled floor of the counting chamber and, after placing the cover-glass firmly in position, ${ }^{2}$ allow an interval of a few minutes to elapse for the corpuscles to settle before making the count. Now place the slide under the microscope and count the number of erythrocytes in a number of squares, counting the corpuscles which are in contact with the upper and the right-hand boundaries of the

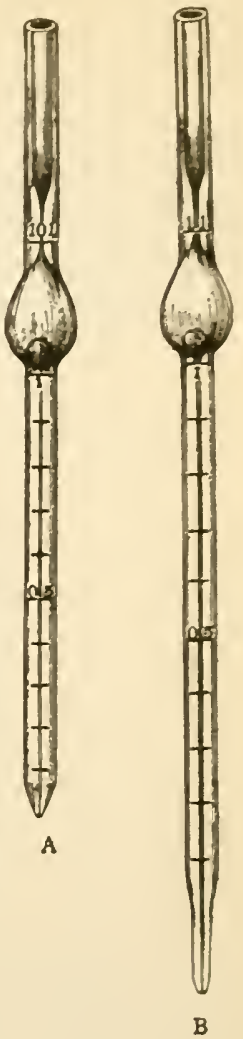

Fig. 96.-ТномаZEISS CAPILLAR Y Pipettes.

A, Erythrocytometer B, Leucocytometer. formula:

1 Toison's solution has the following Methyl-violet.............0.025 gram.

Sodium chloride........... I gram.

Sodium sulphate.......... 8 grams.

Glycerol.

30 grams

Distilled water............ I60 grams.

Sherrington's solution has the following formula:

Methylene-blue........... o. I gram.

Sodium chloride........... I. 2 gram.

Neutral potassium oxalate.... r. 2 gram.

Distilled water.

300.0 grams

${ }^{2}$ If the cover-glass is in accurate apposition to the counting cell Newton's rings may be plainly observed. Eustis (Jour. Am. II ed. Ass'n, 6r, rg84, rgr3) suggests the following technic: "After the usual shaking of the pipette, and expulsion of a few drops of the suspension, a good sized drop is placed on the counting chamber, no particular attention being paid to its size. The cover-glass, which has been previously cleaned, is then rapidly grasped between the thumb and index-finger of the right hand, while the slide is steadied on the table with the left hand. While firm pressure is exerted on the cover-glass it is rapidly slid across the counting chamber, through the drop of suspension on it. The cover-glass will cut through the drop at exactly O.I mm. The excess from the drop will rise on top of the cover-glass and jump across the moat. Newton's rings will be obtained in each instance. The drop on top of the edge of the coverglass is wiped or soaked up with the point of a towel or blotting-paper and the preparation is completed." 
square as belonging to that square. Take the squares in some definite sequence in order that the recounting of the same corpuscles may be avoided. A satisfactory procedure is to begin in the upper right-hand corner and proceed from left to right counting the cells in each individual square. Take the next lower row of squares and count from left to right and so on (see Fig. Ior, page 3II). Of course, all things being equal, the greater the number of squares examined the more accurate the count. It is considered essential under all circumstances, where an accurate count is desired, that the counting chamber shall be filled, at least twice, and the individual counts made in each instance, as indicated above, before the data are deemed satisfactory. Under no conditions should less than 200 squares be examined.

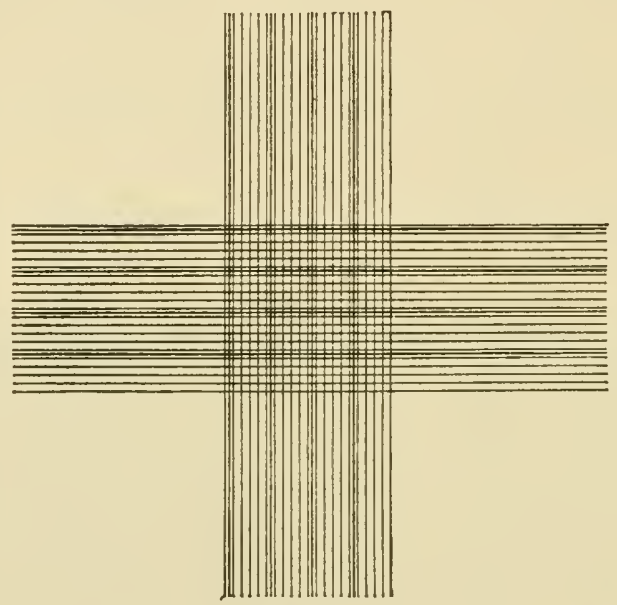

Fig. 97.-Ordinary Ruling of Thoma-Zeiss Counting Chamber. (Da Costa.)

To calculate the number of erythrocytes per cubic millimeter of undiluted blood proceed as follows: Determine the number of corpuscles in any given number of squares and divide this total by the number of squares, thus obtaining the average number of erythrocytes per square. Multiply this average by 4000 to obtain the number of erythrocytes per cubic millimeter of diluted blood, and multiply this product by Ioo or 200 , according to the dilution, to obtain the number of erythrocytes per cubic millimeter of undiluted blood. Thus: $\begin{aligned} & \text { Average number of erythrocytes } \\ & \text { per square }\end{aligned}$
$\quad 4000 \times 200\left(\right.$ or I00) $=\begin{array}{c}\text { Number of erythrocytes per } \\ \text { cubic millimeter. }\end{array}$

Great care should be taken to see that the capillary pipette is properly cleaned. After using, it should be immediately rinsed out with the diluting fluid, then with water, alcohol, and ether in the sequence given. Finally dry air should be drawn through the capillary and a horsehair inserted to prevent the entrance of dust particles.

In counting leucocytes by means of the hemocytometer proceed as follows: As mentioned above, if the diluting fluid is either Toison's or Sherrington's 
solution the leucocytes may be counted in the same specimen of blood in which the erythrocytes are counted. When this is done it is customary to use a slide provided with Zappert's modified ruling (Fig. 98). This method is rather more accurate than the older one of counting the leucocytes in a separate specimen of blood. Furthermore, it is obviously preferable to count both the erythrocytes and the leucocytes from the same blood sample. To insure accuracy the number of leucocytes within the whole ruled region should be determined in duplicate blood samples. This includes the examination of an area eighteen times as great as the old style Thoma-Zeiss central ruling. This region then would correspond to 3600 of the small squares and, if duplicate examinations were made, the total bumber of small squares examined would aggregate 7200 .

The calculation would be as follows: Number of leucocytes in $7200 \times 200 \times 4000 \div 7200=\begin{gathered}\text { Number of leucocytes per cubic } \\ \text { millimeter. }\end{gathered}$
squares squares

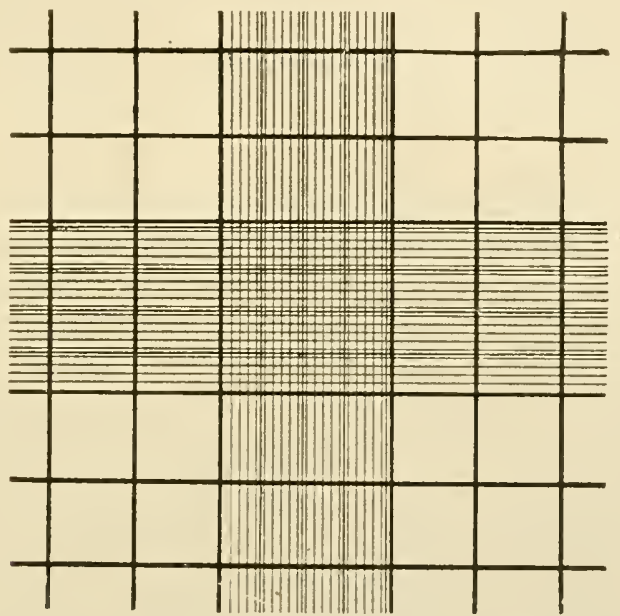

Fig. 98.-Zappert's Modified Ruling of Thoma-Zeiss Countixg Chimber. (Du Cosla.)

If a Zappert slide is not available, a good plan to follow is to place a diaphragm in the tube of the ocular of the microscope consisting of a circle of black cardboard or metal ${ }^{1}$ having a square hole in the center of such a size as to allow of the examination of exactly roo squares or onefourth of a square millimeter at one time. With this arrangement any portion of the specimen may be examined and counted whether within or without the ruled area. In counting by means of this device it is, of course, helpful if the microscope is provided with a mechanical stage, but even without this arrangement, if the observer is carcful to see that the leucocytes at the extreme boundary of one field move to the opposite boundary when the position of the slide is changed, the device may be very satisfactorily employed. The leucocytes should be counted in 36

${ }^{1}$ Ehrlich's mechanical eyepiece with iris diaphragm is also very satisfactory for this purpose. 
of the diaphragm-fields in $d u$ plicate specimens and the calculation made in the same manner as explained above.

If the leucocytes are counted in a separate specimen of blood ordinarily the diluting fluid is $0.3-0.5$ per cent acetic acid, a fluid in which the leucocytes alone remain visible. Under these conditions the dilution is customarily made in the pipette having I I as the final graduation. The capillary portion is of larger caliber and so requires a greater amount of blood to fill it to the 0.5 or I mark than is required in the use of the other form of pipette. In counting the leucocytes according to this method it is customary to draw blood into the pipette up to the I mark and immediately fill the remaining portion of the apparatus to the II graduation with the $0.3-0.5$ per cent acetic acid. It then remains to count the number of leucocytes in the whole central ruled portion of 400 squares. This should be done in duplicate samples and the calculation made as follows:

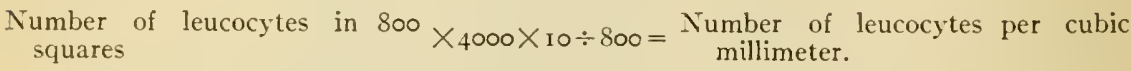

6. Bürker's Hemocytometer. I-This is an improved apparatus ${ }^{2}$ for the more accurate counting of erythrocytes than is possible by the Thoma-Zeiss apparatus. The principles involved are somewhat different from those in force with the latter apparatus. For example, the blood is diluted in a separate vessel, not in the pipette with which the sample is drawn, and furthermore the cover-glass is applied to the counting chamber and clamped in place before the diluted blood is applied to the ruled area. Hayem's solution ${ }^{3}$ is used as the diluting fluid. Toison's solution is not satisfactory for use with the Bürker counting chamber as its viscosity is too great. The corpuscles settle rapidly in Hayem's fluid as the specific gravity of the fluid is IOI 5 whereas that of the erythrocytes is Iogo.

The pipette for measuring the quantity of blood (Fig. 99, upper pipette) has a point which is not ground dull but is polished. This allows of better judgment in deciding whether the column of blood extends to the very tip. The volume of the pipette between tip and mark is $25 \mathrm{cu}$. mm. The mark extends all the way around the tube so that errors of parallax may be avoided.

The pipette for measuring the diluting fluid (Fig. 99, middle pipette) also has a polished point and circular mark and delivers $4975 \mathrm{cu} . \mathrm{mm}$. This volume of diluting fluid with $25 \mathrm{cu}$. mm. of blood gives a dilution of $\mathrm{I}: 200$. Both pipettes are provided with a piece of rubber tubing and mouthpiece.

For transferring the diluted blood from the diluting flask to the chamber a plain pipette provided with a rubber cap is used (Fig. 99, lower pipette). It is filled by pressing the cap slowly with the index-finger, inserting the tip into the liquid and then releasing the pressure.

${ }^{1}$ Bürker: Pflüger's Archiv', I42, 337, I91 I; Mïnch. med. I1'och., 59, pp. I4 and 89, I9I 2.

2 Manufactured by C. Zeiss, Jena.

${ }^{3}$ Hayem's solution has the following formula:

Mercuric chloride

0.25 gram.

Sodium chloride............................ o. 5 gram.

Sodium sulphate................. 2. 5 grams.

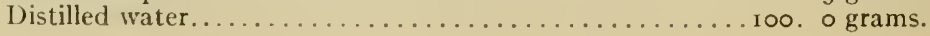


The diluting is done in a small round-bottomed flask as shown in Fig. 99. Several of these flasks should be kept on hand in a wooden rack which will hold them in an upright position. Each flask is provided with a paraffined, or smooth cork stopper.

In the older counting chambers the floor of the chamber is circular and the counting is done in the center of this space. The corpuscles are therefore counted in the center of a capillary, circular film where on account of surface tension their number is slightly greater than elsewhere. This source of error is avoided in the new counting chamber (Fig. 102) in which the floor is represented by the upper surface of a piece of glass $25 \mathrm{~mm}$. long and $5 \mathrm{~mm}$. wide which is rounded off at both ends and divided into two portions by a groove $1.5 \mathrm{~mm}$. wide through the center.

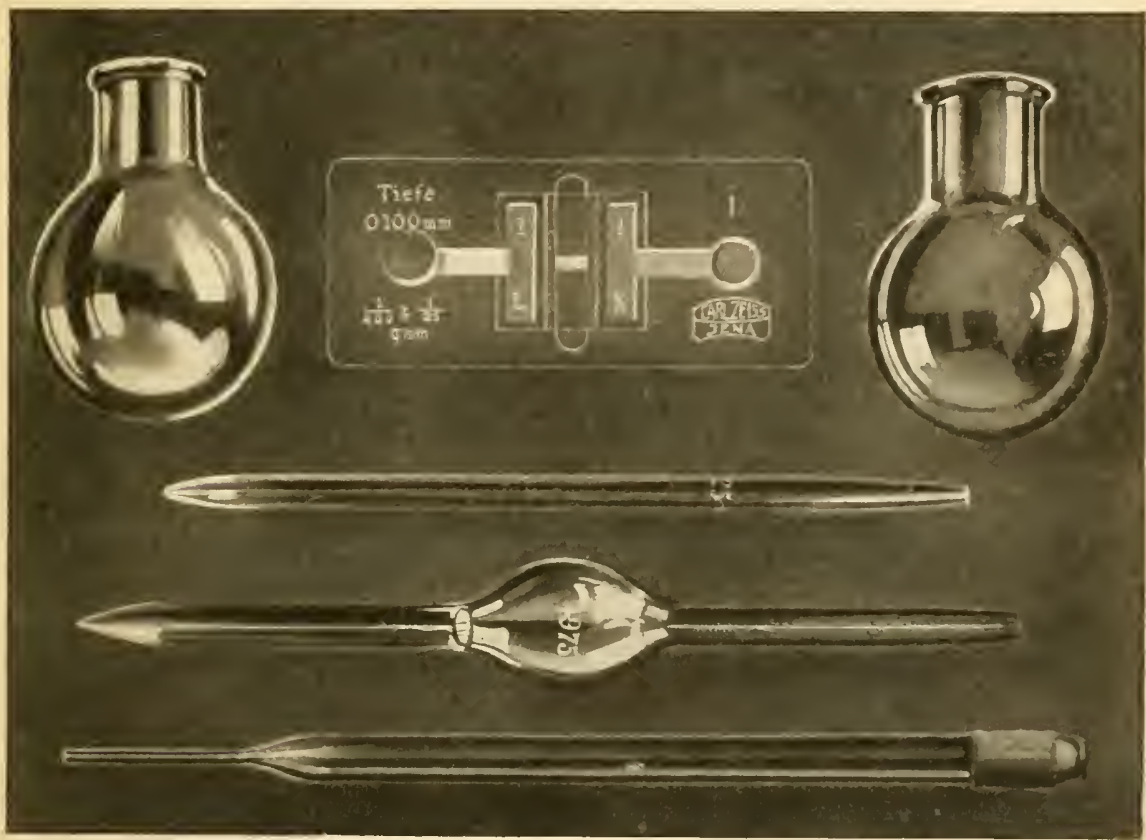

Fig. 99.-Bürker's Pipettes, Mixing Flasks and Counting Chamber.

At each side of this floor piece, separated from it by a groove is a glass plate $(7.5 \mathrm{~mm} . \times 2 \mathrm{Imm}$.) of such height that the space between the floor of the cell and a cover-glass placed across the plates is $0.100 \mathrm{~mm}$. A cover-glass $23 \mathrm{~mm}$. long and $21 \mathrm{~mm}$. wide with rounded polished edges is used so that the rounded ends of the floor piece project beyond it. The chamber is provided with clamps to press the cover-glass firmly upon both plates (Fig. 99).

The ruling on each portion of the floor piece is that shown in Fig. I00, which will be explained below.

Measuring the Diluting Fluid.-Four thousand nine hundred and serenty-five cu. mm. of diluting fluid (Hayem's) are measured out into the diluting llask. To do this the pipette is filled by suction to slightly above the mark and the rubber tube is carefully clamperl off. Then with a soft piece of linen the tip is wiped dry. The meniscus is then accurately adjusted to the mark by lightly touching the point of the pipette to the cleaned tip of the finger. The pipette is then inserted into 
the diluting flask and with the tip nearly touching the bottom of the flask the fluid is allowed to run out. The time of the flow should be about forty seconds and is controlled by placing the tip of the index-finger loosely upon the mouthpiece. The pipette is emptied completely by alternately blowing through it and touching it to the wall of the flask slightly above the level of the liquid. The drops clinging to the wall are united with the bulk of the liquid by a suitable motion of the flask. The flask is then stoppered, care being taken from now on that none of the liquid ever touches the neck of the flask or the stopper.

Taking the Blood Sample.-Usually the best time to draw the blood is before breakfast. For a single determination the author prefers to draw it from the tip of the fourth finger of the left hand. For repeated determinations it is well to change off between third, fourth and fifth fingers of left hand. The temperature of the room should not be below $17^{\circ} \mathrm{C}$. to prevent an undue contraction of the cutaneous vessels. The instrument used to puncture the finger should have a chisel-shaped point which is preferable to the ordinary lancet-shaped point. The

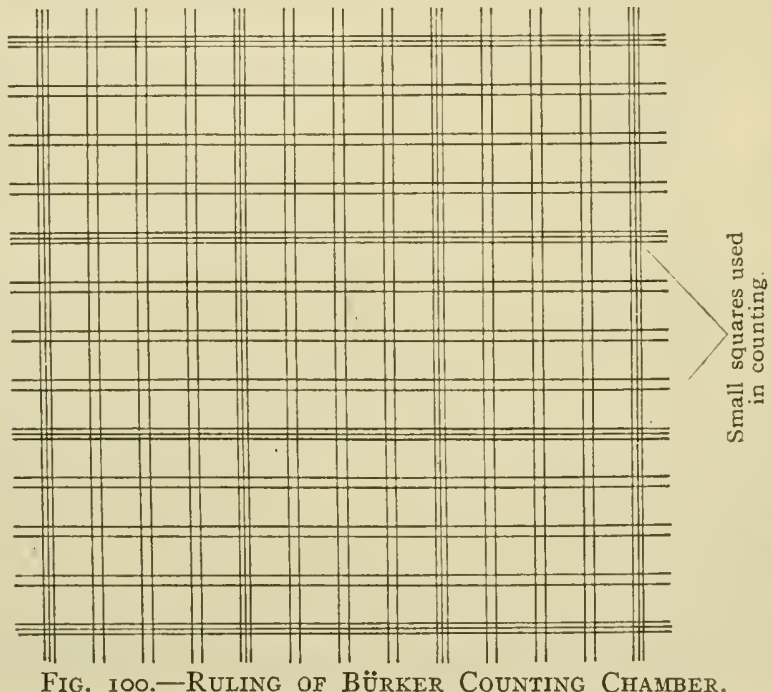

first drop of blood is wiped off. Into the second one the tip of the pipette is inserted and blood is drawn in until the meniscus is even with or a little beyond the mark. The tip is then wiped off without touching the capillary opening and the observer assures himself that the column of blood extends to the very end of the capillary. The meniscus is then accurately adjusted to the mark.

Mixing of the Blood and Diluting Fluids.-The tip of the pipette is now dipped into the diluting fluid which has been measured into the flask and the blood is slowly blown out. The blood having a much higher specific gravity than the Hayem's fluid sinks to the bottom. The pipette is then filled with the pure supernatant diluting fluid and emptied again, care being taken to avoid air bubbles. This is repeated until the blood is removed as completely as possible. To mix the blood and diluting fluid the flask is rotated for two minutes in spiral curves of continually decreasing radius. The motion should be alternately clockwise and counterclockwise. After complete mixing the pipette is rinsed out several times with the diluted blood. 
Transferral of the Diluted Blood to the Chamber.-The counting chamber which has been cleaned with distilled water and alcohol-ether and then wiped dry with a soft cloth as free from lint as possible is placed upon a black surface and carefully brushed with a camel's hair brush. The cover-glass is now placed over the chamber by sliding it over the two glass plates with both thumbs while the index-fingers are pressing it down. By means of the clamps it is held in place firmly so that Newton's rings (if possible of the first order: brown and black) may be seen over the entire area of the plates. The chamber is placed upon the stage of the microscope and is brought into a horizontal position.

Before transferring the diluted blood to the chamber the flask must be shaken for two minutes as described before. The liquid shows a cloudy appearance and must be allowed to stand until the turbidity has become uniform.

One of the plain pipettes described above is now inserted into the diluted blood

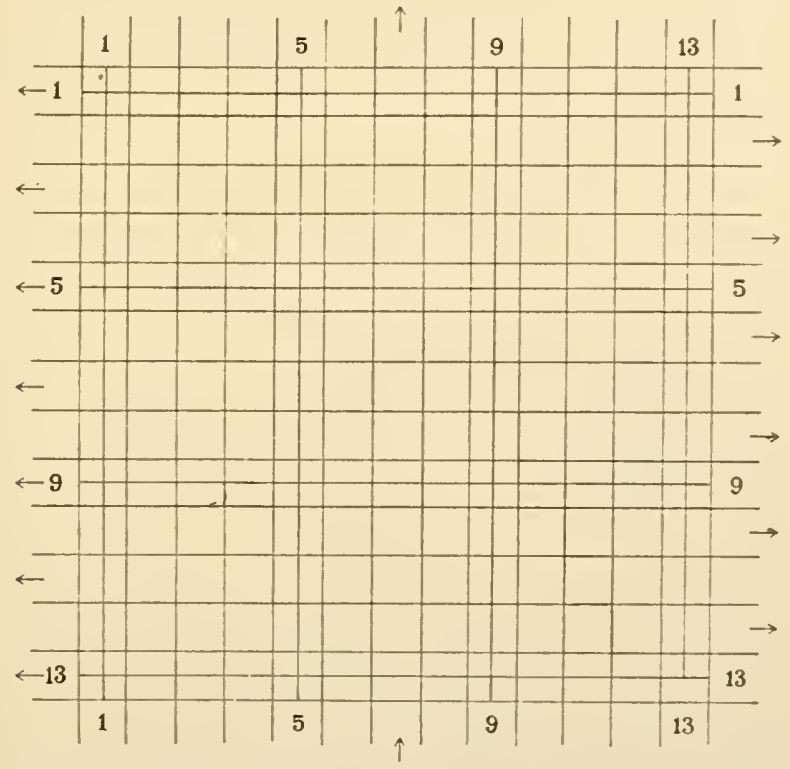

Fig. IOI.-SCHEMA.

while slight pressure is being exerted on the rubber cap. The pressure is released slowly and the liquid rises into the pipette. The point of the pipette is now immediately placed upon one of the projecting ends of the floor plate and very slight pressure is exerted on the rubber cap until the liquid coming from the pipette just reaches the cover-glass when the pressure is released. An instantaneous filling of the capillary space results. The pipette should be emptied immediately, rinsed with distilled water and placed in an upright position in a beaker of water. The other portion of the counting chamber is now filled in the same way with a second pipette and about one minute is allowed for the settling of the corpuscles. During this time the pipettes may be washed with distilled water and ether-alcohol and dried by suction. Occasionally, the pipettes should be cleaned with a horse hair and with concentrated $\mathrm{H}_{2} \mathrm{SO}_{4}$ containing a little $\mathrm{K}_{2} \mathrm{Cr}_{2} \mathrm{O}_{7}$.

To see whether the distribution of the corpuscles has been uniiorm the chamber is illuminated with a wide-open diaphragm and viewed at an angle. If the opacity 
is not uniform in either of the portions of the chamber, that one should not be used for counting. If the counting must be interrupted or requires a long time a moist chamber ${ }^{1}$ should be used to prevent evaporation of the diluting fluid. The diluted blood may be retained in the mixing flasks and duplicate countings obtained after the lapse of twenty-four hours or more according to Bürker.

Counting and Calculation.-A mechanical stage movable in two directions is indispensable. With a magnification of 320 diameters the counting is begun in the left upper corner of the ruling. Proceed from left to right along one row then move from right to left along the next lower row, and so on. Only the small squares are used for counting (see Fig. roo) and the figures are recorded in the schema $a^{2}$ (see Fig. IOI) in which the squares crossed by horizontal or vertical lines correspond to the small squares used for counting. Usually So squares are counted and by recording the figures in the schema the count may be verified and an idea of
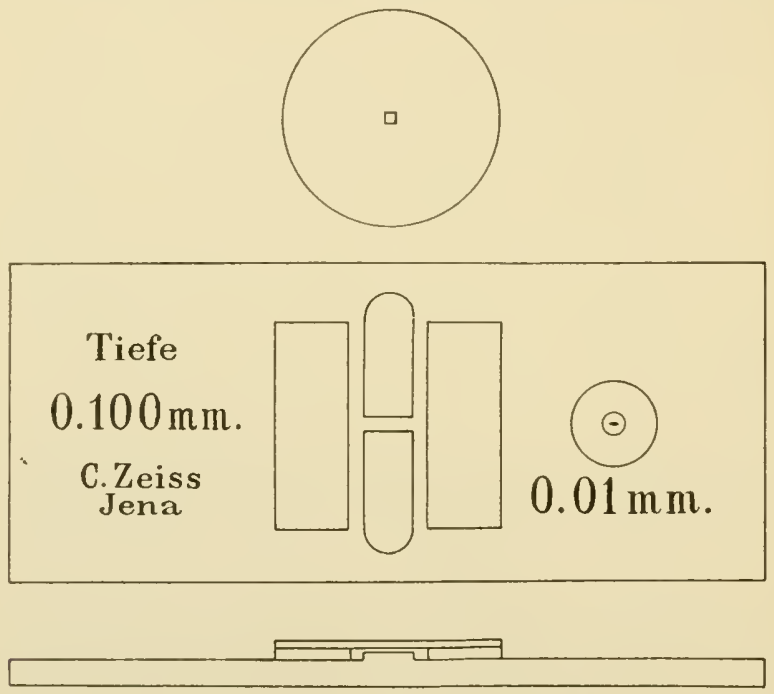

Fig. IO2.-BÜrker Counting Chamber.

the uniformity of the distribution may be formed. Half of the counted squares should be in the one, half in the other portion of the counting chamber. For more accurate measurements more squares may be counted.

The observer will do well not to attempt counting each individual corpuscle in a square. After some practice each typical group of corpuscles will immediately suggest a number. A very common form of grouping is one corpuscle surrounded by four others. This should immediately suggest the number five. In this way the counting will become more rapid and also more reliable.

The calculation is very simple. The number of corpuscles in so squares divided by roo will give the number of millions per cubic millimeter. If, for example, 536 corpuscles have been counted in 80 squares then with a dilution of I : 200 the number of corpuscles per cubic millimeter is 5,360,000. Thus, ${ }_{80}^{536} \times 4000 \times 200=5,360,000$ erythrocytes per cubic millimeter. More than two decimal places are without significance.

1 Bürker: Pflïger's Archiv, I I8, 465, I907.

${ }^{2}$ The firm of H. Laupp in Tübingen has put this schema on the market (in packs of 100 ). 


\section{CHAPTER XVII}

\section{MILK}

MILK is the most satisfactory individual food material elaborated by nature. It contains the three nutrients, protein, fat, and carbohydrate and inorganic salts in such proportion as to render it a very acceptable dietary constituent. It is a specific product of the secretory activity of the mammary gland. It contains, as the principal solids, olein, palmitin, stearin, butyrin, casein, lact-albumin, lacto-globulin, lactose, phosphates of calcium, potassium and magnesium, citrates of sodium and potassium and chloride of calcium. The calcium phosphate of milk is the neutral calcium phosphate, $\mathrm{CaHPO}_{4}{ }^{1}{ }^{1}$ Milk also contains some iron but not enough for the needs of the body if milk is the only source of the iron. It also contains at least traces of lecithin, cholesterol, urea, creatine, creatinine, and the tri-glycerides of caproic, lauric, and myristic acids. According to Osborne and Wakeman ${ }^{2}$ milk contains two phosphatides, one being probably stearyl-oleyl-lecithin.

Recent investigations indicate the presence in milk of some substance or substances of unknown character which are of great nutritional importance. The presence of a growth-promoting substance (vitamine) in butter fat has been demonstrated by McCollom and Davis and by Osborne and Mendel. ${ }^{3}$

By passing milk through a special form of earthenware filter Van Slyke and Bosworth ${ }^{4}$ have obtained a separation of the constituents in milk which are in true solution from those insoluble in water or in suspension. The soluble constituents and the water constitute the milk serum. They suggest the following classification of milk constituents:

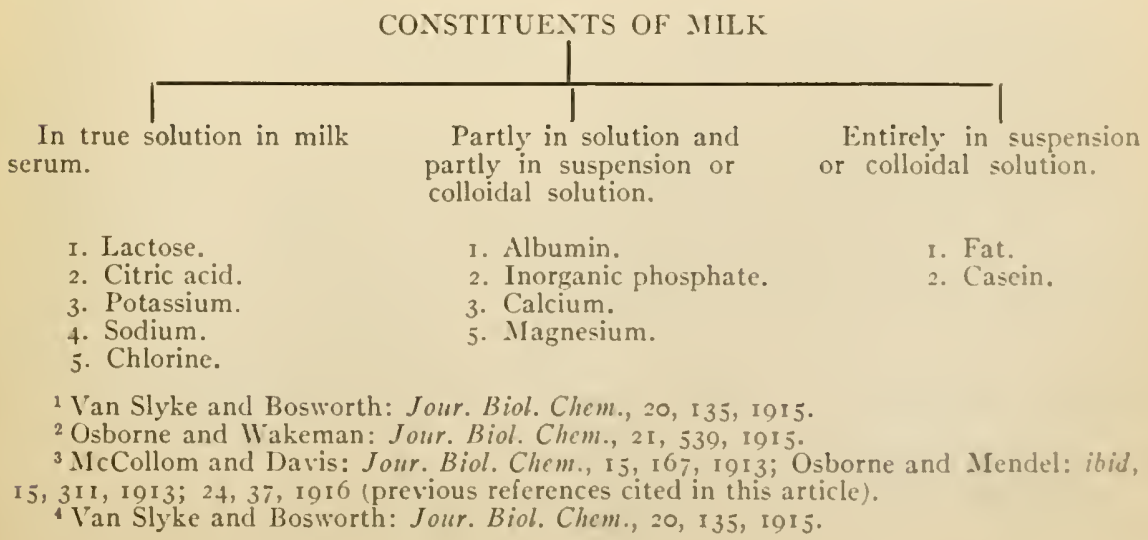

In true solution in milk serum.
I. Lactose,
2. Citric acid.
3. Potassium.
4. Sodium.
5. Chlorine.

Partly in solution and partly in suspension or colloidal solution.

Entirely in suspension or colloidal solution.

1 Van Slyke and Bosworth: Jour. Biol. Chem., 20, 135, 1915.

${ }^{2}$ Osborne and Wakeman: Jour. Biol. Chem., 21, 539, 1915.

${ }^{3}$ MicCollom and Davis: Jour. Biol. Chem., I5, I67, rgi3; Osborne and Mendel: ibid, I5, 311, 1913; 24, 37, 1916 (previous references cited in this article).

Tan Slyke and Bosworth: Jour. Biol. Chem., 20, 135 , I915.

1. Fat.

2. Casein.

1. Albumin.

3. Calcium.

5. Magnesium. 
Fresh milk, both human and cow's, is amphoteric in reaction to litmus and acid to phenolphthalein. The acidity is believed to be due in part at least to soluble acid phosphates. ${ }^{1}$ Upon standing for a sufficiently long time, unsterilized milk sours, i.e., it becomes strongly acid in reaction to litmus due to the production of the optically inactive fermentation lactic acid,

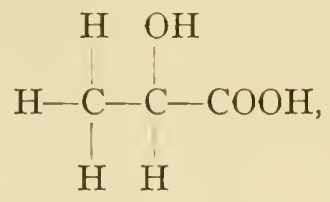

from the lactose contained in it. This is brought about through bacterial activity. The white color is imparted to the milk partly through the fine emulsion of the fat and partly through the medium of the caseinogen in solution. The specific gravity of milk varies somewhat, the average being about I.03O. Its freezing-point is about $-0.56^{\circ} \mathrm{C}$.

This lactic acid fermentation may be brought about by Bact. lactis and other microorganisms. Certain putrefactive bacteria in the human intestines may also cause lactic acid fermentation. The chemical changes in lactic acid fermentation may be indicated thus:

$$
\begin{gathered}
\mathrm{C}_{12} \mathrm{H}_{22} \mathrm{O}_{11}+\mathrm{H}_{2} \mathrm{O} \rightarrow \underset{\text { Lactose. }}{\rightarrow} \underset{\text { Galactose }}{\mathrm{C}_{6} \mathrm{H}_{12} \mathrm{O}_{6}}+\mathrm{C}_{6} \mathrm{H}_{12} \mathrm{O}_{6} \\
\mathrm{Glucose}_{6} \mathrm{H}_{12} \mathrm{O}_{6} \rightarrow 2 \mathrm{C}_{3} \mathrm{H}_{6} \mathrm{O}_{3} \\
\text { Galactose } \\
\text { or Glucose. }
\end{gathered}
$$

Fresh milk does not coagulate on being boiled but a film consisting of a combination of casein and calcium salts forms on the surface. If the film be removed, thus allowing a fresh surface to come into contact with the air, a new film will form indefinitely upon the application of heat. Surface evaporation and the presence of fat facilitate the formation of the film, but are not essential (Rettger $\left.{ }^{1}\right)$. As Jamison and Hert $z^{2}$ have shown, a similar film will form on heating any protein solution containing fat or paraffin. If the milk is of a pronounced acid reaction, through the inception of lactic acid fermentation, or from any other cause, no film will form when heat is applied, but instead a true coagulation will occur. When milk is boiled certain changes occur in its odor and taste. These changes, according to Rettger, ${ }^{3}$ are due to a partial decomposition of the milk proteins and are accompanied by the liberation of a volatile sulphide, probably hydrogen sulphide.

\footnotetext{
${ }^{1}$ Rettger: American Journal of Physiology, 7, 325, 1902.

2 Jamison and Hertz: Journal of Physiology, 27, 26, 1902.

${ }^{3}$ Rettger: American Journal of Physiology, 6, 450, 1902 .
} 
The milk-curdling enzymes of the gastric and the pancreatic juice have the power of splitting the casein of the milk, through a process of hydrolytic cleavage, into soluble paracasein and a peptone-like body. This soluble paracasein then forms a combination with the soluble calcium salts of the milk and an insoluble curd of paracasein results. The clear fluid surrounding the curd is known as whey. This action of rennin may be represented by the following scheme:
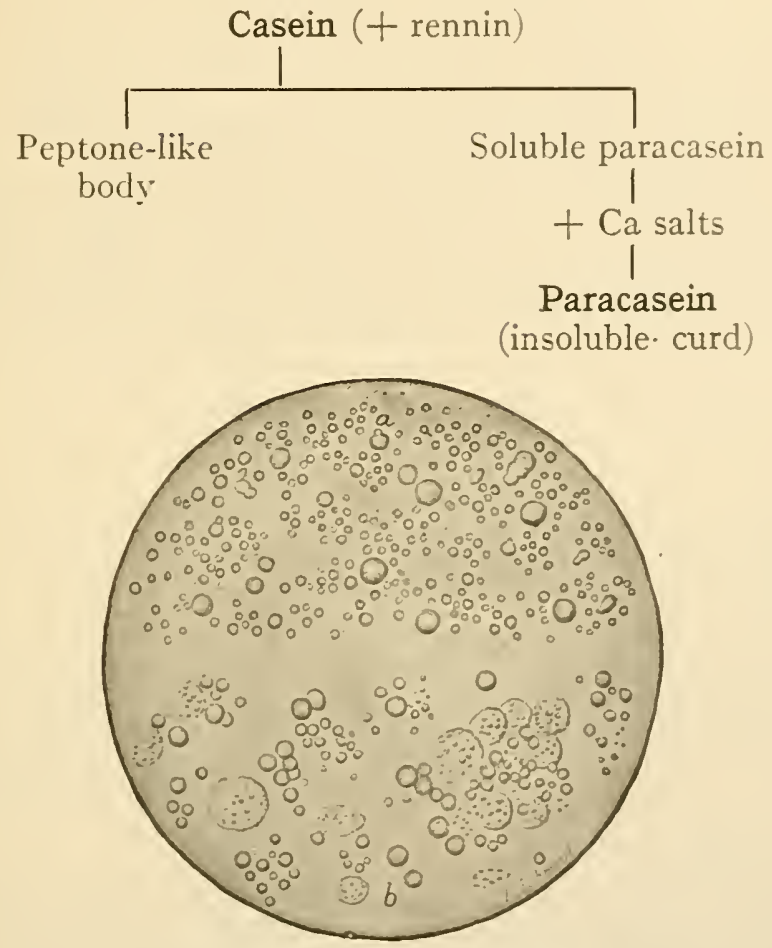

Fig. io3.- Normal Milk and Colostrtir.

$a$, Normal milk; $b$, Colostrum.

There is still considerable confusion of terms when different authorities discuss milk proteins and the action of milk curdling enzymes upon them. The English scientists ${ }^{1}$ quite uniformly call the principal protein of milk caseinogen whereas the insoluble curd formed by rennin is termed casein. On the other hand, the Germans and many Americans give the name casein to the milk protein and paracasein to the product of the action of rennin upon this protein. The confusion of terms may be represented thus:

$$
\text { English }
$$

Caseinogen.

Casein.

$\begin{array}{ll}= & \text { German. } \\ = & \text { Casein. } \\ & \text { Paracasein. }\end{array}$

German.

Paracasein.

${ }^{1}$ Halliburton: Journal of Physiology, I I, $4+$, , I000. 
The most important difference between human milk and cow's milk is in the protein content, although there are also differences in the carbohydrate and ash and likewise striking biological differences difficult to define chemically. It has been shown that the casein of human milk differs from the casein of cow's milk in being more difficult to precipitate by acid or coagulate by gastric rennin. The casein curd (paracasein) also forms in much looser and more flocculent manner than that from cow's milk and is for this reason much more easily digested than the latter. Both human and cow's milk contain important non-nitrogenous substances of an unknown character. Human milk contains the greater quantity of these substances. ${ }^{1}$

The relative composition of human and cow's milk is shown in the following table which embraces data reported by Meigs and Marsh.

COMIPOSITION OF MILK (PER CENT OF IVHOLE MILK) NORMAL VARIATIONS FROM BEGINNING OF SECOND MONTH OF LACTATION

\begin{tabular}{l|l|l} 
Constituent & Cow & Human
\end{tabular}

\begin{tabular}{|c|c|c|}
\hline Water (Avg.) $\ldots \ldots \ldots \ldots \ldots \ldots \ldots \ldots$ & 87.0 & $87 \cdot 5$ \\
\hline Solids (Avg.) .................. & I 3.0 & 12.5 \\
\hline 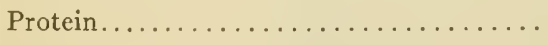 & $4^{-2.5}$ & I. $5^{-0.7}$ \\
\hline Fat.......... & $2-4$ & $2-4$ \\
\hline Sugar........... & $3 \cdot 5^{-5}$ & $6-7 \cdot 5$ \\
\hline Ash. & $0.6-0.7$ & $0.2-0.3$ \\
\hline
\end{tabular}

The above data indicate that human milk contains less protein, more sugar and much less ash than cow's milk. The percentage composition of human milk at different periods is represented in the following table. ${ }^{2}$

PERCENTAGE COMPOSITION OF HUMAN MILK BY PERIODS

\begin{tabular}{|c|c|c|c|c|c|c|c|}
\hline Period & Fat & Sugar & Protein & Casein & Albumin & Ash & $\begin{array}{l}\text { Total } \\
\text { solids }\end{array}$ \\
\hline Colostrum (I-I 2 days).... & 2.83 & $7 \cdot 59$ & 225 & & & 0.31 & I $3 \cdot 4$ \\
\hline Transition ( $12-30$ days).. & $4 \cdot 37$ & $7 \cdot 74$ & I. 56 & & & 0.24 & $13 \cdot 4$ \\
\hline Mature ( $1-9$ mos.) ... & 3.26 & $7 \cdot 50$ & I. I5 & 0.43 & 0.72 & 0.21 & $\{2.2$ \\
\hline Late (10-20 mos.) & 3.16 & $7 \cdot 47$ & I. .07 & 0.32 & 0.75 & 0.20 & $\mathrm{I} 2.2$ \\
\hline
\end{tabular}

${ }^{1}$ Meigs and Marsh: Jour. Biol. Chem., 16, I47, 1913.

'Holt, Courtney and Fales: Am. Jour. Dis. Children, 10, 229, 1915. 
The composition of the ash of milk is shown in the following table reported by Holt, Courtney and Fales. ${ }^{1}$

PERCENTAGE COMPOSITION OF THE ASH OF MILK

\begin{tabular}{|c|c|c|c|c|c|c|}
\hline & $\mathrm{CaO}$ & $\mathrm{MgO}$ & $\mathrm{P}_{2} \mathrm{O}_{5}$ & $\mathrm{Na}_{2} \mathrm{O}$ & $\mathrm{K}_{2} \mathrm{O}$ & $\mathrm{Cl}$ \\
\hline Human milk.... & $23 \cdot 3$ & 3.7 & I 6.6 & 7.2 & 28.3 & 16.5 \\
\hline Cow's milk. & 23.5 & 2.8 & 26.5 & 7.2 & 24.9 & 13.6 \\
\hline
\end{tabular}

It will be observed that the composition of the ash of the two varieties of milk is about the same except for phosphorus. The higher phosphorus content in the case of cow's milk is due principally to the fact that the milk contains a higher percentage of casein or phosphoprotein. It should be borne in mind that cow's milk contains on the average over three times as much ash as human milk. Therefore unless cow's milk has been diluted with more than twice its volume, there is still present as high a concentration of the inorganic constituents as are present in normal human milk. Hence there is no necessity for the addition of any of these constituents in infant feeding.

Interesting data relative to the composition of milk from various sources may be gathered from the following table which was compiled mainly from the results of investigations by Pröscher ${ }^{2}$ and by Abderhalden ${ }^{3}$ in Bunge's laboratory. It will be noted that the composition of the milk varies directly with the length of time needed for the young of the particular species to double in weight.

\begin{tabular}{|c|c|c|c|c|c|}
\hline \multirow{2}{*}{ Species } & \multirow{2}{*}{$\begin{array}{l}\text { Period in which } \\
\text { weight of the } \\
\text { newborn is } \\
\text { doubled (days) }\end{array}$} & \multicolumn{4}{|c|}{ Ioo Parts of milk contain } \\
\hline & & Proteins & Salts & Calcium & $\begin{array}{c}\text { Phosphoric } \\
\text { acid }\end{array}$ \\
\hline Man... & 180 & I. 6 & 0.2 & 0.033 & 0.047 \\
\hline Horse. . & 60 & 2.0 & 0.4 & 0.124 & $0.13 \mathrm{I}$ \\
\hline Cow......... & 47 & $3 \cdot 5$ & 0.7 & 0.160 & 0.197 \\
\hline Goat........ & 22 & 3.7 & $0 . S$ & O. 197 & 0.284 \\
\hline Sheep......... & $I_{5}$ & +9 & 0.8 & 0.245 & 0.293 \\
\hline Pig.... & 14 & 5.2 & $0 . S$ & 0.249 & 0.308 \\
\hline Cat... & $9 \cdot 5$ & 7.0 & I.O & $\ldots \ldots$ & $\ldots \ldots \ldots$ \\
\hline Dog.... & 9 & 7.4 & I. 3 & 0.455 & 0.508 \\
\hline Rabbit...... & 6 & 10.4 & 2.5 & 0.891 & 0.997 \\
\hline
\end{tabular}

The secretion of the mammary glands of the newborn of both sexes is called "witches' milk." The name is centuries old and evidently"

'Holt, Courtney and Fales: Am. Jour. Dis. Children, 10, 229, I915.

${ }^{2}$ Pröscher: Zcil. f. physiol. Chemic, 24, 2S5, ISgS.

3 Abderhalden: Ibid., 26,487 , r899; and 27 , pp. foS and 457 , r S99. 
refers to the mystery of the useless secretion. Basch ${ }^{1}$ has recently suggested that this secretion of "witches milk" is brought about by the passage of hormones (see Chapter on Pancreatic Digestion) from the blood of the mother to the fetus.

Lactose, the principal carbohydrate constituent of milk, is an important member of the disaccharide group. It occurs only in milk, except as it is found in the urine of women during pregnancy, during the nursing period, and soon after weaning; it also occurs in the urine of normal persons after the ingestion of a very large amount of lactose in the food. It is not derived directly from the blood, but is a specific product of the cellular activity of the mammary gland. It has strong reducing power, is dextro-rotatory and forms an osazone with phenylhydrazine. Lactose is not fermentable by pure yeast. For changes which lactose

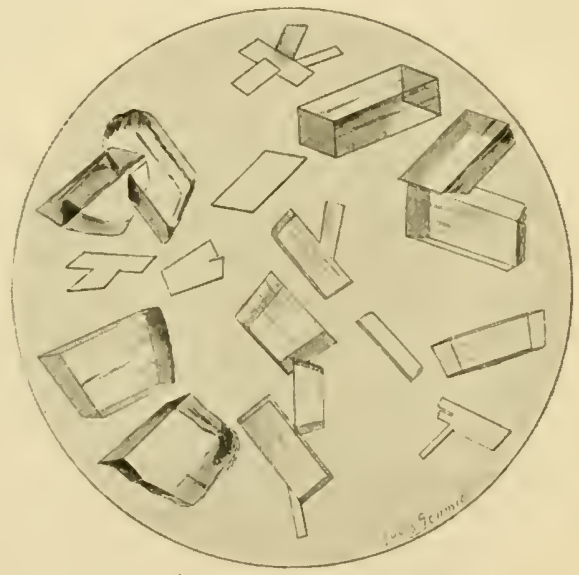

Fig. IO4.-LACTOSE.

undergoes in lactic acid fermentation see page 3I4. The crystalline form of lactose is shown in Fig. I04.

Casein, the principal protein constituent of milk, belongs to the group of phosphoproteins and contains 0.7 per cent of phosphorus. ${ }^{2}$ It has acidic properties and combines with bases to produce salts. ${ }^{3}$ It is probably present in milk in the form of neutral calcium caseinate (Casein $\left.\mathrm{Ca}_{4}\right)^{4}$ It is not coagulable upon boiling and is precipitated from its neutral solution by certain metallic salts as well as upon saturation with sodium chloride or magnesium sulphate. Its acid solution is precipitated by an excess of mineral acid.

Lactalbumin and lacto-globulin, the protein constituents of milk,

2. Basch: Mïnch. med. Woch., 58, 2266, I9I I.

${ }^{2}$ Bosworth and Van Slyke: Jour. Biol. Chem., I9, 67, I914.

${ }^{3}$ Van Slyke and Bosworth: Jour. Biol. Chem., I 4, 207-227, I9I4.

4 Van Slyke and Bosworth: Jour. Biol. Chem., 20, I35, I9I5. 
next in importance to casein, closely resemble serum albumin and serum globulin in their general properties.

Butter (milk fat) consists in large part of olein and palmilin. Stearin, butyrin, caproin and traces of other fats are also present. When butter becomes rancid through the cleavage of certain of its constituent fats by bacteria the odors of caproic and butyric acids are in evidence.

The pigment of the fat of cow's milk is made up of carotin and xanthophylls. The principal pigment is carotin, an unsaturated hydrocarbon pigment which is widely distributed in plants. ${ }^{1}$ The pigment of the fat of human milk is made up of carotin and xanthophylls in about equal proportions. Carotin is also probably the pigment of human fat. The pigment of body fat, blood serum, corpus luteum and skin secretions of the cow is principally carotin.

Colostrum is the name given to the product of the mammary gland secreted for a short time before parturition and during the early period of lactation (see Fig. I03, page 3I5). It is yellowish in color, contains more solid matter than ordinary milk, and has a higher specific gravity (1.040-1.080). The most striking difference between colostrum and ordinary milk is the high percentage of lactalbumin and lacto-globulin in the former. This abnormality in the protein content is responsible for the coagulation of colostrum upon boiling.

Such enzymes as lipase, amylase, galactase, catalase, oxidases, peroxidases, and reductases have been identified in milk, but not all of them in milk of the same species of animal.

Among the principal preservatives used in connection with milk are formaldehyde, hydrogen peroxide, boric acjd, borates, salicylic acid, and salicylates. The use of milk preservatives is illegal in most states.

\section{Experiments ON Milk}

I. Reaction.-Test the reaction of fresh cow's milk to litmus, phenolphthalein and Congo red.

2. Biuret Test.-Make the biuret test according to directions given on page 98.

3. Microscopical Examination.-Examine fresh whole milk, skimmed or centrifugated milk, and colostrum under the microscope. Compare the microscopical appearance with Fig. I03, page 3 I5.

4. Specific Gravity.-Determine the specific gravity of both whole and skimmed milk (see page 324). Which possesses the higher specific gravity? Explain why this is so.

5. Film Formation.-Place ro c.c. of milk in a small beaker and boil a few minutes. Note the formation of a film. Remove the film and heat again. Does

${ }^{1}$ Palmer and Eckles: Jour. Biol. Chcm., 17, 101, I914. 
the film now form? Of what substance is this film composed? The biuret test was positive; why do we not get a coagulation here when we heat to boiling?

6. Coagulation Test.-Place about 5 c.c. of milk in a test-tube, acidify slightly with dilute acetic acid and heat to boiling. Do you get any coagulation? Why?

7. Action of Hot Alkali. - To a little milk in a test-tube add a few drops of potassium hydroxide and heat. A yellow color develops and gradually deepens into a brown. To what is the formation of this color due? (See Moore's Test, Chapter II.)

8. Test for Chlorides. - To about 5 c.c. of milk in a test-tube add a few drops of very dilute nitric acid to form a precipitate. Filter off this precipitate and test the filtrate for chlorides. Does milk contain any chlorides?

9. Guaiac Test.-To about 5 c.c. of water in a test-tube add 3 drops of milk and enough alcoholic solution of guaiac (strength about $I: 60)^{1}$ to cause turbidity. Thoroughly mix the fluids by shaking and observe any change which may gradually take place in the color of the mixture. If no blue color appears in a short time, heat the tube gently below $60^{\circ} \mathrm{C}$. and observe whether the color reaction is hastened. In case a blue color does not appear in the course of a few minutes, add hydrogen peroxide or old turpentine, drop by drop, until the color is observed.

Fresh milk will frequently give this blue color when treated with an alcoholic solution of guaiac without the addition of hydrogen peroxide or old turpentine. Those milks which respond positively, fail to do so after boiling I $^{-20}$ seconds. What substances beside milk respond to this test? See discussion on page 258 .

I0. Differentiation of Human and Cow's Milk (Modification of Bauer's Test) ${ }^{2}$. -Introduce 2 c.c. of fresh human milk into a 50 c.c. test-tube and 2 c.c. of fresh cow's milk into another similar tube. Add to the contents of each tube I drop of a 0.25 per cent aqueous solution of nile-blue sulphate (Gruibler). Shake the tubes gently and permit them to stand undisturbed for 10-30 minutes. The milk assumes a bluish cast in each case. At the end of the 10-minute interval add Io c.c. of ether to the contents of each tube and shake very thoroughly for one minute. The ether extracts the pigment from the human milk, leaving the milk white. In the case of cow's milk the ether does not extract the dye and the milk remains bluish in color.

\section{I. Tests to Differentiate between Raw Milk and Heated Milk.-} (a) Trikresol Peroxidase Reaction (Kastle).-The peroxidase reaction of milk is founded upon the fact that small amounts of raw milk will induce the oxidation of various leuco compounds by hydrogen peroxide. This reaction has been used in a practical way as the most convenient means of differentiating between raw milk and heated milk. Many substances have been employed for this purpose, e.g., guaiac, paraphenylenediamine, ortol, amidol, etc. Kastle has found that a dilute

${ }^{1}$ Buckmaster advises the use of an alcoholic solution of guaiaconic acid instead of an alcoholic solution of guaiac resin. Guaiaconic acid is a constituent of guaiac resin.

${ }^{2}$ Bauer: Monatssch. f. Kinderheil., I I, 474, rgr2-13. 
solution of "trikresol" acts as a sensitizing agent in the peroxidase reaction and offers the following test which is based upon this fact.

Procedure.-To 2-5 c.c. of raw milk in a test-tube add 0.I-0.3 c.c. of $M /$ ro hydrogen peroxide and I c.c. of a I per cent solution of "trikresol." A slight though unmistakable yellow color will be observed to develop throughout the solution. Repeat the test using milk which has been boiled or heated to $80^{\circ} \mathrm{C}$. for ro-20 minutes and cooled, and note that no yellow color is produced.

The color reaction in the case of the raw milk probably results from the oxidation of the cresols by the hydrogen peroxide. The first product of this oxidation ${ }^{2}$ then oxidizes the leuco compound, when such is present, and causes the color observed.

(b) Benzidine Peroxidase Reaclion (Wilkinson and Pelers). ${ }^{3}$-To Io c.c. of the milk to be tested add 2 c.c. of a 4 per cent alcoholic solution of benzidine, sufficient acetic acid to coagulate the milk (usually $2-3$ drops) and finally 2 c.c. of a 3 per cent solution of hydrogen peroxide. Raw milk yields an immediate blue color. In adding the peroxide it is best to permit it to flow slowly down the wall of the vessel containing the mixture instead of allowing it to mix with the milk. Milk which has been heated to $78^{\circ} \mathrm{C}$. or above remains unchanged.

12. Saturation with Magnesium Sulphate.-Place about 5 c.c. of milk in a test-tube and saturate with solid magnesium sulphate. What is this precipitate?

I3. Influence of Gastric Rennin on Milk.-Prepare a series of five tubes as follows :

(a) 5 c.c. of fresh milk +0.2 per cent $\mathrm{HCl}$ (add drop by drop until a precipitate forms).

(b) 5 c.c. of fresh milk +5 drops of rennin solution. ${ }^{4}$

(c) 5 c.c. of fresh milk + Io drops of 0.5 per cent $\mathrm{Na}_{2} \mathrm{CO}_{3}$.

(d) 5 c.c. of fresh milk + Io drops of ammonium oxalate.

(e) 5 c.c. of fresh milk +5 drops of 02 per cent $\mathrm{HCl}$.

Now to each of the tubes (c), (d) and (e) add 5 drops of rennin solution. Place the whole series of five tubes at $40^{\circ} \mathrm{C}$. and after 10-1 5 minutes note what is occurring in the different tubes. Give a reason for each particular result.

14. Preparation of Casein.-Fill a large beaker one-third full of skimmed (or centrifugated) milk and dilute it with an equal volume of water. Add dilute hydrochloric acid until a flocculent precipitate forms. Stir after each acidification and do not add an excess of the acid as the precipitate would dissolve. Allow the precipitate to settle, decant the supernatant fluid, and reserve it for use in later (15-18) experiments. Filter off the precipitate of casein and remove the excess of moisture by pressing it between fllter papers. Transfer the casein to a small beaker, add enough 95 per cent alcohol to cover it and stir for a few moments. Filter, and press the precipitate between filter papers to remove the alcohol. Transfer the casein again to a small dry beaker, cover the precipitate with ether and heat on a water-bath for ten minutes, stirring con-

1 "Trikresol" is the trade name of an antiseptic which contains the three cresols in approximately equal proportions.

${ }_{2}^{2}$ Probably some organic peroxide or quinoid compound.

3 Wilkinson and Peters: Z. Nahr-Genussm., I6, No. 3, p. 172.

Any commercial rennin or rennet preparation or an extract of the gastric mucosa of the pig may be employed. 
tinuously. Filter (reserve the filtrate), and press the precipitate as dry as possible between filter papers. Open the papers and allow the ether to evaporate spontaneously. Grind the precipitate to a powder in a mortar. Upon the casein prepared in this way make the following tests:

(a) Solubility.-Try the solubility in water, sodium chloride, dilute acid and alkali.

(b) Millon's Reaction.-Make the test according to the directions given on page 97 .

(c) Biuret Test.-Make the test according to directions given on page 98 .

(d) Glyoxylic Acid Reaction (Hopkins-Cole).--Make the test according to the directions given on page 98 .

(e) Unoxidized Sulphur.- Test for unoxidized sulphur according to the directions given on page 108 . The sulphur content of casein is rather low, e.g., about 0.7 per cent.

(f) Fusion Test for Phosphorus.-Test for phosphorus by fusion according to directions given on page I29. Casein contains 0.7 per cent of phosphorus.

I5. Coagulable Proteins of Milk.-Place the filtrate from the original casein precipitate in a casserole and heat, on a wire gauze, over a free flame. As the solution concentrates, a coagulum consisting of lactalbumin and lactoglobulin will form. Continue to concentrate the solution until the volume is about onehalf that of the original solution. Filter off the coagulable proteins (reserve the filtrate) and test them as follows:

(a) Millon's Reaction.-Make the test according to the directions given on page 97.

(b) Biuret Test.-Make the test according to the directions given on page 98.

(c) Glyoxylic Acid Reaction (Hopkins-Cole).-Make the test according to the directions given on page 98 .

16. Detection of Calcium Phosphate.-Evaporate the filtrate from the coagulable proteins, on a water-bath, until crystals begin to form. It may be

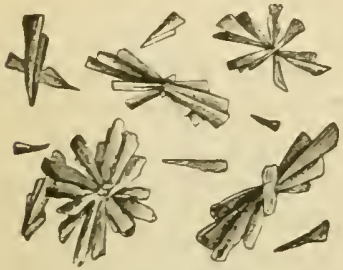

Fig. 105.-CALCIUM Phosph.tite. necessary to concentrate to I5 c.c. before any crystallization will be observed. Cool the solution, filter off the crystals (reserve the filtrate), and test them as follows:

(a) Microscopical Examination.-Examine the crystals and compare them with those in Fig. 105.

(b) Dissolve the crystals in nitric acid. Test part of the acid solution for phosphates. Render the remainder of the solution slightly alkaline with ammonia, then acidify with acetic acid and add ammonium oxalate. Examine the crystals under the microscope and compare them with those in Fig. I34, page 459.

I7. Detection of Lactose.-Concentrate the filtrate from the calcium phosphate until it is of a syrup-like consistency. Allow it to stand over night and observe the formation of crystals of lactose. Make the following experiments.

(a) Microscopical Examination.-Examine the crystals and compare them with those in Fig. 104, page 318.

(b) Fehling's Test. - Try Fehling's test upon the mother liquor.

(c) Phenylhydrazine Test.-Apply the phenylhydrazine test to some of the mother liquor according to the directions given on page 22 . 
I8. Milk Fat.-(a) Evaporate the ether filtrate from the casein (Experiment I3) and observe the fatty residue. The milk fat was carried down with the precipitate of casein and was removed when the latter was treated with ether. If centrifugated milk was used in the preparation of the caseinogen the amount of fat in the ether filtrate may be very small. To secure a larger yield of fat proceed according to directions given under (b) below.

(b) To 25 c.c. of whole milk in an evaporating dish add a little sand or filter paper and evaporate the fluid to dryness on a water-bath. Grind or break up the residue after cooling and extract with ether in a flask. Filter and remove the ether from the filtrate by evaporation. How can you identify fats in the ethereal residue?

I9. Saponification of Butter.-Dissolve a small amount of butter in alcohol made strongly alkaline with potassium hydroxide. Place the alcoholic-potash solution in a casserole, add about roo c.c. of water and boil for Io-I 5 minutes or until the odor of alcohol cannot be detected. Place the casserole in a hood and neutralize the solution with sulphuric acid. Note the odor of volatile fatty acids, particularly butyric acid. Under certain conditions the odor of ethyl butyrate may also be detected.

20. Detection of Preservatives.-(a) Formaldehyde.-In these tests two controls should be run, one with pure milk and one with milk to which a very small amount of formaldehyde has been added.

I. Leach's Hydrochloric Acid Test.-Mix Io c.c. of milk and Io c.c. of concentrated hydrochloric acid containing about 0.002 gram of ferric chloride in a small procelain evaporating dish or casserole and gradually raise the temperature of the mixture, on a water-bath, nearly to the boiling-point, with occasional stirring. If formaldehyde is present a violet color is produced, while a brown color develops in the absence of formaldehyde. In case of doubt the mixture, after having been heated nearly to the boiling-point for about one minute, should be diluted with 50-75 c.c. of water, and the color of the diluted fluid carefully noted, since the violet color if present will quickly disappear. Formaldehyde may be detected by this test when present in the proportion I :250,000.

II. Gallic Acid Test--Acidify 30 c.c. of milk with 2 c.c. of normal sulphuric acid and distil. Add $0.2-0.3$ c.c. of a saturated alcoholic solution of gallic acid to the first 5 c.c. of the distillate, then incline the test-tube and slowly introduce 3-5 c.c. of concentrated sulphuric acid, allowing it to run slowly domn the side of the tube. A green ring, which finally changes to blue, is formed at the juncture of the fluids. This is claimed, by Sherman, to be twice as delicate as either the sulphuric acid or the hydrochloric acid test for formaldehydc.

(b) Salicylic Acid and Salicylates.-Remont's Method. ${ }^{1}$ - Acidify 20 c.c. of milk with sulphuric acid, shake well to break up the curd, add 25 c.c. of ether, mix thoroughly, and allow the mixture to stand. By means of a pipette remove 5 c.c. of the ethereal extract, evaporate it to dryness, boil the residue with ro c.c. of fo per cent alcohol, and cool the alcoholic solution. Make the volume to c.c., filter through a $d r y$ paper if necessary to remove fat, and to 5 c.c. of the filtrate, which represents 2 c.c. of milk, add 2 c.c. of a 2 per cent solution of ferric chloride. The production of a purple or violet color indicates the presence of salicylic acid.

${ }^{1}$ For other tests see Sherman's Organic Analysis, Second Edition, p. 37 . 
This test may form the basis of a quantitative method by diluting the final solution to 50 c.c. and comparing this with standard solutions of salicylic acid. The colorimetric comparisons may be made in a Duboscq colorimeter.

(c) Hydrogen Peroxide.-Add 2-3 drops of a 2 per cent aqueous solution of para-phenylenediamine hydrochloride to IO-I 5 c.c. of milk. If hydrogen peroxide is present a blue color will be produced immediately upon shaking the mixture or after allowing it to stand for a few minutes. It is claimed that hydrogen peroxide may be detected by this test when present in the proportion $I: 40,000$.

(d) Boric Acid and Borates.-To the ash, obtained accord-

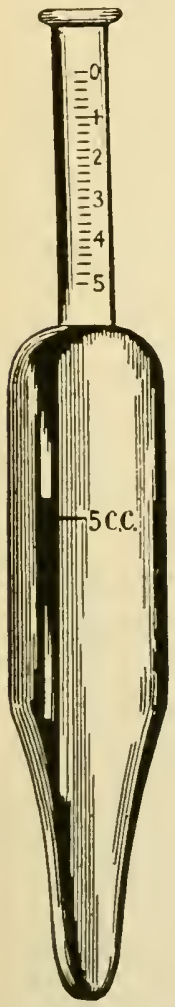

FIG. IO6.-BABCOCK TUBE. ing to the directions given in Experiment 4, page 327 , add 2 drops of dilute hydrochloric acid and I c.c. of water. Place a strip of turmeric paper in the dish and after allowing it to soak for about one minute remove it and allow it to dry in the air. The presence of boric acid is indicated by the production of a deep red color which changes to green or blue upon treatment with a dilute alkali. This test is supposed to show boric acid when present in the proportion I : 8000 .

\section{Quantitative Analysis of Milk}

r. Specific Gravity.-This may be determined conveniently by means of a Soxhlet, Veith, or Quevenne lactometer. A lactometer reading of $32^{\circ}$ denotes a specific gravity of $\mathrm{x.032}$. The determination should be made at about $60^{\circ} \mathrm{F}$. and the lactometer reading corrected by adding or subtracting $0 . \mathrm{I}^{\circ}$ for every degree F. above or below that temperature.

2. Fat.-(a) Babcock's Centrifugal Method. - Principle.-The principle of this method is the destruction of organic matter other than fat by sulphuric acid and the centrifugation of the acid solution in the special tube shown in Fig. Io6 and the subsequent reading of the percentage of fat by means of the tube's graduated neck. The method is one of the most satisfactory in common use and is accurate to within 0.5 per cent.

Procedure.-By means of a special narrow pipette introduce milk into the tube up to the 5 c.c. mark. Now add sufficient sulphuric acid (sp. gr. 1.83I.834) to fill the body of the tube and rotate the tube to secure a homogeneous acid-milk solution. Fill the neck of the tube with an acid-alcohol mixture. ${ }^{2}$ Centrifuge the tube and contents for one to two minutes and read off the percentage of fat by means of the graduated neck of the tube. If the top of the fat

${ }^{3}$ A modification of this method for use with sweetened dairy products, e.g., ice cream, and entailing the use of a different type of centrifuge tube has been proposed by Halverson (Jour. Ind. and Eng. Chem., 5, 403, I9I3). acid.

${ }_{2}$ This mixture consists of equal volumes of amyl alcohol and concentrated hydrochloric 
column is not at zero it may be brought there by the addition of water and a moment's recentrifugation.

In case very rich milk (over 5 per cent fat) is under examination, it may be diluted with an equal volume of water before examination and the fat percentage multiplied by 2. In the examination of cream it is customary to dilute the sample with four volumes of water and multiply the resultant fat value by 5 .

(b) Quantitative Determination of Fat in Milk by the Meigs ${ }^{1}$ Method with Modification and Improved Apparatus by Croll. ${ }^{2}$ - The method as stated by Dr. Meigs is: Approximately ro c.c. of milk is carefully weighed and transferred to an ordinary roo c.c. glass-stoppered graduated cylinder. Twenty c.c. each of distilled water and ether $(0.720)$ are added, the ground-glass stopper tightly inserted in the bottle, and the whole shaken vigorously for five minutes. Then the bottle is carefully unstoppered, 20 c.c. 95 per cent alcohol added, the stopper reinserted and again shaken for five minutes. The bottle is now placed on a table and the contents will separate into two distinct strata, the upper of which contains practically all the fat. This stratum is carefully removed by a small pipette and transferred to a carefully weighed glass evaporating dish. The thin ether layer remaining is washed by the addition of 5 c.c. of ether. This is removed by pipetting off. This washing is repeated four times. On each addition the sides of the bottle should carefully be washed down by the fresh ether. Finally, the pipette is rinsed with a little ether. The evaporating dish with contents is now placed on a safety water-bath and the ether evaporated. The drying is continued in a hot-air oven at a temperature below $100^{\circ} \mathrm{C}$. and finally completed in a desiccator to constant weight.

Croll's modification consists of subsequent repeated extraction of the end-product of evaporation with absolute ether. The com-

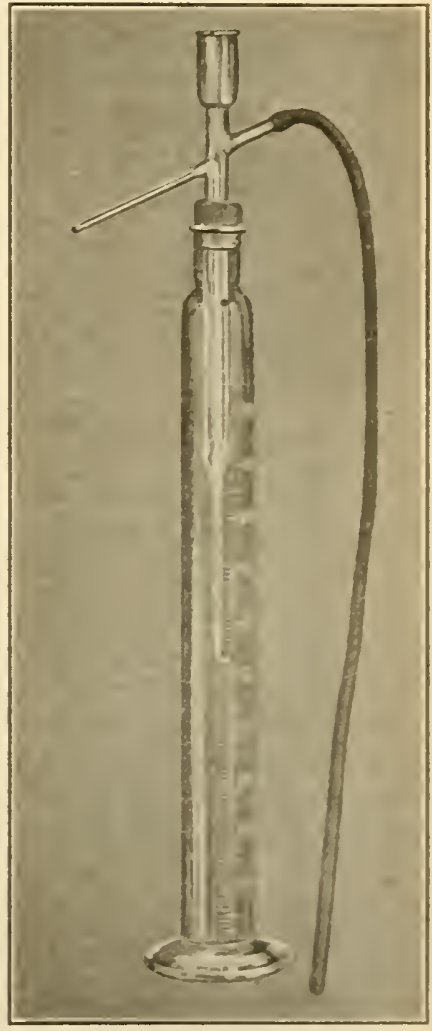

FIG. IO7.-CROLL'S FAT APPARATES. bined extracts are filtered and the small filter paper is washed repeatedly with absolute ether. The combined extracts and washings are evaporated and dried as before and then weighed.

The piece of apparatus shown in Fig. 107, above was also devised by Croll to do away with the use of the pipette. ${ }^{3}$ On closing the top with a finger and blowing into the mouthpiece, the upper stratum is forced out into the dish. The bottle is washed by simply pouring the ether into the tube. This lessens the possibility of accidental loss.

${ }_{1}$ Original paper by Dr. Arthur V. Meigs in Philadelphia Medical Times, July I, ISS2.

${ }^{2}$ Croll: Biochem. Bull., 2, 509, 1913.

${ }^{3}$ If desired a cork with two tubes may be substituted for this somewhat complicated apparatus. 
The accuracy of the method compared with that of the Soxhlet method, using the paper-coil modification and extracting until fresh portions of absolute ether gave no further trace of extractive material, is shown by the average

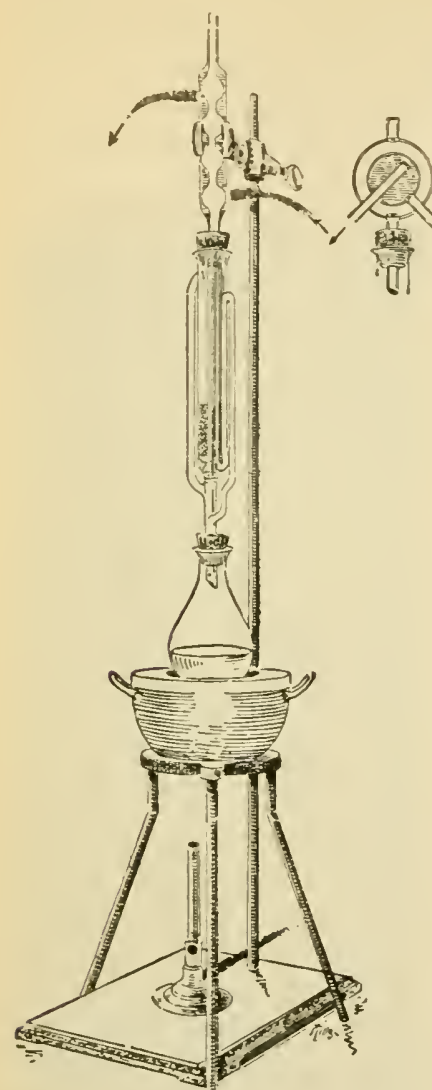
difference on twelve samples of human milk being only 0.017 per cent less than by the Soxhlet and on seven samples cow's milk being only 0.019 per cent less. The extreme differences in case of the human milk were-0.004 per cent and-0.044 per cent and in case of the cow's milk-0.006 per cent and -0.068 per cent.

(c) Adums' Paper-coil Mcthod.-Introduce about 5 c.c. of milk into a small beaker, quickly ascertain the weight to centigrams, stand a fatfree coil $^{1}$ in the beaker and incline the vessel and rotate the coil in order to hasten the absorption of the milk. Immediately upon the complete absorption of the milk remore the coil and again quickly ascertain the weight of the beaker. The difference in the weights of the beaker at the two weighings represents the quantity of milk absorbed by the coil. Dry the coil carefully at a temperature below $100^{\circ} \mathrm{C}$. and extract it with ether for 3-5 hours in a Soxhlet apparatus (Fig. 108). Using a safety water-bath, heat the flask containing the fat 10 constant weight at a temperature below $100^{\circ} \mathrm{C}$.

Calculation.--Divide $\mathrm{t}$ he weight of fat, in grams, by the weight of milk, in grams. The quotient is the percentuge

Fig. io8.-Soxhlet Apparates. of fat contained in the milk examined.

(d) Nephelometric Method of Bloor. ${ }^{2-}$-This method is exactly similar in principle and procedure to the method given for the determination of fat in blood. (See page 295.) One c.c. of milk is ordinarily taken.

(e) Approximate Determination by Feser's Lactoscope.-Milk is opaque mainly because of the suspended fat globules and

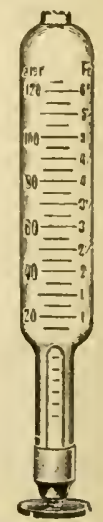
therefore by means of the estimation of this opacity we may obtain data as to the approximate content of fat. Feser's lactoscope (Fig. I09) may be used for this purpose. Proceed as follows: By means of the graduated pipette accompanying the instrument introduce 4 c.c. of milk into the lactoscope. Add water gradually, shaking after each addition, and note the point at which the black lines upon the inner white glass cylinder are distinctly visible. Observe the point on the graduated

1 Very satisfactory coils are manufactured by Schleicher and Schüll.

${ }^{2}$ Bloor: J. Am. Chem. Soc., 36, I300, 1914. 
scale of the lactoscope which is level with the suriace of the diluted milk. This reading represents the percentuge of fat present in the undiluted milk. Pure milis should contain at least 3 per cent of fat

3. Total Solids. ${ }^{1-}$-Introduce $2-5$ grams of milk into a weighed flat-bottomed platinum dish ${ }^{2}$ and quickly ascertain the weight to milligrams. Expel the major portion of the water by heating the open dish on a water-bath and continue the heating in an air-bath or water oven at $97^{\circ}-100^{\circ} \mathrm{C}$. until the weight is constant. (If platinum dishes are employed this residue may be used in the determination of ash according to the method described below.)

Calculation. ${ }^{3}$-Divide the weight of the residue, in grams, by the weight of milk used, in grams. The quotient is the percentage of solids contained in the milk examined.

4. Ash.-Heat the dry solids from 2-5 grams of milk, obtained according to the method just given, over a very low flame ${ }^{4}$ until a white or light gray ash is obtained. Cool the dish in a desiccator and weigh. (This ash may be used in testing for borates according to directions on page 324.)

5. Proteins: Nephelometric Determination of Proteins, Casein, Globulin, and Albumin in Kilk. Method of Kober. ${ }^{5}-$ Principle.-The proteins are precipitated with sulphosalicylic acid and the precipitate estimated nephelometrically (see discussion of nephelometric methods, page 290).

Procedure.-Five c.c. of milk are carefully measured into a 250 c.c. flask and after adding 200 c.c. of distilled water and ro c.c. of decinormal sodium hydroxide solution, water is added to the mark and the mixture shaken. Ten c.c. are put with exactly 2 c.c. of ether in a centrifuge tube which is then tightly stoppered with a cork and vigorously shaken. Allow to separate and withdraw 5 c.c. of the aqueous layer without contamination with ether. Dilute to 50 c.c. Take Io c.c. of this solution and add ro c.c. of 3 per cent sulphosalicylic acid. A suspension of casein is obtained which can be matched accurately with the following standard: I volume ( 5 c.c.) of a o.or per cent casein solution ${ }^{6}$ to which is added 2 volumes (ro c.c.) of 3 per cent sulphosalicylic acid.

The protein obtained with this reagent is not all casein, and in order to obtain the exact amount of casein the casein is precipitated according to the "official

${ }^{1}$ Shackell's method for the vacuum desiccation of frozen preparations may be used where great accuracy is desired (see American Journal of Physiology, 24, 325, I909).

${ }^{2}$ Lead foil dishes, costing only about one dollar per gross, make a very satisfactory substitute for the platinum dishes.

${ }^{3}$ The percentage of total solids may be calculated from the specific gravily and percenlage of fat by means of the following formula which has been proposed by Richmond:

$\mathrm{S}=$ total solids.

$$
\mathrm{S}=0.25 \mathrm{~L}+\mathrm{I} .2 \mathrm{~F}+0 . \mathrm{I}_{4}
$$

$\mathrm{L}=$ lactometer reading.

$\mathrm{F}=$ fat content.

4 Great care should be used in this ignition, the dish at no time being heated above a faint redness, as chlorides may volatilize.

${ }^{6}$ Kober: J. Am. Chem. Soc., 35, I585, I9r3

- Standard Cascin Solution.-Dissolve with stirring 0.I gram of casein or its equivalent in I c.c. of o.I $\mathrm{N} \mathrm{NaOH}$, add 95 c.c. of distilled water, add 2 c.c. of toluenc, shake thoroughly and make up to rooc.c. This is the stock solution which keeps three or four days or longer. The standard solution is made up fresh every day by making Io c.c. of the stock solution up to roo c.c. with water. The standard is controlled by total nitrogen estimations using the factor 6.38 for casein. 
method" or the method of Hart given below and the amount of precipitate obtained in an aliquot portion of the filtrate. by adding 4 volumes of the reagent, is determined nephelometrically. This fraction, for want of a better name called the "globulin and albumin fraction," is subtracted from the gross casein, to give the amount of casein precipitated by the "official method."

The ether used in extracting the fat increases the volume of the solution and hence a factor allowing for this must be used. For ro c.c. of diluted milk and 2 c.c. of ether the factor is 0.910 .

6. Proteins.-Introduce a known weight of milk (5-Io grams) into a 500 c.c. Kjeldahl digestion flask and add 20 c.c. of concentrated sulphuric acid and about 0.2 gram of copper sulphate. Expel the major portion of the water by heating over a low flame and finally use a full flame and allow the mixture to boil one to two hours. Complete the determination according to the directions given under Kjeldahl Method, page 483 .

Calculation.--Multiply the total nitrogen content by the factor $6.37^{1}$ to obtain the protein content of the milk examined.

7. Hart's Casein Method.2-Introduce I0.5 c.c. of milk into a 200 c.c. Erlenmeyer flask and add 75 C.C. of distilled water and I-I.5 C.C. of ro per cent acetic acid. $^{3}$ Mix the contents by giving the flask a vigorous rotary motion. The precipitated casein is now filtered off upon a 9-II cm. filter paper. ${ }^{4}$ Wash out the adsorbed and loosely combined acetic acid by means of cold water. Continue the washing of both the casein on the filter and that adhering to the flask, until the wash water has reached a volume of at least 250 c.c.

Now return the precipitate and paper to the original Erlenmeyer flask, add 75-80 c.c. of neutral (carbon dioxide-free) water, Io c.c. of $\mathrm{N} /$ Io potassium hydroxide and a few drops of phenolphthalein. Stopper the flask and shake it vigorously, by hand or machine, until the casein has been brought into solution. ${ }^{5}$ Rinse the stopper with neutral (carbon dioxide-free) water and titrate the alkaline casein solution at once with $\mathrm{N} /$ ro hydrochloric acid until there is a disappearance of all red color. ${ }^{6}$

Calculation.- Subtract the corrected ${ }^{6}$ acid reading from the ro c.c. of alkali used. The difference is the percentage of casein in the milk. For example, if it takes 6.7 c.c. of $\mathrm{N} /$ Io hydrochloric acid to titrate the alkaline solution to the end point and the check test was equivalent to 20 c.c. N/ Io acid the casein value would be obtained as follows:

$$
\text { ro }-(6.7+0.2)=3.1 \text { per cent casein }
$$

1 The usual factor employed for the calculation of protein from the nitrogen content is 6.25 and is based on the assumption that proteins contain on the average 16 per cent of nitrogen. This special factor of 6.37 is used to calculate the protein content from the total nitrogen, since the principal protein constituents of milk, i.e., casein and lactalbumin, contain about 15.7 per cent of nitrogen.

2 Hart: Jour. Biol. Chem., 6, 445, igog.

In general x.5 c.c. of acetic acid gives a clear solution which filters nicely but occasionally, when the milk has a low casein value it is advisable to use less acetic acid.

'The process of filtration may be retarded through the packing of the casein mass upon the filter paper. In this case conduct a fine stream of cold water against the upper point of contact of filter paper and casein. By this means the casein precipitate is loosened and gathers in the apex of the filter. This procedure is very essential. It is not necessary to remove the casein which adheres to the interior of the flask.

${ }^{5}$ Solution is indicated by the disappearance of the white casein particles which would otherwise settle to the bottom of the flask.

- A check test should be run parallel with the entire determination. Even with special precautions as to neutrality, it is generally found that an acid check of $0.2-0.3$ will be obtained. This check titration should be added to the volume of acid used in titration. 
8. Casein.-Mix about 20 grams of milk with 40 c.c. of a saturated solution of magnesuim sulphate and add the salt in substance until no more will dissolve. The precipitate consists of casein admixed with a little fat and lacto-globulin. Filter off the precipitate, wash it thoroughly with a saturated solution of magnesium sulphate, ${ }^{1}$ transfer the filter paper and precipitate to a Kjeldahl digestion flask, and determine the nitrogen content according to the directions given in a previous experiment (6).

Calculation.-Multiply the total nitrogen by the factor 6.37 to obtain the casein content.

9. Lactalbumin.-To the filtrate and washings from the determination of casein, in Experiment 8, add Almén's² reagent until no more precipitate forms. Filter off the precipitate and determine the nitrogen content according to the directions given under Proteins, page 328 .

Calculation.-Multiply the total nirrogen by the factor 6.37 to obtain the lactalbumin content.

10. Lactose.-To about 350 c.c. of water in a beaker add 20 grams of milk, mix thoroughly, acidify the fluid with about 2 c.c. of Io per cent acetic acid and stir the acidified mixture continuously until a flocculent precipitate forms. At this point the reaction should be distinctly acid to litmus. Heat the solution to boiling for one-half hour, filter, rinse the beaker thoroughly, and wash the precipitated proteins and the adherent fat with hot water. Combine the filtrate and wash water and concentrate the mixture to about i5o c.c. Cool the solution and dilute it to 200 c.c. in a volumetric flask. Titrate this sugar solution according to directions given under Fehling's Method, page 523 or Benedict's Method, page 522 .

Myers $^{3}$ recommends the following procedure for the determination of lactose in milk. One part of milk is mixed with an equal volume of phosphotungstic acid solution ( 70.0 grams acid and 200 c.c. conc. $\mathrm{HCl}$ in I liter of water) and 2-3 parts of water. Mix well, filter until clear, and titrate the clear filtrate against Benedict's solution ( 25 c.c. reducing $67 \mathrm{mg}$. of lactose.)

The milk may also be clarified for the lactose determination by means of aluminum hydroxide ${ }^{4}$ or dialyzed iron. ${ }^{5}$ The dialyzed iron procedure is as follows: Dilute $10 \mathrm{gm}$. of milk to 25 c.c. and add about 3 c.c. of ro per cent colloidal iron solution adding the last portion drop by drop to determine the exact amount necessary. Filter and wash with water to make the clear filtrate roo c.c. Titrate using Benedict's method (see page 522).

The preparation of aluminium hydroxide cream and its use in protein removal are described under Nitrogen Partition, p. 485, Chapter XXVI.

Calculation.-Make the calculation according to directions given under Fehling's Method, page 523, bearing in mind that ro c.c. of Fehling's solution is completely reduced by 0.0676 gram of lactose. ${ }^{6}$

${ }^{1}$ Preserve the filtrate and washings for the determination of lactalbumin (Expt. Q)

${ }^{2}$ Almén's reagent may be prepared by dissolving 5 grams of tannic acid in 240 C.c. of 50 per cent alcohol and adding Io c.c. of 25 per cent acetic acid.

${ }^{3}$ Myers: Mïnch. med. Woch., 59, 1494, 1912.

4 Welker and Marsh: J. Am. Chem. Soc., 35, \$23, 1913.

6 Hill: Jour. Biol. Chem., 20, 175, 1915.

- In case Benedict's method is used it should be remembered that 25 c.c. of the reagent is reduced by 0.067 gram of lactose. 


\section{EPITHELIAL AND CONNECTIVE TISSUES}

\section{EPITHELIAL TISSUE (KERATIN)}

THE albuminoid keratin constitutes the major portion of hair, horn, hoof, feathers, nails, and the epidermal layer of the skin. There is a group of keratins the members of which possess very similar properties. The keratins as a group are insoluble in the usual protein solvents and are not acted upon by the gastric or pancreatic juices. They all respond to the xanthoproteic and Millon reactions and are characterized by containing large amounts of sulphur. Keratin from any of its sources may be prepared in a pure form by treatment, in sequence, with artificial gastric juice, artificial pancreatic juice, boiling alcohol, and boiling ether, from twenty-four to forty-eight hours being devoted to each process.

The percentage composition of some typical keratins is given in the following table:

\begin{tabular}{|c|c|c|c|c|c|c|}
\hline & \multirow{2}{*}{ Source } & \multicolumn{5}{|c|}{ Percentage composition } \\
\hline & & $\mathrm{S}$ & $\mathrm{N}$ & C & $\mathrm{H}$ & 0 \\
\hline \multicolumn{2}{|c|}{ Nails $^{1}$} & 2.80 & $17 \cdot 51$ & 51.00 & 6.94 & 21.75 \\
\hline \multicolumn{2}{|c|}{ Horn ${ }^{2}$} & 3.20 & & 50.86 & 6.94 & \\
\hline \multirow{5}{*}{$\begin{array}{l}\stackrel{\mathscr{E}}{\Xi} \\
\stackrel{\Xi}{\Xi} \\
\stackrel{\Xi}{\Xi}\end{array}$} & Indian $\ldots \ldots \ldots$ & 4.82 & $15 \cdot 40$ & 44.06 & 6.53 & 29.19 \\
\hline & Japanese . . & $4 \cdot 96$ & 14.64 & 42.99 & $5 \cdot 9 \mathrm{I}$ & $3 I \cdot 50$ \\
\hline & Negro........... & 4.84 & 14.90 & $43 \cdot 85$ & 6.37 & 30.04 \\
\hline & Caucasian (adults).. & 5.22 & 15.79 & $44 \cdot 49$ & 6.44 & 28.66 \\
\hline & Caucasian (children) & 4.93 & $14 \cdot 5^{8}$ & 43.23 & 6.46 & 30.80 \\
\hline
\end{tabular}

The composition of human hair is influenced by its color and by the race, sex, age and purity of breeding of the individual. ${ }^{3}$ It may be dif-

${ }^{1}$ Mulder: Versuch einer allgem. physiol. Chem., Braunschweig, I844-5I.

2 Horbaczewski: Ladenburg's Handwörterbuch d. Chem., 3 .

${ }^{3}$ Rutherford and Hawk: Jour. Biol. Chem., 3, 459, 1907. 
ferentiated from all other animal hair or wool by its high content of cystine. Human hair may yield nearly i 2 per cent of this amino-acid. ${ }^{2}$

\section{Experinents on Epithelial Tissue}

\section{Keratin}

Horn shavings or nail parings may be used in the experiments which follow:

I. Solubility.-Test the solubility of keratin in water, dilute and concentrated acid and alkali.

2. Millon's Reaction.

3. Xanthoproteic Reaction.

5. Glyoxylic Acid Reaction (Hopkins-Cole).

6. Test for Unoxidized Sulphur.

\section{CONNECTIVE TISSUE}

\section{WHITE FIBROUS TISSUE}

The principal solid constituent of white fibrous connective tissue is the albuminoid collagen. This body is also found in smaller percentage in cartilage, bone, and ligament, but the collagen from the various sources is not identical in composition. In common with the keratins, collagen is insoluble in the usual protein solvents. It differs from keratin in containing less sulphur. One of the chief characteristics of collagen is, according to Hofmeister, the property of being hydrolyzed by boiling acid or water with the formation of gelatin. Emmett and Gies ${ }^{2}$ claim that under these conditions there is an intramolecular rearrangement of collagen and the resultant gelatin is consequently not the product of hydrolysis. The liberation of ammonia from the collagen during the process apparently confirms this view. Collagen gives Millon's reaction as well as the xanthoproteic and biuret tests.

The form of white fibrous tissue most satisfactory for general experiments is the tendo Achillis of the ox. According to Buerger and Gies $^{3}$ the fresh tissue has the following composition:

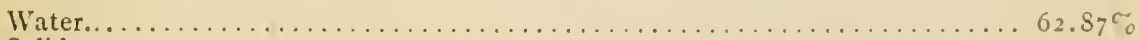

Solids................................... 37 . 3

Inorganic matter............................ 0.47

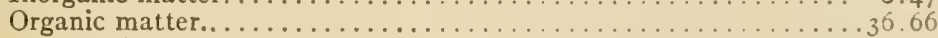

Fatty substance (ether-soluble)........... 1.04

Coagulable protein................... 0.22

Mucoid.............................. I. S

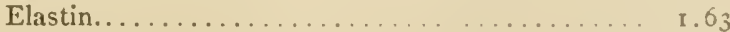

Collagen............................. 31.59

Extractives, etc................... 0.90

1 Buchtala: Zeil. physiol. Chem., 85, 246, 1913.

${ }^{2}$ Emmett and Gies: Jour. Biol. chem., 3, xxxiii (Procecdings), 1907.

${ }^{3}$ Buerger and Bies: Am. Jour. Physiol., 6, 219, 1901. 
The mucoid just mentioned is called tendomucoid ${ }^{1}$ and is a glycoprotein. It possesses properties similar to those of other connectivetissue mucoids, e.g., osseomucoid and chondromucoid.

Gelatin, the body which results from the hydrolysis of collagen (see statement of Emmett and Gies p. 331), is sometimes classed as an albuminoid (see Chapter V). It responds to nearly all the protein tests. It differs from the keratins and collagen in being easily digested and absorbed. Gelatin is not a satisfactory substitute for the protein constituents of a normal diet, however, since a certain portion of its nitrogen is not available for the uses of the organism. Gelatin from cartilage differs from gelatin from other sources in containing a lower percentage of nitrogen. Tyrosine and tryptophane are not numbered among the decomposition products of gelatin, hence it does not respond to Millon's reaction or the glyoxylic acid reaction. Cystine is also absent.

\section{Experiments on White Fibrous Tisste}

The tendo Achillis of the ox may be taken as a satisfactory type of the white fibrous connective tissue.

I. Preparation of Tendomucoid.-Dissect away the fascia from about the tendon and cut the clean tendon into small pieces. Wash the pieces in running water, subjecting them to pressure in order to remove as much as possible of the soluble protein and inorganic salts. This washing is very important. Transfer the washed pieces of tendon to a flask and add 300 c.c. of half-saturated lime water. ${ }^{2}$ Shake the flask at intervals of twenty-four hours. Filter off the pieces of tendon and precipitate the mucoid with dilute hydrochloric acid. Allow the mucoid precipitate to settle, decant the supernatant fluid and filter the remainder. Test the mucoid as follows:

(a) Solubility.-Try the solubility in water, sodium choride, dilute and concentrated acid and alkali.

(b) Biuret Test.-First dissolve the mucoid in potassium hydroxide solution and then add a dilute solution of copper sulphate.

(c) Test for Unoxidized Sulphur.

(d) Hydrolysis of Tendomucoid.-Place the remainder of the mucoid in a small beaker, add about 30 c.c. of water and 2 c.c. of dilute hydrochloric acid and boil until the solution becomes dark brown. Cool the solution, neutralize it with concentrated potassium hydroxide, and test by Fehling's test. With a reduction of Fehling's solution and a positive biuret test what do you conclude regarding the nature of tendomucoid?

- 2. Collagen.-This substance is present in the tendon to the extent of about 32 per cent. Therefore in making the following tests upon the pieces of tendon from which the mucoid, soluble protein, and inorganic salts were removed in the last experiment, we may consider the tests as being made upon collagen.

${ }^{1}$ Cutter and Gies: Am. Jour. Physiol., 6, r55, rgor.

2 Made by mixing equal volumes of salurated lime water and water from the faucet. 
(a) Solubility. - Cut the collagen into very fine pieces and try its solubility in water and dilute and concentrated acid and alkali.

(b) Millon's Reaction.

(c) Biuret Test.

(d) Xanthoproteic Reaction.

(e) Glyoxylic Acid Reaction (Hopkins-Cole).

(f) Test for Unoxidized Sulphur.-Take a large piece of collagen in a testtube and add about 5 c.c. of potassium hydroxide solution. Heat until the collagen is partly decomposed, then add $I-2$ drops of lead acetate and again heat to boiling.

(g) Formation of Gelatin from Collagen.-Transfer the remainder of the pieces of collagen to a casserole, fill the vessel about two-thirds full of water and boil for several hours, adding water at intervals as needed. By this means the collagen is transformed and a body known as gelatin is produced (see page 322).

3. Gelatin.-On the gelatin formed from the transformation of collagen in the above experiment $(\mathrm{g})$, or on gelatin furnished by the instructor make the following tests :

(a) Solubility.-Try the solubility in the ordinary solvents (see page 2I) and in hot water.

(b) Millon's Reaction.

(c) Glyoxylic Acid Reaction (Hopkins-Cole).-Conduct this test according to the modification given on page 107 .

(d) Test for Unoxidized Sulphur.

Make the following tests upon a solution of gelatin in hot water:

(a) Precipitation by Mineral Acids.-Is it precipitated by strong mineral acids such as concentrated hydrochloric acid?

(b) Salting-out Experiment.-Saturate a little of the solution with solid ammonium sulphate. Is the gelatin precipitated? Repeat the experiment with sodium chloride. What is the result?

(c) Precipitation by Metallic Salts. - Is it precipitated by metallic salts such as copper sulphate, mercuric chloride, and lead acetate?

(d) Coagulation Test.-Does it coagulate upon boiling?

(e) Precipitation by Alkaloidal Reagents.-Is it precipitated by such reagents as picric acid, tannic acid, and trichloracetic acid?

(f) Biuret Test.-Does it respond to the biuret test?

(g) Bardach's Reaction.-Does it yield the typical crystals of this reaction? (See page Ior.)

(h) Precipitation by Alcohol.-Fill a test-tube one-half full of 95 per cent alcohol and pour in a small amount of concentrated gelatin solution. Do you get a precipitate? How would you prepare pure gelatin from the tendo Achillis of the ox?

\section{YELLOW ELASTIC TISSUE (ELASTIN)}

The ligamentum muche of the ox may be taken as a satisfactory type of the yellow elastic connective tissue. The principal solid constituent of this tissue is elastin, a member of the albuminoid group. 
In common with the keratins and collagen, elastin is an insoluble body and gives the protein color reactions. It differs from keratin principally in the fact that it may be digested by enzymes and that it contains a very small amount of sulphur.

It has been demonstrated that elastin has the property of adsorbing pepsin from the gastric juice and thus protecting it so the enzyme can function later in the intestine ${ }^{1}$ (see Chapter on Gastric Digestion).

Yellow elastic tissue also contains mucoid and collagen but these are present in much smaller amount than in white fibrous tissue, as may be seen from the following percentage composition of the fresh ligamentum muche of the ox as determined by Vandegrift and Gies. ${ }^{2}$

Water.

Solids. .

Inorganic matter

Organic matter.

Fatty substance (ether-soluble)

Coagulable protein.

Mucoid..

Elastin. . . .

Collagen

Extractives, etc

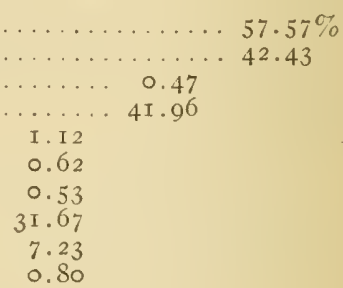

Experiments on Elastin

I. Preparation of Elastin (Richards and Gies). ${ }^{3}$ - Cut the ligament into fine strips, run it through a meat chopper and wash the finely divided material in cold, running water for $24-48$ hours. Add an excess of half-saturated lime water, (see note at the bottom of page 332) and allow the hashed ligament to extract for 48-72 hours. Decant the lime water, remove all traces of alkali by washing in water and then boil in water with repeated renewals until only traces of protein material can be detected in the wash water. Decant the fluid and boil the ligament in ro per cent acetic acid for a few hours. Treat the pieces with 5 per cent hydrochloric acid at room temperature for a similar period, extract again in hot acetic acid and in cold hydrochloric acid. Wash out traces of acid by means of water and then thoroughly dehydrate by boiling alcohol and boiling ether in turn. Dry in an air-bath and grind to a powder in a mortar.

2. Solubility.- Try the solubility of the finely divided elastin, prepared by yourself or furnished by the instructor, in the ordinary solvents (see page 2I). How does its solubility compare with that of collagen?

3. Millon's Reaction.

4. Xanthoproteic Reaction.

5. Biuret Test.

6. Glyoxylic Acid Reaction (Hopkins-Cole).-Conduct this test according to the modification given on page 107 .

7. Test for Unoxidized Sulphur.

${ }^{1}$ Abderhalden and Meyer: Zeit. physiol. Chem., 74, 67, I9II.

2 Vandegrift and Gies: Am. Jour. Physiol., 5, 287, I $90 \mathrm{I}$.

${ }^{3}$ Richards and Gies: Am. Jour. Physiol., 7, 93, 1902. 


\section{CARTILAGE}

The principal solid constituents of the matrix of cartilaginous tissue are chondromucoid, chondroitin-sulphuric acid, chondroalbumoid and collagen. Chondromucoid differs from the mucoids isolated from other connective tissues in the large amount of chondroitin-sulphuric acid obtained upon decomposition. Besides being an important constituent of all forms of cartilage, chondroitin-sulphuric acid has been found in bone, ligament, the mucosa of the pig's stomach, the kidney of the ox, the inner coats of large arteries and in human urine. It may be decomposed through the action of acid and yields a nitrogenous body known as chondroitin and later this body yields chondrosin. Chondrosin is also a nitrogenous body and has the power of reducing Fehling's solution more strongly than dextrose. Levene and La Forge ${ }^{1}$ claim the reducing action of chondrosin to be due to an hexosamine isomeric with glucosamine. Sulphuric acid is a by-product in the formation of chondroitin, and acetic acid is a by-product in the formation of chondrosin.

Chondroalbumoid is similar in some respects to elastin and keratin. It differs from keratin in being soluble in gastric juice and in containing considerably less sulphur than any member of the keratin group. It gives the usual protein color reactions.

\section{Experiments on CARTilage}

I. Preparation of the Cartilage.-Boil the trachea of an ox in water until the cartilage rings may be completely freed from the surrounding tissue. Use the cartilage so obtained in the following experiments:

2. Solubility.-Cut one of the rings into very small pieces and try the solubility of the cartilage in water and dilute and concentrated acid and alkali.

3. Millon's Reaction.

4. Xanthoproteic Reaction.

5. Glyoxylic Acid Reaction (Hopkins-Cole).-Conduct this test according to the modification given on page Io7.

6. Test for Unoxidized Sulphur.

7. Preparation of Cartilage Gelatin.-Cut the remaining cartilage rings into small pieces, place them in a casserole with water and boil for several hours. Filter while the solution is still hot. Observe that the filtrate soon becomes more or less solid. What is the reason for this? Bring a portion of the material into solution by heat and try the following tests:

(a) Biuret Test.

(b) Bardach's Reaction.

(c) Test for Unoxidized Sulphur.

(d) To about 5 c.c. of the solution in a test-tube add a few drops of barium chloride. Do you get a precipitate, and if so to what is the precipitate due?

${ }^{1}$ Levene and La Forge: Proc. Soc. cp. Biol. and . Med., It, 124 , Igr 4 . 
(e) To about 5 c.c. of the solution in a test-tube add a few drops of dilute hydrochloric acid and boil for a few moments. Now add a little barium chloride to this solution. Is the precipitate any larger than that obtained in the preceding expeiment? Why?

(f) To the remainder of the solution add a little dilute hydrochloric acid and boil for a few moments. Cool the solution, neutralize with solid potassium hydroxide, and try Fehling's test. Explain the result.

\section{OSSEOUS TISSUE}

Of the solids of bone about equal parts are organic and inorganic matter. The organic portion, called ossein, may be obtained by removing the inorganic salts through the medium of dilute acid. Ossein is practically the same body which is termed collagen in the other connective tissues, and in common with collagen yields gelatin upon being boiled with dilute mineral acid.

In common with the other connective tissues bone contains a mucoid and an albuminoid. Because of their origin these bodies are called osseomucoid and osseoalbumoid. Osseomucoid, when boiled with hydrochloric acid, yields sulphuric acid and a substance capable of reducing Fehling's solution. The composition of osseomucoid is very similar to that of tendomucoid and chondromucoid (see page II 3 ).

The inorganic basis of the dry, fat-free bone is a chemical substance, not a mixture. This fact is indicated by the uniform composition of the bones of fasting animals as well as by the definite relationship existing between the elements present. Bones of normal and fasting animals of the same species present no profound differences in percentage composition. The percentage composition of the dry, fat-free femurs of two $\operatorname{dogs}^{1}$ after the animals had fasted for IO4 and I4 days respectively was as follows:

\begin{tabular}{|c|c|c|c|c|c|c|}
\hline Dog No. & Length of fast & Ash & $\mathrm{N}$ & $\mathrm{CaO}$ & $\mathrm{MgO}$ & $\mathrm{P}_{2} \mathrm{O}_{5}$ \\
\hline I. & IO4 days & $6 \mathrm{I} .50$ & 4.6 & 33.3 & 0.8 & 12.80 \\
\hline 2. & I4 days & $6 \mathbf{I} .65$ & $4 . \mathbf{I}$ & $33 . \mathrm{I}$ & 0.9 & $\mathbf{I} 2.90$ \\
\hline
\end{tabular}

The marked uniformity in composition notwithstanding the wide variation in the fasting periods is significant. The tensile strength of the femur of the dog has been found to be at least 25,000 pounds to the square inch ${ }^{1}$ whereas that of oak is 10,000 and that of cast iron 20,000 pounds to the square inch.

${ }^{1}$ Johnston and Hawk: Unpublished data. For data on a 117 -day fast by dog No. I, see Howe, Mattill and Hawk: Jour. Biol. Chem., II, I03, I9I2. 


\section{EXPERIMENT on Osseous Tissue}

The percentage composition of normal human bone and of bone from a case of osteomalacia is given in the following table: ${ }^{1}$

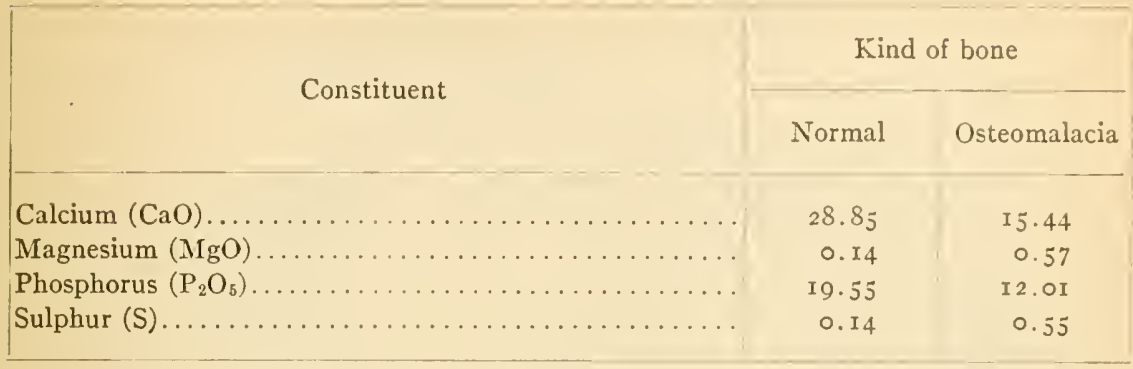

Qualitative Analysis of Bone Ash.-Take I gram of bone ash in a small beaker and add a little dilute nitric acid. What does the effervescence indicate? Stir thoroughly and when the major portion of the ash is dissolved add an equal volume of water and filter. To the acid filtrate add ammonium hydroxide to alkaline reaction. A heavy white precipitate of phosphates results. (What phosphates are precipitated here by the ammonia?) Filter and test the filtrate for chlorides, sulphates, phosphates, and calcium. Add dilute acetic acid to the precipitate on the paper and test a little of this filtrate for calcium and phosphates. Heat the remainder of the filtrate to boiling and add $\left(\mathrm{NH}_{4}\right)_{2} \mathrm{CO}_{3}$ and $\mathrm{NH}_{4} \mathrm{Cl}$ slowly to this hot solution as long as a precipitate forms. Filter off the precipitate of $\mathrm{CaCO}_{3}$ and wash with hot water until free from alkali. ${ }^{2}$ Add a solution of $\mathrm{Na}_{2} \mathrm{HPO}_{4}$, make strongly alkaline with $\mathrm{NH}_{4} \mathrm{OH}$, and note the formation of a white precipitate of ammonium magnesium phosphate $\left(\mathrm{NH}_{4} \mathrm{MgPO}_{4}\right)$ Examine the crystals under the microscope and compare with those shown in Fig. I29, page 408. To the precipitate on the filter paper, which was insoluble in acetic acid add a little dilute hydrochloric acid and test this last filtrate for phosphates and iron.

Reference to the following scheme may facilitate the analysis.

${ }^{1}$ McCrudden: Jour. Biol. Chem., 7, I99, I9ro.

${ }^{2}$ Magnesium is not precipitated here because of presence of $\mathrm{NH}_{4} \mathrm{Cl}$. 


\section{BONE ASH.}

Add dilute nitric acid, stir thoroughly and after the major portion of the ash has been brought into solution add a little distilled water and filter.

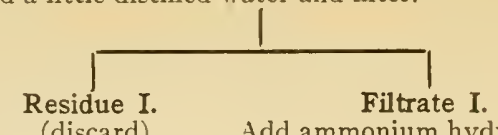

(discard)
Add ammonium hydroxide to alkaline reaction and filter.
Residue III.

Treat on paper with hydrochloric acid.

Filtrate IV.

\section{Test for:}

I. Iron.

2. Phosphates.

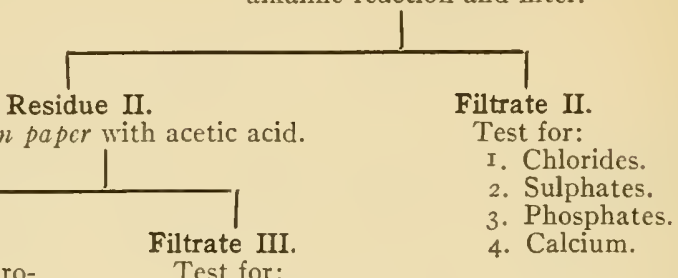

\section{Treat on paper with acetic acid.}

Filtrate III.

Test for:

I. Phosphates.

2. Calcium.

3. Magnesium
Filtrate II.

2. Sulphates,

4. Calcium.

\section{ADIPOSE TISSUE}

Adipose tissue consists almost entirely of a mixture of fats. For discussion and experiments see chapter on Fats, page 176 . 


\section{CHAPTER XIX \\ MUSCULAR TISSUE}

THE muscular tissues are divided physiologically into the voluntary (striated) and the involuntary (non-striated or smooth). In the chemical examination of muscular tissue the voluntary form is generally employed. Muscle contains about 25 per cent of solid matter, of which about four-fifths is protein material and the remaining onefifth extractives and inorganic salts.

The proteins are the most important of the constituents of muscular tissue. In the living muscle we find two proteins, myosinogen and para-myosinogen. These may be shown to be present in muscle plasma expressed from fresh muscles. In common with the plasma of the blood this muscle plasma has the power of coagulating, and the clot formed in this process is called myosin. According to Halliburton ${ }^{1}$ and others in the onset of rigor mortis we have an indication of the formation of this myosin clot within the body. The relation between the proteins of living and dead muscle is represented graphically by Halliburton as follows:

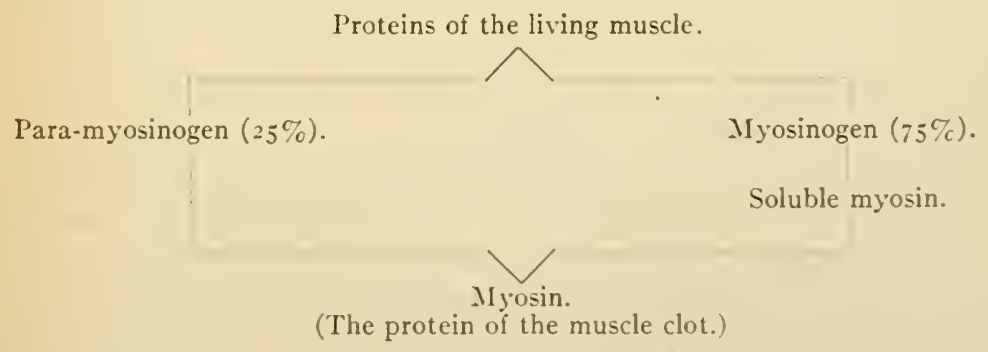

Of the total protein content of living muscle about 75 per cent is made up by the myosinogen and the remaining 25 per cent is paramyosinogen. These proteins may be separated by subjecting the muscle plasma to fractional coagulation in the usual way. Under these conditions the para-myosinogen is found to coagulate at $47^{\circ} \mathrm{C}$. and the myosinogen to coagulate at $56^{\circ} \mathrm{C}$. It is also claimed by some investigators that it is possible to separate these two proteins by the fractional ammonium sulphate method, but the possibility of making

${ }^{1}$ Halliburton: Biochemistry of Muscle and Nerve. 1904. P. 4. 
an accurate separation by this method is somewhat doubtful. It is well established that para-myosinogen is a globulin since it responds to certain of the protein precipitation tests and is insoluble in water. Myosinogen, on the contrary, is not a typical globulin since it is soluble in water. It has been called a pseudo-globulin. Myosin possesses the globulin characteristics. It is insoluble in water but soluble in the other protein solvents and is precipitated from its solution upon saturation with sodium chloride.

Mellanby has reported observations which he claims indicate that there is only one protein in muscle and that rigor mortis is due to the coagulation of this protein under the combined influences of the salt present in the muscle and the lactic acid developed upon the death of the muscle. He further states that the disappearance of rigor is due to the fact that the lactic acid which is continually formed brings this protein into solution. There is a difference of opinion as to whether true rigor ever occurs in connection with non-striated (smooth) ${ }^{1}$ muscle.

Our ideas concerning the cause of rigor have undergone an important revision in recent years. A very attractive theory has been advanced by $\mathrm{Meigs}^{2}$ and experimental confirmation has been accorded it by von Fürth and Lenk. ${ }^{3}$ According to this theory, rigor has no connection with the coagulation of the muscle proteins and may even be hindered or prevented by such coagulation. The cause of rigor, from this new viewpoint, lies in the imbibition of water by the muscle colloids. It is well known that colloids possess the property of absorbing whatever fluid may be in contact with them. Moreover, the capacity of the colloid for water is increased if the fluid is slightly acid in reaction. Therefore the postmortem production of lactic acid facilitates the imbibition of muscle fluid by the muscle colloids. Under such conditions, the fibers swell, become rigid and the condition known as rigor mortis results. The disappearance of rigor is believed to be due to the coagulation of the muscle protein through the agency of the accumulated lactic acid. This change is accompanied by a release of the imbibed water by the colloids, inasmuch as the capacity of a colloid for retaining fluid is lowered by coagulation.

Under the name extractives we class a number of muscle constituents which occur in traces in the tissue and may be extracted by water, alcohol, or ether. There are two classes of these extractives, the nonnitrogenous extractives and the nitrogenous extractives. Grouped under

I Saxl: Beiträge zur chemischen Physiologie und Pathologie, 9, I, 1907.

2 Meigs: American Journal of Physiology, 26, I9I, I9 10.

${ }^{3}$ von Fürth and Lenk: Wiener klinische Wochenschrift, 24, 1079, I9II. 
the non-nitrogenous bodies we have glycogen, dextrin, sugars, lactic acid, inosite, $\mathrm{C}_{6} \mathrm{H}_{6}(\mathrm{OH})_{6}$, and fat. In the class of nitrogenous extractives we have creatine, creatinine, xanthine, hypoxanthine, uric acid, urea, carnine, guanine, phosphocarnic acid, inosinic acid, carnosine, taurine, carnitine, novaine, ignotine, neosine, oblitine, carnomuscarine, and methylguanidine (sce formulas on pp. I 27 and 346). Not all of these extractives are present in the muscles of all species of animals. Other extractives besides those enumerated above have been described and there are undoubtedly still others whose presence remains undetermined. A detailed consideration would, however, be unprofitable in this place.

Glycogen is an important constituent of muscle. The content of this polysaccharide in muscle varies and is markedly decreased by intense muscular activity. It is transformed into sugar and used as fuel. The liver is the organ which stores the reserve supply of glycogen and transforms it into glucose which is passed into the blood stream and so carried to the working muscle where it is synthesized into glycogen. The glycogen thus formed is then changed into glucose as the working muscle may need it.

Glycogen is a polysaccharide and has the same percentage composition as starch and dextrin. It resembles starch in forming an opalescent solution and resembles dextrin in being very soluble, in giving reddish color with iodine and in being dextro-rotatory. Glycogen may be prepared from muscle by extracting with boiling water and then precipitating the glycogen from the aqueous solution by alcohol; dilute or concentrated potassium hydroxide may also be used to extract the glycogen. Glycogen may be prepared in the form of a white, tasteless, amorphous powder. It is completely precipitated from its solution by saturation with solid ammonium sulphate, but is not precipitated by saturation with sodium chloride. It may also be precipitated by alcohol, tannic acid, or ammoniacal basic lead acetate. It has the power of holding cupric hydroxide in solution in alkaline fluids but cannot reduce it. It may be hydrolyzed with the formation of glucose by dilute mineral acids and is readily digested by amylolytic enzymes.

Mendel and Leavenworth have drawn the conclusion, from the examination of embryo pigs, that embryonic structures do not contain exceptionally large amounts of glycogen. The distribution of the glycogen was not observed to differ from that in the adult animal except that the liver of the embryo does not assume its glycogen-storing function early. They further draw the conclusion that the metabolic transformations of glycogen in the embryo and the adult are entirely analogous. 
The lactic acid occurring in the muscular tissue of vertebrates is paralactic or sarcolactic acid, ${ }^{1}$

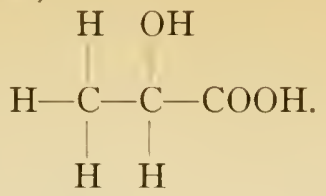

The reaction of an inactive living muscle is alkaline, but upon the death of the muscle, or after the continued activity of a living muscle, the reaction becomes acid, due to the formation of lactic acid. There is a difference of opinion regarding the origin of this lactic acid. Some investigators claim it to arise from the carbohydrates of the muscle, while others ascribe to it a protein origin. The strongest evidence favors a carbohydrate source. ${ }^{2}$

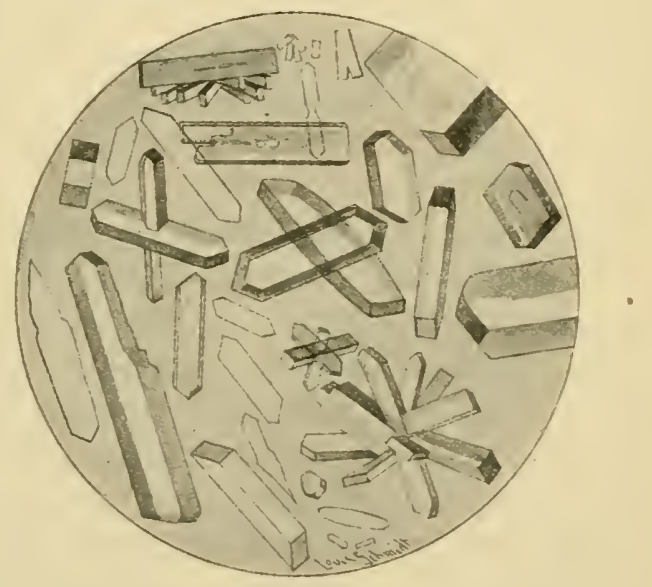

Fig. i io.-Creatine.

Among the nitrogenous extractives of muscle, those which are of the most interest in this connection are creatine and the purine bases, xanthine and hypoxanthine. Creatine is found in varying amounts in the muscles of different species, the muscles of birds having shown the largest amount. It has also been found in the blood, the brain, in transudates and in the thyroid gland. Creatine may be crystallized and forms colorless rhombic prisms (Fig. Iro) which are soluble in warm water and practically insoluble in alcohol and ether. Upon boiling a solution of creatine with dilute hydrochloric acid it is dehydrolyzed and its anhydride creatinine is formed. The theory that the

\footnotetext{
${ }^{1}$ This is dextro-rolatory, whereas fermentation lactic acid (d-l-lactic acid) is optically inactive.

${ }^{2}$ Levene and Meyer: Jour. Biol. Chem., I I, 361, I912.
} 
creatine of ingested meat is transformed into creatinine and excreted in the urine has been proven untenable through the researches of Folin, Klercker, and Wolf and Shaffer. It is now known that under normal conditions the ingestion of creatine in no way influences the excretion of creatinine. In the case of Eck fistula dogs, however, London and Bolyarski ${ }^{1}$ found ingested creatine to increase the output of creatinine in the urine. This finding is of importance as throwing light upon the rôle of the liver in creatine and creatinine metabolism. In this connection it is important to note that there is no normal excretion of endogenous (see page $37 \mathrm{~S}$ ) creatine, a statement proven by the fact that if no creatine be ingested none will be excreted. Folin ${ }^{2}$ has shown that the main bulk of ingested creatine is retained in the body, unless the diet contains a large amount of protein material. In fasting the urine contains considerable creatine, i.e., I $20 \mathrm{mg}$. or more per day. Under certain pathological conditions, e.g., fevers, the urine may contain endogenous creatine which is probably derived from the catabolism of muscular tissue, as Benedict, Mellanby, and Shaffer have suggested. Benedict and Osterberg ${ }^{3}$ believe we may have a high creatine elimination which has no relation to the catabolism of muscle.

McCrudden ${ }^{4}$ reports creatine in the urine in cases of infantilism achondroplasia and cretinism the amount present being increased when the carbohydrate ingestion was increased.

It has been stated that creatine does not occur in non-striated muscle. It has, however, been found in the non-striated muscles of the lamprey the lowest form of vertebrates. ${ }^{5}$

Amberg and Morrill, ${ }^{6}$ Sedgwick: ${ }^{7}$ Rose ${ }^{8}$ and Folin ${ }^{9}$ have shown that creatine is a normal constituent of the urine of infants and children (Io-I $5 \mathrm{mg}$. per day). Folin explains this phenomenon on the basis of the relatively high protein intake, whereas Rose believes it is due to a peculiar carbohydrate metabolism.

Besides being a normal constituent of muscle, xanthine has been found in the brain, spleen, pancreas, thymus, kidneys, testicles, liver, and in the urine. It may be obtained in crystalline form (Fig. II , p. 344), but ordinarily it is amorphous. Xanthine is easily soluble in alkalis, less soluble in water and dilute acids, and entirely insoluble in alcohol and ether.

${ }^{1}$ London and Bolyarskii: Zcil. phys. chem., 62, 465, 1909 .

${ }^{2}$ Folin: Hammarsten Festschrift, p. I 5.

${ }^{3}$ Benedict and Osterberg: Jour. Biol. Chem., IS, 195, I9r4.

4 Mc Crudden: Jour. Expt. Med., I5, 457, I9I 2.

5 Mellanby: Jour. of Physiol., 36,472 , Ioos. Wilson: Jour. Biol. Chem., IS, I7, Igit.

- Amberg and Morrill: Jour. Biol. Chem., 3. 3 I 1, 1907.

${ }^{7}$ Sedgwick: Jour. Am..Med. Ass'n, 55, i i 7 , , so Io.

8 Rose: Jour. Biol. Chem., I0, 265, 19I I.

? Folin: Ibid., II, 253 , I912. 
Hypoxanthine occurs ordinarily in those tissues and fluids which contain xanthine. It has been found, unaccompanied by xanthine, in bone marrow and in milk. Unlike xanthine it may be easily crystallized in the form of small, colorless needles. It is readily soluble in alkalis, acids, and boiling water, less soluble in cold water and practically insoluble in alcohol and ether.

The predominating inorganic salt of muscle is potassium phosphate. Besides this salt we have present chlorides and salts of sodium, calcium, magnesium, and iron. Sulphates are also present in traces.

Mendel and Saiki have made some interesting observations upon the chemical composition of non-striated or smooth (involuntary) mammalian

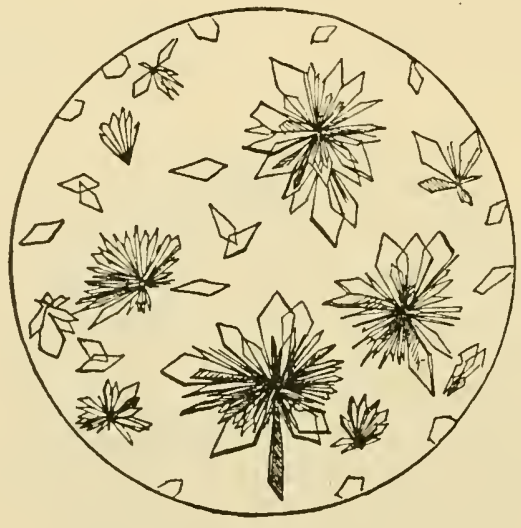

Fig.iri.-Xanthine.

A ter the drawings of Horbaczewski, as represented in Neubauer and Vogel. (Ogden.)

muscle, such as the urinary bladder and the muscular coat of the stomach of the pig. Hypoxanthine was found to be the predominant purine base present. Creatine and paralactic acid were also isolated. These investigators were unable to demonstrate, definitely, the presence of glycogen in the non-striated muscles studied, but state that "the tissues possess the property of transforming glycogen in the characteristic enzymatic way." The most important part of their investigation consists in a rather complete analysis of the inorganic constituents of these muscles. A notable difference in the relative distribution of the various inorganic constituents was observed, a difference which, according to the authors, "can be accounted for in part only by an admixture of lymph." The comparative composition of the inorganic portion of striated and non-striated muscle and of blood serum for comparison is shown in the following table: 
Per Ioo parts of fresh muscle

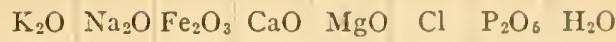

Non-striated muscle (Mendel and Saiki) 0.08 I 0.3280 .0 II 0.0440 .0070 .1710 .18480 .6 Skeletal muscle (Katz)..............306 $0.2100 .0080 .0110 .0470 .0480 .487 \quad 72.9$ Biood serum (Abderhalden)......... $0.0270 .425 \ldots .0 .0120 .0040 .363^{\circ} 0.020$ 9I. 8

An interesting comparative study of the ash of the smooth muscle of the stomach of the frog and the striated muscle from the same animal was very recently reported by Meigs and Ryan. ${ }^{1}$ Their data indicate "that smooth muscle contains somewhat less potassium and phosphorus and somewhat more sodium and chlorine than the striated muscle of the same animal, but that the differences in these respects between the two tissues are not by any means so marked as has sometimes been supposed." Their average figures for each type of muscle follow :

Per roo parts of fresh muscle

Muscle

\begin{tabular}{l|l|l|l|l|l|llll}
$\mathbf{K}$ & $\mathrm{Na}$ & $\mathrm{Fe}$ & $\mathrm{Ca}$ & $\mathrm{Mg}$ & $\mathrm{P}$ & $\mathrm{Cl}$ & $\mathrm{S}$ & Solids & $\mathrm{H}_{2} \mathrm{O}$
\end{tabular}

Striated............ $0.3500 .0540 .010 \quad 0.0280 .0300 .1550 .0660$. I 4 I 20.1379 .87 Smooth.............. 0.3250.0730.00070.0040.0130.1370.1200.161 I7.70 82.30

The preparation from which the above data for smooth muscle were obtained were shown by histological examination to consist in large part of smooth muscle fibers.

Muscular tissue is said to contain a reddish pigment called myohematin, which is a derivative of hemoglobin.

The so-called "fatigue substances" of muscle are carbon dioxide, paralactic acid, and potassium dihydrogen phosphate.

The ordinary commercial "meat extract" is composed principally" of the water-soluble constituents of muscle and contains practically nothing of nutritive value. The protein material to which meat owes its value as an article of diet is ordinarily practically all removed in the preparation of the extract. Occasionally some preparations are found to contain proteose, which is formed from the meat proteins in the process of preparation.

Lusk $^{2}$ has shown that Liebig's extract is without influence upon the

${ }^{1}$ Meigs and Ryan: Journal of Biological Chemistry, Ir, 40I, I9I 2.

2Lusk: Jour. Biol. Chem., I3, I55, I912. 
metabolism (energy) in spite of the glandular activity it is known to induce.

The structural formulas of some of the nitrogenous extractives of muscle are as follows:<smiles>N=C(N)CCCCCCC(=O)O</smiles>

CReatine, $\mathrm{C}_{4} \mathrm{H}_{0} \mathrm{~N}_{8} \mathrm{O}_{2}$.

Methyl-guanidine acetic actd.<smiles>CCCCCCCC(N)=O</smiles>

CREATININE, $\mathrm{C}_{4} \mathrm{H}_{7} \mathrm{~N}_{3} \mathrm{O}$. Crealine anhydride.

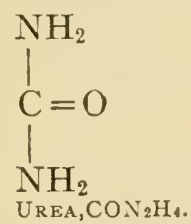

$\mathrm{CH}_{2} \cdot \mathrm{NH}_{2}$<smiles>CCS(=O)(=O)O</smiles>

TAURINE, $\mathrm{C}_{2} \mathrm{H}_{7} \mathrm{NSO}_{3}$.

Amino-ethyl sulphonic acid.

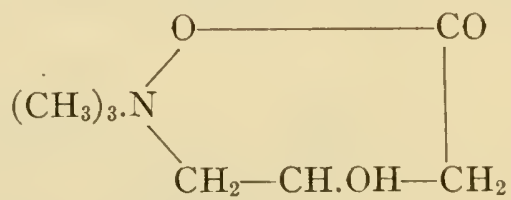

CARNITINe, $\mathrm{C}_{7} \mathrm{H}_{15} \mathrm{NO}_{3}$.

$\gamma$-trimethyloxybutyrobetaine.

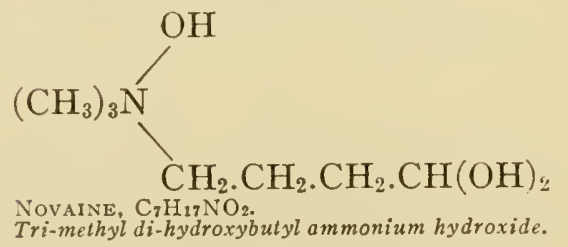

Carnosine, $\mathrm{C}_{9} \mathrm{H}_{14} \mathrm{~N}_{4} \mathrm{O}_{3}$.

Neosine, $\mathrm{C}_{6} \mathrm{H}_{17} \mathrm{NO}_{2}$.

Ignotine, $\mathrm{C}_{9} \mathrm{H}_{14} \mathrm{~N}_{4} \mathrm{O}_{3}$.

Phosphocarnic acid, $\mathrm{C}_{10} \mathrm{H}_{17} \mathrm{~N}_{3} \mathrm{O}_{5}$ or $\mathrm{C}_{10} \mathrm{H}_{15} \mathrm{~N}_{3} \mathrm{O}_{5}$.

Inosinic acid, $(\mathrm{HO})_{2} \cdot \mathrm{PO} . \mathrm{O} \cdot \mathrm{CH}_{2}(\mathrm{CHOH})_{3} \cdot \mathrm{CH}:\left(\mathrm{C}_{5} \mathrm{H}_{3} \mathrm{~N}_{4} \mathrm{O}\right)$.

\section{Purine Bodies. ${ }^{1}$ -}

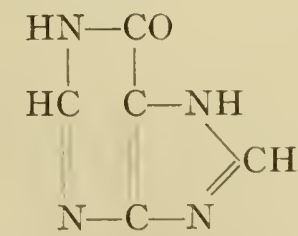

HypoxantiIne, $\mathrm{C}_{6} \mathrm{H}_{4} \mathrm{~N}_{4} \mathrm{O}$. 6-oxypurine.

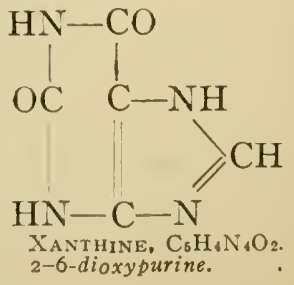

${ }^{1}$ For discussion of the purine bodies which are found as muscle extractive see Chapter VI on Nucleic Acids. 


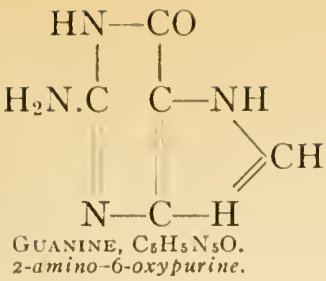

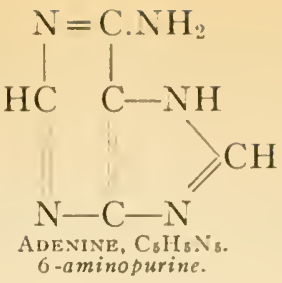

Experiments on Muscular Tissue

I. Experiments on "Living" Muscle

I. Preparation of Muscle Plasma (Halliburton).-Wash out the blood vessels of a freshly killed rabbit with 0.9 per cent sodium chloride. This can best be done by opening the abdomen and inserting a cannula into the aorta. Now remove the skin from the lower limbs, cut away the muscles and divide them into very small pieces by means of a meat chopper. Transfer the pieces of muscle to a mortar and grind them with clean sand and a little ice cold 5 per cent magnesium sulphate. Place in an ice-box over night. Filter off the salted muscle plasma and make the following tests:

(a) Reaction.- Test the reaction to litmus, phenolphthalein, and Congo red. What is the reaction of this fresh muscle plasma?

(b) Fractional Coagulation.-Place a little muscle plasma in a test-tube and arrange the apparatus for fractional coagulation as explained on page I05. Raise the temperature very carefully from $30^{\circ} \mathrm{C}$. and note any changes which may occur and the exact temperature at which such changes take place. When the first protein (para-myosinogen) coagulates filter it off and then heat the clear filtrate as before, being careful to note the exact temperature at which the next coagulation (myosinogen) occurs. There will probably be a preliminary opalescence in each case before the real coagulation occurs. Therefore do not mistake the real coagulation-point and filter at the wrong time. What are the coagulation temperatures of these two proteins? Which protein was present in greater amount?

(c) Formation of the Myosin Clot.-Dilute a portion of the plasma with 3 or 4 times its volume of water and place it on a water-bath or in an incubator at $35^{\circ} \mathrm{C}$. for several hours. A typical myosin clot should form. Note the muscle serum surrounding the clot. . Now test the reaction. Has the reaction changed, and if so to what is the change due? Make a test for lactic acid. What do you conclude?

2. Preparation of Muscle Plasma (v. Fürth). - Remove the blood-free nuscles of a rabbit as explained above. Finely divide by means of a meat chopper and grind in a mortar with a little clean sand and some 0.9 per cent sodium chloride. Wrap portions of the muscle in muslin and press thoroughly by means of a tincture press or lemon squeezer. Filter and make the tests according to the directions given in the last experiment.

3. "Fuchsin-frog" Experiment.-Inject a saturated aqueous solution of Fuchsin "S" into the lymph spaces of a frog two or three times daily for one or two days, in this way thoroughly saturating the tissues with the dye. Pith the animal (insert a heavy wire or blunt needle through the occipito atlantoid membrane), remove the skin from both hind legs and expose the sciatic nerve in one of them. 
Insert a small wire hook through the jaws of the frog and suspend the animal from an ordinary clamp or iron ring. Pass electrodes under the exposed sciatic nerve, and after tying the other leg to prevent any muscular movement, stimulate the exposed nerve by means of make and break shocks from an induction coil. The stimulated leg responds by pronounced muscular contractions, whereas the tied leg remains inactive. Continue the stimulation until the muscles are fatigued. The muscular activity has caused the production of lactic acid and this in turn has reacted with the injected fuchsin to cause a pink or red color to develop. The muscles of the inactive leg still remain unchanged in color.

The normal color of the Fuchsin " $S$ " when injected was red, but upon being absorbed it became colorless through the action of the alkalinity of the blood. Upon stimulating the muscles, however, as above explained, lactic acid was formed and this acid reacted with the fuchsin and again produced the original color of the dye.

\section{Experiments on "Dead" Muscle}

I. Preparation of Myosin.-Take 25 grams of finely divided lean beef which has been carefully washed to remove blood and lymph constituents and place it in a beaker with ro per cent sodium chloride. Stir occasionally for several hours. Strain off the meat pieces by means of cheese cloth, filter the solution and saturate it with sodium chloride in substance. Filter off the precipitate of myosin and make the tests as given below. This filtration will proceed very slowly. Myosin collects as a film on the sides of the filter paper and may be removed and tested before the entire volume of fluid has been filtered. If this precipitate remains for any length of time on the paper in contact with the air it will become transformed into the protean myosan. Test the myosin precipitate as follows:

(a) Solubility.-Try its solubility in water, sodium chloride, dilute acid and alkali. Is myosin an albumin or a globulin?

(b) Xanthoproteic Reaction.-See page 98.

(c) Coagulation Test.-Suspend a little of the myosin in water in a testtube and heat to boiling for a few moments. Now remove the suspended material and try its solubility in ro per cent sodium chloride. What property does this experiment show myosin to possess?

Test the filtrate from the original myosin precipitate as follows:

(a) Biuret Test.-What does this show?

(b) Place a little of the solution in a test-tube and heat to boiling. At the boiling-point add a drop of dilute acetic acid and filter. Test this filtrate for proteose with picric acid. Is any proteose present? Saturate another portion of the filtrate with ammonium sulphate and test for peptone in the usual way (see page 120). Do you find any peptone?

From your experiments on "living" and "dead" muscle what are your ideas regarding the proteins of muscle?

2. Preparation of Glycogen.-Grind a few oysters or scallops ${ }^{1}$ in a mortar

${ }^{2}$ Glycogen may also be prepared from the liver of an animal which has been fed a high carbohydrate diet for I-2 days previously. The best yield of glycogen can, however, generally be obtained from scallops. To secure best yield of glycogen the liver, scallops or oysters should be fresh. If permitted to stand some glycogen will be converted into glucose. 
with sand. Transfer to an evaporating dish, add water, and boil for 20 minutes. Note the opalescence of the solution. At the boiling-point faintly acidify with acetic acid. Why is this acid added? Filter, and divide the filtrate into two parts. Test one part of the filtrate as follows :

(a) Iodine Test. - To 5 c.c. of the solution in a test-tube add $5^{-10}$ drops of iodine solution and 2-3 drops of ro per cent sodium chloride. What do you observe? Is this similar to the iodine test upon any other body with which we have had to deal?

If difficulty is experienced in securing a satisfactory iodine test proceed as follows: Make equal volumes of glycogen solution acid in reaction with hydrochloric acid. Boil one solution to hydrolyze the glycogen. Add equal volumes of iodine solution to each and note the more pronounced iodine reaction in the unhydrolyzed solution.

(b) Reduction Test.-Does the solution reduce Fehling's solution?

(c) Hydrolysis of Glycogen.-Add ro drops of concentrated hydrochloric acid to 10 c.c. of the solution and boil for ro minutes. Cool the solution, neutralize with solid potassium hydroxide and test with Fehling's solution. Does it still fail to reduce Fehling's solution? If you find a reduction how can you prove the identity of the reducing substance?

(d) Influence of Saliva.-Place 5 c.c. of the solution in a test-tube, add 5 drops of saliva and place on the bath-water at $40^{\circ} \mathrm{C}$. for ro minutes. Does this now reduce Fehling's solution?

To the second part of the glycogen filtrate add 3-4 volumes of 95 per cent alcohol. Allow the glycogen precipitate to settle, decant the supernatant fluid, and filter the remainder. Heat the glycogen on a water-bath to remove the alcohol, then subject it to the following tests:

(a) Solubility.- Try its solubility in water and ro per cent sodium chloride solution.

(b) Iodine Test.-Place a small amount of the glycogen in a depression of a test-tablet and add 2-3 drops of dilute iodine solution and a trace of a sodium chloride solution. The same wine-red color is observed as in the iodine test upon the glycogen solution.

3. Testing for Inorganic Constituents.-(a) Examination of Ash of Muscle.Incinerate a small amount of muscular tissue, dissolve the ash in dilute hydrochloric acid. Test for potassium, phosphates, magnesium, calcium and chlorides.

(b) Demonstration of Phosphates and Magnesium in Muscle (Hürthle's Experiment).-Tease a very small piece of frog's muscle on a microscopical slide. Expose the slide to ammonia vapor for a few moments, then adjust a cover-glass, and examine the muscle fibers under the microscope. Note the large number of crystals of ammonium magnesium phosphate, distributed every-

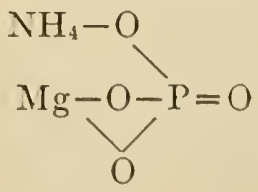

where throughout the muscle fiber, thus demonstrating the abundance of phosphates and magnesium in the muscle (Fig. I29, page 408). 


\section{Separation of Extractives from Muscles}

I. Creatine.-Dissolve about Io grams of a commercial extract of meat in 200 c.c. of warm water (Test for Protein by Biuret and Coagulation Tests, see Chapter V.) Precipitate the inorganic constituents by neutral lead acetate, being careful not to add an excess of the reagent. Write the equations for the reactions taking place here. Allow the precipitate to settle, then filter and remove the excess of lead in the warm filtrate by hydrogen sulphide. Filter while the solution is yet warm, evaporate the clear filtrate to a syrup, and allow it to stand at least 48 hours in a cool place. Crystals of creatine should form at this point. Examine under the microscope (Fig. IIO, page 342). Treat the syrup with 200 c.c. of 88 per cent alcohol, stir well with a glass rod to bring all soluble material into solution, and then filter. The purine bases have been dissolved and are in the filtrate, whereas the creatine crystals were insoluble in the 88 per cent alcohol and remain on the filter paper. Wash the crystals with 88 per cent alcohol, then remove them and bring them into solution in a little hot water. Decolorize the solution by animal charcoal and concentrate it to a small volume. Allow the solution to cool and note the separation of colorless crystals of creatine. ${ }^{1}$

Make the following tests on the crystals:

(a) Microscopical Examination.-Examine some crystals under the microscope and compare the form with those reproduced in Fig. 1 ro, page 342.

(b) Transformation of Creatine into Creatinine.-Dissolve the crystals in about 30 c.c. of hot water. To one-half of the solution in a flask add an equal volume of normal hydrochloric acid and heat on a boiling water-bath for five hours with reflux condenser. The creatine has been changed into creatinine. Apply tests for creatinine as given in Chapter XXII to the original solution as well as to the acidified solution.

Diacetyl Reaction.-To 5 c.c. of a dilute creatine solution add an equal volume of saturated sodium carbonate solution and a few drops of a solution of diacetyl. A pink color should develop. This test has been made the basis of a method for the quantitative determination of creatine. ${ }^{2}$

2. Hypoxanthine.-Evaporate the alcoholic filtrate from the creatine to remove the alcohol. Make the solution ammoniacal and add ammoniacal silver nitrate until precipitation ceases. The precipitate consists principally of hypoxanthine silver and xanthine silver. Collect these silver salts on a filter paper and wash them with water. Place the precipitate and paper in an evaporating dish and boil for one minute with nitric acid having a specific gravity of I.I. Filter while hot through a double paper, wash with the same strength of nitric acid and allow the solution to cool. By this treatment with nitric acid hypoxanthine silver nitrate and xanthine silver nitrate have been formed. The former is insoluble in the cold solution and separates on standing. After standing several hours filter off the hypoxanthine silver nitrate and wash with water until the wash water is only slightly acid in reaction. Examine the crystals of hypoxanthine silver nitrate under the microscope and compare them with those' in Fig. 84 , above. Now wash the crystals from the paper into a beaker with a little water and warm the liquid. Remove the silver by hydrogen sulphide and filter.

${ }^{1}$ For an improved method of preparing pure creatine from creatinine see chapter on Physiological Constituents of the Urine.

2 Walpole: Jour. Physiol., 42, 301, I91. 
By this means hypoxanthine nitrate has been formed and is present in the filtrate. (For crystalline form of hypoxanthine nitrate see Fig. 40, p. 136.) Concentrate on a water-bath to drive off hydrogen sulphide, and render the solution slightly alkaline with ammonia. Warm for a time, to remove the free ammonia, filter,

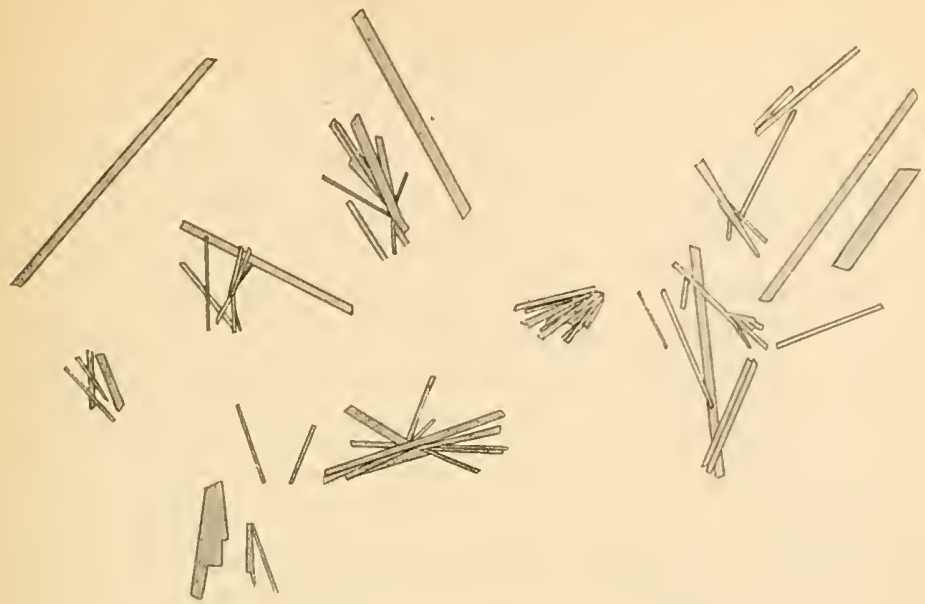

Fig. i 1 2.-Hypoxanthine Silver Nitrate.

(Drawn from a student preparation by Dr. E. F. Hirsch.)

concentrate the filtrate to a small volume and allow it to stand in a cool place. Hypoxanthine should crystallize in small colorless needles. Examine the crystals under the microscope.

3. Xanthine.- To the filtrate from the above experiment containing the

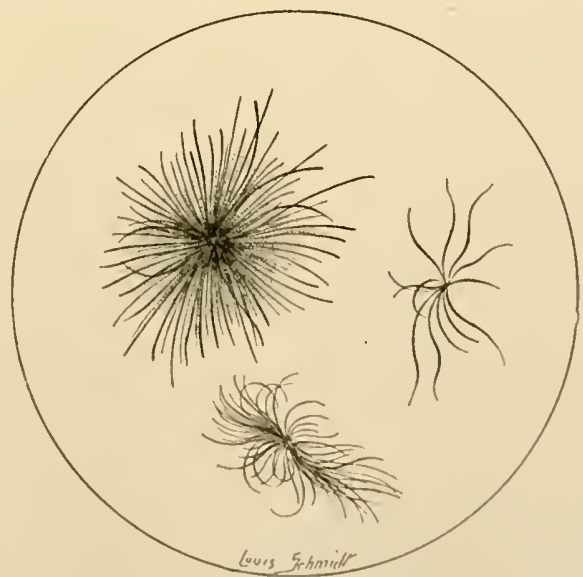

Fig. I13.-Xanthine Silver Nitrite.

xanthine silver nitrate add ammonia in excess. (The crystalline form of xanthine silver nitrate is shown in Fig. II3.) A brownish-red precipitate of xanthine silver forms. Treat this suspended precipitate with hydrogen sulphide (do not use an excess of hydrogen sulphide), warm the mixture for a few mo- 
ments and filter while hot. Concentrate the filtrate to a small volume and put away in a cool place for crystallization (Fig. III, page 344). To obtain xanthine in crystalline form special precautions are generally necessary. Evaporate the solution to dryness and test according to directions given in Chapter VI on Nucleic Acids. 


\section{CHAPTER XI}

\section{NERVOUS TISSUE}

In common with the other solid tissues of the body, nervous tissue contains a large amount of water. The percentage of water present depends upon the particular form of nervous tissue but in all forms it is invariably greater in the gray matter than in the white. Embryonic nervous tissues also contain a larger percentage of water than the tissues of adult life. The gray matter of the brain of the fotus, for instance, contains about 92 per cent of water, whereas the gray matter of the brain of the adult contains but $83-84$ per cent of the fluid.

Among the solid constituents of nervous tissue are proteins, cholesterol, cerebrosides (cerebrin, etc.), lecithin, kephalin, protagon (?), paranucleoprotagon, muclein, neurokeratin, collagen, extractives, and inorganic salts. The proteins are present in the greatest amount and comprise about 5o per cent of the total solids. Three distinct proteins, two globulins, and a nucleoprotein, have been isolated from the nervous tissue. The globulins coagulate at $47^{\circ} \mathrm{C}$. and $70-75^{\circ} \mathrm{C}$., respectively; while the nucleoprotein coagulates at $56-60^{\circ} \mathrm{C}$. This nucleoprotein contains about 0.5 per cent of phosphorus (Halliburton, Levene.) Nervous tissue is composed of a relatively large quantity of a variety of compounds which collectively may be grouped under the term "lipoid"-substances resembling the fats in some of their physical properties and reactions but distinct in their composition. We will class cholesterol, the cerebrosides and the phosphorized fats as lipoids.

The consideration of lipoids (or lipins ${ }^{1}$ ) is assuming added importance. These substances constitute one of the two great groups of tissue colloids, the proteins being the remaining group. So far as structure and chemical properties are concerned the various classes of lipoids are entirely unlike.

The group of phosphorized fats are very important constituents of nervous tissue. The best known members of this group are lecithin protagon (?) and kephalin. Lecithin occurs in larger amount than the other members of the group, has been more thoroughly studied than the others and is apparently of greater importance. Upon decomposition lecithin yields fally acids, glycero-phosphoric acid, and

${ }^{1}$ Rosenbloom and Gies: Biochemical Bulletin, I, 5 I, I9II. The term lipoid was introduced by Overton (Studien über die Narkose, Jena, I90I, Gustav Fischer). 
choline. Each lecithin molecule contains two fatty acid radicals which may be those of the same or different fatty acids. Thus we have different lecithins depending upon the particular fatty acids radicals which are present in the molecule. The formula of a typical lecithin would be the following.

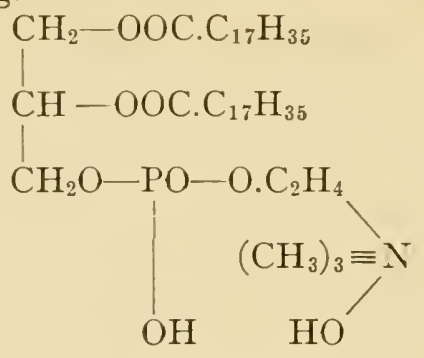

This lecithin would be called distearyl-lecithin or choline-distearylglycero-phosphoric acid. Upon decomposition the molecule splits according to the following reaction:

$$
\underset{\text { Lecithin. }}{\mathrm{C}_{44} \mathrm{H}_{90} \mathrm{NPO}_{9}}+\underset{3 \mathrm{H}_{2} \mathrm{O}}{\rightarrow} \underset{\text { Stearic acid. }}{2 \mathrm{C}_{18} \mathrm{H}_{36} \mathrm{O}_{2}}+\mathrm{Glycero}_{3} \mathrm{H}_{9} \mathrm{PO}_{6}+\underset{\mathrm{C}_{5}}{\mathrm{H}_{15} \mathrm{NO}_{2}} \text {. }
$$

The lecithins are not confined to the nervous tissues but are found in nearly all animal and vegetable tissues. Lecithin is a primary constituent of the cell. It is soluble in chloroform, ether, alcohol, benzene, and carbon disulphide. The chloroform or alcohol-ether solution may be precipitated by acetone. Lecithin may be caused to crystallize in the form of small plates by cooling the alcoholic solution to a low temperature. It has the power of combining with acids and bases, and the hydrochloric acid combination has the power of forming a double salt with platinic chloride.

Choline, as was indicated above, is one of the decomposition products of lecithin. It is trimethyl-hydroxyethyl-ammonium hydroxide and has the following formula:

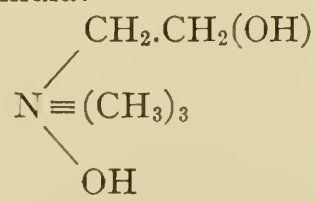

Researches have shown that great importance is to be attached to the detection of choline in the cerebro-spinal fluid and the blood in certain cases of degenerative disease of the nervous system. In this connection tests for choline (see page 357 ) are of interest and value.

Protagon, another nitrogenous phosphorized substance, is a body over which there has been much discussion. Upon decomposition it 
is said by some investigators to yield cerebrin and the decomposition products of lecithin. It has been shown by Posner and Gies ${ }^{1}$ as well as by Rosenheim and Tebb ${ }^{2}$ that protagon is a mixture and has no existence as a chemical individual. $\mathrm{Koch}^{3}$ reported data obtained from purified preparations which indicate that protagon contains at least three substances: "a phosphatide containing cholin, a cerebroside containing sugar, a complex combination of a cholin-free phosphatide with a cerebroside to which an ethereal sulphuric acid group is attached." On the basis of his data, he believed the term protagon to have no chemical significance. He proposed the term sulphatide. Koch's preparation analyzed as follows (per cent):

$\begin{array}{ccccc}\text { Choline } & \text { Sugar } & \text { Nitrogen } & \text { Phosphorus } & \text { Sulphur } \\ \text { I.0 } & \text { I } 2.0 & 2,3 & \text { I.7 } & \text { I.9 }\end{array}$

He suggested the following structure:

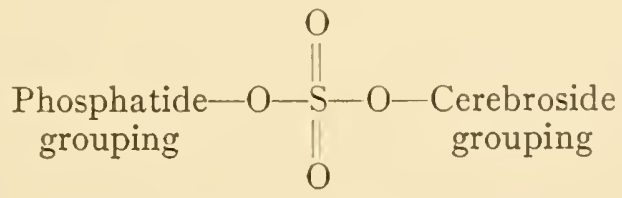

Kephalin is the third member of the group of phosphorized fats. It is precipitated from its acetone-ether extract by alcohol. It contains about 4 per cent of phosphorus and has been given the formula $\mathrm{C}_{42} \mathrm{H}_{79}$ $\mathrm{NPO}_{13}$. Kephalin may be a stage in lecithin metabolism.

The cerebrosides are substances containing nitrogen but no phosphorus, and are important constituents of the white matter of nervous tissue. Certain ones have also been found in the spleen, pus, and in egg yolk. They may be extracted from the tissue by boiling alcohol and are insoluble in cold alcohol, cold and hot ether, and in water and dilute alkalis. The cerebroside termed cerebrin is a mixture containing phrenosin (pseudo-cerebrin or cerebron), a body yielding the carbohydrate galactose on decomposition.

Cholesterol, one of the primary cell constituents, is present in fairly large amount in nervous tissue. It occurs in two forms, i.e., free and combined as an ester. It is claimed ${ }^{4}$ that 99 per cent of the cholesterol of brain tissue (boy) is in the free state. It is a mon-atomic alcohol containing at least one double bond and possesses the formula $\mathrm{C}_{27} \mathrm{H}_{45} \mathrm{OH}$ or $\mathrm{C}_{27} \mathrm{H}_{43} \mathrm{OH}$. There is still some uncertainty as to the exact structure of cholesterol. It may possess a terpene structure. It

I Posner and Gies: Journal of Biological Chemistry, 1, 59, I905-06.

${ }^{2}$ Rosenheim and Tebb: Journal of Physiology, 36 and 37, 1907-S.

${ }^{3}$ Koch: Journal Biological Chemisty, I I, March, I 912 , Proceedings.

4 Lapworth: Jour. Path. Bact., rgIr, p. 256. 
was formerly called a "non-saponifiable fat" but since it is not changed in any way by boiling alkalis it is not a fat. It is soluble in ether, chloroform, benzene, and hot alcohol. It crystallizes in the form of thin, colorless, transparent plates (Fig. 57, page 210). Cholesterol is present in bile, occurs abundantly in one form of biliary calculus. It is also present in blood and its quantitative determination is of clinical importance (see Chapter XVI). It has been found in feces, wool fat, egg yolk, and milk, frequently in the form of its esters of higher fatty acids. It is generally believed that the cholesterol present in the animal body has its origin in the regetable kingdom. Some evidence has been submitted ${ }^{1}$ indicating a synthesis of cholesterol under certain conditions in the animal body. However, it is probable that cholesterol is not readily synthesized in the body. ${ }^{2}$

Paranucleoprotagon is a phosphorized substance originally isolated from brain tissue by Ulpiani and Lelli and recently reinvestigated by Steel and Gies. It is said to possess lecithoprotein characteristics.

Nervous tissue yields about I per cent of ash which is made up in great part of alkaline phosphates and chlorides.

\section{Experinexts on the Lipoids of Nervous Tissue ${ }^{3}$}

I. Preparation of Lecithin. 4 -Treat the finely divided brain of a sheep with ether and allow it to stand in the cold for 48-72 hours. The cold ether will extract lecithin and cholesterol. Filter and add acetone to the filtrate to precipitate the lecithin. Filter off of the lecithin and test it as follows:

(a) Microscopical Examination.-Suspend a small portion in a drop of water on a slide and examine under the microscope.

(b) Osmic Acid Test. ${ }^{5}$ - Treat a small portion with osmic acid. What happens?

(c) Acrolein Test.-Make the acrolein test according to directions on page 180.

(d) Test for Phosphorus.-See page r29, Chapter VI.

2. Preparation of Cholesterol.-Place a small amount of finely divided brain tissue under ether and stir occasionally for one hour. Filter, evaporate the filtrate to dryness on a water-bath, and test the cholesterol according to directions given below. (If it is desired, the ether extract from the so-called protagon,

1 Klein: Biochem. Zeit., 30, 465, 19го.

${ }^{2}$ Gardner and Lander: Proc. Royal Soc., London (B), 87, 229 , I9r3.

3 Preparation of So-called Prolagon.-Divide the brain of a sheep into small pieces, treat with 85 per cent alcohol and warm on a water-bath $45^{\circ} \mathrm{C}$. for two hours. Filter hol into a bottle or strong flask and cool to $0^{\circ} \mathrm{C}$. for one-half hour by means of a freezing mixture. By this procedure both protagon and cholesterol are caused to precipitate. Filter the cold solution rapidly and treat the precipitate on the paper with ice cold ether to dissolve out the cholesterol. The protagon may now be redissolved in warm 85 per cent alcohol from which solution it will precipitate upon cooling.

4 For the preparation of lecithin in purer form see MacLeon: Jour. Path. Bact., I 8, 490, I9I4.

${ }^{5}$ Osmic acid serves to detect fats which contain unsaturated fatty acid radicals, e.g., oleic acid, in their molecule. 
or the ether-acetone filtrate from the lecithin may be used for the isolation of cholesterol. In these cases it is simply necessary to evaporate the solution to dryness on a water-bath.) Upon the cholesterol prepared by either of the above methods make the following tests:

(a) Microscopical Examination.-Examine the crystals under the microscope and compare them with those in Fig. 57, page 210.

(b) $\mathrm{H}_{2} \mathrm{SO}_{4}$ Test (Salkowski).-Dissolve a few crystals of cholesterol in a little chloroform and add an equal volume of concentrated sulphuric acid. A play of colors from bluish-red to cherry-red and purple is noted in the chlorofrom, while the acid assumes a marked green fluorescence.

(c) Acetic Anhydride- $\mathrm{H}_{2} \mathrm{SO}_{4}$ Test (Liebermann-Burchard).-Dissolve a few crystals of cholesterol in 2 c.c. of chloroform in a dry test-tube. Now add ro drops of acetic anhydride and $x-3$ drops of concentrated sulphuric acid. The solution becomes red, then blue, and finally bluish-green in color.

(d) Iodinc-sulphuric Acid Test.-Place a few crystals of cholesterol in one of the depressions of a test-tablet and treat with a drop of concentrated sulphuric acid and a drop of a very dilute solution of iodine. A play of colors, consisting of violet, blue, green, and red, results.

(c) Schiff's Reaction.-To a little cholesterol in an evaporating dish add a few drops of a reagent made by adding I volume of to per cent ferric chloride to 3 volumes of concentrated sulphuric acid. Evaporate to dryness over a low flame and observe the reddish-violet residue which changes to a bluish-violet.

(f) Phosphorus.-Test for phosphorus according to directions given in Chapter II, page I29. Is phosphorus present?

3. Preparation of Cerebrin. - Treat roo grams of finely divided brain tissue, in a flask, with 200 c.c. of 95 per cent alcohol and boil on a water-bath for onehalf hour, keeping the volume constant by adding fresh alcohol as needed or by the use of a reflux condenser. Filter the solution hot and stand the cloudy filtrate away for 24 hours. (If the filtrate is not cloudy concentrate it upon the water-bath until it is so.) Filter off the cerebrin (cerebrin, lecithin, kephalin, cholesterol) and test it as follows :

(a) Microscopical Examination.--Suspend a small portion in a drop of water on a slide and examine under the microscope.

(b) Solubility. - Try the solubility of cerebrin in water, Io per cent sodium chloride and in dilute acid and alkali, and in hot and cold alcohol and hot and cold ether.

(c) Phosphorus.-Test for phosphorus according to directions in Chapter VI, page I29. How does the result compare with that on lecithin?

(d) Place a little cerebrin on platinum foil and warm. Note the odor.

(e) Hydrolysis of Cerebrin.-Place the remaining cerebrin in a small evaporating dish, add equal volumes of water and dilute hydrochloric acid, and boil for one hour. Cool, neutralize with solid potassium hydroxide, filter, and test with Fehling's solution. Is there any reduction, and if so how do you explain it?

4. Tests for Choline. (a) Rosenheim's P'eriodide Test.- P'repare an alcoholic extract of the fluid under examination. and after evaporation apply Rusenheim's iodo-potassium iodicle solution' to a little of the residue. 11 a short time dark

${ }^{1}$ Prepared by dissolving 2 grams of indine and 6 grams of potarsium iodide in roo c c. water. 
brown plates and prisms of choline periodide begin to form and may be detected by means of the microscope. Occasionally they are large enough to be visible to the naked eye. They somewhat resemble crystals of hemin (see page 265). If the slide be permitted to stand, thus allowing the fluid to evaporate, the crystals will disappear and leave brown oily drops. They will reappear, however, upon the addition of fresh iodine solution. v. Staněk claims that this choline compound has the formula $\mathrm{C}_{5} \mathrm{H}_{14}$ NOI.I.

(b) Rosenheim's Bismuth Test.-Extract the fluid under examination with absolute alcohol, evaporate, and reextract the residue. Repeat the extraction several times. Dissolve the final residue in 2-3 c.c. of water and add a drop of Kraut's reagent. ${ }^{1}$ Choline is indicated by the appearance of a bright brick-red precipitate.

1 Dissolve 272 grams of potassium iodide in water and add 80 grams of bismuth subnitrate dissolved in 200 grams of nitric acid (sp. gr. I.I8). Permit the potassium nitrate to crystallize out, then filter it off and make the filtrate up to I liter with water. 


\section{CHAPTER XXI}

\section{URINE: GENERAL CHARACTERISTICS OF NORMAL AND PATHOLOGICAL URINE}

Volume.-The volume of urine excreted by normal individuals during any definite period fluctuates within very wide limits. The average output for twenty-four hours is placed by German writers between 1500 and 2000 c.c. This value is not strictly applicable to conditions in America, however, since it has been found that the average normal excretion of the adult male American falls within the lower values of I000-I200 C.c. The volume-excretion is influenced greatly by the diet, particularly by the ingestion of fluids.

Certain pathological conditions cause the output of urine for any definite period to depart very decidedly from the normal output. Among the pathological conditions in which the volume of urine is increased above normal are the following: Diabetes mellitus, diabetes insipidus, certain diseases of the nervous system, contracted kidney, amyloid degeneration of the kidney, and in convalescence from acute diseases in general. Many drugs such as calomel, digitalis, acetates, and salicylates also increase the volume of the urine excreted. A decrease from the normal is observed in the following pathological conditions: Acute nephritis, diseases of the heart and lungs, fevers, diarrhœa, and vomiting.

Color.-Normal urine ordinarily possesses a yellow tint, the depth of the color being dependent in part upon the density of the fluid. The color of normal urine is due principally to a pigment called urochrome: ${ }^{1}$ traces of hematoporphyrin, urobilin, and uroerythrin have also been detected. Under pathological conditions the urine is subject to pronounced variations in color and may contain many varieties of pigments. Under such circumstances the urine may vary in color from an extremely light yellow to a very dark brown or black. Vogel has constructed a color chart which is of some value for purposes of comparison. The nature and origin of the chief variations in the urinary color are set forth in tabular form by Halliburton as follows:

I Urochrome is believed to be identical with the yellow pigment (lactochrome) of milk whey (Paimer and Coolidge: Jour. Biol. Chem., I7, 25I, I9I4). 


\begin{tabular}{|c|c|c|}
\hline Color & Cause of coloration & Pathological condition \\
\hline Nearly colorless... & $\begin{array}{l}\text { Dilution, or diminution of } \\
\text { normal pigments. }\end{array}$ & $\begin{array}{l}\text { Nervous conditions: hy- } \\
\text { druria, diabetes insipidus, } \\
\text { granular kidney. }\end{array}$ \\
\hline Dark yellow to brown-red. & $\begin{array}{l}\text { Increase of normal, or oc- } \\
\text { currence of pathological, } \\
\text { pigments. Concentrated } \\
\text { urine. }\end{array}$ & Acute febrile diseases. \\
\hline \multirow[t]{2}{*}{ Milky } & Fat globules............. & Chyluria. \\
\hline & Pus corpuscles........ & $\begin{array}{l}\text { Purulent diseases of the } \\
\text { urinary tract. }\end{array}$ \\
\hline Orange & Excreted drugs... & Santonin, crysophanic acid. \\
\hline \multirow[t]{2}{*}{ Red or reddish. } & $\begin{array}{l}\text { Hematoporphyrin............ } \\
\text { Unchanged hemoglobin..... }\end{array}$ & $\begin{array}{l}\text { Hemorrhages, or hemoglo- } \\
\text { binuri. }\end{array}$ \\
\hline & $\begin{array}{l}\text { Pigments in food (logwood, } \\
\text { madder, bilberries, fuchsin). }\end{array}$ & \\
\hline \multirow[t]{4}{*}{ Brown to brown black..... } & Hematin.... & Small hemorrhages. \\
\hline & Methemoglobin.......... & Methemoglobinuria. \\
\hline & Melanin... & Melanotic sarcoma. \\
\hline & Hydrochinol and catechol. & Carbolic-acid poisoning. \\
\hline $\begin{array}{l}\text { Greenish yellow, greenish } \\
\text { brown, approaching black.. }\end{array}$ & Bile-pigments.......... & Jaundice. \\
\hline Dirty green ${ }^{1}$ or blue . & $\begin{array}{l}\text { A dark blue scum on surface, } \\
\text { with a blue deposit, due to } \\
\text { an excess of indigo-forming } \\
\text { substances. }\end{array}$ & $\begin{array}{l}\text { Cholera, typhus; seen espe- } \\
\text { cially when the urine is } \\
\text { putrefying. }\end{array}$ \\
\hline $\begin{array}{l}\text { Brown-yellow to red-brown, } \\
\text { becoming blood-red upon } \\
\text { adding alkalis. }\end{array}$ & $\begin{array}{l}\text { Substances contained in } \\
\text { senna, rhubarb and cheli- } \\
\text { dorium which are intro- } \\
\text { duced into the system. }\end{array}$ & \\
\hline
\end{tabular}

Transparency.-Normal urine is ordinarily perfectly clear and transparent when voided. On standing for a variable time, however, a cloud (nubecula) consisting principally of nucleoprotein or mucoid (see page 396) and epithelial cells forms. A turbidity due to the precipitation of phosphates is normally noted in urine passed after a hearty

1 This dirty green or blue color also occurs after the use of methylene blue in the organism. 
meal. The urine obtained $2-3$ hours after a meal or later is ordinarily free from turbidity. Permanently turbid urines ordinarily arise from pathological conditions.

Odor.-The odor of normal urine is of a faint, aromatic type. The bodies to which this odor is due are not well known, but it is claimed by some investigators to be due, at least in part, to the presence of minute amounts of certain volatile organic acids. Dehn and Hartman ${ }^{1}$ hare recently succeeded in isolating from urine a neutral ill-smelling substance which they call urinod. Its empirical formula is $\mathrm{C}_{6} \mathrm{H}_{8} \mathrm{O}$. Urinod occurs in urine to the extent of only $\mathrm{I}-2$ parts in 100,000 parts of urine. When the urine undergoes decomposition, e.g., in alkaline fermentation, a very unpleasant ammoniacal odor is erolved. All urines are subject to such decomposition if allowed to stand for a sufficiently long time. Under normal conditions the urine very often possesses a peculiar odor due to the ingestion of some certain drug or vegetable. For instance, cubebs, copaiba, myrtol, saffron, tolu, and turpentine each imparts a somewhat specific odor to the urine. After the ingestion of asparagus, the urine also possesses a typical odor due to the formation of methyl mercaplan $\left(\mathrm{CH}_{3} \cdot \mathrm{SH}\right)$ in the intestine.

Frequency of Urination.-The frequency of urination varies greatly in different individuals, but in general is dependent upon the amount of fluid in the bladder. In pathological conditions an inflammatory affection of the urinary tract or any disturbance of the innervation of the bladder will influence the frequency. Affections of the spinal cord which lead to an increased irritability of the bladder or a weakening of the sphincter, or any condition lowering the residual capacity of the bladder, will result in increasing the frequency of urination.

Reaction.-The mixed 24-hour urinary excretion of a normal individual ordinarily possesses an acid reaction to litmus. This acidity in normal cases is represented on the average by a hydrogen ion concentration of $10 \times 10^{-7}$, although it may vary from 0.40 to $\mathrm{I}_{50} \times \mathrm{IO}^{-}{ }^{-}$. The reaction of the urine represents an equilibrium between a large number of acid and basic constituents, both organic and inorganic. which it contains. Organic acids and bases play a part in producing the nurmal reaction, but this is probably, in the main, dependent upon the relative amounts of the mono- and dibasic sodium and potassium phosphates. The monobasic sodium phosphate $\left(\mathrm{NaH}_{2} \mathrm{PO}_{4}\right)$ is acid in reaction. while the dibasic phosphate $\left(\mathrm{Na}_{2} \mathrm{HPO}_{4}\right)$ is alkaline in reaction. The excretion of acid or alkaline phosphate by the kidneys is one of the factors in the regulation of the neutrality of the blood and of the organism in general. The acidity of the urine as determined by titration runs

${ }^{1}$ Dehn and Hartman: Jour. Am. Chem. Soc., 36, 2136 1914. 
in general parallel with the hydrogen ion concentration and seems to be dependent upon the same factors, and in more acid urines mainly on the phosphate content. (For further discussion of acidity see Chapter VIII on Gastric Analysis.)

The mean acidity in cardio-renal diseases is high-about $50 \times 10^{-7}$ as compared with $10 \times 10^{-7}$, the normal mean. In general the acidity tends to be increased in the greater number of pathological disorders.

The composition of the food is perhaps the most important factor in determining the reaction of the urine (see Chapter XXVII on Metabolism for influence of base-forming and acid-forming foods). The reaction ordinarily varies considerably according to the time of day the urine is passed. For instance, for a variable length of time after a meal the urine may be neutral or even alkaline in reaction to litmus,

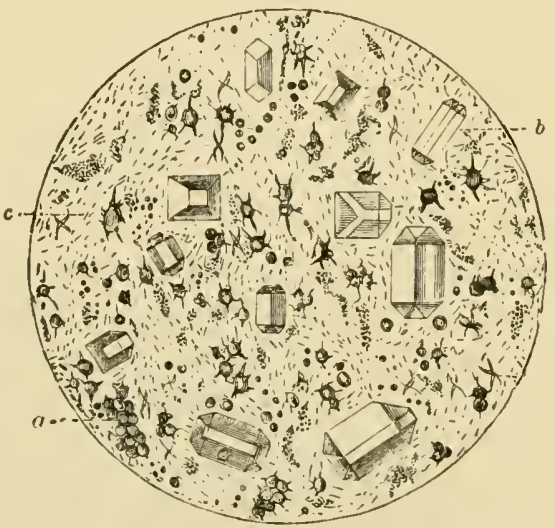

Fig. ir4,-Deposit in Ammontacal Fermentation.

$a$, Acid ammonium urate; $b$, ammonium magnesium phosphate; $c$, bacteria.

owing to the claim of the gastric juice upon the acidic radicals to further the formation of hydrochloric acid for use in carrying out the digestive secretory function. This change in reaction is known as the alkaline tide and is common to perfectly healthy individuals. The urine may also become temporarily alkaline in reaction to litmus, as the result of ingesting alkaline carbonates or certain salts of tartaric and citric acids which may be transformed into carbonates within the organism. Normal urine upon standing for some time becomes alkaline in reaction to litmus, owing to the inception of alkaline or ammoniacal fermentation through the agency of micro-organisms. This fermentation has no especial diagnostic value except in cases where the urine has undergone this change within the organism and is voided in the decomposed state. Ammoniacal fermentation is ordinarily due to cystitis or occurs as the result of infection in the process of catheterization. A microscopical 
examination of such urine (Fig. II4) shows the presence of ammonium magnesium phosphate crystals, amorphous phosphates, and not infrequently ammonium urate.

Ingestion of acid fruits (oranges, lemons, peaches, etc.) causes the formation of alkaline urine. This is due to the fact that the ash of such fruits is alkaline and when the fruits are combusted in the body carbonates are formed. On the other hand, bread, cereals, etc., yield an acid ash and an acid urine.

Occasionally a urine which possesses a normal acidity when voided, upon standing instead of undergoing ammoniacal fermentation as above described, will become more strongly acid in reaction. Such a phenomenon is termed acid fermentation. Accompanying this increased acidity there is ordinarily a deepening of the tint of the urinary color.

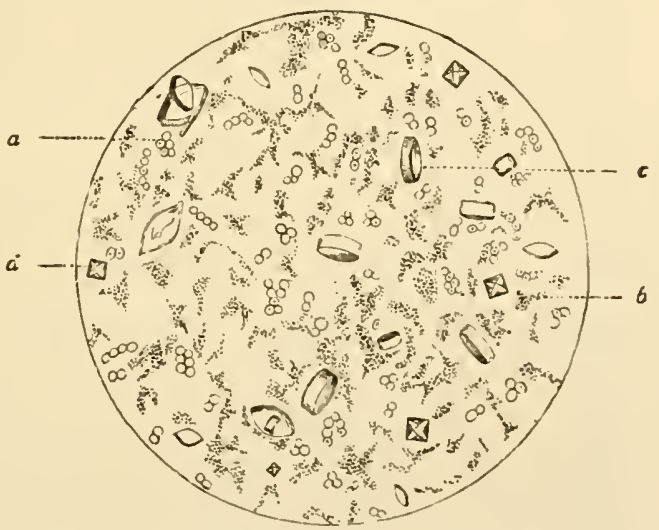

Fig. I15.-Deposit in Acid Fermentation.

$a$, Fungus; $b$, amorphous sodium urate; $e$, uric acid; $d$, calcium oxalate.

Such urines may contain acid urates, uric acid, fungi, and calcium oxalate (Fig. II 5, above). On standing for a sufficiently long time any urine which exhibits acid fermentation will ultimately change in reaction, due to the inception of alkaline fermentation, and will show the microscopical deposits characteristic of such a urine.

Specific Gravity.- The specific gravity of the urine of normal individuals varies ordinarily between $\mathrm{I} .015$ and 1.025 . This value is subject to wide fluctuations under various conditions. For instance, following copious water- or beer-drinking the specific gravity may fall to 1.003 or lower, whereas in cases of excessive perspiration it may rise as high as 1.040 or eren higher. Where a very accurate detcrmination of the specific gravity is desired use is commonly made of the pyknometer or of the Westphal hydrostatic balance. These instruments, however, are not suited for clinical use. The clinical method of deter- 
mining the specific gravity is by means of a urinometer (Fig. I 16). This affords a very rapid method and at the same time is sufficiently accurate for clinical purposes. The urinometer is always calibrated for use at a specific temperature and the observations made at any other temperature must be subjected to a certain correction to obtain the true specific gravity. In making this correction one unit of the last order is added to the observed specific gravity for every three degress above

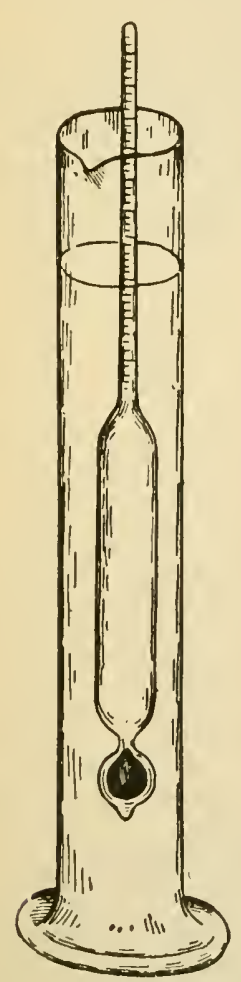

FIG. II6.-URINOMETER AND CyLINDER. the normal temperature and subtracted for every three degrees below the normal temperature. For instance, if in using a urinometer calibrated for $15^{\circ} \mathrm{C}$. the specific gravity of a urine having a temperature of $2 \mathrm{I}^{\circ} \mathrm{C}$. is determined as r.or 8 it is necessary to add to the observed specific gravity two units of the third order to obtain the real specific gravity of the urine. Therefore the true specific gravity, at $15^{\circ} \mathrm{C}$., of a urine having a specific gravity of $\mathrm{I} .0 \mathrm{I} S$ at $2 \mathrm{I}^{\circ} \mathrm{C}$. is I.OI $8+$ $0.002=\mathrm{r} .020$.

Pathologically, the specific gravity may be subjected to very wide variations. This is especially true in diseases of the kidneys. In acute nephritis ordinarily the urine is concentrated and of a high specific gravity, whereas in chronic nephritis the reverse conditions are more apt to prevail. In fact, under most conditions, whether physiological or pathological, the specific gravity of the urine is inversely proportional to the volume excreted. This is not true of diabetes mellitus, however, where the volume of urine is large and the specific gravity is also high, owing to the sugar contained in the urine.

The amount of solids eliminated in the excretion for twenty-four hours may be roughly calculated by means of Long's coefficient, i.e., 2.6. The solid content of rooo c.c. of urine is obtained by multiplying the last two figures of the specific gravity observed at $25^{\circ} \mathrm{C}$. by 2.6 . To determine the amount of solids excreted in twenty-four hours if the volume was I 20 C.c. and the specific gravity was 1.018 the calculation would be as follows:

(a) $18 \times 2.6=46.8$ grams of solid matter in rooo c.c. of urine.

(b) ${ }_{1000}^{46.8 \times I_{20} 0}=52.4$ grams of solid matter in II 20 c.c. of urine.

The coefficient of Häser (2.33) which has been in use for years probably gives values that are inaccurate for conditions existing in America. 
This coefficient was calculated on the basis of the specific gravity determined at a temperature of $15^{\circ} \mathrm{C}$.

Freezing-point (Cryoscopy).- The freezing-point of a solution depends upon the total number of molecules of solid matter dissolved in it. The determination of the osmotic pressure by this method has come to be of some clinical importance, particularly as an aid in the diagnosis of kidney disorders. In this connection it is best to collect the urine from each kidney separately and determine the freezing-point in the individual samples so collected. By this means considerable aid in the diagnosis of renal diseases may be secured. The fluids most frequently examined cryoscopically are the blood (see page 245) and the urine. The freezingpoint is denoted by $\Delta$. The value of $\Delta$ for normal urine varies ordinarily between $-\mathrm{I} .3^{\circ}$ and $-2.3^{\circ} \mathrm{C}$, the freezing-point of pure water being take as $0^{\circ} . \Delta$ is subject to very wide fluctuations under unusual conditions. For instance, following copious water- or beerdrinking $\Delta$ may have as high a value as $-0.2^{\circ} \mathrm{C}$., whereas on a diet containing much salt and deficient in fluids the value of $\Delta$ may be lowered to $-3^{\circ} \mathrm{C}$. or even lower. The freezing point of normal blood is generally about $-0.56^{\circ} \mathrm{C}$. and is not subject to the wide variations noted in the urine, because of the tendency of the organism to maintain the normal osmotic pressure of the blood under all conditions. Variations between $-0.5 \mathrm{I}^{\circ}$ and $0.62^{\circ} \mathrm{C}$. may be due entirely to dietary conditions, but if any marked variation is noted it can, in most cases, be traced to a disordered kidney function.

Freezing-point determinations may be made by means of the Beckmann-Heidenhain apparatus (Fig. II7) or the Zikel pektoscope. The Beckmann-Heidenhain apparatus consists of

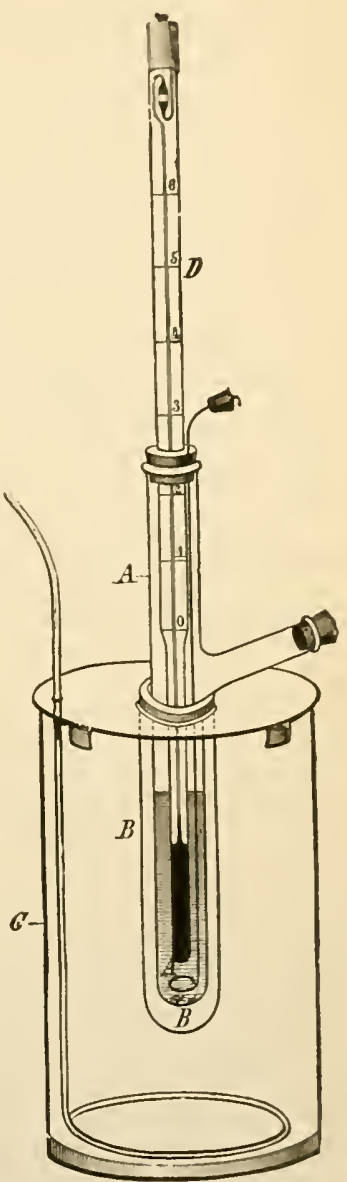

Fig. Ir7.-BECKMANNHeIDENHAIN FREEZINGPOINT Apparatus. (Long.)

$D$, a delicate thermom. eter; $C$, the containing jar; $B$, the outside or air mantle tube; $A$, the tube in which the mixture to be observed is placed. Two stirrers are shown, one for the cooling mixture in the jar and one for the experimental mixture. the following parts: A strong battery jar or beaker (C) furnished with a metal cover which is provided with a circular hole in its center. This strong glass vessel serves to hold the freezing mixture by means 
of which the temperature of the fluid under examination is lowered. A large glass tube (B) designed as an air-jacket, and formed after the manner of a test-tube is introduced through the central aperture in the metal cover and into this air-jacket is lowered a smaller tube (A) containing the fluid to be tested. A very delicate thermometer (D), graduated in hundredths of a degree is introduced into the inner tube and is held in place by means of a cork so that the mercury bulb is immersed in the fluid under examination but does not come into contact with any glass surface. A small platinum wire stirrer serves to keep the fluid under examination well mixed while a larger stirrer is used to manipulate the freezing mixture. (Rock salt and ice in the proportion I:3 form a very satisfactory freezing mixture.)

In making a determination of the freezing-point of a fluid by means of the Beckmann-Heidenhain.apparatus proceed as follows: Place the freezing mixture in the battery jar and add water (if necessary) to secure a temperature not lower than $3^{\circ} \mathrm{C}$. Introduce the fluid to be tested into tube $\mathrm{A}$, place the thermometer and platinum wire stirrer in position, and insert the tube into the air-jacket which has previously been inserted through the metal cover of the battery jar. Manipulate the two stirrers in order to insure an equalization of temperature and observe the course of the mercury column of the thermometer very carefully. The mercury will gradually fall and this gradual lowering of the temperature will be followed by a sudden rise. The point at which the mercury rests after this sudden rise is the freezing-point. This rise is due to the fact that previous to freezing, a fluid is always more or less over-cooled and the thermometer temporarily registers a temperature somewhat below the freezing-point. As the fluid freezes, however, there is a very sudden change in the temperature of the liquid and this change is imparted to the thermometer and causes the rise as indicated. It occasionally occurs that the fluid under examination is very much over-cooled and does not freeze. Under such circumstances a small piece of ice is introduced into it by means of the side tube noted in the figure. This so-called "inoculation" causes the fluid to freeze instantaneously. (For details of the method of determining the freezing-point consult standard works on physical or organic chemistry.)

Electrical Conductivity.-The electrical conductivity of the urine is dependent upon the number of inorganic molecules or ions present, and in this differs from the freezing-point which is dependent upon the total number of molecules both inorganic and organic which are in solution. The conductivity of the urine has been investigated but slightly, but from the data secured it seems that the value generally falls below $\kappa=0.03$. The conductivity of blood serum has been de- 
termined as $\kappa=0.0 \mathrm{I} 2$. Up to the present time the determination of the electrical conductivity of any of the fluids of the body has been put to very slight clinical use. Experience may show the conductivity value to be a more important aid to diagnosis than it is now considered, particularly if it is taken in connection with the determination of the freezing-point. By a combination of these two methods the portion of the osmotic pressure due respectively to electrolytes and nonelectrolytes may be determined. For a discussion of electrical conductivity, the method by which it is determined, and the principles involved consult standard works on physical or electro-chemistry.

Collection and Preservation of the Urine Sample.-If any dependable data are desired regarding the quantitative composition of the urine the examination of the mixed excretion for twenty-four hours is $a b$ solutely necessary. In collecting the urine the bladder may be emptied at a given hour, say $8 \mathrm{~A} . \mathrm{M}$., the urine discarded and all the urine from that hour up to and including that passed the next day at $\delta \mathrm{A}$. M., saved, thoroughly mixed, and a sample taken for analysis. Until recently it was believed that powdered thymol (isopropylmetacresol)

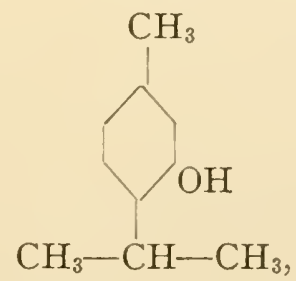

was a very satisfactory preservative since the excess might be removed by filtration, if desired, and it was believed that the small amount which went into solution would have no appreciable influence upon the determination of any of the urinary constituents. It appears however that thymol is not such a satisfactory urinary preservative as was believed. Evidence has been presented showing it to be unsatisfactory for the preservation of urines which contain sugar, acetone or diacetic acid and in which it desired to estimate the quantitative content of these constituents. Claim has also been made that thymol is not a satisfactory preservative for urines that are to be examined quantitatively for phosphates or magnesium. Thymol being a phenol mill cause an inaccuracy when phenols are being determined quantitatively. Urines preserved by thymol will also give a confusing white ring when subjected to the nitric acid test for albumin (see Chapter XXIII).

Toluene is a very satisfactory preservative for urine. In using this preservative simply overlay the urine with the toluene. Rosenbloom ${ }^{1}$

${ }^{1}$ Rosenbloom: New York Medical Journal, 99, 735, I9I4. 
claims that camphor is a very satisfactory urine preservative which does not interfere with the tests for important urinary constituents.

In certain pathological conditions it is desirable to collect the urine passed during the day separately from that passed during the night. When this is done the urine voided between $\delta \mathrm{A}$. M. and $8 \mathrm{P}$. M. may be taken as the day sample and that roided between 8 P. M. and 8 A. M. as the night sample.

The qualitative testing of urine samples collected at random, except in a few specific instances, is of no particular value so far as giving us any accurate knowledge as to the exact urinary characteristics of the individual is concerned. In the great majority of cases the qualitative as well as the quantitative tests should be made upon the mixed excretion for a twenty-four-hour period as well as upon a night sample as ‘above described. 


\section{URINE : PHYSIOLOGICAL CONSTITUENTS}

\section{Organic Physiological Constituents}

Urea.

Uric acid.

Creatinine.

Creatine. ${ }^{2}$

Ethereal sulphuric acids......

Indoxyl-sulphuric acid.

Phenol- and p-cresol-sulphuric acids.

Pyrocatechol-sulphuric acid.

Skatoxyl-sulphuric acid.

Hippuric acid.

Oxalic acid.

Neutral sulphur compounds....

Cystine.

Chondroitin-sulphuric acid.

Thiocyanates.

Taurine derivatives.

Oxyproteic acid.

Alloxyproteic acid.

Uroferric acid.

Allantoin.

Para-oxyphenyl-acetic acid.

Para-oxyphenyl-propionic acid.

Homogentisic acid.

Uroleucic acid.

Oxymandelic acid.

hynurenic acid.

Amino-acicls.

Peptides.

Benzoic acid.

Nucleoprotein.

Oxaluric acid.

Glucose.

${ }^{1}$ It is impossible to make any absolule classification of the physiological and pathological constituents of the urine. A substance may be present in the urine in small a mount physiologically and be sufficiently increased under certain conditions as to be termed a pathological constituent. Therefore it depends, in some instances upon the quantily of a constituent present whether it may be correctly termed a physiological or a pathological constituent.

${ }^{2}$ Normal constituent of urine of adults but found in larger amount in urine of infants and children (see p. 509). 
Enzymes ............ $\left\{\begin{array}{l}\text { Pepsin. } \\ \text { Gastric rennin. } \\ \text { Amylase. }\end{array}\left\{\begin{array}{l}\text { Acetic acid. } \\ \text { Butyric acid. } \\ \text { Formic acid. }\end{array}\right.\right.$

Paralactic acid.

Phenaceturic acid.

Urocanic acid.

Phosphorized compounds..... $\{$ Glycerophosphoric acid.

Phosphocarnic acid.

Urochrome.

Pigments............. Urobilin.

Uroerythrin.

Ptomaines and leucomaines.

Purine Bases............. $\left\{\begin{array}{l}\text { Adenine. } \\ \text { Guanine. } \\ \text { Xanthine. } \\ \text { Epiguanine. } \\ \text { Episarkine. } \\ \text { Hypoxanthine. } \\ \text { Paraxanthine. } \\ \text { Heteroxanthine. } \\ \text { I-Methylxanthine. }\end{array}\right.$

Ammonia.

2. Inorganic Physiological Constituents

Sulphates.

Chlorides.

Phosphates.

Sodium and potassium.

Calcium and magnesium.

Carbonates.

Iron.

Fluorides.

Nitrates.

Silicates.

Hydrogen peroxide.

Normal urine varies widely in composition, being influenced by diet and other factors. The following table represents the composition of a normal urine. ${ }^{1}$

${ }^{1}$ Vierordt: Daten und Tabellen. Jena, I906, p. 330. 
COMPOSITION OF A NORMAL URINE

Volume (24 hours) I 500 c.c.

\begin{tabular}{|c|c|c|}
\hline Constituent & $\begin{array}{c}\text { Absolute } \\
\text { weight, } \\
\text { grams }\end{array}$ & $\begin{array}{l}\text { Approximate } \\
\text { percentage }\end{array}$ \\
\hline 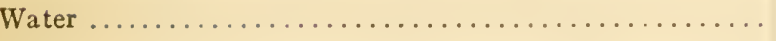 & 1440.00 & 96.0 \\
\hline$\ldots \ldots \ldots \ldots \ldots \ldots \ldots$ & 60.0 & $4 \cdot 0$ \\
\hline 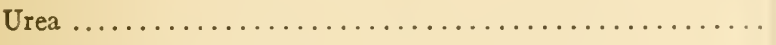 & 35.0 & $2 \cdot 33$ \\
\hline 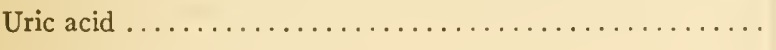 & 0.75 & 0.05 \\
\hline 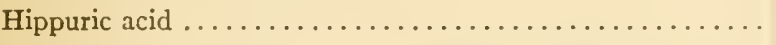 & 0.7 & 0.05 \\
\hline 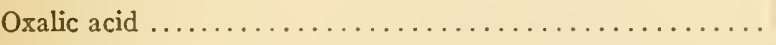 & $0.0 I_{5}$ & $0.00 \mathrm{r}$ \\
\hline Aromatic oxyacids $\ldots \ldots \ldots \ldots \ldots \ldots \ldots$ & 0.06 & 0.004 \\
\hline 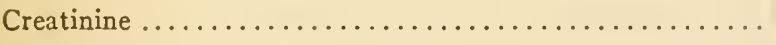 & I. 0 & 0.07 \\
\hline 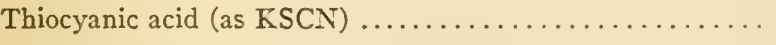 & 0.15 & $0.0 \mathrm{I}$ \\
\hline 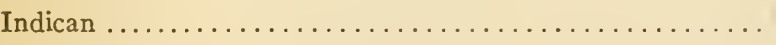 & o.oI & 0.001 \\
\hline 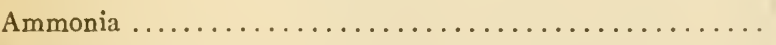 & 0.65 & 0.04 \\
\hline Sodium chloride $\ldots \ldots \ldots \ldots \ldots \ldots \ldots \ldots \ldots \ldots$ & 16.5 & I. I \\
\hline Phosphoric acid $\ldots \ldots \ldots \ldots \ldots \ldots \ldots$ & 2.5 & $0 . x_{5}$ \\
\hline Total sulphuric acid ${ }^{1} \ldots$ & 2.5 & $0 . I_{5}$ \\
\hline Silicic acid . . . . . . . . . . . . & 0.45 & 0.03 \\
\hline Potassium $\left(\mathrm{K}_{2} \mathrm{O}\right) \ldots \ldots \ldots \ldots \ldots \ldots \ldots \ldots \ldots$ & 2.5 & $0 . x_{5}$ \\
\hline 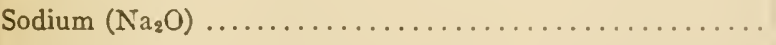 & 5.0 & 0.3 \\
\hline Calcium $(\mathrm{CaO}) \ldots$ & 0.25 & 0.015 \\
\hline Magnesium ( $\mathrm{MgO}) \ldots$ & 0.30 & 0.02 \\
\hline Iron . & 0.005 & 0.0004 \\
\hline
\end{tabular}

${ }^{1}$ For data as to "partition" of sulphur and nitrogen, see Chapter XXVII on Metabolism. 


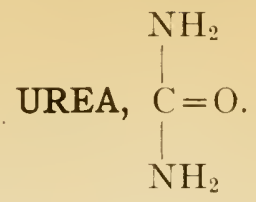

Urea is the principal end-product of the metabolism of protein substances. It was formerly believed that about 90 per cent of the total nitrogen of the urine was present as urea. Folin, however, has shown that the distribution of the nitrogen of the urine among urea and the other nitrogen-containing bodies present depends entirely upon the absolute amount of the total nitrogen excreted. He found that a decrease in the total nitrogen excretion was always accompanied by a decrease in the percentage of the total nitrogen excreted as urea, and that after so regulating the diet of a normal person as to

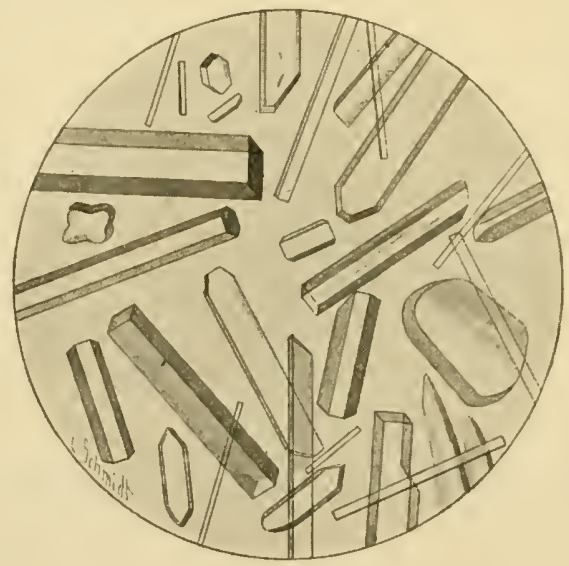

Fig. II 8.-UREA.

cause the excretion of total nitrogen to be reduced to 3-4 grams in 24 hours, only about 60 per cent of this nitrogen appeared in the urine as urea. His experiments also seem to show urea to be the only one of the nitrogenous excretions which is relatively as well as absolutely decreased as a result of decreasing the amount of protein metabolized. This same investigator reports a hospital case in which only 14.7 per cent of the total nitrogen was present as urea and about 40 per cent was present as ammonia. Mörner had previously reported a case in which but 4.4 per cent of the total nitrogen of the urine was present as urea, and 26.7 per cent was present as ammonia.

Urea occurs most abundantly in the urine of man and carnivora and in somewhat smaller amount in the urine of herbivora; the urine of fishes, amphibians, and certain birds also contains a small amount of 
the substance. Urea is also found in nearly all the fluids and in many of the tissues and organs of mammals. The amount excreted, under normal conditions, by an adult man in 24 hours is about $30-35$ grams. The excretion is greatest in amount aiter a diet of meat, and least in amount after a diet consisting of non-nitrogenous foods; this is due to the fact that the urea output is regulated by the protein ingestion. It is true also that a non-nitrogenous diet has a tendency to decrease the metabolism of the tissue proteins and thus cause the output of urea under these conditions to fall below the output of urea observed during starvation. The output of urea is also increased after copious water- or beerdrinking. The increase is probably due primarily to the washing out of the tissues of the urea previously formed, but which had not been removed in the normal processes, and secondarily to a stimulation of protein catabolism.

Urea may be formed in the organism from amino-acids such as leucine, glycocoll, and aspartic acid: it may also be formed from ammonium carbonate $\left(\mathrm{NH}_{4}\right)_{2} \mathrm{CO}_{3}$ or ammonium carbamate, $\mathrm{H}_{4}$ N.O.CO.NH 2 .

There are differences of opinion regarding the transformation of the substances just named into urea, but there is rather conclusive evidence that at least a part of the urea is formed in the liver; it may be formed in other organs or tissues as well.

Urea crystallizes in long, colorless, four- or six-sided, anhydrous, rhombic prisms (Fig. II S), which melt at $132^{\circ} \mathrm{C}$. and are soluble in water or alcohol and insoluble in ether or chloroform. Ii a crystal of urea is heated in a test-tube, it melts and decomposes with the liberation of ammonia. The residue contains cyanuric acid,

and biuret,
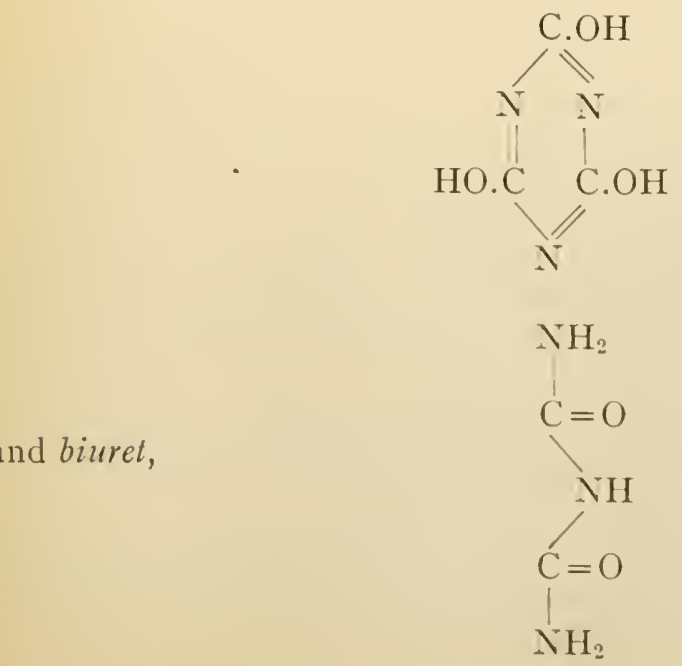
The biuret may be dissolved in water and a reddish-violet color obtained by treating the aqueous solution with copper sulphate and potassium hydroxide (see Biuret Test, page 98). Certain hypochlorites or hypobromites in alkaline solution have the power of decomposing urea into nitrogen, carbon dioxide, and water. Sodium hypobromite brings about this decomposition, as follows:

$$
\mathrm{CO}\left(\mathrm{NH}_{2}\right)_{2}+3 \mathrm{NaOBr} \rightarrow 3 \mathrm{NaBr}+\mathrm{N}_{2}+\mathrm{CO}_{2}+{ }_{2} \mathrm{H}_{2} \mathrm{O} .
$$

This property forms the basis for a clinical quantitative determination of urea (see page 496 ).

The soy bean has been shown to contain an enzyme called urease which has the power to decompose urea with the liberation of ammonia. ${ }^{1}$ This fact is made use of in the quantitative determination of urea (see Chapter XXVI).

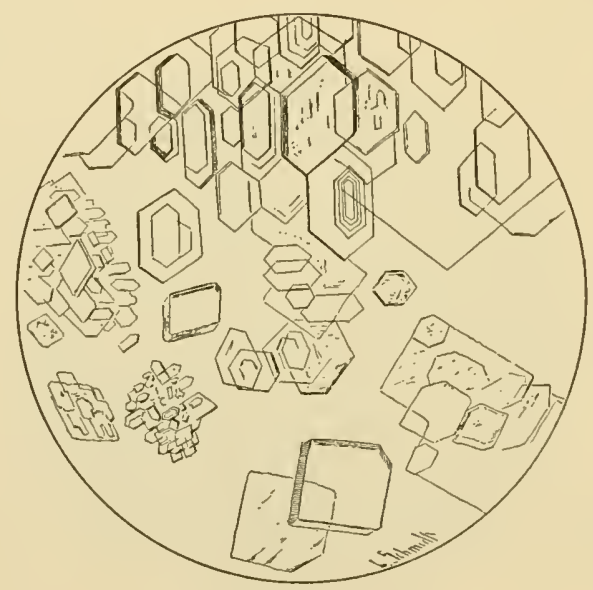

Fig. i ig.-Urea Nitrate.

Urea has the power of forming crystalline compounds with certain acids; urea nitrate and urea oxalate are the most important of these compounds. Urea nitrate, $\mathrm{CO}\left(\mathrm{NH}_{2}\right)_{2} \cdot \mathrm{HNO}_{3}$, crytallizes in colorless, rhombic or six-sided tiles (Fig. II9, above), which are easily soluble in water. Urea oxalate, $\left[\mathrm{CO}\left(\mathrm{NH}_{2}\right)_{2}\right]_{2} \cdot \mathrm{H}_{2} \mathrm{C}_{2} \mathrm{O}_{4}$, crystallizes in the form of rhombic or six-sided prisms or plates (Fig. I2I, page 376): the oxalate differs from the nitrate in being somewhat less soluble in water. The formation of the nitrate and oxalate and the decomposition of urea by the enzyme urease are the most satisfactory methods for the detection of urea.

A decrease in the excretion of urea is observed in many diseases in which the diet is much reduced and in some disorders as a result of

${ }^{1}$ Takeuchi: Jour. College of Agr., Tokyo, Igog, Part I. 
alterations in metabolism, e.g., myxedema, and in others as a result of changes in excretion, as in severe and advanced kidney disease. A pathological increase is found in a large proportion of diseases which are associated with a toxic state. In marked acidosis it may be considerably decreased relative to the total nitrogen (see Ammonia).

\section{EXPERIMENTS ON UREA}

I. Isolation from the Urine. ${ }^{1}$-Place 800 c.c. of urine in a precipitating jar, add 250 c.c. of baryta mixture, ${ }^{2}$ and stir thoroughly. Filter off the precipitate of phosphates, sulphates, urates, and hippurates and evaporate the filtrate on a water-bath to a thick syrup. This syrup contains chlorides, creatinine, organic salts, pigments, and urea. Extract the syrup with warm 95 per cent alcohol and filter again. The filtrate contains the urea contaminated with pigment. Decolorize the filtrate by boiling with animal charcoal, filter again, and stand the filtrate away in a cold place for crystallization. Examine the crystals under the microscope and compare them with those shown in Fig. 118, page 372 .

2. Solubility.-Test the solubility of urea, prepared by yourself or furnished by the instructor, in water and in alcohol and ether.

3. Melting-point.-Determine the melting-point of some pure urea furnished by the instructor. Proceed as follows: Into an ordinary melting-point tube, sealed at one end, introduce powdered urea. Fasten the tube to the bulb of a thermometer as shown in Fig. $\mathrm{I} 2 \mathrm{O}$, and suspend the bulb and its attached tube in a small beaker containing sulphuric acid. Gently raise the temperature of the acid by means of a low flame, stirring the fluid continually, and note the temperature at which the urea begins to melt.

4. Crystalline Form.-Dissolve a crystal of pure urea in a few drops of 95 per cent alcohol and place I-2 drops of the alcoholic solution on a microscopic slide. Allow the alcohol to evaporate spontaneously,

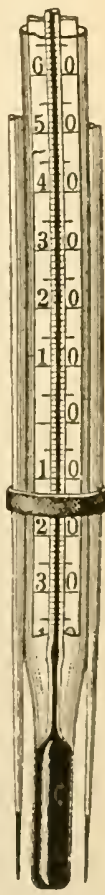

Fig. I 20.-MELTINGPOINT TUBES FASTENED TO BULB OF THERMOMETER. examine the crystals under the microscope, and compare them with those reproduced in Fig. Ir8, page 372. Recrystallize a little urea from water in the same way and compare the crystals with those obtained from the alcoholic solution.

5. Formation of Biuret.-Place a small amount of urea in a dry test-tube and heat carefully in a low flame. The urea melts at $132^{\circ} \mathrm{C}$. and liberates ammonia. Continue heating until the fused mass begins to solidify. Cool the

2 'The method based upon the precipitation by nitric acid is also satisfactory (see Hoppe-Seyler's Handbuch der Physiol. und Pathol. Chem. Anal., Eighth edition, I909, P. I 45).

${ }_{2}$ Baryta mixture consists of a mixture of I volume of a saturated solution of $\mathrm{Ba}\left(\mathrm{NO}_{3}\right)_{2}$; and 2 volumes of a saturated solution of $\mathrm{Ba}(\mathrm{OH})_{2}$. 
tube, dissolve the residue in dilute potassium hydroxide solution, and add very dilute copper sulphate solution (see page 98). The purplish-violet color is due to the presence of biuret which has been formed from the urea through the application of heat as indicated. This is the reaction :
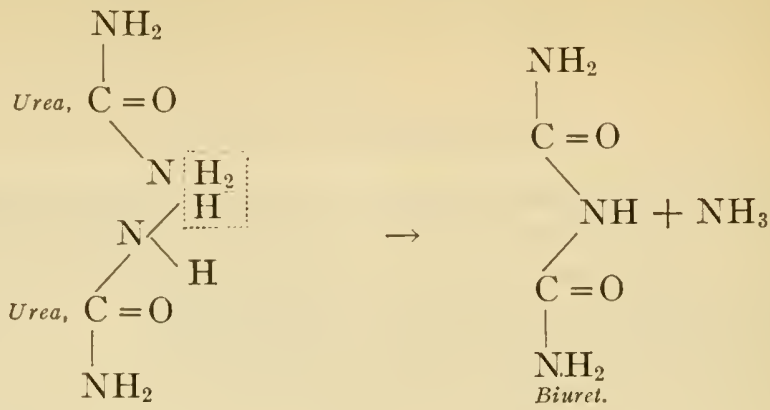

6. Urea Nitrate.-Prepare a concentrated solution of urea by dissolving a little of the substance in a few drops of water. Place a drop of this solution on a microscopic slide, add a drop of concentrated nitric acid, and examine under the microscope. Compare the crystals with those reproduced in Fig. I I9, page 374 .

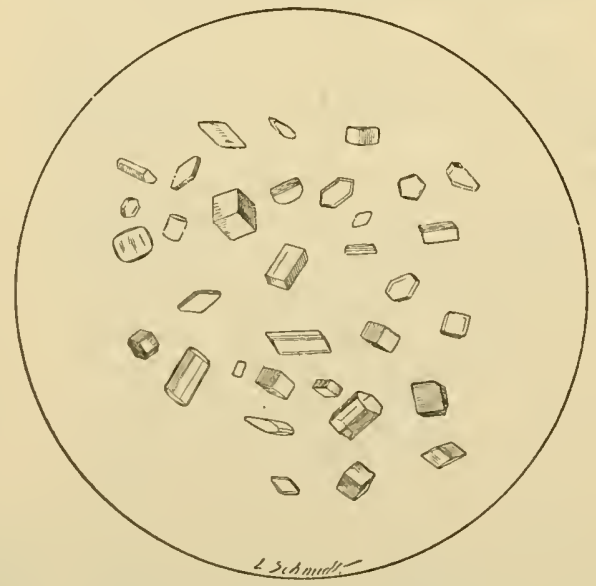

Fig. I 2 I.-UREa Oxalate.

7. Urea Oxalate.- To a drop of a concentrated solution of urea, prepared as described in the last experiment (6), add a drop of a saturated solution of oxalic acid. Examine under the microscope and compare the crystals with those shown in Fig. 121 , above.

8. Decomposition by Sodium Hypobromite.-Into a mixture of 3 c.c. of concentrated sodium hydroxide solution and 2 c.c. of bromine water in a test-tube introduce a crystal of urea or a small amount of concentrated solution of urea. Through the influence of the sodium hypobromite, $\mathrm{NaOBr}$, the urea is decomposed and carbon dioxide and nitrogen are liberated. The carbon dioxide is absorbed by the excess of sodium hydroxide, while the nitrogen is evolved and causes the marked effervescence observed. This property forms the basis for 
one of the methods in common use for the quantitative determination of urea. Write the equation showing the decomposition of urea by sodium hypobromite.

9. Furfural Test. - To a few crystals of urea in a small porcelain dish add $\mathrm{I}-2$ drops of a concentrated aqueous solution of furfural and $I-2$ drops of concentrated hydrochloric acid. Note the appearance of a yellow color which gradually changes into a purple. Allantoin also responds to this test (see page 392).

It is claimed that all ammonium compounds and all compounds containing the amino $\left(-\mathrm{NH}_{2}\right)$ group yield nitrogen when treated with hypobromite as in this test.

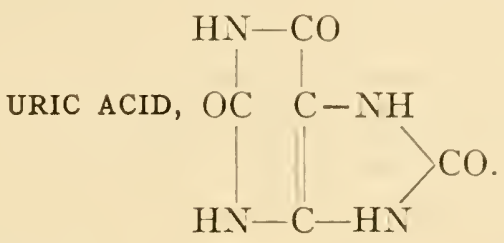

Uric acid is one of the most important of the constituents of the urine. It is generally stated that normally about $0.7 \mathrm{gram}$ is excreted in 24 hours, but that this amount is subject to wide variations, particularly under certain dietary and pathological conditions. It has been shown, however, that the average daily excretion of uric acid for ten men ranging in age from 19 to 29 years and fed a normal mixed diet was 0.597 gram, a value somewhat lower than the generally accepted average of 0.7 gram for such a period. On a purine-free diet the uric acid output may be $0.1-0.5$ gram per day, whereas a high purine diet may yield a daily output of 2 grams. Uric acid is a diureide and consequently upon oxidation may yield two molecules of urea. It acts as a weak dibasic acid and forms two classes of salts, neutral and acid. The neutral potassium and lithium urates are the most easily soluble of the alkali salts; the ammonium urate is difficultly soluble. The acid-alkali urates are more insoluble and form the major portion of the sediment which separates upon cooling the concentrated urine; the alkaline eartin urates are very insoluble. Ordinarily uric acid occurs in the urine in the form of urates and upon acidifying the liquid the uric acid is liberated and deposits in crystalline form. This property forms the basis of one of the older methods for the quantitative determination of uric acid (Heintz Method, Chapter XXVI).

Uric acid is very closely related to the purine bases as may be seen from a comparison of its structural formula with those of the purine bases given on page r 27. According to the purine nomenclature it is designated 2-6-8-trioxypurine. Uric acid forms the principal endproduct of the nitrogenous metabolism of birds and scaly amphibians; in the human organism it occupies the fourth position inasmuch as here 
urea, ammonia, and creatinine are the chief end-products of nitrogenous metabolism. It is generally said that the relation existing between uric acid and urea in human urine under normal conditions varies on the average from $I: 40$ to $\mathrm{I}: \mathrm{ro0}$ and is subject to wider variations under pathological conditions; and further that because of the high content of uric acid in the urine of newborn infants the ratio may be reduced to I: ro or even lower. We now know that this ratio of uric acid to urea is of little significance under any conditions.

In man, uric acid probably results principally from the destruction of nuclein material. It may arise from nuclein or other purine material ingested as food or from the disintegrating cellular matter of the organism. The uric acid resulting from the first process is said to be of $e x$ ogenous origin, whereas the product of the second form of activity is said to be of endogenous origin. As a result of experimentation, Sivén, and Burian and Schur, and Rockwood claim that the amount of endogenous uric acid formed in any given period is fairly constant for each individual under normal conditions, and that it is entirely independent of the total amount of nitrogen eliminated. Folin has taken exception to the statements of these investigators and claims that, following a pronounced decrease in the amount of protein metabolized, the absolute quantity of uric acid is decreased but that this decrease is relatively smaller than the decrease in the total nitrogen excretion and that the per cent of the uric acid nitrogen, in terms of the total nitrogen, is therefore decidedly increased. According to Mareš, ${ }^{1}$ food-stuffs act to increase the endogenous uric acid output by stimulating the digestive glands to activity. That a portion of the endogenous uric acid may arise in this way has recently been shown by Mendel and Stehle. ${ }^{2}$

In birds the formation of uric acid is analogous to the formation of urea in man. In these organisms it is derived principally from the protein material of the tissues and the food and is formed through a process of synthesis which occurs for the most part in the liver; a comparatively small fraction of the total uric acid excretion of birds may result from nuclein material.

When pure, uric acid may be obtained as a white, odorless, and tasteless powder, which is composed principally of small, transparent, crystalline, rhombic plates. Uric acid as it separates from the urine is invariably pigmented, and crystallizes in a large variety of characteristic forms, e.g., dumb-bells, wedges, rhombic prisms, irregular rectangular or hexagonal plates, whetstones, prismatic rosettes, etc. Uric acid is insoluble in alcohol and ether, soluble with difficulty in boiling

1 Mareš: Arch.f.d. ges. Physiol., I34, 59, I9ro.

2 Mendel and Stehle: Jour. Biol. Chem., 22, 215 , I915. 
water ( $1: 1800$ ) and practically insoluble in cold water (I:39,480, at $18^{\circ} \mathrm{C}$.). It is soluble in alkalis, alkali carbonates, boiling glycerol: concentrated sulphuric acid, and in certain organic bases such as ethylamine and piperidine. It is claimed that the uric acid is held in solution in the urine by the urea and disodium hydrogen phosphate present. Uric acid possesses the power of reducing cupric hydroxide in alkaline solution and may thus lead to an erroneous conclusion in testing for sugar in the urine by means of Fehling's or Trommer's test. A white precipitate of cuprous urate is formed if only a small amount of cupric hydroxide is present, but if enough of the copper salt is present the characteristic red or brownish-red precipitate of cuprous oxide is obtained. Uric acid does not possess the power of reducing bismuth in alkaline solution and therefore does not interfere in testing for sugar in the urine by means of Boettger's or Nylander's tests.

In addition to being an important urinary constituent uric acid is normally present in the brain, heart, liver, lungs, pancreas, and spleen; it also occurs in the blood of birds and has been detected in traces in human blood under normal conditions.

Pathologically, the excretion of uric acid is subject to wide variations, but the experimental findings are rather contradictory. It may be stated with certainty, however, that in leukemia, because of the destruction of nuclein material, the uric acid output is increased absolutely as well as relatively to the urea output; under these conditions the ratio between the uric acid and urea may be as low as $\mathrm{I}: 9$, whereas the normal ratio, as we have seen, is $I: 50$ or higher. An actual output of I 2 grams of uric acid per day has been reported in leukemia. In the study of the influence of $\mathrm{X}$-ray on metabolism Edsall and others have reached some interesting conclusions. Edsall found that the excretion of uric acid is usually increased and that in some conditions, particularly in leukemia, it may be greatly increased. The excretion of total nitrogen, phosphates, and other sustances may also be considerably increased.

In gout the kidney is said to lose the power of properly eliminating uric acid and it collects in the blood in abnormally high concentration.

Normal $=\mathrm{I}-3 \mathrm{mg}$. uric acid per $\mathrm{roo}$ grams of blood.

Gout $=3-6 \mathrm{mg}$. uric acid per 100 grams of blood.

In gout the uric acid content of the urine is generally low preceding an attack and increases during the attack. Atophan has been found to increase the uric acid output in gout, apparently due to increased kidney activity.

The uric acid content of the urine is of importance in relation to the formation of uric acid calculi. The administration of alkali carbonates 
and citrates, or the feeding of base-forming foods, by decreasing the acidity of the urine increases its solvent power for uric acid and decreases the liability of formation of this type of calculus. ${ }^{1}$

\section{Experiments on URIC ACID}

I. Isolation from the Urine--Place about 200 c.c. of filtered urine in a beaker, render it acid with 2-Io c.c. of concentrated hydrochloric acid, stir thoroughly, and stand the vessel in a cold place for 24 hours. Examine the pigmented crystals of uric acid under the microscope and compare them with those shown in Fig. I36, page 46r, and Pl. V, opposite.

2. Solubility.-Try the solubility of pure uric acid, furnished by the instructor, in water, dilute acid and alkali and in alcohol, ether and concentrated sulphuric acid.

3. Crystalline Form of Pure Uric Acid.-Place about roo c.c. of water in a small beaker, render it distinctly alkaline with potassium hydroxide solution and

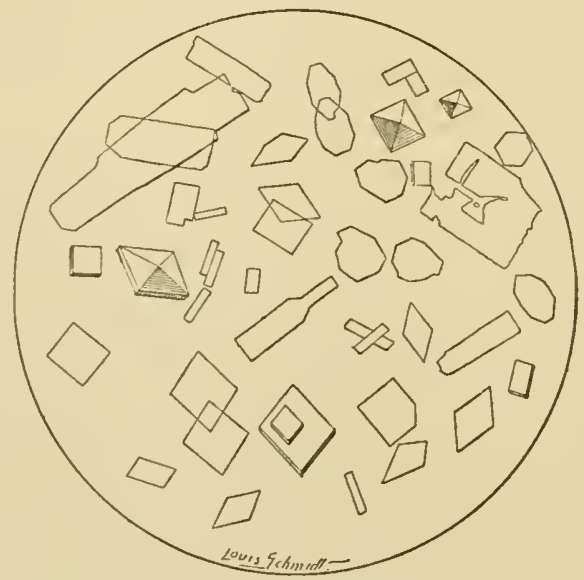

Fig. I 22.-PURE URIC ACID.

add a small amount of pure uric acid, stirring continuously. Cool the solution, render it distinctly acid with hydrochloric acid and allow it to stand in a cool place for crystallization. Examine the crystals under the microscope and compare them with those reproduced in Fig. 122.

4. Murexide Test.-To a small amount of pure uric acid in a small evaporating dish add 2-3 drops of concentrated nitric acid. Evaporate to dryness carefully on a water-bath or over a very low flame. A red or yellow residue remains which turns purplish red after cooling the dish and adding a drop of very dilute ammonium hydroxide. The color is due to the formation of murexide. If potassium hydroxide is used instead of ammonium hydroxide a purplish violet color due to the production of the potassium salt is obtained. The color disappears upon warming; with certain related bodies (purine bases) the color persists under these conditions.

${ }^{1}$ Blatherwick: Arch. Int. Med., I4, 409, I9I4. 
PLATE V.

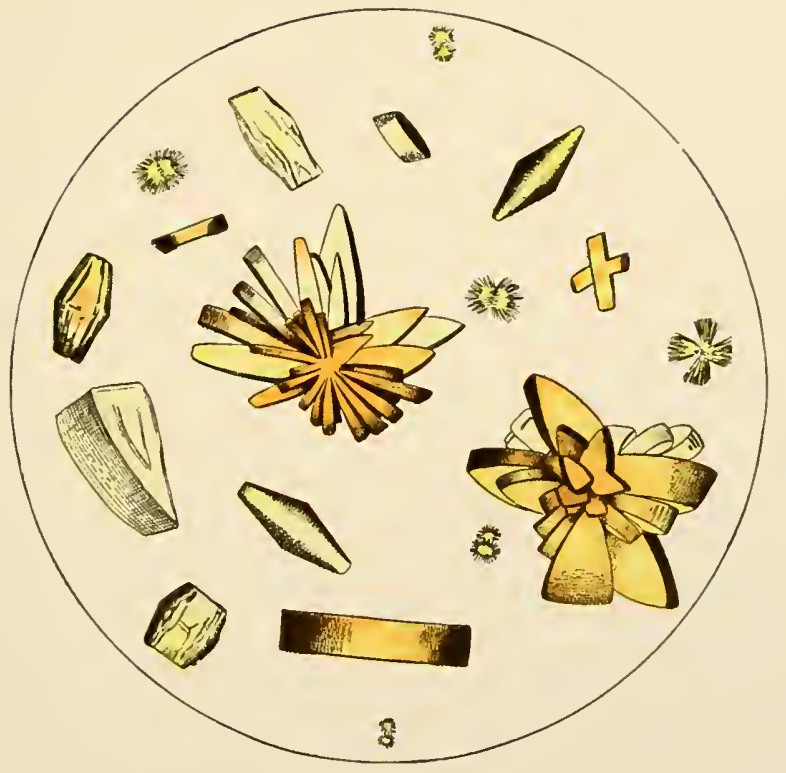

Uric Acid Crystils. Normal Color. (From Purdy, after leyer.) 

In this reaction the uric acid is oxidized to dialuric acid and alloxan. These two substances condense to form alloxantin. This alloxantin reacts with ammonium hydroxide to form purpuric acid. The purple color is due to the formation of ammonium purpurale or murexide.

5. Phosphotungstic Acid Reaction (Folin).-To 20 c.c. of saturated sodium carbonate solution in a small beaker add a small amount of uric acid. Stir the solution until the uric acid has dissolved, then add I c.c. of Folin's uric acid reagent (see Chapter XXVI). A blue color results.

6. Silver Reduction Test (Schiff).-Dissolve a small amount of pure uric acid in sodium carbonate solution and transfer a drop of the resulting mixture to a strip of filter paper saturated with.silver nitrate solution. A yellowish-brown or black coloration due to the formation of reduced silver is produced.

It is claimed that chlorides interfere with this test.

7. Ganassini's Test. ${ }^{1-D i s s o l v e ~ a ~ s m a l l ~ a m o u n t ~ o f ~ u r i c ~ a c i d ~ i n ~ s o d i u m ~ c a r b o n-~}$ ate. Irecipitate the dissolved uric acid by means of zinc chloride, filter off the precipitate, and permit it to stand in contact with the air. A sky-blue color will develop, a color change which may be hastened by sunlight. A similar reaction may be obtained by treating the original precipitate with $\mathrm{K}_{2} \mathrm{~S}_{2} \mathrm{O}_{3}$.

8. Influence upon Fehling's Solution.-Dilute I c.c. of Fehling's solution with 4 c.c. of water and heat to boiling. Now add slowly, a few drops at a time, I-2 c.c. of a concentrated solution of uric acid in potassium hydroxide, heating after each addition. From this experiment what do you conclude regarding the possibility of arriving at an erroneous decision when testing for sugar in the urine by means of Fehling's test?

9. Reduction of Nylander's Reagent.-To 5 c.c. of a solution of uric acid in potassium hydroxide add about one-half a cubic centimeter of Nylander's reagent and heat to boiling for a few moments. Do you obtain the typical black end-reaction signifying the reduction of the bismuth?

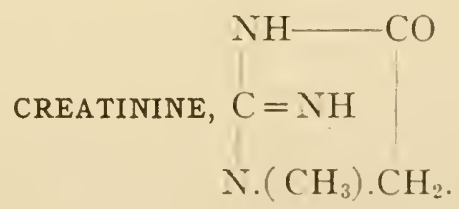

Creatinine is the anhydride of creatine and is a constituent of normal human urine. The theory that creatinine is derived from the creatine of ingested muscular tissue as well as from the creatine of the muscular tissue of the organism has been proven to be incorrect by Folin, Klercker, and Wolf and Shafier. Shaffer believes that creatinine is the result of some special process of normal metabolism which takes place to a large extent, if not entirely, in the muscles. and further that the amount of such creatinine elimination, expressed in milligrams per kilogram body acight, is an index of this special process. ${ }^{2}$ He further

${ }^{1}$ Ganassini: Boll. soc., I008, No. I.

"He proposes to designate as the "creatinine coefficient" the excretion of crealinincnitrogen (mg.) per kilogrum of body aicight. 
states that the muscular efficiency of the individual depends upon the intensity of this process. Under normal conditions about $\mathrm{I}-\mathrm{I} 1 / 4 \mathrm{gram}$ of creatinine is excreted by an adult man in 24 hours, ${ }^{1}$ the exact amount depending in great part upon the nature of the food and decreasing markedly in starvation. Very little that is important is known regarding the excretion of creatinine under pathological conditions. The creatinine content of the urine is said to be increased in typhoid fever, typhus, tetanus, and pneumonia, and to be decreased in anæmia, chlorosis, paralysis, muscular atrophy, advanced degeneration of the kidneys, and in leukemia (myelogeneous, lymphatic and pseudo). An increase

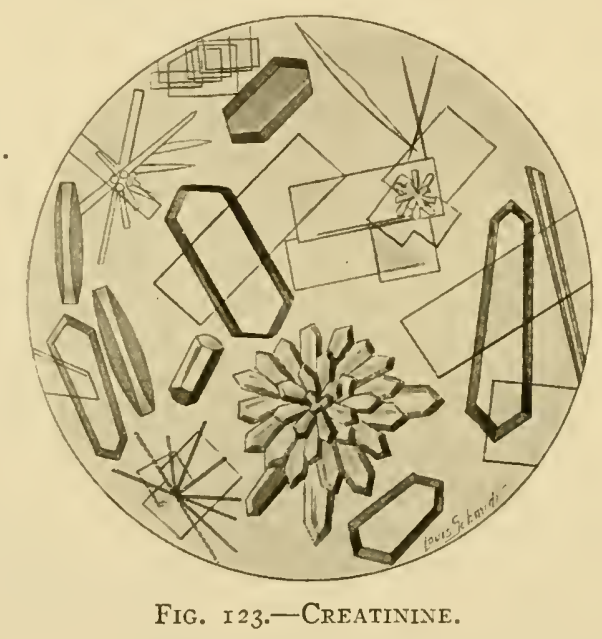

of creatinine was also noted in diabetes, an increase probably due to the creatinine content of the meat eaten. The greater part of the data, however, relating to the variation of the creatinine excretion under pathological conditions are not of much value since in nearly every instance the diet was not sufficiently controlled to permit the collection of reliable data. And further, until the advent of the Folin method (see page 506) there was no accurate method for the quantitative determination of creatinine. Shaffer has called attention to the fact that a low excretion of creatinine is found in the urine of a remarkably large number of pathological subjects, representing a variety of conditions, and that it is therefore evident that the excretion of an abnormally small amount of this substance is by no means peculiar to any one disease. A considerable increase in the creatinine content of the blood has been observed in uremia. ${ }^{2}$

${ }^{1}$ According to Shaffer the amount excreted by strictly normal individuals is between 7 and II mg. of creatinine-nitrogen per kilogram of body weight.

${ }^{2}$ Folin and Denis: Jour. Biol. Chem., 17,487 , x 914.

Myers and Fine: Jour. Biol. Chem., 20, 391, 1914. 
Creatinin ecrystallizes in colorless, glistening monoclinic prisms (Fig. 123, page 382) which are soluble in about I2 parts of cold water; they are more soluble in warm water and in warm alcohol. It forms salts only with strong mineral acids. One of the most important and interesting of the compounds of creatinine is creatinine-zinc chloride, $\left(\mathrm{C}_{4} \mathrm{H}_{7} \mathrm{~N}_{3} \mathrm{O}\right)_{2}-$ $\mathrm{ZnCl}_{2}$, which is formed from an alcoholic solution of creatinine upon treatment with zinc chloride in acid solution. Creatinine has the power of reducing cupric hydroxide in alkaline solution and in this way may interfere with the determination of sugar in the urine. In the reduction by creatinine the blue liquid is first changed to a yellow, and the formation of a brownish-red precipitate of cuprous oxide is brought about only after continuous boiling with an excess of the copper salt. Creatinine does not reduce alkaline bismuth solutions and therefore does not interfere with Nylander's and Boettger's tests.

It has recently been shown by Folin that the absolute quantity of creatinine eliminated in the urine on a meat-free diet is a constant quantity different for different individuals, but wholly independent of quantitative changes in the total amount of nitrogen eliminated. Shaffer has very recently confirmed these findings and has shown that the output of creatinine under these conditions is constant from hour to hour as well as from day to day.

\section{EXPERIMENTS ON CREATININE}

r. Preparation of Pure Creatinine from Urine (Folin-Benedict ${ }^{1}$ ).-To ro liters ${ }^{2}$ of undecomposed urine in a large precipitating jar add with stirring a hot solution of $r 80$ grams of picric acid in 450 c.c. of boiling alcohol. Allow to stand over night and syphon off the supernatant fluid. Pour the residue upon a large Buchner funnel, drain with suction, wash once or twice with cold saturated picric acid and suck dry. Treat the dry or nearly dry picrate in a large mortar or evaporating dish with enough concentrated $\mathrm{HCl}$ to form a moderately thin paste (about 60 c.c. of acid for each 100 grams of picrate) and stir the mixture thoroughly with the pestle for $3^{-5}$ minutes. Filter with suction on a hardened paper, and wash the residue twice with enough water to cover it, sucking as nearly dry as possible each time. Transfer the filtrate to a large flask and neutralize with an excess of solid magnesium oxide (the "heavy" variety is best). Add this oxide in small portions with cooling of the flask under running water between the additions. Neutralization of the acid will be indicated by a bright yellow color of the mixture, or litmus paper may be used to test it. Filter with suction. Wash the residue twice with water. Immediately add a few cubic centimeters of glacial acetic acid to the filtrate to make it strongly acid. Pay no attention to any precipitate that may form, but dilute the solution with about 4 volumes of 95 per

1 Benedict: Jour. Biol. Chem., I S, IS2, IgIf.

Folin: Ibid., I 7463 , I914.

2 If it is simply desired to demonstrate the presence of creatinine, I liter may be employed and the various reagents reduced accordingly: 
cent alcohol. After 15 minutes filter off the slight precipitate which forms. Treat the final filtrate with $30-40$ c.c. of 30 per cent zinc chloride. Stir and let stand over night in a cool place. Pour off the supernatant liquid and collect the creatinine zinc chloride on a Buchner funnel, wash once with water, then thoroughly with 50 per cent alcohol, finally with 95 per cent alcohol and dry. A nearly white, light crystalline powder should be obtained. The yield should be 90-95 per cent of the original creatinine (usually about I.5-I.8 grams of creatinine zinc chloride per liter of urine).

Recrystallize the creatinine-zinc chloride by treating Io grams with roo c.c. of water and about 60 c.c. of normal sulphuric acid, heating the mixture until a clear solution is obtained. Add about 4 grams of purified animal charcoal, continue boiling for about a minute, filter with suction through a small Buchner funnel, pouring the filtrate back on the filter three or four times until it runs through perfectly colorless. Wash residue with hot water and transfer the total filtrate to a beaker and while hot treat with a little strong zinc chloride solution ( 3 c.c.) and with about 7 grams of potassium acetate dissolved in a little water. After ten minutes dilute with an equal volume of alcohol, and allow to stand in a cold place for some hours. Filter off the crystalline product and examine under microscope (see Fig. I24). To remove the small amount of potassium sulphate which it contains stir up with twice its weight of water, filter, wash with a little water and then with alcohol. The preparation should be snow white. Yield, 8590 per cent.

Place the finely powdered recrystallized creatinine zinc chloride in a dry flask and treat with seven times its weight (by volume) of concentrated aqueous ammonia. Warm slightly and agitate gently until a clear solution is obtained, care being taken to drive off no more ammonia during the warming than is necessary to obtain a clear solution. Stopper the flask, allow to cool, place in the ice-box for an hour or more. Pure creatinine crystallizes out. It may be recrystallized from boiling alcohol or concentrated ammonia, but this is usually unnecessary. The product is perfectly pure and can be used as a standard in the quantitative determination of creatine and creatinine. See chapters on Quantitative Analysis of Urine and Blood.

1. Preparation of Creatine.-Creatine may be prepared from creatinine zinc chloride by decomposition with calcium hydrate, the process being one of hydrolysis (Benedict).

One hundred grams of creatinine zinc chloride are treated with about 700 c.c. of water in a large casserole and the mixture heated to boiling; I 50 grams of pure powdered calcium hydrate are then added, with stirring, and the mixture boiled gently for 20 minutes (with occasional stirring). The hot mixture is then filtered with suction, the residue being washed with hot water. The filtrate is then treated with hydrogen sulphide gas for a few minutes and poured through a folded filter to remove the zinc. The filtrate is acidified by the addition of about 5 c.c. of glacial acetic acid and boiled down rapidly to a volume of about 200 c.c. This solution is allowed to stand over night, preferably in a cool place. The next day the crystallized creatine is filtered off with suction, washed with a very little cold water, and then thoroughly washed with alcohol and dried. ${ }^{1}$ This product is then recrystallized by dissolving in about seven times its weight of boiling water and allowing

${ }_{1}^{1}$ The filtrate obtained at this point should be diluted with alcohol and treated with zinc chloride ( 50 c.c. of a 30 per cent solution) for recovery of the unconverted creatinine. 
the solution to cool slowly and stand for some hours. This product should be perfectly pure creatine. If necessary it can be recrystallized with very little loss. The crystallized product should be filtered off, washed with alcohol and ether and dried in air for about half an hour. Thus obtained the creatine contains water of crystallization which it loses very readily upon exposure to air. To prepare creatine which can be weighed with absolute exactness it is necessary to dehydrate this product by" heating for some hours at about $95^{\circ}$.

The yield in this process is about 18 grams of recrystallized creatine, and about 55 grams of creatinine zinc chloride recovered. Longer boiling with lime does not bring about a greater yield, as after the 20-minute point creatine is decomposed almost exactly as fast as it is formed.

Examine the crystals of creatine under the microscope and compare with illustration in Chapter XIX on Muscular Tissue. For other creatine tests see Chapter XIX.

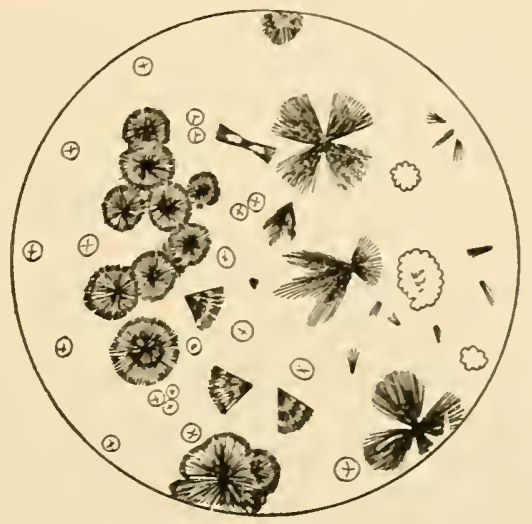

FIg. I 24.-Creatinine-ZiNc Chloride. (Salkowski.)

3. Nitro-prusside Test (Weyl).-Take 5 c.c. of urine in a test-tube, add a few drops of sodium nitro-prusside and render the solution alkaline with potassium hydroxide solution. A ruby-red color results which soon turns yellow. See Legal's test for acetone, page 437.

4. Nitro-prusside-acetic Acid Test (Salkowski).-To the yellow solution obtained in Weyl's test above add an excess of acetic acid and apply heat. A green color results and is in turn displaced by a blue color. A precipitate of Prussian blue may form.

5. Picric Acid Reaction (Jaffe).-Place 5 c.c. of urine in a test-tube, add an aqueous solution of picric acid and render the mixture alkaline with potassium hydroxide solution. A red color is produced which turns yellow if the solution be acidified. Glucose gives a similar red color but only upon the application of heat. This color reaction observed when creatinine in alkaline solution is treated with picric acid is the basic principle of Folin's colorimetric method for the quantitative determination of creatinine (see page 506).

\section{ETHEREAL SULPHATES}

The most important of the ethereal sulphates found in the urine are phenol-sulphuric acid, p-cresol-sulphuric acid, indonyl-sulphuric 
acid, and skatoxyl-sulphuric acid. Pyrocatechol-sulphuric acid also occurs in traces in human urine. The total output of ethereal sulphuric acid $\left(\right.$ as $\left.\mathrm{SO}_{3}\right)$ varies ordinarily from o.I gram to 0.25 gram for 24 hours and comprise $5^{-I} 5$ per cent of the total sulphur. In health the ratio of ethereal sulphuric acid to inorganic sulphuric acid is about $\mathrm{I}: \mathrm{ro}$. These ethereal sulphuric acids originate in part from the phenol, cresol, indole and skatole formed in the putrefaction of protein material in the intestine. The phenol passes to the liver where part of it is conjugated to form phenol potassium sulphate and appears in this form in the urine whereas the indole and skatole undergo a preliminary oxidation to form indoxyl and skatoxyl respectively before their conjugation and elimination.

It was formerly generally considered that each of the ethereal sulphuric acids was formed principally in the putrefaction of protein material in the intestine and that therefore a determination of the total ethereal sulphuric acid content of the urine was an index of the extent to which these putrefactive processes were proceeding within the organism. Folin, however, conducted a series of experiments which seemed to show that the ethereal sulphuric acid content of the urine did not afford an index of the extent of intestinal putrefaction, since these bodies arise only in part from putrefactive processes. He claims that the ethereal sulphuric acid excretion represents a form of sulphur metabolism which is more in evidence upon a diet containing a very small amount of protein or upon a diet containing absolutely no protein. The ethereal sulphuric acid content of the urine diminishes as the total sulphur content diminishes but the percentage decrease is much less. Therefore when considered from the standpoint of the total sulphuric acid content the ethereal sulphuric acid content is not diminished but is increased, although the total sulphuric acid content is diminished. Folin's experiments also seem to show that the indoxyl sulphuric acid (indoxyl potassium sulphate or indican) content of the urine does not originate to any degree from the metabolism of protein material but that it arises in great part from intestinal putrefaction and that the excretion of indoxyl sulphuric acid may alone be taken as a rough index of the extent of putrefactive processes within the intestine. Indoxyl sulphuric acid,

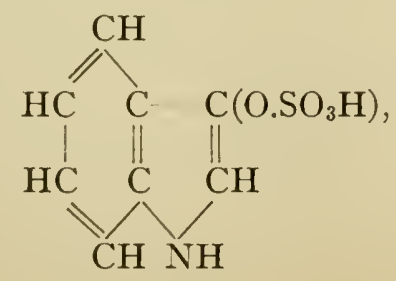


therefore, which occurs in the urine as indoxyl potassium sulphate or indican,

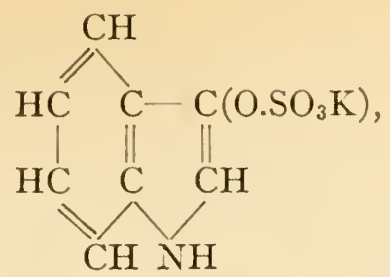

is clinically the most important of the ethereal sulphuric acids. Under normal conditions from 4 to $20 \mathrm{mg}$. of indican are excreted per day. The variations are due mainly to diet, a high meat diet causing an increase and a carbohydrate diet a decrease. Pathologically the greatest increases are found in disorders involving increased putrefaction and stagnation of intestinal contents. Bacterial decomposition of body protein as in gangrene, putrid pus formation, etc., gives rise to an increased indican excretion.

It was formerly believed that the phenol was excreted practically quantitatively in the conjugated form: Recent work of Folin and Denis ${ }^{1}$ seems to indicate that this is not true. Only part of the phenols formed in intestinal putrefaction are excreted in the conjugated form, the remainder being excreted as free phenol. The phenol output tends to vary directly but not proportionally with the protein ingestion. The total phenol excretion of normal men on an ordinary mixed diet a verages around 0.4 gram per day.

\section{TESTS FOR INDICAN ${ }^{2}$}

I. Jaffe's Test.-Nearly fill a test-tube with a mixture composed of equal volumes of concentrated $\mathrm{HCl}$ and the urine under examination. Add 2-3 c.c. of chloroform and a few drops of a calcium hypochlorite solution, place the thumb over the end of the test-tube and shake the tube and contents thoroughly. The chloroform is colored more or less, according to the amount of indican present. Ordinarily a blue color due to the formation of indigo-blue is produced; less frequently a red color due to indigo-red may be noted.

Repeat) this test on some of this same urine to which formaldehyde has been added. Is there any variation in the reaction from what you previously obtained?

This is the reaction (see also pages $2 \mathrm{I}_{2}-2 \mathrm{I} 3$ ):

${ }^{1}$ Folin and Denis: Jour. Biol. Chem., 22, 300, 19:5.

${ }^{2}$ The urine should always be examined $f r e s h$ if this is possible. In any event formaldehyde should never be used as a preservative for such urines as are to be examined for indican by means of any test involving hypochlorite or potassium permanganate. The formaldehyde through its reducing power lowers the oxidizing efficiency of the mixture. The formation of formic acid from the aldehyde may also interfere. 

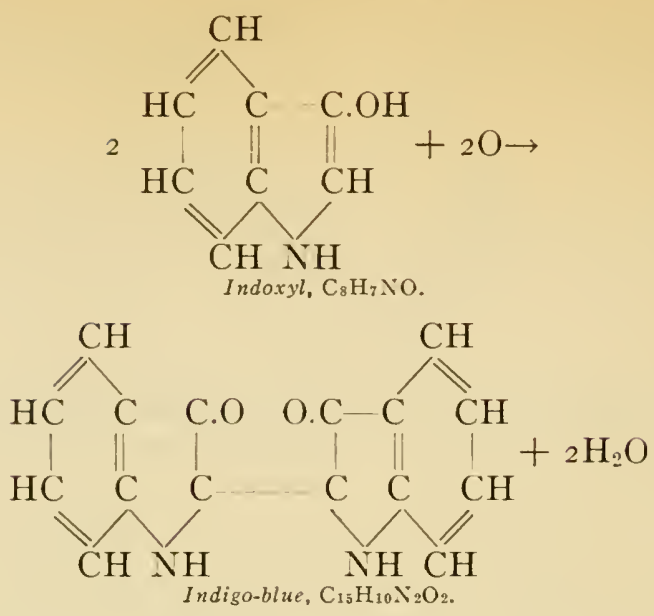

2. Obermayer's Test. - Nearly fill a test-tube with a mixture composed of equal volumes of Obermayer's reagent ${ }^{1}$ and the urine under examination. Add 2-3 c.c. of chloroform, place the thumb over the end of the test-tube and shake thoroughly. How does this compare with Jaffe's test?

3. Jolles' Reaction. ${ }^{2}$-To ro c.c. of urine add I c.c. of a 5 per cent alcoholic thymol solution and shake. Add about ro c.c. of fuming $\mathrm{HCl}$ containing 5 grams of ferric chloride per liter. Shake again carefully and let stand for $\mathbf{5}$ minutes. Add about 4 c.c. of chloroform and extract the pigment by repeated gentle shaking. The chloroform becomes intensely violet. $0.0032 \mathrm{mg}$. of indican can be detected in Io c.c. of urine. It is much the most delicate test for indican.

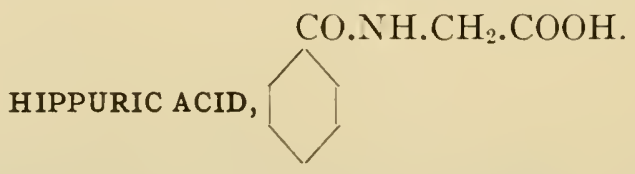

This acid occurs normally in the urine of both the carnivora and herbivora but is much more abundant in the urine of the latter. It is formed by a synthesis of benzoic acid and glycocoll which takes place in the kidneys and elsewhere ${ }^{3}$. The glycocoll comes from decomposition of protein. The benzoic acid thus utilized may come from (I) preformed benzoic acid of fruits and vegetables; (2) other aromatic compounds of fruits and vegetables; (3) aromatic amino-acids (tyrosine and phenylalanine) from the alimentary tract. 'The average excretion of hippuric acid by an adult man for 24 hours under normal conditions is about 0.7 gram. Hippuric acid crystallizes in needles or rhombic prisms (see Fig. I25, p. 389) the particular form depending upon the

\footnotetext{
${ }^{1}$ Obermayer's reagent is prepared by adding $2-4$ grams of ferric chloride to a liter of concentrated $\mathrm{HCl}$ (sp. gr. I.Ig).

${ }^{2}$ Zeit. physiol. Chem., 94, 79, 1915.

${ }^{3}$ Kingsbury and Bell: Jour. Biol. Chem., 21 , 297, 1915.
} 
rapidity of crystallization. Pure hippuric acid melts at $187^{\circ} \mathrm{C}$. 'The most satisfactory method for the isolation of hippuric acid from the urine in crystalline form is that proposed by Roaf (see below). It is easily soluble in alcohol or hot water, and only slightly soluble in ether. The output of hippuric acid is increased in diabetes owing probably to the ingestion of much protein and fruit. Plums, prunes and cranberries in particular increase the hippuric acid output considerably due to their relatively high content of benzoic acid. Hippuric acid is decreased in'fevers and in certain kidney disorders where the synthetic

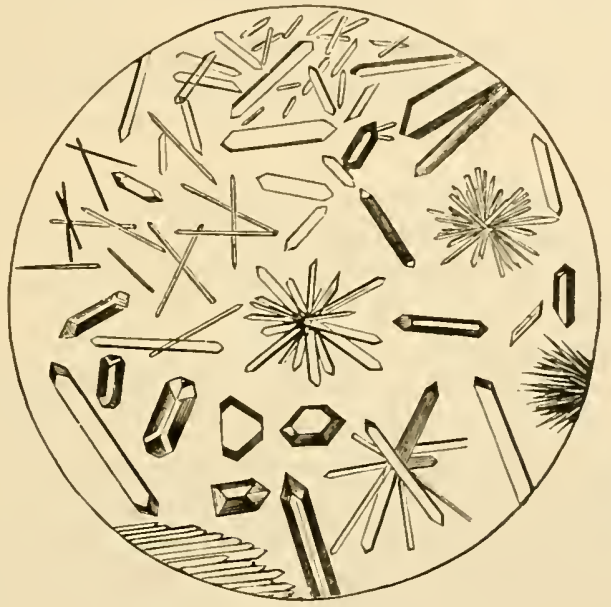

Fig. I 25.-Hippuric ACid.

activity of the renal cells is diminished. It may be determined quantitatively by methods given in Chapter XXVI.

\section{Experiments on Hipturic ACID}

r. Separation from the Urine.-(a) First Method.-Render 500-I000 c.c. of urine of the horse or cow ${ }^{1}$ alkaline with milk of lime, boil for a few moments and filter while hot. Concentrate the filtrate, over a burner, to a small volume. Cool the solution, acidify it strongly with concentrated hydrochloric acid and stand it in a cool place for 24 hours. Filter off the crystals of hippuric acid which have formed and wash them with a little cold water. Remove the crystals from the paper, dissolve them in a very small amount of hot water and percolate the hot solution through thoroughly washed animal charcoal, being careful to wash out the last portion of the hippuric acid solution with hot water. Filter, concentrate

1 If urine of the horse or cow is not available human urine may serve the purpose fully. as well provided means are taken to increase its content of hippuric acid. This mat be conveniently accomplished by ingesting 2 grams of ammonium benzoate at night. (See chapter on Metabolism). 'The fraction of urine passed in the morning will be found to have a high content of hippuric acid. The ammonium benzoate is in no way harmful. In case ammonium benzoate is not available sodium benzoate may be substituted. 
the filtrate to a small volume and stand it aside for crystallization. Examine the crystals under the microscope and compare them with those in Fig. 125, page 389. This method is not as satisfactory as Roaf's method (see below).

(b) Roaf's Method.-Place 500 c.c. of urine of the horse or cow in a casserole or precipitating jar and add an equal volume of a saturated solution of ammonium sulphate ${ }^{1}$ and 7.5 c.c. of concentrated sulphuric acid. Permit the mixture to stand for 24 hours and remove the crystals of hippuric acid by filtration. Purify the crystals by recrystallization according to the directions given above under First Method. Examine the crystals under the microscope and compare them with those given in Fig. 125, page 389 .

If sufficient urine is not available to permit the use of 500 c.c. a smaller volume may be used inasmuch as it is possible, by the above technic, to isolate hippuric acid in crystalline form from as small a volume as $25-50$ c.c. of herbivorous urine. The greater the amount of ammonium sulphate added the more rapid the crystallization until at the saturation point the crystals of hippuric acid sometimes form in about ten minutes.

2. Melting-point.-Determine the melting-point of the hippuric acid prepared in the above experiment (see page 375 ).

3. Solubility.-Test the solubility of hippuric acid in hot and cold water and in alcohol, and ether.

4. Formation of Nitro-Benzene (Lücke's Reaction).-To a little hippuric acid in a small porcelain dish add I-2 c.c. of concentrated $\mathrm{HNO}_{3}$ and evaporate to dryness on a water-bath. Transfer the residue to a dry test-tube, apply heat, and note the odor of the artificial oil of bitter almonds (nitrobenzene).

5. Spiro's Reaction. ${ }^{2}$-Warm the hippuric acid with acetic anhydride, anhydrous sodium acetate and benzaldehyde. After one-half hour permit the solution to cool. Note the formation of crystals of the lactimide of phenylaminocinnamic acid, melting-point $165-166^{\circ}$. Heat some of the crystals with concentrated sodium hydroxide until ammonia is given off. Acidify and note the formation of phenylpyroracemic acid $\left(\mathrm{C}_{6} \mathrm{H}_{6} \mathrm{CH}_{2} \cdot \mathrm{CO} \cdot \mathrm{COOH}\right)$. This acid is soluble in ether.

6. Sublimation.-Place a few crystals of hippuric acid in a dry test-tube and apply heat. The crystals are reduced to an oily fluid which solidifies in a crystalline mass upon cooling. When stronger heat is applied the liquid assumes a red color and finally yields a sublimate of benzoic acid and the odor of hydrocyanic acid.

7. Formation of Ferric Salt.-Render a small amount of a solution of hippuric acid neutral with dilute potassium hydroxide. Now add I-3 drops of neutral ferric chloride solution and note the formation of the ferric salt of hippuric acid as a cream-colored precipitate.

8. Synthesis of Hippuric Acid.-To some of the glycocoll prepared in the last experiment or furnished by the instructor, add a little water, about I c.c. of benzoy] chloride and render alkaline with potassium hydroxide solution. Stopper the tube and shake it until no more heat is evolved. Now render strongly alkaline with potassium hydroxide and shake the mixture until no odor of benzoyl chloride can be detected. Cool, acidify with hydrochloric acid, add an equal volume of petroleum ether, and shake thoroughly to remove the benzoic acid. (Evaporate this solution and note the crystals of benzoic acid. Compare them with those shown in Fig. I27, page 396.) Decant the ethereal solution into a porcelain dish and extract again

${ }^{1}$ I 25 grams of solid ammonium sulphate may be substituted.

${ }^{2}$ Spiro: Zeit. physiol. Chem., 28, I 74, 1899. 
with ether. The hippuric acid remains in the aqueous solution. Filter it off and wash it with a small amount of cold water while still on the filter. Remove it to a small, shallow vessel, dissolve it in a small amount of hot water and set it aside for crystallization. Examine the crystals microscopically and compare them with those in Fig. I25, page 389.

The chemistry of the synthesis is represented thus:

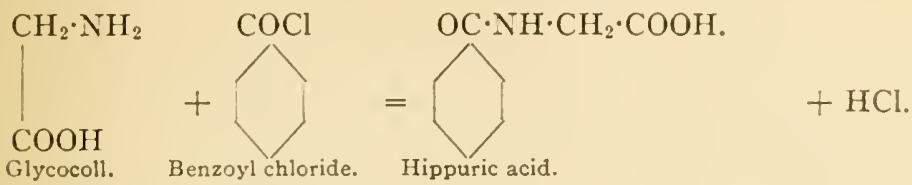

\section{OXALIC ACID, \\ $\mathrm{COOH}$}

Oxalic acid is a constituent of normal urine, about $5_{5}-20 \mathrm{mg}$. being eliminated in 24 hours. It is present in the urine as calcium oxalate which is kept in solution through the medium of the acid phosphates. The origin of the oxalic acid content of the urine is not well understood. It is eliminated, at least in part, unchanged when ingested, therefore since many of the common articles of diet, e.g., asparagus, apples, cabbage, grapes, lettuce, spinach, tomatoes, etc., contain oxalic acid (oxalates) it seems probable that the ingested food supplies a portion of the oxalic acid found in the urine. There is also experimental evidence that part of the oxalic acid of the urine is formed within the organism in the course of protein and fat metabolism. It has also been suggested that oxalic acid may arise from an incomplete combustion of carbohydrates, especially under certain abnormal conditions. Pathologically, oxalic acid is found to be increased in amount in diabetes mellitus, in organic diseases of the liver, and in various other conditions which are accompanied by a derangement of the oxidation mechanism. An abnormal increase of oxalic acid is termed oxaluria. A considerable increase in the content of oxalic acid may be noted unaccompanied by any other apparent symptom. Calcium oxalate crystallizes in at least two distinct forms, dumb-bells and octahedra. (Fig. I34, page 459).

\section{EXPERIMENTS}

Preparation of Calcium Oxalate.-First Method.-Place 200-250 c.c. of urine in a beaker, add 5 c.c. of a saturated solution of calcium chloride, make the urine slightly acid with acetic acid, and stand the beaker aside in a cool place for 24 hours. Examine the sediment under the microscope and compare the crystalline forms with those shown in Fig. I34, page 459.

Second Method.-Proceed as above, replacing the acetic acid by an excess of ammonium hydroxide and filtering off the precipitate of phosphates. 


\section{NEUTRAL SULPHUR COMPOUNDS}

Under this head may be classed such bodies as cystine (see page 75), chondroitin-sulphuric acid, oxyproteic acid, alloxyproteic acid, uroferric acid, methyl mercaptan, ethyl sulphide, thiocyanates and taurine derivatives. The sulphur content of the bodies just enumerated is generally termed unoxidized or neutral sulphur in order that it may not be confused with the acid or oxidized sulphur which occurs in the inorganic sulphuric acid and ethereal sulphuric acid forms. Ordinarily the neutral sulphur content of normal human urine is $5^{-25}$ per cent of the total sulphur content (see "Sulphur Partition" in chapter on Metabolism.) The actual amount excreted may be $0.2-0.4$ grams

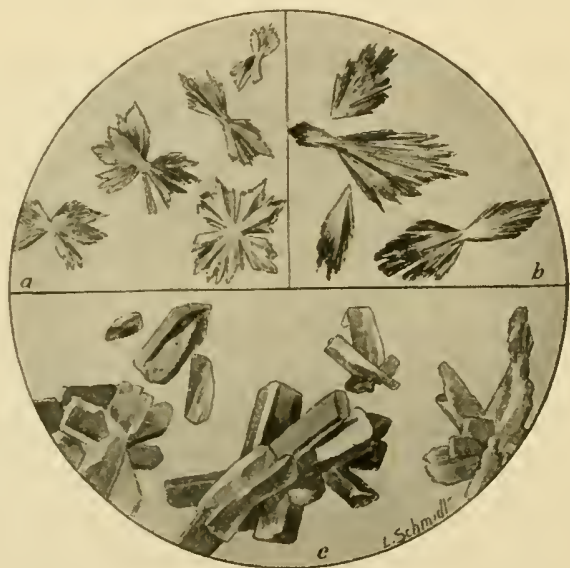

Fig. I 26.-Allantoin, Frou Cat's URine.

$a$ and $b$, Forms in which it crystallized from the urine; $c$, recrystallized allantoin. (Drawn from micro-photographs furnished by Prof. Lafayette B. Mendel of Yale University.)

per day, calculated as $\mathrm{SO}_{3}$. Its origin is mainly endogenous. The excretion is fairly constant for any given individual in spite of dietary changes. In tuberculosis, cancer, cystinuria, etc., the amount may be relatively or absolutely increased.

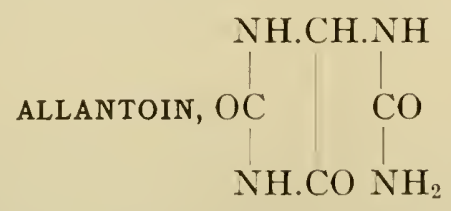

Allantoin is found in the urine of practically all mammals including man. In human urine it occurs in very small amount (5-I5 mg. per day) whereas in the case of all other mammals investigated except anthropoid apes, it is the principal end-product of purine metabolism 
and may constitute 90 per cent or over of the total purine output. ${ }^{1}$ Allantoin is formed by the oxidation of uric acid and the output is increased by the feeding of thymus or pancreas to lower animals. When pure it crystallizes in prisms (Fig. I 26, page 392) and when impure in granules and knobs. Pathologically, it has been found increased in diabetes insipidus and in hysteria with convulsions (Pouchet). Mendel and Dakin ${ }^{2}$ have shown that allantoin is optically inactive notwithstanding the fact that it contains an asymmetric carbon atom. This phenomenon they believe to be due to tautomeric change. Wiechowski has suggested an excellent method for the quantitative determination of allantoin. (See Chapter XXVI.)

\section{EXPERIMENTTS}

I. Separation from the Urine. ${ }^{3}$-Meissner's Method.-Precipitate the urine with baryta water. Neutralize the filtrate carefully with dilute sulphuric acid, filter immediately, and evaporate the filtrate to incipient crystallization. Completely precipitate this warm fluid with 95 per cent alcohol (reserve the precipitate). Decant or filter and precipitate the solution by ether. Combine the ether and alcohol precipitates and extract with cold water or hot alcohol; allantoin remains undissolved. Bring the allantoin into solution in hot water and recrystallize.

2. Preparation from Uric Acid.-Dissolve 4 grams of uric acid in Ioo c.c. of water rendered alkaline with potassium hydroxide. Cool and carefully add 3 grams of potassium permanganate. Filter, immediately acidulate the filtrate with acetic acid and allow it to stand in a cool place over night. Filter off the crystals and wash them with water. Save the wash water and filtrate, unite them and after concentrating to a small volume stand away for crystallization. Now combine all the crystals and recrystallize them from hot water. Use these crystals in the experiments which follow.

3. Microscopical Examination.-Examine the crystals made in the last experiment and compare them with those shown in Fig. I 26.

4. Solubility.-Test the solubility of allantoin in cold and hot water, cold and hot alcohol and in ether.

5. Reaction.-Dissolve a crystal in water and test the reaction to litmus.

6. Furfural Test (Schiff).-Place a few crystals of allantoin on a test-tablet or in a porcelain dish and add $\mathbf{I}-2$ drops of a concentrated aqueous solution of furfural and I-2 drops of concentrated hydrochloric acid. Observe the formation of a yellow color which turns to a light purple if allowed to stand. This test is given by urea but not by uric acid.

7. Murexide Test.-Try this test according to the directions given on page 380. Note that allantoin fails to respond.

8. Reduction of Fehling's Solution.-Make this test in the usual way (see page 4 I6) except that the boiling must be prolonged and excessive. Ultimately

1 Wiechowski: "Die Purinstoffe und das Allantoin " in Neubauer and Huppert's ". I n. lyse des II arns," Wiesbaden, igr 3.

${ }^{2}$ Mendel and Dakin: Jour. Biol. Chem., 7, I 53, 1010.

${ }^{3}$ The urine of the dog after thymus, pancreas, or uric acid feeding may be employed. 
the allantoin will reduce the solution. Compare with the result on uric acid, page $38 \mathrm{r}$.

\section{AMINO-ACIDS}

Certain of these acids are always present in normal urine. The excretion of total amino-acid nitrogen by a normal adult averages $0.4^{-1.0}$ gram per day or about 2-6 per cent of the total nitrogen. Free amino-acid nitrogen (see van Slyke procedure, Chapter IV) is considerably less than this, and ordinarily constitutes $0.5^{-I}$ per cent of the total nitrogen. The amount may be largely increased in disorders associated with tissue waste, e.g., typhoid, acidosis, pronounced atrophy of the liver, etc. For tests on amino-acids see Chapter IV.

\section{AROMATIC OXYACIDS}

Two of the most important of the oxyacids are parahydroxyphenyl-acetic acid,

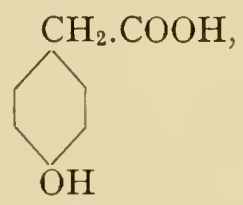

and parahydroxy-phenyl-propionic acid,

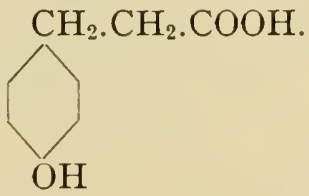

They are products of the putrefaction of protein material and tyrosine is an intermediate stage in their formation. Both these acids for the most part pass unchanged into the urine where they occur normally in very small amount. The content may be increased in the same manner as the phenol content, in particular by acute phosphorus poisoning. A fraction of the total aromatic oxyacid content of the urine is in combination with sulphuric acid, but the greater part is present in the form of salts of sodium and potassium.

Homogentisic Acid or di-hydroxyphenyl-acetic acid,

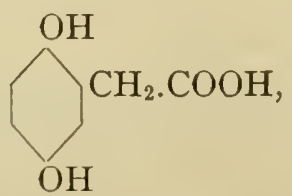

*For a full discussion see Underhill's "The Physiology of the Amino Acids," Yale University Press, November, I9I 5. 
is another important oxyacid sometimes present in the urine. Under the name glycosuric acid it was first isolated from the urine by Prof. John Marshall of the University of Pennsylvania; subsequently Baumann isolated it and determined its chemical constitution. It occurs in cases of alcaptomuria. A urine containing this oxyacid turns greenishbrown from the surface downward when treated with a little sodium hydroxide or ammonia. If the solution be stirred the color very soon becomes dark brown or even black. Homogentisic acid reduces alkaline copper solutions but not alkaline bismuth solutions. Uroleucic acid is similar in its reactions to homogentisic acid.

Hydroxymandelic Acid or parahydroxyphenyl-glycolic acid,

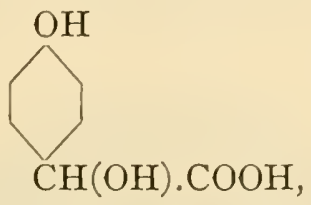

has been detected in the urine in cases of yellow atrophy of the liver.

Kynurenic Acid or $\gamma$-oxy- $\beta$-quinoline carbonic acid,

$\mathrm{CH} \mathrm{COH}$

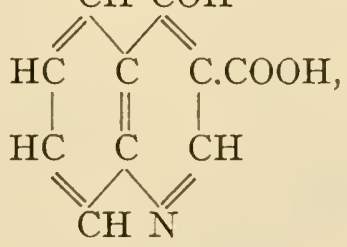

is present in the urine of the dog and has recently been detected by Swain in the urine of the coyote. To isolate it from the urine proceed as follows: Acidify the urine with hydrochloric acid in the proportion I:25. From this acid fluid both the uric acid and the kynurenic acid separate in the course of $24-48$ hours. Filter off the combined crystalline deposit of the two acids, dissolve the kynurenic acid in dilute ammonia (uric acid is insoluble), and reprecipitate it with hydrochloric acid. If a solution containing kynurenic acid be evaporated to dryness with hydrochloric acid and potassium chlorate, a reddish residue is obtained which becomes first brownish green and then emerald green on adding ammonia (Jaffé).

Kynurenic acid may be quantitatively determined by Capaldi's method. ${ }^{1}$

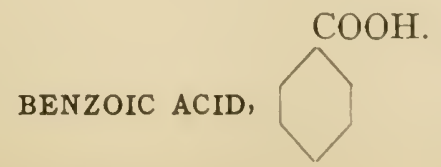

' Zeilschrift filr physiologische Chemie, 23, 92, I897. 
Benzoic acid has been detected in the urine of the rabbit and dog. It is also said to occur in human urine accompanying renal disorders. The benzoic acid probably originates from a fermentative decomposition of the hippuric acid of the urine. Benzoic acid and glycocoll are synthesized in the kidney and elsewhere ${ }^{1}$ to form hippuric acid (see page $5^{8} 5$ ). Certain fruits and berries contain considerable benzoic acid; e.g., cranberries have been shown to contain 0.06 per cent. ${ }^{2}$

\section{EXPERIMENTS}

I. Solubility.-Test the solubility of benzoic acid in water, alcohol, and ether.

2. Crystalline Form.-Recrystallize some benzoic acid from hot water, examine the crystals under the microscope, and compare them with those reproduced in Fig. 127.

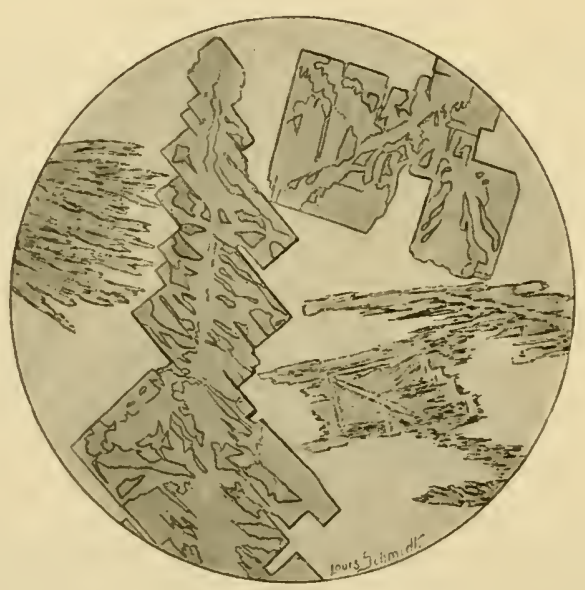

Fig. I 27.-BENzoIC ACID.

3. Sublimation.-Place a little benzoic acid in a test-tube and heat over a flame. Note the odor which is evolved and observe that the acid sublimes in the form of needles.

4. Dissolve a little sodium benzoate in water and add a solution of neutral ferric chloride. Note the production of a brownish-yellow precipitate (salicylic acid gives a reddish-violet color under the same conditions). Add ammonium hydroxide to some of the precipitate. It dissolves and ferric hydroxide is formed. Add a little hydrochloric acid to another portion of the original precipitate and stand the vessel away over night. What do you observe?

\section{NUCLEOPROTEIN}

The nubecula of normal urine has been shown by one investigator to consist of a mucoid containing $\mathrm{I} 2.7$ per cent of nitrogen and 2.3 per cent of sulphur. This body evidently originates in the urinary

${ }^{1}$ Kingsbury and Bell: Jour. Biol. Chem., 21, 297, 1915.

${ }^{2}$ Radin [quoted by Blatherwick (Arch. Int. Med., I 4, 409, 1 914) from unpublished data]. 
passages. It is probably slightly soluble in the urine. Some investigators believe that the body forming the nubecula of normal urine is nucleoprotein and not a mucin or mucoid as stated above. A discussion of nucleoprotein and related bodies occurring in the urine under pathological conditions will be found on page 428 .

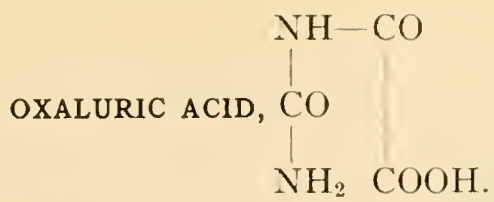

Oxaluric acid is not a constant constituent of normal human urine, and when found occurs only in traces as the ammonium salt. Upon boiling oxaluric acid it splits into oxalic acid and urea.

\section{GLUCOSE}

This sugar occurs in traces in normal urine. It is, however, not present in sufficient concentration to be detected by any of the ordinary tests used in urine analysis. In certain pathological conditions (pp. $4 \mathrm{I} 3$ and 523) the sugar in the urine is notably increased. Folin has recently modified Benedict's sugar test (see Chapter XXIII) so it may be used to demonstrate the sugar content of normal urine. ${ }^{1}$

\section{ENZYMES}

Various types of enzymes produced within the organism are excreted in both the feces and the urine. In this connection it is interesting to note that pepsin, rennin, lipase and an amylase have been positively identified in the urine. The occurrence of trypsin in the urine, at least under normal conditions, is questioned.

\section{VOLATILE FATTY ACIDS}

Acetic, butyric, and formic acids have been found under normal conditions in the urine of man and of certain carnivora as well as in the urine of herbivora. Normally they arise principally from the fermentation of carbohydrates and the putrefaction of proteins. The acids containing the fewest carbon atoms (formic and acetic) are found to be present in larger percentage than those which contain a larger number of such atoms. 'The volatile fatty acids occur in normal urine in traces, the total output for 24 hours according to older investigators varying from 0.008 gram to 0.05 gram.

Pathologically, the excretion of volatile fatty acids is increased in

${ }^{1}$ Folin: Jour. Biol. Chem., 22, 327, 1915. 
diabetes, fevers, and in certain hepatic diseases in which the parenchyma of the liver is seriously affected. Under other pathological conditions the output may be diminished. These variations, however, in the excretion of the volatile fatty acids possess very little diagnostic value.

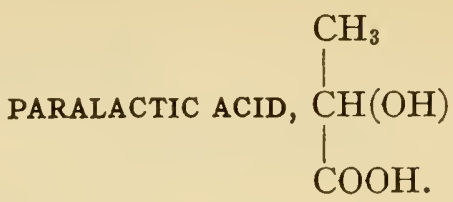

Paralactic acid is supposed to pass into the urine when the supply of oxygen in the organism is diminished through any cause, e.g., in eclampsia, acute yellow atrophy of the liver, carbon-monoxide poisoning, acute phosphorus poisoning, or epileptic attacks. This acid has also been found in the urine of healthy persons following the physical exercise incident to prolonged marching. Paralactic acid has been detected in the urine of birds after the removal of the liver. Underhill reports the occurrence of this acid in the urine of a case of pernicious vomiting of pregnancy.

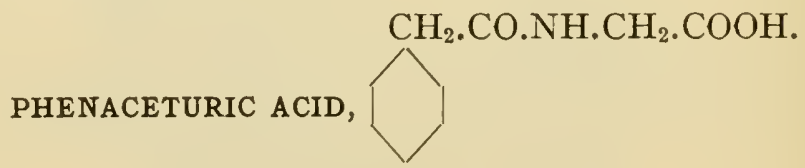

Phenaceturic acid occurs principally in the urine of herbivorous animals. It may be isolated from the urine of the dog after feeding phenylacetic acid. ${ }^{1}$ It is produced in the organism through the synthesis of glycocoll and phenylacetic acid. It is doubtful if it occurs in normal human urine even after the ingestion of phenylacetic acid. It may be decomposed into its component parts by boiling with dilute mineral acids. The crystalline form of phenaceturic acid (small rhombic plates with rounded angles) resembles one form of uric acid crystal.

$$
\mathrm{HC}=\mathrm{C}-\mathrm{CH}=\mathrm{CH} \cdot \mathrm{COOH}
$$

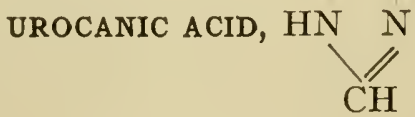

This acid has been found in the urine of dogs, but not in human urine. It is imidazolyl-acrylic acid. Hunter ${ }^{2}$ found it among the pancreatic digestion products of casein. It crystallizes as sickle-shaped crystals.

${ }^{1}$ Sherwin: Dissertation, Tübingen, I9I5.

${ }^{2}$ Hunter: Jour. Biol. Chem., I I, 537, I9I3. 


\section{PHOSPHORIZED COMPOUNDS}

Phosphorus in organic combination has been found in the urine in such bodies as glycerophosphoric acid, which may arise from the decomposition of lecithin, and phosphocarnic acid. It is claimed that on the average about 2.5 per cent of the total phosphorus elimination is in organic combination.

\section{PIGMENTS}

There are at least three pigments normally present in human urine. These pigments are urochrome, urobilin, and uroerythrin.

\section{A. UROCHROME}

This is the principal pigment of normal urine and imparts the characteristic yellow color to that fluid. It is apparently closely related to its associated pigment urobilin since the latter may be readily converted in to urochrome through evaporation of its aqueous-ether solution. Urochrome may be obtained in the form of a brown, amorphous powder which is readily soluble in water and 95 per cent alcohol. It is less soluble in absolute alcohol, acetone, amyl alcohol and acetic ether, and insoluble in benzene, chloroform, and ether. Urochrome is said to be a nitrogenous body (4.2 per cent nitrogen), free from iron. Urochrome is believed to be identical with the yellow pigment, lactochrome, of milk whey. ${ }^{1}$ The chromogen of urochrome, i.e., urochromogen is present in the urine in pulmonary tuberculosis. Its presence is said to be of prognostic value (see page 453).

\section{B. UROBILIN}

Urobilin, which was at one time considered to be the principal pigment of urine, in reality contributes little toward the pigmentation of this fluid. It is claimed that no urobilin is present in freshly voided normal urine but that its precursor, a chromogen called urobilinogen, is present and gives rise to urobilin upon decomposition through the influence of light. It is claimed by some investigators that there are various forms of urobilin, e.g., normal, febrile, physiological, and pathological. Urobilin is said to be very similar to, if not absolutely identical with, hydrobilirubin (see page 222).

Urobilin may be obtained as an amorphous powder which varies in color from brown to reddish-brown, red and reddish-yellow, depending upon the way in which it is prepared. It is easily soluble in ethyl alcohol, amyl alcohol, and chloroform, and slightly soluble in ether,

${ }^{1}$ Palmer and Coolidge: Jour. Biol. Chem., I 7, 25r, I9r4. 
acetic ether, and in water. Its solutions show characteristic absorption bands (see Absorption Spectra, Plate II). Under normal conditions urobilin is derived from the bile pigments in the intestine.

- Urobilin is increased in most acute infectious diseases such as erysipelas, malaria, pneumonia, and scarlet fever. It is also increased in appendicitis, carcinoma of the liver, catarrhal icterus, pernicious anemia, and in cases of poisoning by antifebrin, antipyrin, pyridin, and potassium chlorate. In general it is usually increased when blood destruction is excessive and in disturbances of the liver. It is markedly decreased in phosphorus poisoning.

In liver disease, of any type, urobilinogen occurs in the urine. Its detection is the basis of a specific test for functional liver incapacity.

\section{EXPERIMENTS}

I. Ammoniacal-zinc Chloride Test.-Render some of the urine ammoniacal by the addition of ammonium hydroxide, and after allowing it to stand a short time filter off the precipitate of phosphates and add a few drops of zinc chloride solution to the filtrate. Observe the production of a greenish fluorescence. Examine the fluid by means of the spectroscope and note the absorption band which occupies much the same position as the absorption band of urobilin in acid solution (see Absorption Spectra, Plate II).

2. Ether-absolute Alcohol Test.-Mix urine and pure ether in equal volumes and shake gently in a separatory funnel. Separate the ether extract, evaporate it to dryness, and dissolve the residue in 2-3 c.c. of absolute alcohol. Note the greenish fluorescence. Examine the solution spectroscopically and observe the characteristic absorption band (see Absorption Spectra, Plate II).

3. Ring Test.-Acidify 25 c.c. of urine with $2-3$ drops of concentrated hydrochloric acid, add 5 c.c. of chloroform and shake the mixture. Separate the chloroform, place it in a test-tube, and add carefully 3-5 c.c. of an alcoholic solution of zinc acetate. Observe the formation of a green ring at the zone of contact of the two fluids. If the tube is shaken a fluorescence may be observed.

4. Spectroscopic Examination.-Acidify the urine with hydrochloric acid and allow it to remain exposed to the air for a few moments. By this means if any urobilinogen is present it will be transformed into urobilin. The urine may now be examined by means of the spectroscope. If urobilin is present in the fluid the characteristic absorption band lying between $b$ and $F$ will be observed (see Absorption Spectra, Plate II). It may be found necessary to dilute the urine with water before a distinct absorption band is observed. This test may be modified by acidifying Io c.c. of urine with hydrochloric acid and shaking it gently with 5 c.c. of amyl alcohol. The alcoholic extract when examined spectroscopically will show the characteristic urobilin absorption band. (Note the spectroscopic examination in experiment (I) above.)

5. Iodine Test (Gerhardt). - To 20 c.c. of urine add 3-5 c.c. of chloroform and shake well. Separate the chloroform extract and add to it a few drops of iodine solution (I in KI). Render the mixture alkaline with dilute solution of potassium hydroxide and note the production of a yellow or yellowish-brown color. The solution ordinarily exhibits a greenish fluorescence. 
6. Alcoholic-zinc Chloride Test (Wirsing).-To 20 c.c. of urine add $3-5$ c.c. of chloroform and shake gently. Separate the chloroform extract and add to it a drop of an alcoholic solution of zinc chloride. Note the rose-red color and the greenish fluorescence. If the solution is turbid it may be rendered clear by the addition of a few cubic centimeters of absolute alcohol.

\section{UROERYTHRIN}

This pigment is frequently present in small amount in normal urine. The red color of urinary sediments is due in great part to the presence of Juroerythrin. It is easily soluble in amyl alcohol, slightly soluble in acetic ether, absolute alcohol, or chloroform, and nearly insoluble in water. Dilute solutions of uroerythrin are pink in color while concentrated solutions are orange red or bright red; none of its solutions fluoresce. Uroerythrin is increased in amount after strenuous physical exercise, digestive disturbances, fevers, certain liver disorders, and in various other pathological conditions.

\section{PTOMAINES AND LEUCOMAINES}

These toxic substances are said to be present in small amount in normal urine. Very little is known definitely, however, about them. It is claimed that five different poisons may be detected in the urine, and it is further stated that each of these substances produces a specific and definite symptom when injected intravenously into a rabbit. The resulting symptoms are narcosis, salivation, mydriasis, paralysis, and convulsions. The day urine is principally narcotic and is $2-4$ times as toxic as the night urine which is chiefly productive of convulsions.

\section{PURINE BASES}

The purine bases found in human urine are adenine, carnine, epiguanine, episarkine, guanine, xanthine, heteroxanthine, hypoxanthine, paraxanthine, and I-methylxanthine. The main bulk of the purine base content of the urine is made up of paraxanthine, heteroxanthine and I-methylxanthine, which are derived for the most part from the caffeine, theobromine, and theophylline of the food. The total purine base content is made up of the products of two distinct forms of metabolism, i.e., metabolism of ingested nucleins and purines and metabolism of tissue nuclein material. Purine bases resulting from the first form of metabolism are said to be of exogenous origin, whereas those resulting from the second form of metabolism are said to be of endogenous origin. The daily output of purine bases by the urine is extremely small and varies greatly with the individual ( $16-60 \mathrm{mg}$.). The output is increased after the ingestion of nuclein material as well as after the increased de- 
struction of leucocytes. A well-marked increase accompanies leukemia. Edsall and others have shown that the output of purine bases by the urine is increased as a result of $\mathrm{X}$-ray treatment. The purine bases form a higher percentage of the total purine excretion in the case of the monkey, sheep and goat than in man.

\section{EXPERIMENT}

I. Formation of the Silver Salts.-Add an excess of magnesia mixture ${ }^{1}$ to 25 c.c. of urine. Filter off the precipitate and add ammoniacal silver solution ${ }^{2}$ to the filtrate. A precipitate composed of the silver salts of the various purine bases is produced. The purine bases may be determined quantitatively by means of Krüger and Schmidt's method (see page $5_{13}$ ), or Welker's method (see page 5I5).

\section{Inorganic Physiological Constituents}

\section{Ammonia}

Next to urea, ammonia is the most important of the nitrogenous end-products of protein metabolism. Ordinarily about $2.5-4.5$ per cent of the total nitrogen of the urine is eliminated as ammonia and on the average this would be about 0.7 gram per day. Under normal conditions the ammonia is present in the urine in the form of the chloride, phosphate, or sulphate. This is due to the fact that combinations of this sort are not oxidized in the organism to form urea, but are excreted as such. This explains the increase in the output as ammonia which follows the administration of the ammonium salts of the mineral acids or of the acids themselves. On the other hand, when ammonium acetate and many other ammonium salts of certain organic acids are administered no increase in the output of ammonia occurs since the salt is oxidized and its nitrogen ultimately appears in the urine as urea. Acid-forming foods (see page 580 ) also increase the ammonia output, whereas the administration of alkalies or of base-forming foods decreases the excretion of ammonia

Experiments $^{3}$ indicate that the nitrogen in food protein may in part be replaced by ammonium salts.

Copious water drinking increases the ammonia output. This fact has been interpreted as indicating a stimulation of the gastric secretion. ${ }^{4}$

1 Magnesia mixture may be prepared as follows: Dissolve 175 grams of $\mathrm{MgSO}_{4}$ and 350 grams of $\mathrm{NH}_{4} \mathrm{Cl}$ in 1400 c.c. of distilled water. Add 700 grams of concentrated $\mathrm{NH}_{4}$ $\mathrm{OH}$, mix very thoroughly and preserve the mixture in a glass-stoppered bottle.

${ }^{2}$ Ammoniacal silver solution may be prepared according to directions given on page 593 .

${ }^{3}$ Grafe and Schläpfer: Zeit. physiol. chem., 77, I, I912, experiments by Abderhalden in same journal.

"Wills and Hawk: Jour. Am. Chcm. Soc., 36, 158, 1914. 
The acids formed during the process of protein destruction within the body have an influence upon the excretion of ammonia similar to that exerted by acids which have been administered. Therefore a pathological increase in the output of ammonia is observed in such diseases as are accompanied by an increased and imperfect protein metabolism, and especially in diabetes, in which disease acetoacetic acid and $\beta$-oxybutyric acid are found in the urine in combination with the ammonia.

Folin claims that a pronounced decrease in the extent of protein metabolism, as measured by the total nitrogen in the urine, is frequently accompanied by a decreased elimination of ammonia. The ammonia elimination is therefore probably determined by other factors than the total protein catabolism as such. Furthermore, he believes that a decided decrease in the total nitrogen excretion is always accompanied by a relative increase in the ammonia-nitrogen, provided the food is of a character yielding an alkaline ash.

The quantitative determination of ammonia must be made upon the fresh urine, since upon standing the normal urine will undergo ammoniacal fermentation (see page 362).

\section{EXPERIMENTS}

(See Experiment 2 under Phosphates, page 408.)

\section{Sulphates}

Sulphur in combination is excreted in two forms in the urine: first, as unoxidized, loosely combined or neutral sulphur, and second, as oxidized or acid sulphur. The unoxidized or neutral sulphur is excreted mainly as a constituent of such bodies as cystine, cysteine, taurine, hydrogen sulphide, ethyl sulphide, thiocyanates, sulphonic acids, oxyproteic acid, alloxyproteic acid, and uroferric acid. The amount of ncutral sulphur eliminated is in great measure independent of the extent of protein decomposition or of the total sulphur excretion. In this characteristic it is somewhat similar to the excretion of creatinine. The oxidized sulphur is eliminated in the form of sulphuric acid, principally as salts of sodium, potassiun, calcium, and magnesium; a relatively small amount occurs in the form of cthereal sulphuric acid, i.e., sulphuric acid in combination with such aromatic bodies as phenol, indole, skatole, cresol, pyrocatechol, and hydroquinol. Sulphuric acid in combination with $\mathrm{Na}, \mathrm{K}, \mathrm{Ca}$ or $\mathrm{Mg}$ is sometimes termed inorganic or preformed sulphuric acid, whereas the cthereal sulphuric acid is sometimes called conjugate sulphuric acid. The greater part of the sulphur is climinated in the 
oxidized form, but the absolute percentage of sulphur excreted as the performed, ethereal or loosely combined type depends upon the total quantity of sulphur present; i.e., there is no definite ratio between the three forms of sulphur which will apply under all conditions. The preformed sulphuric acid may be precipitated directly from acidified urine with $\mathrm{BaCl}_{2}$, whereas the ethereal sulphuric acid must undergo a preliminary boiling in the presence of a mineral acid before it can be so precipitated.

The sulphuric acid excreted in the urine arises principally from the oxidation of protein material within the body; a relatively small amount is due to ingested sulphates. Under normal conditions about 2.5 grams of sulphuric acid $\left(\mathrm{SO}_{3}\right)$ are eliminated daily, about $75^{-} 95$ per cent of this being in the form of sulphates. About 90 per cent of this sulphate excretion is in the form of inorganic sulphate and Io per cent as ethereal sulphates. Since the sulphuric acid content of the urine has, for the most part, a protein origin and since one of the most important constituents of the protein molecule is nitrogen, it would be reasonable to suppose that a fairly definite ratio might exist between the excretion of these two elements. However, when we appreciate that the percentage content of $\mathrm{N}$ and $\mathrm{S}$ present in different proteins is subject to rather wide variations, the fixing of a ratio which will express the exact relation existing between these two elements as they appear in the urine as end-products of protein metabolism is practically impossible. It has been suggested that the ratio $5: 1$ expresses this relation in a general way.

Pathologically, the excretion of sulphuric acid by the urine is increased in acute fevers and in all other diseases marked by a stimulated metabolism, whereas a decrease in the sulphuric acid excretion is observed in those diseases which are accompanied by a loss of appetite and a diminished metabolic activity.

\section{EXPERIMENTS}

I. Detection of Inorganic Sulphuric Acid.-Place about ro c.c. of urine in a test-tube, acidify with acetic acid and add some barium chloride solution. A white precipitate of barium sulphate forms.

2. Detection of Ethereal Sulphuric Acid.-Filter off the barium sulphate precipitate formed in the above experiment, add I c.c. of hydrochloric acid and a little barium chloride solution to the filtrate and heat the mixture to boiling for 1-2 minutes. Note the apperarance of a turbidity due to the presence of sulphuric acid which has been separated from the ethereal sulphates and has combined with the barium of the $\mathrm{BaCl}_{2}$ to form $\mathrm{BaSO}_{4}$.

3. Detection of Unoxidized or Neutral Sulphur.-Place about Io c.c. of urine in a test-tube, introduce a small piece of zinc, add sufficient hydrochloric acid 
to cause a gentle evolution of hydrogen, and over the mouth of the tube place a filter paper saturated with lead acetate solution. In a short time the portion of the paper in contact with the vapors within the test-tube becomes blackened due to the formation of lead sulphide. The nascent hydrogen has reacted with the loosely combined or neutral sulphur to form hydrogen sulphide, and this gas coming in contact with the lead acetate paper has caused the production of the black lead sulphide. Sulphur in the form of inorganic or ethereal sulphuric acid does not respond to this test.

4. Calcium Sulphate Crystals.-Place ro c.c. of urine in a test-tube, add Io drops of calcium chloride solution and allow the tube to stand until crystals form. Examine the calcium sulphate crystals under the microscope and compare them with those shown in Fig. I 28.

\section{Chlorides}

Next to urea, the chlorides constitute the chief solid constituent of the urine. The principal chlorides found in the urine are those of sodium, potassium, ammonium, and magnesium, with sodium chloride predominating. The

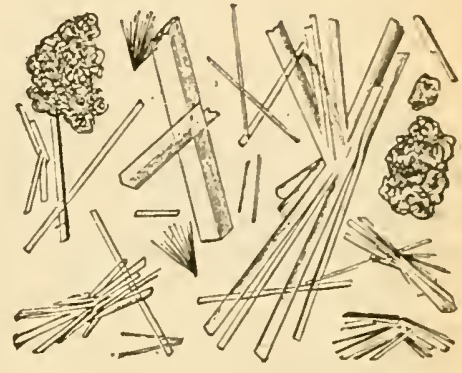

Fig. i 28.-Calciuar Sulphate. (Hensel and Weil.) excretion of chloride is dependent, in great part, upon the nature of the diet, but on the average the daily output is about IO-I 5 grams, expressed as sodium chloride. Copious water drinking increases the output of chlorides considerably. Because of their solubility, chlorides are never found in the urinary sediment.

Since the amount of chlorides excreted in the urine is due primarily to the chloride content of the food ingested, it follows that a decrease in the amount of ingested chloride will likewise cause a decrease in the chloride content of the urine. In cases of actual fasting the chloride content of the urine may be decreased to a slight trace which is derived from the body fluids and tissues. Under these conditions, however, an examination of the blood of the fasting subject will show the percentage of chlorides in this fluid to be approximately normal. This forms a very striking example of the care nature takes to maintain the normal composition of the blood. There is a limit to the power of the body to maintain this equilibrium, however, and if the fasting organism be subjected to the influence of diuretics for a time, a point is reached where the normal composition of the blood can no longer be maintained and a gradual decrease in its chloride content. occurs which finally results in death. Death is supposed to result not so much because of a lack of chlorine as from a deficiency of sodium. This is shown from the fact that potassium chloride, for instance, cannot replace the sodium chloride 
of the blood when the latter is decreased in the manner above stated. When this substitution is attempted the potassium salt is excreted at once in the urine, and death follows as above indicated.

Pathologically the excretion of chlorides may be decreased in some fevers, chronic nephritis, croupous pneumonia, diarrhœa, certain stomach disorders, and in acute articular rheumatism. Any condition accompanied by the formation of an exudate (e.g., pneumonia) will cause a diminished chloride output. In convalescence and with resolution of the exudate the chloride excretion rises again.

\section{EXPERTMENT}

Detection of Chlorides in Urine.-Place about 5 c.c. of urine in a test-tube, render it acid with nitric acid and add a few drops of a solution of silver nitrate. A white precipitate, due to the formation of silver chloride, is produced. This precipitate is soluble in ammonium hydroxide.

\section{Phosphates}

Phosphoric acid exists in the urine in two general forms: First, that in combination with the alkali metals, sodium and potassium, and the radical ammonium; second, that in combination with the alkaline earth metals, calcium and magnesium. Phosphates formed through a union of phosphoric acid with the alkali metals are termed alkaline phosphates, or phosphates of the alkali metals, whereas phosphates formed through a union of phosphoric acid with the alkaline earth metals are termed earthy phosphates, or phosphates of the alkaline earth metals.

Three series of salts are formed by phosphoric acid: Normal, $\mathrm{M}_{3} \mathrm{PO}_{4},{ }^{1}$ mono-hydrogen, $\mathrm{M}_{2} \mathrm{HPO}_{4}$, and di-hydrogen, $\mathrm{MH}_{2} \mathrm{PO}_{4}$. The dihydrogen salts are acid in reaction, and it is claimed that about 60 per cent of the total phosphate content of the urine is in the form of this type ofsalt, and that the acidity of the urine is due in great part to the presence of sodium di-hydrogen phosphate (see page 36r). Henderson" maintains that "determinations of hydrogen ionization in urine and its behavior toward indicators both support the view that in urine there exists a mixture of mono- and di-hydrogen phosphates of sodium, ammonium and other bases."

In bones the phosphates occur principally in the form of the normal salts of calcium and magnesium. The mono-hydrogen salts as a class are alkaline in reaction to litmus, and it is to the presence of di-sodium

${ }^{1} \mathrm{M}$ may be occupied by any of the alkali metals or alkaline earth metals.

${ }^{2}$ Henderson: Am. Jour. Physiol., I 5, 257, I906. 
hydrogen phosphate, $\mathrm{Na}_{2} \mathrm{HPO}_{4}$, that the greater part of the alkalinity of the saliva is due.

The excretion of phosphoric acid is extremely variable, but on the average the total output for 24 hours. is about 2.5 grams, expressed as $\mathrm{P}_{2} \mathrm{O}_{5}$. Ordinarily the total output is mainly in the form of phosphates and is distributed between alkaline phosphates and earthy phosphates approximately in the ratio $2: \mathrm{I}$. The organic phosphorus of the urine constitutes only I-4 per cent of the total phosphorus content. The greater part of this phosphoric acid arises from the ingested food, either from the preformed phosphates or more especially from the phosphorus in organic combination such as we find it in phospho-proteins, mucleoproteins and lecithins; the phosphorus-containing tissues of the body also contribute to the total output of this element. Alkaline phosphates ingested with the food have a tendency to increase the phosphoric acid content of the urine to a greater extent than the earthy phosphates so ingested. This is due, in a measure, to the fact that a portion of the earthy phosphates, under certain conditions, may be precipitated in the intestine and excreted in the feces; this is especially to be noted in the case of herbivorous animals. Since the extent to which the phosphates are absorbed in the intestine depends upon the form in which they are present in the food, under ordinary conditions, there can be no absolute relationship between the urinary output of nitrogen and phosphorus. If the diet is constant, however, from day to day, thus allowing of the preparation of both a nitrogen and a phosphorus balance, ${ }^{1}$ a definite ratio may be established. In experiments upon dogs which were fed an exclusive meat diet, the ratio of nitrogen to phosphorus, in the urine and feces, was found to be $8 . \mathrm{I}: \mathrm{I}$.

It has been demonstrated by recent investigation that the ingestion of inorganic phosphorus compounds may give rise to organic phosphorus compounds such as lecithin, phosphatides, nucleoproteins and phosphoproteins. This is an instance of an organic substance synthesized from an inorganic substance. The experiments have been made principally on ducks ${ }^{2}$ and hens. ${ }^{3}$

Pathologically the excretion of phosphoric acid is increased in such diseases of the bones as diffuse periostosis, osteomalacia, and rickets: according to some investigators, in the early stages of pulmonary tuberculosis, in acute yellow atrophy of the liver, in diseases which are accompanied by an extensive decomposition of nervous tissue, and after

I In metabolism experiments, a statement showing the relation existing between the nitrogen content of the food on the one hand and that of the urine and feces on the other, for a definite period, is termed a nitrogen balance or a "balance of the income and outgo of nitrogen" (see chapter on Metabolism).

2 Fingerling: Biochem. Zeit., 38, 448, rg 2.

3.McCollum and Halpin: Jour. Biol. Chem., II, 47 (Proceedings), 1912. 
sleep induced by potassium bromide or chloral hydrate (Mendel). It is also increased after copious water drinking. A decrease in the excretion of phosphates is at times noted in febrile affections, such as the acute infectious diseases, in pregnancy, in the period during which the fetal bones are forming, and in diseases of the kidneys, because of non-elimination.

The so-called "phosphaturias" many times represent decreased acidity and not increased phosphate content of the urine. Such conditions are, however, significant as indicating a possible tendency to the - formation of phosphatic calculi.

\section{ExPERIMENTS}

I. Formation of "Triple Phosphate."-Place some urine in a beaker, render it alkaline with ammonium hydroxide, add a small amount of magnesium sul-

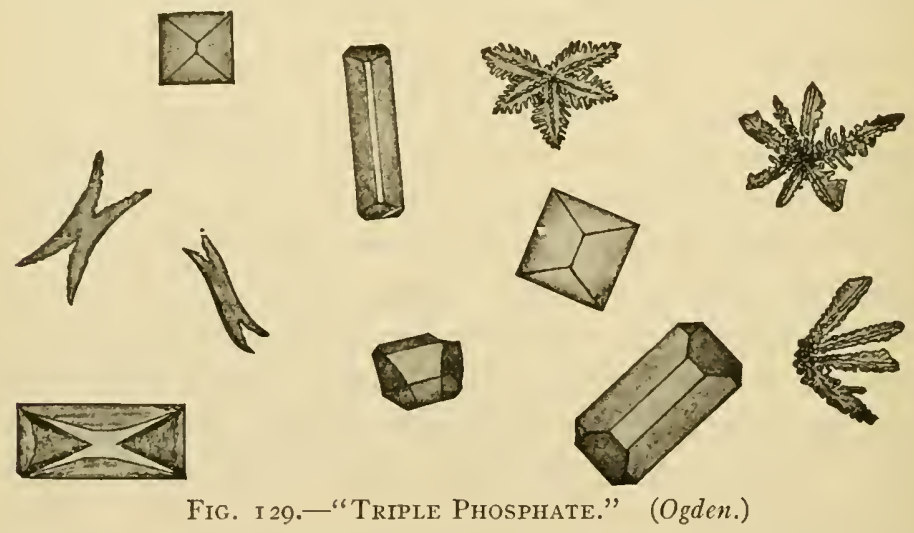

phate solution and allow the beaker to stand in a cool place over night. Crystals of ammonium magnesium phosphate, "triple phosphate," form under these conditions. Examine the crystalline sediment under the microscope and compare the forms of the crystals with those shown in Fig. 129, above.

2. Ammoniacal Fermentation.-Stand some urine aside in a beaker for several days. Ammoniacal fermentation will develop and "triple phosphate" crystals will form.

(a) Examine the sediment under the microscope and compare the crystals with those shown in Fig. I29.

(b) Hold a glass rod dipped in concentrated hydrochloric acid near the surface of the urine. Note the fumes of ammonium chloride.

(c) Insert a strip of red litmus paper in the urine. Permit the paper to dry. Note the gradual restoration of red color, due to volatilization of ammonia (volatile alkali). Run a control test using 0.5 per cent $\mathrm{Na}_{2} \mathrm{CO}_{3}$ (fixed alkali).

3. Detection of Earthy Phosphates.-Place ro c.c. of urine in a test-tube and render it alkaline with ammonium hydroxide. Warm the mixture and note the separation of a precipitate of earthy phosphates. 
4. Detection of Alkaline Phosphates.-Filter off the earthy phosphates as formed in the last experiment, and add a small amount of magnesia mixture (see page 602) to the filtrate. Now warm the mixture and observe the formation of a white precipitate due to the presence of alkaline phosphates. Note the difference in the size of the precipitates of the two forms of phosphates from this same volume of urine. Which form of phosphates was present in the larger amount, earthy or alkaline?

5. Influence upon Fehling's Solution.-Place 2 c.c. of Fehling's solution in a test-tube, dilute it with 4 volumes of water and heat to boiling. Add a solution of sodium dihydrogen phosphate, $\mathrm{NaH}_{2} \mathrm{PO}_{4}$, a small amount at a time, and heat after each addition. What do you observe? What does this observation force you to conclude regarding the interference of phosphates in the testing of diabetic urine by means of Fehling's test?

\section{Sodium and Potassium}

The elements sodium and potassium are always present in the urine. Usually they are combined with such acidic radicals as $\mathrm{Cl}, \mathrm{CO}_{3}, \mathrm{SO}_{4}$ and $\mathrm{PO}_{4}$. The amount of potassium, expressed as $\mathrm{K}_{2} \mathrm{O}$, excreted in 24 hours by an adult, subsisting upon a mixed diet, is on the average 2-3 grams, whereas the amount of sodium, expressed as $\mathrm{Na}_{2} \mathrm{O}$, under the same conditions, is ordinarily $4^{-6}$ grams. The ratio of $\mathrm{K}$ to $\mathrm{Na}$ is generally about $3: 5$. The absolute quantity of these elements excreted depends, of course, in large measure upon the nature of the diet. Because of the non-ingestion of $\mathrm{NaCl}$ and the accompanying destruction of potassium-containing body tissues, the urine during fasting contains more potassium salts than sodium salts.

Pathologically the output of potassium, in its relation to sodium. may be increased during fever; following the crisis, however, the output of this element may be decreased. It may also be increased in conditions associated with acidosis.

\section{Calcium and Magnesium}

The greater part of the calcium and magnesium excreted in the urine is in the form of phosphates. The daily output of calcium, which depends principally upon the nature of the diet, aggregates on the average about $0 . \mathrm{I}-0.4$ gram (expressed as $\mathrm{CaO}$ ) per day. The percentage of calcium salts present in the urine at any one time (10-40 per cent of total calcium output) forms no dependable index as to the absorption of this class of salts, since they are again excreted in to the intestine after absorption. It is therefore impossible to draw any satisfactory conclusions regarding the excretion of calcium unless we obtain accurate analytical data from both the feces and the urine. 
Very little is known positively regarding the actual course of the excretion of the calcium under pathological conditions. An excess is found in some diseases of the bones, e.g., osteomalacia. In others as in rickets the urinary excretion may be very low.

The daily excretion of magnesium by way of the urine usually amounts to between 0.1 and 0.3 gram, expressed as MgO. The amount depends mainly on the diet. About 50 per cent or more of the excreted magnesium is usually eliminated by the kidneys, the remainder passes out in the feces. There may be a retention of magnesium in certain bone disorders accompanying a loss of calcium; in osteomalacia for example. Thus the excretion of calcium and magnesium do not necessarily run parallel.

\section{Carbonates}

Carbonates generally occur in small amount in the urine of man and carnivora under normal conditions, whereas much larger quantities are ordinarily present in the urine of herbivora. The alkaline reaction of the urine of herbivora is dependable in great measure upon the presence of carbonates. In general a urine containing carbonates in appreciable amount is turbid when passed or becomes so shortly after. These bodies ordinarily occur as alkali or alkaline earth compounds and the turbid character of urine containing them is usually due principally to the latter class of substances. The carbonates of the alkaline earths are often found in amorphous urinary sediments.

\section{Iron}

Iron is present in small amount in normal urine. It probably occurs partly in inorganic and partly in organic combination. The iron contained in urinary pigments or chromogens is in organic combination. According to different investigators the iron content of normal urine will probably not average more than I-5 mg. per day. After splenectomy there is an increased loss of iron from the body particularly by way of the feces (Asher).

\section{EXPERIMENT}

Detection of Iron in Urine.-Evaporate a convenient volume (IO-I 5 C.c.) of urine to dryness. Incinerate and dissolve the residue in a few drops of iron-free hydrochloric acid and dilute the acid solution with 5 c.c. of water. Divide the acid solution into two parts and make the following tests: (a) To the first part add a solution of ammonium thiocyanate; a red color indicates the presence of iron. (b) To the second part of the solution add a little potassium ferrocyanide solution; a precipitate of Prussian blue forms upon standing. 


\section{Fluorides, Nitrates, Silicates and Hydrogen Peroxide}

These substances are all found in traces in human urine under normal conditions. Nitrates are undoubtedly introduced into the organism in the water and ingested food. The average excretion of nitrates is about 0.5 gram per day, the output being the largest upon a vegetable diet and smallest upon a meat diet. Nitrites are found only in urine which is undergoing decomposition and are formed from nitrates in the course of ammoniacal fermentation. Hydrogen peroxide has been detected in the urine, but its presence is believed to possess no pathological importance. 


\section{CHAPTER XXIII}

\section{URINE : PATHOLOGICAL CONSTITUENTS ${ }^{1}$}

Glucose.

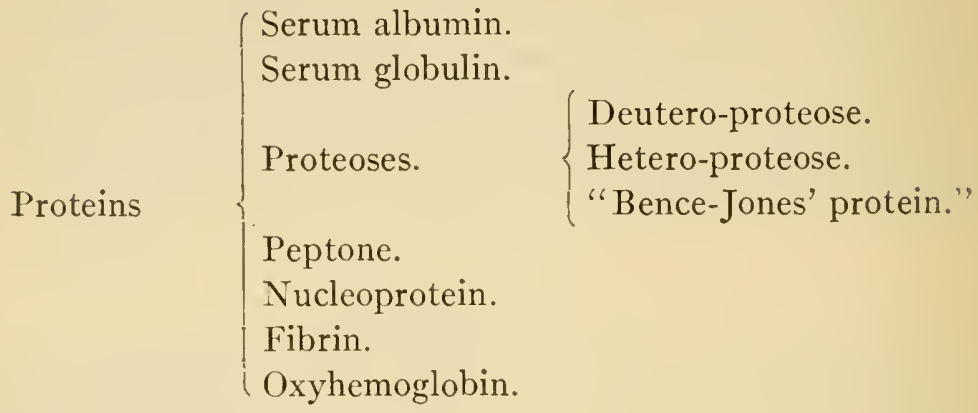

Blood $\left\{\begin{array}{l}\text { Form elements. } \\ \text { Pigment. }\end{array}\right.$

Pus.

Bile. $\left\{\begin{array}{l}\text { Pigments. } \\ \text { Acids. }\end{array}\right.$

Creatine. $^{2}$

Acetone.

Acetoacetic acid.

$\beta$-Hydroxybutyric acid.

Conjugate glycuronates.

Pentoses.

Fat.

Hematoporphyrin.

Lactose.

Galactose.

Fructose.

Arsenic.

Mercury.

Inositol.

Laiose.

Melanin.

Urorosein.

Nephrorosein.

Urochromogen.

Unknown substances.

${ }^{1}$ See note at the bottom of page 369 .

${ }^{2}$ Normal constituent of urine of infants and children. 


\section{GLUCOSE}

Traces of this sugar occur in normal urine, but the amount is not sufficient to be readily detected by the ordinary simple qualitative tests. There are two distinct types of pathological glycosuria, i.e, transitory glycosuria and persistent glycosuria. The transitory type may follow the ingestion of an excess of sugar, causing the assimilation limit to be exceeded, or it may accompany any one of several disorders which cause impairment of the power of assimilating sugar. In the persistent type large amounts of sugar are excreted daily in the urine for long periods of time. Under such circumstances a condition known as diabetes mellitus exists. In this disorder the urine may contain Io per cent of glucose and the average sugar content is $3-5$ per cent. Ordinarily, diabetic urine which contains a high percentage of sugar possesses a faint yellow color, a high specific gravity, and a volume which is above normal. Over roo grams of sugar are daily eliminated in some severe cases of diabetes mellitus.

\section{EXPERIMENTS}

The various tests for glucose in the urine which are embraced in the experiments given herewith are based upon one of the following properties of this sugar:

(I) Its power to reduce the oxides of certain metals in alkaline solution.

(2) Its power to rotate the plane of polarized light.

(3) Its power to form crystalline osazones with phenylhydrazine.

(4) Its ability to ferment with ordinary yeast.

I. Phenylhydrazine Reaction.-Test the urine according to one of the following methods: (a) To a small amount of phenylhydrazine mixture (enough to fill the rounded portion of a small test-tube), furnished by the instructor, ${ }^{1}$ add 5 c.c. of the urine, shake well, and heat on a boiling water-bath for one-half to three-quarters of an hour. Allow the tube to cool slowly (not under the tap) and examine the crystals microscopically (Plate III, opposite page 22). If the solution has become too concentrated in the boiling process it will be light red in color and no crystals will separate until it is diluted with water.

Yellow crystalline bodies called osazones are formed from certain sugars under these conditions, in general each individual sugar giving rise to an osazone of a definite crystalline form which is typical for that sugar.

It is important to remember in this connection that, of the simple sugars of interest in physiological chemistry, glucose and fructose yield the same osazone, with phenylhydrazine. Each ozazone has a definite

1 This mixture is prepared by combining one part of phenylhydrazine-hydrochloride and two parts of sodium acetate, by wight. These are thoroughly mixed in a mortar. 
melting-point, and as a further and more accurate means of identification it may be recrystallized and identified by the determination of its melting-point and nitrogen content. The reaction taking place in the formation of phenylglucosazone is as follows:

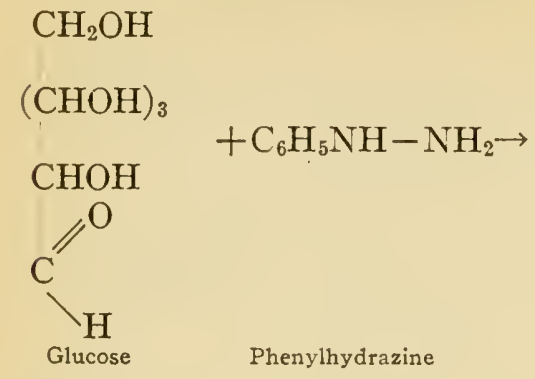

$\mathrm{CH}_{2} \mathrm{OH}$

$(\mathrm{CHOH})_{3}$

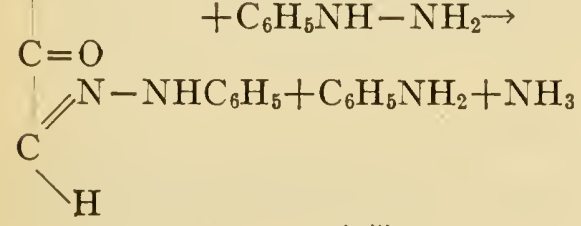

Aniline
$\mathrm{CH}_{2} \mathrm{OH}$

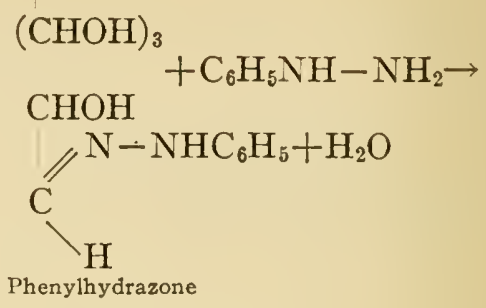

$\mathrm{CH}_{2} \mathrm{OH}$

$(\mathrm{CHOH})_{3}$

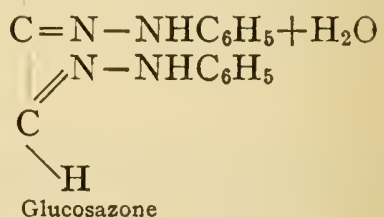

(b) Place 5 c.c. of the urine in a test-tube, add I c.c. of phenylhydrazineacetate solution furnished by the instructor, ${ }^{1}$ and heat on a boiling water-bath for one-half to three-quarters of an hour. Allow the liquid to cool slowly and examine the crystals microscopically (Plate III, opposite page 22).

The phenylhydrazine test has been so modified by Cipollina as to be of use as a rapid clinical test. The directions for this test are given in the next experiment.

2. Cipollina's Test.-Thoroughly mix 4 c.c. of urine, 5 drops of phenylhydrazine (the base) and $1 / 2$ c.c. of glacial acetic acid in a test-tube. Heat the mixture for about one minute over a low flame, shaking the tube continually to prevent loss of fluid by bumping. Add 4-5 drops of potassium hydroxide or sodium hydroxide (sp. gr. I.I6), being certain that the fluid in the test-tube remains acid; heat the mixture again for a moment and then cool the contents of the tube. Ordinarily the crystals form at once, especially if the urine possesses a low specific gravity. If they do not appear immediately allow the tube to stand at least 20 minutes before deciding upon the absence of sugar.

Examine the crystals under the microscope and compare them with those shown in Plate III, opposite page 22.

3. Riegler's Reaction. ${ }^{2}$-Introduce 0.1 gram of phenylhydrazine-hydrochloride and $0.25 \mathrm{gram}$ of sodium acetate into a test-tube, add 20 drops of the urine under

${ }^{1}$ This solution is prepared by mixing one part by volume, in each case of glacial acetic acid, one part of water and two parts of phenylhydrazine (the base).

${ }^{2}$ Riegler: Compt. rend. soc. biol., 66, p. 795 . 
examination, and heat the mixture to boiling. Now introduce ro c.c. of a 3 per cent solution of potassium hydroxide and gently shake the tube and contents. If the urine under examination contains glucose the liquid in the tube will assume a red color. One per cent glucose yields an immediate color whereas 0.05 per cent yields the color only after the lapse of a period of one-half hour from the time the alkali is added. If the color appears after the 30-minute interval the color change is without significance inasmuch as sugar-free urines will respond thus. The reaction is given by all aldehydes and therefore the test cannot be safely employed in testing urines preserved by formaldehyde. Albumin does not interfere with the test.

4. Bottu's Test. ${ }^{1}$-To 8 c.c. of Bottu's reagent ${ }^{2}$ in a test-tube add I c.c. of the urine under examination and mix the liquids by gentle shaking. Now heat the upper portion of the mixture to boiling, add an additional I c.c. of urine and heat the mixture again immediately. The appearance of a blue color accompanied by the precipitation of small particles of indigo blue indicates the presence of glucose in the urine under examination. The test will serve to detect the presence of $0 . \mathrm{I}$ per cent of glucose and is uninfluenced by creatinine or by ammonium salts.

5. Reduction Tests.- To their aldehyde or ketone structure many sugars owe the property of readily reducing the alkaline solutions of the oxides of metals like copper, bismuth, and mercury; they also possess the property of reducing ammoniacal silver solutions with the separation of metallic silver. Upon this property of reduction the most widely used tests for sugars are based. When whitish-blue cupric hydroxide in suspension in an alkaline liquid is heated it is converted into insoluble black cupric oxide, but if a reducing agent like certain sugars be present the cupric hydroxide is reduced to insoluble yellow cuprous hydroxide, which in turn on further heating may be converted into brownish-red or red cuprous oxide. These changes are indicated as follows:
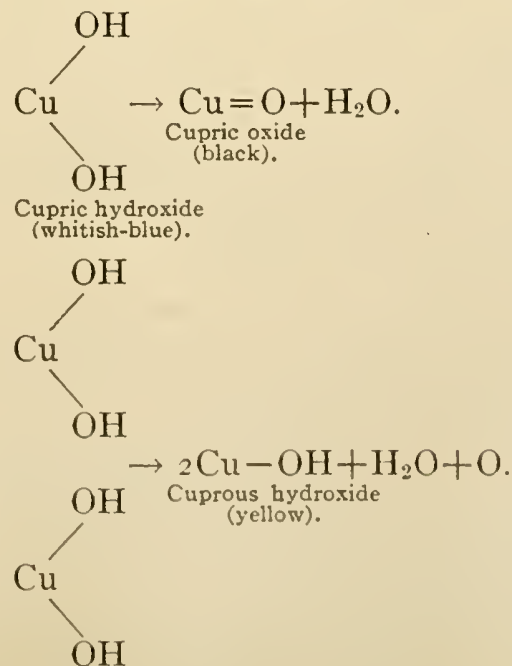

${ }^{1}$ Bottu: Compl. rend. soc. biol., 66, p. 972.

${ }^{2}$ This reagent contains 3.5 grams of $o$-nitrophenylpropiolic acid and 5 c.c. of a freshly prepared ro per cent solution of sodium hydroxide per liter. 


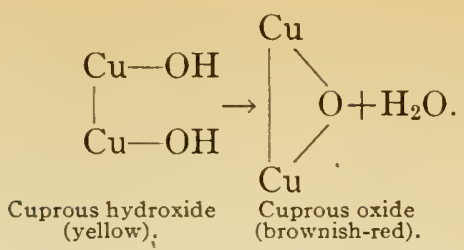

'The chemical equations here discussed are exemplified in Trommer's and Fehling's tests.

(a) Trommer's Test.-To 5 c.c. of urine in a test-tube add one-half its volume of $\mathrm{KOH}$ or $\mathrm{NaOH}$. Mix thoroughly and add, drop by drop, agitating after the addition of each drop, a very dilute solution of copper sulphate. Continue the addition until there is a slight permanent precipitate of cupric hydroxide and in consequence the solution is slightly turbid. Heat, and the cupric hydroxide is reduced to yellow cuprous hydroxide or to brownish-red cuprous oxide.

If the solution of copper sulphate used is too strong, a small brownish-red precipitate produced in the presence of a low percentage of glucose may be entirely masked. On the other hand, if too little copper sulphate is used a light-colored precipitate formed by uric acid and purine bases may obscure the brownish-red precipitate of cuprous oxide. The action of $\mathrm{KOH}$ or $\mathrm{NaOH}$ in the presence of an excess of sugar and insufficient copper will produce a brownish color. Phosphates of the alkaline earths may also be precipitated in the alkaline solution and be mistaken for cuprous hydroxide. Trommer's test is not very satisfactory.

Salkowski ${ }^{1}$ has proposed a modification of the Trommer procedure which he claims is a very accurate sugar test.

(b) Fehling's Test.-To about I c.c. of Fehling's solution ${ }^{2}$ in a test-tube add about 4 c.c. of water, and boil. ${ }^{3}$ [The cupric hydroxide is held in solution by the sodium potassium tartrate (Rochelle salt).] This is done to determine whether the solution will of itself cause the formation of a precipitate of brownish-red cuprous oxide. If such a precipitate forms, the Fehling's solution must not be used. Add urine to the hot Fehling's solution, a few drops at a time, and heat the mixture to boiling after each addition (never add more urine than the original volume of Fehling's solution). The production of yellow cuprous hydroxide or brownish-red cuprous oxide indicates that reduction has taken place. The yellow precipitate is more likely to occur if the urine is added rapidly and in large amount, whereas with a less rapid addition of smaller amounts of urine the brownish-red precipitate is generally formed.

This is a much more satisfactory test than Trommer's, but even this test is not entirely reliable when used to detect sugar in the urine.

\footnotetext{
${ }^{1}$ Salkowski: Zeit. physiol. Chem., 79, I64, I912.

${ }^{2}$ Fehling's solution is composed of two definite solutions-a copper sulphate solution and an alkaline tartrate solution, which may be prepared as follows:

Copper sulphate solution $=34.65$ grams of copper sulphate dissolved in water and made up to 500 c.c.

Alkaline tartrate solution $=\mathrm{r} 25$ grams of potassium hydroxide and 173 grams of Rochelle salt dissolved in water and made up to 500 c.c.

These solutions should be preserved separately in rubber-stoppered bottles and mixed in equal volumes when needed for use. This is done to prevent deterioration.

${ }^{3}$ More dilute Fehling solution should be used in testing urines containing small amounts of sugar. In case of urines containing a high concentration of sugar it may sometimes be desirable to use a larger volume of Fehling's solution.
} 
Such bodies as conjugate glycuronates, uric acid, nucleoprotein, and homogentisic acid, when present in sufficient amount, may produce a result similar to that produced by sugar. Phosphates of the alkaline earths may be precipitated by the alkali of the Fehling's solution and in appearance may be mistaken for the cuprous hydroxide. Cupric hydroxide may also be reduced to cuprous oxide and this in turn be dissolved by creatinine, a normal urinary constituent. This will give the urine under examination a greenish tinge and may obscure the sugar reaction even when a considerable amount of sugar is present. According to Laird ${ }^{1}$ even small amounts of creatinine will retard the reaction velocity of reducing sugars with Fehling's solution.

Conjugate glycuronates are formed after the ingestion of such substances as chloral hydrate, camphor, menthol, thymol, antipyrin, phenol, etc. The chloral hydrate is excreted in the urine as trichlorethylglycuronic acid, $\mathrm{C}_{2} \mathrm{Cl}_{3} \mathrm{H}_{2} \cdot \mathrm{C}_{6} \mathrm{H}_{9} \mathrm{O}_{7}$. This compound reduces Fehling's solution and is levorotatory, whereas glucose also reduces but is dextrorotatory. Therefore by means of a polariscopic test we may differentiate between a "chloral urine" and a "sugar urine."

In testing urine preserved by chloroform a positive test may be obtained in the absence of sugar. This is due to the fact that the hot alkali produces formic acid (a reducing fatty acid) from the chloroform.

Ammonium salts also interfere with Fehling's test. If present in excess the urine should be made alkaline and boiled in order to decompose the ammonium salts.

(c) Benedict's Modifications of Fehling's Test.-First Modification.-To 2 c.c. of Benedict's solution ${ }^{2}$ in a test-tube add 6 c.c. of distilled water and 7-9 drops (not more) of the urine under examination. Boil the mixture vigorously for about r $5-30$ seconds and permit it to cool to room temperature spontaneously. (If desired this process may be repeated, although it is ordinarily unnecessary.) If sugar is present in the solution a precipitate will form which is often bluishgreen or green at first, especially if the percentage of sugar is low, and which usually becomes yellowish upon standing. If the sugar present exceeds 0.06 per cent this precipitate generally forms at or below the boiling-point, whereas if less than 0.06 per cent of sugar is present the precipitate forms more slowly and generally only after the solution has cooled. The greenish precipitate obtained with urines containing small amounts of sugar may be a compound of copper with the sugar or a compound of some constituent of the urine with reduced copper

${ }^{1}$ Laird: Journ. Palh. and Bacl., r6, 398 rgr2.

${ }^{2}$ Benedict's modificd Fehling solution consists of two definite solutions-a copper sulphate solution and an alkaline tartrate solution, which may be prepared as follows:

Copper sulphate solution $=34.65$ grams of copper sulphate dissolved in water and made up to 500 c.c.

Alkaline tartrate solution $=100$ grams of anhydrous sodium carbonate and $173 \mathrm{grams}$ of Rochelle salt dissolved in water and made up to 500 c.c.

These solutions should be preserved separately in rubber-stoppered bottles and mixed in equal volumes when needed for use. This is done to prevent deterioration. 
oxide instead of being a precipitate of cuprous hydroxide or oxide as is the case when the original Fehling solution is reduced.

Benedict claims that, whereas the original Fehling's test will not serve to detect sugar when present in a concentration of less than o.I per cent, that the above modification will serve to detect sugar when present in as small quantity as $0.015^{-0.02}$ per cent. This claim has been corroborated by Harrison. ${ }^{1}$ The modified solution used in the above test differs from the original in that Ioo grams of sodium carbonate is substituted for the 125 grams of potassium hydroxide ordinarily used, thus forming a Fehling solution which is considerably less alkaline than the original. This alteration in the composition of the Fehling solution is of advantage in the detection of sugar in the urine inasmuch as the strong alkalinity of the ordinary Fehling solution has a tendency, when the reagent is boiled with a urine containing a small amount of glucose, to decompose sufficient of the sugar to render the detection of the remaining portion exceedingly difficult by the usual technic. Benedict claims that for this reason the use of his modified solution permits the detection of smaller amounts of sugar than does the use of the ordinary Fehling solution.

Second Modification. ${ }^{2-B e n e d i c t ~ h a s ~ f u r t h e r ~ m o d i f i e d ~ h i s ~ s o l u t i o n ~ a n d ~ h a s ~}$ succeeded in obtaining one which does not deteriorate upon long standing. ${ }^{3}$ The following is the procedure for the detection of glucose in the urine: To 5 c.c. of the reagent in a test-tube add 8 (not more) drops of the urine to be examined. The fluid is then boiled vigorously for from one to two minutes and then allowed to cool spontaneously. In the presence of glucose the entire body of the solution will be filled with a precipitate, which may be red, yellow, or green in color, depending upon the amount of sugar present. If no glucose is present, the solution will either remain perfectly clear, or will show a very faint turbidity, due to precipitated urates.

Even very small quantities of glucose in urine (O.I per cent) yield precipitates of surprising bulk with this reagent, and the positive reaction for glucose is the filling of the entire body of the solution with a precipitate, so that the solution becomes opaque. Since amount

${ }^{1}$ Harrison: Pharm. Jour., 87, 746, I9I I.

2 Benedict: Jour. Am. Mcd. Ass'n, 57, I I93, I9гі.

'Benedict's new solution has the following composition:

Copper sulphate....................... I7. $3 \mathrm{gm}$.

Sodium citrate.......................... I73.0 gm.

Sodium carbonate (anhydrous)................. I00.0 gm.

Distilled water to........................... 1000.0 c.c.

With the aid of heat dissolve the sodium citrate and carbonate in about 600 c.c. of water. Pour (through a folded filter if necessary) into a glass graduate and make up to 850 c.c. Dissolve the copper sulphate in about Ioo c.c. of water and make up to I50 c.c. Pour the carbonate-citrate solution into a large beaker or casserole and add the copper sulphate solution slowly, with constant stirring. The mixed solution is ready for use, and does not deteriorate upon long standing. 
rather than color of the precipitate is made the basis of this test, it may be applied, even for the detection of small quantities of glucose, as readily in artificial light as in daylight. Chloroform does not interfere with this test nor do uric acid or creatinine interfere to such an extent as in the case of Fehling's test.

(d) Haines' Test.-This is a copper reduction test similar in many respects to the Fehling and Benedict reactions. In Haines' solution ${ }^{1}$ the cupric hydroxide is held in solution by glycerol instead of Rochelle salt as in Fehling's solution.

Perform the test as follows: Introduce about 5 c.c. of Haines' solution ${ }^{1}$ into a test-tube and heat to boiling. If no reduction occurs add 6-8 drops of the urine and again bring to a boil. If glucose is present an abundant yellow (cuprous hydroxide) or brownish-red (cuprous oxide) precipitate is thrown down. This test is about as delicate as Fehling's test.

(e) Allen's Modification of Fehling's Test.-The following procedure is recommended: "From 7 to 8 c.c. of the sample of urine to be tested is heated to boiling in a test-tube, and, without separating any precipitate of albumin which may be produced, 5 c.c. of the solution of copper sulphate used for preparing Fehling's solution is added. This produces a precipitate containing uric acid, xanthine, hypoxanthine, phosphates, etc. To render the precipitation complete, however, it is desirable to add to the liquid, when partially cooled, from I to 2 c.c. of a saturated solution of sodium acetate having a feebly acid reaction to litmus. ${ }^{2}$ The liquid is filtered and to the filtrate, which will have a bluish-green color, 5 c.c. of the alkaline tartrate mixture used for preparing Fehling's solution is added, and the liquid boiled for I 5-20 seconds. In the presence of more than 0.25 per cent of sugar, separation of cuprous oxide occurs before the boiling-point is reached; but with smaller quantities precipitation takes place during the cooling of the solution, which becomes greenish, opaque, and suddenly deposits cuprous oxide as a fine brownish-red precipitate."

Mercuric Oxide Reduction Test (Cramer). ${ }^{3}$ - This test depends on the reduction of mercuric oxide in a weakly alkaline solution with the formation of metallic mercury. The degree of alkalinity is an important factor, as the test becomes more sensitive but less specific the greater the alkalinity of the reagent.

Apply the test as follows:

Introduce 3 c.c. of Cramer's "2.5 standard reagent" into a test-tube and heat to boiling. The reagent remains clear but becomes slightly yellow. Add 3 c.c. of urine and heat the mixture to boiling. Remove the tube from the flame, and

${ }^{1}$ Haines solution may be prepared by dissolving 8.314 grams of copper sulphate in 400 c.c. of water adding 40 c.c. of glycerol and 500 c.c. of 5 per cent potassium bydroxide solution.

${ }^{2}$ Sufficient acetic acid should be added to the sodium acetate solution to render it feebly acid to litmus. A saturated solution of sodium ace tate keeps well, but weaker solutions are apt to become mouldy, and then possess the power of reducing Fehling's solution. Hence it is essential in all cases of importance to make a blank test by mixing equal measures of copper sulphate solution, alkaline tartrate solıtion and water, adding a little sodium ace tate solution, and heating the mixture to boiling.

${ }^{3}$ Cramer: Bioch. Jour., 9, I56, 1915.

4 See Chapter II, page 28. 
after 30 seconds acidify with a few drops of acetic acid. Hold the tube over ordinary print. If the urine is normal, a slight but distinct turbidity remains but the print is clearly readable. If sugar is present in the urine above the normal concentration, the contents of the tube darken and on standing a finely divided precipitate of mercury settles to the bottom of the tube. The amount of the precipitate depends upon the concentration of reducing sugar in the urine.

It is claimed that this test is free from fallacies inherent in Fehling's test as the result of the reducing action of uric acid and creatinine. The test is said to be more sensitive than Fehling's test or the bismuth reduction tests, and is particularly suitable for the examination of urines in which the amount of sugar present exceeds the normal amount only slightly.

If the reagent be made more alkaline than indicated, it ceases to be specific for reducing sugars.

(f) Bismuth Reduction Test (Boettger). - To 5 c.c. of urine in a test-tube add I c.c. of $\mathrm{KOH}$ or $\mathrm{NaOH}$ and a very small amount of bismuth subnitrate, and boil. The solution will gradually darken and finally assume a black color due to reduced bismuth. If the test is made with urine containing albumin this must be removed, by boiling and filtering, before applying the test, since with albumin a similar change of color is produced (bismuth sulphide).

(g) Bismuth Reduction Test (Nylander).-To 5 c.c. of urine in a test-tube add one-tenth its volume of Nylander's reagent ${ }^{1}$ and heat for five minutes in a boiling water-bath. ${ }^{2}$ The mixture will darken if reducing sugar is present and upon standing for a few moments a black color will appear.

This color is due to the precipitation of bismuth. If the test is made on urine containing albumin this must be removed, by boiling and filtering, before applying the test, since with albumin a similar change of color is produced. Glucose when present to the extent of 0.08 per cent may be easily detected by this reaction (Rabe ${ }^{3}$ claims that o.oI per cent may be so detected). Uric acid and creatinine which interfere with the Fehling test do not interfere with the Nylander's reaction. It is claimed by Bechold that the bismuth reduction tests give a negative reaction with solutions containing sugar when mercuric chloride or chloroform is present. Other observers ${ }^{4}$ have failed to verify the inhibitory action of the mercuric chloride and have shown that the inhibitory influence of chloroform may be overcome by raising

${ }^{1}$ Nylander's reagent is prepared by digesting 2 grams of bismuth subnitrate and 4 grams of Rochelle salt in roo c.c. of a Io per cent potassium hydroxide solution. The reagent is then cooled and filtered.

${ }^{2}$ Hammarsten suggests that the solution be boiled for $2-5$ minutes (according to the sugar content) over a free flame and the tube then permitted to stand five minutes before drawing conclusions.

Rabe: Apolh. Ztg., 29, 554, I9I4.

"Rehfuss and Hawk: Jour. Biol. Chem., 7, 267, r910; also Zeidlitz: C psala Lakäreforen Forh., N. F., II, Ig06. 
the temperature of the urine to the boiling-point for a period of five minutes previous to making the test.

Urines rich in indican, uroerythrin, urochrome or hematoporphyrin, as well as urines excreted after the ingestion of large amounts of certain medicinal substances, may give a darkening of the Nylander's reagent similar to that of a true sugar reaction. It is a disputed point whether the urine after the administration of urotropin will reduce the Nylander reagent. ${ }^{1}$

Straus $z^{2}$ has recently shown that the urine of diabetics to whom "Iothion" (diiodohydroxypropane) has been administered will give a negative Nylander's reaction and respond positively to the Fehling and polarization tests. "Iothion" also interferes with the Nylander test in vitro whereas KI and I do not.

According to Rustin and Otto the addition of $\mathrm{PtCl}_{4}$ increases the delicacy of Nylander's reaction. They claim that this procedure causes the sugar to be converted quantitatively. No quantitative method has yet been devised, however, based upon this principle.

A positive bismuth reduction test is probably due to the following reactions:

(a)

(b)

$$
\begin{gathered}
\mathrm{Bi}(\mathrm{OH})_{2}(\mathrm{NO})_{3}+\mathrm{KOH} \rightarrow \mathrm{Bi}(\mathrm{OH})_{3}+\mathrm{KNO}_{3} . \\
2 \mathrm{Bi}(\mathrm{OH})_{3}-3 \mathrm{O} \rightarrow \mathrm{Bi}_{2}+3 \mathrm{H}_{2} \mathrm{O} .
\end{gathered}
$$

Bohmansson, ${ }^{3}$ before testing the urine under examination treats it (Io c.c.) with ${ }^{1} 5$ volume of 25 per cent hydrochloric acid and $1 \frac{2}{2}$ volume of boneblack. This mixture is shaken one minute, then filtered, and the neutralized filtrate tested by Nylander's reaction. Bohmansson claims that this procedure removes certain interfering substances. notably urochrome.

6. Fermentation Test.-Rub up in a mortar about 15 c.c. of the urine with a small piece of compressed yeast. Transfer the mixture to a saccharometer (Fig. 3, page 3I) and stand it aside in a warm place for about 12 hours. If glucose is present, alcoholic fermentation will occur and carbon dioxide will collect as a gas in the upper portion of the tube. On the completion of fermentation, introduce, by means of a bent pipette, a little $\mathrm{KOH}$ solution in to the graduated portion, place the thumb tightly over the opening in the apparatus and invert the saccharometer. Remembering that $\mathrm{KOH}$ has the power to absorb $\mathrm{CO}_{2}$ how do you explain the result? ${ }^{4}$

7. Polariscopic Examination.-For directions as to the use of the polariscope see Chapter II.

'Abt: Archives of Pedialrics, 24, 275, 1907; also Weitbrecht: Schuciz. II"och., 47, 577 , I909.

${ }^{2}$ Strausz: Mïnch. med. Hoch., 59, 85 , 19 r2.

${ }^{3}$ Bohmansson: Biochem. Zcil., I0, D. 2 SI.

- The findings of Neuberg and associates indicate that the liberation of carbon dioxide by yeast is not necessarily a criterion of the presence of sugar. The presence of an enzyme called carboxylase has been demonstrated in yeast which has the prover of splicting off $\mathrm{CO}_{2}$ from the carboxyl group of amino- and other aliphatic acids. 


\section{PROTEINS}

Normal urine contains a trace of protein material, but the amount present is so slight as to escape detection by any of the simple tests in general use for the detection of protein urinary constituents. The following are the more important forms of protein material which have been detected in the urine under pathological conditions:

(I) Serum albumin.

(2) Serum globulin.

(3) Proteoses

Deutero-proteose.

Hetero-proteose.

"Bence-Jones' protein."

(4) Peptone.

(5) Nucleoprotein.

(6) Fibrin.

(7) Oxyhemoglobin.

\section{ALBUMIN}

Normal urine contains a trace of albumin which is too slight to be detected by the usual procedures.

Albuminuria is a condition in which serum albumin or serum globulin appears in the urine. There are two distinct forms of albuminuria, i.e., renal albuminuria and accidental albuminuria. Sometimes the terms "true" albuminuria and "false" albuminuria are substituted for those just given. In the renal type the albumin is excreted by the kidneys. This is the more serious form of the malady and at the same time is more frequently encountered than the accidental type. Among the causes of renal albuminuria are altered blood pressure in the kidneys, altered kidney structure, or changes in the composition of the blood entering the kidneys, thus allowing the albumin to diffuse more readily. In the accidental form of albuminuria the albumin is not excreted by the kidneys as is the case in the renal form of the disorder, but arises from the blood, lymph, or some albumin-containing exudate coming into contact with the urine at some point below the kidneys. It has been suggested $^{1}$ that albuminurias may be classed as pre-renal, renal and post-renal. The pre-renal type is illustrated by the albuminuria of heart disease, whereas the post-renal form corresponds to what we have called "accidental" albuminuria.

The determination of albumin may be of assistance in following the course of kidney disturbances, but the results can only be interpreted in the light of other clinical findings.

${ }^{1}$ Bruce: Lancet, May 6, I9II, p. I 205. 


\section{EXPERIMENTS}

(The urine should be filtered before performing these tests.)

Nitric Acid Ring Test (Heller.) - Place 5 c.c. of concentrated $\mathrm{HNO}_{3}$ in a testtube, incline the tube, and by means of a pipette allow the urine to flow slowly down the side. The liquids should stratify with the formation of a white zone of precipitated albumin at the point of juncture.

If the albumin is present in very small amount the white zone may not form until the tube has been allowed to stand for several minutes. If the urine is quite concentrated a white zone, due to uric acid or urates, will form upon treatment with nitric acid as indicated. 'This ring may be easily differentiated from the albumin ring by repeating the test after diluting the urine with 3 or 4 volumes of water, whereupon the ring, if due to uric acid or urates, will not appear. It is ordinarily possible to differentiate between the albumin ring and the uric acid ring without diluting the urine, since the ring, when due to uric acid, has ordinarily a less sharply defined upper border, is generally broader than the albumin ring and frequently is situated in the urine aboie the point of contact with the nitric acid. Concentrated urines also occasionally exhibit the formation, at the point of contact, of a crystalline ring with very sharply defined borders. This is urea nitrate and is easily distinguished from the "fluffy" ring of albumin. If there is any difficulty in differentiation a simple dilution of the urine with water, as above described, will remove the difficulty. Various colored zones, due either to the presence of indican, bile pigments, or to the oxidation of other organic urinary constituents, may form in this test under certain conditions. These colored rings should never be confounded with the white ring which alone denotes the presence of albumin.

After the administration of certain drugs a white precipitate of resin acids may form at the point. of contact of the two fluids and may cause the observer to draw wrong conclusions. This ring, if composed of resin acids, will dissolve in alcohol, whereas the albumin ring will not dissolve in this solvent.

Weinberger has shown that a ring closely resembling the albumin ring is often obtained in urines preserved for a considerable time by thymol when subjected to the nitric acid test. The ring is due to the formation of nitrosothymol and possibly nitrothymol. If the thymol is removed from the urine by extraction with petroleum ether ${ }^{1}$ previous to adding nitric acid, the ring does not form.

An instrument called the albumoscope (horismuscope) has been de-

${ }_{1}^{1}$ Accomplished readily by gently agitating cqual volumes of petroleum ether and the urine under examination for two minulcs in a test-tube before applying the test. 
vised for use in this test and has met with considerable favor. The method of using the albumoscope is described below.

Use of the Albumoscope.-This instrument is intended to facilitate the making of "ring" tests such as Heller's and Roberts'. In making a test about 5 c.c. of the solution under examination is first introduced into the apparatus through the larger arm (see Fig. I30), and the reagent used in the particular test is then introduced through the capillary arm and allowed to flow down underneath the solution under examination. If a reasonable amount of care is taken there is

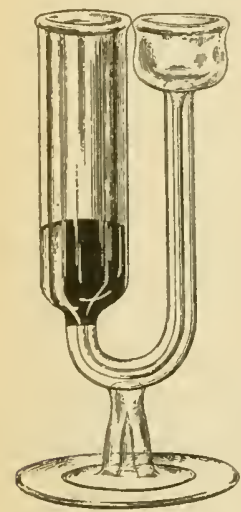

Fig. I30.-ALBUnOSCOPE. no possibility of mixing the two solutions and a definitely defined white "ring" is easily obtained at the zone of contact.

2. Nitric Acid and Magnesium Sulphate Ring Test (Roberts).-Place 5 c.c. of Roberts' reagent ${ }^{1}$ in a testtube, incline the tube, and by means of a pipette allow the urine to flow slowly down the side. The liquids should stratify with the formation of a white zone of precipitated albumin at the point of juncture.

This test is a modification of Heller's ring test and is rather more satisfactory than that test, since the colored rings never form and the consequent confusion is avoided. The albumoscope (see above) may also be used in making this test.

3. Spiegler's Ring Test.-Place 5 c.c. of Spiegler's reagent ${ }^{2}$ in a test-tube, incline the tube and, by means of a pipette, allow 5 c.c. of urine, acidified with acetic acid, to flow slowly down the side. A white zone will form at the point of contact. This is an exceedingly delicate test, in fact too delicate for ordinary clinical purposes, since it serves to detect albumin when present in the merest trace $(1: 250,000)$ and hence most normal urines will give a positive reaction for albumin when this test is applied. Proteose and peptone are also said to respond to this test.

4. Coagulation or Boiling Test.-(a) Heat 5 c.c. of urine to boiling in a testtube. (If the urine is not clear it should be filtered.) A precipitate forming at this point is due either to albumin or to phosphates. Acidify the urine slightly by the addition of $3-5$ drops of very dilute acetic acid, adding the acid drop by drop to the hot solution. If the precipitate is due to phosphates it will disappear under these conditions, whereas if it is due to albumin it will not only fail to disappear but will become more flocculent in character, since the reaction of a

${ }^{1}$ Robert's reagent is composed of I volume of concentrated $\mathrm{HNO}_{3}$ and 5 volumes of a saturated solution of $\mathrm{MgSO}_{4}$.

${ }^{2}$ Spiegler's reagent has the following composition:

Tartaric acid..

20 grams.

Iercuric chloride........................ 40 grams.

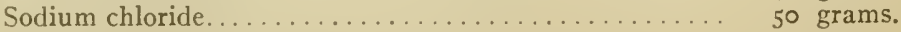

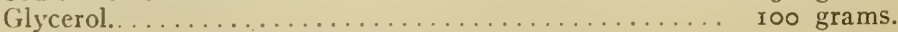

Distilled water......................... 1000 grams. 
fluid must be acid to secure the complete precipitation of the albumin by this coagulation process.

Too much acid should be aroided since it will cause the albumin to go into solution. Certain resin acids may be precipitated by the acid, but the precipitate due to this cause may be easily differentiated from the albumin precipitate by reason of its solubility in alcohol.

(b) A modification of this test in quite general use is as follows: Fill a testtube two-thirds full of urine and gently heat the upper half of the fluid to boiling, being careful that this fluid does not mix with the lower half. A turbidity indicates albumin or phosphates. Acidify the urine slightly by the addition of 3-5 drops of dilute acetic acid, when the turbidity, if due to phosphates, will disappear.

Nitric acid is often used in place of acetic acid in these tests. In case nitric acid is used ordinarily I-2 drops is sufficient.

5. Acetic Acid and Potassium Ferrocyanide Test.-To 5 c.c. of urine in a test-tube add 5-ro drops of acetic acid. Mix well and add potassium ferrocyanide drop by drop, until a precipitate forms.

This is a very delicate test. Schmiedl claims that a precipitate of $\mathrm{Fe}(\mathrm{Cn})_{6} \mathrm{~K}_{2} \mathrm{Zn}$ or $\mathrm{Fe}(\mathrm{Cn})_{6} \mathrm{Zn}_{2}$ is formed when urines containing zinc are subjected to this test and that this precipitate resembles the precipitate secured with protein solutions. In the case of human urine a reaction was obtained when 0.000022 gram of zinc per cubic centimeter was present. Schmiedl further found that the urine collected from rabbits housed in zinc-lined cages possessed a zinc content which was sufficient to yield a ready response to the test.

Proteoses may also be detected by this test. To differentiate albumin from proteose perform the coagulation test (see page 424 ).

6. Tanret's Test.-To 5 c.c. of urine in a test-tube add Tanret's reagent ${ }^{1}$ drop by drop until a turbidity or precipitate forms. This is an exceedingly delicate test. Sometimes the urine is stratified upon the reagent as in Heller's or Roberts' ring test. According to Repiton, urates interfere with the delicacy of this test. Tanret, however, claims that urates do not interfere inasmuch as any precipitate due to urates nay be brought into solution by heat, whereas an albumin precipitate under the same conditions will persist. Tanret further states that mucin interferes with the delicacy of the test and that it should therefore be removed from the urine under examination by acidification with acetic acid and filtration beiore testing for albumin. This test also serves to detect proteoses.

7. Sodium Chloride and Acetic Acid Test.-Mix two volumes of urine and one volume of a saturated solution of sodium chloride in a test-tube, acidify with acetic acid, and heat to boiling. The production of a cloudiness or the formation of a precipitate indicates the presence of albumin. The resin acids may interfere here

${ }^{2}$ Tanret's reagent is prepared as follows: Dissolve $\mathrm{I} .35$ grams of mercuric chloride in 25 c.c. of water, add to this solution 3.32 grams of potassium iodide dissolved in 25 c.c. of water, then make the total solution up to 60 c.c. with water and add 20 c.c. of glacial acetic acid to the mixture. 
as in the ordinary coagulation test (page 424), but they may be easily differentiated from albumin by means of their solubility in alcohol.

\section{GLOBULIN}

Serum globulin is not a constituent of normal urine but frequently occurs in the urine under pathological conditions and is ordinarily associated with serum albumin. In albuminuria globulin in varying amounts often accompanies the albumin, and the clinical significance of the two is very similar. Under certain conditions globulin may occur in the urine unaccompanied by albumin.

\section{EXPERIMENTS}

Globulin will respond to all the tests just outlined under Albumin. If it is desirable to differentiate between albumin and globulin in any urine the following processes may be employed:

I. Saturation with Magnesium Sulphate.-Place 25 c.c. of neutral urine in a small beaker and add pulverized magnesium sulphate in substance to the point of saturation. If the protein present is globulin it will precipitate at this point. If no precipitate is produced acidify the saturated solution with acetic acid and warm gently. Albumin will be precipitated if present.

The above procedure may be used to separate globulin and albumin if present in the same urine. To do this filter off the globulin after it has been precipitated by the magnesium sulphate, then acidify the clear solution and warm gently as directed. Note the formation of the albumin precipitate.

2. Half-saturation with Ammonium Sulphate.-Place 25 c.c. of neutral urine in a small beaker and add an equal volume of a saturated solution of ammonium sulphate. Globulin, if present, will be precipitated. If no precipitate forms add ammonium sulphate in substance to the point of saturation. If albumin is present it will be precipitated upon saturation of the solution as just indicated. This method may also be used to separate globulin and albumin when they occur in the same urine.

Frequently in urine which contains a large amount of urates a precipitate of ammonium urate may occur when the ammonium sulphate solution is added to the urine. This urate precipitate should not be confounded with the precipitate due to globulin. The two precipitates may be differentiated by means of the fact that the urate precipitate ordinarily appears only after the lapse of several minutes whereas the globulin generally precipitates at once.

\section{PROTEOSE AND PEPTONE}

Proteoses, particularly deutero-proteose and hetero-proteose, have frequently been found in the urine under various pathological conditions such as diphtheria, pneumonia, intestinal ulcer, carcinoma, 
dermatitis, osteomalacia, atrophy of the kidneys, and in sarcomata of the bones of the trunk. The presence of proteose in the urine may frequently be demonstrated in any pathological condition in which there is absorption of partially digested pus. "Bence-Jones' protein," a proteose-like substance, is of interest in this connection and its appearance in the urine is believed to be of great diagnostic importance in cases of multiple myeloma or myelogenic osteosarcoma. By some investigators this protein is held to be a variety of hetero-proteose, whereas others claim that it possesses albumin characteristics. The origin of "Bence-Jones" protein" is unknown. Its origin has at various times been ascribed to the blood proteins, the bones or to abnormal metabolism of protein material in the body. It occurs in the urine in about 80 per cent of the cases of multiple myeloma. If its presence is unaccompanied by multiple myeloma it is nearly always associated with some disease of the blood-forming organs or of the bones. When "Bence-Jones' protein" is hydrolyzed it is found to contain all the aminoacids which are characteristic of typical proteins.

Peptone certainly occurs much less frequently as a constituent of the urine than does proteose, in fact most investigators seriously question its presence under any conditions. There are many instances of peptonuria cited in the early literature, but because of the uncertainty in the conception of what really constituted a peptone it is probable that in many cases of so-called peptonuria the protein present was really proteose.

\section{EXPERIMENTS}

I. Phosphotungstic Precipitation Test (v. Aldor).-Acidify ro c.c. of urine with hydrochloric acid, add phosphotungstic acid until no more precipitate forms and centrifugate ${ }^{1}$ the solution. Decant the supernatant fluid, add some absolute alcohol to the precipitate, and centrifugate again. This washing with alcohol is intended to remove the urobilin and hence should be continued so long as the alcohol exhibits any coloration whatever. Now suspend the precipitate in water and add potassium hydroxide to bring it into solution. At this point the solution may be blue in color, in which case decolorization may be secured by gently heating. Apply the biuret test to the cool solution. A positive biuret test indicates the presence of proteoses.

2. Boiling Test.-Make the ordinary coagulation test according to the direction given under Albumin, page 424. If no coagulable protein is found allow the boiled urine to stand and note the gradual appearance, in the cooled fluid, of a flaky precipitate of proteose. Spiegler's reaction may also be applied at this point. A precipitate indicates proteose.

3. Schulte's Method.-Acidify 5o c.c. of urine with dilute acetic acid and filter off any precipitate of nucleoprotein which may form. Now test a few cubic centi-

${ }^{1}$ If not convenient to use a centrifuge the precipitate may be filtered off and washed on the filter paper with alcohol. 
meters of the urine for coagulable protein, by tests 2 and 4 under Albumin, pages 424-5. If coagulable protein is present remove it by coagulation and filtration before proceeding. Introduce 25 c.c. of the urine, freed from coagulable protein, into I 50 c.c. of absolute alcohol and allow it to stand for I $2-24$ hours. Decant the supernatant fluid and dissolve the precipitate in a small amount of hot water. Now filter this solution, and after testing again for nucleoprotein with very dilute acetic acid, try the biuret test. If this test is positive the presence of proteose is indicated. ${ }^{1}$

Urobilin does not ordinarily interfere with this test since it is almost entirely dissolved by the absolute alcohol when the proteose is precipitated.

4. Detection of "Bence-Jones' Protein."-Heat the suspected urine very gently, carefully noting the temperature. At as low a temperature as $40^{\circ} \mathrm{C}$. a turbidity may be observed, and as the temperature is raised to about $60^{\circ} \mathrm{C}$. a flocculent precipitate forms and clings to the sides of the test-tube. If the urine is now acidified very slightly with acetic acjd and the temperature further raised to $100^{\circ} \mathrm{C}$. the precipitate at least partly disappears; it will return upon cooling the tube.

This property of precipitating at so low a temperature and of dissolving at a higher temperature is typical of "Bence-Jones' protein" and may be used to differentiate it from all other forms of protein material occurring in the urine.

\section{NUCLEOPROTEIN}

There has been considerable controversy as to the proper classification for the protein body which forms the "nubecula" of normal urine. By different investigators it has been called mucin, mucoid, phosphoprotein, nucleoalbumin, and nucleoprotein. Of course, according to the modern acceptation of the meanings of these terms they cannot be synonymous. Mucin and mucoid are glycoproteins and hence contain no phosphorus (see page 1 I2), whereas phosphoproteins and nucleoproteins are phosphorized bodies. It may possibly be that both these forms of protein, i.e., the glycoprotein and the phosphorized type, occur in the urine under certain conditions (see page 396). In this connection we will use the term nucleoprotein. The pathological conditions under which the content of nucleoprotein is increased includes all affections of the urinary passages and in particular pyelitis, nephritis, and inflammation of the bladder.

\section{EXPERIMENTS}

I. Detection of Nucleoprotein.-Place Io c.c. of urine in a small beaker, dilute it with three volumes of water to prevent precipitation of urates, and make the reaction very strongly acid with acetic acid. If the urine becomes turbid it is an indication that nucleoprotein is present.

${ }^{1}$ If it is considered desirable to test for peptone the proteose may be removed by saturation with $\left(\mathrm{NH}_{4}\right)_{2} \mathrm{SO}_{4}$ according to the directions given on $\mathrm{p}$. I 20 and the filtrate tested for peptone by the biuret test. 
If the urine under examination contains albumin the greater portion of this substance should be removed by boiling the urine before testing it for the presence of nucleoprotein.

2. Tannic Acid Precipitation Test (Ott).-Mix 25 c.c. of the urine with an equal volume of a saturated solution of sodium chloride and slowly add Almén's reagent. ${ }^{1}$ In the presence of nucleoprotein a voluminous precipitate forms.

\section{BLOOD}

The pathological conditions in which blood occurs in the urine may be classified under the two divisions hematuria and hemoglobinuria. In hematuria we are able to detect not only the hemoglobin but the unruptured corpuscles as well, whereas in hemoglobinuria the pigment alone is present. Hematuria is brought about through blood passing into the urine because of some lesion of the kidney or of the urinary tract below the kidney. Hemoglobinuria is brought about through hemolysis, i.e., the rupturing of the stroma of the erythrocyte and the liberation of the hemoglobin. This may occur in scurvy, typhus, pyemia, purpura, and in other diseases. It may also occur as the result of a burn covering a considerable area of the body, or may be brought about through the action of certain poisons or by the injection of various substances having the power of dissolving the erythrocytes. Transfusion of blood may also cause hemoglobinuria.

Even in true hematuria the erythryocytes may escape detection if the urine is ammoniacal inasmuch as the cells disintegrate under these conditions.

\section{EXPERIMENTS}

I. Potassium Hydroxide Test (Heller).- - Render Io c.c. of urine strongly alkaline with potassium hydroxide solution and heat to boiling. Upon allowing the heated urine to stand a precipitate of phosphates, colored red by the contained hematin, is formed. It is ordinarily well to make a "control " experiment using normal urine, before coming to a final decision.

Certain substances, such as cascara sagrada, rhubarb, santonin, and senna, cause the urine to give a similar reaction. Reactions due to such substances may be differentiated from the true blood reaction by the fact that both the precipitate and the pigment of the former reaction disappear when treated with acetic acid, whereas if the color is due to hematin the acid will only dissolve the precipitate of phosphates and leave the pigment undissolved.

2. Teichmann's Hemin Test.-Place a small drop of the suspected urine or a small amount of the moist sediment on a microscopic slide, add a minute grain of sodium chloride and carefully evaporate to dryness over a low flame. Put a cover-glass in place, run underneath it a drop of glacial acetic acid, and warm gently until the formation of gas bubbles is observed. Cool the preparation, examine under the microscope, and compare the form of the crystals with those

${ }^{1}$ Dissolve 5 grams of tannic acid in 240 c.c. of 50 per cent alcohol and add ro c.c. of 25 per cent acetic acid. 
reproduced in Figs. 78 and 79, page 265. (See Atkinson and Kendall's and Nippe's modifications, page 264.)

3. Heller-Teichmann Reaction.-Produce the pigmented precipitate according to directions given in Heller's test above. If there is a copious precipitate of phosphates and but little pigment the phosphates may be dissolved by treatment with acetic acid and the residue used in the formation of the hemin crystals according to directions in Experiment 2, above.

4. v. Zeynek and Nencki's Hemin Test.-To ro c.c. of the urine under examination add acetone until no more precipitate forms. Filter off the precipitate and extract it with ro c.c. of acetone rendered acid with $2-3$ drops of hydrochloric acid. Place a drop of the resulting colored extract on a slide, immediately place a coverglass in position, and examine under the microscope. Compare the form of the crystals with those shown in Figs. 78 and 79, page 265. Hemin crystals produced by this manipulation are sometimes very minute, thus rendering it difficult to determine the exact form of the crystal.

6. Guaiac Test.-Place 5 c.c. of urine in a test-tube and by means of a pipette introduce a freshly prepared alcoholic solution of guaiac (strength about I :6o) into the fluid until a turbidity results, then add old turpentine or hydrogen peroxide, drop by drop, until a blue color is obtained.

This is a very delicate test when properly performed. Buckmaster has suggested the use of guaiaconic acid instead of the solution of guaiac. The test is positive both before and after boiling the blood for $1^{-20}$ seconds. Pus does not respond after boiling. Old, partly putrefied pus gives the test even without the addition of hydrogen peroxide or old turpentine whereas fresh pus responds upon the addition of hydrogen peroxide. See discussion on page 258 and test on page 262 .

7. Schumm's Modification of the Guaiac Test.-To about 5 c.c. of urine ${ }^{1}$ in a test-tube add about ro drops of a freshly prepared alcoholic solution of guaiac. Agitate the tube gently, add about 20 drops of old turpentine, subject the tube to a thorough shaking, and permit it to stand for about $2-3$ minutes. A blue color indicates the presence of blood in the solution under examination. In case there is not sufficient blood to yield a blue color under these conditions, a few cubic centimeters of alcohol should be added and the tube gently shaken, whereupon a blue coloration will appear in the upper alcohol-turpentine layer.

A control test should always be made using water in place of urine. In the detection of very minute traces of blood only $3-5$ drops of the guaiac solution should be employed.

8. Ortho-Tolidin Test (Ruttan and Hardisty). ${ }^{2}$-To I c.c. of a 4 per cent glacial acetic acid solution of 0 -tolidin ${ }^{3}$ in a test-tube add I c.c. of the solution

${ }^{1}$ Alkaline urine should be made slightly acid with acetic acid as the blue end-reaction is very sensitive to alkali.

${ }^{2}$ Ruttan and Hardisty: Canadian Medical Assn. Journal, Nov., I9I 2, also Biochemical Bull., 2, 225, I913.<smiles>CC(N)C(C)N</smiles> 
under examination and I c.c. of 3 per cent hydrogen peroxide. In the presence of blood a bluish color develops (sometimes rather slowly) and persists for sometime (several hours in some instances).

This test is said to be as sensitive for the detection of occult blood in feces and stomach contents as is the benzidine reaction. It is also claimed to be more satisfactory for urine than any other blood test. The acetic acid solution may be kept for one month with no reduction in delicacy.

9. Benzidine Reaction.-This is one of the most delicate of the reactions for the detection of blood. Different benzidine preparations vary greatly in their sensitiveness, however. Inasmuch as benzidine solutions change readily upon contact with light, it is essential that they be kept in a dark place.

The test is peformed as follows: To a saturated solution of benzidine in alcohol or glacial acetic acid add an equal volume of 3 per cent hydrogen peroxide and I c.c. of the urine under examination. If the mixture is not already acid, render it so with acetic acid, and note the appearance of a blue color. A control test should be made substituting water for the urine.

Often when urines containing a small amount of blood are tested by this reaction, the mixture is rendered so turbid as to make it difficult to decide as to the presence of a faint green color. Such urines should be washed with water before the test is applied to it. The sensitiveness of the benzidine reaction is greater when applied to aqueous solutions than when applied to the urine.

For a modification of this test and further discussion see Chapter $\mathrm{XV}$ on Blood and Lymph.

9. Spectroscopic Examination.-Submit the urine to a spectroscopic examination according to the directions given on page 296 , looking especially for the absorption bands of oxyhemoglobin and methemoglobin (see Absorption Spectra, Plate I).

\section{PUS}

Pus may be present in the urine in inflammatory affections of various types. Such a condition is termed pyuria. Albumin always accompanies the pus. In catarrh of the bladder and in inflammation of the urethra or of the pelvis of the kidney pus is particularly apt to be present in the urine. If a urine of high pus concentration is voided it may indicate the rupturing of an abscess in some part of the genitourinary tract. Pus may be detected by one of the procedures given below.

\section{ExPERIMENTS}

I. Microscopical Detection of Pus. - The characteristic form elements of pus are leucocytes. They may occur in very small number in normal urine. Examine the 
urine (centrifugated if necessary) under the microscope. Any considerable number of pus corpuscles indicates a pathological urine. In acid urine the pus corpuscles appear as round, colorless cells, composed of refractive, granular protoplasm. Sometimes they may exhibit amœboid movements, particularly if the slide containing them be warmed slightly. They are nucleated (one or more nuclei), the nuclei being clearly visible only upon treating the cells with water, acetic acid or some other suitable reagent. In alkaline urine the pus corpuscles are often degenerated. They may occur as swollen, transparent cells, which exhibit no granular structure. If the degeneration has proceeded far enough the nuclei fade and the cell disintegrates and only débris remains.

Sometimes it is almost impossible to differentiate between pus corpuscles and certain types of epithelial cells. In such a case apply one of the following chemical tests.

2. Guaiac Test.-This test is not specific for pus, but is given by certain other substances and particularly by blood (see Chapter XV). Perform the test as follows: Acidify the urine (if alkaline) with acetic acid, filter, ${ }^{1}$ and add tincture of guaiac to the sediment on the paper. If the pus is old, and partly putrefied. it will give a blue color. If no blue color is secured, add old turpentine, or hydrogen peroxide, drop by drop. A blue color formed only under these conditions indicates fresh pus.

As a control test boil some of the urine (or sediment) for $15^{-20}$ seconds and repeat the test. Pus does not respond after boiling. In the case of blood the test is positive both before and after boiling.

3. Potassium Hydroxide Test (Donné).-Separate the sediment from the urine (by decantation, filtration or centrifugation); place a small piece of solid potassium hydroxide on the sediment and stir. If pus is present (and particularly if it be fresh pus and not disintegrated) the sediment will become slimy and tough. If the sediment is mucus it will more or less pass into solution in the concentrated alkali.

\section{BILE}

Both the pigments and the acids of the bile may be detected in the urine under certain pathological conditions. Of the pigments, bilirubin is the only one which has been positively identified in fresh urine; the other pigments, when present, are probably derived from the bilirubin. A urine containing bile may be yellowish-green to brown in color and when shaken foams readily. The staining of the various tissues of the body through the absorption of bile due to occlusion of the bile duct cause a condition known as icterus or jaundice. Bile is always present in the urine under such conditions unless the amount of bile reaching the tissues is extremely small.

\section{EXPERIMENTS}

\section{Tests for Bile Pigments}

Practically all of these tests for bile pigments are based on the oxidation of the pigment by a variety of reagents with the formation

${ }^{1}$ If desired, the urine may be centrifuged and the sediment used in the test. 
of a series of colored derivatives, e.g., biliverdin (green), bilicyanin (blue), choletelin (yellow).

I. Gmelin's Test.-To about 5 c.c. of concentrated nitric acid in a test-tube add an equal volume of urine carefully so that the two fluids do not mix. At the point of contact note the various colored rings, green, blue, violet, red, and reddish-yellow.

2. Rosenbach's Modification of Gmelin's Test.-Filter 5 c.c. of urine through a small filter paper. Introduce a drop of concentrated nitric acid into the cone of the paper and observe the succession of colors as given in Gmelin's test.

3. Nakayama's Reaction. - To 5 c.c. of urine in a test-tube add an equal volume of a ro per cent solution of barium chloride. Centrifugate the mixture, pour off the supernatant fluid, and heat the precipitate with 2 c.c. of Nakayama's reagent. ${ }^{1}$ In the presence of bile pigments the solution assumes a blue or green color.

3. Huppert's Reaction. - Thoroughly shake equal volumes of urine and milk of lime in a test-tube. The pigments unite with the calcium and are precipitated. Filter off the precipitate, wash it with water, and transfer to a small beaker. Add alcohol acidified slightly with hydrochloric acid and warm upon a water-bath until the solution becomes colored an emerald green.

According to Steensma, this procedure may give negative results even in the presence of the pigments, owing to the fact that the acid-alcohol is not a sufficiently strong oxidizing agent. He therefore suggests the addition of a drop of a 0.5 per cent solution of sodium nitrite to the acid-alcohol mixture before warming on the water-bath. Try this modification also.

4. Salkowski's Test--Render 5 c.c. of urine alkaline with a few drops of a io per cent sodium carbonate solution and add a ro per cent solution of calcium chloride, drop by drop, until the supernatant fluid exhibits the normal urinary color when the contents of the test-tube are thoroughly mixed. Filter off the precipitate, and after washing it place it in a second tube with 95 per cent alcohol. Acidify the alcohol with hydrochloric acid and, if necessary, shake the tube to bring the precipitate into solution. Heat the solution to boiling and observe the appearance of a green color which changes through blue and violet to red; if no bile is present the solution does not undergo any color change. This test will frequently exhibit greater delicacy than Gmelin's test. Steensma's suggestions mentioned under Huppert's Reaction, above, apply in connection with this test also.

5. Hammarsten's Reaction.-To about 5 c.c. of Hammarsten 's reagent ${ }^{2}$ in a small evaporating dish add a few drops of urine. A green color is produced. If more of the reagent is now added the play of colors as noted in Gmelin's test may be obtained.

6. Smith's Test.-To $2-3$ c.c. of urine in a test-tube add carefully about 5 c.c. of dilute tincture of iodine $(\mathrm{I}: \mathrm{IO})$ so that the fluids do not mix. 1 green ring is observed at the point of contact.

7. Salkowski-Schippers Reaction.-Neutralize the acidity of ro c.c. of the urine under examination with a few drops of a dilute solution of sodium carbonate, and add 5 drops of a 20 per cent solution of sodium carbonate and Io drops of a 20

${ }^{1}$ Prepared by combining 99 c.c. of alcohol and I c.c. of fuming hydrochloric acid containing 4 grams of ferric chloride per liter.

${ }^{2}$ Hammarsten's reagent is made by mixing x volume of 25 per cent nitric acid and ro volumes of 25 per cent hydrochloric acid and then adding I volume of this acid mixture to 4 volumes of 95 per cent alcohol. 
per cent solution of calcium chloride. Filter off the resultant precipitate upon a hardened filter paper and wash it with water. Remove the precipitate to a small porcelain dish, add 3 c.c. of an acid-alcohol mixture ${ }^{1}$ and a few drops of a dilute solution of sodium nitrite and heat. The production of a green color indicates the presence of bile pigments.

8. Bonanno's Reaction. 2-Place 5-1o c.c. of the urine under examination in a small porcelain evaporating dish and add a few drops of Bonanno's reagent. ${ }^{3}$ If bile is present an emerald-green color will develop. Bonanno says the reaction is not interfered with by any known normal or pathological urinary constituent.

Tests for Bile Acids

r. Sucrose- $\mathrm{H}_{2} \mathrm{SO}_{4}$ Test (Pettenkofer). - To 5 c.c. of urine in a test-tube add 5 drops of a 5 per cent solution of sucrose. Now incline the tube, run about 2-3 c.c. of concentrated sulphuric acid carefully down the side and note the red ring at the point of contact. Upon slightly agitating the contents of the tube the whole soultion gradually assumes a reddish color. As the tube becomes warm, it should be cooled in running water in order that the temperature may not rise above $70^{\circ} \mathrm{C}$.

It is claimed that this test is not satisfactory in the presence of protein and chromogenic substances which yield interfering colors with sulphuric acid.

2. Furfural- $\mathrm{H}_{2} \mathrm{SO}_{4}$ Test (Mylius).-To approximately 5 c.c. of urine in a test-tube add 3 drops of a very dilute ( $\mathrm{I}$ : rooo) aqueous solution of furfural,

$\mathrm{HC}-\mathrm{CH}$

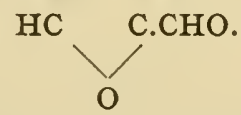

Now incline the tube, run about 2-3 c.c. of concentrated sulphuric acid carefully down the side and note the red ring as above. In this case also, upon shaking the tube, the whole solution is colored red. Keep the temperature below $70^{\circ} \mathrm{C}$. as before.

3. Foam Test (v. Udránsky). - To 5 c.c. of urine in a test-tube add $3-4$ drops of a very dilute ( 1 : 1000) aqueous solution of furfural. Place the thumb over the top of the tube and shake until a thick foam is formed. By means of a small pipette add 2-3 drops of concentrated sulphuric acid to the foam and observe the dark pink coloration produced.

4. Surface Tension Test (Hay).-This test is based upon the principle that bile acids have the property of reducing the surface tension of fluids in which they are contained. The test is performed as follows: Cool about ro c.c. of urine in a test-tube to $17^{\circ} \mathrm{C}$. or lower, and sprinkle a little finely pulverized sulphur upon the surface of the fluid. The presence of bile acids is indicated if the

1 Made by adding 5 c.c. of concentrated hydrochloric acid to 95 c.c. of 96 per cent. alcohol.

${ }^{2}$ Il Tommasi, 2, No. 2r.

${ }^{3}$ This reagent may be prepared by dissolving 2 grams of sodium nitrite in Ioo c.c. of concentrated hydrochloric acid. 
sulphur sinks to the bottom of the liquid, the rapidity with which the sulphur sinks depending upon the amount of bile acids present in the urine. The test is said to react with bile acids when the latter are present in the proportion I : 120,000 . Allen $^{1}$ has recently suggested the quantitative determination of bile acids by a surface tension method.

5. Neukomm's Modification of Pettenkofer's Test.-To a few drops of urine in an evaporating dish add a trace of a dilute sucrose solution and one or more drops of dilute sulphuric acid. Evaporate on a water-bath and observe the development of a violet color at the edge of the evaporating mixture. Discontinue the evaporation as soon as the color is observed.

6. Peptone Test (Oliver).-To 5 c.c. of urine add $2-3$ drops of acetic acid, filtering if necessary. Add an equal volume of a I per cent. solution of Witte's peptone to the acid solution. A precipitate is formed which is insoluble in an excess of acetic acid. This precipitate is a compound of protein and bile acids.

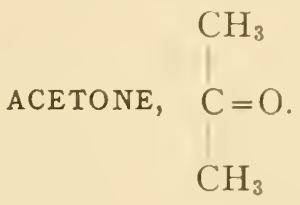

It was formerly very generally believed that acetone appeared in the urine under pathological conditions because of increased protein decomposition. It is now generally thought that, in man, the output of acetone arises principally from the breaking down of fatty tissues or fatty foods within the organism. The quantity of acetone eliminated has been shown to increase when the subject is fed an abundance of fat-containing food as well as during fasting, whereas a replacement of the fat with carbohydrates is followed by a marked decrease in the acetone excretion. If no carbohydrate food is fed the output of acetone bodies increases at once, producing a physiological acidosis (see Chapter XXVII on Metabolism).

Acetone and the closely related bodies, $\beta$-hydroxybutyric acid and acetoacetic acid, are generally classified as the acetone bodies. They are all associated with a deranged metabolic function and may appear in the urine together or separately, depending upon the conditions. Acetone and diacetic acid may occur alone in the urine but $\beta$-hydroxybutyric acid is never found except in conjunction with one or the other of these bodies. Acetone and acetoacetic acid arjse chiefly from the oxidation of $\beta$-hydroxybutyric acid. The relation existing between these three bodies is shown in the following equations.

(a) $\mathrm{CH}_{3} \cdot \mathrm{CH}(\mathrm{OH}) \cdot \mathrm{CH}_{2} \cdot \mathrm{COOH}+\mathrm{O} \rightarrow \mathrm{CH}_{3} \mathrm{CO} \cdot \mathrm{CH}_{2} \cdot \mathrm{COOH}+\mathrm{H}_{2} \mathrm{O}$. $\beta$-hydroxybutyric acid.

(b) $\mathrm{CH}_{3} \mathrm{CO} . \mathrm{CH}_{2} \cdot \mathrm{COOH} \rightarrow \underset{\text { Acetoacetic acid. }}{\left(\mathrm{CH}_{3}\right)_{2} \mathrm{CO}}+\mathrm{CO}_{2}$.

'Allen: Jour. Biol. Chem., 22, 505, 19r 5 . 
Acetone, chemically considered, is a ketone, di-methyl ketone. When pure it is a liquid which possesses a characteristic aromatic fruit-like odor, boils at $56-57^{\circ} \mathrm{C}$. and is miscible with water, alcohol, or ether in all proportions. Acetone is a physiological as well as a pathological constituent of the urine and under normal conditions the daily output (preformed acetone + acetoacetic acid) is about $3-15 \mathrm{mg}$.

Pathologically, the elimination of acetone is often greatly increased and at such times a condition of acetonuria is said to exist. Values from $0.02-6$ grams or higher have been obtained for preformed acetone plus acetone derived from acetoacetic acid. This pathological acetonuria may accompany diabetes mellitus, scarlet fever, typhoid fever, pneumonia, nephritis, phosphorus poisoning, grave anemias, fasting, and a deranged digestive function; it also frequently accompanies auto-intoxication and chloroform and ether anethesia.' The types of acetonuria most frequently met with are those noted in febrile conditions and in advanced cases of diabetes mellitus. The blood in diabetic comas has been found to contain as high as $45 \mathrm{mg}$. of total acetone (acetone + acetoacetic acid) for roo c.c. of blood serum.

\section{EXPERIMENTS}

I. Isolation from the Urine.-In order to facilitate the detection of acetone in the urine, the specimen under examination should be distilled and the tests as given below applied to the resulting distillate. If it is not convenient to distil the urine, the tests may be conducted upon the undistilled fluid. To obtain an acetone distillate proceed as follows: Place Ioo-250 c.c. of urine in a distillation flask or retort and render it acid with acetic acid. Collect about one-third of the original volume of fluid as a distillate, add 5 drops of to per cent hydrochloric acid and redistil about one-half of this volume. With this final distillate conduct the tests as given below.

2. Gunning's Iodoform Test.-To about 5 c.c. of the urine or distillate in a test-tube add a few drops of Lugol's solution ${ }^{1}$ or ordinary iodine solution (I in $\mathrm{KI}$ ) and a few drops of dilute $\mathrm{NH}_{4} \mathrm{OH}$ to form a black precipitate (nitrogen iodide). Allow the tube to stand (the length of time depending upon the content of acetone in the fluid under examination) and note the formation of a yellowish sediment consisting of iodoform. Examine the sediment under the microscope and compare the form of the crystals with those shown in Fig. 8, page 42.

If the crystals are not well formed recrystallize them from ether and examine again. The crystals of iodoform should not be confounded with those of calcium phosphate (Fig. 105, page 322) which may be formed in this test, particularly if made upon the undistilled urine. This test is preferable to Lieben's test (4) since no substance other than acetone will produce iodoform when treated according to the directions for

${ }^{1}$ Lugol's solution may be prepared by dissolving 4 grams of iodine and 6 grams of potassium iodide in 100 c.c. of distilled water. 
this test; both alcohol and aldehyde yield iodoform when tested by Lieben's test.

Gunning's test is rather satisfactory for the detection of acetone, and has been used with good results even upon the undistilled urine. Protein material apparently interferes with the reaction, and when present the urine should be distilled and the distillate used. ${ }^{1}$ In some instances where the amount of acetone present is very small it is necessary to allow the tube to stand 24 hours before making the examination for iodoform crystals. This test serves to detect acetone when present in the ratio $I: 100,000$.

3. Sodium Nitroprusside Test (Legal). - Introduce about 5 c.c. of the urine or distillate into a test-tube, add a few drops of freshly prepared aqueous solution of sodium nitroprusside and render the mixture alkaline with potassium hydroxide. (Be sure to add the nitroprusside before the solution is rendered alkaline.) A ruby-red color, due to creatinine, a normal urinary constituent, is produced (see Weyl's test, page 385). Add an excess of acetic acid and if acetone is present the red color will be intensified, whereas in the absence of acetone a yellow color will result. Make a control test upon normal urine to show that this is so.

A similar red color may be produced by paracresol in urines containing no acetone.

Two hypotheses have been proposed to explain the color reaction between acetone and nitroprusside: (I) The formation of a complex ion of ferropentacyanide with the isonitroso compound of the ketone, or (2) the formation of such an ion with the isonitroamine derivative of the ketone. ${ }^{2}$

4. Iodoform Test (Lieben).--Introduce 5 c.c. of the urine or distillate into a test-tube, render it alkaline with potassium hydroxide and add I-2 c.c. of iodine solution drop by drop. If acetone is present a yellowish precipitate of iodoform will be produced. Identify the iodoform by means of its characteristic odor and its typical crystalline form (see Fig. 8 , page 42 ).

While fully as delicate as Gunning's test (2) this test is not as accurate since, by means of the procedure involved, either alcohol or aldehyde will yield a precipitate of iodoform. This test is especially liable to lead to erroneous deductions when urines from the advanced stages of diabetes are under examination, because of the presence of alcohol formed from the sugar through fermentative processes. ${ }^{3}$ If protein is present in the urine to be tested it may prevent the acetone from responding to the above reaction. It is therefore advisable to use

1 Rosenbloom: Jour. Am. Mcd. Ass'n, 59, 445, I9I 2.

${ }^{2}$ Cambi: Alli. accud. Lincei, 22, 376, 1913.

${ }^{3}$ Welker reports the production of a pink or red color during the application of this test to the distillates from pathological urines which had been preserved with powdered thymol. He found the color to be due to an iodothymol compound which bad been previously prepared synthetically by Messinger and Vortmann. 
the distillate to secure most accurate results. ${ }^{1}$ Sobel ${ }^{2}$ has suggested a quantitative method for acetone based on Lieben's test.

5. Reynolds-Gunning Test.-This test depends upon the solubility of mercuric oxide in acetone and is performed as follows: 'To 5 c.c. of the urine or distillate add a few drops of mercuric chloride, render the solution alkaline with potassium hydroxide and add an equal volume of 95 per cent alcohol. Shake thoroughly in order to bring the major portion of the mercuric oxide into solution and filter. Render the clear filtrate faintly acid with hydrochloric acid and stratify some ammonium sulphide $\left(\mathrm{NH}_{4}\right)_{2} \mathrm{~S}$ upon this acid solution. At the zone of contact a blackish-gray ring of precipitated mercuric sulphide, $\mathrm{HgS}$, will form. Aldehyde also responds to this test. Aldehyde, however, has never been detected in the urine and could be present in this instance only if the acidified urine was distilled too far.

6. Rothera's Reaction. ${ }^{3}$-To 5 -10 c.c. of urine or distillate in a test-tube add a little solid ammonium sulphate, $2-3$ drops of a freshly prepared 5 per cent solution of sodium nitroprusside and $\mathrm{x}-2$ c.c. of concentrated ammonium hydroxide. The development of a permanganate color indicates the presence of acetone.

Hunter ${ }^{4}$ claims that this reaction serves to detect acetoacetic acid rather than acetone. Others claim it detects both acetone and acetoacetic acid.

8. Salicylaldehyde Reaction (Frommer),-Render to c.c. of urine strongly alkaline with potassium hydroxide, add I0-12 drops of a Io per cent solution of salicylaldehyde in absolute alcohol and warm the mixture to about $70^{\circ}$. If acetone be present the fluid becomes yellow, then red, reddish-purple and dark red in turn. The color of the urine is practically unchanged if no acetone is present.

This color is due to the formation of dihydroxydibenzoylacetone through the interaction of salicylaldehyde and acetone.

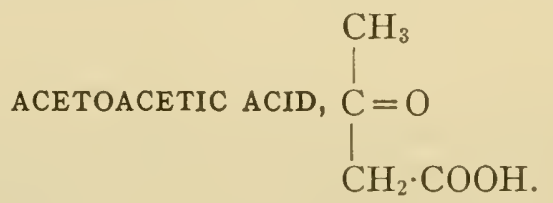

Acetoacetic or diacetic acid occurs in traces in normal urine. The sum of the acetone and the acetoacetic acid excreted in normal urine per day ranges from 3 to $5 \mathrm{mg}$. and ordinarily three-quarters oi this is acetoacetic acid. Under certain pathological conditions it occurs in larger quantities and is rarely found except associated with acetone. It is formed from $\beta$-hydroxybutyric acid, another of the acetone bodies, and upon decomposition yields acetone and carbon dioxide. Acetoaceturia occurs ordinarily under the same conditions as the pathological acetonuria, i.e., in fevers, diabetes, etc. (pp. 435 and 534). If very little acetoacetic acid is formed it may be transformed into acetone, whereas if a larger quantity is produced both

\footnotetext{
${ }^{1}$ Rosenbloom: Jour. Am. Med. Ass'n, 59, 445, IgI 2.

${ }^{2}$ Sobel: Schweiz. A poth. Zig., 52,62, 1914.

'Rothera: Jour. Physiol., 37, 49r, I908.

${ }^{1}$ Hunter: Quart. Jour. Exp. Physiol., 8, I3, IgI4.
} 
acetone and acetoacetic acid may be present in the urine. Acetoaceturia is most frequently observed in children, especially accompanying fevers and digestive disorders; it is perhaps less frequently observed in adults, but when present, particularly in fevers and diabetes it is frequently followed by fatal coma.

Acetoacetic acid is a colorless liquid which is miscible with water, alcohol and ether, in all proportions. It differs from acetone in giving a violet-red or Bordeaux-red color with a dilute solution of ferric chloride.

\section{ExpERTIENTS 1}

r. Le Nobel Reaction. ${ }^{2}$-Make ro c.c. of urine acid with acetic acid, add a few drops of a dilute aqueous solution of sodium nitroprusside and stratify concentrated ammonium hydroxide upon the mixture. In the presence of acetoacetic acid a violet ring forms at once.

Acetone also responds to this test, but the test is more delicate for acetoacetic acid and the response is more prompt.

2. Ferric Chloride Test (Gerhardt). - To 5 c.c. of urine in a test-tube add ferric chloride solution, drop by drop, until no more precipitate forms. In the presence of acetoacetic acid a Bordeaux-red color is produced; this color may be somewhat masked by the precipitate of ferric phosphate, in which case the fluid should be filtered.

A positive result from the above manipulation simply indicates the possible presence of acetoacetic acid. Before making a final decision regarding the presence of this body make the two following control experiments:

(a) Place 5 c.c. of urine in a test-tube, small beaker, or Erlenmeyer flask and boil it vigorously for 3-5 minutes. Cool the vessel and, with the boiled urine, make the test as given above. As has been already stated, acetoacetic acid yields acetone upon decomposition and acetone does not give a Bordeauxred color with ferric chloride. By boiling as indicated above, therefore, any acetoacetic acid present would be decomposed into acetone and carbon dioxide and the test upon the resulting fluid would be negative. If positive, the color is due to the presence of bodies other than acetoacetic acid.

(b) Place 5 c.c. of urine in a test-tube, acidify with $\mathrm{H}_{2} \mathrm{SO}_{4}$, to free acetoacetic acid from its salts, and carefully extract the mixture with ether by shaking. If acetoacetic acid is present it will be extracted by the ether. Now remove the ethereal solution, evaporate it to dryness, dissolve the residue in $\mathrm{r}-2$ c.c. of water and add 3-5 drops of 3 per cent ferric chloride. Acetoacetic acid is indicated by the production of the characteristic Bordeaux-red color.

This color disappears spontaneously in $2.4-4 S$ hours. Such substances as antipyrin, kairin, phenacetin, salicylic acid, salicylates, sodium acetate, thiocyanates, and thallin yield a similar red color

1 To prepare a diacetic acid solution which may be added to urine, if urines containing this acid are not available, proceed as follows: Treat 13 grams of ethyl acetoacetate with 500 c.c. of N/5 sodium hydroxide. Allow to stand for 48 hours to hydrolyze the ester. In preparing urine for tests add $\mathrm{I}$ part of this solution to Io parts of urine.

${ }_{2}^{2}$ Harding and Ruttan: Biochem. Jour., 6, 4+5, I9I 2; also Biochems. Bull., 2, 223, 1983. 
under these conditions, but when due to the presence of any of these substances the color does not disappear spontaneously but may remain permanent for days. Many of these disturbing substances are soluble in benzene or chloroform and may be removed from the urine by this means before extracting with ether as above. Acetoacetic acid is insoluble in benzene or chloroform.

3. Sodium Nitrite-Ferrous Sulphate Reaction (Hurtley).-Place Io c.c. of urine in a large test-tube, add $2 \mathrm{I} / 2$ c.c. of concentrated hydrochloric acid and I c.c. of fresh I per cent sodium nitrite. Shake the tube and permit it to stand for two minutes. Add 15 c.c. of concentrated ammonium hydroxide and 5 c.c. of ro per cent ferrous sulphate. Shake the tube and permit it to stand. Note the slow development of a violet or purple color in the presence of acetoacetic acid.

This test serves to detect acetoacetic acid when present in a dilution of I to 50,000. The concentration of the acetoacetic acid regulates the speed at which the color develops. If the concentration be very low an interval of five hours may elapse before the color appears. The test is believed to be specific for acetoacetic acid.

4. Arnold-Lipliawsky Reaction.-This reaction is somewhat more delicate than Gerhardt's test (2) and serves to detect acetoacetic acid when present in the proportion of $I: 25,000$. It is also negative toward acetone, $\beta$-hydroxybutyric acid and the interfering drugs mentioned as causing erroneous deductions in the application of Gerhardt's test. If the urine under examination is highly pigmented it should be partly decolorized by means of animal charcoal before applying the test as indicated below.

Place 5 c.c. of the urine under examination and an equal volume of the ArnoldLipliawsky reagent ${ }^{1}$ in a test-tube, add a few drops of concentrated ammonia and shake the tube vigorously. Note the production of a brick-red color. 'Take I-2 c.c. of this colored solution, add Io-20 c.c. of hydrochloric acid (sp. gr. I.Ig), 3 c. c. of chloroform, and 2-4 drops of ferric chloride solution and carefully mix the fluids. Acetoacetic acid is indicated by the chloroform assuming a violet or blue color; if acetoacetic acid is absent the color may be yellow or light red.

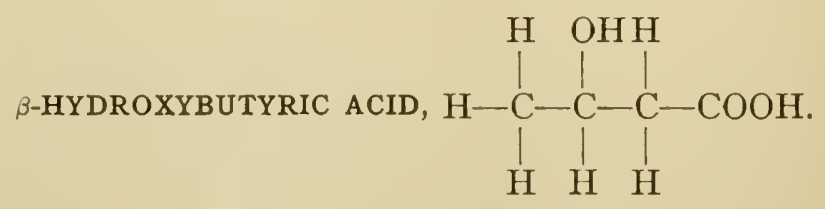

This acid occurs in normal urine in traces, e.g., 20-30 mg. per day. ${ }^{2}$ It is found under certain pathological conditions in larger quantities

${ }^{1}$ This reagent consists of two definite solutions which are ordinarily preserved separately and mixed just before using. The two solutions are prepared as follows:

(a) One per cent aqueous solution of potassium nitrite.

(b) One gram of p-amino-acetophenon dissolved in roo c.c. of distilled water and enough hydrochloric acid (about 2 c.c.) added, drop by drop, to cause the solution, which is at first yellow, to become entirely colorless. An excess of acid must be avoided

Before using, $a$ and $b$ are mixed in the ratio $\mathrm{I}: 2$.

${ }^{2}$ Shaffer and Marriott: Jour. Biol. Chem., 16, 265, r913. 
and then always in conjunction with either acetone or acetoacetic acid. Either of these bodies may be formed from $\beta$-hydroxybutyric acid under proper conditions. It is present in especially large amount in severe cases of diabetes and has also been detected in digestive disturbances, continued fevers, scurvy, measles, and in fasting. It is probable that, in man, $\beta$-hydroxybutyric acid, in common with acetone and acetoacetic acid, arises principally from the breaking down of fatty tissues within the organism. Any condition in which large amounts of acetone and acetoacetic acid, and in severe cases $\beta$-hydroxybutyric acid also, are excreted in the urine is known as an "acidosis." In diabetes the deranged metabolic conditions cause the production of great quantities of these substances which lead to an acid intoxication and ultimately to diabetic coma. In severe diabetes 50-I00 grams or over per day may be excreted. In such conditions the $\beta$-hydroxybutyric acid may constitute 6o-8o per cent of the tolal acetone bodies. In rare cases we may have an excretion of large amounts of $\beta$-hydroxybutyric acid with a low acetone output. An acidosis may also occur under certain physiological conditions (see Chapter XXVII on Metabolism).

Ordinarily $\beta$-hydroxybutyric acid is an odorless, transparent syrup which is levorotatory and easily soluble in water, alcohol, and ether; it may be obtained in crystalline form.

\section{EXPERIMENTS}

I. Black's Reaction. L-Inasmuch as the urinary pigments as well as any contained sugar or acetoacetic acid will interfere with the delicacy of this test when applied to the urine directly, the following preliminary procedure is necessary: Concentrate ro c.c. of the urine under examination to one-third or onefourth of its original volume in an evaporating dish at a gentle heat. Acidify the residue with a few drops of concentrated hydrochloric acid, add sufficient plaster of Paris to make a thick paste and allow the mixture to stand until it begins to "set." It should now be stirred and broken up in the dish by means of a stirring rod with a blunt end. Extract the porous meal thus produced twice with ether by stirring and decantation. Any $\beta$-hydroxybutyric acid present will be extracted by the ether. Evaporate the ether extract spontaneously or on a water-bath, dissolve the residue in water, and neutralize it with barium carbonate. To 5 to Io c.c. of this neutral fluid in a test-tube add 2 to 3 drops of ordinary commercial acid hydrogen peroxide. Mix by shaking and add a few drops of Black's reagent. ${ }^{2}$ Permit the tube to stand and note the gradual development of a rose color which increases to its maximum intensity and then gradually fades. ${ }^{3}$

1 Black: Jour. Biol. Chem., 5, 207, I90S.

${ }^{2}$ Made by dissolving 5 grams of ferric chloride and 0.4 gram of ferrous chloride in roo c.c. of water.

${ }^{3}$ This disappearance of color is due to the further oxidation of the acetcacetic acid. 
In carrying out the test care should be taken to see that the solution is cold and approximately neutral and that a large excess of hydrogen peroxide and Black's reagent are not added. In case but little $\beta$-hydroxybutyric acid is present the color will fail to appear or will be but transitory if the oxidizing agents are added in too great excess. It is preferable to add a few drops of the reagent and at intervals of a few minutes repeat the process until the color undergoes no further increase in intensity. One part of $\beta$-hydroxybutyric acid in 10,000 parts of the solution may be detected by this test.

2. Polariscopic Examination.-Subject some of the urine (free from protein) to the ordinary fermentation test (see page 42I). This will remove glucose and fructose, which would interfere with the polariscopic test. Now examine the fermented fluid in the polariscope and if it is levorotatory the presence of $\beta$-hydroxybutyric acid is indicated. This test is not absolutely reliable, however, since conjugate glycuronates are also levorotatory after fermentation.

\section{CONJUGATE GLYCURONATES}

Glycuronic acid does not occur free in the urine, but is found, for the most part, in combination with phenol. Much smaller quantities are excreted in combination with indoxyl and skatoxyl. The total content of conjugate glycuronates seldom exceeds 0.004 per cent under normal conditions. The output may be very greatly increased as the result of the administration of antipyrin, borneol, camphor, chloral hydrate, menthol, morphine, naphthol, turpentine, etc. The glycuronates as a group are levorotatory whereas glycuronic acid is dextrorotatory. Most of the glycuronates reduce alkaline metallic oxides and so introduce an error in the examination of urine for sugar. Conjugate glycuronates often occur associated with glucose in glycosuria, diabetes mellitus, and in some other disorders. As a class the glycuronates are non-fermentable.

\section{EXPERIMENTS}

I. Naphthoresorcinol Reaction (Tollens).-Introduce 5 c.c. of urine in a test-tube and add 0.5-I c.c. of a I per cent solution of naphthoresorcinol in 95 per cent alcohol, and 5 c.c. of concentrated hydrochloric acid. Raise the temperature gradually to the boiling-point and boil for one minute, shaking the tube continuously. Stand the tube aside four minutes, then cool under the tap. Extract with an equal volume of ether. Glycuronates are indicated by the ether extract assuming a violet-red color. The spectroscope shows this extract to possess two absorption bands, one on the $\mathrm{D}$ line and one to the right of this line.

2. Polariscopic-Fermentation Test.-If glucose is present in the urine tested 
for glycuronates the urine may first be subjected to a polariscopic examination, then fermented and a second polariscopic examination made. The sugar being dextrorotatory and fermentable and the glycuronates being levorotatory and non-fermentable the second polariscopic test will show a levorotation indicative of conjugate glycuronates.

3. Reduction-Polariscopic Test.-Test the urine by Fehling's test. If positive try the Resorcinol- $\mathrm{HCl}$ reaction for fructose. If negative test the optical activity. Levorotation indicates glycuronates.

\section{PENTOSES}

We have two distinct types of pentosuria, i.e., alimentary pentosuria, resulting from the ingestion of large quantities of pentose-rich vegetables such as prunes, cherries, grapes, or plums, and fruit juices, in which condition the pentoses appear only temporarily in the urine; and the chronic form of pentosuria, in which the output of pentoses bears no relation whatever to the quantity and nature of the pentose content of the food eaten. In occurring in these two forms, pentosuria resembles glycosuria (see page 4I3), but it is definitely known that pentosuria bears no relation to diabetes mellitus and there is no generally accepted theory to account for the occurrence of the chronic form of pentosuria. The pentose detected most frequently in the urine is arabinose, the inactive form generally occurring in chronic pentosuria although keto-pentose ${ }^{1}$ may occur in some cases. The levorotatory variety occurs in the alimentary type of the disorder.

\section{EXPERIMENTS}

I. Orcinol-Hydrochloric Acid Reaction (Bial). ${ }^{2}$-To 5 c.c. of Bial's reagent ${ }^{3}$ in a test-tube add 2-3 c.c. of urine and heat the mixture gently until the first bubbles rise to the surface. ${ }^{4}$ Immediately or upon cooling the solution becomes green and a flocculent precipitate of the same color may form.

This test is believed to be more accurate than the original orcinol test. It is claimed that urines containing menthol, kreosolal, etc., respond to the old orcinol reaction, but not to Bial's. If so desired the osazone of the pentose may be formed, then distilled with hydrochloric acid and the distillate tested by Bial's test (Jolles).

2. Phloroglucinol-Hydrochloric Acid Reaction (Tollens).-To equal volumes of urine and hydrochloric acid (sp. gr. I.o9) add a little phloroglucinol and heat the mixture on a boiling water-bath. Pentose, galactose, or glycuronic acid

1 Levene and La Forge: Jour. Biol. Chem., is, 3 I9, I9I4.

${ }^{2}$ Bial: Deul. med. Woch., 28, 252, 1902

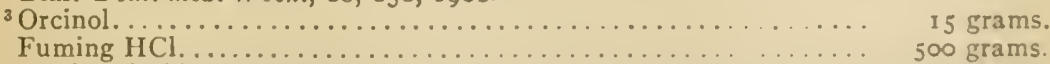

Ferric chloride (ro per cent)..................... 20-30 drops.

4 The test may also be performed by adding the urine to the hol reagent. No further heating should be necessary if pentose is present. 
will be indicated by the appearance of a red color. To differentiate between these bodies examine by the spectroscope and look for the absorption band between $\mathrm{D}$ and $\mathrm{E}$ given by pentoses and glycuronic acid, and then differentiate between the two latter bodies by the melting-points of their osazones.

3. Orcinol Test.-Place equal volumes of urine and hydrochloric acid (sp. gr. I.09) in a test-tube, add a small amount of orcinol, and heat the mixture to boiling. Color changes from red through reddish-blue to green will be noted. When the solution becomes green it should be shaken in a separatory funnel with a little amyl alcohol, and the alcoholic extract examined spectroscopically. An absorption band between $\mathrm{C}$ and $\mathrm{D}$ will be observed.

\section{FAT}

When fat finds its way into the urine through a lesion which brings some portion of the urinary passages into communication with the lymphatic system a condition known as chyluria is established. The turbid or milky appearance of such urine is due to its content of chyle. This disease is encountered most frequently in tropical countries, but is not entirely unknown in more temperate climates. Albumin is a constant constituent of the urine in chyluria. Upon shaking a chylous urine with ether the fat is dissolved by the ether and the urine becomes clearer or entirely clear.

\section{HEMATOPORPHYRIN}

Urine containing this body is occasionally met with in various diseases, but more frequently after the use of quinine, tetronal, trional, and especially sulphonal. Such urines ordinarily possess a reddish tint, the depth of color varying greatly under different conditions.

\section{EXPERIMENTS}

I. Spectroscopic Examination.-To Ioo c.c. of urine add about 20 c.c. of a ro per cent solution of potassium hydroxide or ammonium hydroxide. The precipitate which forms consists principally of earthy phosphates to which the hematoporphyrin adheres and is carried down. Filter off the precipitate, wash it and transfer to a flask and warm with alcohol acidified with hydrochloric acid. By this process the hematoporphyrin is dissolved and on filtering will be found in the filtrate and may be identified by means of the spectroscope (see page 296, and Absorption Spectra, Plate II).

2. Acetic Acid Test.-To roo c.c. of urine add 5 c.c. of glacial acetic acid and allow the mixture to stand 48 hours. Hematoporphyrin deposits in the form of a precipitate.

\section{LACTOSE}

Lactose is rarely found in the urine except as it is excreted by women during pregnancy, during the nursing period, or soon after weaning. 
It is rather difficult to show the presence of lactose in the urine in a satisfactory manner, since the formation of the characteristic lactosazone is not attended with any great measure of success under these conditions. It is, however, comparatively easy to show that it is not glucose, for, while it responds to reduction tests, it does not ferment with pure yeast and does not give a glucosazone. An absolutely conclusive test, of course, is the isolation of the lactose in crystalline form (Fig. I04, page 3I8) from the urine.

On oxidation with nitric acid lactose and galactose yield mucic acid. This test is frequently used in urine examination to differentiate lactose and galactose from other reducing sugars. To differentiate lactose from pentose, since neither ferments, we may apply the Orcinol-HCl test of Bial, see page 443 .

\section{EXPERIMENTS}

I. Mucic Acid Test.-Treat roo c.c. of the urine under examination with 20 c.c. $^{1}$ of concentrated nitric acid and evaporate the mixture in a broad, shallow glass vessel, upon a boiling water-bath until the volume of the solution is only about 20 c.c. At this point the fluid should be clear and a fine white precipitate of mucic acid should separate.

If the percentage of lactose in the urine is low it may be necessary to cool the solution and permit it to stand for some time before the precipitate will form. It is impossible to differentiate between galactose and lactose by means of this test, but the reaction does serve to differentiate these two sugars from all other reducing sugars. A satisfactory differentiation between lactose and galactose in pure solution may be made by means of Barfoed's test, page 30. This test is, however, not suited for urine examination. To differentiate galactose and lactose in urine use the Phloroglucinol-Hydrochloric Acid Reaction of Tollens, see pages 36 and 443 .

2. Rubner's Test.-To ro c.c. of urine in a small beaker add some lead acetate, in substance, heat to boiling, and add $\mathrm{NH}_{4} \mathrm{OH}$ until no more precipitate is dissolved. In the presence of lactose a brick-red or rose-red color develops, whereas glucose gives a coffee-brown color, maltose a light yellow color, and fructose no color at all under the same conditions.

3. Compound Test.-Try the Nylander reaction. If positive try the pheny:hydrazine test. If negative (the lactosazone is not readily forned in urine) apply the fermentation test. If this test is also negative, differentiate between lactose and pentose by Orcinol- $\mathrm{HCl}$ reaction (Bial) and mucic acid tests.

I If the specific gravity of the urine is 1020 or over it is necessary to use $25-35$ c.c. of nitric acid. Under these conditions the mixture should be cvaporated until the remaining volume is approximately equivalent to that of the nitric acid added. 


\section{GALACTOSE}

Galactose has occasionally been detected in the urine, and in particular in that of nursing infants afflicted with a deranged digestive function. Lactose and galactose may be differentiated from other reducing sugars which may be present in the urine by means of the mucic acid test. This test simply consists in the production of mucic acid through oxidation of the sugar with nitric acid.

\section{EXPERIMENTS}

I. Mucic Acid Test.-Treat roo c.c. of the urine under examination with 20 c.c. ${ }^{1}$ of concentrated nitric acid and evaporate the mixture in a broad, shallow glass vessel, upon a boiling water-bath, until the volume of the solution is only 20 c.c. At this point the fluid should be clear and a fine, white precipitate of mucic acid should separate.

If the percentage of galactose present in the urine is low it may be necessary to cool the solution and permit it to stand for some time before the precipitate will form. It is impossible to differentiate between galactose and lactose by means of this test, but the reaction does serve to differentiate these two sugars from all other reducing sugars. A satisfactory differentiation between galactose and lactose may be made by the Phloroglucinol-Hydrochloric Acid Test of Tollens, below.

2. Phloroglucinol-Hydrochloric Acid Reaction (Tollens).-To equal volumes of the urine and hydrochloric acid (sp. gr. I.og) add a little phloroglucinol and heat the mixture on a boiling water-bath. Galactose, pentose, and glycuronic acid will be indicated by the appearance of a red color. Galactose may be differentiated from the two latter substances in that its solutions exhibit no absorption bands upon spectroscopical examination.

\section{FRUCTOSE}

Diabetic urine frequently possesses the power of rotating the plane of polarized light to the left, thus indicating the presence of a levorotatory substance. The levorotation is sometimes due to the presence of fructose, although not necessarily confined to this carbohydrate, since conjugate glycuronates and $\beta$-hydroxybutyric acid, two other levorotatory bodies, are frequently found in the urine of diabetics. Fructose is invariably accompanied by glucose in diabetic urine, but fructosuria has been observed as a separate anomaly. The presence of

${ }^{1}$ If the specific gravity of the urine is ro 20 or over it is necessary to use $25-35$ c.c. of nitric acid. Under these conditions the mixture should be evaporated until the remaining volume is approximately equivalent to that of the nitric acid added. 
fructose may be inferred when the percentage of sugar, as determined by the titration method, is greater than the percentage indicated by the polariscopic examination.

\section{EXPERIMENTS}

I. Borchardt's Reaction.-To about 5 c.c. of urine in a test-tube add an equal volume of 25 per cent hydrochloric acid and a few crystals of resorcinol. Heat to boiling and after the production of a red color, cool the tube under running water and transfer to an evaporating dish or beaker. Make the mixture slightly alkaline with solid potassium hydroxide, return it to a test-tube, add 2-3 c.c. of acetic ether, and shake the tube vigorously. In the presence of fructose the acetic ether is colored yellow.

The only urinary constituents which interfere with the test are nitrites and indican and these interfere only when they are simultancously present. Under these conditions, the urine should be acidified with acetic acid and heated to boiling for one minute to remore the nitrites. In case the indican content is very large, it will impart a blue color to the acetic ether, thus masking the yellow color due to fructose. When such urines are to be examined, the indican should first be removed by Obermayer's test (see page 388). The chloroform should then be discarded, the acid-urine mixture diluted with one-third its volume of water, and the test applied as described above. The urine of patients who have ingested santonin or rhubarb responds to the test. The test will serve to detect fructose when present in a dilution of I $: 2000$, i.e., 0.05 per cent.

2. Resorcinol-Hydrochloric Acid Reaction (Seliwanoff).-To 5 c.c. of Seliwanoff's reagent ${ }^{1}$ in a test-tube add a few drops of the urine under examination and heat the mixture to boiling. The presence of fructose is indicated by the production of a red color and the separation of a red precipitate. The latter may be dissolved in alcohol to which it will impart a striking red color.

If the boiling be prolonged a similar reaction may be obtained with urines containing glucose. This has been explained ${ }^{2}$ in the case of glucose as due to the transformation of the glucose into fructose by the catalytic action of the hydrochloric acid. The precautions necessary for a positive test for fructose are as follows: The concentration of the hydrochloric acid must not be more than i 2 per cent. The reaction (red color) and the precipitate must be observed after not more than $20-30$ seconds of boiling. Glucose must not be present in amounts exceeding 2 per cent. 'The precipitate must be solub!e in alcohol with a bright red color.

1 Seliwanoff's reagent may be prepared by dissolving 0.05 gram of resorcinol in roo c.c. of dilute $(\mathrm{I}: 2)$ hydrochloric acid.

${ }^{2}$ Koenigsfeld: Bioch. Zeit. 38, 3r r, I9r2. 
3. Phenylhydrazine Test.-Make the test according to directions under Glucose, 3, page 22 .

4. Polariscopic Examination.-A simple polariscopic examination, when taken in connection with other ordinary tests, will furnish the requisite data regarding the presence of fructose, provided fructose is not accompanied by other levorotatory substances, such as conjugate glycuronates and $\beta$-hydroxybutyric acid.

\section{ARSENIC}

When any soluble form of arsenic is introduced into the body in any way, it is quickly absorbed and distributed by the blood and lymph. The absorption is influenced by the quantity and quality of the food in the stomach, and the activity of the circulation of the part in contact with the poison. Some of the absorbed arsenic may be returned to the alimentary canal by way of the bile and gastro-intestinal mucous membrane. After absorption it may be deposited in the liver, kidneys, brain, bone, muscles, and walls of the stomacl and intestines. It is eliminated in all of the excretions, but chiefly by the kidneys and through the feces. It does not appear very promptly in the urine but continues to be excreted in the urine over a long period of time, in some cases for several months. The urine may be examined for arsenic by the following methods.

I. Marsh and Marsh-Berzelius Method.-This method has the advantage of serving as a qualitative and quantitative determination, and is a very delicate test; it is, however, long and tedious. The various steps in the analysis are: (I) the destruction of the organic matter in the urine; (2) treatment with sulphuric acid to drive off excess nitric acid and break up nitro-compounds; and (3) application of independent test to the resultant solution. Proceed as follows: The urine, to which is added one-third its volume of nitric acid, is placed in a casserole or evaporating dish and evaporated at $150^{\circ}$ to $160^{\circ}$ to a syrupy consistency. The mass is then allowed to cool and 5 c.c. concentrated sulphuric acid added, and gentle heat applied. The heating must be done cautiously, or deflagration takes place and some of the arsenic is sure to be lost. The mass will liquefy and finally darken, indicating organic matter. Cool and add concentrated nitric acid, I c.c., and apply very gentle heat; copious reddish-bromn fumes are evolved. Gradually raise the temperature until darkening of the solution occurs, then cool, add I c.c. concentrated nitric acid and again apply gentle heat, and repeat the process until the solution fails to darken. Now raise the temperature until white fumes begin to come off. At this temperature excess nitric acid will have been removed and all nitro-com. pounds broken up. The solution at this point is clear and at most a pale straw color. Cool and add a mixture of Io c.c. concentrated sulphuric acid and 40 c.c. water, and test for arsenic using a Marsh apparatus. The apparatus (see Fig. I3I, p. 449) consists of a wide-mouth flask-250 c.c. capacity-fitted with a two-hole stopper. Through one hole is passed the stem of a separatory funnel of 50 to 60 c.c. capacity. Through the other hole a piece of glass tube bent at right angles, which is fitted to a calcium chloride tube, and this in turn to a narrow quartz tube, the distal end of 
which is drawn to a fine bore and bent up almost at a right angle. All joints must be air-tight.

Introduce 30 to 40 grams of arsenic-free granulated zinc into the flask, insert the stopper and through the funnel introduce 50 c.c. dilute sulphuric acid ( $\mathrm{I}$ part to 4 parts water). After a few minutes collect a test-tube of gas by inverting a test-tube over the end of the quartz tube, and test it by igniting. When the gas in the test-tube ignites quietly, light the gas issuing from the quartz tube.

Hold a clean porcelain crucible lid in the flame and note whether any deposit occurs. This precaution must be taken to insure that the chemicals and apparatus are not contaminated with arsenic.

Now introduce the prepared urine solution into the funnel and adjust the flow so that 6 to 8 drops are introduced into the flask per minute. Immediately hold a clean porcelain crucible lid in the flame and at the first evidence of a dark deposit apply heat, using a wing-top burner, to the quartz tube. The arsenic if present will deposit in the quartz tube beyond the flame. Now test the spot on the lid to see if it is arsenic; it should dissolve readily in sodium hypochlorite solution.

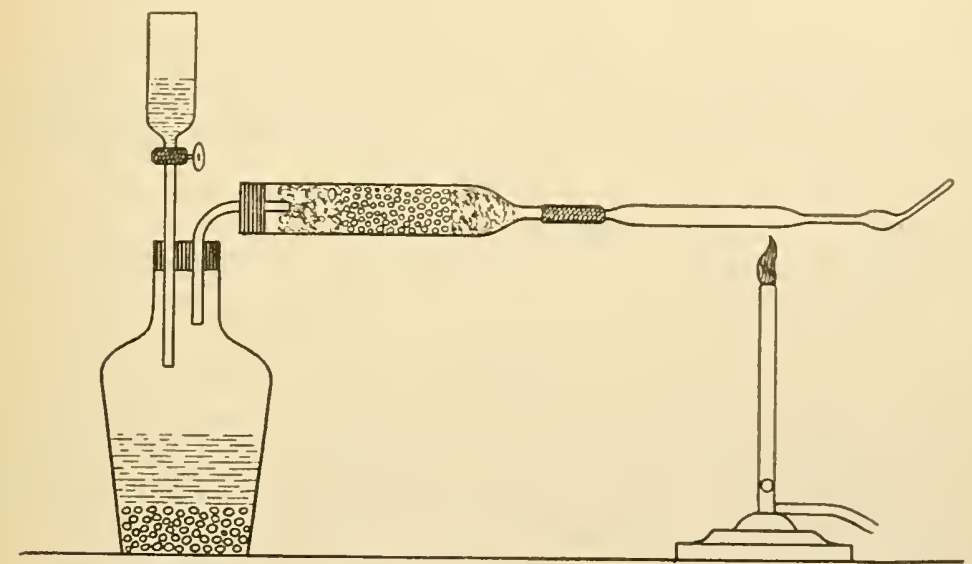

Fig. i3 1 .-MARsh Apparatus.

Continue the operation for two hours, remove the Bunsen burner and again hold the lid in the flame. If no more deposits on the lid, the arsenic has all come over and is deposited in the quartz tube; if deposition occurs, apply the Bunsen again and repeat.

When complete, remove the quartz tube, weigh it after cooling, then dissolve out the arsenic with nitric acid, wash, dry, and weigh again. The difference in weight is the weight of metallic arsenic in the volume of urine taken.

2. Reinsch's Test. - This test is very much simpler, but not so delicate. It has the advantage of application in the presence of organic matter. The test is performed as follows: The urine, acidified with one-fifth its volume of pure hydrochloric acid, is placed in a beaker. A piece of bright copper foil free from arsenic is then introduced, and the urine heated almost to the boiling-point. It is then set aside for six to eight hours. The arsenic is deposited on the copper foil, bluishgray color. The foil is then removed, washed successively in pure water, alcohol, ether, and dried without heat. The foil is then rolled into a scroll and inserted into a $3 \mathrm{~mm}$. bore glass tube 4 inches long, about $\mathrm{I}$ inch from the end. The tube is 
then held in the bunsen flame at an angle of 20 to 25 degrees applying heat where the copper foil is situated. The arsenic volatilizes and is oxidized, and deposits as octahedral crystals of arsenic trioxide on the cooler part of the tube. The crystals can readily be recognized by the microscope and sometimes with a simple magnifying lens.

\section{MERCURY}

The rapidity of absorption of mercury depends upon a number of conditions such as, mode of administration, the nature of the compound and its physical state, the state and condition of the stomach and intestines, the quantity and quality of the food in the stomach and the state of the circulation of the portal of entrance. There is no definite knowledge as to the form in which it is absorbed. Elimination depends upon the state of the excretory organs. It is eliminated as an albuminate in all the excretions of the body, urine, feces, saliva, sweat, tears, and milk. Elimination begins about two hours after introduction. Depending upon the amount introduced and absorbed, the time required for its complete elimination varies from 24 hours to many weeks. Mercury may be detected in the urine by the following methods.

I. Reinsch's Test.-The procedure is carried out in the same manner as for arsenic (see above). A piece of arsenic-free copper foil is introduced into the urine acidified with one-fifth its volume of pure hydrochloric acid. The urine is, however, not heated to boiling, but warmed to $50^{\circ}$ or $60^{\circ}$ and set aside ior 12 hours or preferably 24 hours. Metallic mercury is deposited on the foil as a bright lustrous mirror. The foil is then washed with pure water, alcohol, ether, and dried without heat, rolled into a scroll, inserted into a glass tube and heated in the same manner as under arsenic. The mercury is deposited in the metallic state in the form of globules readily distinguished with the microscope.

2. Amalgamation Test. $-\mathrm{A}$ more rapid method than the above is by amalgamation with zinc. Add 5 grams of zinc dust to the urine and heat for 15 minutes, stirring continuously. Allow the amalgamated zinc to settle and decant the urine. Then wash by decantation several times with pure water, then with alcohol, and finally with ether and dry in air. Now introduce the dry zinc into a narrow dry glass tube sealed at one end. With the Bunsen soften the tube about 2 inches above the zinc and constrict the tube by pulling the ends apart. Introduce a small bit of glass wool or asbestos sufficient to support a small piece of iodine. Introduce the iodine supported by the asbestos at the constriction. Apply heat to the zinc amalgam, and then gently to the region holding the iodine to gently volatilize it, and immediately reapply heat to the zinc. The mercury volatilizes and meeting the iodine vapor unites with it, and is deposited as the red iodide of mercury.

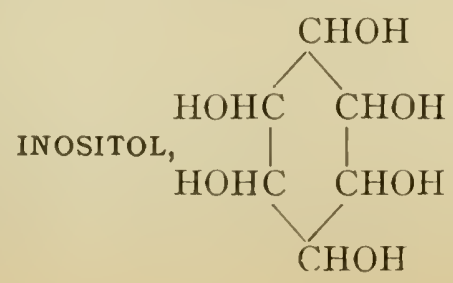


Inositol occasionally occurs in the urine in albuminuria, diabetes mellitus, and diabetes insipidus. It is claimed also that copious waterdrinking causes this substance to appear in the urine. Inositol was at one time considered to be a sugar but is now known to be hexahydroxybenzene, as the above formula indicates. It is an example of a non-carbohydrate in whose molecule the $\mathrm{H}$ and $\mathrm{O}$ are present in the proportion to form water. In other words it has the formula of the hexoses, i.e., $\mathrm{C}_{6} \mathrm{H}_{12} \mathrm{O}_{6}$. Inositol occurs widely distributed in the vegetable kingdom, and because of this fact the theory has been voiced that it represents one of the first stages in the conversion of a carbohydrate into the benzene ring. It is found in the liver. spleen, lungs, brain, kidneys, suprarenal capsules, muscles, leucocytes, testes, and urine under normal conditions.

\section{EXPERIMENT}

I. Detection of Inositol (Scherer).--Acidify the urine with concentrated nitric acid and evaporate nearly to dryness. Add a few drops of ammonium hydroxide and a little calcium chloride solution to the moist residue and evaporate the mixture to dryness. In the presence of inositol (o.oor gram) a bright red color is obtained.

For a more satisfactory test, which is also more time-consuming, see Salkowski's ${ }^{1}$ modification of Scherer's test.

\section{LAIOSE}

This substance is occasionally found in the urine in severe cases of diabetes mellitus. By some investigators laiose is classed with the sugars. It resembles fructose in that it has the property of reducing certain metallic oxides and is levorotatory, but differs from fructose in being amorphous, non-fermentable, and in not possessing a sweet taste.

\section{MELANINS}

These pigments never occur normally in the urine, but are present under certain pathological conditions, their presence being especially associated with melanotic tumors. Ordinarily the freshly passed urine is clear, but upon exposure to the air the color deepens and may at last be very dark brown or black in color. The pigment is probably present in the form of a chromogen or melanogen and upon coming into contact with the air oxidation occurs, causing the transformation of the melanogen into melanin and consequently the darkening of the urine.

It is claimed that melanuria is proof of the formation of a visceral

1 Salkowski: Zeil. physiol. chem., 69, 478 , i 9 го. 
melanotic growth. In many instances, without doubt, urines rich in indican have been wrongly taken as diagnostic proof of melanuria. The pigment melanin is sometimes mistaken for indigo and melanogen for indican. It is comparatively easy to differentiate between indigo and melanin through the solubility of the former in chloroform.

In rare cases melanin is found in urinary sediment in the form of fine amorphous granules.

\section{EXPERIMENTS}

I. Ferric Chloride Reaction (von Jaksch-Pollak).-Add a few drops of ferric chloride solution to to c.c. of urine in a test-tube and note the formation of a gray color. Upon the further addition of the chloride a dark precipitate forms, consisting of phosphates and adhering melanin. An excess of ferric chloride causes the precipitate to dissolve.

This is the most satisfactory test for the indentification of melanin in the urine.

2. Bromine Test (Zeller).-To 50 c.c. of urine in a small beaker add an equal volume of bromine water. In the presence of melanin a yellow precipitate will form and will gradually darken in color, ultimately becoming black.

\section{UROROSEIN}

This is a pigment which is not present in normal urine but may be detected in the urine in various diseases, such as pulmonary tuberculosis, typhoid fever, nephritis, and stomach disorders. Urorosein, in common with various other pigments, does not occur preformed in the urine, but is present in the form of a chromogen, which is transformed into the pigment upon treatment with a mineral acid. Herter ${ }^{1}$ showed this chromogen to be indole acetic acid,

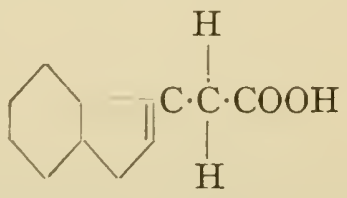

Normal urine responds to the urorosein reaction (see below) if nitrites are present.

\section{EXPERIMENTS}

I. Nitrite-Hydrochloric Acid Test (Urorosein Reaction).-To Io c.c. of urine in a test-tube add 2 c.c. of concentrated hydrochloric acid and a few drops of a I per cent solution of potassium nitrite. A rose-red color indicates urorosein.

The chromogen (indole acetic acid) has been changed into urorosein by oxidation.

${ }^{1}$ Herter: Jour. Biol. Chem., 4, 253 , 1908. 
2. Robin's Reaction.-Acidify ro c.c. of urine with about I 5 drops of concentrated hydrochloric acid. Lpon allowing the acidified urine to stand, a rose-red color will appear if urorosein is present.

3. Nencki and Sieber's Reaction.-To roo c.c. of urine in a beaker add io c.c. of 25 per cent sulphuric acid. Allow the acidified urine to stand and note the appearance of a rose-red color. The pigment may be separated by extraction with amyl alcohol.

\section{NEPHROROSEIN}

This pigment is closely related to urorosein ${ }^{1}$ and like urorosein it is produced from a chromogen when the urine is treated with nitric acid or with concentrated hydrochloric acid and a little sodium nitrite solution. It is sometimes called $\beta$-urorosein to differentiate it from the true urorosein which is termed $\alpha$-urorosein. Nephrorosein occurs only in pathological urines.

\section{UROCHROMOGEN}

This is the chromogen of urochrome, the normal urinary pigment (see Chapter XXI). It is claimed that the urochromogen reaction of the urine is an aid to prognosis and diagnosis of pulmonary tuberculosis. Urochromogen is not present in normal urine. Its presence in pathological urine is due probably to faulty oxidation, i.e., failure to oxidize the chromogen to urochrome. Urochromogen may be detected by oxidizing it to urochrome by means of potassium permaganate. In this process a certain antecedent of urochromogen is also oxidized to urochrome. Whereas the diazo reaction (see page 454 ) is also given by urines containing urochromogen, it is claimed that the diazo reaction does not show the presence of the precursor of urochromogen. Hence the urochromogen reaction is said to be more constant and uniform in its appearance.

\section{ExperinIENT}

Urochromogen Reaction (Weisz).2-Fill a test-tube a little less than onethird full of urine, dilute it with 2 volumes of distilled water and mix thoroughly. Pour one-half the diluted urine into another tube and to one of the tubes add 3 drops of a I per cent solution of potassium permanganate. Shake the tube thoroughly. In the presence of urochromogen a yellow tint will appear in the tube to which permanganate was added.

The reaction is due to the oxidation of urochromogen to urochrome, and is believed to be of value as an aid in prognosis and diagnosis of

1 Arnold: Zeil. physiol. Chem., 7 I.

2 Weisz: Münch. med. Hoch., 58, r 348, I9I.

Vitri: Semana Medica, 20, No. 28, r9r3.

Heflebower: Am. Jour. Sled. Sci., I 43,22 I, I 9 I 2.

Metzger and Watson: Jour. Am. Med. Ass'n., 62, isS6, igrt.

Pignacca: Gazelia d. Osp. c delle Clin., 25, 353, I9 if.

Ferrannini: Riforma med., 31, 479, 1915. 
pulmonary tuberculosis. The presence of sugar, albumin or urobilin in low concentration does not interfere with the test. The test often runs parallel with the diazo reaction (see below.) The test is supposed to be positive when the focus of the lung is so active or extensive as to flood the blood with toxins or to break down the defensive forces of the body. It is claimed, therefore, that this test will differentiate the cases in which the tuberculosis is beyond help from the tuberculin from those in which the body is liable to respond favorably to its action. ${ }^{1}$ Some investigators claim the test is not specific and that a positive reaction will be obtained in many disorders other than tuberculosis. $^{2}$

\section{UNKNOWN SUBSTANCES}

I. Ehrlich's Diazo Reaction.-Place equal volumes of urine and Ehrlich's diazobenzenesulphonic acid reagent ${ }^{3}$ in a test-tube, mix thoroughly by shaking, and quickly add ammonium hydroxide in excess. The test is positive if both the fluid and the foam assume a red color. If the tube is allowed to stand a precipitate forms, the upper portion of which exhibits a blue, green, greenish-black, or violet color. Normal urine gives a brownish-yellow reaction with the above manipulation.

The exact nature of the substance or substances upon whose presence in the urine this reaction depends is not well understood. Some investigators claim that a positive reaction indicates an abnormal decomposition of protein material, whereas others assume it to be due to an increased excretion of alloxyproteic acid, oxyproteic acid, or uroferric acid. Weisz ${ }^{4}$ claims that urochromogen is the principal urinary substance which causes a positive diazo reaction.

The reaction may be taken as a metabolic symptom of certain disorders, which is of value diagnostically only when taken in connection with the other symptoms. The reaction appears principally in the urine in febrile disorders and in particular in the urine in typhoid fever, tuberculosis, and measles. The reaction has also been obtained in the urine in various other disorders such as carcinoma, chronic rheumatism,

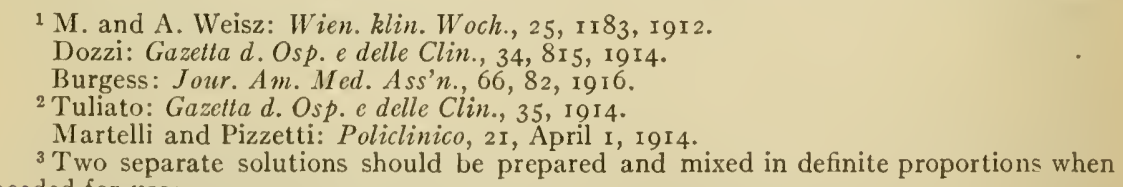
needed for use:

(a) Five grams of sodium nitrite dissolved in I liter of distilled water.

(b) Five grams of sulphanilic acid and 50 c.c. of hydrochloric acid in I liter of distilled water.

Solutions $a$ and $b$ should be preserved in well-stoppered vessels and mixed in the proportion 1 : 50 when required. Green asserts that greater delicacy is secured by mixing the solutions in the proportion I : IOO. The sodium nitrite deteriorates upon standing and becomes unfit for use in the course of a few weeks.

4 Weisz: Wiunch. med. Woch., 58, I 348 , r 9 II. 
diphtheria, erysipelas, pleurisy, pneumonia, scarlet 'fever, syphilis, typhus, etc. The administration of alcohol, chrysarobin, creosote, cresol, dionin, guaiacol, heroin, morphine, naphthalene, opium, phenol, tannic acid, etc., will also cause the urine to give a positive reaction.

The following chemical reactions take place in this test:

(a) $\mathrm{NaNO}_{2}+\mathrm{HCl} \rightarrow \mathrm{HNO}_{2}+\mathrm{NaCl}$

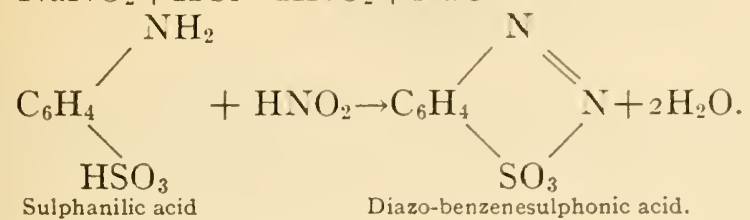

2. Methylene Blue Reaction (Russo). ${ }^{1-}$ To 5 c.c. of urine add 4 drops of a 0 . I per cent solution of methylene blue. In cases of typhoid fever, measles, smallpox and certain other disorders there will be a change in color from blue to green. In normal urine the blue color persists. The test is sometimes used as a substitute for the diazo reaction (see p. 454 ).

\section{PHENOLSULPHONEPHTHALEIN TEST FOR KIDNEY EFFICIENCY}

This test for renal function was devised by Rowntree and Geraghty. ${ }^{2}$ It depends upon the injection into the tissues of a dyestuff which is eliminated rapidly by the normal kidneys, and can be easily estimated quantitatively in the urine.

This dyestuff, phenolsulphonephthalein, is non-irritative to the body either when taken by mouth or when injected into the tissues, ${ }^{3}$ so that it does no harm to an already weakened kidney.

The patient upon whom the test is to be performed is given $300-400$ c.c. of water $20-30$ minutes previously, in order to assure a free flow of urine.

The procedure is as follows: One c.c. of a solution containing $6 \mathrm{mg}$. of phenolsulphonephthalein 4 is injected intramuscularly in the lumbar region, the time of injection being noted. The patient is then catheterized and the urine as it forms thereafter allowed to drop into a beaker containing 2 drops of 25 per cent $\mathrm{NaOH}$. The appearance of a red color in the alkalinized urine indicates beginning excretion of the drug, the normal time being within 5 to ro minutes after its injection.

${ }^{1}$ Russo: Riforma med., No. 19, I905.

Peskow: Semaine med., 103, I9I2.

da Pozzo: Gaz. Osp. Clin., 35, 865 , 19 r4.

${ }^{2}$ Rowntree and Geraghty: Jour. Pharm. and Exper. Therap., 1, 579, 1910: also dreh. Int. Med., March, 1912, p. 284.

3 Abel and Rowntree: Jour. Pharm. and Exper. Therap., I, 231, 19 ro.

- This solution is prepared by adding $0.6 \mathrm{gram}$ phenolsulphonephthalein and o. + c.c. of $2 / \mathrm{N} \mathrm{NaOH}$ to enough 0.75 per cent $\mathrm{NaCl}$ solution to make roo c.c. This gives the monosodium or acid salt which is slightly irritant locally when injected. It is necessary to add 2-3 drops more $2 / \mathrm{N} \mathrm{NaOH}$ which changes the color to a bordeaux red. This preparation is non-irritant. 
Urine is now collected in one-hour samples. In patients with obstruction to the flow of urine from the bladder the retention catheter is stoppered and the urine drawn off at the end of each hour. Other patients may simply be allowed to urinate at the hourly periods.

To each hour sample of urine is added 25 per cent $\mathrm{NaOH}$, drop by drop, until the maximum intensity of color appears. This color will remain constant for an indefinite period of time. Each sample is then placed in a 1000 c.c. volumetric flask and diluted to the mark with distilled water.

Comparison is made in a Duboscq (Hellige or Sargent) colorimeter (see p. 486) with a standard consisting of $3 \mathrm{mg}$. of phenolsulphonephthalein in 1000 c.c. of solution. The cylinder containing the standard may conveniently be placed at the ro mm. mark. Since the volume of each urine sample is the same as that of the standard, the percentage elimination of phenolsulphonephthalein in each may be easily calculated as follows :

Reading of Urine: Reading of Standard : : $100: \mathrm{X}$.

The amount of the drug eliminated normally is $40-60$ per cent during the first hour and $20-25$ per cent during the second hour, or a total of $60-85$ per cent for two hours. The amount of the drug excreted has been found to be independent of the quantity of urine obtained. In case of delayed excretion the collection of hourly samples may be continued until practically all of the drug has been recovered in the urine.

If it is desired to test the function of each kidney separately, ureteral catheterization must be resorted to, the experiment otherwise being performed as above described.

The phenolsulphonephthalein test may be used to indicate the amount of derangement in quantitative functional disturbance of the kidneys, as in chronic interstitial and chronic parenchymatous nephritis or uremia.

McLean ${ }^{1}$ has very recently suggested a method for studying kidney function which is based upon the relationship between the urea content of the blood and the rate at which the urea is excreted by the kidney. It gives similar values to the phenol-sulphonephthalein test. It has an advantage in that it enables one to measure kidney function by a study of an actual normal function of the organ, i.e., urea excretion. The method, however, is more or less complex.

\footnotetext{
${ }^{1}$ McLean: Jour. Am. Med. Assn., 66., 4I5, I9I6.
} 


\section{CHAPTER XXIV}

\section{URINE : ORGANIZED AND UNORGANIZED SEDIMENTS}

THE data obtained from carefully conducted microscopical examinations of the sediment of certain pathological urines are of very great importance diagnostically. Too little emphasis is sometimes placed upon the value of such findings.

The sedimentary constituents may be divided into two classes, i.e., organized and unorganized. The sediment is ordinarily collected

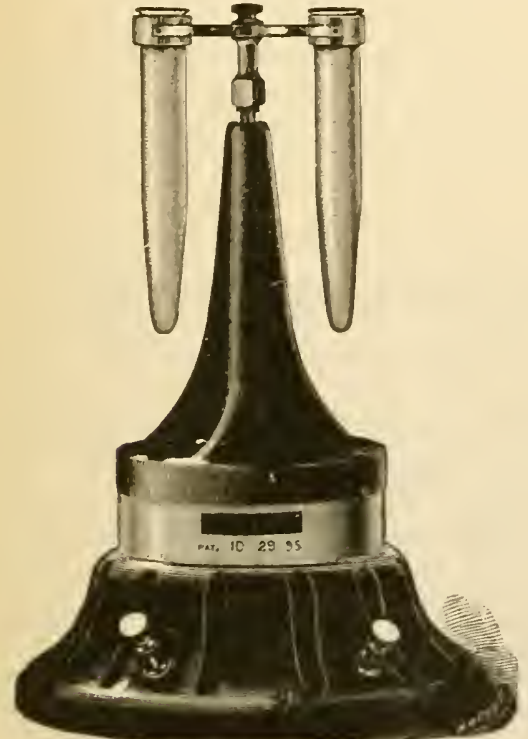

Fig. 132.-The Purdy Electric Centriftge.

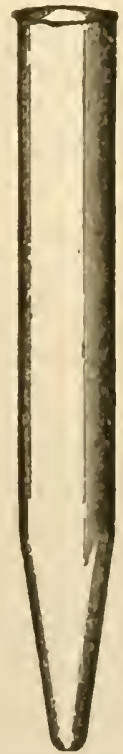

FIG. 133.-SEDJMENT TT'BE FOR THE Purdy Electric Cestriftge.

for examination by means of the centrifuge (Fig. I32). An older . method, and one still in rogue in some quarters, is the so-called gracity method. This simply consists in placing the urine in a conical glass and allowing the sediment to settle. The collection of the sediment by means of the centrifuge, however, is much preferable, since the process of sedimentation may be accomplished by the use of this instrument in a few minutes, and far more perfectly, whereas when the other method is used it is frequently necessary to allow the urine to remain in the con- 
ical glass I 2-24 hours before sufficient sediment can be secured for the microscopical examination.

\section{(a) Unorganized Sediments}

Ammonium magnesium phosphate ("triple phosphate").

Calcium oxalate.

Calcium carbonate.

Calcium phosphate.

Calcium sulphate.

Uric acid.

Urates.

Cystine.

Cholesterol.

Hippuric acid.

Leucine (?) and tyrosine.

Hematoidin and bilirubin.

Magnesium phosphate.

Indigo.

Xanthine.

Melanin.

\section{Ammonium Magnesium Phosphate ("Triple Phosphate").-} Crystals of "triple phosphate" are a characteristic constituent of the sediment when alkaline fermentation of the urine has taken place either before or after being voided. They may even be detected in amphoteric or slightly acid urine provided the ammonium salts are present in large enough quantity. This substance may occur in the sediment in two forms, i.e., prisms and the feathery type. The prismatic form of crystals (Fig. I29, page 408) is the one most commonly observed in the sediment; the feathery form (Fig. I29, page 408) predominates when the urine is made ammoniacal with ammonia.

The sediment of the urine in such disorders as are accompanied by a retention of urine in the lower urinary tract contains "triple phosphate" crystals as a characteristic constituent. The crystals are frequently abundant in the sediment during paraplegia, chronic cystitis, enlarged prostate, and chronic pyelitis.

Calcium Oxalate.-Calcium oxalate is found in the urine in the form of at least two distinct types of crystals, i.e., the dumb-bell type and the octahedral type (Fig. I34, page 459). Either form may occur in the sediment of neutral, alkaline, or acid urine, but both forms are found most frequently in urine having an acid reaction. Occasionally, in alkaline urine, the octahedral form is confounded with "triple phos- 
phate" crystals. They may be differentiated from the phosphate crystals by the fact that they are insoluble in acetic acid.

The presence of calcium oxalate in the urine is not of itself a sign of any abnormality, since it is a constituent of normal urine. It is increased above the normal, however, in such pathological conditions as diabetes

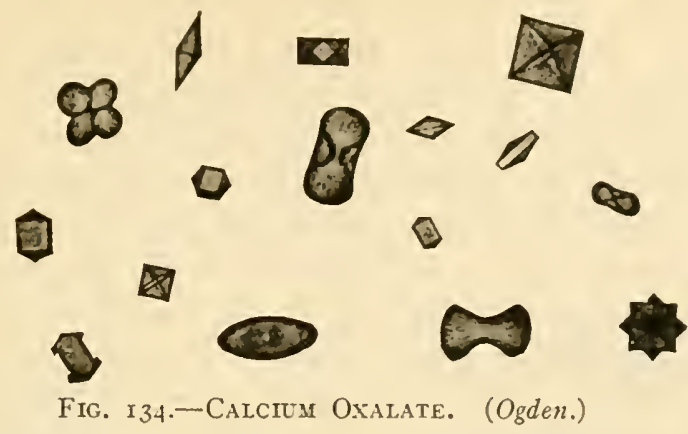

mellitus, in organic diseases of the liver, and in various other conditions which are accompanied by a derangement of digestion or of the oxidation mechanism, such as occurs in certain diseases of the heart and lungs.

Calcium Carbonate.-Calcium carbonate crystals form a typical constituent of the urine of herbivorous animals. They occur less fre-

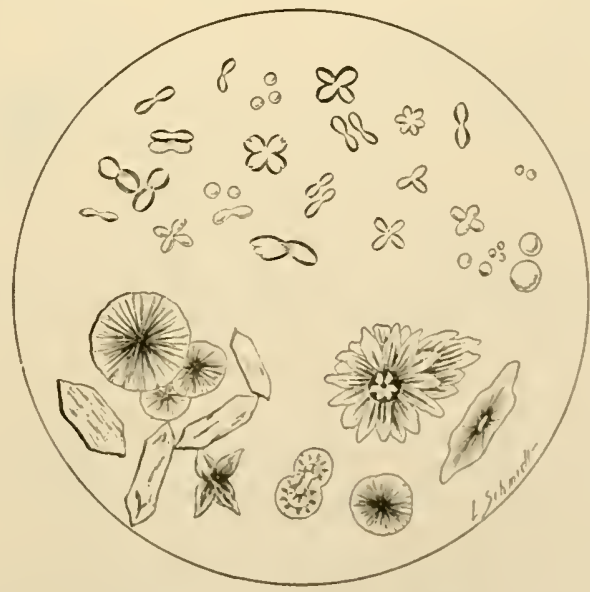

Fig. i35.-Calcium Carbonate.

quently in human urine. The reaction of urine containing these crystals is nearly always alkaline, although they may occur in amphoteric or in slightly acid urine. It generally crystallizes in the form of granules, spherules, or dumb-bells (Fig. 135). The crystals of calcium carbonate may be differentiated from calcium oxalate by the 
fact that they dissolve in acetic acid with the evolution of carbon dioxide gas.

Calcium Phosphate (Stellar Phosphate).-Calcium phosphate may occur in the urine in three forms, i.e., amorphous, granular, or crystalline. The crystals of calcium phosphate are ordinarily pointed, wedgeshaped formations which may occur as individual crystals or grouped together in more or less regularly formed rosettes (Fig. I05, page 322). Acid sodium urate crystals (Fig. $I_{37}$, page 462 ) are often mistaken for crystals of calcium phosphate. We may differentiate between these two crystalline forms by the fact that acetic acid will readily dissolve the phosphate, whereas the urate is much less soluble and when finally brought into solution and recrystallized one is frequently enabled to identify uric acid crystals which have been formed from the acid urate solution. The clinical significance of the occurrence of calcium phosphate crystals in the urinary sediment is similar to that of "triple phosphate" (see page $45^{8}$ ).

Calcium Sulphate.-Crystals of calcium sulphate are of quite rare occurrence in the sediment of urine. Their presence seems to be limited in general to urines which are of a decided acid reaction. Ordinarily it crystallizes in the form of long, thin, colorless needles or prisms (Fig. I 28 , page 405) which may be mistaken for calcium phosphate crystals. There need be no confusion in this respect, however, since the sulphate crystals are insoluble in acetic acid, which reagent readily dissolves the phosphate. As far as is known their occurrence as a constituent of urinary sediment is of very little clinical significance.

Uric Acid.- Uric acid forms a very common constituent of the sediment of urines which are acid in reaction. It occurs in more varied forms than any of the other crystalline sediments (Plate V, opposite page 380 , and Fig. 136), some of the more common varieties of crystals being rhombic prisms, wedges, dumb-bells, whetstones, prismatic rosettes, irregular or hexagonal plates, etc. Crystals of pure uric acid are always colorless (Fig. I22, page 380), but the form occurring in urinary sediments is impure and under the microscope appears pigmented, the depth of color varying from yellow to a dark reddishbrown according to the size and form of the crystal.

The presence of a considerable uric acid sediment does not, of necessity, indicate a pathological condition or a urine of increased uric acid content, since this substance very often occurs as a sediment in urines whose uric acid content is diminished from the normal merely as a result of changes in reaction, etc. Pathologically, uric acid sediments occur in gout, acute febrile conditions, chronic interstitial nephritis, etc. If the microscopical examination is not conclusive, uric acid may be 
differentiated from other crystalline urinary sediments from the fact that $j$ is soluble in alkalis, alkali carbonates, boiling glycerol, concentrated sulphuric acid, and in certain organic bases such as ethylamine and piperidin. It also responds to the murexide test (see page 380), Schiff's reaction (see page 38I) and to Folin's phosphotungstic acid reaction (see page 38 I).

Urates.-The urate sediment may consist of a mixture of the urates of ammonium, calcium, magnesium, potassium, and sodium. 'The ammonium urate may occur in neutral, alkaline, or acid urine, whereas the other forms of urates are confined to the sediments of acid urines. Sodium urate occurs in sediments more abundantly than the other

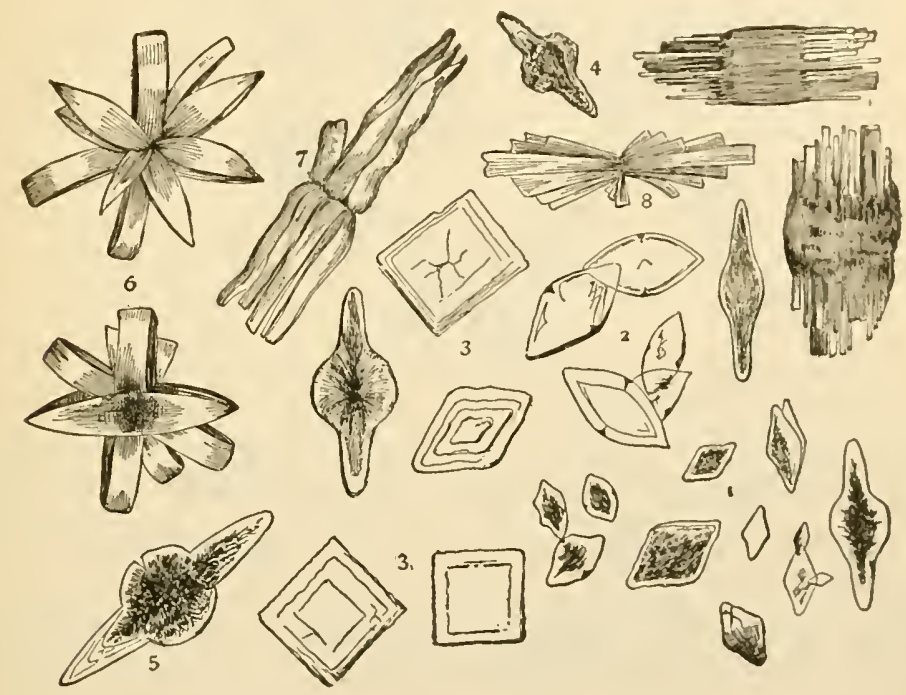

Fig. I36.-VARIOUS Forms OF URIC ACID.

I, Rhombic plates; 2 , whetstone forms; 3,3 , quadrate forms; 4 , 5, prolonged into points; 6,8 , rosettes; 7 , pointed bundles; 9 , barrel forms precipitated by adding hydrochloric acid to urine.

urates. There are two sodium urates, the mono and the di, which may be expressed thus $\mathrm{Na}^{+} \mathrm{H}^{+}>\mathrm{C}_{5} \mathrm{H}_{2} \mathrm{~N}_{4} \mathrm{O}_{3}{ }^{-}$and $\mathrm{Na}^{+}>\mathrm{C}_{5} \mathrm{H}_{2} \mathrm{~N}_{4} \mathrm{O}_{3}{ }^{-}$. Both salts dissociate with the production of an alkaline reaction, the alkalinity being stronger in the case of the di-sodium urate. The so-called quadriurate or hemiurate have no existence as chemical units. ${ }^{1}$ The urates of calcium, magnesium, and potassium are amorphous in character, whereas the urate of ammonium is crystalline. Sodium urate may be either amorphous or crystalline. When crystalline it forms groups of fan-shaped clusters or colorless, prismatic needles (Fig.

1 Taylor: Jour. Biol. Chem., I, I77, 1905. 
I37. Ammonium urate is ordinarily present in the sediment in the burr-like form of the "thorn-apple" crystal, i.e., yellow or reddishbrown spheres, covered with sharp spicules or prisms (Plate VI, opposite). The urates are all soluble in hydrochloric acid or acetic acid and their acid solutions yield crystals of uric acid upon standing. They also respond to the murexide test. The clinical significance of

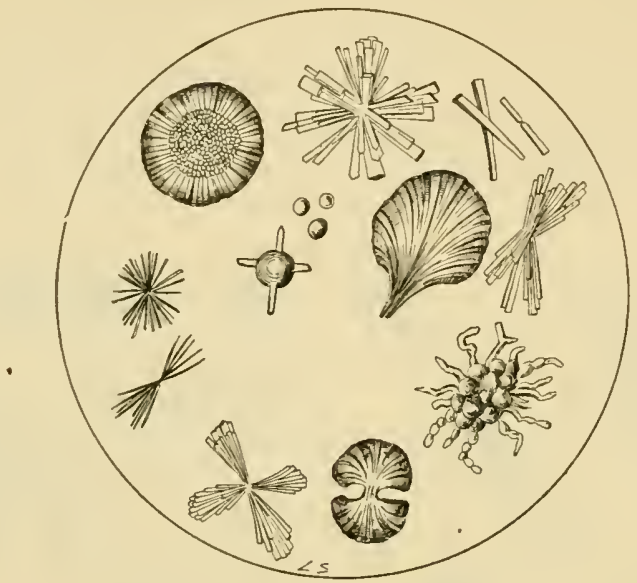

Fig. I37-ACID Sodium URATE.

urate sediments is very similar to that of uric acid. A considerable sediment of amorphous urates does not necessarily indicate a high uric acid content, but ordinarily signifies a concentrated urine having a very strong acidity.

Cystine.-Cystine is one of the rarer of the crystalline urinary sediments. It has been claimed that it occurs more often in the urine of
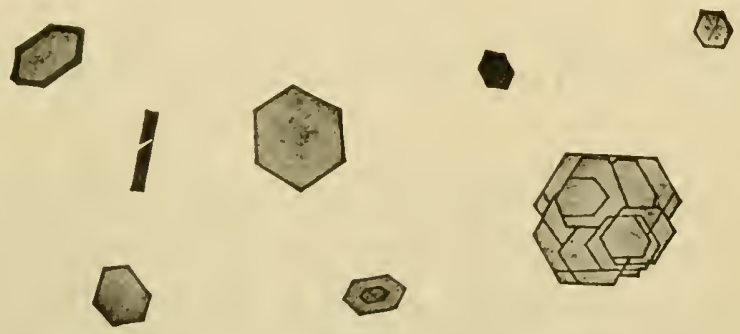

FIG. I38.-CySTINE. (Ogden.)

men than of women. Cystine crystallizes in the form of thin, colorless, hexagonal plates (Fig. 26, page 76, and Fig. 138) which are insoluble in water, alcohol, and acetic acid, and soluble in minerals, acids, alkalis, and especially in ammonia. Cystine may be identified by burning it upon platinum foil, under which condition it does not 
PLATE VI.

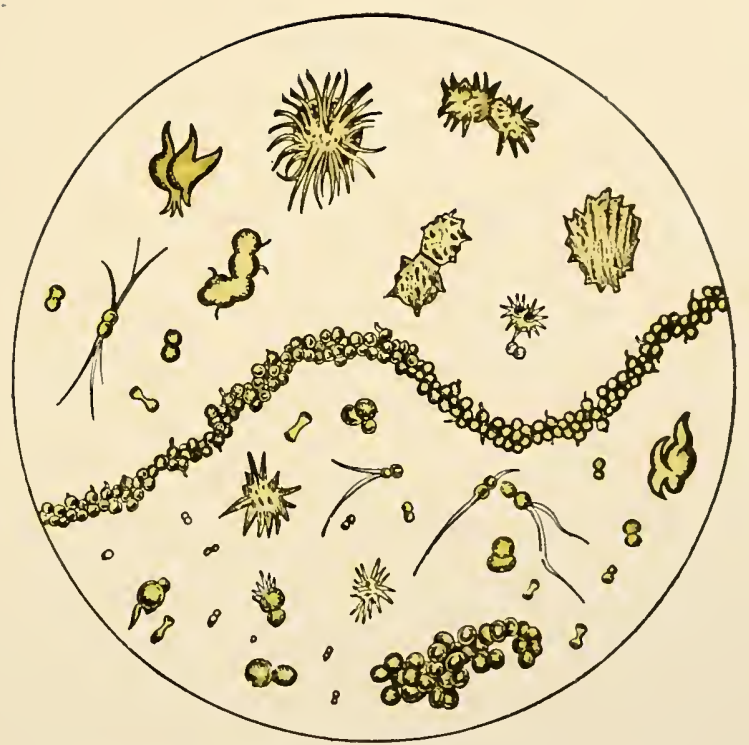

Ammonium Urates, showlng Spherules and Thorn-apple-shaped Cristals. (From Ogden, after Peyer.) 

melt but yields a bluish-green flame. For preparation of Cystine see Chapter IV.

Cholesterol.-Cholesterol crystals have been but rarely detected in urinary sediments. When present they probably arise from a pathological condition of some portion of the urinary tract. Crystals of cholesterol have been found in the sediment in cystitis, pyelitis, chyluria, and nephritis. Ordinarily it crystallizes in large regular and irregular colorless, transparent plates, some of which possess notched corners (Fig. 57, page 210). Frequently, instead of occurring in the sediment, it is found in the form of a film on the surface of the urine.

Hippuric Acid.-This is one of the rare sediments of human urine. It deposits under conditions similar to those which govern the formation of uric acid sediments. The crystals, which are colorless needles or prisms (Fig. I25, page 389) when pure, are invariably pigmented in a manner similar to the uric acid crystals when observed in urinary sediment and because of this fact are frequently confounded with the rarer forms of uric acid. Hippuric acid may be differentiated from uric acid from the fact that it does not respond to the murexide test and is much more
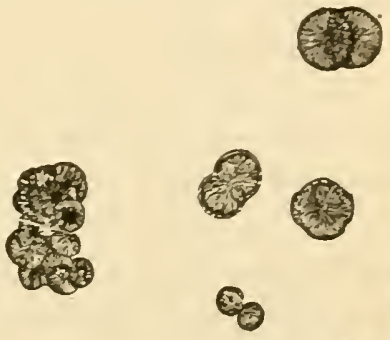

Fig. I39.-CRystals of IMPURE LetCNE. (Ogden.) soluble in water and in ether. The detection of crystals of hippuric acid in the urine has very little clinical significance, since its presence in the sediment depends in most instances very greatly upon the nature of the diet. It is particularly prone to occur in the sediment after the ingestion of certain fruits as well as after the ingestion of benzoic acid (see pages 388 and 585 ).

Leucine and Tyrosine.-Leucine and tyrosine have frequently been detected in the urine, either in solution or as a sediment. Neither of them occurs in the urine ordinarily except in association with the other, i.e., whenever leucine is detected it is more than probable that tyrosine accompanies it. They have been found pathologically in the urine in acute yellow atrophy of the liver, in acute phosphorus poisoning, in cirrhosis of the liver, in severe cases of typhoid fever and small-pox, and in leukemia. In urinary sediments leucine ordinarily crystallizes in characteristic spherical masses which show both radial and concentric striations and are highly refractive (Fig. I39). Some investigators claim that these crystals which are ordinarily called leucine are, in reality, generally urates. This view point has become more general in recent years. For the crystalline form of pure leucine 
obtained as a decomposition product of protein see Fig. 28, page 80. Tyrosine crystallizes in urdinary sediments in the well-known sheaf or tuft formation (Fig. 25, page 76). For other tests on leucine and tyrosine see pages 86 and 87 .

Hematoidin and Bilirubin.-There are divergent opinions regarding the occurrence of these bodies in urinary sediment. Each of them crystallizes in the form of tufts of small needles or in the form of small plates which are ordinarily yellowish-red in color (Fig. 56, page 205). Because the of fact that the crystalline form of the two substances is identical many investigators claim them to be one and the same body. Other investigators claim, that while the crystalline form is the same in each case, there are certain chemical differences which may be brought out very strikingly by properly testing. For instance, it has been claimed that hematoidin may be differentiated from bilirubin through the fact that it gives a momentary color reaction (blue) when nitric acid is brought into contact with it, and, further, that it is not dissolved on treatment with ether or potassium hydroxide. Pathologically, typical crystals of hematoidin or bilirubin have been found in the urinary sediment in jaundice, acute yellow atrophy of the liver, carcinoma of the liver, cirrhosis of the liver, and in phosphorus poisoning, typhoid tever, and scarlatina.

Magnesium Phosphate.-Magnesium phosphate crystals occur rather infrequently in the sediment of urine which is neutral, alkaline, or feebly acid in reaction. It ordinarily crystallizes in elongated, highly refractive, rhombic plates which are soluble in acetic acid.

Indigo.-Indigo crystals are frequently found in urine which has undergone alkaline fermentation. They result from the breaking down of indoxyl-sulphates or indoxyl-glycuronates. Ordinarily indigo deposits as dark blue stellate needles or occurs as amorphous particles or broken fragments. These crystalline or amorphous forms may occur in the sediment or may form a blue film on the surface of the urine. Indigo crystals generally occur in urine which is alkaline in reaction, but they have been detected in acid urine.

Xanthine.-Xanthine is a constituent of normal urine but is found in the sediment in crystalline form very infrequently, and then only in pathological urine. When present in the sediment xanthine generally occurs in the form of whetstone-shaped crystals somewhat similar in form to the whetstone variety of uric acid crystal. They may be differentiated from uric acid by the great ease with which they may be brought into solution in dilute ammonia and on applying heat. Xanthine may also form urinary calculi. The clinical significance of xanthine in urinary sediment is not well understood. 
Melanin.-Melanin is an extremely rare constituent of urinary sediments. Ordinarily in melanuria the melanin remains in solution; if it separates it is generally held in suspension as fine amorphous granules.

\section{(b) Organized Sediments}

Epithelial cells.

Pus cells.

Casts.

$$
\text { Hyaline. }
$$

Granular.

Epithelial.

Blood.

Fatty.

Waxy.

Pus.

Cylindroids.

Erythrocytes.

Spermatozoa.

Urethral filaments.

Tissue débris.

Animal parasites.

Micro-organisms.

Fibrin.

Foreign substances due to contamination.

Epithelial Cells.-The detection of a certain number of these cells in urinary sediment is not, of itself, a pathological sign, since they occur in normal urine. However, in certain pathological conditions they are greatly increased in number, and since different areas of the urinary tract are lined with different forms of epithelial cells, it becomes necessary, when examining urinary sediments, to note not only the relative number of such cells, but at the same time to carefully observe the shape of the various individuals in order to determine, as far as possible, from what portion of the tract they have been derived. Since the different layers of the epithelial lining are composed of cells different in form from those of the associated layers, it is evident that a careful microscopical examination of these cells may tell us the particular layer which is being desquamated. It is frequently a most difficult undertaking, however, to make a clear differentiation between the various forms of epithelial cells present in the sediment. If skilfully done, such a microscopical differentiation may prove to be of verygreat diagnostic aid.

The principal forms of epithelial cells met with in urinary sediments are shown in Fig. 140, page 466. 
Pus Cells.-Pus corpuscles or leucocytes are present in extremely small numbers in normal urine. Any considerable increase in the number, however, ordinarily denotes a pathological condition, generally an acute or chronic inflammatory condition of some portion of the urinary tract. The sudden appearance of a large amount of pus in a sediment denotes the opening of an abscess into the urinary tract. Other form elements, such as epithelial cells, casts, etc., ordinarily accompany pus corpuscles in urinary sediment and a careful examination of these associated elements is necessary in order to form a correct diagnosis as to the origin of the pus. Protein is always present in urine which contains pus.

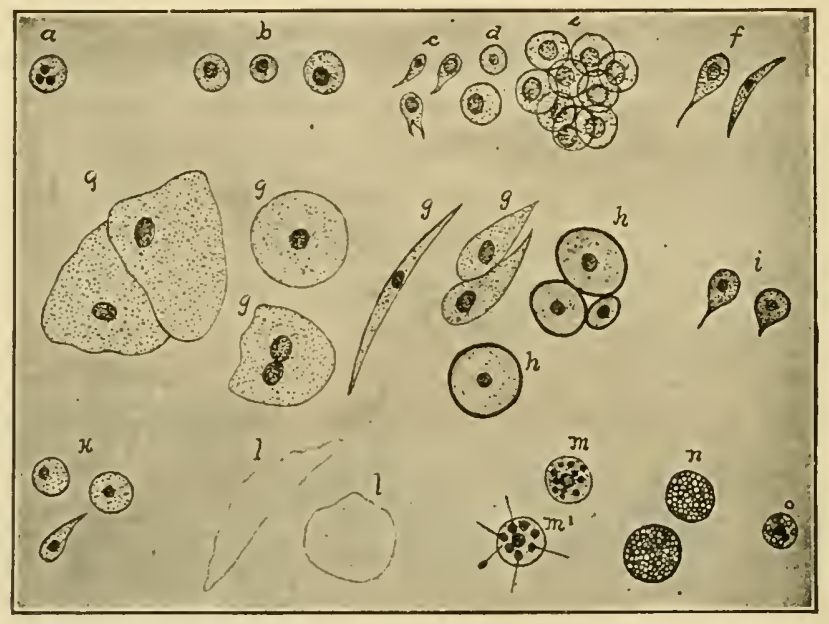

Fig. I40.-Epithelium from Different Areas of the Urinary Tract.

$a$, Leucocyte (for comparison); $b$, renal cells; $c$, superficial pelvic cells; $d$, deep pelvic cells; $e$, cells from calices; $f$, cells from ureter; $g, g, g, g, g$, squamous epithelium from the bladder; $h, h$, neck-of-bladder cells; $i$, epithelium from prostatic urethra; $k$, urethral cells; $l, l$, scaly epithelium; $m, m^{\prime}$, cells from seminal passages; $n$, compound granule cells; $o$, fatty renal cell. (Ogden.)

The appearance which pus corpuscles exhibit under the microscope depends greatly upon the reaction of the urine containing them. In acid urine they generally present the appearance of round, colorless cells composed of refractive, granular protoplasm, and may frequently exhibit ameboid morements, especially if the slide containing them be warmed slightly. They are nucleated (one or more nuclei), the nuclei being clearly visible only upon treating the cells with water, acetic acid, or some other suitable reagent. In urine which has a decided alkaline reaction, on the other hand, the pus corpuscles are often greatly degenerated. They may be seen as swollen, transparent cells, which exhibit no granular structure and as the process of degeneration con- 
tinues the cell outline ceases to be visible, the nuclei fade, and finally only a mass of débris containing isolated nuclei and an occasional cell remains.

It is frequently rather difficult to make a differentiation between pus corpuscles and certain types of epithelial cells which are similar in form. Such confusion may be avoided by the addition of iodine solution ( $\mathrm{I}$ in $\mathrm{KI}$ ), a reagent which stains the pus corpuscles a deep mahogany-brown and transmits to the epithelial cells a light yellow tint. The test proposed by Vitali often gives very satisfactory results. This simply consists in acidifying the urine (if alkaline) with acetic acid, then filtering, and treating the sediment on the filter paper with freshly prepared tincture of guaiac. The presence of pus in the sediment is indicated

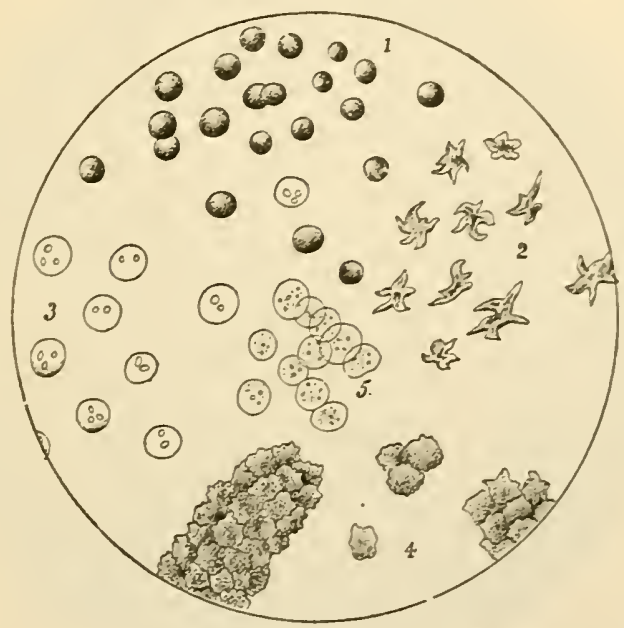

Fig. i4r.-Pus Corpuscles. (After Ulizmann.)

I, Normal; 2, showing amœboid movements; 3 , nuclei rendered distinct by acetic acid; 4 , as observed in chronic pyelitis; 5 , swollen by ammonium carbonate.

if a blue color is observed. Large numbers of pus corpuscles are present in the urinary sediment in gonorrhœa, leucorrhœa, chronic pyelitis, and in abscess of the kidney. In addition to the usual constituents found in leucocytes Mandel and Levene ${ }^{1}$ claim that pus cells contain glucolhionic acid. See Pus tests, page 43 r.

Casts.- These are cylindrical formations, which originate in the uriniferous tubules and are forced out by the pressure of the urine. They vary greatly in size, but in nearly every instance they possess parallel sides and rounded ends. The linding of casts in the urine is very important because of the fact that they gencrally indicale some kidney disorder; if albumin accompanies the casts the indication is

${ }^{1}$ Mandel and Levene: Biochemische Zcilschrift, 4. 75, 100;. 
much accentuated. Casts have been classified according to their microscopical characteristics as follows: $(a)$ hyaline, $(b)$ granular, $(c)$ epithelial, $(d)$ blood, $(e)$ fatty, $(f)$ waxy, $(g)$ pus.

(a) Hyaline Casts. - These are composed of a basic material which is transparent, homogeneous, and very light in color (Fig. I42). In fact, chiefly because of these physical properties, they are the most difficult form of renal casts to detect under the microscope. Frequently such casts are impregnated with deposits of various forms, such as erythrocytes, epithelial cells, fat globules, etc., thus rendering the form of the cast more plainly visible. Staining is often resorted to

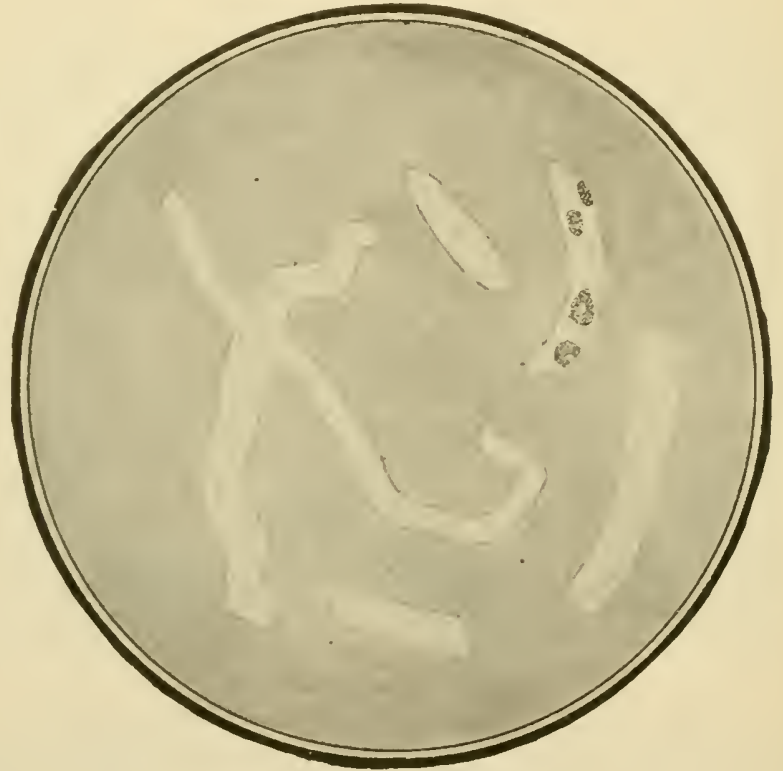

Fig. I 42.-Hyaline Casts.

One cast is impregnated with four renal cells.

in order to render the shape and character of the cast more easily determined. Ordinary iodine solution ( $\mathrm{I}$ in KI) may be used in this connection; many of the aniline dyes are also in common use for this purpose, e.g., gentian-violet, Bismarck-brown, methylene-blue, fuchsin, and eosin. Generally, but not always, albumin is present in urine containing hyaline casts. Hyaline casts are common to all kidney disorders, but occur particularly in the earliest and recovering stages of parenchymatous nephritis and interstitial nephritis.

(b) Gramular Casts.-The common hyaline material is ordinarily the basic substance of this form of cast. The granular material generally consists of albumin, epitheial cells, fat, or disintegrated erythrocytes or 
leucocytes, the character of the cast varying according to the nature and size of the granules (Fig. I43, and Fig. I44, page 470). Thus we have casts of this general type classified as finely granular and coarsely gramular casts. Granular casts, and in particular the finely granular types, occur in the sediment in practically every kidney disorder but are probably especially characteristic of the sediment in inflammatory disorders.

(c) Epithelial Casts.-These are casts bearing upon their surface epithelial cells from the lining of the uriniferous tubules (Fig. I45, page 470). The basic material of this form of cast may be hyaline or

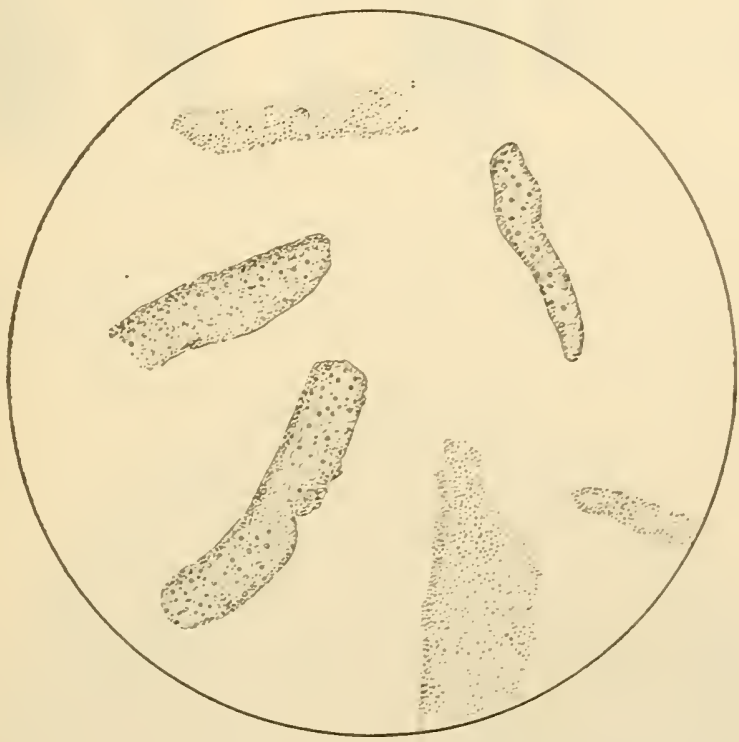

Fig. I43.-Granular Casts. (After Peyer.)

granular in nature. Epithelial casts are particularly abundant in the urinary sediment in acute nephritis.

(d) Blood Casts.-Casts of this type may consist of erythrocytes borne upon a hyaline or a fibrinous basis (Fig. 146, page 470). The occurrence of such casts in the urinary sediment denotes renal hemorrhage and they are considered to be especially characteristic of acute diffuse nephritis and acute congestion of the kidney.

(e) Fatty Casts.--Fatty casts may be formed by the deposition of fat globules or crystals of fatty acid upon the surface of a hyaline or granular cast (Fig. I47, page 47I). In order to constitute a true fatty cast the deposited material must cover the greater part of the surface area of the cast. The presence of fatty casts in urinary sediment in- 
dicates fatty degeneration of the kidney; such casts are particularly characteristic of subacute and chronic inflammation of the kidney.

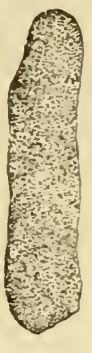

$a$

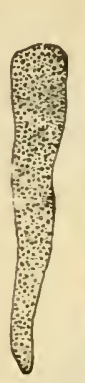

Fig. I 44.-Granular Casts.

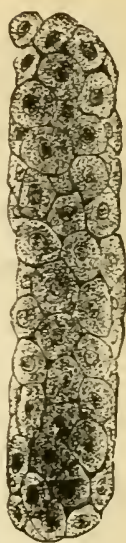

Fig. i 45.-Epithellal Casts.

a Finely granular; $b$, coarsely granular.

(f) Waxy Casts. - These casts possess a basic substance similar to that-which enters into the foundation of the hyaline form of cast. In

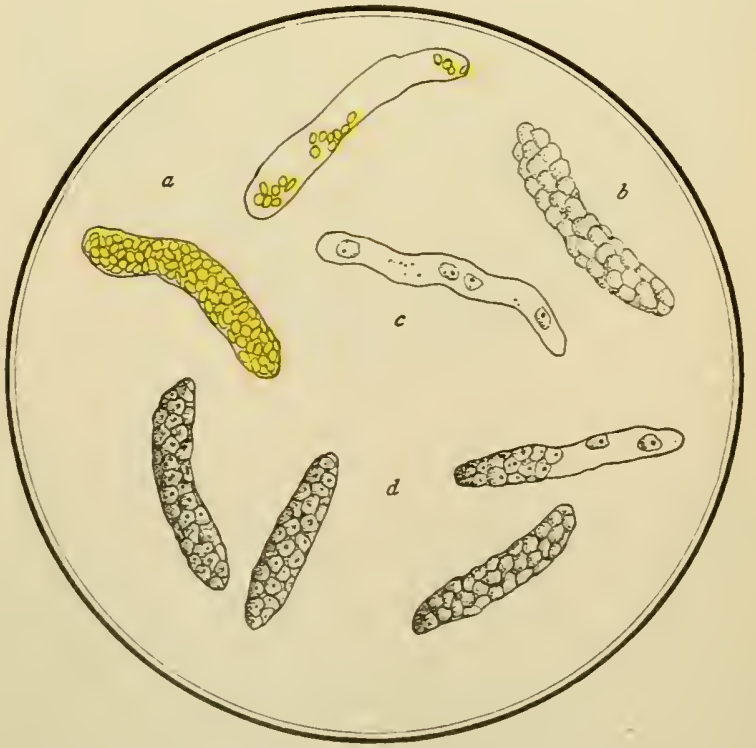

Fig. I 46.-Blood, Pus, Hyaldne and Epithellal Casts.

$a$, Blood casts; $b$, pus cast; $c$, hyaline cast impregnated with renal cells; $d$, epithelial casts.

common with the hyaline type they are colorless, refractive bodies, but differ from this form of cast in being, in general, of greater length and diameter and possessing sharper outlines and a light yellow color 


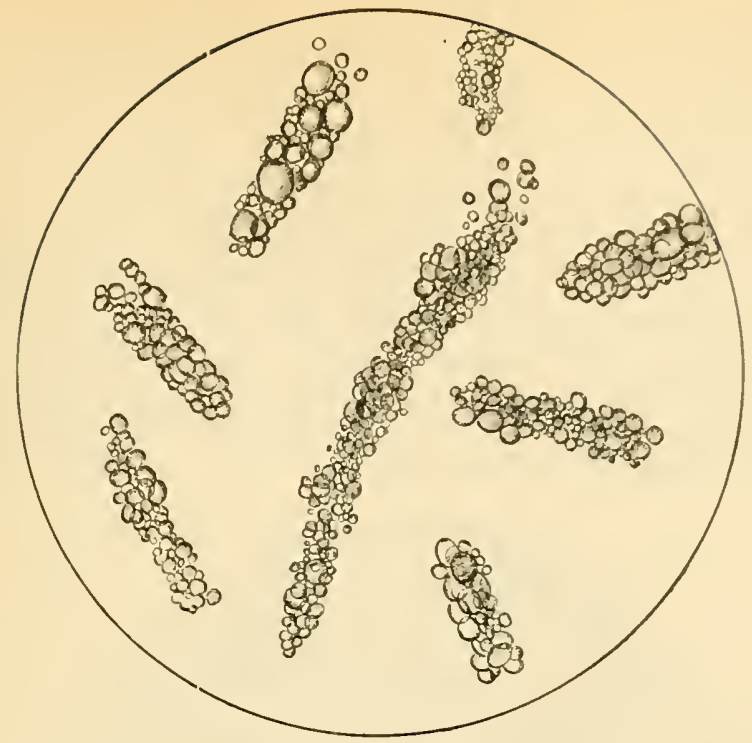

Fig. I 47.-Fatty Casts. (After Peyer.)

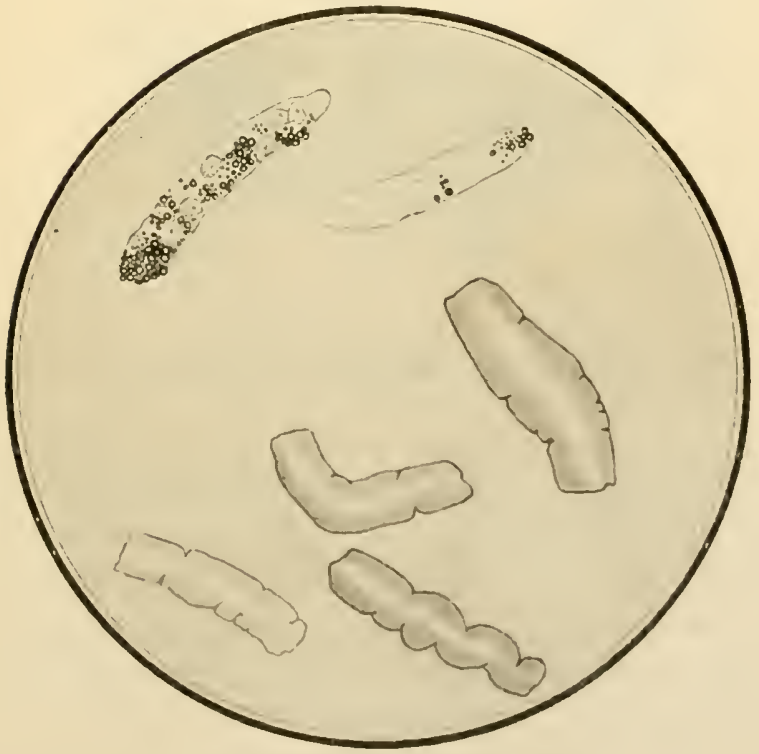

FIG. I48.-FATTY AND WIXY CASTS. $a$, Fatty casts; $b$, Waxy casts. 
(Fig. I48, page 47I). Such casts occur in several forms of nephritis, but do not appear to characterize any particular type of the disorder except amyloid disease, in which they are rather common.

(g) Pus Casts.-Casts whose surface is covered with pus cells or leucocytes are termed pus casts (Fig. I46, p. 470). They are frequently mistaken for epithelial casts. The differentiation between these two types is made very simple, however, by treating the cast with acetic acid which causes the nuclei of the leucocytes to become plainly visible. The true pus cast is quite rare and indicates renal suppuration.

Cylindroids.-These formations may occur in normal or pathological urine and have no particular clinical significance. They are frequently

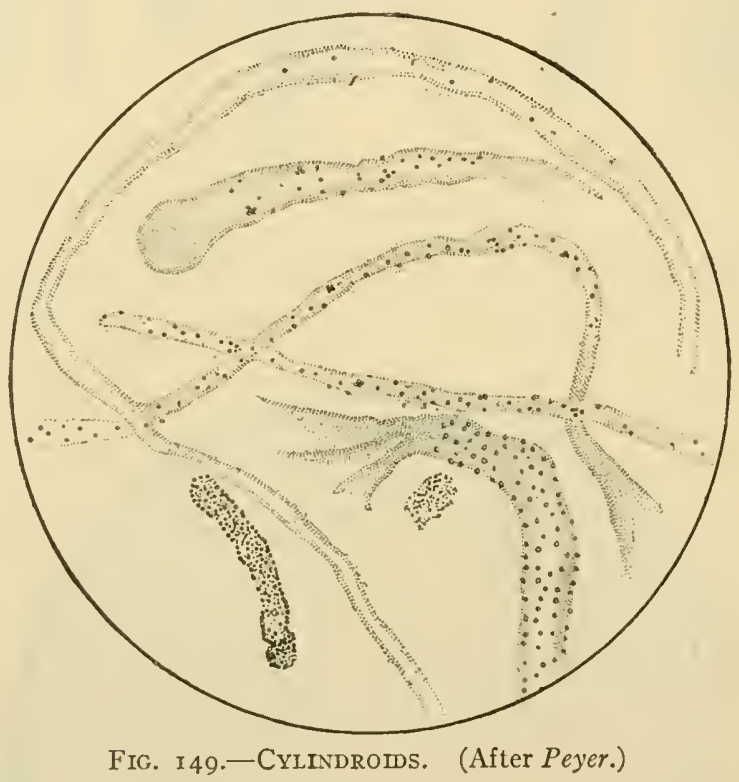

mistaken for true casts, especially the hyaline type, but they are ordinarily flat in structure with a rather smaller diameter than casts, may possess forked or branching ends, and are not composed of homogeneous material as are the hyaline casts. Such "false casts" may become coated with urates, in which event they appear granular in structure. The basic substance of cylindroids is often the nucleoprotein of the urine (Fig. I49, above).

Erythrocytes.-These form elements are present in the urinary sediment in various diseases. They appear as the normal biconcave, yellow erythrocyte (Plate IV, opposite page 249) or may exhibit certain modifications in form, such as the crenated type (Fig. I50) which is often seen in concentrated urine. Under different condi- 
tions they may become swollen sufficiently to entirely erase the biconcave appearance and may even occur in the form of colorless spheres having a smaller diameter than the original disc-shaped corpuscles. Erythrocytes are found in urinary sediment in hemorrhage of the kidney or of the urinary tract, in traumatic hemorrhage, hemorrhage from congestion, and in hemorrhagic diathesis.

Spermatozoa.-Spermatozoa may be detected in the urinary sediment in diseases of the genital organs, as well as after coitus, nocturnal emissions, epileptic, and other convulsive attacks, and sometimes in severe febrile disorders, especially in typhoid fever. In form they consist of an oval body, to which is attached a long, delicate tail (Fig.

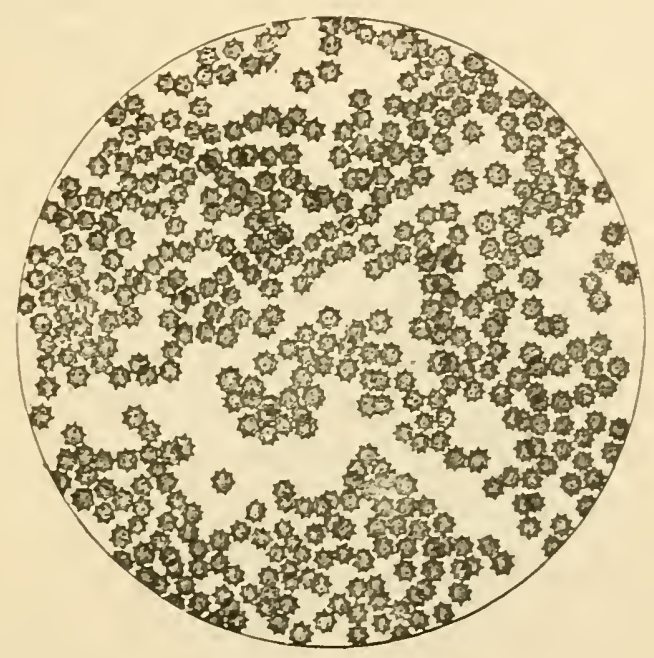

Fig. I 50.-Crenated ERythrocytes.

I5 I, page 474). Upon examination they may show motility or may be motionless.

Urethral Filaments.- These are peculiar thread-like bodies which are sometimes found in urinary sediment. They may occasionally be detected in normal urine and pathologically are found in the sediment in acute and chronic gonnorrhœa and in urethrorrhœa. The ground-substance of these urethral filaments is, in part at least, similar to that of the cylindroids (see page 472). The urine first voided in the morning is best adapted for the examination for filaments. These filaments may ordinarily be removed by a pipette since they are generally macroscopic.

Tissue Debris.-Masses of cells or fragments of tissue are frequently. found in the urinary sediment. They may be found in the sediment in tubercular affections of the kidney and urinary tract or in tumors of these organs. Ordinarily it is necessary to make a histological ex- 
amination of such tissue fragments before coming to a final decision as to their origin.

Animal Parasites.-The cysts, hooklets, and membrane shreds of echinococci are sometimes found in the urinary sediments. Other animal organisms which are more rarely met with in the urine are embryos of the Filaria sanguinis and eggs of the Distoma hematobium and Ascarides. Animal parasites in general occur most frequently in the urine in tropical countries.

Micro-organisms.--Bacteria as well as yeasts and moulds are frequently detected in the urine. Both the pathogenic and non-pathogenic forms of bacteria may occur. The non-pathogenic forms most frequently observed are micrococcus urea, bacillus urea, and staphylococcus urea liquefaciens. Of the pathogenic forms many have been

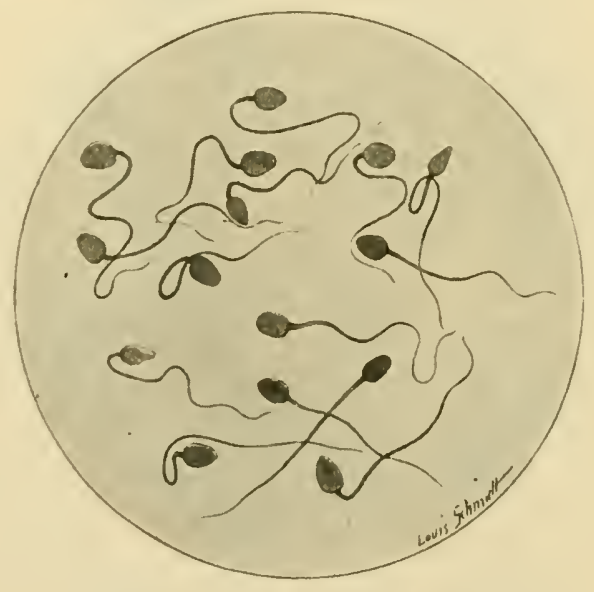

Fig. i 5i.-Humax Spermatozoa.

observed, e.g., Bacterium Coli, typhoid bacillus, tubercle bacillus, gonococcus, bacillus pyocyaneus, and proteus vulgaris. Yeast and moulds are most frequently met with in diabetic urine.

Fibrin.-Following hematuria, fibrin clots are occasionally observed in the urinary sediment. They are generally of a semi-gelatinous consistency and of a very light color, and when examined under the microscope they are seen to be composed of bundles of highly refractive fibers which run parallel.

Foreign Substances Due to Contamination.-Such foreign substances as fibers of silk, linen, or wool; starch granules, hair, fat, and sputum, as well as muscle fibers, vegetable cells, and food particles, are often found in the urine. Care should be taken that these foreign substances are not mistaken for any of the true sedimentary constituents already mentioned. 


\section{CHAP'TER XXV}

\section{URINE : CALCULI}

URINARY calculi, also called concretions, or concrements are solid masses of urinary sediment formed in some part of the urinary tract. They vary in shape and size according to their location, the smaller calculi, termed sand or gravel, in general arising from the kidney or the pelvic portion of the kidney, whereas the large calculi are ordinarily formed in the bladder. There are two general classes of calculi as regards composition, i.e., simple and compound. The simple form is made up of but a single constituent, whereas the compound type contains two or more individual constituents. The structural plan of most calculi consists of an arrangement of concentric rings about a central nucleus, the number of rings frequently being dependent upon the number of individual constituents which enter into the structure of the calculus. However, layers quite different in macroscopical appearance may be almost identical in composition. In case two or more calculi unite to form a single calculus the resultant body will obviously contain as many nuclei as there were individual calculi concerned in its construction. Under certain conditions the growth of a calculus will be principally in only one direction, thus preventing the nucleus from maintaining a central location. The qualitative composition of urinary calculi is dependent, in great part, upon the reaction of the urine, e.g., if the reaction of the urine is acid the calculi present will be composed, in great part at least, of substances that are capable of depositing in acid urine, e.g., uric acid, urates and calcium oxalates.

According to Ultzmann, out of 545 cases of urinary calculus, uric acid and urates formed the nucleus in about SI per cent of the cases; earthy phosphates in about 9 per cent; calcium oxalate in about o per cent; cystine in something over $x$ per cent, while in about 3 per cent of the cases some foreign body comprised the nucleus.

More recent analyses ${ }^{1}$ of twenty-four calculi showed the nucleus in 75 per cent of them to be calcium oxalate (6o per cent) and in 25 per cent to be phosphate ( 56 per cent). All of the calculi contained some uric acid and urates, but only three gave more than so per cent.

${ }^{1}$ Kahn and Rosenbloom: Jour. A m. . Med. Ass'n, 59, 2252, 19 r 3. 
In the chemical examination of urinary calculi the most valuable data are obtained by subjecting each of the concentric layers of the calculus to a separate analysis. Material for examination may be conveniently obtained by sawing the calculus carefully through the nucleus, then separating the various layers, or by scraping off from each layer (without separating the layers) enough powder to conduct the examination as outlined in the scheme (see page 477 ).

\section{Varieties of Calculus}

Uric Acid and Urate Calculi.-Uric acid and urates constitute the nuclei of a large proportion of urinary concretions. Such stones are always colored, the tint varying from a pale yellow to a brownish-red. The surface of such calculi is generally smooth but it may be rough and uneven.

Phosphatic Calculi.-Ordinarily these concretions consist principally of "triple phosphate" and other phosphates of the alkaline earths, with very frequent admixtures of urates and oxalates. The surface of such calculi is generally rough but may occasionally be rather smooth. The calculi are somewhat variable in color, exhibiting gray, white, or yellow tints under different conditions. When composed of earthy phosphates the calculi are characterized by their friability.

Calcium Oxalate Calculi.-This is the hardest form of calculus to deal with, and is rather difficult to crush. They ordinarily occur in two general forms, i.e., the small, smooth concretion which is characterized as the hemp-seed calculus, and the medium-sized or large stone possessing an extremely uneven surface, which is generally classed as a mulberry calculus. This roughened surface of the latter form of calculus is due, in many instances, to protruding calcium oxalate crystals of the octahedral type.

Calcium Carbonate Calculi.-Calcium carbonate concretions are quite common in herbivorous animals, but of exceedingly rare occurrence in man. They are generally small, white, or grayish calculi, spherical in form and possess a hard, smooth surface.

Cystine Calculi.--The cystine calculus is a rare variety of calculus. Ordinarily they occur as small, smooth, oval, or cylindrical concretions which are white or yellow in color and of a rather soft consistency.

Xanthine Calculi.-This form of calculus is somewhat more rare than the cystine type. The color may vary from white to brownishyellow. Very often uric acid and urates are associated with xanthine in this type of calculus. Upon rubbing a xanthine calculus it has the property of assuming a wax-like appearance. 
On Heating the Powder on Platinum Foil, It

Does not burn

The powder when treated with HCl

Joes not effervesce

The powder gently heated, then treated with $\mathrm{HCl}$

The powder when moistened with a little $\mathrm{KOHI}$

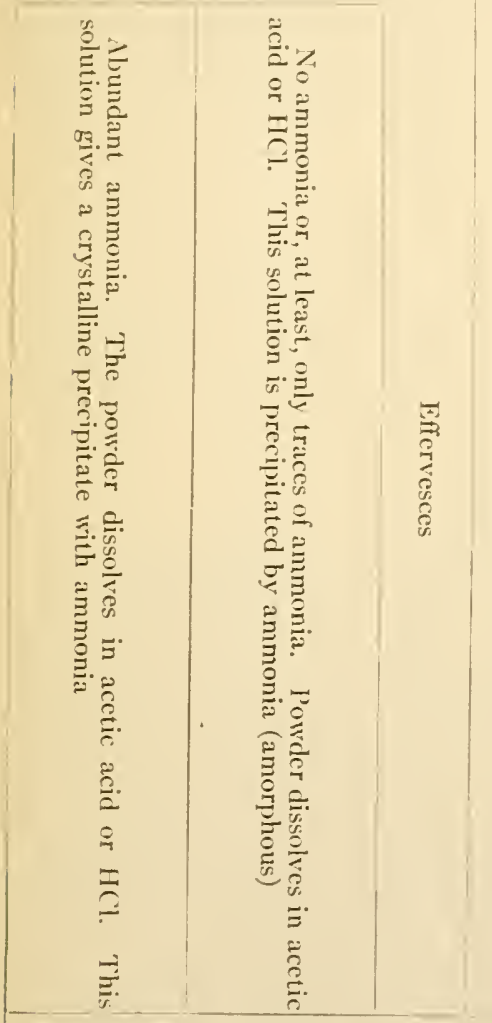

With flame

Docs burn

Without flame
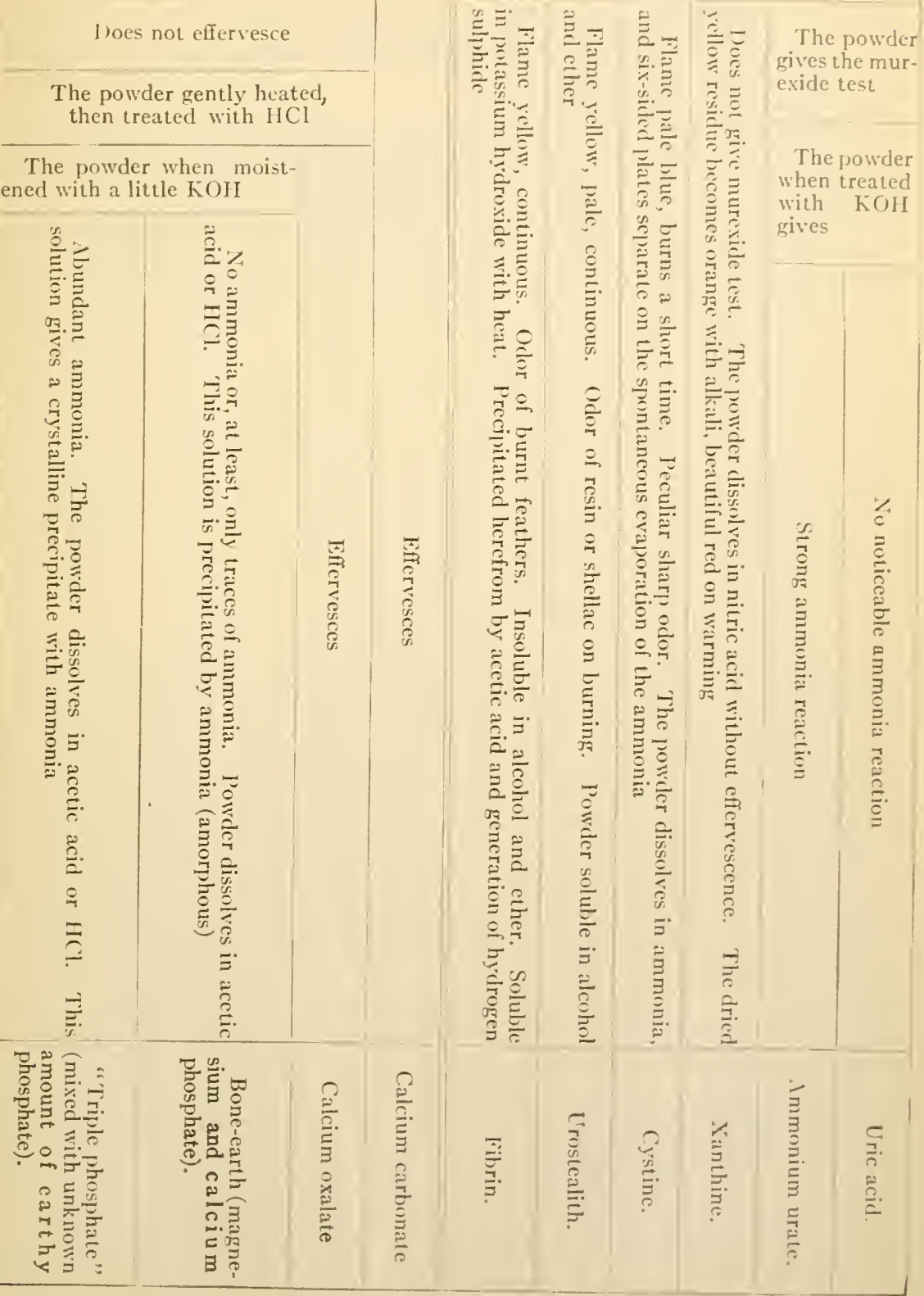
Urostealith Calculi.-This form of calculus is extremely rare. Such concretions are composed principally of fat and fatty acid. When moist they are soft and elastic, but when dried they become brittle. Urostealiths are generally light in color.

Fibrin Calculi.-Fibrin calculi are produced in the process of blood coagulation within the urinary tract. They frequently occur as nuclei of other forms of calculus. They are rarely found.

Cholesterol Calculi.-An extremely rare form of calculus somewhat resembling the cystine type.

Indigo Calculi.-Indigo calculi are extremely rare, only two cases having been reported. One of these indigo calculi is on exhibition in the museum of Jefferson Medical College of Philadelphia.

The scheme, proposed by Heller and given on page 477 , will be found of much assistance in the chemical examination of urinary calculi. 


\section{CHAPTER XXVI}

\section{URINE : QUANTITATIVE ANALYSIS}

IN analyzing a normal or pathological urine quantitatively for any of its constituents it is particularly necessary that the complete and exact 24-hour sample be obtained. For directions with regard to the collection and preservation of urine for analysis see Chapter XXI on General Characteristics of Normal and Pathological Urine. Methods for the determination of the specific gravity of the urine are also there described. Before any urine is taken for analysis its total volume should be measured, using a large graduated cylinder, and this volume is thereafter taken as a basis for the calculations of the daily output of the individual constituents determined. If the urine be pathological it is of course necessary to precede its quantitative analysis by qualitative tests for the pathological constituents.

\section{Acidity by Titration}

Folin's Method.-Principle.-The urine is titrated with standard sodium hydroxide solution, using phenolphthalein as an indicator. Potassium oxalate is added to precipitate the calcium which would otherwise interfere with the end-point due to the precipitation of calcium phosphate on neutralization of the urine. The acidity of the urine as determined in this way is not a correct measure of the true acidity, which is dependent upon the concentration of hydrogen ions. The results obtained do, however, ordinarily show a certain parallelism with the hydrogen ion concentration and are of value for comparative purposes.

Procedure.-Place 25 c.c. of urine in a 200 c.c. Erlenmeyer flask and add 15-20 grams of finely pulverized potassium oxalate and I-2 drops of a I per cent phenolphthalein solution to the fluid. Shake the mixture vigorously for $\mathrm{x}-2$ minutes and titrate it immediately with $\mathrm{N} /$ Io sodium hydroxide until a faint but unmistakable pink remains permanent on further shaking. Take the burette reading and calculate the acidity of the urine under examination.

Calculation.-If y represents the number of cubic centimeters of $\mathrm{N}$ Io sodium hydroxide used and $y^{\prime}$ represents the volume of urine excreted in 24 hours, the total acidity of the 24-hour urine specimen may be calculated by means of the following proportion : 
$25: y:: y^{\prime}: x$ (acidity of 24 -hour urine expressed in cubic centimeters of $N / 10$ sodium hydroxide).

Each cubic centimeter of $\mathrm{N} /$ ro sodium hydroxide contains 0.004 gram of sodium hydroxide, and this is equivalent to 0.0063 gram of oxalic acid. Therefore, in order to express the total acidity of the 24 -hour urine specimen in equivalent grams of sodium hydroxide, multiply the value of $x$, as just determined, by 0.004 , or multiply the value of $x$ by 0.0063 if it is desired to express the total acidity in grams of oxalic acid.

Interpretation--(Under the heading "Interpretation" there will be found, in connection with the various quantitative methods which follow, brief notes as to the possible significance of the results obtained. For some further points (and reference to literature) see the chapters on the Normal and Pathological Constituents of Urine and on Metabolism. Consult text-books on physiological chemistry and clinical diagnosis for complete discussion). The acidity of the urine expressed in cubic centimeters $\mathrm{N} / \mathrm{ro}$ alkali required to neutralize the 24 -hour output varies ordinarily from 200 to 500 under normal conditions with an average of perhaps 350 . It is dependent almost entirely upon the diet, being low on a vegetable (base-forming diet) and high on a diet containing much meat, rice, whole wheat products, fruits containing benzoic acid, as prunes and cranberries, etc. (acidforming foods). On the administration of 15 grams of sodium bicarbonate it may go down to 100 ; the ingestion of much acid-forming food may increase it to 600 . In fasting it may rise in a few days to 800 . It must be borne in inind that acidities of less than 250 usually indicate a true alkalinity of the urine inasmuch as phenolphthalein changes in an alkaline solution. Samples of urine collected shortly after a meal may be alkaline due to the so-called "alkaline tide."

Bacterial decomposition of the urea of the urine occurring in the urinary tract will increase the amount of ammonia and decrease the acidity of the urine. The same change usually occurs in urine left in contact with the air. The acidity of the urine is increased in acidosis, cardio-renal and certain other disorders. The acidity of the urine may be somewhat increased by administration of mineral acids, acid phosphates, or benzoates, but it is much more difficult to increase than to decrease this acidity.

\section{Hydrogen Ion Concentration or True Acidity}

Indicator Method (Henderson and Palmer's Adaptation of Sörensen's Method). ${ }^{1-P r i n c i p l e .-T h e ~ r e a c t i o n ~ o f ~ t h e ~ u r i n e ~ i s ~ e s t i m a t e d ~}$

'Henderson and Palmer: Jour. Biol. Chem., I3, 393, I9I3. 
by matching the colors produced when a few drops of indicator are added respectively to the diluted urine and to standard solutions of known reaction similarly diluted. Similar hydrogen ion concentrations are indicated by similar colors. The indicator must be properly' chosen.

Standard Solutions. - A series of standard solutions of known hydrogen ion concentration must be prepared. 'The solutions as indicated in Table I (page 482) are satisfactory for urine analysis. The table also indicates the $\mathrm{H}$ ion concentration of each solution, the figure given being the logarithm of this concentration $\left(\mathrm{P}_{\mathrm{H}+}\right)$. It is more convenient and rational to express the concentration by this logarithmic notation. True $\mathrm{H}$ ion concentrations corresponding to the logarithmic figures are given in Table II (page 482 ).

The I 3 solutions indicated are made up by mixing equal volumes of their ingredient solutions of the composition indicated. Solutions 4 to $\mathrm{I}_{2}$ are all that are ordinarily required as the normal urinary $\mathrm{H}$ ion concentrations lie between 4.80 and 7.50 and pathological variations are usually within these limits. The mean normal value is almost exactly 6.00 .

Procedure.-Select eleven 250 c.c. flasks of good glass and indistinguishable in color and form. Into each of ten of these introduce Io c.c. of the various standard solutions. Make up to 250 c.c. with distilled water and add to each exactly the same amount of an aqueous solution of sodium alizarine sulphonate (10-r 5 drops). Mix well by inverting. Introduce ro c.c. of the urine to be tested into a similar 250 c.c. flask, dilute and add indicator in exactly the same way as before. Match the color of the diluted urine solution with one of the standard solutions. By consulting Table II (page 482) determine to what $\mathrm{H}$ ion concentration this corresponds. This table points out the indicators to be used for different ranges of acidity. From 5.3-6.7 p-nitrophenol is satisfactory and is used in the same way as alizarin except that it must be present in concentration of 0.08 per cent. Neutral red is used in the same way for acidities from $6.7-7.5$ about I.5 c.c. of the I per cent solution being required. For acidities greater than 5.5 methyl red is used in the following way: Io c.c. portions of the standard solutions are introduced into carefully selected colorless test-tubes and ro c.c. of urine is introduced into another tube. The standard solutions are then colored to match the urine by the addition of small amounts of $p$-nitrophenol, methyl orange, alizarine or bismark brown. Then to standard solutions and urine add o.15 c.c. of a saturated solution in 50 per cent alcohol, of methyl red and match the colors. For concentrations of $7 \cdot 5^{-9.27}$ or less undiluted urine is matched in test-tubes against undiluted standard solutions, using phenolphthalein as an indicator (without previous coloration of standard solution). In all cases estimations are made in duplicate. 
TABLE I

\begin{tabular}{|c|c|c|c|c|c|}
\hline No. & $\mathrm{NaH}_{2} \mathrm{PO}_{4}$ & $\mathrm{Na}_{2} \mathrm{HPO}_{4}$ & $P_{H+}$ & Indicat & \\
\hline $\begin{array}{l}I \\
2\end{array}$ & $0.0001 \mathrm{~N}$ & $\begin{array}{l}0.1000 \mathrm{~N} \\
0.0480 \mathrm{~N}\end{array}$ & $\begin{array}{l}9.27 \\
8.7\end{array}$ & Phenolphthalein & \\
\hline 3 & $0.0001 \mathrm{~N}$ & $0.0120 \mathrm{~N}$ & 8.0 & & \\
\hline 4 & $0.0166 \mathrm{~N}$ & $0.0833 N$ & $7 \cdot 48$ & Neutral red & \\
\hline $\begin{array}{l}5 \\
6\end{array}$ & $0.0010 \mathrm{~N}$ & $0.0060 N$ & $7 \cdot 38$ & & \\
\hline & $0.0010 \mathrm{~N}$ & $0.0023 \mathrm{~N}$ & 6.90 & & \\
\hline & $\mathrm{CH}_{3} \mathrm{COOH}$ & $\mathrm{CH}_{3} \mathrm{COONa}$ & & & \\
\hline 7 & $0.0009 \mathrm{~N}$ & $0.0920 \mathrm{~N}$ & $6.70)$ & & Sodium alizar \\
\hline 9 & $0.0023 N$ & & $\begin{array}{l}6.30 \\
6.00\end{array}$ & & suiphonate \\
\hline 2 & $\begin{array}{l}0.0040 \geqslant \\
0.0092\end{array}$ & $\begin{array}{l}0.0920 \mathrm{~N} \\
0.0920 \mathrm{~N}\end{array}$ & 5.70 & p-Nitrophenol & \\
\hline I & $0.0230=$ & $0.0920 \mathrm{~N}$ & 5.30 & & \\
\hline I & $0.0460 \mathrm{~N}$ & $0.0920 \mathrm{~N}$ & 4.90 & Methyl red & \\
\hline I & $0.0920 \mathrm{~N}$ & $0.0920 \mathrm{~N}$ & $4 \cdot 70$ & & \\
\hline & & & BLE II & & \\
\hline Log & & $\stackrel{+}{\mathrm{H}}$ & & Log & $\stackrel{+}{\mathrm{H}}$ \\
\hline 4.6 & & $250 \times 10^{-7}$ & & 6.4 & $4.0 \times 10^{-7}$ \\
\hline $\begin{array}{l}4.8 \\
5.0\end{array}$ & & $\begin{array}{l}160 \times 10^{-7} \\
100 \times 10^{-7}\end{array}$ & & 6.6 & $2.5 \times 10^{-7}$ \\
\hline 5.2 & & $\begin{array}{r}100 \times 10^{-7} \\
63 \times 10^{-7}\end{array}$ & & 6.8 & I. $6 \times 10^{-7}$ \\
\hline $5 \cdot 4$ & & $\begin{array}{l}03 \times 10^{-7} \\
40 \times 10^{-7}\end{array}$ & & $\begin{array}{l}7.0 \\
7.2\end{array}$ & $\begin{array}{l}1.0 \times 10^{-7} \\
0.63 \times 10^{-7}\end{array}$ \\
\hline 5.6 & & $25 \times 10^{-7}$ & & $\begin{array}{l}7.2 \\
7.4\end{array}$ & $0.40 \times 10^{-7}$ \\
\hline 5.8 & & $16 \times 10^{-7}$ & & 7.6 & $0.25 \times 10^{-7}$ \\
\hline 6.0 & & $10 \times 10^{-7}$ & & 7.8 & $0.16 \times 10^{-7}$ \\
\hline 6.2 & & $6.3 \times 10^{-7}$ & & 8.0 & $0.10 \times 10^{-7}$ \\
\hline
\end{tabular}

Interpretation.- The $\mathrm{H}$ ion concentration of the urine is influenced by the same factors as the titratable acidity (see page 480). The normal values lie between 4.80 and 7.50 with a mean value of almost exactly 6.00. For vegetarians the mean value is about 6.64. In cardio-renal disorders the mean is 5.3 . In most pathological conditions the hydrogen ion concentration is increased.

\section{Total Solids}

I. Drying Method.-Place 5 c.c. of urine in a weighed shallow dish, acidify very slightly with acetic acid ( $\mathrm{I}-3$ drops), and dry it in vacuo in the presence of sulphuric acid to constant weight. Calculate the percentage of solids in the urine sample and the total solids for the 24 -hour period.

Interpretation. - The average excretion of total solids by a normal adult man is about 70 grams. It is largely dependent upon the protein and salts of the diet. It may be decreased in severe nephritis due to impaired excretion, and greatly increased in diabetes with high sugar elimination.

Practically all the methods the technic of which includes evaporation at an increased temperature, either under atmospheric conditions or in vacuo, are attended with error.

Shackell's method ${ }^{1}$ which entails the vacuum desiccation of the frozen sample is extremely satisfactory and should be used in all biological work where the greatest accuracy is desired.

${ }^{1}$ Shackell: American Journal of Physiology, 24, 325, 1909 
2. Calculation by Long's Coefficient. - The quantity of solid material contained in the urine excreted for any 24-hour period may be approximately computed by multiplying the second and third decimal figures of the specific gravity by 2.6. This gives us the number of grams of solid malter in I liler of urine. From this value the total solids for the 24 -hour period may easily be determined.

Calculation.- - If the volume of urine for the 24 hours was I I 20 C.c. and the specific gravity I.OIS, the calculation would be as follows:

(a) $\mathrm{I} 8 \times 2.6=46.5$ grams of solid matter in I liter of urine.

(b) ${ }_{\text {IOco }}^{46.8 \times \text { I } 20}=52.4$ grams of solid matter in I I 20 c.c. of urine.

Long's coefficient was determined for urine whose specific gravity was taken at $25^{\circ} \mathrm{C}$. and is probably more accurate, for conditions obtaining in America, than the older coefficient of Haeser, 2.33 .

Interpretation.-See above.

\section{Total Nitrogen}

I. Kjeldahl Method. ${ }^{1-P r i n c i p l e .-T h e ~ p r i n c i p l e ~ o f ~ t h i s ~ m e t h o d ~ i s ~}$ the conversion of the various nitrogenous bodies of the urine into ammonium sulphate by boiling with concentrated sulphuric acid, the subsequent decomposition of the ammonium sulphate by means of a fixed alkali $(\mathrm{NaOH})$ and the collection of the liberated ammonia in an acid of known strength. Finally, this partly neutralized acid solution is titrated with an alkali of known strength and the nitrogen content of the urine under examination computed.

Procedure.-Place 5 c.c. of urine in a 500 c.c. longnecked Jena glass Kjeldahl flask, add 20 c.c. of concentrated sulphuric acid and about 0.2 gram of copper sulphate

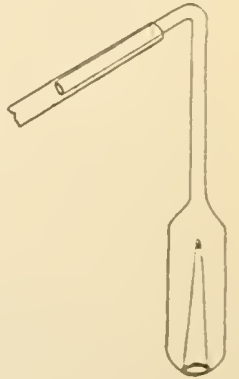

FIG. 152,-FOLIN FUME ABSORBER. and boil the mixture for some time after it is colorless (aboutjone hour). If a suitable hood or fume chamber is not available the sulphuric acid vapors may be carried away by suction. Connect the outlet tube of a 2-3 liter wash bottle filled with caustic soda solution with a suction pump. The inlet tube is connected with a Folin fume absorption tube such as illustrated in Fig. 152 . If such a tube is not at hand a small funnel may be attached. The absorption tube is placed loosely over the mouth of the digestion flask and a constant current of air drawn through the apparatus.

Allow the flask to cool and dilute the contents with about 200 c.c. of ammonia-free water. Add a little more of a concentrated solution of $\mathrm{NaOH}$ than is necessary to neutralize the sulphuric $\mathrm{acid}^{2}$ and introduce into the flask a little coarse pumice stone or a few pieces of granulated zinc, ${ }^{3}$ to prevent bumping, and a

1 There are numerous modifications of the original $\mathrm{K}$ jeldahl method; the one described here, however, has given excellent satisfaction and is recommended for the determination of the nitrogen content of urine.

2 This concentrated sodium hydroxide solution should be prepared in quantity and "check" tests made to determine the volume of the solution necessary to neutralize the volume (2o c.c.) of concentrated sulphuric acid used.

Powdered zinc may be substituted. 
small piece of paraffin to lessen the tendency to froth. By means of a safetytube connect the flask with a condenser so arranged that the delivery-tube passes into a vessel containing a known volume (the volume used depending upon the nitrogen content of the urine) of $\mathrm{N} /$ Io sulphuric acid, using care that the end of the delivery-tube reaches beneath the surface of the fluid. ${ }^{1}$ Mix the contents of the distillation flask very thoroughly by shaking and distil the mixture until its volume has diminished about one-half. Titrate the partly neutralized $\mathrm{N} /$ Io sulphuric acid solution by means of $\mathrm{N} /$ Io sodium hydroxide, using congo red as indicator, and calculate the content of nitrogen of the urine examined.

Calculation.- Subtract the number of cubic centimeters of $\mathrm{N} /$ ro sodium hydroxide used in the titration from the number of cubic centimeters of $\mathrm{N} / \mathrm{ro}$ sulphuric acid taken. The remainder is equivalent to the number of cubic centimeters of $\mathrm{N} /$ Io sulphuric acid, neutralized by the ammonia of the urine. One c.c. of $\mathrm{N} /$ Io sulphuric acid is equivalent to $0.0014 \mathrm{gram}$ of nitrogen. Therefore, if $y$ represents the volume of urine used in the determination, and $y^{\prime}$ the number of cubic centimeters of $\mathrm{N} /$ ro sulphuric acid neutralized by the ammonia of the urine, we have the following proportion:

$y: 100:: y^{\prime} \times 0.0014: x$ (percentage of nitrogen in the urine examined).

Calculate the quantity of nitrogen in the 24 -hour urine specimen.

Interpretation. - An adult of medium size on a mixed diet will usually excrete I2-I 8 grams of nitrogen per day. It varies, however, almost directly with the protein ingestion and hence usually runs parallel to the excretion of urea (see page 493). In a normal adult the total nitrogen of the feces and of the urine will often be almost exactly equal to the total nitrogen of the food. Such a condition is called "nitrogen equilibrium." The feces usually contain very little nitrogen. (See also Ammonia, Creatinine, etc.)

Calculation of Percentage Nitrogen Distribution.-In modern metabolism studies where the various forms of nitrogen are determined, in addition to the total nitrogen as yielded by the Kjeldahl method, it is customary to indicate what portion of the total nitrogen was present in the form of each of the individual nitrogenous constituents. These percentage values are secured by dividing the weight (grams) of nitrogen excreted for the day in the form of each individual nitrogenous constituent by the weight of the total nitrogen output for the same period. For example, if the total nitrogen excretion is $9.8 \mathrm{I} 4$ grams and the excretion of urea-nitrogen is 8.520 grams and the excretions of nitrogen in the forms of ammonia and creatinine are $0.27 \mathrm{I}$ gram and 0.639 gram respectively, the percentage distribution for these forms of nitrogen would be calculated as follows:

8.520 grams urea-nitrogen $\div 9.814$ grams total nitrogen $=84.3$ per cent

$0.27 \mathrm{I}$ gram ammonia-nitrogen $\div 9.8 \mathrm{I} 4$ grams total nitrogen $=2.7$ per cent

0.639 gram creatinine-nitrogen $\div 9.814$ grams total nitrogen $=6.5$ per cent

1 This delivery-tube should be of large caliber in order to avoid the "sucking back" of the fluid. 
Nitrogen Partition in Urines Containing Albumin.--If the urine to be tested contains albumin this must be removed before an attempt at a nitrogen partition is made. This may be done by heating to boiling, acidifying with acetic acid to coagulate the protein, filtering and making up the filtrate to the original volume of the urine. If very small amounts of albumin are present this is attended with difficulty. In these cases Tracy and Welker ${ }^{1}$ have suggested the use of aluminium hydroxide cream. It apprently removes none of the nitrogenous constituents of normal urine.

Procedure.-One liter of urine (containing not over I per cent of albumin) is mixed with one liter of aluminium cream $^{2}$ and filtered.

2. Folin-Farmer Microchemical Method. ${ }^{3}$-Principle.-This method belongs with the so-called microchemical methods inasmuch as it is adapted to the determination of amounts of nitrogen in the neighborhood of I mg. while in the ordinary Kjeldahl procedure $30-100 \mathrm{mg}$. of nitrogen are generally manipulated. One c.c. of diluted urine is decomposed with sulphuric acid as in the Kjeldahl method, the ammonia formed is set free by the addition of alkali and carried over into an acid solution by means of a current of air. The ammonia solution is then treated with the Nessler-Winkler reagent and the color produced compared with that of a standard solution of an ammonium salt treated in the same way.

Colorimeter.-For this method as well as for a number of other methods commonly used in urinary and blood analysis an instrument known as a colorimeter is required. Through its aid we are able accurately to measure the respective depths of color in two solutions and hence to calculate the comparative amounts of substances which form colored compounds in a quantitative manner. The most satisfactory instrument for this purpose is the Duboscq colorimeter (see Fig. $\mathrm{r}_{53}$, page 486). This enables the two colored solutions to be compared in the same optical field and with a degree of accuracy of about $r$ per cent. The later type of the Duboscq colorimeter with cylinders instead of prisms movable is to be preferred, particularly as this type may be readily adapted to the comparison of cloudy solutions or suspensions, the instrument thus modified being called a nephelometer (see Fig. $S_{3}$, page 29r). In this later form of colorimeter the depths of the colored

${ }^{1}$ Tracy and Welker: Jour. Biol. Chem. 22, 55, r915; For other applications of aluminium hydroxide precipitation of colloids, sec Welker and Marshall, J. A m. Chem. Soc., $25,820,1913$.

${ }^{2}$ Aluminium Hydroxide Cream.-To a I per cent solution of ammonium alum at room temperature add a slight excess of a I per cent solution of ammonium hyclroxide. II ash by decantation until the wash water shows only the faintest trace of residue on evaporation. Stronger solutions should not be used.

${ }^{3}$ Folin and Farmer: Jour. Biol. Chem., I I, 493, I912. 
solutions through which the light passes are regulated by raising or lowering the cups and are accurately indicated in millimeters on a vernier scale at the back of the instrument. The standard solution is placed at any convenient depth and the color of the solution to be examined is matched with it by raising or lowering cups. When the color is of the same intensity as the standard the depth of the solution is read. The amounts of the colored substance in solution are inversely proportional to the depths of the columns of fluid. Thus if the standard is set at Io $\mathrm{mm}$. and the solution under examination has the same color

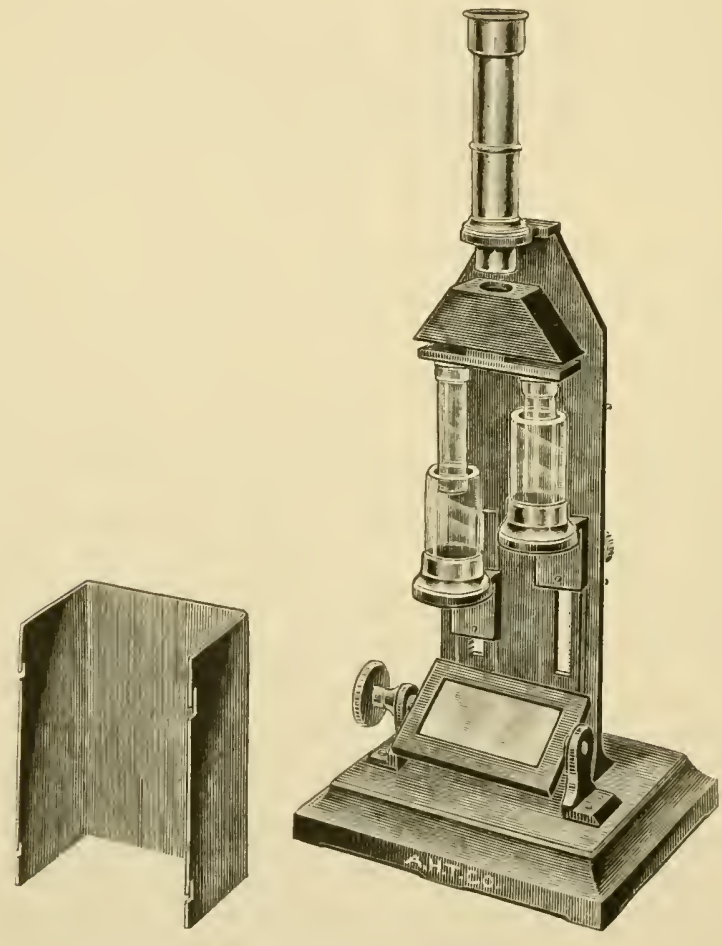

Fig. I53.-DuboscQ Colorimeter.

density at $20 \mathrm{~mm}$. the latter has just one-half the concentration of the standard.

A large number of other colorimeters have been devised and may be used in place of the Duboscq. Most of these though less expensive than this instrument are also less accurate. The Hellige colorimeter has been recommended, particularly for clinical determinations by Myers and Fine. ${ }^{1}$ Kober has devised a combined colorimeter and nephelometer which may be obtained in this country (see page 293). For merely approximate determinations the color

${ }^{1}$ Myers and Fine: Posl-graduate, I914-19r 5. 
comparisons may be made directly with a series of colored standards of varying strengths made up in exactly similar test-tubes or small flasks.

Procedure.-Introduce 5 c.c. of urine into a 50 c.c. volumetric flask if the specific gravity of the urine is over ror 8 , or into a 25 c.c. flask if the specific gravity is less than ror $8 .^{1}$ Fill the flask to the mark with distilled water and invert it several times in order to guarantee thorough mixing. Transfer I c.c. ${ }^{2}$ of the diluted urine to a large $(20-25 \mathrm{~mm} . \times 200 \mathrm{~mm}$.) Jena-glass test-tube. Add to this I c.c. of concentrated sulphuric acid, I gram of potassium sulphate, I drop of 5 per cent copper sulphate solution and a small, clean, quartz pebble or

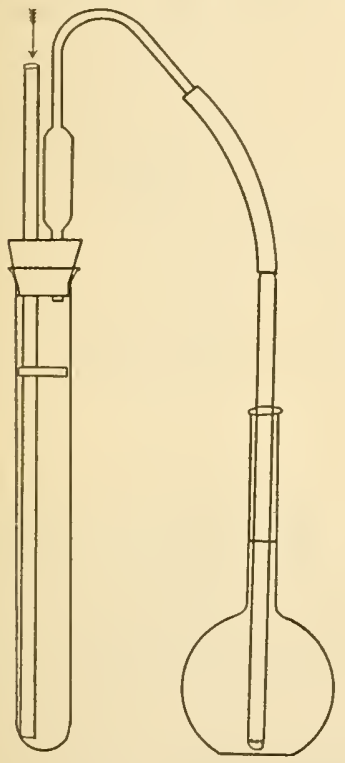

FIG. I54.

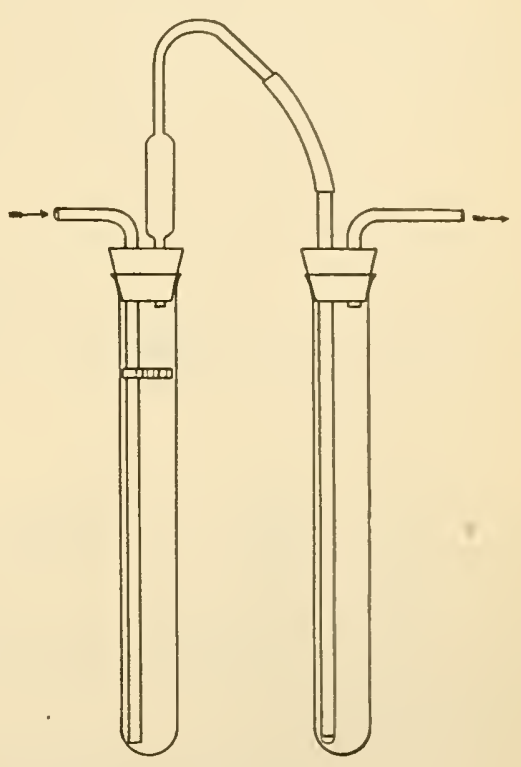

F1G. 155 .

Figs. I54 AND I55.-Forms of Apparatus USEd in Methods of Folix AND ASSOCiates for Determination of Total Nitrogex, Urea and AmMonia. (From Jour. Biol. Chem. vol. II, I9I2.)

glass bead. (The pebble or bead is added to prevent bumping.) Boil the mixture over a micro-burner ${ }^{3}$ for about six minutes, i.e., about two minutes after the mixture has become colorless. Allow to cool until the digestion mixture begins to become viscous. This ordinarily takes about three minutes, but in any event the mixture must not be permitted to solidify. Add about 6 c.c. of water (a few drops at a time, at first, then more rapidly) to prevent solidification. To this acid solution add an excess of sodium hydroxide ( 3 c.c. of a saturated solution is sufficient) and aspirate the liberated ammonia by means of a rapid air current

1 The purpose is to so dilute the urine that I c.c. of the diluted fluid shall contain $0.75^{-}$ I.5 mg. of nitrogen.

3 This measurement should be made by means of a modified Ostwald pipette (see Ostwald-Luther: Physiko-Chemische Messungen, 2d. ed., p. 135). Such pipettes may be obtained from Eimer and Amend, New York.

3 type of burner which has proven satisfactory is Eimer and Anend's No. 2587.

- Either a vacuum pump or compressed air or a force pump may be used. The compressed air method is rather the more convenient inasmuch as the ammonia may be collected directly in a volumetric flask. Inasmuch as the necks of such flasks (100 c.c.) are 
into a volumetric flask (roo c.c.) containing about 20 c.c. of ammonia-free water

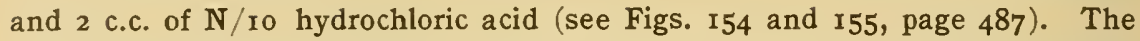
air current should be only moderately rapid for the first two minutes but at the end of this two-minute period the current should be run at its maximum speed for an interval of eight minutes.

Disconnect the flask, dilute the contents to about 60 c.c. with ammoniafree water and dilute similarly $\mathrm{I} \mathrm{mg}$. of nitrogen in the form of ammonium sulphate $^{1}$ in a second volumetric flask. Nesslerize both solutions as nearly as possible at the same time with 5 c.c. of Nessler-Winkler solution ${ }^{2}$ diluted, immediately before using, with about 25 c.c. of ammonia-free water to avoid turbidity. Immediately fill the two flasks to the mark with ammonia-free water, mix well and determine the relative intensity of the two colors by means of a Duboscq colorimeter. ${ }^{3}$

The color of the unknown should be adjusted to that of the standard both from above and below the level of the latter. The matching of the colors is ordinarily very easy. It is desirable to make the readings by diffused daylight if possible. If electric light must be used, a sheet of smooth white paper should be interposed between the colorimeter and the source of light.

Calculation.-The reading of the standard divided by the reading of the unknown gives the nitrogen in milligrams in the volume of the urine taken. Calculate the total nitrogen output for the 24 -hour period.

Interpretation.- See page 484 .

3. Bock and Benedict's Modification of the Folin-Farmer Procedure.Bock and Benedict ${ }^{4}$ have found distillation of the ammonia more accurate than

not large enough to permit of the use of a two-hole rubber stopper when suction is used, the ammonia should be collected in one of the Jena test-tubes previously described which contains 2 c.c. of $\mathrm{N} / \mathrm{I}$ o hydrochloric acid and about 5 c.c. of ammonia-free water. The ammonium salt is then transferred to the volumetric flask with $40-50$ c.c. of water and Nesslerized as described.

${ }^{1}$ Care should be taken to secure the pure salt. All ammonium salts contain pyridine bases which titrate like ammonia but do not react with Nessler's reagent. Pure ammonium sulphate may be prepared by decomposing a high-grade ammonium salt with sodium hydroxide and passing the liberated ammonia into pure sulphuric acid. The salt is then precipitated by means of alcohol, then brought into solution in water and re-precipitated by alcohol. The final product should be dried in a desiccator over sulphuric acid. Dr. H. L. Emerson of Boston prepares a salt which is very satisfactory for use in this method. According to Bock and Benedict, Kahlbaum's "Zur Analyse" ammonium chloride is satisfactory.

${ }^{2}$ Chem. Zeit., I899, p. 54r. The Nessler-Winkler solution has the following formula:

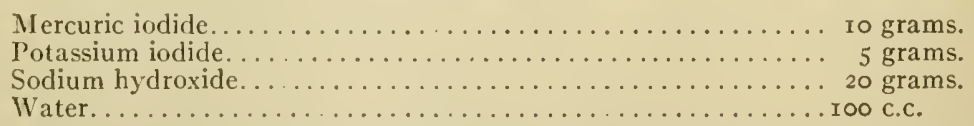

The mercuric iodide is rubbed up in a small porcelain mortar with water, then washed into a flask and the potassium iodide added. The sodium hydroxide is dissolved in the remaining water and the cooled solution added to the above mixture. The solution cleared by standing is preserved in a dark bottle.

The 25 c.c. portion of the diluted reagent should be added about one-third at a time to the contents of the flask. It is very essential that the dilution of the reagent takes place immediately preceding its use, inasmuch as the diluted reagent deteriorates in a few minutes as is indicated by the formation of a brick-red precipitate. Fortunately the reagent does not decompose in this manner in the presence of the ammonium salt.

3 The standard may be set at any desired depth but a very satisfactory depth is $20 \mathrm{~mm}$. The depth should be uniform throughout any series of comparative tests.

4 Bock and Benedict: Jour. Biol. Chem., 20, 47, I9I 5. 
aspiration. They connect the large Jena test-tube in which the digestion was carried out with a small Liebig condenser (made from a piece of glass tubing 30 by $150 \mathrm{~mm}$. with two-hole rubber stoppers at each end through which pass the inlet and outlet tubes and the condenser tube itself). See Fig. I56. The lower end of the condenser is connected with a glass tube (or better an old pipette, to prevent back suction) which reaches nearly to the bottom of the volumetric flask used as a receiver. The distillation tube also has a two-hole rubber stopper. It is connected with the condenser and also carries a long straight tube which reaches nearly to the bottom of the test-tube, and is closed above with a piece of rubber tubing and a pinch-cock. The digestion is carried out just as in the Folin-Farmer method (see page 485 ) and when partially cool 7 c.c. of water are added. Into the long tube passing through the stopper suck 3 c.c. of saturated sodium hydroxide solution and close the pinch-cock. Insert the stopper, connect with the condenser

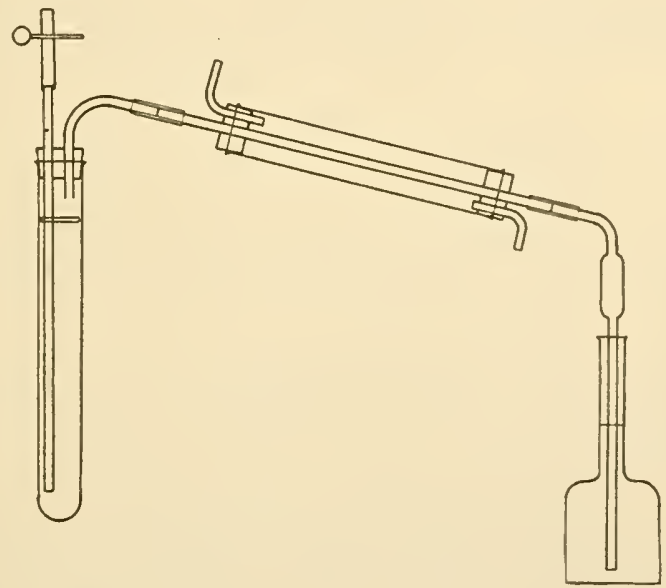

Fig. I56.-BOCK AND Benedict Apparatus.

and allow the alkali to run into the test-tube. The fluids are mixed by blowing a few bubbles of air through the apparatus. The test-tube is then heated to vigorous boiling (over a large free flame), the distillation being continued until a separation of salts occurs in the test-tube and the mixture begins to bump. This distillation requires about two minutes. The test-tube is then disconnected from the condenser and the latter washed down with a few cubic centimeters of water. The liquid in the receiving flask is diluted and Nesslerized as in the Folin-Farmer method (see page 485 ).

Bock and Benedict, while holding the distillation procedure to be more accurate than aspiration, do not consider that the colorimetric method is equivalent to the standard Kjeldahl proceclure in accuracy or reliability, although usually it agrees with the latter method within about 2-3 per cent, and is indispensable where very small amounts of nitrogen are to be determined. According to Folin ${ }^{1}$ and others the method is capable of greater accuracy than this, and the aspiration

${ }^{1}$ Folin: Jour. Biol. Chcm., 2r, I95, I9I5. 
procedure gives satisfactory results. The method should be checked up carefully by each new learner of the method, using pure solutions. Outside air is better than laboratory air for aspiration purposes. Care is needed in using the pipettes, which should be of the Ostwald type and accurate. In using them allow the pipette to drain against the side of the vessel for ro seconds and then blow out clean so that nothing is left behind in the tip. The reagents used must be as free as possible from ammonia and must be checked up, particularly the sulphuric acid and potassium sulphate. Those who have trouble in using a colorimeter may substitute titration with $\mathrm{N} / 50$ hydrochloric acid using alizarin, or better methyl red, as an indicator.

4. Gulick's Modification of the Folin-Farmer Colorimetric Method. ${ }^{1-}$-Principle.-By using small amounts of sulphuric acid and potassium sulphate it is possi-

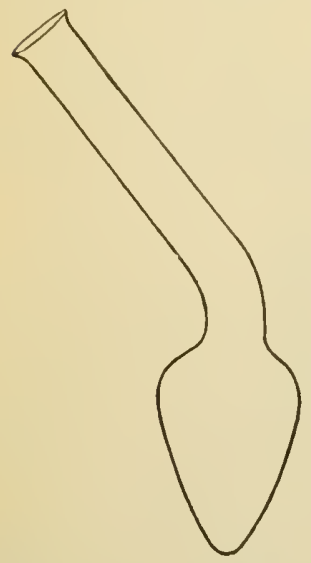

Frg. I 57.-GUL1CK MICRO-OXIDATION FLASK. ble to Nesslerize the products of the digestion directly without the necessity of previous aspiration of the ammonia.

Procedure.-Dilute the sample of urine to an exact multiple of its original volume (about 4 -10 times) with acid mixture, ${ }^{2}$ so that 0.5 c.c. of the dilution will contain between 0.4 and $0.7 \mathrm{mg}$. of nitrogen. With an Ostwald pipette introduce 0.5 c.c. of this mixture into the bulb of a micro-oxidation flask. The type illustrated in Fig. I57, with a bulb capacity of about I5 c.c., is the most convenient. Add also a small spherical glass bead or scrap of platinum or, better, a piece of platinum wire $4-5$ $\mathrm{mm}$. long bent into a tight spiral. Agitate continually while boiling off the water over a micro-burner (about one minute). Set up to heat over a very small flame well guarded against the wind. A luminous flame 6-7 $\mathrm{mm}$. high coming from a burner tube of about $3 \mathrm{~mm}$. outside diameter may be used. The digestion bulb is set at a slant so that the mouth is directed upward and the heat applied at the tip. Heat for at least one minute after the acid becomes clear white. The boiling and oxidation require about 6-ro minutes. As soon as the glass is cool enough to bear water add sufficient ammonia-free water to dissolve the contents, and rinse quantitatively into a 50 c.c. volumetric flask.

Introduce into a second 50 c.c. flask $0.5 \mathrm{mg}$. of nitrogen in the form of pure ammonium sulphate. Fill both flasks to about 40 c.c. with ammonia-free water. Into each of the flasks then inject 5 c.c. of the modified Winkler solution ${ }^{3}$ in a vigorous stream from a pipette. Fill to the mark and mix thoroughly. Compare

${ }^{1}$ Gulick: Jour. Biol. Chem., r8, 541, I9I4.

${ }^{2}$ Acid Oxidizing Mixture.-To I 25 c.c. of ammonia-free water add 40 c.c. of sulphuric acid, 5 c.c. of a saturated solution of mercuric chloride and $20 \mathrm{gm}$. of potassium sulphate. Then make up to 200 c.c. with ammonia-free water.

${ }^{3}$ Modified W'inkler Solution.-Dissolve 40 grams of sodium hydroxide in about 200 c.c. of ammonia-free water. Mix I 5 grams of mercuric iodide and ro grams of potassium iodide and dissolve in about I5 c.c. of water. Transfer with the aid of the alkali to a 500 c.c. volumetric flask and make up to 500 c.c. with ammonia-free water. Transfer to an Erlenmeyer flask and let stand 24 hours to settle. 
at once or at least within an hour in a colorimeter, using a depth of standard of 20-30 $\mathrm{mm}$.

\section{Urea}

I. Urease Methods.-Principle.-These methods depend upon the principle that the enzyme urease is able, at ordinary temperatures, to transform urea, quickly and completely, into ammonium carbonate. Takeuchi ${ }^{1}$ in 1909 discovered the presence of this enzyme in the soja or soy bean. The application of this enzyme to the determination of urea in urine, blood, etc., was first proposed by Marshall, ${ }^{2}$ whose methods have been modified by Van Slyke and Cullen. ${ }^{3}$ These latter investigators prepared a permanent preparation of the enzyme, in a water-soluble form, the use of which makes more convenient the rapid and accurate determination of urea in urine, blood and other biological fluids.

The urease method is probably the most satisfactory of all methods for the determination of urea. Other nitrogenous constituents such as allantoin are not decomposed by urease. The method involves no carefully regulated heating procedures, and is applicable to diabetic urines.

The procedure for the determination in urine consists in treating the urine sample with urease, aerating the ammonia formed into fiftiethnormal acid, and titrating the excess of acid with fiftieth-normal alkali. (For colorimetric procedure see page 492.)

Preparation of Solid Urease. ${ }^{-}$-Digest one part of soy bean meal with five parts of water at room temperature, with occasional stirring, for an hour, and clear the solution by filtration through paper pulp or centrifugation. Pour this extract slowly; with stirring, into at least ro volumes of acetone. The acetone dehydrates the enzyme preparation. Filter, dry in vacuum, and powder. The activity of the preparation is retained indefinitely. Thus prepared it is not perfectly soluble in water, but this fact interferes in no way with its use.

Standardization of the Enzyme Preparation.-Make up accurately a 3 per cent solution of pure urea. Treat this solution exactly as the urine is treated in the following method, using $1 / 2$ c.c. of the solution. The ammonia formed should neutralize 25 c.c. of $\mathrm{N} / 50$ acid. If it does so the preparation is of suficient strength to use as indicated. If not, more of the preparation must be used for a determination.

The ground soy bean may also be used directly in this determination. It should pass through a 20-mesh sieve. Rose and Coleman for their micro-procedure

1 Takeuchi: Journ. Coll. Agr. Tokyo, 1909, Part s.

${ }^{2}$.1larshall, E. K., Jr.: J. Biol. Chem., I4, 283, I913; I5, 495, I913; I5; 4 8;, I9I3; I7, 351,1914 .

${ }^{3}$ Van Slyke, D. D., and Cullen, G. E.: $J$. A m. I Ied. Ass'n, 62, 155s, ror4. See, also, J. Biol. Chem., I9, I4I, 1914.

4 Van Slyke and Cullen: Jour. Biol. Chem., I0, 2II, IOI4. Satisfactory preparations of Urease may be obtained from the Arlington Chemical Company, Yonkers, N. Y., and from Hynson, Westcott and Co., Baltimore. 
(see below) use $0.2-0.4$ gram of bean flour acting in a water-bath at $50-60^{\circ}$ for five minutes. In their macro-method, using 5 c.c. of urine they dilute with 30 c.c. of water warmed to $50-60^{\circ}$ and then add 5 grams of the soy bean flour and let stand for 30 minutes. They then add 5 c.c. of saturated sodium carbonate solution and aerate as usual.

(a) Procedure of Van Slyke and Cullen.-Dilute 5 c.c .of urine to 50 c.c. with ammonia-free water. Measure 5 c.c. of the diluted urine into Tube "A" (see Fig. I58), add I drop of caprylic alcohol (to prevent frothing), and I c.c. of enzyme solution." Close " $\mathrm{A}$ " with stopper shown in figure, and let the tube stand 15 minutes for the enzyme to act. Measure into Tube " $B$ " 25 c.c. of N/50 $\mathrm{HCl}$ or $\mathrm{H}_{2} \mathrm{SO}_{4}$. Add I drop of caprylic alcohol and I drop of a I per cent alizarin solution, ${ }^{2}$ as indicator. Connect " $A$ " and " $B$ " as shown in the figure. At the

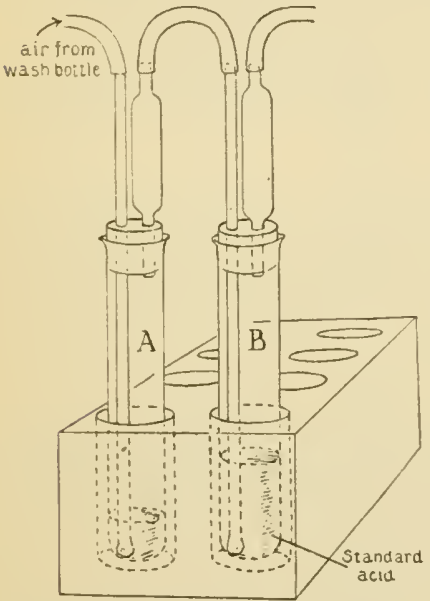

FIG. I5S.-VAN SLYKE AND CUllen APParatus. end of 15 minutes aspirate for about one-half minute to remove any ammonia present in the free condition in "A." After this aspiration, open " $A$ " and introduce 5 c.c. of saturated potassium carbonate. Close "A" at once and aspirate until all the ammonia has been removed from " $A$ " and carried over into the acid in "B." The time needed for the aspiration varies for different pumps from 5 to 30 minutes, and should be determined by trial for the particular apparatus used. At the end of the time needed for the aeration, ${ }^{3}$ the pump is disconnected (care being taken to avoid back suction) and the excess acid in "B" is titrated by means of fiftieth-normal alkali.

Calculations. - The number of cubic centimeters of fiftieth-normal acid neutralized is multiplied by the factor 0.056 to give the number of grams of urea-plus ammonia-nitrogen in 100 c.c. of the urine. The ammonia alone may be determined at the same time as the ammonia plus urea, using the same technic except that 5 c.c. of the undiluted urine, no urease, and the factor 0.0056 are used for the determination of ammonia alone. The ammonia tubes are run in the same series as those for the urea determination, using the same air current for all.

(b) Colorimetric Modification.--Rose and Coleman ${ }^{4}$ suggest the colorimetric determination of the ammonia which is carried over by the aspiration, rather than titration of the excess of acid. They Nesslerize the solution in "B," and compare the color produced with the color of a Nesslerized solution of known ammonia content, as in the Folin-Farmer method for total nitrogen. If this procedure is followed, the amount of urea and ammonia nitrogen in the solution acted upon by the

1 The enzyme solution is prepared by dissolving 2 grams of the enzyme preparation, 0.6 gram of dipotassium-hydrogen phosphate, and 0.4 gram of monopotassium-dihydrogen phosphate in ro c.c. of water. Solution is aided by stirring with a glass rod. The slightly opalescent solution should be covered with toluol and may be kept for two weeks without losing activity.

${ }^{2}$ Folin states that methyl red is preferable to alizarin for ammonia titrations.

${ }^{3}$ See Fiske (Jour. Biol. Chem. 23, 455, I9 I5) and Van Slyke and Cullen (Jour. Biol. Chem., 24, 117, I916) for discussion of details of method.

"Rose and Coleman: Biochem. Bull., 3, 4ri, I9r4. 
urease must not exceed $2 \mathrm{mg}$. This procedure has been found useful where small quantities of urea are to be estimated.

Interpretation.-The mean average daily excretion of urea by normal adults is usually placed at about $30-35$ grams but is very closely dependent upon the protein ingestion and hence may vary widely. It is of significance only when the amount of nitrogen ingested is known with some degree of accuracy. In disorders associated with increased tissue catabolism as in fevers, the excretion of urea is increased. It may be decreased in pronounced kidney and liver disorders due to decreased formation and decreased power of elimination, but these findings are not constant.

The per cent of the total nitrogen of the urine occurring as urea varies on the average from So-9o. On a high protein diet it is nearer 90 per cent; on a very low nitrogen but high calorie diet it may not be over 60 per cent. In marked acidosis it may be considerably decreased relative to the total nitrogen (see ammonia).

(c) Marshall's Urease Method. ${ }^{1}$-Principle.-This is a simple clinical method for the determination of urea in urine. It differs from the preceding method in that instead of aspirating off the ammonia formed from the urea by the action of the urease, it is titrated directly in the urine mixture, thus simplifying the procedure. The method is nearly as accurate as the preceding, for normal urine the error being only about 2 per cent which is very satisfactory for a rapid clinical procedure. For diabetic urines the aeration procedure should be used as such urines contain substances which render the titration inaccurate.

Procedure.-Two 5 c.c. portions of the urine are measured into flasks of 200-300 c.c. capacity and diluted with distilled water to about 100-I25 c.c. One c.c. of a ro per cent solution of urease prepared as described on page $49 \mathrm{I}$ is added to one flask, a few drops of toluene to each and the solution allowed to remain, well stoppered, at room temperature over night (or five hours). The fluid in each flask is titrated to a distinct pink color with $\mathrm{N} /$ Io hydrochloric acid using methyl orange as an indicator. A few cubic centimeters of the enzyme solution used should also be titrated to determine the amount of N/ro hydrochloric acid required to neutralize I c.c.

Calculation.-The amount of hydrochloric acid required for the contents of the flask containing the urine and enzyme solution, less the amount used for 5 c.c. of urine alone and that previously determined for I c.c. of enyzme solution, corresponds to the urea originally present in the sample of urine. Since I c.c. of $\mathrm{N} / \mathrm{ro} \mathrm{HCl}$ is equivalent to $3 \mathrm{mg}$. of urea, the number of cubic centimeters required, multiplied by 0.6 gives the value of urea expressed in grams per liter of urine.

Interpretation.-See above.

${ }^{1}$ Marshall: Jour. Biol. Chem., 14, 2S 3 , 1913. 
2. Benedict's Method. ${ }^{2}$-Principle.-The urea is decomposed by heating with a mixture of potassium bisulphate and zinc sulphate. The fact that the hydrolyzing agent is a salt and that the digestion takes place in the practical absence of water seem to insure less decomposition of substances other than urea. The ammonia formed is distilled off and determined in the usual manner.

Procedure.-Five c.c. of urine are introduced into a rather wide Jena glass test-tube, about 3 grams of potassium bisulphate and I-2 grams of zinc sulphate ${ }^{2}$ added, a small quantity of powdered pumice and a bit of paraffin are introduced and the mixture boiled almost to dryness either over a free flame or by immersion in a sulphuric acid bath at about $130^{\circ}$. The tubes are then weighted (a screw clamp is convenient) and immersed for three-fourths of their length in a bath of sulphuric acid at a temperature of $162-165^{\circ}$ (not lower) for one hour.

The contents of the tube are then washed into an 800 c.c. Kjeldahl distillation flask, diluted to about 400 c.c. with water, made alkaline by the addition of $15^{-20}$ c.c. of Io per cent $\mathrm{KOH}$ (or 25 c.c. I 5 per cent $\mathrm{Na}_{2} \mathrm{CO}_{3}$ ) and distilled as usual in the $\mathrm{Kjeldahl}$ method (page $48_{3}$ ). The value obtained must be corrected for ammonia by a separate determination of the latter.

Welker ${ }^{3}$ has suggested an electrical bath for use in the first part of this method.

3. Microchemical Method of Folin and Pettibone.4-Principle.The urine is heated with potassium acetate and acetic acid to hydrolyze the urea. The desired temperature is maintained with the aid of a temperature indicator. The ammonia formed is aspirated off and determined colorimetrically with Nessler-Winkler reagent.

Procedure.-Dilute the urine so that I c.c. contains $0.75^{-I .5} \mathrm{mg}$. of urea nitrogen. Generally dilutions of $\mathrm{I}: 20$ or $\mathrm{I}: \mathrm{IO}$, depending on the concentration, are satisfactory. By means of an Ostwald pipette (see page 487) introduce I c.c. of the diluted urine into a large dry Jena test-tube $(20-25 \mathrm{~mm}$. by $200 \mathrm{~mm}$.) which already contains 7 grams of dry ammonia-free potassium acetate ${ }^{5}$ (free from lumps), I c.c. of 50 per centacetic acid, a small sand pebble or a little powdered zinc (not zinc dust) to prevent bumping during boiling and a temperature indicator. ${ }^{\circ}$

I Benedict: Jour. Biol. Chem., 8, 405, IgI I.

${ }^{2}$ An excess of zinc salt is to be avoided as too large quantity tends to cause slight frothing during the final distillation.

3 Welker: Biochem. Bull., I, 439, Igr 2.

- Folin and Pettibone: Jour. Biol. Chem., II , 5I3, IgI 2.

5 A satisfactory preparation containing less than I per cent of moisture and free from ammonia may be obtained from J. T. Baker Chemical Co., Phillipsburg, N. J

" "This temperature indicator consists of powdered chloride-iodide of mercury ( $\mathrm{HgICl}$ ) inclosed in a sealed glass bulb not over I $\mathrm{mm}$. in diameter. This salt is bright red at ordinary temperatures. At I I $8^{\circ} \mathrm{C}$. it turns yellow and melts to a clear dark red liquid at $155^{\circ} \mathrm{C}$. It solidifies again at about $148^{\circ} \mathrm{C}$. and resumes its red color gradually only in the course of about 24 hours. The melting-point temperature, $153^{\circ} \mathrm{C}$., is fortunately a temperature very readily obtained and maintained by means of potassium acetate and as the acetate begins to cake and solidify at $160^{\circ}-161^{\circ} \mathrm{C}$, there is no danger in this combination of having either too high or too low a temperature without its being unmistakably apparent.

The $\mathrm{HgICl}$ may be prepared by heating, in a dry state, intimately mixed mercuric chloride and mercuric iodide in molecular proportions at $150^{\circ}-160^{\circ} \mathrm{C}$. for $6-8$ hours. At the end of the heating the product should be powdered and used as it is for it cannot be purified by the use of solvents. It should be kept dry until sealed up as indicated." These tem. perature indicators may be obtained ready prepared in tubes from Eimer and Amend New York. 
Close the test-tube by means of a rubber stopper carrying an empty narrow "calcium chloride tube" ( $1.5 \mathrm{~cm}$. by $25 \mathrm{~cm}$., without bulb) as a condenser. Suspend the test-tube and condenser above a micro-burner (see page 487 ) by means of a burette clamp or some similar device in such a way that they may be easily raised or lowered. Heat gently, using a bottomless beaker or some similar device as a wind shield if needed. The acetate will soon dissolve (two minutes) and the mixture begin to boil. At this point the indicator begins to melt showing that the desired temperature $\left(153-160^{\circ} \mathrm{C}\right.$.) has been reached. Continue the boiling in a gentle, even manner for ten minutes at the end of which time the decomposition of the urea is complete. Remove the apparatus from the flame and dilute the contents with 5 c.c. of water. ${ }^{1}$ Add an excess of alkali (2 c.c. of a saturated solution of sodium hydroxide or potassium carbonate) and remove the liberated ammonia by means of a strong air current (see page 487). The ammonia may be caught in a roo c.c. volumetric flask which contains about 35 c.c. of ammonia-free water and 2 c.c. of $\mathrm{N} / \mathrm{IO}$ acid. With a strong air current this process requires only about ten minutes. Determine the ammonia colorimetrically against I mg. of nitrogen in the form of ammonium sulphate. For the colorimetric procedure see the total nitrogen determination page 485 .

4. Method of Folin and Denis. ${ }^{2}$-Principlc.--Sugar interferes with the decomposition of urea. This was formerly believed to be due to the formation of nitrogenous "melanins," 3 but is more probably due to the formation of definite, stable ureids. ${ }^{4}$ This difficulty may be overcome by proper dilution of the urine thus preventing the formation of the ureids. Because of this great dilution titration procedures are inapplicable, and the colorimetric procedure is applied.

Procedure.-Dilute I C.c. of the urine with 20 to 100 volumes of ammonia-free water and decompose I c.c. of this dilute urine with potassium acetate and acetic acid as described under the method of Folin and Pettibone on page 494.

By means of an air current remove the ammonia to a second test-tube which contains about 2 c.c. of water and 0.5 c.c. of N/Io hydrochloric acid. Add to the contents of this tube about 2 c.c. of water and 3 c.c. of the diluted ( $1: 5$ ) NesslerWinkler solution (page 490). Wash this colored solution into a Io c.c. volumetric flask and dilute it to the mark with ammonia-free water. Transfer the entire volume to a dry cylinder of a Duboscq colorimeter and determine the depth of color against a standard containing I mg. of nitrogen per Ioo c.c. of solution. For the detailed colorimetric procedure see the method for total nitrogen, page 494 .

5. Method of Folin. ${ }^{5}$ - Principle. - The urea is decomposed by heating with magnesium chloride and hydrochloric acid. The ammonia is distilled off and determined by titration. This method was one of the first accurate methods for the determination of urea. Allantoin is also included in the results from this method but occurs only in minute quantities in human urine. The method is not applicable to urines containing sugar as ureides are formed. For such urines the method of Folin and Denis (above) or the urease method using aspiration (page f9l) may be employed. The earlier Mörner-Sjöqvist method ${ }^{6}$ has been combined with that

1 This water should be added by means of a pipette through the calcium chloride tube so as to rinse the sides of the tube and the bottom of the rubber stopper from any possible traces of ammonium acetate. Not more than 5 c.c. of water should be used for this purpose.

${ }^{2}$ Folin and Denis: Jour. Biol. Chem., II, 520, I9I2.

${ }^{3}$ Mörner: Skand. Arch. Pleysiol., I 4, 3 I 9.

- Folin: Am. Jour. Physiol., 13, 46, 1905.

5 Folin: Am. Jour. Physiol., I 3, 46, I 905.

- Mörner: Skand. Arch. Pliysial., I4, 297, I903. See also Fourth Edition of this book. 
of the Folin procedure in such a way as to render the latter applicable to urines containing sugar, to remove certain interfering substances such as allantoin and make the separate determination of ammonia unnecessary. The method is, however, time-consuming and the other methods mentioned are on this account to be preferred for the analysis of diabetic urines.

Procedure.-Place 5 c.c. of urine in a 200 c.c. Erlenmeyer flask and add to it 5 c.c. of concentrated hydrochloric acid, 20 grams of crystallized magnesium chloride, a piece of paraffin the size of a hazel nut, and $2-3$ drops of a I per cent aqueous solution of "alizarin red." Insert a Folin safety

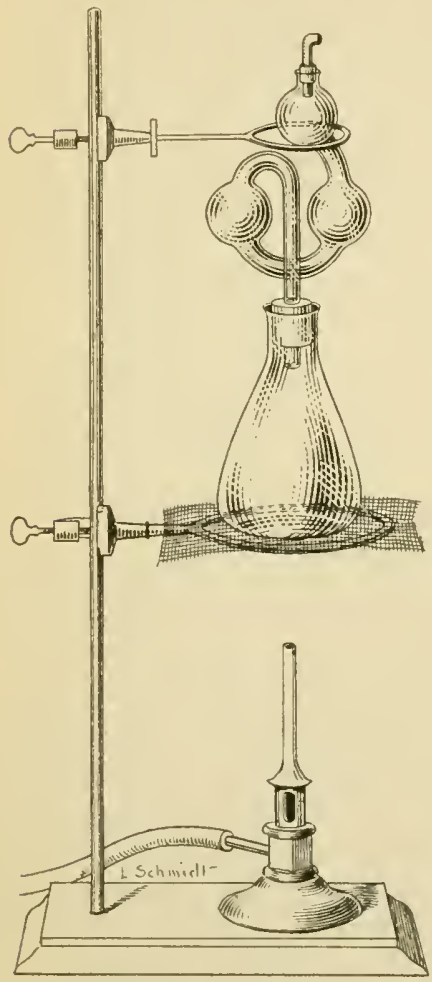

FIG. I 59.-FoLIN'S UREA APPARATUS. tube (Fig. I59) into the neck of the flask and boil the mixture until each drop of reflow from the safety tube produces a very perceptible bump; the heat is then reduced somewhat and continued one and one-half hours. The contents of the flask must not remain alkaline, and to obviate this, at the first appearance of a reddish tinge in the contents of the flask a few drops of the acid distillate are shaken back into the flask. At the end of one and one-half hours the contents of the vessel are transferred to a I-liter flask with about 700 c.c. of distilled water, about 20 c.c. of Io per cent potassium hydroxide or sodium hydroxide solution is added and the mixture distilled into a known volume of $\mathrm{N} /$ Io sulphuric acid until the contents of the flask are nearly dry or until the distillate fails to give an alkaline reaction to litmus, showing the absence of ammonia. The time devoted to this process is ordinarily about an hour. Boil the distillate a few moments to free it from $\mathrm{CO}_{2}$, then cool and titrate the mixture with $\mathrm{N} /$ Io sodium hydroxide, using "alizarin red" as indicator.

A "check" experiment should always be made to determine the original ammonia content of the urine and of the magnesium chloride, if it is not absolutely pure, which of course should be subtracted from the total amount of ammonia as determined by the above process.

6. Hypobromite Methods.-Principle.-The hypobromite methods are based upon the fact that urea is decomposed by a solution of sodium hypobromite with the formation of carbon dioxide and gaseous nitrogen according to the following equation:

$$
\mathrm{CO}(\mathrm{NH})_{2}+3 \mathrm{NaOBr}=\mathrm{N}_{2}+\mathrm{CO}_{2}+{ }_{2} \mathrm{H}_{2} \mathrm{O}+3 \mathrm{NaBr}
$$

The carbon dioxide is retained by the alkaline solution and the nitrogen gas is collected and measured. On account of its simplicity this has been the most widely used of clinical methods for the determination of urea. It possesses, however, sevcral essential inaccuracies among which may be mentioned the fact that all of the urea nitrogen is not liberated, while on the other hand substances always present 
in urine such as ammonia, creatinine, and uric acid yield nitrogen more or less rapidly when the urine is treated with hypobromite. The measurement of the gas as ordinarily carried out involves considerable crrors. 'The results are usually high, and may be excessively so. Robinson and Muller have suggested that the ureometer be shaken for five minutes to hasten the reaction and that the results as obtained be multiplied by the factor 0.917 to insure more accurate results. They use the Doremus-Hinds apparatus.

(a) Hypobromite Method (using the Doremus-II inds Ureometer). - The DoremusHinds ureometer (Fig. I60) is one of the simplest and cheapest forms of apparatus in general use for the determination of urea by the hypobromite process. It is, however, much less accurate than those types of apparatus which involve the measurement of the nitrogen in a gas burette over water with equalization of pressure. In using this apparatus proceed as follows: Fill the side tube $B$ and the lumen of the stop-cock $\mathrm{C}$ with the urine under examination. Carefully wash out tube $\mathrm{A}$ with water and introduce into it sodium hypobromite solution, ${ }^{3}$ being careful to fill the bulb sufficiently full to prevent the entrance of air into the graduated portion. Now allow I c.c. of urine ${ }^{2}$ to flow from tube $B$ into tube $A$, and after the evolution of gas bubbles has ceased (Io-20 minutes) take the reading of the graduated scale on tube $\mathrm{A}$.

Calculation.-Observe the reading on the graduated scale of tube $A$. This tube is so graduated as to represent the weight of urea, in grams, per cubic centimeter of urine. If we wish to compute the percentage of urea present this may be done very readily by simply moving the decimal point two places to the right; e.g., if the reading is 0.02 gram the urine contains 2 per cent of urea.

Interpretation.-See page 493.

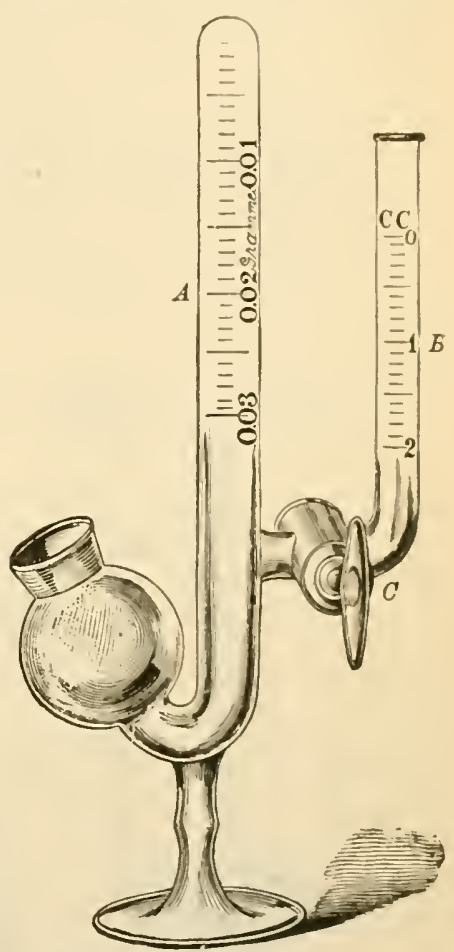

Fig. 160--DOREaIt's-HINDS UREOMETER.

(b) IIypobromite Method (using Marshall's Crea Apparatus).-Place the thumb over the side opening of the bulbed-tube of the apparatus (Fig. I62) and carefully fill the tube with sodium hypobromite solution. ${ }^{3}$ Close the opening in the end of the tube with a rubber stopper, incline the tube to allow air-bubbles to escape, and

1 Robinson and Muller: Jour. Am. Med. Ass'n, 62, 5I 4, I9I 4.

${ }^{2}$ If the content of urea in the urine under examination is large, the urine may be diluted with water before determining the urea. If this is done it must of course be taken into consideration in computing the content of urea.

${ }^{3}$ The ingredients of the sodium hypobromite solution should be prepared in the form of two separate solutions. When needed for use mix one volume of solution $a$, one volume of solution $b$, and 3 volumes of water.

(a) Dissolve 125 grams of sodium bromide in water, add 125 grams of bromine and make the total volume of the solution I liter.

(b) A solution of sodium hydroxide having a specific gravity of I.250. This is approximately a 22.5 per cent solution.

Preserve both solutions in rubber-stoppered bottles. 
finally invert the tube and fix the stoppered end in the saucer-shaped vessel. By means of the graduated pipette rapidly introduce I c.c. of urine ${ }^{1}$ into the hypobromite solution through the side opening of the bulbed-tube. Withdraw the pipette immediately after the urine has been introduced. When the decomposition of the urea is completed (10-20 minutes), gently tap the bulbed-tube with the finger in order to dislodge any gas bubbles which may have collected on the inner surface of the glass. The atmospheric pressure should now be equalized by attaching the funnel-tube to the bulbed-tube at the side opening and introducing hypobromite solution into it until the columns of liquid in the two tubes are uniform in height. The graduated scale of the bulbed-tube should now be read in order to determine the number of cubic centimeters of nitrogen gas evolved. By means of the appended formula the weight of the urea present in the urine under examination may be computed.

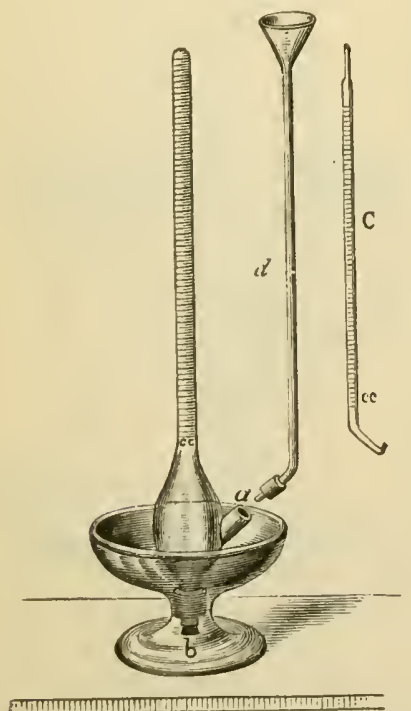

Fig. i6r.-Marshall Urea Apparatus. (Tyson.)

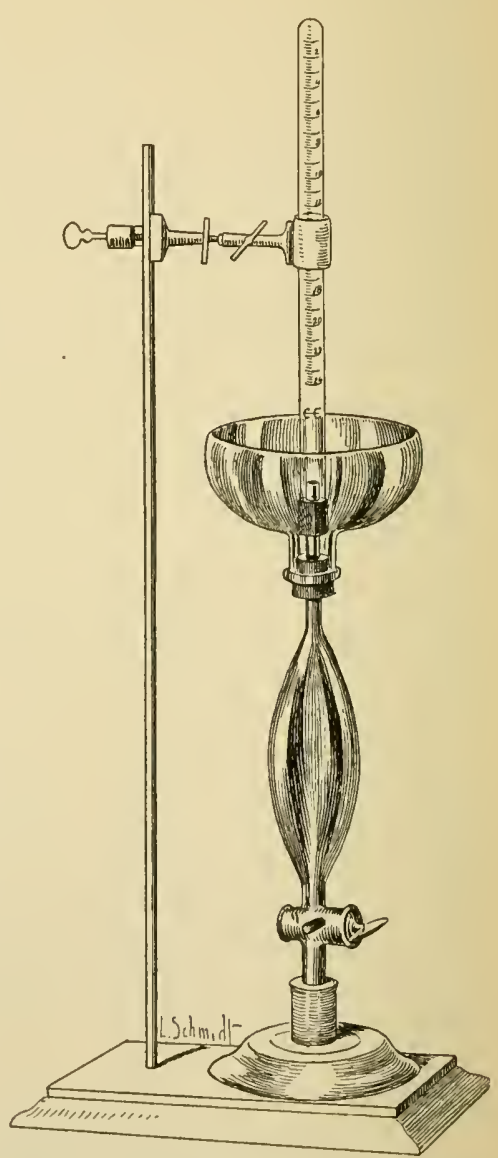

Fig. I62.-HÜFNER UREA APPARATUS.

$a$, Bulbed measuring tube; $b$, saucershaped vessel; $c$, graduated pipette; $d$, funnel-tube.

Calculation. ${ }^{2}-$ By properly substituting in the following formula, the weight of urea, in grams, contained in the volume of urine decomposed (I c.c. or more) may readily be determined:

${ }^{1}$ Ordinarily I c.c. of urine is sufficient; more may be used, however, if its content of urea is very low.

${ }^{2} 0.003665=$ coefficient of expansion of gases for $I^{\circ} \mathrm{C} . \quad 354.5=$ number of c.c. of nitrogen gas evolved from I gram of urea. 
$w=\frac{i(p-T)}{354.5 \times 760(1+0.003665 t)}$.

$w=$ weight of urea, in grams.

$v=$ observed volume of nitrogen expressed in cubic centimeters.

$p=$ barometric pressure expressed in millimeters of mercury.

$T=$ tension of aqueous vapor ${ }^{1}$ for temperature $t$.

$t=$ temperature (Centigrade).

If we wish to calculate the percentage of urea we may do so by means of the following proportion in which $y$ represents the volume of urine used and $w$ denotes the weight of the urea contained in the volume $y$ :

$$
y: w:: x: \text { (pcrcentage of urea). }
$$

Sodium hypobromite solution may also be employed for the determination of urea in the apparatus devised by Hüfner, which is pictured in Fig. I62.

Interpretation.-See page 493.

\section{Ammonia}

I. Folin's Method.-Principle.-The ammonia of the urine is set free by the addition of an alkali and this ammonia is then carried over

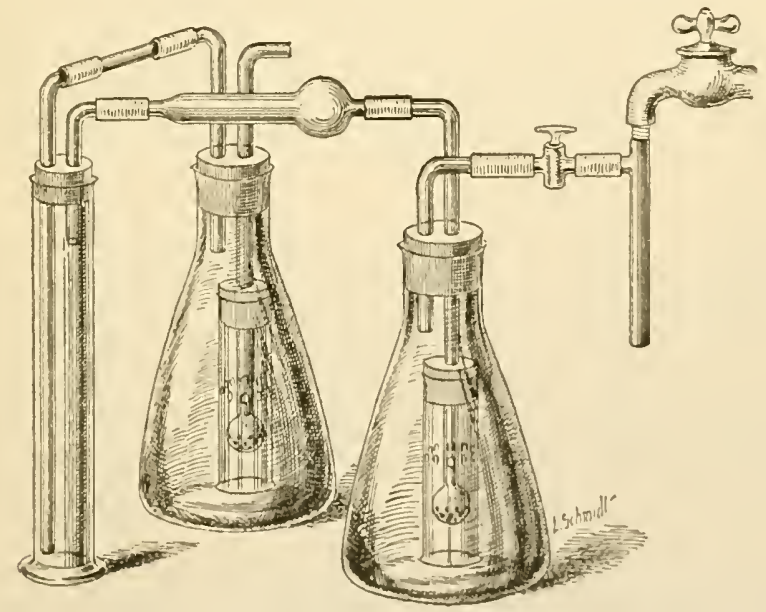

Fig. 163.-Folin Auronia Apparatus.

by an air current into a flask containing a measured amount of standard acid. The excess acid is then titrated. The necessity for distillation is avoided. table:

${ }^{1}$ The values of $T$ for the temperatures ordinarily met with are given in the following
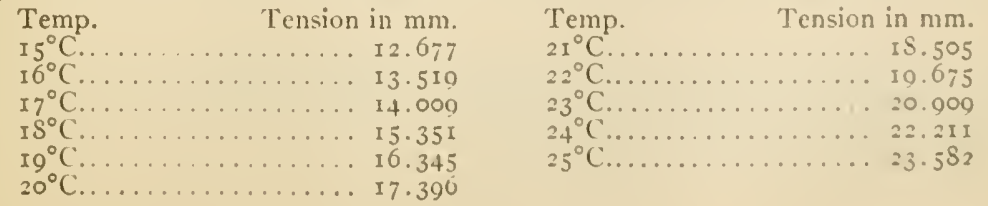
Procedure.-Place 25 c.c. of urine in an aerometer cylinder, 30-40 cm. in height (Fig. I63, p. 499), add about I gram of dry sodium carbonate and introduce some crude petroleum to prevent foaming. Insert into the neck of the cylinder a rubber stopper provided with two perforations, into each of which passes a glass tube, one of which reaches below the surface of the liquid. The shorter tube (Io cm. in length) is connected with a calcium chloride tube filled with cotton, and this tube is in turn joined to a glass tube extending to the bottom of a 500 c.c. wide-mouthed flask which is intended to absorb the ammonia and for this purpose should contain 20 c.c. of $\mathrm{N} /$ ro sulphuric acid, 200 c.c. of ammonia-free

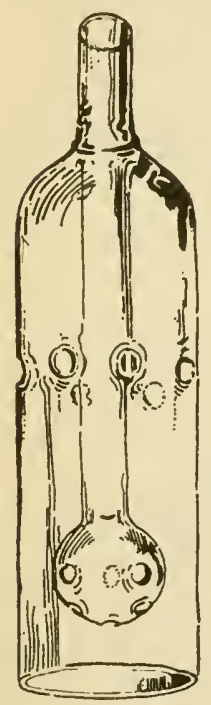

FIG. I64.-FOLIN IMPROVED ABSORPTION TLBE. distilled water and a few drops of an indicator (alizarin red or Congo red). To insure the complete absorption of the ammonia the absorption flask is provided with a Folin improved absorption tube (Fig. I64), wbich is very effective in causing the air passing from the cylinder to come into intimate contact with the acid in the absorption flask. In order to exclude any error due to the presence of ammonia in the air a similar absorption apparatus to the one just described is attached to the other side of the aerometer cylinder, thus insuring the passage of ammonia-free air into the cylinder. With an ordinary filter pump and good water pressure the last trace of ammonia should be removed from the cylinder in about one and one-half hours. ${ }^{1}$ The number of cubic centimeters of the $N /$ ro sulphuric acid neutralized by the ammonia of the urine may be determined by direct titration with $\mathrm{N} /$ Io sodium hydroxide.

Steele $^{2}$ has suggested a modification for use on urines containing triple phosphate sediments. In this modification $0.5^{-1.0}$ gram of $\mathrm{NaOH}$ and about I5 grams of $\mathrm{NaCl}$ are substituted for the $\mathrm{Na}_{2} \mathrm{CO}_{3}$ of the Folin method. The use of sodium hydroxide and chloride instead of carbonate has also been recommended by other workers ${ }^{3}$ as a general procedure, inasmuch as triple phosphate crystals are almost always formed on adding sodium carbonate and these are decomposed with some difficulty by sodium carbonate but readily by the hydroxide. It has not been shown that the use of sodium hydroxide in this manner brings about the decomposition of any other urinary nitrogen compounds.

Calculation.-Subtract the number of cubic centimeters of $\mathrm{N} /$ Io sodium hydroxide used in the titration from the number of cubic centimeters of $\mathrm{N} /$ ro sulphuric acid taken. The remainder is the number of cubic centimeters of $\mathrm{N} /$ Io sulphuric acid neutralized by the $\mathrm{NH}_{3}$ of the urine. One c.c. of $\mathrm{N} /$ Io sulphuric

1 With any given filter pump a "check" test should be made with urine or, better, with a solution of an ammonium salt of known strength to determine how long the air current must be maintained to remove all the ammonia from 25 c.c. of the solution.

${ }^{2}$ Steele: Jour. Biol. Chem., 8, 365, I910.

${ }^{3}$ Benedict and Osterberg: Biochem. Bull., 3, 4I, I9I3.

Shulansky and Gies: Biochcm. Bull., 3, 45, 1913. 
acid is equivalent to 0.0017 gram of $\mathrm{NH}_{3}$. Therefore if $y$ represents the volume of urine used in the determination and $\mathrm{y}^{\prime}$ the number of cubic centimeters of $\mathrm{N} /$ Io sulphuric acid neutralized by the $\mathrm{NH}_{3}$ of the urine, we have the following proportion:

$\mathrm{y}^{\prime}: 100:: \mathrm{y}^{\prime} \times 0.0017: \mathrm{x}$ (percentage of $\mathrm{NH}_{3}$ in the urine examined).

Calculate the quantity of $\mathrm{NH}_{3}$ in the 24-hour urine specimen.

Interpretation. - The average daily output of ammonia in the urine is about 0.7 gram, amounting to $2.5-4.5$ per cent of the total nitrogen excretion. It is increased by the ingestion of acids or acid-forming foods and decreased by the ingestion of alkalis or base-forming foods. In acidosis it may be very greatly increased, being excreted in combination with hydro-oxybutyric and other acids. Values of 5 grams have been noted. It is at the same time increased relative to total nitrogen and urea. In pronounced liver disorders the same thing is noted, as ammonia is not so completely transformed into urea before excretion.

2. Micro-chemical Method of Folin and MacCallum. ${ }^{1}$ - Principle This method is a combination of the aeration procedure for ammonia with its colorimetric determination by means of Nessler-Winkler solution. It gives satisfactory results, but is probably not as accurate as the regular Folin procedure where the amount of substance for analysis is not limited.

Procedure.-By means of Ostwald pipettes introduce I-5 c.c. of urine ${ }^{2}$ into a Jena test-tube $(20-25 \mathrm{~mm}$. by $200 \mathrm{~mm}$.) and add to the urine a few drops of a solution containing ro per cent of potassium carbonate and 15 per cent of potassium oxalate. To prevent foaming add a few drops of kerosene or heavy, crude machine oil. Pass a strong air current (see page 487 ) through the mixture until the ammonia has been entirely removed. ${ }^{3}$ Collect the ammonia in a roo c.c. volumetric flask containing about 20 c.c. of ammonia-free water and 2 c.c. of $\mathrm{N} /$ Io acid.

Nesslerize as described in the method for total nitrogen, page 488 , and compare with I mg. of nitrogen obtained from a standard ammonium sulphate solution and similarly Nesslerized.

It has been noted that a trace of something capable of giving a color with the Nessler-Winkler solution continues to come long after all the ammonia has been removed from the urine. The nature of this substance has not yet been determined. In actual determinations by this method, the influence of this unknown substance, because of the small volume of urine used, is entirely negligible.

1 Folin and MacCallum: Jour. Biol. Chem., ז1, 523, I9 2.

2 The volume of urine taken should contain $0.75^{-1} .5 \mathrm{mg}$. of ammonia nitrogen. With normal urines 2 c.c. will generally yield the desired amount. With very dilute urines 5 c.c. may be required, while with diabetic urines rich in ammonium sales I c.c. may be excessive, thus requiring dilution.

${ }^{3}$ Ordinarily a period of ten minutes is sufficiently long. 
3. Formol Titration Method (Malfatti). ${ }^{1}$-Principle.-This method is based on the reaction taking place when formalin solution is added to a solution containing ammonium salts (see Amino-acid Nitrogen, below). An acid reaction is produced in the mixture, which is then titrated with standard alkali using phenolphthalein as an indicator. Amino-acids give the same reaction so that the result of the titration represents ammonia + amino-acid nitrogen. This method may be used for the rapid clinical estimation of these forms of nitrogen as a substitute for an ammonia determination, but the results do not represent ammonia as is sometimes stated.

Procedure.-To 25 c.c. of urine in a 200 c.c. Erlenmeyer flask add $15^{-20}$ grams of finely pulverized potassium oxalate, a few drops of phenolphthalein, and titrate to a faint but permanent pink color with $\mathrm{N} /$ ro $\mathrm{NaOH}$. (The urine mixture just after neutralization in the urinary acidity determination (see page 479) may be used.) Then add ro c.c. of neutral formalin solution (see aminoacid nitrogen), mix well and titrate with $\mathrm{N} /$ Io sodium hydroxide to a permanent pink color.

Calculation.-One c.c. of $\mathrm{N} /$ Io sodium hydroxide is equivalent to I.7 $\mathrm{mg}$. of ammonia. Multiply the number of cubic centimeters of $\mathrm{N} /$ ro alkali used by 1.7 and by 4 to get the number of milligrams of ammonia + aminoacid nitrogen (expressed as ammonia) in roo c.c. of the urine examined.

\section{Amino-Acid Nitrogen}

I. Henriques-Sörensen Formol Titration Meth̆od. ${ }^{2}-$ Principle.-A solution containing amino-acids is nearly neutral in reaction. If formaldehyde be added, however, the following reaction takes place with the formation of methylene derivatives which an more strongly acid in reaction due to the destruction of the basic properties of the amino groups. The carboxyl groups may then be titrated using phenolphthalein as an indicator.

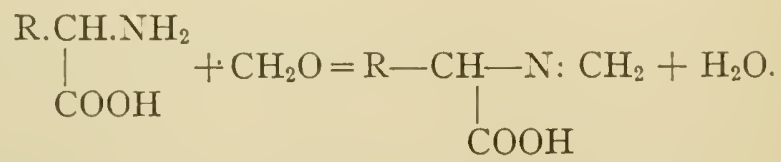

The acidity as shown by the titration is a measure of the amount of amino-acid nitrogen present. Ammonia likewise reacts with formaldehyde in a similar manner as is shown in the following equation:

$$
{ }_{4} \mathrm{NH}_{4} \mathrm{Cl}+6 \mathrm{CH}_{2} \mathrm{O}=\mathrm{N}_{4}\left(\mathrm{CH}_{2}\right)_{6}+6 \mathrm{H}_{2} \mathrm{O}+4 \mathrm{HCl} \text {. }
$$

Hence the formol titration in the presence of ammonia gives results which include both amino-acid and ammonia nitrogen. Ammonia

1 Malfatti: Z. anal. Chem., 47, 273, I908.

${ }^{2}$ Henriques and Sörensen: Zeit. phy'siol. chem., 64, I 20, 1909. 
may be determined and a correction applied, or the ammonia may be removed by means of phosphotungstic acid. Phosphates also interfere by obscuring the end-point and are removed by the addition of barium salts.

It must be borne in mind that polypeptides and still more complex protein derivatives likewise react with formol to a certain degree so that the results do not strictly represent "amino-acid nitrogen."

The method is, with some modifications involving the preparation of the solution to be titrated, applicable in the determination of aminoacids in any medium, e.g., urine, protein digests, etc. When poorly dissociated acids, e.g. some fatty acids, are present, these will in part be included in the result and lead to values which are too high. Certain of the amino-acids when present in large amounts will give erroneous results, but in the ordinary urine or digest these errors are either negligible or compensate each other. In the titration of colored solutions the control solution which is necessary in this method must be colored to correspond with the color of the unknown solution.

Procedure.-The determination of the amino-acids is carried out as follows: The solution to be analyzed, if carbonates, phosphates and ammonia are absent, is made neutral to litmus (paper) and the solution titrated with formaldehyde as below. ${ }^{1}$ In case carbonates, phosphates or ammonia are present a preliminary treatment is necessary which will vary according to the quantity of ammonia present.

(a) For Small Amounts of Ammonia.-Applicable to most urines. Fifty c.c. of the material under examination is pipetted into a 100 c.c. measuring flask and I c.c. phenolphthalein solution ${ }^{2}$ and 2 grams of solid barium chloride are added; the whole is shaken, to saturate the solution with barium chloride; saturated barium hydroxide solution is added until the red color of the phenolphthalein develops and then an excess of 5 c.c. is added. The flask is filled to the graduation mark with water, shaken and permitted to stand for I 5 minutes, after which it is filtered through a dry filter. Eighty c.c. of the clear red filtrate (which corresponds to 40 c.c. of the liquid under examination) are placed in a roo c.c. measuring flask, neutralized to litmus and diluted to Ioo c.c. with freshly boiled water. Equal portions of this solution, 40 c.c. (equivalent to I 6 c.c. of the original solution), may be taken for analysis, one for the formol trtration and the other for the determination of ammonia nitrogen. ${ }^{3}$

(b) For Large Amounts of Ammonia.-After the treatment with phenolphthalein, barium chloride, and barium hydroxide, and the solution has been diluted to Ioo c.c. as in (a) above, the ammonia is distilled off, in vacuo.t

${ }^{1}$ As a standard of comparison the litmus paper used for neutralization is contrasted with a similar piece dipped in a phosphate solution having a neutral reaction $\left(\mathrm{N}, 15 \mathrm{KH}_{2} \mathrm{PO}\right.$ and $\mathrm{M} / \mathrm{I}_{5} \mathrm{Na}_{2} \mathrm{HPO}_{4}$ ).

${ }^{2}$ A solution of 0.5 gram of phenolphthalein in 50 c.c. of alcohol and 50 c.c. of water.

${ }^{3}$ The determination of ammonia may be dispensed with in case a separate determination is made.

- For particulars with regard to the distillation, etc., see Henriques and Sörensen: Zeil. physiol. Chem., 64, 137, 1909. 
In case the solution is deeply colored, as in protein digests, it may be necessary to decolorize ${ }^{1}$ before the titration is attempted.

Final Titration.-For the final titration a volume of from 20-40 c.c. which contains approximately 0.025 gram of nitrogen is the most desirable. A control solution is run composed of an equal volume of boiled distilled water and 20 c.c. of the formaldehyde mixture. ${ }^{2}$ This control solution is colored ${ }^{3}$ so that its tint matches that of the solution to be titrated.

To this control is added about half the volume of $\mathrm{N} / 5$ alkali which will be used in the titration of the solution under investigation and it is then titrated with $\mathrm{N} / 5$ acid to a faint red (first stage). ${ }^{4}$

An additional drop of $\mathrm{N} / 5$ alkali is added, which imparts a distinct red to the solution (second stage).

The solution to be analyzed is now titrated to the color produced in the second stage of the control. The formaldehyde mixture is now added; Io c.c. for each 20 c.c. of the solution, and the mixture again titrated to the second stage with $\mathrm{N} / \mathbf{5}$ alkali. ${ }^{5}$

Two drops of the $\mathrm{N} / 5$ alkali are now added to the control solution which assumes a deep red color (third stage). Fifth normal alkali is now added to the solution under examination until it assumes a color corresponding to the third stage of the control. This completes the titration.

Calculation.-The calculations are similar to those which pertain to any acidimetry procedure. Each cubic centimeter of an $\mathrm{N} / 5$ alkali or acid solution is equivalent to 0.0028 gram of nitrogen. An example will illustrate the procedure: 40 c.c. of solution ( 16 c.c. of urine) required 5. Io c.c. $\mathrm{N} / 5 \mathrm{NaOH}$; control, 0.10 c.c. $\mathrm{N} / 5 \mathrm{NaOH}$; total required for amino-acids 5.00 c.c. equivalent to 0.014 gram of nitrogen. Ammonia nitrogen in 16 c.c. of urine 0.007 gram N. Then $0.014-0.007=0.007$ gram amino-acid nitrogen in 16 c.c. of urine.

Interpretation. - The excretion of total amino-acid nitrogen by a normal adult averages between 0.4 to 1.0 gram per day or from 2 to 6 per cent of the total nitrogen. Free amino-acid nitrogen (see Van Slyke procedure) is considerably less than this, ordinarily 0.5 to 1.0 per cent of the total nitrogen. The amount may be largely increased in disorders associated with tissue waste as typhoid, in pronounced atrophy of the liver, acidosis, etc.

2. Benedict-Murlin Modification. ${ }^{6}$-Principle.-In this method the ammonia is removed by means of phosphotungstic acid, and excess acid as well as carbonates and phosphates carried down with barium.

${ }^{1}$ For methods see Jessen-Hansen, Abderhalden's Arbeits Methoden, vol. 6, p. 202, 19r 2.

2 The formaldehyde solution is freshly prepared for each set of determinations as follows: to 50 c.c. of commercial formaldehyde (formol) (3c-40 per cent) add I c.c. of the phenolphthalein solution. $\mathrm{N} / 5$ alkali is then added until the mixture acquires a faint red color. The volume of the formaldehyde used will vary with the volume of the solution to be analyzed; approximately ro c.c. of the formalin solution are added for each 20 c.c. of the unknown solution.

${ }^{3}$ Solution of Bismark brown is very satisfactory for urines. Tropæolin O, Tropæolin $\mathrm{OO}, p$-nitro-phenol, methyl orange or alizarin sulphonate, may be used.

${ }_{4}$ This procedure is recommended in order that the final volume of the control and the unknown solutions shall be approximately the same when the process is complete.

${ }^{5}$ This is best accomplished by adding alkali until the color is deeper than that of the control, then acid again until lighter and finally alkali to the desired color.

${ }^{6}$ Benedict and Murlin: Jour. Biol. Chem., 16, 385, 1913. 
Procedure.-Measure into a 500 c.c. Erlenmeyer flask 200 c.c. of a 24 -hour urine which has been diluted to 2000 c.c. (or its equivalent). Add an equal volume of ro per cent phosphotungstic acid (Merck $)^{1}$ in 2 per cent $\mathrm{HCl}$. Let stand at least three hours, better over night. Pour off 250 c.c. of the clear fluid, arld I c.c. of a 0.5 per cent solution of phenolphthalein and then barium hydroxide in substance until the whole fluid turns decidedly pink. The barium hydroxide should be added a very little at a time. Let stand one hour. Filter off two roo c.c. samples $(=50$ c.c. urine). Neutralize these samples to litmus (using good quality litmus paper) with $\mathrm{N} / 5 \mathrm{HCl}$. Add at once $10-20$ c.c. of neutral formalin ${ }^{2}$ and titrate cautiously to a deep red color, i.e., until the drop produces no additional color with $\mathrm{N} / \mathrm{ro}$ $\mathrm{NaOH}$. Deduct from the result thus obtained the amount of $\mathrm{N} / \mathrm{IO} \mathrm{NaOH}$ necessary to produce the same depth of color in an equal quantity of water, freed from carbon dioxide by boiling and cooling, and to which an equal volume of neutral formalin has been added.

Calculation.-One c.c. of $\mathrm{N} / \mathrm{r} \circ \mathrm{NaOH}$ is equivalent to $\mathrm{I}+\mathrm{mg}$. of amino-acid nitrogen. Multiply the number of cubic centimeters of $\mathrm{N} /$ ro $\mathrm{NaOH}$ used (after deducting for control as indicated above) by $\mathrm{I}_{4} 4$ and by 2 (as the equivalent of 50 c.c. of urine was used) to obtain the number of milligrams of amino-acid nitrogen in 100 c.c. of the urine.

Interpretation.-See page 504 .

3. Method of Frey-Gigon. ${ }^{3-}$-Principle.-The ammonia is removed from the urine by aspiration after treatment with barium hydroxide and the formol titration performed in the usual manner.

Procedure.-Treat 50 c.c. of urine in an aerometer cylinder such as used in the Folin ammonia method, with 20 c.c. of saturated barium hydroxide solution and I5 c.c. of alcohol. Aspirate for two or three hours, using a slow current of air. The ammonia is carried off. (It may be collected in standard acid solution and determined as in the Folin method (see page 499) if desired.) Transfer the urine mixture quantitatively to a 250 c.c. flask and make to mark with distilled water. Shake well, allow to settle, filter. Take roo c.c. of the filtrate and just neutralize with $\mathrm{N} / 5$ hydrochloric acid, using rosolic acid as an indicator. Then to another Ioo c.c. portion of the filtrate add an amount of the standard acid equal to that added to the first portion. Next add Io c.c. of neutral formalin solution, a few drops of phenolphthalein and titrate in the usual manner to a red-violet color. Calculate as in the preceding method.

The amino-acid nitrogen may also be approximately determined by carrying out the titration for ammonia + and amino-acid nitrogen as given under Ammonia, page 502 , making a separate determination of ammonia, and subtracting the latter result from the former.

4. Van Slyke's Method for Total Amino-Acid Nitrogen. 1 Take 25 c.c. of urine $^{5}$ and mix with I c.c. of concentrated sulphuric acid and heat in an autoclave at $180^{\circ}$ (oil bath temperature) for one and one-half hours. Transfer to a 50 c.c. flask and add 2 grams powdered calcium hydroxide. Shake thoroughly, make up to 50 c.c. and filter through a dry folded filter. Transfer 20 c.c. of the

${ }^{1}$ Kahlbaum's preparation is a very different substance.

${ }^{2}$ To 50 c.c. commercial formalin solution (30-40 per cent) add I c.c. of phenolphthalein solution and then $\mathrm{N} / 5 \mathrm{NaOH}$ to a very faint pink color. The solution should be freshly prepared.

${ }^{3}$ Frey and Gigon: Biochem. Zcil., 22, 309, 1909.

"Van Slyke: Jour. Biol. Chcm., r6, 125, I913.

${ }^{5}$ See (Van Slyke: Proc. Soc. Exp. Biol. and ifed., 13, 63, 1915) for treatment of urines containing glucose or albumin. 
filtrate to a Jena glass evaporating dish and concentrate to dryness on the waterbath. This requires about half an hour. The residue is moistened with I c.c. of 50 per cent acetic acid to bring the calcium hydroxide and carbonate into solution, and is then washed into a 1o c.c. flask and filled up to the mark. One can use the entire solution for determination of the amino-nitrogen in the large amino-apparatus, or use 2 c.c. portions for the micro-apparatus. (See Van Slyke Apparatus, Figs. 34 and 35, p. 88 in Chapter IV on Proteins.)

The length of time which the nitrous acid solution should be shaken in order to drive off all the amino-nitrogen depends somewhat on the temperature. When the latter is $15-20^{\circ}$ the time should be five to four minutes; for $20-25^{\circ}$ it is three minutes, for $25-30^{\circ}$, two and a half to two minutes. It is preferable that the solution should be shaken vigorously with a motor and the time kept down to these limits, for the sake not only of rapidity but of accuracy.

Van Slyke's Method for Free Amino-Acid Nitrogen.-To 25 c.c. of urine ${ }^{1}$ in a 50 c.c. flask add urease solution and allow to stand for one and one-half times the interval which has been found necessary to effect the maximum decomposition of urea, as observed by titration of the ammonia. The last traces of urea are decomposed. At the end of the digestion period ro c.c. of a ro per cent suspension of calcium hydroxide are added, the mixture shaken and made up to 50 c.c. Then filter, evaporate, and complete the determination according to the method outlined under total amino-acid nitrogen, above.

\section{Creatinine}

Folin's Colorimetric Method.-Principle.--This method is based upon the characteristic property possessed by creatinine, of yielding a certain definite color-reaction in the presence of picric acid in alkaline solution.

Procedure.-Place 1o c.c of urine in a 500 c.c. volumetric flask, add $15 \mathrm{cc}$. of a saturated solution of picric acid and 5 c.c. of a Io per cent solution of sodium hydroxide, shake thoroughly and allow the mixture to stand for five minutes. During this interval pour a little $\mathrm{N} / 2$ potassium bichromate solution ${ }^{2}$ into each of the two cylinders of the colorimeter (Duboscq's, see Fig. I53, p. 486) and carefully adjust the depth of the solution in one of the cylincers to the $8 \mathrm{~mm}$. mark. A few preliminary colorimetric readings may now be made with the solution in the other cylinder, in order to insure greater accuracy in the subsequent examination of the solution of unknown strength. Obviously the two solutions of potassium bichromate are identical in color and in their examination no two readings should differ more than $0.1-0.2 \mathrm{~mm}$. from the true value $(8 \mathrm{~mm}$.). Four or more readings should be made in each case and an average taken of all of them exclusive of the first reading, which is apt to be less accurate than the succeeding readings. In time as one becomes proficient in the technic it is perfectly safe to take the average of the first two readings.

At the end of the five-minute interval already mentioned, the contents of the 500 c.c. flask are diluted to the 500 c.c. mark, the bichromate solution is thoroughly rinsed out of one of the cyclinders and replaced with the solution thus prepared and a number of colorimetric readings are immediately made.

${ }^{1}$ See note 5 , page 505 .

${ }^{2}$ This solution contains 24.55 grams of potassium bichromate to the liter A pure creatinine standard is to be preferred, see p. 507 . 
Ordinarily ro c.c. of urine is used in the determination by this method, but if the content of creatinine is above $15 \mathrm{mg}$. or below $5 \mathrm{mg}$. the determination should be repeated with a volume of urine selected according to the content of creatinine. This variation in the volume of urine according to the content of creatinine is quite essential, since the method loses in accuracy when more than $15 \mathrm{mg}$. or less than $5 \mathrm{mg}$. of creatinine is present in the solution of unknown strength.

Calculation.- By experiment it has been determined that ro mg. of pure creatinine, when brought into solution and diluted to 500 c.c. as explained in the above method, yields a mixture 8 . I mm. of which possesses the same colorimetric value as $8 \mathrm{~mm}$. of a $\mathrm{N} / 2$ solution of potassium bichromate. Bearing this in mind the computation is readily made by means of the following proportion in which y represents the number of millimeters of the solution of unknown strength equivalent to the $8 \mathrm{~mm}$. of the potassium bichromate solution:

\section{$y: 8.1:: 10: x$ (mg. of creatinine in the quantity of urine used).}

This proportion may be used for the calculation no matter what volume of urine $(5$, ro, or 15 c.c.) is used in the determination. The 10 represents $10 \mathrm{mg}$. of creatinine which gives a color equal to $8.1 \mathrm{~mm}$., whether dissolved in 5 , ro, or 15 c.c. of fluid.

Calculate the quantity of creatinine in the 24 -hour urine specimen.

Interpretation. - The daily excretion of creatinine by an adult of medium weight averages about $\mathrm{I}^{1} / 4$ grams. The value is nearly constant from day to day for a given individual being influenced by the diet hardly at all unless this contains much preformed creatinine (as in case of a heavy meat diet). The excretion of creatinine is to a certain extent a measure of muscular efficiency and of the amount of active muscle tissue in the body. Relative to body weight less creatinine is excreted by obese persons.

Creatinine excretion is decreased in disorders associated with muscular atrophy and muscular weakness. It increases with increased tissue cetabolism as in fever.

By the "creatinine coefficient" is meant the number of milligrams of creatinine-nitrogen excreted daily per kilo of body weight. This varies under normal conditions from $7^{-\mathrm{I} I \text {. }}$

Use of Pure Creatinine Standards. - Instead of using as a standard a potassium dichromate solution as above indicated, a solution of pure creatinine is to be recommended. By using this certain arbitrary factors are eliminated and the method becomes of more general applicability. The standard need not be set at a definite mark as is necessary in the case of dichromate and temperature and time have less influence on the accuracy of the results. A stock solution of pure creatinine (made according to Benedict's directions; see Chapter XXII on Physiological Constituents of Urine) is made by dissolving I gram of the substance in sufticient N/10 HCI to make a liter. This solution contains I mg. of creatinine per cubic centimeter. In carrying out the determination treat ro c.c. of the stock solution in the same way and at the same time as the ro c.c. sample of urine. Compare in the colorimeter. The calculation s simple. The reading of the standard divided by' the reading of the 
urine gives directly the number of milligrams of creatinine per cubic centimeter of urine.

Folin's Microchemical Modification. ${ }^{1-P r i n c i p l e .-T h e ~ p r i n c i p l e ~ i s ~ t h e ~ s a m e ~}$ as that of the original colorimetric method (see page 506). This procedure is to be recommended particularly where only small amounts of material are available.

Procedure.--One c.c. of the standard creatinine (see above) solution (I mg. per c.c.) is measured into a Ioo c.c. volumetric flask and I c.c. of urine into another; 20 c.c. of saturated picric acid solution (measured with a cylinder) are added to each and then 1.5 c.C. of a ro per cent solution of sodium hydroxide. At the end of ten minutes the flasks are filled up to the mark with tap water and the color of the unknown is determined. The reading of the standard divided by the reading of the unknown gives directly the number of miliigrams of creatinine in the amount of urine taken for analysis.

3. Shaffer's Modification for the Determination of Creatinine in Very Dilute Solutions. ${ }^{2}$ The regular Folin procedure is not accurate when applied to urines containing less than $20 \mathrm{mg}$. of creatinine per roo c.c. By a slight modification it becomes applicable to creatinine solutions containing as little as I mg. or less per Ioo c.c.

Procedure.-To the solution under examination add an equal volume of saturated picric acid solution and one-tenth this volume of Io per cent sodium hydroxide solution. After standing 6-1o minutes the liquid is diluted to a definite volume depending upon the intensity of the color developed. With very dilute solutions one may add solid picric acid equivalent to half saturation ( 0.6 per cent) and when dissolved, one-twentieth the volume of sodium hydroxide. Provided the creatinine solution itself has not sufficient color to interfere, the results by this method appear to be as accurate as the original procedure. The colorimetric readings and calculations are made in the same way as in the preceding methods.

\section{Creatine}

Folin-Benedict Method. ${ }^{3}$ - Principle.-Creatinine on boiling with acid is transformed into creatinine. By determining the content of creatinine before and after the acid treatment we are able to calculate the amount of creatinine originally present in the urine. The Folin colorimetric method (page 506) is used for determining the creatinine in both cases. The method is not applicable to diabetic urines.

Procedure.-Introduce into a small flask or beaker Io c.c. of the urine to be examined. (If Io c.c. contains more than 12 or less than $7 \mathrm{mg}$. of total creatinine use a correspondingly smaller or larger volume of urine.) Add from ro-20 c.c. of normal $\mathrm{HCl}$, and a pinch or two of powdered or granulated lead. Boil the mixure over a free flame as slowly or as rapidly as may be desired, until very nearly down to dryness, when the heating should be continued to dryness either on the water-bath or very easily by simply holding the vessel in the hand and heating carefully for a moment or two. Let the residue stand on the waterbath for a few minutes until most of the excess of hydrochloric acid gas has been

${ }^{1}$ Folin: Jour. Biol. Chem., x 7, 469, r9 44.

${ }^{2}$ Shaffer: Jour. Biol. Chem., 18,525 , 19x4.

${ }^{3}$ Benedict: Jour. Biol. Chem., 18, r9x, 1914. 
expelled, after which dissolve it in about ro c.c. of hot water and rinse the solution quantitatively through a plug of cotton or glass wool (to remove all metallic lead) into a 500 c.c. volumetric flask. Add 20-25 c.c. of a saturated picric acid solution and about 7-8 c.c. of a ro per cent $\mathrm{NaOH}$ solution, which contains 5 per cent of Rochelle salt. ${ }^{1} \quad$ At the end of five minutes fill to the mark with water and read in the colorimeter just as in the case of creatinine (see page 506).

Calculation.-Calculate the creatinine content of the solution in the same manner as given under Creatinine (page 507). From the value thus obtained subtract the value for the creatinine content of the urine before dehydration. The difference will be the creatine content of the original urine in terms of creatinine.

Interpretation.-Creatine occurs only in very small amounts in the urine of normal adults, but is found in larger amounts in that of children (Io to $50^{\circ} \mathrm{mg}$. per day). Creatine ingestion in adults has little effect on the urinary excretion. In fasting, the amount is markedly increased (it may amount to $100 \mathrm{mg}$. or more per day). Creatine also appears in the urine after high water ingestion. It is found in many pathological conditions associated with malnutrition and disintegration of muscular tissue, in fever, etc. Very large amounts have been found in cases of carcinoma of the liver.

2. Folin-Benedict and Myers Method. ${ }^{2}$-To 20 c.c. of urine in a 50 c.c. volumetric flask, add 20 c.c. of normal hydrochloric acid and place the flask in an autoclave at a temperature of $\operatorname{II} 7-\mathrm{I}_{2} 0^{\circ} \mathrm{C}$. for one-half hour. Add distilled water until the volume of the acid-urine mixture is exactly 50 c.c., close the flask by means of a stopper, and shake it thoroughly. Approximately neutralize 25 c.c. of this mixture, introduce it into a 500 c.c. volumetric flask and determine its creatinine content according to Folin's Colorimetric Method (see page 506).

For calculation and interpretation see the foregoing method.

3. Method of Folin. ${ }^{3}$-Water-bath Procedure.-Heat Io c.c. of urine with 5 c.c. of normal hydrochloric acid on the boiling water-bath or at $90^{\circ} \mathrm{C}$. for three hours. The creatine is transformed into creatinine. Some darkening takes place but this does not interfere because of the subsequent dilution. The mixture is made up to 50 c.c., 25 c.c. of this is taken, neutralized, and creatinine plus creatine determined just as in the case of creatinine alone. The creatine is obtained by difference. This procedure may be used for diabetic urines which is not the case with the autoclave procedure nor with the Benedict modification. It is perhaps not quite so accurate as the autoclave procedure.

4. Microchemical Modification of Folin. ${ }^{4-B y}$ greatly diluting the urine the time required for the conversion of creatine to creatinine is decreased, and picric acid can be substituted for mineral acid.

Procedure.-Enough urine to give $0.7-1.5 \mathrm{mg}$. of creatinine is measured into a weighed Erlenmeyer Jena flask (capacity 200 c.c.) ; 20 c.c. of saturated picric acid solution, about 30 c.c. of water, and a few very small pebbles to promote even boiling are added and the mixture is gently boiled, preferably over a micro-burner

${ }_{1}$ The Rochelle salt should be present to prevent any formation of turbidity, which otherwise may occur, due to the presence of traces of dissolved lead.

"Benedict and Myers: A m. I. Phys., is, 397, s 907 .

${ }^{3}$ Folin: Zeitschr. f. physiol. Chem., 4I, 222, igo

4 Folin: Jour. Biol. Chem., I7, 469 , igr 4 . 
for about one hour. At the end of this time the heat is increased and the solution is boiled down to rather less than 20 c.c. The flask is transferred to the scales and enough water is added to make the total solution equal to $20-25$ grams. The solution is cooled in running water, I.5 C.C. of ro per cent sodium hydroxide are added, and the total creatinine is determined as in the preformed creatinine determination using I mg. of creatinine as a standard. The method has been found to give good results in the presence of glucose and other sugars.

Morris ${ }^{1}$ has suggested that in the case of diabetic urines the total creatinine be determined after precipitation of the creatine and creatinine with picric acid. The method is not recommended as a regular procedure.

\section{Uric Acid}

I. Microchemical Colorimetric Method.-Benedict and Hitchcock Modification of the Folin-Macallum-Denis Procedure.-The principle of the method depends upon the fact, first noted by Folin and Macallum ${ }^{2}$ and further investigated by Folin and Denis, ${ }^{3}$ that uric acid gives, with phosphotungstic acid and alkali, a deep blue color the depth of which is proportional to the amount of uric acid present. Since certain other substances present in urine produce a similar blue color with the phosphotungstic acid, it is necessary to separate the uric acid from them. This is accomplished by precipitation as the silver salt. The silver urate is subsequently dissolved and treated with the uric acid reagent.

Benedict and Hitchcock ${ }^{4}$ have examined the method of Folin and Denis and have suggested a number of important modifications.

Procedure.-Measure such an amount of urine as will contain from 0.7 to $\mathrm{r} .3 \mathrm{mg}$. of uric acid ( 2 to 4 c.c. is usually the correct amount) into a centrifuge tube, dilute with water to about 5 c.c., and add 15 to 20 drops of an ammoniacal silver magnesium solution. ${ }^{5}$ Mix the contents of the tube with a small stirring rod and centrifuge the tube for one or two minutes. Pour off the supernatant liquid, as completely as possible, by inverting the tube, allowing it to drain a moment, and then touching the inside of the lip of the tube with a towel or piece of filter paper. Add to the residue in the tube two drops of a 5 per cent solution of potassium cyanide to dissolve the silver urate, stir the mixture thoroughly with a thin rod, for half a minute, add a few drops ( 0.5 to I.o c.c.) of water, and stir again. ${ }^{6}$ Two c.c. of the uric acid reagent ${ }^{7}$ are added and the mixture stirred

1 Morris: Jour. Biol. Chem., 21, 201, I9I5.

2 Folin and Macallum: J. Biol. Chem., 13, 363, I9 2.

${ }^{3}$ Folin and Denis: J. Biol. Chem., I4, 95, 1913; ibid., I3, 469, I9I3.

4 Benedict and Hitchcock: J. Biol. Chem., 20, 6r9, I9 I5; Benedict: ibid., 20, 629, I9 I 5.

- This solution has the following composition:

3 per cent silver lactate solution. .................. 7 . c.c.

Magnesia mixture........................... 30 c.c.

Concentrated ammonium hydroxide solution.............. roo c.c.

- At this point perfectly clear solutions are obtained with pure uric acid solutions in phos. phate mixture or in pyridin. With urines some magnesium ammonium phosphate is precipitated with the uric acid, which does not dissolve in the cyanide. After adding the two subsequent reagents, however, a perfectly clear solution is obtained.

${ }^{7}$ Preparation of the Uric Acid Reagent.-Place Ioo grams of sodium tungstate, 80 c.c. of 85 per cent phosphoric acid, and $750 \mathrm{c.c}$. of distilled water in a liter flask. Boil the mixture with a reflux condenser for two hours, cool and dilute to I liter, filtering if necessary. 
again, after which add Io c.c. of 20 per cent sodium carbonate solution, ${ }^{1}$ transfer quantitatively to a 50 c.c. flask, and at the end of about one-half minute, dilute to mark. Compare this solution in the Duboscq colorimeter (page. 486) with a simultaneously prepared solution obtained by treating 5 c.c. of the standard uric acid solution, ${ }^{2}$ contained in a 50 c.c. flask, with 2 drops of the potassium cyanide solution, 2 c.c. of the uric acid reagent, 10 c.c. of 20 per cent sodium carbonate solution, and diluting to the mark at the end of about one-half minute. The standard solution is best set at a height of $15 \mathrm{~mm}$. in the colorimeter.

Calculation.-The reading of the standard divided by the reading of the urine gives the number of milligrams of uric acid in the amount of sample taken.

Interpretation.-For adults on a mixed diet the average excretion of uric acid is about 0.7 gram. It arises from the purines of ingested food (exogenous uric acid) and from purines derived from the body tissues by disintegration of nuclein material (endogenous uric acid). Exogenous uric acid depending entirely upon the diet is greatly increased by the ingestion of purine-rich foods (meat, liver, sweetbreads, etc.) and reduced to a very low level on purine-free foods, e.g., milk, eggs, etc. (see Chapter XXVII). Endogenous uric acid is influenced by exercise and by the diet (protein foods particularly giving rise to increases). It appears to be partly the result of gastro-intestinal secretory activity. On a purine-free diet the average excretion is $0.1-0.5$ gram. On a high purine diet the uric acid output may be 2 grams per day.

In gout the uric acid content of the urine is low preceding an attack and increases during the attack, this fall and rise being more or less characteristic. The excretion rises after atophan administration apparently due to increased kidney activity. In leukemia the excretion is extremely high due to nuclear destruction. The uric acid content of the urine is of importance in relation to the formation of uric acid calculi. The administration of alkali carbonates and citrates by decreasing the acidity of the urine increases its solvent power for uric acid, and decreases the liability of the formation of this type of calculus.

3. Folin-Shaffer Method. ${ }^{3}$ - Principle.-The uric acid is precipitated as ammonium urate by the addition of ammonia, the precipitate filtered off, washed and titrated with potassium permanganate. A prelininary

${ }^{1}$ Sodium Carbonate Solution.-Dissolve 200 grams of anhydrous sodium carbonate in warm water and make up to $\mathrm{r}$ liter.

${ }^{2}$ Standard Uric Acid Solution.-The solution of uric acid in phosphate solution is very readily prepared, does not need to be standardized, and appears to keep indefinitely. It is prepared in the following manner. Dissolve 9 grams of pure crystallized disodium hydrogen phosphate, together with I gram of crystallized sodium dihydrogen phosphate, in 200 to 300 c.c. of hot water, and filter if the solution is not perfectly clear. Make this filtrate up to about 500 c.c. with hot water, and pour this hot or warm (and perfectly clear) solution upon exactly $200 \mathrm{mg}$. of pure uric acid suspended in a few cubic centimeters of water in a liter volumetric Dask. Agitate the mixture for a few minutes until the uric acid completely dissolves. Cool, add exaclly I.4 c.c. of glacial acetic acid, dilute to the mark, and mix. Add about 5 c.c. of chloroform to prevent the growth of bacteria or moulds in the solution. Five c.c. of this solution contains exactly i mg. of uric acid.

${ }^{3}$ Folin and Shaffer: Zcit. physiol. Chcm., 32, 552, 190r. 
treatment with an ammonium sulphate-uranium acetate solution is for the purpose of removing interfering organic substances. The method gives accurate results.

Procedure.-Introduce Ioo c.c. ${ }^{1}$ of urine into an Erlenmeyer flask, add 25 c.c. of the Folin-Shaffer reagent ${ }^{2}$ and after shaking the flask to thoroughly mix the fluids allow the mixture to stand, ${ }^{3}$ with or without further stirring, until the precipitate has settled (5-10 minutes). Filter, transfer roo c.c. of the filtrate to a 200 c.c. Erlenmeyer flask, add 5 c.c. of concentrated ammonium hydroxide and allow the mixture to stand for 24 hours. Transfer the precipitated ammonium urate quantitatively to a filter paper, ${ }^{4}$ using ro per cent ammonium sulphate to remove the final traces of the urate from the flask. Wash the precipitate approximately free from chlorides by means of ro per cent ammonium sulphate solution, ${ }^{5}$ remove the paper from the funnel, open it, and by means of hot water rinse the precipitate back through the funnel into the flask in which the urate was originally precipitated. The volume of fluid at this point should be about I00 c.c. Cool the solution to room temperature, add I5 c.c. of concentrated sulphuric acid and titrate at once with $\mathrm{N} / 20$ potassium permanganate, $\mathrm{K}_{2} \mathrm{Mn}_{2} \mathrm{O}_{8}$, solution. The first tinge of pink color which extends throughout the fluid after the addition of two drops of the permanganate solution, while stirring with a glass rod, should be taken as the end-reaction. Take the burette reading and compute the percentage of uric acid present in the urine under examination.

Calculation.-Each cubic centimeter of $\mathrm{N} / 20$ potassium permanganate solution is equivalent to $3.75 \mathrm{mg}$. (0.00375 gram) of uric acid. The roo c.c. from which the ammonium urate was precipitated is equivalent to only four-fifths of the roo c.c. of urine originally taken; therefore we must take five-fourths of the burette reading in order to ascertain the number of cubic centimeters of the permanganate solution required to titrate roo c.c. of the original urine to the correct end point. If $y$ represents the number of cubic centimeters of the permanganate solution required, we may make the following calculation:

$$
\mathrm{y} \times 0.00375=\text { weight of uric acid in } 100 \text { c.c. of urine. }
$$

Because of the solubility of the ammonium urate a correction of $3 \mathrm{mg}$. should be added to the final result.

Calculate the quantity of uric acid in the 24-hour urine specimen.

4. Heintz Method.-This is a very simple method and was the first one in general use for the quantitative determination of uric acid. It is less accurate than the methods just described. The procedure is as follows: Place roo c.c. of filtered urine in a beaker, add 5 c.c. of concentrated hydrochloric acid, stir the fluid thoroughly, and stand it away in a cool place for 24 hours. Filter off the uric acid crystals upon a washed, dried and weighed filter paper and wash them with cold distilled water, a few cubic centimeters at a time, until the chlorides are removed.

${ }^{1}$ It is preferable to use more than roo c.c. of urine if the fluid has a specific gravity less than 1.020 .

${ }^{2}$ The Folin-Shaffer reagent consists of 500 grams cf ammonium sulphate, 5 grams of uranium acetate and 60 c.c. of ro per cent acetic acid in 650 c.c. of distilled water.

${ }^{3}$ The mixture should not be allowed to stand for too long a time at this point, since uric acid may be lost through precipitation.

4 The Schleicher and Schüll hardened papers or the Baker and Adamson washed, askless variety are very satisfactory for this purpose.

${ }^{5}$ This washing may be conveniently done by decantation if desired, thus retaining the major portion of the precipitate in the flask. 
Now wash, in turn, with alcohol and with ether and finally dry the paper and crys tals to constant weight at $110^{\circ} \mathrm{C}$. In the process of washing the uric acid free from chlorides an error is introduced, since every cubic centimeter of water so used dissolves 0.00004 gram of uric acid. ${ }^{1}$ For this reason a correction is necessary. It has been suggested that the pigment of the crystals is equivalent in weight to the amount of uric acid dissolved by the first 30 c.c. of water, and this factor should be taken into account in the computation of the percentage of uric acid.

Calculation.-Since roo c.c. of urine was used the corrected weight of the uric acid crystals, in grams, will express the percentage of uric acid present.

5. Krüger-Schmidt Method.-Krüger and Schmidt have devised a method for the combined determination of uric acid and the other purine bodies of urine. This procedure is described under Purine Bases, below: A modification of this method by Hunter is also given.

6. Ruhemann's Uricometer Method.-Principle.-When iodine solution is added to urine an amount of iodine is absorbed roughly proportional to the amount of the uric acid present. A special graduated tube is required and carbon disulphide is used to indicate the presence of excess iodine.

Procedure.-Fill the tube (see Fig. I65) to the lowest mark S with carbon disulphide (the bottom of the meniscus should rest on the line). Then add iodine solution ( 1.5 grams iodine, I.5 grams potassium iodide, I 5 grams absolute alcohol, and ${ }_{5}$ grams distilled water) to the mark J. Add urine to mark 2.45 (2.6 c.c.). Insert the glass stopper and shake. The carbon disulphide will show the dark brown color of iodine. Add more urine gradually with continued shaking until only a violet-pink color remains in the disulphide after shaking. Then add drop by drop with vigorous shaking until the disulphide becomes colorless. Read on the scale the amount of uric acid in parts per thousand of urine.

When the uric acid content of the urine is low this method gives reasonably accurate results from the clinical standpoint. ${ }^{2}$ At a level of 0.3 gram per day the probable error will not exceed IO-I 5 per cent. Above I gram no limit can safely be placed on the degree of error in pathological cases especially where albumin, diacetic acid, purine bases and certain drugs are likely to be present. The results by this method are ordinarily high.

\section{Purine Bases}

I. Krüger and Schmidt's Method.-Principle.-This method serves for the determination of both uric acid

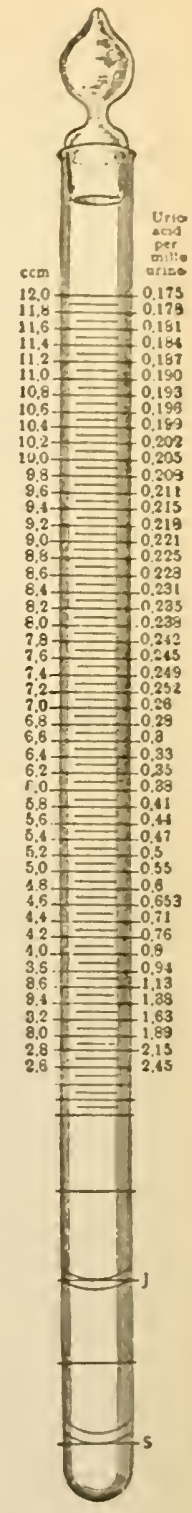

FIG. $165 .-$ RTHEMAN'S U'RICOMETER. and the purine bases. The principle involved is the precipitation of both the uric acid and the purine bases in combination with copper

${ }^{1}$ His and Paul: Zeit. physiol. Chem., 3r, I, I900.

${ }^{2}$ Bradley and Bunta: Jour. A m. J I cd. Assn., Jan. 4, I9:3. 
oxide and the subsequent decomposition of this precipitate by means of sodium sulphide. The uric acid is then precipitated by means of hydrochloric acid and the purine bases are separated from the filtrate in the form of their copper or silver compounds. The nitrogen content of the precipitates of uric acid and purine bases is then determined by means of the Kjeldahl method (see page 483 ) and the corresponding values for uric acid and purine bases calculated.

Procedure.-To 400 c.c. of albumin-free urine ${ }^{1}$ in a liter flask ${ }^{2}$, add 24 grams of sodium acetate, 40 c.c. of a solution of sodium bisulphite ${ }^{3}$ and heat the mixture to boiling. Add 40-80 c.c. ${ }^{4}$ of a 10 per cent solution of copper sulphate and maintain the temperature of the mixture at the boiling-point for at least three minutes. Filter off the flocculent precipitate, wash it with hot water until the wash water is colorless, and return the washed precipitate to the flask by puncturing the tip of the filter paper and washing the precipitate through by means of hot water. Add water until the volume in the flask is approximately 200 c.c., heat the mixture to boiling and decompose the precipitate of copper oxide by the addition of 30 c.c. of sodium sulphide solution. ${ }^{5}$ After decomposition is complete, the mixture should be acidified with acetic acid and heated to boiling until the separating sulphur collects in a mass. Filter the hot fluid by means of a filter-pump, wash with hot water, add ro c.c. of Io per cent hydrochloric acid and evaporate the filtrate in a porcelain dish until the total volume has been reduced to about ro c.c. Permit this residue to stand about two hours to allow for the separation of the uric acid, leaving the purine bases in solution. Filter off the precipitate of uric acid, using a small filter paper, and wash the uric acid, with water made acid with sulphuric acid, until the total volume of the original filtrate and the wash water aggregates 75 c.c. Determine the nitrogen content of the precipitate by means of the Kjeldahl method (see page 483 ), and calculate the uric acid equivalent. ${ }^{6}$

Render the filtrate from the uric acid crystals alkaline with sodium hydroxide, add acetic acid until faintly acid and heat to $70^{\circ} \mathrm{C}$. Now add I c.c. of a ro per cent solution of acetic acid and ro c.c. of a suspension of manganese dioxide ${ }^{7}$ to oxidize the traces of uric acid which remain in the solution. Agitate the mixture for one minute, add ro c.c. of the sodium bisulphite solution ${ }^{8}$ and 5 c.c. of a ro per cent solution of copper sulphate and heat the mixture to boiling for

${ }^{1}$ If albumin is present, the urine should be heated to boiling, acidified with acetic acid, and filtered.

${ }^{2}$ The total volume of urine for the 24 hours should be sufficiently diluted with water to make the total volume of the solution $1600-2000$ C.c.

${ }^{3}$ A solution containing 50 grams of Kahlbaum's commercial sodium bisulphite in 100 c.c. of water.

The exact amount depending upon the content of the purine bases.

${ }^{-} \mathrm{This}$ is made by saturating a I per cent solution of sodium hydroxide with hydrogen sulphide gas and adding an equal volume of I per cent sodium hydroxide.

Ordinarily the addition of 30 c.c. of this solution is sufficient, but the presence of an excess of sulphide should be proven by adding a drop of lead acetate to a drop of the solution. Under these conditions a dark brown color will show the presence of an excess of sodium sulphide.

'This may be done by multiplying the nitrogen value by three and adding $31 / 2 \mathrm{mg}$. to the product as a correction for the uric acid remaining in solution in the 75 c.c.

${ }^{7}$ Made by heating a 0.5 per cent solution of potassium permanganate with a little alcohol until it is decolorized.

${ }^{8}$ To dissolve the excess of manganese dioxide. 
three minutes. Filter off the precipitate, wash it with hot water, and determine its nitrogen content by means of the Kjeldahl method (see page 483). Inasmuch as the composition and proportion of the purine bases present in urine is variable, no factor can be applied. The result as regards these bases must therefore be expressed in terms of nitrogen.

Benedict and Saiki ${ }^{1}$ report cases in which the total purine nitrogen by this method. was less than the uric-acid nitrogen as determined by the Folin-Shaffer method. The inaccuracy was found to lie in the Krüger and Schmidt method. To obviate this they advise the addition of 20 c.c. of glacial acetic acid for each 300 c.c. of urine employed, the acid being added before the first precipitation.

Interpretation. - The amount of purine bases cxcreted by a normal man is small and variable. Values from I6-60 $\mathrm{mg}$. have been found. The purine base nitrogen is of course only a fraction of this. The amount excreted is influenced by the diet somewhat in the same way as is the excretion of uric acid being also increased in disorders associated with increased uric acid excretion such as leukemia. The purine bases form a higher percentage of the total purine excretion in the case of the monkey, sheep, and goat than in the case of man.

2. Hunter and Givens' Modification of Kriger-Schmidt Method. ${ }^{2}$ Principle.-The Krüger-Schmidt process is combined with the microchemical colorimetric method for uric acid (see page 510).

Procedure.-The first copper-purine precipitate as obtained in the KrigerSchmidt procedure is suspended in about 200 c.c. of water, to which there is added about I c.c. of concentrated hydrochloric acid. The mixture is vigorously boiled, whereupon the whole or greater part of the precipitate goes into solution. Removal of the copper is effected by treatment with hydrogen sulphide in the heat, and excess of the sulphide is completely expelled by renewed boiling. Filtration under suction, and thorough washing of flask and filter result in a filtrate which is perfectly clear and nearly colorless. This is concentrated if necessary, and made up to a convenient volume which must of course be sufficiently large to retain, when cool, the uric acid in solution. Of this an aliquot part is utilized directly for the colorimetric determination of uric acid. In the remainder the residual uric acid is destroyed and bases determined according to the regular Krüger-Schmidt procedure. This modification is recommended particularly where the amount of uric acid present is minute.

3. Welker's Modification of the Methods of Arnstein and of Salkowski. ${ }^{3}$ Principle.-The phosphates are removed by treatment with magnesia mixture. The purine bases and uric acid are then thrown down as their silver salts and the nitrogen content of this precipitate determined.

Procedure.-Four hundred c.c. of urine, free from protein, are treated with roo c.c. of magnesia mixture and 600 c.c. of water. This is then filtered and of the clear filtrate a measured quantity (600-800 c.c.) is treated with an excess (ro c.c.) of a 3 per cent silver nitrate solution. Concentrated ammonium hydroxide is added in small quantities, with stirring, until all the chlorides have dissolved.

1 Benedict and Saiki: Jour. Biol. Chem., 7, 27, I 909.

${ }^{3}$ Hunter and Givens: Jour. Biol. Chem., 17, 37, I914.

${ }^{3}$ Dittman and Welker: New Fork Med. Jour., May-June, rgog. 
Allow the flocculent precipitate of the silver purine compounds to settle to the bottom, then pass the supernatant liquid through the filter before disturbing the precipitate. Finally transfer the precipitate quantitatively to the paper which must be of known nitrogen content. The precipitate is washed with dilute (I per cent) ammonium hydroxide. The paper with the precipitate is then transferred to a Kjeldahl flask and about roo c.c. of water and a small quantity (about o.I gram) of magnesium oxide are added. The water is then boiled until all the ammonia has been driven off. Test the steam with litmus paper.

The material in the flask is then digested by means of the usual K jeldahl method (see page ${ }_{4} 8_{3}$ ). The digestion must be watched carefully at the time the sulphuric acid reaches sufficient concentration to affect the filter paper, inasmuch as the $\mathrm{SO}_{2}$ produced causes considerable frothing. The total nitrogen (purine base, uric acid and filter-paper nitrogen) is now determined in the usual way (see K jeldahl Method, page 483). This result minus the uric acid and filter-paper nitrogen will give the figure for the purine-base nitrogen.

4. Salkowski's Method.-Principle.-The purin bases and uric acid are precipitated as silver-magnesium salts, these compounds decomposed with hydrogen sulphide, and the uric acid precipitated by means of sulphuric acid. The purin bases in the filtrate are again precipitated as their silver salts and the silver content of the precipitate determined after ignition by titration with thiocyanate.

Procedure.-Place 400-600 c.c. of protein-free urine in a beaker. Introduce into another beaker $30-50$ c.c. of an ammoniacal silver nitrate solution ${ }^{1}$ wih $30-50$ c.c. of magnesia mixture, ${ }^{2}$ add some ammonium hydroxide and if necessary some ammonium chloride to clear the solution. Now add this solution to the urine, stirring continually with a glass rod, and allow the mixture to stand for one-half hour. Collect the precipitate on a filter paper, wash it with dilute ammonium hydroxide, and finally wash it back into the original beaker. Suspend the precipitate in 600-800 c.c. of water, add a few drops of hydrochloric acid and decompose it by means of hydrogen sulphide. Now heat the solution to boiling, filter while hot and evaporate the filtrate to dryness on a water-bath. Extract the residue with $20-30$ c.c. of hot 3 per cent sulphuric acid and allow the extract to stand 24 hours. Filter off the uric acid, wash it, make the filtrate ammoniacal and precipitate the purine bases again with silver nitrate. Collect this precipitate on a small-sized chlorine-free filter paper, wash, dry, and incinerate it in the usual manner. Now dissolve the ash in nitric acid and titrate with ammonium thiocyanate according to the Volhard-Arnold method (see page 556). Calculate the content of purine bases in the urine examined, bearing in mind that in an equal mixture of the silver salts of the purine bases, such as we have here, one part of silver corresponds to 0.277 gram of nitrogen or to $0.738 \mathrm{I}$ gram of the bases.

For interpretation see page 5 I 5 .

\section{Purine Nitrogen}

Hall's Purinometer. ${ }^{3}$-By means of the instrument shown in Fig. I66, urine may be examined for total purine nitrogen, i.e., nitrogen in the form of purine bases,

${ }^{1}$ Prepared by dissolving 26 grams of silver nitrate in about 500 c.c. of water, adding enough ammonium hydroxide to redissolve the precipitate which forms upon the first addition of the ammonia and making the balance of the mixture up to I liter with water.

${ }^{2}$ Directions for preparation may be found on p. 602 .

${ }^{3}$ Hall: The Purine Bodies, Philadelphia, r904. 
urates and uric acid. The method does not give an absolutely accurate measure of the purine values. It is, however, of considerable service clinically. The principle of the method is the preliminary precipitation of the phosphates present followed by the precipitation of the purine bodies in the form of their silver compounds by means of an ammoniacal silver nitrate solution. The volume of this silver precipitate is then determined and its nitrogen value interpolated by means of a table of equivalent values.

Procedure.-Collect the 24 -hour urine and mix it thoroughly. Take roo c.c. of the urine and if albumin is present make slightly acid with acetic acid and boil and filter. Close the stopcock of the instrument and introduce 90 c.c. of urine and 20 c.c. of a modified magnesia mixture. ${ }^{1}$ Turn the stopcock and permit the precipitated phosphates to pass into the lower chamber of the instrument. After an interval of ten minutes has elapsed the stopcock should be closed and sufficient ammoniacal silver nitrate solution ${ }^{2}$ added to make the total volume in the upper chamber roo c.c. The precipitate of the silver compounds of the purine bodies should be pale yellow. Any silver chloride present may be brought into solution in the strong ammoniacal solution by the repeated inversion of the purinometer. In case the chloride does not dissolve it should be brought into solution by the addition of further ammonium hydroxide. Place the purinometer in a dark room for 24 hours and at the end of this time read the volume of the purine precipitate. Interpolate the value in terms of purine nitrogen by means of the following table:

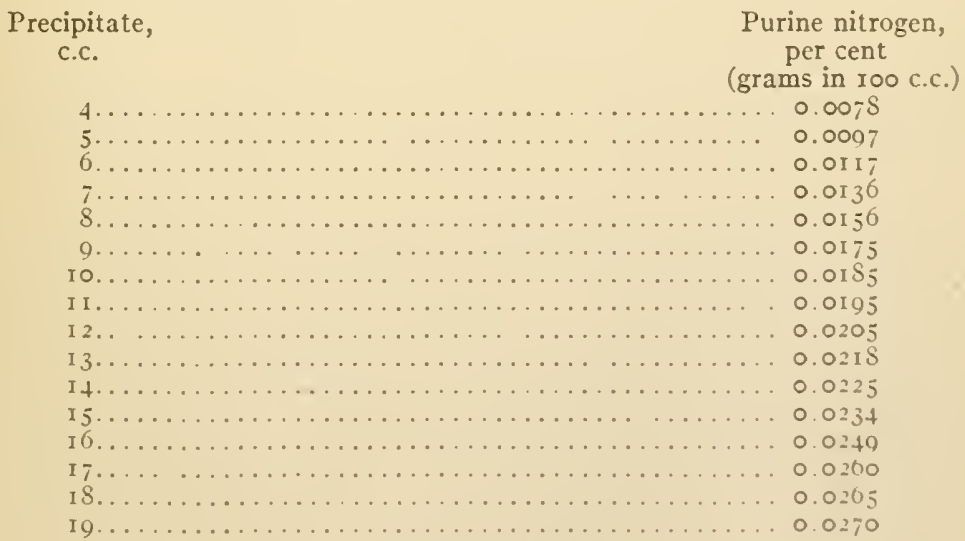

I This is prepared as follows: Dissolve ro grams of magnesium chloride in about 75 c.c. of water and add to grams of ammonium chloride. Introduce too c.c. uf concentrated ammonium hydroxide into this mixture. If a precipitate forms adel ammonium hydroxide until a clear solution is obtained. Nake the volume 200 c.c. by means of water and add ro grams of purified talcum.

${ }^{2}$ This solution has the following formula:

Silver nitrate.

Ammonium hydroxide (sp. gr. o.po).

I gram.

Talcum

100 c.c.

Distilled water.

5 grams. roo c.c. 


\begin{tabular}{|c|c|}
\hline $\begin{array}{l}\text { Precipitate, } \\
\text { c.c. }\end{array}$ & $\begin{array}{l}\text { Purine nitrogen, } \\
\text { per cent } \\
\text { (grams in } 100 \text { c.c.) }\end{array}$ \\
\hline 20.. & $\ldots \ldots 0.0275$ \\
\hline $2 \mathrm{I}$. & $\ldots .0 .0283$ \\
\hline 22. & $\ldots .0 .0286$ \\
\hline 23. & 0.0299 \\
\hline 24. & $\cdots 0.0312$ \\
\hline 25. & 0.0325 \\
\hline
\end{tabular}

Calculation.-Multiply the purine nitrogen percentage by the total volume of urine and divide by roo to obtain the total purine nitrogen value. For example, if the precipitate was found to be 12 c.c. and the total volume of the 24 -hour urine was I 300 c.c. the calculation would be as follows:

$$
\begin{aligned}
& \text { I } 2 \text { c.c. }=0.0205 \text { per cent purine nitrogen. } \\
& 0.0205 \times 13.0=0.2665 \text { gram purine nitrogen. }
\end{aligned}
$$

Interpretation.-The endogenous purine nitrogen excretion (on purine-free diet) averages about 0.15 gram per day, though variations are considerable. As uric acid makes up about nine-tenths of this the amount varies much as uric acid varies. The exogenous purine nitrogen excretion may be largely influenced by the methyl purines of tea, coffee, and cocoa which are excreted unchanged, as well as by the purine bases which are normally oxidized to uric acid (see Uric Acid, page 5II).

\section{Allantoin}

I. Method of Wiechowski-Handovsky. ${ }^{1-P r i n c i p l e .-T h e ~ u r i n e ~ i s ~ p r e c i p i t a t e d ~}$ with phosphotungstic acid and lead acetate and in the presence of chlorides with silver acetate. The heavy metals are removed with hydrogen sulphide. The allantoin is then precipitated as a mercuric compound and the amount of mercury and hence of allantoin in the precipitate determined by titration with ammonium thiocyanate. This method, though rather tedious, is probably the most accurate method for the determination of allantoin.

Procedure.-The urine is diluted to about I per cent urea. As rabbit urine contains in the day's output about 2-4 grams of urea, and that of other herbivora usually forms about a 4 per cent urea solution, it is usually desirable to dilute $3-4$ times. A greater dilution is not desirable. The urine is treated with I per cent of sulphuric acid and about 3 c.c. of acetic acid for each day's volume. Test a small quantity of the urine to determine the amount of 50 per cent phosphotungstic acid required to completely precipitate it. The bulk of the urine is then treated on this basis with sufficient solid phosphotungstic acid to precipitate it completely. Stir to dissolve the acid and allow to stand for several hours. Filter with suction, first lining the filter with infusorial earth by rubbing up a little of the substance with some of the urine mixture and filtering with suction. To some ordinary lead oxide in a mortar add a small amount of the filtrate and stir until it becomes warm, then add the rest of the filtrate and stir, adding more oxide if necessary until the solution reacts alkaline due to the formation of basic lead acetate.

Filter again. The filtrate should give no precipitate with basic lead acetate. A measured volume of the filtrate is then treated with measured volumes of acetic acid and silver nitrate solution to completely precipitate any chlorides present. Filter again preferably through infusorial earth. Pass hydrogen sulphide through

1 Handovsky: Zeit. physiol. Chem., 90, 2 I I, rgr4.

Wiechowski: Neubauer-Huppert: Analyse des Harns, Wiesbaden, rg13, p. ro76. 
the filtrate until the heavy metals are completely precipitated. Filter again. Pass a current of air through the filtrate to remove all hydrogen sulphide (test with lead acetate paper). Neutralize this final filtrate with calcium carbonate and remove the carbon dioxide with a current of air.

The neutral filtrate is then treated with an amount of allantoin reagent in excess of that required to precipitate the allantoin as indicated by a preliminary test. (The allantoin reagent is a solution containing 5 per cent mercuric acetate and 20 per cent of sodium acetate. The reagent when used must be clear.)

Allow to stand for half an hour (not too long) and then filter. A measured amount of the filtrate is taken and treated with about ro c.c. of iron-ammonium alum and the red solution decolorized with dilute sulphuric acid. (The solution is not completely decolorized but retains a faint greenish tint.) Any calcium sulphate precipitate formed at this point may be disregarded. Titrate with $\mathrm{N} /$ ro ammonium thiocyanate solution to a yellow color, which increases in intensity on the addition of $\mathrm{I}-2$ drops more of solution. The titration should not be carried out in artificial light and some practice is required to get the exact end point. The thiocyanate solution should be standardized occasionally against standard silver nitrate solution.

Calculation.-One c.c. of $\mathrm{N} / \mathrm{I}$ o $\mathrm{NH}_{4} \mathrm{SCN}^{\top}$ solution corresponds to 0.00436 gram of allantoin. The limit of error of the method is about $5 \mathrm{mg}$. for the daily output of allantoin. In the calculation of course all losses through filtration, etc., must be considered.

For the considerable modifications required in carrying out the determination on human urine with its very low content of allantoin see the section by Wiechowski in Neubauer-Huppert. ${ }^{1}$

Interpretation.-Allantoin may be found in very small amounts in human urine ( 5 - 5 mg. per day), appearing to be mainly, though not entirely, exogenous in origin. It forms, however, the principal end-product of the purine metabolism of practically all mammals other than man and the anthropoid apes. Thus over go per cent of the purine-allantoin nitrogen excretion of the dog, the cow, and the pig occurs in this form. In these animals its origin is from exogenous and endogenous purines, and its excretion is influenced by much the same factors as is that of uric acid in man.

2. Determination by Difference.-Method of Plimmer and Skelton. ${ }^{2}$ - Allantoin is transformed into urea and determined as such in the acid-magnesium chloride method of Folin for urea in urine (page 495). Urease, however, has no effect upon allantoin. Therefore, determine the urea + allantoin of the urine according to Folin's procedure (see Urea), and also determine the true urea according to the urease method (see Urea). The difference between the results thus obtained represents allantoin. If sugar is present it must be removed before applying Folin's procedure.

\section{Hippuric Acid}

I. Method of Folin and Flanders. ${ }^{3}$ - Principle.- The hippuric acid is hydrolyzed to benzoic acid in alkaline solution and then the solution is boiled with strong nitric acid to remove pigments and emulsifying

1 Wiechowski: Neubater-Huppert: Analyse des Harns, Wiesbaden, 19r3, p. 10;6.

2 Plimmer and Skelton: Bioch. J., 8, 70 and 64I, I9I4.

${ }^{3}$ Folin and Flanders Jour. Biol. Chem, I1, 257, I912. 
substances. The benzoic acid is extracted with chloroform and titrated with sodium ethylate.

Procedure.-Measure roo c.c. of urine into a porcelain evaporating dish by means of a pipette. Add ro c.c. of 5 per cent $\mathrm{NaOH}$ and evaporate to dryness on the steam-bath. Transfer the residue to a 500 c.c. Kjeldahl flask by means of 25 c.c. of water and 25 c.c. of concentrated $\mathrm{HNO}_{3}$. Add 0.2 gram of copper nitrate, a couple of pebbles or glass pearls and boil very gently for four and onehalf hours over a micro-burner. Fit the necks of the flasks with condensers of the Hopkins type made from large test-tubes fitted with two-hole rubber stoppers, the inlet tubes extending near the bottom of the test-tubes while the outlet tube is shorter. These condensers should fit rather loosely. A good current of water flowing through the condensers prevents loss of benzoic acid or change in concentration of the nitric acid.

After cooling, rinse the condensers down with 25 c.c. of water and transfer the contents of the flask to a 500 c.c. separatory funnel, with the aid of 25 c.c. more of water. The total volume of the solution is now roo c.c. Add to the solution sufficient ammonium sulphate to just saturate it (about 55 grams). Make four extractions with freely washed chloroform, using $50,35,25$, and 25 c.c. portions. The first two portions may be used to further rinse out the Kjeldahl flask.

Collect the successive portions of chloroform in another separatory funnel. Add to the combined extracts Ioo c.c. of a saturated solution of pure sodium chloride, to each liter of which has been added 0.5 c.c. of concentrated $\mathrm{HCl}$. Shake well, draw the chloroform into a dry 500 c.c. Erlenmeyer flask and titrate with $\mathrm{N} / \mathrm{ro}$ sodium alcoholate, ${ }^{3}$ using 4 or 5 drops of phenolphthalein as an indicator. The first distinct end point should be taken, although it may fade on standing a short time.

Calculation.-Multiply the number of cubic centimeters of alcoholate used by the factor for hippuric acid as determined by standardization to obtain the amount of hippuric acid in the 100 c.c. of urine used. One c.c. of exactly N/1o sodium alcoholate is equivalent to $0.0179 \mathrm{gram}$ of hippuric acid. Calculate the daily output of hippuric acid from the 24 -hour volume.

Interpretation.-The average excretion of hippuric acid by a normal adult man is about 0.7 gram per day. The amount is increased by the ingestion of benzoic acid or fruits containing it (plums, prunes, cranberries). It arises in part apparently from putrefaction products formed in the intestine. In herbivora it is often the most abundant nitrogenous constituent of the urine.

2. Dakin's Methods. ${ }^{2}$ - Preliminary Procedure.-Place I 50 c.c. (or more) of the urine under examination in a porcelain evaporating dish and evaporate almost to dryness upon a water-bath. Add about I gram of sodium dihydrogen phosphate,

${ }^{1}$ The sodium alcoholate is made by dissolving 2.3 grams of cleaned metallic sodium in $I$ liter of absolute alcohol. It is advisable that it be slightly weaker rather than stronger than tenth-normal. It may be standardized against pure benzoic acid in washed chloroform. It may also be standardized against $\mathrm{N} / \mathrm{Io} \mathrm{HCl}$ provided the alcoholate solution contains not more than traces of carbonate.

${ }^{2}$ Private communication to the author from Dr. H. D. Dakin. 
about 25 grams of gypsum $\left(\mathrm{CaSO}_{4}, 2 \mathrm{H}_{2} \mathrm{O}\right)$ and rub up with a pestle and stir with a spatula until a uniform mixture results. Dry the powder thus produced in a wateroven for about two hours, at the end of which period it should be rubbed up a second time, to remove lumps, and transferred to a Schleicher and Schüll "cxtraction shell" and extracted in a Soxhlet apparatus in the usual way (see page 326 ). The extraction medium is ethyl acetate and the flask containing the acetate should be strongly heated over a sand-bath ${ }^{1}$ for about two hours. The ethyl acetate extract is now transferred to a separatory funnel, and the original flask rinsed with sufficient fresh ethyl acetate to make the total volume in the separatory funnel ${ }^{2}$ about 100 c.c. Wash the ethyl acetate solution five times with a saturated solution of sodium chloride, using 8 c.c. of the sodium chloride solution at each extraction, shaking vigorously and removing the sodium chloride extract in each case before adding fresh sodium chloride solution. The sodium chloride removes the urea completely and the hippuric acid is then determined in the urea-free solution by the following volumetric or gravimetric procedure:

I. Volumetric Dctcrmination.-Transfer the urea-free ethyl acetate solution, prepared as described above, to a $\mathrm{K}$ jeldahl flask, add about 25 c.c. of water, a small piece of pumice stone to prevent bumping, attach a condenser and distil off the acetate $^{3}$ over a free flame. After practically all of the ethyl acetate has been distilled off, the nitrogen in the remaining solution should be determined by means of the Kjeldahl method (sec page $48_{3}$ ).

The main source of error in this method is the fact that any nitrogen present in the form of phenaceturic acid or indole acetic acid is determined as hippuric acid nitrogen. The error from this source is, however, usually trifling.

Calculation.--Calculate as usual for nitrogen determinations, remembering that I c.c. of $\mathrm{N} / \mathrm{IO}$ sulphuric acid is equivalent to 0.0179 gram hippuric acid.

\section{Interpretation.-See page 520.}

2. Gravimetric Determination.-The urea-free ethyl acetate solution contained in the separatory funnel, after washing with sodium chloride solution, as described under Preliminary Procedure, page 520 , is washed with 5 c.c. of distilled water to remove the major portion of the sodium chloride. Transfer the solution from the separatory funnel to a round-bottomed flask and subject it to a steam distillation in the usual way. A slow current of stean should be used while the ethyl acetate is being distilled off and later a more rapid current may be employed. The distillation should be continued for 20 minutes. Now add about O. I gram of charcoal to the aqueous solution which is heated to boiling and filtered hot. Evaporate the solution in a weighed Jena glass dish on a water-bath until the volume of the solution is reduced to about 3 c.c. Stand the dish in a warm place until evaporation is complete and a crystalline residue remains. Wash the residue, in turn, with z c.c. of dry ether and I c.c. of water, dry it in an air-bath at $100^{\circ} \mathrm{C}$. and weigh. If it is so desired the residue may be recrystallized from a little hot water and the melting-point determined. Pure hippuric acid melts at $I S 7^{\circ} \mathrm{C}$. Contamination with phenaceturic acid may be detected both by the melting-point and the microscopical charactcristics.

1 w water-bath cannot be substituted inasmuch as the resultant extraction would be too slowi.

${ }^{2}$ This ethyl acetate solution contains hippuric acid, urea, and other substances.

${ }^{3}$ The ethyl acetate after separation from the watery layer of the distillate may be dried over calcium chloride and used again. 


\section{Glucose}

I. Benedict's Method. ${ }^{1-P r i n c i p l e-B e n e d i c t ' s ~ r e a g e n t ~ f o r ~ t h e ~ e s t i-~}$ mation of reducing sugars contains potassium thiocyanate as well as copper sulphate, and in the presence of the former a white precipitate of cuprous thiocyanate is formed on reduction instead of the usual red precipitate of cuprous oxide. The small amount of potassium ferrocyanide also aids in keeping cuprous oxide in solution. As the precipitate formed is white the loss of all blue tint in the solution, indicating complete reduction of the copper, is readily observed. The alkali used is sodium carbonate, which has the advantage over the hydroxides in that there is less danger of destruction of small amounts of sugar. The solution also has the great advantage of being stable for an indefinite length of time. The method is recommended for simplicity and accuracy.

Procedure.--The urine, ro c.c. of which should be diluted with water to 100 c.c. (unless the sugar content is believed to be low, when it may be used undiluted), is poured into a 50 c.c. burette up to the zero mark. Twenty-five c.c. of the reagent ${ }^{2}$ are measured with a pipette into a porcelain evaporation dish $(25-30 \mathrm{~cm}$. in diameter), ro to 20 grams of crystallized sodium carbonate (or one-half the weight of the anhydrous salt) are added, together with a small quantity of powdered pumice stone or talcum, and the mixture heated to boiling over a free flame until the carbonate has entirely dissolved. The diluted urine is now run in from the burette, rather rapidly, until a chalk-white precipitate forms and the blue color of the mixture begins to lessen perceptibly, after which the solution from the burette must be run in a few drops at a time, until the disappearance of the last trace of blue color, which marks the end point. The solution must be kept vigorously boiling throughout the entire titration. If the mixture becomes too concentrated during the process, water may be added from time to time to replace the volume lost by evaporation.

Calculation.-The calculation of the percentage of sugar in the original sample of urine is very simple. The 25 c.c. of copper solution are reduced by exactly 50 $\mathrm{mg}$. of glucose. Therefore the volume run out of the burette to effect the reduction contained $50 \mathrm{mg}$. of the sugar. When the urine is diluted $\mathrm{r}: \mathrm{ro}$, as in the

\footnotetext{
${ }^{1}$ Benedict: Jour. Am. Med. Ass'n, 57, Ir93, I9r r.

${ }^{2}$ Copper sulphate (crystallized) .................... 18.0 grams.

Sodium carbonate (crystallized, one-half the weight of the the anhydrous salt may be used $) \ldots \ldots \ldots \ldots \ldots \ldots \ldots$ 200.0 grams.

Sodium or potassium citrate.................. 200.0 grams.

Potassium thiocyanate .................... I25.0 grams.

Potassium ferrocyanide ( 5 per cent solution)........... 5.0 c.c.

Distilled water to make a total volume of.............. I000.० c.c.
}

With the aid of heat dissolve the carbonate, citrate and thiocyanate in enough water to make about 800 c.c. of the mixture and filter if necessary. Dissolve the copper sulphate separately in about 100 c.c. of water and pour the solution slowly into the other liquid, with constant stirring. Add the ferrocyanide solution, cool and dilute to exactly I liter. Of the various constituents, the copper salt only need be weighed with exactness. Twenty-five c.c. of the reagent are reduced by $50 \mathrm{mg}$. of glucose. 
usual titration of diabetic urines, the formula for calculating the per cent of the sugar is the following:

$$
\mathrm{X}^{0.05^{\circ}} \times 1000=\text { per cent in original sample, wherein } \mathrm{X}
$$

is the number of cubic centimeters of the diluted urine required to reduce 25 c.c. of the copper solution.

In the use of this method chloroform must not be present during the titration. If used as a preservative in the urine it may be removed by boiling a sample for a few minutes, and then diluting to its original volume.

Interpretation.-Sugar in the urine in amounts sufficient to be detected by the commonly employed qualitative tests indicates a pathological condition, unless very large amounts of sugar have been ingested just previously, in which case the condition is spoken of as an alimentary glycosuria. Persistent glycosuria thus indicates diabetes mellitus, a disorder in which the amount of sugar may rise as high as roper cent and averages $3-5$ per cent. The volume of urine excreted per day is usually also large and the absolute sugar excretion may thus be very great (roo grams of glucose per day are not uncommon). The quantitative methods for the estimation of sugar in urine enable us to determine the severity of the disorder as well as to follow its course under treatment, etc.

2. Fehling's Method.-Principle-Diluted urine is run into a measured amount of Fehling's solution at the boiling-point until all of the copper it contains is reduced as indicated by the loss of blue color. This method has several disadvantages over Benedict's method. The end point is difficult to determine and the mixed solution is unstable. It gives less accurate results.

Procedure.-Place Io c.c. of the urine under examination in a Ioo c.c. volumetric flask and make the volume up to Ioo c.c. with distilled water. (If the urine contains less than 0.5 per cent of sugar it may be used without dilution. A concentration of about 0.5 per cent is the most satisfactory for this titration.) Thoroughly mix this diluted urine by pouring it into a beaker and stirring with a glass rod, then transfer a portion of it to a burette which is properly supported in a clamp.

Now place ro c.c. of Fehling's solution ${ }^{1}$ in a small beaker, dilute it with approximately 40 c.c. of distilled water, heat to boiling, and observe whether decomposition of the Fehling's solution itself has occurred as indicated by the production of a turbidity. If such turbidity is produced the Fehling's solution is unfit for use. Clamp the burette containing the dilute urine immediately over the beaker and carefully allow from $0.5^{-I}$ c.c. of the diluted urine to flow into the boiling Fehling's solution. Bring the solution to the boiling-point after each addition of urine and continue running the urine from the burette, $0.5^{-I}$ c.c. at a time, as indicated, until the Fehling's solution is completely reduced, i.e., until all the cupric of p. 26 .

${ }^{1}$ Directions for the preparation of Fehling's solution are given in a note at the bottom 
oxide in solution has been precipitated as cuprous oxide. This point will be indicated by the absolute disappearance of all blue color. When this end point is reached note the number of cubic centimeters of diluted urine used in the process and calculate the percentage of dextrose present, in the sample of urine analyzed, according to the method given below.

This is a satisfactory method, the main objection to its use being the uncertainty attending the determination of the end-reaction, i.e., the difficulty with which the exact point where the blue color finally disappears is noted. Several means of accurately fixing this point have been suggested, but they are practically all open to objection. As good a "check" as any, perhaps, is to filter a few drops of the solution through a double paper, after the blue color has apparently disappeared, acidify the filtrate with acetic acid and add potassium ferrocyanide. If the copper of the Fehling's solution has been completely reduced, there will be no color reaction, whereas the production of a brown color indicates the presence of unreduced copper. Harrison has recently suggested the following procedure to determine the exact end point: To about I c.c. of a starch iodide solution ${ }^{1}$ in a test-tube add 2-3 drops of acetic acid and introduce into the acidified mixture $I-2$ drops of the solution to be tested. Unreduced copper will be indicated by the production of a purplish-red or blue color due to the liberation of iodine.

It is ordinarily customary to make at least three determinations by Fehling's method before coming to a final conclusion regarding the sugar content of the urine under examination.

Calculation.-Ten c.c. of Fehling's solution is completely reduced by 0.05 gram of dextrose. ${ }^{2}$ If y represents the number of cubic centimeters of undiluted urine (obtained by dividing the burette reading by 10) necessary to reduce the Io c.c. of Fehling's solution, we have the following proportion.

$$
y: 0.05:: 100: x \text { (percentage of dextrose). }
$$

Interpretation.-See page 523 .

3. Bang's Method. ${ }^{3}$ - Principle.-The solution to be tested is boiled with alkaline cupric chloride solution containing thiocyanate and potassium chloride. The cupric salt under these conditions is reduced to the cuprous form without any precipitation taking place. The reduced copper is titrated with standard iodine solution using starch as an indicator. The titration reaction is as follows:

$$
\mathrm{CuCl}+\mathrm{I}+\mathrm{K}_{2} \mathrm{CO}_{3}=\mathrm{CuCO}_{3}+\mathrm{KCl}+\mathrm{KI}
$$

Procedure.-A roo c.c. Jena flask with a straight neck the flange of which has been cut off is used for the boiling procedure. Over the neck of the flask place

1 The starch-iodide solution may be prepared as follows: Mix 0.I gram of starch with cold water in a mortar and pour the suspended starch granules into $75-100$ c.c. of boiling water, stirring continuously. Cool the starch paste, add $20-25$ grams of potassium iodide and dilute the mixture to 250 c.c. This solution deteriorates upon standing, and therefore must be freshly prepared as needed.

2 The values for certain other sugars are as follows:

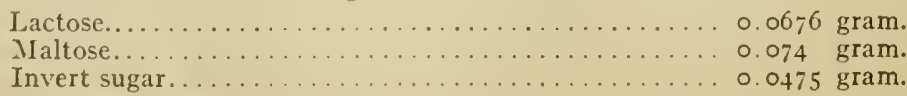

${ }^{3}$ Bang: Biochem. Zeit., 49, I, I9I3. 
a piece of tight fitting rubber tubing sufficiently long (about 2 inches), so that it may be provided with a pinch cock or clamp to shut off the contents of the flask from the outside air (see Fig. $8 \mathrm{I}$, page 28I).

Introduce into the flask 0.I to 2.0 c.c. (or more) of the sugar solution containing not more than $10 \mathrm{mg}$. of glucose. Then add 55 c.c. of the diluted copper solution. ${ }^{1}$ Heat over an asbestos gauze with a flame standardized to bring the solution to the boiling-point in from $31 / 2-33 / 4$ minutes. Boil for exactly 3 minutes, being prepared to close the flask with the pinch-cock at the end of the 3 minutes. Remove from the flame and at once cool under the tap to room temperature. Remove the rubber tubing, add to the contents of the flask $1 / 2-I$ c.c. of the starch solution (I gram of soluble starch in 100 c.c. of saturated $\mathrm{KCl}$ solution, which keeps indefinitely). Titrate with the standard iodine solution ${ }^{1}$ run in from an accurate burette with a glass stopcock. When the iodine starch color appears throughout the solution rotate gently and let stand a few seconds. The end point is reached when the blue color endures for 10-20 seconds. It is preferable to carry out the titration in an atmosphere of carbon dioxide, maintained by means of a delivery tube hung over the side of the flask. Otherwise the titration must be carried out rapidly to prevent reoxidation by the oxygen of the air.

Calculation.-Divide the number of cubic centimeters of $\mathrm{N} / 100$ iodine solution used in the titration by 2.7 to obtain the number of milligrams of glucose in the amount of solution used.

This method is suitable for urine analysis. The urine must however be free from albumin and as urine contains substances reacting slowly with iodine the end point must be taken when the blue color persists for about ro seconds and any slow decolorization disregarded.

\section{Interpretation.- See page 523 .}

4. Peters' Method."- Principle.-The sugar solution is boiled with an alkaline copper solution under rigidly standardized conditions and after filtration the unreduced copper is determined by adding potassium iodide and titrating the liberated iodine with standard thiosulphate solution.

Procedure.-1. The Heating Power.-It is necessary to standardize the heating power of the flame used in the reduction process. A 200 c.c. Erlenmeyer flask of Jena glass and of about $6 \mathrm{~cm}$. basal diameter is used. This bears a two-hole rubber stopper, one hole of which carries a thermometer. The lower end of the thermome-

${ }^{1}$ Copper solution.-Stock Solution.-Dissolve first I 60 grams potassium bicarbonate, 100 grams potassium carbonate and 66 grams of potassium chloride with about 700 c.c. of distilled water in a liter flask. Pure salts must be used in each case. As the bicarbonate is difficulty soluble it should be finely powdered and brought into solution first, preferably with warming to $30^{\circ} \mathrm{C}$. The $\mathrm{K} \mathrm{Cl}$ is then dissolved and finally, with cooling, the carbonate. Then add roo c.c. of a 4.4 per cent solution of pure crystalline $\mathrm{CuSO}_{4} \cdot \mathrm{II}_{2} \mathrm{O}$. Fill to the mark with distilled water. Jix without strong shaking and let stand for 24 hours before using.

Dilute Copper Solution.-Dilute 300 c.c. of the stock solution to 1000 c.C. Mix with only gentle shaking. Allow to stand for several hours before using.

Standard Iodine Solution. - The N/100 iodine solution is made by dilution of $N / 10$ iodine solution (see appendix) with boiled out distilled water. The solution is stable for three months if kept in a dark bottle. It may also be prepared daily from $\mathrm{KI}$ and $\mathrm{KIO}$. Introduce into a Ioo c.c. Alask about i c.c. of 2 per cent $K \mathrm{KO}_{3}$, and $2-2.5$ grams of $\mathrm{KI}$ and then exactly ro c.c. of $\mathrm{N} / \mathrm{Io} \mathrm{HCl}$. The $\mathrm{HCl}$ liberates an equivalent amount of iodine (sulphuric acid is less desirable). Make to roo c.c. with distilled water and mix.

2 Peters: J. Am. Cliem. Soc., 34, 92S, I I 2; 34, 422, I9I2. 
ter should extend to about $2 \mathrm{~mm}$. from the bottom of the flask and the $35^{\circ}$ mark on the thermometer stem should be visible above the stopper. The flask is placed on an asbestos gauze supported by an adjustable ring stand. A Bunsen or Méker burner is used and is placed at from $3-5 \mathrm{~cm}$. beneath the lower surface of the asbestos gauze. Use a large flame and allow the ring and gauze to become thoroughly heated. Then place the flask, into which 60 c.c. of distilled water has been introduced, in the center of the gauze and observe the time required for the temperature in the flask to rise from 35 to $95^{\circ} \mathrm{C}$. By several trials the flame and position of the gauze are so adjusted that it requires (within a few seconds either way) just I 20 seconds for the temperature to rise from $35-95^{\circ} \mathrm{C}$. This standard flame is then used in the determinations which follow.

B. The Reduction Process.-Into a 200 c.c. Jena Erlenmeyer flask fitted as above, introduce 20 c.c. of alkaline tartrate solution, ${ }^{1} 20$ c.c. of the copper sulphate solution ${ }^{1}$ and the sugar solution to be analyzed which should first be made up to 20 c.c. so that the total volume of the fluid in the reduction flask is always 60 c.c. Place the flask over the standard flame and note when the temperature of the mixture reaches $95^{\circ} \mathrm{C}$. Allow to boil for exactly 20 seconds after the temperature reaches $95^{\circ}$. Then the flask is promptly removed with the stopper still in place and held under the tap for a moment to stop the reduction but not to cool the mixture more than a few degrees below the boiling-point. Filter hot through a previously prepared Gooch crucible with a heavy asbestos mat through which sufficient of a suspension of talcum has been filtered so that with suction, not a stream but a rapid succession of drops comes through the filter. (A calcium chloride tube packed with glass wool and asbestos may also be used.) The suction flask should have a capacity of about 200 c.c. so that the titration may be carried out in it directly. Wash the filter with a fine stream of water using not more than $15-20$ c.c. of water in all. To the filtrate add 4 c.c. of concentrated sulphuric acid and cool to $20^{\circ} \mathrm{C}$. Add $6-7$ c.c. of a saturated solution of potassium iodide. Titrate the liberated iodine with standard thiosulphate solution, ${ }^{2}$ adding a few drops of a solution of soluble starch as an indicator near the close of the titration. The "spot test" may be used in determining the end point. As long as a drop of thiosulphate produces a perceptible white area in falling upon the quiet solution the end point has not been reached. The chocolate-brown color of the mixture changes to a light cream white as the last necessary drop of thiosulphate is added.

A blank should be run in exactly the same way but with the omission of the sugar solution.

Calculation.-Subtract the number of cubic centimeters of thiosulphate required for the titration of unreduced copper from the number of cubic centimeters required for the blank. This gives the amount of thiosulphate equivalent to copper

I Copper Solution.-Dissolve 34,639 grams of highest purity crystallized copper sulphate (Kahlbaum's "zur Analyse mit Garantieschein) in water to make 500 c.c.

Alkaline Tartrate Solution.-Dissolve 173 grams of sodium potassium tartrate and I 25 grams of potassium hydroxide in water to make 500 c.c.

${ }^{2}$ N/5 Sodium Thiosulphate.-Dissolve about 50 grams of ordinary c.p. sodium thiosulphate or exactly 49.66 grams of the pure, dry, recrystallized salt, in enough boiled-out distilled water to make a liter. Allow to stand for several days. The solution should be standardized against the copper solution prepared as above. For this purpose introduce 20 c.c. of the copper solution into a 200 c.c. Erlenmeyer flask, add 20 c.c. of 30 per cent acetic acid and 40 c.c. of water. Add about 7 grams of a saturated solution of KI and titrate with the thiosulphate using starch as an indicator. Calculate the equivalent of I c.c. of thiosulphate in $\mathrm{Cu}$. One c.c. of the copper sulphate solution contains $17.647 \mathrm{mg}$. of $\mathrm{Cu}$. The thiosulphate remains constant for some months. It should be kept in a dark bottle. 
reduced. Multiply this result by the value of I c.c. of thiosulphate expressed as $\mathrm{Cu}$, and obtain the number of milligrams of copper reduced. Then by consulting the table of values (below) determine the weight of glucose equivalent to this amount of copper.

Cole ${ }^{1}$ has determined the copper values for lactose using this method exactly as outlined. He has also suggesterl the following empirical formula which agrees well with the values derived from his table:

Mg. anhydrous lactose $=1.25+0.7-5 \times \mathrm{mg} . \mathrm{Cu}$.

Interpretation.-See page 523.

TABLE FOR THE DETERMINATION OF GLUCOSE

According to Peters

\begin{tabular}{|c|c|c|c|c|c|}
\hline Glucose & $\begin{array}{l}\text { Copper, } \\
\text { mg. }\end{array}$ & $\frac{\text { Glucose }}{\mathrm{Cu}}$ ratio & Glucose & $\begin{array}{l}\text { Copper, } \\
\text { mg. }\end{array}$ & $\begin{array}{l}\text { Glucose } \\
\mathrm{Cu}\end{array}$ \\
\hline I & I. 2 & 0.833 & 60 & I I $5 \cdot 5$ & 0.522 \\
\hline 2 & 2.8 & 0.714 & 70 & 134.4 & 0.522 \\
\hline 5 & 8.2 & 0.610 & So & 152.9 & 0.522 \\
\hline 8 & 13.8 & 0.580 & 90 & I 71.0 & 0.522 \\
\hline Io & 17.4 & 0.575 & 100 & I9I. 6 & 0.522 \\
\hline I 5 & $27 \cdot 7$ & 0.542 & I IO & 208.9 & 0.527 \\
\hline 20 & $37 \cdot I$ & 0.539 & I 20 & 228.1 & 0.526 \\
\hline 25 & 48.1 & 0.522 & 135 & 255.0 & 0.529 \\
\hline 30 & $57 \cdot 3$ & 0.522 & $I 50$ & 280.8 & 0.534 \\
\hline 35 & 67.6 & 0.522 & $16_{5}$ & 306.8 & 0.538 \\
\hline 40 & 76.2 & 0.522 & ISo & 330.5 & $0.5+5$ \\
\hline 45 & 86.0 & 0.522 & 200 & 349.6 & 0.572 \\
\hline 50 & 96.0 & 0.522 & & & \\
\hline
\end{tabular}

5. Bertrand's Method. ${ }^{2}$ Principle.-The sugar is boiled with alkaline copper sulphate solution and the precipitated cuprous oxide filtered off, dissolved in an acid solution of ferric sulphate and the resultant ferrous salt titrated with potassium permanganate. This method of titrating cuprous oxide may be conveniently used where other reduction procedures such as that of Peters or of Munson and Walker, ${ }^{3}$ have been employed. In this case the tables corresponding to the particular method and not the Bertrand tables must be consulted in calculating the sugar equivalent.

The following reactions are involved in the Bertrand titration:

$$
\begin{gathered}
\mathrm{Cu}_{2} \mathrm{O}+\mathrm{Fe}_{2}\left(\mathrm{SO}_{4}\right)_{3}+\mathrm{H}_{2} \mathrm{SO}_{4}={ }_{2} \mathrm{CuSO}_{4}+{ }_{2} \mathrm{FeSO}_{4}+\mathrm{H}_{2} \mathrm{O} \\
\mathrm{IOFeSO}_{4}+2 \mathrm{KMnO}_{4}+8 \mathrm{H}_{2} \mathrm{SO}_{4}={ }_{5} \mathrm{Fe}_{2}\left(\mathrm{SO}_{4}\right)_{3}+\mathrm{K}_{2} \mathrm{SO}_{4}+2 \mathrm{MnSO}_{4}+8 \mathrm{H}_{2} \mathrm{O} .
\end{gathered}
$$

Procedure.-Introduce into an Erlenmeyer flask of 150 c.c. capacity, 20 c.c. of the sugar solution (of a concentration of 0.5 per cent or less), 20 c.c. of the copper

${ }^{1}$ Cole: Biochem. J., 8, 134, 1914.

${ }^{2}$ Bertrand: Bull. Soc. Chim. de France, $35,12 S_{5}, 1906$.

Munson and Walker: Bull. 10\%, U. S. Dept. of Agriculture. 
TABLE FOR THE DETERMINATION OF REDUCING SUGARS According to Bertrand

\begin{tabular}{|c|c|c|c|c|c|}
\hline Sugar in mg. & $\begin{array}{l}\text { Glucose } \\
\mathrm{Cu}\end{array}$ & $\begin{array}{c}\text { Invert sugar } \\
\mathrm{Cu}\end{array}$ & $\begin{array}{l}\text { Galactose } \\
\text { I Cu }\end{array}$ & $\begin{array}{l}\text { Maltose } \\
\qquad \mathrm{Cu}\end{array}$ & $\begin{array}{l}\text { Lactose } \\
\mathrm{Cu}\end{array}$ \\
\hline IO & 20.4 & 20.6 & 19.3 & I I . 2 & $\mathrm{I} 4 \cdot 4$ \\
\hline II & 22.4 & 22.6 & 2 I. 2 & I 2.3 & I 5.8 \\
\hline I 2 & $24 \cdot 3$ & $2+.6$ & 23.0 & I 3.4 & 17.2 \\
\hline I 3 & 26.3 & 26.5 & $24 \cdot 9$ & $14 \cdot 5$ & I 8.6 \\
\hline 14 & $28 \cdot 3$ & 28.5 & 26.7 & I 5.6 & 20.0 \\
\hline I5 & 30.2 & 30.5 & 28.6 & I6.7 & $2 I \cdot 4$ \\
\hline 16 & 32.2 & 32.5 & 30.5 & I 7.8 & 22.8 \\
\hline 17 & $34 \cdot 2$ & $34 \cdot 5$ & $32 \cdot 3$ & 18.9 & $24 \cdot 2$ \\
\hline 18 & 36.2 & 36.4 & $34 \cdot 2$ & 20.0 & 25.6 \\
\hline I9 & 38.1 & 38.4 & 36.0 & $2 I \cdot I$ & 27.0 \\
\hline 20 & 40. $x$ & 40.4 & 37.9 & 22.2 & 28.4 \\
\hline $2 \mathrm{I}$ & 42.0 & $4^{2} \cdot 3$ & $39 \cdot 7$ & $23 \cdot 3$ & 29.8 \\
\hline 22 & $43 \cdot 9$ & $44 \cdot 2$ & 4 I. 6 & $24 \cdot 4$ & $3 \mathrm{I} . \mathrm{I}$ \\
\hline 23 & 45.8 & $46 . I$ & $43 \cdot 4$ & $25 \cdot 5$ & $32 \cdot 5$ \\
\hline 24 & 47.7 & 48.0 & 45.2 & 26.6 & 33.9 \\
\hline 25 & $49 \cdot 6$ & 49.8 & 47.0 & $27 \cdot 7$ & $35 \cdot 2$ \\
\hline 26 & $51 \cdot 5$ & $5 \mathrm{x} \cdot 7$ & 48.9 & 28.9 & 36.6 \\
\hline 27 & $53 \cdot 4$ & 53.6 & 50.7 & 30.0 & 38.0 \\
\hline 28 & $55 \cdot 3$ & $55 \cdot 5$ & 52.5 & 3 I.I & $39 \cdot 4$ \\
\hline 29 & $57 \cdot 2$ & $57 \cdot 4$ & $54 \cdot 4$ & 32.2 & 40.7 \\
\hline 30 & 59.1 & $59 \cdot 3$ & 56.2 & $33 \cdot 3$ & $42 . I$ \\
\hline $3 I$ & 60.9 & $6 I . I$ & 58.0 & $34: 4$ & $43 \cdot 4$ \\
\hline 32 & 62.8 & $6_{3} .0$ & 59.7 & 35.5 & 44.8 \\
\hline 33 & 64.6 & 64.8 & $61 \cdot 5$ & 36.5 & $46 . I$ \\
\hline 34 & 66.5 & 66.7 & $63 \cdot 3$ & 37.6 & $47 \cdot 4$ \\
\hline 35 & 68.3 & 68.5 & 65.0 & 38.7 & 48.7 \\
\hline 36 & 70.1 & 70.3 & 66.8 & 39.8 & 50.1 \\
\hline 37 & 72.0 & 72.2 & 68.6 & 40.9 & $5 \mathrm{I} \cdot 4$ \\
\hline 38 & 73.8 & 74.0 & 70.4 & 41.9 & 52.7 \\
\hline 39 & $75 \cdot 5$ & 75.9 & 72.1 & 43.0 & 54.1 \\
\hline 40 & $77 \cdot 5$ & $77 \cdot 7$ & 73.9 & $44 \cdot I$ & $55 \cdot 4$ \\
\hline 1 $4 I$ & $79 \cdot 3$ & $79 \cdot 5$ & 75.6 & $45 \cdot 2$ & $56 \cdot 7$ \\
\hline 42 & $8 I . I$ & 8 I. 2 & $77 \cdot 4$ & 46.3 & $5^{8.0}$ \\
\hline 43 & 82.9 & 83.0 & 79.1 & 47.4 & $59 \cdot 3$ \\
\hline 44 & $84 \cdot 7$ & 84.8 & So. 8 & 48.5 & 60.6 \\
\hline 45 & 86.4 & 86.5 & 82.5 & $49 \cdot 5$ & 61.9 \\
\hline $4^{6}$ & 88.2 & 88.3 & $8+\cdot 3$ & 50.6 & $63 \cdot 3$ \\
\hline 47 & 90.0 & $90 . I$ & 86.0 & 51.7 & 64.6 \\
\hline $4^{8}$ & 91.8 & $9 \mathbf{I} .9$ & 87.8 & 52.8 & $65 \cdot 9$ \\
\hline 49 & 93.6 & 93.6 & 89.5 & 53.9 & 67.2 \\
\hline 50 & $95 \cdot 4$ & $95 \cdot 4$ & 91.2 & 55.0 & 68.5 \\
\hline $5 \mathrm{I}$ & 97.1 & $97 . I$ & 92.9 & $56 . I$ & 69.8 \\
\hline 52 & 98.9 & 98.8 & 94.6 & 57.1 & 71.1 \\
\hline 53 & 100.6 & 100.6 & 96.3 & 58.2 & 72.4 \\
\hline 54 & IO $2 \cdot 3$ & 102.3 & 98.0 & $59 \cdot 3$ & $73 \cdot 7$ \\
\hline 55 & IO4.I & $10_{4} .0$ & 99.7 & 60.3 & 74.9 \\
\hline 56 & 105.8 & 105.7 & 101.5 & $6 \mathrm{I} .4$ & 76.2 \\
\hline
\end{tabular}


TABLE FOR THE DETERMINATION OF REDUCING SUGARS.-(Conlinued) According to Bertrand

\begin{tabular}{|c|c|c|c|c|c|}
\hline Sugar in $\mathrm{mg}$. & $\begin{array}{c}\text { Glucose } \\
\mathrm{Cu}\end{array}$ & $\begin{array}{c}\text { Invert sugar } \\
\mathrm{Cu}\end{array}$ & $\begin{array}{c}\text { Galactose } \\
\mathrm{Cu}\end{array}$ & $\begin{array}{l}\text { Ialtose } \\
\mathrm{Cu}\end{array}$ & $\begin{array}{c}\text { Lactose } \\
\mathrm{Cu}\end{array}$ \\
\hline 57 & 107.6 & 107.4 & 103.2 & 62.5 & 77.5 \\
\hline $5^{8}$ & $109 \cdot 3$ & 109.2 & 104.9 & $63 \cdot 5$ & 78.8 \\
\hline 59 & I I I. I & I I 0.9 & 106.6 & 64.6 & 80.1 \\
\hline 60 & II 2.8 & I 2.6 & 108.3 & 65.7 & 81.4 \\
\hline $6 I$ & II $4 \cdot 5$ & II $4 \cdot 3$ & I I0.0 & 66.8 & 82.7 \\
\hline 62 & II 6.2 & I 15.9 & III. 6 & 67.9 & 83.9 \\
\hline 63 & II 7.9 & II 7.6 & I $3 \cdot 3$ & 68.9 & 85.2 \\
\hline 64 & II 9.6 & 119.2 & I 15.0 & 70.0 & 86.5 \\
\hline 65 & I 21.3 & I 20.9 & I I 6.6 & $7 \mathrm{IIX}$ & 87.7 \\
\hline 66 & 123.0 & I 22.6 & IIS. 3 & 72.2 & $89 \circ$ \\
\hline 67 & 124.7 & I 24.2 & I 20.0 & $73 \cdot 3$ & 90.3 \\
\hline 68 & $\mathrm{I} 26.4$ & I 25.9 & I 21.7 & $74 \cdot 3$ & 91.6 \\
\hline 69 & I 28.1 & I 27.5 & I $23 \cdot 3$ & $75 \cdot 4$ & 92.8 \\
\hline 70 & I 29.8 & $\mathrm{I} 29.2$ & 125.0 & 76.5 & 94.1 \\
\hline 71 & I3I. 4 & I 30.8 & I 26.6 & 77.6 & 95.4 \\
\hline 72 & I33.I & 132.4 & 128.3 & 78.6 & 96.6 \\
\hline 73 & $134 \cdot 7$ & 134.0 & 130.0 & $79 \cdot 7$ & 97.9 \\
\hline 74 & I 36.3 & 135.6 & I $3 I .5$ & 80.8 & $99 . I$ \\
\hline 75 & 137.9 & 137.2 & I $33 . \mathrm{I}$ & $8 \mathrm{I} .8$ & 100.4 \\
\hline 76 & I 39.6 & 138.9 & I 34.8 & 82.9 & 101.7 \\
\hline 77 & I 4 I. 2 & 140.5 & I 36.4 & 84.0 & 102.9 \\
\hline 78 & 142.8 & 142.1 & I 38.0 & 85.1 & 104.2 \\
\hline 79 & 144.5 & I 43.7 & I 39.7 & S6.I & 105.4 \\
\hline So & 146.1 & $I+5 \cdot 3$ & I 4 I. 3 & $S_{7.2}$ & 106.7 \\
\hline 8I & 147.7 & 146.9 & $\mathrm{I}_{4} 2.9$ & $83 \cdot 3$ & 107.9 \\
\hline 82 & $149 \cdot 3$ & 148.5 & 144.6 & 89.4 & 109.2 \\
\hline 83 & 150.9 & 150.0 & 146.2 & 90.4 & IIO. 4 \\
\hline 84 & I 52.5 & 151.6 & 147.8 & 91.5 & I I $\mathrm{x} \cdot 7$ \\
\hline $8_{5}$ & I 54.0 & I 53.2 & I 49.4 & 92.6 & I 12.9 \\
\hline 86 & I 55.6 & $15+. S$ & I5I.I & 93.7 & II 4.1 \\
\hline 87 & I 57.2 & 156.4 & 152.7 & 94.5 & 115.4 \\
\hline 88 & 158.8 & 157.9 & I $5+\cdot 3$ & $95 \cdot 3$ & II 6.6 \\
\hline 89 & 160.4 & 159.5 & I 56.0 & 96.9 & 117.9 \\
\hline 90 & I62.0 & I6I.I & I 57.6 & 98.0 & IIg. I \\
\hline $9 I$ & $\mathrm{I} 63.6$ & 162.6 & 159.2 & 99.0 & 120.3 \\
\hline 92 & I65.2 & 164.2 & 160.5 & 100.1 & 121. 6 \\
\hline 93 & I 66.7 & 165.7 & 162.4 & IOI. I & 122.8 \\
\hline 94 & I 68.3 & $167 \cdot 3$ & 164.0 & 102.2 & 124.0 \\
\hline 95 & 169.9 & 168.8 & 165.6 & 103.2 & 125.2 \\
\hline 96 & 171.5 & 170.3 & 167.2 & 104.2 & 126.5 \\
\hline 97 & 173.1 & 171.9 & I68.S & $105 \cdot 3$ & 127.7 \\
\hline 98 & I 74.6 & I 73.4 & 170.4 & 106.3 & 128.9 \\
\hline 99 & 176.2 & 175.0 & 172.0 & 107.4 & $1,30.2$ \\
\hline 100 & 177.8 & 176.5 & 173.6 & 108.4 & 1314 \\
\hline
\end{tabular}


solution $^{1}$ and 20 c.c. of the alkaline tartrate solution. ${ }^{1}$ Heat to boiling over an asbestos gauze and boil gently for exactly three minutes. Let stand a moment that the copper oxide may settle and then filter with suction through a Gooch crucible with a heavy asbestos mat (or a calcium chloride tube with successive layers of glass wool, coarse asbestos and fine asbestos wool) into a flask of about I 50 c.c. capacity. Wash the residue in the flask twice by decantation with a little hot water pouring the supernatant liquid through the filter. Throw away the clear filtrate, rinse the flask and replace it. To the flask containing the cuprous oxide add 5-20 c.c. of the acid ferric sulphate solution. ${ }^{1}$ A green solution containing ferrous sulphate is formed. Pour through the filter together with a little more of the acid solution if necessary to completely dissolve the copper oxide. Wash flask and filter with a little water. Titrate the filtrate with standard potassium permanganate solution ${ }^{1}$ to a rose color. The procedure should be carried out as rapidly as possible.

Calculation.-Multiply the number of cubic centimeters of permanganate used by its copper equivalent as determined by standardization, and from the table (page 528-529) obtain the corresponding value for the sugar under examination. Interpretation-See page 523 .

6. Fermentation Method.-Principle.-This method consists in the measurement of the volume of carbon dioxide evolved when the dextrose of the urine undergoes fermentation with yeast. None of the various methods whose manipulation is based upon this principle is absolutely accurate. The method in which Einhorn's saccharometer (Fig. 3, page 3I) is the apparatus employed is perhaps as satisfactory as any for clinical purposes.

Procedure.-Place about $I_{5}$ c.c. of urine in a mortar, add about I gram of yeast ( $1 / \mathrm{I} 6$ of the ordinary cake of compressed yeast) and carefully crush the latter by means of a pestle. Transfer the mixture to the saccharometer, being careful to note that the graduated tube is completely filled and that no air bubbles gather at the top. Allow the apparatus to stand in a warm place $\left(30^{\circ} \mathrm{C}\right.$.) for 12 hours and observe the percentage of dextrose as indicated by the graduated scale of the instrument. Both the percentage of dextrose and the number of cubic centimeters of carbon dioxide are indicated by the graduations on the side of the saccharometer tube.

The fermentation method becomes a much more accurate procedure if the saccharometer of Lohnstein is used.2

1 (a) Copper Sulphate Solution.-Forty grams of pure crystallized copper sulphate are dissolved in water to make a liter.

(b) Alkaline Tartrate Solution.-Dissolve 200 grams of Rochelle salts and $\times 50$ grams of $\mathrm{NaOH}$ in water to make rooo c.c.

(c) Acid Ferric Sulphate Solution.-Dissolve 50 grams of ferric sulphate in about 200 c.c. of water and pour into this a mixture of 200 c.c. of concentrated sulphuric acid diluted with about 400 c.c. of water. Mix and make up to rooo c.c.

(d) Potassium Permanganate Solution.-Dissolve 5 grams of potassium permanganate in water to make 1000 c.c. Standardization.-Dissolve 0.250 gram of ammonium oxalate in 50-100 c.c. of water, add I-2 c.c. of concentrated sulphuric acid and titrate with the permanganate to a rose color. About 22 c.c. will be required. Multiply the number of grams of oxalate used by 0.895 to get the equivalent in $\mathrm{Cu}$ of the number of cubic centimeters of permanganate used. Calculate the $\mathrm{Cu}$ value of I c.c.

${ }^{2}$ Lohnstein: Wiinch. med. Woch., I\$99, 167 r. 
The availability of the fermentation procedure as a quantitative aid has been appreciably lowered through the important findings of Neuberg and Associates. " They show that yeast has the property of splitting off carbon dioxide from the carboxyl group of amino-and other aliphatic acids. The active agent in this "sugar-free fermentation" is an enzyme called carboxylase. Inasmuch as amino-acids are always present in the urine, the error from this source is apparent.

7. Polariscopic Examination.-Before subjecting urine to a polariscopic examination the slightly acid fluid should be decolorized as thoroughly as possible by the addition of a little lead acetate. The urine should be well stirred and then filtered through a filter paper which has not been previously moistened. In this way a perfectly clear and almost colorless liquid is obtained.

In determining dextrose by means of the polariscope it should be borne in mind that this carbohydrate is often accompanied by other optically active substances, such as proteins, fructose, $\beta$-hydroxybutyric acid, and conjugate glycuronates which may introduce an error into the polariscopic reading; the method is, however, sufficiently accurate for practical purposes.

For directions as to the manipulation of the polariscope see page $3 \mathrm{I}$.

Below are given the specific rotations of some physiologically important sugars as well as of certain other optically active substances the possible presence of which must be borne in mind in determining glucose polarimetrically in urine.

Specific Rotation

Glucose
Maltose
Isomaltose
Lactose
Pentose (i-ara-
$\quad$ binose).

Specific Rotation

Fructose
$\beta$-Hydroxybutyric
acid.
Conjugated Gly-
curonic Acids.$$
-92.25
$$$$
-24.12
$$

Levorotatory in varying degrees.

\section{Protein}

I. Scherer's Coagulation Method.-The content of coagulable protein may be accurately determined as follows : Place 50 c.c. of urine in a small beaker and raise the temperature of the fluid to about $40^{\circ} \mathrm{C}$. upon a water-bath. Add dilute acetic acid, drop by drop, to the warm urine, to precipitate the protein which will separate in a flocculent form. Care should be taken not to add too much acid; ordinarily less than 20 drops is sufficient. The temperature of the water in the water-bath should now be raised to the boiling-point and maintained there for a few minutes in order to insure the complete coagulation of the protein present. Now filter the urine ${ }^{2}$ through a previously washed, dried, and weighed

${ }^{1}$ Neuberg and Associates: Biochem. Zeilschr., vol. $3 \mathrm{r}$ and 36 , $10 \mathrm{Ir}$.

2 If it is desired the precipitate may be liltered off on an unweighed paper, and its nitrogen content determined by the Kjeldahl method (see $\left.\mathrm{p} .4 s_{3}\right)$. In order to arrive at correct figures for the protein content it is then simply necessary to multiply the total nitrogen content by 6.25 (see p. 328). Correction should be made for the nitrogen conient of the filter paper used unless this factor is negligible. 
filter paper, wash the precipitated protein, in turn, with hot water, 95 per cent alcohol, and with ether, and dry the paper and precipitate, to constant weight, in an air-bath at $110^{\circ} \mathrm{C}$. Subtract the weight of the filter paper from the combined weight of the paper and precipitate and calculate the percentage of protein in the urine specimen.

Calculation.-To determine the percentage of protein present in the urine

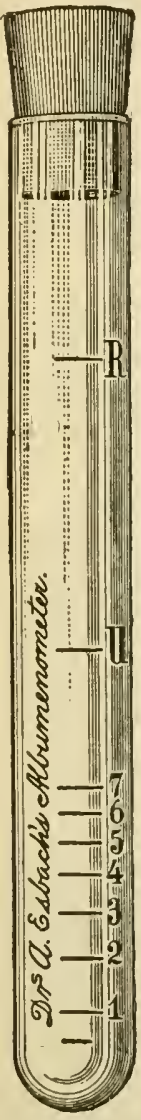

Fig. $167 .-$ EsBaCH'S ALBUMINOMETER. under examination, multiply the weight of the precipitate, expressed in grams, by 2 .

Interpretation.-The amount of albumin occurring in the urine is not necessarily an index of the severity or type of the disorder giving rise to it. Hence no significant figures can be given. Normal human urine probably contains a trace of albumin which is too slight to be detected or determined by the usual procedures. The determination of albumin may be of assistance in following the course of kidney disturbances, but the results can be interpreted only in the light of other clinical findings.

2. Esbach's Method.-This method depends upon the precipitation of protein by Esbach's reagent ${ }^{1}$ and the apparatus used in the estimation is Esbach's albuminometer (Fig. I67). In making a determination fill the albuminometer to the point $U$ with urine, then introduce the reagent until the point $\mathbf{R}$ is reached. Now stopper the tube, invert it slowly several times in order to insure the thorough mixing of the fluids, and stand the tube aside for 24 hours. Creatinine, resin, acids, etc., are precipitated in this method, and for this and other reasons it is not as accurate as the coagulation method. It is, however, extensively used clinically. According to Sahli ${ }^{2}$ the method is "accurate approximately to one part per I000," whereas Pfeiffer ${ }^{3}$ claims it is not accurate for less than onehalf or for more than five parts per 1000 .

Calculation.-The graduations on the albuminometer indicate grams of protein per liter of urine. Thus, if the protein precipitate is level with the figure 3 of the graduated scale, this denotes that the urine examined contains 3 grams of protein to the liter. To express the amount of protein in per cent simply move the decimal point one place to the left. In the case under consideration the urine contains 0.3 per cent protein.

Interpretation.-See above.

3. Kwilecki's Modification of Esbach's Method.4-Add Io drops of a Io per cent solution of $\mathrm{FeCl}_{3}$ to the acid urine before introducing the Esbach's reagent.

${ }^{1}$ Esbach's reagent is prepared by dissolving Io grams of picric acid and 20 grams of citric acid in I liter of water.

${ }^{2}$ Sahli: Lehrbuch d. klin. Untersuchungs-Methoden, 5th Aufl, I 909.

3 Pfeiffer: Berl. klin. Woch., 49, I I 4, I9r2.

4 Kwilecki: M ünch med. Woch., 56, p. I330. 
Warm the tube and contents in a water-bath at $72^{\circ} \mathrm{C}$. for $5^{-6}$ minutes and make the reading.

4. Turbidity Method of Folin and Denis. ${ }^{1-P r i n c i p l e .-T h e ~ a l b u m i n ~ o f ~ t h e ~}$ urine is precipitated with sulphosalicylic acid and the turbidity produced compared with that of a standard protein solution.

Procedure.-To about 75 c.c. of water in each of two roo c.c. volumetric flasks is added 5 c.c. of a 25 per cent solution of sulphosalicylic acid. To one flask is then added 5 c.c. of the standard protein solution containing ro $\mathrm{mg}$. of albumin and to the other is added the albuminous urine I c.c. at a time (by means of an Ostwald pipette) until the turbidity obtained seems to be reasonably near that of the standard. The two flasks are then filled up to the mark with water, cautiously inverted a few times to secure mixing, and are then ready for the quantitative comparison just as in colorimetric work. The standard must invariably be read against itself. The standard ${ }^{2}$ containing $10 \mathrm{mg}$. of protein is set at $20 \mathrm{~mm}$. The unknown must not read less than io nor more than 30.

Calculation.-Dividing 200 by the product of the reading of the unknown and the number of cubic centimeters of urine taken gives the albumin in milligrams per cubic centimeter of urine. The albuminous suspensions must not be shaken but mixed very carefully. The method is fairly accurate and requires but a few minutes if a standard solution is at hand. The method is not applicable to urines deeplycolored with blood or bile but may be used for albuminous fluids other than urine if such fluids are not highly pigmented. It must be borne in mind that different proteins, as serum albumin and serum globulin, may give markedly different degrees of turbidity under the same conditions. ${ }^{3}$

Interpretation.-See page 532 .

\section{Acetone and Acetoacetic Acid}

I. Folin-Hart Method.-Principle.-This method as well as the two following serve the purpose of determining the acetone, and acetoacetic acid together in terms of acetone. Acetoacetic acid on heating is decomposed with the formation of acetone. In this method the acetone preformed and that derived from the acetoacetic acid are carried over by means of an air current into a solution of iodine in potassium hydroxide (alkaline hypoiodite solution). The acetone is here absorbed and retained as iodoform. The excess iodine over that necessary to combine with the acetone is determined by titration with sodium thiosulphate. The method is simple and accurate if directions are carefully followed.

${ }^{1}$ Folin and Denis: Jour. Biol. Chem., IS, 273, I914.

${ }^{2}$ Standard Albumin solution. Fresh blood serum free from hemoglobin is used. $25-35$ c.c. of the serum are diluted with a 15 per cent solution of chemically pure sodium chloride to about ${ }_{5} 00$ c.c. The solution is mixed and filtered. By means of nitrogen determinations the protein content of the filtrate is determined (protein $=N \times 6.25$ ) and on the basis of the figure obtained the solution is diluted with 5 per cent sodium chloride solution so that it contains $2 \mathrm{mg}$. of protein per cubic centimeter. It is best to saturate the albumin solution with chloroform. The solution keeps for months.

${ }^{3}$ Marshall and Banks: Proceedings of the American Philosophical Society; 54,$1 ; 6$, I9I5. 
Procedure.-Introduce into a wide-mouth bottle 200 c.c. of water, an accurately measured excess of $\mathrm{N} /$ ro iodine solution ${ }^{1}$ and an excess of 40 per cent potassium hydroxide. Prepare an aerometer cylinder containing alkaline hypoiodite solution to absorb any acetone which may be present in the air of the laboratory, and between the cylinder and bottle suspend a test-tube about 2 inches in diamter. This large test-tube should contain 20 c.c. of the urine under examination, Io drops or a Io per cent solution of phosphoric acid, Io grams of sodium chloride and a little petroleum, and should be raised sufficiently high to facilitate the easy application of heat to its bottom portion. The connections on the side of the tube should be provided with bulb-tubes containing cotton. When the apparatus is arranged as described, it should be connected with a Chapman pump and an air current passed through for twenty-five minutes. During this period the contents of the test-tube are heated just to the boiling-point and after an interval of five minutes again heated in the same manner. By this means the diacetic acid is converted into acetone and at the end of the twenty-five-minute period this acetone, as well as the preformed acetone, will have been removed from the urine to the absorption bottle and there retained as iodoform.

The contents of the absorption bottle should now be acidified with concentrated hydrochloric acid, ${ }^{2}$ and titrated with $\mathrm{N} /$ ro sodium thiosulphate and starch as in the Messinger-Huppert method (see below).

Interpretation.- Normal adults on a mixed diet excrete on the average $3^{-}{ }_{5} \mathrm{mg}$. of combined acetone and acetoacetic acid per day and anything over $20 \mathrm{mg}$. is usually pathological. The amount is considerably increased in fasting and on a carbohydrate-free diet due to the development of acidosis. In severe diabetic acidosis values up to 6 grams per day or even higher may be noted. It is sometimes found in large amounts in intoxications associated with pregnancy. It may be found in increased amounts in the urine in a great variety of pathological conditions. Quantitative estimation enables us to follow the course of the acidosis. Ammonia excretion is also largely increased in

${ }_{1}$ Proceed as follows in order to obtain a rough idea regarding the amount of $\mathrm{N} /$ ro iodine solution to be used: Introduce into a test-tube ro c.c. of the urine under examination and I c.c. of a solution of ferric chloride made by dissolving Ioo grams of ferric chloride in roo c.c. of distilled water. After permitting the mixture to stand for two minutes, compare the color with that of an equal volume of the ferric chloride solution in a testtube of similar diameter. If the two solutions be of approximately the same color intensity, 20 c.c. of the urine under examination will yield sufficient acetone to require nearly ro c.c. of $\mathrm{N} /$ ro iodine solution. In case the -mixture is darker in color than is the ferric chloride solution, the former should be diluted with distilled water until it is of approximately the same intensity as the ferric chloride solution. From this data the amount of $N /$ ro iodine solution required may be roughly estimated by means of the following table:

Urine c.c. Ferric chloride

\begin{tabular}{|c|c|}
\hline Water & $\mathrm{N} /$ ro Iodine required c.c. \\
$\mid \ldots \ldots \ldots \ldots \ldots \ldots$ & \\
10 & 10 \\
20 & 35 \\
30 & 40 \\
\end{tabular}

${ }^{2}$ An excess of iodine is indicated by the development of a brown color. 
these conditions, being used in the neutralization of the excess acids formed in the body. Usually about three-quarters of the combined acetone and acetoacetic acid excretion is in the form of acetoacetic acid, but the proportion is not constant.

Acidosis is due mainly to a disturbance in the metabolism of fats. The fatty acids are ordinarily oxidized through hydroxybutyric acid to acetoacetic acid which is in turn oxidized through formic and acetic acids to carbon dioxide and water. When fat catabolism is increased to such an extent that the body cannot bring about complete oxidation of the products formed, a considerable portion of the acetoacetic acid instead of being oxidized in this way is transformed into acetone (a process taking place but to a slight extent normally). This acetone as well as acetoacetic acid and in more severe cases $\beta$-hydroxybutyric acid will then be eliminated to varying degrees in the urine.

The relation of the acetone bodies is indicated in the following scheme:

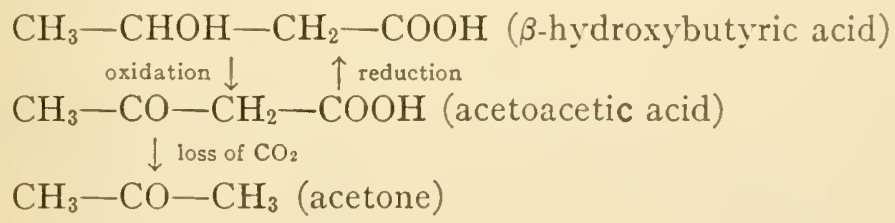

In fasting the decomposition of fat is increased due to the lack of carbohydrate material and acidosis develops. The same holds true for a carbohydrate-free diet. Apparently also fat is much less readily oxidized in the presence of a carbohydrate deficiency.

2. Messinger-Huppert Method.-Principle.-This method differs from the preceding in that distillation of the acetone is substituted for aspiration. It is an accurate method of determining acetone and diacetic acid together.

Procedure.-Place roo c.c. of urine in a distillation flask and add 2 c.c. of 50 per cent acetic acid. Connect the flask with a condenser, properly arrange a receiver, attach a terminal series of bulbs containing water, and distil over about nine-tenths of the urine mixture. Remove the recciver, attach another, and subject the residual portion of the mixture to a second distillation. Test this fluid for acetone and if the presence of acetone is indicated add about roo c.c. of water to the residue and again distil. Treat the united acetone distillates with I c.c. of dilute ( 2 per cent) sulphuric acid and redistil, collecting this second distillate in a glass-stoppered flask. During distillation, however, the glass stopper is replaced by a cork with a double perforation, the glass tube from one perforation passing to the condenser, while the bulbs containing water, before mentioned, are attached by means of the tube in the other perforation. Allow the distillation process to proceed until practically all of the fluid has passed over, then remove the receiving flask and insert the glass stopper. Now treat the distillate carefully with ro c.c. 
of a $\mathrm{N} /$ Io solution of iodine and add an excess of sodium hydroxide solution ( $15^{-25}$ c.c. of 20 per cent). Stopper the flask and shake it for about one minute. Add I-2 drops of concentrated hydrochloric acid, and note the production of a brown color at the point of contact if an excess of iodine is present. In case there is no such excess, the solution should be treated with $\mathrm{N} /$ ro iodine solution until an excess is obtained. Retitrate this excess of iodine with N/Io sodium thiosulphate solution until a light yellow color is observed. At this point a few cubic centimeters of starch paste should be added and the mixture again titrated until no blue color is visible. This is the end-reaction.

Calculation.-Subtract the number of cubic centimeters of $\mathrm{N} /$ ro thiosulphate solution used from the volume of $\mathrm{N}$ / Io iodine solution employed. Since I c.c. of the iodine solution is equivalent to $0.967 \mathrm{mg}$. of acetone, and since I c.c. of the thiosulphate solution is equivalent to I c.c. of the iodine solution, if we multiply the remainder from the above subtraction by 0.967 we will obtain the number of milligrams of acetone in the roo c.c. of urine examined.

Calculate the quantity of acetone in the twenty-four-hour urine specimen.

Interpretation.-See page 534 .

3. Method of Scott-Wilson. ${ }^{1}$-Principle.-The urine is distilled with acid and the acetone (preformed and from acetoacetic acid by

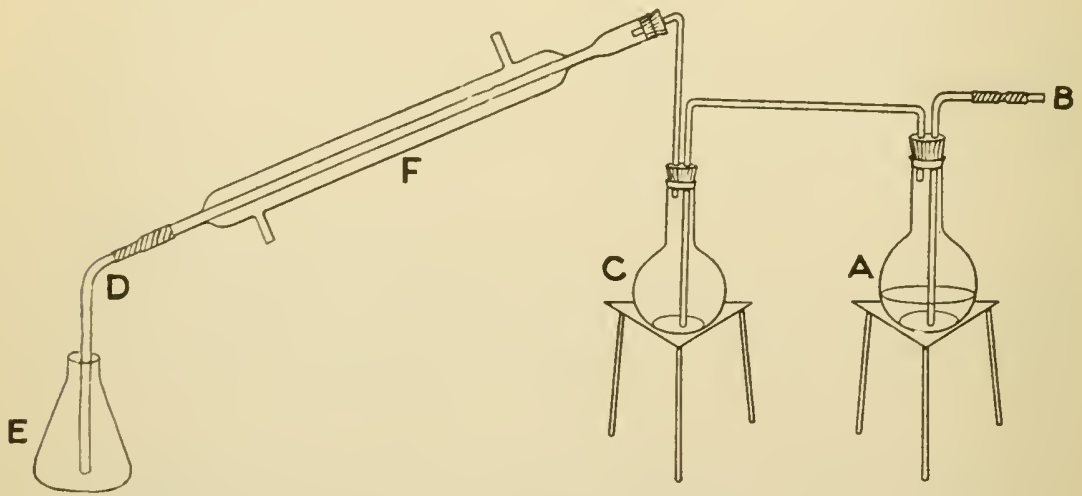

Fig. э68.-ScotT-Wilson Apparatus.

hydrolysis) collected in an alkaline solution of basic mercuric cyanide. A precipitate of keto-mercuric cyanide is formed according to the following equation:

$$
\mathrm{C}_{3} \mathrm{H}_{6} \mathrm{O}+{ }_{2} \mathrm{Hg}(\mathrm{CN})_{2}+{ }_{3} \mathrm{HgO}=\mathrm{C}_{3} \mathrm{OHg}_{5} \mathrm{C}_{4} \mathrm{~N}_{4}+{ }_{3} \mathrm{H}_{2} \mathrm{O}
$$

The precipitate is filtered off and its mercury content determined by titration with potassium thiocyanate solution. The method is adapted to the determination of acetone in small amounts, and has the advantage over the iodoform method that it is not affected by alcohol.

Procedure.-The apparatus for the distillation of acetone is set up as in the illustration (Fig. I68). Introduce roo c.c. of the normal urine (or a lesser quan-

${ }^{1}$ Scott-Wilson: Jour. Physiol., 42, 444, rgrr. 
tity of an abnormal urine high in acetone) into flask A, together with 25 grams anhydrous sodium sulphate and I c.c. of concentrated sulphuric acid. Ten c.c. of 40 per cent sodium hydroxide solution are placed in flask $\mathrm{C}$ and ro c.c. or more of the mercuric cyanide solution ${ }^{1}$ in the Erlenmeyer flask $E$, the tube $D$ being arranged so as to dip beneath the surface of the reagent.

If more than $0.4 \mathrm{mg}$. of acetone is present in the amount of urine taken, the quantity of reagent should be proportionately increased. It is desirable to make a test to determine the approximate amount of acetone bodies as outlined under the Folin-Hart method (page 534). Connect up the apparatus closing B with a glass-rod plug, and light the burners under $\mathrm{A}$ and $\mathrm{C}$. The sodium hydroxide solution in $\mathrm{C}$ must boil before the urine in $\mathrm{A}$ as otherwise condensation would take place in $\mathrm{C}$. Keep the hydroxide solution just at the boiling point and allow A to boil briskly. Note the first appearance of turbidity in flask $\mathrm{E}$ and distil for five minutes from that time. Remove the plug at B and extinguish the burners. Remove tube $\mathrm{D}$ and rinse with distilled water into flask $\mathrm{E}$. Allow to stand for ten minutes.

Filter off the precipitate on an asbestos mat in a Gooch crucible. The pores of the filter should have been partially closed by filtering a suspension of talc through it. If the first portions of the filtrate are not clear, pass them through the filter again. Transfer any precipitate adherent to the sides of the flask to the filter with the aid of water and wash the filter thoroughly with water. Transfer the asbestos and precipitate to an Erlenmeyer flask by means of a glass rod. Any precipitate adherent to the crucible or glass rod is washed into the flask with a jet of "acid mixture" (nitric acid 40 parts, sulphuric acid 5 parts and water 55 parts). About Io c.c. of this acid mixture should be used altogether. Then add I c.c. of $\mathrm{N} / 5$ potassium permanganate solution. ${ }^{2}$ Boil until the brown color given by the permanganate has completely disappeared. This should occur in one to two minutes but if the decolorization occurs more rapidly (a few seconds), insufficient permanganate has been added and another cubic centimeter or so should be run in so that the color is not dispelled on two minutes boiling. Clear with a few drops of yellow nitric acid.

Cool the completely decolorized solution under the tap and add 2 c.c. of a saturated solution of ferric alum. Titrate with standard potassium thiocyanate solution ${ }^{3}$ until a brownish tinge appears throughout the solution. Care should be taken with the end point as the addition of several drops after the end point has been reached will not perceptibly darken the color.

Calculation.-One c.c. of the thiocyanate solution if made up exactly is equivalent to I $\mathrm{mg}$. of $\mathrm{Hg}$ or $0.058 \mathrm{mg}$. of acetone. Multiply the number of cubic centimeters of thiocyanate used by its equivalent of mercury in milligrams and by 0.058 to get the amount of acetone and acetoacetic acid (expressed as acetone) in the amount of urine analyzed. Calculate the daily output from the 24-hour volume.

${ }^{1}$ Scott-H'ilson Reagent.-Dissolve 0.5 gram mercuric cyanide and o grams sodium hydroxide in 60 c.c. of water and then run in with constant stirring 20 c.c. of 0.7268 per cent silver nitrate solution. (The silver nitrate solution is made by taking I part of standard silver nitrate solution ( 1 c.c. $=10 \mathrm{mg} . \mathrm{NaCl}$ ) and 3 parts of water.)

2 Made by dissolving 6.324 grams of potassium permanganate in water and making up to a liter.

${ }^{3}$ Make up an approximately o.I per cent solution of potassium thiocyanate and standardize it against mercuric nitrate or silver nitrate. It is convenient to have the solution of such a strength that I c.c. $=$ I mg. of IIg. 
Interpretation.-See page 534 .

\section{Acetone}

Folin's Method.-Principle.-The preformed acetone is aspirated from the urine mixture at room temperature to prevent decomposition of acetoacetic acid. The acetone is collected in alkaline hypoiodite solution as in the Folin-Hart method (page 533). Iodoform is formed quantitatively and the excess of iodine is titrated with sodium thiosulphate.

Procedure.- The same type of apparatus is.used in this method as that described in Folin's method for the determination of ammonia (see page 499). Introduce $20-25$ c.c. of the urine under examination into the aerometer cylinder and add ro drops of ro per cent phosphoric acid, ${ }^{1} 8$-10 grams of sodium chloride, ${ }^{2}$ and a little petroleum. Introduce into an absorption flask, ${ }^{3}$ such as is used in the ammonia determination (see page 499), I50 c.c. of water, ro c.c. of a 40 per cent solution of potassium hydroxide, and an excess of a $\mathrm{N} /$ ro iodine solution. Connect the flask with the aerometer cylinder, attach a Chapman pump, and permit an air current, slightly less rapid than that used for the determination of ammonia, to be drawn through the solution for 20-25 minutes. All of the acetone will, at this point, have been converted into iodoform in the absorption flask. Add ro c.c. of concentrated hydrochloric acid (a volume equivalent to that of the strong alkali originally added), to the contents of the latter and titrate the excess of iodine by means of $\mathrm{N} /$ ro sodium thiosulphate solution and starch, as in the Messinger-Huppert method (see page 535).

Folin has further made suggestions regarding the simultaneous determination of acetone and ammonia by the use of the same air current. ${ }^{4}$ This is an important consideration for the clinician inasmuch as urines which contain acetone and acetoacetic acid are generally those from which the ammonia data are also desired. The procedure for the combination method is as follows: Arrange the ammonia apparatus as usual (see page 499), and to the aerometer of the ammonia apparatus attach the acetone apparatus set up as described above. Regulate the air current with special reference to the determination of acetone and at the end of $20-25$ minutes disconnect the acetone apparatus and complete the determination of the acetone as just described. The air current is not interrupted, and after having run one and one-half hours the ammonia apparatus is detached and the ammonia determination completed as described on page 500 .

If data regarding acetoacetic acid are desired, the result obtained by Folin's method may be subtracted from the result obtained by the Messinger-Huppert method (see page 535), inasmuch as the latter method determines both acetone and acetoacetic acid. Under all conditions the determination of acetone should be as expeditious as possible. This is essential, not only because of the fact that any

${ }_{1}$ Oxalic acid $(0.2-0.3$ gram $)$ may be substituted if desired.

2 Acetone is insoluble in a saturated solution of sodium chloride.

${ }^{3}$ Folin's improved absorption tube (see Fig. 128, p. 500) should be used in this connection inasmuch as the original type embracing the use of a rubber stopper is unsatisfactory because of the solvent action of alkaline hypoiodite on rubber.

- These determinations may even be made on the same sample of urine if the sample is too small for the double determination. 
acetoacetic acid present in the urine will become transformed into acetone, but also because of the rapid spontaneous decomposition of the alkaline hypoiodite solution used in the determination of the acetone. It has been claimed that alkaline hypoiodite solutions are almost completely converted into iodate solutions in one-holf hour. Folin states, however, that the transformation is not so rapid as this, but he nevertheless emphasizes the necessity of rapidity of manipulation. At the same time it should be remembered that the air current must not be as rapid as for ammonia, inasmuch as the alkaline hypoiodite solution will not absorb all the acetone under those conditions.

Interpretation.-Usually about one-fourth of the total acetone and acetoacetic acid excretion is in the form of acetone, but the proportion varies considerably. See Acetone and Acetoacetic Acid.

\section{Acetoacetic Acid}

r. Folin-Hart Method.-Arrange the apparatus as described under the Folin-Hart method for the determination of acetone and acetoacetic acid (see page 533). Start the air current in the usual way and permit it to run 25 minutes without the application of heat to the urine under examination. Under these conditions the preformed acetone present in the solution is all removed (see page 538). Immediately attach a freshly prepared absorption bottle or introduce fresh alkaline hypoiodite solution into the original bottle. Apply heat to the large test-tube as already described (see page 533), in order to convert the acetoacetic acid into acetone, permit the air current to continue for the usual 25-minute period, and determine the acetoacetic acid value in terms of acetone by the usual titration procedure (see page 536 ).

Interpretation.-Ordinarily about three-fourths of the total acetone and acetoacetic acid excretion occurs in the form of acetoacetic acid, which, however, is readily transformed into acetone with loss of carbon dioxide. See Acetone and Acetoacetic Acid.

2. Folin-Messinger-Huppert and Folin-Scott-Wilson Method.--Determine the combined acetone and a cetoacetic acid, in terms of acetone, by the MessingerHuppert method or the Scott-Wilson method (see pages 535 and 536), and subsequently determine the acetone by Folin's method (see page 538). Subtract the value determined by the second method from that obtained in either of the first two methods to secure data regarding the acetoacetic acid content of the urine, in terms of acetone.

\section{Acetone, Acetoacetic Acid, and $\beta$-Hydroxybutyric Acid}

\section{Method of Shaffer and Marriott. ${ }^{1}$ - Principle.-By this procedure} the combined acetone and acetoacetic acid is determined in the same sample of urine used in the determination of $\beta$-hydroxybutyric acid. The preformed acetone and the acetoacetic acid are distilled off to.

\footnotetext{
1 Shaffer and Marriott: Jour. Biol. Chem., 16, 265, 1015.
} 
gether as acetone and determined by the iodine titration method. The $\beta$-hydroxybutyric acid remains in the residue from distillation and is oxidized by means of potassium bichromate. The product of the oxidation is acetone which is distilled off and determined as such.

Procedure.-Determination of Acetone and Acetoacetic Acid.-Measure from $25-100$ c.c. $^{1}$ or more of urine (usually 50 c.c.) with a pipette into a 500 c.c. volumetric flask containing 200-300 c.c. of water. Add basic lead acetate solution (U. S. P.) in volume equal to that of the urine used ${ }^{2}$ and mix well. Add concentrated ammonium hydroxide in amount equal to about one-half that of the lead acetate solution. Dilute the contents of the flask to the mark with water, shake, and after standing a few minutes filter the liquid, preferably through a folded filter. Measure 200 c.c. of the filtrate into a round bottom flask (80o c.c. or liter Kjeldahl flasks are convenient) dilute with water to about 600 c.c. and add 15 c.c. of concentrated sulphuric acid and a little talc or a boiling stone. Distil until about 200 c.c. of distillate have been collected. The tube of the condenser should dip beneath the surface of the water in the receiving flask so that no loss of acetone will occur. The distilling flask must also be fitted with a dropping tube or dropping funnel so water may be run in from time to time and the volume of liquid in the flask kept from becoming less than $400-500$ c.c. A good condenser should be used, but it is not necessary to cool the distillate in ice.

The distillate thus obtained is transferred to a second Kjeldahl flask and Io c.c. of Io per cent $\mathrm{NaOH}$ added. It is then redistilled for about 20 minutes. ${ }^{3}$ This second distillate is then titrated with standard iodine and thiosulphate solution as in the method for acetone and acetoacetic acid previously given (see Messinger-Huppert method, page 535), and the calculation made in the same way. The result gives the combined acetone and acetoacetic acid content of the urine expressed as acetone.

Determination of the $\beta$-Hydroxybutyric Acid.-The flask containing the residue from the first distillation above is used in the determination of the $\beta$-hydroxybutyric acid. A new receiver is arranged as before with the tip from the condenser dipping beneath the surface of the water. The distillation is then continued and water added whenever necessary to keep the volume between 400 and 600 c.c. A dilute solution (I per cent) of potassium bichromate is added during the distillation. At first 20 c.c. of this I per cent solution is added slowly through the dropping tube and then Io c.c. portions every 15-20 minutes until

1 The amount used depends upon the expected yield of $\beta$-oxybutyric acid. In the case of urines which give a strong ferric chloride reaction for diacetic acid, or when $5^{-10}$ grams or more of $\beta$-oxybutyric acid is expected, it is unnecessary to use more than $25-50$ c.c. of urine. However, in case only a trace of $\beta$-oxybutyric acid is expected, the volume should be much larger as indicated. Under all conditions, the amount specified is sufficient for duplicate determinations. It is desirable to use such a volume of urine as contains the proper amount of $\beta$-oxybutyric acid to yield $25-50 \mathrm{mg}$. of acetone.

${ }^{2}$ If the urine contains but little or no sugar only half the amount or less of lead acetate should be used.

${ }^{3}$ In many instances when a high degree of accuracy is not required this redistillation may be omitted and the first distillate titrated directly. The results so obtained are slightly higher than those after redistillation from alkali. The object of the redistillation is to get rid of fatty acids of which formic acid is one of the most troublesome. 
the whole has been added.' Should the liquid become markedly green the bichromate must be added at correspondingly shorter intervals and in amount sufficient to maintain a slight red-yellow color of the chromic acid which may be detected even in the presence of the green. Continue the distillation with moderate boiling for from two to three hours. The distillate which should be collected in a liter flask to avoid transference is again distilled for about 20 minutes after adding Io c.c. of Io per cent sodium hydroxide and 25 c.c. of 3 per cent hydrogen peroxide. The flask must be heated cautiously until the peroxide has been decomposed. This final distillate is titrated with standard iodine and thiosulphate in the usual manner (see page 536) and the result expressed as hydroxybutyric acid. One c.c. of $\mathrm{N} / \mathrm{ro}$ iodine solution is equivalent to 1.736 mg. of hydroxybutyric acid. One c.c. of the $1.035 \mathrm{~N} /$ Io iodine which is recommended for acetone titrations is equivalent to $1.793 \mathrm{mg}$. of hydroxybutyric acid. About Io per cent should be added to the results for $\beta$-hydroxybutyric acid as obtained by this method as the yield of acetone is only about 90 per cent of the theoretical. This error appears to be practically constant, so that satisfactory results may be obtained by correction.

Interpretation. - $\beta$-Hydroxybutyric acid may occur in normal human urine to the extent of $20-30 \mathrm{mg}$. per day. In fasting or on a carbohydrate-free diet very large amounts may be excreted (up to 20 grams per day). In severe diabetes mellitus the largest amounts are found. Excretions of 50 or even 100 grams or over per day have been noted. It is always present in the urine when large amounts of acetone are present. In severe diabetes it is usually the most abundant of the acetone bodies making up from 60-80 per cent of the total. The ratio is, however, by no means constant and it should be borne in mind that in rare cases large amounts of $\beta$-hydroxybutyric acid may be eliminated although the acetone excretion is very low. (See Acetone and Acetoacetic Acid.)

2. Nephelometric Methods for Acetone, Acetoacetic Acid, and $\beta$-Hydroxybutyric Acid.-Folin and Denis² have suggested nephelometric methods for the determination of the acetone bodies in urine. The methods are similar to those used by Marriott for blood analysis (see Chapter XVI) but the air current is used instead of distillation.

\section{$\beta$-Hydroxybutyric Acid}

I. Black's Method.-Principle.-The urine is mixed with plaster of Paris to form a coarse meal and extracted with ether to obtain the hydroxybutyric acid which is then determined by meins of the polariscope.

${ }^{1}$ From 0.5 gram to $\mathrm{I}$ gram of bichromate will usually be sulficient, and not more than I gram should be addled unless the liquid turns green indicating a great reduction to chromium sulphate. Very rarely 2 or 3 grams of bichromate may be necessary, especially if the sugar has not been completely removed.

${ }^{2}$ Folin and Denis: Jour. Biol. Chem., 18, 263, ro14. 
Procedure.- Render 50 c.c. of the urine under examination faintly alkaline with sodium carbonate and evaporate to one-third the original volume. Concentrate to about ro c.c. on a water-bath, cool the residue, acidify it with a few drops of concentrated hydrochloric acid ${ }^{1}$ and add plaster of Paris to form a thick paste. Permit the mixture to stand until it begins to "set," then break it up with a stout glass rod having a blunt end and reduce the material to the consistency of a fairly dry coarse meal. ${ }^{2}$ Transfer the meal to a Soxhlet apparatus and extract with ether for six to ten hours. At the end of this period evaporate the ether-extract either spontaneously or in an air current. Dissolve the residue in water, add a little boneblack, if necessary, filter until a clear solution is obtained and make up the filtrate to a known volume (25 c.c. or less) with water. The $\beta$-hydroxybutyric acid should then be determined by means of the polariscope. Its specific rotation is -24.12 .

This method is satisfactory where the amount of hydroxybutyric acid present is not too small. Errors due to incomplete extraction of the acid are partly counterbalanced by the extraction of other levorotatory substances.

Interpretation.-See page 54I.

2. Method of Schaffer and Marriott.-See page 539.

\section{Indican}

Ellinger's Method.-Principle.-This method for the quantitative determination of indican is based upon the principle underlying Jaffe's qualitative test for indican. The urine after removal of interfering substances with basic lead acetate is treated with Obermayer's reagent to oxidize the indican to indigo. The indigo is extracted with chloroform, the chloroform evaporated off and the residue titrated with potassium permanganate. The method is not very accurate but is as satisfactory as any.

Procedure. - To 50 c.c. of urine ${ }^{3}$ in a small beaker or casserole add 5 c.c. of basic lead acetate solution, "mix thoroughly, and filter. Transfer 40 c.c. of the filtrate to a separatory funnel, add an equal volume of Obermayer's reagent (see page 388) and 20 c.c. of chloroform, and extract in the usual manner. This extraction with chloroform should be repeated until the chloroform solution remains colorless. Shake up the combined chloroform extracts two or three times with distilled water in a separating funnel and complete the purification by extracting with very dilute sodium hydroxide ( $\mathrm{I}:$ I000). Remove all traces of alkali by washing with water. Now filter the combined chloroform extracts through a dry filter paper into a dry Erlenmeyer flask. Distil off the chloroform, heat the residue on a boiling water-bath for five minutes in the open

1 The residue should give a distinct red color with litmus paper.

${ }^{2}$ Before this is accomplished it may, in some cases, be necessary to add a little more plaster of Paris.

${ }^{3}$ If the urine under examination is neutral or alkaline in reaction it may be made faintly acid with acetic acid before adding the basic lead acetate.

4 For preparation of basic lead acetate solution see Appendix. 
flask, and wash the dried residue with hot water. ${ }^{1}$ Add ro c.c. of concentrated sulphuric acid to the washed residue, heat on the water-bath for five to ten minutes, dilute with 100 c.c. of water, and titrate the blue solution with a very dilute solution of potassium permanganate. ${ }^{2}$ The end point is indicated by the dissipation of all the blue color from the solution and the formation of a pale yellow color.

Beautiful plates of indigo blue sometimes appear in the chloroform extract of urines containing abundant indican. In urines preserved by thymol the determination of indican is interfered with unless great care is taken in washing the chloroform extract with dilute alkali. Care should be taken, therefore, to make the indican determination upon fresh urine, before the addition of the preservative.

Plasencia ${ }^{3}$ has suggested a method which is shorter than Ellinger's and according to its sponsor, just as accurate.

Calculation.-One cubic centimeter of the diluted permanganate solution is equivalent to about $0.15 \mathrm{mg}$. of indigo. Ellinger claims that one-sixth of the amount determined must be added to the value obtained by titration in order to secure accurate data. This correction should always be made.

Interpretation. - From 4-20 $\mathrm{mg}$. of indican are excreted per day by normal men. In normal individuals the variations are dependent mainly upon the diet. A meat diet increases the indican excretion, while a milk or carbohydrate-rich diet decreases it. Pathologically the greatest increases are found in disorders involving increased putrefaction and stagnation of intestinal contents. Bacterial decomposition of body protein as in gangrene, putrid pus formation, etc., gives rise to increases.

\section{Phenols}

Colorimetric Method of Folin and Denis. ${ }^{4}$-Principle.-This method is based upon the fact that phenols yield with a solution of phosphotungstic-phosphomolybdic acid and alkali a deep blue color the depth of which is proportional to the amount of such substances present. Traces of protein, which may be present in the urine, and uric acid give a blue color with the reagent and are removed by precipitation with an ammoniacal silver solution and colloidal iron as a preliminary to the determination of the phenols.

Procedure.-Removal of Interfering Substances.-Place ro c.c. of ordinary urine, or 20 c.c. of a dilute urine in a 50 c.c. volumetric flask. To this add an

${ }^{1}$ The washing should be continued until the wash water is no longer colored. Uriinarily two or three washings are sullicient. If a separation of indigo particles takes place during the washing process, the wash water sloould be filtered, the indigo extracted with chloroform, and the usual method applied from this point.

${ }^{2} \mathrm{~A}$ "stock solution" of potassium permanganate containing 3 grams per liter shoulı be prepared, and when needed for titration purposes a suitable volume of this solution should be diluted with 40 volumes of water. The potassium permanganate solution may be standardized with pure indigo.

${ }^{3}$ Plasencia: Revista de iledicina y Cirugia., 17, r, 19 г.

- Folin and Denis: J. Biol. Clem., 22, 305, ror 5. 
acid silver lactate solution (from 2 to 20 c.c. of a 3 per cent solution of silver lactate in 3 per cent lactic acid) until no further precipitate is obtained. Add a few drops of colloidal iron, shake the flask, dilute to mark with distilled water, shake again, and filter the contents through a dry filter. Phenols are not precipitated by this procedure but are recovered quantitatively in the filtrate. Transfer 25 c.c. of the filtrate to a 50 c.c. volumetric flask, and add a sufficient quantity of saturated sodium chloride solution, containing Io c.c. of strong hydrochloric acid per liter, to precipitate all the silver. Fill the flask to the mark with distilled water, mix thoroughly, and filter through a dry filter. This filtrate, which contains half the phenol from the urine taken for analysis, is used for the determination of free and total phenols.

Free Phenols.-Place 20 c.c. of the filtrate mentioned above in a 50 c.c. volumetric flask, add 5 c.c. of the phosphotungstic-phosphomolybdic acid reagent ${ }^{1}$ and 15 c.c. of a saturated solution of sodium carbonate. Dilute to volume with luke warm water $\left(30-35^{\circ} \mathrm{C}\right.$.), mix thoroughly and after allowing to stand for 20 minutes compare the deep blue color in the Duboscq colorimeter (see Fig. I53, page 486) against a standard solution of phenol (see below) similarly treated.

Total Phenols (Free and Conjugated).-Place 20 c.c. of the same filtrate used for the determination of free phenols in a large test-tube, add Io drops of concentrated hydrochloric acid, cover the tube with a small funnel, heat rapidly to boiling over a free flame, and then place in a boiling water-bath for ten minutes. This process serves to decompose the conjugated phenols. At the end of the ten minutes, remove the tube, cool, and transfer the contents to a 100 c.c. volumetric flask. Add ro c.c. of the phosphotungstic-phosphomolybdic reagent, 25 c.c. of saturated sodium carbonate solution, dilute to mark with luke warm water $\left(30-35^{\circ} \mathrm{C}\right.$.), mix thoroughly, allow to stand for 20 minutes, and read in the Duboscq colorimeter (see page 486 ) against a standard solution of phenol (see below).

Standard Solution of Phenol.-The standard used is a solution of pure phenol in $\mathrm{N} / \mathrm{I} 00$ hydrochloric acid containing I $\mathrm{mg}$. of phenol in Io c.c., standardized by means of the iodometric titration. The preparation is carried out as follows: Make a phenol solution in $\mathrm{N} / \mathrm{I}$ o hydrochloric acid, which contains approximately $\mathrm{I} \mathrm{mg}$. of crystallized phenol per cubic centimeter. Transfer 25 c.c. of this solution to a 250 c.c. flask, add 50 c.c. of $\mathrm{N} /$ Io sodium hydroxide, heat to $65^{\circ} \mathrm{C}$., add 25 c.c. of $\mathrm{N} /$ Io iodine solution, stopper the flask, and let stand at room temperature 30 or 40 minutes. Add 5 c.c. of concentrated hydrochloric acid and titrate the excess of iodine with $\mathrm{N}$ / Io thiosulphate solution. Each cubic centimeter of $\mathrm{N} /$ Io iodine solution corresponds to $1.567 \mathrm{mg}$. of phenol. On the basis of the result dilute the phenol solution so that Io c.c. contain I mg. of phenol. Five c.c. of this solution (equivalent to $0.5 \mathrm{mg}$. of phenol), when Io c.c. of the phosphotungstic phosphomolybdic reagent and 25 c.c. of saturated sodium carbonate solution are added, and the whole made up with water at about $30^{\circ} \mathrm{C}$. to Ioo c.c., give when set in the colorimeter at $20 \mathrm{~mm}$. a convenient standard.

Calculation.- The filtrate used for the determination of free and total phenols

1 This reagent is prepared as follows: Boil together for two hours (using a reflux condenser) Ioo grams of sodium tungstate, 20 grams of phosphomolybdic acid (or an equivalent of molybdic acid), 50 c.c. of phosphoric acid ( 85 per cent.), and 75 c.c. of distilled water. After the period of heating, cool, dilute to I liter with distilled water, and filter if necessary. 
contains the phenols from one-half the amount of urine analyzed. The actual determination of phenols, both free and total, is made upon a two-fifths portion of this filtrate and this amount of filtrate contains the phenols from one-fifth of the amount of urine analyzed. In the determination of free phenols the colored solution is diluted to only half that of the standard while in the determination of total phenols the dilution is the same as that of the standard.

Hence,

and

$$
\frac{\mathbf{R}_{1}}{\mathbf{R}_{2} \times 4}=\text { milligrams of free phenol }
$$

$$
\frac{\mathbf{R}_{1}}{\mathbf{R}_{2} \times 2}=\text { milligrams of total phenol }
$$

in 2 c.c. or 4 c.c. of urine according to whether Io c.c. or 20 c.c. of urine was taken for analysis, when $R_{1}$ is taken as the reading obtained with the standard solution, and $R_{2}$ is taken as the reading obtained with the unknown.

Interpretation.-By this method total phenol excretions of from $0.2-0.5$ gram per day have been noted in normal individuals. These results are much higher than figures previously obtained by other methods. The free phenols varied from 0.I-0.3 gram per day. The total phenol excretion appears to vary directly but not proportionately with the protein intake. The amount of conjugated phenol indicates the extent to which the phenols have been detoxicated. The excretion of phenols is increased in gastro-intestinal disorders associated with increased putrefaction. It is increased by the ingestion of phenols or of benzene.

\section{Oxalic Acid}

Salkowski-Autenrieth and Barth Method.-Principle. -The oxalic acid is precipitated by means of $\mathrm{CaCl}_{2}$. From the solution of this precipitate in hydrochloric acid the oxalic acid is extracted with ether and reprecipitated as calcium oxalate.

Procedure.-Place the 24-hour urine specimen in a precipitating jar, add an excess of calcium chloride, render the urine strongly ammoniacal, stir it well, and allow it to stand $18-20$ hours. Filter off the precipitate, wash it with a small amount of water and dissolve it in about 30 c.c. of a hot 15 per cent solution of hydrochloric acid. By means of a separatory funnel extract the solution with I 50 c.c. of ether which contains 3 per cent of alcohol, repeating the extraction four or five times with fresh portions of ether. Unite the ethercal extracts, allow them to stand for an hour in a flask, and then filter through a $d r y$ filter paper. Add 5 c.c. of water to the filtrate, to prevent the formation of diethyl oxalate when the solution is heated, and distil off the cther. If necessary, decolorize the liquid with animal charcoal and filter. Concentrate the filtrate to $3-5$ c.c., add a little calcium chloride solution, make it ammoniacal, and after a few minutes render it slightly acid with acetic acid. Allow the acidified solution to stand several hours, collect 
the precipitate of calcium oxalate on a washed filter paper, ${ }^{1}$ wash. incinerate strongly (to $\mathrm{CaO}$ ), and weigh in the usual manner.

Calculation.-Since 56 parts of $\mathrm{CaO}^{1}$ are equivalent to 90 parts of oxalic acid, the quantity of oxalic acid in the volume of urine taken may be determined by multiplying the weight of $\mathrm{CaO}$ by the factor $\mathrm{I} .607 \mathrm{I}$.

Interpretation.-From I 5-20 $\mathrm{mg}$. of oxalic acid are excreted by a normal adult on an ordinary mixed diet. It arises from oxalates of the food ingested and from fat and protein metabolism. It is increased by the ingestion of apples, grapes, cabbage, etc., although most of the ingested oxalate is destroyed. It is increased in disturbances of metabolism associated with decreased oxidation, according to certain observers. The term "oxaluria" has been largely a misnomer.

\section{Sulphur}

\section{(a) Gravimetric Procedures}

I. Total Sulphates.-Folin's Method.-Principle.-The sulphuric acid of the conjugated sulphates is set free by boiling with acid. The total sulphates are then precipitated with barium chloride.

Procedure.-Place 25 c.c. of urine in a 200-250 c.c. Erlenmeyer flask, add 20 c.c. of dilute hydrochloric acid ${ }^{2}$ (I volume of concentrated $\mathrm{HCl}$ to 4 volumes of water) and gently boil the mixture for 20-30 minutes. To minimize the loss of water by evaporation the mouth of the flask should be covered with a small watch glass during the boiling process. Cool the flask for 2-3 minutes in running water, and dilute the contents to about I50 c.c. by means of cold water. Add ro c.c. of a 5 per cent solution of barium chloride slowly, drop by drop, to the cold solution. ${ }^{3}$ The contents of the flask should not be stirred or shaken during the addition of the barium chloride. Allow the mixture to stand at least one hour, then shake up the solution and filter it through a weighed Gooch crucible. ${ }^{4}$

Wash the precipitate of $\mathrm{BaSO}_{4}$ with about 250 c.c. of cold water, dry it in an air-bath or over a very low flame, then ignite, ${ }^{5} \mathrm{cool}$ and weigh.

Calculation.- Subtract the weight of the Gooch crucible from the weight of the crucible and the $\mathrm{BaSO}_{4}$ percipitate to obtain the weight of the precipitate.

${ }^{1}$ Schleicher and Schüll, No. 589 , is satisfactory.

${ }^{2}$ If it is desired, 50 c.c. of urine and 4 c.c. of concentrated acid may be used instead.

${ }^{3} \mathrm{~A}$ dropper or capillary funnel made from an ordinary calcium chloride tube and so constructed as to deliver Io c.c. in 2-3 minutes is recommended for use in adding the barium chloride.

${ }^{4}$ If a Gooch crucible is not available, the precipitate of $\mathrm{BaSO}_{4}$ may be filtered off upon a washed filter paper (Schleicher \& Schüll's, No. 589, blue ribbon), and after washing the precipitate with about 250 c.c. of cold water the paper and precipitate may be dried in an air-bath or over a low flame. The ignition may then be carried out in the usual way in the ordinary platinum or porcelain crucible. In this case correction must be made for the weight of the ash of the filter paper used.

${ }^{5}$ Care must be taken in the ignition of precipitates in Gooch crucibles. The flame should never be applied directly to the perforaled bottom or to the sides of the crucible, since such manipulation is invariably attended by mechanical losses. The crucibles should always be provided with lids and tight bottoms during the ignition. In case porcelain Gooch crucibles, whose bottoms are not provided with a non-perforated cap, are used, the crucible may be placed upon the lid of an ordinary platinum crucible during ignition. The lid should be supported on a triangle, the crucible placed upon the lid and the flame applied to the improvised bottom. Ignition should be complete in ro minutes if no organic matter is present. 
The weight of $\mathrm{SO}_{3}{ }^{1}$ in the volume of urine taken may be determined by means of the following proportion.

Mol. Wt. Wt. of Mol, wt.

$\mathrm{BaSO}_{4}: \mathrm{BaSO}_{4}:: \mathrm{SO}_{3}: \mathrm{x}$ (wt. of $\mathrm{SO}_{3}$ in grams).

Representing the weight of the $\mathrm{BaSO}_{4}$ precipitate by y and substituting the proper molecular weights, we have the following proportion:

$233.43: y:: 80.06: x$ (wt. of $\mathrm{SO}_{3}$ in grams in the quantity of urine used).

Calculate the quantity of $\mathrm{SO}_{3}$ in the twenty-four-hour specimen of urine.

To express the result in percentage of $\mathrm{SO}_{3}$ simply divide the value of $\mathrm{x}$, as just determined, by the quantity of urine used.

Interpretation.-The total sulphate excretion (ethereal and inorganic sulphates) by a normal adult on a mixed diet is usually between I.5 and 3.0 gram of $\mathrm{SO}_{3}$ with an average of about 2.0 gram. The sulphuric acid is derived but to a slight extent ordinarily from ingested sulphates, being mainly dependent on the sulphur of the protein ingested and will consequently vary widely with the protein content of the diet. From 75 to 95 per cent of the total sulphur of the urine is ordinarily found as sulphate, the proportion being greatest on a high protein diet. The sulphate excretion is increased in all conditions associated with increased decomposition of body protein as in acute fevers and decreased whenever there is a decrease in metabolic activity.

2. Inorganic Sulphates.-Folin's Method.-Place 25 c.c. of urine and Ioo c.c. of water in a 200-250 c.c. Erlenmeyer flask and acidify the diluted urine with ro c.c. of dilute hydrochloric acid (I volume of concentrated $\mathrm{HCl}$ to 4 volumes of water). In case the urine is dilute 50 c.c. may be used instead of 25 c.c. and the volume of water reduced proportionately. Add ro c.c. of 5 per cent barium chloride slowly, drop by drop, to the cold solution and from this point proceed as indicated in the method for the determination of Total Sulphates, page 546.

Calculate the quantity of inorganic sulphates, expressed as $\mathrm{SO}_{3}$, in the twentyfour-hour urine specimen.

Calculation.-Calculate according to the directions given under Total Sulphates, above.

Interpretation.-On an average about 90 per cent of the total sulphates of the urine exists as inorganic sulphates but the proportion of the sulphates existing in this form varies widely, being greater on a high protein diet than on a very low protein diet. The amount varies with the total sulphates (which see).

3. Ethereal Sulphates.-Folin's Method.-Principle.-The inorganic sulphates are removed with barium chloride and the conjugated sulphates then determined after hydrolysis.

Procedure.-Place 25 c.c. of urine in an Erlenmeyer flask of suitable size,

${ }^{1}$ It is considered preferable by many investigators to express all sulphur values in terms of $\mathrm{S}$ rather than $\mathrm{SO}_{3}$. 
dilute it with 75 c.c. of water and acidify the mixture with 30 c.c. of dilute hydrochloric acid (I volume of concentrated $\mathrm{HCl}$ to 4 volumes of water). To the cold solution add 20 c.c. of a 5 per cent solution of barium chloride, drop by drop. ${ }^{1}$ Allow the mixture to stand about one hour, then filter it through a dry filter paper. ${ }^{2}$ Collect 125 c.c. of the filtrate and boil it gently for at least one-half hour. Cool the solution, filter off the precipitate of $\mathrm{BaSO}_{4}$, wash, dry and ignite it according to the directions given on page 546 .

Calculation. - The weight of the $\mathrm{BaSO}_{4}$ precipitate should be multiplied by 2 since only one-half ( 125 c.c.) of the total volume (250 c.c.) of fluid was precipitated by the barium chloride. The remaining calculation should be made according to directions given under Total Sulphates, page 546.

Calculate the quantity of ethereal sulphates, expressed as $\mathrm{SO}_{3}$, in the twentyfour-hour urine specimen.

Interpretation.-The excretion of ethereal sulphates (expressed as $\mathrm{SO}_{3}$ ) varies ordinarily from $\mathrm{O}$.I to 0.25 gram per day comprising from 5 to 5 per cent of the total sulphur excretion. The absolute amount of ethereal sulphate increases with increase in the protein of the diet and particularly with increase of putrefactive processes in the intestine or elsewhere. The amount excreted cannot howerer be taken as an index of the extent of intestinal putrefaction.

4. Total Sulphur.-Benedict's Method. ${ }^{3}$ - Principle.-The urine is evaporated and ignited with a solution of copper nitrate and potassium chlorate. Organic matter is thus destroyed and all unoxidized sulphur is oxidized to the sulphate form and can be readily precipitated with barium chloride in the usual manner. The method is very convenient and accurate.

Ten c.c. of urine are measured into a small $(7-8 \mathrm{~cm}$.) porcelain evaporating dish and 5 c.c. ${ }^{4}$ of Benedict's sulphur reagent ${ }^{5}$ added. The contents of the dish are evaporated over a free flame which is regulated to keep the solution just below the boiling-point, so that there can be no loss through spattering. When dryness is reached the flame is raised slightly until the entire residue has blackened. The

1 See note (3) at the bottom of p. 546.

${ }^{2}$ This precipitate consists of the inorganic sulphates. If it is desired, this $\mathrm{BaSO}_{4}$ precipitate may be collected in a Gooch crucible or on an ordinary quantitative filter paper and a determination of inorganic sulphates made, using the same technic as that suggested above. In this way we are enabled to determine the inorganic and ethereal sulphates in the same sample of urine.

${ }^{3}$ Benedict: Journal of Biological Chemistry, 6, 363, I909.

1 If the urine is concentrated the quantity should be slightly increased.

${ }^{5}$ Crystallized copper nitrate, sulphur-free or of known sulphur content....200 grams. Sodium or potassium chlorate....................... 50 grams

Distilled water to....................................

Denis has suggested the use of the following solution:

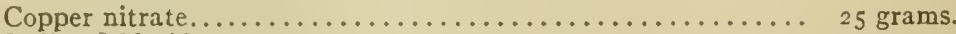

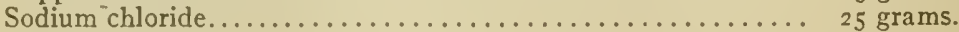

Ammonium nitrate............................. ro grams.

Water to make............................ Ioo c.c.

The procedure is the same as the above except that 25 c.c. of urine and 5 c.c. of reagent are taken. It gives accurate results. 
flame is then turned up in two stages to the full heat of the bunsen burner and the contents of the dish thus heated to redness for ten minutes after the black residue (which first fuses) has become dry. This heating is to decompose the last traces of nitrate (and chlorate). The flame is then removed and the dish allowed to cool more or less completely. Ten to 20 c.c. of dilute ( $I: 4)$ hydrochloric acid is then added to the residue in the dish, which is then warmed gently until the contents have completely dissolved and a perfectly clear, sparkling solution is obtained. This dissolving of the residue requires scarcely two minutes. With the aid of a stirring rod the solution is washed into ${ }^{1}$ a small Erlenmeyer flask, diluted with cold, distilled water to roo-r 50 c.c., ro c.c. of ro per cent barium chloride solution added drop by drop, and the solution allowed to stand for about an hour. It is then shaken up and filtered as usual through a weighed Gooch crucible. Controls should be run on the oxidizing mixture.

Calculation.-Make the calculation according to directions given under Total Sulphates, page 546. Calculate the quantity of sulphur expressed as $\mathrm{SO}_{3}$ or $\mathrm{S}$, present in the twenty-four-hour urine specimen.

Interpretation. - The total sulphur $\left(\mathrm{SO}_{3}\right)$ excretion averages about 2.5 grams per day. It runs more or less parallel with the decomposition of endogenous and exogenous protein and a definite ratio between the excretion of total $N$ and total $S$ might be expected. It has been suggested that the ratio $5: x$ expresses this relation in a general way but no constant value can be given. See Total Sulphates.

5. Total Sulphur.-Osborne-Folin Method.-Principle.-This method depends on the destruction of organic matter by means of sodium peroxide. It is employed particularly for the determination of sulphur in foods and feces. Benedict's procedure (see above) is simpler and fully as satisfactory for urine.

Place 25 c.c. of urine ${ }^{2}$ in a 200-250 c.c. nickel crucible and add about 3 grams of sodium peroxide. Evaporate the mixture to a syrup upon a steam water-bath and heat it carefully over an alcohol flame until it solidifies (1 5 minutes). Now remove the crucible from the flame and allow it to cool. Moisten the residue with $\mathrm{I}-2 \mathrm{c}$.c. of water, ${ }^{3}$ sprinkle about $7-8$ grams of sodium peroxide over the contents of the crucible and fuse the mass over an alcohol flame for about ro minutes. Allow the crucible to cool for a few minutes, add about roo c.c. of water to the contents and heat at least one-half hour over an alcohol flame to dissolve the alkali and decompose the sodium peroxide. Next rinse the mixture into a 400-450 c.c. Erlenmeyer flask, by means of hot water, and dilute it to about 250 c.c. Heat the solution nearly to the boiling-point and add concentrated hydrochloric acid slowly until the nickelic oxide, derived from the crucible, is just brought into solution. ' A few ninutes' boiling should now yield a clear solution. In case too little peroxide or too much water was added for the final fusion a clear solution will not be obtained. In this event cool the solution and remove the insoluble matter by filtration.

'Sometimes the porcelain glaze cracks during heating, in which case the sulution should be filtered into the flask.

${ }^{2}$ If the urine is very dilute 50 c.c. may be used.

${ }^{3}$ This moistening of the residue with a small amount of water is very essential and should not be neglecter.

4 About IS C.c. of acid are required for $S$ grams of sodium peroxide. 
To the clear solution add 5 c.c. of very dilute alcohol (about I8-20 per cent) and continue the boiling for a few minutes. The alcohol is added to remove the chlorine which was formed when the solution was acidified. Add ro c.c. of a Io per cent solution of barium chloride, slowly, drop by drop, ${ }^{1}$ to the liquid. Allow the precipitated solution to stand in the cold two days and then filter and continue the manipulation according to the directions given under Total Sulphates, page 546 .

Calculation.-Make the calculation according to directions given under Total Sulphates, page 546. Calculate the quantity of sulphur, expressed as $\mathrm{SO}_{3}$ or $\mathrm{S}$, present in the twenty-four-hour urine specimen.

$$
\text { Interpretation.-See page } 549 \text {. }
$$

\section{(b) Volumetric Procedures}

6. Volumetric Determination of Ethereal and Inorganic Sulphates. - Method of Rosenheim and Drummond. ${ }^{2}$-Principle.-Thesulphates of the urine are precipitated by means of benzidine solution, the precipitate of benzidine sulphate being filtered off and the sulphuric acid of the compound titrated with $\mathrm{N}$ / Io $\mathrm{KOH}$ using phenolphthalein as an indicator. This is possible because the benzidine is a very weak base and its sulphate readily dissociates. It is necessary that excess of $\mathrm{HCl}$ be avoided in the precipitation process.

Procedure.-(a) Inorganic Sulphates.-Preparation of the benzidine solution. Rub 4 grams of benzidine (Kahlbaum) into a fine paste with about ro c.c. of water and transfer to a 2 -liter flask with the aid of about 500 c.c. of water. Add 5 c.c. of concentrated $\mathrm{HCl}$ (sp. gr. I.I9) and make up to 2 liters with distilled water. One hundred and fifty c.c. of this solution, which keeps indefinitely, are sufficient to precipitate o.I gram $\mathrm{H}_{2} \mathrm{SO}_{4}$.

Measure 25 c.c. of urine into a 250 c.c. Erlenmeyer flask and acidify with dilute hydrochloric acid $(\mathrm{I}: 4)$ until the reaction is distinctly acid to Congo red paper. Usually $\mathrm{I}-2$ c.c. of dilute acid are required. One hundred c.c. of the benzidine solution, as prepared above, are then run in and the precipitate, which forms in a few seconds, allowed to settle for ten minutes. Filter with suction and wash the precipitate with ro-20 c.c. of water saturated with benzidine sulphate. ${ }^{3}$ Transfer the precipitate and filter paper to the original precipitation flask with about 50 c.c. of water and titrate hot with $\mathrm{N} /$ ro $\mathrm{KOH}$, after first adding a few drops of saturated alcoholic solution of phenolphthalein.

Calculation.-One c.c. of N/ro KOH corresponds to $4.9 \mathrm{mg}$. $\mathrm{H}_{2} \mathrm{SO}_{4}$ or $4.0 \mathrm{mg}$. of $\mathrm{SO}_{3}$. Multiply the number of cubic centimeters of $\mathrm{N} / \mathrm{ro} \mathrm{KOH}$ required by 4.9 and by 4 to get the amount of $\mathrm{H}_{2} \mathrm{SO}_{4}$ in 100 c.c. of the urine analyzed.

1 See note (3) at the bottom of p. 546.

${ }^{2}$ Rosenheim and Drummond: Biochem. J., 8, I43, Igr4.

${ }^{3}$ In order to obtain accurate results it is most important that the precipitate should be finely suspended in water before titration and this again entails certain precautions during filtration so as to prevent the caking together of the precipitate. The authors use a funnel of $6 \mathrm{~cm}$. diameter and a perforated porcelain plate $\left(5^{-7} \mathrm{~mm}\right.$.) covered either with paper pulp or with a well-fitting filter paper. Do not allow the precipitate to be sucked dry on the filter. The final filtrate should show no acid reaction to Congo red. 
(b) Total Sulphates (Inorganic and Ethereal).-Measure 25 c.c. of urine into an Erlenmeyer flask, add 2-2.5 c.c. of dilute $\mathrm{HCl}$ (I :4) and 20 c.c. of water and boil for $\mathbf{r}^{-20}$ minutes. The ethereal sulphates are hydrolized. ${ }^{1}$ Allow the solution to cool and then precipitate the sulphate with benzidine as in the determination of inorganic sulphates. The titration and calculation are also carried out in the same way.

(c) Ethereal Sulphates.-Determine the total sulphates and inorganic sulphates as indicated above. Subtract the amount of inorganic sulphate from that of the total sulphate and obtain the amount of ethereal sulphate present.

(d) Total Sulphur.-According to Rosenheim and Drummond ${ }^{2}$ the benzidine method may be employed for the estimation of total sulphur in the solution obtained on the oxidation of urine by the Wolf-Osterberg ${ }^{3}$ modification of Benedict's method. This modification involves the use of larger quantities of urine than the Benedict method or a reduction in accuracy and hence probably has no advantages over Benedict's original procedure. See below for modification of Raiziss and Dubin.

(e) Neutral Sulphur.-Neutral sulphur is most readily determined by difference. Subtract from the total sulphur as determined by one of the methods given above the amount of total sulphates. The difference corresponds to the neutral sulphur of the urine sample examined.

Interpretation.- The neutral sulphur of the urine is made up of cystine and related bodies, thiocyanate, oxyproteic acids, etc. It makes up ordinarily from 5-25 per cent of the total sulphur of the urine, or on the average 0.2 to 0.4 gram per day calculated as $\mathrm{SO}_{3}$. The absolute amount is fairly constant for a given individual through wide variations of protein intake, indicating that its origin is mainly endogenous, that is, that it arises principally from the decomposition of tissue protein. On this account the percentage of the total sulphur excretion existing in the neutral form may rise to 25 per cent on a very low protein diet and decrease to 5 per cent on a high protein diet, the absolute amount remaining nearly constant. In fasting percentages as high as 70 have been noted. In many disorders as tuberculosis. cancer, cystinuria, etc., the amount may be relatively and in some cases absolutely increased but no fixed relations have been determined for the various conditions.

7. Total Sulphur.-Method of Raiziss and Dubin. LPrinciple.-The urine is oxidized by Benedict's method (page 548 ) the sulphur precipitated as benzidine sulphate and the benzidine titrated with $\mathrm{N} /$ ro permanganate solution. Very small amounts of sulphur may be determined in this way.

Procedure. - To 2 c.c. of urine in an $8 \mathrm{~cm}$. porcelain lish, add 0.5 c.c. of Benedict's reagent (page 548) and evaporate to dryness on the water-batth. Heat the dish

I $A$ larger amount of $\mathrm{HCl}$ may be used (20 c.c. of the dilute acid) if desired. In this case it is necessary to neutralize the solution carefully after boiling and again add diluec $\mathrm{HCl}$ until the reaction is acid to Congo red.

${ }^{2}$ Rosenheim and Drummond: Bioch. Jour., \&, I +3, 1014.

${ }^{3}$ Wolf and Osterberg: Bioch. Zcit., 20, 420, 1010.

"Raiziss and Dubin: Jour. Biol. Chim., is, 297, 19it. 
carefully over a small flame till the contents are black, and then heat to redness for only two minutes. Cool, add 2 c.c. of hydrochloric acid ( $1: 4)$ and warm. Neutralize the clear solution with $\mathrm{NaOH}$ (Io per cent) and again acidify with I drop of $\mathrm{HCl}$ $(I: 4)$. Add 25 c.c. of a solution of benzidine hydrochloride ${ }^{1}$ with stirring. Let stand I 5 minutes and then filter off on an asbestos filter. Transfer the precipitate to the filter by means of the filtrate and wash with 5 c.c. of cold water, drop by drop. Put the filter into a 500 c.c. Erlenmeyer flask, add I c.c. of $\mathrm{NaOH}$ (Io per cent) and 200 c.c. of water. Boil the suspension for five minutes and then cool to room temperature. Add 20 c.c. concentrated sulphuric acid and titrate the warm solution at once with $\mathrm{N}$ / Io potassium permanganate solution till a distinct pink coloration is obtained, which should last 20 seconds. In titrating at first add only about 0.5 c.c. at a time and toward the end only 2 drops at a time, waiting till the color disappears before further addition of permanganate solution. As the titration progresses it will be noticed that the yellow color gradually disappears, the solution turning practically colorless. It is at this stage of the titration that care should be taken in adding only 2 drops of permanganate at a time.

Calculation.-One c.c. of $\mathrm{N} /$ ro potassium permanganate is equivalent to 0.099 mg. of sulphur. Multiply the number of cubic centimeters of permanganate used by 0.099 and divide by 2 in order to obtain the weight of sulphur in I c.c. of urine. Calculate the day's sulphur output.

Interpretation.-See page 549 .

\section{Phosphorus}

I. Total Phosphates.-Uranium Acetate Method.-Principle.Standard uranium acetate is run into a measured quantity of urine until all of the phosphate has been precipitated as insoluble uranium phosphate. An excess of uranium is indicated by a reddish coloration with potassium ferrocyanide. This method is accurate and gives practically the total phosphorus of urine inasmuch as the latter exists generally almost entirely as phosphates.

Procedure.-To 50 c.c. of urine in a small beaker or Erlenmeyer flask add 5 c.c. of a special sodium acetate solution ${ }^{2}$ and heat the mixture to the boilingpoint. From a burette, run into the hot mixture, drop by drop, a standard solution of uranium acetate ${ }^{3}$ until a precipitate ceases to form and a drop of the mixture when removed by means of a glass rod and brought into contact with a

${ }^{1}$ Six and seven-tenths grams of benzidine (Merck reagent) are put in a $r$-liter flask, 29 c.c. of hydrochloric acid (sp. gr. I. I2) added and the solution diluted up to the mark.

${ }^{2}$ The sodium acetate solution is prepared by dissolving 100 grams of sodium acetate in 800 c.c. of distilled water, adding Ioo c.c. of 30 per cent acetic acid to the solution, and making the volume of the mixture up to I liter with water.

${ }^{3}$ Uranium Acetate Solution.-Dissolve about 35.0 grams of uranium acetate in I liter of water with the aid of heat and 3-4 c.c. of glacial acetic acid. Let stand a few days and filter. Standardize against a phosphate solution containing 0.005 gram of $\mathrm{P}_{2} \mathrm{O}_{5}$ per cubic centimeter. For this purpose dissolve 14.72I grams of pure air-dry sodium ammonium phosphate $\left(\mathrm{NaNH}_{4} \mathrm{HPO}_{4}+{ }_{4} \mathrm{H}_{2} \mathrm{O}\right)$ in water to make a liter. To 20 c.c. of this phosphate solution in a 200 c.c. beaker add 30 c.c. of water and 5 c.c. of sodium acetate solution (see above) and titrate with the uranium solution to the correct end reaction as indicated in the method above. If exactly 20 c.c. of uranium solution are required I c.c. of the solution is equivalent to 0.005 gram $\mathrm{P}_{2} \mathrm{O}_{5}$. If stronger than this dilute accordingly and check again by titration. 
drop of a solution of potassium ferrocyanide on a porcelain test-tablet produces instantaneously a brownish-red coloration. ${ }^{1}$ Take the burette reading and calculate the $\mathrm{P}_{2} \mathrm{O}_{5}$ content of the urine under examination.

Calculation.-Multiply the number of cubic centimeters of uranium acetate solution used by 0.005 to determine the number of grams of $\mathrm{P}_{2} \mathrm{O}_{3}$ in the 50 c.c. of urine used. To express the result in percentage of $\mathrm{P}_{2} \mathrm{O}_{5}$ multiply the value just obtained by 2 , e.g., if 50 c.c. of urine contained 0.074 gram of $\mathrm{P}_{2} \mathrm{O}_{5}$ it would be equivalent to 0.148 per cent.

Calculate, in terms of $\mathrm{P}_{2} \mathrm{O}_{5}$, the total phosphate content of the 24 hour urine specimen.

Interpretation. - The excretion of phosphoric acid is extremely variable but on the average the total output for the 24 hours is about 2.5 grams expressed as $\mathrm{P}_{2} \mathrm{O}_{5}$. Ordinarily the total output is mainly in the form of phosphates and is distributed between alkaline and earthy phosphates in the ratio of 2 : I but this is likewise inconstant. The greater part of the phosphate excretion arises from the ingested food, either from the preformed phosphates or more especially from the organic combinations as phospho- and nucleoproteins. The excretion is consequently very largely dependent upon the phosphorus content of the diet. Some of the phosphoric acid results from the breakdown of the tissues of the body, and this endogenous phosphoric acid excretion is increased in conditions of increased metabolism as in fevers. The findings in pathological conditions have been somewhat contradictory due to lack of control of dict. The so-called "phosphaturias" nearly always represent decreased acidity and not increased phosphate content of the urine. Such conditions are, however, significant as indicating a possible tendency to the formation of phosphatic calculi.

2. Earthy Phosphates.-Principle.-The earthy phosphates are precipitated by making the urine alkaline. The precipitate is filtered off, dissolved in acid and titrated with uranium acetate.

Procedure.-To roo c.c. of urine in a beaker add an excess of ammonium hydroxide and allow the mixture to stand $12-24$ hours. Under these conditions the phosphoric acid in combination with the alkaline earths, calcium and magnesium, is precipitated as phosphates of these metals. Collect the precipitate on a filter paper and wash it with very dilute ammonium hydroxide. Pierce the paper, and remove the precipitate by means of hot water. Bring the phosphates into solution by adding a small amount of dilute acetic acid to the warm solution. Make the volume up to 50 c.c. with water, add 5 c.c. of sodium acetate solution, and determine the $\mathrm{P}_{2} \mathrm{O}_{3}$ content of the mixture according to the directions given under the previous method.

Calculation.-Multiply the number of cubic centimeters of uranium acetate solution used by 0.005 to determine the number of grams of $\mathrm{P}_{\approx} \mathrm{O}_{5}$ in the 100 c.c.

${ }^{1}$ A ro per cent solution of potassium ferrocyanide is satistactory. 
of urine used. Since 100 c.c. of urine was taken this value also expresses the percentage of $\mathrm{P}_{2} \mathrm{O}_{5}$ present.

Calculate the quantity of earthy phosphates, in terms of $\mathrm{P}_{2} \mathrm{O}_{5}$, present in the 24-hour urine specimen.

The quantity of phosphoric acid present in combination with the alkali metals may be determined by subtracting the content of earthy phosphates from the total phosphates.

Interpretation.-Ordinarily the earthy phosphates make up from 30-40 per cent of the total phosphate excretion. The amount varies with the excretion of calcium and magnesium (which see).

3. Total Phosphorus.-(a) Volumetric Procedure-Principle.-The organic matter is destroyed by digestion with a mixture of sulphuric and nitric acids or some other oxidizing agent. The phosphorus is then precipitated as the phosphomolybdate and determined gravimetrically or volumetrically.

Preparation of the Solution.--Pipette ro c.c. of urine (or an amount of substance containing about $20 \mathrm{mg}$. of $\mathrm{P}_{2} \mathrm{O}_{5}$ ) into a Kjeldahl flask. Add ro c.c. of a mixture of equal parts of concentrated $\mathrm{H}_{2} \mathrm{SO}_{4}$ and concentrated $\mathrm{HNO}_{3}$. Digest over a low flame until red fumes cease to come off. If the mixture darkens due to the charring action of the sulphuric acid, add nitric acid from a separatory funnel a few drops at a time and continue the digestion. When the mixture remains clear on evaporation to the point where white sulphuric fumes come off the digestion is completed by heating for $10-15$ minutes longer. Cool and transfer the solution to a 400 c.c. Erlenmeyer flask with the aid of enough water to make a total volume of about 75 c.c. ${ }^{1}$

Instead of oxidizing the material as described above it may be ignited with magnesia to destroy organic matter. About 2 grams of the solid substance or 25 c.c. of urine (previously evaporated nearly to dryness) are mixed with a little more than an equal bulk of magnesium oxide in a porcelain dish of about 30 c.c. capacity. Five c.c. of magnesium nitrate solution (see Reagents and Solutions, page 602) are added and the mixture heated very gently at first, then gradually to bright redness. The mixture is cooled and transferred with water to a 250 c.c. flask. An excess (20-30 c.c.) of $\mathrm{HCl}$ are added and the mixture boiled a few minutes. Remove from the flame and add at once enough barium chloride solution to precipitate any sulphate present. Cool, make to mark, filter and take an aliquot for analysis.

Precipitation of the Phosphomolybdate.-Neutralize the solution with ammonia, make slightly acid with nitric acid, and add 5 grams of ammonium nitrate in substance (or 25 c.c. of a 60 per cent solution). Heat on a water-bath to $60-65^{\circ} \mathrm{C}$. (not higher) and add $30-40$ c.c. of molybdate solution, ${ }^{2}$ stir and let

${ }^{1}$ In the case of urine it is possible to neutralize this acid solution with ammonia, make it acid with acetic acid and titrate with uranium acetate as in the preceding method.

${ }_{2}^{2}$ Made by adding 5 c.c. of concentrated $\mathrm{HNO}_{3}$ to Ioo c.c. of the ordinary molybdate solution (see Reagents and Solutions, page 602). 
stand for about I5 minutes at $60-65^{\circ}$. Filter at once through a small paper, washing the precipitate twice by decantation with 2 per cent ammonium nitrate solution using about 25 c.c. each time, stirring up the precipitate well in each case, and allowing to settle. Transfer the precipitate to the filter and wash with 2 per cent ammonium nitrate solution until two fillings of the filter (collected separately) do not greatly diminish the color produced with phenolphthalein by I drop of the standard alkali.

Titration of the Phosphomolybdate.-Transfer the precipitate and filter back to the original beaker and dissolve in a small excess of $\mathrm{N} / 5 \mathrm{NaOH}$ (about 2-3 c.c. more than required to completely dissolve the yellow precipitate). Add about 100 c.c. of boiled and cooled water and a few drops of phenolphthalein as an indicator (a red color should be observed indicating excess of $\mathrm{NaOH}$ ) and titrate the excess of $\mathrm{NaOH}$ with $\mathrm{N} / \mathrm{IO}$ acid.

Calculation.-Divide the number of cubic centimeters of $\mathrm{N} /$ Io acid required by 2 and subtract from the number of cubic centimeters of $\mathrm{N} / 5 \mathrm{NaOH}$ used. This gives the number of cubic centimeters of $\mathrm{N} / 5 \mathrm{NaOH}$ required. Multiply by 1.268 (the equivalent of I c.c. of $\mathrm{N} / 5 \mathrm{NaOH}$ in $\mathrm{P}_{2} \mathrm{O}_{5}$ ) and obtain the number of milligrams of $\mathrm{P}_{2} \mathrm{O}_{5}$ in $x_{0}$ c.c. of the urine analyzed. Calculate the daily output of $\mathrm{P}_{2} \mathrm{O}_{5}$ in this case from the 24 -hour volume.

Interpretation.-Nearly all of the phosphorus of the urine exists as alkali and earthy phosphates. Consequently the total phosphorus varies in the same way as the total phosphates (which see). A small portion of the phosphorus of the urine may exist in organic combination though never in a reduced form. This organically bound phosphate may amount to from I-4 per cent of the total phosphorus excretion. Little is known with regard to the compounds in which it occurs. Possibly some glycerophosphoric acid may occur either free or as lecithin.

Gravimetric Modification.-The phosphorus may be determined somewhat more accurately by substituting a gravimetric procedure for the above titration. In this case the washed phosphomolybdate precipitate is dissolved on the filter paper with ammonium hydroxide and hot water to make a volume of not more than Ioo c.c. Nearly neutralize with $\mathrm{HCl}$, cool, and add about ro c.c. of magnesia mixture (see Appendix) from a burette. Add slowly (about I drop per second) stirring vigorously. After 15 minutes add I2 c.c. of ammonium hydroxide solution (sp. gr. 0.9o). Let stand for some time (two hours is usually enough) then filter and wash the precipitate with 2.5 per cent ammonia until practically free from chlorides. Ignite to whiteness or to a grayish-white ash and weigh. Multiply the weight of magnesium pyrophosphate thus obtained by 0.637 to get the weight of $\mathrm{P}_{2} \mathrm{O}_{5}$.

Calculation.-Calculate as explained above.

${ }^{1}$ It is better to use a special filter tube of about $11 / 4$ inches diameter (similar to a Gooch filtering tube) in which is placed a perforated porcelain plate ${ }^{1}{ }_{s}$ inches in diameter, covered with a layer of asbestos $\mathrm{I} / 8$ inch thick. Filtration is carried out with suction and is very rapid. Ordinary Gooch crucibles lined with asbestos may also be used but are not so satisfactory. 'The asbestos used should be spccially prepared (sce Appendix). For a good discussion of the details of procedure and sources of error of this volumetric method see Hibbard: J. Ind. Eng. Chem., 5, 998, ror3. 


\section{Chlorides}

I. Volhard-Arnold Method.-Principle.-The urine is acidified with nitric acid and the chlorides precipitated with a measured excess of standard silver nitrate solution. The silver chloride formed is filtered off and in the filtrate the excess silver nitrate is titrated back with standard ammonium thiocyanate solution. Ferric ammonium sulphate is used as an indicator. A red color due to the formation of ferric thiocyanate indicates that an excess of thiocyanate is present and that the end point has been reached.

Procedure.-Place ro c.c. of urine in a roo c.c. volumetric flask, add 20-30 drops of nitric acid (sp. gr. I.2) and 2 c.c. of a cold saturated solution of ferric alum. If necessary, at this point a few drops of 8 per cent solution of potassium permanganate may be added to dissipate the red color. Now slowly run in a known volume of the standard silver nitrate ${ }^{1}$ solution ( 20 c.c. is ordinarily used) in order to precipitate the chlorine and insure the presence of an excess of silver nitrate. The mixture should be continually shaken during the addition of the standard solution. Allow the flask to stand 10 minutes, then fill it to the 100 c.c. graduation with distilled water and thoroughly mix the contents. Now filter the mixture through a dry filter paper, collect 50 c.c. of the filtrate and titrate it with standardized ammonium thiocyanate solution. ${ }^{2}$ The first permanent tinge of red-brown indicates the end point. Take the burette reading and compute the weight of sodium chloride in the Io c.c. of urine used.

Calculation.-The number of cubic centimeters of ammonium thiocyanate solution used indicates the excess of standard silver nitrate solution in the 50 c.c. of filtrate titrated. Multiply this reading by 2, inasmuch as only one-half of the filtrate was employed, and subtract this product from the number of cubic centimeters of silver nitrate ( 20 c.c.) originally used, in order to obtain the actual number of cubic centimeters of silver nitrate utilized in the precipitation of the chlorides in the ro c.c. of urine employed.

To obtain the weight in grams of the sodium chloride in the ro c.c. of urine used, multiply the number of cubic centimeters of the standard silver nitrate solution, actually utilized in the precipitation, by o.oro. If it is desired to express the result in percentage of sodium chloride move the decimal point one place to the right.

In a similar manner the weight, or percentage of chlorine may be computed using the factor 0.006 instead of 0.010 .

${ }^{2}$ Standard silver nitrate solution may be prepared by dissolving 29.042 grams of silver nitrate in I liter of distilled water. Each cubic centimeter of this solution is equivalent to 0.0 ro gram of sodium chloride or to 0.006 gram of chlorine.

2 This solution is made of such a strength that $x$ c.c. of it is equal to $\mathrm{I}$ c.c. of the standard silver nitrate solution used. To prepare the solution dissolve $\mathrm{x} 3$ grams of ammonium thiocyanate, $\mathbf{N H S}{ }_{4} \mathrm{CN}$, in a little less than a liter of water. In a small flask place 20 c.c. of the standard silver nitrate solution, 5 c.c. of the ferric alum solution and 4 c.c. of nitric acid (sp. gr. I.2), add water to make the total volume roo c.c. and thoroughly mix the contents of the flask. Now run in the ammonium thiocyanate solution from a burette until a permanent red-brown tinge is produced. This is the end-reaction and indicates that the last trace of silver nitrate has been precipitated. Take the burette reading and calculate the amount of water necessary to use in diluting the ammonium thiocyanate in order that Io c.c. of this solution may be exactly equal to ro c.c. of the silver nitrate solution. Make this dilution and titrate again to be certain that the solution is of the proper strength. 
Calculate the quantity of sodium chloride and chlorine in the 24 -hour urine specimen.

Interpretation.-From Io I 5 grams of chlorine, expressed as sorlium chloride, are excreted per day, on the average, by normal adults. The amount is, however, closely dependent upon the chloride content of the food ingested. In fasting, the chloride excretion falls rapidly to a very minimal quantity. On high water ingestion it is increased. In pneumonia and certain other acute infectious diseases the excretion of chlorides may be markedly diminished particularly during the periods in which exudates are forming. In convalescence and with resolution of the exudates the chlorine excretion rises again. A decrease has also been noted in nephritis associated with edema.

2. Volhard-Harvey Method. ${ }^{1-}$-Principle.-This procedure differs from the Volhard-Arnold method in that the excess of silver nitrate is titrated directly without filtering and hence in the presence of the silver chloride. The procedure is thus more rapid but the exact end point is more difficult to determine.

Procedure.-Introduce 5 c.c. of urine into a small porcelain evaporating dish or casserole and dilute with about 20 c.c. of distilled water. Precipitate the chlorides by the addition of ro c.c. of standard silver nitrate solution ${ }^{2}$ and add 2 c.c. of acidified indicator. ${ }^{3}$ Now run in a standard ammonium thiocyanate solution $^{4}$ from a burette until a faint red-brown tint is visible throughout the mixture. This point may be determined readily by permitting the precipitate to settle somewhat. Calculate the sodium chloride value as indicated below.

(If a red tint is produced when the first drop of thiocyanate is added an additional ro c.c. of the standard silver nitrate solution must be introduced. The titration should then proceed as above described and proper allowance made in the calculation for the extra volume of silver nitrate employed.)

Calculation.-Since 2 c.c. of the ammonium thiocyanate solution is equivalent to I c.c. of the silver nitrate solution, divide the burette reading by 2 and subtract the quotient from Io c.c., the quantity of silver nitrate solution taken. This value is the number of cubic centimeters of silver nitrate solution actually

${ }^{1}$ Harvey: Archives of Inlernal Medicine, 6, I 2, 1910.

${ }^{2}$ See p. 556 .

${ }^{3}$ This is prepared as follows: To 30 c.c. of distilled water add 70 c.c. of 33 per cent nitric acid (sp. gr. 1.2) and dissolve 100 grams of crystalline ferric ammonium sulphate in this dilute acid solution. Filter and use the filtrate which is a saturated solution of the iron salt. This single reagent takes the place of the nitric acid and ferric alum as used in Volhard-Arnold method, and insures the use of the proper quantity of acid.

- This is a solution of ammonium thiocyanate of such a strength that 2 c.c. is equivalent to I c.c. of the silver nitrate solution. First make a concentrated solution by dissolving 13 grams in I liter of water. To determine the requisite dilution to make such a solution that 2 c.c. shall be equivalent to I c.c. of the silver nitrate solution proceed as follows: Introduce ro c.c. of the silver nitrate solution into a small porcelain evaporating dish or casserole, add $30-50$ c.c. of distilled water, 2 c.c. of the acid indicator and titrate as described above with the ammonium thiocyanate solution. The total volume of the concentrated thiocyanate solution excluding that used in this titration is divided by Io, and the result multiplied by the difference between this burette reading and 20 c.c. This will give the volume of distilled water which must be added to the concentrated thiocyanate solution to render 2 c.c. equivalent to I C.C. of the silver nitrate solution. 
used in the precipitation of the chlorides. As I c.c. of the silver nitrate solution is equivalent to 0.01 gram of sodium chloride, the number of cubic centimeters of silver nitrate solution used multiplied by 0.01 gram will give the weight of sodium chloride in the 5 c.c. portion of urine used. The weight of chlorine may be computed by using the factor 0.006 instead of 0.0 r.

Calculate the weight of sodium chloride and chlorine in the 24 -hour urine specimen.

A "short cut" method of calculating the 24-hour output of sodium chloride consists in subtracting the burette reading from 20 c.c., multiplying this value by the total urine volume and pointing off three places.

\section{Interpretation.--See above.}

3. Dehn-Clark Method ${ }^{1}$ - Principle.-In this method any organic matter which may interfere with the titrimetric determination of the chlorides is destroyed by oxidation with sodium peroxide. The chlorides are then determined by the Volhard-Arnold titration procedure.

Procedure.-To ro c.c. of urine in a 75-100 c.c. casserole, add 1.0-1.2 grams of sodium peroxide and evaporate the mixture to dryness on a boiling water-bath. In case the residue is not pure white, thus indicating that insufficient sodium peroxide has been added, the residue should be moistened with distilled water, additional sodium peroxide added, and the mixture again evaporated to dryness. When the oxidation is complete, treat the mass with ro-20 c.c. of distilled water and stir until it has practically all been brought into solution. Then introduce a bit of litmus paper and add dilute nitric acid ( $\mathrm{r}: \mathrm{r}$ ) until the litmus paper turns red and all effervescence ceases. Now place the casserole on a hot plate or on a gauze and heat the contents almost to the boiling-point. ${ }^{2}$ To the hot solution add a standard solution of silver nitrate (see page 556 ) in slight excess. ${ }^{3}$ Filter off the silver chloride while the solution is still hot and wash the precipitate thoroughly with distilled water. To the filtrate, add I c.c. of a saturated solution of ferric ammonium sulphate and then titrate with a standard solution of ammonium thiocyanate (see page $55^{6}$ ) until the clear, slightly yellow fluid (or the opalescent, milky fluid, in case there is much excess of silver nitrate) changes to a slight reddish-brown color. The color of the end point varies with the individual. The exact end point reached is not so important as is the securing of the same end point in a series of determinations as that obtained in the standardization of the standard solutions used.

Calculation.- The standard solution of silver nitrate should be made up so that I c.c. equals 0.010 gram of sodium chloride and I c.c. of the ammonium thiocyanate should be equivalent to I c.c. of the silver nitrate solution. The calculation is then exactly the same as in the Volhard-Arnold method (page 556) except that the burette reading is not multiplied by 2 .

Interpretation.-See page 557 .

4. Mohr's Method.-Principle.-The organic matter of the urine is destroyed by ignition with potassium nitrate. To the solution of the ash potassium chromate is added and then standard silver nitrate solution run in from a burette. The first

${ }^{1}$ Private communication to the author from Mr. S. C. Clark.

${ }^{2}$ If there is a slight precipitate, due to silicic acid from the casserole, this is filtered off and the filtrate collected in a 200 c.c. beaker.

${ }^{3}$ This point is most easily recognized by keeping the solution hot and in constant agitation while adding the silver nitrate so that the silver chloride formed coagulates and sinks, leaving a clear, supernatant fluid. 
excess of silver nitrate over that necessary to precipitate the chlorides reacts with the chromate to form red silver chromate $\left(\mathrm{Ag}_{2} \mathrm{CrO}_{4}\right)$. This indicates the end point. The method is accurate when applied to the ash of urine in this manner.

Procedure.-To Io c.c. of urine in a small platinum or porcelain crucible or dish add about 2 grams of chlorine-free potassium nitrate and evaporate to dryness at $100^{\circ} \mathrm{C}$. (The evaporation may be conducted over a low fla me provided care is taken to prevent loss by spurting.) By means of crucible tongs hold the crucible or dish over a free flame until all carbonaceous matter has disappeared and the fused mass is slightly yellow in color. Cool the residue somewhat and bring it into solution in a small amount ( $15-25$ c.c.) of distilled water acidified with about ro drops of nitric acid. Transfer the solution to a small beaker, being sure to rinse out the crucible or dish very carefully. Test the reaction of the fluid, and if not already acid in reaction to litmus, render it slightly acid with nitric acid. Now neutralize the solution by the addition of calcium carbonate ${ }^{1}$ in substance, add $2-5$ drops of neutral potassjum chromate solution to the mixture, and titrate with a standard silver nitrate solution.

This standard solution should be run in from a burette, stirring the liquid in the beaker after each addition. The end-reaction is reached when the yellow color of the solution changes to a slight orange-red. At this point take the burette reading and compute the percentage of chlorine and sodium chloride in the urine examined.

Calculation.-The calculation is made exactly as in the Tolhard-Arnold method, page 556, except that the reading is not multiplied by two.

Calculate the quantity of sodium chloride and chlorine in the twenty-fourhour urine specimen.

Interpretation.-See page 557 .

\section{Calcium and Magnesium}

McCrudden's Methods. ${ }^{2}$-Principle.-Urine contains magnesium, phosphates and a small amount of iron, each of which will interfere with the accurate determination of its calcium content if proper conditions of acidity are not maintained during the precipitation. In the following method the proper acidity is attained through the use of sodium acetate and hydrochloric acid, and this with slow addition of the ammonium oxalate reduces the danger of occlusion of magnesium oxalate, calcium phosphate, or ferric phosphate in the calcium oxalate precipitate.

The calcium oxalate precipitate is either ignited and weighed as $\mathrm{CaO}$ or determined volumetrically by titration with potassium permanganate. Magnesium is determined in the filtrate from the calcium determination after destruction of the organic matter. It is determined in the usual way by ignition of the magnesium ammonium phosphate precipitate and weighing as the pyrophosphate.

${ }^{1}$ The cessation of effervescence and the presence of some undecomposed calcium carbonate at the bottom of the vessel are the indications of neutralization.

${ }^{2}$ McCrudden: Jour. Biol. Chem., 7, 83, I9ro; 10, 187, I9II. 
Lyman has suggested a nephelometric method for the determination - of calcium in urine and feces. ${ }^{1}$

Procedure for Calcium.- If the urine is alkaline make it neutral or slightly acid and filter. Take 200 c.c. of the filtered urine for analysis. If it is only faintly acid to litmus paper add ro drops of concentrated hydrochloric acid (sp. gr. I.20). If the urine is strongly acid it may be made just alkaline with ammonia and then just acid with hydrochloric acid after which the ro drops of concentrated hydrochloric acid are added. Then add ro c.c. of 2.5 per cent oxalic acid. Run in slowly with stirring 8 c.c. of 20 per cent sodium acetate. Allow to stand over night at room temperature or shake vigorously for ten minutes. Filter off the precipitate of calcium oxalate on a small paper and wash free from chlorides with 0.5 per cent ammonium oxalate solution. The precipitate may then be dried, ignited to constant weight and weighed as calcium oxide or it may be manipulated volumetrically as described below.

Volumetric Procedure.-If free from uric acid the calcium oxalate precipitate may be washed three times with distilled water, filling the filter about twothirds full and allowing it to drain completely before adding more. A hole is made in the paper and the calcium oxalate washed into the flask. The volume of the fluid is brought up to about 50 c.c. and Io c.c. of concentrated sulphuric acid added. Titrate with standard potassium permanganate solution to a pink color which endures for at least a minute.

Calculation.-One c.c. of $\mathrm{N} / \mathrm{ro}$ permanganate solution is equivalent to 2.8 mg. of $\mathrm{CaO}$. Calculate the daily output of calcium expressed as $\mathrm{CaO}$.

Interpretation.-The average urinary excretion of calcium by normal adults lies between o.I to 0.4 gram (expressed as $\mathrm{CaO}$ ) per day. It is dependent very largely upon the amount of calcium in the diet. From Io to 40 per cent of the ingested calcium ordinarily is excreted by this channel, the greater part being eliminated by the feces. The proportion is dependent particularly on the amount of calcium in the food. If the calcium ingestion is very high the per cent of the total excretion taking place by way of the kidneys will be low, and vice versa. As excretion takes place by way of the intestine as well as by the kidneys no conclusions can be drawn from urinary analyses alone. The excretion of calcium may be greatly increased in certain bone disorders as osteomalacia. In others as in rickets the urinary excretion may be very low.

Procedure for Magnesium.-Transfer the filtrate from the determination of calcium as above to a porcelain dish, add about 20 c.c. of concentrated nitric acid and evaporate to dryness. Heat the residue over a free flame until the ammonium salts are destroyed and the residue fuses. After cooling take the residue up with water and a little hydrochloric acid and filter if necessary. Dilute to about 80 c.c., nearly neutralize with ammonia and cool. Add a slight excess of sodium acid phosphate and then ammonia drop by drop with constant stirring until the solution is alkaline and then add enough more slowly with

\footnotetext{
'Lyman: Jour. Biol. Chem., 21, 55 I, I9r 5.
} 
constant stirring to make the solution contain one-fourth its bulk of dilute ammonia (sp. gr. 0.96). Allow to stand over night. Filter and wash free from chlorides with alcoholic ammonia solution (I part alcohol, I part dilute ammonia, 3 parts water). The precipitate with filter paper is incinerated slowly and carefully with good supply of air to prevent reduction, in the usual manner, and ignited and weighed as the pyrophosphate.

Calculation.-To obtain the weight of $\mathrm{MgO}$ multiply the weight of magnesium pyrophosphate by 0.3624 .

Interpretation. - The daily excretion of magnesium by way of the urine usually amounts to between O.I and $0.3 \mathrm{gram}$ (expressed as $\mathrm{MgO}$ ). The amount depends mainly upon the diet. Usually 50 per cent or more of the excreted magnesium is eliminated by the kidneys, the remainder passing out in the feces. The proportion varies, however, and it is impossible to draw any conclusions from the urinary output alone. There may be a retention of magnesium in certain bone disorders accompanying a loss of calcium; in osteomalacia for example. Thus the excretions of calcium and magnesium do not necessarily run parallel.

Determination of Calcium in Ash of Foods or Feces.-Ignite the material in a crucible to a white ash and dissolve the ash with the aid of a little hydrochloric acid. Bring the volume of the ash solution to $75^{-1} 50$ c.c. Make just alkaline with strong ammonia added drop by drop (using litmus paper or alizarin as an indicator). Add concentrated $\mathrm{HCl}$ drop by drop until just acid to litmus. Then add Io drops of concentrated $\mathrm{HCl}$ (sp. gr. 1.20), and Io c.c. of 2.5 per cent oxalic acid. Either of two procedures may then be followed. (a) The solution is boiled until the precipitated calcium oxalate is coarsely crystalline, and then an excess of 3 per cent ammonium oxalate is slowly added to the boiling solution and the boiling continued until the precipitate is coarsely crystalline. (If but little calcium is present nothing will precipitate at this point and it is not necessary to add oxalate.) Or (b) the flask closed with a rubber stopper is shaken vigorously for ten minutes, in excess of 3 per cent ammonium oxalate is then added. Cool to room temperature. Add 8 c.c. of 20 per cent sodium acetate solution. (In case of ash of feces add I 5 c.c.) The solution may either be $(a)$ allowed to stand over night or (b) stoppered and vigorously shaken for ten minutes. The calcium oxalate is filtered off on a small ash-free paper and washed free from chlorides with 0.5 per cent ammonium oxalate solution. Either of two procedures may next be followed. (a) The precipitate and filter are dried, burned in a platinum or porcelain crucible to constant weight as $\mathrm{CaO}$. (b) The precipitate is washed three times with cold distilled water, as given under the method for urine and the oxalate titrated with potassium permanganate.

Magnesium is determined in the filtrate from calcium just as given above.

\section{Sodium and Potassium}

Determination of Combined Sodium and Potassium. From 50 to 100 c.c. of urine, depending upon the specific gravity, are oxidized in a Kjeidahl tlask with nitric and sulphuric acids as in the Neumann procedure for total phosphorus (see page 554). To remove the sulphuric acid as completely as possible transfer with 36 
the aid of a little water to a platinum dish and evaporate to dryness over a free flame. (The alkalies are in the form of sulphate and do not volatilize.) Dissolve the residue in hot water with the aid of a little dilute hydrochloric acid. Heat to boiling and add barium chloride solution until no more precipitate forms. Thile still hot add an excess of ammonia and ammonium carbonate. The barium chloride precipitates the sulphates and part of the phosphates: the ammonia in the presence of excess barium precipitates the rest of the phosphates, and the carbonate precipitates the calcium and most of the magnesium, as well as the excess barium. Filter and wash the precipitate well with hot water containing a few drops of ammonia. Evaporate filtrate and washings to dryness and heat the residue to dull redness for a moment. Redissolve in water and treat again with ammonia and ammonium carbonate to remove any remaining alkaline earth metals. Filter and wash as before. Transfer filtrate and washings to a weighed platinum dish, add a few drops of hydrochloric acid and evaporate to dryness. Heat the residue gently to remove ammonium salts and then to dull redness for a moment. Desiccate and weigh. Reheat to constant weight which represents the combined chlorides of sodium and potassium. The reagents used in the determination must be tested and found free from alkali metals or a correction made for the alkali metals present in the reagents used. The sodium is determined by difference after potassium has been estimated by the method given below:

Potassium.-Dissolve the alkali chlorides from the preceding determination in a little water and add a slight excess of Io per cent platinic chloride over that necessary to precipitate all of the alkali present calculated as sodium chloride (about I 7 c.c. being required for each gram of sodium chloride). Evaporate to a syrupy consistency on the water-bath and add about 50 c.c. of So.per cent alcohol. Stir occasionally for a few minutes. This operation must be carried out in the absence of ammonia rapors. Filter through a weighed Gooch crucible, washing the precipitate with 80 per cent alcohol first thoroughly by decantation and then on the filter, for some time after the filtrate is colorless. Dry at IIO-II $5^{\circ} \mathrm{C}$. and weigh.

Calculation.-Multiply the weight of potassium platinic chloride by 0.194 I to obtain the amount of $\mathrm{K}_{2} \mathrm{O}$ present. Express as $\mathrm{KCl}$ by using instead of this factor the factor 0.30712 . Subtract from the weight of total alkali chlorides as determined in the preceding method, the weight of potassium chloride as calculated and obtain the amount of sodium chloride present.

Interpretation.-The average alkali excretion of an adult on a mixed diet is about 2-3 grams of potassium expressed as $\mathrm{K}_{2} \mathrm{O}$ and $4^{-6}$ grams of sodium expressed as $\mathrm{Na}_{2} \mathrm{O}$. The ratio of $\mathrm{Na}$ to $\mathrm{K}$ is thus about $5: 3$. Both the ratio and the absolute amounts of these elements excreted are, however, largely dependent upon the salt content of the diet. Because of the non-ingestion of sodium chloride and the accompanying destruction of potassium-containing body tissues, the urine during fasting contains more potassium than sodium salts. The excretion of the bases, particularly $\mathrm{K}$, nıay be increased in fevers and in acidosis.

\section{Iron}

Method of Wolter. ${ }^{1}$ - Principle. - The urine is ashed, the ash dissolved, and the iron present oxidized to the ferric form by means of hydrogen peroxide. The iron is then determined iodometrically.

'Wolter: Bioch. Zeit., 24, IoS, Iq 10. 
Procedure.-The 24-hour specimen of urine is treated with 30 c.c. of concentrated iron-free nitric acid and then evaporated to low volume in a large evaporating dish on the water-bath. Transfer to a small evaporating dish. Heat to drymess on the sand bath and then char, using a small flame. Transfer the charred mass by means of a glass spatula to a crucible. The remaining material in the evaporating dish is transferred with the aid of a little hot water and a rubber "policeman" to it second crucible. Evaporate to dryness on the water-bath and then ash the material in both crucibles. Dissolve the ash in about 30 c.c. of iron-frce hydrochloric acirl, transfer to an Erlenmeyer flask, add 2 c.c. of hydrogen peroxide and boil for threequarters of an hour. After cooling, 2 grams of potassium iodide and a few drops of fresh starch paste are added. The liberated iodine is titrated with $N /$ Ioo thiosulphate solution. Controls should be run on reagents. A correction of $0.32 \mathrm{mg}$. is usually necessary for the undecomposed hydrogen peroxide. The thiosulphate solution is made up as necded from an N/Io stock solution by dilution. It is standarrlized against an iron solution containing $2 \mathrm{mg}$. of iron in ro c.c. The number of cubic centimeters of thiosulphate used in titration of the iodine set free from the ash solution is multiplied by the iron equivalent of I c.c. of the thiosulphate (about $0.2 \mathrm{mg}$.) to obtain the total amount of iron in the 24 -hour specimen of urine. From I-5 $\mathrm{mg}$. of iron are usually excreted per day. 


\section{CHAPTER XXVII}

\section{METABOLISM}

Metabolism is a part of that complex series of processes grouped together under the head of Nutrition. It embraces a consideration of those changes taking place in the body other than those customarily classified as secretion, digestion, excretion, etc. Metabolism may be defined as all chemical and physical changes which occur in living matter and which constitute the basis of the material phenomena of life. This conception of metabolism holds for the simple individual cell of the amœba as well as for the complex mechanism of the human body. There are two types of metabolism, one constructive, the other destructive. The constructive metabolism is termed anabolism; the destructive metabolism is termed catabolism.

Thus:

$$
\text { Metabolism }\left\{\begin{array}{l}
\text { Anabolism (constructive metabolism). } \\
\text { Catabolism (destructive metabolism). }
\end{array}\right.
$$

In general we may say that the main bulk of the food-stuffs of the diet, i.e., protein, fat and carbohydrate, is transformed in the gastro-intestinal tract and that the end-products of this transformation are carried to the cells of the body and there built up by anabolic (synthetic) processes into cell structure or stored as a reserve to be used as required. All living cells undergo wear and tear in the course of their life cycle. By catabolic (cleavage) processes therefore a portion of the living cell substance or of the stored material is reduced to simpler fragments and these are eliminated from the body after having yielded the bulk of their energy in the form of heat or mechanical work. It is apparent, therefore, that the chemical sicke of metabolism is closely associated with the physical side. Each of the three types of food-stuffs (protein, fat and carbohydrate) is concerned with the upkeep of the tissues and with the liberation of energy. It is true, however, that the main burden of the upkeep falls upon the proteins whereas the combustion of fats and carbohydrates yields the major portion of the required energy. The above facts are embraced in the following scheme: 
THE CELL

PROTEIN, FAT,

CARBOHYDRATE
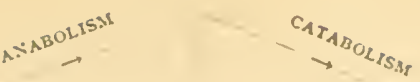

END-PRODUCTS

COMBUSTION

Without doubt both anabolic and catabolic processes are going on incessantly within every individual living cell. At one time the anabolic phase will be more prominent; at another the catabolic activity will be in the ascendency. It should also be borne in mind that metabolism implies a transformation of energy as well as an exchange of malerials.

For further brief discussions of certain phases of metabolism see the following experiments. A detailed discussion being out of place in this volume, the reader is referred to the following books:

(I) Taylor's "Digestion and Metabolism," Lea and Febiger, I9 2.

(2) Sherman's "Chemistry of Food and Nutrition," Macmillan.

(3) Osler \& McCrae's "Modern Medicine," Vol. II, Second Edition, I9I4, Lea and Febiger. The author's section on "General Consideration of Metabolism," pages 549-673.

\section{METABOLISM EXPERIMENTS}

\section{Metabolism Procedures Involving the Manipulation of the Urine}

I. Collection and Preservation of the Urine.-In metabolism tests, such as those given in this chapter the accurate collection of the urine for the exact 24-hour period is of the utmost importance. Proceed as follows: Empty the bladder at a given hour, e.g., 8 a.m. and discard the urine. Prepare a thoroughly clean bottle of proper size, introduce into it sufficient toluene to cover the bottom of the bottle and use this bottle for the collection of all urine voided from $8 \mathrm{a} . \mathrm{m}$. until $8 \mathrm{a} . \mathrm{m}$. the next day. During the day, when not actually in use for the introduction of a urine fraction, the bottle should be kept in a refrigerator or cold room in order that the sample may not deteriorate before it is examined. Measure the volume of the sample and determine its specific gravity (see Chapter XXI) and reaction before proceeding to the quantitative estimation of any specilic urinary constituents.

2. Complete Analysis of Urine.-Ingest an ordinary mixed diet (or any special diet) and collect the urine accurately for a 24 -hour period (see above). Measure the volume of the sample, determine the specific gravity and preserve the urine (see above) until the following constituents have been determined (for Methods 
of Analysis, see Chapter XXVI). Total solids, titratable acidity, hydrogen ion concentration, total nitrogen, amino-acid nitrogen, ammonia, urea, uric acid, creatinine, total sulphur, ethereal sulphates, inorganic sulphates, neutral sulphur (by difference) total phosphates and sodium chloride.

Calculate the nitrogen and sulphur "partitions," i.e., the percentage of the total nitrogen and sulphur which occur in the different forms and tabulate the data from the complete analysis. Compare your results with those listed in the table on pages $37 \mathrm{I}$ and 578 .

3. Hyperglycemia Produced by Carbohydrate Ingestion.-The average glucose content of normal blood is somewhat less than o.I per cent. This is increased (hyperglycemia) on the ingestion of carbohydrate food. The increase is noted more quickly after the ingestion of

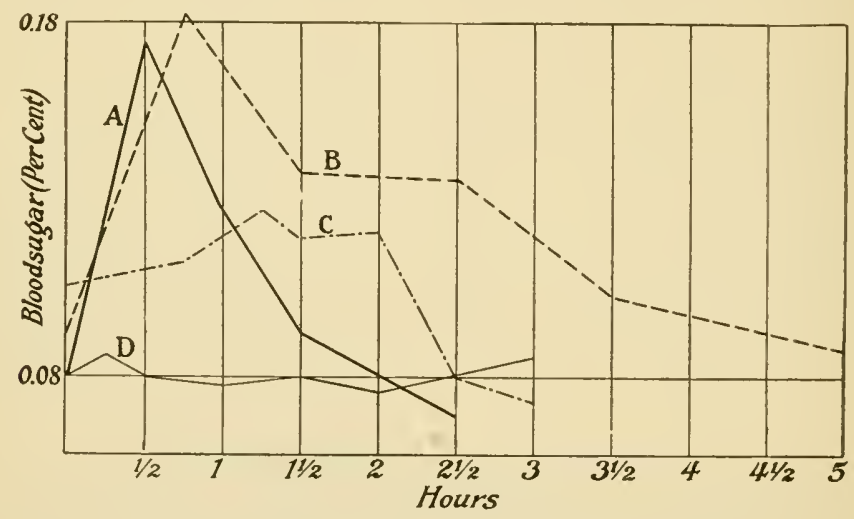

Fig. i69.-Blood Sugar as Influexced by Diet.

$A=$ glucose; $B=$ starch;

$C=$ starch and fat; $D=$ fat.

monosaccharides than after the ingestion of the more complex carbohydrates. After the ingestion of roo grams of glucose or starch an increase in the sugar of the blood sometimes occurs in five minutes. ${ }^{1}$ (See Fig. 169.)

(a) Influence of Glucose.--In the morning before breakfast, or three to five hours after breakfast, determine the normal sugar content of your blood by means of some accurate micromethod. (See Chapter XVI.) Ingest roo grams of glucose dissolved in 250 c.c. of water, and again determine the blood sugar level at intervals of 5,15 and 30 minutes and one, two and three hours. (Plot a curve similar to the one shown in Fig. 169. The urine may also be examined for sugar at intervals of one hour after the sugar ingestion.

Repeat the experiment on another day using 250 grams of glucose and compare the results with those obtained after the ingestion of 100 grams. Explain your findings. If desired this experiment may be combined with the ones on "Alimentary Glycosuria," and "Carbohydrate in Feces," see pages 568 and 59I.

${ }^{1}$ Jacobson: Bioch. Zeil., 56, 47x, I913. 
(b) Influence of Starch.-Repeat the experiment as given above for glucose except that 170 grams of white bread or 100 grams of starch made into a paste: are substituted for the 100 grams of glucose.

The experiment may be repeated as described above using an increased amount of starch.

The various experiments may be conducted on patients suffering from diabetes mellitus if such are available and instructive data collected. The alimentary hyperglycemia will generally be more prolonged than in the case of normal subjects.

4. Influence of Physical Exercise upon Blood Sugar.-After strenuous physical exertion by a normally nourished individual there is an increase in the sugar concentration of the blood. ${ }^{2}$ Similar increases are not shown by resting individuals similarly nourished nor by fasting individuals after strenuous physical exercise. This point is illustrated in the following protocol:

INFLUENCE OF PHYSICAL EXERCISE ON BLOOD SUGAR (Normal Man)

\begin{tabular}{|c|c|c|c|}
\hline Day & Experimental conditions & $\begin{array}{c}\text { Blood } \\
\text { examined, } \\
\text { hour }\end{array}$ & $\begin{array}{l}\text { Blood } \\
\text { sugar, } \\
\text { per cent }\end{array}$ \\
\hline \multirow{2}{*}{ I } & Normal. & 7 A. M. & 0.043 \\
\hline & Marched 8 miles in 2 hours; a te 200 grams sucrose. & I 2 A. M. & 0.080 \\
\hline \multirow{2}{*}{2} & Normal. & 7 A. M. & 0.045 \\
\hline & Complete rest; ate 200 grams sucrose. & I2 A. M. & 0.055 \\
\hline \multirow{2}{*}{3} & Fasting and complete rest. & 7. A. M. & $0.04 i$ \\
\hline & Fasting and complete rest. & I2 A. MI. & 0.045 \\
\hline \multirow{2}{*}{4} & Fasting. & iA. M. & 0.047 \\
\hline & Fasting and 2-hour march ( 8 miles). & $\mathrm{I} 2 \mathrm{~A} . \mathrm{M}$. & 0.052 \\
\hline
\end{tabular}

The increase in blood sugar under the influence of exercise occurs rather sooner in the diabetic organism. Typical data follow:

Experiment.-Ingest a simple uniform diet (see Experiment 34, page 592) for five days taking the first meal after 12 o'clock (noon) and the last one before ro P. M. On the morning of the second day $(7$ A. M.) determine the sugar in your blood (see methods, Chapter XII). About three hours later take a brisk walk for $\delta$ miles covering the distance in about two hours and consume 200 grams sucrose during the walk. Make a second analysis of the blood sugar. On the third day analyze your blood for sugar at 7 A. M. and again at 100 n, remaining quict in the meantime. The fourth day should be passed without physical exertion whereas on the second day between Io $\mathrm{A}$. M. and I2 M. a brisk S-mile walk is taken but no

${ }^{1}$ In making starch paste, rub up the dry starch in a mortar with cold water and pour the suspended starch granules into boiling water and stir.

2. Ioraczewski: Bioch. Zeil, 7 1, 268, 1915. 
sucrose ingested. Sugar analyses should be made at 7 A. M. and I 2 M. each day. INFLUENCE OF EXERCISE ON BLOOD SUGAR (Diabetes Patient)

\begin{tabular}{|c|c|c|c|}
\hline Day & $\begin{array}{c}\text { Blood } \\
\text { examined, } \\
\text { hour }\end{array}$ & $\begin{array}{l}\text { Blood } \\
\text { sugar, } \\
\text { per } \\
\text { cent }\end{array}$ & Experimental conditions \\
\hline I & 8 A. M. & 0.062 & $\begin{array}{l}\text { Rest. Diet consisting of } 2500 \text { grams milk, } 300 \text { grams } \\
\text { bread, } 50 \text { grams fat. }\end{array}$ \\
\hline \multirow{2}{*}{2} & 8 A. M. & 0.120 & Eight-mile march ( 2 hours). Diet as above. \\
\hline & 3 P. M. & 0.085 & \\
\hline \multirow{2}{*}{3} & 8 A. M. & 0.055 & Rest. Diet as on first day. \\
\hline & 3 P. M. & c.050 & Rest. Diet as on first day. \\
\hline \multirow{2}{*}{4} & 8 A. M. & 0.050 & Rest. Diet as on first day. \\
\hline & 3 P. M. & 0.055 & Rest. Diet as on first day. \\
\hline 5 & 8 A. M. & 0.084 & Eight-mile march ( 2 hours). Diet as on first day. \\
\hline
\end{tabular}

Calculate your results, tabulate them and compare them with those given above.

5. Alimentary Glycosuria.-Normal urine contains a trace of glucose but not enough to permit detection by the ordinary tests used in urinary analysis. If more glucose is ingested than can be absorbed and assimilated by the body the excess will be eliminated in the urine. The "assimilation limit" for the glucose has been exceeded, and a transient alimentary glycosuria results. To demonstrate this, glycosuria proceed as follows: Before breakfast or luncheon empty the bladder and test the urine for sugar by any reliable test (see Chapter XXIII). If the test is negative, ingest along with the other articles of diet, 250 grams of glucose, sucrose, or lactose dissolved in water. Empty the bladder at the end of every hour for a period of three hours and test the urine for reducing sugar and the sugar ingested.

Was there any glycosuria and if so how soon after the sugar ingestion did it appear? If no glycosuria resulted repeat the test on a subsequent day using a larger quantity of sugar. If desired, the sugar in the urine may be determined quantitatively by one of the methods given in Chapter XXVI.

This experiment may be made more complete by making determinations of blood sugar at short intervals as described in Experiment 3, page 566. If desired, data on glycosuria, hyperglycemia and carbohydrate in feces (page 59I) may be collected from one experiment.

6. Absorption of Carbohydrate as Influenced by Fat Ingestion.-When fat is eaten along with carbohydrate food the absorption of the latter is somewhat delayed. This has been shown experimentally. ${ }^{1}$ To demonstrate the point proceed as follows: Determine the content of sugar in the blood at various intervals

${ }^{1}$ Jacobson: Bioch. Zeit., 56, 47 I, I9r3. 
after the ingestion of 170 grams of white bread as described in Experiment 3 (b). page 567. Plot a curve for these values similar to the one shown in Fig. 169, page 566. On a later day repeat the experiment and ingest 170 grams of white wread and 85 grams of butter. Plot the curve for these blood sugar concentrations along with blood sugar values obtained after the ingestion of white bread as described above. Has the fat exerted any influence upon the absorption of the carbohydrate? Repeat the above experiment on a case of diabetes mellitus if such is available and note that fat exerts the same influence upon carbohydrate absorption as it excrts in the normal human body.

7. Time Relations of Protein Metabolism.-It is a well-known physiological fact that an interval elapses between the ingestion of protein food and the appearance in the urine of certain products representing the complete catabolism of this food. For example, if one ingests an excess of protein material an interval elapses before the urine gives evidence of the complete excretion of certain products representative of the catabolism of the protein. Urea is the chief of these. The term "nitrogen lag" has been used to designate the period elapsing between the ingestion of protein and the excretion in the urine of a quantity of nitrogen equivalent to that contained in the protein.

Experiment.-Ingest a simple uniform diet whose exact composition has been determined by analysis or whose approximate composition has been estimated. (See table below.) Continue this diet from one to four days. Collect the urine in two-hour periods from 7 A. M. to II P. M. and in an eight-hour period between II P. M. and 7 A. M. Analyze each specimen for total nitrogen or urea. At the end of this preliminary period add to the uniform diet, at one meal, a weighed quantity (150-250 grams) of lean meat specially prepared and analyzed. Collect the urine in periods as before and determine total nitrogen or urea. Calculate the total nitrogen or urea excretion; tabulate the data and plot curves showing the course of the nitrogen excretion on the various days of the experiment. How long was the "nitrogen lag?"

COMPOSITION OF COMMON FOODS

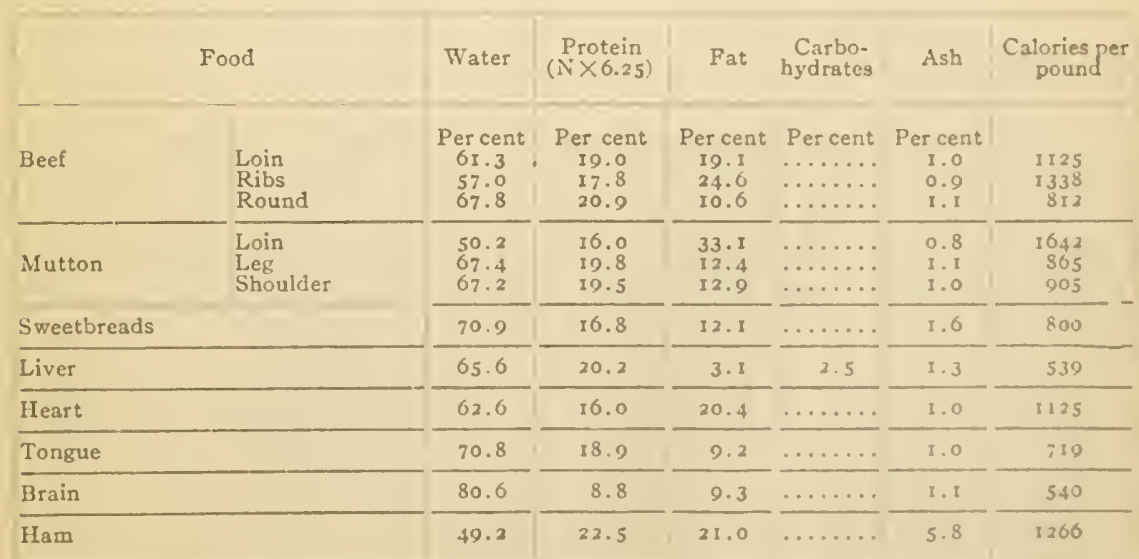

'Sherman's "Food Products," Macmillan, I914. 


\begin{tabular}{|c|c|c|c|c|c|c|c|}
\hline Foo & & Water & $\begin{array}{l}\text { Protein } \\
(\mathrm{N} \times 6.25)\end{array}$ & Fat & $\begin{array}{l}\text { Carbo- } \\
\text { hydrates }\end{array}$ & Ash & $\begin{array}{l}\text { Calories per } \\
\text { pound }\end{array}$ \\
\hline Chicken & $\begin{array}{l}\text { Broilers } \\
\text { Fowl }\end{array}$ & $\begin{array}{c}\text { Per cent } \\
74.8 \\
63.7\end{array}$ & $\begin{array}{l}\text { Percent } \\
21.5 \\
19.3\end{array}$ & $\begin{array}{c}\text { Per cent } \\
2.5 \\
16.3 \\
\end{array}$ & $\begin{array}{l}\text { Per cent } \\
\cdots \cdots \cdots \\
\cdots \cdots \cdots\end{array}$ & $\begin{array}{c}\text { Per cent } \\
\text { I.I } \\
\mathbf{I} .0\end{array}$ & $\begin{array}{r}492 \\
1016\end{array}$ \\
\hline Fish & $\begin{array}{l}\text { Halibut } \\
\text { Salmon }\end{array}$ & $\begin{array}{l}75.4 \\
64.6\end{array}$ & $\begin{array}{l}18.6 \\
22.0\end{array}$ & $\begin{array}{r}5.2 \\
12.8 \\
\end{array}$ & $\begin{array}{l}\ldots \ldots \\
\cdots \ldots \ldots \\
\cdots \ldots\end{array}$ & $\begin{array}{l}\text { I. } 0 \\
\text { I. } 4\end{array}$ & $\begin{array}{l}550 \\
922\end{array}$ \\
\hline Cereals & $\begin{array}{l}\text { Oatmeal (boiled) } \\
\text { Rice (boiled) } \\
\text { Shredded Wheat } \\
\end{array}$ & $\begin{array}{r}84.5 \\
72.5 \\
8.1 \\
\end{array}$ & $\begin{array}{r}2.8 \\
2.8 \\
10.5 \\
\end{array}$ & $\begin{array}{l}0.5 \\
0.1 \\
1.4 \\
\end{array}$ & $\begin{array}{l}11.5 \\
24.4 \\
77.9^{1} \\
\end{array}$ & $\begin{array}{l}0.7 \\
0.2 \\
2.1 \\
\end{array}$ & $\begin{array}{r}280 \\
498 \\
1660 \\
\end{array}$ \\
\hline Crackers & $\begin{array}{l}\text { Graham } \\
\text { Oatmeal } \\
\text { Soda }\end{array}$ & $\begin{array}{l}5.4 \\
6.3 \\
5.9\end{array}$ & $\begin{array}{r}10.0 \\
11.8 \\
9.8\end{array}$ & $\begin{array}{r}9.4 \\
\text { II.I } \\
9.1 \\
\end{array}$ & $\begin{array}{l}73.8^{1} \\
69.0^{1} \\
73.1^{1}\end{array}$ & $\begin{array}{l}\text { I. } 4 \\
\text { I. } 8 \\
\text { 2. I }\end{array}$ & $\begin{array}{l}1904 \\
1920 \\
1875\end{array}$ \\
\hline Bread & $\begin{array}{l}\text { White } \\
\text { Graham }\end{array}$ & $\begin{array}{l}35.3 \\
35.7\end{array}$ & $\begin{array}{l}9.2 \\
8.9\end{array}$ & $\begin{array}{l}1.3 \\
1.8\end{array}$ & $\begin{array}{l}53 . I^{1} \\
52 . I^{1}\end{array}$ & $\begin{array}{l}\mathrm{I} . \mathrm{I} \\
\mathrm{I} . \mathrm{S}\end{array}$ & $\begin{array}{l}\text { I I } 82 \\
\text { I I } 89\end{array}$ \\
\hline Potatoes & $\begin{array}{l}\text { Boiled } \\
\text { Mashed } \\
\text { Chips } \\
\text { Sweet }\end{array}$ & $\begin{array}{r}75.5 \\
75.1 \\
2.2 \\
51.9\end{array}$ & $\begin{array}{l}2.5 \\
2.6 \\
6.8 \\
3.0\end{array}$ & $\begin{array}{r}0.1 \\
3.0 \\
39.8 \\
2.1\end{array}$ & $\begin{array}{l}20.9^{1} \\
17.8 \\
46.7 \\
42.1\end{array}$ & $\begin{array}{l}\text { I. } 0 \\
\text { I. } 5 \\
4.5 \\
0.9\end{array}$ & $\begin{array}{r}429 \\
493 \\
2598 \\
903\end{array}$ \\
\hline Eggs (hen) & $\begin{array}{l}\text { White } \\
\text { Yolk } \\
\text { Entire edible por- } \\
\text { tion. }\end{array}$ & $\begin{array}{l}86.2 \\
49.5 \\
73.7\end{array}$ & $\begin{array}{l}12.3 \\
15.7 \\
\text { I } 3.4\end{array}$ & $\begin{array}{r}0.2 \\
33.3 \\
10.5\end{array}$ & $\begin{array}{l}\ldots \ldots \cdots \\
\cdots \cdots \cdots \\
\cdots \cdots\end{array}$ & $\begin{array}{l}0.6 \\
\text { I. I } \\
\text { I. } 0\end{array}$ & $\begin{array}{r}231 \\
1643 \\
672 \\
\end{array}$ \\
\hline Milk & & 87.0 & $3 \cdot 3$ & 4.0 & 5.0 & 0.7 & 314 \\
\hline Butter & & 12.7 & I. 3 & 84.0 & $\ldots \ldots \ldots$ & $1.9^{2}$ & 3450 \\
\hline Peanut butter & & $2 . I$ & $29 \cdot 3$ & 46.5 & I 7.1 & 5.0 & $274 \mathrm{I}$ \\
\hline Soups & $\begin{array}{l}\text { Consommé } \\
\text { Tomato } \\
\text { Pea } \\
\text { Celery (cream) }\end{array}$ & $\begin{array}{l}96.0 \\
90.0 \\
86.9 \\
88.6\end{array}$ & $\begin{array}{l}2.5 \\
1.8 \\
3.6 \\
2.1 \\
\end{array}$ & $\begin{array}{r}\ldots . . \\
\text { I. I } \\
0.7 \\
2.8 \\
\end{array}$ & $\begin{array}{l}0.4 \\
5.6 \\
7.6 \\
5.0 \\
\end{array}$ & $\begin{array}{l}\text { I. I } \\
\text { I. } 5 \\
\text { I. } \\
\text { I. } \\
\end{array}$ & $\begin{array}{r}53 \\
179 \\
232 \\
243 \\
\end{array}$ \\
\hline Tapioca pudding & & $64 \cdot 5$ & 3.3 & 3.2 & 28.2 & 0.8 & 702 \\
\hline Doughnuts & & 18.3 & 6.7 & 21.0 & $53 . I^{1}$ & 0.9 & 1942 \\
\hline Ginger snaps & & 6.3 & 6.5 & 8.6 & $76.0^{1}$ & 2.6 & I 848 \\
\hline Peas (cooked) & & 73.8 & 6.7 & 3.4 & $\mathbf{I} 4.6$ & I. 5 & 525 \\
\hline Lettuce & & $94 \cdot 7$ & I. 2 & 0.3 & $2.9^{1}$ & 0.9 & 87 \\
\hline Apples & & 84.6 & 0.4 & 0.5 & $14.2^{1}$ & 0.3 & 285 \\
\hline Oranges & & 86.9 & 0.8 & 0.2 & II. 6 & 0.5 & 233 \\
\hline Bananas & & $75 \cdot 3$ & 1.3 & 0.6 & $22.0^{1}$ & 0.8 & 447 \\
\hline Figs & & 79.1 & $\mathrm{I} .5$ & $\ldots \ldots$ & 18.8 & 0.6 & 368 \\
\hline Sandwiches & $\begin{array}{l}\text { Egg } \\
\text { Chicken }\end{array}$ & $\begin{array}{l}4 \mathrm{I} \cdot 4 \\
48 \cdot 5 \\
\end{array}$ & $\begin{array}{r}9.6 \\
\mathrm{I} 2.3 \\
\end{array}$ & $\begin{array}{r}12.7 \\
5.4 \\
\end{array}$ & $\begin{array}{l}34 \cdot 5 \\
32.1 \\
\end{array}$ & $\begin{array}{l}\text { I. } 8 \\
\text { I. } 7 \\
\end{array}$ & $\begin{array}{l}1319 \\
1026 \\
\end{array}$ \\
\hline Cheese (American) & & 30.0 & 28.8 & 35.9 & 0.3 & $5.0^{3}$ & 1990 \\
\hline
\end{tabular}

A less accurate experiment than the above but one which yields interesting data may be carried out as follows:

Ingest a simple diet whose nitrogen content can be estimated with some degree of accuracy (see table above). Collect the urine in two-hour periods from 7 A. M. to II P. M. and in an eight-hour period from II P. M. to

${ }^{1}$ Percentage of fiber, included under carbohydrate: shredded wheat (1.7), Graham crackers (I.5), oatmeal crackers (I.9), soda crackers (0.3), white bread (0.5), Graham bread (I.I), boiled potatoes (0.6), doughnuts (0.7), ginger snaps $(0.7)$, lettuce $(0.7)$, apples (I.2), bananas (I.O).

${ }^{2}$ Including salt.

${ }^{3}$ Including salt and sugar. 
7 A. M. and analyze for total nitrogen or urea. The next day ingest the same diet plus I50-250 grams of lean meat whose nitrogen content has been determined by analysis or estimated. Collect the urine as upon the previous day and determine its total nitrogen or urea content. Plot curves showing the course of the nitrogen or urea excretion on each of the days. How soon after the ingestion of the large quantity of meat did you note an increase in the nitrogen or urea excretion? How many hours after the meal was the maximum quantity of nitrogen or urea excreted?

8. Influence of Purine-free and High Purine Diets. - The uric acid of the body has a two-fold origin, i.e., it may arise from the metabolism of the purine (nuclein) material of body tissue (glandular organs in particular) or it may arise from the ingestion of purine (nuclein) material. That uric acid which arises from the first source is called endogenous while that which arises from the second source is termed exogenous. Secretory activity may also act to increase the endogenous uric acid output. The urine will therefore contain uric acid even though no precursor of the acid be ingested. We may also increase the uric acid output markedly by ingesting a high purine diet. However, no matter how much purine material is eaten only a small part of this purine material reappears in the urine as uric acid. In gout it is claimed there is an accumulation of uric acid in the blood due to the fact that the kidney has lost the ability to maintain the normal blood uric acid level. In this disease the excretion of uric acid may be low before an attack and increase considerably during an attack. The excretion of exogenous uric acid in gout is also much slower than normal.

Experiment.-Ingest a purine-free diet containing about 16 grams of nitrogen and consisting of egg, cheese, milk, starch, fruit, sugar and water for a period of two days (for purine content of foods, see table, page 572). Determine or estimate the nitrogen content (see table, page 569) and during the next two days substitute sweetbreads, thymus or liver for all the nitrogen of the diet maintaining the calorific value of the diet the same as before. Return to the original purine-free diet for a third interval of two days. During the final period of two days feed a diet of sweetbreads or liver containing 50 per cent more nitrogen than that of the first sweetbread period. Collect the urine for each of the eight days of the experiment and determine uric acid, and total nitrogen or urea. Note the rise in the uric acid output during the sweetbread periods. The uric acid output on the purine-free diet is endogenous in origin. Tabulate your results. The following data were secured by Taylor and Rose ${ }^{1}$ in a similar but much more carefully controlled test than that just outlined.

1 Taylor and Rose: Jour. Biol. Chcm., I4, 419 , 19:3. 


\section{INFLUENCE OF PURINE-FREE AND HIGH PURINE DIETS}

(Daily Output)

\begin{tabular}{|c|c|c|c|c|}
\hline $\begin{array}{l}\text { Urinary constituents determined } \\
\text { (grams) }\end{array}$ & $\begin{array}{l}\text { Purine-free } \\
\text { diet }\end{array}$ & $\begin{array}{l}\text { Purine diet } \\
\text { (medium) }\end{array}$ & $\begin{array}{l}\text { Purine diet } \\
\text { (increased) }\end{array}$ & $\begin{array}{l}\text { Purine-free } \\
\text { diet }\end{array}$ \\
\hline Uric acid..... & 0.09 & 0.14 & 0.24 & 0.07 \\
\hline Total nitrogen.............. & 8.9 & 8.7 & $9 . I$ & 8.8 \\
\hline Urea $\mathrm{N}\left(+\mathrm{NH}_{3}\right) \ldots \ldots \ldots$ & $7 \cdot 3$ & 7.1 & 7.1 & 7.05 \\
\hline Creatinine & 1.57 & I. 49 & I. $5 \mathrm{I}$ & \\
\hline
\end{tabular}

PURINE CONTENT OF FOODS

(Percentage purine base nitrogen)

\begin{tabular}{|c|c|c|c|c|c|c|c|}
\hline \multirow{3}{*}{$\begin{array}{c}\text { Food } \\
\text { Beef, ........... }\end{array}$} & \multicolumn{3}{|c|}{ Analyzed by } & \multirow[b]{2}{*}{8} & \multicolumn{3}{|c|}{ Analyzed by } \\
\hline & Vogel $^{2}$ & Hall $^{3}$ & $\begin{array}{l}\text { Bessau } \\
\text { and } \\
\text { Schmid }^{4}\end{array}$ & & Vogel $^{2}$ & $\mathrm{Hall}^{3}$ & $\begin{array}{l}\text { Bessau } \\
\text { and } \\
\text { Schmid }\end{array}$ \\
\hline & 0.059 & 0.052 & 0.037 & Lettuce. . & & .. & 0.003 \\
\hline Liver. . & 0.099 & 0.110 & 0.093 & Cucumbers..... & &. & None. \\
\hline Mutton... & & $\ldots \ldots$ & 0.026 & Rye bread...... & 0.014 & . & Trace. \\
\hline Tongue.. & & & 0.055 & White bread.... & 0.008 & None. & None. \\
\hline Chicken. . & - & 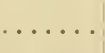 & 0.029 & Milk... & 0.0002 & 0.0002 & None. \\
\hline Thymus..... & 0.398 & 0.403 & 0.330 & Eggs..... & None. & None. & None. \\
\hline Cod fish... & 0.040 & 0.023 & 0.038 & Cheese... & 0.0004 & & None. \\
\hline Potatoes.... & 0.001 & 0.0007 & 0.009 & Rice..... . & 0.0004 & None. & None. \\
\hline Celery... & & . . & 0.005 & Tapioca... & & & None. \\
\hline Peas. . & 0.016 & 0.016 & 0.018 & Oatmeal... & & & None. \\
\hline Spinach.. & 0.022 & & 0.024 & Onions.... & & & None. \\
\hline Hominy & 0.004 & & & Tomatoes. & None. & & None. \\
\hline
\end{tabular}

1 Other purine-free foods not listed here are fruits, butter, cream and starch.

2 Vogel: Milnch. med. Woch., 58, 2433, I9I1.

${ }^{3}$ Hall: "The Purine Bodies," Philadelphia, rgo4.

${ }^{4}$ Bessau and Schmid: Therap. Monatsch., March, Igro. 
9. A Study of Endogenous Uric Acid Output.-The uric acid in the urine is said to have two sources, i.e., from the purine material of the tissues and from the purine material ingested. The former is endogenous uric acid, the latter exogenous uric acid. ${ }^{1}$ The output of uric acid on the purine-free diet in Experiments $57 \mathrm{I}$ and 574 is endogenous.

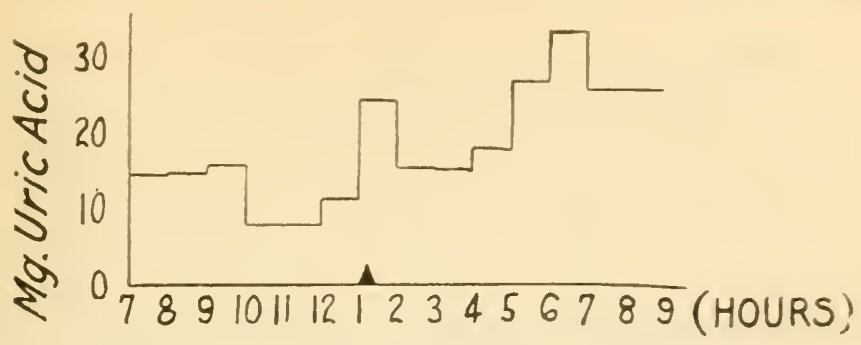

Fig. i70-Ixfluexice of Protern Ingestiox ox Exdogexoes Uric Acid Odtpet. GlUtex (130 GRAMS) INGESTED AT I P.AI. (ISendel \& Slehle: Jour. Biol. Chem., 22, 215 , I915.)

Mares ${ }^{2}$ claims that food-stuffs act to increase the endogenous uric acid output by stimulating the digestive glands to activity. A similar finding is reported by Mendel and Stehle. ${ }^{3}$ The food-stuff having the most pronounced influence was protein. Pilocarpine which stimulates the digestive glands was found to increase the endogenous uric acid output whereas atropine which inhibits secretory activity was found to decrease the output of endogenous uric acid.

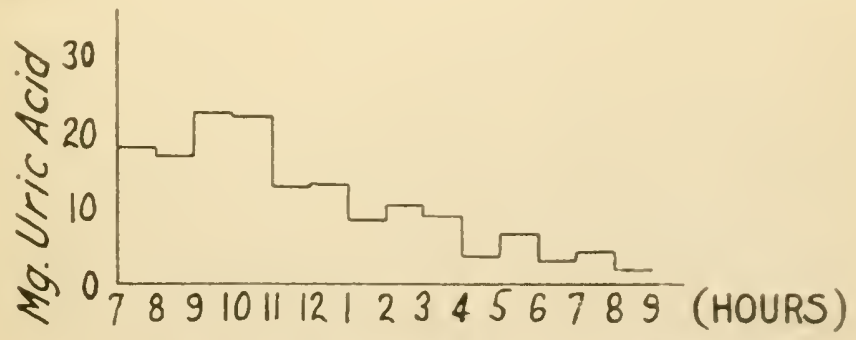

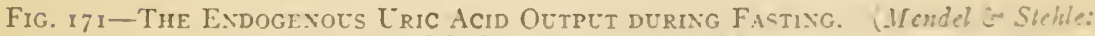
Jour. Biol. Chem., 22, 215, 1915.

The influence of protein upon the endogenous uric acid excretion is shown by the chart in Fig. I70. The fasting output by the same individual is shown, for comparison, in Fig. I71.

Experiment.-Ingest a purine-free diet consisting of milk, egg, fruit, cheese, butter, sugar and bread for one day. Continue the diet for breakfast and luncheon the next day but eat nothing after I 2 o'clock noon, until 12 o'clock noon the

${ }^{1}$ Burian and Schur: Zeil. physiol. Chem., 43, 532, 1904-5.

2 Mlares: Arch.f. d. ges. Physiol., 134, 59, 1910.

3 Mendl andd Stehle: Jour. Biol. Chem, 22, $235,1915$. 
following day, i.e., the third day of the experiment. At that time ingest $125^{-150}$ grams of gluten or some other purine-free protein preparation. On the fourth day of the experiment eat nothing until 9 P. M.

Collect the urine each day in hour periods from 7 A. M. to 9 P. M. and analyze for uric acid (see methods in Chapter XXVI). Chart your data similarly to those shown in Figs. I70 and I7I, page 573, and compare them with the findings there recorded.

Io. The Rate of Purine Excretion.-The purine material ingested by the average normal person and which is not transformed in the body will be eliminated in about 24 hours. In the case of persons afficted with gout the purine elimination is delayed. The establishment of this delayed purine elimination is often of diagnostic assistance.

Demonstrate the rate of purine excretion as follows: Ingest a purine-free diet consisting of egg, milk, cheese, starch, sugar, fruit and water for two days and follow this by a day in which sweetbreads, thymus or liver is substituted for one of the meals of the day (see table page $\mathbf{5 7 2}$ for purine content of foods). Finish the experiment by ingesting the original purine-free diet for two days. Collect each day's urine and analyze for uric acid. How soon after the sweetbread ingestion was the original plane of endogenous uric acid elimination reestablished? If one desires to locate this time more definitely the urine may be collected in short periods (one to two hours) and the uric acid content of each specimen determined. Particularly instructive data may be collected by performing the above experiment on a gout patient and upon a normal person for comparison.

II. A Study of Creatinine Elimination.-It has been established that a normal person ingesting a creatinine-free diet will excrete a uniform quantity of creatinine from day to day. The daily excretion of an adult man of average weight ranges from I-I.5 grams. For data as to creatinine excretion of a $60 \mathrm{~kg}$. man see Taylor and Rose's figures in table on page 572. The creatinine excretion depends primarily on the active mass of protoplasmic tissue, and therefore, it is generally true that a fat man will show a lower creatinine output than a lean man of like body weight. For further discussion of creatinine see Chapter XXII.

Experiment.-Ingest an ordinary mixed diet (non-meat) for a period of three days varying the character of the diet daily. Collect the urine and analyze for creatinine. (See Chapter XXVI for methods of analysis.)

Did the creatinine elimination change with the change in diet?

I2. Influence of Water.-It has been demonstrated that increased water ingestion influences many of the functions and activities of the human body. ${ }^{1}$ The increase in protein catabolism which accompanies high-water intake is shown in the following clata collected from

${ }^{2}$ Hawk: The relationship of water to certain life processes and more especially to nutrition. Read before American Philosophical Society, Philadelphia, Feb., Igr4. (See Bioch. Bull., 3, 420, I9I4). 
an experiment upon a normal man. ${ }^{1}$ In this experiment the water ingestion at meals was increased 3 liters per day during the Water Period.

INFLUENCE OF HIGH-WATER INTAKE UPON URINE VOLUME AND NITROGEN PARTITION

\begin{tabular}{|c|c|c|c|c|c|c|}
\hline $\begin{array}{l}\text { Day of } \\
\text { experi- } \\
\text { ment }\end{array}$ & $\begin{array}{c}\text { Urine } \\
\text { volume }\end{array}$ & Nitrogen & $\begin{array}{l}\text { Urea- } \\
\text { nitrogen }\end{array}$ & $\begin{array}{c}\text { Ammonia- } \\
\text { nitrogen }\end{array}$ & $\begin{array}{c}\text { Creatinine- } \\
\text { nitrogen }\end{array}$ & $\begin{array}{l}\text { Creatine- } \\
\text { nitrogen }\end{array}$ \\
\hline \multicolumn{7}{|c|}{ Preliminary Period } \\
\hline & c. c. & grams & grams & grams & grams & grams \\
\hline 4 & 830 & I 2.987 & I I. $33 \mathrm{~S}$ & 0.288 & 0.629 & \\
\hline 5 & 920 & I 2.084 & I. I $47^{6}$ & 0.305 & 0.6 Ig & $\ldots$ \\
\hline 6 & 880 & $x_{3} \cdot x_{3}$ & I I . 568 & 0.369 & $0.65 \mathrm{I}$ & \\
\hline
\end{tabular}

Water Period

\begin{tabular}{|c|c|c|c|c|c|c|}
\hline 7 & 3440 & I4. I6I & 12.596 & 0.486 & 0.610 & 0.063 \\
\hline 8 & 3840 & I $3.49 \mathrm{I}$ & I I. $5^{-8_{3}}$ & 0.499 & $0.6 \times 6$ & 0.024 \\
\hline 9 & 3670 & I 2.98 I & II . 2 I 2 & 0.553 & $0.5^{89}$ & 0.102 \\
\hline Io & 3610 & I 2.976 & I. I. 455 & 0.485 & 0.608 & 0.055 \\
\hline II & 4020 & I3. 138 & I I . 879 & $0.45^{6}$ & $0.5^{89}$ & 0. 128 \\
\hline
\end{tabular}

The above data indicate an increased catabolism of protein material as is shown by an increased output of total nitrogen upon the first and second days (days 7 and 8 ) of the Water Period. Part of this increase may, however, have been due to a "flushing" of the tissues rather than to increased catabolism of protein structures.

Experiments-(a) Relation of Water Intake to Volume and Specific Gravity of the Urine.-Ingest an ordinary mixed diet for two days. Collect the urine in 24hour periods. During the first day ingest very little fluid of any kind either at meals or between meals. On the second day ingest as much water as you can without physical inconvenience. A person of average size should have no difficulty in drinking $5^{-6}$ quarts per day.

Measure the volume of each day's urine and take the specific gravity. Note the pronounced increase in volume and the low specific gravity of the urine under the influence of high-water ingestion.

(b) Influence on Protein Catabolism. - That water stimulates protein catabolism. may easily be demonstrated as follows: Ingest a uniform diet (milk, crackers, butter, peanut butter and water) for a period of four days. During the first two days ingest your customary volume of water per day. During the last two days increase the water ingestion to 5-6 liters per day. Collect urine in 24-hour periods and analyze for total nitrogen by Kjeldahl method (see Chapter XXVI and Note on page 589). Note the increased excretion of nitrogen under the influece of highwater intake. If time permits other nitrogenous urinary constituents may be determined (see table above).

${ }^{1}$ Fowler and Hawk: Jour. Expl. Med., I 2, 388, I9io. 
I3 "Salt-free" Diet.-In order to be properly nourished we must ingest a certain amount of inorganic matter daily. If we fail to do this our metabolic processes become abnormal and the urine is one index of this abnormality. ${ }^{1}$

Experiment.-Ingest an ordinary mixed diet containing an ample salt content for a period of two days. Follow this period by the ingestion of a diet which has had its salt content reduced to a very low value. ${ }^{2}$ Sugar and olive oil or nonsalted butter may supply the bulk of the calorific part of the diet and dialyzed egg white or casein or commercial protein preparations, e.g., plasmon, gluten or glidine may supply the protein. Ingest such a diet for three days. (This is an "acidforming" diet, see page 580.) Collect the urine and analyze for sodium chloride, acidity, ammonia and total nitrogen. Compare the data from the normal days with those obtained when the "salt-free" diet was ingested. Test the urine (Chapter XXVI) and blood (Chapter XVI) for acetone. An acidosis follows the ingestion of a salt-free diet for a sufficient length of time.

Did you feel perfectly normal during the interval you were ingesting the "salt-free" diet?

14. Salt-rich Diet.-On an ordinary mixed diet a normal adult will daily excrete IO-I 5 grams of chloride, expressed as sodium chloride, in the urine. On a salt-free diet this excretion decreases, whereas if the diet contains an excessive quantity of sodium chloride this excess will be promptly excreted in the urine. Normal feces contain very little sodium chloride even after excessive sodium chloride ingestion (see Experiment 33).

Experiment.-Ingest an ordinary mixed diet for two days. On each of the following two days take a similar diet plus a weighed amount (e.g., Io grams) of sodium chloride. Collect the urine for the four days in 24-hour samples, preserve and analyze for sodium chloride (for methods see Chapter XXVI). What proportion of the added chloride was recovered?

If it is desired to make the experiment quantitative in character ingest a uniform diet (see Experiment, page 592) each day instead of the ordinary mixed diet, and examine urine and feces (see Experiment 33 ) for chloride.

I5. Acidosis.-Acidosis may be induced in a normal person by the ingestion of a "salt-free" diet such as described in Experiment I3, above, or by the ingestion of a carbohydrate-free diet. The acidosis appears somewhat earlier under the latter conditions. The noncarbohydrate diet is rather better suited for the demonstration of acidosis because of its greater palatability. When carbohydrates are ingested there is an oxidation of fatty acids to carbon dioxide and water. When no carbohydrates are ingested a portion of the fatty acids are converted into acetone bodies. These are difficult to oxidize and are excreted as such. The ketonuria (excretion of acetone and

'Taylor: University of California Publications, Pathology, I.

${ }^{2}$ It is practically impossible to secure an absolutely "salt-free" diet. 
diacetic acid) is particularly pronounced. The following table shows the data obtained in an actual case of the withdrawal of carbohydrate food from the diet of a normal man (von Noorden).

ACIDOSIS ACCOMPANYING CARBOHYDRATE WITHDRAWAL

\begin{tabular}{|c|c|c|}
\hline Day & Diet & $\begin{array}{c}\text { Excretion of acetone bodies cal- } \\
\text { culated as } \beta \text {-hydroxybutyric } \\
\text { acid (grams) }\end{array}$ \\
\hline I & Protein, fat and corbohydrate. & None. \\
\hline 2 & Protein and fat. & 0.8 \\
\hline 3 & Protein and fat. & 1.9 \\
\hline 4 & Protein and fat. & 8.7 \\
\hline 5 & Protein and fat. & 20.0 \\
\hline 6 & Protein, fat and carbohydraie. & 2.2 \\
\hline
\end{tabular}

Experiment.-Ingest an ordinary mixed diet for one day. Follow this by a period of two to four days in which no digestible carbohydrate is eaten. (A diet of meat, eggs, butter, agar-agar and water has a very low digestible carbohydrate value.) Collect the urine for each day of the experiment, examine it qualitatively for acetone bodies (see tests in Chapter XXIII). If present, determine the total acetone bodies quantitatively (for methods see Chapter XXVI). The blood may also be examined (see Chapter XVI). Did the withdrawal of carbohydrate food cause an acidosis or ketonuria? How did it compare with the acidosis in the above table?

I6. "Alkaline Tide."-For a time after a meal the normal acid reaction of the urine may be changed to neutral or alkaline. This has been explained as due to the withdrawal of hydrogen ions to manufacture the hydrochloric acid of the gastric juice.

Experiment.-Ingest an ordinary mixed diet. Urinate just before dinner and note the reaction of the urine to litmus. If acid, determine the hydrogen ion concentration by the method given in Chapter XXVI. (If alkaline, discard the urine and make the test on another day.) After eating a heavy dinner (meats) collect the urine at intervals of a half-hour and take the reaction to litmus and determine the hydrogen ion concentration as before. Did your urine change in reaction after the meal and if so how long a period elapsed between the meal and the occurrence of the maximum change in reaction?

I7. The "Partition" of Urinary Nitrogen and Sulphur as Influenced by Diet.-It was first shown by Folin ${ }^{1}$ that the percentage of the total nitrogen and total sulphur of the urine which appeared in the form of any particular nitrogenous constituent or in any particular form of

${ }^{2}$ Folin: Amer. Jour. Physiol., I3, II8, I905. 
sulphur was regulated directly by the extent of the total nitrogen and sulphur elimination. This point is well illustrated in the following table which contains data regarding the so-called "partition" or "distribution" of the urinary nitrogen and sulphur.

THE NITROGEN AND SULPHUR “PARTITIONS" AS INFLUENCED BY DIET"

\begin{tabular}{|c|c|c|c|c|c|c|}
\hline \multirow[b]{2}{*}{ Constituent of the urine } & \multicolumn{3}{|c|}{ Normal protein diet } & \multicolumn{3}{|c|}{ Starch-cream diet } \\
\hline & 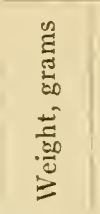 & 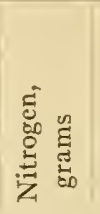 & 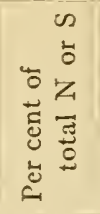 & 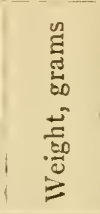 & 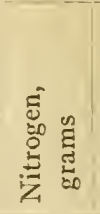 & 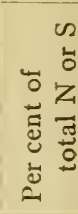 \\
\hline Urea......... & 31.6 & $14 \cdot 7$ & $87 \cdot 5$ & 4.72 & 2.2 & 6 I.7 \\
\hline Ammonia... & 0.6 & 0.49 & 3.0 & $0.5 \mathrm{I}$ & $0.4^{2}$ & II. 3 \\
\hline Creatinine... & I. 55 & 0.58 & 3.6 & I. $6 \mathrm{I}$ & 0.60 & I7. 2 \\
\hline Uric acid. . & 0.54 & O. 18 & I. I & 0.27 & 0.09 & 2.5 \\
\hline Undetermined. & . & 0.85 & $4 \cdot 9$ & $\cdots \cdots$ & 0.27 & $7 \cdot 5$ \\
\hline Total N....... & & I6.8 & 100.0 & $\cdots \cdots$ & 3.6 & 100.0 \\
\hline Inorganic $\mathrm{SO}_{3} .$. & 3.27 & & 90.0 & 0.46 & & 60.5 \\
\hline Ethereal $\mathrm{SO}_{3} \ldots$ & 0.19 & & 5.2 & o. ro & & 13.2 \\
\hline Neutral $\mathrm{SO}_{3} \ldots \ldots \ldots \ldots \ldots \ldots \ldots \ldots$ & O. I 8 & & 4.8 & 0.20 & 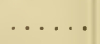 & 26.3 \\
\hline Total SO & 3.64 & & 100.0 & 0.76 & & 100.0 \\
\hline
\end{tabular}

It will be observed from an examination of this table that a normal protein diet which gave 16.8 grams of urinary nitrogen yielded 87.5 per cent of this nitrogen as urea, 3 per cent as ammonia, 3.6 per cent as creatinine and I.I per cent as uric acid; whereas a "non-protein diet" (starch and cream containing about I gram of nitrogen) which gave only 3.6 grams of urinary nitrogen yielded only 6 I.7 per cent of this nitrogen as urea but gave a greatly increased percentage output in the case of each of the other nitrogenous constituents mentioned, e.g., II.3 per cent as ammonia, I 7.2 per cent as creatinine and 2.5 per cent as uric acid. The percentage output of neutral sulphur was also greatly increased.

It will furthermore be observed that the actual daily output of

${ }^{1}$ Folin: Am. Journ. Physiol., I3, II8, I905. 
certain of the constituents is uninfluenced by the amount of protein ingested. Among these are creatinine and neutral sulphur. On the other hand the output of inorganic sulphur and urea is more or less directly proportional to the protein ingestion. The observation of such facts as these led Folin to formulate his theory of protein metabolism. ${ }^{1}$

Experiment.-During a period of two or three days ingest an ordinary mixed diet containing I0o-I25 grams of protein (I6-20 grams of nitrogen) per day. Collect the urine accurately in 24-hour periods (page 565) preserve it and analyze the urine of the second and third days for total nitrogen, urea, creatinine, total sulphur, inorganic sulphates, ethereal sulphates and neutral sulphur (by difference). For methods of analysis see Chapter XXVI. Follow this period by one of three days in which a diet of starch and cream having a similar calorific value is ingested. Analyze the urine for the second and third days as indicated above. Calculate your results and tabulate as shown in the table on page 578 . How did the change in the diet alter the metabolism of nitrogen and sulphur?

In calculating the calorific value of a diet make use of the following values:

I gram protein .................. large calories

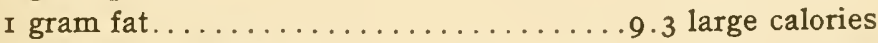

I gram carbohydrate ............. I large calories.

18. Protein-Sparing Action of Carbohydrate and Fat.-The non-nitrogenous nutrients, carbohydrate and fat, have the power to diminish the extent of the catabolism of protein in the normal human body. In other words they are said to "spare" protein. This point is illustrated in data reported by von Noorden and Dieters, which are tabulated below.

PROTEIN-SPARING ACTION OF CARBOHYDRATE AND FAT

\begin{tabular}{|c|c|c|} 
Nitrogen ingested & Nitrogen in urine \\
\hline I2.6 grams +200 grams sucrose. & 9.0 grams. & I3 per cent reduction in protein \\
catabolism.
\end{tabular}

It will be observed that the addition of 200 grams of sucrose to the diet was accompanied by a decrease of $I_{3}$ per cent in the amount of protein catabolized. It has been established that carbohydrates are more efficient "protein sparers" than are the fats. For example Voit found carbohydrate to produce a 9 per cent decrease in protein catabolism whereas fats produced only a 7 per cent decrease.

Experiment.-Ingest a uniform diet of known or estimated nitrogen content for a period of four days. Collect and preserve the urine accurately (see page $5_{5}$ ) in 24-hour samples and analyze the excretion of the third and fourth day's for total nitrogen. On the fifth day add 200 grams of sucrose to the diel. Analyze this urine

${ }^{1}$ The author's article on "General Considerations of Metabolism" in "Modern Medicine" (Osler and McCrae) 2nd Edition, I9I4, p. 594. See also Folin: American Journol Physiol., 13, I18, I905. 
also for total nitrogen. Calculate your results and tabulate the data as shown in table on page 579 .

Did the sucrose influence the catabolism of protein in your body?

\section{I9. Hydrogen Ion Concentration of the Urine as Influenced by} the Ingestion of Acid-Forming and Base-Forming Foods.-It has been demonstrated by Sherman and Gettler ${ }^{1}$ that vegetables and fruits, on burning, leave an ash in which the basic elements (sodium, potassium, calcium and magnesium) predominate, whereas cereals, meats and fish foods leave an ash in which the acid-forming elements (chlorine, sulphur and phosphorus) predominate. A list of acid-forming and baseforming foods is given in the following table.

\section{EXCESS OF ACID-FORMING OR BASE-FORMING ELEMENTS IN FOODS (Sherman and Gettler)}

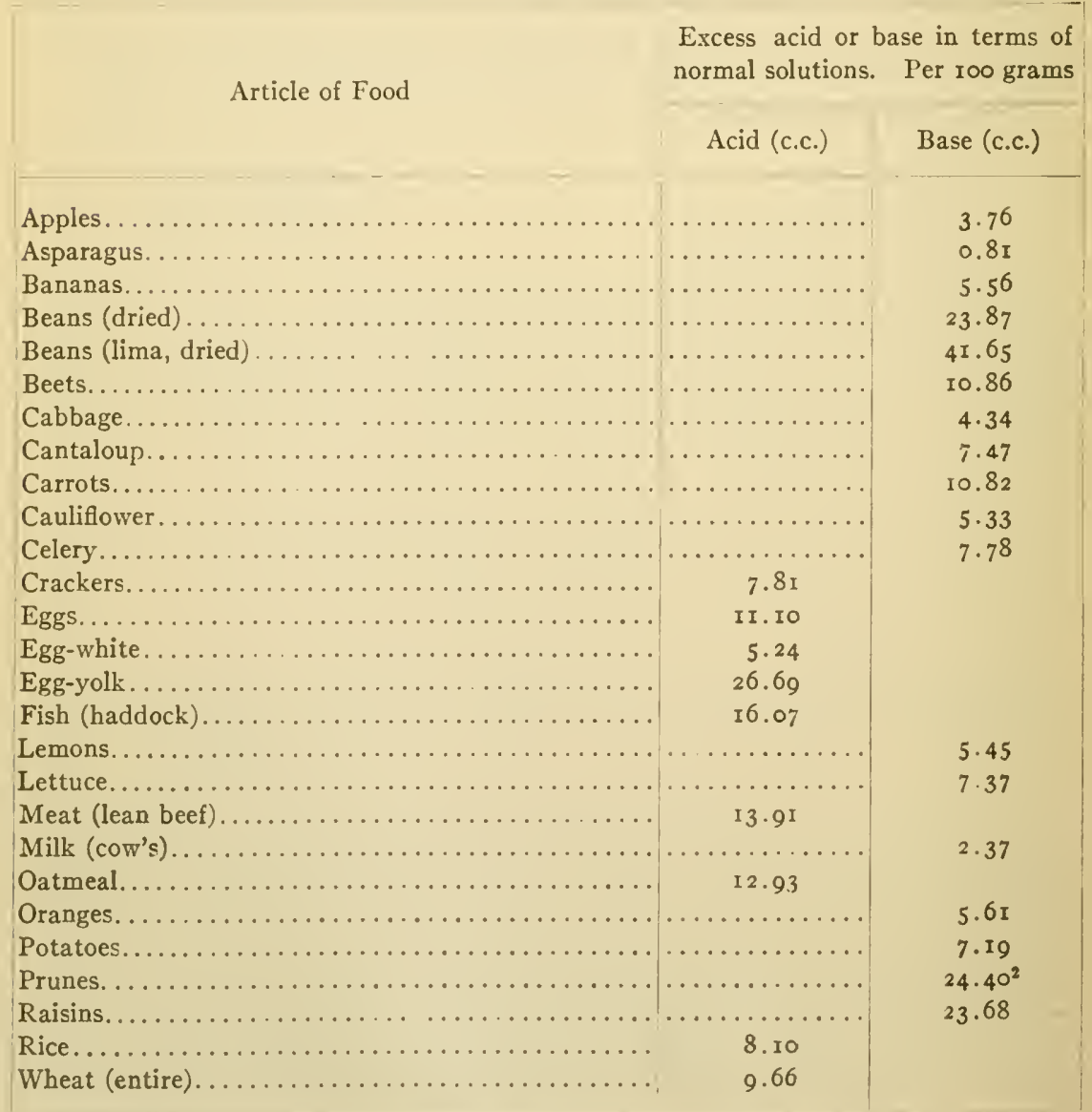

1 Sherman and Gettler: Jour. Biol. Chem., Ir, 323, r912.

${ }^{2}$ Prunes, plums and cranberries yield an alkaline ash but serve to mcrease the hydrogen ion concentration of the urine because of their benzoic acid content, this acid being synthesized with glycocoll in the kidney and elsewhere to form hippuric acid. 
The above data indicate that potatoes, oranges, raisins, apples, bananas and cantaloups are important base-forming foods. Among the most important acid-forming foods are found rice, whole wheat bread, oatmeal, meats and eggs. Certain fruits, $c . g .$, cranberries, ${ }^{1}$ prunes and plums yield a basic ash but are acid-forming foods.

This is due to the fact that they contain benzoic acid which is synthesized with glycocoll in the body to produce hippuric acid (see page $5^{85}$ ). It is worthy of note that some plant foods are base-formers and others are acid-formers. It is also an important fact that acid fruits yield a basic ash (see page 580 ).

The normal diet should contain sufficient base-forming elements to neutralize the acids formed. If these acids are not neutralized by the basic elements in the diet they will be neutralized by the fixed bases of the tissues of the body and a seriously deranged metabolism may result. (See experiment on "salt-free diet," page 576.) Organic salts of the alkalis (e.g., sodium bicarbonate or sodium acetate) are often given therapeutically. They reduce the $H$ ion concentration of the urine: the same result so far as urine reaction is concerned may be secured by feeding properly selected base-forming foods. The ingestion of sodium dihydrogen phosphate $\left(\mathrm{NaH}_{2} \mathrm{PO}_{4}\right)$ will increase the acidity of the urine: a like result may be produced by feeding properly selected acid-forming foods. Anything which produces an increase in the $\mathrm{H}$ ion concentration of the urine will produce an increase in the ammonia output.

On a mixed diet the $\mathrm{H}$ ion concentration of the urine ${ }^{2}$ has been found to be $6 \pm$ o.I. ${ }^{3}$ In nephritis the $H$ ion concentration of the urine may be increased to 5.33 or higher. Alkalis have been used with apparent success in the treatment of nephritis. ${ }^{4}$ It is evident that baseforming foods properly selected should be suitable dietary articles for nephritics. ${ }^{1}$ For a detailed discussion of acid-forming and base-forming foods see article by Blatherwick. ${ }^{5}$

Experiment.-Ingest a uniform diet consisting of milk, crackers, butter, peanut butter, and water in desired quantities for a period of three days. Follow this by a period of six days during the first three of which considerable quantities of acid-forming foods (see table page 580) are added to the diet. During the second

${ }^{1}$ Radin reports this berry to contain 0.06 per cent benzoic acid (Blatherwick: Arch. Int. Med., $\left.\mathrm{I}_{4}, 409, \mathrm{I}_{91}\right)$.

${ }^{2}$ Henderson and Palmer: Jour. Biol. Chem., I4, SI, ror3.

${ }^{3} \mathrm{H}$ ion concentration may be expressed as gram of ionized $\mathrm{H}$ per liter of water. A neutral solution has a $\mathrm{H}$ ion concentration of $\mathrm{I} \times \mathrm{IO}^{-7}$, or $0.000,000, \mathrm{I}$ gram per liter. It is often customary to express the $H$ ion concentration according to Sörensen's logarithmic notation. For example instead of expressing the $\mathrm{H}$ ion concentration of a neutral solution as $I \times \mathrm{IO}^{-7}$ he expresses it as 7.00. An increasing $\mathrm{H}$ ion concentration decreases this value and an increasing $\mathrm{OH}$ ion concentration increases the value.

${ }^{4}$ Fisher: Nephritis, New York, IgI 2.

${ }^{5}$ Blatherwick: Arch. Int. II cd., I 4,409 , I9I 4 . 
half of the period (days four to six) add an abundance of base-forming foods to the diet. Distilled water should be used for drinking purposes and a uniform volume should be ingested daily. Collect the urine in 24-hour periods, preserve and analyze for $\mathrm{H}$ ion concentration, titratable acidity and ammonia (for methods see Chapter XXVI). Compare your results with those tabulated in the table below.

REACTION OF URINE AS INFLUENCED BY DIET ${ }^{1}$

\begin{tabular}{|c|c|c|c|c|c|c|c|c|}
\hline \multirow[b]{2}{*}{$\begin{array}{c}\text { Determi- } \\
\text { nation. }\end{array}$} & \multicolumn{2}{|c|}{$\begin{array}{l}\text { Basal } \\
\text { diets² }\end{array}$} & I & 2 & 3 & 4 & 5 & 6 \\
\hline & $\begin{array}{l}\text { No. I } \\
5 \\
\text { days }\end{array}$ & $\begin{array}{c}\text { No. } 2 \\
5 \\
\text { days }\end{array}$ & $\begin{array}{l}\text { Baked pota- } \\
\text { toes ( } 750 \\
\text { grams per } \\
\text { day) +basal } \\
\text { diet No. I } \\
\text { (6 days) }\end{array}$ & $\begin{array}{l}\text { Rice (210 } \\
\text { grams per } \\
\text { day) + basal } \\
\text { diet No. I } \\
\text { (4 days) }\end{array}$ & $\begin{array}{c}\text { Cranberry } \\
\text { sauce (300- } \\
600 \text { grams } \\
\text { per day)+ } \\
\text { basal diet, } \\
\text { No. I } \\
\text { (6 days) }\end{array}$ & $\begin{array}{l}\text { Breads } \\
\text { (whole } \\
\text { wheat) } 450 \\
\text { grams for I } \\
\text { day +basal } \\
\text { diet, No. I }\end{array}$ & $\begin{array}{c}\text { Prunes } \\
\text { (330-550 } \\
\text { grams per } \\
\text { day) + basal } \\
\text { diet. No. }{ }^{2} \\
\text { (3 days) }\end{array}$ & $\begin{array}{l}\text { Cantaloup } \\
\text { (260 grams } \\
\text { per day) }+ \\
\text { basal diet. } \\
\text { No. } 2 \\
\text { (5 days) }\end{array}$ \\
\hline $\begin{array}{l}\mathrm{H} \text { ion con- } \\
\text { centration. }\end{array}$ & 7.19 & 5.57 & 7.74 & $\frac{7.48-6.90}{7.14}$ & $\underbrace{6.30-5.70}_{6.19}$ & $\begin{array}{c}6.80 \\
\text { (Previous } \\
\text { day } \quad 6.90 \text { ) }\end{array}$ & $\underbrace{5.30-4.80}_{5.07}$ & $\frac{5.30-7.38}{6.70}$ \\
\hline $\begin{array}{l}\text { Titratable } \\
\text { acidity } \\
\text { (c.c. N/Io }\end{array}$ & 275 & 474 & $\frac{196-216}{203}$ & $\underbrace{166-297}_{233}$ & $\underbrace{39 I-488}_{407}$ & $\begin{array}{c}350 \\
\text { (Previous } \\
\text { day 297) }\end{array}$ & $\frac{570-540-578}{563}$ & $\underbrace{466-250}_{328}$ \\
\hline $\begin{array}{l}\text { Ammonia } \\
\stackrel{\text { (grams) }}{ }\end{array}$ & 0.310 & 0.464 & $\frac{0.221-0.248}{0.238}$ & $\frac{0.166-0.25 I}{0.198}$ & $\frac{0.219-0.391}{0.305}$ & $\begin{array}{l}0.280 \\
\text { (Previous } \\
\text { day } 0.25 \mathrm{I} \text { ) }\end{array}$ & $\frac{0.602-0.729}{0.654}$ & $\frac{0.513-0.220}{0.310}$ \\
\hline
\end{tabular}

\section{Hydrogen Ion Concentration of the Urine as Influenced by} Alkali and Acid Ingestion.--The ingestion of certain organic salts of the alkalis, e.g., sodium citrate and sodium bicarbonate will cause a decrease in the hydrogen ion concentration of the urine. The ingestion of acids

\section{INFLUENCE OF INGESTED SODIUM BICARBONATE ON H ION CONCENTRATION OF URINE}

\begin{tabular}{|c|c|c|c|c|c|c|c|}
\hline \multirow{2}{*}{$\begin{array}{l}\text { Experiment } \\
\text { Number }\end{array}$} & \multirow{2}{*}{$\begin{array}{l}\text { Sodium } \\
\text { Bicarbonate, } \\
\text { Grams }\end{array}$} & \multirow{2}{*}{$\begin{array}{l}\text { Hydrogen Ion Concen- } \\
\text { tration before Bicarbon- } \\
\text { ate Ingestion }\end{array}$} & \multicolumn{5}{|c|}{$\begin{array}{l}\text { Time of Collection of Specimen } \\
\text { of Urine and H Ion Concen- } \\
\text { tration }\end{array}$} \\
\hline & & & $\begin{array}{l}\text { I I. OO } \\
\text { A. M. }\end{array}$ & $\begin{array}{l}\text { I } 2.00 \\
\text { noon }\end{array}$ & $\begin{array}{l}\circ \mathrm{I} .00 \\
\text { P. M. }\end{array}$ & $\begin{array}{l}2.00 \\
\text { P. M. }\end{array}$ & $\begin{array}{l}3.00 \\
\text { P. MI }\end{array}$ \\
\hline I & 4 & $7 \cdot 40$ & 8.30 & $7 \cdot 48$ & $7 \cdot 4^{8}$ & 7.40 & 5.85 \\
\hline 2 & 8 & $5 \cdot 40$ & 8.50 & 8.30 & $6.5^{\circ}$ & $6.5^{\circ}$ & 7.40 \\
\hline 3 & I 2 & $5 \cdot 30$ & 8.70 & 8.70 & 8.70 & 8.70 & 8.70 \\
\hline 4 & 8 & 7.40 & 8.50 & 8.70 & 8.50 & 8.50 & $8.5^{\circ}$ \\
\hline 5 & 8 & 5.85 & $\ldots$ & . . . . & 8.70 & 8.70 & 8.30 \\
\hline 6 & 8 & 6.70 & 7.48 & 8.70 & 8.50 & 8.70 & 8.50 \\
\hline
\end{tabular}

${ }^{1}$ Tabulated from data reported by Blatherwick (Arch. Int. Med., I4, 409, I9I4). Experiments all made on the same subject (B).

${ }_{2}$ Basal diet $\mathrm{No}$. I contained 100 grams Graham crackers, 25 grams butter, 400 c.c. whole milk ingested at each of the three daily meals. One apple and one soft boiled egg added at supper. In diet No. 2 whole wheat crackers were substituted for the Graham crackers.

This day was preceded by $\mathrm{NaHCO}_{3}$ ingestion for three days and by rice ingestion for four days.

"This diet followed immediately after the diet of prunes (see 5). 
(either organic or inorganic) or acid salts, e.g., sodium dihydrogen phosphate will increase the hydrogen ion concentration of the urine. The alkalis are much more effective in producing changes in reaction than are the acids. The influence of ingested alkali (sodium bicarbonate) is shown in the foregoing table containing data submitted by Henderson and Palmer. ${ }^{1}$

Blatherwick ${ }^{2}$ reports a decrease in ammonia nitrogen output from 0.256 gram to 0.072 gram, and an accompanying decreased acidity under the influence of bicarbonate ingestion ( 25 grams in two days).

The influence of ingested acid (benzoic) is shown in the following data reported by Blatherwick. ${ }^{2}$

INFLUENCE OF BENZOIC ACID INGESTION ${ }^{3}$

\begin{tabular}{|c|c|c|c}
\hline Day & $\begin{array}{c}\text { Titratable acidity } \\
\text { (c.c. N/I0) }\end{array}$ & H ion concentration & Ammonia N (grams) \\
\hline $\mathrm{I}$ & 392 & $6 . \mathrm{I}_{5}$ & 0.292 \\
\hline 2 & 410 & 6.15 & 0.374 \\
\hline 3 & 443 & 6.00 & 0.422 \\
\hline 4 & 434 & 6.00 & 0.408 \\
\hline 5 & 468 & 5.70 & $0.4 \mathrm{I} 8$ \\
\hline
\end{tabular}

(For further discussion of dietary alterations of urine reaction see preceding experiment.)

Experiments.-(a) Influence of Alkali.-Ingest a uniform diet consisting of milk, crackers, butter, peanut butter and distilled water for a period of two days. During the next two days take the same diet and ingest 24 grams of sodium bicarbonate between meals ( 12 in A. M. and I2 in P. M.). Collect the urine in 24-hour periods and analyze it for titratable acidity, $\mathrm{H}$ ion concentration and ammonia. Compare your results with those shown in table on page 582.

If desired the bicarbonate may be given in one dose of 8-12 grams and the urine collected in hourly specimens for the next five hours and each specimen analyzed. Data from such experiments are shown in table on page 582 .

(b) Influence of Acid.-Proceed as above except that I gram of benzoic acid (in capsule) is ingested before each meal of the experimental period.

The experiment may also be varied by ingesting ro grams of sodium dihydrogen phosphate early in the day and collecting the urine in hourly fractions or in one 24-hour sample.

${ }^{1}$ Henderson and Palmer: Jour. Biol. Chem., r4, 8 r, Igr3.

${ }^{2}$ See p. $58 \mathrm{r}$.

${ }^{3}$ One gram of benzoic acid in a capsule before each meal. Basal diet No. I described on page 582 was used. 
From your experiments what do you conclude as to the relative efficiency of acid and alkali in altering the reaction of the urine?

\section{Influence of a High Calorie Non-Nitrogenous Diet.-If an} individual fasts there is a combustion of a certain amount of protein tissue each day of the fast. The destruction of such tissue is rather low on the first day due to the fact that the glycogen stores of the body are being utilized to furnish the necessary energy. If an individual instead of fasting, ingests a diet of high calorific value and very low in nitrogen the output of nitrogen in the urine of the third or fourth day will be less than on the third or fourth day in fasting. This is due to the fact that the body derives sufficient energy from the high calorie diet and there is less destruction of protein body tissues than occurs in fasting. For a discussion of energy value of foods see "Determination of Fuel Value of Foods," below, and the table on page 569.

Experiment.-Ingest a high calorie diet which is very low in nitrogen or actually non-nitrogenous. A satisfactory diet may include sugar, butter, starch, cream, agar-agar and water. (For energy values see below and table, page 568.) Ingest such a diet for three days. Collect the urine in 24-hour periods, preserve and analyze it for total nitrogen, acidity and ammonia. Note the low nitrogen excretion on the third day as compared with the nitrogen output of the third day of fasting. If so desired, you may (at some later date) fast for three days and repeat the above analyses for comparison.

Determination of Fuel Value of Food.-When organic substances are oxidized or burned in the human body they liberate a certain amount of heat. This calorific energy or heat value varies according to the type of organic matter undergoing oxidation. Thus the proteins, fats and carbohydrates of the diet when they are burned in the body yield different quantities of heat per unit of substance than do organic acids, alcohol, etc. The energy values of pure protein fat and carbohydrate are the following:

$\begin{array}{ll}\text { Protein } & =4 . I \text { large calories per gram. } \\ \text { Fat } & =9.3 \text { large calories per gram. } \\ \text { Carbohydrate } & =4 . I \text { large calories per gram. }\end{array}$

In arriving at the energy value of any given diet it is customary to burn weighed samples of the various foods in an oxygen atmosphere in an apparatus called a bomb calorimeter (see Fig. I72, page 586). By this means we may determine how much heat is liberated when the ingested food is oxidized in the body. A correction must be made for the incompletely oxidized substances of the urine and feces. A large mass of data concerning the heat value of foods has been collected and tabulated, and it is therefore possible to arrive at an approximate idea of the energy value of a diet by calculation (see table, page 569). The bomb colorimeter shown in Fig. I 72, page 586, is one of the most satisfactory for actual determination of the heat of combustion of organic substances.

22. Metabolism in Fasting.-The metabolism of a fasting man is entirely different from the metabolism of a well-nourished person. 
The collection and analysis of the urine during a short fast (three to seven days) will demonstrate many important facts. The following table, which contains data from fasting tests made in the author's laboratory, ${ }^{1}$ illustrates some of the points in which fasting metabolism differs from normal metabolism:

METABOLISM IN FASTING

\begin{tabular}{|c|cccccccc}
\hline $\begin{array}{c}\text { Day } \\
\text { of }\end{array}$ & Body & Total & Ammonia & Creatine & Acidity. & $\mathrm{P}_{2} \mathrm{O}_{5}$ & Chloride \\
period & $\mathrm{kg}$. & $\mathrm{N}$ & $\mathrm{N}$ & $\mathrm{N}$ & c.c. $\mathrm{N} / \mathbf{I} 0$ & grams & grams, \\
\hline
\end{tabular}

Preliminary Feeding Period

\begin{tabular}{l|lllllll} 
I-4 Av. 74.16 & 10.430 & 0.II2 & None & 238.6 & 2.768 & 9.007
\end{tabular}

Fasting Period

$\begin{array}{lllllllll}\mathrm{I} & 73.32 & 10.072 & 0.288 & 0.269 & 328.9 & 2.616 & 5.035 \\ 2 & 71.98 & 15.072 & 0.642 & 0.073 & 677.1 & 2.509 & 3.231 \\ 3 & 70.92 & 14.463 & 0.862 & 0.089 & 770.4 & 2.851 & 2.539 \\ 4 & 70.24 & 13.080 & 1.201 & 0.068 & 664.2 & 2.490 & 1.253 \\ 5 & 69.61 & 11.801 & 1.266 & 0.033 & 525.0 & 2.376 & 1.474 \\ 6 & 69.12 & 11.214 & 1.373 & 0.022 & 462.4 & 1.186 & 1.132 \\ 7 & 68.70 & 10.734 & 1.371 & 0.003 & 438.9 & 0.955 & 1.137\end{array}$

Abstinence from food for a few days can in no way operate to the disadvantage of a normal person. In fact individuals affected with certain types of gastro-intestinal disorders are benefited by fasting. The fasting treatment ${ }^{2}$ is also being used with success in cases of diabetes mellitus.

In order to determine experimentally how the fasting metabolism differs from normal metabolism proceeds as follows: Ingest an ordinary mixed diet and collect your urine (see page 565) for a day. Measure the volume and analyze the sample for total nitrogen, ammonia, creatine, sodium chloride, total phosphates and acidity $^{3}$ (for methods see Chapter XXVI). For the next few days (three to seven as desired) ingest nothing but water and collect the urine accurately and analyze for the constituents enumerated above. Tabulate your results and compare them with those given in the table above.

23. Synthesis of Hippuric Acid in Human Body.-Hippuric Acid is present in human urine in small amount, about 0.7 gram being excreted

1 The chloride, phosphate and acidity determinations were collected during one sevenday fast and the other data collected during a difierent fast on the same man. (See Howe, Mattill and Hawk: Jour. Amer. Chem. Soc., 33, 568, I9r1; and IVilson and Hark: Jour. A mcr. Chem. Soc., 36, 137, 1914.)

2 Allen: Amer. Jour. Med. Sci., I5O, 4 So, ror 5.

${ }^{3}$ A more accurate experiment may be carried out by ingesting a uniform diet of known composition (see page 569 ) for a few days before the fast. 
per day. The urine of herbivorous animals contains much larger quantities. This acid is formed in the animal body, by synthesis from benzoic acid and glycocoll which takes place in the kidneys and elsewhere. ${ }^{1}$

Experiment.-Ingest 2 grams of sodium benzoate or ammonium benzoate before retiring at night. Collect the first fraction of urine voided the next morning. The benzoate has been synthesized with glycocoll to form hippuric acid.

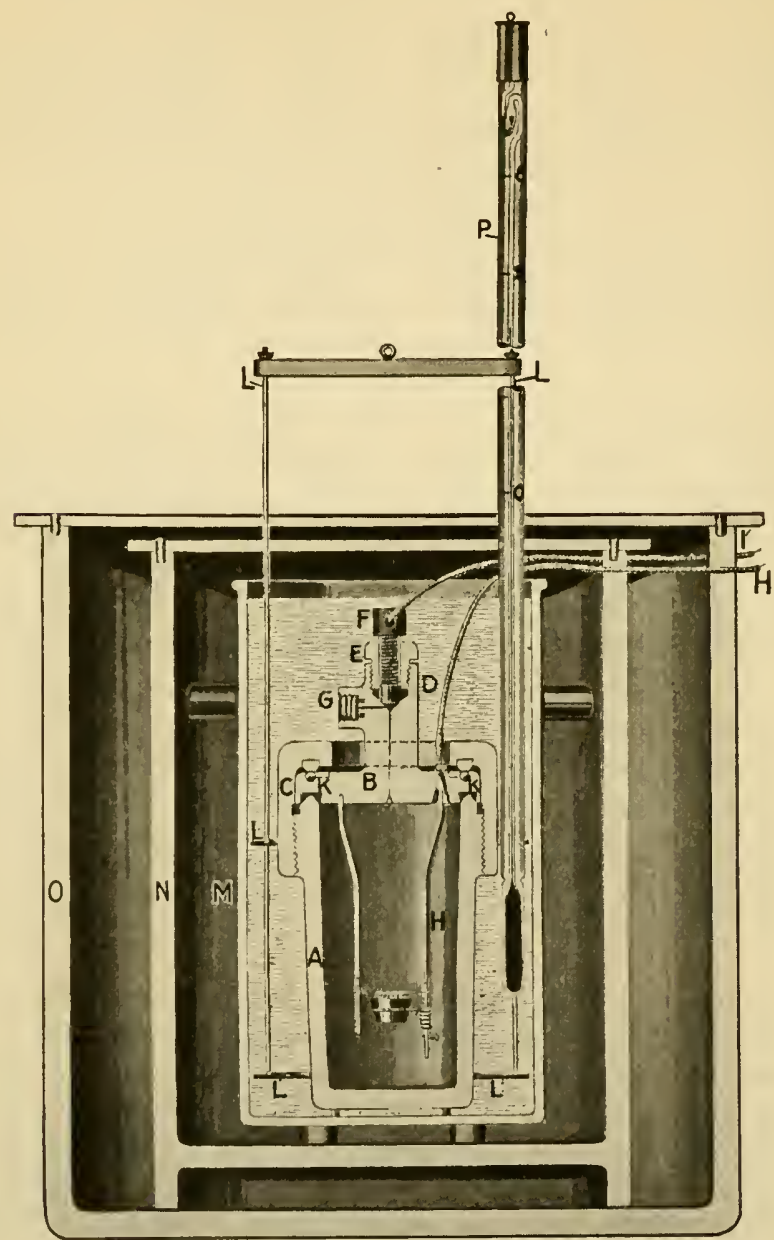

Fig. i72.-Berthelot-Atwater Bomb Calorimeter.

The urine will therefore be found to contain much more of this acid than is normally present. Isolate the hippuric acid by one of the following methods :

(a) First Method.-Render the urine alkaline with milk of lime, boil for a few moments and filter while hot. Concentrate the filtrate, over a burner, to a small volume. Cool the solution, acidify it strongly with concentrated hydrochloric acid and stand it in a cool place for 24 hours. Filter off the crystals of hippuric acid which have formed and wash them with a little cold water. Remove the crystals

\footnotetext{
${ }^{1}$ Kingsbury and Bell: Jour. Biol. Chem., 21, 297, 19r5.
} 
from the paper, dissolve them in a very small amount of hot water and percolate the hot solution through thoroughly washed animal charcoal, being careful to wash out the last portion of the hippuric acid solution with hot water. Filter, concentrate the filtrate to a small volume and stand it aside for crystallization. Examine the crystals under the microscope and compare them with those in Fig. I 25, page 389. This method is not as satisfactory as Roaf's method (see below).

(b) Roaf's Method.-Place the urine in a casserole or precipitating jar and add an equal volume of a saturated solution of ammonium sulphate and r.j c.c. of concentrated sulphuric acid per roo c.c. of urine. Permit the mixture to stand for twenty-four hours and remove the crystals of hippuric acid by filtration. Purify the crystals by recrystallization according to the directions given above under First Method. Examine the crystals under the microscope and compare them with those given in Fig. I 25, page 389 .

It is possible, by the above technic, to isolate hippuric acid in crystalline form from as small a volume as $25-50$ c.c. of herbivorous urine. The greater the amount of ammonium sulphate added the more rapid the crystallization until at the saturation point the crystals of hippuric acid sometimes form in about ten minutes.

\section{METABOLISM PROCEDURES INVOLVING THE MANIPULATION OF THE FECES ${ }^{1}$}

24. "Separation" of Feces.-In order to differentiate the feces which correspond to the food ingested during any given interval it is customary to cause the person under observation to ingest some substance, at the beginning and end of the period in question, which shall sufficiently differ in color and consistency from the surrounding feces as to render such differentiation comparatively easy. Two "markers" very widely used in such tests are wood charcoal and carmine. In making an actual separation of feces in a metabolism experiment proceed as follows: Just preceding or in the early part of the first meal (usually breakfast) of the metabolism test, ingest a gclatine capsule (No. o) containing $0.2-0.3$ gram of carmine or charcoal. From this time collect all stools in flat-bottom porcelain dishes and examine for the presence of the "marker." All fecal matter containing portions of the marker may be considered as representing the diet in question. This fecal matter should be retained and preserved (see page jSS). Just before or in the early part of the first meal (usually breakfast) following the end of the metabolism test a second "marker" in a gelatine capsule should be ingested. The feces should be carefully inspected until the marker makes its appearance. Retain all fecal matter uncolored by the marker, reject the remainder. Frequent difficulties are encountered in the practical separation of feces, but the character of such difficulties will be most satisfactorily impressed by the performance of actual separations.

'For other practical work on feces see Chapter XIV. 


\section{Collection and Preservation of Feces and the Mixing and} Weighing for Analysis.-The older methods in vogue in metabolism work embraced the analysis of dried feces. Various investigators later demonstrated that the drying of feces was accompanied by losses and changes of some of the organic constituents of the feces. ${ }^{1}$ Therefore the chemical examination of all stools wherever possible should be made on the fresh feces. If a study is being made which extends over several days and it is desired to economize time and effort in the chemical examination the daily fecal output or an aliquot portion of each stool may be collected in a friction-top can or pail of suitable size and preserved by thymol and refrigeration. ${ }^{2}$ This method has been found satisfactory when the feces are to be examined for inorganic constituents or total nitrogen. For the determination of fat, carbohydrate, etc., the fresh stool should be employed.

In the preservation of feces for the determination of total nitrogen the following simple procedure may be used: Introduce each stool into a weighed friction-top can or pail and place the vessel in a cold room or refrigerator. $^{3}$ At the end of the period mix the feces thoroughly and analyze weighed portions. In case individual stools are analyzed, the stool should be collected in a weighed flat-bottom porcelain dish. ${ }^{4}$ After mixing the feces very thoroughly the weight of dish, spatula and feces is determined and the weight of the feces secured by difference. ${ }^{5}$ A portion of the well-mixed feces is then introduced into a large weighing bottle containing a glass hoe. Desired amounts of feces are then removed for analysis and the exact weight of such amounts obtained by difference.

26. Bacterial Nitrogen in Feces.-About 50 per cent. of the total nitrogen of the feces is made up of bacterial cells (see Chapter XIV on Feces). To demonstrate this point proceed as follows:

(a) Ingest an ordinary mixed diet. Collect a representative stool from this diet and after mixing it thoroughly separate the bacterial cells from a weighed portion as described in Chapter XIV. After examining some of the suspension under the microscope and noting the bacterial cells determine the bacterial nitrogen in

' Zaitschek: Pfiigers Arch., 98, 595, I903.

Schimidzu: Bioch. Zeit., 28, 237, rgrr.

König: Landw. Vers. Stat., 38, 230.

Frear and Holter: Report, Penn. State College, p. r23, r8gr.

Emmett and Grindley: Jour. Am. Chem. Soc., 3r, 570, rgog.

${ }^{2}$ Howe, Rutherford and Hawk: Jour. Amer. Chem. Soc., 32, r683, rgro. This procedure is not satisfactory if fat is to be determined (Smith, Miller and Hawk: Jour. Biol. Chem., 21, 395, 1915). Such feces shows an hydrolysis of fat to fatty acid and a decrease in total fat.

${ }^{3}$ The author uses a brine $\operatorname{tank}$ at $-12^{\circ} \mathrm{C}$. in which the feces are quickly frozen.

4 The spatula for mixing the feces should be weighed with the dish.

${ }^{5}$ In case it is desired an aliquol part of each stool may be placed in a friction-top can or pail and preserved as a "composite sample" for the period. 
the entire volume of suspension by the Kjeldahl method ${ }^{1}$ (see Chapter XXVI) Also determine the total nitrogen in weighed portions of the original feces by the Kjeldahl method. What percentage of the total nitrogen of the feces is bacterial nitrogen?

(b) If it is desired to determine the actual amount of nitrogen which is excreted daily in the feces in the form of bacterial cells, proceed as follows: Ingest an ordinary mixed diet for a period of three days. Separate the feces for this period according to directions given on page 587 , using charcoal for the first separation and carmine for the second or vice versa. Preserve the feces for the period according to directions given on page 588. Mix the weighed feces thoroughly and analyze for bacterial nitrogen and total nitrogen according to directions given elsewhere ${ }^{2}$ (see Chapters XIV and XXVI). Calculate the actual output of bacterial nitrogen per day and the percentage of the total nitrogen of the feces which was excreted per day in the form of bacterial nitrogen.

27. "Metabolic Product" Nitrogen in Feces.-A certain quota of the fecal nitrogen is due to the presence of residues of digestive secretions, epithelial cells, bacteria, etc. The nitrogen in these forms has been called "metabolic nitrogen." To determine this form of nitrogen proceed as follows: Ingest a non-nitrogenous diet for a period of two days. The diet may include desired quantities of starch, cream, sugar, butter, water and sodium chloride. About 15 grams of agar-agar should be added to the diet to prevent constipation and to insure the evacuation of approximately the normal quantity of feces. (For influence of agar-agar see Experiment 28.) To separate the feces properly ingest a capsule of carmine at the beginning of the test and one of charcoal at the end (see page 587). Preserve the feces as described on page 588. After mixing the feces thoroughly determine the nitrogen in weighed quantities by the Kjeldahl method ${ }^{1}$ according to directions given in Chapter XXVI. Calculate the quantity of nitrogen eliminated per day. Inasmuch as no nitrogen was ingested the nitrogen present in the feces is of metabolic origin, i.e., it is made up principally of nitrogen in the form of cells, digestive secretions and bacteria.

\section{Influence of Indigestible Non-Nitrogenous Material upon} Fecal Output.-This may be demonstrated by agar-agar ingestion. This indigestible hemicellulose has the property of absorbing water readily and therefore when ingested it increases the bulk of the feces considerably. This fact is made use of in some forms of constipation and in the determination of metabolic product nitrogen (see Experiment 27).

Experiment.-Ingest a uniform diet for four days. Divide the interval into periods of two days each, ${ }^{3}$ and "separate" the feces by charcoal or carmine (see Experiment 24). On the third and fourth days ingest ro grams of agar-agar at each meal. Collect the feces for each two-day period (see Experiment 24, page 587 ), and note the increase in the daily excretion under the influence of the agar ingestion. What was the increase per gram of agar?

${ }^{1}$ In the oxidation process use ro grams of potassium sulphate instead of the copper sulphate. The remainder of the procedure is the same as for urine.

${ }^{2}$ More accurate results will be secured if the bacterial nitrogen is determined on each individual stool in the fresh condition.

${ }^{3}$ Longer periods are desirable where great accuracy is desired. 
29. Protein Utilization.-By "protein utilization" is meant the percentage of the ingested protein which is actually absorbed and assimilated.

This may be determined by the following procedure: Ingest any diet of known nitrogen content for a period of three days ${ }^{1}$ (see table, page 569). Collect all feces from the diet making the "separations" as directed on page 587 , using carmine as the initial "marker" and charcoal as the final "marker" or vice versa. Preserve the feces as directed on page 588. Mix the total feces thoroughly and determine the nitrogen by the Kjeldahl method (see Chapter XXVI and note on page 589). The approximate nitrogen utilization may be calculated as follows : (Food nitrogen - Feces nitrogen) $\times$ I00 Food nitrogen

$=$ Approximate percentage nitrogen utilization. If it is desired to ascertain the actual percentage of the ingested nitrogen which has been utilized by the body we must make a correction for metabolic nitrogen. In doing this proceed as follows: Ingest a non-nitrogenous diet as described on page 589 for a period of two days, using sufficient agar-agar to insure a daily fecal output which shall approximate in weight that obtained when the regular protein diet was ingested. ${ }^{2}$ Separate and preserve the feces as directed on page 588. Mix thoroughly and analyze for nitrogen according to the Kjeldahl method (see Chapter XXVI and note on page 589). Calculate the actual percentage utilization of the diet as follows:

[Food Nitrogen $-($ Fecal nitrogen - metabolic nitrogen $)] \times 100$

Food nitrogen

$=$ Actual percentage nitrogen utilization. If urinary nitrogen is determined the above data enable us to prepare a nitrogen balance (see Experiment 34, page 592).

30. Influence of Defective Mastication on Food Residues in Feces.-Rapid eating accompanied by defective mastication leads to the appearance of relatively large macroscopic food residues in the feces. Under some conditions, however, protein utilization (see above) may be as satisfactory during food "bolting" as when the food is very thoroughly masticated. ${ }^{3}$ This problem may be studied by the following method:

(a) Ingest a diet containing meat and be certain to masticate the diet very thoroughly. Collect a stool, examine macroscopically; mix carefully and examine microscopically (see page 229).

(b) Ingest a diet similar to that employed in above experiment (a). "Bolt" the food, i.e., ingest it practically without mastication. Examine the feces as above. Note the difference in the macroscopical and microscopical findings under $(a)$ and $(b)$.

If the nitrogen of food and feces is determined we may calculate the protein utilization (see Experiment 29). By the additional determination of urinary nitrogen, we may prepare a nitrogen balance (see Experiment 34, page 592).

1 See note 3 , p. 589 .

${ }^{2}$ It is frequently difficult to so regulate the agar-agar intake as to secure the proper fecal output. In such an event the proper value for metabolic nitrogen must be obtained by calculation. For example if 89.1 grams of feces were excreted per day on the protein diet, and 166.5 grams per day (with a nitrogen value of 0.5 gram) when agar was employed, the actual value for metabolic product nitrogen may be obtained by the following proportion, assuming that the content of metabolic nitrogen is proportional to the weight of feces excreted: 89.1 : I66.5: : $x: 0.5 . \quad x=0.268$ gram metabolic nitrogen per day.

${ }^{3}$ Foster and Hawk: Jour. Amer. Chem. Soc., 37, I347, I9I 5. 
31. Fat in Feces. - A normal adult will digest and absorb at least 90 per cent of the fat in the diet when the amount ingested does not exceed roo grams. If the diet contains an excessive amount of fat, e.g., 300 grams per day, considerable appears in the feces. In pancreatic diseases and such conditions as are accompanied by a decrease in bile flow the digestion and assimilation of fat is lessened.

Experiments.- (a) Ingest an ordinary mixed diet containing an average amount of fat per day, e.g., 75-roo grams. Collect a stool and examine it microscopically as directed in Chapter XIV. (b) Now ingest a diet containing an excessive quantity of fat, e.g., 300 grams per day. Separate the feces and subject a representative sample of the feces from the high fat diet to microscopical examination. (c) If it is desired the fat may be extracted from some of the stool by applying the principle involved in the quantitative determination of fat in the Saxon method (see Chapter XIV). Evaporate the ether extract and identify the fat in the residue by tests given in Chapter IX.

32. Carbohydrate in Feces.-Under normal conditions the great bulk of the soluble carbohydrate in the food is absorbed from the intestine even when the ingestion is high. Hence the content of soluble carbohydrate in feces is low. To demonstrate this proceed as follows:

(a) Ingest for three days an ordinary mixed diet to which Ioo grams of glucose or sucrose is daily added. Separate and preserve the feces (see page 588) and when the final "marker" appears extract an aliquot portion of the total mixed feces ${ }^{1}$ with water, decolorize with boneblack, filter, and after making the filtrate up to a known volume determine the sugar by Benedict's method (see page 522). Calculate the soluble carbohydrate content of the feces for the three-day interval. (b) Proceed as above with the exception that at least 250 grams of sugar should be added to the diet instead of 100 as in (a).

How did the daily excretion of soluble carbohydrate in (a) compare with that in (b)? Why is this so? If a diet of known carbohydrate content is fed this experiment will give us accurate data as to soluble carbohydrate utilization (see Protein Utilization, page 590). If it is desired this experiment may be combined with the hyperglycemia and glycosuria experiments on pages 566 and 568 . See also Experiment 36, page 593.

33. Inorganic Elements in the Feces.-The salts of sodium and potassium being very soluble are almost completely absorbed. from the intestine. The same is true of the chlorides including that of sodium which is of greatest importance. Hence the alkali metals and chlorides are excreted mainly in the urine and are found only in very small amounts in the feces even when large amounts are ingested. With calcium, magnesium, iron and phosphate conditions are different. Not only are salts of calcium, magnesium and iron less readily absorbed but they are excreted to a large extent by way of the intestinal mucosa rather than by the kidney's. Ordinarily about 90 per cent of ingested calciun is

${ }^{2}$ If time permits it is more satisfactory to analyze each individual stool in fres/s condition. 
eliminated by way of the feces and a little less than half of the magnesium. From 20-30 per cent of the phosphorus ingested is usually found in the feces.

Experiments.-(a) Ingest for a period of three days an ordinary mixed diet without added salt and containing no milk. Separate the feces for the period (see page 587) and retain a portion of the well-mixed feces for analysis.

(b) Proceed as above with the exception that there is added to the mixed diet ro grams of common salt and a quart of milk (containing about 1.6 grams of $\mathrm{CaO}$, 0.2 gram $\mathrm{MgO}, \mathrm{r} .4$ grams of chloride expressed as sodium chloride, and 2.2 grams $\mathrm{P}_{2} \mathrm{O}_{5}$ ). Mix feces well and reserve part for analysis.

Ash ro gram samples of the feces from the above diets. Dissolve with the aid of a little dilute nitric acid, filter and make up to 100 c.c. Determine in aliquot portions of this solution: (I) Chlorides by Volhard method. (2) Calcium and magnesium by McCrudden's method. (3) Phosphorus by uranium titration. (For details of analytical methods see Chapter XXVI.) Calculate the percentages of the added $\mathrm{Ca}, \mathrm{Mg}, \mathrm{P}$, and $\mathrm{Cl}$ which are recovered from the feces.

For a more detailed study of chloride excretion combine this experiment and Experiment 14 (see Experiment 13).

\section{METABOLISM PROCEDURES INVOLVING THE MANIPULATION OF BOTH URINE AND FECES}

34 Preparation of a Metabolic Balance.-This test entails the analysis of the food ingested and of the urine and feces excreted, i.e., a study of the income and outgo. Proceed as follows:

Select a diet which is simple, i.e., consists of few constituents, and which lends itself readily to accurate chemical analysis. A good type of diet for ordinary metabolism experiments of this sort consists of crackers (graham or soda), BALANCE OF CALCIUM, MAGNESIUM, PHOSPHORUS, SULPHUR, AND NITROGEN IN ACROMEGALY

\begin{tabular}{|c|c|c|c|c|c|}
\hline & $\begin{array}{c}\text { Calcium } \\
\text { oxide }\end{array}$ & $\begin{array}{l}\text { Magnesium } \\
\text { oxide }\end{array}$ & $\begin{array}{l}\text { Phosphoric } \\
\text { anhydride }\end{array}$ & Sulphur & Nitrogen \\
\hline & \multicolumn{5}{|c|}{ Grams } \\
\hline Ingestion (daily) ............ & I. 494 & 0.486 & 3.192 & 1.190 & 18.84 \\
\hline Excretion (urine)... . & 0.159 & 0.160 & I. 701 & I. .006 & 17.60 \\
\hline Excretion (feces)......... & I. .093 & 0.226 & I. .002 & 0.135 & I. 10 \\
\hline Excretion (total).... & I. 252 & 0.386 & 2.703 & I. I4 I & 18.70 \\
\hline Retention (daily). & 0.242 & 0.100 & 0.489 & 0.049 & 0.14 \\
\hline Retention (per cent). & 16.2 & 20.6 & $15 \cdot 3$ & $4 \cdot I$ & 0.7 \\
\hline
\end{tabular}


milk, butter, water and agar-agar (to prevent constipation). Meat specially prepared in quantity sufficient for an entire experiment may also be utilized. Ingest uniform quantities of these dietary constituents each day for a period of three days. ${ }^{1}$ Make an accurate collection of the urine passed during this interval (see page 565). Separate the feces representing the three-day period (see page 587), and analyze foods, urine and feces. The balances ordinarily prepared are those for nitrogen, sulphur, phosphorus and calcium. Analytical methods for the determination of these elements may be found in Chapter XXVI.

The foregoing table includes balances obtained in a metabolism test on acromegaly. ${ }^{2}$

35. Excretion of Urinary and Fecal Chloride after a High Chloride Ingestion.Combine the procedures outlined under Experiments 14 and 33, pages 576 and 59 I.

36. A Study of the Elimination of Carbohydrate in Urine and Feces after Excessive Carbohydrate Ingestion.-Combine the procedures outlined in Experiments 5 and 32 , pages 568 and $59 \mathrm{r}$.

${ }^{1}$ See note 3 , p. 589 .

${ }^{2}$ Bergeim, Stewart and Hawk: Jour. Expt., Med., 20, 2 I8, I9I4. 


\section{REAGENTS AND SOLUTIONS}

Alizarin. ${ }^{1}$ - A I per cent solution of alizarin mono-sodium sulphonate in water.

Almen's Reagent. ${ }^{2}-$ Dissolve 5 grams of tannic acid in 240 c.c. of 50 per cent alcohol and add Io c.c. of 25 per cent acetic acid.

Aluminium Hydroxide Cream. ${ }^{3}$ - To a I per cent solution of ammonium alum at room temperature add a slight excess of a I per cent solution of ammonium hydroxide. Wash by decantation until the wash water shows only the faintest trace of residue on evaporation.

Ammoniacal Silver Solution. ${ }^{4}$-Dissolve 26 grams of silver nitrate in about 500 c.c. of water, add enough ammonium hydroxide to redissolve the precipitate which forms upon the first addition of the ammonium hydroxide and make the volume of the mixture up to I liter with water.

Ammonium Thiocyanate Solution. ${ }^{5}$ - This solution is made of such a strength that I c.c. of it is equal to I c.c. of the standard silver nitrate solution mentioned below. To prepare the solution dissolve $\mathrm{I} 2.9$ grams of ammonium thiocyanate, $\mathrm{NH}_{4} \mathrm{SCN}$, in a little less than a liter of water. In a small flask place 20 c.c. of the standard silver nitrate solution, 5 c.c. of a cold saturated solution of ferric alum and 4 c.c. of nitric acid (sp. gr. I.2), add water to make the total volume roo c.c., and thoroughly mix the contents of the flask. Now run in the ammonium thiocyanate solution from a burette until a permanent red-brown tinge is produced. This is the end-reaction and indicates that the last trace of silver nitrate has been precipitated. Take the burette reading and calculate the amount of water necessary to use in diluting the ammonium thiocyanate in order that Io c.c. of this solution may be exactly equal to ro c.c. of the silver nitrate solution. Make the dilution and titrate again to be certain that the solution is of the proper strength.

Arnold-Lipliawsky Reagent. ${ }^{6}$ - This reagent consists of two definite solutions which are ordinarily preserved separately and mixed just before using. The two solutions are prepared as follows:

(a) One per cent aqueous solution of potassium nitrate.

\footnotetext{
1 Indicator in various procedures, pp. 174 and $48 \mathrm{I}$.

2 Ott's precipitation test, p. 429 . Determination of lactalbumin, p. 329 .

3 Removal of protein in various methods, pp. 329,485 .

- Salkowski's method, p. 5 r6.

6 Volhard-Arnold method, p. 556, and Dehn-Clark method, p. 557.

B Arnold-Lipliawsky reaction, p. 440.
} 
(b) One gram of $p$-amino-acetophenon dissolved in roo c.c. of distilled water and enough hydrochloric acid (about 2 c.c.) added drop by drop, to cause the solution, which is at first yellow, to become entirely colorless. An excess of acid must be avoided.

Asbestos for Suction Filters. ${ }^{1}$ The asbestos is shredded, placed in a wide mouth flask and covered with Io per cent $\mathrm{HCl}$. Heat on waterbath for five hours. Filter on Buchner funnel, wash free from acid, return to the flask, cover with 5 per cent $\mathrm{NaOH}$ and heat on water-bath for three hours. Filter, wash free from alkali, then with dilute acid and finally with water until free from acid. Suspend in a large volume of water, allow to settle for five minutes. Pour off the upper two-thirds and discard. Repeat the washing of the desired coarse portion several times until the supernatant liquid remains nearly clear.

Bang's Sugar Reagents. ${ }^{2}$ - (a) Acid $\mathrm{KCl}$ Solution.-Consisting of 1360 c.c. of saturated $\mathrm{KCl}$ to which is added 640 c.c. of water and I. 5 c.c. 25 per cent. $\mathrm{HCl}$.

(b) Stock Copper Solution.-Introduce into a rooo c.c. flask 700 c.c. of boiled and cooled water. Warm to about $30^{\circ} \mathrm{C}$. and add I 60 grams of pure potassium bicarbonate in powder form. When dissolved and 66 grams of pure $\mathrm{KCl}$. Cool and then add 100 grams potassium carbonate. Finally add roo c.c. of 4.4. per cent. solution of pure crystalline copper sulphate. Let stand a short time, then make to mark with boiled water. Allow to stand a day or so before using.

(c) N/200 I Solution.-Made fresh each day. Dilute $\mathrm{N} /$ Io I solution 20 times. Or make as follows: Introduce into a roo c.c. flask 2 grams KI, I-2 c.c. of 2 per cent $\mathrm{KIO}_{3}$ solution and 5 c.c. of $\mathrm{N} /$ IO $\mathrm{HCl}$. Make to mark with boiled and cooled distilled water.

(d) Starch Solution.-A I per cent. solution of Kahlbaum's soluble starch in a saturated $\mathrm{KCl}$ solution.

(e) Dilute Copper Solution.-Dilute 300 c.c. of the stock solution to rooo c.c. Mix with only gentle shaking. Let stand several hours before using.

Barfoed's Solution. ${ }^{3}$-Dissolve 4.5 grams of neutral, crystallized copper acetate in roo c.c. of water and add I. 2 c.c. of 50 per cent acetic acid.

Baryta Mixture. ${ }^{4}$ - A mixture consisting of I rolume of a saturated solution of barium nitrate and 2 volumes of a saturated solution of barium hydroxide.

${ }^{1}$ See methods entailing use of Gooch crucibles.

${ }^{2}$ Determination of sugar, pages $2 S O$ and 524 .

${ }^{3}$ Barfoed's test, p. 30 .

4 Isolation of urea from urine, p. 375. 
Basic Lead Acetate Solution. ${ }^{1-}$ This solution possesses the following formula:

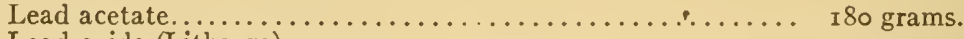

Lead oxide (Litharge)............................. I Io grams.

Distilled water to make......................... I

Dissolve the lead acetate in about 700 c.c. of distilled water, with boiling. Add this hot solution to the finely powdered lead oxide and boil for onehalf hour with occasional stirring. Cool, filter and add sufficient distilled water to the filtrate to make the weight $\mathrm{I} \mathrm{kg}$.

Benedict's Solutions. ${ }^{2}$-First Modification.-Benedict's modified Fehling solution consists of two definite solutions-a copper sulphate solution and an alkaline tartrate solution, which may be prepared as follows:

Copper sulphate solution $=34.65$ grams of copper sulphate dissolved in water and made up to 500 c.c.

Alkaline tartrate solution $=$ Ioo grams of anhydrous sodium carbonate and I73 grams of Rochelle salt dissolved in water and made up to roo c.c.

These solutions should be preserved separately in rubber-stoppered bottles and mixed in equal volumes when needed for use. This is done to prevent deterioration.

Second Modification.-Benedict has further modified his solution and has succeeded in obtaining one which does not deteriorate upon long standing. It has the following composition:

Copper sulphate........................ I 7.3 grams.

Sodium citrate........................... I73.0 grams.

Sodium carbonate......................... I00.0 grams.

Distilled water to make I liter.

With the aid of heat dissolve the sodium citrate and carbonate in about 600 c.c. of water. Pour (through a folded filter paper if necessary) into a glass graduate and make up to 850 c.c. Dissolve the copper sulphate in about roo c.c. of water and make up to I50 c.c. Pour the carbonate-citrate solution into a large beaker or casserole and add the copper sulphate solution slowly, with constant stirring. The mixed solution is ready for use and does not deteriorate upon long standing.

\section{Benedict's Sugar Reagent. ${ }^{3}$}

Copper sulphate (crystallized).............................. anhydrous salt may be used) $\ldots \ldots \ldots \ldots \ldots \ldots \ldots \ldots$

Sodium or potassium citrate...........................

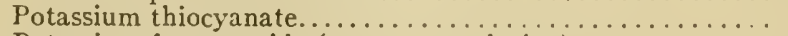

Potassium ferrocyanide ( 5 per cent solution).............

Potassium ferrocyanide ( 5 per cent solution) ........... 5.0 c.c.
Distilled water to make a total volume of.......... I000.0 c.c.

${ }^{1}$ Indican determination, p. 542.

${ }^{2}$ Benedict's modifications of Fehling's test, pp. 27 and 417 .

${ }^{3}$ Quantitative determination of sugar, p. 522.

ז8. 0 grams.

200.0 grams. 200.0 grams. I 25.0 grams. 
With the aid of heat dissolve the carbonate, citrate and thiocyanate in enough water to make about 800 c.c. of the mixture and filter if necessary.

Dissolve the copper sulphate separately in about Ioo c.c. of water and pour the solution slowly into the other liquid, with constant stirring. Add the ferrocyanide solution, cool and dilute to exactly r liter. Of the various constituents, the copper salt only need be weighed with exactness. Twenty-five c.c. of the reagent are reduced by $50 \mathrm{mg}$. of glucose.

\section{Benedict's Sulphur Reagent.}

Crystallized copper nitrate, sulphur-free or of known sulphur

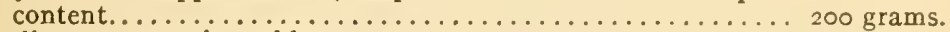

Sodium or potassium chlorate.................... 50 grams.

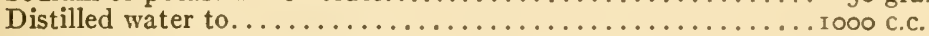

Benzidine Solutions for Volumetric Sulphur Determinations. - (a) Rosenheim and Drummond.-Rub 4 grams of benzidine (Kahlbaum) into a fine paste with about ro c.c. of water and transfer to a 2 -liter flask with the aid of about 500 c.c. of water. Add 500 c.c. of concentrated $\mathrm{HCl}$ (sp. gr. r.I9) and make up to 2 liters with distilled water. I50 c.c. of this solution, which keeps indefinitely, are sufficient to precipitate o.I gram $\mathrm{H}_{2} \mathrm{SO}_{4}$.

(b) Raiziss and Dubin.-Put 6.7 grams of benzidine (Merck Reagent) in a r-liter flask, add 29 c.c. of hydrochloric acid (sp. gr. I.r2) and dilute up to the mark.

Bertrand Sugar Reagents. ${ }^{1}-(a)$ Copper Sulphate Solution.-Forty grams of pure crystallized copper sulphate are dissolved in water to make a liter.

(b) Dissolve 200 grams of Rochelle salts and 150 grams of $\mathrm{NaOH}$ in water to make rooo c.c.

(c) Acid Ferric Sulphate Solution.-Dissolve 50 grams of ferric sulphate in about 200 C.c. of water and pour into this a mixture of 200 c.c. of concentrated sulphuric acid diluted with about 400 c.c. of water. Mix and make to rooo c.c.

(d) Potassium Permanganate Solution.-Dissolve 5 grams of potassium permanganate in water to make rooo c.c. Standardization.Dissolve 0.250 gram of ammonium oxalate in 50-roo c.c. of water add I-2 c.c. of concentrated sulphuric acid and titrate with the permanganate to a rose color. Multiply the number of grams of oxalate used by 0.895 to get the equivalent in $\mathrm{Cu}$ of the number of cubic centimeters of permanganate used. Calculate the $\mathrm{Cu}$ value of $\mathrm{I}$ c.c.

${ }^{1}$ Determination of sugar, p. 527 . 


\section{Bial's Reagent. ${ }^{1}$}

Orcinol............................... I.5 grams.

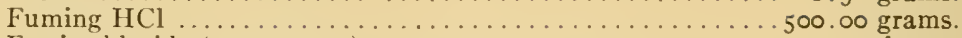

Ferric chloride (ro per cent).................... 20-30 drops.

Biuret Reagent, Gies. ${ }^{2}$ - This reagent consists of Io per cent $\mathrm{KOH}$ solution to which 25 c.c. of 3 per cent $\mathrm{CuSO}_{4}$ solution per liter has been added. This imparts a slight though distinct blue color to the clear liquid.

Biuret Paper (Kantor and Gies). ${ }^{2}$ - Immerse filter paper in Gies' Biuret Reagent (above) then dry and cut into strips.

Black's Reagent. ${ }^{3}$ - Made by dissolving 5 grams of ferric chloride and 0.4 gram of ferrous chloride in roo c.c. of water.

Blood Serum.-This may easily be obtained in quantity by the procedure described under Hemagglutination in the chapter on Blood.

Boas' Reagent. ${ }^{4}$-Dissolve 5 grams of resorcinol and 3 grams of sucrose in 100 c.c. of 50 per cent alcohol.

Bonnano's Reagent.-Dissolve 2 grams of sodium nitrite in Ioo c.c. of concentrated hydrochloric acid.

Bottu's Reagent.-To 3.5 grams of 0 -nitrophenylpropiolic acid add 5 c.c. of a freshly prepared ro per cent solution of sodium hydroxide and make the volume of the solution I liter with distilled water.

Carmine-Fibrin. ${ }^{5}$ - Prepared by running fibrin through a meat chopper, washing carefully and placing in $1 / 2$ per cent ammoniacal carmine solution (very little excess ammonia should be present) until the maximum coloration of the fibrin (dark red) is obtained. The fibrin is then washed in water and in water acidified with acetic acid. It is preserved under glycerol.

Chloride Reagents for Blood Analysis. ${ }^{6}$-(a) An acid M/29.25 solution of silver nitrate, I c.c. of which is equivalent to $2 \mathrm{mg}$. of $\mathrm{NaCl}$.

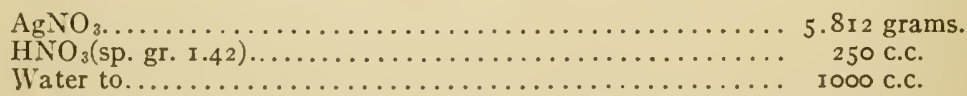

(b) A solution of $\mathrm{M} / 5^{8} .5$ potassium iodide, I c.c. of which is equivalent to I mg. of $\mathrm{NaCl}$.

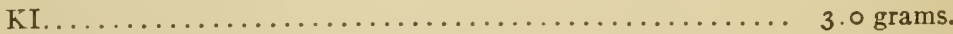

Water to............................. I000 c.c.

This solution is standardized against the silver solution by adding 5 c.c. of the latter to 5 c.c. of solution $(c)$ and titrating with the iodide solution

1 Test for pentose, p. 37 .

2 Protein tests, p. I00.

'Black's reaction, p. $44 \mathrm{I}$.

${ }^{4}$ Test for free acid, p. I54.

$\checkmark$ Tests on proteases, p. I 2 .

${ }^{6}$ Method of McLean and Van Slyke, p. 286. 
to the blue end point. The iodide solution is then diluted to such a degree that ro c.c. are exactly equivalent to 5 c.c. of the silver solution.

(c) A solution used as an indicator, to regulate acidity, and provide an oxidizing agent.

Sodium citrate $\left(\mathrm{Na}_{3} \mathrm{C}_{0} \mathrm{H}_{6} \mathrm{O}_{7}+5 \frac{1}{2} \mathrm{H}_{2} \mathrm{O}\right)$

Sodium nitrite.

Soluble starch...

Water to.....
446.0 grams.

20.0 grams.

2.5 grams.

I000.0 c.c.

The starch is first dissolved with the aid of heat in about 500 c.c. of the water. The citrate and nitrite are then added, and the mixture is heated until all is dissolved. The solution while still hot is filtered through cotton, the filter washed with hot water, the filtrate allowed to cool, and made up to rooo c.c. The solution keeps indefinitely.

Combined Hydrochloric Acid (Protein Salt).-To prepare so-called combined hydrochloric acid simply add a soluble protein such as Witte's peptone to free hydrochloric acid of the desired strength until it no longer responds to free acid tests (see chapter on Gastric Digestion). For example, if 0.2 per cent combined acid is required the protein would be added to 0.2 per cent free hydrochloric acid.

Strictly speaking there is no such thing as "combined" acid in this sense. When the protein is added a protein salt of the acid is formed which ionizes differently from the free acid.

Congo Red. ${ }^{1}$-Dissolve 0.5 gram of Congo red in 90 c.c. of water and add ro c.c. of 95 per cent alcohol.

Congo Red-Fibrin.-This may be prepared by placing fibrin in faintly alkaline Congo red solution and heating to $80^{\circ} \mathrm{C}$. The fibrin is then washed and preserved under glycerol.

Creatinine, Standard Solution for Colorimetric Method. ${ }^{2}$-Dissolve I gram of pure creatinine in Iooo c.c. of $\mathrm{N} / \mathrm{I}$ o $\mathrm{HCl}$. The solution contains I mg. of creatinine per cubic centimeter.

Cross and Bevan's Reagent.-Combine two parts of concentrated hydrochloric acid and one part of zinc chloride by weight.

Ehrlich's Diazo Reagent. ${ }^{3}$ - Two separate solutions should be prepared and mixed in definite proportions when needed for use.

(a) Five grams of sodium nitrite dissolved in I liter of distilled water.

(b) Five grams of sulphanilic acid and 50 c.c. of hydrochloric acid in I. liter of distilled water.

Solutions $(a)$ and (b) should be preserved in well-stoppered vessels and mixed in the proportion I: 50 when required. Green asserts that

1 Test for free acid, p. 153 .

${ }^{2}$ Determination of creatinine, p. 506.

3 Ehrlich's diazo reaction, p. 454. 
greater delicacy is secured by mixing the solutions in the proportion I:IOO. The sodium nitrite deteriorates upon standing and becomes unfit for use in the course of a few weeks.

Esbach's Reagent. ${ }^{1-D i s s o l v e ~}$ Io grams of picric acid and 20 grams of citric acid in I liter of water.

Fehling's Solution. ${ }^{2}-$ Fehling's solution is composed of two definite solutions-a copper sulphate solution and an alkaline tartrate solution, which may be prepared as follows:

Copper sulphate solution $=34.65$ grams of copper sulphate dissolved in water and made up to 500 c.c.

Alkaline tartrate solution $=I 25$ grams of potassium hydroxide and I 73 grams of Rochelle salt dissolved in water and made up to 500 c.c.

These solutions should be preserved separately in rubber-stoppered bottles and mixed in equal volumes when needed for use. This is done to prevent deterioration.

Ferric Alum Solution. ${ }^{3}-$ A cold saturated solution.

Folin-Shaffer Reagent. ${ }^{4}$ - This reagent consists of 500 grams of ammonium sulphate, 5 grams of uranium acetate, and 60 c.c. of ro per cent acetic acid in 650 c.c. of distilled water.

Formalin Solution (Neutral). ${ }^{5}$ - To 50 c.c. of commercial formaldehyde solution (30-40 per cent) add I c.c. of phenolphthalein solution and then standard alkali solution until the mixture assumes a faint red color. The solution should be freshly prepared for each set of determinations.

Furfural Solution. ${ }^{6}$-Add I c.c. of furfural to rooo c.c. of distilled water.

Gallic Acid Solution. ${ }^{7}$-A saturated alcoholic solution.

Guaiac Solution. ${ }^{8}$-Dissolve 0.5 gram of guaiac resin in 30 c.c. of 95 per cent alcohol.

Gulick's Acid Oxidizing Mixture. ${ }^{9}$ - To I25 c.c. of ammonia free water add 40 c.c. of sulphuric acid, 5 c.c. of a saturated solution of mercuric chloride, and 20 grams of potassium sulphate. Then make up to 200 c.c. with ammonia-free water.

Gulick's Modified Winkler Solution. ${ }^{10}$-Dissolve 40 grams of sodium

1 Esbach's method, p. 532.

${ }^{2}$ Fehling's method, p. 523. Fehling's test, pp. 26 and 416 .

3 Volhard-Arnold method, p. 556.

${ }^{4}$ Folin-Shaffer method, p. 5 II.

5 Formol titration procedure, p. 502.

${ }^{6}$ Mylius's modification of Pettenkofer's test, pp. 208 and 434. v. Udránsky's test, pp. 208 and 434.

${ }^{7}$ Gallic acid test, p. 323

${ }^{8}$ Guaiac test, pp. I5, 235,262 , and 432 .

9 Determination of total nitrogen, p. 490.

${ }^{10}$ Determination of total nitrogen, p. 490 . 
hydroxide in about 200 c.c. of ammonia-free water. Mix $\mathrm{r}_{5}$ grams of mercuric iodide and ro grams of potassium iodide and dissolve in about I 5 c.c. of water. Transfer with the aid of the alkali to a 500 c.c. volumetric flask and make up to 500 c.c. with ammonia-free water. Transfer to an Erlenmeyer flask and let stand 24 hours to settle.

Günzberg's Reagent. ${ }^{1-D i s s o l v e ~} 2$ grams of phloroglucinol and I gram of vanillin in too c.c. of 95 per cent alcohol.

Haines' Solution. ${ }^{2}$ - This solution may be prepared by dissolving 8.314 grams of copper sulphate in 400 c.c. of water adding 40 c.c. of glycerol and 500 c.c. of 5 per cent potassium hydroxide solution.

Hammarsten's Reagent. ${ }^{3}$-Mix I volume of 25 per cent nitric acid and ig volumes of 25 per cent hydrochloric acid and add I volume of this acid mixture to 4 volumes of 95 per cent alcohol. It is preferable that the acid mixture be prepared in advance and allowed to stand until yellow in color before adding it to the alcohol.

Hayem's Solution.-This solution has the following formula:

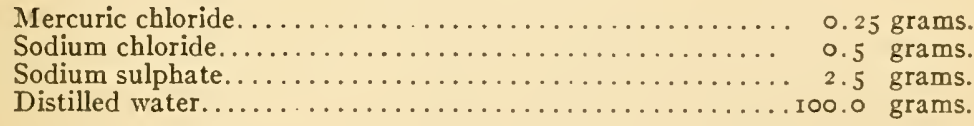

Hopkins-Cole Reagent. ${ }^{4}$-To I liter of a saturated solution of oxalic acid add 60 grams of sodium amalgam and allow the mixture to stand until the evolution of gas ceases. Filter and dilute with 2-3 volumes of water.

Hopkins-Cole Reagent (Benedict's Modification).-Ten grams of powdered magnesium are placed in a large Erlenmeyer flask and shaken up with enough distilled water to liberally cover the magnesium. Two hundred and fifty c.c. of a cold, saturated solution of oxalic acid is now added slowly. The reaction proceeds very rapidly and with the liberation of much heat, so that the flask should be cooled under running water during the addition of the acid. The contents of the flask are shaken after the addition of the last portion of the acid and then poured upon a filter, to remove the insoluble magnesium oxalate. A little wash water is poured through the filter, the filtrate acidified with acetic acid to prevent the partial precipitation of the magnesium on long standing, and made up to a liter with distilled water. This solution contains only the magnesium salt of glyoxylic acid.

Hypobromite Solution. ${ }^{5}$ - The ingredients of this solution should

${ }^{1}$ Test for free acid, p. I54.

${ }^{2}$ Haines' test, p. 4 rg.

${ }^{3}$ Hammarsten's reaction, pp. 207 and 433.

- Hopkins-Cole reaction, p. 98 .

5 Methods for determination of urea, p. 496. 
be prepared in the form of two separate solutions which may be united as needed.

(a) Dissolve 125 grams of sodium bromide in water, add 125 grams of bromine and make the total volume of the solution I liter.

(b) A solution of sodium hydroxide having a specific gravity of I.25. This is approximately a 22.5 per cent solution.

Preserve both solutions in rubber-stoppered bottles and when needed for use mix I volume of solution (a), I volume of solution (b), and 3 volumes of water.

Iodine Solution (N/IO). ${ }^{1}$-Weigh out 12.685 grams of pure resublimed iodine into a small weighing bottle using a porcelain spatula. Dissolve 18 grams of pure KI in about I50 c.c. of water. Transfer the iodine to a liter flask washing out the last traces with some of the KI solution, which is then poured into the flask. Stopper and shake occasionally until dissolved. If necessary a few more crystals of KI may be added to aid solution. Dilute to the mark and mix well. Keep in glass-stoppered bottle in cool dark place. Standardize at once against $\mathrm{N} /$ ro sodium thoisulphate solution. Measure out accurately 25 c.c. of the iodine solution into an Erlenmeyer flask, run in sodium thiosulphate until the color is pale yellow, then add a few cubic centimeters of a I per cent solution of starch (preferably soluble starch) and titrate to disappearance of blue color. Care should be taken near the end point.

Iodine Solution. ${ }^{2}$ - Prepare a 2 per cent solution of potassium iodide and add sufficient iodine to color it a deep yellow.

Iodine-Zinc Chloride Reagent. ${ }^{3}-$ Dissolve 20 grams of zinc chloride in 8.5 c.c. of water. Cool, and introduce iodine solution (3 grams KI+ r. 5 gram I in 60 c.c. of water) drop by drop until iodine begins to precipitate.

Kraut's Reagent. ${ }^{4}$-Dissolve 272 grams of potassium iodide in water and add 80 grams of bismuth subnitrate dissolved in 200 grams of nitric acid (sp. gr. r.r8). Permit the potassium nitrate to crystallize out, then filter it off and make the filtrate up to I liter with water.

Lead Acetate, Basic. (See Basic Lead Acetate.)

Lugol's Solution. ${ }^{5}$-Dissolve 4 grams of iodine and 6 grams of potassium iodide in roo c.c. of distilled water.

Magnesia Mixture. ${ }^{6}$-Dissolve 175 grams of magnesium sulphate and 350 grams of ammonium chloride in 1400 c.c. of distilled water.

1 Determination of acetone and acetoacetic acid, p. 533 .

2 Iodine test, p. 45.

${ }^{3}$ Amyloid formation, p. 49.

- Rosenheim's bismuth test for choline, p. 357.

5 Gunning's iodoform test, page 436 , and Bardach's reaction, p. ror.

6 Method for determination of total phosphorus, p. 554. 
Add 700 grams of concentrated ammonium hydroxide, mix thoroughly, and preserve the mixture in a glass-stoppered bottle.

Magnesium Nitrate Solution for Ignition. ${ }^{1-D i s s o l v e ~} 320$ grams of calcined magnesia in nitric acid, avoiding an excess of the latter; then add a little calcined magnesia in excess; boil; filter from the excess of magnesia, ferric oxide etc., and dilute with water to 2 liters.

Methyl Red. ${ }^{2}$-Saturated solution in 50 per cent alcohol.

Millon's Reagent. ${ }^{3}$-Digest I part (by weight) of mercury with 2 parts (by weight) of nitric acid (sp. gr. I.42) and dilute the resulting solution with 2 volumes of water.

Molisch's Reagent. ${ }^{4-A}$ I 5 per cent alcoholic solution of $\alpha$-naphthol.

Molybdate Solution. ${ }^{5}$-Dissolve 100 grams of molybdic acid in I44 c.c. of ammonium hydroxide (sp. gr. 0.90 ) and 27 I c.c. of water; slowly and with constant stirring pour the solution thus obtained into 489 c.c. of nitric acid (sp. gr. I.42) and II 48 c.c. of water. Keep the mixture in a warm place for several days, or until a portion heated to $40^{\circ} \mathrm{C}$. deposits no yellow precipitate of ammonium phosphomolybdate. Decant the solution from any sediment and preserve in glass-stoppered bottles.

Mörner's Reagent. ${ }^{6}$ - Thoroughly mix I volume of formalin, 45 volumes of distilled water, and 55 volumes of concentrated sulphuric acid.

Nakayama's Reagent. ${ }^{7}$-Prepared by combining 99 c.c. of alcohol and I c.c. of fuming hydrochloric acid containing 4 grams of ferric chloride per liter.

$\alpha$-Naphthol Solution. ${ }^{8}$-Dissolve I gram of $\alpha$-naphthol in Ioo c.c of 95 per cent. alcohol.

\section{Nessler-Winkler Solution.}

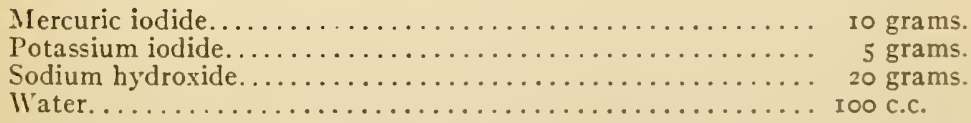

The mercuric iodide is rubbed up in a small porcelain mortar with water, then washed into a flask and the potassium iodide added. The sodium hydroxide is dissolved in the remaining water and the cooled solution added to the above mixture. The solution cleared by standing is preserved in a dark bottle.

\footnotetext{
1 Determination of phosphorus, p. 554 .

${ }^{2}$ Determination of $\mathrm{H}$ ion concentration, pp. I 58 and 480 .

${ }^{3}$ Millon's reaction, p. 97.

Molisch's reaction, p. 21 .

- Detection and determination of phosphorus, pp. I 29 and 554.

- Mörner's test, p. 86.

7 Nakayama's reaction, pp. 207 and 433 .

${ }^{8}$ Oxidases p. I 4. For other $\alpha$-naphthol solution see Molisch reaclion.
} 
Neutral Olive Oil. ${ }^{1-}$-Shake ordinary olive oil with a Io per cent solution of sodium carbonate, extract the mixture with ether, and remove the ether by evaporation. The residue is neutral olive oil.

Neutral Red. ${ }^{1-A}$ I per cent solution in 50 per cent alcohol.

p-Nitrophenol. ${ }^{2}$ - A I per cent solution in 50 per cent alcohol.

Nylander's Reagent. ${ }^{3}$-Digest 2 grams of bismuth subnitrate and 4 grams of Rochelle salt in IO० c.c. of a Io per cent solution of potassium hydroxide. The reagent should then be cooled and filtered.

Obermayer's Reagent. ${ }^{4}-$ Add $2-4$ grams of ferric chlorid to a liter of hydrochloric acid (sp. gr. I.rg).

Oxalated Plasma. ${ }^{5}$-Allow arterial blood to run into an equal volume of 0.2 per cent ammonium oxalate solution.

Para-dimethylaminobenzaldehyde Solution. ${ }^{6}$ - This solution is made by dissolving 5 grams of para-dimethylaminobenzaldehyde in roo c.c. of Io per cent sulphuric acid.

Para-phenylenediamine Hydrochloride Solution. ${ }^{7}$ - Two grams dissolved in Ioo c.c. of water.

Peters' Sugar Reagents. ${ }^{8}$ - (a) Copper Solution.-Dissolve 34.639 grams of highest purity crystallized copper sulphate (such as Kahlbaum's "zur analyse mit garantieschein") in water to make 500 c.c.

(b) Alkaline Tartrate Solution.-Dissolve I73 grams of sodium potassium tartrate and $\mathbf{2} 25$ grams of potassium hydroxide in water to make 500 c.c.

(c) N/5 Sodium Thiosulphate.-Dissolve about 50 grams of ordinary c.p. sodium thiosulphate or exactly 49.66 grams of the pure, dry, recrystallized salt, in enough boiled out distilled water to make a liter. Allow to stand for several days. The solution should be standardized against the copper solution prepared as above. For this purpose introduce 20 c.c. of the copper solution into a 200 c.c. Erlenmeyer flask, add 20 c.c. of strong acetic acid ( 30 per cent) and 40 c.c. of water. Add about 7 grams of a saturated solution of $\mathrm{KI}$ and titrate with the thiosulphate using starch as an indicator. Calculate the equivalent of I c.c. of thiosulphate in $\mathrm{Cu}$. One c.c. of the copper sulphate solution contains $\mathrm{I} 7.647 \mathrm{mg}$. of $\mathrm{Cu}$. The thiosulphate remains constant for some months. It should be kept in a dark bottle.

\footnotetext{
1 Emulsification of fats, p. I 80 .

${ }^{2}$ Determination of $\mathrm{H}$ ion concentration, $\mathrm{pp}, \mathrm{I}_{5} 8$ and 480 .

${ }^{3}$ Nylander's test, pp. 29 and 420.

- Obermayer's test, p. 388 .

5 Experiments on blood plasma, p. 268.

'Herter's para-dimethylaminobenzaldehyde reaction, p. 2 I9.

7 Detection of hydrogen peroxide, p. 323 .

8 Determination of sugar, p. 525 .
} 
Phenolphthalein. ${ }^{1-D i s s o l v e ~ I ~ g r a m ~ o f ~ p h e n o l p h t h a l e i n ~ i n ~ r o o ~ c . c . ~}$ of 95 per cent alcohol.

Permanganate Solution (Alkaline) for Van Slyke Method. ${ }^{2}$ - The alkaline permanganate solution contains 50 grams of potassium permanganate and 25 grams of potassium hydroxide per liter.

Potassium Permanganate Standard (N/Io) Solution.-Dissolve $3 . I 62$ grams of pure potassium permanganate in a liter of distilled water, allow to stand a few clays, and filter through glass wool. Standardize against $\mathrm{N}$ / Io oxalic acid solution or against pure dry sodium or potassium oxalate. One c.c. of $\mathrm{N} /$ io permanganate is equivalent to $7.0 \mathrm{mg}$. of sodium oxalate.

Phenylhydrazine Mixture. ${ }^{3}$ - This mixture is prepared by combining I part of phenylhydrazine-hydrochloride and 2 parts of sodium acetate by weight. These are thoroughly mixed in a mortar.

Phenylhydrazine-Acetate Solution. ${ }^{4}$-This solution is prepared by mixing I volume of glacial acetic acid, I volume of water, and 2 volumes of phenylhydrazine (the base).

Picramic Acid.-Permanent Standard for Lewis-Benedict Blood Sugar Method. ${ }^{5}$-A solution of picramic acid makes a very satisfactory permanent standard. The color is identical in quality with that formed in the method and its solution keeps perfectly. The formula of the permanent standard is:

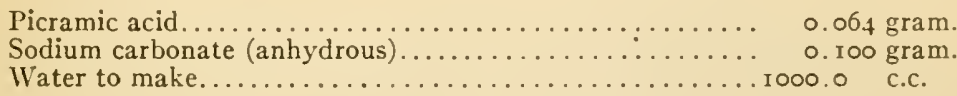

Dissolve the picramic acid with the aid of heat in $25-50$ c.c. of distilled water which has been made alkaline with sodium carbonate. Cool and dilute to I liter. This solution has the same intensity of color as that obtained by the proposed method with $0.64 \mathrm{mg}$. of sugar when the final volume of the reaction fluid is made ro c.c. The solution should be standardized against pure glucose. A satisfactory preparation of picramic acid may be obtained from the J. T. Baker Chemical Co., Phillipsburg, N. J.

Roberts' Reagent. ${ }^{6}$ - Mix I volume of concentrated nitric acid and 5 volumes of a saturated solution of magnesium sulphate.

Rosenheim's Iodo-Potassium Iodide Solution. ${ }^{7}$-Dissolve 2 grams of iodine and 6 grams of potassium iodide in roo c.c. of water.

\footnotetext{
1 Töpfer's method, p. I 74 .

${ }^{2}$ Determination of a mino-acid nitrogen p. SS.

3 Phenylhydrazine reaction, pp. 22 and 413.

- Phenylhydrazine reaction, pp. 22 and 413.

$\checkmark$ Determination of sugar in blood, p. 279.

- Roberts' ring test, pp. $\mathrm{IO}_{4}$ and 424.

' Rosenheim's periodide test, p. 273.
} 
Sahli's Reagent. ${ }^{-}$This reagent consists of a mixture of equal parts of a 48 per cent solution of potassium iodide and an 8 per cent solution of potassium iodate.

Salted Plasma. ${ }^{2}$-Allow arterial blood to run into an equal volume of a saturated solution of sodium sulphate or a ro per cent solution of sodium chloride. Keep the mixture in the cold room for about 24 hours.

Schweitzer's Reagent. ${ }^{3}$-Add potassium hydroxide to a solution of copper sulphate which contains some ammonium chloride. Filter off the precipitate of cupric hydroxide, wash it, and bring 3 grams of the moist cupric hydroxide into solution in a liter of 20 per cent ammonium hydroxide.

Scott-Wilson Acetone Reagents. ${ }^{4}$ - (a) Mercury Reagent.-This reagent is made up as follows: Mercuric cyanide Io grams, sodium hydroxide $I 80$ grams, water $\mathrm{I} 200$ c.c. The solution is agitated in a flask and 400 c.c. of a 0.7268 per cent solution of silver nitrate slowly run in. Let stand three days and decant supernatant liquid. The silver nitrate solution is made by taking I part of standard silver nitrate solution (I c.c. $=$ Io $\mathrm{mg} . \mathrm{NaCl}$ ) and 3 parts of water.

(b) Standard Potassium Thiocyanate Solution.-Make up an approximately o.I per cent solution of potassium thiocyanate and standardize it against mercuric nitrate or silver nitrate. It is convenient to have the solution of such strength that I c.c. $=$ I mg. of $\mathrm{Hg}$.

(c) Acid Mixture.-Nitric acid 40 parts, sulphuric acid 5 parts, and water 55 parts.

(d) Permanganate $\mathrm{N} / 5$ Solution.-Dissolve 6.324 grams of potassium permanganate in water and make up to a liter.

Seliwanoff's Reagent. ${ }^{5}-$ Dissolve 0.05 gram of resorcinol in Ioo c.c. of dilute $(\mathrm{I}: 2)$ hydrochloric acid.

Sherrington's Solution. ${ }^{6}$ - This solution possesses the following formula:

Methylene-blue.

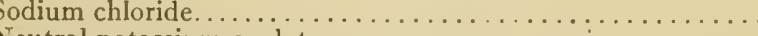

Neutral potassium oxalate.

O. I gram.

Distilled water

I. 2 grams.

I. 2 grams.

300.0 grams.

Silver Nitrate Solution. ${ }^{7}$-Dissolve 29.042 grams of silver nitrate in I liter of distilled water. Each cubic centimeter of this solution is

1 Determination of free acid, p. 164.

${ }^{2}$ Experiments on blood plasma, p. 268.

${ }^{3}$ Schweitzer's solubility test, p. 49.

'Determination of acetone and acetoacetic acid, pp. 284 and 536.

5 Seliwanoff's reaction, pp. 35 and 447.

6 "Blood counting," p. 305. p. 558 .

7 Volhard-Arnold method, p. 556, Mohr's method, p. 558, and Dehn-Clark method, 
equivalent to 0.01 gram of sodium chloride or to 0.006 gram of chlorine.

Sodium Acetate Solution. ${ }^{1-D}$-Dssolve Ioo grams of sodium acetate in 800 c.c. of distilled water, add Ioo c.c. of 30 per cent acetic acid to the solution, and make the volume of the mixture up to I liter with distilled water.

Sodium Alcoholate (N/Io) Solution. ${ }^{2}$ - The sodium alcoholate is made by dissolving 2.3 grams of cleaned metallic sodium in I liter of absolute alcohol. It is advisable that it be slightly weaker than stronger than tenth-normal. It may be standardized against pure benzoic acid in washed chloroform. It may also be standardized against $\mathrm{N} /$ Io $\mathrm{HCl}$ provided the alcoholate solution contains not more than traces of carbonate.

Sodium Alizarin Sulphonate. ${ }^{3}$ - Dissolve I gram of sodium alizarin sulphonate in Ioo c.c. of water.

Sodium Sulphide Solution. ${ }^{4}$-Saturate a I per cent solution of sodium hydroxide with hydrogen sulphide gas and add an equal volume of I per cent sodium hydroxide.

Sodium Thiosulphate Standard (N/Io) Solution. ${ }^{5}$ - Weigh out 25 grams of ordinary c.p. sodium thiosulphate or 24.83 grams of the pure dry recrystallized salt. Dissolve in water and dilute to a liter. Boiled distilled water must be used. Keep in a bottle with a siphon arrangement and carrying a soda lime tube to exclude $\mathrm{CO}_{2}$.

It is best standardized against acid potassium iodate $\mathrm{KH}\left(\mathrm{IO}_{3}\right)_{2}$. Weight out accurately 0.3249 gram of acid potassium iodate. Dissolve in 50 c.c. of water, heating gently if necessary. Transfer the solution to a Ioo c.c. flask, rinsing the beaker carefully and make to mark with water. This solution is exactly decinormal. Pipette out 25 c.c. into an Erlenmeyer flask, add I gram of potassium iodide dissolved in a little water, and a few cubic centimeters of dilute hydrochloric acid. Titrate immediately with the thiosulphate solution. When the solution becomes pale yellow add a few cubic centimeter of I per cent solution of soluble starch and titrate to loss of blue color.

Solera's Test Paper. ${ }^{6}$ - Saturate a good quality of filter paper with 0.5 per cent starch paste to which has been added sufficient iodic acid to make a I per cent solution of iodic acid and allow the paper to dry in the air. Cut it in strips of suitable size and preserve for use.

\footnotetext{
1 Uranium acetate method, p. 552.

2 Determination of hippuric acid, p. 519.

${ }^{3}$ Töpfer's method, p. I 7 .

- Kriger and Schmidt's method, p. 5 I 3.

5 Determination of acetone and acetoacetic acid, p. 533.

'Solera's reaction, p. 59.
} 
Spiegler's Reagent. ${ }^{1}$ - This reagent has the following composition:

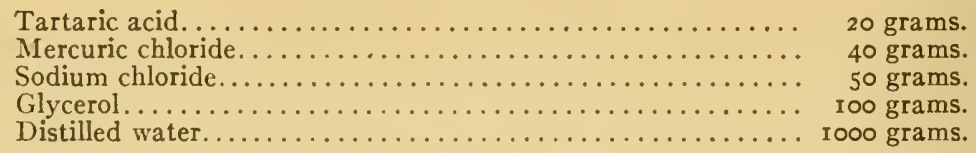

Starch Iodide Solution. ${ }^{2}$ - Mix o.I gram of starch powder with cold water in a mortar and pour the suspended starch granules into 75roo c.c. of boiling water, stirring continuously. Cool the starch paste, add 20-25 grams of potassium iodide and dilute the mixture to 50 c.c. This solution deteriorates upon standing, and therefore must be freshly prepared as needed.

Starch Paste.-Grind 2 grams of starch powder in a mortar with a small amount of water. Bring 200 c.c. of water to the boiling-point and add the starch mixture from the mortar with continuous stirring. Bring again to the boiling-point and allow it to cool. This makes an approximate I per cent starch paste which is a very satisfactory strength for general use.

Stokes' Reagent. ${ }^{3}$ - A solution containing 2 per cent ferrous sulphate and 3 per cent tartaric acid. When needed for use a small amount should be placed in a test-tube and ammonium hydroxide added until the precipitate which forms on the first addition of the hydroxide has entirely dissolved. This produces ammonium ferrotartrate, which is a reducing agent.

Suspension of Manganese Dioxide. ${ }^{4}$-Made by heating a 0.5 per cent solution of potassium permanganate with a little alcohol until it is decolorized.

Tanret's Reagent. ${ }^{5}$-Dissolve 1.35 grams of mercuric chloride in 25 c.c. of water, add to this solution 3.32 grams of potassium iodide dissolved in 25 c.c. of water, then make the total solution up to 60 c.c. with distilled water and add 20 c.c. of glacial acetic acid to the mixture.

Tincture of Iodine. ${ }^{6}$-Dissolve 70 grams of iodine and 50 grams of postassium iodide in I liter of 95 per cent alcohol.

Toison's Solution. ${ }^{7}$-This solution has the following formula:

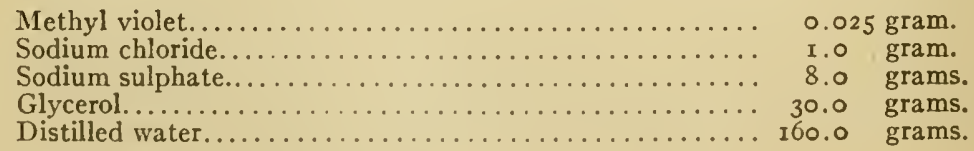

1 Spiegler's ring test, pp. I04 and 424 .

${ }^{2}$ Fehling's method, p. 523.

${ }^{3}$ Hemoglobin, p. 296. Hemochromogen, p. 299.

- Krüger and Schmidt's method, p. 5 I 3.

- Tanret's test, pp. I04 and 425 .

- Smith's test, pp. 268 and 433 .

7 "Blood counting," p. 305. 
Töpfer's Reagent. ${ }^{1}$-Dissolve 0.5 gram of di-methylaminoazobenzene in roo c.c. of 95 per cent alcohol.

Tropæolin OO. ${ }^{2}$-Dissolve 0.05 gram of tropæolin OO in rooc.c. of 50 per cent alcohol.

Uffelmann's Reagent. ${ }^{3}$-Add a 5 per cent solution of ferric chloride to a I per cent solution of carbolic acid until an amethyst-blue color is obtained.

Uranium Acetate Solution. ${ }^{4}$-Dissolve about 35.0 grams of uranium acetate in I liter of water with the aid of heat and 3-4 c.c. of glacial acetic acid. Let stand a few days and filter. Standardize against a phosphate solution containing 0.005 gram of $\mathrm{P}_{2} \mathrm{O}_{5}$ per cubic centimeter. For this purpose dissolve 14.721 grams of pure air-dry sodium ammonium phosphate $\left(\mathrm{NaNH}_{4} \mathrm{HPO}_{4}+{ }_{4} \mathrm{H}_{2} \mathrm{O}\right)$ in water to make a liter. To 20 c.c. of this phosphate solution in a 200 c.c. beaker add 30 c.c. of water and 5 c.c. of sodium acetate solution (see above) and titrate with the uranium solution to the correct end reaction as indicated in the method proper, page $55^{2}$. If exactly 20 c.c. of uranium solution are required I c.c. of the solution is equivalent to 0.005 gram $\mathrm{P}_{2} \mathrm{O}_{5}$. If stronger than this dilute accordingly and check again by titration.

Urease. ${ }^{5}$ - (a) Soy Bean Meal.-Grind the soy bean to a powder which will pass through a 20 -mesh sieve.

(b) Solid Urease Preparation.-Digest I part of soy bean meal with 5 parts of water at room temperature, with occasional stirring for an hour, and clear the solution by filtration through paper pulp or centrifugation. Pour this extract slowly, with stirring, into at least rovolumes of acetone. The acetone dehydrates the enzyme preparation. Filter, dry in vacuum and powder. For standardization procedure see the determination of urea in urine.

(c) Enzyme Solution.-Dissolve 2 grams of urease, prepared as above, together with 0.6 gram of di-potassium-hydrogen phosphate and 0.4 gram of mono-potassium-dihydrogen phosphate in ro c.c. of water. The solution may be kept under toluol for two weeks, without losing activity.

\section{Uric Acid Reagents. ${ }^{6-(a)}$ Silver Magnesium Solution.-}

3 per cent silver lactate solution................... 70 c.c.

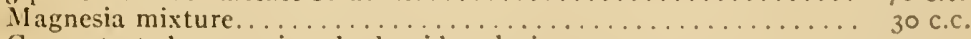

Concentrated ammonium hydroxide solution.................. soo c.c.

1 Töpfer's method, p. 174 .

${ }^{2}$ 'Test for free acid, p. I 54 .

${ }^{8}$ Uffelmann's reaction, p. 170.

4 Phosphate determination, p. 552 .

5 Determination of urea, P. $49 \mathrm{r}$.

${ }^{6}$ Determination of uric acid, p. 274 and p. 510. 
(b) Uric Acid Reagent for Colorimetric Method.-Place roo grams of sodium tungstate, 80 c.c. of 85 per cent phosphoric acid, and 750 c.c. of distilled water in a liter flask. Boil the mixture with a reflux condenser for two hours, cool and dilute to I liter, filtering if necessary.

(c) Sodium Carbonate Solution.-Dissolve 200 grams anhydrous sodium carbonate in warm water and make up to I liter.

(d) Uric Acid Formaldehyde Standard.-Place I gram of uric acid in a liter volumetric flask and dissolve with 200 c.c. of a 0.4 per cent lithium carbonate solution. Add to the solution 40 c.c. of 40 per cent formaldehyde solution, shake the mixture and allow to stand for a few minutes. Acidify the clear solution with 20 c.c. of normal acetic acid and dilute to mark with distilled water The solution should remain perfectly clear and the next day (not before) may be standardized against a freshly prepared solution of uric acid in lithium carbonate. The color produced by 5 c.c. of this solution corresponds very closely to that produced from I $\mathrm{mg}$. of pure uric acid. The colorimeter reading obtained from the solution when thus compared with pure uric acid ( I mg.) is, of course, thereafter used as the standard value corresponding to I $\mathrm{mg}$. of uric acid.

(e) Uric Acid Standard in Phosphate Solution.-Dissolve 9 grams of pure crystallized disodium hydrogen phosphate, together with I gram of crystallized sodium dihydrogen phosphate, in 200 to 300 c.c. of hot water, and filter if the solution is not perfectly clear. Make this filtrate up to about 500 c.c. with hot water, and pour this hot or warm (and perfectly clear) solution upon exactly $200 \mathrm{mg}$. of pure uric acid suspended in a few cubic centimeters of water in a liter volumetric flask. Agitate the mixture for a few minutes until the uric acid completely dissolves. Cool, add exactly r.4 c.c. of glacial acetic acid, dilute to the mark, and mix. Add about 5 c.c. of chloroform to prevent the growth of bacteria or moulds in the solution. Five c.c. of this solution contain exactly I $\mathrm{mg}$. of uric acid.

The solution of uric acid in the phosphate solution is very readily prepared, does not need to be standardized, and appears to keep indefinitely. 
INTERNATIONAL ITONIC WEIGHTS, I9I6

\begin{tabular}{|c|c|c|c|c|}
\hline & \multicolumn{2}{|c|}{$0=16}$. & \multicolumn{2}{|r|}{$0=16}$. \\
\hline Aluminium & $\mathrm{Al}$ & 27.1 & Manganese........... & 54.93 \\
\hline Antimony. & $\mathrm{Sb}$ & 120.2 & Mercury............... & 200.6 \\
\hline Arsenic... & As & 74.96 & Molybdenum......... & 96.0 \\
\hline Barium... & $\mathrm{Ba}$ & 137.37 & Nickel.... & $5^{8} .68$ \\
\hline Bismuth... & $\mathrm{Bi}$ & 208.0 & Nitrogen.............. N & 14.01 \\
\hline Boron....... & $\mathrm{B}$ & I1.0 & Osmium ............. & 190.9 \\
\hline Bromine.... & $\mathrm{Br}$ & 79.92 & $\ldots \ldots \ldots \ldots$ & 10.00 \\
\hline Cadmium. & $\mathrm{Cd}$ & I 12.40 & Palladium............ & 106.7 \\
\hline Calcium.. & $\mathrm{Ca}$ & 40.07 & Phosphorus........... P & 31.04 \\
\hline Carbon... & $\mathrm{C}$ & 12.005 & Platinum...... & 195.2 \\
\hline Chlorine... & $\mathrm{Cl}$ & $35 \cdot 4^{6}$ & Potassium........... $\mathrm{K}$ & 39.10 \\
\hline Chromium. . & $\mathrm{Cr}$ & 52.0 & Radium........... R & 226.0 \\
\hline Cobalt.... & Co & 58.97 & Selenium............ & 79.2 \\
\hline Copper.. & $\mathrm{Cu}$ & $63 \cdot 57$ & Silicon.... & $28 \cdot 3$ \\
\hline Fluorine. . & $\mathrm{F}$ & 19.0 & Silver..... & 107.88 \\
\hline Glucinum. . & Gl & $9 \cdot 1$ & Sodium............ $N$ & 23.00 \\
\hline Gold ........ & $\mathrm{Au}$ & 197.2 & Strontium........... Sr & $8_{7} \cdot 6_{3}$ \\
\hline Hydrogen... & $\mathrm{H}$ & I. .008 & Sulphur. . . . . . . . & 32.06 \\
\hline Iodine... & I & I 26.92 & Tantalum............ T & I 81.5 \\
\hline Iridium. . & Ir & 193.1 & Tellurium........... & $127 \cdot 5$ \\
\hline Iron..... & $\mathrm{Fe}$ & 55.84 & Tin........ & 118.7 \\
\hline Lanthanum. & $\mathrm{La}$ & 39.0 & Titanium.... & 48.1 \\
\hline Lead........ & $\mathrm{Pb}$ & 20720 & Tungsten............ II & 184.0 \\
\hline Lithium... & $\mathrm{Li}$ & 6.94 & Uranium............ U & 238.2 \\
\hline Magnesium & $\mathrm{Mg}$ & $24 \cdot 32$ & Zinc............... Z & $65 \cdot 37$ \\
\hline
\end{tabular}





\section{INDEX}

\section{Main references are in heavy-faced type.}

Abderhalden test for pregnancy, principle of, 3

Absorption of carbohydrate as influenced by fat ingestion, 568

Acacia solution, formation of emulsion by, 178

Acetoacetic acid, 270,284, 412,438, 539

Arnold-Lipliawsky test for, 440

formula for, 438

Gerhardt's test for, 439

Hurtley's reaction for, 440

Le Nobel reaction for, 439

quantitative determination of, $284,294.539$

Acetone, 270, 284, 294, 412, 435, 538

bodies, $270,284,435,533$

determination of in blood, 284

of in urine, 533

formula for, 435

Frommer's test for, 438

Gunning's jodoform test for, 436

Legal's iodoform test for, 437

Lieben's test for, 437

quantitative determination of, $284,294,538$

Reynolds-Gunning test for, 438

Rothera's reaction for, 438

Acholic stool, 223

Achroo-dextrins, 43, 56

$\alpha$-achroo-dextrin, 56

$\boldsymbol{\beta}$-achroo-dextrin, 56

$\gamma$-achroo-dextrin, 56

Acid, acetic, 335, 370, 397

acetoacetic, $270,284,\{12,438,539$

alloxyprotcic, 369,392

amino-acetic, 69,71

amino-butyric, 214

amino-ethyl-sulphonic, 204, $3 \nmid 6$

$\alpha$-amino- $\beta$-hydroxy-propionic, 69,73

$\alpha$-amino- $\beta$-imidazol-propionic, 68,77

$\alpha$-amino-iso-butyl-acetic, 69,79

$\alpha$-amino- $\beta$-methyl- $\beta$-ethyl-propionic, 68,80

$\alpha$-amino-normal glutaric, 69,82

$\alpha$-amino-propionic, 68, 72

amino-succinic, 69,82

amino-valerianic, $2 \times 4$

$\alpha$-amino-iso-valerianic (see Valine), 60,78

$\alpha$-diamino- $\beta$-dithiolactyl, 69,75

aspartic, 69,82

benzoic, $72,369,390,395,583,586$

butyric, $8,319,323,370,397$

caproic, 3 I 3,3 ro

carbamic, 247

cholic, 204

chondroitin-sulphuric, 335, 369, 392

citric, 313

combined hydrochloric (protein salt), 56, 140 , 175

cyanuric, 373

$\alpha-\epsilon$-diamino-caproic, 68,80
Acid, diaminotrihydroxydodecanoic, 68,85

diazo-benzene-sulphonic, 454

ethereal sulphuric, 2 I2, 369, 385

fatty, $176,178,182,212,370,397$

formic, $26,370,397$

free hydrochloric, 56, 140, $355,164,175$

glucothionic, 467

glutamic, 69,82

glycerophosphoric, 353,354,370, 399

glycocholic, 204

glycosuric, 395

glycuronic, $36,38,442$

glyoxylic, 98

guanidine- $\alpha$-amino-valerianic, 68,79

hippuric, $72,369,388,390,396,585$

homogentisic, $26,369,394,417$

hydroxymandelic, 369. 395

iminazolpropionic, 214

indolacetic, 214,452

indole- $\alpha$-amino-propionic, 68,77

indoxyl-sulphuric, $212,369,385$

inosinic, $34 \mathrm{r}, 346$

kynurenic, 369, 394

lactic. $40,140,159,170,314,340,342$

lauric, 313

mucic, $36,40,445,446$

myristic, 313

nucleic. 94, 1 23, I 24, 130-133

osmic, $35^{6}$

oxalic, $369,391,545$

oxaluric, 369,397

oxy- $\alpha$-pyrrolidine-carboxy-lic, 68,85

oxyproteic, 369.392

palmitic, $176,177,38 \mathrm{r}, 182$

para-cresolesulphuric, 369,385

para-oxyphenyl-acetic, 212,2 I $4,360,394$

para-oxy- $\beta$-pheny- $\alpha$-amino-propionic, 69,74

86

para-oxypheny-propionic, $212,214,369,394$

paralactic, 247, 314, 342, 370,398

phenaccturic, 370,398

phenol-sulphuric, 369, 385

phenylacetic, 214

phenyl- $\alpha$-amino propionic. 69,73

phenylpropionic, $2 \mathrm{I} 4$

phosphocarnic, 34 I, $346,370,399$

phosphoric, 406

pyrocatechin-sulphuric, 369,385

$\alpha$-pyrrolidine-carboxylic, 68,85

sarcolactic, 342

skatole acetic, 77

skatole carbonic, 217,220

skatoxyl-sulphuric, 369,385

stearic, 177, 354

succinic, 214

sulphanilic, 455 
Acid, tannic, 45, 48, 103

taurocholic, 204

trichlorethylglucuronic, 417

uric, $26,127,247,274,341,369,37 \mathrm{I}, 377,458$, $460,475,510$

urocanic, 370,398

uroferric, 369,392

uroleucic, 369

volatile fatty, 212, 215, 370, 397

Acid albuminate. See Acid metaprotein.

Acid-forming foods, influence of on hydrogen ion concentration of urine, 580

Acid infraprotein. See Acid metaprotein.

Acid metaprotein, 95, 115,116

coagulation of, I I 6

experiments $r$, II 6

precipitati. , $\therefore, 1 \mathrm{I} 6$

preparation of, 116

solubility of, I I 6

sulphur content of, I I 6

Acidity of gastric juice, quantitative determination of, $148,159,162$

urine, cause of, $36 \mathrm{r}, 406$

quantitative determination of, by hydrogen ion concentration, 840 by titration, 479

Acidosis, 441, 576

metabolism in, 576

Acid-hematin, 254, 269, 299

Acree-Rosenheim formaldehyde reaction, Ioo

Acrolein, formation of, from olive oil, 180 from glycerol, 183

Activation, 6, 187

Activation by calcium salts, I 87

Adam's paper coil method for determination of fat in milk, 326

Adaptation, 57

Adenase, 4

Adenine, 4, I 25, I 26, I 27, $132,347,370,401$

Adipocere, 179

Adrenaline, determination of in adrenals, 290

Agar-agar. 20, 50, 225, 580

Agglutination, 250, 262

Alanine, $68,72,2 \mathrm{I} 4$

Albumin, egg, 93, 107

crystallized, preparation of, 106 powdered, preparation of, 107 tests on, 107

serum, 93, 245, 268, 4I 2, 422 。

solution, preparation of, 97

Albumin in urine, 412,422

acetic acid and potassium ferrocyanide test for, 425

coagulation or boiling test for, 424

determination of, 531

Heller's ring test for, 423

Roberts' ring test for, 42.4

sodium chloride and acetic acid test for, 425

Spiegler's ring test for, $42 \mathrm{~J}$

Tanret's test for, 425

tests for, 423

Albumins, 93, 95, 96

Albuminates. See Metaproteins.

Albuminates, formation of, by netallic salts, 102

Albuminoids, 93, I I 2, 330

Albumoscope, $\mathrm{IO}_{3}, \mathbf{4 2 3}$

Albumoses (see Proteoses, p. I I 8 )

Alcohol-soluble proteins (see Prolamins, p. II I)
Alcoholic-zinc chloride test for urobilin, 40 I

Aldehyde, 25, 42

Aldehyde group, 39

Aldehyde test for alcohol, 42

v. Aldor's method of detecting proteose in urine, 427

Aldose, 19

Aliphatic nucleus, 65, 68

Alizarin yellow $R$, use of, as indicator, 157 , 158

Alkali albuminate. See Alkali metaprotein.

Alkali-hematin, 254, 298

Alkali metaprotein, 95, I15, I 16

experiments on, I 6

precipitation of, II 6

preparation of, II 6

sulphur content of, I I 6

Alkaline tide, 362, 577

demonstration of, 577

Allantoin, 369, 392

crystalline form of, 392

experiments on, 393

formula for, 392

preparation of, from uric acid, 393

quantitative determination of, by Wiechowski-Handovsky method, 5 I 8 by difference, 5 I 9

separation of, from urine, 393

Allen's modification of Fehling's test, 419

Almén's reagent, preparation of, 429

Alloxy proteic acid, 369, 392

Aloin-turpentine test for "occult blood," 235

Aluminium hydroxide, use of, in removal of protein, 276, 329

Amalgamation test for mercury, 450

Amandin, 93

Amide nitrogen, 64

A mino acids, $65,67,68,186,195,248,270,369$, 394

group, 65, 88, 99

preparation of, in crystalline form, 85,87

$\alpha$-amino- $\beta$-hydroxy-propionic acid, 69,73

$\alpha$-amino- $\beta$-imidazol-propionic acid, 68,77

$\alpha$-amino-iso-butyl-acetic acid, 69,78

$\alpha$-amino-normal-glutaric acid, 69, 82

Amino-butyric acid, 2 I 4

Amino-nitrogen, quantitative determination of,

$88,91,277,502$

Amino-succinic acid, 69,82

A mino-valerianic acid, 214

$\alpha$-amino-iso-valerianic acid, 69,78

Ammonia, 64, 67, 71, 107

in blood, 247,270

quantitative determination of, 278

in urine, $370,402,501,575$

quantitative determination of, 490

Ammoniacal cupric hydroxide, solubility test, 49

Ammoniacal silver solution, preparation of, 59.4

Ammoniacal-zinc chloride test for urobilin, 400

Ammonium benzoate, synthesis of, to form

hippuric acid, 585

Ammonium magnesium phosphate ("Triple phosphate"), 363, 408

in urinary sediments, 458

Ammonium purpurate, 38 I

Ammonium urate, $363,377,461,511$ crystalline form of, Plate VI, opposite p. 462

Amphopeptone, 95, II9 
Amygdalin, 4

Amylase, pancreatic, 4, II, 187, 192

digestion of dry starch by, 188, 192 inulin by, 192

experiments on, II, I9I

influence of bile upon action of, 192

most favorable temperature for action of, 192

salivary, $4,10,55,140$

activity of, in stomach, 57,140

experiments on, 10,59

inhibition of activity of, 57, 6 I

nature of action of, 56

products of action of, 56

vegetable, 4 , Io

Amylases, 4, 10, 55, 187

experiments on, I0, 59, 191, 239

Amyloid, 49, 113

Amylolytic enzymes, Sce Amylases.

quantitative determination of activity of, 192,239

Anabolism, 564

Animal parasites in feces, 227

in urinary sediments, $465,47$.

Anti-enzymes, 9

experiments on, 17

Antimony pentachloride as cellulose solvent, 5o

Antimony trichloride as cellulose solvent, 49

Antipepsin, 9, I 7

Antipeptone, 95, II9

Antirennin, 9

Antithrombin, 257

Antitrypsin, 9, 18

A porrhegmas, 214

Arabinose, 19, 37, 4.13

Bial's reaction for, 37,443

orcinol test on, 38,444

phenylhydrazine test on, 38

Tollens' reaction on, 38,443

Arginase, 4

Arginine, 4, 67, 68, 79, 186

Arnold-Lipliawsky reaction for acetoacetic acid, 440

reagent, preparation of, 440

Aromatic oxyacids, 369,394

Arsenic in urine, detection of, 448

determination of, 448

Ascaris, 17, 18

Ash of milk, quantitative determination of, 327

Asparagine, 82

formula for, 82

Aspartic acid, $65,67,69,82$ crystalline form of, $8 \mathrm{i}$ formula for, 82

Assimilation limit, 2 I

Assimilation limit of dextrose, 25, 413.568

Atkinson and Kendall's hemin test, 266

Autolytic enzymes, 3

Azolitmin, use of, as indicator, is 8

Babcock fat method, 324

tubc, 324

Bacteria in feces, 221, 224, 225, 588 quantitative determination oi, 241

Bacterial nitrogen in feces, 226, 588 determination of, 241

Balance, metabolic preparation of, 592 calciun, 502
Balance, magnesium, 592

nitrogen, 592

phosphorus, 592

sulphur, 592

Bang reduction fiask, 28 I

Bang's method for estimation of sugar in blood. 280

Bang's method for estimation of sugar in urine, 324

Bardach's reaction, I OI

Barfoed's reagent, preparation of, 30, 200, 595

Barfoed's test for monosaccharides, 30

Baryta mixture, preparation of, 375,595

Base-forming foods, influence of on hydrogen ion

concentration of urine, 362,580

Basic lead acctate solution, 542, 596

Bayberry tallow, saponification of, 18 I source of, 18

Bayberry wax. See Bayberry tallow, I $8 \mathrm{I}$

Bead test (Einhorn), 238

Beckmann-Heidenhain apparatus, 365

"Bence-Jones' protein," detection of, 428

Benedict creatine preparation, 384

Benedict and Bock's method for quantitative determination of total nitrogen, 488

Benedict and Lewis method for sugar in blood, 279

Benedict-Folin creatinine preparation, 383

Benedict-Folin method for creatine in urine, 508

Benedict-Hitchcock method for uric acid in urine, 510

Benedict-Murlin method for amino-acid nitrogen in urine, $\mathbf{5 0 4}$

Benedict-Pearce method for sugar in blood, 280

Benedict's method for quantitative determination of sugar, 522

Benedict's method for quantitative determination of sulphur, 548

Benedict's method for quantitative determination of urea, 494

Benedict's method for uric acid in blood, 275

Benedict's modifications of Fehling's test, 27,417 solution, for use in quantitative determination of sugar, preparation of, 522. 596 solutions, preparation of, $27,417,596$ sulphur reagent, preparation of, 597

Benzidine peroxidase reaction (Wilkinson and Peters), 321

Benzidine reaction for blood, I71. 233, 263, 43 I

Benzoic acid, 72, 369, 390, 395, 583, 585

crystalline form of. 396

experiments upon, 396

formula for, 395

solubility of, 390

sublimation of, 396

Bergeim's modification of the Herter-Foster method for indole in feces, 242

Bergeim's phosphonuclease theory for the orizin of hydrochloric acid of gastric juice, $\mathrm{I}+\mathrm{t}$

Berthelot-Atwater bomb calorimeter. \$SO

Bertrand's method for sugar determination, $5 \geq 7$

Bial's reaction for pentoses, 37.443

Bial's reagent, preparation of, $37,4+3$

Bile, $171,202,412,432$

analysis of, 203.

constituents of, 203

daily secretion of, 20,3

freezing-puint of, 203

influence on digestion. gastric, 147

pancreatic, 178 , 100 
Bile, inorganic constituents of, 203, 209 nucleoprotein of, 203, 207 reaction of, 202207

secretion of, 202

specific gravity of, 203.

Bile acids, 204

Hay's test for, 209

Mylius's test for, 208

Neukomm's test for, 209

Oliver's test for, 209

Pettenkofer's test for, 208 tests for, 208

v. Udránsky's test for, 208

Bile acids in feces, detection of, 236

Bile acids in urine, 412,434

Hay's test for, 434

Mylius's test for, 434

Neukomm's test for, 435

Oliver's test for, 435

Pettenkofer's test for, 434

tests for, 434

v. Udránsky's test for, 434

Bile pigments, 203, 204, 207

Gmelin's test for, 207

Hammarsten's reaction for, 207

Huppert's reaction for, 207

Nakayama's reaction for, 207

Rosenbach's test for, 207

Smith's test for, 208

tests for, 207

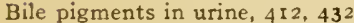

Gmelin's test for, 433

Hammarsten's reaction for, 433

Huppert's reaction for, 433

Nakayama's reaction for, 433

Rosenbach's test for, 433

Salkowski-Schipper's reaction for, 433

Salkowski's test for, 433

Smith's test for, 433

tests for, 432

Bile salts, 204, 434

crystallization of, 204, 209

Biliary calculi, 206, 209

analysis of, 209

Bilicyanin, 204

Bilifuscin, 204

Bilihumin, 204

Biliprasin, 204

Bilirubin, 204

crystalline form of, 205

in urinary sediments, 458,464

Biliverdin, 204, 206

"Biological" blood test, 258

Bismuth, influence on color of feces, 222,238

Bismuth reduction tests, 29,420

Bismuth test for choline, 358

Biuret, 99. 373, 375

formation of, from urca, 99,373

Biuret paper of Kantor and Gies, 100

Biuret potassium cupric hydroxide. Sce Cupripotassium biuret, 99

test, 98

Posner's modification of, 100

Biuret reagent (Gics), preparation of, 300

Black's method for determination of $\beta$-hydroxy-

butyric acid, 54 I

reaction for $\beta$-hydroxybutyric acid, 44 I
Black's reagent, preparation of, 44 I

Blood, $171,225,233,245,270,412,429$ acetoacetic acid in, 270284 acetone in, $247,270,284$ agglutination of, 250,262 amino-acid nitrogen in, 270 amino-acids in, 247,248 ammonia in, 270,278

Bordet test for, 258

Blood, cholesterol in, 247, 270, 285 coagulation of, 257

Howell's theory of, 257

composition of normal and pathological, 270 constituents of, 247,248

creatine in, 248, 270, 276

creatinine in, $248,270,276$

crystallization of oxyhemoglobin of, 250,266

defibrinated, 257,260

detection of, $171,233,263,429$

determination of acetone in, 284, 294

acetoacetic acid in, 284,294

amino acid nitrogen in, 277

ammonia in, 278

chlorides in, 286

cholesterol in, 285

creatine in, 276

creatinine in, 276

fat in, 295

$\beta$-hydroxybutyric acid in, 284, 294

hydrogen ion concentration in, 288

non-protein nitrogen in, 271

sugar in, 279

total nitrogen in, 279

total solids in, 288

urea in, 274

uric acid in, 274

erythrocytes of, $249,261,472$

experiments on, 260,270

fat in, 247, 295

form elements of, 249

guaiac test for, $235,258,262,430$

hemin test for, 264,420

B-hydroxybutyric acid in, 270, 284

in arthritis, 270,271

in cholelithiasis, $247,270,271$

in diabetes, 270, 271

in gout, 270,271

in lipemia, 270, 271

in nephritis, 270,271

in uremia, 270,271

in urine, 412,429

leucocytes of, 255

medico-legal tests for, 257,203

menstrual, 257

microscopical examination of, $249,260,261$ 269

non-protein nitrogen of, 270

determination of, 27

nucleoprotein of, 246,24 ;

"occult," in feces, 225, 233

ortho-tolidin test for, $171,233,263,430$

oxyhemoglobin of, 250,296

pigment of, 250

plaques, 256

plasma, 245, 268

platelets, 256

plates, 256

preparation of hematin from, 266 
Blood preparation of "laky," 245, 268

reaction of, 245,260

serum, 247, 267

specific gravity of, 245,260

spectroscopic examination of, 296

sugar in, 247, 270, $27 \mathrm{I}$

test for iron in, 261

total amount of, 245

v. Zeynek and Nencki's hemin test for, 266

Blood analysis, 270

Blood casts in urine, $465,+69$

Blood corpuscles, 249,255,261, 472

"counting," 304, 308

Blood dust, 245, 256

Blood in urine, 412,429

benzidine reaction for, $43 \mathrm{r}$

guaiac test for, 430

Teichmann's hemin test for, 429

Heller's test for, 729

Heller-Teichmann reaction for, 430

ortho-tolidin test for, 430

Schumm's modification of guaiac test for, 430

spectroscopic examination of, 431

tests for, 429

v. Zeynek and Nencki's hemin test for, 430

Blood plasma, 245, 268

constituents of, 245

effect of calcium on oxalated, 268

experiments on, 268

preparation of fibrinogen from, 268

oxalated, 268

salted, 268

Blood serum, 247, 267

coagulation temperature of, 267

constituents of, 247,267

experiments on, 267

precipitation of proteins of, 267

separation of albumin and globulin of, 268

sodium chloride in, 268 sugar in, 268

Blood stains, examination of, 269

Bloor's nephelometer, cut of, 29 I

Boas' reagent, as indicator, 155 preparation of, 155

Bock and Benedict's apparatus, cut of, 489

Bock and Benedict's microchemical method for total nitrogen in urine, 488

Boettger's test for sugar, 29,420

"Bolting" of food, influence of, on food residues

in feces, 590

Bomb calorimcter, Berthelot-Atwater, 586

Bonanno's reaction, 208, 434

Bonanno's reagent, preparation of, 208, 434

Bone, constituents of, $336-338$

ossein of, preparation of, 336

quantitative composition of, 236,237

Bone ash, scheme for analysis of, 338

Borchardt's reaction for fructose, 35, 447

Bordet test, detection of human blood by, 258

Boric acid and borates in milk, detection of, 324

Bottu's reagent, preparation of, 23, 415

Bottu's test, 23, 415

Bromelin, 5

Bromine test for melanin, 452 for tryptophane, 180

Buccal glands, 54
Buffy coat, formation of, 247

Bunge's mass action theory, 140

Bürker's hemocytometer, 308

Butter, composition of, 319

Butyric acid, 8, 319,321, 370, 397

Butyrin, 177,313

Bynin, 93, II I

\section{Cadaverin, 81,212}

Calcium in urine, 370,409

quantitative determination of, 559

balance, preparation of, 592

carbonate in urinary sediments, 458,459

caseinate, 318

in bone, detection of, $337^{-8}$

in feces, estimation of, 592

oxalate, 391,458

in urinary sediments, 458

paracasein, I 43,3 I 5

phosphate in urinary sediments, 458,460

in bone, detection of, $337-8$

in milk, 313.322

sulphate in urinary sediments, 458,460

Calculi, biliary, 206, 209

urinary, 475

calcium carbonate in, 476

oxalate in, 476

cholesterol in, 478

cystine in, 476

fibrin in, 478

indigo in, 478

phosphates in, 476

uric acid and urates in, 476

urostealiths in, 479

xanthine in, 476

Calliphora, larvæ of, formation of fat from protein by, 179

Cambogia, influence of, on color of feces, 238

Calomel, influence on color of stool, 222, 238

Camphor as urine preservative, 368

Cane sugar (sce Sucrose, p. 41)

Canton silk, 60

Caproic acid, 313, 319

Carbamic acid, 247

Carbocyclic nucleus, $65,68-9$

Carbohydrosis, 4

Carbohydrates, I 9

absorption of, as influenced by fat ingestion. 568

classification of, in

composition of, 19

control of putrefaction by, 213

in fcces, estimation of, 591

protein-sparing, action of, 579

review of, 5 I

scheme for detection of, 53

variation in solubility of, 20

Carbonates in urine, 370,410

Carbon moicty of protein molecule, 1;0

Carbon monoxide, hemoglobin, 254, 255, 297 potassium iodide, test for, 298 tannin test for, 298

Carboxylase, 4, 10, 31, 42 I

Carmine, use in feces separation, 239, 587

Carmine-fibrin, preparation of, 12

Carnine, $3.4 t$

Carnitine, 341

formula for, 346 
Carnomuscarine, $34 \mathrm{I}$

Carnosine, 34I, 346

Carotin, 3 I9

Cartilage, 335

constituents of, 335

experiments on, 335

Hopkins-Cole reaction on, 335

Millon's reaction on, 335

preparation of gelatin from, 335

solubility of, 335

unoxidized sulphur in, 335

xanthoproteic test on, 335

Casein, 67, I43, 313, 3 I5, 318

decomposition of, 67

quantitative determination of, $327-329$

soluble, I 28,236

action of rennin upon, 143,3 I3

biuret test on, 322

Millon's test on, 322

precipitation of, $32 \mathrm{I}$

preparation of, 32 I

quantitative determination of, Hart's method

for, 327

solubility of, 322

test for phosphorus in, 322

test for unoxidized sulphur in, 322

Caseinate, calcium, 318

Caseinogen. See Casein.

Casts, 465,467

blood, 465,469

epithelial, 465,469

fatty, 465,469

granular, 465,468

hyaline, 465,468

pus, 465,472

waxy, 465,470

Casts in urinary sediments, 465,467

Catabolism, 564

Cat gut, 146

Catalase, 5, 15

animal, 16

experiments on, 16

quantitative determination of, I6 vegetable, I6

Catalysis, 2

Cell, 565

Cellulose, 20, 48

action of Schweitzer's reagent on, 49

hydrolysis of, 49

iodine test on, 49

solubility of, 49

solvents, 49

utilization by animals, 48

Cellulose group, 20

Cerebrin (cerebroside, 353, 355, 357

experiments on, 357

hydrolysis of, 357

microscopical examination of, 357

preparation of, 357

solubility of, 357

Cercbro-spinal fluid, choline in, 354

Cercbrosides, 353,355

Charcot-Leyden crystals, 225 form of, 225

Chlorides in blood, 270,286

in urine, $370,405,556$

detection of, 406

quantitative determination of, 556
Cholecyanin, 206

Choleprasin, 204

Cholera-red reaction for indole, 218

Cholesterol, 203, 2 I0, 247, 270, 27 r, 285, 313, 353 , 355

crystalline form of, 210

formula for, 355

in blood, 247, 270, 27x, 285

determination of, 285

iodine-sulphuric acid test for, 210,357

isolation of, from biliary calculi, 209

Liebermann-Burchard test for, 210,357

occurrence of, in urinary sediments, 458,463

origin of, 356

preparation of, from nervous tissue, 356

Salkowski's test for, 210,357

Schiff's reaction for, 210,357

tests for, 210,357

Choletelin, 204

Choline, 21 2, 354, 357

as putrefaction product, 2 I 2

formula for, 354

tests for, 357

Chondrigen, I 13

Chondroalbumoid, 335

Chondromucoid, I 13,335

Chondroitin, 335

Chondroitin-sulphuric acid, 3.35, 369, 392

Chondrosin, 335

reducing action of, 335

Chromoproteins (see Hemoglobins), I 13

Chyle, 260

Cipollina's test, 23, $\Delta \mathrm{I} 4$

Clark's modification of Dehn's method for determination of chlorides, 558

Cleavage products of protein (see Decomposition products), 64, 68-69

Clupeine, 67, 94

Coagulases, 4

Coagulated proteins, 95, ir6 biuret test on, I 8

digestion of, I 18

formation of, II 6

Hopkins-Cole reaction on, I I 8

Millon's reaction on, $x \times 8$

solubility of, 118

xanthoproteic reaction on, 118

Coagulation of blood, 256

Howell's theory of, 257

Coagulation of proteins, I 16

changes in composition during, 117

fractional, 105, 117

Coagulation temperature of proteins, I05, x 77 . apparatus used in determining, 106 method employed in determining, 105

Co-cnzyme, 7

Cochineal, use of, 158

Collagen, 93, 112, 331, 332

experiments on, 332

percentage of, in ligament, 334

in tendon, 331

production of gelatin from, 333

solubility of, 333

transformation of, $3.32,333$

Collection and preservation of feces in metabolism experiments, 588

of urine in metabolism experiments, 565

Collodion dialyzer, 24 
Colloidal solution, 3 I 3

Colloids, 313,340

tissue, 340

Colostrum, 315, 316, 319

microscopical appearance of, 315

Combined hydrochloric acid (protein salt), 56

140,175

preparation of, 599

tests for, 175

Combustion of foodstuffs, 584

Composition of common foods (Table), 369

Compound test for lactose in urine, 445

Congealing-point of fat, I 84

Congo red, as indicator, I53, I5 1

preparation of, 599

Congo-red fibrin, preparation of, I 2

Conjugated proteins, 9s, I I 2

classes of, 94, II 2

nomenclature of, 94, II 2

occurrence of, 94, II 2

Conjugate glycuronates, $26,4 \mathrm{I} 7, \mathbf{4 4 2}$ naphthoresorcinol reaction for, $\Delta \downarrow^{2}$ polariscopic-fermentation test for, 4.12 reduction-polariscopic test for, 4.13

Connective tissue, 330

Constipation, aid in, 51, 223

Cowie's guaiac test, 235

Cramer's mercuric oxide test for reducing sugar. 28,419

Creatine, 247, 248, 270, 276, 34I, 342, 350, 369, 384,412

crystalline form of, $3 t^{2}$

diacetyl reaction for, 350

formula for, 346

quantitative determination of, 276, 508

separation of, from meat extract, $35^{\circ}$

transformation into creatinine, 350

Creatinine, 26, 248, 270,369, 38 r

coefficient, definition of, 294

crystalline form of, 382

daily excretion of, 382

elimination, a study of, 574

experiments on, 383

formula for, $38 \mathrm{I}$

from creatine, 350

Jaffe's reaction for, 385

quantitative determination of, $2 ; 6,506$

Salkowski's test for, 385

separation of, from urine, 383

Weyl's test for, 385

Creatinine-zinc chloride, formation of, $38_{3}, 38_{5}$

Cresol, para, 2 I 2

tests for, 210

Cross and Bevan's reagent, 49 preparation of, 49 solubility test, 40

Cryoscopy, 365

Cul-de-sac, 139

Cupri-potassium biuret, formation of, 9 ?

Cyanuric acid, 373

$$
\text { formula for, } 99
$$

formula for, 373

Cylindroids in urinary sediments, 465.472

a-Cyprinine, 07

Cystine, 65, 67 60, 75, 87, 458, 4h2

crystalline form of $-i$

detection of, 402

formula for, 76
Cystine in hair, 87

in urinary sediments, 458,462

preparation of, in crystalline form, 37

Cytosine, 125, 126, 128, 133

Wheeler-Johnson reaction for, I 33

Dakin's methods for quantitatve determination of hippuric acid, 520

Dare's hemoglobinometer, 302

description of, 302

determination of hemoglobin by, 302

Deaminases, 4

Decomposition products of proteins, $63,67,68$. 69,71

crystalline forms of, 7284

experiments on, 8587

isolation of, $85-87$

Defensive enzymes, 3

Degradation products of protein (see Decomposition products) $63,67-69,7$ I

Dehn-Clark method for chlorides, 558

Delusive feeding experiments, 138

Derived proteins, 94. II 4

Detection of preservatives in milk, 323

boric acid and borates, 324

formaldehyde, 323

hydrogen peroxide, 324

salicylic acid and salicvlates, 323

Determination of ace toacetic acid in blood, 284. 294 in urine, 533

acetone in blood, 284,294

in urine. 538

acetone and diacetic in blood, 284, 294

in urine, 533

acidity of urine, by titration, 479

by hydrogen ion concentration, $4: 0$

albumin in urine, 531

allantoin in urine, 518

amino acid nitrogen, $88,9 \mathrm{r}, 27 \overline{7}, 502$

in blood, 277

in protein hydrolysis, $64,88,91$

in urinc, 502

of ammonia in urine, 499

amylolytic activity, 192

ash of milk, 327

calcium in urine, 559

casein of milk, 32;-329

catalase, 16

chlorides in blood, 286

in urine, 550

cholesterol in blood, 285

creatine in blood, 276

in urine, 508

creatinine in blood, 270

in urine, 500

diacetic acid in blood, 284, 294 in urine, 533

fat in blood, 205

in fcces, 243

in milk, 324

fecal amylase, 230

fecal tacteria, 241

glucose in urine, 523

hippuric acd in urine, 510

hydrogen ion concentration of bl ned, $28 \mathrm{~s}$ of urine, 4 sin

B-hydroxybutyric acha in blond, $28_{4} .208$ in urine, 530.541 
Determination, indican in urine, $\mathbf{5 4 2}$ indole in feces, 242 iron in urine, 591 lactalbumin in milk, 329 lactose in milk, 329 magnesium in urine, $\mathbf{5 5 9}$ nitrogen in urine, $483-491$ partition in urine, 484,577 oxalic acid in urine, 545 $\beta$-oxybutyric acid in blood, 284, 294 in urine, 539,54

peptic activity, 165

phenols in urine, 543

phosphorus in urine, 552 potassium in urine, $56 \mathrm{I}$ protein in milk, 327,328 protein in urine, 531 purine bases in urine, 513 purine nitrogen in urine, 516 sodium in urine, $56 \mathrm{I}$ sulphur in urine, 546 total solids in milk, 327 total solids in urine, 483 tryptic activity, 169 urea in blood, 274 in urine, 491-499 uric acid in blood, 274 in urine, $510-513$

Deuteroproteose, 95,119

Dextrin, 20, 43, 47, 56 achroo-, 43,56 $\alpha$-achroo-, 56 8 -achroo-, 56 $\gamma$-achroo-, 56 erythro-, 43,56 action of tannic acid on 48

diffusibility of, 48

Fehling's test on, 48

hydrolysis of, 48

iodine test on, 47

solubility of, 47

Dextrosazone, crystalline form of, Plate III, opposite p. 22

Dextrose (see Glucose).

Diacetic acid (see Acetoacetic acid), 270, 284. $412,438,539$

Diamino acid nitrogen, 64

Diaminotrihydroxydodecanoic acid, 65, 67-69, 85 $\alpha-\epsilon$-di-amino-caproic acid, 68,80

Dialysis, 24

Dialyzers, preparation of, 24

Diastase (see Vegetable amylase), 4, 10

Diazo-benzene-sulphonic acid, 454 reagent, preparation of, 454

Diazo reaction (Ehrlich's), 454

Differentiation between pepsin and pepsinogen, 141,145

Digestion, gastric, 138 intestinal, 195 pancreatic, 185 salivary, 54

Di-iodo-hydroxypropane (Iothion), 30, 42 I

Di-methyl-amino-azobenzene (see Töpfer's reagent), 175

2,5-dinitrohydroquinol, use of, 158

Dipeptides, 66, 95

Disaccharides, 19, 38 classification of, 19
Dissociation products of protein (see Decomposition products, 63 ).

Donnés pus test, 432

Doremus-Hinds ureometer, 497

Drying method for determination of total solids in urine, 482

Duodenum, epithelial cells of, 185

Earthy phosphates in urine, 406,408 quantitative determination of, 553

Edestan, 94, II4 experiments on, II 5

Edestin, 67, 93, 109 coagulation of, 109 crystalline forms of, 109 decomposition of, 67 microscopical examination of, 109 Millon's test on, 109 preparation of, 109 solubility of, 109 tests on crystallized, 109 filtrate of, 110

Ehrlich's diazo-benzene-sulphonic acid reagent, preparation of, 454

Ehrlich's diazo reaction, 454

Ehrlich's mechanical eye-piece, use of, 307

Einhorn's bead test, 238

Einhorn's saccharometer, 31

Elastin, 93, 331, 333, 334 adsorption of pepsin by, 334 experiments on, 334 preparation of, 334 solubility of, 334

Electrical conductivity of urine, 366

Electrolytes, influence on enzyme activity, 7,188

Embryos, glycogen in, 341

Emulsin, 4

Energy metabolism, 564, 584

Enterokinase, 187,196 demonstration of, 197

Enzymes, 1

activation of, 6 adsorption of, 6 classification of, 4 defensive, 3 definition of, 2 experiments on, to influence of electrolytes, 7,188 list of, 4 preparation of, 5, $11,13,14$ properties of, 6 reference books, 10

Epiguanine, 370, 401

Epinepherine, determination of in adrenal glands, 290

Episarkine, 370, 40I

Epithelial cells in urinary sediments, 465 casts in urinary sediments, 465,469

Epithelial tissue, 330 experiments on, 331

Epstein's sugar method, 282 apparatus for, 283

Erepsin, 5, 195, 198 experiments on, 198

Erythrocytes, 249, 261, 472 counting the, 304,308 diameter of, 249 form of, 249 
Erythrocytes, influence of osmotic pressure on, $26 \mathrm{t}$

in urinary sediments, 465,472

number of, per cubic millimeter, 249,250

of different species, 249

stroma of, 249

variation in number of, 250

Erythro-dextrin, 43, 56

Esbach's albuminometer, 532

method for determination of albumin, 532 reagent, preparation of, 532

Ester, definition of, 176

hydrochloric acid, of hematin, 266

sulphuric acid, of hematin, 266

Ethereal sulphates, 369,385 quantitative determination of, $547,55 \mathrm{r}$

Ethereal sulphuric acid, 21 2, 369, 385

Ethyl butyrate test for pancreatic lipase, 193 sulphide, 392

Euglobulin, 246

Excelsin, 109

crystalline form of. 110

Extractives of muscular tissue, 340 nitrogenous, 34 I

non-nitrogenous, $34 \mathrm{l}$

Fasting, feces in, 227 metabolism in, 584

Fatigue substances of muscle, 345

Fats, 176

absorption of, 178,188

apparatus for determination of meltingpoint of, 183

chemical composition of, 176,177

congealing-point of, 184

crystallization of, I 78, I $8 \mathrm{I}$

digestion of, 178,188

emulsification of, 178,180

experiments on, 180

formation of, from protein, 179-180

formation of acrolein from, I8o

hydrogenation of, 177

hydrolysis of, 177

influence of, on carbohydrate absorption, 568

in milk, 313, 319, 323

in urine, $412,444,465,469$

melting-point of, 183

nomenclature of, 176,177

occurrence of, 176

permanent emulsions of, 178,180

protein-sparing, action of, 579

quantitative determination of, in milk, 324

rancid, 178

reaction of, 178

saponification of, $177,181,182$

solubility of, 178,180

transitory emulsions of, 178,180

Fat-splitting enzymes (see Lipases, 5, 13, 178, 188, 193)

Fatty acid, 176, 178, 182, 212, 370, 397

Fatty casts in urinary sediments, 465,469

Fatty degeneration, 179

Fecal amylase, quantitative determination of, 239

Fecal bacteria, 221, 224, 225, 241, 588 quantitative determination of, 241

Feces, 221, 587, 592 agar-agar, influence of, $223-224,589$ albumin and globulin in, 237
Feces, bacteria in, 22 1, 224, 228, 241, 588 bacterial nitrogen in, demonstration of, 588 bile acids in, 236

bilirubin in, 222, 236

blood in, 225, 233

carbohydrate in, estimation of, 59 I

casein in, 230

cholesterol in, 224, 232

collection and preservation in metabolism experiments, 588

color of, influence of drugs and foods upon, 238

daily excretion of, 221,589

enzymes of, 226,239

experiments on, $229,587,592$

"fasting," 227

fat in, determination of, 243

food residues in, 227,590

form and consistency of, 223

hydrobilirubin in, 222, 235, 237

hydrogen ion concentration of, 223

indole in, 223, 237

determination of, 242

influence of defective mastication on, 590

inorganic constituents of, 225, 237, 591

elements of, demonstiation of, 591

macroscopic constituents of, 224

metabolic product, nitrogen in, 227589

microscopic constituents of, 224

microscopical examination of, 229

nitrogen of, 227

nucleoprotein in, 236

odor of, 223

parasites and ova in, 227

pigment of, 222

proteose and peptone in, 237

reaction of, 223

Scybala form of, 223

separation of, importance of, 224,587

separation of, experiment on, 239,587

Fehling's method for determination of glucose,

in blood, 279

in urine, 523

Benedict's modifications of, 522 solution, preparation of, 600 test, $26,+16$

Allen's modification of, 419

Benedict's modifications of, 27,417

Ferments, classification of, if

Fermentation, lactic acid, 170,3 I 4

"sugar-free," \$, 10, 31, 421

Fermentation method for determination of glucose, 530

Fermentation test, 31,421

Ferric chloride test for thiocyanate in saliva, 59 for melanin in urine, 452

Fibrin, 257, 268, 465,474

carmine, preparation of, 12

congo-red, preparation of, 12

in urinary sediments, 465,474

separation of, from blood, 247,257

solubility of, 268

Fibrin ferment, 247,257

Fibrin-heteroproteose, 68

Fibrinogen, 247, 256, 257, 268

Fibroin, Tussah silk, 68

Fischer apparatus, 75 photograph of, 75 
Fleischl's hemometer, 300

description of, 299

determination of hemoglobin by, 299

Fleischl-Miescher hemometer, 30 I

Fluorides in urine, $370,4 \mathrm{I} \mathrm{I}$

Fly-maggots, experiments on, 179

Foam test for bile acids, 208, 434

Folin-Hart method for determination of combined acetone and acetoacetic acid, 533 for determination of acetoacetic acid539

Folin-Messinger-Huppert method for determination of acetoacetic acid, 539

Folin's method for determination of acetone in urine, 538

acidity of urine, by titration, 479

ammonia, 499

creatinine, in blood, 276

in urine, 506,508

ethereal sulphates, 547

inorganic sulphates, 547

total sulphates, 546

urea, in urine, 495

of preparing cystine, 87

test for uric acid, $38 \mathrm{I}$

Folin and Denis' method for determination of uric acid 'in blood, 274

Folin and Denis' method for determination of uric acid in urine, modification of, 5 Io

Folin and Denis' method for phenols in urine, 543

Folin and Denis' method for urea, 495

Folin and Denis' nephelometric method for albumin in urine, 533

Folin and Denis' nephelometric method for acetone bodies in urine, $54 \mathrm{I}$

Folin and Flanders' method for hippuric acid in urine, 5 I 9

Folin and Macallum's microchemical method for ammonia, 501

Folin and Pettitone's microchemical method for urea in urine, 494

Folin, Benedict and Myers' method for determination of creatine, 509

Folin-Benedict method for determination of creatine, 508

Folin-Farmer microchemical method for total nitrogen in urine, 485

Folin-Farmer microchemical method, Bock and Benedict's modification of, 488

Folin-Farmer microchemical method, Gulick's modification of, 490

Folin-Shaffer method for determination of uric acid, 5 II

Foods, composition of, 569 purine content of, 572

Foreign substances in urinary sediment, 465,474

Formaldehyde, as milk preservative, 323 reaction (Konto), 218

Formaldehyde- $\mathrm{H}_{2} \mathrm{SO}_{4}$ test (Mörner), 86

Formation of methyl-phenylfructosazone, 35

Form elements of blood, 245

Formic acid, 26, 370, 397

Formol titration method of Benedict-Murlin, 504 of Malfatti, 502

of Sörensen, 502

Fractional coagulation of protcins, 105, II 7

method of gastric analysis, 148,159

Free hydrochloric acid, 56, I 40, 155
Free hydrochloric acid, tests for, 155

Freezing-point of bile, 203

blood, 245

milk, 3 I 4

pancreatic juice, I 86

urine, 365

Frey-Gigon method for amino-acid nitrogen in urine, 505

Fructose, Borchardt's reaction for, 35

in urine, 446

methyl-phenylhydrazine test for, 35

Seliwanoff's reaction for, 35

Fuchsin-frog experiment, 347

Fuld and Levison's method for peptic activity, 167

Fundus glands, 139

Furfural, formation of, 22 solution, preparation of, 600

Fusion mixture, preparation of, 129

Galactans, 20, 50

Galactase, 359

Galactose, I9, 36, 314, 446

experiments on, 36

Gallic acid test for formaldehyde, 323

Ganassini's test, 38 I

Gastric acidity and the use of indicators, 152

Gastric analysis, 148, 174 detection of bile in, 17

of blood in, 171

of food rests in, 173

of lactic acid in, 170

of mucus in, 173

determination of free acidity in, 164

of peptic activity in, 165

of total acidity in, 162

of tryptic activity in, 169

examination of samples in, 162

fractional method of, 148,159

introduction of the tube in, 159

Rehfuss tube, use of in, 148,159

retention meal in, 161

test meals in, $16 \mathrm{I}$

use of indicators in, 152

Gastric digestion, 138

conditions essential for, 145

general experiments on, 145

influence of bile on, 147

influence of different temperatures on, 145

influence of water on, 138

most favorable acidity for, I 45

power of different acids in, 146

products of, 14I, I 44

Gastric fistula, 138

Gastric juice, I 39

acidity of, 140,15

influence of water on, 138 of regurgitation on, 140,151

analysis of, 148,174

artificial, preparation of, I 44

composition of, 125,15 I

enzymes of, 139

lactic acid in, test for, I 70

origin of hydrochloric acid of, 140

quantitative analysis of, 148,174

quantity of, 138

reaction of, 139 
Gastric juice, secretion of, 138,139 influence of water on, 138 specific gravity of, 139

Gastric lipase, 139, 143

Gastric protease, 12

Gastric rennin, 139, I 43, I $47,315,321$ action of, upon casein, $143,197,315,321$ experiments on, 147,321 influence of, upon milk, $1+7,321$ in gastric juice, absence of, 143 nature of action of, $1+3,3$ is occurrence of, $1 .+3$

Gastric residuum, 160 composition of, $\mathrm{I} 5 \mathrm{I}$

Gelatin, 67, 68, 332, 333

coagulation of, 333

decomposition of, 67,68

experiments on, 333

formation of, 333

Hopkins-Cole reaction on, 333

Gelatin, Millon's reaction on, 333

precipitation of, by alcohol. 333

alkaloidal reagents, 333

metallic salts, 333

by mineral acids, 333

preparation of, from cartilage, 335 from collagen, 333

salting-out of, 333

solubility of, 333

Gerhardt's test for acetoacetic acid, 439

Gerhardt's test for urobilin, 400

Gies' biuret reagent, preparation of, Ioo

Gliadin, 93, II I, 1 12

decomposition of, 67

preparation of, 112

tests on, II 2

Globin, 67, 93, 113, 250 decomposition of, 67

Globulins, 93, 108

experiments on, 109

preparation of, 109

serum, $93,246,412,422$

in urine, 412,422

tests for, 423

vegetable, 109

Glucoproteins (see Glycoproteins, pp. 94, 112 )

Glucose, Allen's modification of Fehling's test for, 419

Barfoed's test on, 30

Benedict's modification of Fehling's test, 27. 417

Boettger's test on, 29, 420

Bottu's test on, 23, 415

Cipollina's test on, 23, 414

Cramer's mercuric oxide test on, 28, 419

diffusibility of, 24

experiments on, $2 \mathrm{I}, 4 \mathrm{I} 3$

Fehling's test on, 26, 416

fermentation of, 31,421

formula for, 21

klycosuria produced by, 568

Haines test on, 419

hyperglycemia produced by, 566

iodine test on, 24

Molisch's reaction on, 21

Moore's test on, 24

Nylander's test on, 20, +20

phenylhydrazine test on, 22, 413
Glucose, quantitative determination of, 522 reduction tests on, 25, 415

Riegler's reaction, 23,414

solubility of, 2 I

Trommer's test on, 25, 416

Glucosidases, 4

$\alpha$-Glucosides, 4

$\beta$-Glucosides, 4

Glucosazone, crystalline form of, Plate III, opposite p. 22

Glucothionic acid, 467

Glutamic acid, $69,8 \mathbf{8}, 186$

formula for, 82

Glutelins, 93, I 10

tests on, II

Gluten, preparation of, III tests on, II I

Glutenin, 93, 110,111 preparation of, I I I tests on, III

Glyccrol, $176,177,178,182$

borax fusion test on, 183

experiments on, 182

formula for, 177

hypochlorite-crcinol reaction for, 183

Glycerol extract of pig's stomach, preparation of, 144

Glycerophosphoric acid, 353, 354, 370, 399

Glycocholic acid, 204

Glycocholic acid group, $204^{\circ}$

Glycocoll, 65, 69, 71, 204

crystalline form of, 211

formula for, 71, 20.4

preparation of, 2 I I

Glycocoll ester hydrochloride, crystalline form of, 72

Glycogen, 20, 47, 34I, 348

experiments on, 349

hydrolysis of, 349

in embryos, $34 \mathrm{I}$

influence of saliva on, 349

iodine test on, 349

preparation of, 348

Glycogenase, 4

Glycolytic enzymes, 3,4

Glycoproteins, 94, 112-I13, 332

experiments on, 332

hydrolysis of, 332

Glycosuria, alimentary, 2I, 568

by glucose ingestion, $2 \pi, 568$

Glycosuric acid, 395

Glycuronates, conjugate, 26, +17, 442 tests for, $44^{2}$

Glycuronic acid, 36, 38, 442

Glycyl-glycine, formation of, ;0

Glycyl-tryptophane test, 195, 199

G]yoxylic acid, 98

formula for, 98

reaction (Hopkins-Colc), 98

Gmelin's test for bile pigments, 207, $\$ 33$

Rosenbach's modification of, $207,+33$

Gout, blood in, 248,270,27t, 379

Granular casts in urinary sediment, 465,467

Granulose, 43

Green stools, cause of, 222, 238

Gross' method for quantitative decermination of

tryptic activity, 191

Guaiac solution, preparation of, 600 
Guaiac test on blood, 235, 258, 262, 430

on feces, 235

on milk, 320

on pus, 432

on urine, 430

Guaiac test, Schumm's modification of, 262

Guanase, $\Delta$

Guanidine- $\alpha$-amino-valerianic acid, 68,79

Guanidine-residue, 64

Guanine, 4, 125-1 27, 133, 341

Guanine chloride, crystalline form of, 135

Gulick's colorimetric method for total nitrogen in urine, 490

Gulick's micro-oxidation flask, 490

Gum arabic, 20, 50, 5I

Gums and vegetable mucilage group of carbohydrates, 20

Gunning's iodoform test for acetone, 436

Günzberg's reagent, as indicator, 155 preparation of, 155

Haine's test on sugar, 4 I9

Hair, human, $33^{\circ}$

Hammerschlag's method for determination of specific gravity of blood, 260

Hammarsten's reaction, 207, 433 reagent, preparation of, 207,433

Harding and MacLean's method for determination of amino-acid nitrogen, 9 I

Hart's casein method, 328

Häser's coefficient, 364,483

Hayem's solution, 601

Hay's test for bile acids, 209, 434

Heintz method for determination of uric acid, 5 I 2

Helicoprotein, 94

Heller's test for blood in urine, 429

Heller's ring test for protein, Io3

Heller-Teichmann reaction for blood in urine, 430

Hemagglutination, 250, 262

Hemagglutinin, 250, 262

Hematein test for blood in feces, 234

Hematin, 113, 250, 254, 266

acid-, 254, 299

alkali-, 254, 298

preparation of, 266

reduced alkali-, 299

Hematoidin, 205, 222, 224

crystalline form of, 205, 222

in urinary sediments, 458,464

Hematoporphyrin, 205, 254, 359, 4I 2, 444 in urine, $359,4 \mathrm{I} 2,444$

Hematuria, 429

Hemicellulose, 20, 50 experiments on, 5 I utilization of, by animals, 50

Hemin crystals, form of, 265 tests, 264, 266, 429

Hemiurate, $46 \mathrm{I}$

Hemochromogen, II 3, 250, 254, 269, 299

Hemocyanin, 94, 113

Hemocytometer, Bürker's, 308 Thoma-Zeiss, 304

Hemoconein (see Blood dust, 245, 256)

Hemoglobin, 94, II 3, 249, 250, 254, 255, '262,'266, 296

carbon monoxide, 254, 297

decomposition of, 250

derivatives of, relationship of, 254
Hemoglobin, diffusion of, 262

met, 254, 298

oxy, 250, 254, 266, 296

quantitative determination of, 299-304 reduced, 254, 296

Hemoglobins, 94, I 13

Hemoglobinuria, 429

Hemolysis, 245, 26I

Henderson and Palmer's method for determina. tion of hydrogen ion concentration, 480

Herter's naphthaquinone reaction for indole, 2 I8

Herter's para-dimethylaminobenzaldehyde reac. tion, 2 I 9

Heterocyclic nucleus, 65, 68, 69

Heteroproteose, 96 I I 9

Heteroxanthine, 370, 401

Hexone bases, 80

Hexosans, 20

Hexoses, 19, 20

Hippuric acid, $72,369,388,390,396,585$

cystalline form of, 389

Dakin's method for quantitative determination of, 520

experiments on, 389,585

formula for, 388

in urinary sediments, 463

Lücke's reaction for, 390

melting-point of, 389

Roaf's method for crystallization of, 390

separation of, from urine, 389

solubility of 390

sublimation of, 390

synthesis of, 390,585

demonstration of, 585

Histidine, 65,77

hydrochloride, crystalline form of, 78

Knoop's color reaction for, 78

Histones, 93

Hoffmann's reaction for tyrosine, 86

Homogentisic acid, 26, 369, 394, 41 7 formula for, 394

Hopkin's thiophene reaction for lactic acid, I7 I

Hopkins-Cole reaction, 98 on solutions, 98 on solids, I07

Hopkins-Cole reagent, preparation of, 98

Hopkins-Cole reagent (Benedict modification), preparation of, 98

Hordein, 93, II I

Horismascope (see Albumoscope, 103, 423)

Hormones definition and discussion of, 185,3 r 8 in blood, 185

Hüfner's urea apparatus, 498

Human fat, composition of, 178

hair, composition of, 330

milk, differentiation from cow's, 316,320

Hunter and Givens' modification of Krüger and Schmidt's method, 5 I 5

Huppert's reaction for bile pigments, 207, 433

Harthle's experiment, 349

Hurtley's test for acetoacetic acid, 440

Hyaline casts in urinary sediments, 465,468

Hydrobilirubin, detection of, in feces, 222,235 extraction of, 235

Hydrochloric acid of the gastric juice, I 40, 15 origin of, theories as to, 140 seat of formation of, 139 
Hydrochloric acid test for formaldehyde (Leach), 323

acid-zinc chloride solubility test, 49

Hydrogen ion concentration and titratable acidity, $155,479-480$

mode of expressing, I 52 of blood, determination of, 288 of urine, determination of, 480 as influenced by diet, 580

by acids, 582,583

by alkali, 582,583

comparison of, with titratable acidity, 155,158

determination of, by means of indicators, 158,480

of McClendon's electrode, 149

Hydrogen peroxide in urine, 369, $41 \mathrm{I}$

detection of, in milk, 324

Hydrogenated fat, $I 77$

Hydrogenation, definition of, 177

Hydrolysis of cellulose, 48,49

cerebrin, 357

dextrin, 47,48

glycogen, 349

inulin, 46

proteins, 64

starch, 43,45

sucrose, 41,42

$\beta$-Hydroxybutyric acid, 270, 271, 284, 295, 412, 440, $54 \mathrm{I}$

Black's method for determination of, 54 I

Black's reaction for, $44^{\text {r }}$

formula for, 440

in blood, 270, 284, 295

origin of, $44 \mathrm{I}$

polariscopic examination for, 4.42

quantitative determination of, in blood, 284,295

in urine, $539,54 \mathrm{I}$

Shaffer and Marriott's method for determination of, 539

Hy peracidity, I40, I64

curve, I64

Hypercholesterolemia, 247, 270, 27 I

Hyperglycemia, 270, 566

produced by carbohydrate ingestion, $\mathbf{5 6 6}$

Hypoacidity, 140

Hypobromite methods for determination of urea in urine, 496

Hypobromite solution, preparation of, $60 \mathrm{r}$

Hypochlorite-orcinol reaction for glycerol, 183

Hypoxanthine, I 27, 34I, 346, 350, 370, $40 \mathrm{I}$

chloride, crystalline form of, 136

formula for, 127,346

oxidase, 127

Hypoxanthine silver nitrate, crystalline form of, $35 \mathrm{I}$

Ichthulin, 94

Ignotine, 34 I, 346 formula for, 346

Imide bonds, 70

Iminazolethylamine, 214

Iminazolpropionic acid, 214

Indican, $212,386,542$

formula for, 212,387
Indican, Jaffe's test for, 387

Jolles' reaction for, 388

Obermayer's test for, 388

origin of, 212,386

Indicator method for determination of hydrogen ion concentration, 158,480

solutions, preparation of, 158 use of, 158

Indicators, experiments on, 152

table of, 153,158

tabulation of results of tests on, 154

use of, 152

in gastric analysis, 152

Indigo-blue, 213,388

formula for, 213,388

Indigo in urinary sediments, 458,464

Indolacetic acid, 21.4, 432

Indole, $212,218,223,237$

formula for, 212

origin of, 212

in feces, quantitative determination of, by

Bergeim's method, 242

test for, 218,237

$\beta$-Indole- $\alpha$-amino-propionic acid, 68, 77

Indolpropionic acid, 214

Indoxyl, 212

formula for, 2 I 2,388

origin of, $2 \mathrm{I} 2$

potassium sulphate (see Indican, p. 212 , 387,542

Indoxyl-sulphuric acid, 2 I2, 369, 385

formula for, 2 I2, 386

Influence of purine-free and high purine diets, $57 \mathrm{I}$

Infraproteins (see Metaproteins, 94, I I5, II6)

Inorganic elements in feces, absorption of, 591 physiological constituents of urine, 370, 402

Inosinic acid, 341,346

formula for, 346

Inositol, 19, 412, 450

formula for, 450

in urine, $4 \mathrm{I} 2,450$

Intestinal digestion, 195

juice, 195

enzymes of, 195, 196, 198-20I

preparation of, 199-20I

Inulase, 4,46

Inulin, 20, 46

action of amylolytic enzymes on, 46

Fehling's test on, 46

hydrolysis of, 46

iodine test on, 46

reducing power of, 46

solubility of. 46

sources of, 46

Inversion, $4 \mathrm{r},+3$

Invertase (see Sucrase, 4, 41, 195, 199)

Invertases, experiments on, 199

Invertin (sce Sucrase, 4, 41, 195, 199)

Inverting enzymes, 3

Invert sugar, 4 I

Iodide of dextrin, 45

of starch, 47

Iodine absorption test, $\mathrm{I}_{3}$

test for starch and dextrin, 45, 46, 47, 49, 5 I. 56

for urobilin, 400

Iodine-sulphuric acid test for cholesterol, 210,357

Iodine-zinc-chloride reaction, 49 
Iodoform test for alcohol, 42

for acetone (Lieben), 437

Iodothymol compound, 437

"Iothion," 30, 421

Iron, reduced, influence on color of feces, 238 in blood, $26 \mathrm{I}$

detection of, 26 I

in bone ash, 338

detection of, 338

in protein, 63

in urine, $410,562,591$

detection of, 410

determination of, 591

Isoleucine, 65,80

Isomaltose, 19,40

Isopropylmetacresol (see Thymol)

Isovalerianic acid, 2 I 4

Jacoby-Solms method, I6 7

Jaffe's reaction for creatinine, 382

Jaffe's test for indican, 387

v. Jaksch-Pollak reaction for melanin, 452

Jejunum, epithelial cells of, I 85

Jolles' reaction for indican, 388

Juice, gastric, 138,150

pancreatic, 185

intestinal, 195

Kantor and Gies's biuret paper, 100

Kastle's peroxidase reaction, 320

Kephalin, 353, 355

Kephyr, 40

Keratin, 93, 112,330

experiments on, 33 I

solubility of, 330

sources of, 330

sulphur content of, 330

Ketone, 25

Ketose, I9

Kidney efficiency test, 455

Kjeldahl method for determination of nitrogen, 483

Kjeldahl-Folin-Farmer nitrogen method, 485

Knoop's color reaction for histidine, 78

Konto's reaction for indole, 218

Koumyss, 40

Kraut's reagent, preparation of, 602

Kreosotal, 443

Krüger and Schmidt's method for the quantita. tive determination of purine bases, 5 I 3

of uric acid, 5 I 3

Kwilecki's modification of Esbach's method, 532

Kynurenic acid, 369, 395

formula for, 395

isolation of, from urine, 395

quantitative determination of, 395

Laccase, 5

Lactalbumin, 93,3 เ3, 318

quantitative determination of, 329

Lactase, $4,196,200$

experiments on, 200

Lactic acid, 40, I 70,342

ether-ferric chloride test (Strauss) for, 170

fermentation, 40, 3 I 4

ferric chloride test (Kelling) for, 170

Hopkins' thiophene reaction for, I I I
Lactic acid in muscular tissue, 342

in stomach contents, 170

tests for, I 70 .

Uffelmann's test for, 170

Lactochrome, 319, 399

Lacto-globulin, 313,318

Lactometer, determination of specific gravity of milk by, 324

Lactosazone, crystalline form of, Plate III, opposite p. 22

Lactoscope, Feser's, 326

Lactose, 19, 40, 318

experiments on, 40

fermentation of, 40,318

in urine, 412,444

quantitative determination of, 329

Laiose in urine, $45 \mathrm{I}$

"Laked" blood, 245, 26 I

Laky blood, 26 I

Lanolin, 178,188

Lauric acid, 313

Leach's hydrochloric acid test for formaldehyde, 323

Lecithin, 94, 247, 353

acrolein test on, 356

decomposition of, 354

experiments on, 356

formula for, $35 \Delta$

microscopical examination of, 356

osmic acid test on, 356

preparation of, 356

test for phosphorus in, 356

Lecithoproteins, 94, I I 4

Legal's reaction for indole, 218

test for acetone, 437

Le Nobel reaction for diacetic acid, 439

Lenzmann-Kober nephelometer, cut of, 293

Leucine, 67, 69, 79, 85, 87

crystalline form of impure, 463

pure, 80

experiments on, 87

formula for, 79

in urinary sediments, 463

microscopical examination of, 87

separation of, from tyrosine, 86

solubility of, 87

sublimation of, 87

Leucocytes, 245, 255

counting the, 306

number of, per cubic millimeter, 255

size of, 255

variation in number of, 255

Leucocytosis, 255

Leucosin, 103

Leucyl-alanyl-glycine, formation of, 70

Leucyl-glycyl-alanine, 57

Leucyl-leucine, formation of, 70

Levo- $\alpha$-proline, 83

Levulosazone, crystalline form of, Plate III, opposite p. 22

Levulose, (see Fructose), 19, 34

Lichenin, 20, 47

Lieben's test for acetone, 437

Lieberkühn's jelly (see Alkali metaprotein, p. I I6)

Liebermann-Burchard test for cholesterol, 210,357

Liebermann's reaction, 100

Linoleic acid, I 77

Lipase, gastric, I39, I43 
Lipase, pancreatic, 5, I3, I 78, 188,193

experiments on, 13,193

ethyl-butyrate test for, 193

litmus-milk test for, 193

Lipases, 5, 13, 178, 188, I93

autolytic, 5

experiments on, 13, 193

pancreatic, 13

vegretable, 13

Lipemia, blood in, 270,271

Lipeses, 9

Lipins, 353

Lipoids of nervous tissue, 353.356

Lipolytic enzymes (see Lipases, p. 5, 13, 178, 188, 193)

"Litmus-milk" test for pancreatic lipasc, 193

Long's coefficient, 364,483

Lücke's reaction for hippuric acid, 390

Lugol's solution preparation of, 602

Lymph, 245. 259

Lysine, 68, 80, I42, I 86

Lysine picrate, crystalline form of, 8 I

McClendon's electrode, determination of $\mathrm{H}$ ion conc. by, 149

Magnesia mixture, preparation of, 602

Magnesium balancc, preparation of,592

in bone, detection of, 337,338

in urine, 370,409

quantitative determination of, 559

phosphate in urinary sediments, 458 , 464

Malfatti's formol titration method for ammonia in urine, 502

Maltase, 4, 57, I $96,20 \mathrm{I}$

experiments on, 20 r

Maltosazone, crystalline form of, Plate III, opposite p. 22

Maltose, 19, 39

experiments on, 39

structure of, 39

Marsh apparatus, 449

cut of, 449

method for arsenic, 4.48

Marshall's hypobromite urea apparatus, 498

Marshall's clinical urease method for estimation of urea in urine, 493

Mastication, defective, influence of, on food residues in feces, 227,590

Mauvein, use of, as indicator, 158

McCrudden's method for determination of calcium, 559

of magnesium, 559

McLean and Van Slyke's method for determination of chlorides in blood, 286

Melanin in urine, $412,45 \mathrm{I}$ tests for, 452 urinary sediments, 458,465

Melting-point apparatus, 183 of fats, determination of, 183

Mercuric oxide test for reducing sugar, 28,419

Mercury in urine, 450 detection of, 450

Metabolism, 564 experiments, 565

balance of income and outgo in, prepa. ration of, 592
Metabolism experiments, collection and preservation of feces in, 588

urine in, 565

separation of feces in, 587

in acidosis, 576

in fasting, 584

in gout, 571,574

influence of acids on, 582

of alkalies on, 582

of defective mastication on, 590

of digestion on, 577

of fats and carbohydrates as protein sparers in, 570

of high calorie, non-nitrogenous diet on, 584

indigestible, non-nitrogenous material on, 589

of water on, 574

of acid-forming and base-forming foods, 580

of ammonium benzoate, 585

of carbohydrates, 566-568, 591, 593

of energy, 579, 584

of fat, 591

of inorganic elements, 391, 593

of nitrogen and sulphur as influenced by diet, 577

of proteins, 569,590

time relations of, 569

of purines, $571-574$

on "salt-free" diet, 576

on salt-rich diet, 576

relation of bacterial nitrogen of feces to, 588

of metabolic nitrogen of feces to, 589

study of creatinine elimination in, 57.4

Menstrual blood, 257

Messinger-Huppert method for determination of combined acetone and diacetic acid, 535

Metaproteins, 94, II 5

acid, 94, II 5, I I 6

alkali, 94, I I 5, I 16

experiments on, 116

precipitation of, 116

sulphur content of, I I 6

Methemoglobin, 254, 298

Methylene blue, $I+6$ reaction (Russo), 455

Methyl-mercaptan, 2 I 2

Methyl orange, use of, as indicator, is $\mathrm{s}$ red, use of, as indicator, 158

violet, use of, as indicator, 158

Methyl-pentose (see Rhamnose, p. 19)

Methylphenylhydrazine, 35

Methylphenylfructosazone, formation of, 35

I-methylxanthin, 370, 401

Nett's method for determination of peptic activity, 165

Mett's tubes, preparation of, 166

Mlicro-organisms in urinary sediments, 405,474

in feces, 221, 225

in intestine, 212,225

Mlilk, 313

ash of human and eow's, 310, 317

easein of, $313,315,318$

citrates in, 313

composition of human and enws, 316,317

detection of calcium phosphate in, 322

lactose in, 322

preservatives in, 323 
Milk, difference between human and cow's, 316 , 317,320

experiments on, 319

formation of film on, 314,319

freezing-point of, $3 \mathrm{I}_{4}$

guaiac test on, 320

human and cows, differentiation, 3 I 6,320

influence of rennin on, 147,32 I

isolation of fat from, 323,324

Kastle's peroxidase reaction on, 320

lactose in, $313,318,322$

crystalline form of, 318

fermentation of, 314

microscopical appearance of, 3I5, 3 I 9

preparation of casein from, 321

properties of casein of, 318,322

quantitative analysis of, 324

reaction of, 314, 319

separation of coagulable proteins of, 322

serum, 3 I 3

specific gravity of, 314, 319

unknown constituents of, 3 I 3

Millon's reaction, 97

reagent, preparation of, 97

Mohr's method for determination of chlorides, 558

Molisch's reaction, 2 I

Molybdate solution, preparation of, 603

Monamino acid nitrogen, 64

Monosaccharides, I9, 20

Barfoed's test for, 30

classification of, I9

Mörner-Sjōqvist-Folin method for determination of urea, 495

Mörner's reagent, preparation of, 86 test for tyrosine, 86

Motor and functional activities of the stomach, 146 .

Mucic acid, $36,40,445,446$ test, $36,40,445,4.46$

Mucin, 55, 58, 94, II 3

biuret test on, 59

hydrolysis of, 59

isolation of, from saliva, 59

Mnlon's reaction on, 59

Mucins, 55, 58, 94, 113

Mucoid, 94, II3, 331, 332

experiments on, 332

hydrolysis of, 332

in urine, 397,428

preparation of, from tendon, 332

Mucoids, 94, II3, 331, 332

Murexide test, 380

Muscle plasma, 339, 347

formation of myosin clot in, 339,347

fractional coagulation of, 339,347

preparation of, 347

reaction of, 342,347

Muscular tissue, 339

ash of, smooth and striated, 345

commercial extracts of, 345

experiments on "dead," 348

"living," 347

extractives of, 341,345

fatigue substances of, 345

formulas of nitrogenous extractives of, 346

glycogen in, $34 \mathrm{I}, 348$

involuntary, 339
Muscular tissue, lactic acid in, $340,342,348$ magnesium in, demonstration, 349 nonstriated, 339

phosphate in, demonstration of, 349 pigment of, 345 preparation of glycogen from, 348 muscle plasma from, 347

Muscular tissue, proteins of, 339,348

reaction of living, 342,348

rigor mortis of, 339,340

separation of extractives from, 350

striated, 339

voluntary, 339

Myohematin, 345

Myosan, 94, 348

formation of, 348

Myosin, 339, 348

biuret test on, 348

coagulation of, 348

preparation of, 348

solubility of, 348

Myosinogen, 339, 347

Myristic acid, 313

Myristin, 177

Myrtle wax (see Bayberry tallow, 182)

Nakayama's reaction for bile pigments, 207, 433 reagent, preparation of, 207,433

$\alpha-$ Naphthol reaction, 2 I

Naphthoresorcinol reaction for glycuronates (Tollens), 442

Nencki and Sieber's reaction for urorosein, 453

Neosine, 34r

formula for, 346

Nephelometer, Bloors, cut of, 29 I

description of, 290

Lenzmann-Kober, cut of, 293

Nephelometric methods, 290, 326, 327, 533, $54 \mathrm{I}$

Nephelometric, determination of, acetone bodies in blood, 294

acetone and diacetic acid in urine, $54 \mathrm{I}$

fat in blood, 295

in milk, 326

$\beta$-hydroxybutyric acid in urine, $54 \mathrm{I}$

proteins in milk, 327

in urine, 533

Nephritis, blood in, $248,270,27$ I

Nephrorosein in urine, 453

Nervous tissue, 353

constituents of, 353

experiments on lipoids of, 356

lipoids of, 353,356

percentage of water in, 353

phosphorized fats of, 353

proteins of, 353

Nessler-Winkler solution, 603

Neurine, 2 I 2

Neumann's method for total phosphorus, 554

Neurokeratin, 353

Neutral fats, I77, 178, I 80

Neutral olive oil, preparation of, 180

Neutral red, use of, I 58

Neutral sulphur compounds, 369,392

Nippe's hemin test, 264

Nitrates in urine, $370,4 \mathrm{II}$

Nitric acid test (Heller), 103

for phenol, 220

Nitric acid- $\mathrm{MgSO}_{4}$ test (Roberts), IO4 
Nitrilase, 8

Nitrilesc, 8

Nitrites in saliva, test for, 59

Nitrogen, 63. 64

forms of in protein molccule, 64

importance of, in sustaining life, 64

in urine, quantitative determination of, 483

Nitrogen distribution, calculation of, 48.4

Nitrogen iodide, formation of, 436

Nitrogen "lag," 560

$$
\text { "partition," 485, } 577
$$

Nitrogenous extractives of muscular tissuc, 341 , 350

formulas for, 346

p-.itrophenol, use of, as indicator, 158

Nitroprusside reaction for indole (Legal), 218

Nitroprusside test for creatinine (Weyl), 385

Nitroprusside-acetic acid test for creatinine (Salkowski), 385

Nitroso-indole nitrate test, 219

Nitrosothymol, formation of in Heller's test, 423

Non-ritrogenous extractives of muscular tissue, 341

Non-protein nitrogen of blood, 248, 270, $27 \mathrm{I}$

Normal urine, 359, 369, $37 \mathrm{I}$ characteristics of, 359 constituents of, $369,37 \mathrm{I}$

Novaine, 341 cxperiments on, $375-410$ formula for, 3.46

Nubecula, 396,428

Nucleases, 5 experiments on, 134

Nucleic acid, 124-128, I31-137 decomposition of, $12+-126$ experiments on, $131-137$ from yeast, formula for, 125

Nucleicacidase, 5,126

Nucleins, 123, 124, 144, 571

Nucleoproteins, 94, I1 $2,123,128-131,396,428$ decomposition of, $123-124$

experiments on, $128-13 \mathrm{I}$

from yeast, 128

preparation of, I 28

protein, carbohydrate and phosphoric radicals in, 130

tests on, 129

thymus, preparation of, 130 experiments on, 130

in bile, 203, 207

in feces, 236

in nervous tissue, 353

in urine, $369,396,412,428$ test for, 428

occurrence of, 123

Ott's precipitation test for, $\$ 28$

Nucleosidasc, 5, 126 .

Nucleoside, 125

Nucleotidasc, 5, 126

Nucleotide, I 25

Nylander reaction, 29, 420

Nylander reagent, preparation of, 29,420

Obermajor's test for indican, 388 reagent, preparation of, 388

Oblitine, $34 \mathrm{r}$

"Occult" blood in feces, 225, 233 tests for, 233
Olein, 177,313

Olive oil, 180

emulsification of, 180

ncutral, preparation of, i 80

Oliver's peptone test for bile acids, $209, \$ 35$

Optical methods, $3 \mathrm{r}-3+4$

Orcinol- $\mathrm{HCl}$ reaction (Bial), $3 \pi, 4+3$

Orcinol test, 38,444

Organic physiological constituents of urine, 360

Organized ferments, $I$

Organized urinary sediments, $45 \%, 465$

Ornithine, 65, 21.4

Ortho-tolidin test for blood, I 7 I, 233, 263, 430

Osborne-Folin method for determination of total sulphur in urine, 5.49

Ossein, 336 preparation for, 336

Osseoalbumoid, 336

Osseomucoid, $94,153,336$ chemical composition of, 113

Osseous tissue, 336 experiment on, 337

Ott's precipitation test for detection of nucleoprotein in urine, 428

Ovalbumin, 93

Ovoglobulin, 93

Oxalated plasma, preparation of, 268

Oxalic acid, 369, 391, 545

formula for, 39 I

in urine, $369,39 \mathrm{r}$

quantitative determination of, $5+5$

Oxaluria, 39 I

Oxaluric acid, 369,397

Oxamide, 99

Oxidases, 5, 14, 319 experiments on, 14

Oxyacids, 212,217,220,369, 394 tests for, 220

$\beta$-Oxybutyric acid (see $\beta$-hydroxybutyric acid, $270,271,284,295,412,440,54 I)$

Oxy'hemoglobin, 64, 250, 254, 255

Reichert's method for crystallization of, 266 crystalline forms of, $25 \mathrm{I}-254$

Oxymandelic acid, 369, 395

Oxyproline, 65, 67, 85

Oxyproteic acid, $369,392,454$

Palmitic acid, $176,177, I 8 \mathrm{r}, 182$ crystalline form of, I 82 experiments on, 182

formula for, 177

preparation of, 182

Palmitin, 176, 3 I9

Pancreatic amylase, $4,11,187,192$ digestion of dry starch by, 188, 192 inulin by, 192

experiments on, $I \mathbb{I}, 19 I$

influcnce of bile upon action of, 192

most favorablc temperature for action of, I 92

Pancrsatic digestion, 185

general experiments on, 190 products of, 186, r 89

Pancreatic insufficiency, Schmidt's nuclei test for, 237

Pancreatic juice, I $23,185,186$, I 87 artificial, preparation of, 189 daily excretion of, I 86 
Pancreatic juice, enzymes of, 186

freezing-point of, I 86

mechanism of, secretion of, 185

reaction of, 186

solid content of, I 86

specific gravity of, 186

Pancreatic lipase, 5, I $3,178,188$

experiments on, 13,193

ethyl-butyrate test for, I 93

litmus-milk test for, 193

Pancreatic protease (see Trypsin, pp. 5, 12, I86)

Pancreatic rennin, 5, 189, 194

experiments on, I94

Papain, 5, I3

Papayotin (see Papain)

Paracasein, 315

Para-cresol-sulphuric acid, 369,385

Paradimethylamino benzaldehyde solution, preparation of, 604

Paralactic acid, 247,314, 342,370, 398

Paramyosinogen, 339

Paranucleoprotagon, 353. 356

Paraoxyphenylacetic acid, 212, 2 I 4, 369, 394

Paraoxy- $\beta$-phenyl- $\alpha$-amino-propionic acid, 69,74 . 86

Paraoxyphenylpropionic acid, 212, 214, 369, 394

Paraphenylenediamine hydrochloride, 324

Parasites, 227, 465, 474

Paraxanthine, 370, 401

Parietal cells, I 39

Parotid glands, characteristics of saliva secreted by, 54

Pathological constituents of urine, 4 I 2

Pathological urine, 359, 412

constituents of, 412

experiments on, 413

"Partition" of nitrogen and sulphur in urine, 577

Pektoscope, 365

Pentamethylenediamine, 214

Pentapeptides, 66, 95

Pentoses, 19, 37

experiments on, 37

in urine, $+12,443$

tests for, 443

Pentosans, 20, 36, 50

Pepsin (see Gastric Protease), 5, I2, I 4 I, I 65

action of, influence of bile upon, I47

influence of diffcrent acids upon, 146

influence of metallic salts upon, 146 temperature upon, I 45

conditions essential for action of, I 45

differentiation of, from pepsinogen, I 4 I, 145

digestive properties of, I4I

formation of, I 4 I

most favorable acidity for action of, 145

presence of, in intestine, I 42

proteolytic action of, $14 \mathrm{I}$

Pepsin-hydrochloric acid, 145

Pepsin-rennin controversy, I 43

Pepsinogen, 6, I 4 I, I 44

differentiation of, from pepsin, I4I, I45

formation of, I4I

extract of, preparation of, 144

Peptases, 5

Peptic activity, Fuld and Levison's method for, determination of, 167

Mett's method for the determination of, I 65
Peptic activity, Rose's method for determination of, I67

Peptic proteolysis, I 4 I

products of, I4 I

relation of, to tryptic proteolysis, 142

Peptides, 66, 71, 95, 120

Peptone, 65, 7I, 95, I1 8

ampho, 95, I19

anti, I 19

differentiation of, from proteoses, I I 9

experiments on, II 9, I 20

in urine, 412,426

tests for, 427

separation of, from proteoses, 120

Peptone test for bile acids (Oliver), 209, 435

Periodide test for choline, 357

Peroxidases, 5, I 4, I 5, 32 I

Peters' method for sugar determination, 525

Pettenkofer's test for bile acids, 208, 434

Mylius's modification of, 208 , 43.4

Neukomm's modification of 209, 435

Phenaceturic acid, 370,398

Phenol, 213, 387

excretion of, 387.545

quantitative determination of, in urine, 543 tests for, 219

Phenolphthalein as indicator, $153,157,158$, I62, 479

preparation of, 158,605

test for blood in feces, 234

Phenolsulphonephthalein test for kidney effciency, 455

Phenol-sulphuric acid, 369, 385

Phenylacetic acid, 2I4

Phenyl- $\alpha$-amino propionic acid, 69, 73

Phenylalanine, 65, 67, 69, 73

Phenylethylamine, 214

Phenylglucosazone, 22 and plate opposite

Phenylhydrazine, 22

acetate solution, preparation of, 23

mixture, preparation of, 22

reaction, $22,23,413$

Cipollina's modification of, 23, 4I 4

Phenyllactosazone, crystalline form of, Plate III, opposite p. 22

Phenylmaltosazone, crystalline form of Plate III, opposite p. 22

Phenylpotassium sulphate, 386

Phenylpropionic acid, 214

Phloroglucinol- $\mathrm{HCl}$ reaction, 36. 38, 443. 446

Phosphate solutions and hydrogen ion concentration, I54, I56, I57, I59, 36 I, 48 I

Phosphates in urine, $36 \mathrm{r}, 406,553$ detection of, 408

experiments on, 408

quantitative determination of, 552

Phosphatase, 9

Phosphatese, 9

Phosphatides, 203,296, 353

Phosphocarnic acid, 34I, 346,370, 399

Phosphonuclease, I 36

Phosphoprotcins, 94, 95, I I 3, 3 I 8

Phosphorized compounds in urine, 370,399

Phosphorus in urine, determination of, 552 organic, test for, 129

Phosphotungstic acid reaction (Folin), 38 I 
Phosphotungstic precipitation test for proteose (v. Aldor), 427

Physiological constituents of urine, 369

Phytase, 5

Phytin, 5

Picric acid reaction for creatinine (Jaffe), 385

Pigments of urine, $359,370,399,451$

Pine wood test for indole, 219

Piria's test for tyrosine, 86

Plasma of blood, 245,268

of muscle, 339,347

Plasmaphæresis, 249

Polariscope, use of, 3 I

in detection of conjugate glycuronates, 443

in determination of dextrose, 3 I, 53 I

$\beta$-hydroxybutyric acid, $442,54 I$

Polypeptides, 66, 7I, 95

Polysaccharides, 20, 43

classification of, 20

properties of, 43

Posner's modification of biuret test, 100

Potassium in urine, 370,409

quantitative determination of, 562

Potassium hydroxide test for blood in urine (Heller), 429

indoxyl-sulphate (see Indican, pp. 212, 387, $5+2)$

formula for, 212,387

origin of, 212,386

tests for, 387

Primary protein derivatives, 94, II 4

Primary proteoses, I I9

Products of protein hydrolysis, 66, 67, 7 I

Prolamins, 93, I I I

classification of, 93

preparation of, I 12

tests on, I I 2

Proline, 65, 67, 68, 83, 111, I42, 186 crystalline form of copper salt of, $8+$ crystalline form of lævo- $\alpha-, 8$,

Prosecretin, 185

Protagon, 353, 354

preparation of, 356

structure of, 355

Protamines, classification of, 93, 95

Proteans, 94, 114

Protease, gastric, 5, 12, 141 experiments on, I2, I 44

pancreatic, $5,12,186$

experiments on, 12,189

vegetable, 5. 13

Proteases, 5, 12

experiments on, 12

Protective enzymes (sec defensive enzymes), 3

Protein content of foods, 569

derivatives, primary, $65,9.4,114$

sccondary, 65, 94, 118

metabolism, time relations of, 569

influence of water on, 575

utilization, determination of, 590

Protein-coagulating enzymes, $5,143,189,256,315$

Protein-cystine, 77

Protein-sparing action of fat and carbohydrate, 579

Proteins, 63, 92, $\$ 12, \$ 22$

acetic acid and potassium ferro-cyanide test for, 104
Proteins, Acree-Rosenheim test on, 100

action of alkalordal reagents on, 103

of metallic salts on, 103

mineral acids, alkalies and organic acids on, 103

Bardach's reaction on, 10 r

biuret test on, 98

chart for use in review oi, $12 t$

chemical composition of, 63

classification of, 93,95

coagulated, 94, 116

biuret test on, I 18

formation of, II 6

Hopkins-Cole reaction on, I I 8

Millon's reaction on, 118

solubility of, 118

xanthoproteic reaction on, II 8

quantitative determination of, 327

coagulation, influence of salts upon, II 7

coagulation or boiling test for, 105

color reactions of, 97

conjugated, $94,95,112,123$

classes of, 94, 112

experiments on, 59, 113, 128, 296, 321

nomenclature of, 94, 112

occurrence of, 94, 112

decomposition of, $67,67,68$

by hydrolysis, 65

by oxidation, 64

products of, $64,67,68,71$

experiments on, 85

separation of, 85

study of, $64,67,85$

derived, 94,114

formation of fat from, 179

formulas of, 64

Heller's ring test on, $\mathrm{r}_{3}$

Hopkins-Cole reaction on, 98

importance of, to life, 63

in urine, 412,422

determination of, $53 \mathrm{I}$

test for, 423

Liebermann's reaction on, I00

Millon's reaction on, 97

of milk, 315,318

molecular weights of, 64

Posner's reaction on, 100

precipitation of, by alcohol, I06

alkaloidal reagents, 103

metallic salts, 103

mineral acids, 103

precipitation reactions of, 102

quantitative determination of, in milk, 328

review of, 121

Robert's ring test on, 104

salting-out experiments on, 105

salts of, 102

scheme for separation of, 122

simple, 93, 95

synthesis of, 66,70

xanthoproteic reaction on, 98

Proteolysis, peptic, $1+2$

tryptic, 142, 186

Proteolytic enzymes (sce Proteases, p. 12)

Proteose, 64, 94, 95, 118

v. Aldor's method for detection of, $\$ 27$

biuret test on, 120

coagulation test on, 120 
Proteose, deutero, 94, 95, I 20

differentiation of, from peptone, I 20

experiments on, II 9,120

hetero, 95, 120

in urine, 412,426

test for, 427

potassium ferrocyanide and acetic test on,

I 20

powder, preparation of, $\mathrm{I} 20$

precipitation of, by nitric acid, 120

by picric acid, I20

by potassio-mercuric iodide, I 20

by trichloracetic acid, I 20

primary, 120

proto, 95, 96, I 20

Schulte's method for detection of, 427

secondary, 120

separation of, from peptones, 120

Protoproteose, 95, 96, 120

Proteoses and peptones, 95, 96, II9, 120 separation of, I 20

tests on, $\mathrm{x} 20$

Proteose-peptone, II

Proteose-peptone, coagulation test on, $\mathrm{r} 20$

experiments on, II 9

Millon's reaction on, II9

precipitation of, by nitric acid, $\mathbf{I} 20$

by picric acid, 120

Prothrombin, 256, 257

Pseudo-globulin, 246

Ptomaines and leucomaines in urine, 370, $40 \mathrm{I}$

Ptyalin (see Salivary amylase, I, 4, I0, 55, I87)

Purinases, 127,134

experiments on, $\mathrm{I} 34$

Purine bases, I25, 126, I32, 346, 370, $40 \mathrm{I}$ formulas for, 127

in urine, quantitative determination of, $5 \mathrm{r} 3$ tests on, 132,133

content of foods, 572

excretion, rate of, 574

oxidases, $5,127,134$

Purines, amino, 127,132 oxy, 127,132

Purine-free and high purine diets, influence of, $57 \mathrm{I}$

Pus casts in urinary sediments, 465,472

cells in urinary sediments, 472

in urine, $43 \mathrm{I}$

tests for, 431,432

Putrefaction, control of, by carbohydrate, 213 indican as an index of, 212,386

Putrefaction mixture, preparation of a, 214 products, 212

experiments on, $2 \mathrm{I} 4$

most important, 2 I 2

tests for, 2 I 8

Putrescine, 2 I 2

Pyloric glands, 138

Pyrimidine bases, I25, I26, 128

experiments on, 133

formulas for, 128

Pyrocatechol-sulphuric acid, 369, 386

$\alpha$-pyrrolidine-carboxylic acid (see Proline, pp. 65.

$67,68,83$, I I 1, I 42, I 86 )

Pyuria, 43I

Quadriurate, 46I

Qualitative analysis of the products of salivary digestion, 60
Quantitative analysis of blood, 270

of gastric juice, 148

of milk, 324

of urine, 479

Quevenne lactometer, determination of specific gravity of milk by, 324

Raffinose, I9, 42

Raiziss and Dubin's volumetric methods for total sulphur, $55 \mathrm{I}$

Rancid fat, 178

Raw and heated milk tests, 320

Reaction of the urine, $36 \mathrm{I}, 479,580$

Reagents and solutions, 594-6ro,

Reduced alkali-hematin, 299

Reduced hemoglobin, 296

Reductases, 3 I 9

Rehfuss stomach tube, cut of $I_{4} 9$

$$
\text { use of, 1 49, I } 59
$$

Reichert's method for crystallization of oxyhemoglobin, 266

Reinsch test for arsenic, 449

for mercury, $45^{\circ}$

Remont's method for detection of salicylic acid and salicylates, 323

Rennin, gastric, I39, I43, I47, 31 5, 32 I

action of, upon casein, I43, 147, 315, $32 \mathrm{I}$

experiments on, $147,32 \mathrm{I}$

influence of, upon milk, I 47, 321

in gastric juice, absence of, 143

nature of action of, 143,315

occurrence of, 143

Rennin, pancreatic, 5, 189,194

experiments on, 194

Rennin-pepsin controversy, 143

Resorcinol- $\mathrm{HCl}$ reaction, 35,447

Respiration, chemistry of, 255

Retention meal in gastric analysis, I6I

Reticulin, I I 2

Reversibility of enzyme action, 8,57

Reynolds-Gunning test for acetone, 438

Rhamnose, I9, 38

Rhubarb, influence of, on color of feces, 238

Ricin, 13, 262

Riegler's reaction, 23, 4 I4

Rigor mortis, 339,340

Ring test for urobilin, 400

Roaf's method for crystallizing hippuric acid, 390 , 587

Robert's ring test for protein, 104, 424 reagent, preparation of, 104,424

Robin's reaction for urorosein, 453

Rosolic acid, use of, as indicator, 158,513

Rosenheim's bismuth test for choline, 358

Rosenheim's periodide test for choline, 357

Rosenheim and Drummond's volumetric methods

for sulphates and total sulphur, 550-55 I

Rose's method for determination of pepsin, $x 67$

Rothera's reaction for acetone, 438

Rubner's test for lactose in urine, 445

Ruhemann's uricometer, cut of, 513 use of, 5 I 3

Russo's reaction, 455

Ruttan and Hardisty's orthotolidin test for blood, $171,233,263,430$

Saccharide group, 20

Saccharose (see Sucrose, I9, 4I) 
Sahli's desmoid reaction, 146

reagent, 164

Salicylaldehyde reaction for acetone (Frommer), 438

Saliva, 54

alkalinity of, 55

amount of, 55

bacteria in, 58

biuret test on, 58

calcium in, 59

chlorides in, 59

constituents of, 55

digestion of dry starch by, 60

digestion of inulin by, 60

digestion of starch paste by, 56, 59

dilution of, influence on digestion, 60

enzymes contained in, $\mathbf{5 5}$

excretion of potassium iodide in, 61

inorganic matter in, tests for, 59

Millon's reaction on, $5^{8}$

mucin from, preparation of, 59

nitrites in, test for, 59

phosphates in, test for, 59

potassium thiocyanate in, 59

reaction of, 55,58

secretion of, 54

specific gravity of, 55,58

sulphates in, test for, 59

tests on, 58

thiocyanates in, $\mathbf{5 5}, 59$

tripeptide-splitting enzymes in, 57

Salivary amylase, I, 4, 10, 55, I87

activity of, in stomach, 57,188

inhibition of activity of, 57

nature of action of, 56,57

products of action of, 56

scheme showing, 56

Salivary digestion, 54

graphic representation of, 56

influence of acids and alkalis on, 56, 6I

dilution on, 57, 60

metallic salts on, $6 \mathrm{r}$

temperature on, 60

nature of action of acids and alkalis on, 61 qualitative analysis of products of, 60

Salivary digestion in stomach, 57,188

glands, 54

stimuli, 54

Salkorwsk-Autenrieth-Barth method for determination of oxalic acid in urine, 545

Salkowski's method for determination of purine bases, 516

Salkowski-Schippers reaction for bile pigments, 208,433

Salkowski's test for cholesterol, 210,357 for creatinine, 385

Salmine, $67,68,60,04,95$

"Salt-free" diet, metabolism on a, 576

Salted plasma, preparation of, 268

Salting-out experiments on proteins, 105, I 22

Santonin, influence of on color of feces, 238

Saponification, $x 77,18 \mathrm{r}, 182$

of bayberry tallow, $18 \mathrm{I}$

of lard, 182

Sarcolactic acid, 342

Scallops, preparation of glycogen from, 348

Schalfijew's method for preparation of hemin, 266
Schema for "blood counting," 3 II

Scheme for analysis of biliary calculi, 209

bone ash, 338

stomach contents, I 59

urinary calculi, 477

separation of carbohydrates, 53

of proteins, 122

Scherer's coagulation method for determination of albumin in urine, $53 \mathrm{I}$

Schiff's reaction for cholesterol, 2 ro, 357 ior uric acid, 381

Schmidt diet, composition of, 228

Schmidt's nuclei test for pancreatic insufficiency. 237

Schmidt's test for hydrobilirubin, 235

Schulte's method for detection of proteose in urine, 427

Schumm's modification of the guaiac test, 262

Schütz's law, statement of, 9, I 66

Schweitzer's reagent, action of, on cellulose, 49 preparation of, 49

Scleroproteins, (see Albuminoids), 93, II

Scombrine, 67, 94

Scombrone, 93, 95

Scott-Wilson apparatus, cut of, 536

Scott.Wilson method for acetone and diacetic acid in blood, 28 , in urine, 536

reagent 537

Scybala, 50, 223

Secondary protein derivatives, $65,94,118$

Secondary proteoses, 120

Secretin, 185

Seliwanoff's reaction, 35,447 reagent, preparation of, 35,447

Senna, influence of, on color of feces, 238

Separation of feces, importance of, in nutrition and metabolism experiments, 224,587

Serine, 65, 67, 69,73 crystalline form of, 73

formula for, 73

Serum albumin, 93,245,268, 412, 422 in urine, 412,422 test for, 423

Serum, blood, 245,267 milk, 313

Serum globulin, 93, 246, 412, 422 in urine, 412,422 test for, 423

Shackell's method for vacuum desiccation, 482

Shaffer and Marriott's method for acetone and diacetic acid, 539

Shaffer and Marriott's method for determination of $\beta$-hydroxybut yric acid, 539

Shcrrington's solution, preparation of, 305

Silicates in urine, 370,411

Silver reduction test for uric acid (Schiff), 35I

Skatole, 212,213,214, 219 tests for, 210

Skatole-carbonic acid, 217,220 test for, 220

Smith's test for bile pigments, 208, 433

Soap, salting-out of, 18 insoluble, preparation of, $1 \leqslant 2$

Sodium and potassium in urine, 370, 409, 561 quantitative determination of, 561

Sodium alizarin sulphowate as indicator, 153,154 , $157,158,174,48 \mathrm{r}$ 
Sodium alizarin sulphonate, preparation of, I54, 158

Sodium chloride, crystalline form, 267

Sodium chloride in urine, $409,556,56 \mathrm{I}, 576$

Sodium hypobromite solution, preparation of, $60 \mathrm{I}$

Sodium nitrite-ferrous sulphate reaction for diacetic acid (Hurtley), 440

Sodium nitroprusside test for acetone, 437

Sodium sulphide solution, preparation of, 607

Solera's reaction for detection of thiocyanate in saliva, 59

test paper, preparation of, 59

Soluble starch, II, 43, 56

as indicator, $165,280,286,524,533$

Sörensen's formol titration method for amino nitrogen, 502

indicator method for hydrogen ion concentration, 158,480

Soxhlet apparatus for extraction of fat, 326

Soxhlet lactometer, determination of specific gravity of milk by, 324

Specificity of enzyme action, 7

Spectroscope, use of in detection of blood, 297

Spermatozoa in urinary sediments, 473 microscopical appearance of human, 474

Spiegler's ring test for protein, 104, 424 reagent, preparation of, 104, 424

Spiro's reaction for hippuric acid, 390

Spongin, 69

Standard ammonium thiocyanate solution, preparation of, 594

creatinine solution, 599

iodine solution, 602

potassium permanganate, 605

picramic acid, 605

silver nitrate solution, preparation of, 606

sodium alcoholate, 607

sodium thiosulphate, 604,607

uranium acetate solution, preparation of, 609

uric acid solution, 6 10

Starch, 20, 43

action of alcohol on iodide of, 45

action of alkali on iodide of, 45

heat on iodide of, 46

dry, digestion of, by pancreatic amylase, I 88 , I 92

dry, digestion of, by salivary amylase, 60

experiments on, 43

hyperglycemia produced by, 567

iodine test for, 45

microscopical characteristics of, 43,44

microscopical examination of, 45

potato, preparation of, 43

soluble, II, 43, 56

soluble starch as indicator, $165,280,286$,

$$
524,533
$$

solubility of, 43

various forms of, 44

Starch group, 20

Starch paste, action of tannic acid on, 45

diffusibility of, 45

digestion of, by pancreatic amylase, 188 191

by salivary amylase, I0, 56, 59

Fehling's test on, 45

hydrolysis of, 45

iodic acid paper, 59

preparation of, 45
Steapsin (see Pancreatic lipase, 5, 13, 178, 188, I93

Stearic acid, 177,354

Stearin, $177,178,313$

Stellar phosphate, 322,460

Stercobilin, 222

Stokes' reagent, action of, 296 preparation of, 296

Stomach contents, lactic acid in tests for, 170 examination of, 159 peptide-splitting enzyme in, 142, 199 removal of, I6I

tube, Rehfuss, I 49

Stomach, motor and functional activities of, 159

Stone-cystine, 77

Sturine, 67, 68, 93

Sublingual glands, characteristics of saliva secreted by, 54

Submaxillary glands, characteristics of saliva secreted by, 54

Substrate, 3

Succinic acid, 214

Sucrase, 4, 14, 195, 200

experiments on, I4, 200

vegetable, 14

Sucrose, I9, 4 I

experiments on, 42

inversion of, 42

production of alcohol from, 42

structure of, $4 \mathrm{I}$

Sucrose- $\mathrm{H}_{2} \mathrm{SO}_{4}$ test (Pettenkofer), 208, 434

Sulphanilic acid, 455

Sulphates in saliva, test for, 59

Sulphates in urine, $370,403,546$

ethereal, 404, $55 \mathrm{I}$

quantitative determination of, 547

experiments on, 404

inorganic, 404,550

quantitative determination of, 547

total, quantitative determination of, 546 , 551

Sulphocyanides (see Thiocyanates, 55, 59, 369, 392)

Sulphur in protein, 63,108

acid, fo8

in urine, gravimetric determination of, $5+6$ volumetric determination of, 550

lead blackening, 108

loosely combined, I08

mercaptan, 108

neutral, 108, 404

oxidized, 108

"partition," in urine, 577

tests for, 108

unoxidized, 108

Sulphuric acid test (Piria), 86

Surface tension test for bile acids (Hay), 209, 434

Suspension of manganese dioxide, $5 \mathrm{I} 3$

Synthesis of hippuric acid, 585 demonstration of, 585

Tallow bayberry, saponification of, $18 \mathrm{r}$

Tallquist's hemoglobin scale, determination of hemoglobin by, 304

Tannic acid, influence of, on dextrin, 48 on starch, 45 precipitation test for proteose (Ott), 429 
Tannin test for carbon monoxide hemoglobin, 298

Tanret's reagent, preparation of, $104,+25$

Tanret's test, 104, 425

Tartar, formation of, 55

Taurine, 204, 210,34I, 346,369, 392

derivatives, 369,392

formula for, 204

microscopical appearance, 2 I I

preparation of, 210

Taurocholic acid, 204 group, 204

Teichmann's crystals, form of (see Hemin crystals, p. 265)

test, $264,266,+29$

Tendomucoid, 94, I1 3,332

biurct test on, 332

chemical composition of, II 3

hydrolysis of, 3.32

loosely combined sulphur in, test for, 332

preparation of, 332

solubiiity of, 332

Test meals, 16

Ewald, I6r

water, 161

retention, I6I

Tetrapeptides, 66, 95

Tetramethylene-diamine, 2 I 4

Tetranucleotide, I 25

Thiocyanates in saliva, significance of, $\mathbf{5} 5$

ferric chloride test for, 59

Solera's reaction for, 59

Thiocyanates in urine, 369,392

Thiophene reaction, I 7 I

Thoma-Zeiss hemocytometer, 304

Thrombin, 247, 257

Thromboplastine, 25\%

Thymol, formula for, 367

interference in Heller's ring test, 423

determination of sugar, acetone bodies, phosphates and magnesium in urine, 367

interference of, in Lieben's acetone test, 437 use of, as preservative, 367

Thymolphthalein, use of, as indicator, 157,158

Thymus histone, 93

Thymus nucleic acid, I24, 131 preparation of, I3I tests on, I32

Time :clations of protein metabolism, 569

Tincture of iodine, preparation of, 608

Tissue, adipose, experiments on, I 76, 338

connective, 330

white fibrous, 331

composition of. 331

experiments on, 332

yellow elastic, 333

composition of, 334

experiments on, 334

epithelial, 330

experiments on 331

muscular. 339

experiments on, 347

nervous, 353

experiments on, 356

osseous, 336

experiments on, 337

Tissue débris in urinary sedıments, 465,473
Titanium tetrachloride as cellulose sulvent, 49

Toison's solution, preparation of, 603

o-Tolidin test for blood, $233,263.430$

Tollen's reaction for conjugate gly"curonates, 142 arabinose, 37

galactose, 36. 446

pentoses in urine, 443

Topper's method for quantitative analy"sis of gastric juice, 174

Tópfer's reagent, as indicator, $153,154,16 \div 175$ preparation of, 175

Total solids, of milk, quantitative determination of, 327

of urine, quantitative determination of, 483

Total sulphur of urine, quantitative determination of, $5+8$

phosphorus of urine, quantitative determination of, 554

Trichloracetic acid, precipitation of protein by. 103, 120, 272

Tricresol-peroxidase reaction (Kastle), 320

Trimethyl-oxyethyl-ammonium hydroxide (see

Choline, 2 I2, 354, 35\%)

Trioses, 19

Tripeptides, 66.95

Triple phosphate, $362,408,458,4: 6$ crystalline form of, 408 formation of, 408

Trisaccharides, 19, 42

Trommer's test, 25

Tropæolin O, use of, as indicato:, 157 preparation of, 158

Tropaolin OO, use of, as indicator, 153, 154, 155. 157, 159

preparation of, 155,158

Tropeolin OOO, use of, as indicator, I5T preparation of, 158

Trypsin (see also Pancreatic protease, 5, I2, 186 ) action of, upon proteins, 66, I86, I 89

experiments on, 190

influence of alkalis and mineral acids upon, I86

in stomach. $15 \mathrm{r}, 169$

determination of, 160

nature of, 186

Trypsinogen, 6, 186

activation of, 6. IS6, 196

Tryptic digestion, 66, 186

influence of bile on, 190

most farorable reaction for, 19 I temperature for, 192

products of, $66,186,189$

Tryptic proteolysis, 142,186

Tryptophane, 65, 67,68,77, 98, 199

bromine water test for, 199

formula for, if

group in the protein molecule, 93

Hopkins-Cole reaction for, 98

mercury compound of, preparation of, 120 occurrence of, as a decomposition product of protein, $65,67,68,7 i$

occurrence of, as an end-product of pancreatic digestion, 186. IS

Tuberculosis, urochromogen reaction for, 453

Tussah silk fibroin, 69

Tyrosinase, 5

Tyrosine, 5, 65,67,69, 74, 56, 97, 156, 463 
Tyrosine, crystalline form of, 76 experiments on, 86

formula for, 77

Hoffmann's reaction for, 86

in urinary sediments, 463

microscopical examination of, 86

Mörner's test for, 86

occurrence of, $65,67,69,186$

Piria's test for, 86

salts of, 77

separation of, from leucine, 85

solubility of, 86

sublimation of, 86

Tyrosine-sulphuric acid, 86

v. Udránsky's test for bile acids, 208, 434

Uffelmann's reagent, preparation of, 170 reaction for lactic acid, 170

Unknown substances in urinc, 454

Unorganized ferments, I sediments in turine, 457,458

Unsaturated acids, 177

Uranium acetate method for determination of total phosphates in urine, $\mathbf{5 5 2}$

Uracil, I25, I28, I33

Wheeler-Johnson reaction for, 133

Urate, ammonium, crystalline form of, Plate VI, opposite p. 462

sodium, crystalline form of, 462

Urates in urinary sediments, 461

Urea, 248, 274, 34 I, 372, $49 \mathrm{I}$ crystalline form of, 372

decomposition of, by sodium hypobromite, 374,496

excretion of, 372,374

experiments on, 375

formation of, 373

formula for, 372

furfural test for, 377

isolation of, from the urine, 375

melting-point of, 375

quantitative determination of, in blood, 274

Urea nitrate, 374,376 in urine, $491-499$

crystalline form of, 374

formula for, 37.4

oxalate, 374,376

crystalline form of, 376

formula for, 374

Urease, 4

decomposition of urea by, $49 \mathrm{I}$

preparation of, $49 \mathrm{I}$

quantitative determination of urea by, 274 . $49 \mathrm{I}$

Uremia, blood in, $270,27 \mathrm{I}$

Urethral filaments in urinary sediments, 465 . 473

Uric acid, 26, 127, 133, 137, 248, 270, 274, 341,369. $377-381,460,476,510,571,573$

calculi, 476

crystalline form of, pure, 380

endogenous, 378,573

exogenous, $378,57 \mathrm{I}$

experiments on, 380

formula for, 377

Gânassini's test, 38 I

in blood, $248,270,274$

in leukæmia, 379
Uric acid in urinary sediments, 460

crystalline form of, Plate $V$, opposite p. 380,461

isolation of, from the urine, 380

metabolism, 57 I, 573

murexide test for, 380

origin of, 378

quantitative determination of, in blood,

Benedict's method, 275

Folin-Denis method, 274

in urine, microchemical colorimetric method, 510

Folin-Shaffer method, 5 II

Heintz method, 512

Krüger-Schmidt method, 513

Uricometer method, 5 I3

quantitative determination of, Krüger and Schmidt's method for, 5 I 3

Hunter and Givens' modification of, 515 reducing power of, $26,379,4 \mathrm{I} 6,4 \mathrm{I} 7$

Ruhemann's uricometer method for, 5 I3

Schiff's reaction for, 38 i

Uricase, 5, 127

experiments on, 137

Uricolytic enzymes, $3,5,127$

experiments on, 137

Urinary calculi, 475

calcium carbonate in, 476

oxalate in, 476

cholesterol in, 478

compound, 475

cystine in, 476

fibrin in, 478

indigo in, 478

phosphates in', 476

scheme for chemical analysis of, 477

simple, 475

uric acid and urates in, 476

urostealiths in, 478

xanthine in, 476

Urinary concrements (see Urinary calculi, p."475)

Urinary sediments, 457

ammonium magnesium phosphate in 458

animal parasites in, 465,474

calcium carbonate in, 458, 459

oxalate in, 458

phosphate in, 458,460

sulphate in, 458,460

casts in, 465,467

cholesterol in, 458,463

collection of, 457

cylindroids in, 465,472

cystine in, 458,462

epithelial cells in, 465

erythrocytes in, 465,472

fibrin in, 465,474

foreign substances in, 465,474

hematoidin and bilirubin in, 458,464

hippuric acid in, 458,463

indigo in, 458,464

leucine and tyrosine in, 458,463

magnesium phosphate in, 458,464

melanin in, 458,465

micro-organisms in, 465,474

organized, 457,465

pus cells in, 465,466 
Urinary sediments, spermatozoa in, 465, 474 tissue debris in, 465,473

unorganized, 457,458

urates in, 458, 461

urethral filaments in, 465,473

uric acid in, 458,460

xanthine in, 458,464

Urination, frequency of, 36 I

Urine, 359-563

acetoacetic acid in, $412,438,539$

acctone in, 4 i2, 435, 538

acidity of, $36 \mathrm{I}, 479,577,580,582$

acid fermentation of, 363

albumin in, $412,422,531$

alkaline fermentation of, 362,408

allantoin in, 369, 392, 518

amino-acids in, 369, 394, 502

ammonia in, $369,402,499$

aromatic oxyacids in, 369,394

benzoic acid in, 369, 395

bile in, 412,432

blood in, 412,429

calcium in, 370, 409, 559

carbonates in, 370,410

chlorides in, 370,405

collection of, 367

collection and preservation of, in metabolism

tests, 367,565

color of, 359

complete analysis of, 565

conjugate glycuronates in, 412,442

creatine in, $369,384,508,509$

creatinine in, $369,381,506,574$

dextrose in (see glucose, $412,413,522$ )

diacetic acid in (see acetoacetic acid, 412,438 , 539)

electrical conductivity of, 366

enzymes in, 370,397

ethereal sulphuric acid in, $369,385,547$

fat in, 412,444

fluorides in, 370,411

freezing-point of, 365

fructose in, 412,446

galactose in, 412,446

general characteristics of, 359

globulin in, 412,426

glucose in, $412,4 \times 3,522$

Hăser's coefficient for solids in, 364, 483

hematoporphyrin in, 412,444

hippuric acid in, $369,388,519$

hydrogen ion concentration of, 36 I, 480,580

as influenced by diet, 580

by acid and alkali, 582

hydrogen peroxide in, $370,41 \mathrm{I}$

$\beta$-hydroxybutyric acid in, $412,440,539$

inorganic physiological constituents of, 370 , 402

inositol in, 412,450

iron in, 370, 410, 562

lactose in, 412,444

levulose in (sce fructose, 412,446 )

laiose in, 412,451

leucomaines in, $370,40 x$

Long's cocfficient for solids in, 364,483

magnesium in, $370,409,559$

melanin in, 412,451

neutral sulphur compounds in, 369, 392, $55 \mathrm{r}$, 578
Urine, nitrates in, 370,411

nucleoprotein in, 360, 396, 412, 428

odor of, 36 I

organic physiological constituents of, 360

oxalic acid in, 369,391

oxaluric acid in, 360,397

$\beta$-oxybutyric acid in (see hydroxybutyric acid, 412, 440, 539)

pathological constituents of, 4 I2

paralactic acid in, 370,398

pentoses in, 412,443

peptone in, 412,426

phenaceturic acid in, 370,398

phosphates in, $370,406,552$

phosphorized compounds in, 370, 399

physiological constituents of, 369

pigments of, $359,370,399$

potassium in, $370,409,56 \mathrm{I}$

proteins in, 412,422

proteoses in, $412,422,426$

ptomaines in, 370,401

purine bases in, $370,401,513$

quantitative analysis of, 479-563

reaction of, $361,479,580$ as influenced by diet, 580

by acids and alkalies, 582

silicates in, 370,41 I

sodium in, $370,409,561$

solids of, 364,483

specific gravity of, 363

sulphates in, $370,403,546$

transparency of, 360

unknown substances in, 412,454

urea in, $369,372,49 \mathrm{I}$

uric acid in, $369,377,5$ Io

urinod in, $36 \mathrm{I}$

urorosein in, 412,452

volatile fatty acids in, 370,397

volume of, 359

Urinod, 36 I

Urobilin, 359, 370, 399

tests for, 400

Urobilinogen, 399

Urocanic acid, 398

Urochrome, 359, 370,399, 42 I

Urochromogen, 412,453 reaction (Weisz) for tuberculosis, 453

Uroerythrin, 359, 370, 399, 42 I

Uroferric acid, 369, 392, 454

Uroleucic acid, 369, 395

Urorosein, 4 I2, 452

reaction, 452

tests for, 452

Valine, $65,67,69,78$

Van Slyke's method for determination of total amino-acid nitrogen, in urine, 505

in blood, 277

in protein hydrolysis, 88

Vais Slyke and Cullen's method for urea in blood, 274

for urea in urine, 492

Van S!yke and McLean's method for chlorides in blood, 286

Vegetable anylase, \&, II

lipase, 5, 13 
Vegetable protease, 13

sucrase, 14, 195, 199

Vegetable globulins, 93, 95, 108

Vegetable gums, 20

Veith lactometer, determination of specific gravity of milk by, 324

Viscosity test, 59

Vitamines, 3 I 3

Vitellin, 94, 95

Volatile fatty acids, 2 I2, 2 I 5, 370, 397

Volhard-Arnold method for determination of chlorides, 556

Volhard-Harvey method for determination of chlorides, 557

Volume of the urine, 359

Water at meals, influence of, 138, 185, 402, 574 softened, 57

Water test meal, 16 I

Water, influence of on metabolism, 574

Wax myrtle, I8 I

Waxy casts in urinary sediments, 465,470

Weber's guaiac test for blood in feces, 235

Weinland, formation of fat from protein, 179

Weisz's urochromogen reaction for tuberculosis, 453

Welker's electrical bath, 494 modified method for purine bases, 5 I 5

Welker and Marsh method for deproteinizing milk, 329

Welker and Tracy method for deproteinizing urine, 485

Weyl's test for creatinine, 385

Wheeler-Johnson reaction for uracil and cytosine, 133

White fibrous connective tissue, 33 I experiments on, 332

Wiechowski-Handovsky method for determination of allantoin in urine, 518

Wilkinson and Peters' test, 32 I

Wirsing's test for urobilin, $40 \mathrm{I}$

Witchs' milk, 317

Wohlgemuths' method for quantitative determination of amylolytic activity, 192 Author's modification of, 239
Wolter's method for determination of iron in urine, 562

Xanthine, 126, 127, 132, 346, 354, 40I

bases (see Purine bases, pp. 126, 346, 40I)

crystalline form of, 344

formula for, 127,346

in urinary sediments, 458,464

isolation of, from meat extract, $35 \mathrm{I}$

silver nitrate, $35 \mathrm{I}$

crystalline form of, 35 I

test, 35 I

tests for, 132

Weidel's reaction for, 132

Xanthophylls, 3 I9

Xanthoproteic reaction, 98

Xanthinoxidase, 5

Xylose, 20, 38

orcinol reaction on, 38

phenylhydrazine reaction on, 38

Tollens' reaction on, 38

Yeast, enzymes of, 2

fermentation by, 42

nucleoprotein of, 128

preparation of, 128

tests on, 129

nucleic acid of, 125, 130

formula for, 125

preparation of, 130

tests on, 130

Yellow elastic connective tissue, 333

composition of, 334

experiments on, 334

Zappert slide, 307

Zein, 67, 69, 93, 95, II I

decomposition of, 67,69

Zeller's test for melanin, 452

v. Zeynek and Nencki's hemin test, 266, 430

Zikel pektoscope, 365

Zymase, classification of, 4

preparation of, 2

Zymo-exciter, 7

Zymogen, 6, 186 


$$
\cdot
$$







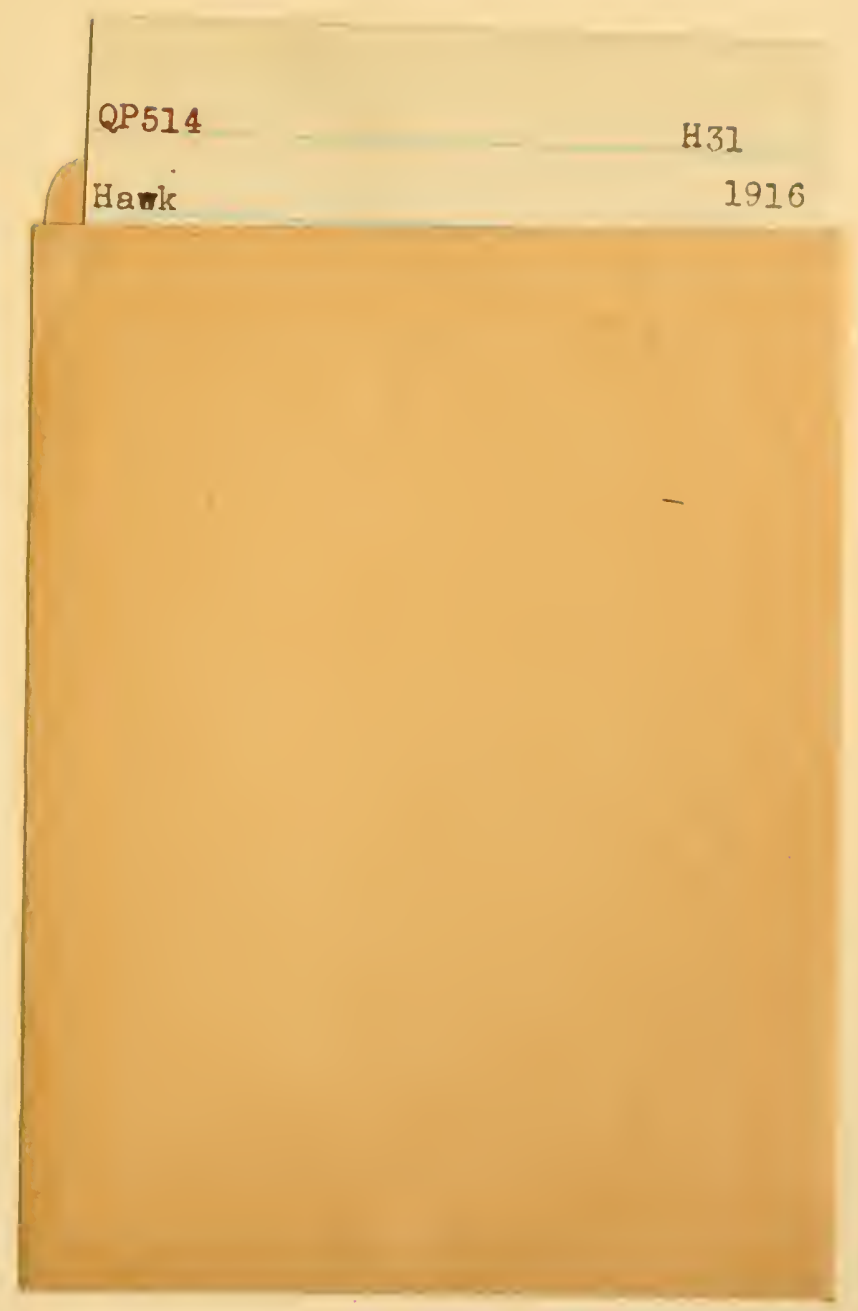


Her.

fis

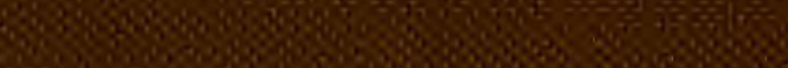

W.2.

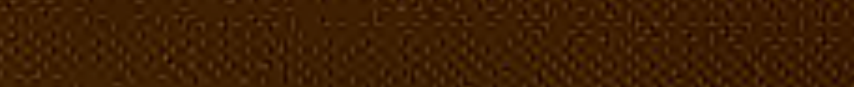

F.

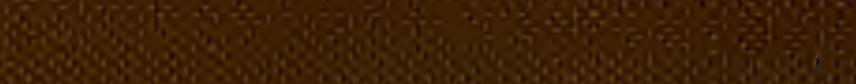

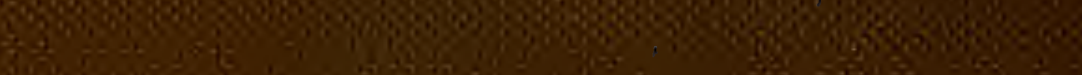

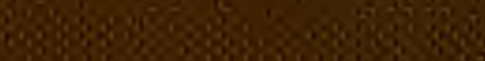

24.

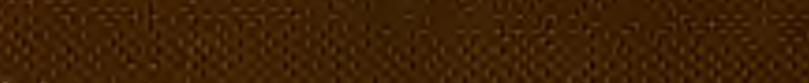
8.

Gerp

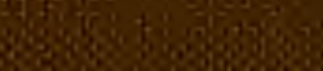

6row

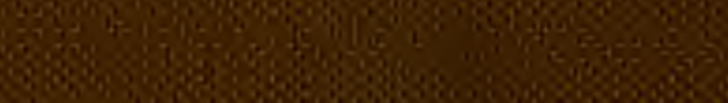

-

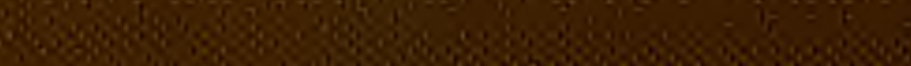

630. 